\title{
MEMBERSHIP
}

\section{EXPRESSION OF INTENT.}

In the application of Statute 5, it is the current intention of the Executive Committee that. Individual Members of the Union in countries that cease participation retain full individual membership.

This chapter is composed as follows:

1. List of adhering countries ${ }^{*}$ )

2. Membership of commissions

3. Geographical repartition ${ }^{(\star \star)}$ of members

. within adhering countries

. within non adhering countries

4. Alphabetical list of members

(*) The situation of countries which is published corresponds to the situation as of December 31, 1991.

$(* *)$ This repartition is done on the basis of the country in which IAU members exercise their work and not their citizenship. 
1. List of Adhering Organizations

The year of adherence and approximate number of IAU members residing in the different Adhering Countries are indicated (as of December 1991).

(*) Associate National Members as opposed to Full National Members (Article 4 of the Statutes).

USSR (which joined the Union in 1935) is replaced by the following states in which individual IAU members are working:

Member.s

Armenia 18

Azerbaidzhan.

Estonia

Georgia

Kazakhstan

9

Latvia

9

Lithuania

Russia

264

Tajikistan

Year of

Members

Adherence

Algeria

1988

1

Commissariat à la Recherche

( CRAAG)

BP 63

Bouzareah

Alger

Argentina

Presidencia de la Nacion

1927

65

Secretaria de Ciencia/Tecnologia

Consejo $\mathrm{NCl}$ de Investigaciones

Cientificas y Técnicas (CONICET)

Rivadavia 1917

1033 Buenos Aires

Australia

1939

173

Australian Academy of Sciences

Attn Executive Secretary

Box 783

Canberra City, ACT 2601 
Austria

Bundestministerium

für Wissenschaft und Forschung

Minoritenplatz 5

1010 wien

Belgium

Administration Affaires Communes

ETS Scientifiques de L'Etat

Bd Pacheco 6e Etage

B 1000 Brussels

Brazil

1961

87

Conselho $\mathrm{NCl}$ Desenvolvimento

Científico/Tecnológico - CNPq

Av W3 Norte Quadra 507 B

Caixa Postal 11-1142

70740 Brasilia DF

Butgaria

Bulgarian Sciences Academy

1957

48

7 November Street 1

1000 Sofia

Canada

National Research Council

1957

206

International Affairs

Montreal Road

Ottawa Ontario K1A 0R6

Chile

Dr. Claudio Anguita, Chairman

1947

43

Chilean Ntl Astronomy Cttee

Univ. Chile. Faculd. Ciencias

Casilla 360

Santiago de Chile

China Nanjing

1935

292

Chinese Astronomical Society

Purple Mountain Observatory

Academia Sinica

Nanjing

China Taipei

1959

19

Vice President

Academia Sinica

Taiwan

Taipei 11529

Colombia

Mrs. M. A. Velasco

Facultad de Sciencias

Universidad Ncl de Colombia

Apartado Aereo 5997

Observatorio Astronomico Ncl

Bogota DE 
Cuba

Academia Ciencias de Cuba

Capitolio NCl

La Habana

Czechoslovakia

Czech \& Slovak National IAU Committee Observatory Ondrejov

25165 Ondrejov

Denmark

Kge Danske Vidensk Selskab

$\mathrm{H} C$ Andersen Boulevard 35

DK 1553 København V

Egypt AR

Academy Scientific Research

Scientific \& Cultural Relations

101 Kasr El-Einy Street

Cairo

Finl and

Delegation of the Finnish Academy

of Sciences \& Letters

Mariankatu 5

SF 00170 Helsinki

France

Académie des Sciences

COFUSI

23. quai Conti

F 75006 Paris

Germany

President, German Council

1951

of German Observatories

Universitäts Sternwarte

Geismarlandstrasse 11

D 3400 Göttingen

Greece

Academy of Athens

28 Panepistimiou Street

GR 10679 Athens

Hungary

Hungarian Academy of Sciences

Box 6

Roosevelt Ter 9

H 1361 Budapest V

I cel and

Ministry of Education

Science Institute

Universtiy of Iceland

Hverfisgötu 4-6

IS 101 Reykjavik 
Indian Ntl Science Academy

Bahadur Shah Zafar Marg

New Delhi 110002

Indonesia

Scientific and Technologic Cooperation

1979

9

Indonesian Institute of Sciences

Lembaga I Imu Pengetahuan Indones

J1. Jend. Gatot Subroto No. 10

Jakarta Selatan

Iran

1969

9

University of Tehran

office of International Relation

Tehran

Iraq

1976

8

Council for Sc Research

Astronomy \& Space Res Center

Box 255

Baghdad

Irel and

The Royal Irish Academy

19 Dawson Street

Dublin 2

Israel

Israel Sciences Academy and Humanities

Albert Einstein Square

Talbieh

Jerusalem 91040

Italy

Consiglio Nzle Ricerche

Servizio Relazioni Intern

Piazzale Aldo Moro 7

I 00100 Roma

Japan

Science Council of Japan

1920

350

22-34 Roppongi 7 chome

Minato-ku

Tokyo 106

Korea DPR

Academy of Sciences of DPRK

Pyongyang

Korea RP

Korean Astronomical Society

Dpt of Astronomy

College of Natural Sciences

Seoul National University

Seoul 151 
Malaysia $(*)$

National Space Science Education Center

Prime Minister's Department

c/o Islamic Center

Jalan Perdana

50519 Kuala Lumpur

Mexico

Instituto de Astronomia UNAM

1921

55

Apartado Posta 770264

Cd. Universitaria

Mexico 04510 DF

Morocco

CNCPRST

52 Charia Omar Ibn Khattab

B.P. 1346 RP

Agda 7

Netherlands

1922

162

Koninklijke Nederlandse Akademie

van Wetenschappen

Kloveniersburgwal 29

$\mathrm{NL} 1011 \mathrm{JV}$ Amsterdam

New Zealand

1964

22

Mrs. S M Usher

Assistant Executive Officer

Royal Society of New Zealand

Box 598

Wellington

Norway

Norske Videnskaps-Akademi

1922

20

Drammensveien 78

N 0271 0slo 2

Peru (*)

Ing Carlos del Rio Cabrera

President

Camilo Carrillo 1189 Piso

Lima 11

Pol and

1922

94

Polskiej Akademi i Nauk

Palac Kultury I Nauki

Skrytka pocztowa 24

00901 Warsaw

Portugal.

Secçao Portuguesa Unioes Intern.

Astronomica/Geodesica/Geofisica

SPUIAGG

Praça de Estrela

P 1200 Lisboa 
M8

Saudi Arabia

1988

King Abdulaziz City

for Science \& Technology

Directorate of Technology Transfer

and International Cooperation

PO Box 6086

Riyadh 11442

South Africa

1938

35

South African ICSU Secretariat

Foundation for Research Dv1pt

Box 2600

Pretoria 0001

Spain

Comision $\mathrm{NCl}$ Astronomia

1922

161

Instituto Géografico/Cadastral

General Ibañez 3

E Madrid 3

Sweden

The Foreign Secretary

1925

84

The Royal Swedish Academy of Sciences

Box 50005

S 10405 Stockholm

Switzerland

1923

55

Schweizerische Akademie

der Naturwissenschatfe

Bärenplatz 2

Postfach 8120

CH 3001 Bern

Turkey

1961

47

Türk Astronomi Dernegi Baskani

The Dean of the Faculty

of Arts \& Sciences

Middle East Technical University

06531 Ankara

UK

The Royal Society

ICSU Information officer

6 Carlton House Terrace

London SW1Y 5AG

Uruguay

Ministerio Relaciones Exteriores

1920

481

Avenida 18 de Julio 1205

Montevideo

USA

Office of International Affairs

National Academy of Sciences

FO 2020

2101. Constitution Avenue NW

Washington DC 20418 
Vatican City State

Governatorato Citta Vaticano

Castel Gandolfo 3

$\checkmark 00120$ Citta del Vaticano

Venezuela

Centro de Investigaciones

de Astronomia CIDA

Apartado Postal 264

Merida 5101 A

Yugoslavia

Savez Drustava Matematicara,

Fisica i Astronoma Jugoslavije

Institut za Matermatiku i Fisiku

Cetinjski put bb

8100 Titograd

Total members in Adhering Countries

1935

39

1953

7

5

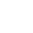


2. Membership of commissions

Number of Members

4 Ephemerides/Ephémérides $\ldots \ldots \ldots \ldots \ldots \ldots \ldots \ldots \ldots \ldots \ldots \ldots \ldots \ldots \ldots \ldots$

5 Documentation \& Astronomical Data/ .................... 114 Documentation \& données astronomiques

6 Astronomical Telegrams/Télégrammes astronomiques $\ldots \ldots \ldots \ldots \ldots .19$

7 Celestial Mechanics/Mécanique céleste $\ldots \ldots \ldots \ldots \ldots \ldots \ldots \ldots \ldots$

8 Positional Astronomy/Astronomie de position $\ldots \ldots \ldots \ldots \ldots \ldots . \ldots$

9 Instruments \& Techniques/Instruments \& techniques ......... 263

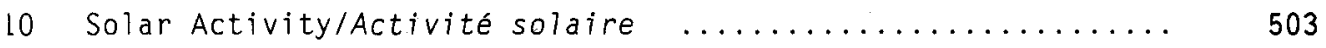

12 Solar Radiation \& Structure/Radiation \& structure solaires ... 342

L4 Atomic \& Molecular Data/Données atomiques \& moléculaires ..... 177

15 Physical Study of Comets. Minor Planets \& Meteorites/ ....... 275

Etude physique des comètes, des petites planètes

\& des météorites

I6 Physical Study of Planets \& Satellites/ ................ 235

Etude physique des planetes \& satelitites

19 Rotation of the Earth/Rotation de la Terre .............. 129

20 Positions \& Motions of Minor Planets, Comets \& Satellites/ ... 181

Positions \& mouvements des petites planètes.

des comètes \& des météorites

21 Light of the Night Sky/Lumière du ciel nocturne $\ldots \ldots \ldots \ldots \ldots .120$

$\geq 2$ Meteors \& Interplanetary Dust/Météores $\ldots \ldots \ldots \ldots \ldots \ldots \ldots \ldots$

\& poussière interplanétaire

24 Photographic Astrometry/Astrometrie photographique $\ldots \ldots \ldots \ldots .140$

25 Stellar Photometry \& Polarimetry/ ................. 198

Photométrie \& polarimétrie stellaires.

26 Double \& Multiple Stars/Etoiles doubles \& multiples ........ 102

$? 7$ Variable Stars/Etoiles variables $\ldots \ldots \ldots \ldots \ldots \ldots \ldots \ldots \ldots \ldots \ldots \ldots$

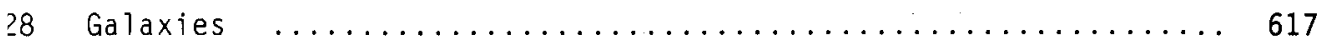

29 Stellar Spectra/Spectres stellaires $\ldots \ldots \ldots \ldots \ldots \ldots \ldots \ldots \ldots . \ldots \ldots$

30 Radial Velocities/Vitesses radiales $\ldots \ldots \ldots \ldots \ldots \ldots \ldots \ldots \ldots . \ldots \ldots$

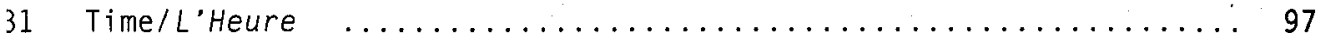


33. Structure \& Dynamics of the Galactic System/

Structure \& dynamique du système galactique

34 Interstellar Matter/Matière interstellaire

35 Stellar Constitution/Constitution des étoiles

Theory of Stellar Atmospheres/

Theorie des atmosphères stellaires

37 Star Clusters \& Associations/Amas stellaires \& associations .. 205

38 Exchange of Astronomers/Echange d'astronomes $\ldots \ldots \ldots \ldots \ldots \ldots 29$

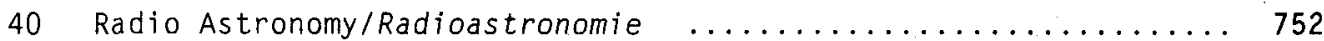

41 History of Astronomy/Histoire de 7 'Astronomie $\ldots \ldots \ldots \ldots \ldots \ldots 11$

42 Close Binary Stars/Etoiles binaires serrées $\ldots \ldots \ldots \ldots \ldots \ldots \ldots 345$

44 Astronomy from Space/L'Astronomie à partir de l'Espace $\ldots . . . .316$

45 Stellar Classification/Classification stellaire ............ 119

46. Teaching of Astronomy/Enseignement de l'Astronomie $\ldots \ldots \ldots \ldots$

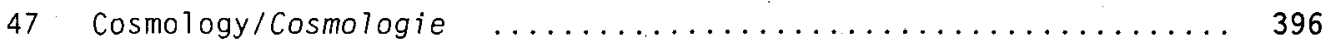

48 High Energy Astrophysics/Astrophysique des hautes energies ... 389

49 The Interplanetary Plasma \& the Heliosphere/ $\ldots \ldots \ldots \ldots \ldots \ldots 117$

50 Protection of Existing \& Potential Observatory Sites/ ........ 64 Protection des sites dobservatoires existants \& potentiels

51 Bioastronomy: Search for Extraterrestrial Life/ ............ 254 Bioastronomie: recherche de la vie extraterrestre 


\section{COMPOSITION OF COMMISSION $04 \quad 1991-1994$}

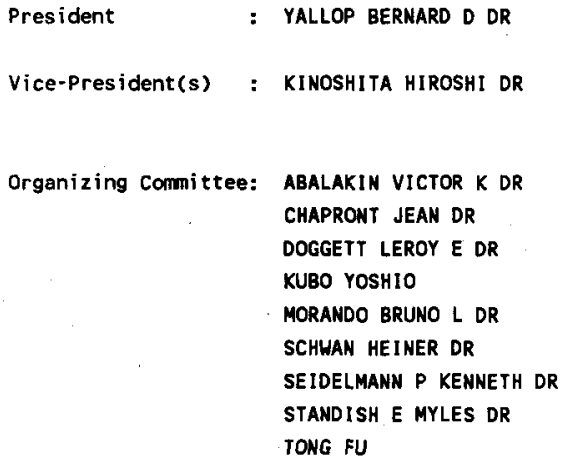




$\begin{array}{ll}\text { President } & \text { : HAUCK BERNARD PROF } \\ \text { Vice-President(s) } & \text { : DLUZHNEVSKAYA O B DR }\end{array}$

Organizing Committee: CREZE MICHEL DR

-DUBOIS PASCAL DR

-GROSBOL PREBEN JOHNSON DR

- - HECK ANDRE OR

LI QI-BIN

- MEAD JAYLEE MONTAGUE DR

- SCHMADEL LUTZ D DR

-TURNER KenNeTH C DR

WARREN WAYNE H JR DR

- WESTERHOUT GART DR

WIILKINS GEORGE A DR

Members:

ABALAKIN VICTOR $K$ DR ALVAREZ PEDRO DR BAKER NORMAN H PROF BESSELL MICHAEL S DR CHU YAOQUAN

DAVIS MORRIS S PROF DEWHIRST DAVID W OR DUCATI JORGE RICARDO DR GARSTANG ROY H PROF HANISCH ROBERT J OR HEINRICH INGE HUANG BI -KUN KADLA ZDENKA I DR KHARIN A S DR LANTOS PIERRE DR LINDE PETER DR LORTET MARIE CLAIRE MATZ STEVEN MICHEAL DR MCNAMARA DELBERT H DR MERMILLIOO JEAN-CLAUDE DR OCHSENBEIN FRANCOIS DR PATUREL GEORGES PIZZICHINI GRAZIELLA QUINTANA HERNAN DR REMY BATTIAU LILIANE G A ROESSIGER SIEGFRIED DR SARASSO MARIA DR SCHMIDT $K$ H DR SHAKESHAFT JOHN R DR SPITE FRANCOIS $M D R$ TSVETKOV MILCHO $K$ DR WAYMAN PATRICK A PROF WENGER MARC WRIGHT ALAN E DR
ABT HELPUT A DR ANDERNACH HEINZ DR BENACCHIO LEOPOLDO BIDELMAN WILLIAM P PROF COGAN BRUCE C DR DAVIS ROBERT J DR DICKEL HELENE $R$ DR DUNCOMBE RAYNOR L DR GRIFFIN ROGER F DR HARVEL CHRISTOPHER ALVIN HEINTZ WULFF $D$ DR JASCHEK CARLOS O R PROF KALBERLA PETER KLECZEK JOSIP DR LEDERLE TRUDPERT DR LIU JINMING LYNGA GOSTA DR MCLEAN BRIAN JOHN MEADOWS A JACK PROF MITTON SIMON DR PAMYATNIKH A A DR PECKER JEAN-CLAUDE PROF POLECHOVA PAVLA DR RAIMOND ERNST DR RENSON P F M DR ROMAN NANCY G DR SCHILBACH ELENA DR SEDMAK GIORGIO PROF SHCHERBINA-SAMOJLOVA I DR SOKOLSKY ANDREJ G DR TERASHITA YOICHI PROF UESUGI AKIRA DR WEIDEMANN VOLKER PROF WIELEN ROLAND PROF OR WU ZHIREN DR
ALBRECHT MIGUEL A DR A'HEARN MICHAEL $F$ DR BENN CHRIS R DR BOUSKA JIRI DR COLUZZI REGINA DR DE BOER KLAAS SJOERDS DR DIXON ROBERT S DR EGRET DANIEL DR GUIBERT JEAN DR HEFELE MERBERT PH D HELOU GEORGE DR JENKNER HELMUT DR KAPLAN GEORGE H DR KRISTENSEN LEIF KAHL LEQUEUX JAMES DR LONSOALE CAROL J DR LYUBIMKOV LEONID S DR MCNALLY DEREK DR MEIN PIERRE NISHIMURA SHIRO DR PASINETTI LAURA E PROF PHILIP A G DAVIS PUCILLO MAURO DR RATHATUNGA KAVAN U RIEGLER GUENTER R DR RUSSO GUIDO DR SCHLUETER A PROF DR SERRANO ALFONSO DR TRITTON SUSAN BARBARA WALLACE PATRICK T MR HELLS DOMALD C III DR WORLEY CHARLES E DR 
COMPOSITION OF COMMISSION $06 \quad 1991-1994$

\author{
President \\ : GRINDLAY JONATHAN E DR \\ Vice-President(s)
}

Organizing Committee: ISOBE SYUZO DR

MARSDEN BRIAN G DR

ROEMER ELIZABETH PROF

\section{Members:}

AKSNES KAARE DR

BIRAUD FRANCOIS DR

FILIPPENKO ALEXEI $\checkmark$ DR KOZAI YOSHIHIDE PROF

NAKANO SYUICHI

GILMORE ALAN C MR

LIU JINMING

POUNDS KENNETH A PROF

SHAROV A S DR

THOLEM DAVID J DR

CANDY MICHAEL P MR HERS JAN MR MRKOS ANTONIN DR ROSINO LEONIDA PROF 


$\begin{array}{ll}\text { President } & : \text { DEPRIT ANDRE PROF } \\ \text { Vice-President(s) } & \text { : FERRAZ-MELLO S PROF DR }\end{array}$

Organizing Committee:-BHATNAGAR K B DR

- CHAPRONT JEAN DR FROESCHLE CLAUDE DR

- he MIAO-FU

- henRaRd JacQues PROF KHOLSHEVNIKOV $K$ V DR KINOSHITA HIROSHI. DR LIESKE JAY H DR MILANI ANDREA PEALE STANTON J PROF ROY ARCHIE E PROF SOKOLSKY ANDREJ G DR

Members:

ABAD ALBERTO J DR

AKIM EFRAIM L OR

ALEXANDER MURRAY E DR

AOKI SHINKO PROF

BARBERIS BRUNO

BENEST DANIEL DR

BORDERIES NICOLE

BRETAGNON PIERRE DR

BROUCKE ROGER DR

CALAME ODILE DR

CARPINO MARIO DR

CHEN ZHEN

CONTOPOULOS GEORGE PROF

CUI DOU-XING

DEMIN V G PROF DR

DORMAND JOHN RICHARD DR

DUNCOMBE RAYNOR L DR

EDELMAN COLETTE DR

ELIPE SANCHEZ ANTONIO

FARINELLA PAOLO DR

FIALA ALAN D DR

GALLETTO DIONIGI

GASKA STANISLAW DR

GOMES RODNEY D S DR

GREBENIKOV E A PROF DR

HADJIDEMETRIOU JOHN D

HEGGIE DOUGLAS C DR

HORI GENICHIRO PROF

IVANOVA VIOLETA DR

ABALAKIN VICTOR K DR
AKSENOV E P PROF DR
ALTAVISTA CARLOS A DR
BAGHOS BALEGH B DR
BATRAKOV YU V DR
BETTIS DALE G PROF
BOZIS GEORGE PROF
BRIEVA EDUARDO PROF
BRUMBERG VICTOR A DR
CANDY MICHAEL P MR
CEFOLA PAUL J DR
CHOI KYU-HONG
COOK ALAN H PROF
DANBY J M ANTHONY DR
DIKOVA SMILIANA D
DOURNEAU GERARD DR
DURIEZ LUC DR
EICHHORN HEINRICH K DR
EMELIANOV NIKOLAJ V DR
FERNANDEZ SILVIA M DR
FONG CHU-GANG
GAPOSCHKIN EDHARD M DR
GIACAGLIA GIORGIO E PROF
GONZALEZ CAMACHO ANTONIO
GREENBERG RICHARD DR
HAMID S EL DIN PROF
HELALI YHYA E OR
HUANG CHENG DR
IZVEKOV $V$ A DR

AHMED MOSTAFA DR

AKSNES KAARE DR

ANTONACOPOULOS GREG PROF

BALMINO GEORGES G DR

BEC-BORSENBERGER ANNICK

BOIGEY FRANCOISE

BRANHAM RICHARD L JR

BRCOKES CLIVE J DR

BRUNINI ADRIAN DR

CARANI COLAS NICHOLAS DR. CHAPRONT-TOUZE MICHELLE

CID PALACIOS RAFAEL PROF COUNSELMAN CHARLES C PROF DAVIS MORRIS S PROF DOGGETT LEROY E DR DROZYNER ANDRZEJ DVORAK RUDOLF DR EL BAKKALI LARBI DR ERDI B OR FERRER MARTINEZ SEBASTIAN GALIBINA I V OR GARFINKEL BORIS DR GOLDREICH P DR gOUDAS CONSTANTINE L PROF GROUSHINSKY N P PROF DR HANSLMEIER ARNOLD HENON MICHEL C DR HUANG TIANYI

JANICZEK PAUL M DR 


\begin{tabular}{|c|c|c|}
\hline JEFFERYS WILLIAM H DR & JOURNET ALAIN & JOVANOVIC BOZIDAR \\
\hline JUPP ALAN H DR & KAMMEYER PETER C DR & KATSIS DEMETRIUS DR \\
\hline KAULA WILLIAM M PROF & KING-HELE DESMOND G DR & KLOKOCNIK JAROSLAV DR \\
\hline KNEZEVIC ZORAN & KOVALEVSKY JEAN DR & KOZAI YOSHIHIDE PROF \\
\hline KRASINSKY GEORGE A DR & KUSTAANHEIMO PAUL E PROF & LALA PETR DR \\
\hline LASKAR JACQUES DR & LAZOVIC JOVAN P PROF & LEMAITRE ANNE OR \\
\hline LIAO XINHAO DR & LISSAUER JACK J DR & LU BEN-KUI \\
\hline LUNDQUIST CHARLES A DR & MACIEJEWSKI ANDRZEJ J DR & MAGNARADZE NINA G DR \\
\hline MARCHAL CHRISTIAN DR & MARKELLLOS VASSILIS V DR & MARSDEN BRIAN G DR \\
\hline MATAS VLADIMIR R DR & MAVRAGANIS A G PROF & MEIRE RAPHAEL \\
\hline MELBOURNE WILLIAM G DR & MERMAN G A DR & MESSAGE PHILIP J OR \\
\hline MIGNARD FRANCOIS DR & MIKKOLA SEPPO DR & MIOC VASILE DR \\
\hline MOONS MICHELE B M M & MORANDO BRUNO L DR & MULHOLLAND J DERRAL DR \\
\hline MUSEN PETER DR & MYACHIN VLADIMIR F DR & NACOZY PAUL E DR \\
\hline NAHON FERNAND PROF & NOBILI ANNA M & NOSKOV BORIS N DR \\
\hline NOVOSELOV V S PROF DR & OESTERWINTER CLAUS & OMAROV TUKEN B PROF \\
\hline ORELLANA ROSA BEATRIZ DR & ORUS JUAN J PROF & OSORIO JOSE J S P PROF \\
\hline O'HANDLEY DOUGLAS A DR & PAL ARPAD PROF DR & PAUHELS T DR \\
\hline PETIT JEAN-MARC DR & PETROVSKAYA M S DR & PIERCE A KEITH DR \\
\hline POPOVIC BOZIDAR PROF DR & ROBINSON WILLIAM J DR & ROORIGUEZ-VILLAMIL R DR \\
\hline RYABOV YU A PROF DR & SAGNIER JEAN-LOUIS DR & SANSATURIO MARIA E DR \\
\hline SCHOLL HANS DR & SCHUBART JOACHIM DR & SCONZO PASQUALE DR \\
\hline SEGAN STEVO & SEHNAL LADISLAV DR & SEIDELMANN P KENNETH OR \\
\hline SEIN-ECHALUCE M LUISA DR & SESSIN WAGNER DR & SHAPIRO IRWIN I PROF \\
\hline SHARAF SH G DR & SIDLICHOVSKY MILOS DR & SIMA ZDISLAV DR \\
\hline SIMON JEAN-LOUIS MR & SINCLAIR ANDREW T DR & SIRY JOSEPH W \\
\hline SKRIPNICHENKO VLADIMIR DR & SOFFEL MICHAEL DR & STANDISH E MYLES DR \\
\hline STELLMACHER IRENE DR & SULTANOV G F ACAD & SUN YI-SUI \\
\hline SZEBEHELY VICTOR G PROF & TABORDA JOSE ROSA DR & TATEVYAN S K DR \\
\hline TAHADROS MAHER JACOUB DR & TAYLOR DONALD BOGGIA DR & THIRY YVES R PROF \\
\hline TONG FU & VALSECCHI GIOVANNI B DR & VALTONEN MAURI J PROF \\
\hline VARVOGLIS H DR & VASHKOV'YAK SOF'YA N DR & VEILLET CHRISTIAN \\
\hline VIEIRA MARTINS ROBERTO DR & VILHENA DE MORAES R DR & HALCH JEAN-JACQUES \\
\hline WALKER IAN WALTER & WHIPPLE ARTHUR L DR & WILLIAMS CAROL A \\
\hline WNUK EDWIN & WU LIAN-DA & $X U$ JI-HONG DR \\
\hline XU PINXIN & YAROV-YAROVOJ M S DR & YI ZHAO-HUA \\
\hline YOKOYAMA TADASHI DR & YOSHIDA HARUO & YOSHIDA JUNZO PROF \\
\hline YUASA MANABU DR & ZAFIROPOULOS BASIL DR & ZARE KHALIL DR \\
\hline ZHANG SHENG-PAN & ZHENG JIA-QING & ZHENG XUE-TANG \\
\hline ZHOU HONG-NAN & $\mathrm{ZHI}$ & \\
\hline
\end{tabular}


$\begin{array}{ll}\text { President } & : \text { MORRISON LESLIE V DR } \\ \text { Vice-President(s) } & : \text { SMITH CLAYTON A JR DR }\end{array}$

Organizing Committee: BENEVIDES SOARES P DR CORBIN THOMAS ELBERT DR HELMER LEIF

HU NING-SHENG

KOVALEVSKY JEAN DR

LINDEGREN LENNART OR

LOPEZ JOSE A ING

MIYAMOTO MASANORI DR

NOEL FERNANDO

POLOZHENTSEV DIMITRIJ DR

REQUIEME YVES DR

SCHWAN HEINER DR

YOSHIZAWA MASANORI DR

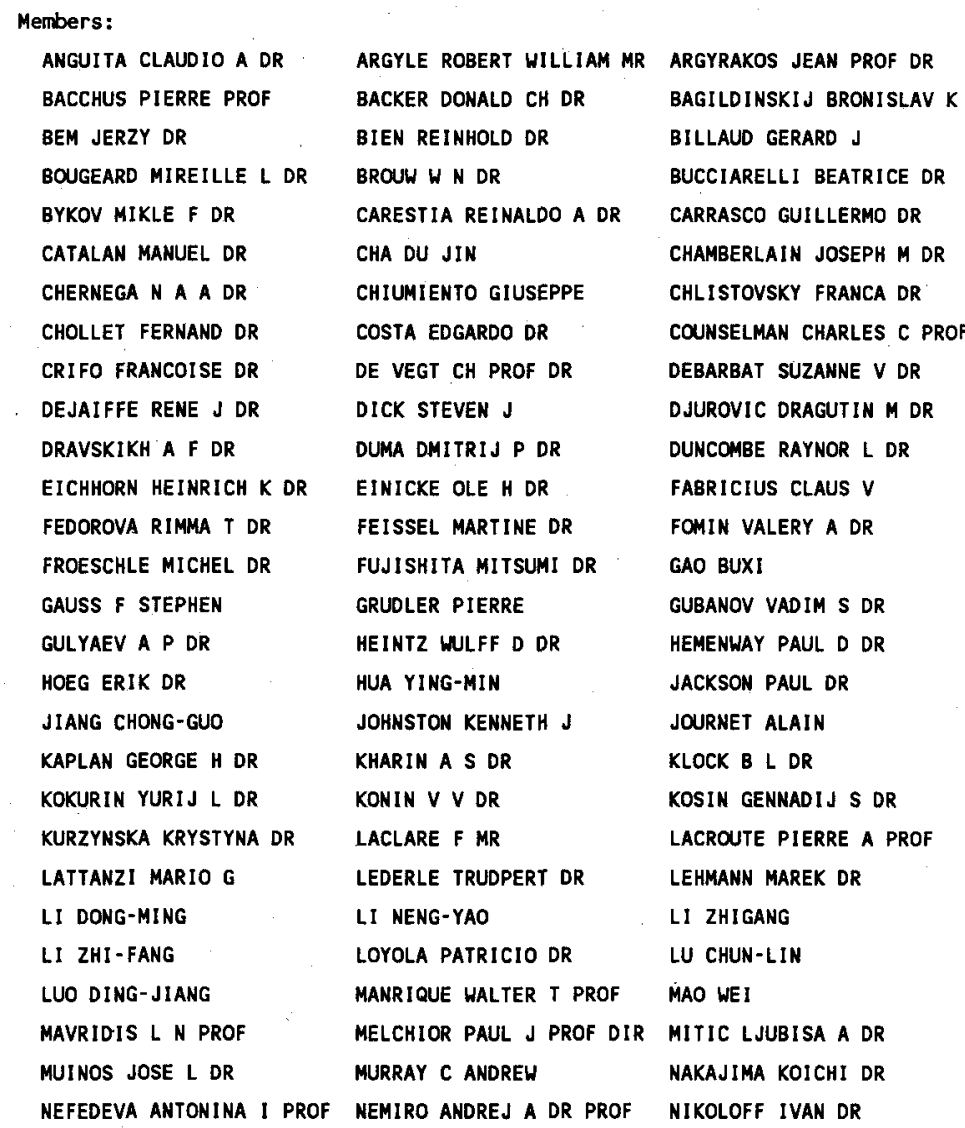




\begin{tabular}{|c|c|c|}
\hline OLSEN FOGH H J & OSORIO JOSE J S P PROF & PAKVOR IVAN \\
\hline PERRYMAN MICHAEL A C DR & PETROV G M DR & PHAM-VAN JACQUELINE MME \\
\hline PILOWSKI K PROF OR & PINIGIN GENNADIJ I DR & POLNITZKY GERHARD DR \\
\hline POMA ANGELO DR & PROVERBIO EDOARDO PROF & PUGLIANO ANTONIO PROF \\
\hline QI GUAN RONG & QIAN ZHI-HAN DR & QUIJANO LUIS \\
\hline RAIMOND ERHST DR & REIZ ANDERS PROF & REYNOLDS JOHN DR \\
\hline ROESER SIEGFRIED DR & ROUSSEAU JEAN-MICHEL MR & RUSSELL JANE L DR \\
\hline RUSU I OR & SADZAKOV SOFIJA DR & SALETIC DUSAN \\
\hline SANCHEZ MANUEL & SARASSO MARIA DR & SATO KOICHI DR \\
\hline SCHMEIDLER F PROF DR & SEVARLIC BRANISLAV M PROF & SHEN KAIXIAN \\
\hline SHI GUANG-CHEN & SIMS KENNETH P DR & SOEDERHJELM STAFFAN DR \\
\hline SOLARIC NIKOLA & SOMA MITSURU DR & SPOELSTRA T A TH DR \\
\hline STANGE LOTHAR & STONE RONALD CECIL & TAFF LAURENCE G DR \\
\hline THOBURN CHRISTIME & THOMAS DAVID V DR & TURON C DR \\
\hline VAN ALTENA WILLIAM \& PROF & VAN LEEUWEN FLOOR DR & VON DER HEIDE JOHANN DR \\
\hline WALLACE PATRICK T MR & WALTER HANS G DR & WESTERHOUT GART DR \\
\hline WIELEN ROLAND PROF DR & XIA YI-FEI & XIE LIANGYUN \\
\hline XU BANG-XIN & Xu $T$ & YAMAZAKI AKIRA DR \\
\hline YASUDA HARUO PRC & YAT & YE SHU-HUA \\
\hline YU KYUNG-LOH PROF & ZHANG HUI & \\
\hline
\end{tabular}


Organizing committee: CULLUM MARTIN DR DAVIS JOHN PROF HUMPHRIES COLIN M DR

MCLEAN IAN S DR MERKLE FRITZ DR TANGO WILLIAM J DR WEST RICHARD M DR

Members:

ABLES HAROLD D DR

ALBRECHT RUDOLF DR

ARNAUD JEAN PAUL

AI GUOXIANG

ALVAREZ PEDRO DR

ASHOK N M DR

BABA NAOSHI DR

BAO KEREN

BAFFA CARLO DR

BARCIA ALBERTO DR

BARWIG HEINZ

BEER REINHARD DR

BLITZSTEIN WILLIAM DR

BORGNINO JULIEN DR

BRECKINRIDGE JAMES B DR

BURTON $W$ BUTLER DR

CHRISTY JAMES WALTER DR

COOKE JOHN ALAN

CURRIE DOUGLAS G DR

DESAI JYOTINDRA N

DOKUCHAEVA OLGA D DR

DREHER JOHN W

EDNIN ROGER $P$

FABRICANT DANIEL G

FLETCHER J MURRAY

FORT BERNARD P DR

GALAN MAXIMINO J

GAY JEAN DR

GLASS IAN STEWART DR

GRIFFITHS RICHARD E DR

GRUNDMANN WALTER

HADLEY BRIAN $W$

HANISCH ROBERT J DR

HARMER DIANNE L MRS

HEWITT ANTHONY $V$ DR

HOOGHOUDT B G IR

HU NING-SHENG

ILYAS MOHAMMAD DR
BARROSO JR JAIR

BAUM WILLIAM A DR

BENSAMMAR SLIMANE DR

BONANNO GIOVANNI DR

BOYCE PETER B DR

BREJDO IZABELLA I DR

CAO CHANGXIN

CLARKE DAVID DR

CORNEJO ALEJANDRO A DR

DALL'OGLIO GIORGIO DR

DIEGO FRANCISCO DR

DOUGLAS NIGEL DR

DUCHESNE MAURICE DR

ENGELS DIETER DR

FEHRENBACH CHARLES PROF

FOMENKO ALEXANDR F DR

FOY RENAUD DR

GAO BILIE

GIBSON DAVID MICHAEL DR

GONG SHOU-SHEN

GRIGORJEV VICTOR M DR

GUIBERT JEAN DR

HALLAM KENHETH L DR.

HAO YUN-XIANG

HECKATHORN HARRY M

HILLIARD R DR

HOUGH JAMES DR

HUANG TIE-QIN

JAYARAJAN A P MR
AIME C DR

APARICI JUAN DR ASSUS PIERRE DR BABCOCK HORACE W DR BARANNE A DR

BARUCH JOHN DR BECKLIN ERIC E DR BINGHAM RICHARO G DR BONMEAU DANIEL BRAULT JAMES W DR BRIDGELAND MICHAEL DR CHELLI ALAIN COHEN RICHARD S CRAWFORD DAVID L DR DAN XHI-XIANG DOBRONRAVIN PETER DR DRAVINS DAINIS PROF DUNKELMAN LAWRENCE ENGVOLD OODBJOERN DR FELENBOK PAUL DR FORD W KENT JR DR FU DELIAN GAUSS F STEPHEN GILLINGHAM PETER MR GRAY PETER MURRAY GROSBOL PREBEN JOHNSON DR GUTCKE DIETRICH HAMMERSCHLAG ROBERT H DR HARMER CHARLES F W MR HEUDIER JEAN-LOUIS OR HONEYCUTT R KENT PROF HU JING-YAO HYSOM EDMUND J JEFFERS STANLEY DR 


\begin{tabular}{|c|c|c|}
\hline JENKNER HELMUT DR & JIANG SHI-YANG & JONES BARBARA \\
\hline JOSEPH CHARLES LYNN DR & KARACHENTSEV I D DR & KARPINSKIJ VADIM N DR \\
\hline KIPPER TONU DR & KISSELL KENNETH E DR & KLOCK B L DR \\
\hline KLOCOK LUBOMIR DR & KOEHLER H PROF DR & KOEHLER PETER \\
\hline KOPYLOV I M DR & KOROVYAKOVSKIJ YURIJ P DR & KOVACHEV B J DR \\
\hline KREIOL TOBIAS J N & KUEHNE CHRISTOPH $F$ & KULKARNI PRABHAKAR $\vee$ PROF \\
\hline LABEYRIE ANTOINE DR & LAQUES PIERRE DR & LASKER BARRY M OR \\
\hline LEMAITRE GERARD R DR & LI DEPEI & LI TING \\
\hline LI ZHIGAMG & LIVINGSTON WILLIAM C & LOCHMAN JAN \\
\hline LU RUWEI DR & LYNCH DAVID $K$ & MACK PETER DR \\
\hline MAHRA H S OR & MAILLARD JEAN-PIERRE DR & MAJOR JOHN DR \\
\hline MALIN DAVID F MR & MALKAMAEKI LAURI J DR & MARTINS DONALD HENRY DR \\
\hline MATZ STEVEN MICHEAL DR & MCGREGOR PETER JOHN DR & MCMULLAN DENNIS DR \\
\hline MEGEVAND DENIS DR & MEINEL ADEN B PROF & MENG XINMIN \\
\hline MERTZ LAWRENCE N DR & MIKHELSON NIKOLAJ N OR & MILLIKAN ALLAN G MR \\
\hline MINAROVJECH MILAN & MORGAN BRIAN LEALAN & MORRIS MICHAEL C \\
\hline MORTON DONALD C DR & MURRAY STEPHEN S DR & MAKAI YOSHIHIRO \\
\hline NELSON JERRY E DR & NEWTON GAVIN DR & NIEMI AIMO \\
\hline NISHIMURA SHIRO DR & NUNES ROGERIO S DE SOUSA & OOGERS GRAHAM J DR \\
\hline OHTSUBO JUNJI DR & O'DELL CHARLES R DR & PASIAN FABIO \\
\hline PENHY ALAN JOHN DR & PERRYMAN MICHAEL A C DR & PETFORD A DAVID DR \\
\hline PETROV PETER P DR & PICAT JEAN-PIERRE DR & PRJTCHET CHRISTOPHER J DR \\
\hline PROKOF'EVA VALENTINA $\vee$ DR & PUCILLO MAURO DR & QIU PUZHANG ASS PROF \\
\hline RACINE RENE DR & RAKOS KARL D PROF & RAMSEY LAURENCE W DR \\
\hline REAY NEWRICK K DR & REDFERN MICHAEL R DR & RICHARDSON E HARVEY DR \\
\hline RING JAMES PROF & ROBERTSON NORNA DR & ROBINSON LLOYD B DR \\
\hline RCODIER CLAUDE DR & ROODIER FRANCOIS PROF & ROSCH JEAN PROF \\
\hline ROUNTREE JANET DR & RUDER HANNS & RUPPRECHT GERO DR \\
\hline RUSCONI LUIGIA DR & RYLOV VALERIJ S DR & SAULT ROBERT DR \\
\hline SAXENA A K DR & SCHROEDER DANIEL I PROF & SCHULTZ ALFRED BERNARD DR \\
\hline SCHULTZ G V DR & SCHUMANN JOERG DIETER DR & SEOMAK GIORGIO PROF \\
\hline SERVAN BERMARD & SHAKHBAZYAN YURIJ L DR. & SHCHEGLOV P V DR \\
\hline SHEN CHANGJUN & SHEN PARN-AN & SHIVANANDAN KANDIAH DR \\
\hline SIM MARY E MISS & SLOVAK MARK HAINES DR & SMITH CHARLES DITTO \\
\hline SMYTH MICHAEL J DR & SNEZHKO LEONID I & STESHENKO N V DR \\
\hline STESHENKO N V DR & STOREY JOHN W V DR & SU DING-QIANG \\
\hline SWINGS JEAN-PIERRE DR & TRAUB WESLEY ARTHUR & TUEG HELMUT DR \\
\hline TULL ROBERT $G$ & ULICH BOBBY LEE & VAKILI FARROKH DR \\
\hline VALNICEK BORIS DR & VALTONEN MAURI J PROF & VAN CITTERS GORDON W DR \\
\hline VELKOV KIRIL & VLADIMIROV SIMEON & VRBA FREDERICK J DR \\
\hline WALKER ALISTAIR ROBIN DR & WALKER DAVID DOUGLAS DR & WALKER GORDON A H PROF \\
\hline HALKER MERLE F PROF & WALLACE PATRICK T MR & WAMPLER E JOSEPH PROF \\
\hline HANG LAN-JUAN & WANG YANAN & WANG YIMING \\
\hline HANG ZHENG MING & WARD HENRY DR & WATSON FREDERICK GARNETT \\
\hline WEISS WERNER W DR & WESTPHAL JAMES A PROF & WILCOCK WILLIAM L PROF \\
\hline WINDHORST ROGIER A DR & HLERICK GERARD DR & WOEHL HUBERTUS DR \\
\hline MORDEN SIMON P DR & HORSWICK SUSAN & W LIN-XJANG \\
\hline WYLLER ARNE A PROF & WYNNE CHARLES G PROF & YANG SHI JIE \\
\hline YAO ZHENG-QIU & YE BINXUN & ZACHAROV IGOR DR \\
\hline ZEALEY WILLIAM J DR & ZHANG XIU ZHONG & ZHANG YOUYI \\
\hline ZHOU BIFANG DR & IGHONG & \\
\hline
\end{tabular}




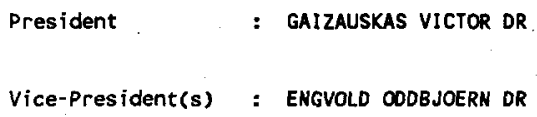

- SCHUESSLER MANFRED DR

- Stepanov alexander $V$ DR

- tANDBERG-HANSSEN EINAR A

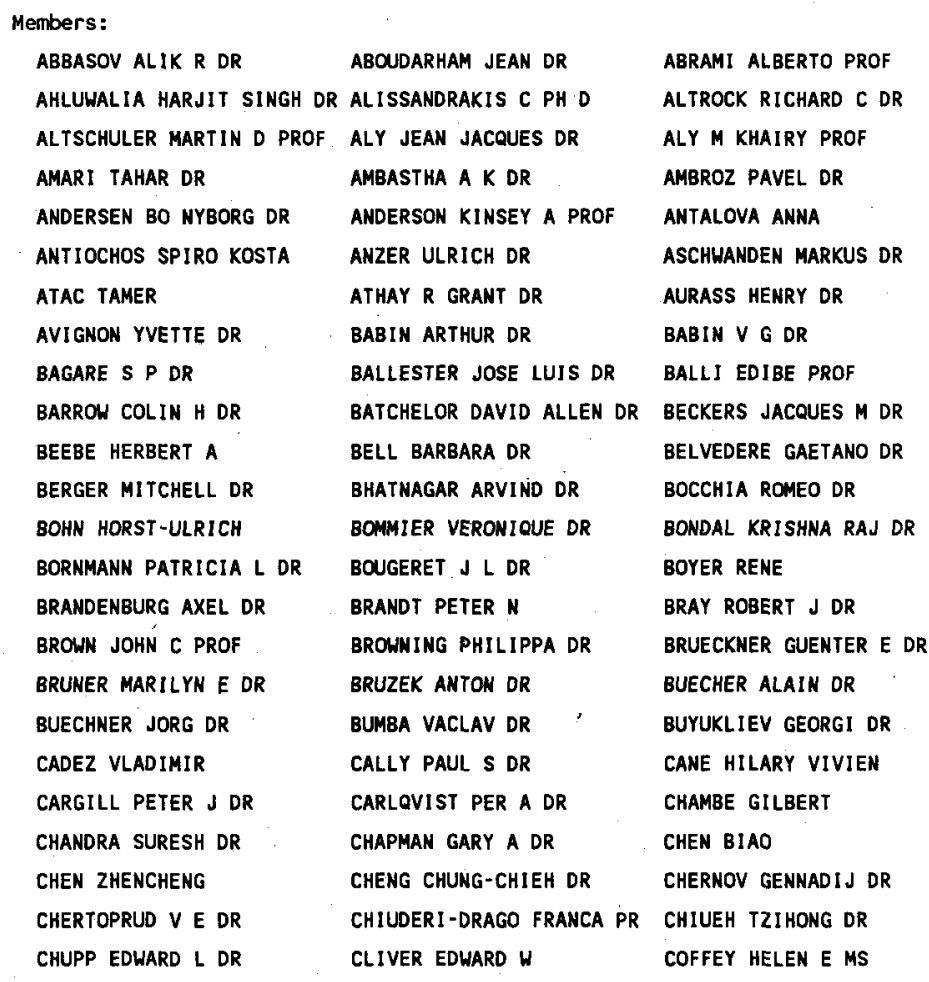




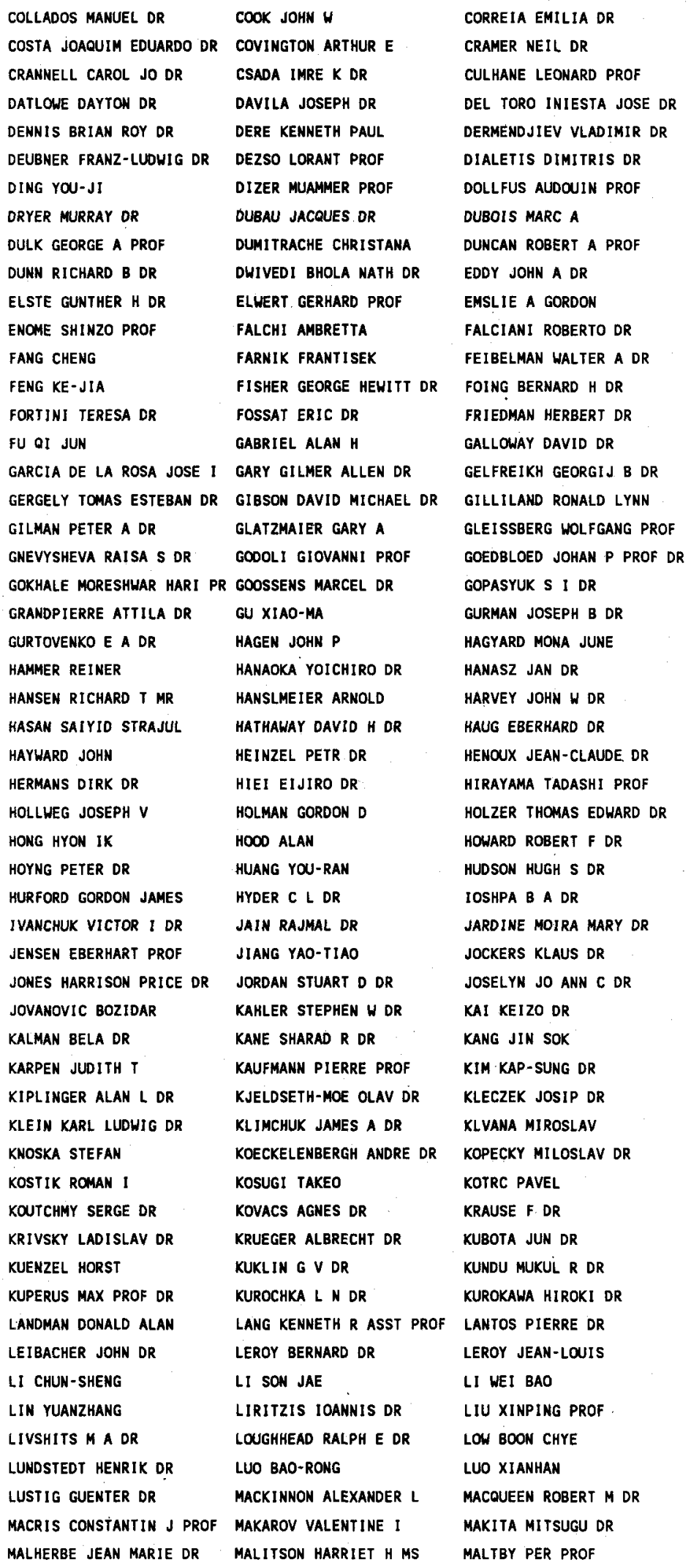




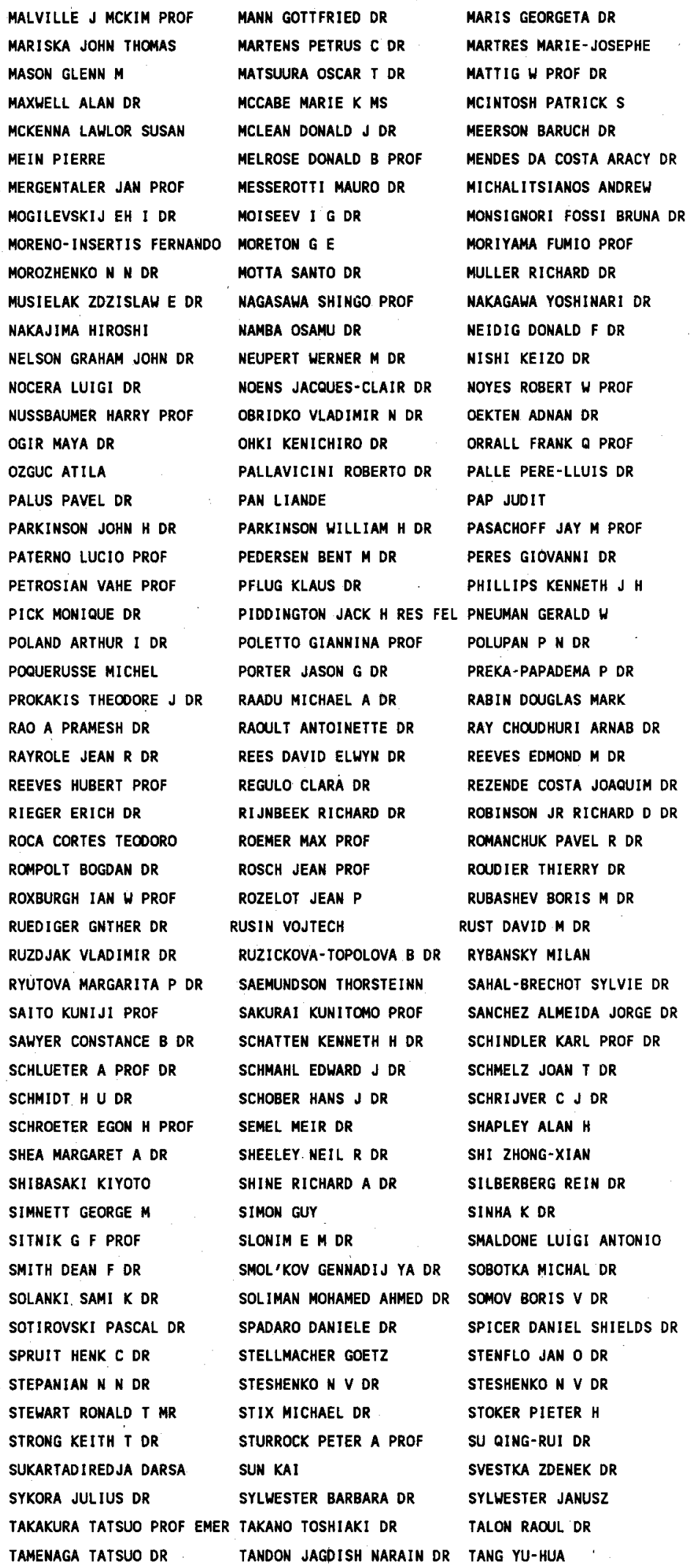




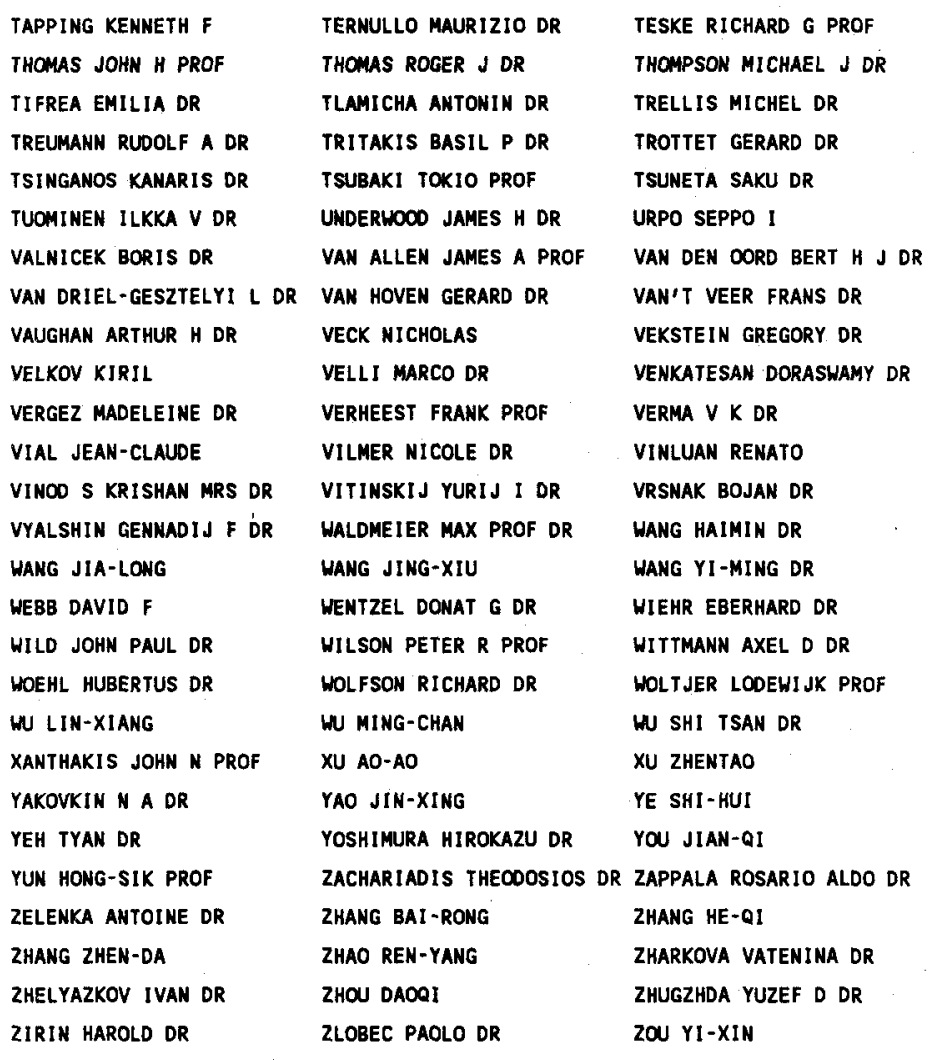


$\begin{array}{ll}\text { President } & : \text { STENFLO JAN O DR } \\ \text { Vice-President(s) } & \text { : DEUBNER FRANZ-LUDWIG DR }\end{array}$

Organizing Committee: AYRES THOMAS R

HARVEY JOHN $W$ DR

KARPINSKIJ VADIM N DR

LANDI DEGL'IMNOCENTI E PR

NORDLUND AKE DR

PASACHOFF JAY M PROF

SIVARAMAN $K$ R DR

STAUDE JUERGEN DR

WANG JING-XIU

HILSON PETER R PROF

YOSHIMURA HIROKAZU DR

\begin{tabular}{|c|c|c|}
\hline \multicolumn{3}{|l|}{ embers: } \\
\hline ABOUDARHAM JEAN DR & ACTON LOREN W DR & ADAM MADGE G DR \\
\hline AI GUOXIANG & AIME C DR & ALISSANDRAKIS C PH D \\
\hline ALTROCK RICHARD C DR & ALTSCHULER MARTIN D PROF & ANDERSEN BO NYBORG DR \\
\hline ANDO HIROYASU DR & ANSARI S M RAZAULLAH PROF & ANTIA H M DR \\
\hline ARNAUD JEAN PAUL & ATHAY R GRANT DR & BALIUNAS SALLIE L \\
\hline BALTHASAR HORST DR & BEARD DAVID B DR & BECKERS JACQUES $M$ DR \\
\hline BECKMAN JOHN E PROF & BEEBE HERBERT A & BEL NICOLE J DR \\
\hline BENFORD GREGORY DR & BHATNAGAR ARVIND DR & BHATTACHARYYA J C PROF \\
\hline BILLINGS DONALD E PROF & BLACKWELL DONALD E PROF & BLAMONT JACQUES E PROF \\
\hline BOCCHIA ROMEO DR & BOEHM KARL-HEINZ PROF & BOEHM-VITENSE ERIKA PROF \\
\hline BOHN HORST-ULRICH & BOMMIER VEROHIQUE DR & BONNET ROGER M DR \\
\hline BOOK DAVID L & BORNMANM PATRICIA L DR & BOUGERET J L DR \\
\hline BRANDT PETER N & BRAULT JAMES $W$ DR & BRAY ROBERT J DR \\
\hline BRECKINRIDGE JAMES B DR & BRUECKNER GUENTER E DR & BRUNER MARILYN E DR \\
\hline BRUNING DAVID H DR & BRUZEK ANTON DR & BUMBA VACLAV DR \\
\hline CADEZ VLADIMIR & CAVALLINI FABIO & CEPPATELLI GUIDO DR \\
\hline CHAMBE GILBERT & CHAN KWING LAM & CHAPMAN GARY A DR \\
\hline CHEN BIAO & CHENG CHUNG-CHIEH DR & CHISTYAKOV VLADIMIR E DR \\
\hline CHRISTENSEN-DALSGAARD J & CHVOJKOVA WOYK E DR & CLARK THOMAS ALAN DR \\
\hline COLLADOS MANUEL DR & COOK JOHN W & COX ARTHUR N DR \\
\hline CRAIG IAN JONATHAN D DR & CRAM LAURENCE EDWARD PROF & CRAMER NEIL DR \\
\hline DARA HELEN DR & DE JAGER CORNELIS PROF & DEL TORO INIESTA JOSE DR \\
\hline DELACHE PHILIPPE J DR & DELBOUILLE LUC PROF & DELIYANNIS JEAN DR \\
\hline DEMARQUE P PROF & DEMING LEO DRAKE OR & DEZSO LORANT PROF \\
\hline DOGAN NADIR PROF & DRAVINS DAINIS PROF & DUMONT SIMONE DR \\
\hline DUNKELMAN LAWRENCE & DUNN RICHARD B DR & DUVALL THOMAS L JR \\
\hline EINAUDI GIORGIO & ELLIOTT IAN DR & ELSTE GUNTHER H DR \\
\hline EPSTEIN GABRIEL LEO & ESSER RUTH DR & EVANS $J V D R$ \\
\hline FALCIANI ROBERTO DR & FANG CHENG & FELDMAN URI \\
\hline FIALA ALAN D DR & FISHER GEORGE HEWITT DR & FOFI MASSIMO DR \\
\hline
\end{tabular}


FOMICHEV VALERI $V$ DR FOUKAL PETER $V$ DR FROEHLICH CLAUS GARCIA-BERRO ENRIQUE DR GOOOLI GIOVAMMI PROF GOMEZ MARIA THERESA DR GORDON CHARLOTTE PROF GURTOVENKO E A DR HEINZEL PETR DR HILDNER ERNEST DR HOANG BINH DY DR HOTINLI METIN DR HOYNG PETER DR JACKSON BERNARD $V$ DR JONES HARRISON PRICE DR JOSHI G C DR

KARLICKY MARIAN KAUFMANM PIERRE PROF KHETSURIANI TSIALA S DR KNOLKER MICHAEL DR KOSTIK ROMAN I KOUTCHMY SERGE DR KRUEGER ALBRECHT DR KULCAR LADISLAV DR KUROCHKA L N DR LANDI DEGL'INMOCENTI M LANTOS PIERRE DR LEROY JEAN-LOUIS LIVINGSTON WILLIAM C LOUGHHEAD RALPH E DR MAKAROV VALENTINE I MARIK MIKLOS OR MARMOLINO CIRO MCKENNA LAWLOR SUSAN MERGENTALER JAN PROF MICHARD RAYMOND DR MONSIGNORI FOSSI BRUNA DR MOORE RONALD L DR MORIYAMA FUMIO PROF MULLER RICHARD DR NECKELL HEINZ DR NICOLET MARCEL PROF ORRALL FRANK Q PROF PALLE PERE-LLUIS DR PAPATHANASOGLOU D DR PEYTURAUX ROGER H PROF PIERCE A KEITH DR PROKAKIS THECDORE J DR RAOULT ANTOINETTE DR REGULO CLARA DR ROBERTI GIUSEPPE DR ROLAND GINETTE DR RUSIN VOJTECH SAKAI JUNICHI SANCHEZ ALMEIDA JORGE DR SCHMIDT WOLFGANG DR GABRIEL ALAN H

GAUR V P GOPALSHAMY N DR GREVESSE $N$ DR HAGYARD MONA JUNE HEJNA LADISLAV DR HILL FRANK DR HOUSE LEWIS L DR ILLING RAINER M E JORDAN CAROLE DR. KARPEN JUDITH T KOTOV VALERY DR KUNDU MUKUL. R DR LE I BACHER JOHN DR LIN YUANZHANG LOCKE JACK L DR LUEST REIMAR PROF MARILLI ETTORE OR MEIN PIERRE MEUE R DR MIHALAS DIMITRI DR MUNRO RICHARD H DR NISHI KEIZO DR PALUS PAVEL DR PFLUG KLAUS DR POQUERUSSE MICHEL RABIN DOUGLAS MARK ROUDIER THIERRY DR RUTTEN ROBERT J DR SAKURAI TAKASHI DR SCHMITT DIETER DR
FONTENLA JUAN MANUEL DR FRAZIER EDWARD N OR GOKDOGAN NUZHET PROF HOLWEGER HARTMUT PROF JEFFERIES JOHN T DR KALKOFEN WOLFGANG DR KAWAGUCHI ICHIRO PROF KLEIN KARL LUDWIG DR KONONOVICH EDWARD V DR KOYAMA SHIM PROF DR KUBICELA ALEKSANDAR DR LA BONTE BARRY JAMES LANDMAN DONALD ALAN MAKAROVA ELENA A DR MATSUSHIMA SATOSHI DR MOURADIAN ZADIG M DR MESIS ANASTASIOS DR OSTER LUDWIG F PROF DR PARKINSON WILLIAM H DR REES DAVID ELWYN DR RIGHINI-COHEN GIOVANNA DR RIGUTTI MARIO PROF ROCA CORTES TEODORO SAUVAL A JACOUES DR GU XIAO-MA
FOSSAT ERIC DR FRIEDMAN HERBERT DR GAIZAUSKAS VICTOR DR GLATZMAIER GARY A GOLDMAN MARTIN $V$ GOPASYUK S I DR HAMMER REINER HIEI EIJIRO DR HIRAYAMA TADASHI PROF HORTON BRIAN H DR HOWARD ROBERT F DR JABBAR SABEH RHAMAN JEFFERIES STUART DR JORDAN STUART D DR KALMAN BELA DR KATO SHOJI PROF KEIL STEPHEN L KNEER FRANZ DR KOPECKY MILOSLAV DR KOTRC PAVEL

KRAEMER GERHARD DR KUKLIN G V OR KUPERUS MAX PROF DR LABS DIETRICH PROF LANDOLFI MARCO LEIGHTON R B PROF LINSKY JEFFREY L DR LOPEZ-ARROYO M LUSTIG GUENTER DR MAKITA MITSUGU DR MARISKA JOHN THOMAS MATTIG W PROF DR MELROSE DONALD B PROF MEYER FRIEDRICH DR MILKEY ROBERT W DR MORENO-INSERTIS FERNANDO MUELLER EDITH A PROF NAMBA OSAMU DR NICOLAS KENNETH ROBERT NOYES ROBERT W PROF OWOCKI STANLEY PETER DR PANDE MAHESH CHANDRA DR PECKER JEAN-CLAUDE PROF PHILLIPS KENWETH $J$ H PRIEST ERIC R PROF RADICK RICHARD R DR REEVES EDMOND $M$ DR ROODIER FRANCOIS PROF ROVIRA MARTA GRACIELA RYBANSKY MILAN SAMAIN DENYS DR SCHMAHL EDWARD J DR SCHOBER HANS J DR 
SCHUESSLER MANFRED DR

SEMEL MEIR DR

SHEELEY NEIL R DR

SIMON GEORGE W DR

SINHA $K$ DR

SKUMANICH ANDRE PROF

SOLANKI SAMI $K$ DR

SOUFFRIN PIERRE B DR

STEBBINS ROBIN

SUEMATSU YOSHINORI DR

TANDBERG-HANSSEN EINAR A

THOMAS RICHARD N OR

TRIPATHI B M DR

TSIROPOULA GEORGIA DR

UNNO WASABURO PROF

VASILEVA GALINA J DR

VIAL JEAN-CLAUDE

VOLONTE SERGE DR

WANG ZHEN-YI

WENTZEL DONAT G OR

WORDEN SIMON P DR

WYLLER ARNE A PROF

YUN HONG-SIK PROF

ZHOU DAOOI

ZIRKER JACK B OR
SCHUARTZ STEVEN JAY SEVERINO GIUSEPPE

SHEN LONG-XIANG

SIMON GUY

SITNIK G F PROF

SMITH PETER L DR

SONG MU-TAO

SPICER DANIEL SHIELDS DR

STEFFEN MATTHIAS DR

SVESTKA ZDENEK DR

TEPLITSKAYA R B DR

TORELLI M DR

TRUJILLO BUENO JAVIER DR

TSUBAKI TOKIO PROF

WS UNDO DR

VAUGHAN ARTHUR H DR

VILMER NICOLE DR

WUKICEVIC $K$ M PROF DR

WARHICK JAMES $W$ DR

WITTMANM AXEL D DR

WU HSIN-HENG DR

YOU JIAN-OI

ZARRO DOMINIC M DR

ZHUGZHDA YUZEF D DR

ZWAAN CORNELIS PROF DR
SEATON MICHAEL J PROF SHALLIS MICHAEL J DR SHINE RICHARD A DR SINGH JAGDEV DR SITTERLY CHARLOTTE M OR SOBOLEV VLADISLAV M DR SOTIROVSKI PASCAL DR STATHOPOULOU MARIA DR STIX MICHAEL DR SWENSSON JOHN W DR THOMAS JOHN H PROF TOUSEY RICHARD DR TSAP T T DR UCHIDA YUTAKA PROF VAN HOVEN GERARD DR VENKATAKR ISHNAN $P$ DR VITINSKIJ YURIJ I DR WALDMEIER MAX PROF DR WEISS NIGEL $O D R$ HOEHL HUBERTUS OR WU LIN-XIANG YOUSSEF NAHED H PROF ZELENKA ANTOINÉ DR ZIRIN HAROLD DR 
COMPOSITION OF COMMISSION $14 \quad 1991-1994$

\author{
President : HIESE NOLFGANG $L$ DR \\ Vice-President(s) : PARKINSON WILLIAM H DR
}

Organizing Committee: ADELMAN SAUL J DR

DUBAU JACQUES DR

GABRIEL ALAN H

KATO TAKAKO DR

NICHOLLS RALPH W PROF

NUSSBAUMER HARRY PROF

RUDZIKAS ZENONAS B

SAHAL-BRECHOT SYLVIE DR

SMITH PETER L DR

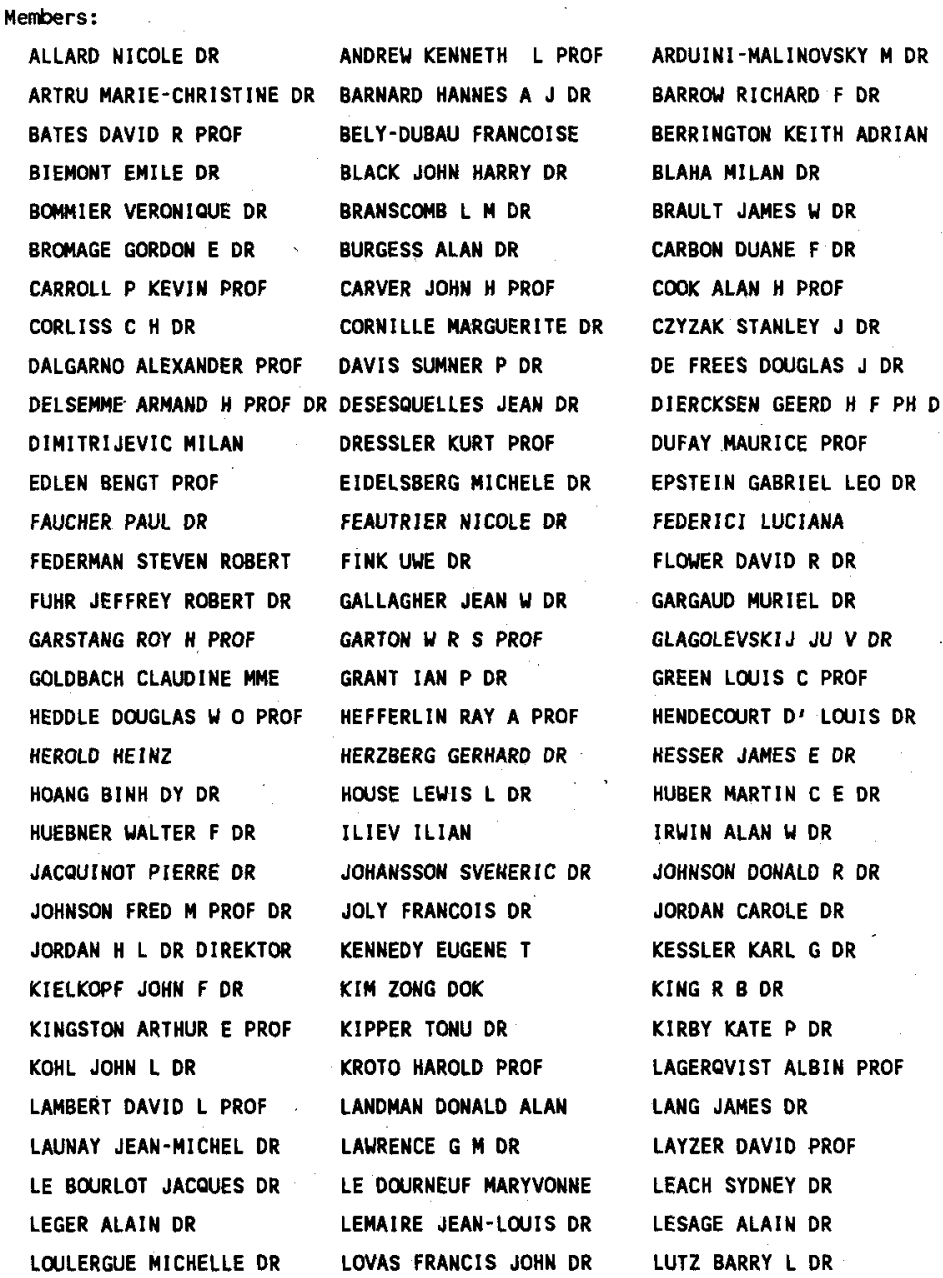




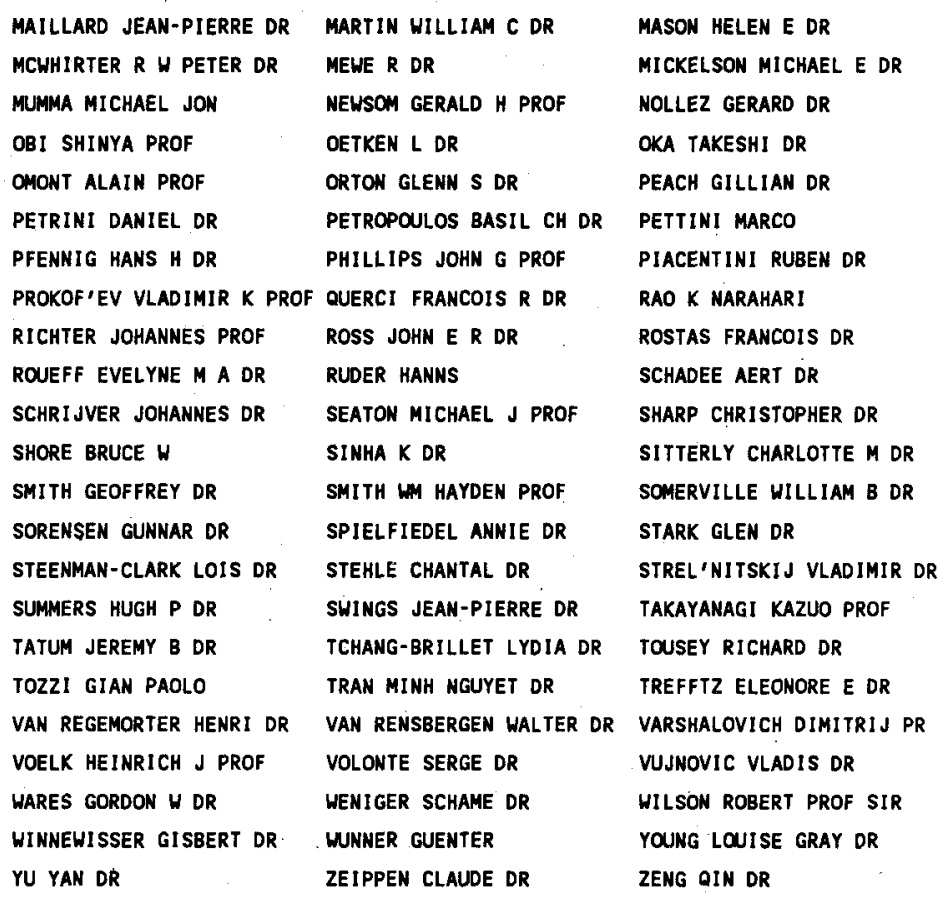


President $\quad$ : HARRIS ALAN WILLIAM DR

Vice-President(s) : A'HEARN MICHAEL F DR

Organizing Committee: BARUCCI MARIA A DR

BELTON MICHAEL J S DR

BRANDT JOHN C DR

GRUEN EBERHARD DR

HUEBNER HALTER F DR

HUGHES DAVID $W$ DR

KELLER HORST UWE DR

KISELEV NIKOLAI N DR

KNEZEVIC ZORAN

KRESAK LUBOR DR

LUPISHKO DMITRIJ $F$

RAHE JURGEN PROF

RICKMAN HANS DR

STEEL DUNCAN I DR

TEDESCO EDWARD $F$

ZAPPALA VINCENZO PROF

\section{Members:}

aLLEgRE CLAUDE PROF ARPIGNY CLAUDE PROF BAILEY MARK EDWARD BINZEL RICHARD P DR BOCKELEE-MORVAN DOMINIQUE BOEHNHARDT HERMANN DR BONELL EDWARD L G DR BROWNLEE DONALD E PROF BURLAGA LEONARD F DR CANDY MICHAEL. P MR CARRUTHERS GEORGE $R$ DR CELLINO ALBERTO DR CHANDRASEKHAR T OR CHEN DAO-HAN CLAIREMIDI JACQUES DR CLUBE $S \vee M$ DR COMBI MICHAEL $R$ DR CROVISIER JACQUES DANKS ANTHONY C DR DE SANCIIS GLOVANMI DELSEMME ARMAND H PROF DR DERMOTT STAMLEY F DI MARTINO MARIO DRYER MURRAY DR ANDRIENKO DMITRY A DR AXFORD $W$ IAN PROF BARKER EDWIN S DR BIRCH PETER MR BRECHER AVIVA DR PROF BRUNK WILLIAM E DR BURNS JOSEPH A PROF CAPACCIONI FABRIZIO DR CARSENTY URI DR CEPLECHA ZDENEK DR CHAPMAN CLARK R DR CHEREDNICHENKO $V$ I DR CLAYTON GEOFFREY C DR COCHRAN ANITA L DR COSMOVICI BATALLI C DR CRUIKSHANK DALE $P$ DR DE ALMEIDA AMAURY A DR DEBEHOGNE HENRI DR SC ERSHKOVICH ALEXANDER PROF EVIATAR AHARON PROF FECHTIG HUGO DR FERNANDEZ JULIO A DR FERRIN IGNACIO
DONI BERTRAM D DZHAPIASHVILI VICTOR P DR ENCRENAZ THERESE DR FELDMAN PAUL DONALD DR DOSSIN F DR FARINELLA PAOLO DR FESTOU MICHEL C DR
ARNOLD JAMES R DR BABADZHANOV PULAT B DR BEARD DAVID B DR BLAMONT JACQUES E PROF BOUSKA JIRI DR BROWN ROBERT HAMILTON BURATTI BONNIE J DR CAMPINS HUMBERTO DR CAPRIA MARIA TERESA DR CARUSI ANDREA CERRONI PRISCILLA DR CHAPMAN ROBERT D DR CHERNYKH N S DR CLAYTON ROBERT N DR COCHRAN WILLIAM DAVID DR CRISTESCU CORNELIA G DR CUYPERS JAN OR DE PATER IMKE DEGEWI J JOHAN DR DEUTSCHMAN WILLIAM A DR FERMAMDEZ JEAN-CLAUDE DR 
FORTI GIUSEPPE DR FULCHIGNONI MARCELLLO PROF GEISS JOHANNES PROF GIOVANE FRANK GREENBERG J MAYO DR GRUDZINSKA STEFANIA DR HALLIDAY IAN DR HARTMANN WILLIAM $K$ HASER LEO N K DR HERZBERG GERHARD DR IBADINOV KHURSANDKUL DR I SOBE SYUZO DR JOCKERS KLAUS DR KEAY COLIN S L PROF KOEBERL CHRISTIAN DR KOWAL CHARLES THOMAS KRISTENSEN LEIF KAHL LANCASTER BROWN PETER LARSON STEPHEN M LEVASSEUR-REGOURD A C PR LINOSEY CHARLES ALLAN LIU LIN-ZHONG LUEST RHEA DR LYTTLETON RAYMOND A PROF MARSDEN BRIAN G DR MCCORD THOMAS 8 DR MCFADOEN LUCY ANN DR MEISEL DAVID D DR MILANI ANDREA MILLIS ROBERT L DR MOROZ V I PROF DR MUKAI TADASHI DR NAPIER WILLIAM M DR NEWBURN RAY L JR O'DELL CHARLES R DR PARISOT JEAN-PAUL PILCHER CARL BERNARD DR PRIALNIK-KOVETZ DINA DR REMY BATTIAU LILIANE G A SAGDEEV ROALD 2 DR SCHLEICHER DAVID G DR SCHMIDT MAARTEN PROF SCHUBART JOACHIM DR SHIMIZU MIKIO PROF SHOR VIKTOR A DR SMITH BRADFORD A PROF SOLC MARTIN

SURDEJ JEAN M G SZEGO KAROLY DR TATUM JEREMY B DR TOMITA KOICHIRO MR VAN FLANDERN THOMAS DR VEVERKA JOSEPH DR HALLIS MAX $K$ DR WATANABE JUN-ICHI DR
FROESCHLE CHRISTIANE D DR FUJIHARA AKIRA DR F GAMELGAARD PETER MAG SCI GEHRELS TOM PROF GERARD ERIC DR GIBSON JAMES GRADIE JONATHAN CAREY GREEN SIMON F GREENBERG RICHARD DR GROSSMAN LAHRENCE PROF GUSTAFSON BO A $S$ HAJDUK ANTON DR HANMER MARTHA S DR HAPKE BRUCE W DR HARWIT MARTIN PROF HAUPT HERMANN F PROF HASEGAWA ICHIRO DR HELIN ELEANOR FRANCIS HUNTRESS WESLEY T DR IRVINE WILLIAM M PROF JACKSON WILLIAM M. DR KARTTUNEN HANNU DR KNACKE ROGER F DR KONOPLEVA VARVARA $P$ DR KOHOUTEK LUBOS DR KRISHNA SWAMY K S DR KRESAKOVA MARGITA DR LAGERKVIST CLAES - INGVAR

LAMY PHILIPPE DR LANE ARTHUR LONME 'DR LARSON HAROLD P DR LEBOFSKY LARRY ALLEN LEE THYPHOON LILLIE CHARLES F DR

LILLER WILLIAM DR LISSAUER JACK J DR LIPSCHUTZ MICHAEL E DR LIU ZONGLI LUMME KARI A DR LOPES-GAUTIER ROSALY DR LUTZ BARRY L DR MALAISE DANIEL J DR MATSON DENNIS L DR MCCROSKY RICHARD E DR MCKENWA LAWLOR SUSAN MARAN STEPHEN $P$ DR MATSUURA OSCAR T DR MCDONNELL J A M PROF MENDIS DEVAMITTA ASOKA DR MICHALOWSKI TADEUSZ DR MILET BERNARD L DR MOEKLMANN DIEDRICH MORRISON DAVID PROF MUMMA MICHAEL JON NEFF JOHN S NIEDNER MALCOLM 8 DR O'KEEFE JOHN A DR PELLAS PAUL DR PILLINGER COLIN DR MILLER FREEMAN D PROF MOORE ELLIOTT P PROF MRKOS ANTONIN DR NAKAMURA TSUKO DR NEUKUM G DR NOLL KEITH STEPHEN DR PAOLICCHI PAOLO DR PEREZ-DE-TEJADA H A DR PITTICH EDUARD $M$ DR REITSEMA HAROLD J PROISY PAUL E DR ROEMER ELIZABETH PROF SCALTRITI FRANCO DR SCHMIDT H U DR SCHOLL HANS DR SHARP CHRISTOPHER DR SHOEMAKER EUGENE M SIVARAMAN $K R$ DR SNYDER LEWIS E STOHL JAN DR SYKES MARK VINCENT DR TAKEDA HIDENORI DR TANABE HIROYOSHI DR TERENT JEVA ALEXANDRA $K$ DR THOLEN DAVID J DR TOTH IMRE DR VALSECCHI GIOVANNI B DR VANYSEK VLADIMIR PROF VILAS FAITH DR WANG SI-CHAO VEEDER GLENH J DR WALKER ALISTAIR ROBIN DR WASSON JOHN $T$ WDOWIAK THOMAS J DR 
WEHINGER PETER A DR WETHERILL GEORGE W WILLIAMS IWAN P PROF WOOLFSON MICHAEL M PROF YABUSHITA SHIN A PROF ZARNECKI JAN CHARLES DR
WEIDENSCHILLING S J DR WHIPPLE FRED L DR WISNIEUSKI WIESLAW $Z$ WOSZCZYK ANDRZEJ PROF YAVNEL ALEXANDER A DR ZELLNER BENJAMIN H DR
WEISSMAN PAUL ROBERT WILKENING LAUREL L DR WOOD JOHN A DR WYCKOFF SUSAN DR YEOMANS DONALD $K D R$ 


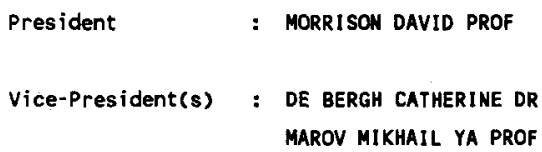

\begin{tabular}{|c|c|c|}
\hline AKABANE TOKUHIDE DR & APPLEBY JOHN F & ARTHUR DAVID W G \\
\hline ATREYA SUSHIL $K$ & BARROW COLIN H DR & BATSON RAYMOND MILNER DR \\
\hline BATTANER EDUARDO DR & BAUM WILLIAM A DR & BEEBE RETA FAYE DR \\
\hline BEER REINHARD DR & BENDER PETER L DR & BERGE GLENN L DR \\
\hline BERGSTRALH JAY T DR & BERTAUX J L DR & BHATIA R K DR \\
\hline BINZEL RICHARD P DR & BLAMONT JACQUES E PROF & BOBROV M S DR \\
\hline BONDARENKO L N DR & BOSMA PIETER B DR & BOSS ALAN P DR \\
\hline OYCE PETER B DR & BRECHER AVIVA OR PROF & BROADFOOT A LYLE OR \\
\hline BROWN ROBERT HAMILTON & BRUNK WILLIAM E DR & BURATTI BONNIE J DR \\
\hline BURNS JOSEPH A PROF & CALAME OOILE DR & CALDWELL JOHN JAMES \\
\hline CAMERON WINIFRED S MRS & CAMICHEL HENRI DR & CAMPBELL DONALD B \\
\hline CAPRIA MARIA TERESA DR & CARSMARU MARIA M DR & CATALANO SANTO DR \\
\hline CHAMBERLAIN JOSEPH $W$ PROF & CHAPMAN CLARK R DR & CHEN DAO-HAN \\
\hline CLAIREMIDI JACQUES DR & COCHRAN ANITA L DR & COLOMBO G PROF DR \\
\hline COMBI MICHAEL R DR & CONNES JANINE DR & COUNSELMAN CHARLES C PROF \\
\hline DE PATER IMKE & DEGEHIJ JOHAN DR & DERMOTT STANLEY $F$ \\
\hline DICKEL JOHN R & DICKEY JEAN O'BRIEN & DOLLFUS AUDOUIN PROF \\
\hline DRAKE FRANK D PROF & DROSSART PIERRE DR & DURRANCE SAMUEL T DR \\
\hline DZHAPIASHVILI VICTOR P DR & ELLIOT JAMES L DR & ELSTON WOLFGANG E PROF \\
\hline EL-BAZ FAROUK DR & ESHLEMAN VON R PROF & ESPOSITO LARRY $W$ \\
\hline FARINELLA PAOLO DR & FIELDER GILBERT DR & FINK UWE DR \\
\hline FOX KENNETH DR & FOX WE MR & FUJIWARA AKIRA DR \\
\hline GEAKE JOHN E DR & GEHRELS TOM PROF & GEISS JOHANNES PROF \\
\hline GERARD JEAN-CLAUDE M C DR & GICLAS HENR & GIERASCH PETER J DR \\
\hline GOLD THOMAS F & GOLDRE & GOLDSTEIN RICHARD M DR \\
\hline OOOY R M & GORENSTEIN & GOUDAS CONSTANTINE L PROF \\
\hline REEN JACK PROF & GROSSMAN LAURENCE PROF & GUERIN PIERRE OR \\
\hline UEST JOHN E D & GULKIS SAMU: & GURSHTEIN ALEXANDER A OR \\
\hline
\end{tabular}




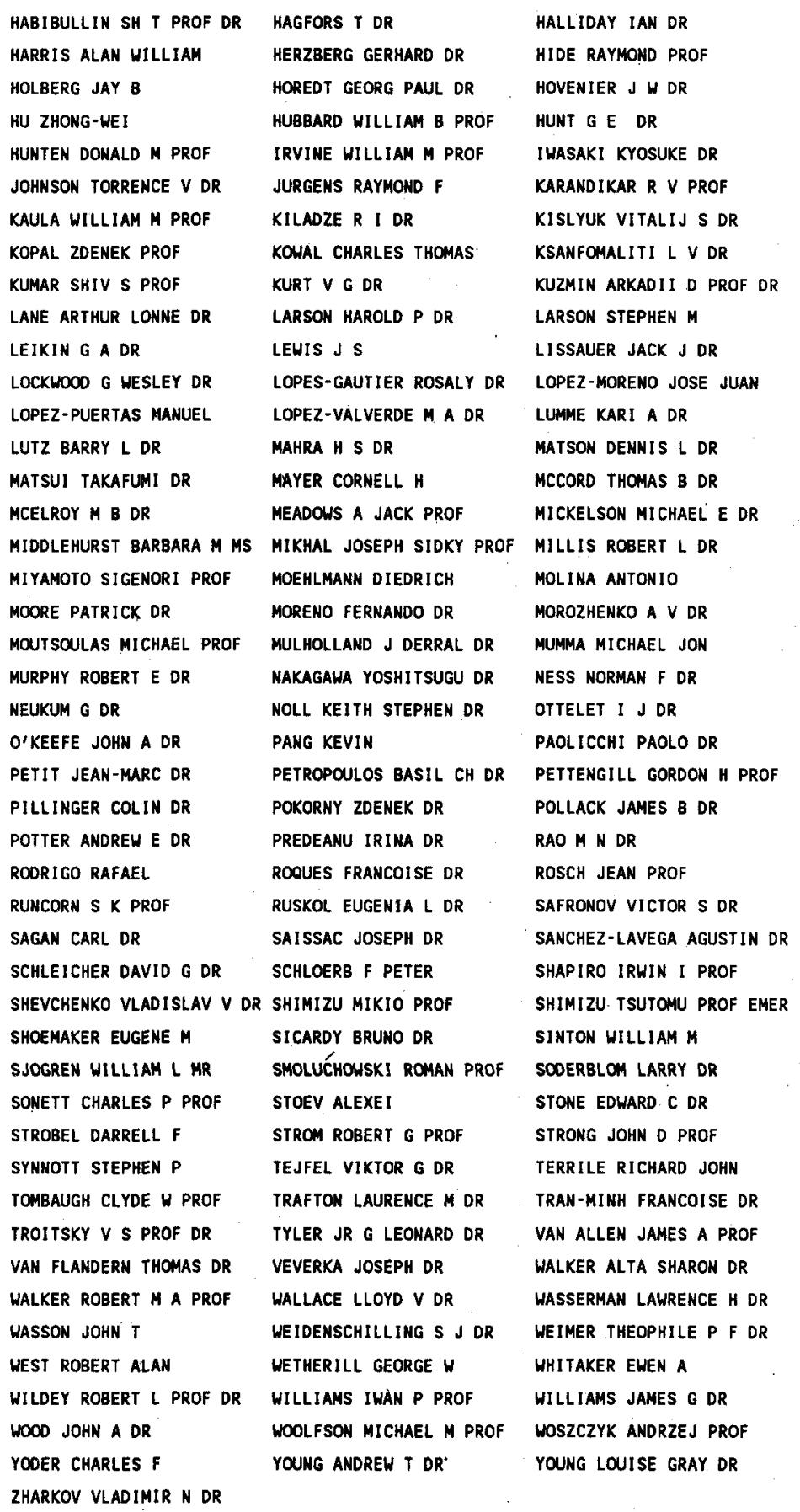




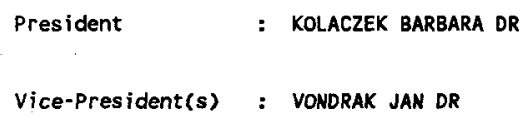

\section{CHIUMIENTO GIUSEPPE}

DEJAIFFE RENE J DR

EL-SHAHAWY MOHAMAD PROF

FONG CHU-GANG

GAO BUXI

GUINOT BERNARD R PROF

HEFTY JAN DR

HUA YING-MIN

KAKUTA CHUICHI DR

KNOWLES STEPHEN H DR

LI ZHIAN DR

LUO DING-JIANG

MARKOWITZ WILLIAM OR

MELCHIOR PAUL J PROF DIR

MONET ALICE $K$ B DR

NAUMOV VITALIJ A DR

OOE MASATSUGU DR

PILKINGTON JOHN D H DR

PROVERBIO EDOARDO PROF

RUDER HANNS

RYKHLOVA LIDIJA V DR

SATO KOICHI OR

SEVARLIC BRANISLAV M PROF

SIDORENKOV NIKOLAY S

STANILA GEORGE DR
BANG YONG GOL

BENDER PETER L DR

BOUCHER CLAUDE DR

BRZEZINSKI ALEXSANDER DR DEBARBAT SUZANNE $V$ DR DJUROVIC DRAGUTIN M DR ENSLIN HEINZ DR FUSISHITA MITSUMI DR GAPOSCHKIN EDWARD M DR HALL R GLENN DR HEMMLEB GERHARD DR IIJIMA SHIGETAKA PROF KAMEYA OSAMU DR LEHMANN MAREK DR LIESKE JAY H DR LUO SHI - FANG MATSAKIS DEMETRIOS N MERRIAM JAMES B MORGAN PETER DR NEWHALL $X \times D R$ PAQUET PAUL EG DR POMA ANGELO DR RANDIC LEO PROF DR RUNCORN S K PROF SADZAKOV SOFIJA DR SCHUTZ BOB EWALO SEVILLA MIGUEL J DR SMITH HUMPHRY M STEPHENSON F RICHARD DR 


\section{SUGAHA CHIKARA DR} TARADY VLADIMIR K DR TSUBOKAWA IETSUNE DR HANG ZHENG MING WINKLER GERNOT M R DR XIAO NAI - YUAN

YANG FUMIN YOKOYAMA KOICHI DR ZHAO MING

ZHU YONG-HE
SUN YONGXIANG

TORAO MASAHISA

VEILLET CHRISTIAN

WILKINS GEORGE A DR

WU SHOU-XIAN

XU JIA-YAN

YATSKIV YA S DR

YUMI SHIGERU PROF DR

ZHENG DA-WEI
TAPLEY BYRON D DR

TSAO MO PROF

VICENTE RAIMUNDO O PROF

WILLIAMS JAMES $G D R$

XIA JIONGYU

$X U$ TONG-QI

YE SHU-HUA

ZHANG GUO-DONG

ZHU YAOZHONG DR 


\author{
President : CARUSI ANDREA \\ Vice-President(s) : YEOMANS DOHALD K DR
}

Organizing committee: AKSWES KAARE DR

ARLOT JEAN-EUDES

KRESAK LUBOR DR

MARSDEN BRIAN G DR

RICKMAN HANS DR

SHOR VIKTOR A DR

WASSERMAN LAWRENCE H DR

WEST RICHARD M DR

ZHANG JIA-XIANG

Members:

ABALAKIN VICTOR $K$ DR

A'HEARN MICHAEL F DR

BAILEY MARK EDHARD

BENEST DANIEL DR

BLOW GRAHAM L

BRANHAM RICHARD L JR

CANDY MICHAEL P MR

CHIO CHOL ZONG

DE PASCUAL MARTINEZ M DR DE SANCTIS GIOVANNI

DELSEMME ARMAND H PROF DR DIRIKIS M A DR

DONNISON JOHN RICHARD DR

DUNHAM DAVID W

DQURNEAU GERARD DR

DVORAK RUDOLF DR

EDMONDSON FRANK $K$ PROF

EVDOKIMOV YU $\checkmark D R$

FERRERI WALTER

FREITAS MOURAO R R DR

GARFINKEL BORIS DR

GICLAS HENRY L MR

HAHN GERHARD J DR

HARRIS ALAN WILLIAM OR

HE MIAO-FU

HENRARD JACQUES PROF

HURNIK HIERONIM PROF

IVANOVA VIOLETA DR

KIANG TAO PROF

KLEMOLA ARNOLD R DR

KOSAI HIROKI

KRISTENSEN LEIF KAHL

LIESKE JAY H DR

LOVAS MIKLOS

MANARA ALESSANDRO A DR

MESSAGE PHILIP J DR

MILLIS ROBERT L DR
ELLIOT JAMES L DR

FERNANDEZ JULIO A DR

FORTI GIUSEPPE DR

FROESCHLE CLAUDE DR

GEHRELS TOM PROF

GILMORE ALAN C MR

HARPER DAVID DR

HELIN ELEANOR FRANCIS

HERS JAN MR

IANNA PHILIP A

IZVEKOV V A DR

KINOSHITA HIROSHI DR

KNEZEVIC ZORAN

KOWAL CHARLES THOMAS

LAGERKVIST CLAES-INGVAR

LINDBLAD BERTIL. A DR

MACHADO LUIZ E DA SILVA

MCCROSKY RICHARD E DR

MILANI ANDREA

MINTZ BLANCO BETTY MRS
HASEGAWA ICHIRO DR
BABADZHANOV PULAT B DR BEC-BORSENBERGER ANNICK

BLANCO CARLO DR

BOWELL EDWARD L G DR

CALAME OOILE DR

CHERNYKH N S DR

CRISTESCU CORNELIA G DR

DEBEHOGNE HENRI DR SC

DOLLFUS AUDOUIN PROF

DOVAL JORGE PEREZ DR

EDELMAN COLETTE DR

ELST ERIC HALTER DR

FERRAZ-MELLO $S$ PROF DR

FRANKLIN FRED A DR

FURUKAWA KIICHIRO DR

GIBSON JAMES

GREENBERG RICHARD DR HARRINGTON ROBERT S DR HAUPT HERMANN $F$ PROF HEMENWAY PAUL D DR HEUDIER JEAN-LOUIS DR ISOBE SYUZO DR KHATISASHVILI ALFEZ SH DR KISSELEVA TAMARA $P$ KOHOUTEK LUBOS DR KOZAI YOSHIHIDE PROF

LAZZARO DANIELA DR LOMB NICHOLAS RALPH DR MAHRA H S DR MCNAUGHT ROBERT H MILET BERNARD L DR MORANDO BRUNO L DR 


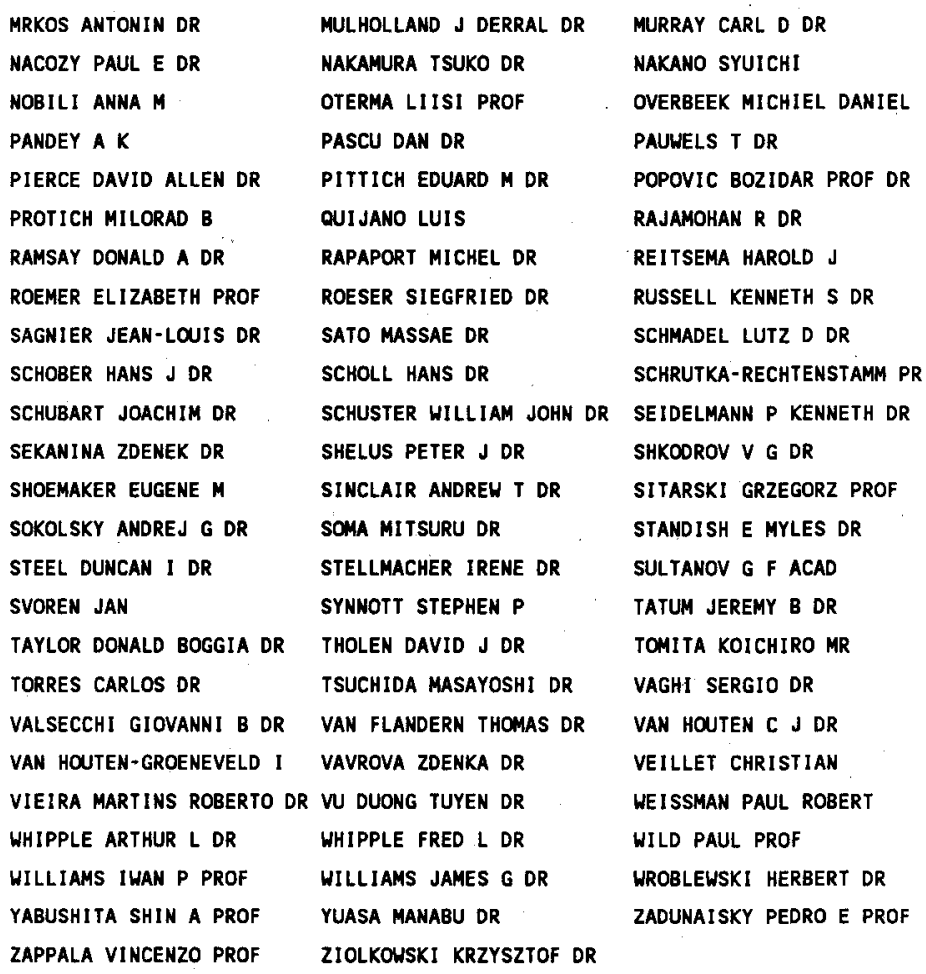


Vice-President(s) : HAUSER MICHAEL G DR

Organizing Committee: BOWYER C STUART PROF DUMONT RENE DR GALPERIN YU I PROF HONG SEUNG SOO DR HOUCK JAMES $R$ LAMY PHILIPPE DR LEINERT CHRISTOPH DR LEVASSEUR-REGOURD A C PR MUKAI TADASHI DR

\begin{tabular}{|c|c|c|}
\hline \multicolumn{3}{|l|}{ lembers: } \\
\hline ANDERSON KINSEY A PROF & ANGIONE RONALD J DR & BAGGALEY WILLIAM J PROF \\
\hline BANOS COSMAS J DR & BATES DAVID R PROF & BELKOVICH O I DR \\
\hline BLACKWELL DONALD E PROF & BLAMONT JACQUES E PROF & BROADFOOT A LYLE DR \\
\hline CHAMBERLAIN JOSEPH W PROF & CLAIREMIDI JACQUES DR & DACHS JOACHIM PROF DR \\
\hline DERMOTT STANLEY $F$ & DIVARI N B DR & DUBIN MAURICE DR \\
\hline DUFAY MAURICE PROF & DUNKELMAN LAWRENCE & ELSAESSER HANS PROF \\
\hline FECHTIG HUGO DR & FELDMAN PAUL DONALD DR & FISHKOVA LUISA M PROF \\
\hline FUJIWARA AKIRA DR & GADSDEN MICHAEL DR & GIOVANE FRANK \\
\hline GREENBERG J MAYO DR & GRUEN EBERHARD DR & GUSTAFSON BO A S \\
\hline HALLIDAY IAN DR & HARHIT MARTIN PROF & HAUG ULRICH PROF \\
\hline HENDECOURT $D^{\prime}$ LOUIS DR & HENRY RICHARD C PROF & HOFMANN WILFRJED DR \\
\hline IVANOV-KHOLOONY G S DR & JACKSON BERNARD $\vee D R$ & JAMES JOHN $F$ MR \\
\hline JARRETT ALAN H PROF & JOUBERT MARTINE & KAPLAN J DR \\
\hline KARANDIKAR R V PROF & KARYGINA ZOYA V DR & KOUTCHMY SERGE DR \\
\hline KULKARN I PRABHAKAR V PROF & LEGER ALAIN DR & LILLIE CHARLES F DR \\
\hline LOPEZ-GONZALEZ MARIA J DR & LOPEZ-MORENO JOSE JUAN & LOPEZ-PUERTAS MANUEL \\
\hline LUMME KARI A DR & MAIHARA TOSHINORI DR & MATHER JOHN CROMUELL \\
\hline MATSUMOTO TOSHIO DR & MATTILA KALEVI OR & MAUCHERAT J DR \\
\hline MCDONNELL J A M PROF & MISCONI NEBIL YOUSIF DR & MOLINA ANTONIO \\
\hline MORGAN DAVID H DR & MUINONEN KARRI DR & MUKAI SONOYO DR \\
\hline NAWAR SAMIR DR & NEUZIL LUDEK DR & NEY EDWARD P PROF \\
\hline NICOLET MARCEL PROF & NISHIMURA TETSUO DR & PARESCE FRANCESCO DR \\
\hline PERRIN JEAN MARIE DR & PFLEIDERER JORG PROF & PITZ ECKHART DR \\
\hline RADOSKI HENRY R DR & RAPAPORT MICHEL DR & RIPKEN HARTMUT $W$ DR \\
\hline ROACH FRANKLIN E & ROBLEY R DR & ROORIGO RAFAEL \\
\hline ROOSEN ROBERT G DR & ROOSEN ROBERT G DR & ROZHKOVSKIJ DIMITRIJ A \\
\hline SANCHEZ FRANCISCO PROF & SANCHEZ-SAAVEDRA M LUISA & SAXENA P P DR \\
\hline SCHWEHM GERHARD DR & SHAROV A S DR & SHEFOV NICOLAI N \\
\hline SOBERMAN ROBERT $K$ DR & SPARROW JAMES G DR & STAUDE HANS JAKOB PH D \\
\hline TANABE HIROYOSHI DR & TOLLER GARY N DR & TOROSHLIDZE TEIMURAZ I OR \\
\hline TRUTSE YU L DR & TYSON JOHN A, & VAN ALLEN JAMES A PROF \\
\hline VAN DE HULST H & VRTILEK & WALLACE LLOYD V DR \\
\hline
\end{tabular}


WALLIS MAX K DR WENIGER SCHAME DR WITT ADOLF N DR YAMAKOSHI KAZUO

ZERULL REINER H DR
WEILL GILBERT M DR WESSON PAUL S DR WOLSTENCROFT RAMON D DR YAMAMOTO TETSUO DR
WEINBERG J L DR

WILSON P DR

WOOLFSON MICHAEL M PROF

YAMASHITA KOJUN DR 

CEPLECHA ZDENEK DR HASEGAWA ICHIRO DR JONES JAMES DR KEAY COLIN S L PROF KOEBERL CHRISTIAN DR SOBERMAN ROBERT $K$ DR STEEL DUNCAN I DR TEDESCO EDHARD $F$

\begin{tabular}{|c|c|c|}
\hline \multicolumn{3}{|l|}{ embers: } \\
\hline ABBOTT WILLIAM N DR & BAGGALEY HILLIAM J PROF & BEARD DAVID B DR \\
\hline BELKOVICH O I DR & BHANDARI N DR & BIBARSOV RAVIL'SH DR \\
\hline BLACKWELL ALAN TREVOR & BROWNLEE DONALD E PROF & CARUSI ANDREA \\
\hline CEVOLANI GIORDANO & CLIFTON KENNETH ST & CLUBE S V M DR \\
\hline DJORGOVSKI STANISLAV DR & DUBIN MAURICE DR & ELFORD WILLIAM GRAHAM DR \\
\hline FECHTIG HUGO DR & FORTI GIUSEPPE DR & GLASS BILLY PRICE DR \\
\hline GOSWAMI JN DR & GRUEN EBERHARD DR & GUSTAFSON BO A S \\
\hline HAJDUK ANTON DR & HAJDUKOVA MARIA & HALLIDAY IAN DR \\
\hline HANNER MARTHA S DR & HARVEY GALE A DR & HAWKES ROBERT LEWIS DR \\
\hline HAWKINS GERALD S DR & HELIN ELEANOR FRANCIS & HEY JAMES STANLEY DR \\
\hline HODGE PAUL W PROF & HONG SEUNG SOO DR & HUGHES DAVID W DR \\
\hline JACCHIA LUIGI G DR & JENHISON ROGER C PROF & KAISER THOMAS R PROF \\
\hline KAPISINSKY IGOR & KASHSCHEEV B L PROF DR & KOSTYLEV K V DR \\
\hline KRAMER KH N DR & KRESAK LUBOR DR & KRESAKOVA MARGITA DR \\
\hline KRUCHINENKO VITALIY G & KVIZ ZDENEX DR & LAMY PHILIPPE DR \\
\hline LEBEDINETS VLADIMIR N DR & LEVASSEUR-REGOURD A C PR & LINDBLAD BERTIL A DR \\
\hline LOVELL SIR BERNARD PROF & MARVIN URSULA B DR & MASON JOHN WILLIAM DR \\
\hline MCCROSKY RICHARD E DR & MCDONNELL J A M PROF & MCINTOSH BRUCE A DR \\
\hline MEISEL DAVID D OR & MILES HOWARD G MR & MISCONI NEBIL YOUSIF DR \\
\hline MURRAY C ANDREW & MAKAZAWA KIYOSHI DR & NAPIER WILLIAM M DR \\
\hline NEWBURN RAY L JR & NUTH JOSEPH A III & O'KEEFE JOHN A DR \\
\hline PADEVET VLADIMIR DR & PECINA PETR & PILLINGER COLIN DR \\
\hline PLAVEC ZDENKA DR & POLNITZKY GERHARD DR & PORUBCAN VLADIMIR DR \\
\hline QUESADA VINICIO DR & RAJCHL JAROSLAV DR & REVELLE DOUGLAS ORSON DR \\
\hline RIPKEN HARTMUT $W$ DR & ROOSEN ROBERT G DR & RUSSELL JOHN A PROF \\
\hline SEKANINA ZDENEK DR & SHAO CHENG-YUAAN & SHESTAKA IVAN S DR \\
\hline SIMEK MILOS OR & SVESTKA JIRI DR & TERENT JEVA ALEXANDRA $K$ DR \\
\hline TOMITA KOICHIRO MR & VERNIANI FRANCO PROF & WANG DE-CHANG \\
\hline WEINBERG J L DR & WETHERILL GEORGE W & WHIPPLE FRED L DR \\
\hline WOOD JOHN A DR & WOOLFSON MICHAEL M PROF & XU PINXIN \\
\hline YAMAKOSHI KAZUO & YAVNEL ALEXANDER A DR & YEOMANS DONALD $K$ DR \\
\hline VOLANKOVA JUDI & & \\
\hline
\end{tabular}


COMPOSITION OF COMMISSION $24 \quad 1991-1994$

\author{
President : DE VEGT CH PROF DR \\ Vice-President(s) : TURON C DR
}

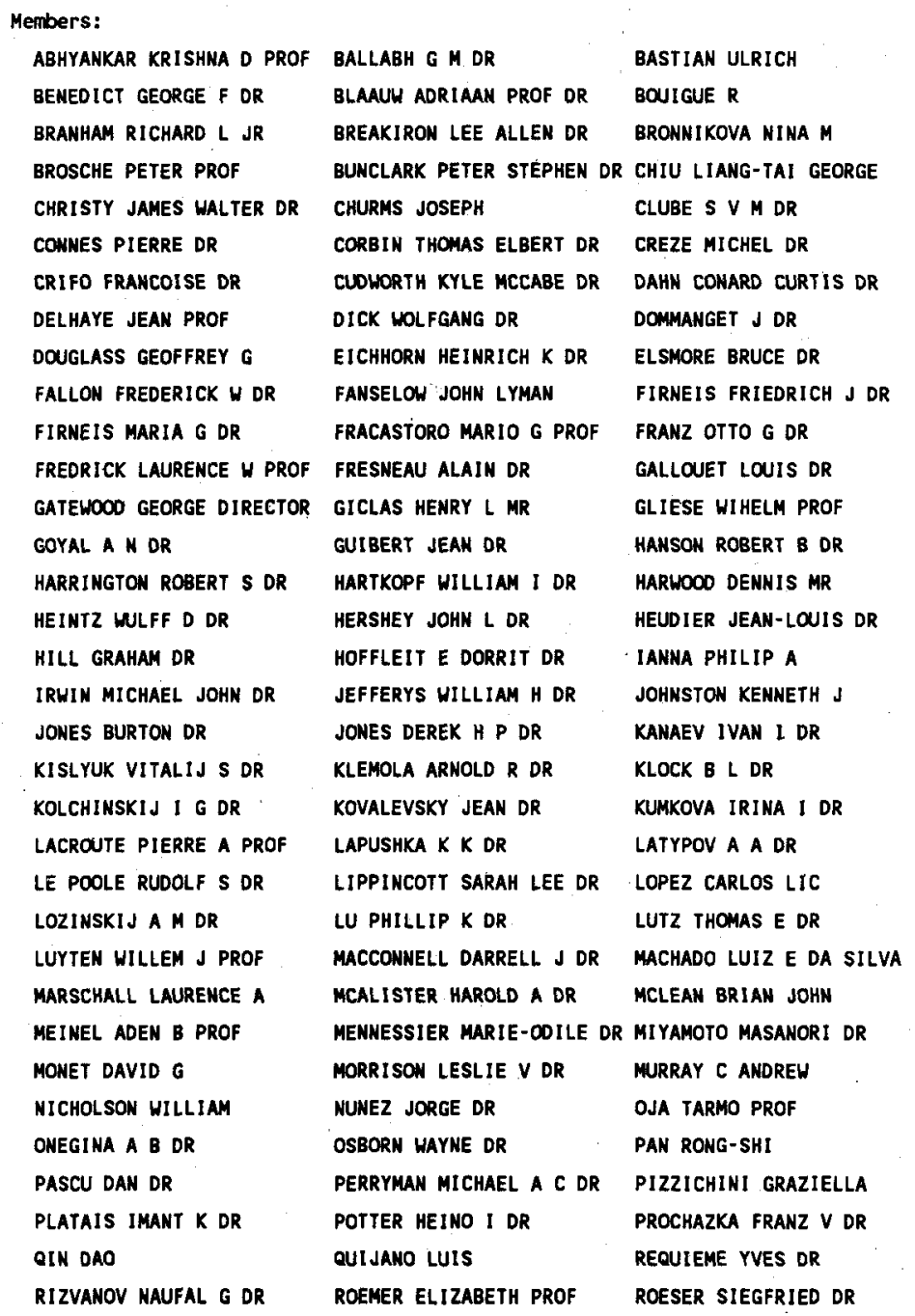


M44

$\begin{array}{lll}\text { RUDER HANNS } & \text { RUSSELL JANE L DR } & \text { SANDERS H L PROF } \\ \text { SCHILBACH ELENA DR } & \text { SHELUS PETER J DR } & \text { SHI GUANG-CHEN } \\ \text { SIMS KENNETH P DR } & \text { SMITH CLAYTON A JR DR } & \text { STEIN. JOHN WILLIAM } \\ \text { STEINERT KLAUS GUENTER DR STONE ROHALD CECIL } & \text { STRAND KAJ AA DR } \\ \text { THOMAS DAVID V DR } & \text { UPGREN ARTHUR R DR } & \text { VALBOUSQUET ARMAND DR } \\ \text { VAN DE KAMP PETER } & \text { VILKKI ERKKI U } & \text { WALTER HANS G DR } \\ \text { WAN LAI } & \text { WASSERMAN LAHRENCE H DR } & \text { WESSELINK ADRIAAN J OR } \\ \text { WESTERHOUT GART OR } & \text { WHITE GRAEME LINDSAY DR } & \text { WILLIAMS CAROL A } \\ \text { WORLEY CHARLES E DR } & \text { WROBLEWSKI HERBERT DR } & \text { YANG TING-GAO } \\ \text { YOUNIS SAAD M } & \text { ZACHARIAS NORBERT DR }\end{array}$


COMPOSITION OF COMMISSION $25 \quad$ 1991-1994

\begin{abstract}
President : YOUNG ANDREW T DR
Vice-President(s) : LANDSTREET JOHN D PROF
\end{abstract}

Organizing Committee: ADELMAN SAUL J DR

BREGER MICHEL PROF DR

GENET R M DR

KNUDE JENS KIRKESKOV DR

LANDOLT ARLO U PROF

LUB JAN DR

MCLEAN IAN S DR

MENZIES JOHN W DR

MOFFETT THOMAS J PROF

STERKEN CHRISTIAAN LEO DR

STRAIZYS $V$ PROF DR

VRBA FREDERICK J DR

Members:

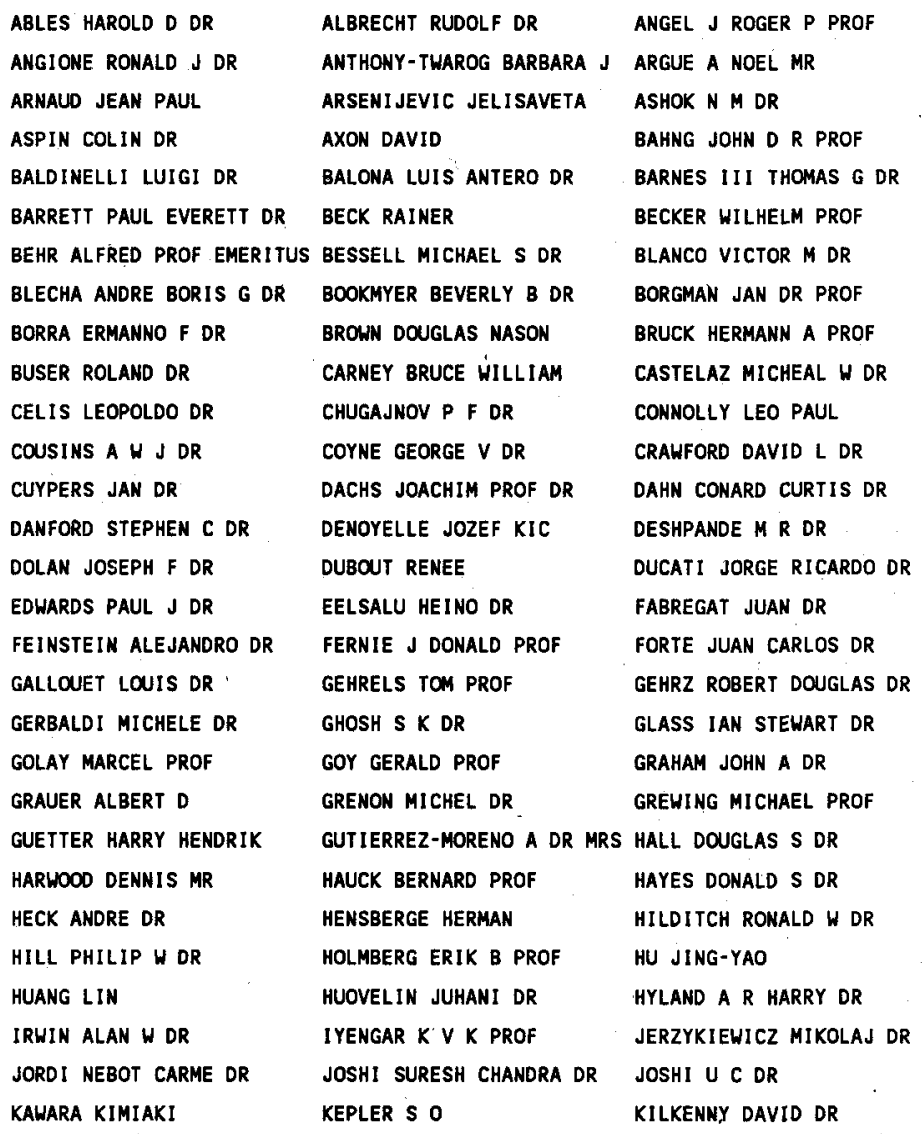




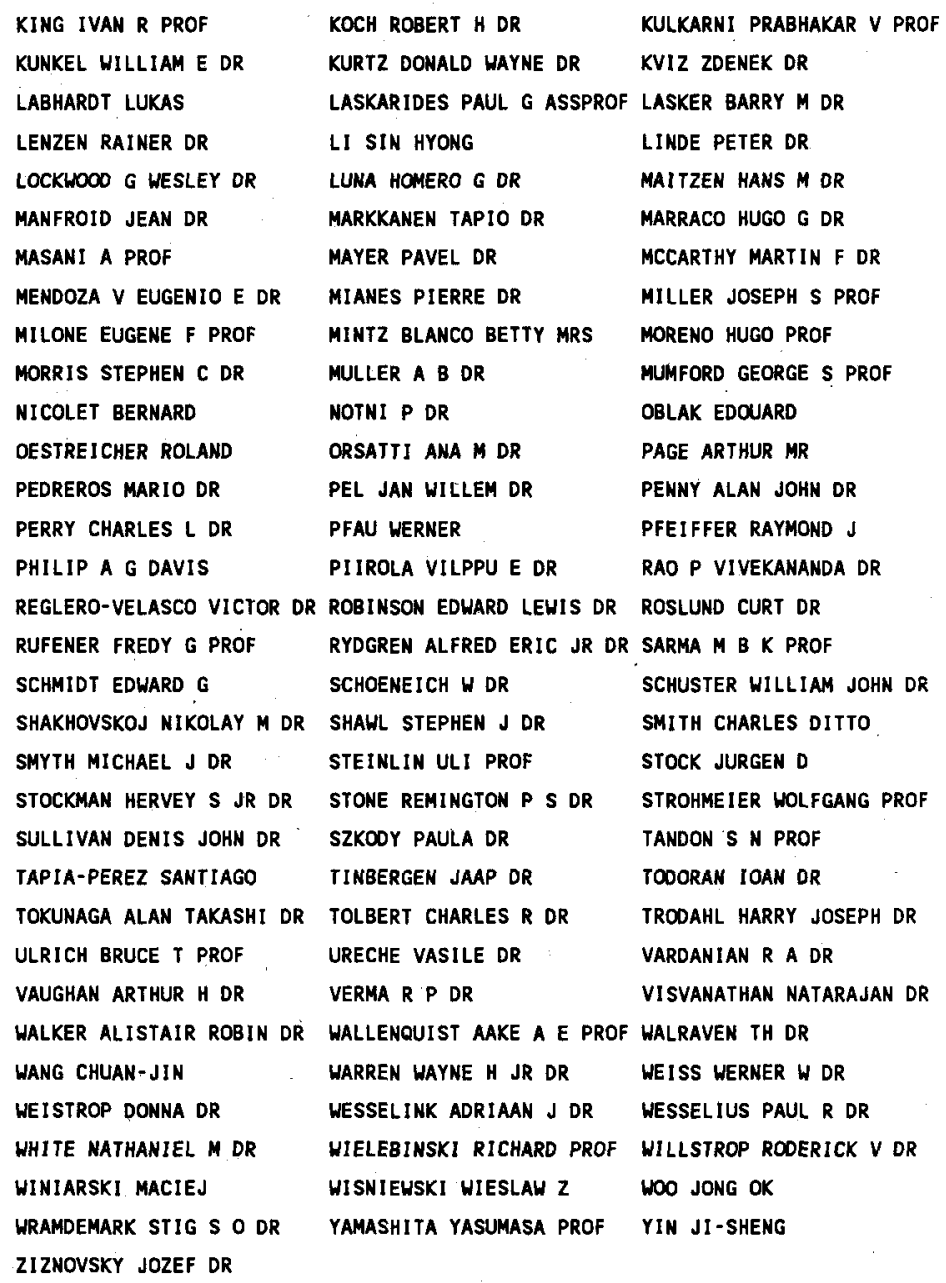


COMPOSITION OF COMMISSION 26 1991-1994

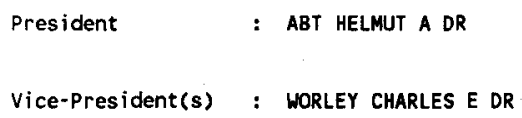

\begin{tabular}{|c|c|c|}
\hline lembers: & & \\
\hline ALLEN CHRISTINE & QGUE A NOEL MR & ARMSTRONG JOHN THOMAS DR \\
\hline BACCHUS PIERRE PROF & BAGNUOLO WILLIAM G JR DR & BAIZE PAULL DR \\
\hline BATTEN ALAN H DR & EAVERS WILLET I DR & BONNEAU DANIEL \\
\hline BROSCHE PETER PROF & CABRITA EZEQUIEL DR & CAMPBELL ALISON DR \\
\hline CESTER BRUNO PROF & CHEN ZHEN & COUTEAU PAUL PROF \\
\hline CULVER ROGER BRUCE DR & DADAEV ALEKSANDR N DR & DOCOBO DURANTEZ JOSE A \\
\hline DOMMANGET J DR & DOUGLASS GEOFFREY G & DUNHAM DAVIO $W$ \\
\hline EICHHORN HEINRICH K DR & FERRER OSVALDO EDUARDO DR & FLETCHER J MURRAY \\
\hline FRACASTORO MARIO G PROF & FRANZ OTTO G DR & FREDRICK LAURENCE W PROF \\
\hline FREITAS MOURAO R R DR & FURENLID INGEMAR K DR & GATEWOOD GEORGE DIRECTOR \\
\hline GAUDENZI SILVIA DR & GEYER EDWARD H PROF DR & GLIESE WIHELM PROF \\
\hline HALBWACHS JEAH LOUIS DR & HARRINGTON ROBERT S DR & HARTKOPF WILLIAM I DR \\
\hline HERSHEY JOHN L DR & HIDAJAT BAMBANG PROF DR & HILL GRAHAM DR \\
\hline HOLDEN FRANK & IANMA PHILIP A & ISHIDA GORO DR \\
\hline JASCHEK CARLOS O R PROF & KISELYYOV ALEXEJ A DR & KOPAL ZDENEK PROF \\
\hline KUMSISHVILI J I OR & LAMPENS PATRICIA DR & LATHAM DAVID W DR \\
\hline LATTANZI MARIO G & LEINERT CHRISTOPH DR & LING J DR \\
\hline LIPPINCOTI SARAH LEE DR & LODEN KERSTIN R DR & LOOEN LARS OLOF PROF \\
\hline LUYTEN WILLEN J PROF & MEYER CLAUDE DR & MIKKOLLA SEPPO DR \\
\hline MOHAN CHANDER DR & MORBEY CHRISTOPHER L & MORBIDELLI ROBERTO DR \\
\hline MOREL PIERRE JACQUES DR & MULLER 'PAUL & OBLAK EDOUARD \\
\hline OSWALT TERRY D DR & PANNUNZIO RENATO & PETERSON DEANE M DR \\
\hline POPOVIC GEORGIJE DR & POVEDA ARCADIO DR & RAKOS KARL D PROF \\
\hline RUSSELL JANE L DR & SALUKVADZE G N DR & SCARDIA MARCO \\
\hline SCHMIDTKE PAUL C DR & SHUL'BERG A M DR & SINACHOPOULOS D DR \\
\hline SMAK JOSEPH I PROF & SOWELL JAMES ROBERT DR & STEIN JOHN WILLIAM \\
\hline STRAND KAJ AA DR & SZABADOS LASZLOO PH D & TOKOVININ ANDREJ A DR \\
\hline TRIMBLE VIRGINIA L DR & TSAY WEAN-SHUN DR & UPGREN ARTHUR R DR \\
\hline VALBOUSOUET ARMAND DR & VALTONEN MAURI J PROF & VAN ALTENA WILLIAM F PROF \\
\hline VAN DE KAMP PETER & VAN DER HUCHT KAREL A DR & WALKER RICHARD L \\
\hline WEIS EDWARD W DR & WIETH-KNUDSEN NIELS P DR & YAN LIN-SHAN \\
\hline
\end{tabular}




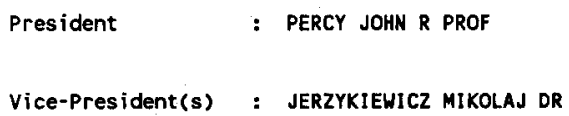

AIZENMAN MORRIS L DR

ALPAR ALI DR

ANTONELLO ELIO

ARSENI JEVIC JELISAVETA

BAADE DIETRICH DR BALONA LUIS ANTERO DR BASTIEN PIERRE DR BAUER WENDY HAGEN BELSERENE EMILIA P BERTHOMIEU GABRIELLE DR BOCHONKO D RICHARD DR BOPP BERNARD W DR BOYARCHUK A A DR BURKI GILBERT PROF BUTLER DENNIS DR CATCHPOLE ROBIN M DR CHRISTY ROBERT F DR CONNOLLY LEO PAUL COUTTS-CLEMENT CHRISTINE CUYPERS JAN DR DELGADO ANTONIO JESUS DICKENS ROBERT $J$ DR DUNLOP STORM EDWARDS PAUL J DR ESKIOGLU A NIHAT EVREN SERDAR DR FEIBELMAN WALTER A DR FITCH WALTER S DR GAHM GOESTA $F$ DR GASCOIGNE S C B DR
ALBINSON JAMES DR ANDO HIROYASU DR ARELLANO FERRO ARMANDO ASTERIADIS GEORGIOS DR BAGLIN ANNIE DR BARTOLINI CORRADO BATESON FRANK M OBE DR BEDOGNI ROBERTO BELVEDERE GAETANO DR BESSELL MICHAEL S DR BOLTON C THOMAS PROF BOULON JACQUES J DR BOYARCHUK MARGARITA E DR BUSKO IVO C DR BYRNE PATRICK - B DR CHAVIRA ENRIQUE SR CHUGAJNOV P F DR CONTADAKIS MICHAEL E DR COX ARTHUR N DR DANFORD STEPHEN C DR DEMERS SERGE DR DJORGOVSKI STAHISLAV DR DUPUY DAVID L DR EFREMOV YURY N DR EVANS ANEURIN FADEYEV YURI A FERLAND GARY JOSEPH FRIEDJUNG MICHAEL DR GALLAGHER III JOHN S DR GENET R M DR
ALFARO EMILIO JAVIER ANTIPOVA LYUDMILA DR ARKHIPOVA V P DR AVGOLOUPIS STAVROS DR BAKER NORMAN H PROF BARUIG HEINZ BATH GEOFFREY T DR BELMONTE AVILES J A DR BENSON PRISCILLA J OR BIANCHINI ANTONIO DR BOND HOWARD E DR BONEN GEORGE H DR BRONN DOUGLAS NASON BUTLER C JOHN DR CAMERON ANOREW COLLIER DR CHEREPASHCHUK A M PROF COHEN MARTIN DR COULSON IAIN M DR CUTISPOTO GIUSEPPE DR DE GROOT MART DR DEUPREE ROBERT G DR DOWNES RONALD A DR DZIEMBOWSKI WOJCIECH PROF EL-BASSUNY ALAWY A A DR EVANS NANCY REMAGE DR FEAST MICHAEL W PROF FERNIE J DONALD PROF FROLOV M S DR GARRIDO RAFAEL GEYER EDWARD H PROF DR 
GIEREN WOLFGANG P DR GOOOLI GIOVANNI PROF GOUGH DOUGLAS O OR GRASDALEN GARY L DR GUERRERO GIANANTONIO DR GURSKY HERBERT DR HAISCH BERNHARD MICHAEL HANSEN CARL J PROF HERBIG GEORGE H DR HESSER JAMES E DR HOFFLEIT E DORRIT DR HUENEMOERDER DAVID P DR IIJIMA TAKASHI DR JEFFERY CHRISTOPHER S DR JONES ALBERT F MR KAROVSKA MARGARITA DR KIM CHULLEEE DR KIPPENHAHN RUDOLF PROF KOLLATH ZOLTAN DR KRAUTTER JOACHIM DR KRZEMINSKI WOJCIECH DR KUMSISHVILI J I DR LAGO MARIA TERESA $V T$ PR LASKARIDES PAUL $G$ ASSPROF LEUNG KAM CHING PROF LOCKWOOD G WESLEY DR LUB JAN DR MAFFEI PAOLO PROF MAKARENKO EKATERINA N DR MARGRAVE THOMAS EWING JR MATTHEUS JAYMIE MCGRAW JOHN T DR METZ KLAUS DR MIRZOYAN L. V DR PROF MORRISON NANCY DUNLAP DR NATHER R EOWARD NICOLOV NIKOLAI S DR OOGERS GRAHAM J DR OSWALT TERRY D DR PAPARO MARGIT OR PARTHASARATHY M OR PETERSEN J O DR PIIROLA VILPPU E DR PRINGLE JAMES E DR PUGACH ALEXANDER F DR RENSON P F M DR RODGERS ALEX $W$ DR ROMANOV YURI S DR RUSSEV RUSCHO DR SAMYAL ASHIT DR SATO NAONOBU PROF SCHLEGEL ERIC MATTHEW DR SCHOEMBS ROLF DR SCUFLAIRE RICHARD DR SHARMA DHARMA PAL DR
GIES DOUGLAS R DR GOETZ WOLDEMAR DR GOUPIL MARIE JOSE GRIMIN VLADIMIR P DR GUINAN EDWARD FRANCIS DR HACKWELL JOHN A DR HALL DOUGLAS S DR HARMANEC PETR DR HERR RICHARD B DR HILL HENRY ALLEN DR HOUK NANCY DR HUTCHINGS JOHN B DR JABLONSKI FRANCISCO DR JEWELL PHILIP R OR KADOURI TALIB HADI KARP ALAN HERSH DR KIM TU HWAN KJURKCHIEVA DIANA DR KOPYLOV I M DR KREIMER JERZY MAREK DR KUBIAK MARCIN A DR KUNKEL WILLIAM E DR LANDOLT ARLO U PROF LAZARO CARLOS OR LITTLE-MARENIN IRENE R DR LIU ZONGLI LONGMORE ANDREW J MADORE BARRY FRANCIS DR MAHMOND FAROUK $M$ A B DR MANNINO GIUSEPPE PROF MARTIN WILLIAM L DR MAVRIDIS L N PROF MCNAMARA DELBERT H DR MILONE EUGENE F PROF MOFFETT THOMAS J PROF MUMFORD GEORGE $S$ PROF NEFF JOHN S NIKOLOV ANDREJ DR OLAH KATALIN DR O'DONOGHUE DARRAGH DR PAPOUSEK JIRI PATERNO LUCIO PROF PETROV PETER P DR POP VASILE DR PROVOST JANIME DR RAKOS KARL D PROF RICHTER G A DR ROORIGUEZ ELOY DR ROSINO LEONIDA PROF SADIK AZIZ R DR SAREYAN JEAN-PIERRE DR SAWYER-HOGG HELEN B DR SCHMIDT EDHARD G SCHWARTZ PHILIP R DR SEEDS MICHAEL AUGUST DR SHERWOOD WILLIAM A DR KEPLER S O
GLAGOLEVSKIJ JU V DR GORBATSKY VITALIJ G PROF GRAHAM JOHN A DR GRYGAR JIRI DR GURM HARDEV S PROF HAEFNER REINHOLD DR HAMDY M A M PROF HEISER ARNOLD M DR HERS JAN MR HILL PHILIP $W$ DR HOWELL STEVE BRUCE DR IBEN ICKO JR PROF JARZEBOWSKI TADEUSZ DR JIANG SHI - YANG KANYO SAMDOR DR KIPLINGER ALAN L DR KOEN MARTHINUS DR KRAFT ROBERT P PROF KRISCIUNAS KEVIN DR KUHI LEONARD $V$ PROF KWEE K K DR LANEY CLIFTON D DR LEITE SCHEID PAULO DR LOPEZ DE COCA M D P DR MAEDER ANDRE PROF MAHRA H S DR MANTEGAZZA LUCIANO MASANI A PROF MAYALL MARGARET W. MENNESSIER MARIE-ODILE DR MILONE LUIS A DR MOHAN CHANDER DR MURDIN PAUL G DR NIARCHOS PANAYIOTIS PH D NUGIS TIIT OPOLSKI ANTONI PROF PAPALOIZOU JOHN C B DR PARSAMYAN ELMA S DR PAVLOVSKI KRESIMIR PETTERSEN BJOERN RAGNVALD POPOVA MALINA D PROF DR PSKOVSKIJ JU P DR RAO N KAMESWARA ROBINSON EDWARD LEWIS DR ROMANO GIULIANO PROF ROUNTREE JANET DR SAMDMANN WILLIAM HENRY SARMA M B $K$ PROF SCHAEFER BRADLEY E DR SCHNEIDER HARTMUT DR SCHWARZENBERG-CZERNY A SHARA MICHAEL DR SHOBBROOK ROBERT R DR 
SINVHAL SHAMBHU DAYAL DR SOL IMAN MOHAMED AHMED DR STELLINGWERF ROBERT F DR STOBIE ROBERT S DR STROM KAREN M SZATMARY KAROLY DR TAMMANN G ANDREAS THOMPSON KEITH DR TREMKO JOZEF DR TURNER DAVID G DR USHER PETER D DR VAN GENDEREN A M DR VOGT NIKOLAUS DR WALKER MERLE F PROF WALRAVEN TH DR WEBBINK RONALD F DR WEISS WERNER W DR WESSELINK ADRIAAN J OR WILLSON LEE ANNE DR WISHIEWSKI WIESLAH $Z$ XIONG DA-RUN ZUCKERMAN BEN M DR
SMAK JOSEPH. I PROF SRIVASTAVA RAM KUMAR DR STEPIEN KAZIMIERZ DR STRASSMEIER KLAUS G DR STROM STEPHEN E SZECSENYI-NAGY GABOR DR DR TEMPESTI PIERO PROF TJIN-A-DJIE HERMAN R E DR TORRES CARLOS ALBERTO DR TSIOUMIS ALEXANDROS DR TUTUKOV A V DR VALTIER JEAN-CLAUDE DR VERHEEST FRANK PROF WAELKENS CHRISTOFFL WALKER WILLIAM S G WARNER BRIAN PROF WEHLAU AMELIA DR HELCH DOUGLAS L DR WHITELOCK PATRICIA ANN OR WILLJAMON RICHARD M WILSON LIONEL DR WOOD PETER R DR YAO BAO-AN
SMEYERS PAUL PROF STARRFIELD SUMMER PROF STERKEN CHRISTIIAAN LEO DR STROHMEIER WOLFGANG PROF SZABADOS LASZLO PH D SZKOOY PAULA DR TERZAN AGOP DR TSVETKOV MILCHO $K$ DR TYLEMDA ROMUALD DR VAN AGT S L TH J DR VIOTTI ROBERTO DR WALKER EDWARD N MR WALLERSTEIN GEORGE PROF WATSON ROBERT DR WEHLAU WILLIAM H PROF WENZEL H DR HING ROBERT F PROF WRIGHT FRANCES $W$ DR ZSOLDOS ENDRE DR 
COMPOSITION OF COMMISSION $28 \quad 1991-1994$

$\begin{array}{ll}\text { President } & \text { : KHACHIKIAN E YE PROF } \\ \text { Vice-President(s) } & \text { : TRIMBLE VIRGINIA L DR }\end{array}$

Organizing Comittee: BERTOLA FRANCESCO PROF BRUZUAL GUSTAVO

CHEN JIAN-SHENG

ELLIS RICHARD S

FREEMAN KENNETH C PROF

GALLAGHER III JOHN S DR

ISRAEL FRANK P DR

OKAMURA SADANORI DR

TAMMANN $G$ ANDREAS PROF DR

ULRICH MARIE-HELENE D DR

Members:

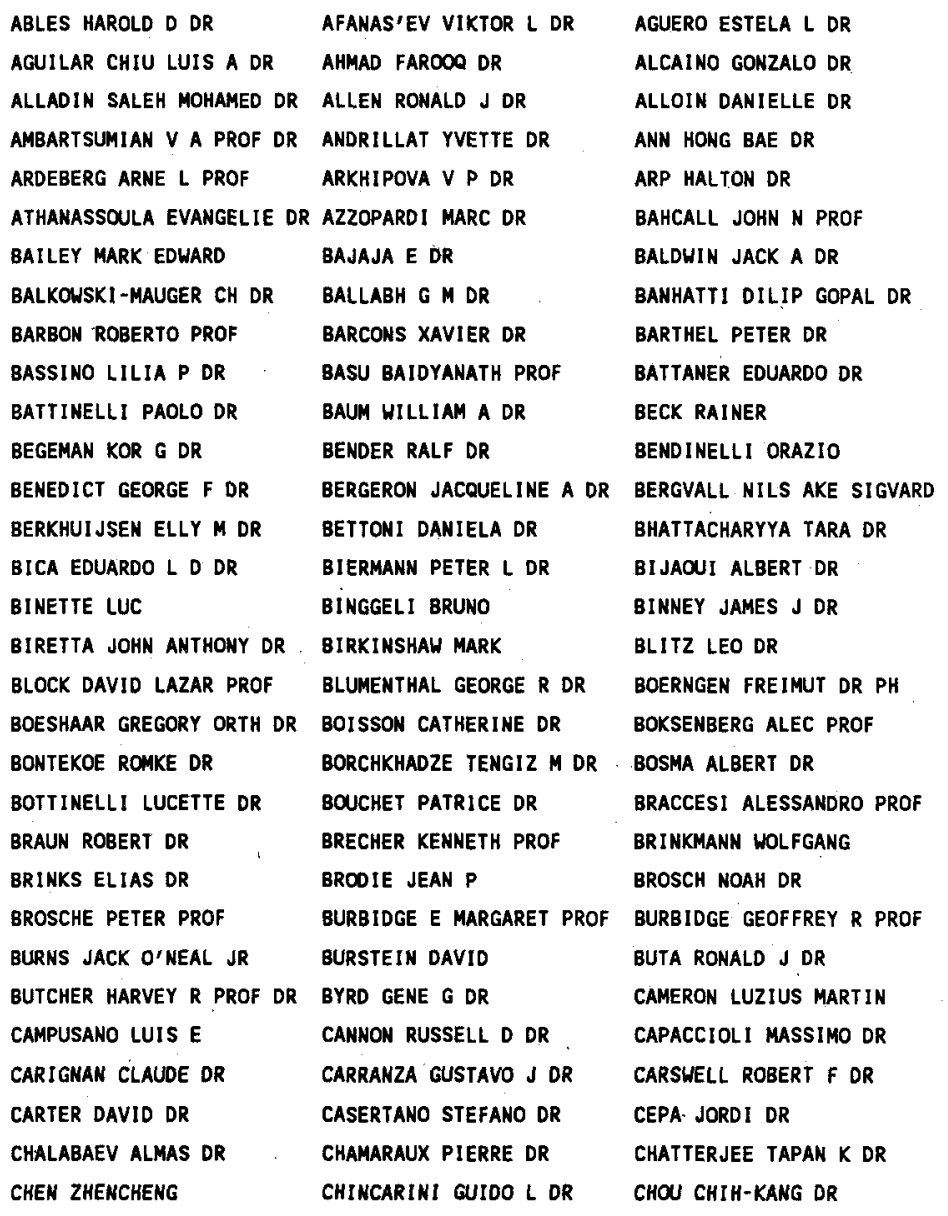




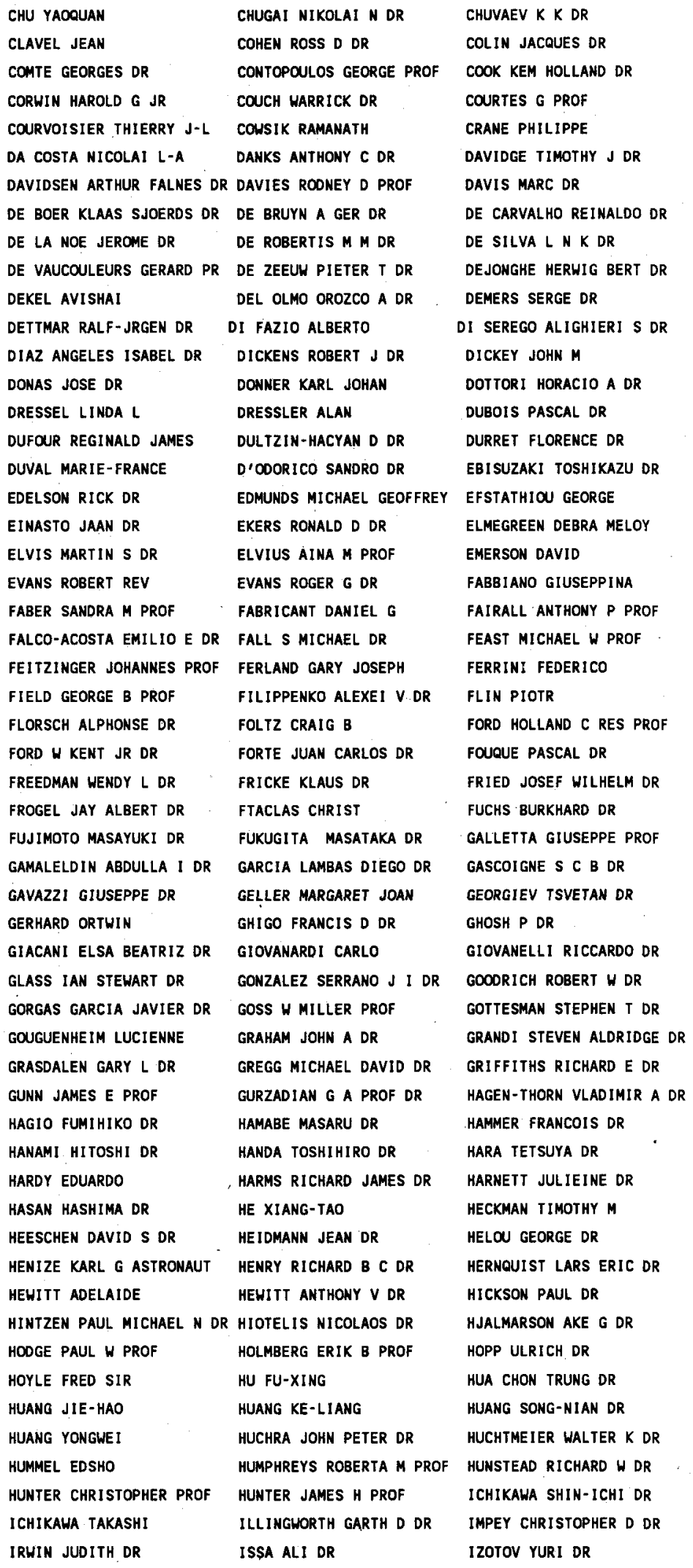


JAFFE WALTER JOSEPH DR JOSHI MOHAN N PROF JUGAKU JUN DR KALINKOV MARIN $P$ DR KANEKO NOBORU DR KAROJI HIROSHI DR KEEL WILLIAM C KERR FRANK J DR KIRSHMER ROBERT PAUL DR KOCHHAR R K OR KOJOIAN GABRIEL DR KOO DAVID C-Y DR KRISHNA GOPAL KUNCHEV PETER DR LAFON JEAN-PIERRE J DR LAUBERTS ANDRIS DR LAYZER DAVID PROF LELIEVRE GERARD DR LI QI-BIN

LILLY SIMON J DR LIPOVETSKY $\vee$ A LO KWOK-YUNG DR LOPEZ ROSARIO DR LORTET MARIE CLAIRE LUGGER PHYLLIS M LYNOS BEVERLY T DR MACALPINE GORDON M MARCELIN MICHEL MARTINET LOUIS PROF MAURICE ERIC N MCCREA WILLIAM SIR MEIKLE WILLIAM P $S$ MILET BERNARD $\perp$ DR MILLER JOSEPH S PROF MINEVA VENETA DR MOORHOOD ALAN F M MOSS CHRISTOPHER DR MURRAY STEPHEN S DR NARLIKAR JAYANT $V$ PROF NOGUCHI MASAFUMI OR NORMAN COLIN A PROF OKE J BEVERLEY PROF OSMAN ANAS MOHAMED PROF O'CONNELL ROBERT WEST DR PAGE THORNTON L DR

PAN RONG-SHI PASTORIZA MIRIANI G DR PENG QIU-HE PEREZ FOURNON ISMAEL DR PETERSON CHARLES JOHN DR PFENNIGER DANIEL DR PISMIS DE RECILLAS PARIS PRABHU TUSHAR $P$ PREVOT-BURNICHON M L DR PRONIK I I OR
JOG CHANDA J OR

JOSHI U C DR

JUNKKARINEN VESA T DR

KALLOGLIAN ARSEN T DR

KAPAHI VIJAY $K$ DR

KATGERT PETER DR

KELLERMANH KENNETH I DR

KING IVAN R PROF

KLEIN ULRICH

KODAIRA KEIICHI PROF

KOLLATSCHNY WOLFRAM DR

KORMENDY JOHN DR

KRON RICHARD G

KUNTH DANIEL

LARSON RICHARD B PROF

LAUSBERG ANDRE DR

LE FEVRE OLIVIER DR

LEQUEUX JAMES DR

LI XIAO-OING

LIN CHIA C PROF

LIU RU-LIANG

LONSDALE CAROL J DR

LORD STEVEN DONALD DR

LOW FRANK J DR

LUMINET JEAN-PIERRE

LYNOS ROGER $C$ OR

MACCHETTO FERDINANDO DR MARQUES DOS SANTOS P PROF MARTIN MARIA CRISTINA DR MASEGOSA GALLEGO J DR MAVRIDES STAMATIA DR MEDIAVILLA EVENCIO DR MEISENHEIMER KLAUS DR MILEY G $K$ DR MILLER RICHARD H DR MIRABEL IGOR FELIX DR MORENO EDMUNDO DR MOULD JEREMY $R$ MUZZIO JUAN C PROF NAVARRO JULIO FERNANDO DR NITYANANDA R DR NOONAN THOMAS W PROF NULSEN PAUL DR OLEAK H DR OSTERBROCK DONALD E PROF O'DEA CHRISTOPHER P DR PALMER PHILIP DR PAPAYANMOPOULOS TH DR PATUREL GEORGES PEREA-DUARTE JAIME D DR PETERS WILLIAM L III DR PETROSIAN ARTASHES R DR PHILLIPS MARK $M$ OR POPOV VASIL NIKOLOV PRENDERGAST KEVIN H PROF PRIEUR JEAN-LOUIS DR PRONIK V I DR
JONES THOMAS WALTER DR JOY MARSHALL J DR KALAFI MANOUCHER KANDRUP HENRY EMIL DR KARACHENTSEV I D DR KAUFMAN MICHELE DR KENNICUTT ROBERT C JR KINMAN THOMAS D DR KNAPP GILLIAN R DR KOGOSHVILI NATELA G KONTIZAS EVANGELOS DR KRAAN-KORTEWEG RENEE C DR KRUMM NATHAN ALLYN KUSTAANHEIMO PAUL E PROF LASKER BARRY M DR LAURENCE ANDREW DR LEACOCK ROBERT JAY LI JING LILLER WILLIAM DR LINDBLAD PER OLOF PROF LIU YONG-ZHEN LOOSE HANS-HERMANN DR LORENZ HILMAR LUCEY JOHN DR LYNDEN-BELL DONALD PROF MA ER MATHEWSON DONALO S PROF MCBREEN BRIAN PHILIP DR MEIER DAVID L MENON T K PROF MILLER HUGH R PROF MOLES MARIANO J DR MORGAN WILLIAM W PROF MUNOZ-TUNON CASIANA NAKAI NAOMASA DR NOREAU LOUIS DR - oemLer augustus JR DR CORT JAN H PROF OWEN FRAZER NELSON DR PACHOLCZYK ANDRZEJ G PROF. PALUMBO GIORGIO G C DR PARKER QUENTIN DR PEIMBERT MANUEL DR PEREZ ENRIQUE DR PETERSON BRADLEY MICHAEL PETROV GEORGY TRENDAFILOV PICKLES ANDREW JOHN DR POVEDA ARCADIO DR PRESS WILLIAM H DR PRITCHET CHRISTOPHER J DR PROUST DOMINIQUE MILLS BERNARD Y PROF 


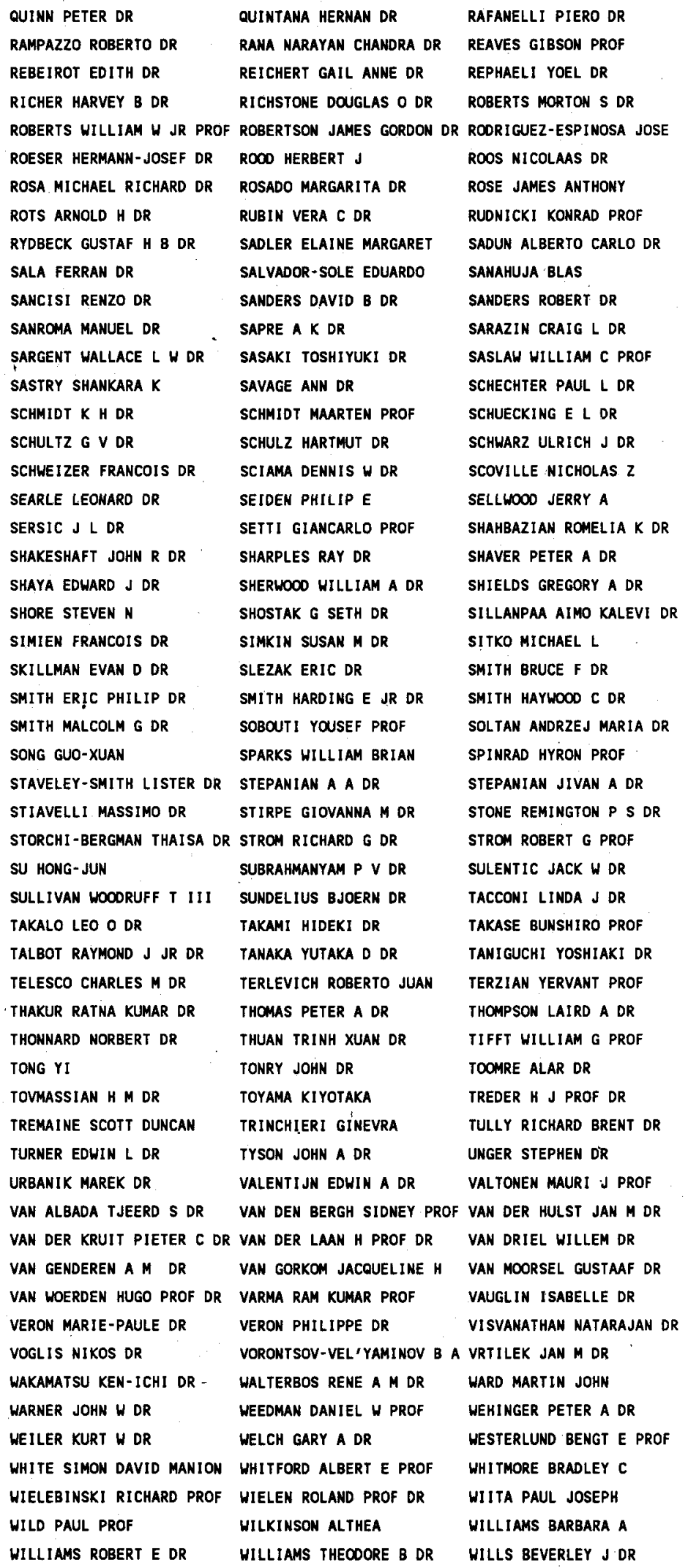


WILLS DEREK DR WLERICK GERARD DR WROBEL JOAN MARIE DR YAMAGATA TOMOHIKO DR ZASOV ANATOLE V DR ZHOU YOU-YUAN
WILSON ALBERT G DR WOOSLEY S E PROF. WYNN-WILLIAMS C G DR YOUNG JUDITH SHARN ZAVATTI FRANCO ZINN ROBERT J DR
WINOHORST ROGIER A DR WORRALL DIANA MARY XIA XIAOYANG DR ZAMORANO JAIME DR ZEILINGER WERNER W DR 
$\begin{array}{ll}\text { President } & \text { : LAMBERT DAVID L.PROF } \\ \text { Vice-President(s) } & : \text { BESSELL MICHAEL S DR }\end{array}$

Organizing committee: BAADE DIETRICH DR BARBUY BEATRIZ DR BOESGAARD ANN M PROF CASSATELLA ANGELO DR CONTI PETER S DR GERBALDI MICHELE DR GUSTAFSSON BENGT DR NUGIS TIIT PILACHOWSKI CATHERINE DR SADAKANE KOZO DR

Members:

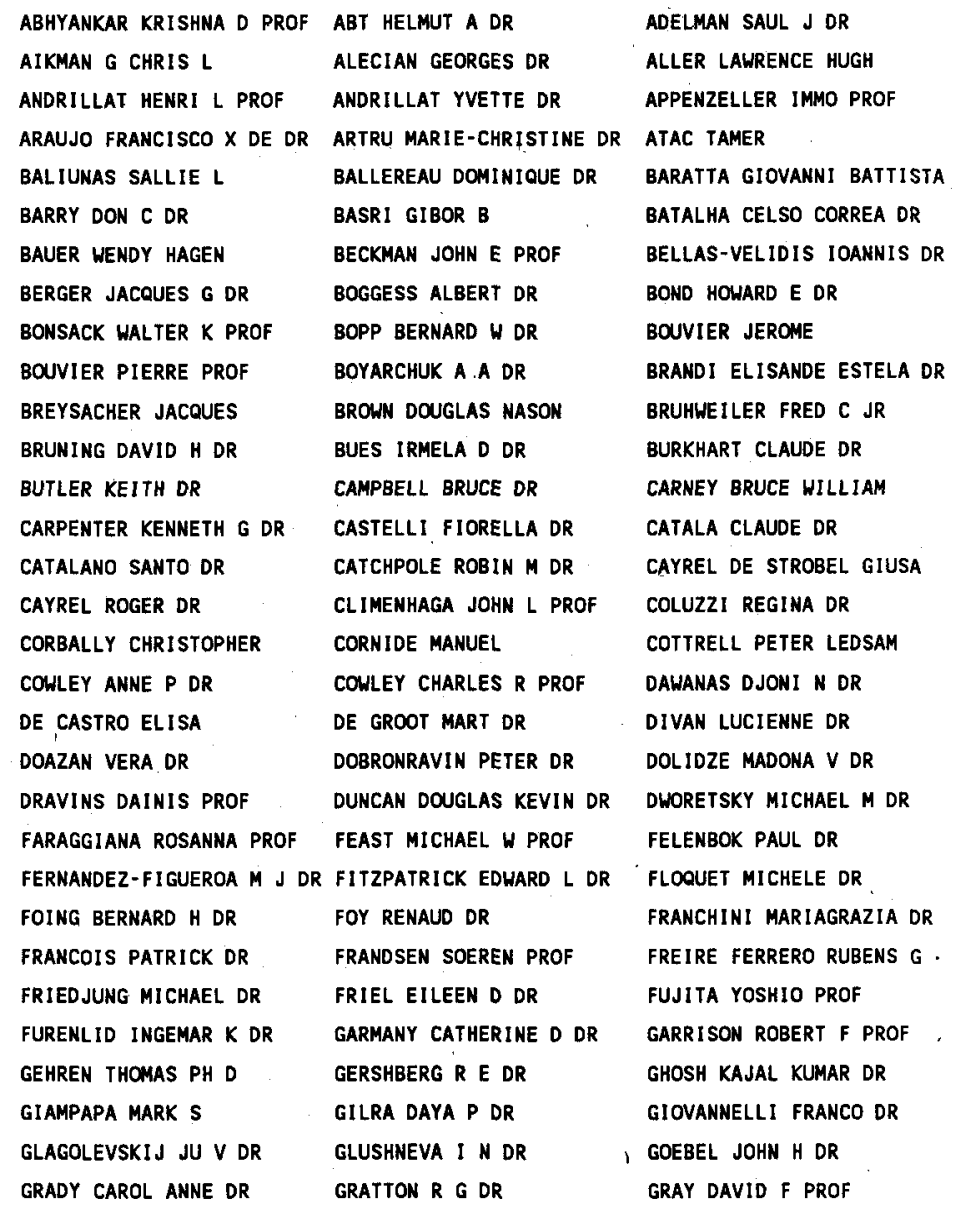




\begin{tabular}{|c|c|c|}
\hline 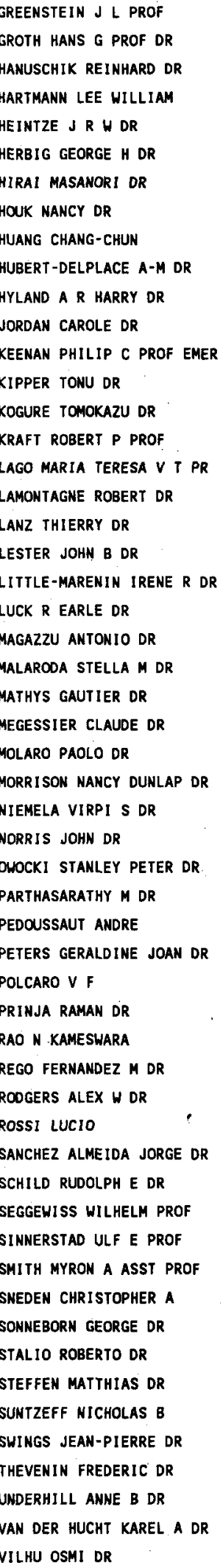 & 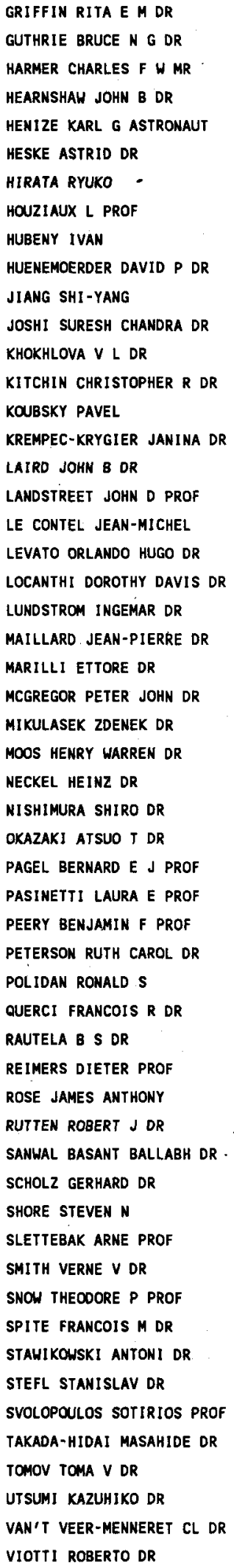 & 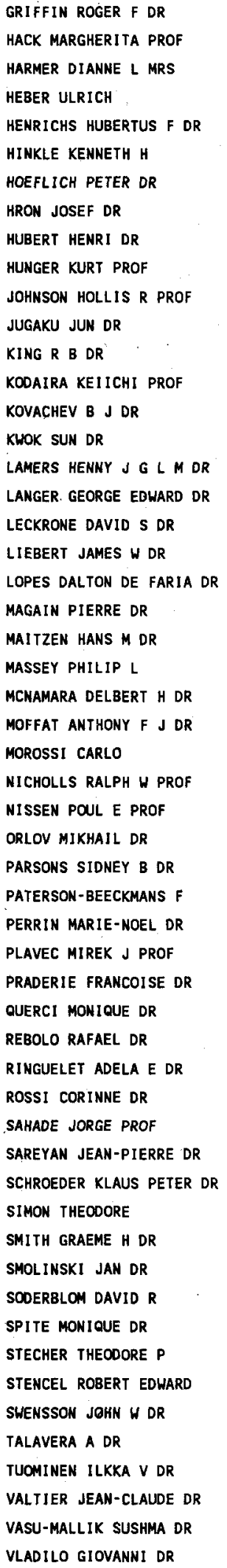 \\
\hline
\end{tabular}


M58

VOGT NIKOLAUS DR WALLERSTEIN GEORGE PROF WEHINGER PETER A DR

WENIGER SCHAME DR WOLF BERNHARD PH D WYCKOFF SUSAN DR ZVERKO JURAJ DR
VOGT STEVEN SCOTT WATERUORTH MICHAEL DR WEHLAU. HILLIAM H PROF WILLIAMS PEREDUR M DR WOLFF SIDNEY C DR YAMASHITA YASUMASA PROF
VREUX JEAN MARIE DR WEGNER GARY ALAN WEISS WERNER $N$ DR WING ROBERT F PROF WOOD III H J DR ZOREC JEAN DR 
COMPOSITION OF COMMISSION $30 \quad 1991-1994$

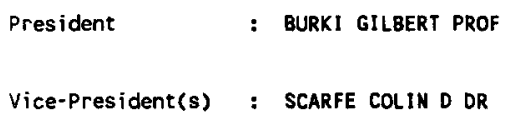

ABT HELMUT A DR

BARBIER-BROSSAT M DR

BEERS TIMOTHY C DR

BREGER MICHEL PROF DR

CARNEY BRUCE HILLIAM

CRAMPTON DAVID DR

DE JONGE J K DR

EDMONDSON FRANK $K$ PROF

FEKEL FRANCIS $C$

FOLTZ CRAIG B

GILMORE GERARD FRANCIS

GRIFFIN ROGER F DR

HEWETT PAUL

HRIVNAK BRUCE J

HUCHRA JOHN PETER DR

KADOURI TALIB HADI

LEVATO ORLANDO HUGO DR

MARSCHALL LAURENCE A

MAURICE ERIC N

MCCLURE ROBERT D PROF

MERMIILLIOD JEAN-CLAUDE DR MEYLAN GEORGES OR

MORRELL NIDIA DR

PEDOUSSAUT ANDRE

PETERSON RUTH CAROL DR

PRESTON GEORGE W DR

RATNATUNGA KAVAN U

RUBIN VERA C DR.

SMITH MYRON A ASST PROF

STOCK JURGEN D

VAN DESSEL EDWIN LUDO DR

WILLSTROP RODERICK $\vee$ DR

\begin{tabular}{|c|c|}
\hline AZZOPARDI MARC DR & BALONA LUIS ANTERO DR \\
\hline BATTEN ALAN H DR & BEAVERS WILLET I OR \\
\hline BERTIAU FLOR C PROF & BOULON JACQUES J DR \\
\hline BURMAGE ROBERT & CAMPBELL BRUCE DR \\
\hline CARQUILLAT JEAN-MICHEL & COCHRAN WILLIAM DAVID DR \\
\hline DAVIS MARC DR & DAVIS ROBERT J DR \\
\hline DE VAUCOULEURS GERARD PR & DUFLOT MARCELLE DR \\
\hline EELSALU HEINO DR & FEHRENBACH CHARLES PROF \\
\hline FLETCHER \ MURRAY & FLORSCH ALPHONSE DR \\
\hline GEORGELIN YVON P DR & GIESEKING FRANK DR \\
\hline GIOVANELLI RICCARDO DR & GOUGUENHEIM LUCIENNE \\
\hline HALBWACHS JEAN LOUIS DR & HEINTZE J R W DR \\
\hline HILDITCH RONALD W DR & HILL GRAHAM DR \\
\hline HUANG CHANG-CHUN & HUBE DOUGLAS P DR \\
\hline IMBERT MAURICE DR & IRWIN ALAN W DR \\
\hline KARACHENTSEV I D DR & KRAFT ROBERT P PROF \\
\hline LEWIS BRIAN MURRAY DR & LINDGREN HARRI \\
\hline MARTIN NICOLE DR & MATHIEU ROBERT D DR \\
\hline MAYOR MICHEL PROF & MAZEH TSEVI DR \\
\hline MCMILLAN ROBERT S DR & MELNICK GARY J \\
\hline MEYLAN GEORGES OR & MORBEY CHRISTOPHER L \\
\hline NORDSTROEM BIRGITTA DR & OETKEN L DR \\
\hline PELLEGRINI PAULO S S DR & PERRY CHARLES L DR \\
\hline PHILIP A G DAVIS & POPOV VICTOR S DR \\
\hline PREVOT LOUIS DR & QUINTANA HERNAN DR \\
\hline REBEIROT EDITH DR & ROMANOV YURI S DR \\
\hline SAMUS NIKOLAI N DR & SANWAL N B DR \\
\hline SOLIVELLA GLADYS R LIC & STEFANIK ROBERT DR \\
\hline TOKOVININ ANDREJ A DR & TONRY JOHN DR \\
\hline WALKER GORDON A H PROF & WEGNER GARY ALAN \\
\hline
\end{tabular}




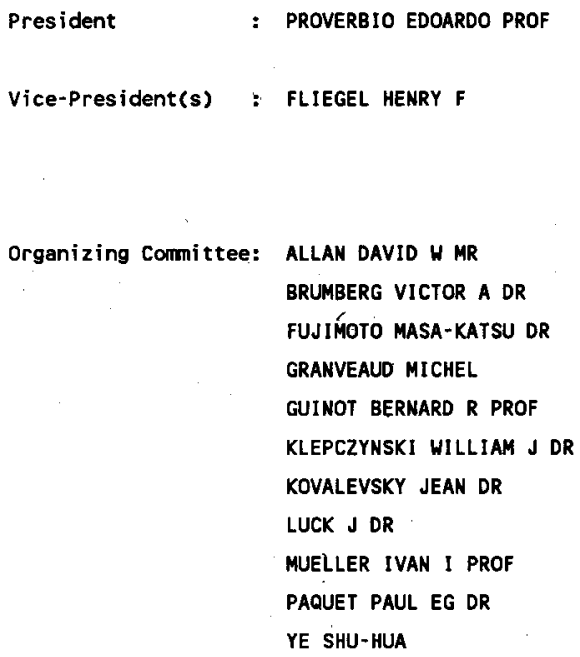


COMPOSITION OF COMMISSION $33 \quad 1991-1994$

$\begin{array}{ll}\text { President } & \text { : BLITZ LEO DR } \\ \text { Vice-President(s) } & \text { : BINNEY JAMES J DR }\end{array}$

Organizing Committee: BLOEMEN JOHANNES B G M DR

CESARSKY CATHERINE J DR

FRIDMAN ALEKSEY M DR

GILMORE GERARD FRANCIS

KALNAJS AGRIS J DR

MATTEUCCI FRANCESCA DR

MAYOR MICHEL PROF

MORRIS MARK ROOT DR

YOSHII YUZURU DR

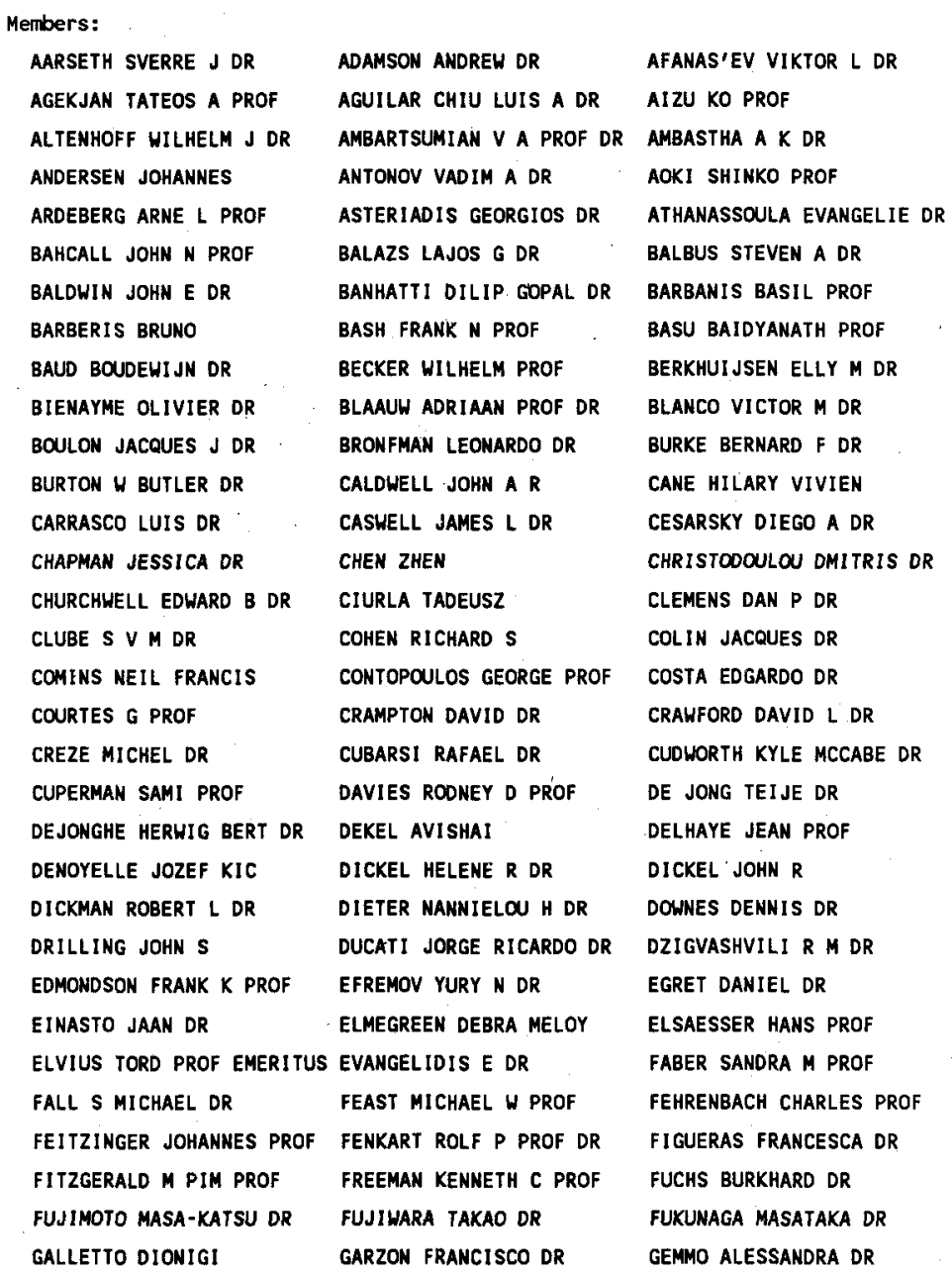


GENKIN IGOR L PROF DR GLIESE WIHELM PROF GOTTESMAN STEPHEN T DR GUPTA SUNIL $K$ DR HABING H J DR HANAMI HITOSHI DR HAUKINS MICHAEL $R S$ HENON MICHEL C DR HOBBS ROBERT W DR HUANG SONG-NIAN DR HUMPHREYS ROBERTA M PROF INAGAKI SHOGO DR ISOBE SYUZO DR IWANOWSKA WILHELMINA PROF JAHREISS HARTMUT OR JIANG DONG-RONG JONAS JUSTIN LEONARD KALANDADZE N B DR KERR FRANK $J$ DR KIMMAN THOMAS D DR KOLESNIK IGOR G DR KULSRUD RUSSELL M DR LARSON RICHARD B PROF LEE HYUNG MOK DR LI JING LINDBLAD PER OLOF PROF LODEN LARS OLOF PROF LUYTEN WILLEM J PROF MACCONNELL DARRELL I DR MARK JAMES WAI-KEE DR MATHEWSON DONALD S PROF MCGREGOR PETER JOHN DR MEZGER PETER G PROF MIRABEL IGOR FELIX DR. MOFFAT ANTHONY F J DR MUENCH GUIDO PROF NAHON FERNAND PROF NINKOVIC SLOBCOAN NORDSTROEM BIRGITTA DR OJA TARMO PROF OLLONGREN A PROF DR PALMER PATRICK E PROF PAPAYANMOPOULOS TH DR PEIMBERT MANUEL DR PESCH PETER DR PILOWSKI K PROF DR POLYMILIS CHRONIS DR QIAN ZHONG-YU RATNATUNGA KAVAN U REIF KLAUS DR ROBERTS WILLIAM W JR PROF ROHLFS K PROF DR RUELAS-MAYORGA R A DR SAAR ENN DR SANDQVIST AAGE DR
GEORGELIN YVON P DR GOLOREICH P DR GRAYZECK EDWIN J DR GYLDENKERNE KJELD DR HAKKILA JON ERIC DR HARTKOPF WILLIAM I DR HAYLI AVRAM PROF HERBST WILLIAM DR HORI GENICHIRO PROF HUGHES VICTOR A PROF HUNTER CHRISTOPHER PROF INNANEN KIMMO A PROF ISRAEL FRANK $P$ DR IYE MASANORI DR JASCHEK CARLOS O R PROF JOG CHANDA J DR JONES DEREK H P DR KASUMOV FIKRET $K O$ DR KHARADZE E K PROF KLARE GERHARD DR KOLESNIK L. N DR KUTUZOV S A DR LATHAM DAVID W DR LEE SANG GAK LIEBERT JAMES $W$ OR LOCKMAN FELIX J LU PHILLIP K DR LYMDEN-BELL DONALD PROF MACRAE DONALD A PROF MAROCHNIK L S PROF DR MAVRIDIS L, N PROF MEATHERINGHAM STEPHEN DR MIKKOLA SEPPO DR MIRZOYAN L V DR PROF MONET DAVID $G$ MURRAY C ANDREW NECKEL TH DR NISHIDA MINORU PROF NORMAN COLIN A PROF OKUDA HARUYUKI DR PROF OORT JAN H PROF PALOUS JAN DR PAULS THOMAS ALBERT DR PEREK LUBOS DR PHILIP A G DAVIS PISMIS DE RECILLAS PARIS PRICE R MARCUS DR RABOLLI MONICA DR REBEIROT EDITH DR RIEGEL KURT W DR ROBIN ANNIE C DR RONG JIAN-XIANG RUIZ MARIA TERESA DR SALA FERRAN DR SANZ I SUBIRANA JAUME DR
GEORGELIN YVONNE M DR GORDON MARK A DR GRENON MICHEL DR HABE ASAO HAMAJIMA KIYOTOSHI DR HAUG ULRICH PROF HEILES CARL PROF. HERMAN JACOBUS DR HRON JOSEF DR HULSBOSCH A N M DR IKEUCHI SATORU DR IRHIN JOHN B PROF IWANISZEWSKA CECYLIA OR JACKSON PETER DOUGLAS DR JASNIEWICZ GERARD DR JOHNSON HUGH M DR KABURAKI MASAKI PROF KATO SHOJI PROF KING IVAN R PROF KMAPP GILLIAN R DR KORMENDY JOHN DR LAFON JEAN-PIERRE J DR LECAR MYRON DR LEISAWITZ DAVID DR LIN CHIA C PROF LODEN KERSTIN R DR LUNEL MADELEINE DR LYNGA GOSTA DR MANCHESTER RICHARD N DR MARTINET LOUIS PROF MCCARTHY MARTIN F DR MENNESSIER MARIE-OOILE DR MILLER RICHARD H DR MIYAMOTO MASANORI DR MONNET GUY J DR MUZZIO JUAN C PROF NELSON ALISTAIR H DR NISHIDA MITSUGU OBLAK EDOUARD OLANO CARLOS ALBERTO DR OSTRIKER JEREMIAH P PROF PANDEY A $K$

PAVLOVSKAYA E D DR PERRY CHARLES L DR PIER JEFFREY R DR POLYACHENKO VALERIJ L DR PRIESTER WOLFGANG PROF RAHARTO MOEDJI REID NEILL ROBERTS MORTON S DR ROBINSON BRIAN J DR RUBIN VERA C DR RYBICKI GEORGE B DR SANCHEZ-SAAVEDRA M LUISA SARGENT ANNEILA I 
SCHECHTER PAUL L DR SCHMIDT MAARTEN PROF SEIMENIS JOHN OR SHAROV A S DR SHU FRANK H PROF SLETTEBAK ARNE PROF SONG GUO-XUAN

SPIEGEL E DR STEINLIN ULI PROF STROBEL ANDRZEJ DR SYGNET JEAN FRANCOIS DR TERZIDES CHARALAMBOS OR TOBIN WILLIAM TOOMRE ALAR DR TOSA MAKOTO DR TURON C DR

SCHMIDT HANS PROF SCHMIDT $K$ H DR SCHMIDT-KALER TH PROF SELLWOOD JERRY A SEGGEWISS WILHELM PROF SHANE WILLIAM W DR SHER DAVID DR SHUTER WILLIAM L H DR SOBOUTI YOUSEF PROF SPARKE LINDA STECKER FLOYD $W$ DR STEPHENSON $C$ BRUCE PROF STURCH CONRAD R DR SZEBEHELY VICTOR G PROF THE PIK-SIN PROF TOMISAKA KOHJI DR TOOMRE JURI TREFZGER CHARLES F DR UPGREN ARTHUR $R$ OR VAN DER KRUIT PIETER C DR VAN WOERDEN HUGO PROF DR VEGA E IRENE DR VETESNIK MIROSLAV DR WEAVER HAROLD F PROF WESTERLUND BENGT E PROF WHITEOAK J B DR WIELEN ROLAMD PROF DR WRAMDEMARK STIG S O DR YOUNIS SAAD M ZHANG BIN

\section{VENUGOPAL $V$ R DR} VOROSHILOV $V$ I DR WEISTROP DONNA DR WHITE RAYMOND E DR WHITTET DOUGLAS C B DR WOLT JER LOOEHIJK PROF WYSE ROSEMARY F DR YUAN CHI PROF ZHAO JUN-LIANG
SHIMIZU TSUTOMU PROF EMER SIMONSON S CHRISTIAN DR SOLOMON PHILIP M DR SPERGEL DAVID N DR STEFANOVITCH-GOMEZ A E OR STIBBS DOUGLAS W N PROF SVOLOPOULOS SOTIRIOS PROF TAMMANN $G$ ANDREAS PROF DR THIELHEIM KLAUS O DR TONG YI

TORRA JORDI DR TSIOUMIS ALEXANDROS DR VALTONEN MAURI I PROF VANDERVOORT PETER O DR VERSCHUUR GERRIT L PROF WAYMAN PATRTCK A PROF WESTERHOUT GART DR WHITELOCK PATRICIA ANN DR WIELEBINSKI RICHARD PROF WOOOWARD PAUL $R$ DR XIANG DELIN ZACHILAS LOUKÁS DR 


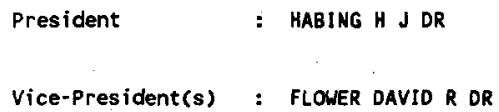

\begin{tabular}{|c|c|c|}
\hline $\begin{array}{l}\text { AANNESTAD PER ARNE DR } \\
\text { AIAD A PROF }\end{array}$ & $\begin{array}{l}\text { ACKER AGNES PROF DR } \\
\text { AITKEN DAVID K DR }\end{array}$ & $\begin{array}{l}\text { ADAMS FRED DR } \\
\text { AKABANE KENJI A PROF }\end{array}$ \\
\hline COLEA JAVIER D & LDROVANDI S M VIEGAS DR & ALLER LAGRENCE HUGH \\
\hline TENHOFF WILHEL & ANANTHARAMAIAH $K R$ DR & ANDREW BRYAN H DR \\
\hline NORIESSE CORNELIS & ANDRILLAT HENRI $L$ PROF & ANDRILLAT YVETTE DR \\
\hline NGLADA GUIL & ARKHIPOVA V & ARNY THOMAS T DR \\
\hline VERY LORNE W DR & AXFORD W IAN PROF & AZCARATE ISMAEL N DR \\
\hline AARS JACOB W M DR & BAART EDHARD E PROF & BACHILLER RAFAEL DR \\
\hline ALDHIN JOHN & BALUTEAU JEAN-PAUL DR & BANIA THOMAS MICHAEL \\
\hline ARLOW MICHAEL J DR & BARNES AARON DR & BARRETT ALAN H PROF \\
\hline BASH FRANK N PROF & BAUDRY ALAIN DR & BECKLIN ERIC E DR \\
\hline BECKMAN JOHN E PROF & BECKWITH STEVEN $\vee W$ & BEDOGNI ROBERTO \\
\hline EL NICOLE J DR & BENAYDOUN JEAN-JACQUES DR & BERGERON JACQUELINE A DR \\
\hline BERKHUI JSEN ELLY & BERNAT ANDREW PLOUS DR & BERTOUT CLAUDE \\
\hline ВНATT & BIANCHI LUCIA & BIEGING JOHN HAROLD DR \\
\hline IGNELL $R$ CARL DR & BINETTE LUC & KURT PH D \\
\hline BLACK JOHN HARRY DR & BLADES JOHN CHRIS DR & BLAIR GUY NORMAN DR \\
\hline BLESS ROBERT C PROF & BLITZ LEO DR & BOCHKAREV NIKOLAY G DR \\
\hline BODE MICHAEL $F$ & BOOENHEIMER PETER PROF & BOESHAAR GREGORY ORTH DR \\
\hline BOGGESS ALBERT DR & BOHLIN RALPH C DR & BOISSE PATRICK DR \\
\hline BOLAND WILFRIED & BORGMAN JAN DR PROF & BOULANGER FRANCOIS \\
\hline BOUVIER JEROME & BRAND PETER W JLDR & BRAUNSFURTH EDWARD PH D \\
\hline BREITSCHWERDT D & MANN WOLFGANG & BROMAGE GORDON E DR \\
\hline BROWN RONALD D F & LENT IN & BURGESS ALAN DR \\
\hline BURKE & MICHAEL G DR & BURTON $N$ BUTLER DR \\
\hline BYSTROVA NATALIJA & JORGE DR & CAPLAN JAMES \\
\hline CAPPA DE NICOLAU C & TTI EI & CAPUZZO DOLCEITA ROBERTO \\
\hline $0-1$ & HERS & A HECTOR \\
\hline CASWELL $J$ & DR & SOLA MONICA \\
\hline
\end{tabular}




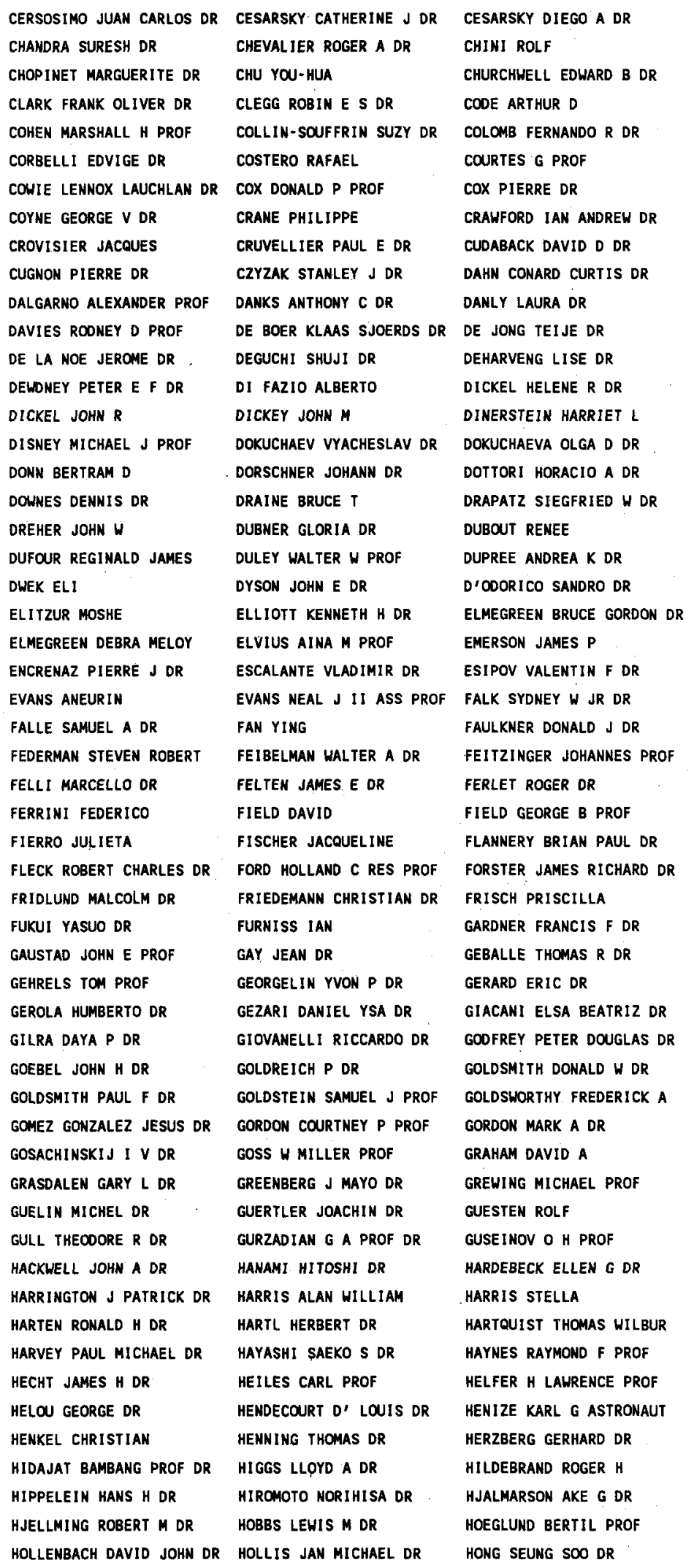




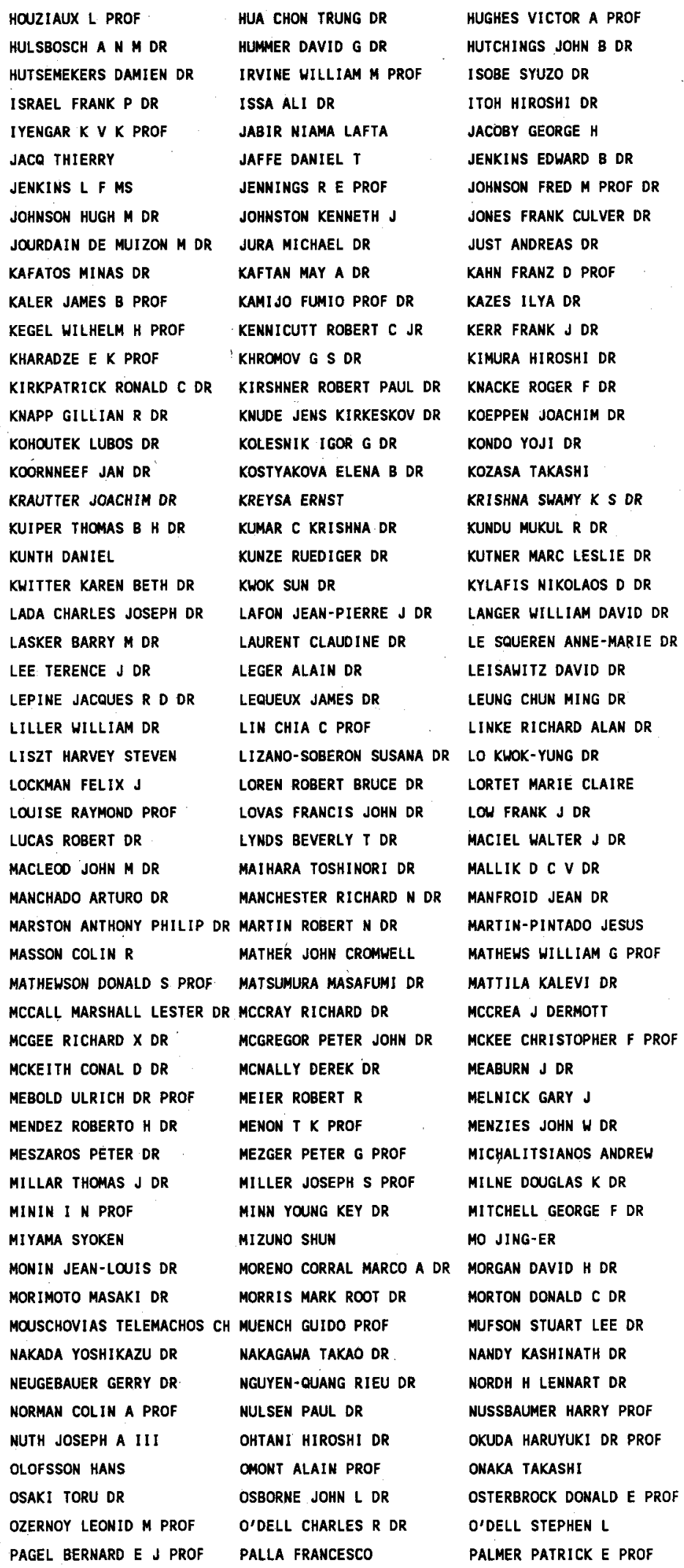


PANAGIA NINO DR

PAULS THOMAS ALBERT DR

PENA MIRIAM DR

PERAULT MICHEL

PETERS NILLIAM L III DR

PHILLIPS THOMAS GOULD DR

POEPPEL WOLFGANG $G L$ DR

PREITE-MARTINEZ ANDREA DR PR

PSKOVSKIJ JU P DR

RADHAKRISHMAN $V$ PROF

RAYMOND JOHH CHARLES

REYNOLDS RONALD J DR

ROBBINS R ROBERT PROF

ROBINSON BRIAN J DR

ROELFSEMA PETER DR

ROGERS ALAN E E DR

ROSE WILLIAM $K$ DR

ROXBURGH IAN W PROF

RUSSELL STEPHEN DR

SAHU KAILASH C DR

SANCHEZ-SAAVEDRA M LUISA

SANDQVIST AAGE DR

SARMA N V G PROF

SAVAGE BLAIR D DR

SCARROIT STANLEY M DR

SCHERB FRANK PROF

SCHMIDT-KALER TH PROF

SCHULZ ROLF ANDREAS

SCHLARZ ULRICH J DR

SEATON MICHAEL J PROF

SHANE WILLIAM $W$ DR

SHARPLESS STEWART PROF

SHCHEGLOV $P \vee D R$

SHU FRANK H PROF

SHUSTOV BORIS M DR

SILK JOSEPH I PROF

SINGH PATAN DEEN DR

SKILLING JOHN DR

SMITH HOWARD ALAN

SNELL RONALD L

SOFIA SABATINO PROF

SOLOMON PHILIṔ M DR

STAHLER SETVEN W DR

STENHOLM BJOERN DR

SUH KYUNG-WON DR

TAKAKUBO KEIYA PROF

TANAKA MASUOO DR

TENORIO-TAGLE G DR

THE PIK-SIN PROF

THRONSON HARLEY ANDREW JR TOKAREV YURIJ $V$ DR

TORRES-PEIMBERT SILVIA DR TOSI MONICA

TREFFERS RICHARD R

TURNER BARRY E DR

ULRICH MARIE-HELENE D DR

VAN DER LAAN H PROF DR

URASIN LIRIK A DR

VAN DISHOECK EWINE F DR

VAN WOERDEN HUGO PROF OR VANDEN BOUT PAUL A
PARKER EUGENE N

PEIMBERT MANUEL DR

PEQUIGNOT DANIEL

PERSI PAOLO

PHILLIPS JOHN PETER

PISMIS DE RECILLAS PARIS

PRASAD SHEO S

PRONIK I I DR

OIN ZHI-HAI

RAWLINGS JONATHAN DR

RENGARAJAN T N DR

RIGHINI-COHEN GIOVANNA DR

ROBERTS WILLIAM W JR PROF

ROCHE PATRICK F DR

ROGER ROBERT S DR

ROSADO MARGARITA DR

ROUAN DANIEL DR

ROZYCZKA MICHAL

SABBADIN FRANCO DR

SALPETER EDHIN E PROF

SANDELL GORAN HANS L DR

SARGENT ANMEILA I

SATO SHUJI DR

SCALO JOHN MICHAEL

SCHATZMAN EVRY PROF

SCHMIDT THOMAS DR

SCHULTZ G V DR

SCHWARTZ RICHARD D

SCOVILLE NICHOLAS $Z$

SHAH GHANSHYAM A DR

SHAPIRO STUART L

SHAWL STEPHEN : J DR

SHIELDS GREGORY A DR

SHULL PETER OTTO DR

SILBERBERG REIN DR

SIMONS STUART DR

SIVAN JEAN-PIERRE DR

SMITH CRAIG H DR

SMITH ROBERT G DR

SOBOLEV $V$ V DR

SOLC MARTIN

SPITZER LYMAN JR DR

STECHER THEOOORE $P$

SU BUMEI

SUZUKI YOSHIMASA PROF

TAMURA SHINICHI DR

TAYLOR KENNETH N R PROF

THADDEUS PATRICK PROF

THONNARD NORBERT DR

TORRELLES JOSE M DR

TOUNES CHARLES HARD DR

TURNER KENNETH C DR

VAN DE HULST H C PROF DR

VAN GORKOM JACQUELINE H

VANYSEK VLADIMIR PROF 


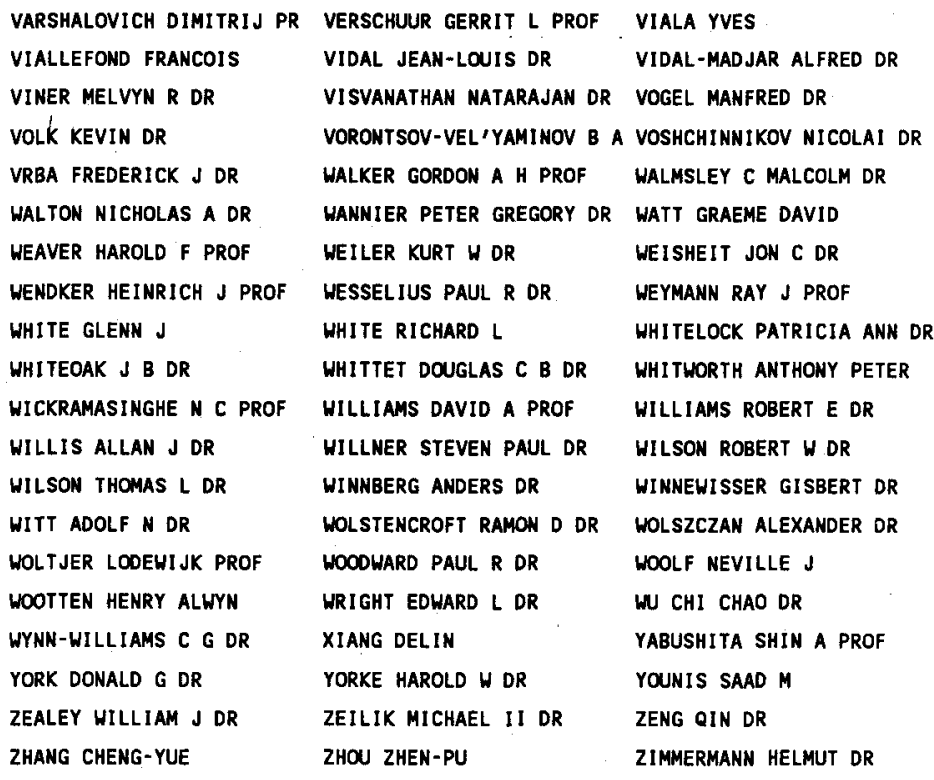




\author{
President : DEMARQUE P PROF \\ vice-President(s) : CHIOSI CESARE S OR
}

Organizing Committee: CANAL RAMON M DR

IBEN ICKO JR PROF

MAEDER ANDRE PROF

MICHAUD GEORGES J DR

NOMOTO KEN'ICHI OR

RENZINI ALVIO PROF

TUTUKOV A V DR

VANDENBERG DON DR

VAUCLAIR GERARD D DR

Members :

\begin{tabular}{|c|c|c|}
\hline AIAD A PROF & AIZENMAN MORRIS L DR & ANAND S P S DR \\
\hline ANGELOV IRAJKO & ANTIA H M DR & APPENZELLER IMMO PROF \\
\hline ARAI KENZO DR & ARIMOTO NOBUO DR & ARNETT W DAVID PROF \\
\hline ARNOULD MARCEL L DR & AUDOUZE JEAN PROF & BAGLIN ANNIE DR \\
\hline BAKER NORMAN H PROF & BAYM GORDON ALAN DR & BEAUDET GILLES DR \\
\hline ECKER STEPHEN A & BELMONTE AVILES J A DR & BENZ WILLY \\
\hline ERTHOMIEU GABRIELLE DR & BISNOVATYI-KOGAN G S DR & BLUDMAN SIDNEY A PROF \\
\hline SOCCHIA ROMEO DR & BOOENHEIMER PETER PROF & BOEHM KARL-HEINZ PROF \\
\hline BONDI HERMANN PROF SIR & BONO G & BOSS ALAN P DR \\
\hline BROWNLEE ROBERT R OR & BUCHLER J ROBERT PROF & BURBIDGE GEOFFREY R PROF \\
\hline CALLEBAUT DIRK $K$ DR & CALOI VITTORIA DR & CAMERON ALASTAIR G $W$ PROF \\
\hline CAPUTO FILIPPINA DR & CARSON T R DR & CASTELLANI VITTORIO PROF \\
\hline CASTOR JOHN I DR & CAUGHLAN GEORGEANME $R$ & CHAN KWING LAM \\
\hline CHAN ROBERTO DR & CHANDRASEKHAR S PROF & CHECHETKIN VALERIJ M DR \\
\hline CHEVALIER CLAUDE DR & CHITRE SHASHIKUMAR M DR & CHIU HONG-YEE DR \\
\hline CHKHIKVADZE IAKOB N & CHRISTENSEN-DALSGAARD J & CHRISTY ROBERT F DR \\
\hline COHEN JEFFREY M DR & CONNOLLY LEO PAUL & COWAN JOHN J DR \\
\hline DAS MRINAL KANTI & DAVIS CECIL G JR & DE GREVE JEAN-PIERRE DR \\
\hline DE JAGER CORNELIS PROF & DE LOORE CAMIEL PROF & DE MEDEIROS JOSE RENAN DR \\
\hline DEARBORN DAVID PAUL $S$ DR & DEINZER $U$ PROF DR & DESPAIN KEITH HOWARD DR \\
\hline DEUPREE ROBERT G DR & DINGENS $P$ PROF DR & DLUZHNEVSKAYA O B DR \\
\hline DURISEN RICHARD H DR & DZIEMBOWSKI WOJCIECH PROF & D'ANTONA FRANCESCA DR \\
\hline EDWARDS ALAN CH DR & EDWARDS TERRY $W$ & EGGLETON PETER P DR \\
\hline EMINZADE T A DR & ENDAL ANDREW S DR & EPSTEIN ISADORE PROF \\
\hline ERGMA & ERIGUCHI YOSHIHARU DR & EZER-ERYURT DILHAN PROF \\
\hline FADEYEV YURI A & FAULKNER DONALD J DR & FAULKNER JOHN PROF \\
\hline FERNANZ MARGARITA DR & FLANMERY BRIAN PAUL DR & FONTAINE GILLES DR \\
\hline FORBES $J$ & ERIC DR & FOWLER WILLIAM A PROF \\
\hline FRANTSMAN YU L DR & FUJIMOTO MASAYUKI DR & GABRIEL MAURICE $R$ DR \\
\hline GALLINO & GEROYANWIS VASSILIS S DR & GHEIDARI S NASSIRI DR \\
\hline GIANNONE PIETRO PROF & GIMENEZ ALVARO & GINGOLD ROBERT ARTHUR DR \\
\hline IRIDHAR SUNETRA DR & GLATZMAIER GARY A & GONG \\
\hline
\end{tabular}




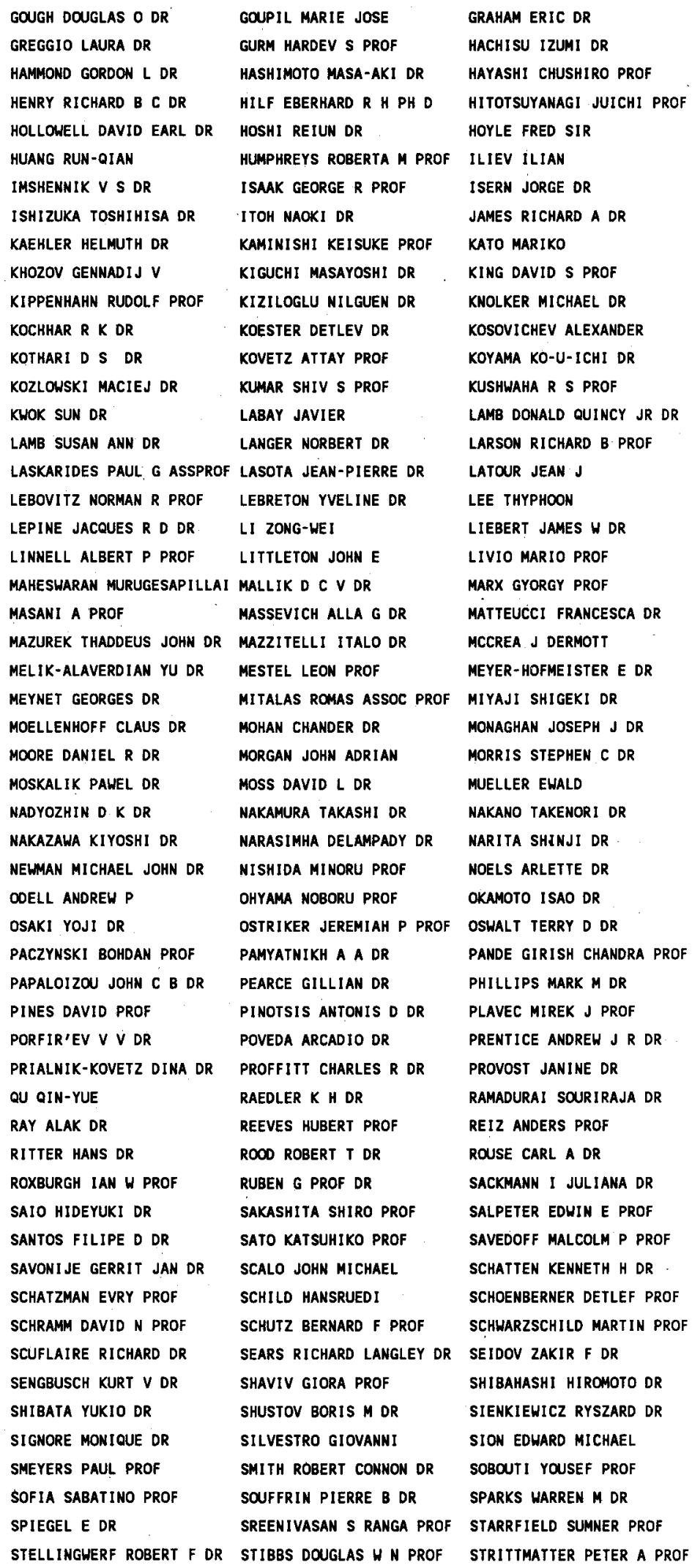


SUDA KAZUO PROF SWEIGART ALLEN $\vee$ DR TASSOUL MONIQUE DR THOMAS HANS-CHRISTOPH DR TOHLINE JOEL EDWARD TRURAN JAMES $W$ JR UCHIDA JUICHI DR WS UNDO DR VAN DER RAAY HERMAN B VARDYA M S DR VILKOVISKIJ EMMANUIL Y DR WARD RICHARD A DR WEBBINK RONALD F DR WEISS NIGEL O DR WILSON ROBERT E PROF WOOSLEY S E PROF YUNGELSON LEV R ZIOLKOWSKI JANUSZ, DR TOOMRE JURI VILA SAMUEL C PROF XIONG DA-RUN ZAHN JEAN-PAUL DR
SUGIMOTO DAIICHIRO PROF TAAM RONALD EVERETT DR TAYLER ROGER J PROF THOMPSON MICHAEL J DR TSCHARNUTER WERNER M DR ULRICH ROGER. $K$ PROF VAN DEN HEUVEL EDWARD P J VAN DER BORGHT RENE PROF VAN HORN HUGH M PROF WEIGERT ALFRED PROF WHEELER J CRAIG PROF WINKLER KARL-HEINZ A DR
SWEET PETER A PROF TAKAHARA MARIKO THIELEMANM FRIEDRICH-KARL TJIN-A-DJIE HERMAN R E DR TRIMBLE VIRGINIA L DR TUONINEN ILKKA V DR UNNO HASABURO PROF VAN RIPER KENNETH A DR VILHU OSMI DR WEAVER THOMAS A DR HEISS ACHIM DR WILLSON LEE ANNE DR WOOD PETER R DR YORKE HAROLD W DR ZHEVAKIN S A PROF DR 
Organizing Committee:-CUNY YVETTE J DR DRAVINS DAINIS PROF

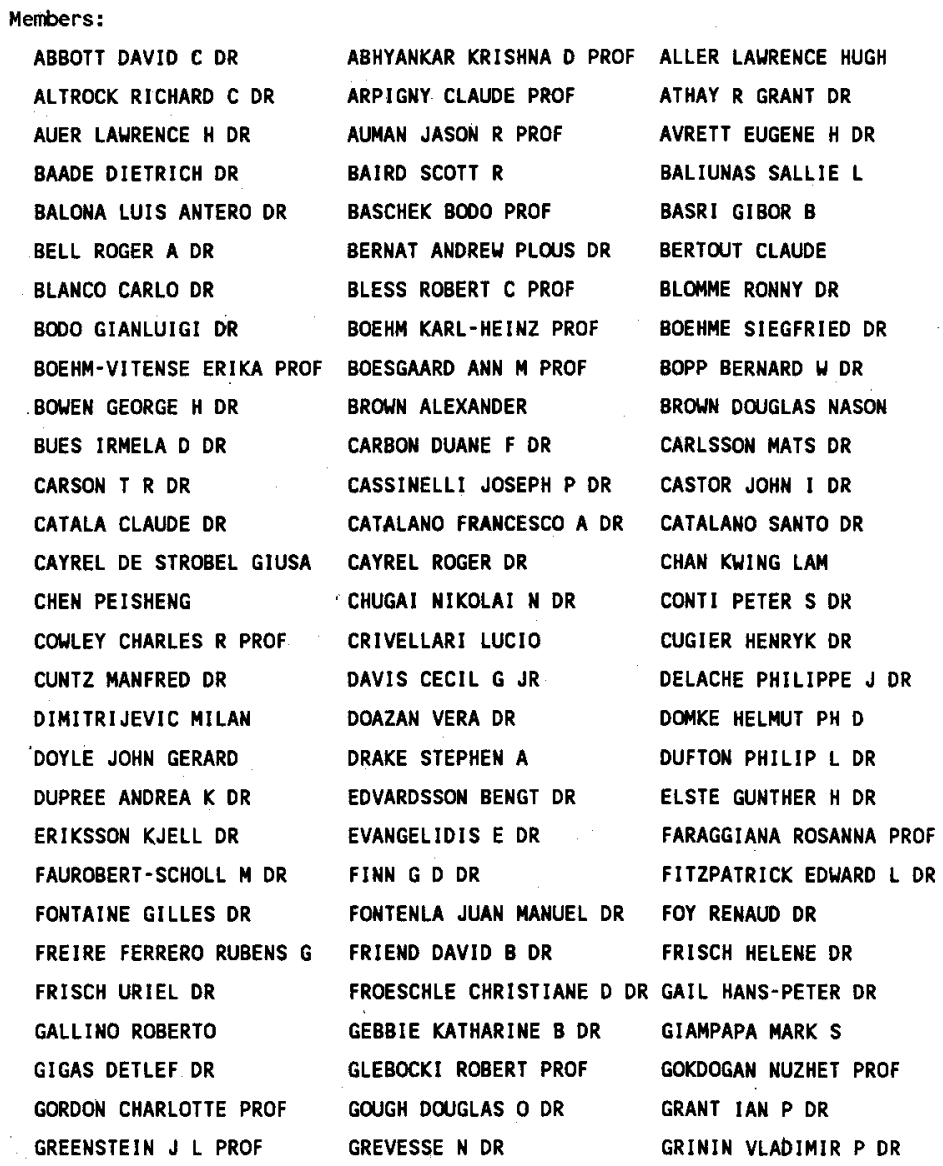


GROTH HANS G PROF DR HACK MARGHERITA PROF HAMMAN WOLF-RAINER HEARN ANTHONY G DR HEKELA JAN DR HOARE MELVIN DR HOLZER THOMAS EDWARD DR HUBENY IVAN HUSFELLD DIRK DR JEFFERIES JOHN T DR KADOURI TALIB HADI KARP ALAN HERSH DR KODAIRA KEIICHI PROF KONDO YOJI DR KRISHNA SWAMY $K S$ DR KUMAR SHIV S PROF. LAMBERT DAVID L PROF LEIBACHER JOHN DR LIU CAIPIN LYUBIMKOV LEONID S DR MASSAGLIA SILVANO MATSUSHIMA SATOSHI DR MIYAMOTO SIGENORI PROF MUKAI SONOYO DR NAGIRNER DMITRIJ I DR NEFF JOHN S ORRALL FRANK Q PROF O'MARA BERNARD J PROF PASINETTI LAURA E PROF PHILLIPS JOHN G PROF PORNCHAI P.-TANAKUN PULS JOAHIM DR RACHKOVSKY D N DR RODONO MARCELLO DR RUCINSKI SLAWOMIR M DR RYBICKI GEORGE $B$ DR SCHARMER GOERAN BJARNE SCHMUTZ WERMER SCHR I JVER C J DR SHIPMAN HENRY L DR SIMONNEAU EDUARDO DR SNEZHKO LEONID I SOUFFRIN PIERRE B DR SPITE MONIQUE DR STEFFEN MATTHIAS DR STIBBS DOUGLAS $W$ N PROF SZECSENYI-NAGY GABOR DR THEJLL PETER ANDREAS DR TRAVING GERHARD PROF UESUGI AKIRA DR UNNO WASABURO PROF VAN'T VEER FRANS DR VARDYA M S DR VIIK TONU DR WATANABE TETSUYA
GUSSMANM E A DR HAISCH BERNHARD MICHAEL. HARTMANM LEE WILLIAM HEASLEY JAMES NORTON HEROLD HEINZ HOEFLICH PETER DR HOTINLI METIN DR HUMMER DAVID G DR HUTCHINGS JOHN B DR JOHNSON HOLLIS R PROF KAMP LUCAS WILLEM DR KHOKHLOVA V L DR KOESTER DETLEV DR KONTIZAS EVANGELOS DR KUDRITZKI ROLF-PETER PH D KUHI LEONARD V PROF KURUCZ ROBERT L DR KUSHUAHA R S PROF LAMERS HENNY J G L M DR LANDSTREET JOHN D PROF LIEBERT JAMES $W$ DR LUCK R EARLE DR MADEJ JERZY MATHYS GAUTIER DR MICHAUD GEORGES J DR MNATSAKANIAN MAMIKON A DR MUENCH GUIDO PROF MUSIELAK ZDZISLAW E DR NARASIMHA DELAMPADY DR NIKOGHOSSIAN ARTHUR G DR OWOCKI STANLEY PETER DR PAGEL BERNARD E J PROF PECKER JEAN-CLAUDE PROF PINTO PHILIP ALFRED DR POTTASCH STUART R PROF QUERCI FRANCOIS R DR RAMSEY LAWRENCE $W$ DR ROSS JOHN E R DR RUTTEN ROBERT J DR SAITO KUNIJI PROF SCHMALBERGER DONALD C DR SCHOENBERNER DETLEF PROF SEDLMAYER ERWIN DR SIMON KLAUS PETER LINNELL ALBERT P PROF LUTTERMOSER DONALD DR MARLBOROUGH J M PROF MATSUMOTO MASAMICHI PROF MIHALAS DIMITRI DR

MUTSCHLECNER J PAUL DR NARIAI KYOJI PROF NORDLUND AKE DR OXENIUS JOACHIM DR PANEK ROBERT J DR PETERS GERALDINE JOAN DR PISKUNOV NIKOLAI E DR PRADERIE FRANCOISE DR QUERCI MONIQUE DR REIMERS DIETER PROF ROVIRA MARTA GRACIELA RYABCHIKOVA TANYA DR SAKHIBULLIN NAIL A DR SCHMID-BURGK J DR PROF SCHOLZ M PROF SHINE RICHARD A DR SIMON THEODORE SITNIK G F PROF SKUMANICH ANDRE PROF SNIJDERS MATTHEUS A J DR SOBOLEV $\checkmark V$ DR SPIEGEL E DR SPITE FRANCOIS M DR SPRUIT HENK C DR STALIO ROBERTO DR STEIN ROBERT F ASSOC PROF STEPIEN KAZIMIERZ DR STROM STEPHEN E SWIHART THOMAS L DR TAKEDA YOICHI DR THOMAS RICHARD N DR TARAFDAR SHANKAR P DR TOOMRE JURI TUONINEN ILKKA $\vee$ DR UENO SUEO PROF ULMSCHNEIDER PETER PROF VAKILI FARROKH DR UNDERHILL ANNE B DR VAN REGEMORTER HENRI DR VAN'T VEER-MENNERET CL DR VARDAVAS ILIAS MIHAIL VASU-MALLIK SUSHMA DR VILHU OSMI DR VAUGHAN ARTHUR H DR HATERS LAURENS B F M DR WEBER STEPHEN VANCE 
MEIDEMANN VOLKER PROF HICKRAMASINGHE N C PROF WOEHL HUBERTUS DR

WYLLER ARNE A PROF

ZAHN JEAN-PAUL DR
WELLLANM PETER PROF DR WILSON PETER R PROF WOLFF SIDNEY C DR YANOVITSKIJ EDGARD G DR
WHITE RICHARD L

WILSON S J

WRIGHT KENMETH O DR YORKE HAROLD W DR 
COMPOSITION OF COMMISSION $37 \quad 1991-1994$

\begin{abstract}
President $\quad:$ MERMILLIOO JEAN-CLAUDE DR
Vice-President(s) : FEINSTEIN ALEJANDRO DR
\end{abstract}

Organizing Committee: CLARIA JUAH DR

DA COSTA GARY STEWART DR

HARRIS GRETCHEN L H DR

JANES KENNETH A DR

MEYLAN GEORGES DR

VANDENBERG DON DR

ZHAO JUN-LIANG

Members:

\begin{tabular}{|c|c|c|}
\hline $\begin{array}{l}\text { AARSETH SVERRE J DR } \\
\text { ALAD A PROF }\end{array}$ & $\begin{array}{l}\text { ABOU-EL-ELLA MOHAMED S DR } \\
\text { ALCAINO GONZALO DR }\end{array}$ & $\begin{array}{l}\text { AGEKJAN TATEOS A PROF } \\
\text { ALFARO EMILIO JAVIER }\end{array}$ \\
\hline ALKSNIS ANDREJS DR & ALLEN CHRISTIME & APARICIO ANTONIO OR \\
\hline ARMANDROFF TAFT E DR & AURIERE MICHEL & BALAZS BELA A PROF DR \\
\hline BECKER WILHELM PROF & BELL ROGER A DR & BIJAOUI ALBERT DR \\
\hline BLAAUW ADRIAAN PROF DR & BOUVIER PIERRE PROF & BUONANNO ROBERTO \\
\hline BURKHEAD MARTIN S & BUTLER DENNIS DR & BYRD GENE G DR \\
\hline CALLEBAUT DIRK K DR & CALOI VITTORIA DR & CANNON RUSSELL O DR \\
\hline CAPUTO FILIPPINA DR & CAPUZZO DOLCETTA ROBERTO & CARNEY BRUCE WILLIAM \\
\hline CASTELLANI VITTORIO PROF & CHAVARRIA-K CARLOS & CHIOSI CESARE S DR \\
\hline CHRISTIAN CAROL ANN & CHUN MUN-SUK OR & COLIN JACQUES DR \\
\hline CUDWORTH KYLE MCCABE DR & CUFFEY J MR & DANFORD STEPHEN C DR \\
\hline DAPERGOLAS A DR & DAUBE-KURZEMNIECE I A DR & DE JONGHE HERWIG BERT DR \\
\hline DEMARQUE P PROF & DEMERS SERGE DR & DI FAZIO ALBERTO \\
\hline DICKENS ROBERT J DR & DLUZHNEVSKAYA O B DR & D'ANTONA FRANCESCA DR \\
\hline EFREMOV YURY N DR & EINASTO JAAN DR & ELMEGREEN BRUCE GORDON DR \\
\hline EL-BASSUNY ALAWY A A DR & FALL S MICHAEL DR & FEAST MICHAEL W PROF \\
\hline FITZGERALD M PIM PROF & FORBES DOUGLAS DR & FORTE JUAN CARLOS DR \\
\hline FREEMAN KENNETH C PROF & FUSI PECCI FLAVIO & GASCOIGNE S C B DR \\
\hline GEISLER DOUGLAS P DR & GOLAY MARCEL PROF & GRATTON R G DR \\
\hline GREEN ELIZABETH M OR & GRIFFITHS WILLIAM $K$ & GRINDLAY JONATHAN E DR \\
\hline GRUBISSICH C PROF OR & GUETTER HARRY HENDRIK & HANES DAVID A DR \\
\hline HARRIS HUGH C & HARRIS WILLIAM E DR & HARVEL CHRISTOPHER ALVIN \\
\hline HASSAN S M PROF & HATZIDIMITRIOU DESPINA DR & HAWARDEN TIMOTHY G DR \\
\hline HAZEN MARTHA L DR & HEGGIE DQUGLAS C DR & HENON MICHEL C DR \\
\hline HERBST WILLIAM DR & HESSER JAMES E DR & HEUDIER JEAN-LOUIS DR \\
\hline HILLS JACK G DR & HUT PIET & IBEN ICKO JR PROF \\
\hline ILLINGWORTH GARTH D DR & INAGAKI SHOGO DR & ISHIDA KEIICHI PROF \\
\hline JONES DEREK H P DR & JOSHI U C DR & KADLA ZDENKA I DR \\
\hline KAMP LUCAS WILLEM DR & KANDRUP HENRY EMIL DR & KILAMBI G C DR \\
\hline KING IVAN R PROF & KONTIZAS EVANGELOS DR & KONTIZAS MARY DR \\
\hline KRAFT ROBERT P PROF & KRON GERALD E & KUN MARIA DR \\
\hline LADA CHARLES JOSEPH & LANDOLT ARLO U PROF & LAPASSET EMILIO DR \\
\hline LARSSON-LEANDER G PROF & LATHAM DAVID $W$ OR & LAVAL ANNIE DR \\
\hline
\end{tabular}




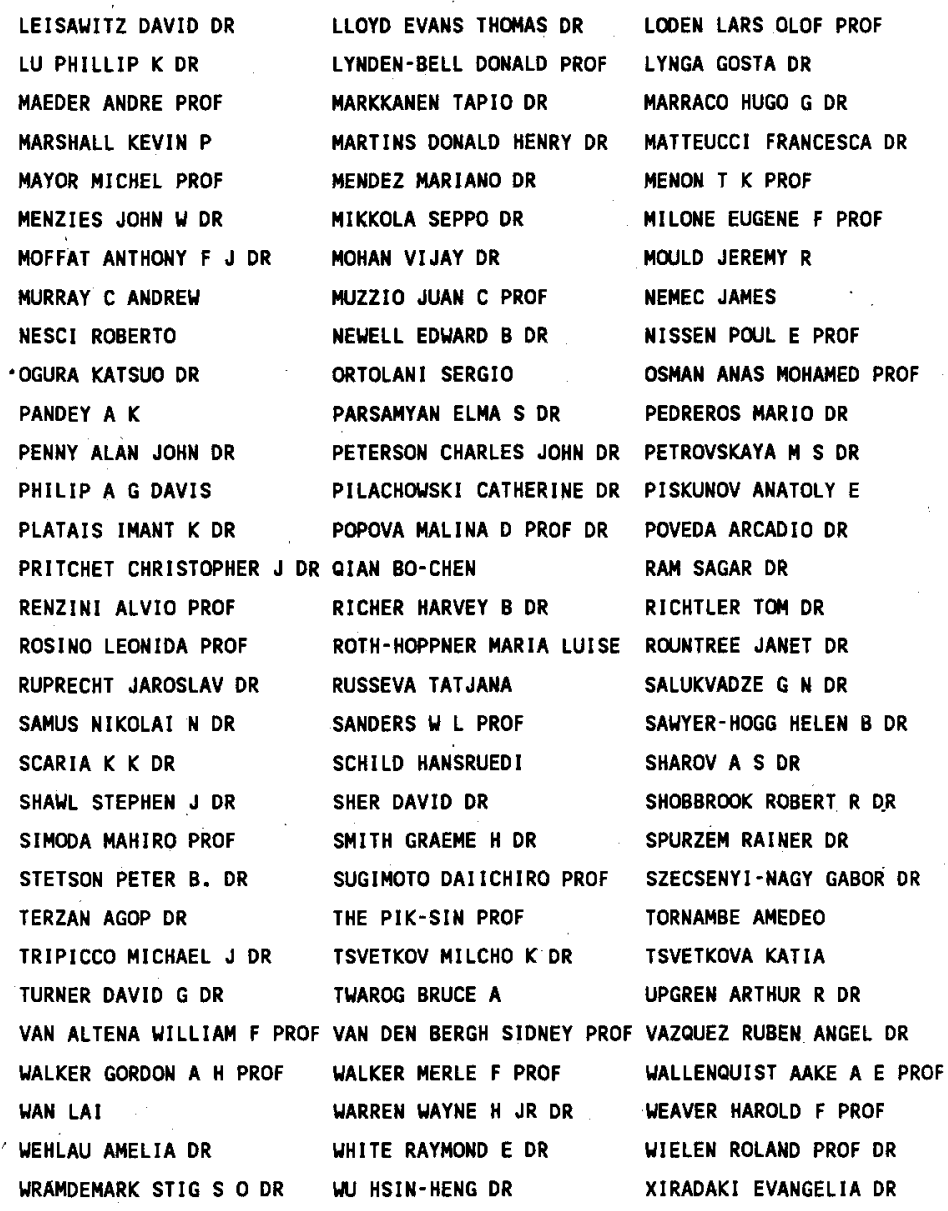

ZINN ROBERT J DR 
$\begin{array}{ll}\text { President } & \text { : SAHADE JORGE PROF } \\ \text { Vice-President(s) : JORGENSEN HENNING E PROF }\end{array}$

Organizing Committee: CHITRE DATTAKUMAR M DR DUCATI JORGE RICARDO DR KRISHNA GOPAL

LEUNG KAM CHING PROF MORIMOTO MASAKI DR ROBERTS MORTON S DR SMITH F GRAHAM PROF

Menbers:

ALY M KHAIRYY PROF CACCIN BRUNO KOZAI YOSHIHIDE PROF MUELLER EDITH A PROF OKOYE SAMUEL E PROF TOLBERT CHARLES R DR WOOD F BRADSHAW PROF
AL-SABTI ABDUL ADIM DR
BOYARCHUK A A DR FLORSCH ALPHONSE DR HAUPT HERMANN F PROF MACRAE DONALD A PROF MARIK MIKLOS DR NHA IL-SEONG DR NINKOVIC SLOBODAN ROUTLY PAUL M DR SWARUP GOVIND PROF VAN DEN HEUVEL EDHARD P J WANG SHOU-GUAN YE SHU-HUA 


\author{
President : MORIMOTO MASAKI DR \\ Vice-President(s) : HHITEOAK J B DR
}

Organizing Committee: BAART EDWARD E PROF

BAATH LARS B DR

BACKER DONALD CH DR

COLOMB FERNANDO $R$ DR

OAVIS RICHARO J OR

FANTI ROBERTO

GERARD ERIC DR

GOMEZ GONZALEZ JESUS DR

GOSACHINSKIJ I $\vee$ DR

GUESTEN ROLF

MEZGER PETER G PROF

MORAN JAMES M DR

VAN DER HULST JAN M DR

VELUSAMY T DR

YE SHU-HUA

MEmberS:
ABDULLA SHAKER ABDUL AZIZ AE
ADE PETER A R DR
AKUJOR CHIDI E
ALLEN RONALD J DR
ALTENHOFF WILHELM J DR
ANDREW BRYAN H DR
ASCHWANDEN MARKUS DR
AVERY LORNE W DR
BAARS JACOB $W$ M DR
BALASUBRAMANIAN $V$ DR
BALL LEWIS DR
BANHATTI DILIP GOPAL DR
BARTEL NORBERT HARALD DR
BASH FRANK N PROF
BAUDRY ALAIN DR
BENNETT CHARLES L DR
BERGE GLENN L DR
BHONSLE RAJARAM V PROF
BIGNELL R CARL DR
BIRKINSHAW MARK
BLOEMHOF ERIC E DR
BOLTON JOHN G
BOS ALBERT DR
BRACEHELL RONALD N PROF
BRIDLE ALAN H PROF
BRONFMAN LEONARDO DR

Members:
ABRAMI ALBERTO PROF AKABANE KENJI A PROF ALEXANDER PAUL DR ALLER MARGO F DR ANDERNACH HEINZ DR ARNAL MARCELO EDMUNDO DR AURASS HENRY DR AXON DAVID BAGRI DURGADAS S BALKLAVS A E DR BALONEK THOMAS J DR BARROW COLIN H DR BARVAINIS RICHARD DR BATTY MICHAEL DR BENM CHRIS R DR BENZ ARNOLO DR BHANDARI RAJENDRA DR BIERMANN PETER L DR BIRETTA JOHN ANTHONY DR BLANDFORD ROGER DAVID DR BLAIR DAVID GERALD BOCKELEE-MORVAN DOMINIQUE BOISCHOT ANDRE DR BOOIH ROY S PROF BORIAKOFF VALENTIN BOTTINELLI LUCETTE DR BRAUDE SEMION YA PROF AG BRINKS ELIAS DR BROTEN NORMAN W
BOWERS PHILLIP $F$ BREgMAN Jacob D IR BROOERICK JOHN DR BROUW W N DR 


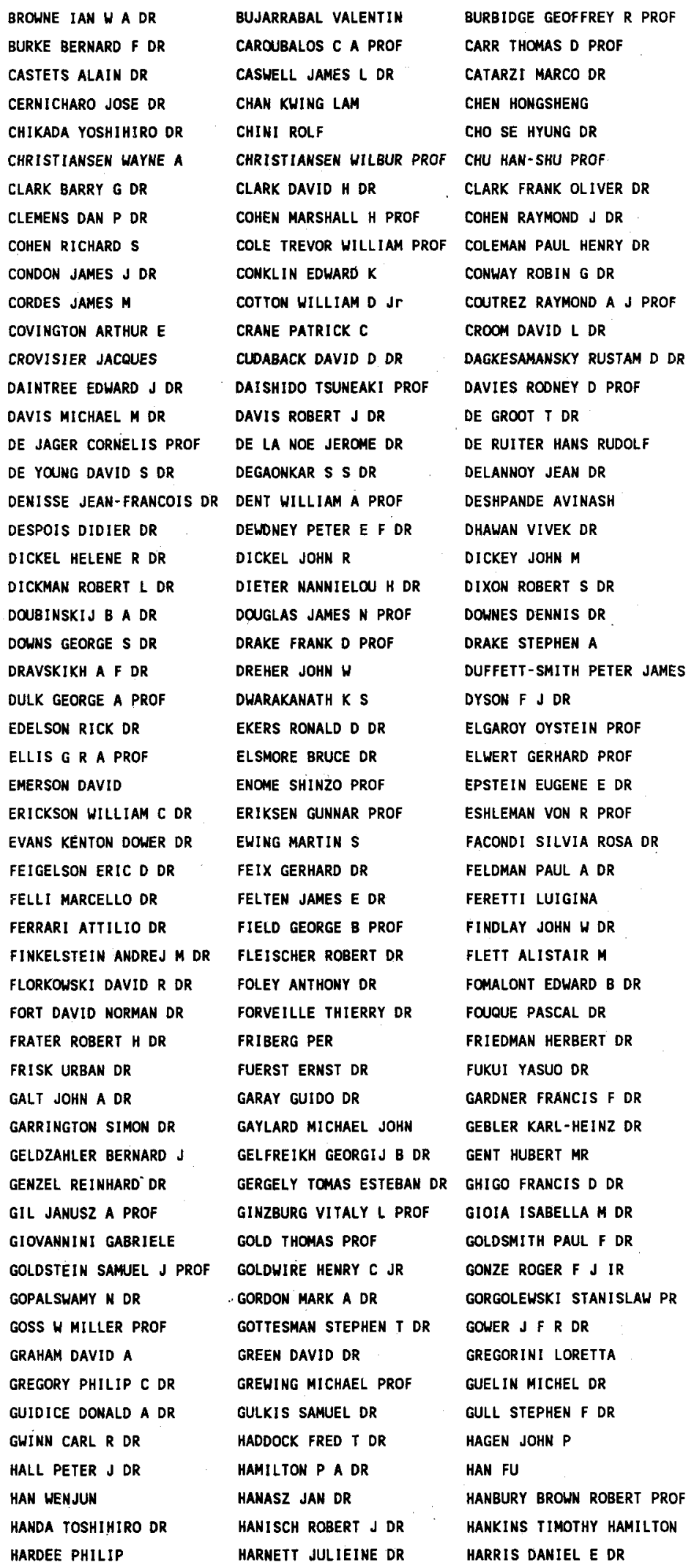




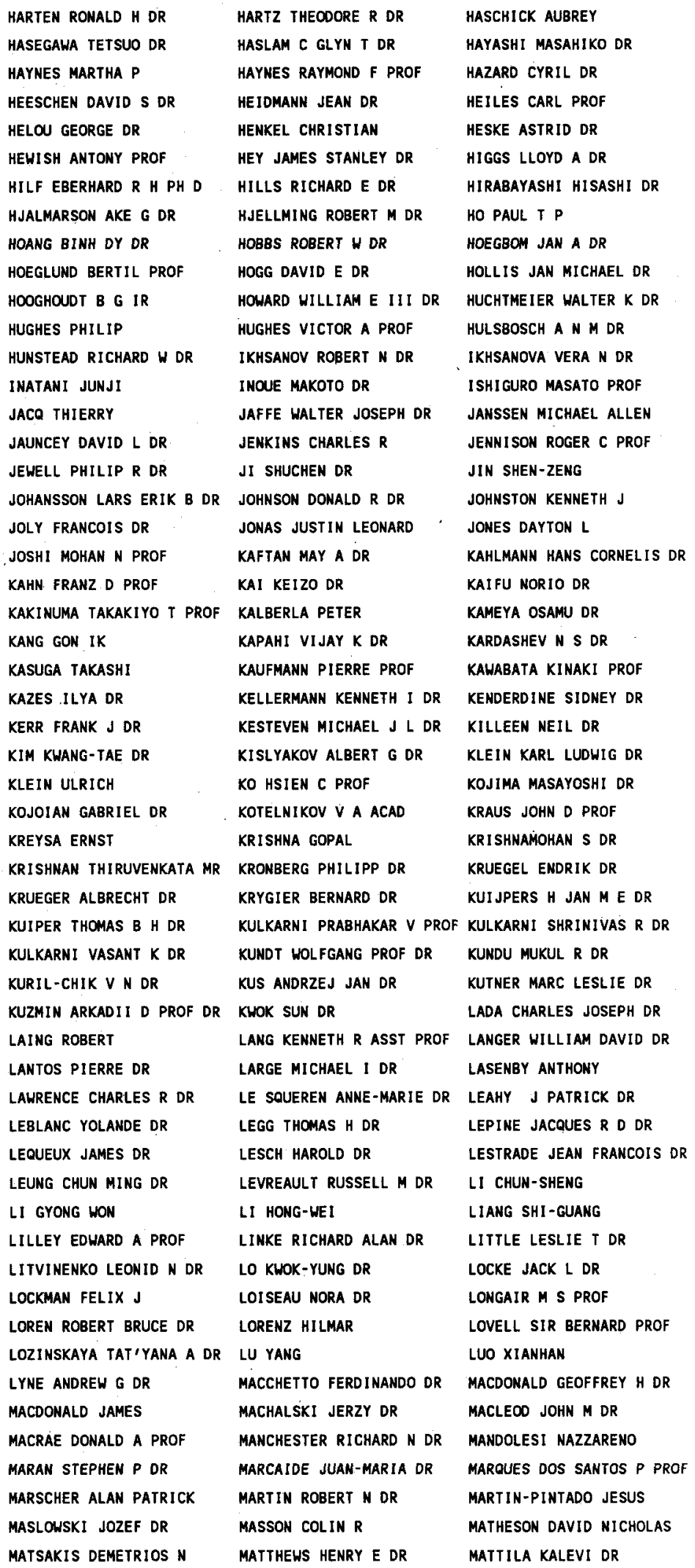




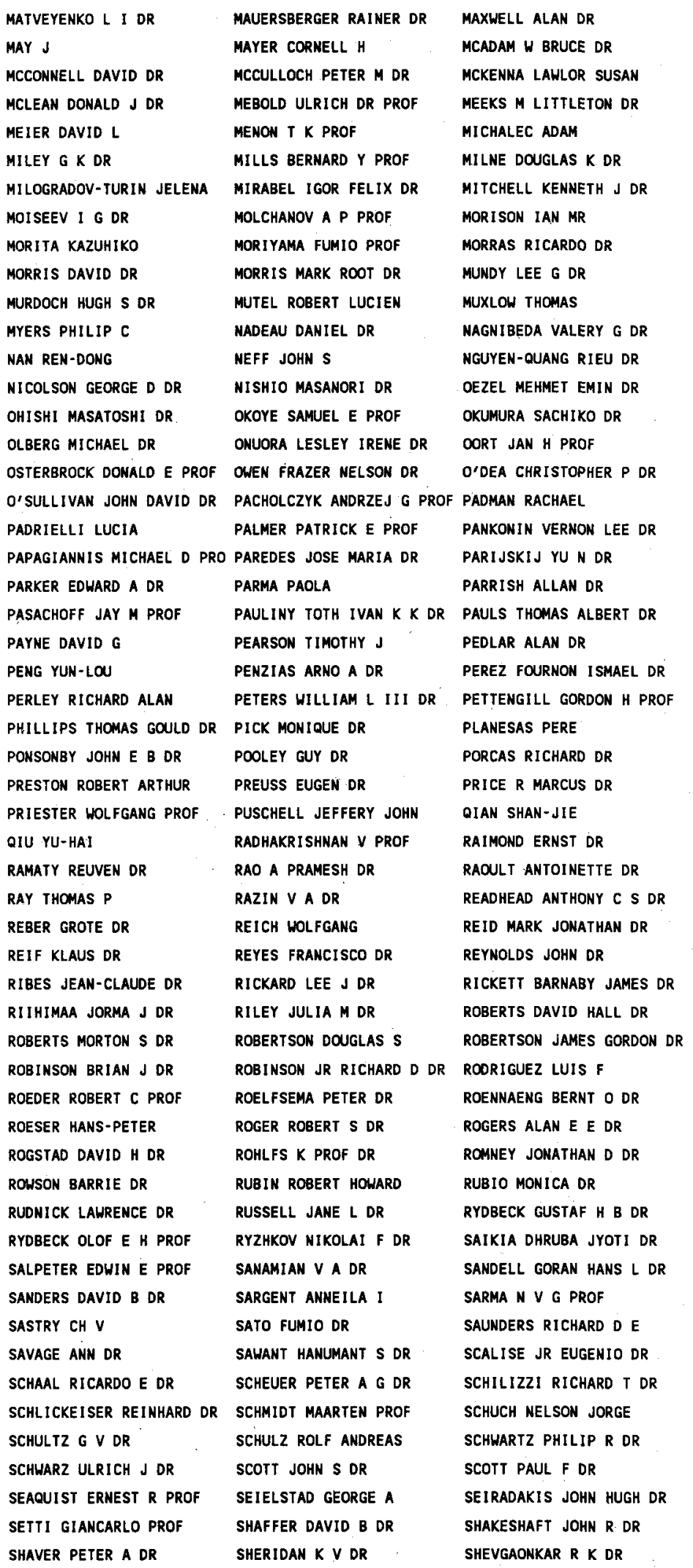




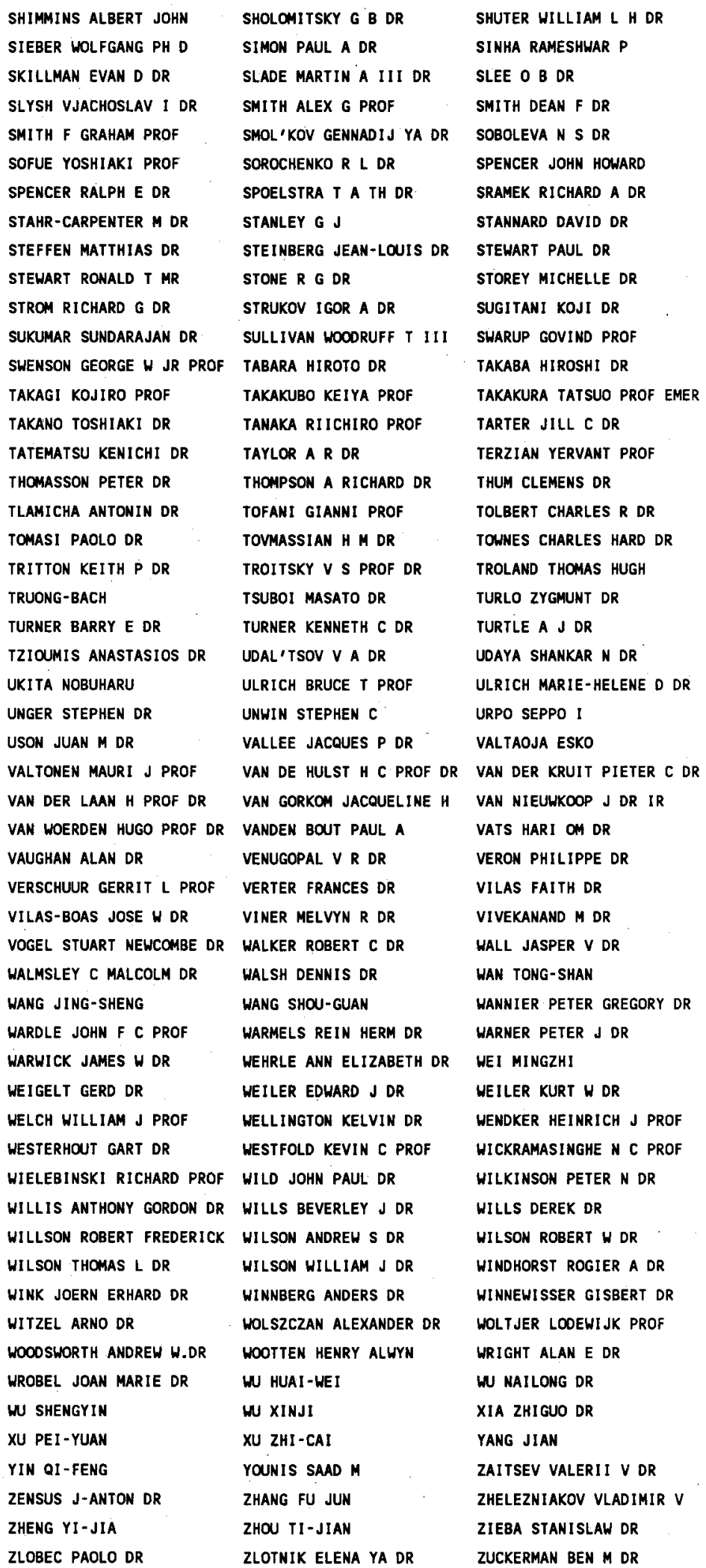


COMPOSITION OF COMMISSION 4 ! 1991-1994

$\begin{array}{ll}\text { President } & \text { : DEBARBAT SUZANNE V DR } \\ \text { Vice-President(s) } & \text { : ANSARI S M RAZAULLAH PROF }\end{array}$

Organizing Committee: DICK STEVEN J

FIRNEIS MARIA G DR

MOESGAARD KRISTIAN P

NORTH JOHN DAVID PROF

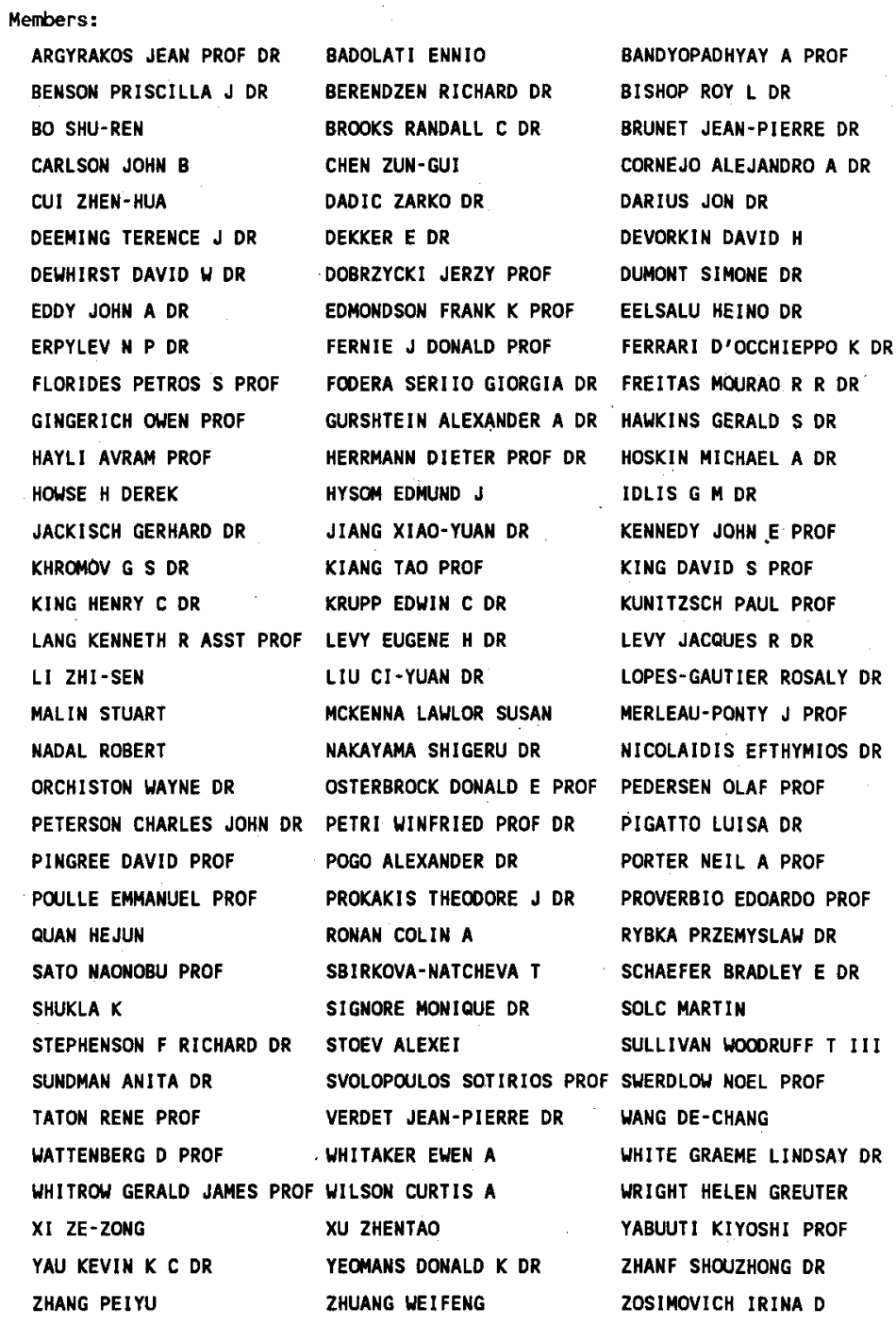




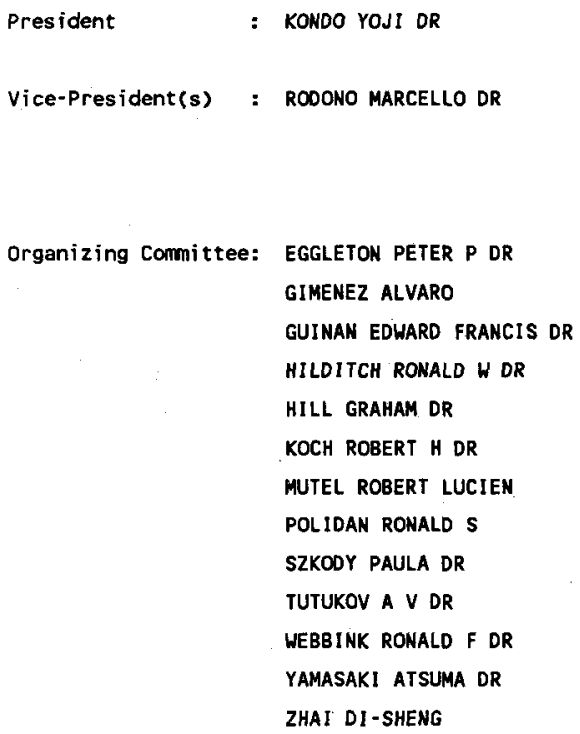

$$
\text { FERRER OSVALDO EDUARDO DR }
$$
firmani claudio a prof FREDRICK LAURENCE $W$ PROF FRIEDJUNG MICHAEL DR GARMANY CATHERINE D DR GIOVANNELLI FRANCO DR GRYGAR JIRI DR GEYER EDUARD H PROF DR GIURICIN GIULIANO

ANDERSEN JOHANNES AQUILANO ROBERTO OSCAR DR BARTOLINI CORRADO BATTEN ALAN H DR BLITZSTEIN WILLIAM DR BOOKMYER BEVERLY B DR BRANDI ELISANDE ESTELA DR BROWNLEE ROBERT R DR BUNWER ALAN N DR CATALANO SANTO DR CHAMMUGAM GANESAR PROF CHEN KWAN-YU PROF CHOI KYU-HONG CLAUSEN JENS VIGGO LEKTOR CRISTALDI SALVATORE DR DE GRDOT MART DR DELGADO ANTONIO JESUS DOUGHTY NOEL A DR DURISEN RICHARD H DR EATON JOEL A DR FAULKNER JOHN PROF FERRARI D'OCCHIEPPO $K$ DR FLANNERY BRIAN PAUL DR FRANTSMAN YU L DR GARCIA DE MARIA J M DR GIANNONE PIETRO PROF GOLDMAN ITZHAK DR GULLIVER AUSTIN FRASER DR GURSKY HERBERT OR 


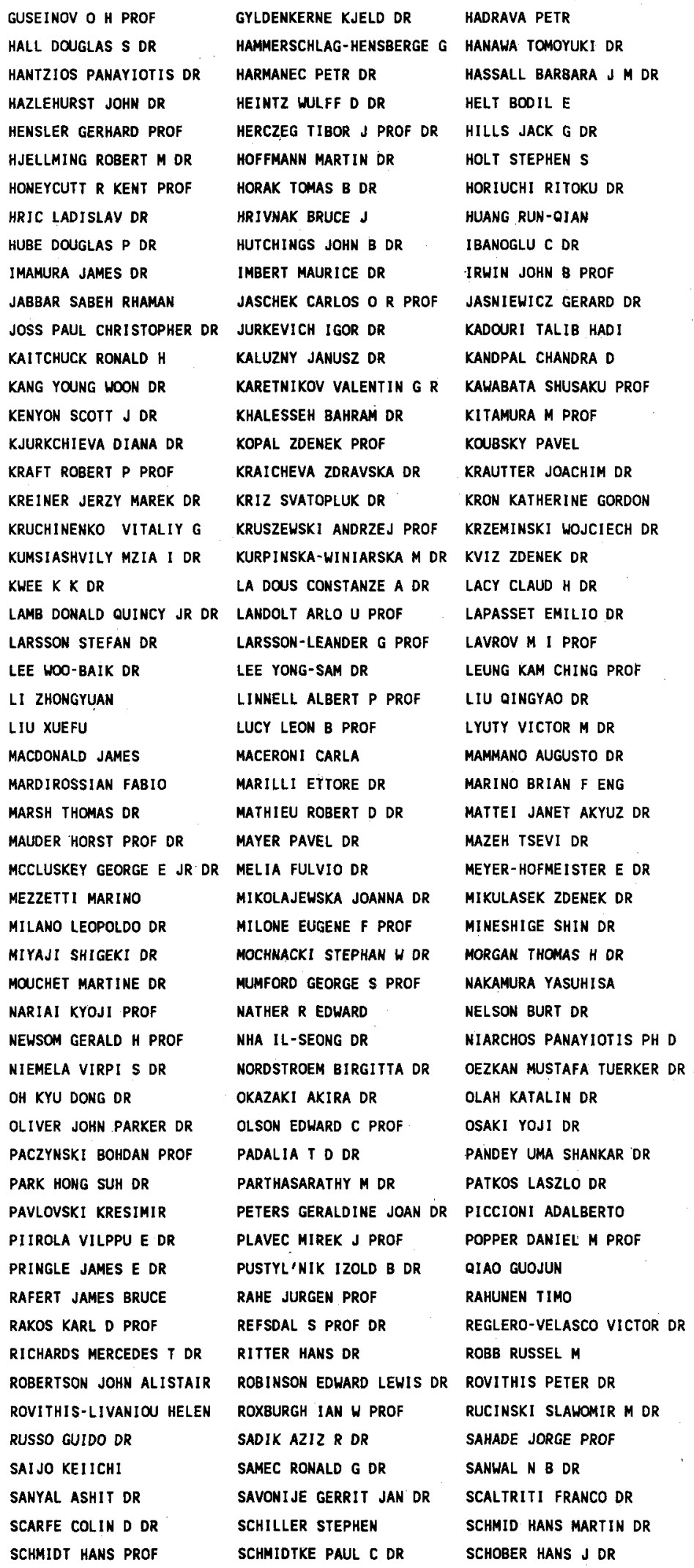




\begin{tabular}{|c|c|c|}
\hline $\begin{array}{l}\text { SCHOEFFEL EBERHARD F DR } \\
\text { SHAFTER ALLEN W DR }\end{array}$ & $\begin{array}{l}\text { SEGGEWISS WILHELM PROF } \\
\text { SHAKURA NICHOLAJ I DR }\end{array}$ & $\begin{array}{l}\text { SEMENIUK IRENA DR } \\
\text { SHAVIV GIORA PROF }\end{array}$ \\
\hline SHEN LIANG-ZHAO & SHU FRANK H PROF & SHUL'BERG A M DR \\
\hline SIMA ZDISLAV DR & SIMMONS JOHN FRANCIS L & SINVHAL SHAMBHU DAYAL DR \\
\hline SION EDWARD MICHAEL & SISTERO ROBERTO F DR & SLOVAK MARK HAINES DR \\
\hline SMAK JOSEPH I PROF & SMITH ROBERT CONNON DR & SOBIESKI STANLEY DR \\
\hline SOEDERHJELM STAFFAN DR & SOLHEIM JAN ERIK & SPARKS HARREN M DR \\
\hline SRIVASTAVA J B DR & SRIVASTAVA RAM KUMAR DR & STAGG CHRISTOPHER DR \\
\hline STARRFIELD SUMNER PROF & STEEL DUNCAN I DR & STEIMAN-CAMERON THOMAS DR \\
\hline STEINER JOAO E DR & STENCEL ROBERT EDWARD & STROHMEIER WOLFGANG PROF. \\
\hline SUGIMOTO DAIICHIRO PROF & SUNDMAN ANITA DR & SVECHNIKOVA MARIA A DR \\
\hline SZABADOS LASZLO PH D & SZAFRANIEC ROZALIA DR & TAAM RONALD EVERETT DR \\
\hline TAN HUISONG & TEAYS TERRY J DR & THOMPSON KEITH DR \\
\hline TODORAN IOAN DR & TOUT CHRISTOPHER DR & TREMKO JOZEF DR \\
\hline TRIMBLE VIRGINIA L DR & URECHE VASILE DR & VAN DEN HEUVEL EDWARD $P J$ \\
\hline VAN HAMME WALTER & VAN PARADIJS JOHANNES DR & VAN'T VEER FRANS DR \\
\hline VAZ LUIZ PAULO RIBEIRO & VETESNIK MIROSLAV DR & WADE RICHARD ALAN DR \\
\hline WALKER RICHARD L & WALKER WILLIAM S G & WALTER KURT PROF DR \\
\hline WARD MARTIN JOHN & WARGAU WALTER F DR & WARNER BRIAN PROF \\
\hline WEHLAU WILLIAM H PROF & WEIGERT ALFRED PROF & WEILER EDWARD J DR \\
\hline WELLLMANN PETER PROF DR & WESSELINK ADRIAAN J DR & WEST DONALD K DR \\
\hline WHEELER J CRAIG PROF & WILLIAMON RICHARD M & WILLIAMS GLEN A DR \\
\hline WILLIAMS ROBERT E DR & WILSON ROBERT E PROF & WOOD DAVID B DR \\
\hline WOOD F BRADSHAW PROF & WOOD JANET H DR & WRIGHT KENNETH O DR \\
\hline ZEILIK MICHAEL II DR & ZHANG ER-HO DR & ZHANG JINTONG \\
\hline ZHOU DAOQI & ZHOU HONG-NAN & ZHU CI-SHENG \\
\hline ZIOLKOWSKI JANUSZ DR & ZUIDERHI JK EDHARDUS. J & ZWITTER TOMAZ \\
\hline
\end{tabular}


COMPOSITION OF COMMISSION $44 \quad 1991-1994$

\begin{abstract}
President $\quad$ : TRUEMPER JOACHIM PROF
Vice-President(s) : FAZIO GIOVANHI G DR
\end{abstract}

Organizing Committee: - BURKE BERNARD F DR

HUTCHINGS JOHN B DR

JENKINS EDHARD B DR

JORDAN STUART D DR

SAVAGE BLAIR D DR

HAMSTEKER WILLEM DR

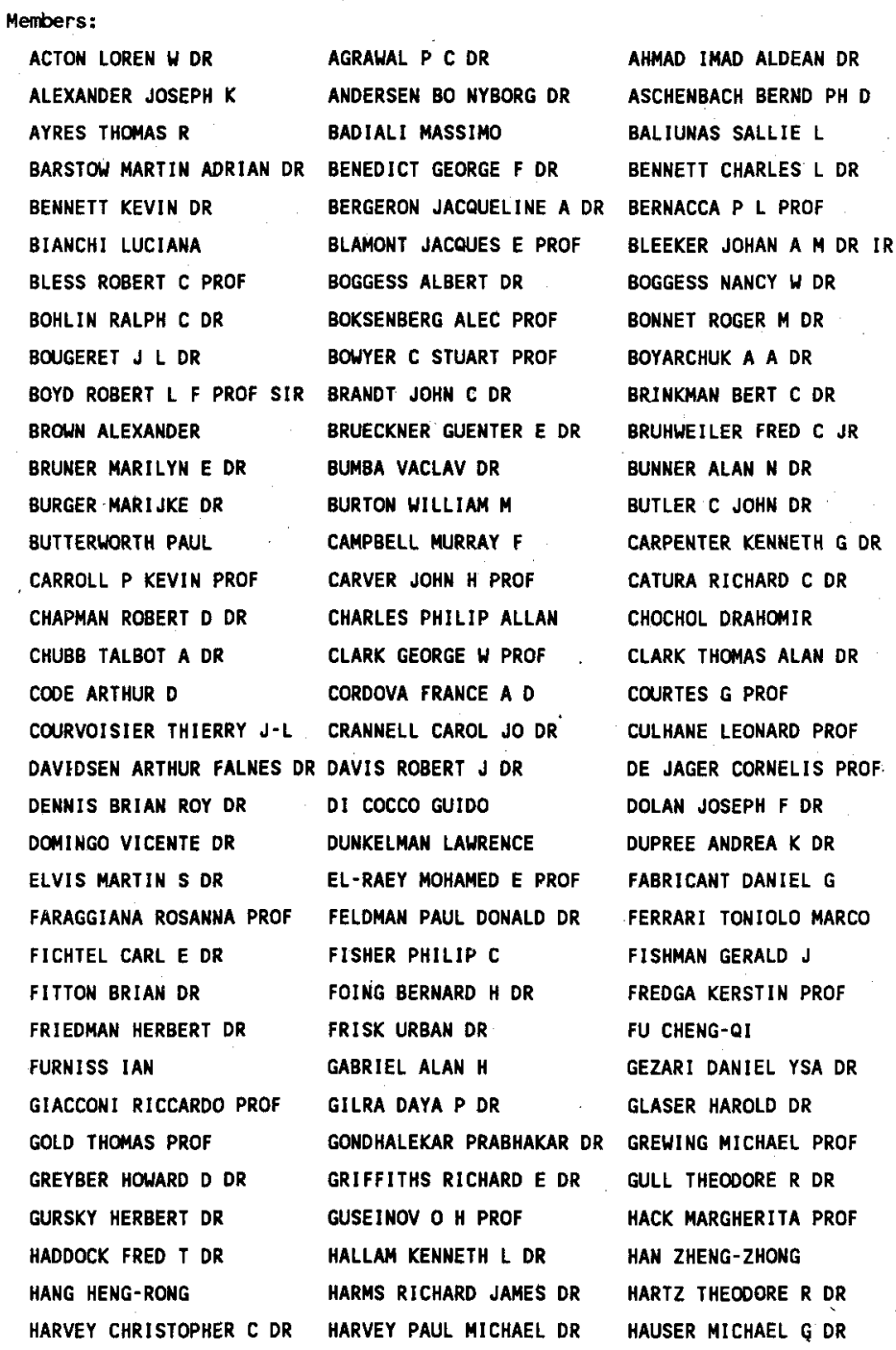




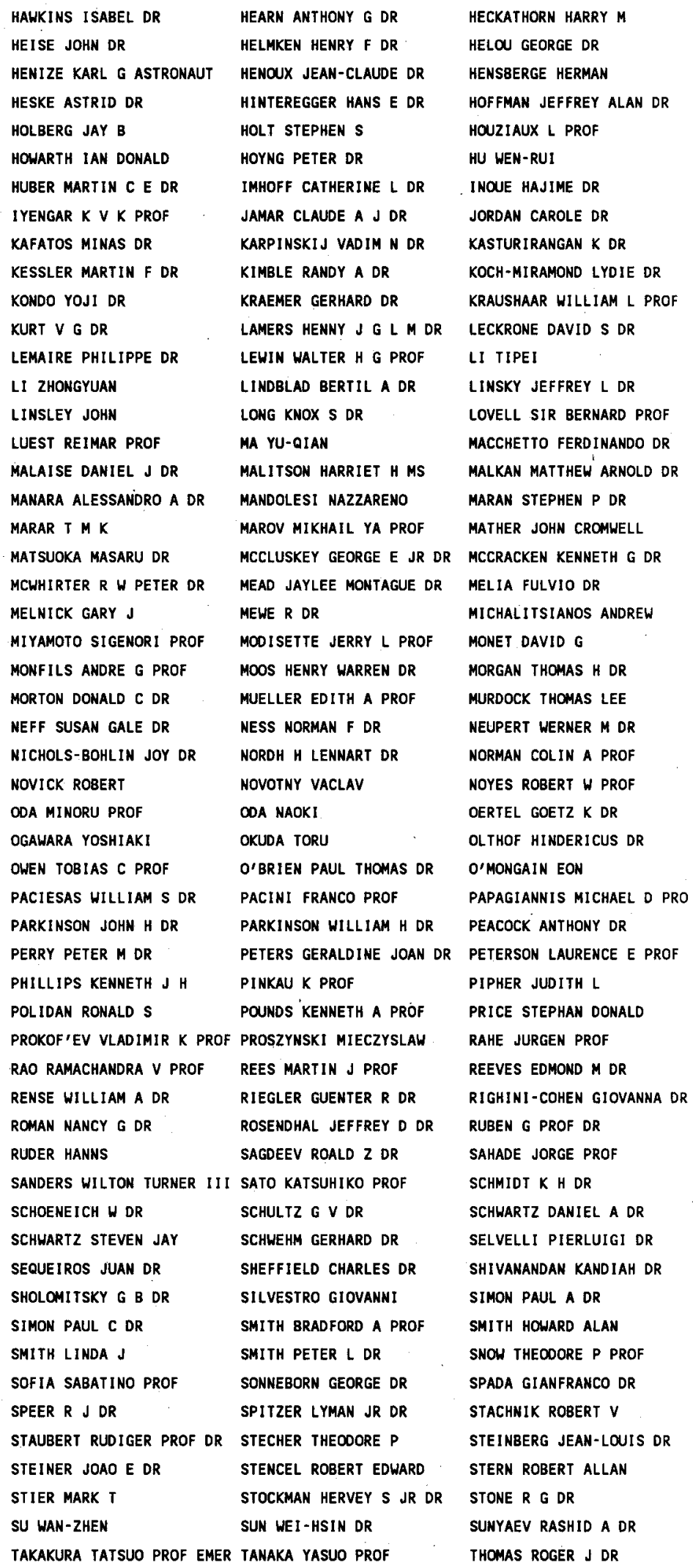


TOVMASSIAN H M DR UNDERHILL ANME B DR VALNICEK BORIS DR VAN DE HULST H C PROF DR VAN DER HUCHT KAREL A VAN SPEYBROECK LEON P DR VIAL JEAN-CLAUDE VILHU OSMI DR WALSH DENNIS DR WEHRLE ANY ELIZABETH DR WEINBERG I L DR WILLIS ALLAN J DR WINKLER CHRISTOPH DR WUNER GUENTER ZOMBECK MARTIN $V$ DR
TRAUB WESLEY ARTHUR VALTONEN MAURI J PROF VIOTTI ROBERTO DR WANG SHUI WEILER EDWARD J DR MESSELIUS PAUL $R$ DR HILLNER STEVEN PAUL DR WRAY JAMES D DR YAMASHITA KOJUN DR ZOU HUI-CHENG UNDERHOOD JAMES H DR
TSUNEMI HIROSHI DR UPSON HALTER L II OR VAN BEEK FRANK PROF DR VAN DUINEN $R$ J DR VIDAL-MADJAR ALFRED DR VRTILEK SAEQA DIL DR WARNER JOHN W DR WEILER KURT W DR WESTPHAL JAMES A PROF WILSON ROBERT PROF SIR WU CHI CHAO DR ZARNECKI JAN CHARLES DR 


\author{
President : MACCONNELL DARRELL J DR \\ Vice-President(s) : LEVATO ORLANDO HUGO DR
}

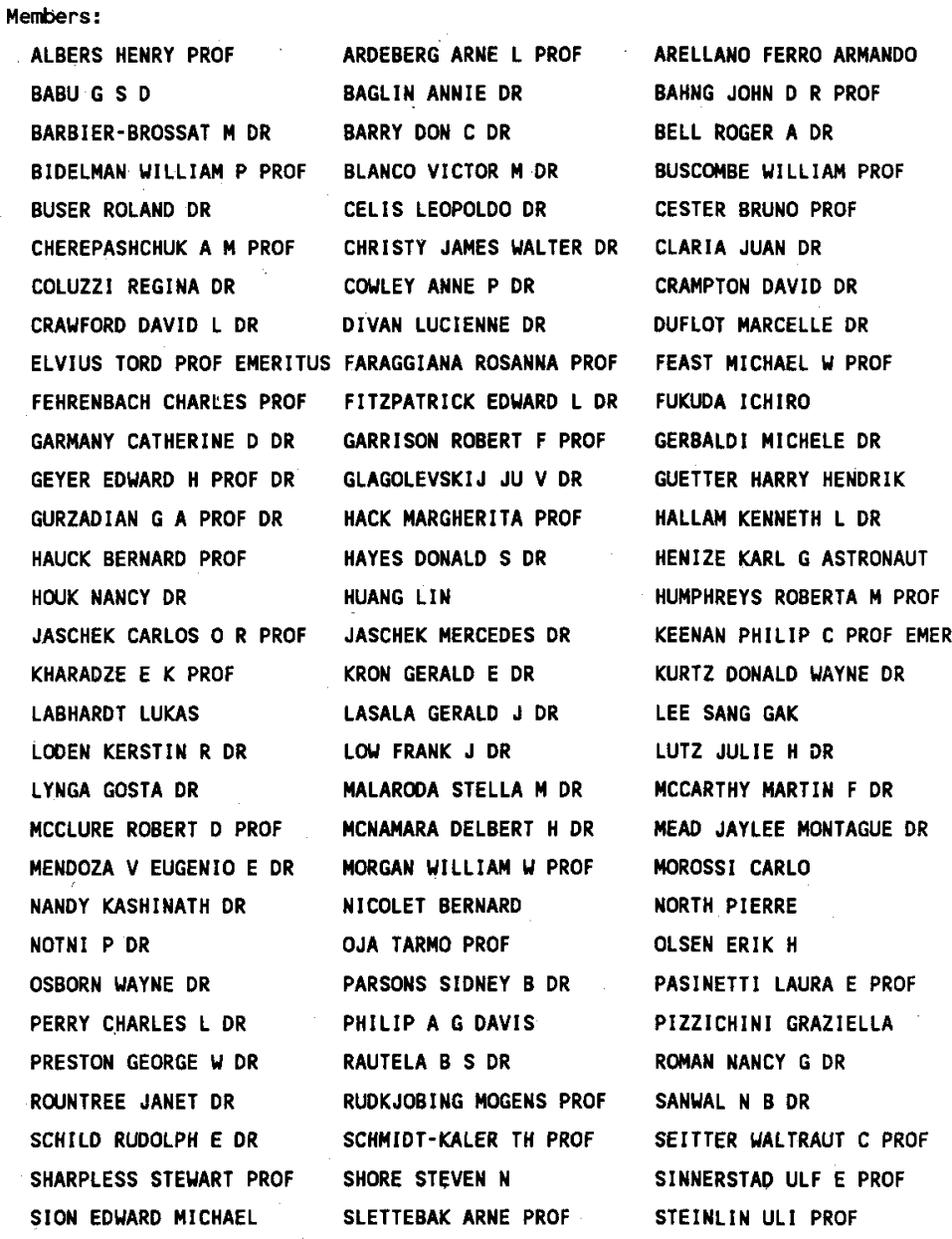


STEPHENSON C BRUCE PROF

STROBEL ANDRZEJ DR

WARREN WAYNE $H$ JR DR

WILLIAMS JOHN A DR

YAMASHITA YASUMASA PROF
STOCK JURGEN D

UPGREN ARTHUR R DR

WESSELIUS PAUL $R$ DR

WU HSIN-HENG DR

YOSS KENNETH M DR
STRAIZYS V PROF DR

WALKER GORDON A H PROF

WESTERLUND BENGT E PROF

WYCKOFF SUSAN DR 
M92

COMPOSITION OF COMMISSION 46 1991-1994

$\begin{array}{ll}\text { President } & \text { : GOUGUENHEIM LUCIENNE } \\ \text { Vice-President(s) : PERCY JOHN R PROF }\end{array}$

Organizing Committee: FIERRO JULIETA

GERBALDI MICHELE DR

HOUZIAUX L PROF

ISOBE SYUZO DR

IWANISZEUSKA CECYLIA DR

PASACHOFF JAY M PROF

ROBBINS R ROBERT PROF

SANDQVIST AAGE DR

MENTZEL DONAT G DR

Members:

\begin{tabular}{|c|c|c|}
\hline ACKER AGNES PROF DR & AIAD A PROF & ANDRILLAT HENRI L PROF \\
\hline ANSARI S M RAZAULLLAH ṔROF & BACALOV MIHAIL & BENSON PRISCILLAA J DR \\
\hline BRAES L L E DR & BRIEVA EDUARDO PROF & BROSCH NOAH DR \\
\hline BRUCK MARY T DR & BUDDING EDWIN DR & BUSCOMBE WILLIAM PROF \\
\hline CALVET NURIA DR & CATALA POCH M A & CHAMBERLAIN JOSEPH M DR \\
\hline COOINA LANDABERRY SAYD J & COUPER HEATHER MISS & CUI ZHEN-HUA \\
\hline DARIUS JON DR & DUPUY DAVID L DR & DUVAL MARIE-FRANCE \\
\hline EMERSON DAVID & FAIRALL ANTHONY P PROF & FENG KE-JIA \\
\hline FERNANDEZ JUL IO A DR & FERNANDEZ -FIGUEROA M J DR & FERRAZ-MELLO S PROF DR \\
\hline FIENBERG RICHARD T DR & FLECK ROBERT CHARLES DR & GALLINO ROBERTO \\
\hline GINGERICH OWEN PROF & GURM HARDEV S PROF & HAUPT HERMANN F PROF \\
\hline HAYHOOD J & HEMENHAY MARY KAY M DR & HEUDIER JEAN-LOUIS DR \\
\hline HIDAJAT BAMBANG PROF DR & HOFF DARREL BARTON & ILYAS MOHAMMAD DR \\
\hline IMPEY CHRISTOPHER D DR & JARRETT ALAN H PROF & JONES BARRIE $W$ DR \\
\hline $\begin{array}{l}\text { KELLER HUWE ULRICH DR } \\
\text { KONONOVICH EDWARD V DR }\end{array}$ & $\begin{array}{l}\text { KENNEDY JOHN E PROF } \\
\text { KOURGANOFF VLADIMIR PROF }\end{array}$ & $\begin{array}{l}\text { KITCHIN CHRISTOPHER R DR } \\
\text { KREINER JERZY MAREK DR }\end{array}$ \\
\hline KRUPP EDWIN $C$ & LAGO MARIA TERESA $\vee T$ PR & LAI SEBASTIANA \\
\hline LI QI-BIN & LITTLE-MARENIN IRENE R DR & LOMB NICHOLAS RALPH DR \\
\hline MA XING-YUAN & MACIEL WALTER J DR & MADDISON RONALD CH DR \\
\hline MARSH JULIAN C & MARTINET LOUIS PROF & MAVRIDIS L N PROF \\
\hline MAZA JOSE & MCCARTHY MARTIN F DR & MCNALLY DEREK DR \\
\hline MEIDAV MEIR DR & MOMCHEV GOSPOOIN & MOREELS GUY DR \\
\hline MUZZIO JUAN C PROF & NARLIKAR JAYANT $V$ PROF & NHA IL-SEONG DR \\
\hline NICOLOV NIKOLAI S DR & NOELS ARLETTE DR & OJA HEIKKI DR \\
\hline OKOYE SAMUEL E PROF & OLSEN FOGH H J & ONUORA LESLEY IRENE DR \\
\hline OSBORN WAYNE DR & OSORIO JOSE & OTHMAN MAZLAN \\
\hline OWAKI NAOAKI DR & PANDEY UMA SHANKAR DR & PARISOT JEAN-PAUL \\
\hline POKORNY ZDENEK DR & PRABHAKARAN NAYAR S R DR & PROVERBIO EDOARDO PROF \\
\hline RAMADURAI SOURIRAJA DR & ROBINSON LEIF J & RODGERS ALEX W DR \\
\hline ROSLUND CURT DR & CHIE E PROF & - SAFKO JOHN L \\
\hline SANAHUJAA BLAS & SAXENA P P DR & SBIRKOVA-NATCHEVA \\
\hline
\end{tabular}


SCHLEICHER DAVID G DR SCHMITTER EDWARD F DR SHEN CHUN-SHAN

SOLHEIM JAN ERIK STOEV ALEXE I

SZECSENYI-NAGY GABOR DR TORRES-PEIMBERT SILVIA DR TROCHE-BOGGINO A E DR VLADIMIROV SIMEON WEST RICHARD M DR ZEALEY WILLIAM J DR
SCHLOSSER WOLFHARD PROF SCHROEDER DANIEL J PROF SHIPMAN HENRY L DR STEFL VLADIMIR SUKARTADIRED JA DARSA SZOSTAK ROLAND DR WUJWOVIC VLADIS DR WILLIAMON RICHARD M ZEILIK MICHAEL II DR
SCHMIDT THOMAS DR SEEDS MICHAEL AUGUST DR SIROKY JAROMIR DR STENHOLM BJOERN DR SVESTKA JIRI DR TABORDA JOSE ROSA DR VAUCLAIR SYLVIE D DR WANG SHUNDE DR WOO JONG OK ZIMMERMANN HELMUT DR 


\begin{tabular}{|c|c|}
\hline ARGENTINA & FEINSTEIN ALEJANDRO DR \\
\hline AUSTRALIA & RODGERS ALEX $W$ DR \\
\hline AUSTRIA & HANSLMEIER ARNOLD \\
\hline BELGIUM & NOELS ARLETTE DR \\
\hline BRAZIL & MACIEL WALIER J DR \\
\hline BULGARIA & NICOLOV NIKOLAI S OR \\
\hline CANADA & BOCHONKO D RICHARD DR \\
\hline CHILE & MAZA JOSE \\
\hline CHINA PR & FENG KE-JIA \\
\hline CHINA R & SHEN CHUN-SHAN \\
\hline COLOMBIA & BRIEVA EDUARDO PROF \\
\hline CZECHOSLOVAKIA & SIROKY JAROMIR DR \\
\hline DENMARK & OLSEN FOGH H J \\
\hline EGYPT & AIAD A PROF \\
\hline FINLAND & OJA HEIKKI DR \\
\hline FRANCE & GOUGUENHEIM LUCIENME \\
\hline GERMANY & SCHLOSSER WOLFHARD PROF \\
\hline GREECE & MAVRIDIS L N PROF \\
\hline HUNGARY & SZECSENYI-NAGY GABOR DR \\
\hline INDIA & RAMADURAI SOURIRAJA DR \\
\hline I NDONES IA & HIDAJAT BAMBANG PROF DR \\
\hline IRELAND & HAYWOOD J \\
\hline ITALY & PROVERBIO EDOARDO PROF \\
\hline JAPAN & ISOBE SYUZO DR \\
\hline KOREA $R$ & WOO JONG OK \\
\hline MALAYSIA & OTHMAN MAZLAN \\
\hline MEXICO & TORRES-PEIMBERT SILVIA OR \\
\hline NETHERLANDS & BRAES $L L E D R$ \\
\hline NEW ZEALAND & BUDDING EDWIN DR \\
\hline NIGERIA & OKOYE SAMUEL E PROF \\
\hline NORWAY & SOLHEIM JAN ERIK \\
\hline PARAGUAY & TROCHE-BOGGINO A E DR \\
\hline POLAND & IWANISZEWSKA CECYLIA DR \\
\hline PORTUGAL & OSORIO JOSE J S P PROF \\
\hline RUMANIA & BOTEZ ELVIRA DR \\
\hline SOUTH AFRICA & FAIRALL ANTHONY P PROF \\
\hline SPAIN & CATALA POCH M A \\
\hline SWEDEN & SANDQVIST AAGE DR \\
\hline SWITZERLAND & MARTINET LOUIS PROF \\
\hline UK & CLARKE DAVID OR \\
\hline URUGUAY & FERMANDEZ JULIO A DR \\
\hline USA & PASACHOFF JAY M PROF \\
\hline VATICAN CITY STATE & MCCARTHY MARTIN F DR \\
\hline VENEZUELA & CALVET NURIA DR \\
\hline YUGOSLAVIA & MILOGRADOV-TURIN JELENA \\
\hline (EX USSR) & KONONOVICH EDWARD $\vee D R$ \\
\hline
\end{tabular}


COMPOSITION OF COMMISSION $47 \quad 1991-1994$

President : PARTRIDGE ROBERT B PROF

Vice-President(s) : NARLIKAR JAYANT V PROF

Organizing Committee: BOND JOHN RICHARD

DRESSLER ALAN

GELLER MARGARET JOAN.

JAUNCEY DAVID L. DR

REES MARTIN J PROF

REEVES HUBERT PROF

SATO KATSUHIKO PROF

SHANDARIN SERGEI F DR

SHAVER PETER A DR

SZALAY ALEX DR

Members:

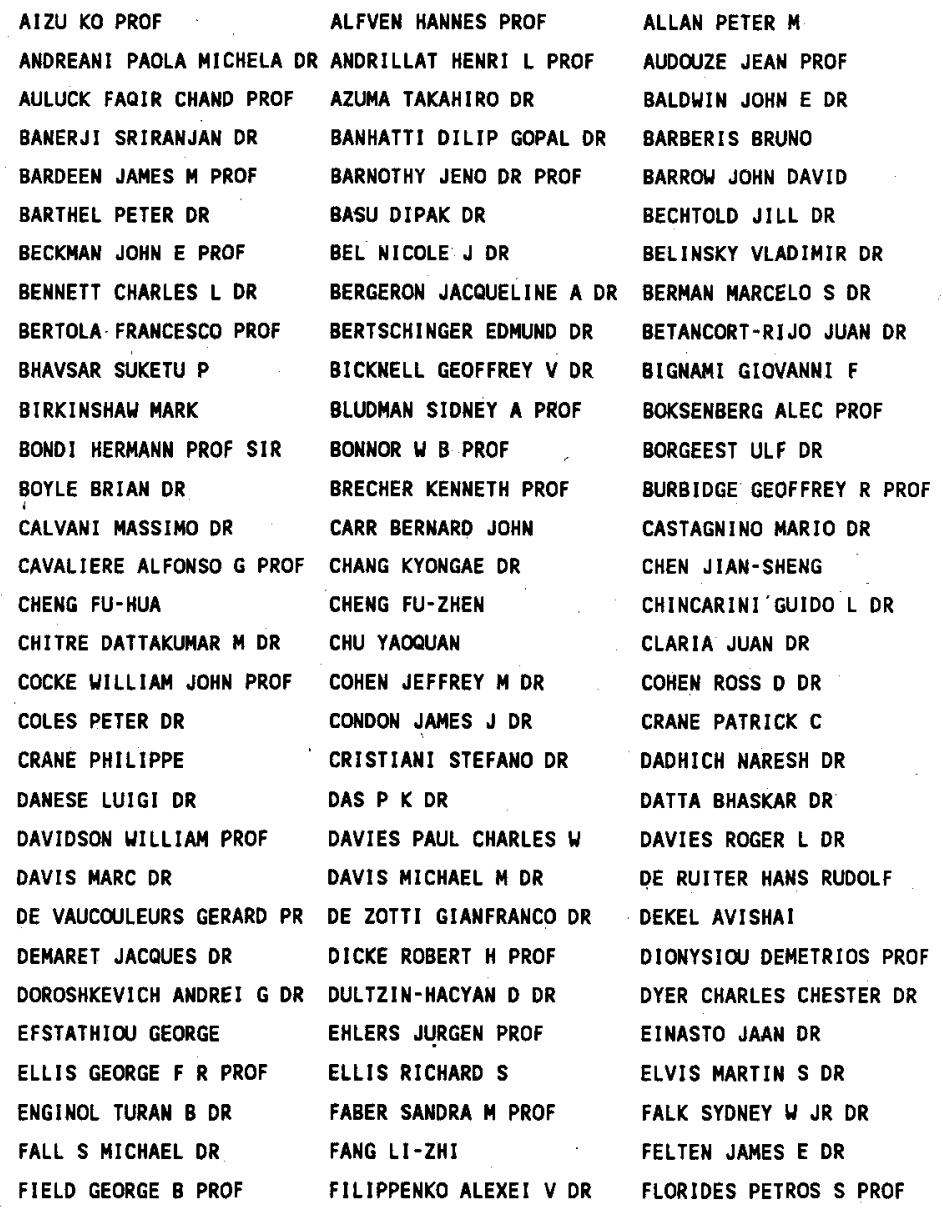




\begin{tabular}{|c|c|c|}
\hline $\begin{array}{l}\text { FOCARDI PAOLA OR } \\
\text { FORMAN WILLIAM RICHARD DR }\end{array}$ & $\begin{array}{l}\text { FONG RICHARD } \\
\text { FOUQUE PASCAL DR }\end{array}$ & $\begin{array}{l}\text { FORD HOLLAND C RES PROF } \\
\text { FRANCESCHINI ALBERTO }\end{array}$ \\
\hline FRENK CARLOS $\$$ & FUJIMOTO MITSUAKI DR & FUKUGITA MASATAKA DR \\
\hline FUKUI TAKAO DR & GALLETTO DIONIGI & GARRISON ROBERT F PROF \\
\hline GIALLONGO EMANUELE DR & GIURICIN GIULIANO & GOOART ODON PROF \\
\hline GOLD THOMAS PROF & GOLOSMITH DONALD W OR & GONG SHU-MO \\
\hline GORET PHILIPPE DR & GOSSET ERIC DR & GOUDA NAOTERU DR \\
\hline GREGORY STEPHEN ALBERT DR & GREYBER HONARD D DR & GRISHCHUK L P DR \\
\hline GUDMUNDSSON EINAR H & GUNN JAMES E PROF & HACYAN SHAHEN DR \\
\hline HAGEN HANS-JUERGEN DR & MARA KEN NOSUKE DR & HARDY EDUARDO \\
\hline HARMS RICHARD JAMES DR & HARRISON EDWARD R PROF & HAWKING STEPHEN $U$ PROF \\
\hline HAYASHI CHUSHIRO PROF & HE XIANG-TAO & HEAVENS ALAN DR \\
\hline HEIDMANN JEAN DR & HELLER MICHAEL PROF & HEWETT PAUL. \\
\hline HEWITT ADELAIDE & HOYLE FRED SIR & HU ESTHER M DR \\
\hline HUCHRA JOHN PETER DR & ICKE VINCENT DR & IKEUCHI SATORU DR \\
\hline IMPEY CHRISTOPHER D DR & ISHIHARA HIDEKI DR & IYER B R DR \\
\hline JAROSZYNSKI MICHAL & JIANG SHUDING & JONES BERMARD $J T$ DR \\
\hline JOSHI MOHAN N PROF & JUNKKARINEN VESA T DR & JUSZKIEWICZ ROMAN \\
\hline KANDRUP HENRY EMIL DR & KAPOOR RAMESH CHANDER & KARACHENTSEV I O DR \\
\hline KASPER U DR & KATO SHOJI PROF & KAWABATA KINAKI PROF \\
\hline KAYSER RAINER DR & KELLERMANN KENNETH I DR & KEMBHAVI AJIT $\mathrm{K}$ \\
\hline KIM JIK SU & KOOAMA HIDEO & KOKKOTAS KONSTANTINOS DR \\
\hline KOLB EDWARD W DR & KOO DAVID C-Y DR & KORMENDY JOHN DR \\
\hline KOVETZ ATTAY PROF & KOZLOVSKY B Z DR & KRASINSKI ANDRZEJ PROF \\
\hline KRISS GERARD A DR & KUNTH DANIEL & KUSTAANHEIMO PAUL E PROF \\
\hline LACEY CEDRIC DR & LACHIEZE-REY MARC & LAHAV OFER DR \\
\hline LAKE KAYLL WILLIAM DR & LASOTA JEAN-PIERRE DR & LAUSBERG ANDRE DR \\
\hline LAYZER DAVID PROF & LEQUEUX JAMES DR & LI ZHI-FANG \\
\hline LIEBSCHER DIERCK-E DR & LILJE PER VIDAR BARTH DR & LILLY SIMON J DR \\
\hline LIU LIAO & LIU YONG-ZHEN & LONGAIR M S PROF \\
\hline LONSDALE CAROL J DR & LU TAN & LUCCHIN FRANCESCO \\
\hline LUMINET JEAN-PIERRE & LYNDEN-BELL DONALD PROF & MACCALLUM MALCOLM A H \\
\hline MADDOX STEPHEN DR & MAEDA KEI-ICHI DR & MANDOLESI NAZZARENO \\
\hline MANOZHOS ANOREJ $V$ OR & MARANO BRUNO & MAROIROSSIAN FABIO \\
\hline MAREK JOHN & MARTINEZ-GONZALEZ E DR & MATERNE JUERGEN DR \\
\hline MATHER JOHN CROMWELL' & MATSUMOTO TOSHIO DR & MATZNER RICHARD A PROF \\
\hline MAVRIDES STAMATIA DR & MCCREA J DERMOTT & MCCREA WILLIAM SIR \\
\hline MELOTT ADRIAN L PROF & MERAT PARVIZ & MERIGHI ROBERTO DR \\
\hline MESZAROS ATTILA DR & MESZAROS PETER DR & MEYER DAVID M DR \\
\hline MEYLAN GEORGES DR & MEZZEITI MARINO & MISNER CHARLES W PROF \\
\hline MORRISON PHILIP PROF & MULLER RICHARD A & NAMBU YASUSADA DR \\
\hline NARASIMHA DELAMPADY DR & NEEMAN YUVAL PROF & NISHIDA MINORU PROF \\
\hline NOERDLINGER PETER D PROF & NOONAN THOMAS W PROF & NORMAN COLIN A PROF \\
\hline NOTIALE LAURENT & NOVELLO MARIO DR & NOVIKOV I D DR \\
\hline NOVOTNY JAN DR & OEMLER AUGUSTUS JR DR & OKOYE SAMUEL E PROF \\
\hline OLOWIN RONALD PAUL DR & OMNES ROLAND PROF & ONUORA LESLEY IRENE DR \\
\hline CORT JAN H PROF & OZERNOY LEONID M PROF & OZSVATH I PROF \\
\hline O'CONNELL ROBERT WEST DR & PACHNER JAROSLAV PROF & PADMANABHAN I DR \\
\hline PADRIELLI LUCIA & PAGE DON NELSON & ONG-SHI \\
\hline PEACOCK JOHN ANDREW & PECKER JEAN-CLAUDE PROF & PEEBLES P JAMES E \\
\hline PENZIAS ARNO A DR & PERRYMAN MICHAEL A C DR & PERSIDES SOTIRIOS C \\
\hline PETERSON BRUCE A DR & PETROSIAN VAHE PROF & PRESS UILLIAM H DR \\
\hline PUGET JEAN-LOUP DR & QU QI & RAMELLA MASSIMO \\
\hline RAULINGS STEVEN DR & RAYCHAUDHURI AMALKUMAR DR & REFSDAL S PROF DR \\
\hline RINDLER WOLFGANG PROF & RIVOLO ARTHUR REX & ROBERTS DAVID HALL DR \\
\hline
\end{tabular}




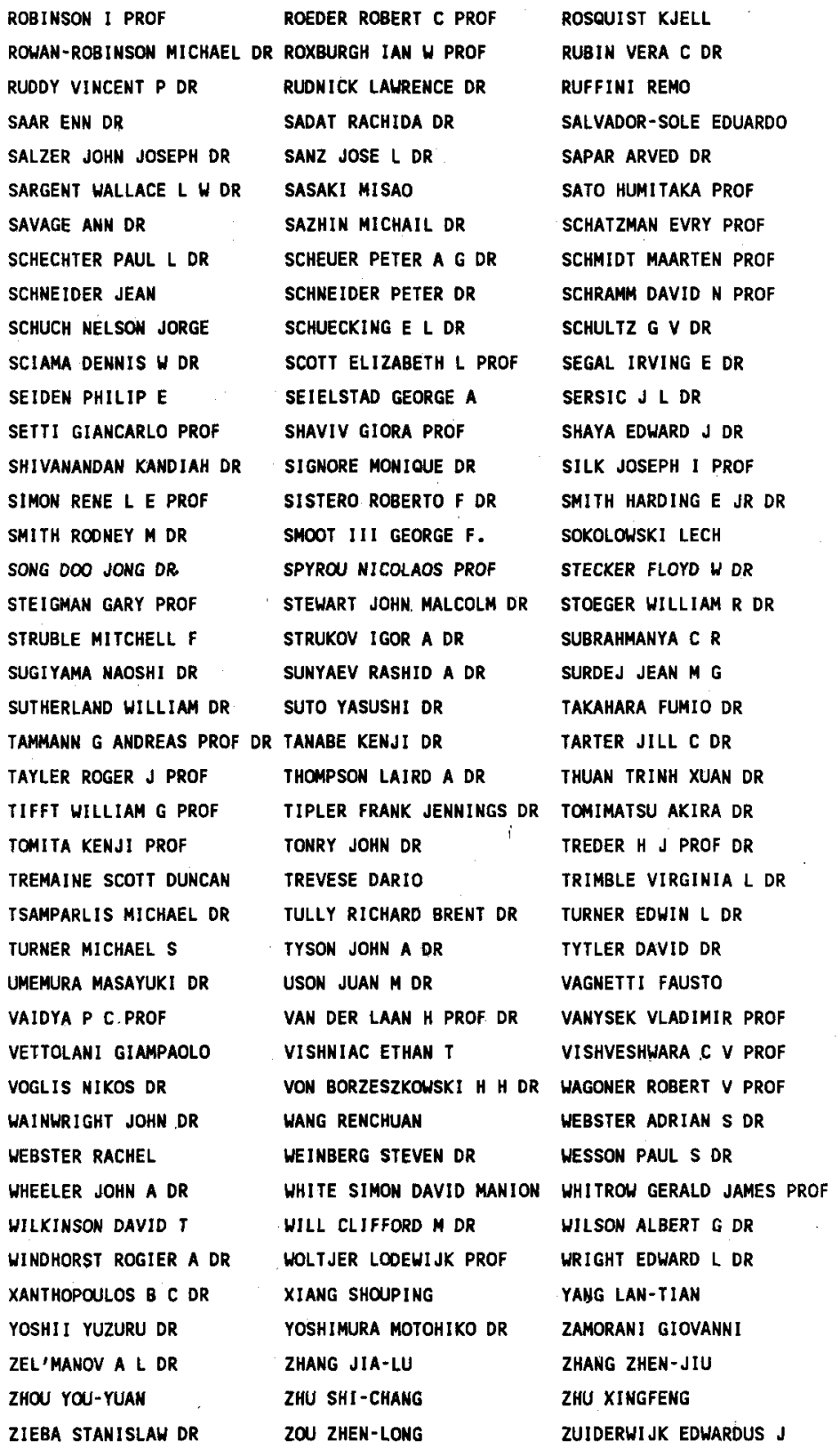




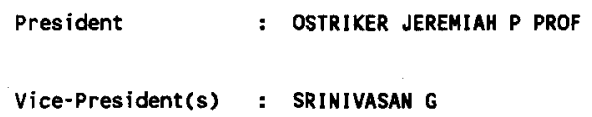

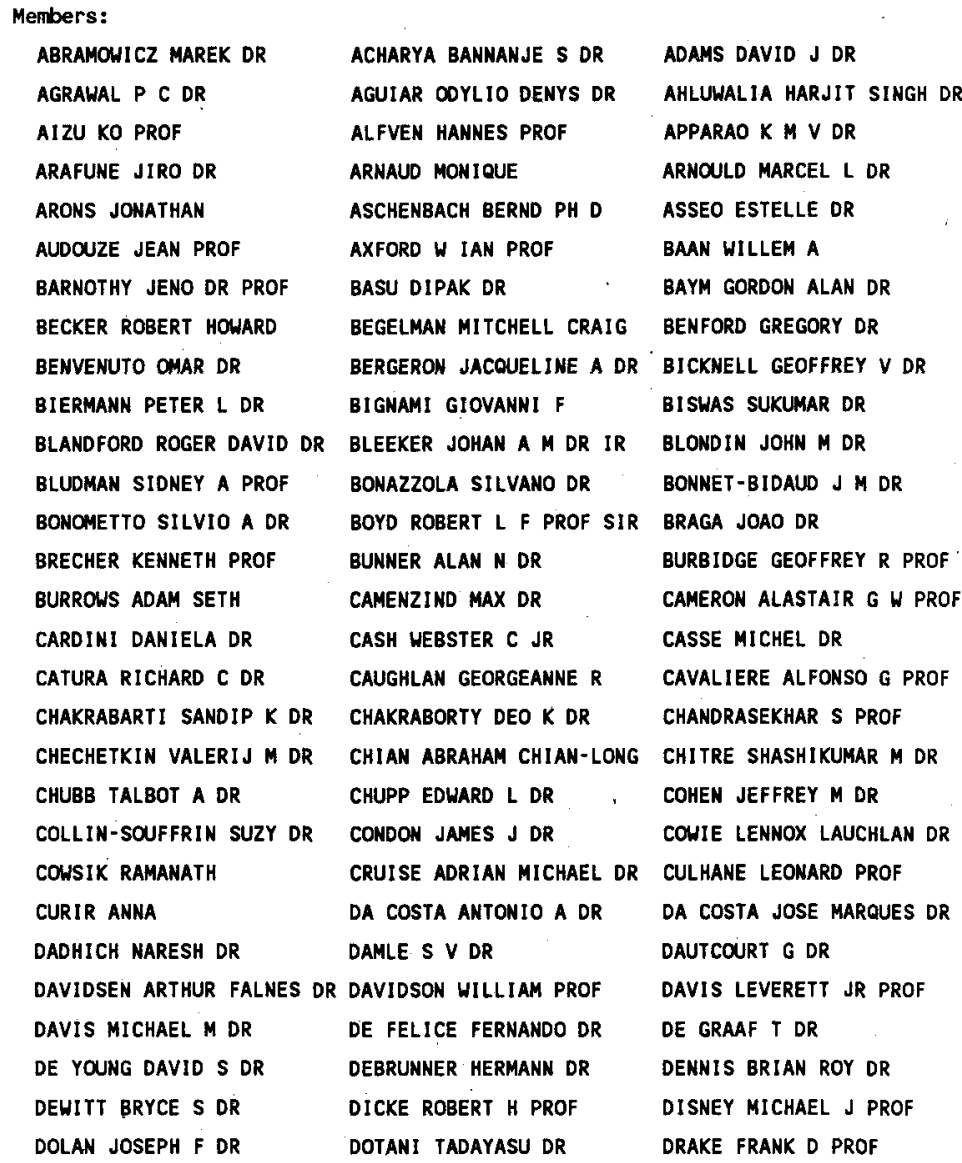


DRURY LUKE O'CONNOR DR

DUTHIE JOSEPH G PROF

EICHLER DAVID DR

EMANUELE ALESSANDRO DR

FANG LI-ZHI

FENTON K B DR

FIELD GEORGE B PROF

FOWLER WILLIAM A PROF

FRANK JUHAN

GAISSER THOMAS $K$

GINZBURG VITALY L PROF

GONZALES-A WALTER D DR

GRENIER ISABELLE DR

GRIFFITHS RICHARD E DR

GURSKY HERBERT DR

HANG HENG-RONG

HAWKING STEPHEN W PROF

HELFAND DAVID JOHN

HOF FMAN JEFFREY ALAN DR

HUANG KE-LIANG

I NOUE HAJIME DR

ITO KENSAI A PROF

JAFFE WALTER JOSEPH DR

JONES THOMAS WALTER DR

KAFKA PETER

KATZ JONATHAN I

KEMBHAVI AJIT $K$

KIRK JOHN DR

KOCH-MIRAMOND LYDIE DR

KOSHIBA MASA-TOSHI DR

KOZLOWSKI MACIEJ DR

KULSRUD RUSSELL M DR

KUSUNOSE MASAAKI DR

LAMB SUSAN ANM DR

LATTIMER JAMES M DR

LI TIPEI

LIANG EDISON P DR

LONGAIR M S PROF

LU TAN

LYNDEN-BELL DONALD PROF

MACCAGNI DARIO

MARTIN INACIO MALMONGE DR

MATSUMOTO RYOJI DR

MAZUREK THADDEUS JOHN DR

MEDINA JOSE DR

MELROSE DONALD B PROF

MEYER FRIEDRICH DR

MICHEL F CURTIS PROF

MIYAMOTO SIGENORI PROF

MURAKAMI TOSHIO

NITYANANDA R DR

NOVICK ROBERT

OEZEL MEHMET EMIN DR

OKOYE SAMUEL E PROF

O'SULLIVAN DENIS $F$
DUORAH HIRA LAL DR EDELSON RICK DR

EILEK JEAN

EVANS $\mathrm{H}$ DOYLE

FAZIO GIOVANHI G DR FERRARI ATTILIO DR

FISHER PHILIP C

FRANCESCHINI ALBERTO

FRANSSON CLAES

GALEOTTI PIERO PROF

GOLD THOMAS PROF

GREENHILL JOHN DR

GREWING MICHAEL PROF

GRINOLAY JONATHAN E DR

GUSEINOV O H PROF

HARWIT MARTIN PROF

HAYMES ROBERT C PROF

HENRIKSEN RICHARD N DR

HOLLOWAY NIGEL J DR

HUNT LESLIE DR

IPSER JAMES R PROF

ITOH MASAYUKI DR

JOKIPII J R PROF

JOSS PAUL CHRISTOPHER DR

KAHN FRANZ D PROF

KELLERRANN KENMETH I DR

KII TSUNEO DR

KLINKHAMER FRANS DR

KOLB EDHARD W DR

KOUPELIS THEODOROS DR

KREISEL E PROF

KUNDT WOLFGANG PROF DR

LAMB DONALD QUINCY JR DR

LAMPTON MICHAEL

LEA SUSAN MAUREEN DR

LI YUAN-JIE

LINSLEY JOHN

LOVELACE RICHARD V E DR

LUEST REIMAR PROF

MA YU-OIAN

MACCHETTO FERDINANDO DR

MASON GLENN M

MATSUOKA MASARU DR

MCBREEN BRIAN PHILIP DR

MEIER DAVID L

MESTEL LEON PROF

MEYER JEAN-PAUL DR

MILLER JOHN C DR

MOON SHIN HAENG DR

MAIDENOV VICTOR O

NOMOTO KEN'ICHI DR

MULSEN PAUL DR

OGALARA YOSHIAKI

OZERNOY LEONID M PROF
DUROUCHOUX PHILIPPE

EDWARDS PAUL $J$ DR

ELVIS MARTIN S DR

FABIAN ANDREW C DR

FELTEN JAMES E DR

FICHTEL CARL E DR

FORMAN WILLIAM RICHARD DR

FRANDSEN SOEREN PROF

FRIEDMAN HERBERT DR

GARMIRE GORDON P PROF

GOLDSMITH DONALD W DR

GREISEN KENMETH I PROF

GREYBER HOWARD D DR

GUNN JAMES E PROF

HALL ANDREW NORMAN

HAUBOLD HANS JOACHIM

HEISE JOHN DR

HENRY RICHARD C PROF

HOYLE FRED SIR

ICHIMARU SETSUO DR

ISRAEL ' HERNER PROF

JACKSON JOHN CHARLES DR

JONES FRANK CULVER DR

JULIUSSON EINAR DR

KAPOOR RAMESH CHANDER

KELLOGG EOWIN M DR

KILLEEN NEIL DR

KOCHAROV GRANT E PROF

KONDO MASAAKI DR

KOYAMA KATSUJI

KRISTIANSSON KRISTER PROF

KURT V G DR

LAMB FREDERICK $K$ PROF

LASHER GORDON JEWETT OR

LI QI-BIN

LI ZONG-WEI

LIU RU-LIANG

LU JU FU DR

LUMINET JEAN-PIERRE

MACCACARO TOMMASO DR

MAGGIO ANTONIO DR

MASON KEITH ONEN

MATZ STEVEN MICHEAL DR

MCCRAY RICHARD DR

MEIKSIN AVERY ABRAHAM DR

MESZAROS PETER DR

MICELA GIUSEPPINA DR

MIYAJI SHIGEKI DR

MORRISON PHILIP PROF

NEEMAN YUVAL PROF

NORMAN COLIN A PROF

OOA MINORU PROF

OKEKE PIUS N DR

O'CONNELL ROBERT F PROF 


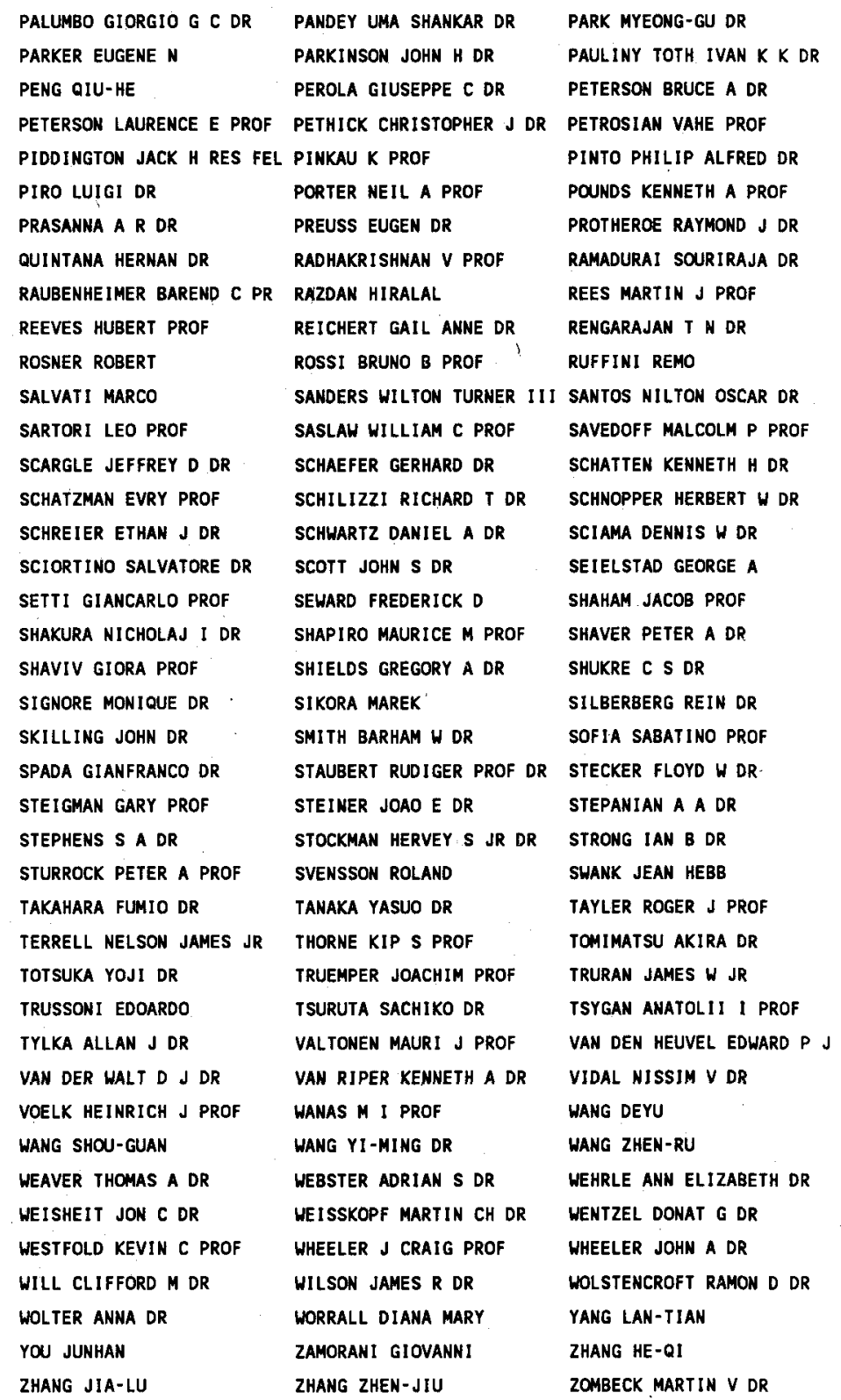




\author{
President : BUTI BIMLA PROF \\ Vice-President(s) : RJPKEN HARTMUT W DR
}

Organizing Committee: BURLAGA LEONARD F DR

KELLER HORST UHE DR

NAKAGAWA YOSHINARI OR

PERKINS FRANCIS W DR

RAADU MICHAEL A DR

SAGDEEV ROALD 2 OR

SONETT CHARLES P PROF

SUESS STEVEN $T$ DR

VINOD $S$ KRISHAN MRS DR

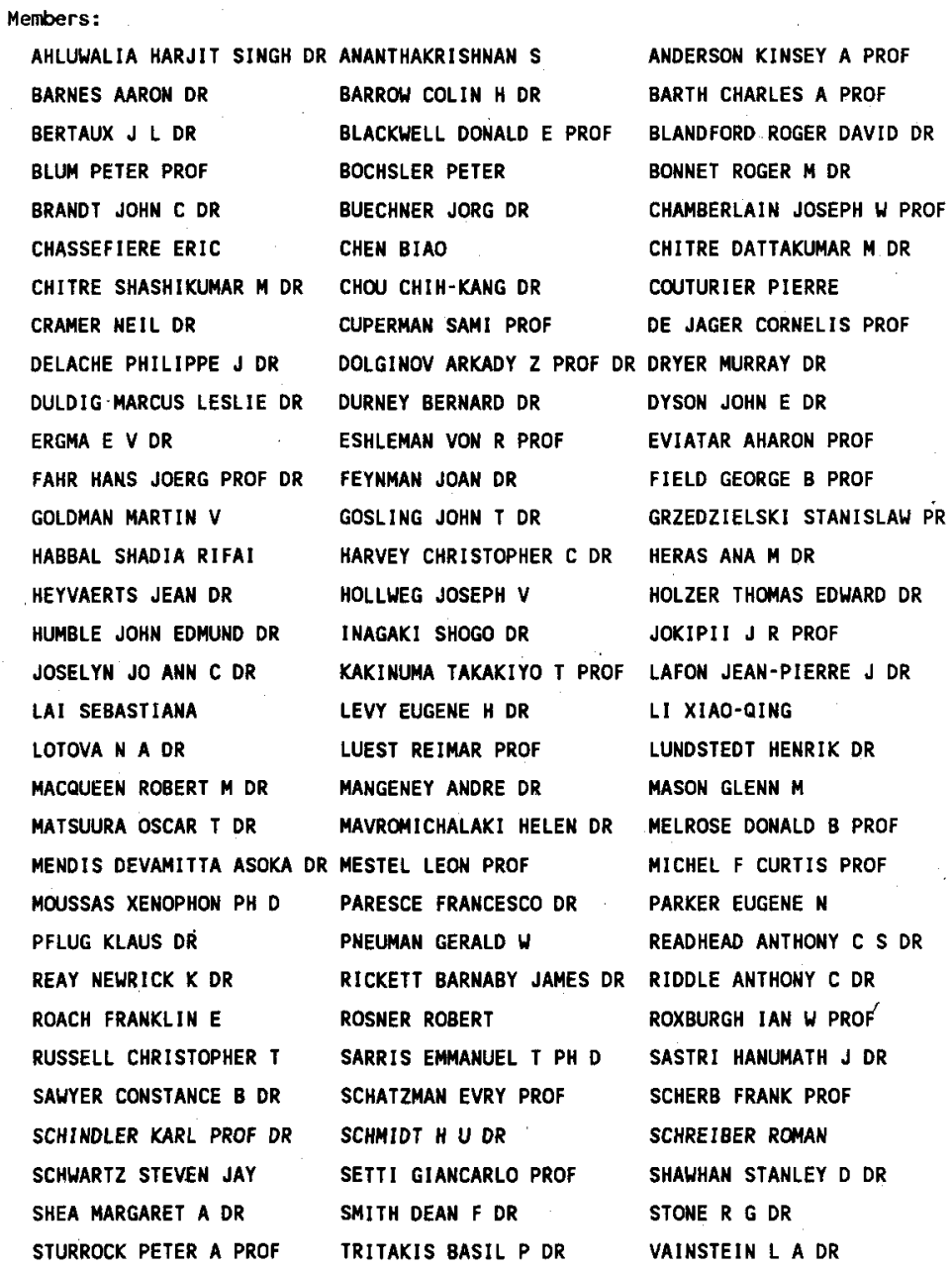


M102

VAN ALLEN JAMES A PROF

WANG SHUNDE DR

WELLER CHARLES S DR

YEH TYAN DR
VERHEEST FRANK PROF

WANG YI-MING DR

HILD JOHN PAUL DR
VUCETICH HECTOR DR WATAMABE TAKASHI DR

WU SHI TSAN DR 


\author{
$\begin{array}{ll}\text { President } & \text { : BUTI BIMLA PROF }\end{array}$ \\ Vice-President(s) : RIPKEN HARTMUT W DR
} CUPERMAN SAMI PROF DURNEY BERNARD DR INAGAKI SHOGO DR LEVY EUGENE H DR PNEUMAN GERALD W SCHMIDT H U DR SMITH DEAN F DR

\author{
BURLAGA LEONARD F DR \\ KELLER HORST UWE OR \\ NAKAGAWA YOSHINARI DR \\ PERKINS FRANCIS $W$ DR \\ RAADU MICHAEL A DR \\ SAGDEEV ROALD Z DR \\ SONETT CHARLES P PROF \\ SUESS STEVEN $T$ DR \\ VINOO S KRISHAN MRS DR
}

ANDERSON KINSEY A PROF BARTH CHARLES A PROF BLANDFORD ROGER DAVID DR BONMET ROGER M OR CHAMBERLAIN JOSEPH W PROF CHITRE DATTAKUMAR M DR COUTURIER PIERRE DE JAGER CORNELIS PROF DOLGINOV ARKADY Z PROF. DR DRYER MURRAY DR DYSON JOHN E DR EVIATAR AHARON PROF FIELD GEORGE B PROF GRZEDZIELSKI STANISLAW PR HERAS ANA M OR HOLZER THOMAS EDWARD' DR JOKIPII J R PROF KAKINUMA TAKAKIYO T PROF LAFON JEAN-PIERRE J DR LI XIAO-QING LUNDSTEDT HENRIK DR MASON GLENN M MELROSE DONALD 8 PROF MICHEL F CURTIS PROF PARKER EUGENE N READHEAD ANTHONY C S DR RICKETT BARNABY JAMES DR RIDDLE ANTHONY C DR ROSNER ROBERT ROXBURGH IAN W PROF SARRIS EMMANUEL T PH D SASTRI HANUMATH J DR SCHATZMAN EVRY PROF SCHERB FRANK PROF SETII GIANCARLO PROF TRITAKIS BASIL P DR

\section{SCHREIBER ROMAN} SHAWHAN STANLEY D DR STONE R G DR VAINSTEIN $L A D R$ 
M104

VAN ALLEN JAMES A PROF

WANG SHUNDE DR

WELLER CHARLES S DR

YEH TYAN DR
VERHEEST FRANK PROF

HANG YI-MING DR

WILD JOHN PAUL DR
VUCETICH HECTOR DR WATANABE TAKASHI DR

WU SHI TSAN DR 
COMPOSITION OF COMMISSION $50 \quad 1991-1994$

$\begin{array}{ll}\text { President } & : \text { MURDIN PAUL G DR } \\ \text { Vice-President(s) } & \text { : BHATTACHARYYA J C PROF }\end{array}$

Organizing Committee: ARDEBERG ARNE L PROF

BLANCO VICTOR M DR

COSTERO RAFAEL

CRAMFORD DAVID L DR

DAVIS JOHN PROF

GERGELY TOMAS ESTEBAN DR

ISOBE SYUZO DR

KOVALEVSKY JEAN DR

MCNALLY DEREK DR

SHCHEGLOV $P \vee D R$

\section{Members:}

\begin{tabular}{|c|c|c|}
\hline $\begin{array}{l}\text { ALY M KHAIRY PROF } \\
\text { BARRETO LUIZ MUNIZ PROF }\end{array}$ & $\begin{array}{l}\text { ARIAS DE GREIFF J PROF } \\
\text { BENSAMMAR SLIMANE DR }\end{array}$ & $\begin{array}{l}\text { ARSENIJEVIC JELISAVETA } \\
\text { BLANCO CARLO DR }\end{array}$ \\
\hline BROWN ROBERT HAMILTON & BURSTEIN DAVID & CAYREL ROGER DR \\
\hline OYNE GEORGE V DR & DAWE JOHN ALAN DR & DOMMANGET J DR \\
\hline UNKELMAN LAWRENCE & EDWARDS PAUL $J$ DR & GALAN MAXIMINO J \\
\hline IBSON DAVID MICHAEL DR & GOEBEL ERNST DR & HELMER LEIF \\
\hline IDAJAT BAMBANG PROF DR & HOAG ARTHUR A DR & HUANG YIN-LIANG \\
\hline IANG SHI-YANG & KAHLMANN HANS CORNELIS DR & KOZAI YOSHIHIDE PROF \\
\hline EIBOWITZ ELIA M DR & MAHRA H S DR & MARKKANEN TAPIO DR \\
\hline IARX SIEGFRIED DR & MATTIG W PROF DR & MCCARTHY MARTIN F DR \\
\hline IENZIES JOHN W DR & NELSON BURT OR & OEZEL MEHMET EMIN DR \\
\hline SORIO JOSE J S P PROF & OWEN FRAZER NELSON DR & PANKONIN VERNON LEE DR \\
\hline SANCHEZ FRANCISCO PROF & SCHILIZZI RICHARD T DR & SMITH F GRAHAM PROF \\
\hline SPOELSTRA T A TH DR & TORRES CARLOS ALBERTO DR & TORRES CARLOS DR \\
\hline TREMKO 'JOZEF D & UPGREN ARTHUR R DR & VAN DEN BERGH SIDNEY PROF \\
\hline IALKER MERLE $F$ & WAYMAN PATRICK A PROF & WHITEOAK J B DR \\
\hline 10OLF NEVILLE & LOSZCZYK ANDRZEJ PROF & WU MING-CHAN \\
\hline
\end{tabular}

ZHANG BAI-RONG 


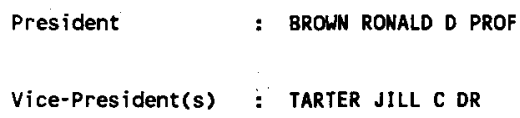

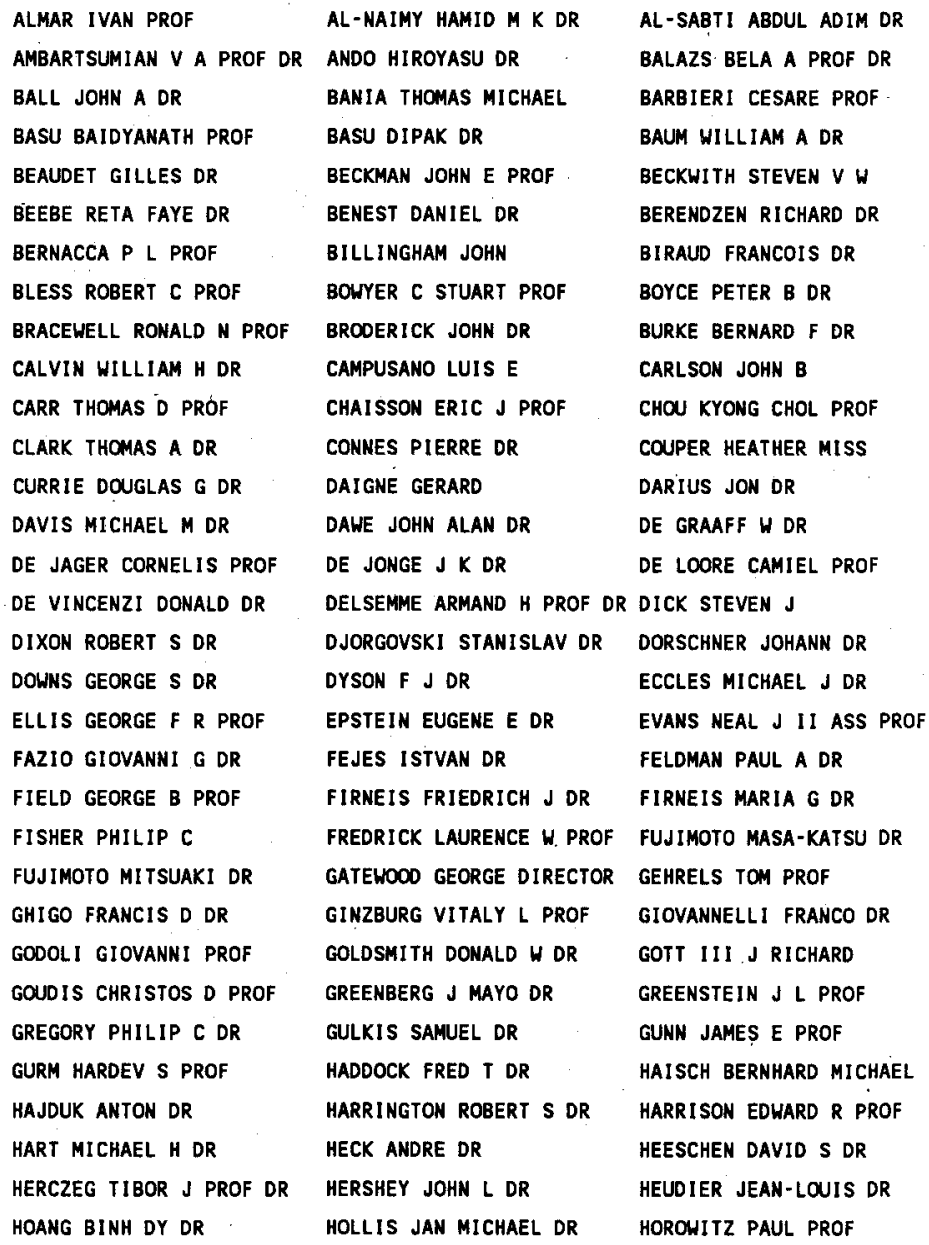




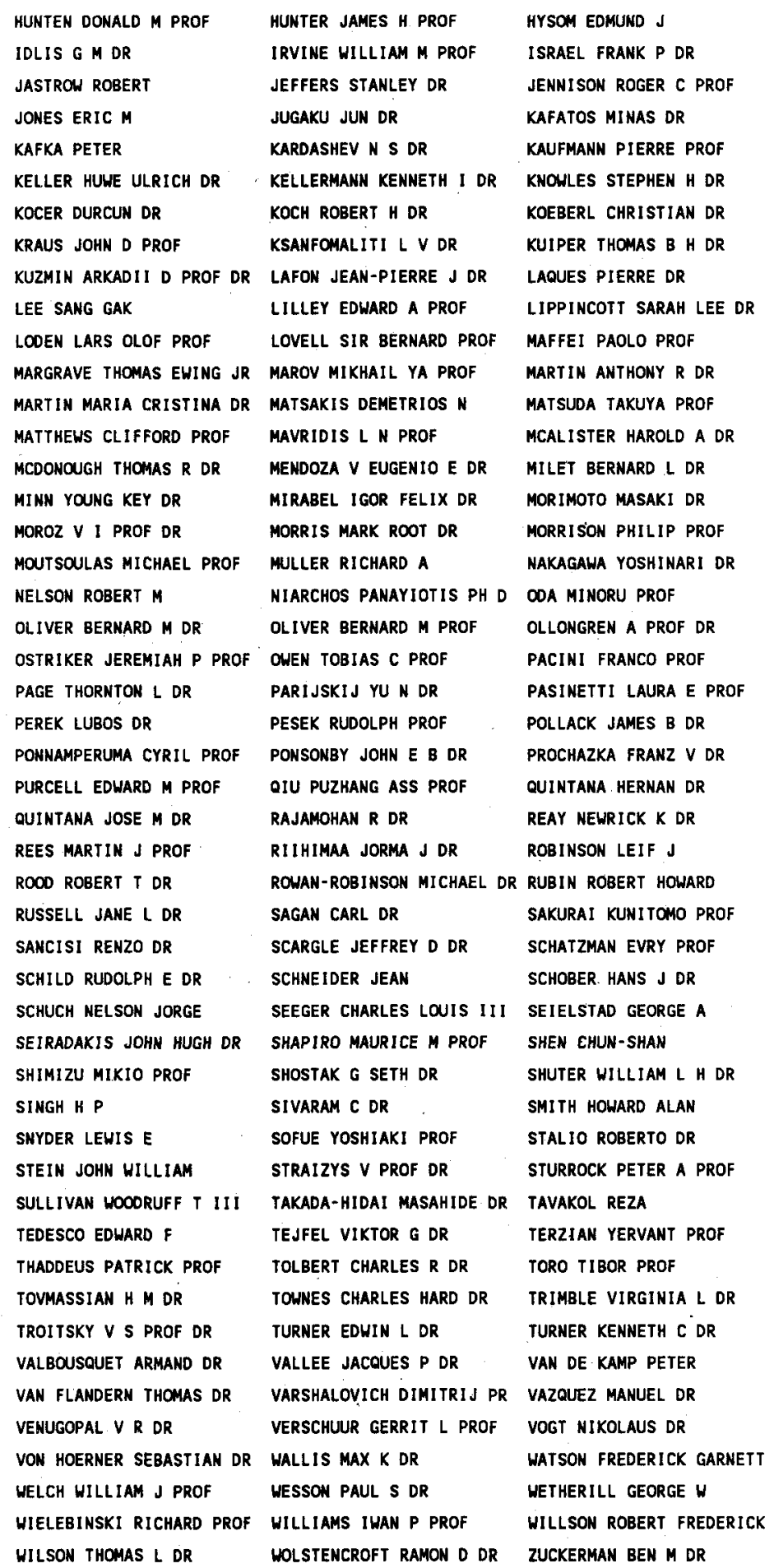


M108

COMPOSITION OF COMMISSION HGPSN 1991-1994

President : AKSNES KAARE DR

Organizing Committee: BRAHIC ANDRE DR

FULCHIGNONI MARCELLO PROF

MAROV MIKHAIL YA PROF

MORRISON DAVID PROF

OWEN TOBIAS C PROF

SHEVCHENKO VLADISLAV V DR

SMITH BRADFORD A PROF

SODERBLOM DAVIO R 
3. Geographical repartition of members

within adhering countries

Country : ALGERIA

SADAT RACHIDA DR

Country : ARGENTINA
AGUERO ESTELA I DR ARIAS ELISA FELICITAS BRANHAM RICHARD L JR CARRANZA GUSTAVO J DR CLARIA JUAN DR FEINSTEIN ALEJANDRO DR FiLLOY EMILIO MANUEL E E GIACANI ELSA BEATRIZ DR LANDI - DESSY J DR LOPEZ CARLOS LIC LOPEZ-GARCIA FRANCISCO DR MALAROOA STELLA M DR MARRACO HUGO G DR MENDEZ ROBERTO H DR MORRELL NIDIA DR OLANO CARLOS ALBERTO DR PEROOMO RAUL

RABOLLI MONICA DR SAHADE JORGE PROF SOLIVELLA GLADYS R LIC VUCETICH HECTOR OR BASSINO LILIA P DR
ALTAVISTA CARLOS A DR AZCARATE ISMAEL N DR CAPPA DE NICOLAU CRISTINA CASTAGNINO MARIO DR COLOMB FERNANDO R DR FERNANDEZ SILVIA $M$ DR FORTE JUAN CARLOS DR HERNANDEZ CARLOS ALBERTO LAPASSET EMILIO DR LOPEZ GARCIA ZULEMA L DR LUNA HOMERO $G$ DR MANRIQUE WALTER T PROF MARTIN MARIA CRISTINA DR MILONE LUIS A DR MUZZIO JUAN C PROF ORELLANA ROSA BEATRIZ DR PIACENTINI RUBEN DR RINGUELET ADELA E DR SERSIC J L DR VAZQUEZ RUBEN ANGEL DR ZADUNAISKY PEDRO E PROF BENVENUTO OMAR DR

Members 
ABLES JOHN G DR BAILEY JEREMY A BESSELL MICHAEL S DR BLAIR DAVID GERALD BOWEN EDWARD G DR BROWW $W$ N DR CALLY PAUL S DR CANNON RUSSELL D DR CASWELL JAMES L DR COGAN BRUCE C DR CRAM LAWRENCE EDWARD PROF DAVIS JOHN PROF DULDIG MARCUS LESLIE DR EDWARDS PAUL J DR

ELLIS G R A PROF

FAULKNER DONALD J DR FREEMAN KENNETH C PROF GASCOIGNE S C B DR GODFREY PETER DOUGLAS DR GRAY PETER MURRAY GREGG MICHAEL DAVID DR HARNETT JULIEINE DR HAYNES RAYMOND F PROF HUMBLE JOHN EDMUND DR JAUNCEY DAVID L DR KESTEVEN MICHAEL J L DR LAMBECK KURT PROF LOUGHHEAD RALPH E DR MALIN DAVID F MR MCADAM W BRUCE DR MCCULLOCH PETER M DR MCLEAN DONALO J DR MELROSE DONALD B PROF MINNET HARRY $C$ MR MORGAN PETER DR NELSON GRAHAM JOHN DR NORRIS JOHN DR o'mara berNard J PRof PETERSON BRUCE A DR PROTHEROE RAYMOND J DR REES DAVID ELWYN DR ROBINSON BRIAN J DR RODGERS ALEX $H$ DR SADLER ELAINE MARGARET SHARMA DHARMA PAL DR SHOBBROOK ROBERT R DR SMITH CRAIG H DR SPARROW JAMES G OR STEWART RONALD T MR STOREY MICHELLE DR
AITKEN DAVID $K$ DR

BALL LEHIS DR

BICKNELL GEOFFREY $\vee$ DR

BOLTON JOHN G

BOYLE BRIAN DR

BROWN RONALD D PROF

CANDY MICHAEL P MR

CARTER DAVID DR

CHAPMAN JESSICA DR

COLE TREVOR WILLIAM PROF

CRAMER MEIL DR

DAWE JOHN ALAN DR

DUNCAN ROBERT A PROF

EKERS RONALD D DR

ERICKSON WILLIAM C DR

FENTON $K$ B DR

GALLOWAY DAVID DR

GILLINGHAM PETER MR

GOLLNOW H DR

GREEN ELIZABETH M DR

HALL PETER J DR

HARWOOO DENNIS MR

HORTON BRIAN H DR . HUNSTEAD RICHARD W DR KALNAJS AGRIS J DR KILLEEN NEIL DR LARGE MICHAEL I DR LUCK J DR MANCHESTER RICHARD N DR MCCONNELL DAVID DR MCGEE RICHARD $X$ DR MCNAUGHT ROBERT H MILLS BERNARD Y PROF MONAGHAN JOSEPH J DR MULLALY RICHARD F DR NEWELL EDWARD B DR NORRIS RAYMOND PAUL O'SULLIVAN JOHN DAVID DR PIDDINGTON JACK H RES FEL QUINN PETER DR REYNOLDS JOHN DR ROBINSON GARRY DR ROSS JOHN E R DR SAULT ROBERT DR SHERIDAN $K \vee D R$ SIMS KENNETH $P$ DR SMITH LINDSEY F DR STAVELEY-SMITH LISTER OR STIBBS DOUGLAS $W N$ PROF TANGO WILLIAM J DR
ALLEN DAVID A DR BATTY MICHAEL DR BIRCH PETER MR BOOTH ANDREW J BRAY ROBERT J DR BURTON MICHAEL G DR CANE HILARY VIVIEN CARVER JOHN H PROF CHRIST IANSEN WILBUR PROF COUCH HARRICK DR DA COSTA GARY STEWART DR DOPITA MICHAEL ANDREW DR DURRANT CHRISTOPHER J DR ELFORD WILLIAM GRAKAM OR EVANS ROBERT REV FRATER ROBERT H DR GARDNER FRANCIS F DR GINGOLD ROBERT ARTHUR DR GOTTLIEB KURT GREENHILL JOHN DR HAMILTON P A DR HATZIDIMITRIOU DESPINA DR HOSKING ROGER I PROF HYLAND A R HARRY DR KEAY COLIN S L. PROF KVIZ ZOENEK OR LOMB NICHOLAS RALPH DR LYNGA GOSTA DR MATHEWSON DONALD S PROF MCCRACKEN KENNETH G DR MCGREGOR PETER JOHN OR MEATHERINGHAM STEPHEN DR MILNE DOUGLAS $K$ DR MORETON G E MURDOCH HUGH S DR NIKOLOFF IVAN DR NULSEN PAUL DR PAGE ARTHUR MR PRENTICE ANDREW J R DR REBER GROTE DR ROBERTSON JAMES GORDON DR ROBINSON JR RICHARD D DR RUSSELL KENNETH S DR SAVAGE ANN DR SHIMMINS ALBERT JOHM

SLEE O B DR SMITH ROBERT G DR STEEL DUNCAN I DR STOREY JOHN $W V$ DR TAYLOR KEITH DR 
Country : AUSTRALIA (Follow on)

TAYLOR KENNETH N R PROF
TURTLE A J DR
VAUGHAN ALAN DR
WATSON FREDERICK GARNETT
WESTFOLD KEVIN C PROF
WICKRAMASINGHE D T DR
WILSON PETER R PROF
ZAMBON GIULIO DR

THOMPSON KEITH DR TZIOUMIS AMASTASIOS DR VISVANATHAN NATARAJAN DR WATSON ROBERT DR WHITE GRAEME LINDSAY DR WILD JOHN PAUL DR WOOD PETER R DR ZEALEY WILLIAM J DR
TUOHY IAN R DR VAN DER BORGHT RENE PROF WATERHORTH MICHAEL DR WELLLINGTON KELVIN DR WHITEOAK J B DR WILSON BRIAN G PROF WRIGHT ALAN E DR BREGER MICHEL PROF DR FERRARI D OCCHIEPPO $K$ DR GOEBEL ERNST DR HAUPT HERMANN F PROF KOEBERL CHRISTIAN DR PFLEIDERER JORG PROF RAKOS KARL D PROF SCHROLL ALFRED DR STRASSMEIER KLAUS G DR STIFT MARTIN JOHANNES DR WEISS HERNER W DR

\section{Country : AZERBAIDZHAN}

ABBASOV ALIK R OR GUSEINOV O H PROF SEIDOV ZAKIR F DR
ASLANOV I A DR GUSEJNOV RAGIM EH DR SULTANOV G F ACAD
EMINZADE T A DR

KASUMOV FIKRET $K$ O DR 


\begin{tabular}{ll} 
ARNOULD MARCEL L DR & ARPIGNY CLAUDE PROF \\
BERTIAU FLOR C PROF & BIEMONT EMILE DR \\
BOSMAN-CRESPIN DENISE & BRIHAYE CHARLES C A DR \\
CALLEBAUT DIRK K DR & COUTREZ RAYMOND A J PROF \\
CUYPERS JAN DR & DE GREVE JEAN-PIERRE DR \\
DEBEHOGNE HENRI DR SC & DEHANT VERONIQUE DR \\
DEJONGHE HERWIG BERT DR & DELBOUILLE LUC PROF \\
DEMARET JACQUES DR & DENIS CARLO DR \\
DINGENS P PROF DR & DOMMANGET J DR \\
ELST ERIC WALTER DR & GABRIEL MAURICE R OR \\
GODART ODON PROF & GONZE ROGER F J IR \\
GOSSET ERIC DR & GREVESSE N DR \\
HENSBERGE HERMAN & HOUZIAUX L PROF \\
JAMAR CLAUDE A J DR & KOECKELENBERGH ANDRE DR \\
LAUSBERG ANDRE DR & LEMAITRE ANNE OR \\
MALAISE DANIEL J DR & MANFROID JEAN DR \\
MELCHIOR PAUL J PROF DIR & MOERDI JK HILLY G DR \\
MOONS MICHELE B M M & NICOLET MARCEL PROF \\
OITELET I J DR & OXENIUS JOACHIM DR \\
PAUWELS T DR & PERDANG JEAN M DR \\
RENSON P F M DR & ROBE H A G DR \\
SAUVAL A JACQUES DR & SCUFLAIRE RICHARD DR \\
SIMON RENE L E PROF & SINACHOPOULOS D DR \\
STERKEN CHRISTIAAN LEO DR & STEYAERT HERMAN PROF DR \\
SVALGAARD LEIF DR & SWINGS JEAN-PIERRE DR \\
VAN RENSBERGEN WALTER DR & VERBEEK PAUL DR \\
VREUX JEAN MARIE DR & WAELKENS CHRISTOFFEL \\
& \\
\hline & BOUNTYY : BRAZIL \\
\hline
\end{tabular}

ABRAHAM ZULEMA DR ALDROVANDI S M VIEGAS DR BARRETO LUIZ MUNIZ PROF BENEVIDES SOARES P DR BRAGA JOAO DR CAPELATO HUGO VICENTE DR CODINA LANDABERRY SAYO J DA COSTA JOSE MARQUES DR DA SILVA LICIO DR DE CARVALHO REINALDO DR DE SOUZA RONALDO DR FERRAZ-MELLO S PROF OR GOMES ALERCIO $M$ DR GRIJO DE OLIVEIRA A K DR JANOT - PACHECO EDUARDO DR KEPLER $S O$ LEITE SCHEID PAULO DR MACHADO LUIZ E DA SILVA MARTIN INACIO MALMONGE DR
AGUIAR OOYLIO DENYS DR ARAUJO FRANCISCO X DE DR BARROSO JR JAIR BERMAN MARCELO S DR CHAN ROBERTO DR CORREIA EMILIA DR DA COSTA NICOLAI L-A DAMINELI NETO AUGUSTO DR DE FREITAS PACHECO J A DR DOTTORI HORACIO A DR FREITAS MOURAO R R DR GOMIDE FERNANDO DE MELLO GRUENHALD RUTH DR JAYANTHI UDAYA B DR KOTANYI CHRISTOPHE DR LEPINE JACQUES R D DR MACIEL WALTER J DR MATSUURA OSCAR T DR BRUNINI ADRIAN DR
BAECK NICOLE A L DR BLOMME RONMY DR BURGER MARIJKE DR CUGNON PIERRE DR DE LOORE CAMIEL PROF DEJAIFFE RENE $J$ DR DELCROIX ANDRE $J S$ DR DENOYELLE JOZEF KIC DOSSIN F DR GERARD JEAN-CLAUDE M C DR GOOSSENS MARCEL DR HENRARD JACQUES PROF HUTSEMEKERS DAMIEN DR LAMPENS PATRICIA DR MAGAIM PIERRE DR MEIRE RAPHAEL MONFILS ANDRE G PROF NOELS ARLETTE DR PAQUET PAUL EG DR REMY BATTIAU LILIANE G A ROLAND GINETIE DR SIMON PAUL C DR SMEYERS PAUL PROF SURDEJ JEAN M G VAN DESSEL EDWIN LUDO DR VERHEEST FRANK PROF ZANDER RODOLPHE DR
ALDROVANDI RUBEN DR BARBUY BEATRIZ OR BATALHA CELSO CORREA DR BICA EDUARDO L D DR BUSKO IVO C DR CHIAN ABRAHAM CHIAN-LONG COSTA JOAQUIM EDUARDO DR DA ROCHA VIEIRA E DR DE ALMEIDA AMAURY A DR DE LA REZA RAMIRO DR DUCATI JORGE RICARDO DR GIACAGLIA GIORGIO E PROF GONZALES-A WALTER D DR JABLONSKI FRANCISCO DR KAUFMANN PIERRE PROF LAZZARO DANIELA DR LOPES DALTON DE FARIA DR MARQUES DOS SANTOS P PROF MENDES DA COSTA ARACY DR 
Country : BRAZIL (Follow on)

\begin{tabular}{|c|c|c|}
\hline NICOLACI DA COSTA L-A & NOVELLO MARIO DR & OPHER REUVEN PROF \\
\hline PALMEIRA RICARDO A R DR & PASTORIZA MIRIANI G DR & PELLEGRINI PAULOO S S DR \\
\hline PIAZZA LILIANA RIZZO & QUARTA MARIA LUCIA & QUAST GERMANO RODRIGO \\
\hline RAO K RAMANUJA DR & REZENDE COSTA JOAQUIM DR & SANTOS NILTON OSCAR DR \\
\hline SATO MASSAE DR & SAWANT HANUMANT S DR & SCALISE JR EUGENIO DR \\
\hline SCHAAL RICARDO E DR & SCHUCH NELSON JORGE & SESSIN WAGNER DR \\
\hline SINGH PATAN DEEN DR & STEINER JOAO E DR & STORCHI-BERGMAN THAISA DR \\
\hline TAKAGI SHIGETSUGU DR & TORRES CARLOS ALBERTO DR & TSUCHIDA MASAYOSHI DR \\
\hline VAZ LUIZ PAULO RIBEIRO & VIEIRA MARTINS ROBERTO DR & VILAS-BOAS JOSE W DR \\
\hline VILHENA DE MORAES $R$ DR & VILLELA THYRSO NETO DR & YOKOYAMA TADASHI DR \\
\hline Cour & BULGA & \\
\hline BACALOV MIHAIL & BONEV BONU K MR & BUYUKLIEV GEORGI DR \\
\hline DERMENDJIEV VLADIMIR DR. & DIKOVA SMILIANA D & DOBRITSCHEV V M MR \\
\hline FILIPOV LATCHEZAR & GEORGIEV TSVETAN DR & GOLEV VALERY K DR \\
\hline ILIEV ILIAN & IVANOV GEORGI R DR & IVANOVA VIOLETA DR \\
\hline KALINKOV MARIN P DR & KJURKCHIEVA DIANA DR & KOLEV DIMITAR ZDRAVKOV \\
\hline KOVACHEV B J DR & KRAICHEVA ZDRAVSKA DR & KUNCHEV PETER DR \\
\hline MINEVA VENETA DR & MOMCHEV GOSPODIN & NICOLOV NIKOLAI S DR \\
\hline NIKOLOV ANDREJ DR & PANOV KIRIL DR & PETROV GEORGY TRENDAFILOV \\
\hline PETROV NIKOLAI & POPOV VASIL NIKOLOV & POPOVA MALINA D PROF DR \\
\hline RADOSLAVOVA ISVETANKA & RAIKOVA DONKA DR & RUSSEV RUSCHO DR \\
\hline RUSSEVA TATJANA & SBIRKOVA-NATCHEVA T & SERAFIMOV KIRIL B ACAD \\
\hline SHKOOROV V G DR & SPASOVA MEOKA MARIMOVA & STOEV ALEXEI \\
\hline TOMOV ALEXANDER NIKOLOV & TOMOV TOMA V DR & TSVETKOV MILCHO K DR \\
\hline TSVETKOV ISVETAN DR & TSVETKOVA KATIA & UMLENSKI VASIL \\
\hline VELKOV KIRIL & VLADIMIROV SIMEON & YANKULOVA IVANKA DR \\
\hline ZHEKOV SVETOZAR A DR & ZHELYAZKOV IVAN DR & ZLATEV SLAVEY \\
\hline
\end{tabular}




\begin{tabular}{|c|c|}
\hline AIKMAN G CHRIS L & ANDREW BRYAN H DR \\
\hline AVERY LORNE $Y$ DR & BARKER PAUL K DR \\
\hline BASTIEN PIERRE DR & BATTEN ALAN H DR \\
\hline BELL MORLEY B & BINETTE LUC \\
\hline BLACKWELL ALAN TREVOR & BOCHONKO D RICHARD OR \\
\hline BOND JOHN RICHARD & BORRA ERMANNO F DR \\
\hline BROOKS RANDALL C DR & BROTEN NORMAN $H$ \\
\hline CALDWELL JOHN JAMES & CAMPBELL BRUCE OR \\
\hline CARIGNAM CLAUDE OR & CARLBERG RAYMOND GARY OR \\
\hline CLARK THOMAS ALAN DR & CLARKE THOMAS R DR \\
\hline CLIMENHAGA JOHN $L$ PROF & CLUTTON-BROCK MARTIN DR \\
\hline COUTTS-CLEMENT CHRISTINE & COVINGTON ARTHUR E \\
\hline CRAMPTON DAVID DR & DAVIDGE TIMOTHY J DR \\
\hline DEMERS SERGE DR & DELDNEY PETER E F DR \\
\hline DOUGLAS R J MR & DULEY WALTER W PROF \\
\hline EVANS NANCY REMAGE DR & FAHLMAN GREGORY G DR \\
\hline FERNIE J DONALD PROF & FICH MICHEL DR \\
\hline FLETCHER J MURRAY & FONTAINE GILLES DR \\
\hline FRIEL EILEEN D DR & GAETZ TiERRANCE J DR \\
\hline GALT JOHN A DR & GARRISON ROBERT F PROF \\
\hline GOWER J F R DR & GRAY DAVID F PROF \\
\hline GRIFFITH JOHN S PROF & GRUNDMANN WALTER \\
\hline HALLIDAY IAN DR & HANES DAVID A DR \\
\hline HARRIS GRETCHEN L H DR & HARRIS WILLIAM E DR \\
\hline HARTWICK F DAVID A DR & HARTZ THEODORE R DR \\
\hline HENRIKSEN RICHARD N DR & HERZBERG GERHARD DR \\
\hline HICKSON PAUL DR & HIGGS LLOYD A DR \\
\hline HUBE DOUGLAS P DR & HUGHES VICTOR A PROF \\
\hline INNANEN KIMMO A PROF & IRWIN ALAN W DR \\
\hline ISRAEL WERNER PROF & JEFFERS STANLEY DR \\
\hline JONES JAMES DR & KAMPER KARL W DR \\
\hline KOEHLER JAMES A PROF & KRONBERG PHILIPP DR \\
\hline LAKE KAYLL WILLIAM DR & LAMONTAGNE ROBERT DR \\
\hline LANDSTREET JOHN D PROF & LAPOINTE S M DR \\
\hline LEGG THOMAS H DR & LESTER JOHN B DR \\
\hline LOCKE JACK L DR & LOUE ROBERT P DR \\
\hline MACRAE DONALD A PROF & MADORE BARRY FRANCIS DR \\
\hline MARLBOROUGH J M PROF & MARTIN PETER G PROF \\
\hline MCCALL MARSHALL LESTER DR & MCCLURE ROBERT D PROF \\
\hline MCDONALD J K PETRIE DR & MCINTOSH BRUCE A DR \\
\hline MENON T K PROF & MERRIAM JAMES B \\
\hline MILONE EUGENE F PROF & MITALAS ROMAS ASSOC PROF \\
\hline MOCHNACKI STEPHAN $W$ DR & MOFFAT ANTHONY $F J D R$ \\
\hline MOORHEAD JAMES M DR & MORBEY CHRISTOPHER L \\
\hline MORTON DONALD C DR & NADEAU DANIEL DR \\
\hline NEMEC JAMES & NICHOLLS RALPH W PROF \\
\hline ODGERS GRAHAM J DR & PACHNER JAROSLAV PROF \\
\hline PEDREROS MARIO DR & PERCY JOHN R PROF \\
\hline POECKERT ROLAND H DR & POPELAR JOSEF DR \\
\hline PRYCE MAURICE H L DR & PURTON CHRISTOPHER R DR \\
\hline
\end{tabular}

AUMAN JASON R PROF BARNARD HANNES A J DR BEAUDET GILLES DR BISHOP ROY L DR BOLTON C THOMAS PROF BRANDIE GEORGE $W$ DR BURKE J ANTHONY DR CANNON WAYNE H DR CHAU WAI $Y$ PROF CLEMENT MAURICE J PROF COSTAIN CECIL C DR CRABTREE DENNIS DR DE ROBERTIS M M DR DOHERTY LORNE H DR DYER CHARLES CHESTER DR FELDMAN PAUL A DR FITZGERALD M PIM PROF FORBES DOUGLAS DR GAIZAUSKAS VICTOR DR GOWER ANN C DR GREGORY PHILIP C DR GULLIVER AUSTIN FRASER DR HARDY EDUARDO HARROWER GEORGE A DR HAWKES ROBERT LEWIS OR HESSER JAMES E DR HILL GRAHAM DR HUTCHINGS JOHH B DR IRWIN JUDITH DR JONCAS GILLES DR KENNEDY JOHN E PROF KHOK SUN DR LANDECKER THOMAS L DR LEAHY DENIS A DR LILLY SIMON J DR MACLEOO JOHN M DR MANN PATRICK J DR MATTHEWS JAYMIE MCCUTCHEON WILLIAM H PROF MEIKSIN AVERY ABRAHAM DR MICHAUD GEORGES J DR MITCHELL GEORGE F DR MOFFAT JOHN W OR MORRIS STEPHEN C DR NAQVI S I H PROF NOREAU LOUIS DR PATHRIA RAJ $K$ PROF PINEAULT SERGE DR PRITCHET CHRISTOPHER J DR RACINE RENE DR 
Country : CANADA (Follow on)

RAMSAY DONALD A DR RICHARDSON $E$ HARVEY DR ROCHESTER MICHAEL G PROF ROTTENBERG J A DR RUCINSKI SLAWOMIR M DR SCRIMGER J NORMAN DR SMYLIE DOUGLAS E DR STETSON PETER B. DR TAPPING KENNETH $F$ TATUM JEREMY B DR TURNER DAVID G DR VAN DEN BERGH SIDNEY PROF VINER MELVYN R DR WALKER GORDON A H PROF WEHLAU WILLIAM H PROF WESEMAEL FRANCOIS DR WOODSWORTH ANDREW W.DR YEE HOWARD K C DR ZHANG SHENG-PAN
REED B CAMERON DR RICHER HARVEY B DR ROGER ROBERT S DR ROUTLEDGE DAVID DR SAWYER-HOGG HELEN B DR SEAQUIST ERNEST R PROF SREENIVASAN S RANGA PROF SUKUMAR SUNDARAJAN DR TASSOUL JEAN-LOUIS PROF TAYLOR A R DR UNDERHILL ANNE B DR VANDENBERG DON DR VOLK KEVIN DR WEBSTER RACHEL WELCH DOUGLAS L DR WESSON PAUL $S$ DR WOOLSEY E G YEN JUI-LIN PROF ZHUANG QIXIANG Country : CHILE

$\begin{array}{ll}\text { ALCAINO GONZALO DR } & \text { ALVAREZ HECTOR DR } \\ \text { APARICI JUAN DR } & \text { BALDHIN JACK A DR } \\ \text { BOUCHEI PATRICE DR } & \text { BRONFMAN LEONARDO DR } \\ \text { CARRASCO GUILLERMO DR } & \text { CELIS LEOPOLDO DR } \\ \text { FAUNDEZ-ABANS M DR } & \text { FOUQUE PASCAL DR } \\ \text { GEISLER DOUGLAS P DR } & \text { GIEREN HOLFGANG P DR } \\ \text { INGERSON THOMAS DR } & \text { KRZEMINSKI WOJCIECH DR } \\ \text { LILLER WILLIAM DR } & \text { LOYOLA PATRICIO DR } \\ \text { MAY J } & \text { MAZA JOSE } \\ \text { MINTZ BLANCO BETTY MRS } & \text { MORENO HUGO PROF } \\ \text { PHILLIPS MARK M DR } & \text { QUINTANA HERNAN DR } \\ \text { ROTH MIGUEL R DR } & \text { RUBIO MONICA DR } \\ \text { SCHWARZ HUGO E } & \text { SUNTZEFF NICHOLAS B } \\ \text { VOGT NIKOLAUS DR } & \text { HALKER ALISTAIR ROBIN DR } \\ \text { WROBLEWSKI HERBERT DR } & \end{array}$

RICE JOHN B DR

ROBB RUSSEL $M$ ROGERS CHRISTOPHER DR ROY JEAN-RENE SCARFE COLIN D DR SHUTER WILLIAM L H DR STAGG CHRISTOPHER DR SUTHERLAND PETER G DR TASSOUL MONIQUE DR TREMAINE SCOTT DUNCAN VALLEE JACQUES P DR VENKATESAN DORASWAMY DR WAINURIGHT JOHN DR WEHLAU AMELIA DR WELCH GARY A DR WILLIS ANTHONY GORDON DR WRIGHT KENNETH O DR ZHANG CHENG-YUE
ANGUITA CLAUDIO A DR BLANCO VICTOR M DR CAMPUSANO LUIS E COSTA EDGARDO DR GARAY GUIDO DR GUTIERREZ-MORENO A DR MRS KUNKEL WILLIAM E DR MATHYS GAUTIER DR MELNICK JORGE NOEL FERNANDO REIPURTH BO RUIZ MARIA TERESA DR TORRES CARLOS DR WILLIAMS ROBERT E DR 


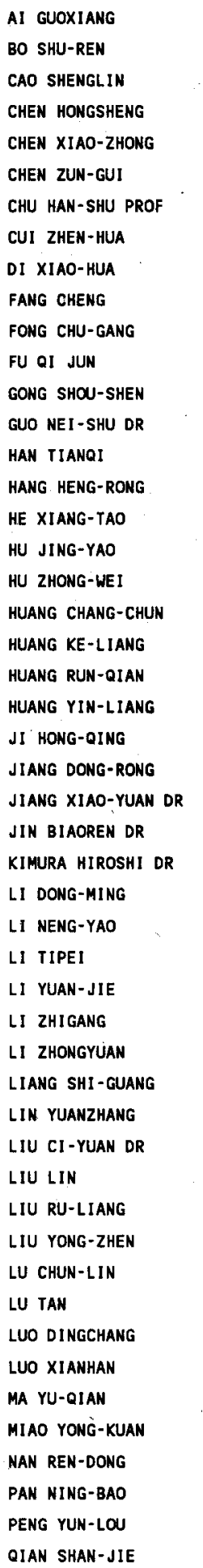

BAO KEREN

CAO CHANGXIN

CHEN BIAO

CHEN JIAN-SHENG

CHEN ZHEN

CHENG FU-HUA

CHU YAOQUAN

DAN XHI-XIANG

DING YOU-JI

FENG HESHENG

FU CHENG-QI

GAO BILIE

GONG SHU-MO

GUO QUAN SHI

HAN WENJUN

HAO YUN-XIANG

HSIANG YAN-YU

HU NING-SHENG

HUA YIMG-MIN

HUANG CHENG DR

HUANG KUN-YI

HUANG TIANYI

HUANG YONGWEI

JI SHUCHEN DR

JIANG SHI-YANG

JIANG YAO-TIAO

JIN SHEN-ZENG

LI CHUN-SHENG

LI HONG-WEI

LI QI-BIN

LI WEI BAO

LI ZHENG-XIN DR

LI ZHI-FANG

LI ZONG-HEI

LIANG ZHONG-HUAN

LIU BAO-LIN

LIU JIRMING

LIU LIN-ZHONG

LIU XINPING PROF

LIU ZONGLI

LU JU FU DR

LU YANG

LUO DING-JIANG

MA ER

MAO WEI

MIAO YONG-RUI

PAN JUN-HUA

PAN RONG-SHI

OI GUAN RONG

QIAN ZHI-HAN DR
BIAN YU-LIN

CAO LIHONG

CHEN DAO-HAN

CHEN PEISHENG

CHEN ZHENCHENG

CHENG FU-ZHEN

CUI DOU-XING

DENG ZUGAN DR

FAN YING

FENG KE-JIA

FU DELIAN

GAO BUXI

GU XIAO-MA

HAN FU

HAN ZHENG-ZHONG

HE MIAO-FU

HU FU-XING

HU WEN-RUI

HUANG BI-KUN

HUANG JIE-HAO

HUANG LIN

HUANG TIE-QIN

HUANG YOU-RAN

JIANG CHONG-GUO

JIANG SHUDING

JIANG ZHAOJI

JIN WEN-JING

LI DEPEI

LI JING

LI TING

LI XIAO-QING

LI ZHIAN DR

LI ZHI-SEN

LI ZONG-YUN

LIAO XINHAO DR

LIU CAIPIN

LIU LIAO

LIU OINGYAO DR

LIU XUEFU

LU BEN-KUI

LU RUWEI DR

LUO BAO-RONG

LUO SHI -FANG

MA XING-YUAN

MENG XINMIN

MO JING-ER

PAN LIANDE

PENG QIU-HE

QIAN BO-CHEN

QIAN ZHONG-YU 
Country : CHINA PR (Follow on)

\begin{tabular}{|c|c|c|}
\hline QIAO GUOJUN & QIN DAO & QIN SONG-NIAN \\
\hline QIN ZHI-HAI & QIU PUZHANG ASS PROF & QIU YU-HAI \\
\hline QU QIN-YUE & QUAN HEJUN & RONG JIAN-XIANG \\
\hline SHEN CHANGJUN & SHEN KAIXIAN & SHEN LIANG-ZHAO \\
\hline SHEN LONG-XIANG & SHEN PARN-AN & SHI GUANG-CHEN \\
\hline SHI ZHONG-XIAN & SONG GUO-XUAN & SONG JIN-AN \\
\hline SONG MU-TAO & SU BUMEI & SU DING-QIANG \\
\hline SU HONG-JUN & SU OING-RUI DR & SU WAN-ZHEN \\
\hline SUN JIN & SUN KAI & SUN YI-SUI \\
\hline SUN YONGXIANG & TAN HUISONG & TANG YU-HUA \\
\hline TONG FU & TONG YI & WAN LAI \\
\hline WAN TONG-SHAN & WANG CHUAN-JIN & WANG DEYU \\
\hline WANG DE-CHANG & WANG JIA-JI & WANG JIA-LONG \\
\hline WANG JING-SHENG & WANG JING-XIU & WANG LAN-JUAN \\
\hline WANG RENCHUAN & WANG SHOU-GUAN & WANG SHUI \\
\hline WANG SHUNDE DR & WANG SI-CHAO & WANG YANAN \\
\hline WANG YIMING & WANG ZHENG MING & WANG ZHEN-RU \\
\hline WANG ZHEN-YI & WEI MINGZHI & WU HUAI-WEI \\
\hline WU LIAN-DA & W LIN-XIANG & WU MING-CHAN \\
\hline WU NAILONG OR & WU SHENGYIN & WU SHOU-XIAN \\
\hline W XINJI & WU ZHIREN DR & XI ZE-ZONG \\
\hline XIA JIONGYU & XIA XIAOYANG DR & XIA YI-FEI \\
\hline XIAN DING-ZHANG & XIANG DELIN & XIANG SHOUPING \\
\hline XIAO NAI-YUAN & XIE GUANG-ZHONG & XIE LIANGYUN \\
\hline XIONG DA-RUN & $X U A O-A O$ & XU BANG-XIN \\
\hline XU JIA-YAN & XU JI-HONG DR & XU PEI - YUAN \\
\hline XU PINXIN & XU TONG-OI & XU ZHENTAO \\
\hline$X U Z H I-C A I$ & YAN LIN-SHAN & YANG FUMIN \\
\hline YANG JIAN & YANG LAN-TIAN & YANG SHI JIE \\
\hline YANG TING-GAO & YAO BAO-AN & YAO JIN-XING \\
\hline YE BINXUN & YE SHI-HUI & YE SHU-HUA \\
\hline YE WENWEI & YI ZHAO-HUA & YIN JI-SHENG \\
\hline YIN QI - FENG & YOU JIAN-QI & YOU JUNHAN \\
\hline YUE ZENG-YUAN & ZENG QIN DR. & ZHAI DI-SHENG \\
\hline ZHAI ZAOCHENG & ZHANG BAI-RONG & ZHANG BIN \\
\hline ZHANG FU JUN & ZHANG GUO-DONG & ZHANG HE-QI \\
\hline ZHANG HUI & ZHANG JIA-LU & ZHANG JIA-XIANG \\
\hline ZHANG JINTONG & ZHANG PEIYU & ZHANG XIU ZHONG \\
\hline ZHANG YOUYI & ZHANG ZHEN-DA & ZHANG ZHEN-JIU \\
\hline ZHAO GANG & ZHAO JUN-LIANG & ZHAO MING \\
\hline ZHAO REN-YANG & ZHENG DA-WEI & ZHENG XUE-TANG \\
\hline ZHENG YING & ZHENG YI-JIA & ZHOU BIFANG DR \\
\hline ZHOU DAOQI & ZHOU HONG-NAN & ZHOU TI-JIAN \\
\hline ZHOU YOU-YUAN & ZHOU ZHEN-PU & ZHU CI-SHENG \\
\hline ZHU NENGHONG & ZHU SHI-CHANG & ZHU WEN-YAO \\
\hline ZHU XINGFENG & ZHU YAOZHOHG DR & ZHU YONG-HE \\
\hline ZHUANG WEIFENG & ZOU HUI-CHENG & ZOU YI-XIN \\
\hline
\end{tabular}


M118

Country : CHINA R

CHIUEH TZIHONG DR

FU-SHONG KUO HUANG YI-LONG DR

LING CHIH-BING DR SHEN CHUN-SHAN

TSAI CHANG-HSIEN DIRECTOR

WU HSIN-HENG DR
CHOU CHIH-KANG DR

HSIANG-KUANG TSENG

HHANG WOEI-YANN $P$ PROF

NEE TSU-WEI DR

SUN WEI-HSIN DR

TSAO MO PROF

Country : COLOMBIA

BRIEVA EDUARDO PROF

Country : CUBA

ALVAREZ POMARES A O DR GARCIA EDUARDO DEL POZO
BOYTEL JORGE DEL PINO DR RAMOS ISABEL FERRO OR
CHOU DEAN-YI DR HUANG YINN-NIEN DR LEE THYPHOON NG KIN-WANG TING YEOU-TSWEN TSAY WEAN-SHUN DR
DOVAL JORGE PEREZ DR TABOADA RAMON RODRIGUEZ 


\begin{tabular}{|c|c|c|}
\hline AMBROZ PAVEL DR & ANTALOVA ANNA & BICAK JIRI DR \\
\hline BOUSKA JIRI DR & BUMBA VACLAV DR & BURSA MILAN DR \\
\hline CEPLECHA ZDENEK DR & CHOCHOL DRAHOMIR & CHVOJKOVA WOYK E DR \\
\hline FARNIK FRANTISEK & FISCHER STANISLAV DR & GRYGAR JIRI DR \\
\hline HADRAVA PETR & HAJDUK ANTON DR & HAJDUKOVA MARIA \\
\hline HANDLIROVA DAGMAR OR & HARMANEC PETR DR & HEFTY JAN DR \\
\hline HEINZEL PETR DR & HEJNA LADISLAV DR & HEKELA JAN DR \\
\hline HORAK TOMAS B DR & HORAK ZDENEK PROF DR & HORSKY JAN PROF \\
\hline HRIC LADISLAV DR & HUDEC RENE DR & KAPISINSKY IGOR \\
\hline KARLICKY MARIAH & KLECZEK JOSIP DR & KLOCOK LUBOMIR DR \\
\hline KLOKOCNIK JAROSLAV DR & KLVANA MIROSLAV & KNOSKA STEFAN \\
\hline KOPECKY MILOSLAV DR & KOTRC PAVEL & KOUBSKY PAVEL \\
\hline KRESAK LUBOR DR & KRESAKOVA MARGITA DR & KRIVSKY LADISLAV DR \\
\hline KRIZ SVATOPLUK DR & KULCAR LADISLAV DR & LETFUS VOJTECH DR \\
\hline LOCHMAN JAN & MAYER PAVEL DR & MESZAROS ATTILA DR \\
\hline MIKULASEK ZDENEK DR & MINAROVJECH MILAN & MRKOS ANTONIN DR \\
\hline NEUZIL LUDEK DR & NOVOTNY JAN DR & NOVOTNY VACLAV \\
\hline ONDERLICKA BEDRICH DR & OUHRABKA MIROSLAV DR & PADEVET VLADIMIR DR \\
\hline PALOUS JAN DR & PALUS PAVEL DR & PAPOUSEK JIRI \\
\hline PECINA PETR & PEREK LUBOS DR & PESEK RUDOLPH PROF \\
\hline PITTICH EDUARD M DR & POKORNY ZOENEK DR & POLECHOVA PAVLA DR \\
\hline PORUBCAN VLADIMIR DR & RAJCHL JAROSLAV DR & RUPRECHT JAROSLAV DR \\
\hline RUSIN VOJTECH & RUZICKOVA-TOPOLOVA B DR & RYBANSKY MILAN \\
\hline SEHNAL LADISLAV DR & SIDLICHOVSKY MILOS DR & SIMA ZOISLAV DR \\
\hline SIMEK MILOS DR & SIROKY JAROMIR DR & SOBOTKA MICHAL DR \\
\hline SOLC IVAN DR & SOLC MARTIN & STEFL VLADIMIR \\
\hline STOHL JAN. OR & SVESTKA JIRI OR & SVOREN JAN \\
\hline SYKORA JULIUS DR & TLAMICHA ANTONIN DR & TREMKO JOZEF DR \\
\hline VALNICEK BORIS DR & VANYSEK VLADIMIR PROF & VAVROVA ZDENKA DR \\
\hline VETESNIK MIROSLAV DR & VONDRAK JAN DR & WEBROVA LUDMILA DR \\
\hline ZACHAROV IGOR DR & Z1ZNOVSKY JOZEF DR & ZVERKO JURAJ DR \\
\hline
\end{tabular}




ANDERSEN JOHANNES
CHRISTENSEN PER R DR
EINICKE OLE H DR
FRANDSEN SOEREN PROF
HANSEN LEIF LECTURER
HOEG ERIK DR
JONES JANET E DR
KJAERGAARD PER DR
KUSTAANHEIMO PAUL E PROF
MADSEN JES
NOROLUND AKE DR
OLSEN ERIK H
PEOERSEN HOLGER OR
PETHICK CHRISTOPHER J DR
SCHNOPPER HERBERT $W$ DR
THEJLL PETER ANDREAS DR
WESTERGAARD NIELS J DR

BAERENTZEN JORN CHR ISTENSEN-DALSGAARD J FABRICIUS CLAUS $V$ GAMMELGAARD PETER MAG SCI HELMER LEIF JOHANSEN KAREN T LCEKTOR JORGENSEN HENNING E PROF KNUDE JENS K.IRKESKOV DR LILJE PER VIDAR BARTH DR MOESGAARD KRISTIAN $P$ NORDSTROEM BIRGITTA DR OLSEN FOGH H J PEDERSEN OLAF PROF REIZ ANDERS PROF SOMMER-LARSEN JESPER DR THOMSEN BJARNE B LECT WIETH-KNUDSEN NIELS P DR

Country : EGYPT

\begin{abstract}
ABDEL HADY AHMED DR ABULAZM MOHAMED SAMIR DR AIAD A PROF AWADALLA NABIL SHOUKRY DR EL NAWAWAY M S DR EL-SHAHAWY MOHAMAD PROF GHOBROS ROSHDY AZER PROF HASSAN S M PROF KAMEL OSMAN M PROF MIKHAIL FAHMY I PROF DR OSMAN ANAS MOHAMED PROF SOLIMAN MOHAMED AHMED DR YOUSEF SHAHINAZ M DR
\end{abstract}

ABDELKAHI M ABUBAKR DR AHMED IMAM IBRAHIM PROF ALY M KHAIRY PROF BAGHOS BALEGH B DR EL-BASSUNY ALAHY A A DR GALAL A A PROF HAMDY M A M PROF HELALI YHYA E DR MAHMOUD FAROUK M A B DR MIKHAL JOSEPH SIOKY PROF SHALTOUT MESALAM A M PROF TAWADROS MAHER JACOUB DR YOUSSEF NAHED H PROF

Country : ESTONIA

\author{
EELSALU HEINO DR \\ KIPPER TONU DR \\ SAAR ENN DR \\ VEISMANN UNO DR
}

\author{
EINASTO JAAN DR \\ NUGIS TIIT \\ SAPAR ARVED DR \\ VIIK TONU DR
}

BRANDENBURG AXEL DR CLAUSEN JENS VIGGO LEKTOR FLORENTIN-NIELSEN RALPH GYLDENKERNE KJELD DR HELT BODIL E JONES BERNARD J T DR JORGENSEN UFFE GRAE DR KRISTENSEN LEIF KAHL LUND NIELS NISSEN POUL E PROF NORGAARD-NIELSEN HANS U PAGEL BERNARD E J PROF PETERSEN $J O$ DR RUDKJOBING MOGENS PROF SORENSEN GUNNAR DR ULFBECK OLE DR

ABOU-EL-ELLA MOHAMED S DR AHMED MOSTAFA DR AWAD MERVAT EL-SAID DR BAKRY ABDEL AZIZ DR EL-RAEY MOHAMED E PROF GAMALELDIN ABDULLA I DR HAMID S EL DIN PROF ISSA ALI DR MARIE M A DR NAWAR SAMIR DR SHARAF MOHAMED ADEL PROF WANAS M I PROF

ERGMA E V DR PUSTYL'HIK IZOLD B DR UUS UNDO DR 
Country : FINLAND

DONNER KARL JOHAN HUOVELIN JUHANI DR KULTIMA JOHANMES MATTILA KALEVI DR OJA HEIKKI DR RAHUNEN TIMO SILLANPAA AIMO KALEVI DR TIURI MARTTI PROF VALTAOJA ESKO VILHU OSMI DR
HAEMEEN ANTTILA KAARLE A HAIKALA LAURI $K$ JAAKKOLA TOIVO S LUMAE KARI A DR MIKKOLA SEPPO DR OTERMA LIISI PROF RAITALA JOUKO T TAKALO LEO O DR TUOMINEN ILKKA $V$ DR VALTAOJA LEENA DR ZHENG JIA-QING
KARTTUNEN HANNU DR MARKKANEN TAPIO DR NIEMI AIMO PIIROLA VILPPU E DR RIIHIMAA JORMA J DR TEERIKORPI VELI PEKKA DR URPO SEPPO I VALTONEN MAURI J PROF 


ABOUDARHAM JEAN DR
AIME C DR
ALLEGRE CLAUDE PROF '
AMARI TAHAR DR
ARDUINI -MALINOVSKY M DR
ARNAUD JEAN PAUL
ARTZNER GUY

ATHANASSOULA EVANGELIE DR AUGARDE RENEE DR AVIGNON YVETTE DR BAGLIN ANNIE DR BALLEREAU DOMINIQUE DR BARANNE A OR BARUCCI MARIA A DR BEL NICOLE J DR BENEST DANIEL DR BERGER CHRISTIANE DR BERRUYER-DESIROTTE N DR BERTOUT CLAUDE

BILLAUD GERARD J

BLAZIT ALAIN DR BOIGEY FRANCOISE BOISSON CATHERINE DR BONMEAU DANIEL BORGNINO JULIEN DR BOUCHER CLAUDE OR BOUGERET $J L$ DR BOULESTEIX JACQUES BOYER RENE BRIOT DANIELLE DR BRYANT JOHN DR BURNAGE ROBERT CANAVAGgIA RENEE DR CARQUILLAT JEAN-MICHEL CASTETS ALAIN DR CAYREL ROGER DR CESARSKY CATHERINE J DR CHAMARAUX PIERRE DR CHAPRONT-TOUZE MICHELLE CHOLLET FERNAND DR COLIN JACQUES DR COMBES MICHEL CONNES PIERRE DR COURTES G PROF COX PIERRE DR CROVISIER JACQUES DAIGNE GERARD DE BERGH CATHERINE DR DEBARBAT SUZANNE $V$ DR DELABOUDINIERE $J-P$
ACXER AGNES PROF DR ALECIAN GEORGES DR ALLOIN DANIELLE DR ANDRILLAT HENRI L PROF ARIMOTO NOBUO DR ARNAUD MONIQUE ASSEO ESTELLLE DR AUBIER MONIQUE G DR AURIERE MICHEL AZZOPARDI MARC DR BAIZE PAUL DR BALMINO GEORGES G DR BARBIER-BROSSAT M DR BAUDRY ALAIN DR BELY-DUBAU FRANCOISE BENSAMMAR SLIMANE DR BERGER JACQUES G DR BERTAUX J L DR BIENAYME OLIVIER DR BIRAUD FRANCOIS DR BOCCHIA ROMEO DR BOISCHOT ANDRE DR BOMMIER VERONIQUE DR BONNET ROGER M DR BOSMA ALBERT DR BOUCHET FRANCOIS R DR BOUIGUE R

BOULON JACQUES J DR BRAHIC ANDRE DR BRUNET JEAN-PIERRE DR BUECHER ALAIN DR CALAME OOILE DR CAPITAINE NICOLE CASOLI FABIENNE DR CATALA CLAUDE DR CAZENAVE ANNY DR CESARSKY DIEGO A DR CHAMBE GILBERT CHASSEFIERE ERIC CHOPINET MARGUERITE DR COLLIN-SOUFFRIN SUZY DR COMTE GEORGES DR CORNILLE MARGUERITE DR COUTEAU PAUL PROF CREZE MICHEL DR CRUVELLIER PAUL E DR DAPPEN HERNER DE LA NOE JEROME DR DEHARVENG JEAN-MICHEL DR DELACHE PHILIPPE J DR
AGRINIER BERNARD L MR ALLARD NICOLE DR ALY JEAN JACQUES DR ANDRILLAT YVETTE DR ARLOT JEAN-EUDES ARTRU MARIE-CHRISTINE DR ASSUS PIERRE DR AUDOUZE JEAN PROF AUVERGNE MICHEL BACCHUS PIERRE PROF BALKOWSKI - MAUGER CH DR BALUTEAU JEAN-PAUL DR BARLIER FRANCOIS E DR BEC-BORSENBERGER ANNICK BENAYDOUN JEAN-JACQUES DR BERGEAT JACQUES G DR BERGERON JACQUELINE A DR BERTHOMIEU GABRIELLE DR BIJAOUI ALBERT DR BLAMONT JACQUES E PROF BOCKELEE-MORVAN DOMINIQUE BOISSE PATRICK DR BONAZZOLA SILVANO DR BONNET-BIDAUD J M DR BOTTINELLI LUCETTE DR BOUGEARD MIREILLE L DR BOULANGER FRANCOIS BOUVIER JEROME BRETAGNON PIERRE DR BRUSTON PAUL DR BURKHART CLALDE DR CAMICHEL HENRI DR CAPLAN JAMES CASSE MICHEL DR CAYREL DE STROBEL GIUSA CELNIKIER LUDWIK DR CHALABAEV ALMAS DR CHAPRONT JEAN DR CHEVALIER CLAUDE DR CLAIREMIDI JACQUES DR COMBES FRANCOISE DR CONNES JANINE DR COUPINOT GERARD DR COUTURIER PIERRE CRIFO FRANCOISE DR CUNY YVETTE J DR DAVOUST EMMANUEL DE LAPPARENT-GURRIET V DR DEHARVENG LISE DR DELANNOY JEAN DR 
Country : FRANCE (Follow on)

\begin{tabular}{|c|c|c|}
\hline DELHAYE JEAN PROF & DEMARCO JEAN ING & DENISSE JEAN-FRANCOIS DR \\
\hline DENNEFELD MICHEL & DESESQUELLES JEAN DR & DESPOIS DIDIER DR \\
\hline DIVAN LUCIENAE DR & DOAZAN VERA DR & DOLEZ NOEL DR \\
\hline DOLLFUS AUDOUIN PROF & DONAS JOSE DR & DOURNEAU GERARD DR \\
\hline DOWNES DENNIS DR & DROSSART PIERRE DR & DUBAU JACQUES DR \\
\hline DUBOIS MARC A & DUBOIS PASCAL DR & DUBOUT RENEE \\
\hline DUCHESNE MAURICE DR & DUFAY MAURICE PROF & DUFLOT MARCELLE DR \\
\hline DUMONT RENE DR & DUMONT SIMONE DR & DURIEZ LUC DR \\
\hline DUROUCHOUX PHILIPPE & DURRET FLORENCE DR & DUVAL MARIE-FRANCE \\
\hline DUVERT GILLES DR & EDELMAN COLETTE DR & EGRET DANIEL DR \\
\hline EIDELSBERG MICHELE DR & ENCRENAZ PIERRE J DR & ENCRENAZ THERESE DR \\
\hline FAUCHER PAUL DR & FAUROBERT-SCHOLL M DR & FEAUTRIER NICOLE DR \\
\hline FEHRENBACH CHARLES PROF & FEISSEL MARTINE OR & FELENBOK PAUL DR \\
\hline FERLET ROGER DR & FERNANDEZ JEAN-CLAUDE DR & FERRANDO PHILIPPE DR \\
\hline FESTOU MICHEL C DR & FLOQUET MICHELE DR & FLORSCH ALPHONSE DR \\
\hline FOING BERMARD H DR & FORT BERNARD P DR & FORVEILLE THIERRY DR \\
\hline FOSSAT ERIC DR & FOY RENAUD DR & FRANCOIS PATRICK DR \\
\hline FREIRE FERRERO RUBENS G & FRESNEAU ALAIN DR & FRIEDJUNG MICHAEL DR \\
\hline FRINGANT ANNE-MARIE DR & FRISCH HELENE DR & FRISCH URIEL DR \\
\hline FROESCHLE CHRISTIANE D DR & FROESCHLE CLAUDE DR & FROESCHLE MICHEL DR \\
\hline GABRIEL ALAN H & GAIGNEBET JEAN DR & GALLOUET LOUIS DR \\
\hline GAMBIS DANIEL DR & GARGAUD MURIEL DR & GARNIER ROBERT ING \\
\hline GAUTIER DANIEL & GAY JEAN DR & GENOVA FRANCOISE DR \\
\hline GEORGELIN YVON P DR & GEORGELIN YVONNE M DR & GERARD ERIC DR \\
\hline GERBAL DANIEL DR & GERBALDI MICHELE DR & GERIN MARYVONNE DR \\
\hline GILLET D DR & GIRAUD EDMOND & GOLDBACH CLAUDINE MME \\
\hline GONCZI GEORGES & GORDON CHARLOTTE PROF & GORET PHILIPPE DR \\
\hline GOUGUENHEIM LUCIENNE & GOUPIL MARIE JOSE & GOUTTEBROZE PIERRE DR \\
\hline GRANVEAUD MICHEL & GREC GERARD & GRENIER ISABELLE DR \\
\hline GRENIER SUZANNE & GREVE ALBERT DR & GRUDLER PIERRE \\
\hline GRY CECILE DR & GUELIN MICHEL DR & GUERIN PIERRE DR \\
\hline GUIBERT JEAN DR & GUINOT BERNARD R PROF & HALBWACHS JEAN LOUIS DR \\
\hline HAMMER FRANCOIS DR & HARVEY CHRISTOPHER C DR & HAYLI AVRAM PROF \\
\hline HECK ANDRE DR & HECQUET JOSETTE OR & HEIDMANN JEAN DR \\
\hline HENDECOURT DI LOUIS DR & HENON MICHEL C DR & HENOUX JEAN-CLAUDE DR \\
\hline HEUDIER JEAN-LOUIS DR & HEYVAERTS JEAN DR & HOANG BINH DY DR \\
\hline HUA CHON TRUNG DR & HUBERT HENRI DR & HUBERT-DELPLACE A-M OR \\
\hline IMBERT MAURICE DR & IRIGOYEN MAYLIS & ISRAEL GUY MARCEL DR \\
\hline JACO THIERRY & JACQUINOT PIERRE DR & JASCHEK CARLOS O R PROF \\
\hline JASCHEK MERCEDES DR & JASNIEWICZ GERARD DR & JOLY FRANCOIS OR \\
\hline JOLY MONIQUE & JOUBERT MARTINE & JOURNET ALAIN \\
\hline JUNG JEAN DR & KAHANE CLAUDINE DR & KANDEL ROBERT S DR \\
\hline KAZES ILYA OR & KLEIH KARL LUOWIG OR & KOCH-MIRAMOND LYYDIE OR \\
\hline KOURGANOFF VLADIMIR PROF & KOUTCHMY SERGE DR & KOVALEVSKY JEAN DR \\
\hline RRIKORIAN RALPH OR & KUNTH DANIEL & LABEYRIE ANTOINE DR \\
\hline LABEYRIE JACQUES DR & LACHIEZE-REY MARC & LACLARE F MR \\
\hline LACROUTE PIERRE A PROF & LAFFINEUR MARIUS MR & LAFON JEAN-PIERRE J DR \\
\hline LALLEMENT ROSINE DR & LAMY PHILIPPE DR & LANHES ANDRE DR \\
\hline LANTOS PIERRE DR & LAOUES PIERRE DR & LASKAR JACQUES OR \\
\hline LASOTA JEAN-PIERRE DR & LATOUR JEAN J & LAUNAY JEAN-MICHEL DR \\
\hline
\end{tabular}


LAURENT CLAUDINE DR LE BORGNE JEAN FRANCOIS LE DQURNEUF MARYVONNE LEACH SYDNEY DR LEFEBVRE MICHEL DR LELIEVRE GERARD DR LEMAITRE GERARD R DR LEQUEUX JAMES DR LESAGE ALAIN DR LEVY JACQUES $R$ DR LOUISE RAYMOND PROF LUMINET JEAN-PIERRE MAILLARD JEAN-PIERRE DR MARCELIN MICHEL MARTIN FRANCOIS DR MASNOU FRANCOISE DR MAUCHERAT J DR MAVRIDES STAMATIA DR MEGESSIER CLAUDE DR MEKARNIA DJAMEL DR MENNESSIER MARIE-OOILE DR MERCIER CLAUDE DR MEYER JEAN-PAUL DR MIGNARD FRANCOIS DR MILLIARD BRUNO MONIN JEAN-LOUIS DR MONTMERLE THIERRY DR MOREL PIERRE JACQUES DR MOURADIAN ZADIG M DR MULLER RICHARD DR NGUYEN-QUANG RIEU DR NOLLEZ GERARD DR OCHSENBEIN FRANCOIS DR PARCELIER PIERRE DR PAUL JACQUES DR PEDOUSSAUt ANDRE PELLETIER GUY DR PR PERRIER CHRISTIAN DR PETIT GERARD DR PETRINI DANIEL DR PICAT JEAN-PIERRE DR POOUERUSSE MICHEL POYET JEAN-PIERRE DR PREVOT LOUIS DR PROISY PAUL E DR PUGET JEAN-LOUP DR RABBIA YVES DR RAYROLE JEAN R DR REINISCH GILBERT DR RIBES JEAN-CLAUDE DR
LAVAL ANMIE OR LE BOURLOT JACQUES DR LE FEVRE OLIVIER DR LEBLANC YOLANDE DR LEFEVRE JEAN DR LEMAIRE JEAN-LOUIS DR LENA PIERRE J PROF LEROY BERNARD DR LESTRADE JEAN FRANCOIS DR LORTET MARIE CLAIRE LOULERGUE MICHELLE DR LUNEL MADELEIME DR MALHERBE JEAN MARIE DR MARCHAL CHRISTIAN DR MARTIN NICOLE DR MASNOU J L DR MAURICE ERIC N MAZURE ALAIN DR MEIN NICOLE DR MELLIER YANNICK DR MERAT PARVIZ MERLEAU-PONTY J PROF MIANES PIERRE DR MILET BERNARD L DR MIRABEL IGOR FELIX DR MONNET GUY J DR MORANDO BRUNO L DR MORRIS DAVID DR MULHOLLAND J DERRAL DR NADAL ROBERT

NITTMAN JOHANN NOTTALE LAURENT OMNES ROLAND PROF PARISOT JEAN-PAUL PECKER JEAN-CLAUDE PROF PELLAS PAUL DR PEQUIGNOT DANIEL PERRIN JEAN MARIE DR PETIT JEAN-MARC DR PEYTURAUX ROGER H PROF PICK MONIQUE DR POULLE EMMANUEL PROF PRADERIE FRANCOISE DR PREVOT-BURNICHON M L DR PROUST DOMINIQUE QUERCI FRANCOIS R DR RAOULT ANTOINETTE DR REBEIROT EDITH OR REQUIEME YVES DR RICORT GILBERT DR
LAZAREFF BERNARD DR LE CONTEL JEAN-MICHEL LE SQUEREN ANNE-MARIE DR LEBRETON YVELINE DR LEGER ALAIN DR LEMAIRE PHILIPPE DR LEORAT JACQUES DR LEROY JEAN-LOUIS LEVASSEUR-REGOURD A C PR LOSCO LUCETTE DR LUCAS ROBERT OR MAGHAN CHRISTIAN DR MANGENEY ANDRE DR MARIOTTI JEAN MARIE DR MARTRES MARIE-JOSEPHE MATHEZ GUY MAURON NICOLAS DR MCCARROLL RONALD PROF MEIN PIERRE MENEGUZZI MAURICE M DR MERAT PARVIZ DR MEYER CLAUDE DR MICHARD RAYMOND OR MILLET JEAN DR MOCHKOVITCH ROBERT DR MONTES CARLOS DR MOREELS GUY DR MOUCHET MARTINE DR MULLER PAUL NAHON FERNAND PROF NOENS JACQUES-CLAIR DR OBLAK EDOUARD OMONT ALAIN PROF PATUREL GEORGES PEDERSEN BENT M DR PELLET ANDRE PERAULT MICHEL PERRIN MARIE-NOEL DR PETON ALAIN DR PHAM-VAN JACQUELINE MME PINEAU DES FORETS G DR POUQUET ANNICK DR PRANTZOS NIKOS DR PRIEUR JEAN-LOUIS DR PROVOST JANINE DR QUERCI MONIQUE DR RAPAPORT MICHEL DR REEVES HUBERT PROF RIBES ELIZABETH DR ROBILLOT JEAN-MAURICE DR 
ROBIN ANNIE C DR ROQUES FRANCOISE DR ROSTAS FRANCOIS DR ROUDIER THIERRY OR ROUSSEAU JEAN-MICHEL MR SAGNIER JEAN-LOUIS DR SAMAIN DENYS DR SCHATZMAN EVRY PROF SCHNEIDER JEAN

\section{SEMEL MEIR DR}

SICARDY BRUNO DR

\section{SIMON GUY}

SIMONNEAU EDUARDO DR

\section{SOL HELENE OR}

\section{SOUFFRIN PIERRE B DR}

\begin{abstract}
SPITE FRANCOIS M DR
\end{abstract}

\begin{abstract}
STEENMAN-CLARK LOIS DR
\end{abstract}
STEINBERG JEAN-LOUIS OR SYGNET JEAN FRANCOIS DR TATON RENE PROF TERZAN AGOP DR THIRY YVES R PROF TRAN-MINH FRANCOISE DR TRUONG-BACH VAKILI FARROKH DR VALTIER JEAN-CLAUDE DR VAN'T VEER-MENNERET CL DR VAUCLAIR SYLVIE D DR VELLI MARCO DR VERON MARIE-PAULE DR VIALA YVES VIDAL-MADJAR ALFRED DR VILMER NICOLE DR VU DUONG TUYEN DR WEIMER THEOPHILE P F DR WINK JOERN ERHARD DR ZAHN JEAN-PAULL OR
ROBLEY R DR ROOUES SYLVIE OR ROTHENFLUG ROBERT DR ROUEFF EVELYNE M A OR ROZELOT JEAN P SAHAL-BRECHOT SYLVIE DR SAREYAN JEAN-PIERRE DR SCHEIDECKER JEAN-PAULL DR SCHOLL HANS DR SERVAN BERNARD SIGNORE MONIQUE DR SIMON JEAN-LOUIS MR SIVAN JEAN-PIERRE DR SORU-ESCAUT IRINA MRS SOULIE GUY SPITE MONIQUE DR STEFANOVITCH-GOMEZ A E DR STELLMACHER GOETZ TALON RAOUL DR TCHANG-BRILLET LYDIA DR TEXEREAU JEAN $M$ THOMAS CLAUDINE DR TRELLIS MICHEL DR TULLY JOHN A DR VALBOUSQUET ARMAND DR VAN REGEMORTER HENRI DR VAPILLON LOIC J DR VAUGLIN ISABELLE DR VERDET JEAN-PIERRE DR VERON PHILIPPE DR VIALLEFOND FRANCOIS VIGIER JEAN-PIERRE OR VITON MAURICE DR WUILLEMIN ANDRE DR WENGER MARC WLERICK GERARD DR ZEIPPEN CLAUDE DR
ROCCA-VOLMERANGE BRIGITTE -

ROSCH JEAN PROF

ROUAN DANIEL DR ROUSSEAU JEANINE DR RYTER CHARLES E DR SAISSAC JOSEPH DR SCHAEFFER RICHARD DR SCHMIEDER BRIGITTE DR SCHUMACHER GERARD DR SIBILLE FRANCOIS SIMIEN FRANCOIS DR SIMON PAUL A DR SLEZAK ERIC DR SOTIROVSKI PASCAL OR SPIELFIEDEL ANNIE DR STASINSKA GRAZYNA DR STEHLE CHANTAL DR STELLMACHER IRENE DR TARRAB IRENE TERRIEN JEAN THEVENIN FREDERIC DR TRAN MINH NGUYET DR TROTTET GERARD DR TURON C DR VALIRON PIERRE DR VAN'T VEER FRANS DR VAUCLAIR GERARD P DR VEILLET CHRISTIAN VERGEZ MADELEINE DR VIAL JEAN-CLAUDE VIDAL JEAN-LOUIS DR VILKKI ERKKI U VOLONTE SERGE DR WALCH JEAN-JACQUES WENIGER SCHAME DR NOLTJER LOOEWI JK PROF ZOREC JEAN DR

BARTAYA R A DR
DOLIDZE MADONA V DR
FISHKOVA LUISA M PROF
KHATISASHVILI ALFEZ SH DR
KOGOSHVILI MATELA G
MAGNARADZE NINA G DR

BORCHKHADZE TENGIZ M DR
DZHAPIASHVILI VICTOR P DR
KALANDADZE N B DR
KHETSURIANI TSIALA S DR
KUMSIASHVILY MZIA I DR
SALUKVADZE G N DR

CHKHIKVADZE IAKOB N DZIGVASHVILI R M DR KHARADZE E $K$ PROF KILADZE R I DR KUMSISHVILI J I DR TOROSHLIDZE TEIMURAZ I DR 
ALBRECHT MIGUEL A DR ANZER ULRICH DR

ARP HALTON DR

AXFORD W IAN PROF

BAHNER KLAUS DR

BARUIG HEINZ

BECK H G

BECKHITH STEVEN $V W$

BENVENUTI PIERO DR

BIEN REINHOLD DR

BLUM PETER PROF

BOEHNHARDT HERMANN DR

BOHN HORST-ULRICH

BOSCHAN PETER DR

BRAUNSFURTH EDWARD PH D

BREYSACHER JACQUES

BRUCH ALBERT

BUES IRMELA D DR

CARSENTY URI DR

CRANE PHILIPPE

DANZIGER I JOHN DR

DE VEGT CH PROF DR

DEUBNER FRANZ-LUDWIG DR

DOMKE HELMUT PH D

DRAPATZ SIEGFRIED W DR

DUSCHL WOLFGANG J DR

EL EID MOUNIB DR

ENARD DANIEL OR

FAHR HANS JOERG PROF DR

FEIX GERHARD DR

FRIED JOSEF WILHELM DR

FUERST ERNST DR

GEFFERT MICHAEL DR

GERHARD ORTWIN

GLATZEL WOLFGANG DR

GOETZ WOLDEMAR DR

GRAHL BERND H DR

GROSBOL PREBEN JOHNSON OR

GROTH HANS G PROF DR

GUESTEN ROLF

HACHENBERG OTTO PROF DR

HAGEN HANS-JUERGEN DR

HANUSCHIK REINHARD DR

HARVEY GALE A DR

HAUBOLD HANS JOACHIM

HAUPT WOLFGANG DR

HEFELE HERBERT PH D

HEMPE KLAUS

HENSLER GERHARD PROF

HERRMANN DIETER PROF DR
ALBRECHT RUDOLF DR APPENZELLER IMMO PROF ASCHENBACH BERND PH D BAADE DIETRICH DR BALTHASAR HORST DR BASCHEK BODO PROF BECK RAINER BEHR ALFRED PROF EMERITUS BERKHUIJSEN ELLYY M DR BIERMANN PETER L DR BOEHME ANMELIES DR BOERNER GERHARD DR BOHRMANN ALFRED PROF BRANDT PETER N BREINHORST ROBERT A DR BRINKMANH WOLFGANG BRUZEK ANTON DR BUTLER KEITH DR CHE-BOHNENSTENGEL ANNE CULLLUM MARTIN OR DAUTCOURT G DR DEINZER W PROF. DR DICK WOLFGANG OR DORENHENDT KLAUS DR DRECHSEL HORST DR D 'ODORICO SANDRO DR ELSAESSER HANS PROF ENGELS DIETER DR FECHTIG HUGO DR FOSBURY ROBERT A E DR FRIEDEMANN CHRISTIAN DR GAIL HANS-PETER DR GEHREN THOMAS PH D GEYER EDHARD H PROF DR GLEISSBERG WOLFGANG PROF GONDOLATSCH FRIEDRICH PRF GREHING MICHAEL PROF GROSSMANN-DOERTH U DR GRUEN EBERHARD DR GUSSMANN E A DR HAEFNER REIHHOLD DR HAMMAN WOLF-RALNER HARRIS ALAN WILLIAM HASER LEO N K DR HAUG EBERHARD DR HAZLEHURST JOHN DR HEINRICH INGE HENKEL CHRISTIAN HERMAN JACOBUS DR HILF EBERHARD R H PH D
ALTENHOFF HILHELM $J$ DR ARNAL MARCELO EDMUNDO OR AURASS HENRY DR BAESSGEN MARTIN DR BARROW COLIN H DR BASTIAN ULRICH BECKERS JACQUES M DR BENDER RALF DR BEUERMANN KLAUS P PROF BIRKLE KURT PH D BOEHME SIEGFRIED DR BOERNGEN FREIMUT DR PH BORGEEST ULF DR BRAUNINGER HEINRICH DR BREITSCHWERDT DIETER DR BROSCHE PETER PROF BUECHNER JORG DR CAMENZIND MAX DR CHINI ROLF DACHS JOACHIM PROF DR DE BOER KL.AAS SJOERDS DR DETTMAR RALF-JURGEN DR DIERCKSEN GEERD H F PH D DORSCHNER JOHANN DR DUERBECK HILMAR W OR EHLERS JURGEN PROF ELGERT GERHARD PROF ENSLIN HEINZ DR FEITZINGER JOHANNES PROF FRICKE KLAUS DR FUCHS BURKHARD DR GEBLER KARL-HEINZ DR GENZEL REINHARD DR GIESEKING FRANK DR GLIESE WIHELM PROF GRAHAM DAVID A GROOTE DETLEF GROTEN ERHIN PROF GUERTLER JOACHIN DR GUTCKE DIETRICH HAERENDEL G DR HAMMER REINER HARTQUIST THOMAS WILBUR HASLAM C GLYN T DR HAUG ULRICH PROF HEBER ULRICH HEMMLEB GERHARD DR HENHING THOMAS DR HEROLD HEINZ HILLEBRANDT WOLFGANG PH D 
Country : GERMANY (Follow on)

HIPPELEIN HANS H DR HOFFMANN MARTIN DR HOPP ULRICH DR HUCHTMEIER WALTER $K$ DR IP WING- HUEN JAEGER FRIEDRICH W PROF JOCKERS KLAUS DR KAEHLER HELMUTH OR KANBACH GOTTFRIED DR KAYSER RAINER DR KELLER HUWE ULRICH DR KIRK JOHN DR KNEER FRANZ DR KOEHLER PETER KOLLATSCHNY WOLFRAM DR KRAUSE F DR KREYSA ERNST KUDRITZKI ROLF-PETER PH D KUENZELL HORST KUNZE RUEDIGER DR LANGER NORBERT DR LEMKE DIETRICH DR LIEBSCHER DIERCK-E OR LUCY LEON B PROF MANN GOTTFRIED DR MATERNE JUERGEN DR MAUERSBERGER RAINER DR MEISENHEIMER KLAUS DR MEURS EVERT DR MEZGER PETER G PROF MOORHOOD ALAN F M MUNDT REINHARD DR NESIS ANASTASIOS DR OEGELMAN HAKKI B DR OLEAK H DR PETRI WINFRIED PROF DR PFLUG KLAUS DR PINKAU K PROF PORCAS RICHARD DR PULS JOAHIM DR REICH WOLFGANG RICHTER G A DR RIEGER ERICH DR ROEMER MAX PROF ROESER SIEGFRIED DR ROSA DOROTHEA DR RUBEN G PROF DR RUPPRECHT GERO DR SCHEFFLER HELMUT PROF SCHINDLER KARL PROF DR
HIRTH HOLFGANG ERNST PH D HOEFLICH PETER DR HOFMANN WILFRIED DR HOREDT. GEORG PAUL DR HUNGER KURT PROF ISSERSTEDT JOERG DR JAHREISS HARTMUT DR JORDAN H L DR DIREKTOR KAFKA PETER

KASPER U DR

KEGEL WILHELM H PROF KENDZIORRA ECKHARD DR KLARE GERHARD DR KMOLKER MICHAEL DR KOEPPEN JOACHIM DR KOZASA TAKASH I KRAUTTER JOACHIM DR KRUEGELL ENDRIK DR KUEHNE CHRISTOPH $F$ KUNDT WOLFGANG PROF DR LABS DIETRICH PROF LEDERLE TRUDPERT DR LENZEN RAINER DR LOOSE MANS-HERMANN OR LUEST REIMAR PROF MARX SIEGFRIED DR MATTIG W PROF DR MEBOLD ULRICH DR PROF MERKLE FRITZ DR MEYER FRIEDRICH OR MOEHLMANN DIEDRICH MUELLER EUALD NECKEL HEINZ DR NEUKUM $G$ DR OESTREICHER ROLAND PAULDRACH ADALBERT $W A$ DR PFAU WERNER PIERRE MARGUERITE DR PITZ ECKHART DR PREUSS EUGEN DR RAEDLER $K$ H DR REIF KLAUS DR RICHTER JOHANNES PROF RIPKEN HARTMUT $W$ DR ROESER HANS-PETER ROESSIGER SIEGFRIED DR ROSA MICHAEL RICHARD DR RUDER HANNS SCHAEFER GERHARD DR SCHILBACH ELENA DR SCHLICKEISER REINHARD DR
HOLHEGER HARTMUT PROF HOUSE FRANKLIN C DR HUSFELD DIRK DR JACKISCH GERHARD DR JENSCH A JUST ANDREAS DR KALBERLA PETER KAUFMANN JENS PETER DR KELLER HORST UWE DR KIPPENHAHN RUDOLF PROF KLEIN ULRICH KOEHLER H PROF OR KOHOUTEK LUBOS DR KRAEMER GERHARD DR KREISEL E PROF KRUEGER ALBRECHT DR KUEHR HELMUT KUNITZSCH PAUL PROF LAMLA ERICH E DR LEINERT CHRISTOPH DR LESCH HAROLD DR LORENZ HILMAR LUEST RHEA DR MATAS VLADIMIR R DR MAUDER HORST PROF DR MEINIG MANFRED DR METZ KLAUS DR MEYER-HOFME ISTER E DR MOELLENHOFF CLAUS DR MUENCH GUIDO PROF NECKEL TH DR NOTNI $P$ DR OETKEN L DR PAUL INY TOTH IVAN K K DR PFENMIG HANS H DR PILOWSKI K PROF DR POHL ECKHARD DR PRIESTER WOLFGANG PROF REFSDAL S PROF DR REIMERS DIETER PROF RICHTLER TOM DR RITTER HANS DR ROESER HERMANN-JOSEF DR ROHLFS K PROF DR ROTH-HOPPNER MARIA LUISE RUEDIGER GUWTHER DR SCHAIFERS KARL DR SCHILLER KARL PROF DR SCHLOSSER WOLFHARD PROF 


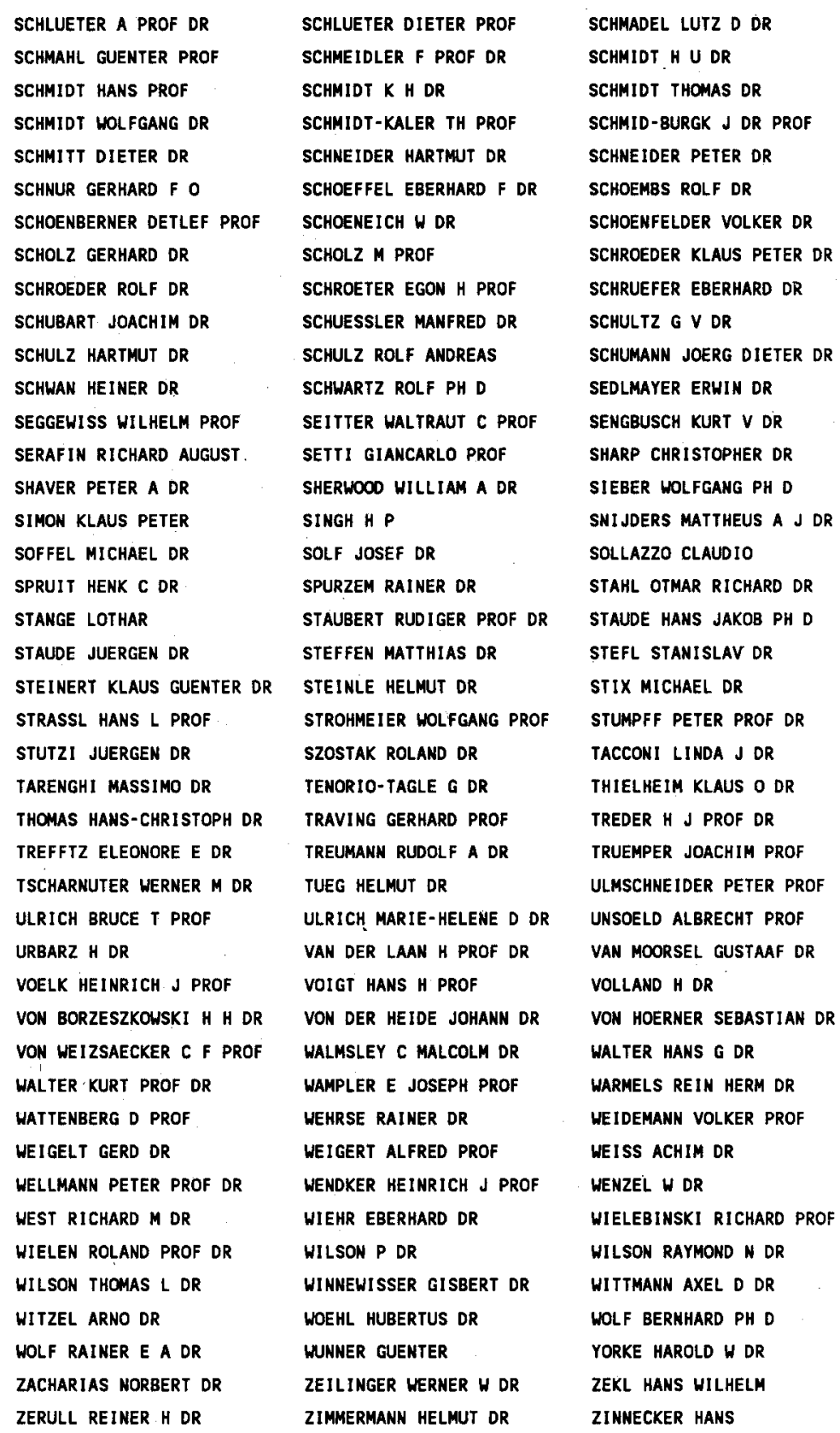


ABBOTT WILLIAM N DR ANTONOPOULOU E DR ASTERIADIS GEORGIOS DR BANOS GEORGE J PROF BOZIS GEORGE PROF CONTADAKIS MICHAEL E DR DAPERGOLAS A DR DIALETIS DIMITRIS DR GOUDAS CONSTANTINE L PROF HANTZIOS PANAYIOTIS DR KAZANTZIS PANAYOTIS DR KONTIZAS MARY DR

LIRITZIS IOANNIS DR MAVRAGANIS A G PROF MERZANIDES CONSTANTINOS NIARCHOS PANAYIOTIS PH D PAPATHANASOGLOU D DR PETROPOULOS BASIL CH DR POULAKOS CONSTANTINE DR ROVITHIS PETER DR SARRIS EMMANUEL T PH D SPITHAS ELEFTERIOS N DR SVOLOPOULOS SOTIRIOS PROF TRITAKIS BASIL $P$ DR TSINGANOS KANARIS DR VARDAVAS ILIAS MIHAIL VENTURA JOSEPH DR VOGLIS NIKOS DR XIRADAKI EVANGELIA DR ZAFIROPOULOS BASIL DR

\begin{tabular}{ll} 
ALISSANDRAKIS C PH D & ANTONACOPOULOS GREG PROF \\
ARABELOS DIMITRIOS DR & ARGYRAKOS JEAN PROF DR \\
AVGOLOUPIS STAVROS DR & BANOS COSMAS J OR \\
BARBANIS BASIL PROF & BELLAS-VELIDIS IOANNIS DR \\
CARANICOLAS NICHOLAS DR. & CAROUBALOS C A PROF \\
CONTOPOULOS GEORGE PROF & DANEZIS EMMANUEL DR \\
DARA HELEN DR & OELIYANNIS JEAN DR \\
DIONYSIOU DEMETRIOS PROF & GEROYANNIS VASSILIS S DR \\
GOUDIS CHRISTOS D PROF & HADJIDEMETRIOU JOHN D \\
HIOTELIS NICOLAOS DR & KATSIS DEMETRIUS DR \\
KOKKOTAS KONSTANTINOS DR & KONTIZAS EVANGELOS DR \\
XYLAFIS NIKOLAOS D DR & LASKARIDES PAUL G ASSPROF \\
MACRIS CONSTANTIN J PROF & MARKELLOS VASSILIS V DR \\
MAVRIDIS L N PROF & MAVROMICHALAKI HELEN DR \\
MOUSSAS XENOPHON PH D & MOUTSOULAS MICHAEL PROF \\
NICOLAIDIS EFTHYMIOS DR & PAPAELIAS PHILIP DR \\
PAPAYANNOPOULOS TH DR & PERSIDES SOTIRIOS C \\
PINOTSIS ANTONIS D DR & POLYMILIS CHRONIS OR \\
PREKA-PAPADEMA P DR & PROKAKIS THEOOORE J DR \\
ROVITHIS-LIVANIOU HELEN & SARRIS ELEFTHERIOS PH D \\
SEIMENIS JOHN DR & SEIRADAKIS JOHN HUGH DR \\
SPYROU NICOLAOS PROF & STATHOPOULOU MARIA DR \\
TERZIOES CHARALAMBOS DR & THEODOSSIOU EFSTRATIOS DR \\
TSAMPARLIS MICHAEL DR & TSIKOUDI VASSILIKI PH D \\
TSIOUMIS ALEXANDROS DR & TSIROPOULA GEORGIA DR \\
VARVOGLIS H DR & VEIS GEORGE PH D \\
VLACHOS DEMETRIUS G PROF & VLAHOS LOUKAS DR \\
XANTHAKIS JOHN N PROF & XANTHOPOULOS B C DR \\
2ACHARIADIS THEOOOSIOS DR & ZACHILAS LOUKAS DR \\
ZIKIDES MICHAEL C OR & \\
\hline
\end{tabular}




ALMAR IVAN PROF
BARLAI KATALIN DR
ERDI B DR
GRANDPIERRE ATIILA OR
ILLES ALMAR ERZSEBET DR
KANYO SANDOR DR
KOVACS AGNES DR
LOVAS MIKLOS
OLAH KATALIN DR
SZABADOS LASZLO PH D
SZECSENYI-NAGY GABOR DR
TOTH IMRE DR

BALAZS LAJOS G DR CSADA IMRE $K$ DR FEJES ISTVAN DR HORVATH ANDRAS DR JANKOVICS ISTVAN DR KELEMEN JANOS KOVACS GEZA DR MARIK MIKLOS DR PAPARO MARGIT DR SZALAY ALEX DR SZEGO KAROLY DR VERES FERENC

Country : ICELAND

GUDMUNDSSON EINAR H

JULIUSSON EINAR DR

Country : INDIA

ABHYANKAR KRISHNA D PROF

AHMAD FAROOQ DR

AMBASTHA A K DR

ANTIA H M DR

AULUCK FAQIR CHAND PROF

BALASUBRAMANIAN $V$ DR

BANERJI SRIRANJAN DR

BASU DIPAK DR

BHAT CHAMAN LAL DR

BHATIA R K DR

BHATNAGAR ASHOK KUMAR

BHATTACHARYA DIPANKAR

BHONSLE RAJARAM V PROF

BONDAL KRISHNA RAJ DR

CHAKRABORTY DEO $K$ DR

CHAUBEY UMA SHANKAR DR DADHICH NARESH OR

DAS P K DR

DESAI JYOTINDRA N

DHAWAN VIVEK DR

DWARAKANATH $K S$

GHOSH KAJAL KUMAR DR

GIRIDHAR SUNETRA DR

GOSWAMI J N DR

GURM HARDEV S PROF

IYER B R DR

JAYARAJAN A P MR

JOSHI MOHAN N PROF

KANDPAL CHANDRA D
ACHARYA BANNANJE S DR

ALLADIN SALEH MOHAMED DR ANANTHAKRISHMAN $S$

APPARAO K M $V$ DR

BABU G S D

BALLABH G M DR

BANHATTI DILIP GOPAL DR BHANDARI N DR

BHAT MARAYANA $P$ DR

BHATIA $\checkmark$ B DR

BHATNAGAR K B DR

BHATTACHARYYA \& C PROF

BISWAS SUKUMAR OR

BUTI BIMLA PROF

CHANDRA SURESH DR

CHITRE SHASHIKUMAR M DR

DAMLE S V DR

DATTA BHASKAR DR

DESHPANDE AVINASH

DUORAH HIRA LAL DR

DWIVEDI BHOLA NATH DR

GHOSH P DR

GOKHALE MORESHWAR HARI PR

GOYAL A N DR

HASAN SAIYID STRAJUL

JAIN RAJMAL DR

JOG CHANDA J DR

JOSHI SURESH CHANDRA DR

KAPAHI VIJAY $K$ DR
BARCZA SZABOLLS DR DEZSO LORANT PROF GERLEI OTTO ILL MARTON J DR KALMAN BELA OR KOLLATH ZOLTAN DR KUN MARIA DR MARX GYORGY PROF PATKOS LASZLO DR SZATMARY KAROLY DR SZEIDL BELA OR ZSOLDOS ENDRE DR

SAEMUNDSON THORSTEINN

AGRAWAL P C DR ALURKAR S K DR ANSARI S M RAZAULLAH PROF ASHOK N M DR BAGARE S P DR BANDYOPADHYAY A PROF BASU BAIDYANATH PROF BHANDARI RAJENDRA DR BHATIA PREM K DR BHATNAGAR ARVIND DR BHATT H C DR BHATTACHARYYA TARA DR BOODAPATI G ANANDARAO DR CHAKRABARTI SANDIP $K$ DR CHANDRASEKHAR T DR COWSIK RAMANATH DAS MRINAL KANTI DEGAONKAR S S DR DESHPANDE M $R$ DR DURGAPRASAD N DR GAUR $V P$

GHOSH S K DR GOPALA RAO U V MR GUPTA SUNIL $K$ DR IYENGAR $K \vee K$ PROF JAIN SURENDRA DR JOSHI G C DR JOSHI U C DR KAPOOR RAMESH CHANDER 
Country : INDIA (Follow on)

\begin{tabular}{|c|c|c|}
\hline KARANDIKAR R V PROF & KASTURIRANGAN K DR & KEMBHAVI AJIT K \\
\hline KILAMBI G C DR & KOCHHAR R K DR & KOTHARI D S DR \\
\hline KRISHNA GOPAL & KRISHNA SWAMY K S DR & KRISHNAMOHAN S DR \\
\hline KRISHNAN THIRUVENKATA MR & KULKARNI PRABHAKAR V PROF & KULKARNI VASANT K DR \\
\hline KUSHWAHA R S PROF & LAHIRI N C & LAL DEVENDRA \\
\hline MAHRA H S DR & MALLIK D C V DR & MANCHANDA R K DR \\
\hline MARAR T M K & MATHUR B S DR & MITRA A P DR \\
\hline MOHAN CHANDER DR & MOHAN VIJAY DR & NARANAN S PROF \\
\hline NARASIMHA DELAMPADY DR & NARAYANA J V & NARLIKAR JAYANT $\vee$ PROF \\
\hline NITYANANDA R DR & PADALIA T D DR & PADMANABHAN T DR \\
\hline PANDE GIRISH CHANDRA PROF & PANDE MAHESH CHANDRA DR & PANDEY A $K$ \\
\hline PANDEY $\mathbf{S} \mathrm{K}$. & PANDEY UMA SHANKAR DR & PARTHASARATHY M DR \\
\hline PATI A $K$ & PERAIAH ANNAMANENI DR & PRABHAKARAN NAYAR S R DR \\
\hline PRABHU TUSHAR $P$ & PRASANNA A R OR & PRATAP R DR \\
\hline PUNETHA LALIT MOHAN DR & RADHAKRISHNAN V PROF & RAGHAVAN NIRUPAMA DR \\
\hline RAJAMOHAN R DR & RAJU P K DR & RAKSHIT H PROF \\
\hline RAM SAGAR DR & RAMADURAI SOURIRAJA DR & RAMAMURTHY SHAMINATHAN \\
\hline RAMANA MURTHY $P V$ OR & RANA NARAYAN CHANDRA DR & RAO A PRAMESH DR \\
\hline RAO M N DR & RAO N KAMESHARA & RAO P VIVEKANANDA DR \\
\hline RAO RAMACHANDRA $V$ PROF & RAUTELA B S DR & RAY ALAK DR \\
\hline RAY CHOUDHURI ARMAB DR & RAYCHAUDHURI AMALKUMAR DR & RAZDAN HIRALAL \\
\hline RENGARAJAN T N DR & SAHA SWAPAN KUMAR DR & SAIKIA DHRUBA JYOTI DR \\
\hline SANWAL BASANT BALLABH DR & SANWAL N B DR & SAPRE A K DR \\
\hline SARMA M B K PROF & SARMA N V G PROF & SASTRI HANUMATH J DR \\
\hline SASTRY CH V & SASTRY SHANKARA $K$ & SAXENA A K DR \\
\hline SAXENA P P DR & SCARIA K K DR & SEN S N DR \\
\hline SHAH GHANSHYAM A DR & SHEVGAONKAR R K DR & SHUKLA K \\
\hline SHUKRE C S DR & SINGH JAGDEV DR & SIMGH KULINDER PAL DR \\
\hline SINHA $K$ DR & SINHA RAMESHWAR $P$ & SINVHAL SHAMBHU DAYAL DR \\
\hline SIVARAM C DR & SIVARAMAN K R DR & SREEKANTAN B V DR \\
\hline SRINIVASAN G & SRIVASTAVA J B DR & SRIVASTAVA RAM KUMAR DR \\
\hline STEPHENS S A DR & SUBRAHMANYA C $R$ & SUBRAHMANYAM $P V D R$ \\
\hline SUBRAMANIAN KANDASWAMY DR & SWARUP GOVIND PROF & TALWAR SATYA P DR \\
\hline TANDON JAGDISH NARAIN DR & TANDON S N PROF & TARAFDAR SHANKAR P DR \\
\hline THAKUR RATNA KUMAR DR & TONWAR SURESH C PROF & TREHAN SURINDAR $K$ PROF \\
\hline TRIPATHI B M DR & UDAYA SHANKAR N DR & VAIDYA P C PROF \\
\hline VARDYA M S DR & VARMA RAM KUMAR PROF & VASU-MALLIK SUSHMA DR \\
\hline VATS HARI ON DR & VELUSAMY T DR & VENUGOPAL V R OR \\
\hline VERMA $R P$ OR & VERMA SATYA DEV DR & VERMA V K OR \\
\hline $\begin{array}{l}\text { VINOO S KRISHAN MRS DR } \\
\text { VIVEXANANDA RAO }\end{array}$ & VISHVESHWARA C $\vee$ PROF & VIVEKANAND M DR \\
\hline Country & In & \\
\hline DAHAMAS DJONI $N$ DR & HIDAJAT BAMBANG PROF DR & IBRAHIM JORGA \\
\hline RADIMAN IRATIUS & RAHARTO MOEDJI & SIREGAR SURYADI DR \\
\hline SUKARTAD IRED JA DARSA & SUTANTYO HINARDI & WIRAMIHAROJA SUHARDJA DR \\
\hline
\end{tabular}




\section{ADJABSHIRIZADEH ALI} KALAFI MANOUCHER MALAKPUR IRADJ DR
ARDEBILI M REZA DR XHALESSEH BAHRAM DR SOBOUTI YOUSEF PROF

Country : IRAQ

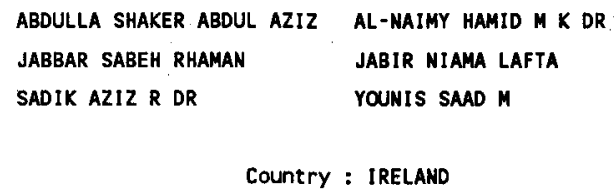

GHEIDARI S NASSIRI DR KIASATPOOR AHMAD PROF TEHERANY D

AL-SABTI ABDUL ADIM DR KADOURI TALIB HADI

DRURY LUKE O'CONNOR DR FEGAN DAVID J DR

HAYWOOO J

KIANG TAO PROF MCKEITH NIALL ENDA DR O'MONGAIN EON RAY IHOMAS P RUSSELL STEPHEN DR

BRAUN ARIE

DEKEL AVISHAI FINZI ARRIGO DR GOLDSMITH S DR HORUITZ GERALD PROF KATZ JOSEPH DR LEIBONITZ ELIA M DR MEERSON BARUCH DR NEEMAN YUVAL PROF OHRING GEORGE PROF RAKAVY GIDEON PROF SACK NOAM DR SHAVIV GIORA PROF VAGER ZEEV DR 
Country : ITALY

\begin{tabular}{|c|c|c|}
\hline ABRAMI ALBERTO PROF & ABRAMOWICZ MAREK DR & AIELLOO SANTI OR \\
\hline ALTAMORE ALDO & ANDREANI PAOLA MICHELA DR & ANGELETTI LUCIO DR \\
\hline ANILE ANGELO M & ANTONELLO ELIO & ANTONUCCI ESTER OR \\
\hline AURIEMMA GIULIO DR & BADIALI MASSIMO & BADOLATI ENNIO \\
\hline BAFFA CARLO DR & BALDINELLI LUIGI DR & BALLARIO M C PROF \\
\hline BANDIERA RINO DR & BANNI ALDO DR & BARATTA GIOVANNI BATTISTA \\
\hline BARBARO G DR & BARBERIS BRUNO & BARBIERI CESARE PROF \\
\hline BARBON ROBERTO PROF & BARLETTI RAFFAELE ENG & BARONE FABRIZIO DR \\
\hline BARTOLINI CORRADO & BATTINELLI PAOLO DR & BATTISTINI PIERLUIGI DR \\
\hline BEDOGNI ROBERTO & BELVEDERE GAETANO DR & BENACCHIO LEOPOLDO \\
\hline BENDINELLI ORAZIO & BERNACCA P L PROF & BERTELLI GIANPAOLO DR \\
\hline BERTIN GIUSEPPE PROF & BERTOLA FRANCESCO PROF & BETTONI DANIELA DR \\
\hline BIANCHI LUCIANA & BIANCHINI ANTONIO DR & BIGNAMI GIOVANNI $F$ \\
\hline BLANCO CARLO DR & BODO GIANLUIGI DR & BONACCINI DOMENICO DR \\
\hline BONANNO GIOVANNI DR & BONIFAZI ANGELO DR & BONO GIUSEPPE DR \\
\hline BONOLI FABRIZIO & BONOMETTO SILVIO A DR & BRACCESI ALESSANDRO PROF \\
\hline BRINI DOMENICO PROF & BROCATO ENZO DR & BROGLIA PIETRO DR \\
\hline BUONANNO ROBERTO & BUSON LUCIO M DR & BUSSO MAURIZIO \\
\hline CACCIANI ALESSANDRO PROF & CACCIARI CARLA DR & CACCIN BRUNO \\
\hline CALOI VITTORIA DR & CALVANI MASSIMO DR & CANTU ALBERTO M DR \\
\hline CAPACCIOLI MASSIMO DR & CAPACCIONI FABRIZIO DR & CAPPELLARO ENRICO DR \\
\hline CAPRIA MARIA TERESA DR & CAPRIOLI GIUSEPPE PROF & CAPUTO FILIPPINA DR \\
\hline CAPUZZO DOLCETTA ROBERTO & CARDINI DANIELA DR & CARPINO MARIO DR \\
\hline CARUSI ANDREA & CASSATELLA ANGELO DR & CASTELLANI VITTORIO PROF \\
\hline CASTELLI FIORELLA DR & CATALANO FRANCESCO A DR & CATALANO SANTO DR \\
\hline CATARZI MARCO DR & CAVALIERE ALFONSO G PROF & CAVALLINI FABIO \\
\hline CAZZOLA PAOLO DR & CELLINO ALBERTO DR & CEPPATELLI GUIDO DR \\
\hline CERRONI PRISCILLA DR & CERRUTI-SOLA MONICA & CESTER BRUNO PROF \\
\hline CEVOLANI GIORDANO & CHINCARINI GUIDO L DR & CHIOSI CESARE S DR \\
\hline CHIUDERI CLAUDIO PROF & CHIUDERI -DRAGO FRANCA PR & CHIUMIENTO GIUSEPPE \\
\hline CHLISTOVSKY FRANCA DR & CIATTI FRANCO DR & COLOMBO G PROF DR \\
\hline COLUZZI REGINA DR & COMORETTO GIOVANNI & CONCONI PAOLO DR \\
\hline CORADINI ANGIOLETTA & CORBELLI EDVIGE DR & COSMOVICI BATALLI C DR \\
\hline COSTA ENRICO & CRISTALDI SALVATORE DR & CRISTIANI STEFANO DR \\
\hline CRIVELLARI LUCIO & CUGUSI LEONINO DR & CURIR ANNA \\
\hline CUTISPOTO GIUSEPPE DR & DALLAPORTA N PROF & DALL 'OGLIO GIORGIO DR \\
\hline DANESE LUIGI DR & DE BIASE GIUSEPPE A DR & DE FELICE FERNANDO DR \\
\hline DE RUITER HANS RUDOLF & DE SABBATA V PROF DR & DE SANCTIS GIOVANNI \\
\hline DE ZOTII GIANFRANCO DR & DELLA VALLE MASSIMO DR & DELLI SANTI SAVERIO \\
\hline DI COCCO GUIDO & DI FAZIO ALBERTO & DI MARTINO MARIO \\
\hline DI SEREGO ALIGHIERI S DR & DI TULLIO GRAZIELLA DR & D'ANTONA FRANCESCA DR \\
\hline EINAUDI GIORGIO & ELLIS GEORGE $F$ R PROF & EMANUELE ALESSANDRO OR \\
\hline FACONDI SILVIA ROSA DR & FALCHI AMBRETTA & FALCIANI ROBERTO OR \\
\hline FALOMO RENATO DR & FANTI CARLA GIOVANNINI & FANTI ROBERTO \\
\hline FARAGGIANA ROSANNA PROF & FARINELLA PAOLO DR & FEDERICI LUCIANA \\
\hline FELLI MARCELLO DR & FERETTI LUIGINA & FERLUGA STENO DR \\
\hline FERRARI ATTILIO DR & FERRARI TONIOLO MARCO & FERRERI WALTER \\
\hline FERRINI FEDERICO & FICARRA ANTONINO DR & FOCARDI PAOLA DR \\
\hline FODERA SERIIO GIORGIA DR & FOF I MASSIMO DR & FORTI GIUSEPPE DR \\
\hline FORTINI TERESA DR & FRACASTORO MARIO G PROF & FRANCESCHINI ALBERTO \\
\hline
\end{tabular}


FRANCHINI MARIAGRAZIA DR FUSI PECCI FLAVIO GALLETTO DIONIGI GALLINO ROBERTO GEMMO ALESSANDRA DR GIANNONE PIETRO PROF GIOVANNELLI FRANCO DR GODOLI GIOVANNI PROF GREGGIO LAURA DR GRUEFF GAVRIL OR HACK MARGHERITA PROF IIJIMA TAKASHI DR

LAI SEBASTIANA LANDINI MASSIMO PROF LATTANZI MARIO $G$ LISI FRANCO DR MACERONI CARLA MAGGIO ANTONIO DR MAMMANO AUGUSTO DR MANDOLESI NAZZARENO MANTOVANI FRANCO MARDIROSSIAN FABIO MARMOLINO CIRO MASSAGLIA SILVANO MAZZONI MASSIMO DR MESSEROTTI MAURO DR MICELA GIUSEPPINA DR MISSANA MARCO DR MONSIGNORI FOSSI BRUNA DR MOTTA SANTO DR NATTA ANTONELLLA DR NOBILI L DR OCCHIONERO FRANCO PROF PACINI FRANCO PROF PALLA FRANCESCO PANMUNZIO RENATO PASIAN FABIO PATERNO LUCIO PROF PERINOTTO MARIO PROF PETTINI MARCO PIGATTO LUISA OR PIRO LUIGI DR PIZZICHINI GRAZIELLA POMA ANGELO DR PREITE-MARTINEZ ANDREA DR PUGLIANO ANTONIO PROF RAMELLA MASSIMO RENZINI ALVIO PROF ROBERTI GIUSEPPE DR ROSINO LEONIDA PROF
FULCHIGNONI MARCELLO PROF GALEOTTI PIERO PROF GALLIANO PIER GIORGIO GAUDENZI SILVIA DR GIACHETTI RICCARDO PROF GIANNUZZI MARIA A DR GIOVANNINI GABRIELE GOMEZ MARIA THERESA DR GREGORINI LORETTA GUARNIERI ADRIANO DR HUANG SONG-NIAN DR KRANJC ALDO DR LANDI DEGL 'INHOCENTI E PR LANDOLFI MARCO LESCHIUTTA S PROF LUCCHIN FRANCESCO MAFFEI PAOLO PROF MAGNI GIANFRANCO MANARA ALESSANDRO A DR MANNINO GIUSEPPE PROF MARANO BRUNO MARGONI RINO MARTINI ALDO DR MATTEUCCI FRANCESCA DR MAZZUCCONI FABRIZIO DR MESSINA ANTONIO MILANI ANDREa MISSANA MATALE PROF MORBIDELLI ROBERTO OR MUREDDU' LEONARDO DR NESCI ROBERTO NOCERA LUIGI DR OLIVA ERNESTO DR PADRIELLI LUCIA PALLAVICINI ROBERTO DR PAOLICCHI PAOLO DR PASINETTI LAURA E PROF PATRIARCHI PATRIZIO DR PEROLA GIUSEPPE C DR PICCA DOMENICO DR PINTO GIROLAMO PROF PIRRONELLO VALERIO POLCARO V F PORCEDDU IGNAZIO E P DR PROVERBIO EDOARDO PROF QUESADA VINICIO DR RAMPAZZO ROBERTO DR RIGHINI ALBERTO PROF RODONO MARCELLO DR ROSSI CORINNE DR
FUSCO-FEMIANO ROBERTO GALLETTA GIUSEPPE PROF GALLIANO PIER GJORGIO GAVAZZI GIUSEPPE DR GIALLONGO EMANUELE DR gIOVANAROI CARLO GIURICIN GIULIANO GRATTON R G DR GRUBISSICH C PROF DR GUERRERO GIANANTONIO DR HUNT LESLIE DR la padula cesare LANDI DEGL'INMOCENTI M LARI CARLO DR LISEAU RENE DR MACCAGNI DARIO MAGAZZU ANTONIO DR MALAGNINI MARIA LUCIA MANCUSO SANTI PROF MANTEGAZZA LUCIANO MARASCHI LAURA DR MARILLI ETTORE DR MASANI A PROF MAZZITELLI ITALO DR MERIGHI ROBERTO DR MEZZETTI MARINO MILANO LEOPOLDO DR MOLARO PAOLO DR MOROSSI CARLO NATALI GIULIANO DR NOBILI ANNA $M$ NOCI GIANCARLO PROF ORTOLANI SERGIO PALAgI fRANCESCO PALUMBO GIORGIO G C DR PARMA PAOLA PASTORI LIVIO PERES GIOVANHI DR PERSI PAOLO PICCIONI ADALBERTO PIOTTO GIAMPAOLLO PIZZELLA G DR POLETTO GIANMINA PROF PORETTI ENNIO PUCILLO MAURO DR RAFANELLI PIERO DR RANIERI MARCELLO RIGUTTI MARIO PROF ROMANO GIULIANO PROF ROSSI LUCIO 
Country : ITALY (Follow on)

\begin{tabular}{|c|c|c|}
\hline RUFFINI REMO & RUSCONI LUIGIA DR & RUSSO GUIDO DR \\
\hline $\begin{array}{l}\text { SABBAD IN FRANCO DR } \\
\text { SALVATI MARCO }\end{array}$ & $\begin{array}{l}\text { SAGGION ANTONIO PROF } \\
\text { SANTAMARIA RAFFAELE DR }\end{array}$ & $\begin{array}{l}\text { SALINARI PIERO } \\
\text { SANTIN PAOLO DR }\end{array}$ \\
\hline SARASSO MARIA DR & SCALTRITI FRANCO DR & SCARDIA MARCO \\
\hline SCIAMA DENNIS W DR & SCIORTINO SALVATORE DR & SECCO LUIGI DR \\
\hline SEDMAK GIORGIO PROF & SELVELLI PIERLUIGI DR & SEMENZATO ROBERTO \\
\hline SERIO SALVATORE DR & SEVER INO GIUSEPPE & SILVESTRO GIOVANNI \\
\hline SMALDONE LUIGI ANTONIO & SMRIGLIO FILIPPO PROF & SPADA GIANFRANCO DR \\
\hline SPADARO DANIELE DR & STAGMI RUGGERO & STALIO ROBERTO DR \\
\hline STANGA RUGGERO & STIAVELLI MASSIMO DR & STIRPE GIOVANNA M DR \\
\hline STRAFELLA FRANCESCO & STRAZZULLLA GIOVANMI & TAFFARA SALVATORE PROF \\
\hline TAGLIAFERRI GIUSEPPE PROF & TANZELLA-NITTI GIUSEPPE & TANZI ENRICO G \\
\hline TEMPESTI PIERO PROF & TERNULLO MAURIZIO DR & TOFANI GIANNI PROF \\
\hline TOMASI PAOLO DR & TORELLI M DR & TORNAMBE AMEDEO \\
\hline TORRICELLI GUIDETTA DR & TOSI MONICA & TOZZI GIAN PAOLO \\
\hline TREVESE DARIO & TRINCHIERI GINEVRA & TRUSSONI EDOARDO \\
\hline TURATTO MASSIMO DR & UBERTINI PIETRO & URAS SILVANO DR \\
\hline VAGNETTI FAUSTO & VAIANA GIUSEPPE S DR & VALSECCHI GIOVANNI B DR \\
\hline VERGNANO A PROF & VERNIANI FRANCO PROF & VETTOLANI GIAMPAOLO \\
\hline VIETRI MARIO DR & VIGOTTI MARIO & VIOTTI ROBERTO DR \\
\hline VIRGOPIA NICOLA PROF & VITTONE ALBERTO ANGELO & VITTORIO NICOLA \\
\hline VLADILO GIOVANNI DR & WOLTER ANMA DR & ZAMORANI GIOVANMI \\
\hline ZANINETTI LORENZO & ZAPPALA ROSARIO ALDO DR & ZAPPALA VINCENZO PROF \\
\hline ZAVATTI FRANCO & 2ITELLI VALENTINA DR & ZLOBEC PAOLO DR \\
\hline
\end{tabular}


AIZU KO PROF ANDO HIROYASU DR ARAI KENZO DR CHIKADA YOSHIHIRO DR DOTANI TADAYASU DR ERIGUCHI YOSHIHARU DR FUJIMOTO MITSUAKI OR FUJIUARA AKIRA DR FUKUE JUN DR FUKUI YASUO DR FURUKAWA KIICHIRO DR HACHISU IZUMI DR HAMADA TETSUO PROF HANAOKA YOICHIRO DR HARA KEN NOSUKE DR HASEGAWA ICHIRO DR HAYASHI CHUSHIRO PROF HIEI EIJIRO DR HIRATA RYUKO HITOTSUYANAGI JUICHI PROF HOSHI REIUN DR ICHIKAWA SHIN-ICHI DR IIJIMA SHIGETAKA PROF INATANI JUNJI INOUE TAKESHI PROF ISHIDA KEIICHI PROF ISHIZAWA TOSHIAKI A PROF ITO KENSAI A PROF ITOH NAOKI DR JUGAKU JUN DR KAIFU NORIO DR KAMEYA OSAMU DR KANEKO NOBORU DR KATO MARIKO KAWABATA KINAKI PROF KAWAGUCHI ICHIRO PROF KIGUCHI MASAYOSHI DR KINOSHITA HIROSHI DR KITAMURA SEIICHI DR KODAIRA KEIICHI PROF KOJIMA MASAYOSHI DR KOSAI HIROKI KOYAMA KATSUJI KOZAI YOSHIHIDE PROF KUNIEDA HIDEYO DR MAEDA KOITIRO MAKINO FUMIYOSHI DR MANABE SEIJI DR MATSUMOTO MASAMICHI PROF MATSUMURA MASAFUMI DR
AKABANE KENJI A PROF AOKI SHINKO PROF AZUMA TAKAHIRO DR DAISHIDO TSUNEAKI PROF EBISUZAKI TOSHIKAZU DR FUJIMOTO MASAYUKI DR FUJISHITA MITSUMI OR FUJIWARA TAKAO DR FUKUGITA MASATAKA DR FUKUNAGA MASATAKA DR GOUDA NAOTERU DR HAGIO FUMIHIKO DR HAMAJIMA KIYOTOSHI DR HANAWA TOMOYUKI DR HARA TADAYOSHI DR HASEGAWA TETSUO DR HAYASHI MASAHIKO DR HIRABAYASHI HISASH] DR HIRAYAMA TADASHI PROF HORI GENICHIRO PROF HOSOKAWA YOSHIMASA H PROF ICHIKAWA TAKASHI IKEUCHI SATORU DR INOUE HAJIME DR IRIYAMA JUN DR ISHIGURO MASATO PROF ISHIZUKA TOSHIHISA DR ITOH HIROSHI DR IWASAKI KYOSUKE DR KABURAKI MASAKI PROF KAKINUMA TAKAKIYO T PROF KAMI JO FUMIO PROF OR KAROJI HIROSHI DR KATO SHOJI PROF KAWABATA KIYOSHI KAWARA KIMIAKI KII TSUNEO DR KITAMOTO SHUNJI DR KOBAYASHI EISUKE DR KODAMA HIDEO KONDO MASAAKI DR KOSHIBA MASA-TOSHI DR KOYAMA KO-U-ICHI DR KUBO YOSHIO KUROKAWA HIROKI DR MAEHARA HIOEO DR MAKISHIMA KAZUO MATSUDA TAKUYA PROF MATSUMOTO RYOJI DR MATSUOKA MASARU OR
AKABANE TOKUHIDE DR ARAFUNE JIRO DR BABA NAOSHI DR DEGUCHI SHUJI OR ENOME SHINZO PROF FUJIMOTO MASA-KATSU DR FUJITA YOSHIO PROF FUKUDA ICHIRO FUKUI TAKAO DR FUKUSHIMA TOSHIO DR HABE ASAO HAMABE MASARU DR HANAMI HITOSHI DR HANDA TOŠHIHIRO DR HARA TETSUYA DR HASHIMOTO MASA-AKI DR HAYASHI SAEKO S DR HIRAI MASANORI DR HIROMOTO NORIHISA DR HORIUCHI RITOKU DR HOSOYAMA KENMOSHUKE DR ICHIMARU SETSUO DR INAGAKI SHOGO DR INOUE MAKOTO DR ISHIDA GORO DR ISHIHARA HIDEKI DR ISOBE SYUZO DR ITOH MASAYUKI DR IYE MASANORI DR KAI KEIZO OR KAKUTA CHUICHI DR KAMINISHI KEISUKE PROF KASUGA TAKASHI KATO TAKAKO DR KAHABATA SHUSAKU PROF KAWATA YOSHIYUKI DR KIKUCHI SADAEMON PROF KITAMURA M PROF KOBAYASHI YUKISAYU KOGURE TOMOKAZU DR KONDO MASAYUKI DR KOSUGI TAKEO KOYAMA SHIN PROF OR KUBOTA JUN DR MAEDA KEI-ICHI DR MAIHARA TOSHINORI DR MAKITA MITSUGU DR MATSUI TAKAFUMI DR MATSUMOTO TOSHIO DR MIKAMI TAKAO DR 
Country : JAPAN (Follow on)

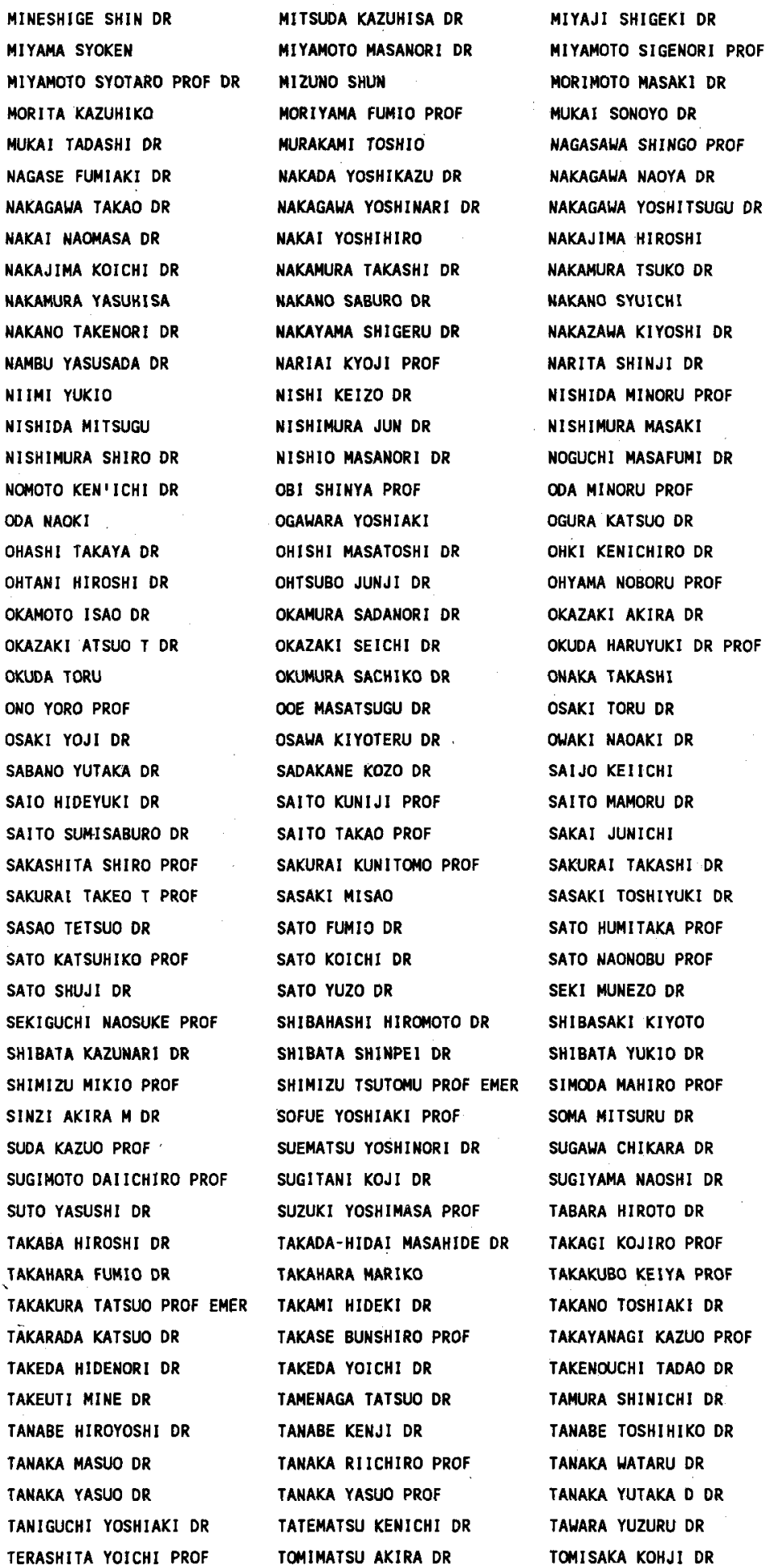




TOMITA KENJI PROF
TOSA MAKOTO DR
TOYAMA KIYOTAKA
TSUBOKAWA IETSUNE DR
TSUNEMI HIROSHI DR
UCHIDA YUTAKA PROF
UKITA NOBUHARU
UTSUMI KAZUHIKO DR
WASHIMI HARUICHI DR
WATANABE TETSUYA
YAMAGATA TOMOHIKO DR
YAMAMOTO TETSUO DR
YAMASHITA YASUMASA PROF
YOKOSAWA MASAYOSHI DR
YOSHIDA HARUO
YOSHIMURA HIROKAZU DR
YUASA MANABU DR

TOMITA KENJI PROF TOMITA KOICHIRO MR TOSHIKI AIKAHA DR TSUBAKI TOKIO PROF TSUCHIYA ATSUSHI DR PROF TSUNETA SAKU DR UENO SUEO PROF UMEMURA MASAYUKI OR WAKAMATSU KEN-ICHI DR WATANABE JUN-ICHI DR YABUSHITA SHIN A PROF YAMAGUCHI SHICHIRO YAMASAKI ATSUMA DR YAMAZAKI AKIRA DR YOKOYAMA KOICHI DR YOSHIDA JUNZO PROF YOSHIMURA MOTOHIKO DR YUMI SHIGERU PROF DR

\section{Country : KAZAKHSTAN}

DENISYUK EDVARD $K$ DR KHARITONOV ANDREJ $V$ DR ROZHKOVSKIJ DIMITRIJ A
GENKIN IGOR L PROF DR OBASHEV SAKEN O DR TEJFEL VIKTOR G DR

Country : KOREA DPR

BAEK CHANG RYONG

CHA GI UNG

DONG IL ZUN

KANG JIN SOK

KIM YONG UK

LI GI MAN

LI J $Y$
BANG YONG GOL

CHIO CHOL ZONG

HONG HYON IK

KIM JIK SU

KIM YUL

LI GYONG WON

LI SIN HYONG
TORAO MASAHISA

TOTSUKA YOJI DR TSUBOI MASATO OR TSUJI TAKASHI UCHIDA JUICHI DR UESUGI AKIRA DR UNWO WASABURO PROF WAKO KOJIRO DR WATANABE TAKASHI DR YABUUTI KIYOSHI PROF YAMAKOSHI KAZUO YAMASHITA KOJUN DR YASUDA HARUO PROF DR YONEYAMA TADAOKI DR YOSHII YUZURU DR YOSHIZAMA MASANORI DR

KARYGINA ZOYA V DR OMAROV TUKEN B PROF VILKOVISKIJ EMMANUIL Y DR
CHA DU JIN CHOI WON CHOL KANG GON IK KIM YONG HYOK DR KIM ZONG DOK LI HYOK HO LI SON JAE 
ANN HONG BAE DR CHOE SEUNG URN DR CHUN MUN-SUK OR KANG YOUNG WOON DR XIM KWANG-TAE DR LEE SANG GAK LEE YONG-SAM DR NHA IL-SEONG DR PARK MYEONG-GU DR SONG DOO JONG DR YU KYUNG-LOH PROF
CHANG KYONGAE DR CHOI KYU-HONG HONG SEUNG SOO OR KIM CHULHEE DR KIM TU HWAN LEE SEE-WOO DR MINA YOUNG KEY DR OH KYU DONG DR PARK SEOK JAE DR SUH KYUNG-WON DR YUN HONG-SIK PROF
Country : LATVIA

\begin{abstract}
ABELE MARIS $K$ DR DAUBE-KURZEMNIECE I A OR LAPUSHKA K K DR
\end{abstract}

ALKSNIS ANDREJS DR DIRIKIS M A OR

PLATAIS IMANT $K$ DR

Country : LITHUANIA

RUDZIKAS ZENONAS B

STRAIZYS V PROF DR

Country : MALAYSIA

ILYAS MOHAMMAD DR

MAHAT ROSLI H DR

MOHD ZAMBRI ZAINUDDIN DR

OTHMAN MAZLAN

Country: MEXICO

\section{ALLEN CHRISTINE}

CANTO JORGE DR

CHATTERJEE TAPAN K DR

CHELLI ALAIN

CRUZ-GONZALEZ IRENE

ECHEVARRIA JUAN DR

FIERRO JULIETA

GALINDO TREJO JESUS DR

HACYAN SHAHEN DR

KOENIGSBERGER GLORIA

MALACARA DANIEL

OBREGON DIAZ OCTAVIO J DR

PENA MIRIAM DR

PEREZ-PERAZA JORGE DR

RECILLAS-CRUZ ELSA DR

RUELAS-MAYORGA R A DR

SERRANO ALFONSO DR

WARMAN JOSEF DR
MENDOZA V EUGENIO E DR

\author{
ARELLANO FERRO ARMANDO \\ CARDONA OCTAVIO DR \\ CHAVARRIA-K CARLOS \\ CORNE JO ALE JANDRO A DR \\ DALTABUIT ENRIQUE DR \\ ECHEVERRIA ROMAN JUAN $M$ \\ FIRMANI CLAUDIO A PROF \\ GARCIA-BARRETO JOSE A \\ HERRERA MIGUEL ANGEL DR \\ LIZANO-SOBERON SUSANA DR \\ MARTINEZ MARIO DR \\ MORENO CORRAL MARCO A DR \\ PEIMBERT MANUEL DR \\ PENICHE ROSARIO DR \\ PISMIS DE RECILLAS PARIS \\ RODRIGUEZ LUIS $F$ \\ SARMIENTO-GALAN A F DR \\ TAPIA MAURICIO DR
}

CHO SE HYUNG DR CHOU KYONG CHOL PROF HYUN JONG-JUNE PROF KIM KAP-SUNG DR LEE HYUNG MOK DR LEE WOO-BAIK DR MOON SHIN HAENG DR PARK HONG SUH DR SHIM WOON-TAIK PROF WOO JONG OK
BALKLAVS A E DR FRANTSMAN YU L DR ZHAGAR YOURI H DR
ZOANAVICIUS KAZIMERAS DR MAJID ABDUL BIN A H DR

BISIACCHI GIANFRANCO DR CARRASCO LUIS DR CHAVIRA ENRIQUE SR COSTERO RAFAEL DE. LA HERRAN $V$ JOSE ENG ESCALANTE VLADIMIR OR FRANCO JOSE DR GONZALEZ G KLAPP JAIME DR LOPEZ JOSE ALBERTO DR MENOEZ MANUEL DR MORENO EDMUNDO DR PENA JOSE PEREZ-DE-TEJADA H A DR POVEDA ARCADIO DR ROSADO MARGARITA DR SCHUSTER WILLIAM JOHN DR TORRES-PEIMBERT SILVIA DR 
EL BAKKALI LARBI DR

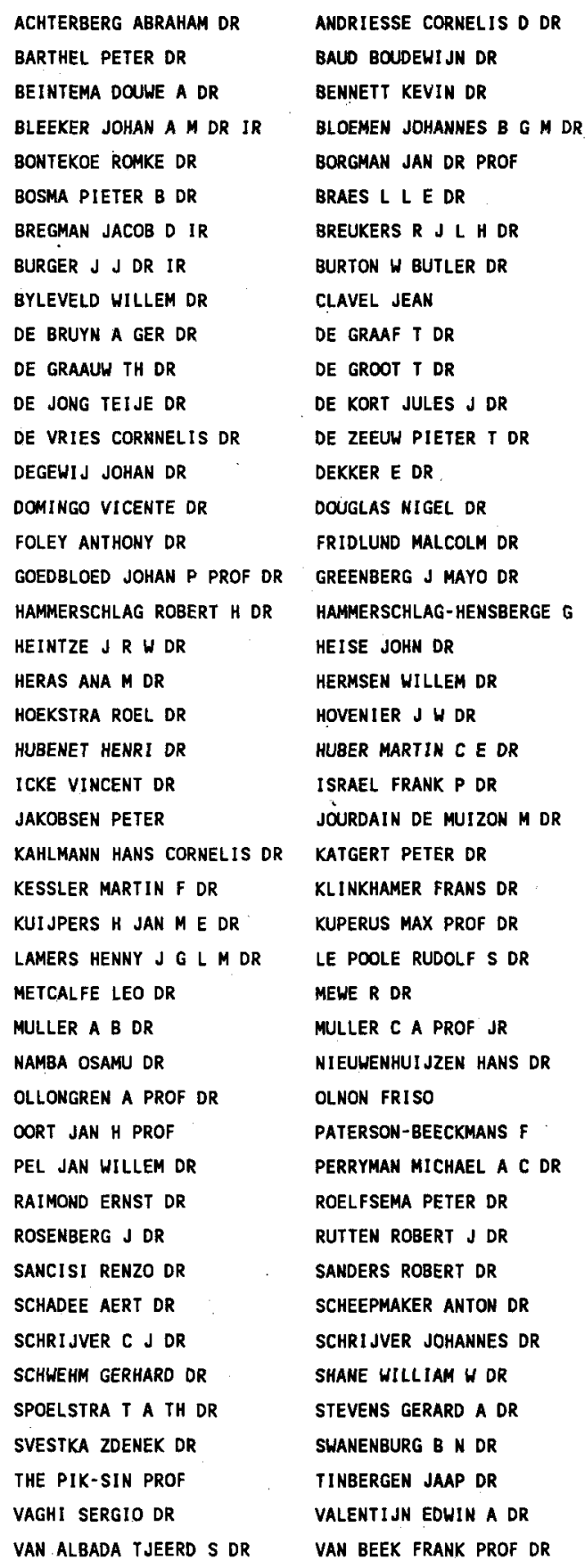

ATAMASIJEVIC IVAN DR BEGEMAN KOR G DR BLAAUW ADRIAAN PROF DR BOLAND HILFRIED BOS ALBERT DR BRAUN ROBERT DR BRINKMAN BERT C DR BUTCHER HARVEY $R$ PROF DR COLEMAN PAUL HENRY DR DE GRAAFF $W$ DR DE JAGER CORNELIS PROF DE KORTE PIETER A J DR DEERENBERG A J M DR DEUL ERIK DR FITTON BRIAN OR FRITZOVA-SVESTKA L OR HABING H J DR HEARN ANTHONY G DR HENR ICHS HUBERTUS F DR HESKE ASTRID DR HOYNG PETER DR HULSBOSCH A N M DR IVES JOHN CHRISTOPHER MR KAASTRA JELLE S DR, KATGERT-MERKELIJN J K DR KRAAN-KORTENEG RENEE C DR KWEE K K DR LUB JAN DR MILEY G K DR MURPHY BRIAN WILLIAM DR NORTH JOHN DAVID PROF OLTHOF HINDERICUS DR PEACOCK ANTHONY OR POTTASCH STUART R PROF RDOS NICOLAAS DR SAHU KAILASH C DR SAVONIJE GERRIT JAN DR SCHILIZZI RICHARD I DR SCHWARZ ULRICH J DR SMIT J A PROF STROM RICHARD G DR TAKENS ROELF JAN DR TJIN-A-DJIE HERMAN R E DR VAN AGT $S L T H 」 D R$ VAN BUEREN HENDRIK G PROF 
Country : NETHERLANDS (Follow on)

VAN DE HULST H C PROF DR VAN DEN HEUVEL EDWARD $P$ J VAN DER HULST JAN M DR VAN OIGGELEN J DR VAN DRIEL-GESZTELYI L DR VAN HERK G VAN NIEUWKOOP J DR IR VERBUNT FRANCISCUS DR WIJNBERGEN JAN DR
VAN DE KAMP PETER

VAN DEN OORD BERT H J DR VAN DER KLIS MICHIEL DR VAN DISHOECK EWINE F DR VAN DUINEN $R$ J DR VAN HOUTEN C I DR VAN PARADIJS JOHANNES DR WATERS LAURENS B F M DR WINKLER CHRISTOPH DR
VAN DE STADT HERMAN DR VAN DER HUCHT KAREL A DR VAN DER KRUIT PIETER C DR VAN DRIEL WILLEM DR VAN GENDEREN A M DR VAN HOUTEN-GROENEVELD I VAN WOERDEN HUGQ PROF DR WESSELIUS PAUL R DR ZWAAN CORNELIS PROF DR

Country : NEW ZEALAND

ALEXANDER MURRAY E DR BATESON FRANK M OBE DR COTTRELL PETER LEDSAM DOUGHTY NOEL, A DR JONES ALBERT $F$ MR ORCHISTON WAYNE DR SULLIVAN DENIS JOHN DR WALKER WILLIAM S G

\author{
ALLEN WILLIAM \\ BLOW GRAHAM L \\ CRAIG IAN JONATHAN D DR \\ GILMORE ALAN C MR \\ KERR ROY P PROF \\ RUMSEY NORMAN J \\ TOBIN WILLIAM
}

BAGGALEY WILLIAM J PROF BUDDING EDWIN DR DODD RICHARD J DR HEARNSHAW JOHN B DR MARINO BRIAN F ENG SCHATTEN KENNETH H DR TRODAHL HARRY JOSEPH DR

$$
\text { Country : NORHAY }
$$

\section{AKSNES KAARE DR} CARLSSON MATS DR ERIKSEN GUNNAR PROF HAVNES OVE DR LEER EGIL PROF PETTERSEN BJOERN RAGNVALD STABELL ROLF DR

\begin{abstract}
ANDERSEN 80 NYBORG DR ELGAROY OYSTEIN PROF ESSER RUTH DR JENSEN EBERHART PROF MALTBY PER PROF
\end{abstract} RINGNES TRULS S DR TRULSEN JAN $K$ PROF
BRAHDE ROLF ENGVOLD OODBJOERN DR HAUGE OIVIND DR KJELDSETH-MOE OLAV DR OESTGAARO ERLEND SOLHEIM JAN ERIK 
BEM JERZY DR CHOLONIEUSSKI JACEK DR CZERNY BOZENA DR DROZYNER ANDRZEJ GASKA STANISLAH DR GORGOLEWSKI STANISLAW PR GRZEDZIELSKI STANISLAW PR HELLER MICHAEL PROF IWANOWSKA WILHELMINA PROF JAROSZYNSKI MICHAL JUSZKIEWICZ ROMAN KOZIEL KAROL PROF DR XREINER JERZY MAREK DR KRUSZEWSKI ANDRZEJ PROF KURPINSKA-WINIARSKA M DR KUSUNOSE MASAAKI DR MACIEJEWSKI ANDRZEJ J DR MERGENTALER JAN PROF MIETELSKI JAN S DR MOSKALIK PAWEL, OR PROSZYNSKI MIECZYSLAW RUDAK BRONISLAW SCHREIBER ROMAN SIENKIEWICZ RYSZARD DR SITARSKI GRZEGORZ PROF SOKOLOWSKI LECH STEPIEN KAZIMIERZ DR SYLWESTER JANUSZ TYLENDA ROMUALD DR USOWICS JERZY BOGDAN DR WOSZCZYK ANDRZEJ PROF ZIOLKOWSKI KRZYSZTOF DR
BORKOWSKI KAZIMIERZ M DR CIURLA TADEUSZ DOBRZYCKI JERZY PROF DZIEMBOWSKI WOJCIECH PROF GIL JANUSZ a PROF GRABOWSKI BOLESLAW DR haENSEL PAWEL DR HURNIK HIERONIM PROF JAKIMIEC JERZY PROF JARZEBOWSKI TADEUSZ DR KALUZNY JANUSZ DR KOZLOWSKI MACIEJ DR KRELOWSKI JACEK DR KRYGIER BERNARD DR KURZYNSKA KRYSTYNA DR LEHMANN MAREK DR MADEJ JERZY MICHALEC ADAM MIKOLAJEHSKA JOANNA DR OPOLSKI ANTONI PROF ROMPOLT BOGDAN DR RUDNICKI KONRAD PROF SCHWARZENBERG-CZERNY A SIKORA MAREK SMAK JOSEPH I PROF SOLTAN ANDRZEJ MARIA DR STROBEL ANDRZEJ DR SZAFRANIEC ROZALIA DR UDALSKI ANDRZEJ DR WINIARSKI MACIEJ ZIEBA STANISLAW DR
BRZEZINSKI ALEKSANDER DR CUGIER HENRYK DR DOMINSKI IRENEUSZ DR FLIN PIOTR GLEBOCKI ROBERT PROF GRUDZINSKA STEFANIA DR HANASZ JAN DR IWANISZEWSKA CECYLIA DR JAKS WALDEMAR DR JERZYKIEWICZ MIKOLAJ DR KOLACZEK BARBARA DR KRASINSKI ANDRZEJ PROF KREMPEC-KRYGIER JANINA DR KUBIAK MARCIN A DR KUS ANDRZEJ JAN DR MACHALSKI JERZY DR MASLOWSKI JOZEF DR MICHALOWSKI TADEUSZ DR MOCZKO JANUSZ DR PACZYNSKI BOHDAN PROF ROZYCZKA MICHAL RYBKA PRZEMYSLAW DR SEMENIUK IRENA DR SIKORSKI JERZY DR SMOLINSKI JAN DR STAWIKOWSKI ANTONI DR SYLUESTER BARBARA DR TURLO ZYGMUNT DR URBANIK MAREK DR WNUK EDWIN ZIOLKOWSKI JANUSZ DR

Country : PORTUGAL

CABRITA EZEQUIEL DR DA COSTA ANTONIO A DR LAGO MARIA TERESA $V$ T PR NUNES ROGERIO S DE SOUSA SANTOS FILIPE D DR VICENTE RAIMUNDO O PROF
CAMPOS L M BRAGA DA COSTA DA SILVA A V C S MAGALHAES ANTONIO A $S$ ENG OSORIO JOSE J S P PROF TABORDA JOSE ROSA DR
COELHO BALSA MARIO C DR DOS REIS M PROF MARQUES MANUEL N DR PASCOAL ANTONIO J B SCI TAVARES $J T L D R$ 
ABALAKIN VICTOR $K$ DR AGEKJAN TATEOS A PROF ANTIPOVA LYUDMILA DR BABADZHANIANC MICHAIL DR BALEGA YURI YU DR, BELINSKY VLADIMIR DR BISNOVATYI-KOGAN G S DR BOCHKAREV NIKOLAY G DR BOYARCHUK MARGARITA E DR BRUMBERG VICTOR A DR CHEREPASHCHUK A M PROF CHISTYAKOV VLADIMIR E DR DAGKESAMANSKY RUSTAM D DR DLUZHNEVSXAYA O B DR DOLGINOV ARKADY $Z$ PROF DR DRAVSKIKH A F DR EMEL IANOV NIKOLAJ $V$ DR EVDOKIMOV YU $V$ DR FOMENKO ALEXANDR F DR FOMINOV ALEXANDR $M$ DR FURSENKO $M$ A DR GELFREIKH GEORGIJ B OR GLEBOVA NINA I DR GNEVYSHEVA RAISA S DR GREBENIKOV E A PROF DR GROUSHINSKY N P PROF DR GULYAEV RUDOLF A DR HAGEN-THORN VLADIMIR A DR IKHSANOVA VERA $N$ DR IVANOV VSEVOLOD $\checkmark$ DR PROF KADLA ZDENKA I DR KARDASHEV N $S$ DR KHOLSHEVNIKOV $K \vee D R$ KIM IRADIA S KISSELEVA TAMARA $P$ KOLESOV A K DR KORCHAK A A DR KOSTINA LIDIJA D DR KOTELNIKOV V A ACAD KSANFOMALITI L $V$ DR KUMKOVA IRINA I DR KUTUZOV S A DR LAVRUKHINA A $K$ PROF DR LIPOVETSKY V A LOZINSKAYA TAT'YANA A DR MAKAROVA ELENA A DR MAROV MIKHAIL YA PROF MERMAN G A DR MININ I N PROF MOLCHANOV A P PROF
AFANASJEVA PRASKOVYA H DR AKIM EFRAJM L DR ANTONOV VADIM A DR BABIN V G DR BATRAKOV YU $V$ DR BELKOVICH O I DR BLINOV N S DR BONDARENKO L N DR BREJDO IZABELLA I DR BYSTROVA NATALIJA V DR CHERNOV GENNADIJ DR CHUGAI NIKOLAI N DR DANILOV VLADIMIR M DR DOKUCHAEV VYACHESLAV DR DOROSHKEVICH ANDREI G DR EFREMOV YU I DR ERPYLEV N P DR FADEYEV YURI A FOMICHEV VALERI $V$ DR FRIDMAN ALEKSEY M DR GALIBINA I $V$ DR GINZBURG VITALY L PROF GLUSHNEVA I N DR GORBATSKY VITALIJ G PROF GRIGORJEV VICTOR M DR GUBANOV VADIM S DR GURSHTEIN ALEXANDER A DR IDLIS G M DR IMSHENNIK $V S$ DR IVANOV-KHOLOONY G S DR KANAEV IVAN I DR KARPINSKIJ VADIM N DR KHOZOV GENMADIJ $V$ KISELYOV ALEXEJ A DR KOCHAROV GRANT E PROF KONONOVICH EDWARD $V$ DR KOROVYAKOVSKIJ YURIJ P DR KOSTYAKOVA ELENA B DR KRASINSKY GEORGE A DR KUKLIN G V DR KURIL-CHIK V N DR KUZMIN ARKADII D PROF OR LEBEDINETS VLADIMIR N DR LIVSHITS M A DR LOZINSKIJ A M DR MANDZHOS ANDREJ $V D R$ MASSEVICH ALLA G DR MERMAN NATALIA $\checkmark$ DR MITROFANOVA LYUDMILA A DR MOROZ V I PROF DR
AFANAS'EV VIKTOR L DR AKSENOV E P PROF DR ARKHIPOVA $\vee P$ DR BAGILDINSKIJ BRONISLAV K BAZILEVSKY ALEXANDR T BELYAEV NIKOLAJ A DR BOBROV M S DR BOYARCHUK A A DR BRONNIKOVA NINA M CHECHETKIN VALERIJ M DR CHERTOPRUD $V$ E DR DADAEV ALEKSANDR N DR DEMIN V G PROF DR DOKUCHAEVA OLGA D DR DOUBINSKIJ B A DR EFREMOV YURY N DR ESIPOV VALENTIN F DR FINKELSTEIN ANDREJ M DR FOMIN VALERY A DR FROLOV M S DR GALPERIN YU I PROF GLAGOLEVSKI」JU V DR GNEDIH YURIJ N DR GOSACHINSKIJ I V DR GRISHCHUK $L P D R$ GULYAEV A P DR HABIBULLIN SH T PROF DR IKHSANOV ROBERT N DR IOSHPA B A DR IZVEKOV $\checkmark$ A DR KARACHENTSEV I D DR KHOKHLOVA V L DR KHROMOV G S OR KISLYAKOV ALBERT G DR KOKURIN YURIJ L DR KOPYLOV I M DR KOSIN GENNADIJ S DR KOSTYLEV $K \vee D R$ KRASSOVSKY $V I$ I KUMAJGOROOSKAYA RAISA DR KURT $\checkmark$ G DR LAVROV M I PROF LEIKIN G A DR LOTOVA N A DR MAKAROV VALENTINE I MAROCHNIK L S PROF DR MATVEYENKO L I DR MIKHELSON NIKOLAJ N DR MOGILEVSKIJ EH I DR MYACHIN VLADIMIR F DR 


\begin{tabular}{|c|c|c|}
\hline NADYOZHIN D K DR & NAGIRNER DMITRIJ I DR & NAGNIBEDA VALERY G DR \\
\hline NAIDENOV VICTOR O & NAUMOV VITALIJ A DR & NEFEDEVA ANTONINA I PROF \\
\hline NEMIRO ANDREJ A DR PROF & NIKITIN A A DR & NOSKOV BORIS N DR \\
\hline NOVIKOV I D DR & NOVIKOV SERGEJ B DR & NOVOSELOV V S PROF DR \\
\hline OBRIDKO VLADIMIR N DR & PAMYATNIKH A A DR & PARIJSKIJ N N PROF \\
\hline PARIJSKIJ YU N DR & PAVLOVSKAYA E D DR & PETROV GENNADIJ M \\
\hline PETROV GEORGIJ I PROF DR & PETROVSKAYA M S DR & PISKUNOV ANATOLY E \\
\hline PISKUNOV NIKOLAI E DR & POLOZHENTSEV DIMITRIJ DR & POLYACHENKO VALERIJ L DR \\
\hline POPOV VICTOR S DR & PORFIR'EV V V DR & POTTER HEINO I DR \\
\hline PRODAN Y I DR & PROKOF'EVA IRINA A DR & PSKOVSKIJ JU P DR \\
\hline PUSHKIN SERGEY B DR & RAZIN V A DR & RIZVANOV NAUFAL G DR \\
\hline RUBASHEV BORIS M DR & RUSKOL EUGENIA L DR & RYABCHIKOVA TANYA DR \\
\hline RYABOV YU A PROF DR & RYKHLOVA LIDIJA V DR & RYLOV VALERIJ S DR \\
\hline RYUTOVA MÁRGARITA P DR & RYZHKOV NIKOLAI F DR & RZHIGA OLEG N DR \\
\hline SAFRONOV VICTOR S DR & SAGDEEV ROALD $Z$ DR & SAKHIBULLIN NAIL A DR \\
\hline SAMUS NIKOLAI N DR & SAZHIN MICHAIL DR & SHAKURA NICHOLAJ I DR \\
\hline SHANDARIN SERGEI F DR & SHARAF SH G DR & SHAROV A S DR \\
\hline SHCHEGLOV P V DR & SHCHEGOLEV DIMITRIJ E DR & SHCHERBINA-SAMOJLOVA I DR \\
\hline SHEFFER EUGENE $K$ DR & SHEFOV NICOLAI N & SHEVCHENKO VLADISLAV V DR \\
\hline SHIRYAEV ALEXANDER A DR & SHISHOV VLADIMIR I DR & SHOLOMITSKY G B DR \\
\hline SHOR VIKTOR A DR & SHULOV OLEG S DR & SHUSTOV BORIS M DR \\
\hline SIDORENKOV NIKOLAY S & SITNIK G F PROF & SKRIPNICHENKO VLADIMIR DR \\
\hline SLYSH VJACHOSLAV I DR & SMOL 'KOV GENNADIJ YA DR & SNEZHKO LEONID I \\
\hline SOBOLEV V V DR & SOBOLEV VLADISLAV M DR & SOBOLEVA N S DR \\
\hline SOCHILINA ALLA S DR & SOKOLSKY ANDREJ G DR & SOMOV BORIS $V$ DR \\
\hline SOROCHENKO R L DR & STANKEVICH KAZIMIR S DR & STREL'NITSKIJ VLADIMIR DR \\
\hline STRUKOV IGOR A DR & SUNYAEV RASHID A DR & SVECHNIKOVA MARIA A DR \\
\hline TATEVYAN S K DR & TEPLITSKAYA R B DR & TERENT JEVA ALEXANDRA K DR \\
\hline TOKAREV YURIJ V DR & TOKOVININ ANDREJ A DR & IROITSKY V S PROF DR \\
\hline TRUTSE YU L DR & TSEYTLIN NAUM M & ISYGAN ANATOLII I PROF \\
\hline TUTUKOV A V DR & UDAL'TSOV V A DR & URASIN LIRIK A DR \\
\hline VAINSTEIN L A OR & VARSHALOVICH DIMITRIJ PR & VASHKOV'YAK SOF'YA N DR \\
\hline VASILEVA GALINA J DR & VEKSTEIN GREGORY DR & VITINSKIJ YURIJ I DR \\
\hline VITYAZEVV VENEAMIN V DR & VORONTSOV-VEL'YAMINOV B A & VOSHCHINNIKOV NICOLAI DR \\
\hline VYALSHIN GENNADIJ F DR & YAROV-YAROVOJ M S DR & YAVNEL ALEXANDER A DR \\
\hline YUNGELSON LEV R & ZAITSEV VALERII $V$ DR & ZASOV ANATOLE $V$ DR \\
\hline ZEL'MANOV A L DR & ZHARKOV VLADIMIR N DR & ZHELEZNIAKOV VLADIMIR V \\
\hline ZHEVAKIN S A PROF DR & ZHUGZHDA YUZEF D DR & ZLOINIK ELENA YA DR \\
\hline
\end{tabular}


Country : SAUDI ARABIA

BOYDAG-YILDIZDOGDU F S DR BROSTERHUS E B F DR NIAZY ADNAN MOHAMMAD DR
HAMZAOGLU ESAI Ė H DR TUFEKCIOGLU ZEKI DR

Country : SOUTH AFRICA

BAART EDWARD E PROF

CALDWELL JOHN A R

COUSINS A $W$ DR

ENGELBRECHT CHRISTIAN DR

FEAST MICHAEL U PROF

HERS JAN MR

JONAS JUSTIN LEONARD

KURTZ DONALD WAYNE DR

MENZIES JOHN $W$ DR

O DONOGHUE DARRAGH DR

VAN DER WALT D J DR

WARNER BRIAN PROF
BALONA LUIS ANTERO DR

CHURMS JOSEPH

DE JAGER GERHARD PROF

EVANGELIDIS E DR

GAYLARD MICHAEL JOHN

HIRST WILLIAM P

KILKENNY DAVID DR

LANEY CLIFTON D DR

NICOLSON GEORGE D DR

RAUBENHEIMER BAREND C PR

WALRAVEN TH DR

WHITELOCK PATRICIA ANN DR
BLOCK DAVID LAZAR PROF

CILLIE G G PROF

DE JAGER OCKER C DR FAIRALL ANTHONY P PROF GLASS IAN STEWART DR JARRETT ALAN H PROF KOEN MARTHINUS DR LLOYD EVANS THOMAS DR OVERBEEK MICHIEL DANIEL STOKER PIETER H WARGAU WALTER F DR 


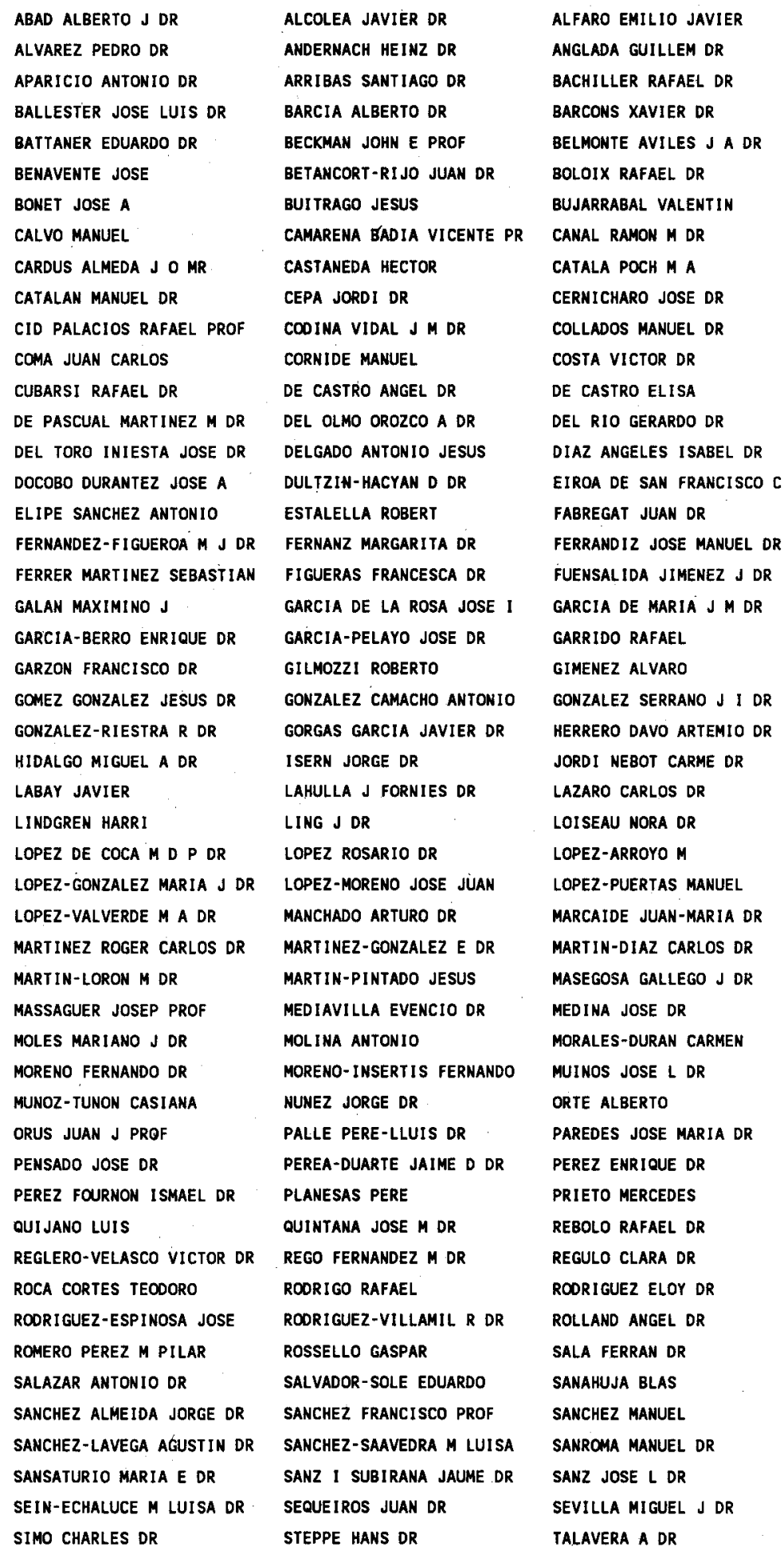


Country : SPAIN (Follow on)

THUM CLEMENS OR

TORROJA J PROF

VILCHEZ MEDINA JOSE M DR

ZAMBRANO ALEJAMDRO DR
TORRA JORDI DR

TRUJILLO BUENO JAVIER DR

VIVES TEODORO JOSE DR

ZAMORANO JAIME DR
TORRELLES JOSE M DR

VAZQUEZ MANUEL DR

WAMSTEKER HILLEM DR

Country : SUEDEN

ADOLFSSON TORD DR

BAATH LARS B DR

BOOTH ROY S PROF

DRAVINS DAINIS PROF

ELLDER JOEL DR

ERIKSSON KJELL DR

FREDGA KERSTIN PROF

GUSTAFSSON BENGT DR

HJALMARSON AKE G DR

HOLMBERG ERIK B PROF

JOHANSSON LENNART DR

KRISTENSON HENRIK DR

LAGERQVIST ALBIN PROF

LAUBERTS ANDRIS DR

LINDBLAD BERTIL A DR

LINDEGREN LENNART DR

LUNDSTEDT HENRIK DR

NILSON PETER DR

OEHMAN YNGVE PROF

OLOFSSON HANS

RICKMAN HANS DR

ROSQUIST KJELL

SAHAI RAGHVENDRA OR

SCHARMER GOERAN BJARNE

STENHOLM BJOERN DR

SUNDMAN ANITA DR

VAN GRONINGEN ERNST DR

WIEDLING TOR OR

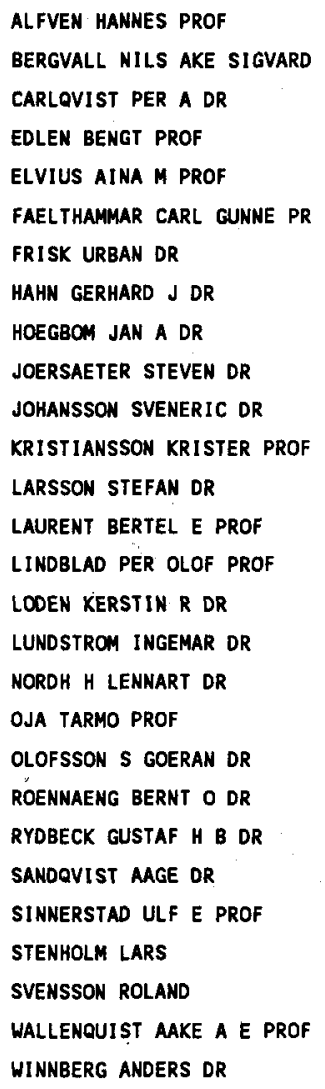

ARDEBERG ARNE L PROF

BJORNSSON CLAES-INGVAR

CATO B TORGNY DR

EDVARDSSON BENGT DR

ELVIUS TORD PROF EMERITUS

FRANSSON CLAES

GAHM GOESTA F OR

HANSSON NILS DR

HOEGLUND BERTIL PROF

JOHANSSON LARS ERIK B DR

KOLLBERG ERIK L PROF

LAGERKVIST CLAES-INGVAR

LARSSON-LEANDER G PROF

LEHNERT B P PROF

LINDE PETER DR

LOOEN LARS OLOF PROF

LYTIKENS EJNAR DR

NYMAN LARS-AKE DR

OLBERG MICHAEL DR

RAADU MICHAEL A DR

ROSLUND CURT DR

RYDBECK OLOF E H PROF

SCHALEN CARL PROF

SOEDERHJELM STAFFAN DR

SUNDELIUS BJOERN DR

SWENSSON JOHN W OR

WESTERLUND BENGT E PROF

WRAMDEMARK STIG S O DR 


$\begin{array}{ll}\text { BARTHOLDI PAUL DR } & \text { BECKER WILHELM PROF } \\ \text { BEUTLER GERHARD PROF } & \text { BINGGELI BRUNO } \\ \text { BOCHSLER PETER } & \text { BONANOMI JACQUES OR } \\ \text { BURKI GILBERT PROF } & \text { BUSER ROLAND DR } \\ \text { CHMIELEWSKI YVES DR } & \text { COURVOISIER THIERRY J-L } \\ \text { DEBRUNNER HERMANN DR } & \text { DRESSLER KURT PROF } \\ \text { FENKART ROLF P PROF DR } & \text { FROEHLICH CLAUS } \\ \text { GOLAY MARCEL PROF } & \text { GOY GERALD PROF } \\ \text { HAUCK BERNARD PROF } & \text { LABHARDT LUKAS } \\ \text { MAETZLER CHRISTIAN DR } & \text { MAGUN ANDREAS DR } \\ \text { MAYOR MICHEL PROF } & \text { MEGEVAND DENIS DR } \\ \text { MEYNET GEORGES DR } & \text { MUELLER EDITH A PROF } \\ \text { NORTH PIERRE } & \text { NUSSBAUMER HARRY PROF } \\ \text { RUFENER FREDY G PROF } & \text { SCHANDA ERWIN PROF } \\ \text { SCHMUTZ WERNER } & \text { SCHULER HALTER DR } \\ \text { SPAENHAUER ANDREAS MARTIN } & \text { STEINLIN ULI PROF } \\ \text { TAMMANM G ANDREAS PROF DR } & \text { TREFZGER CHARLES F DR } \\ \text { WALDMEIER MAX PROF DR } & \text { WILD PAUL PROF } \\ \text { ZELENKA ANTOINE DR } & \end{array}$

BARTHOLDI PAUL DR BOCHSLER PETER BURKI GILBERT PROF
BENZ ARNOLD DR BLECHA ANDRE BORIS G DR BOUVIER PIERRE PROF CAMERON LUZIUS MARTIN DE MEDEIROS JOSE RENAN DR DUERST JOHANNES DR GEISS JOHANNES PROF GRENON MICHEL DR MAEDER ANDRE PROF MARTINET LOUIS PROF MERMILLIOO JEAN-CLAUDE DR NICOLET BERNARD PFENMIGER DANIEL DR SCHMID HANS MARTIN OR SOLANKI SAMI K DR STENFLO JAN O DR VOGEL MANFRED DR XIA ZHIGUO DR
Country : TADZHIKISTAN

BABADZHANOV PULAT B DR

BIBARSOV RAVIL'SH DR KISELEV NIKOLAI N DR

\begin{tabular}{ll} 
& \multicolumn{1}{c}{ CoUntTY $:$ TURKEY } \\
\cline { 2 - 2 } & \\
AKCAYLI MELEK M A DR & AKYOL MUSTAFA UNAL PROF \\
ASLAN ZEKI DR & ATAC TAMER \\
AYDIN CEMAL PROF DR & BALLI EDIBE PROF \\
BOZKURT SUKRU DR & DEMIRCAN OSMAN DR \\
DIZER MUAMMER PROF & DOGAN NADIR PROF \\
ENGINOL TURAN B DR & ERCAN E NIHAL \\
ESKIOGLU A NIHAT & EVREN SERDAR DR \\
GOELBASI ORHAN DR & GOKDOGAN NUZHET PROF \\
GULMEN OMUR DR & HAZER S DR \\
IBANOGLU C DR & KANDEMIR GUELCIN \\
KIRAL ADNAN PROF & KIRBIYIK HALIL DR \\
KIZILOGLU UEMIT DR & KOCER DURCUN DR \\
MENTESE HUSEYIN DR & OEKTEN ADNAN DR \\
OEZKAN MUSTAFA TUERKER DR & OZGUC ATILA \\
SEZER CENGIZ DR & TEKTUNALI H GOKMEN DR \\
YILMAZ FATMA DR & YILMAZ NIHAL DR \\
\hline
\end{tabular}

\author{
ALPAR ALI DR \\ AVCIOGLU KAMURAN PROF DR \\ BOLCAL CETIN DR \\ DERMAN I ETHEM DR \\ ENGIN SEMANUR PROF. \\ ERTAN A YENER DR \\ EZER-ERYURT DILHAN PROF \\ GUDUR N DR \\ HOTINLI METIN DR \\ KARAALI SALIH DR \\ KIZILOGLU NILGUEN DR \\ MARSOGLU A DR \\ OEZEL MERMET EMIN DR \\ PEKUENLUE E RENHAN DR \\ TUNCA ZEYNEL DR
}




\begin{tabular}{|c|c|c|}
\hline AARSETH SVERRE J DR & ADAM MADGE G DR & ADAMS DAVID J DR \\
\hline ADAMSON AMDREW DR & ADE PETER A R DR & ALBINSON JAMES DR \\
\hline ALEXANDER JOHN B & ALEXANDER PAUL DR & ALLAN PETER $M$ \\
\hline ALLEN ANTHONY JOHN DR & ANDERSON BRYAN DR & ANDREWS DAVID A DR \\
\hline ANDREUS PETER J DR & ARDAVAN HOUSHANG DR & ARGUE A NOEL MR \\
\hline ARGYLE ROBERT WILLIAM MR & ATHERTON PAUL DAVID & AXON DAVID \\
\hline BAILEY MARK EDWARD & BALDWIN JOHN E DR & BARLOW MICHAEL J DR \\
\hline BAROCAS VINICIO PROF & BARROW JOHN DAVID & BARROW RICHARD F DR \\
\hline BARSTOU MARTIN ADRIAN DR & BARUCH JOHN DR & BASTIN JOHN A PROF \\
\hline BATES BRIAN DR & BATES DAVID R PROF & BATH GEOFFREY T DR \\
\hline BEALE JOHN S DR & BEGGS DENIS H MR & BELL BURNELL $S$ JOCELYN DR \\
\hline BELL KENNETH LLOYD DR & BELL KENNETH LLOYD DR & BELL STEVEN DR \\
\hline BENN CHRIS R DR & BERGER MITCHELL DR & BERRINGTON KEITH ADRIAN \\
\hline BINGHAM RICHARD G DR & BINNEY JAMES $J$ DR & BLACKMAN CLINTON PAUL DR \\
\hline BLACKWELL DONALD E PROF & BOOE MICHAEL $F$ & BOKSENBERG ALEC PROF \\
\hline BONDI HERMANN PROF SIR & BONNOR W B PROF & BOYD ROBERT L F PROF SIR \\
\hline BRAND PETER $W J L D R$ & BRANDUARDI-RAYMONT G & BRANSON NICHOLAS J B A DR \\
\hline BRIDGELAND MICHAEL DR & BROMAGE GORDON E DR & BROOKES CLIVE J DR \\
\hline BROWN JOHN C PROF & BROWNE IAN $W$ A DR & BROWNING PHILIPPA DR \\
\hline BRUCK HERMANN A PROF & BRUCK MARY T DR & BUNCLARK PETER STEPHEN DR \\
\hline BURGESS ALAN DR & BURGESS DAVID D PROF & BURTON WILLIAM $M$ \\
\hline BUTCHINS SYDNEY ADAIR & BUTLER C JOHN DR & BYRNE PATRICK B DR \\
\hline CALLANAN PAUL DR & CAMERON AMDREW COLLIER DR & CAMPBELL JAMES $\boldsymbol{H}$ \\
\hline CARR BERNARO JOHN & CARSON T R DR & CARSWELL ROBERT F DR \\
\hline CATCHPOLE ROBIN M DR & CHARLES PHILIP ALLAN & CLARK DAVIO H DR \\
\hline CLARKE DAVID DR & CLEGG PETER E DR & CLEGG ROBIN E S DR \\
\hline CLUBE $S \vee M D R$ & COHEN RAYMOND J DR & COLES PETER DR \\
\hline CONHAY ROBIN G DR & COOK ALAN H PROF & COOKE B A DR \\
\hline COOKE JOHN ALAN & COUPER HEATHER MISS & CRAUFORD IAN ANDREW DR \\
\hline CROOM DAVID L DR & CRUISE ADRIAN MICHAEL DR & CULHANE LEONARD PROF \\
\hline CZERNY MICHAL DR & DAINTREE EDHARD J DR & DARIUS JON DR \\
\hline OAVIDSON HILLIAM PROF & DAVIES PAUL CHARLES $W$ & DAVIES ROONEY D PROF \\
\hline OAVIS RICHARD J OR & DE GROOT MART DR & DENNISON P A DR \\
\hline DEWHIRST DAVID W DR & DICKENS ROBERT J DR & DIEGO FRANCISCO DR \\
\hline DISNEY MICHAEL J PROF & DONNISON JOHN RICHARD DR & DORMAND JOHN RICHARD DR \\
\hline DOUNES ANN JULIET B & DOYLE JOHN GERARD & DREW JANET \\
\hline DUFFETT-SMITH PETER JAMES & DUFTON PHILIP L DR & DUNLOP STORM \\
\hline DWORETSKY MICHAEL M DR & DYSON JOHN E DR & ECCLES MICHAEL J DR \\
\hline EDMUNDS MICHAEL GEOFFREY & EDWIN ROGER $P$ & EFSTATHIOU GEORGE \\
\hline EGGLETON PETER P DR & ELLIOTT KENNETH H DR & ELLIS RICHARD S \\
\hline ELSMORE BRUCE DR & EMERSON DAVID & EMERSON JAMES $P$ \\
\hline EVANS ANEURIN & EVANS KENTON DOWER DR & EVANS ROGER G DR \\
\hline FABIAN ANDREN C DR & FALLE SAMUEL A DR & FAMELL DEREK R DR \\
\hline FIELD DAVID & FIELDER GILBERT DR & FLETT ALISTAIR M \\
\hline FLONER DAVID R DR & FONG RICHARD & FOX WE MR \\
\hline FRENK CARLOS $S$ & FURNISS IAN & GADSDEN MICHAEL DR \\
\hline GARRINGTON SIMON DR & GARTON W R S PROF & GEAKE JOHN E DR \\
\hline GENT HUBERT MR & GIETZEN JOSEPH $W$ & GILMORE GERARD FRANCIS \\
\hline GLENCROSS HILLIAM M & GODWIN JON GUNHAR DR. & GOLDSWORTHY FREDERICK A \\
\hline GONDHALEKAR PRABHAKAR DR & GOUGH DOUGLAS O DR & GRAINGER JOHN F OR \\
\hline
\end{tabular}




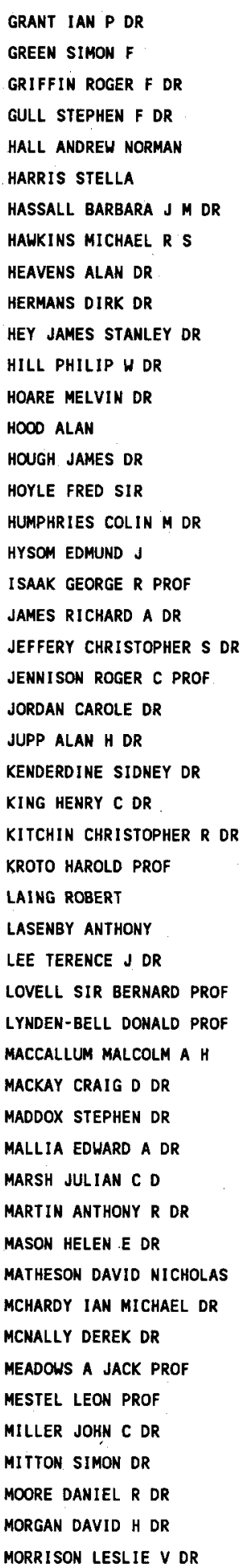

GREEN DAVID DR

GRIFFIN MATTHEW J DR

GRIFFITHS WILLIAM $K$

GUTHRIE BRUCE N G DR HANBURY BROWN ROBERT PROF HARRISON RICHARD A DR HAWARDEN TIMOTHY G DR HAYWARD JOHN HEDDLE DOUGLAS W O PROF HEUETT PAUL

HIDE RAYMOND PROF HILLS RICHARD E DR HOLDEN FRANK HOOD ALAN DR HOWARTH IAN DONALD HUGHES DAVID $W$ DR HUNT G E DR IRELAND JOHN G DR JACKSON JOHN CHARLES DR JAMESON RICHARD F DR JENKINS CHARLES $R$ JONES BARRIE $W$ DR JORDEN PAUL RICHARD KAHN FRANZ D PROF KIBBLEWHITE EDWARD J DR KINGSTON ARTHUR E PROF KOPAL ZDENEK PROF LACEY CEDRIC DR LANCASTER BROWN PETER LAWRENCE ANDREW DR LITTLE LESLIE T DR LUCEY JOHN DR LYME ANDREH G DR MACDONALD GEOFFREY H DR MACKINNON ALEXANDER L MAJOR JOHN DR MAREK JOHN MARSH THOMAS DR MARTIN DEREK H PROF MASON JOHN WILLIAM DR MCCREA HILLIAM SIR MCKEITH CONAL D DR MCWHIRTER R W PETER DR MEIXLE WILLIAM P S MILES HOWARD G MR MILLS ALLAN A DR MOFFAIT HENRY KEITH PROF MOORE PATRICK DR MORISON IAN MR MOSS CHRISTOPHER DR
GREEN ROBIN M DR GRIFFIN RITA E M DR GUEST JOHN E DR HADLEY BRIAN W HARPER DAVID DR HARTLEY KENNETH F DR HAWKING STEPHEN W PROF HAZARD CYRIL DR HEGGIE DOUGLAS C DR HEWISH ANTONY PROF HILDITCH RONALD W DR HILTON JOHN DR HOLLOWAY NIGEL J DR HOSKIN MICHAEL A DR HOWSE H DEREK HUMMEL EDSHO HUTCHEON RICHARD J DR IRHIN MICHAEL JOHN DR JAMES JOHN F MR JARDINE MOIRA MARY DR JENNINGS R E PROF JONES DEREK H P DR JOSEPH ROBERT D DR KAISER THOMAS $R$ PROF KING ANDREW R DR KING-HELE DESMOND G DR KOSOVICHEV ALEXANDER LAHAV OFER DR LANG JAMES DR LEAHY J PATRICK DR LONGAIR M S PROF LYNAS-GRAY ANTHONY E LYTTLETON RAYMOND A PROF MACGILLIVRAY HARVEY T DR MADDISON RONALD CH DR MALIN STUART MARSDEN PHILIP L PROF MARSHALL KEVIN P MARTIN WILLIAM L DR MASON KEITH OWEN MCDONNELL J A M PROF MCMULLAN DENNIS DR MEABURN J DR MESSAGE PHILIP J DR MILLAR THOMAS J DR MITTON JACQUELINE MONTEIRO TANIA S DR MORGAN BRIAN LEALAN MORRIS MICHAEL C MOSS DAVID L DR 
Country : UK (follow on)

\begin{tabular}{|c|c|c|}
\hline MURDIN PAUL G DR & MURRAY C ANDREH & MURRAY CARL D DR \\
\hline MURRAY JOHN B DR & MUXLOW THOMAS & NANDY KASHINATH DR \\
\hline NAPIER WILLIAM M DR & NAVARRO JULIO FERNANDO DR & NELSON ALISTAIR H DR \\
\hline NEWTON GAVIN DR & NICHOLSON WILLIAM & OSBORNE JOHN L DR \\
\hline O'BRIEN PAUL THOMAS DR & PADMAN RACHAEL & PAGE CLIVE G DR \\
\hline PALMER PHILIP DR & PAPALOIZOU JOHN C B DR & PARKER EDWARD A DR \\
\hline PARKER QUENTIN DR & PARKINSON JOHN H DR & PATNAIK ALOK DR. \\
\hline PAXTON HAROLD J B $R$ & PEACH GILLIAN DR & PEACH JOHN V DR \\
\hline PEACOCK JOHN AMDREH & PEARCE GILLJAN DR & PEDLAR ALAN DR \\
\hline PENNY ALAN JOHN DR & PENSTON MARGARET & PERRY JUDITH J DR \\
\hline PETFORD A DAVID DR & PETTINI MAX & PHILLIPS JOHN PETER \\
\hline PHILLIPS KENNETH J H & PIKE CHRISTOPHER DAVID & PILKINGTON JOHN D H DR \\
\hline PILLINGER COLIN DR & PONMAN TREVOR DR & PONSONBY JOHN E B DR \\
\hline POOLEY GUY DR & POUNDS KENWETH A PROF & PRIEST ERIC R PROF \\
\hline PRINGLE JAMES E DR & PRINJA RAMAN DR & PYE JOHN P DR \\
\hline QUENBY JOHN J DR & RACKHAM THOMAS W DR & RAINE DEREK J DR \\
\hline RAPLEY CHRISTOPHER G DR & RAWLINGS JONATHAN DR & RAWLINGS STEVEN DR \\
\hline REAY NEWRICK K DR & REES MARTIN J PROF & RICHARDSON KEVIN J \\
\hline RIJNBEEK RICHARD DR & RILEY JULIA M DR & RING JAMES PROF \\
\hline ROBERISON JOHN ALISTAIR & ROBERTSON NORNA DR & ROBINSON WILLIAM J DR \\
\hline ROBSON IAN E DR & ROCHE PATRICK F DR & RONAN COLIN A \\
\hline ROWAN-ROBINSON MICHAEL DR & ROWSON BARRIE DR & ROXBURGH IAN W PROF \\
\hline ROY ARCHIE E PROF & RUNCORN S K PROF & SANFORD PETER WILLIAM MR \\
\hline SAUNDERS RICHARD D E & SCARROTT STANLEY M DR & SCHEUER PETER A G DR \\
\hline SCHILD HANSRUEDI & SCHUTZ BERNARD F PROF & SCHWARTZ STEVEN JAY \\
\hline SCOTT PAUL F DR & SEATON MICHAEL J PROF & SEYMOUR P A H \\
\hline SHAKESHAFT JOHN R DR & SHALLLIS MICHAEL J DR & SHARPLES RAY DR \\
\hline SIM MARY E MISS & SIMMONS JOHN FRANCIS L & SIMNEIT GEORGE M \\
\hline SIMONS STUART DR & SINCLAIR ANDREW T DR & SISSON GEORGE M MR. \\
\hline SKILLEN IAN DR & SKILLING JOHN DR & SKINNER GERALD DR \\
\hline SMITH F GRAHAM PROF & SMITH GEOFFREY DR & SMITH HUMPHRY M \\
\hline SMITH LINDA J & SMITH ROBERT CONNON DR & SMITH ROONEY M DR \\
\hline SMYTH MICHAEL J DR & SOMERVILLE HILLIAM B DR & SORENSEN SOREN-AKSEL DR \\
\hline SPARKS WILLIAM BRIAN & SPEER R J DR & SPENCER RALPH E DR \\
\hline STANNARD DAVID DR & STEPHENSON F RICHARD DR & STEWART JOHN MALCOLM DR \\
\hline STEWART PAUL DR & STICKLAND DAVID J DR & STOBIE ROBERT S DR \\
\hline SUMMERS HUGH P OR & SUTHERLAND WILLIAM DR & SWEET PETER A PROF \\
\hline SYKES-HART AVRIL B DR & TAVAKOL REZA & TAYLER ROGER J PROF \\
\hline TAYLOR DONALD BOGGIA OR & TER HAAR DIRK & TERLEVICH ELENA DR \\
\hline TERLEVICH ROBERTO JUAN & THOBURN CHRISTINE & THOMAS DAVID V DR \\
\hline THOMAS PETER A OR & THOMASSON PETER DR & THOMPSON G I DR \\
\hline THOMPSON MICHAEL J DR & TOUT CHRISTOPHER DR & TOZER DAVID C DR \\
\hline TRITTON KEITH P DR & TRITTON SUSAN BARBARA & TURNER MARTIN J L DR \\
\hline TWISS R Q DR & TWORKOWSKI ANDRZEJ S & UNGER STEPHEN DR \\
\hline VAN BREDA IAN G DR & VAN DER RAAY HERMAN B & VAN LEEUWEN FLOOR DR \\
\hline VECK NICHOLAS & WALKER DAVID DOUGLAS DR & WALKER EDWARD N MR \\
\hline WALKER HELEN $J$ & WALKER IAN WALTER & WALL JASPER $\vee D R$ \\
\hline WALLACE PATRICK & WALLIS MAX K DR & WALSH DENNIS DR \\
\hline WALTON NICHOLAS A DR & WARD HENRY DR & WARD MARTIN JOHN \\
\hline WARNER PETER J DR & WARWICK ROBERT S DR & WATSON MICHEAL G DR \\
\hline
\end{tabular}




WATT GRAEME DAVID
WHITE GLENN J
WICKRAMASINGHE N C PROF
WILKINSON ALTHEA
WILLIAMS IWAN P PROF
WILLMORE A PETER PROF
WILSON MICHAEL JOHN DR
WOLSTENCROFT RAMON D DR
WORRALL GORDON DR
YALLOP BERNARD D DR
ZUIDERWIJK EOWARDUS J.

Country : UKRAINE
WEISS NIGEL O DR WHITROW GERALD JAMES PROF WILCOCK WILLIAM L PROF WILKINSON PETER N DR HILLIAMS PEREDUR M DR HILLSTROP ROOERICK V DR WILSON ROBERT PROF SIR WOOO ROGER DR WORSWICK SUSAN YAU KEVIN K C DR
WELLGATE G BERNARD MR WHITWORTH ANTHONY PETER WILKINS GEORGE A DR WILLIAMS DAVID A PROF WILLIS ALLAN J DR WILSON LIONEL DR WOLFENDALE ARNOLD W PROF WOOLFSON MICHAEL M PROF WYNME CHARLES G PROF ZARNECKI JAN CHARLES DR

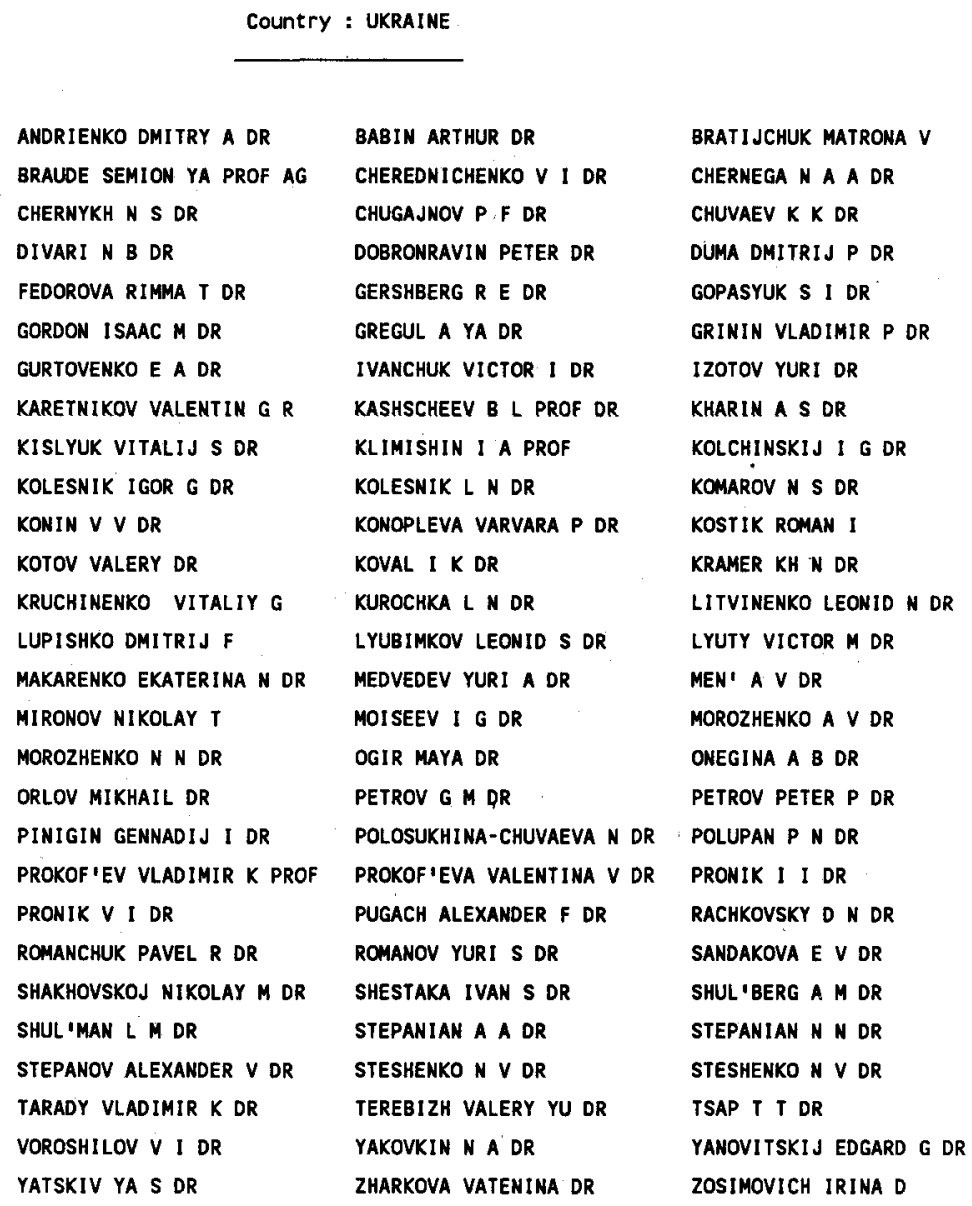


CountrY : URUGUAY

FERNANDEZ JULLO A DR

Country : USA

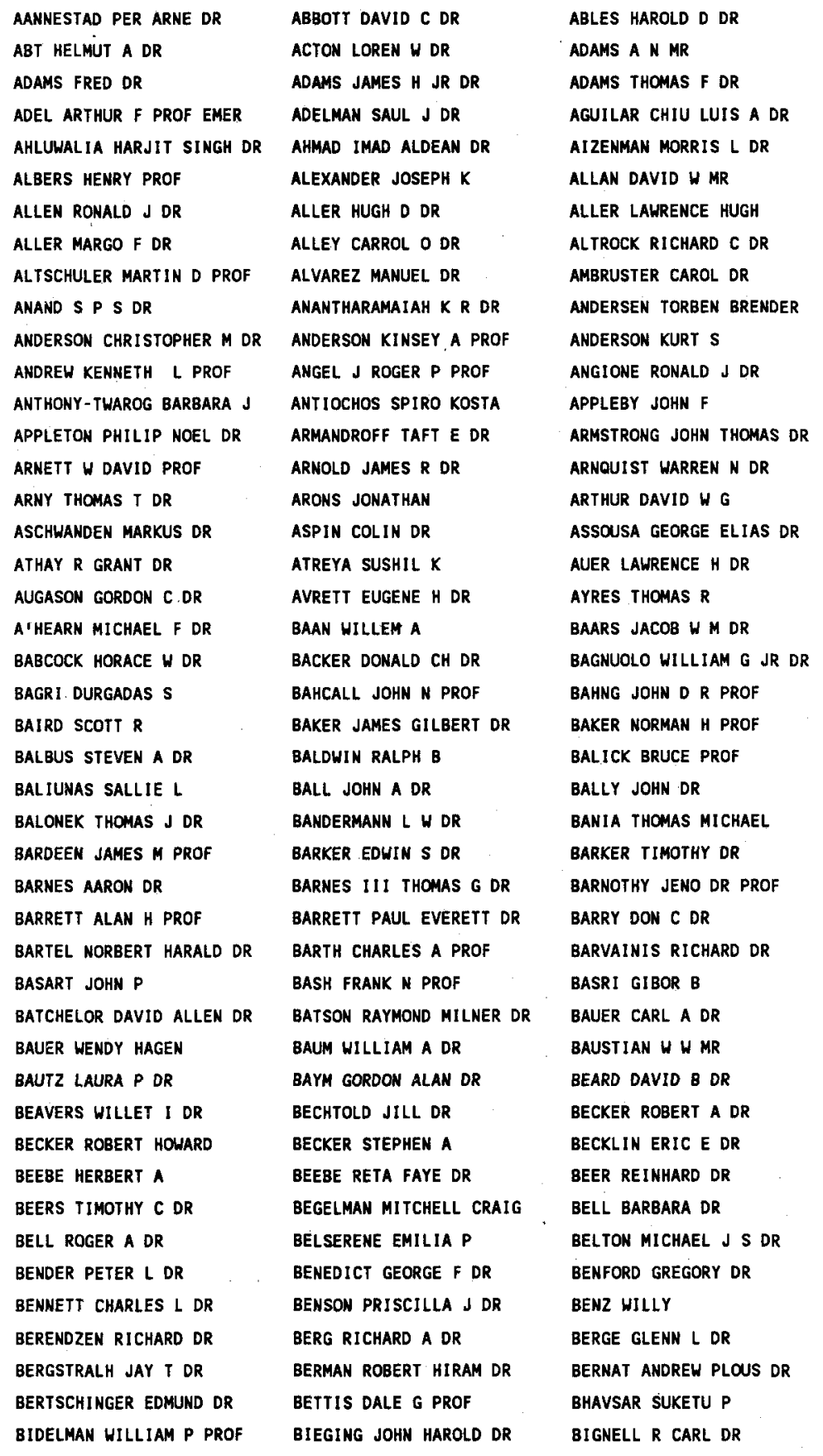


BILLINGHAN JOHN BIRETTA JOHN ANTHONY DR BLADES JOHN CHRIS DR BLAIR WILLIAM P DR BLESS ROBERT C PROF BLOEMHOF ERIC E DR BLUMENTHAL GEORGE R DR BOEHM-VITENSE ERIKA PROF BOGGESS ALBERT DR BOHLIN J DAVID DR BOLEY FORREST I

BOOK DAVID L

BOPP BERNARD $W$ DR BORIAKOFF VALENTIN BOWELL EOWARD L G DR BOWYER C STUART PROF BRACEWELL RONALD N PROF BRANDT JOHN C DR BREAKIRON LEE ALLEN DR BRECKINRIDGE JAMES B DR BRINKS ELIAS DR BRODIE JEAN $P$ BROWN DOUGLAS NASON BROWN ROBERT L DR BRUCATO ROBERT J BRUHWEILER FRED C. JR BRUNK WILLIAM E DR BUFF JAMES $S$ DR BURATTI BONNIE J DR BURKE BERNARD F DR BURNS JACK O'NEAL JR BURSTEIN DAVID BUTLER DENNIS DR BYRD GENE G DR CALVIN WILLIAM H DR CAMPBELL ALISON DR CAMPBELL MURRAY $F$ CANIZARES CLAUDE R PROF CARBON DUANE F DR CARLETON NATHANIEL P DR CAROFF LAWRENCE J CARR THOMAS D PROF CASERTANO STEFANO DR CASTELAZ MICHEAL $W$ DR CATON DANIEL $B$ DR CEFOLA PAUL J DR CHAISSON ERIC J PROF CHAMBLISS CARLSON $R$ DR CHANDRASEKHAR $S$ PROF CHAPMAN GARY A DR
BILLINGS DONALD E PROF BIRKINSHAW MARK BLAHA MILAN DR BLANDFORD ROGER DAVID DR BLITZ LEO DR BLONDIN JOHN M DR BOOENHEIMER PETER PROF BOESGAARD ANN M PROF BOGGESS NANCY $W$ DR BOHLIN RALPH C DR BOND HOWARD E DR BOOKBINDER JAY A DR BORD DONALD JOHN BORNMANN PATRICIA L DR BOWEN GEORGE H DR BOYCE PETER B DR BRADSTREET DAVID H DR BRANSCOMB L M DR BRECHER AVIVA DR PROF BREGMAN JOEL $N$ BROADFOOT A LYLE DR BROUCKE ROGER DR BROWN HARRISON DR BROWNLEE DONALD E PROF BRUECKNER GUENTER E DR BRUNER MARILYN E DR BUCCIARELLI BEATRICE DR BUHL DAVID DR BURBIDGE E MARGARET PROF BURKHEAD MARTIN $S$ BURNS JOSEPH A PROF BUSCOMBE WILLIAM PROF BUTTERWORTH PAUL CAHN JULIUS H PROF CAMERON ALASTAIR G W PROF CAMPBELL BELVA G S OR CAMPINS HUMBERTO DR CAPEN CHARLES F CARDELLI JASON A DR CARLSON JOHN B CARPENTER KENNETH G DR CARRUTHERS GEORGE R DR CASH WEBSTER C JR CASTELLI JOHN $P$ CATURA RICHARD C DR CENTRELla JOAN M DR CHAMBERLAIN JOSEPH M DR CHAN KHING LAM CHANMUGAM GANESAR PROF CHAPMAN ROBERT D DR
BINZEL RICHARD P DR BLACK JOKA HARRY DR BLAIR GUY NORMAN DR BLASIUS KARL RICHARD DR BLITZSTEIN HILLIAM DR BLUDMAN SIDNEY A PROF BOEHM KARL-HEINZ PROF BOESHAAR GREGORY ORTH DR BOHANNAN BRUCE EDWARD BOLDT ELIHU DR BONSACK WALTER $K$ PROF BOOKMYER BEVERLY B DR BORDERIES NICOLE BOSS ALAN P DR BOWERS PHILLIP F BOYNTON PAUL EDWARD DR BRANCH DAVID R DR BRAULT JAMES $W$ DR BRECHER KENNETH PROF BRIDLE ALAN H PROF BROOERICK JOHN DR BROWN ALEXANDER BROWN ROBERT HAMILTON BROWNLEE ROBERT R DR BRUGEL EDWARD $W$ DR BRUNING DAVID H DR BUCHLER J ROBERT PROF BUNNER ALAN N DR BURBIDGE GEOFFREY $R$ PROF BURLAGA LEONARO F DR BURROWS ADAM SETH BUTA RONALD J DR BYARD PAUL L DR CAILlaUlt JEAN PIERRE DR CAMERON WINIFRED S MRS CAMPBELL DONALD 8 CANFIELD RICHARD C DR CAPRIOTTI EUGENE R DR CARGILL PETER J DR CARNEY BRUCE HILLIAM CARPENTER LLOYD DR CARTER WILLIAM EUGENE CASSINELLI JOSEPH P DR CASTOR JOHN I DR CAUGHLAN GEORGEANNE $R$ CHAFFEE FREDERIC H DR CHAMBERLAIN JOSEPH W PROF CHANDRA SUBHASH CHAPMAN CLARK R DR CHEN KHAN-YU PROF 
Country : USA (Follow on)

CHENG CHUNG-CHIEH DR CHIU HONG-YEE DR CHRISTIANSEN WAYNE A CHRISTY ROBERT F DR CHUPP EDWARD L DR CLARK BARRY G DR CLARK THOMAS A DR CLAYTON DONALD D PROF CLEMENS DAN P DR CLIVER EDWARD $W$ COCKE WILLIAM JOHN PROF COFFEY HELEN E MS COHEN LEON PROF COHEN RICHARD S COLBURN DAVID S DR COMBI MICHAEL $R$ DR CONKLIN EDHARD $K$ COOK JOHN H CORBIN THOMAS ELBERT DR CORLISS C H DR. COULSON IAIN M DR COWIE LENNOX LAUCHLAN DR COX ARTHUR N DR CRANE PATRICK $C$ CROCKER DEBORAH ANN OR CUDABACK DAVID D OR CULVER ROGER BRUCE DR CZYZAK STANLEY J DR DANBY J M ANTHONY DR DANLY LAURA DR DAVIDSEN ARTHUR FALNES DR DAVIES ROGER L DR DAVIS LEVERETT JR PROF DAVIS MORRIS S PROF DE FREES DOUGLAS J DR DE VAUCOULEURS GERARD PR DEARBORN DAVID PAUL $S$ DR DEMARQUE P PROF DENAISON EDHIN W OR DERE KENNETH PAUL DEUPREE ROBERT G DR DEVORKIN DAVID H DEWITT-MORETTE CECILE PR DICKE ROBERT H PROF DICKEY JEAN O'BRIEN DICKMAN ROBERT L DR DINERSTEIN HARRIET L DOGGETT LEROY E DR DONN BERTRAM D DOUGLASS GEOFFREY G
CHEVALIER ROger A DR CHIU LIANG-TAI GEORGE CHRISTODOULOU DMITRIS DR CHU YOU-HUA CHURCHWELL EDHARD B DR CLARK FRANK OLIVER DR CLARKE JOHN T CLAYTON GEOFFREY C DR CLIFTON KENMETH ST COCHRAN ANITA L DR COOE ARTHUR D COHEN JEFFREY M DR COHEN MARSHALL H PROF COHEN ROSS D OR COLGATE STIRLING A DR COMINS NEIL FRANCIS CONNOLLY LEO PAUL COOK KEM HOLLAND DR CORDES JAMES M CORUIN HAROLD G JR COUNSELMAN CHARLES C PROF COWLEY ANME P DR COX DONALD P PROF CRANNELL CAROL JO DR CRUIKSHANK DALE P DR CUDWORTH KYLE MCCABE DR CUNTZ MANFRED DR DAHN CONARD CURTIS OR DANFORD STEPHEN C DR DATLOWE DAYTON DR DAVIDSON KRIS DR DAVILA JOSEPH DR DAVIS MARE DR DAVIS ROBERT J DR DE JONGE J $K$ DR DE VIMCENZI DONALD DR DEEMING TERENCE J DR DEMING LEO DRAKE DR DENT WILLIAM A PROF DERMOTT STANLEY $F$ DEUTSCHMAN WILLIAM A DR DEWITT BRYCE S DR DIAMOND PHILIP JOHN DR DICKEL HELENE $R$ DR DICKEY JOHN $M$ DICKMAN STEVEN $R$ DIXON ROBERT S DR DOHERTY LONELL R PROF DOSCHEK GEORGE A DR DOWNES RONALD A DR
CHITRE DATTAKUMAR M DR CHRISTIAN CAROL ANN CHRISTY JAMES WALTER DR CHUBB TALBOT A DR CLARK ALFRED JR PROF CLARK GEORGE W PROF CLAUSSEN MARK $J$ DR' CLAYTOW ROBERT N DR CLINE THOMAS L DR COCHRAN WILLIAM DAVID DR COFFEEN DAVID L DR COHEN JUDITH DR COHEN MARTIN DR COHN HALDAN N COLLINS GEORGE H II PROF CONDON JAMES J DR CONTI PETER S DR CORBALLY CHRISTOPHER CORDOVA FRANCE A D COTTON WILLIAM D Jr COWAN JOHN J DR COULEY CHARLES R PROF CRAINE ERIC RICHARD DR CRAWFORD DAVID L DR CRUTCHER RICHARD M DR CUFFEY J MR CURRIE DOUGLAS G DR DALGARNO ALEXANDER PROF DANKS ANTHONY C DR DAVID LAURENCE P DR DAVIES MERTON E MR DAVIS CECIL G JR DAVIS MICHAEL M DR DAVIS SUMNER $P D R$ DE PATER IMKE DE YOUNG DAVID $S$ DR DELSEMME ARMAND H PROF DR DENNIS BRIAN ROY OR DEPRIT ANDRE PROF DESPAIN KEITH HOWARD DR DEVINMEY EDWARD J DR DEWITT JOHN H JR DICK STEVEN $d$ DICKEL JOHN R DICKINSON DALE F DR DIETER NANNIELOU H OR DJORGOVSKI STANISLAV DR DOLAN JOSEPH F DR DOUGLAS JAMES N PROF DOWNS GEORGE S DR 
DOYLE LAURANCE R DR DRAKE STEPHEN A DRESSLER ALAN DRYER MURRAY DR DULK GEORGE A PROF DUNHAM DAVID $W$ DUPREE ANDREA $K$ DR DURNEY BERNARD DR DUVALL THOMAS L JR DYER EDHARD $R$ DR EDDY JOHN A DR EDWARDS ALAN CH DR EICHLER DAVID DR ELLIOT JAMES L DR ELSTE GUNTHER H DR EL-BAZ FAROUK DR EPPS HARLAND WARREN PROF EPSTEIN ISADORE PROF ESKRIDGE PAUL B DR EIZEL PAUL B DR EVANS JOHN $W$ DR EWEN HAROLD I DR FABER SANDRA M PROF FALGARONE EDITH FALLER JAMES E PROF FANSELOW JOHN LYMAN FAZIO GIOVANNI G DR FEIGELSON ERIC D DR FELDMAN UR! FERLAND GARY JOSEPH FICHTEL CARL E DR FIENBERG RICHARD T DR FINK UWE DR FISCHEL DAVID DR FISHER J RICHARD FISHMAN GERALD J FIX JOHN D DR FLEISCHER ROBERT DR FOGARTY WILLIAM G DR FONTENLA JUAN MANUEL DR FORD HOLLAND C RES PROF FORREST WILLIAM JOHN FOUKAL PETER $V$ DR FRANK JUHAN FRAZIER EDWARD N DR FRENCH RICHARD G FRIEDMAN HERBERT DR FRISCH PRISCILLA FRYE GLENN M PROF FURENLID INGEMAR $K$ OR
DRAINE BRUCE T DREHER JOHN W DREVER RONALD W P DR DUBIN MAURICE DR DUNCAN DOUGLAS KEVIN DR DUNKELMAN LAURENCE DUPUY DAVID L DR DURRANCE SAMUEL T DR DWEK ELI

DYSON F J DR

EDELSON RICK DR

EDWARDS TERRY $W$

EILEK JEAN

ELMEGREEN BRUCE GORDON DR ELSTON WOLFGANG E PROF EMSLIE A GORDON EPSTEIN EUGENE E DR EPSTEIN RICHARD I DR ESPOSITO F PAUL PROF EUBANKS THOMAS M DR EVANS NEAL J II ASS PROF EWING MARTIN $S$. FABRICANT DANIEL G FALK SYDNEY $W$ JR DR FALLON FREDERICK $W$ OR FAULKNER JOHN PROF FEDERMAN STEVEN ROBERT FEKEL FRANCIS C FELDMAN URI DR FEYMMAN JOAN DR FIEDLER RALPH L DR FILIPPENKO ALEXEI $V$ DR FINN G D DR FISCHER JACQUELINE FISHER PHILIP C FITCH WALTER S DR FLANNERY BRIAN PAUL DR FLIEGEL HENRY $F$ FOLTZ CRAIG B FORBES J E DR FORD W KENT JR DR FORSTER JAMES RICHARD DR FOWLER WILLIAM A PROF

FRANKLIN FRED A DR FREDRICK LAURENCE W PROF FRIBERG PER FRIEDMAN SCOTT DAVID DR FROGEL JAY ALBERT DR FTACLAS CHRIST GAISSER THOMAS $K$
DRAKE FRANK D PROF DRESSEL LINDA L DRILLING JOHN S DUFOUR REGINALO JAKES DUNCOMBE RAYNOR L DR DUNN RICHARD B DR DURISEN RICHARD H DR DUTHIE JOSEPH G PROF DYCK M DR

EATON JOEL A DR EDMONDSON FRANK $K$ PROF EICHHORN HEINRICH $K$ DR ELITZUR MOSHE ELMEGREEN DEBRA MELOY ELVIS MARTIN S DR ENDAL ANDREH S DR EPSTEIN GABRIEL LEO DR ESHLEMAN VON R PROF ESPOSITO LARRY W EVANS J V DR EVANS W DOYLE FABBIANO GIUSEPPINA FALCO-ACOSTA EMILIO E DR FALL S MICHAEL DR FANG LI-ZHI FAY THEOOORE D DR FEIBELMAN WALTER A DR FELDMAN PAUL DONALD DR FELTEN JAMES E DR FIALA ALAN D DR FIELD GEORGE B PROF FINDLAY JOHN $W$ DR FIROR JOHN $W$ DR FISHER GEORGE HEWITT DR FISHER RICHARD R DR FITZPATRICK EDWARD L DR FLECK ROBERT CHARLES DR FLORKOWSKI DAVID R OR FOMALONT EDUARD B DR FORBES TERRY $G$ DR FORMAN WILLIAM RICHARD DR FORT DAVID NORMAN DR FOX KENNETH DR FRANZ OTTO G DR FREEDMAN WENDY $L$ DR FRIEDLANDER MICHAEL PROF FRIEND DAVID B DR FROST KENNETH J DR FUHR JEFFREY ROBERT DR GALLAGHER III JOHN S DR 
' Country : USA (follow on)

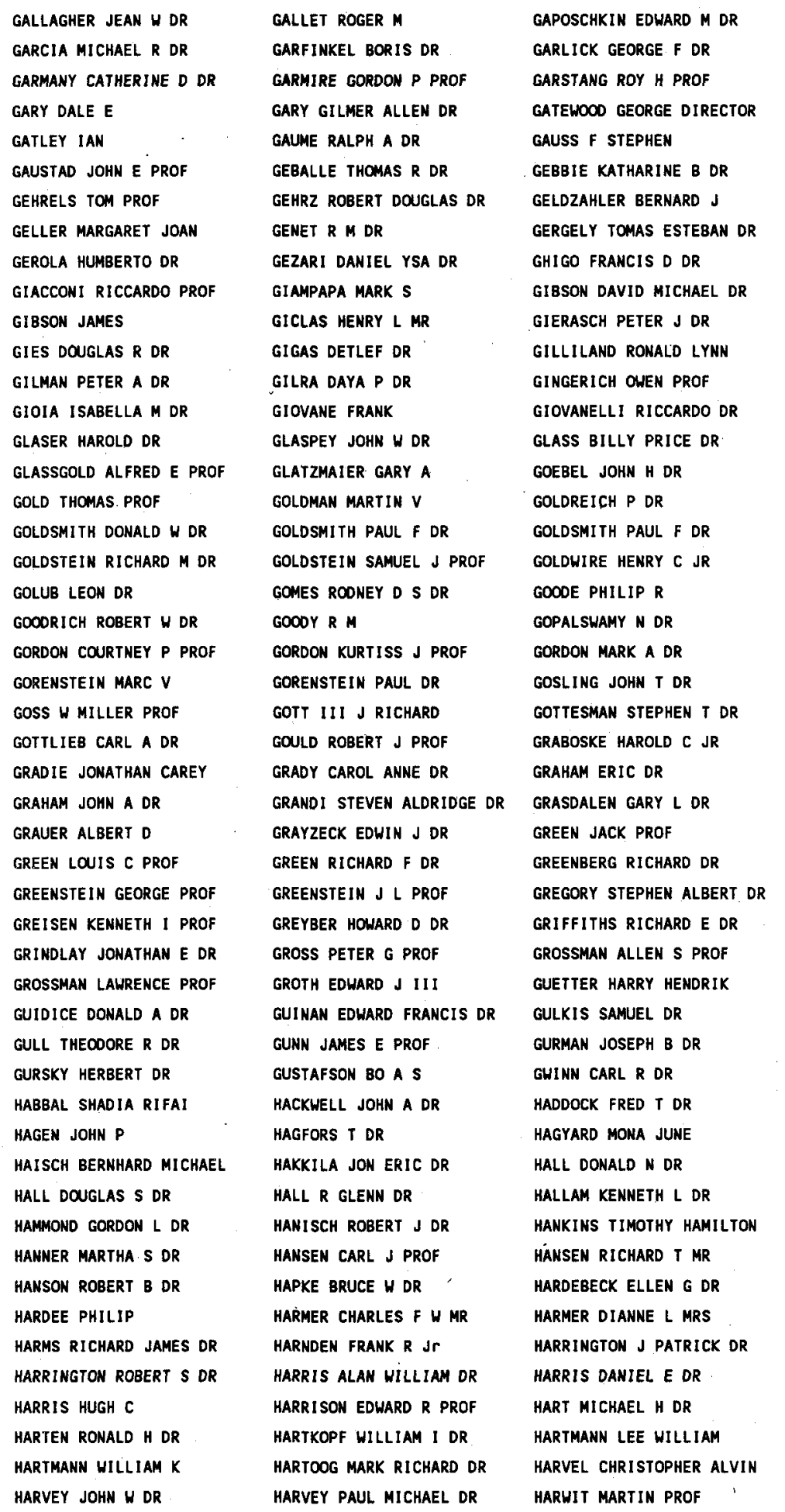


HASAN HASHIMA DR HATHAWAY DAVID H DR HAVLEN ROBERT J DR HAYES DONALD S DR HAZEN MARTHA L DR HECHT JAMES $H$ DR HEDEMAN E RUTH MISS HEGYI DENNIS J ASSOC PROF HEISER ARNOLD M DR HELIN ELEANOR FRANCIS HELOU GEORGE DR HENIZE KARL G ASTRONAUT HERBIG GEORGE H DR HERCZEG TIBOR J PROF DR HERSHEY JOHN L DR HEWITT ANTHONY $V$ DR HILDNER ERNEST DR HILLIARD $R$ DR HINTEREGGER HANS E DR' HO PAUL $T P$ HOBBS ROBERT W DR HOFF DARREL BARTON HOGAN CRAIG J DR HOLLENBACH DAVID JOHN DR HOLLWEG JOSEPH V HOLZER THOMAS EDWARD DR HOROWITZ PAUL PROF HOUSE LEWIS L DR HOUARD HILLIAM E III DR HU ESTHER M DR HUCHRA JOHN PETER DR HUENEMOERDER DAVID P DR HUGHES SHAUN HUMPHREYS ROBERTA M PROF HUNTER CHRISTOPHER PROF HUNTRESS WESLEY T DR HYDER C L DR ILLING RAINER M E IMHOFF CATHERINE L DR IRVINE WILLIAM M PROF JACKSON BERNARD $V$ DR JACOBS KENNETH C DR JAFFE DANIEL T JANICZEK PAUL M DR JEFFERIES JOHN T DR JENKINS EDWARD B DR JENMER DAVID C DR JOHNSON FRED M PROF DR JOHNSON TORRENCE $V$ DR JONES BARBARA

\section{HASCHICK AUBREY \\ HATZES ARTIE P DR \\ HAWKINS GERALD S OR \\ HAYMES ROBERT C PROF \\ HEAP SARA $R$ DR \\ HECKATHORN MARRY M \\ HEESCHEN DAVID S DR \\ HEILES CARL PROF}

HELFAND DAVID JOHM

HELLWIG HELMUT WILHELM DR

HEMENWAY MARY KAY M DR

HENRY RICHARD B C DR

HERBST ERIC DR

HERNQUIST LARS ERIC DR

HERTZ PAUL L DR

HIBBS ALBERT R MGR PLANS

HILL FRANK DR

HILLS JACK G DR

HINTZEN PAUL MICHAEL N DR

HOAG ARTHUR A DR

HODGE PAUL W PROF

HOFFLEIT E DORRIT DR

HOGG DAVID E DR

HOLLIS JAN MICHAEL DR

HOLMAN GORDON D

HONEYCUTT R KENT PROF

HOUCK JAMES $R$

HOWARD ROBERT F DR

HONELL STEVE BRUCE DR

HUBBARD WILLIAM B PROF

HUDSON HUGH S DR

HUGHES JOHN $P$ DR

HUGUENIN G RICHARD

HUNDHAUSEN ARTHUR DR

HUNTER DEIDRE ANN

HURFORD GORDON JAMES

IANNA PHILIP A

ILLINGWORTH GARTH D DR

IMPEY CHRISTOPHER D DR

IRHIN JOHN B PROF

JACKSON PETER DOUGLAS DR

JACOBSEN THEODOR S PROF

JAFFE WALTER JOSEPH DR

JANSSEN MICHAEL ALLEN

JEFFERIES STUART DR

JENKINS L $F$ MS

JEWELL. PHILIP R DR

JOHNSON HOLLIS R PROF

JOHNSTON KENNETH J

JONES BURTON DR
HASEGAWA TATSUHIKO DR HAUSER MICHAEL G DR HAUKINS ISABEL DR HAYNES MARTHA $P$ HEASLEY JAMES NORTON HECKMAN TIMOTHY M HEFFERLIN RAY A PROF. HEINTZ WULFF D DR HELFER H LAURENCE PROF HELMKEN HENRY F DR HEMENWAY PAUL D DR HENRY RICHARD C PROF HERBST WILLIAM DR HERR RICHARD B DR HEUITT ADELAIDE HILDEBRAND ROGER H HILL HEMRY ALLEN DR HINKLE KENNETH H HJELLMING ROBERT M DR HOBBS LEWIS M DR HOESSEL JOHN GREG HOFFMAN JEFFREY ALAN DR HOLBERG JAY B HOLLOWELL DAVID EARL DR HOLT STEPHEN $S$ HOOGHOUDT B G IR HOUK NANCY DR HOWARD W MICHAEL DR HRIVWAK BRUCE J HUBENY IVAN HUEBNER WALTER $F$ DR HUGHES PHILIP HUMMER DAVID G DR HUNTEN DONALD M PROF HUNTER JAMES H PROF HUT PIET IBEN ICKO JR PROF IMAMURA JAMES DR IPSER JAMES $R$ PROF JACCHIA LUIGI G DR JACKSON WILLIAM M DR JACOBY GEORGE H JANES KENNETH A DR JASTROW ROBERT JEFFERYS WILLIAM H DR JENKNER HELMUT DR JOHNSON DONALD R DR JOHNSON HUGH M DR JOKIPII J R PROF JONES DAYTON L 
Country : USA (follow on)

JONES ERIC M

JONES THOMAS WALTER OR JOSEPH CHARLES LYNN DR JUOGE PHILIP DR JURGENS RAYMOND $F$ KAFTAN MAY A DR KALER JAMES $B$ PROF KAMP LUCAS ÚILLEM DR KAPLAN GEORGE H DR KAROVSKA MARGARITA DR KATZ JONATHAN I KAWALER STEVEN D DR KEIL KLAUS DR KELLER GEOFFREY KENNICUTT ROBERT C JR KERR FRANK J DR KIELKOPF JOHN F DR KING IVAN R PROF KINMAN THOMAS D DR KIRBY KATE P DR KISSELL KENNETH E DR KLEIN RICHARD I DR KLEMPERER $W K$ DR KLINGLESMITH DANIEL A DR KNACKE ROGER F DR KNOWLES STEPHEN H DR KOCH ROBERT H DR KOJOLAN GABRIEL DR KONIGL ARIEH DR KOPP ROGER A DR KOUVELIOTOU CHRYSSA DR KOWAL CHARLES THOMAS KRAUSHAAR WILLIAM L PROF KRISCIUNAS KEVIN DR KROGDAHL W S DR KRON KATHERINE GORDON KRUPP EDWIN C DR KUIPER THOMAS B H DR KUMAR C KRISHNA OR KUNDU MUKUL R DR KUTNER MARC LESLIE DR LA BONTE BARRY JAMES LACY JOHN H DR LALA PETR DR LAMB RICHARD C DR LAMPTON MICHAEL. LANDMAN DONALD ALAN LANE ARTHUR LONNE OR LANGER WILLIAM DAVID DR LARSON RICHARD B PROF
JONES FRANK CULVER DR JORDAN STUART D DR JOSS PAUL CHRISTOPHER DR JUNKKARINEN VESA T DR JURKEVICH IGOR DR KAHLER STEPHEN W DR KALKOFEN WOLFGANG OR KANDRUP HENRY EMIL DR KAPLAN J DR

KARP ALAN HERSH DR KAUFMAN MICHELE DR KEEL. HILLIAM C KEIL. STEPHEN L KELLERMANN KENMETH I DR KENT STEPHEN M KESSLER KARL G DR KIMBLE RANDY A DR KING R B DR KINNEY ANNE L DR KIRKPATRICK RONALD C DR KLARMANN JOSEPH PROF KLEINMANN DQUGLAS E DR KLEPCZYNSKI WILLIAM J DR KLIORE ARVYDAS JOSEPH DR KNAPP GILLIAN R DR KO HSIEN C PROF KOESTER DETLEV DR KOLB EDWARD $W$ DR KOO DAVID C-Y DR KORMENDY JOHN DR KOVAR N S DR KRAFT ROBERT P PROF KREIDL TOBIAS J N KRISS GERARD A DR KROLIK JULIAN H KRON RICHARD G KUHI LEONARD $V$ PROF KULKARNI SHRINIVAS R DR KUMAR SHAILENDRA KURFESS JAMES D KUTTER G SIEGFRIED DR LA DOUS CONSTANZE A DR LADA CHARLES JOSEPH DR LAMB DONALD QUINCY JR DR LAMB SUSAN ANN DR LANOE KENMETH PROF LANDOLT ARLO U PROF LANG KENNETH R ASST PROF LANZ THIERRY DR LARSON STEPHEN $M$
JONES HARRISON PRICE DR JOSELYN JO ANN C DR JOY MARSHALL $J$ DR JURA MICHAEL DR KAFATOS MINAS DR KAITCHUCK RONALD H KAMMEYER PETER C DR KANE SHARAD R DR KAPLAN LEWIS D DR KARPEN JUDITH T KAULA WILLIAM M PROF KEENAN PHILIP C PROF EMER KELLER CHARLES $F$ KELLOGG EDHIN M DR KENYON SCOTT J DR KHARE BISHUN N DR KING DAVID $S$ PROF KING ROBERT WILSON JR DR KIPLINGER ALAN L DR KIRSHNER ROBERT PAUL DR KLEIN MICHAEL J DR KLEMOLA ARNOLD R DR KLIMCHUK JAMES A DR KLOCK B L DR KNIFFEN DONALD A DR KOCH DAVID $G$ KOHL JOHN L DR KONDO YOJI DR KOORNNEEF JAN DR KOUPELIS THEODOROS DR KOVAR ROBERT P DR KRAUS JOHN D PROF KRIEGER ALLEN S DR KRISTIAN JEROME DR KRON GERALD E DR KRUMM NATHAN ALLYN KUHN JEFFERY RICHARD DR KULSRUD RUSSELL M DR KUMAR SHIV S PROF KURUCZ ROBERT L DR KWITTER KAREN BETH DR LACY CLAUD H DR LAIRD JOHN B DR LAMB FREDERICK $K$ PROF LAMBERT DAVID L PROF LANDECKER PETER BRUCE DR LANE ADAIR. $P$

LANGER GEORGE EDWARD DR LARSON HAROLD P DR LASALA GERALD J OR 
LASHER GORDON JEWETT DR

LATTIMER JAMES M DR .

LAWRENCE G M DR

LEA SUSAN MAUREEN DR LEBOVITZ NORMAN R PROF

LEE PAUL D DR

LEISAWITZ DAVID DR LEUNG CHUN MING DR LEVISON HAROLD F DR LEWIN WALTER H G PROF LI NED C DR LIDDELL U MR

LILLEY EDWARD A PROF

LIN DOUGLAS N C DR LINGENFELTER RICHARD E LINSKY JEFFREY L DR LIPSCHUTZ MICHAEL E DR LITTLETON JOHN E LIU SOU-YANG DR LOCANTHI DOROTHY DAVIS DR LONG KNOX $S$ DR LOPES-GAUTIER ROSALY DR LOVAS FRANCIS JOHN DR LOW FRANK J DR LUCKE PETER 8 DR LUTTERMOSER DONALD DR LUTZ THOMAS E DR LYNDS BEVERLY T DR. MACCACARO TOMMASO DR MACDONALD JAMES MACY WILLIAM WRAY DR MALINA ROGER FRANK DR MALKAN MATTHEW ARNOLD DR MARAN STEPHEN P DR MARISKA JOHN THOMAS MARKOWITZ WILLIAM DR MARSDEN BRIAN G DR MARTĖNS PETRUS C DR MARTINS DONALD HENRY DR MASSEY PHILIP L MATHEWS WILLIAM G PROF MATSAKIS DEMETRIOS N MATTEI JANET AKYUZ DR MATTHEWS THOMAS A DR MAX CLAIRE E DR MAYALL NICHOLAS U ASTRON MAZUREK THADDEUS JOHN DR MCCAMMON DAN MCCLINTOCK JEFFREY E DR MCCRAY RICHARD DR
LASKER BARRY M OR LAUTMAN D A DR LAURIE DAVID G LEACOCK ROBERT JAY LECAR MYRON DR LEIBACHER JOHN DR LEPP STEPHEN H DR LEUNG KAM CHING PROF LEVREAULT RUSSELL M OR LEWIS BRIAN MURRAY DR LIANG EDISON P DR LIEBERT JAMES $W D R$ LILLIE CHARLES F DR LINCOLN J VIRGINIA MISS LINKE RICHARD ALAN DR LINSLEY JOHN LISSAUER JACK J DR LITTLE-MARENIN IRENE R DR LIVINGSTON WILLIAM C LOCKMAN FELIX J LONGMORE ANDREW J LORD STEVEN DONALD DR LOVELACE RICHARD $V$ E DR LU PHILLIP $K$ DR LUGGER PHYLLIS M LUTZ BARRY L DR LUYTEN WILLEM J PROF LYNDS ROGER C DR MACCHETTO FERDINANDO DR MACK PETER DR MAGALHAES ANTONIO MARIO MALITSON HARRIET H MS MALVILLE J MCKIM PROF MARGON BRUCE H PROF MARK JAMES WAI-KEE OR MARSCHALL LAURENCE A MARSHALL HERMAN LEE DR MARTIN ROBERT N DR MARVIN URSULA B DR MASSON COLIN R MATHIEU ROBERT D OR MATSON DENNIS $L$ DR MATTHEUS CLIFFORD PROF MATZ STEVEN MICHEAL DR MAXWELL ALAN DR MAYER CORMELL H MCALISTER HAROLD A DR MCCARTHY DENNIS D DR MCCLUSKEY GEORGE E JR DR MCCROSKY RICHARD E DR
LATHAM DAVID H DR LAURENCE CHARLES R DR LAYZER DAVID PROF LEBOFSKY LARRY ALLEN LECKRONE DAVID S DR LEIGHTON R B PROF LESTER DANIEL F DR LEVINE RANDOLPH H DR LEVY EUGENE H DR LEWIS J S LIBBRECHT K G OR LIESKE JAY H OR LIN CHIA C PROF LINDSEY CHARLES ALLAN LINNELL ALBERT P PROF LIPPINCOTT SARAH LEE DR LISZT HARVEY STEVEN LITVAK MARVIN M DR LO KWOK-YUNG DR LOCKWOOD G WESLEY DR LONSDALE CAROL J DR LOREN ROBERT BRUCE DR LOW BOON CHYE LUCK R EARLE DR LUNDQUIST CHARLES A DR LUTZ JULIE H DR LYNCH DAVID $K$ MACALPINE GORDON M MACCONNELL DARRELL J DR MACQUEEN ROBERT M DR MAGNANI LORIS ALBERTO DR MALKAMAEKI LAURI J DR MANSFIELD VICTOR N PROF MARGRAVE THOMAS EWING JR MARKERT THOMAS H DR MARSCHER ALAN PATRICK MARSTON ANTHONY PHILIP DR MARTIN WILLIAM C OR MASON GLENN M MATHER JOHN CRONWELL MATHIS JOHN S PROF MATSUSHIMA SATOSHI DR MATTHEWS HENRY E DR MATZNER RICHARD A PROF MAYALL MARGARET $W$ MAYFIELD EARLE B DR MCCABE MARIE $K$ MS MCCLAIN EOHARD $F$ MCCORD THOMAS B DR MCDONALD FRANK B DR 
Country : USA (Follow on)

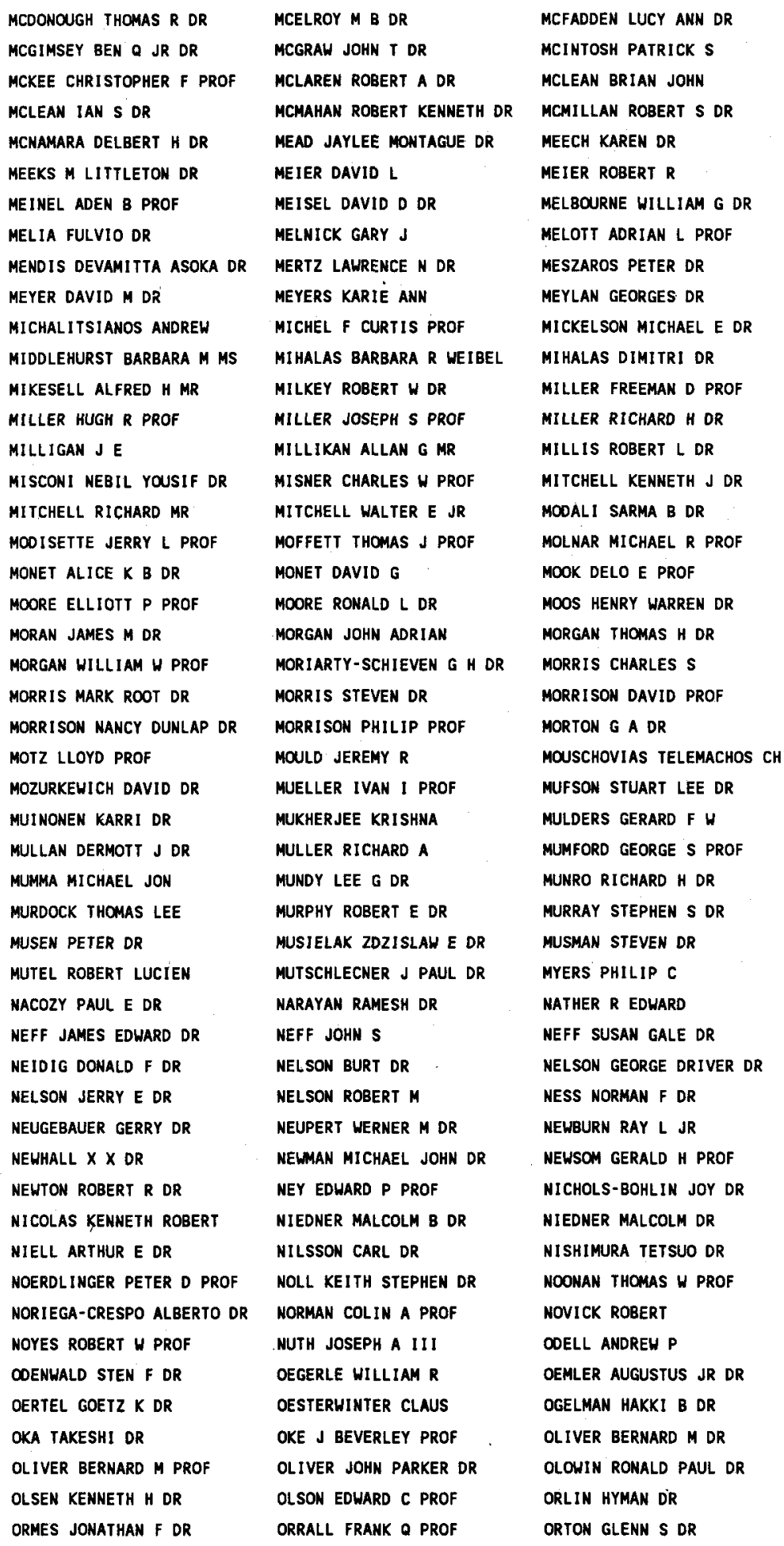




OSBORN WAYNE DR
OSTERBROCK DONALD E PROF
ONEN FRAZER NELSON DR
OZERNOY LEONID M PROF
O'CONMELL ROBERT WEST DR
O'DELL STEPHEN L
O'LEARY BRIAN T
PAGE DON NELSON
PAN XIAO-PEI
PANG KEVIN

PAPAgIanNIS MICHAEL. D PRO

PARISE RONALD A DR

PARKINSON TRUMAN DR

PARSONS SIDNEY B DR

PASCU DAN DR

PEALE STANTON J PROF

PEERY BENJAMIN F PROF

PERKINS FRANCIS $W$ DR

PERRY PETER M DR

PETERS WILLIAM L III DR

PETERSON DEANE M DR

PETRO LARRY DAVID

PFEIFFER RAYMOND J

PHILLIPS THOMAS GOULD DR

PIERCE A KEITH DR

PILCHER CARL BERNARD DR

PINTO PHILIP ALFRED DR

PLAVEC ZDENKA DR

POLAND ARTHUR I DR

PONNAMPERUMA CYRIL PROF

POTTER ANOREW E OR

PRAVDO STEVEN H

PRESTON GEORGE W DR

PRICE R MARCUS DR

PROBSTEIN R F OR

PRYOR CARLTON PHILIP DR

PUSCHELL JEFFERY JOHN

RABIN DOUGLAS MARK

RAFERT JAMES BRUCE

RAMSEY LAWRENCE W DR

RAO K NARAHARI

READHEAD ANTHONY C S DR

REEVES EDMOND M DR

REID NEILL

REVELLE DOUGLAS ORSON DR

REYNOLDS RONALD J DR

RICHARDS MERCEDES T DR

RICKARD JAMES JOSEPH DR

RICKETT BARNABY JAMES DR

RIEGLER GUENTER $R$ DR
OSMER PATRICK $S$ DR

OSTRIKER JEREMIAH P PROF

ONEN TOBIAS C PROF

OZSVATH I PROF

O'DEA CHRISTOPHER P DR

o' HANDLEY DOUGLAS A DR

PACHOLCZYK ANDRZEJ G PROF

PAGE THORNTON L DR

PANAGIA NINO DR

PANKONIN VERNON LEE DR

PAPALIOLIOS COSTAS DR

PARKER EUGENE N

PARKINSON WILLIAM H DR

PARTRIDGE ROBERT B PROF

PAULS THOMAS ALBERT DR

PEARSON TIMOTHY J

PELLERIN JR CHARLES J DR

PERLEY RICHARD ALAN

PESCH PETER DR

PETERSON BRADLEY MICHAEL

PETERSON LAURENCE E PROF

PETROSIAN VAHE PROF

PHILIP A G DAVIS

PICKLES ANDREW JOHN DR

PIERCE DAVID ALLEN OR

PINES DAVID PROF

PIPHER JUDITH L

PMEUMAN GERALD $\mathrm{H}$

POLIDAN RONALD S

POPPER DANIEL M PROF

PRADHAN ANIL DR

PRENDERGAST KEVIN H PROF

PRESTON ROBERT ARTHUR

PRICE STEPHAN DONALD

PROFFIIT CHARLES R DR

PUETTER RICHARD C DR

PYPER SMITH DIANE M DR

RADICK RICHARD R DR

RAHE JURGEN PROF

RANK DAVID M PROF

RATNATUNGA KAVAN U

REASENBERG ROBERT D DR

REICHERT GAIL ANNE DR

REITSEMA HAROLD J

REYES FRANCISCO DR

REYNOLDS STEPHEN $P$

RICHARDSON R S

RICKARO LEE J DR

RIDDLE ANTHONY $C$ DR

RIGHINI-COHEN GIOVANNA DR
OSTER LUDWIG F PROF DR OSHALT TERRY D DR OWOCKI STANLEY PETER DR O CONNELL ROBERT F PROF O'DELL CHARLES R DR O'KEEFE JOHN A DR PACIESAS WILLIAM S DR PALMER PATRICK E PROF PANEK ROBERT J DR PAP JUDIT PARESCE FRANCESCO DR PARKER ROBERT A $R$ PARRISH ALLAN DR PASACHOFF JAY M PROF PAYNE DAVID G

PEEBLES $P$ JaMes $E$ PENZIAS ARNO A OR PERRY CHARLES L DR PETERS GERALDINE JOAN DR PETERSON CHARLES JOHN OR PETERSON RUTH CAROL DR PETTENGILL GORDON H PROF PHILLIPS JOHN G PROF PIER JEFFREY $R$ DR PILACHOWSKI CATHERINE DR PINGREE DAVID PROF PLAVEC MIREK J PROF POGO ALEXANDER DR POLLACK JAMES B DR PORTER JASON G OR PRASAD SHEO $S$ PRESS WILLIAM H DR PRICE MICHAEL J DR PRINCE HELEN DOOSON PROF PROTHEROE HILLIAM M PROF PURCELL EDHARD M PROF QUIRK WILLIAM J DR RADOSKI HENRY R DR RAMATY REUVEN DR RANKIN JOANNA M DR RAYMOND JOHN CHARLES REAVES GIBSON PROF REID MARK JONATHAN DR RENSE WILLIAM A DR REYNOLDS JOHN H PROF RHOOES EDHARD J JR RICHSTONE DOUGLAS O DR RICKER GEORGE $R$ DR RIEGEL KURT W OR RINDLER WOLFGANG PROF 
Country : USA (Follow on)

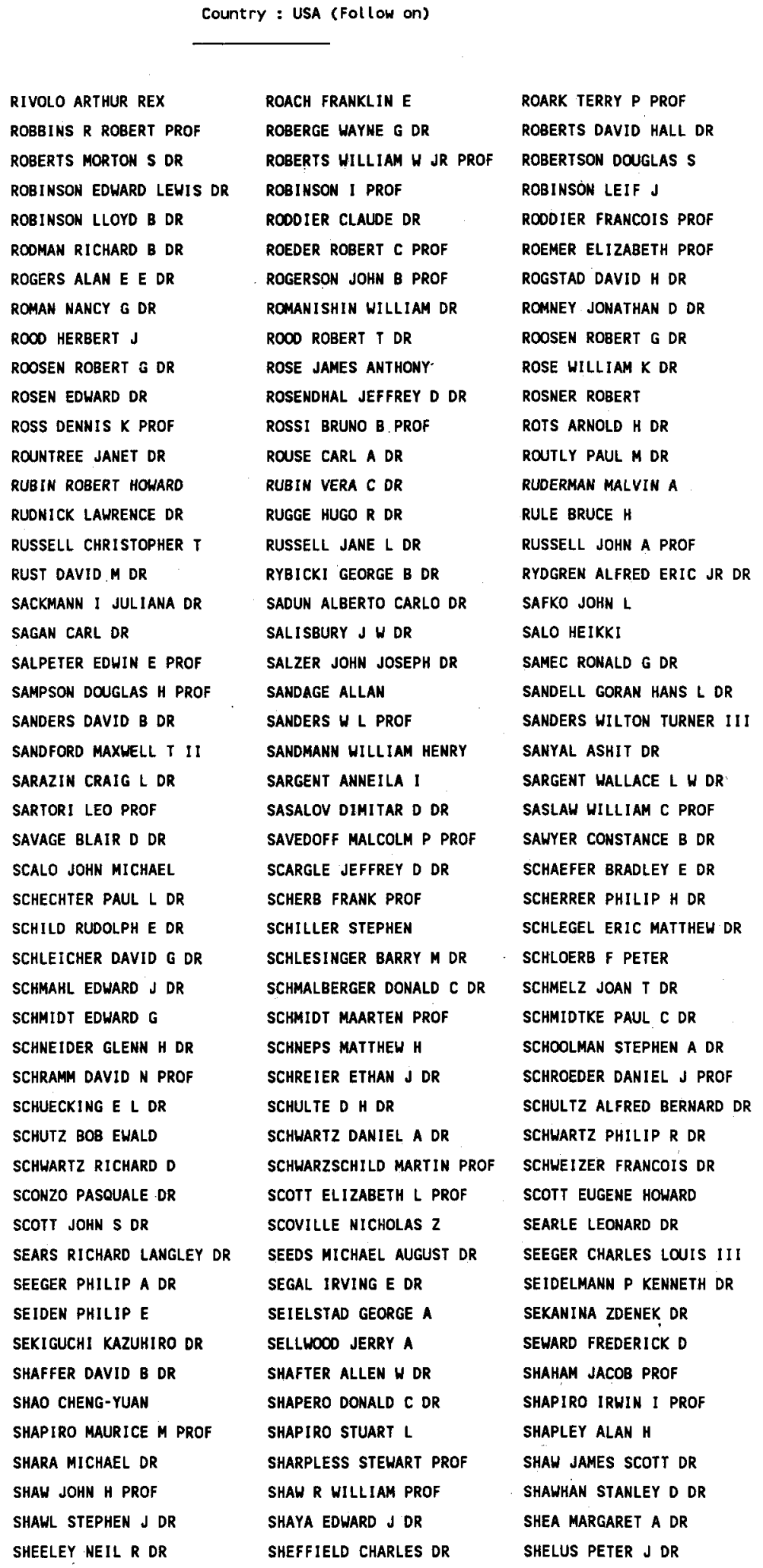


SHEN BENJAMIN S P PROF SHINE RICHARD A DR SHOEMAKER EUGENE M SHOSTAK G SETH DR SHULL PETER OTTO DR SILVERBERG ERIC C DR SIMON MICHAL PROF SIMONSON S CHRISTIAN DR SIRY JOSEPH W SJOGREN WILLIAM L MR SKUMANICH ANDRE PROF SLOVAK MARK HAINES DR SMITH BARHAM W DR SMITH CHARLES DITTO SMITH ELSKE V P DR SMITH HARDING E JR DR SMITH HOWARD ALAN SMITH PETER L DR SMOLUCHOWSKI ROMAN PROF SNELL RONALD L SOBERMAN ROBERT K DR SODERBLON LARRY DR SOLOMON PHILIP M DR SOWELL JAMES ROBERT DR SPENCER JOHN HOWARD SPIEGEL E DR SRAMEK RICHARD A DR STAHLER SETVEN $W$ DR STANFORD SPENCER A STARK GLEN DR STECHER THEODORE $P$ STEIGER W R PROF STEIN JOHN WILLIAM STEINOLFSON RICHARD S DR STEPHENSON C BRUCE PROF STIER MARK $T$ STOCKTON ALAN N DR STONE REMINGTON P S DR STRITTMATIER PETER A PROF STROM ROBERT G PROF STRONG JOHN D PROF STRUCK-MARCELL CURTIS J STURROCK PETER A PROF SULLIVAN WOOORUFF T III SWEITZER JAMES STUART DR SWIHART THOMAS L DR SZEBEHELY VICTOR G PROF TADEMARU EUGENE DR TANDBERG-HANSSEN EINAR A TARNSTRON GUY DR
SHER DAVID DR SHIPMAN HENRY L DR SHORE BRUCE $W$ SHU FRANK H PROF SILBERBERG REIN DR SIMKIN SUSAN M DR SIMON NORMAN R PROF SINTON WILLIAM M SITKO MICHAEL L SKALAFURIS ANGELO J SLADE MARTIN A III DR SMITH ALEX G PROF SMITH BRADFORD A PROF SMITH CLAYTON A JR DR SMITH ERIC PHILIP DR SMITH HAYWOOD C DR SMITH MALCOLM G DR SMITH VERNE $V$ DR SMOOT III GEORGE $F$. SNOW THEODORE P PROF SOBIESKI STANLEY DR SOFIA SABATINO PROF SONETT CHARLES P PROF SPARKE LINDA SPERGEL DAVID N DR SPINRAD HYRON PROF STACEY GORDON J DR STAHR-CARPENTER M DR STANLEY G J STARRFIELD SUMNER PROF STECKER FLOYD W DR STEIGMAN GARY PROF STEIN ROBERT F ASSOC PROF STELLINGWERF ROBERT F DR STEPINSKI TOMASZ DR STINEBRING DANIEL $R$ STONE EDWARD C DR STONE RONALD CECIL STROBEL DARRELL F STROM STEPHEN E STRONG KEITH T DR STRYKER LINDA L SUESS STEVEN $T$. DR SWANK JEAN HEBB SWENSON GEORGE W JR PROF SYKES MARK VINCENT DR SZKOOY PAULA DR TAFF LAUREMCE $G$ DR TAPIA-PEREZ SANTIAGO TARTER C BRUCE DR
SHIELDS GREGORY A DR SHIVANANDAN KANDIAH DR SHORE STEVEN N SHUULL JOHN MICHAEL SILK JOSEPH I PROF SIMON GEORGE W DR SIMON THEOOORE SION EDWARD MICHAEL SITTERLY CHARLOTTE M DR SKILLMAN EVAN D DR SLETTEBAK ARNE PROF SMITH ANDREW M DR SMITH BRUCE $F$ DR SMITH DEAN F DR SMITH GRAEME H DR SMITH HORACE A SMITH MYRON A ASST PROF SMITH WM HAYOEN PROF SNEDEN CHR ISTOPHER A SNYDER LEWIS E SCDERBLOM DAVID R SOIFER BARUCH T DR SONNEBORN GEORGE DR SPARKS WARREN M DR SPICER DANIEL SHIELDS DR SPITZER LYMAN JR OR STACHNIK ROBERT $V$ STANDISH E MYLES DR STARK ANTONY A STEBBINS ROBIN STEFANIK ROBERT DR STEIMAN-CAMERON THOMAS DR STEIN HAYNE A PROF STENCEL ROBERT EDHARD STERN ROBERT ALLAN STOCKMAN HERVEY S JR DR STONE R G DR STRAND KAJ AA DR STROM KAREN M STRONG IAN B DR STRUBLE MITCHELL $F$ STURCH CONRAD R DR SULENTIC JACK $W D R$ SHEIGART ALLEN V DR SWERDLOW NOEL PROF SYWNOTT STEPHEN $P$ TAAM RONALD EVERETT DR TALBOT RAYMOND J JR DR TAPLEY BYRON D DR TARTER JILL C DR 
Country : USA (Follow on)

TAYLOR DONALD J DR

TEDESCO EDWARD $F$

TERRILE RICHARD JOHN

TEUBEN PETER J DR

THOLEN DAVID J DR

THOMAS ROGER $J$ DR

THOMPSON ROOGER I PROF

IHORSTENSEN JOHN $R$

TIFFT WILLIAM G PROF

TOHLINE JOEL EDWARD

TOLLER GARY N DR

TONRY JOHN DR

TOUSEY RICHARD DR

TRAUB WESLEY ARTHUR

TRIMBLE VIRGINIA L DR

TRURAN JAMES $W$ JR

TUCKER WALLACE H DR

TURNER BARRY E DR

TURNER MICHAEL S

TYLKA ALLAN J DR

ULICH BOBBY LEE

UNDERWDOO JAMES H DR

UPGREN ARTHUR $R$ DR

URRY CLAUDIA MEGAN DR

VAN ALLEN JAMES A PROF

VAN BREUGEL WIL

VAN FLANDERN THOMAS DR

VAN HORN HUGH M PROF

VAN SPEYBROECK LEON P DR

VAUGHAN ARTHUR H DR

VERSCHUUR GERRIT L PROF

VEVERKA JOSEPH DR

VISHNIAC ETHAN T

VORPAHL JOAN A DR

VRTILEK SAEOA DIL DR

WADE RICHARD ALAM DR

WAGNER WILLIAM J DR

WALKER ALTA SHARON DR

WALKER RICHARD L

HALLACE LLOYD V DR

WALTER FREDERICX M

WANG YI-MING DR

WARD WILLIAM R DR

WARNER JOHN W DR

WASSERMAN LAWRENCE H DR

WDOWIAK THOMAS J DR

WEBB DAVID $F$

WEBER STEPHEN VANCE

WEEKES TREVOR $C$ DR

WEHRLE ANN ELIZABETH DR
TAYLOR JOSEPH H PROF

TELESCO CHARLES M DR

TERZIAN YERVANT PROF

THADDEUS PATRICK PROF

THOMAS JOHN H PROF

THOMPSON A RICHARD OR

THONNARD NORBERT DR

THRONSON HARLEY ANDREW JR

TIMOTHY J GETHYN DR

TOKUNAGA ALAN TAKASHI DR

TOMASKO MARTIN G DR

TOOMRE ALAR DR

TOWNES CHARLES HARD DR

TREFFERS RICHARD $R$

TRIPICCO MICHAEL J DR

TSURUTA SACHIKO DR

TULL ROBERT G

TURNER EDWIN L DR

TWAROG BRUCE $A$

TYSON JOHN A DR

ULMER MELVILLE P PROF

UNWIN STEPHEN $C$

UPSON WALTER L II DR

USHER PETER D DR

VAN ALTENA WILLIIAM F PROF

VAN CITTERS GORDON W DR

VAN GORKOM JACQUELINE H

VAN HOVEN GERARD DR

VANDEN BOUT PAUL A

VEEDER GLENN J DR

VERTER FRANCES DR

VILA SAMUEL C PROF

VOGEL STUART NEWCOMBE DR

VRBA FREDERICK J DR

WACKERNAGEL H BEAT DR

WAGNER RAYMOND L DR

WAGONER ROBERT $\checkmark$ PROF

HALKER ARTHUR B C JR PROF

HALKER ROBERT C DR

WALLACE RICHARD $K$

WALTERBOS RENE A M DR

WANNIER PETER GREGORY DR

WARDLE JOHN F C PROF

WARREN WAYNE H JR DR

WASSON JOHN T

WEAVER HAROLD F PROF

WEBBER JOHN C DR

WEBSTER ADRIAN S DR

WEGNER GARY ALAN

WEIDENSCHILLING S J DR
TEAYS TERRY J DR

TERRELL NELSON JAMES JR

TESKE RICHARD G PROF

THIELEMANN FRIEDRICH-KARL

THOMAS RICHARD N DR

THOMPSON LAIRD A DR

THORNE KIP S PROF

THUAN TRINH XUAN DR

TIPLER FRANK JENNINGS DR

TOLBERT CHARLES R DR

TOMBAUGH CLYDE W PROF

TOOMRE JURI

TRAFTON LAURENCE M DR

TREXLER JAMES $H$ MR

TROLAND THOMAS HUGH

TSVETANOV ZLATAN IVANOV

TULLY RICHARD BRENT DR

TURNER KENNETH C DR

TYLER JR G LEONARD DR

TYTLER DAVID DR

ULRICH ROGER $K$ PROF

UOMOTO ALAN $K$ DR

UPTON E K L DR

USON JUAN M DR

VAN BLERKOM DAVID J PROF

VAN DORN BRADT HALE DR

VAN HAMME WALTER

VAN RIPER KENNETH A DR

VANDERVOORT PETER O OR

VENKATAKRISHNAN P DR

VESECKY J F DR

VILAS FAITH DR

VOGT STEVEN SCOTT

VRTILEK JAN M DR

WADDINGTON C JAKE PROF

HAGNER ROBERT M DR

WALBORN NOLAN $R$ DR

WALKER MERLE F PROF

WALKER ROBERT M A PROF

WALLERSTEIN GEORGE PROF

WANG HAIMIN DR

WARD RICHARD A DR

WARES GORDON $W D R$

HARHICK JAMES $W D R$

WATSON WILLIAM D PROF

WEAVER THOMAS A DR

WEBBINK RONALD F DR

WEEDMAN DANIEL W PROF

WEHINGER PETER A DR

WEILER EDHARD J DR 
WEILER KURT W DR WEINBERG STEVEN DR WEISHEIT JON C DR WEISTROP DONNA DR WELLS DONALD C III DR WEST DONALD $K$ DR WESTPHAL JAMES A PROF WHEELER I CRAIG PROF WHIPPLE FRED L DR WHITE ORAN R DR WHITE RICHARD E WHITFORD ALBERT E PROF WHITNEY CHARLES A PROF WIDING KENNETH $G$ OR WILDEY ROBERT L PROF DR HILKINSON DAVID T WILLIAMS BARBARA A WILLIAMS JAMES $G D R$ WILLNER STEVEN PAUL DR WILLSON LEE ANNE DR WILSON ANDREW S DR WILSON ROBERT E PROF WINCKLER JOHN R PROF WINGET DONALD E WINKLER PAUL FRANK DR WITT ADOLF N DR WOLFF SIDNEY C DR WOLSZCZAN ALEXANDER DR WOOD III H J DR WOODWARD PAUL $R$ DR WOOTTEN HENRY ALWYN WORRALL DIANA MARY WRIGHT FRANCES W DR WRIGHT MELVYN C H DR WU SHI TSAN DR WYNN-WILLIAMS C G DR YANG KE-JUN YEOMANS DONALD $K$ DR YOSS KENNETH $M$ DR YOUNG JUDITH SHARN YUAN CHI PROF ZARRO DOMINIC M OR ZENSUS J-ANTON DR ZINN ROBERT J DR ZOMBECK MARTIN $V$ DR
WEILL GILBERT M DR WEIS EDHARD W DR WEISSKOPF MARTIN CH DR MELCH HILLIAM J PROF WENTZEL DONAT G DR WEST ROBERT ALAN WETHERILL GEORGE W UHEELER JOHM A DR WHITAKER EWEN A WHITE R STEPHEN PROF WHITE RICHARD L WHITMORE BRADLEY C WHITTET DOUGLAS C B DR WIESE WOLFGANG L DR WILKENING LAUREL L DR WILL CLIFFORD M DR WILLIAMS CAROL A WILLIAMS JOHN A DR WILLS BEVERLEY $J D R$ WILLSON ROBERT FREDERICK WILSON CURIIS A WILSON ROBERT W DR WINDHORST ROGIER A DR WINKLER GERNOT M R DR WISNIEWSKI WIESLAW 2 WITTEN LOUIS PROF WOLFSON C JACOB WOOO DAVID B DR $\$ 000$ JANET H DR WOOLF NEVILLE J WORDEN SIMON P DR WRAY JAMES D DR WRIGHT HELEN GREUTER WROBEL JOAN MARIE DR WYCKOFF SUSAN DR WYSE ROSEMARY F DR YAPLEE B S YOOER CHARLES F YOUNG ANDREW T DR YOUNG LOUISE GRAY DR ZABRISKIE F R PROF ZEILIK MICHAEL II DR ZHANF SHOUZHONG DR ZIRIN HAROLD OR ZUCKERMAN BEN M DR
WE I NBERG J L DR WEISBERG JOEL MARK WEISSMAN PAUL ROBERT WELLER CHARLES S DR WESSELINK ADRIAAN J DR WESTERHOUT GART DR WEYMANN RAY J PROF UHIPPLE ARTHUR L OR WHITE NATHANIEL M DR WHITE RAYMOND E DR WHITE SIMON DAVID MANION WHITNEY BALFOUR $S$ WHITTLE D MARK DR WIITA PAUL JOSEPH WILKES BELINDA J WILLIAMON RICHARD M WILLIAMS GLEN A DR WILLIAMS THEOOORE' B DR WILLS DEREK DR WILSON ALBERT G DR WILSON JAMES'R DR WILSON HILLIAM J OR WING ROBERT F PROF WINKLER KARL-HEINZ A DR WITHBROE GEORGE L DR WOLFE ARTHUR M PROF WOLFSON RICHARD DR WOOO F BRADSHAL PROF WOOD JOHN A DR WOOSLEY S E PROF WORLEY CHARLES E DR WRIGHT EDWARD L DR WRIGHT JAMES P DR WU CHI CHAO DR WYLLER ARNE A PROF YAHIL AMOS DR YEH TYAN DR YORK DONALO G DR YOUNG ARTHUR DR YU YAN DR ZARE KHALIL DR ZELLLNER BENJAMIN H DR ZHANG ER-HO DR ZIRKER JACK B DR 


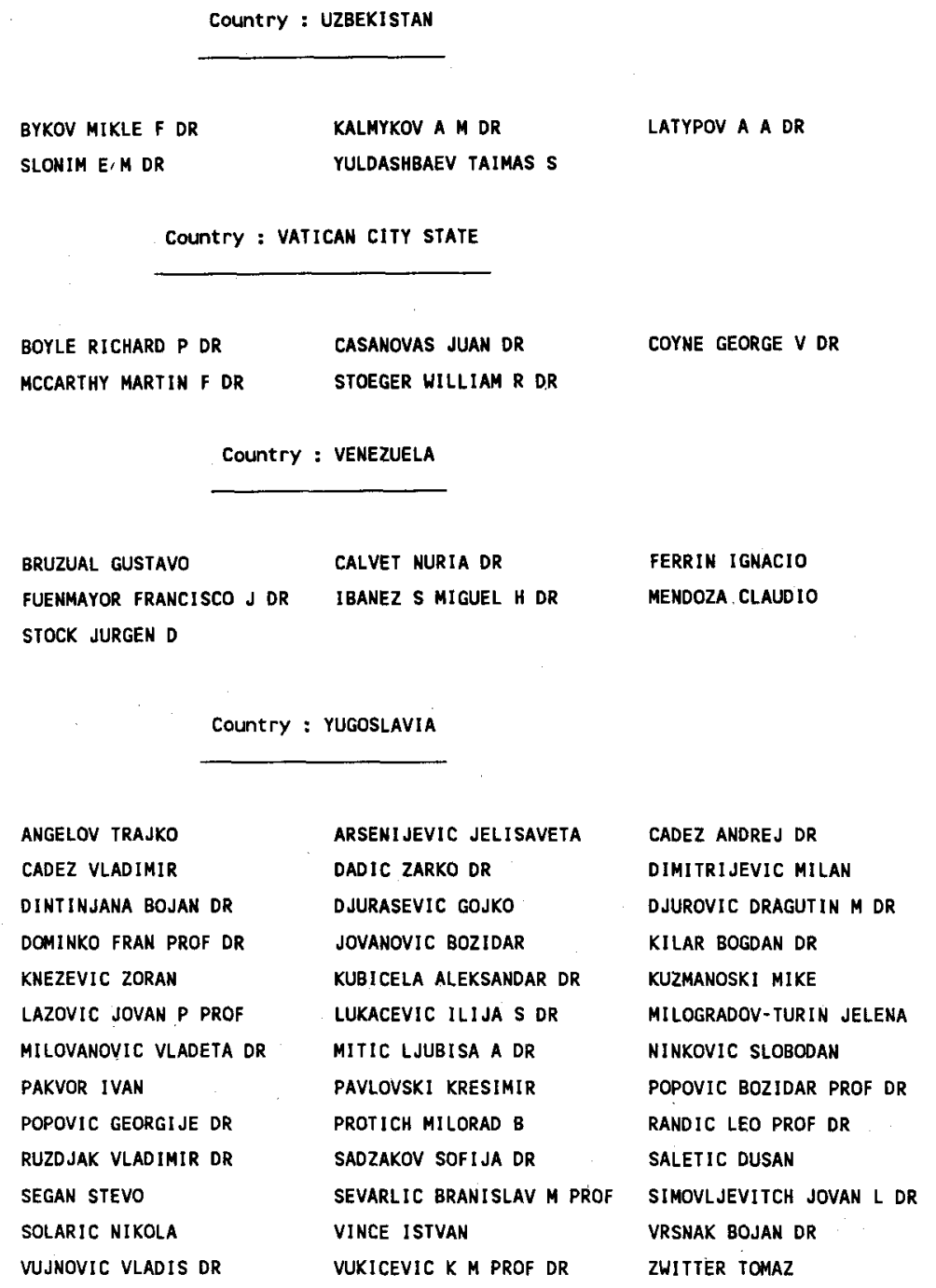

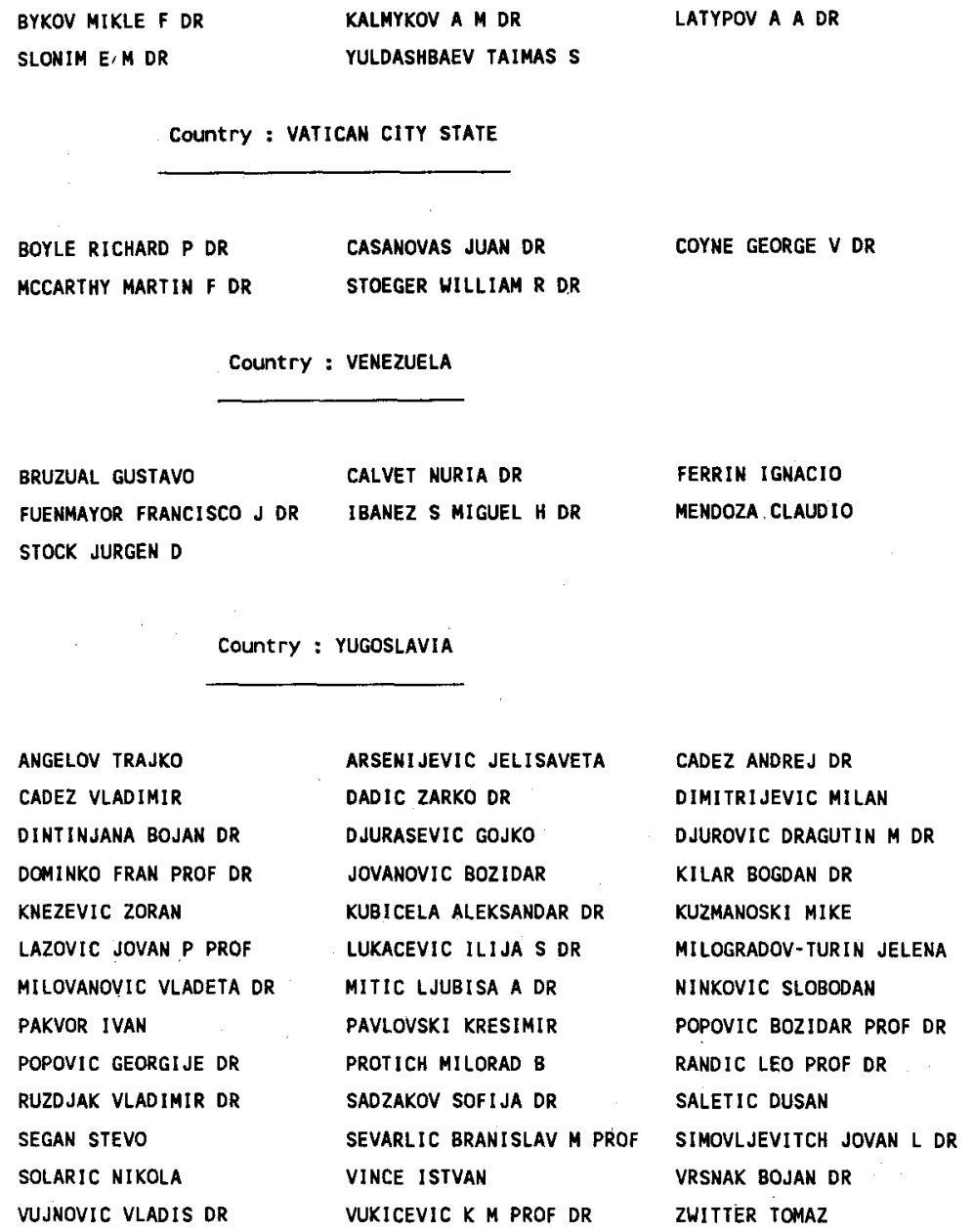


3. Geographical repartition of members

Members

- within non adhering countries

\section{Country : HONG KONG}

YU XIN ALFRED DR

Country : LEBANON

PLASSARD J DR

Country : NIGERIA

AKUJOR CHIDI E

OKEKE PIUS N DR

OKOYE SAMUEL E PROF

ONUORA LESLEY IRENE DR

SCHMITTER EDWARD F DR

Country : PAKISTAN

QUAMAR JAWAID

\section{Country : PARAGUAY}

TROCHE-BOGGINO A E DR

Country : PHILIPPINES

VINLUAN RENATO

Country : RUMANIA

BOTEZ ELVIRA DR

DINESCU A DR

LUMGU NICOLAIE DR

MIOC VASILE DR

PAL ARPAD PROF DR

RUSU I DR

TIFREA EMILIA DR

URECHE VASILE OR

$\begin{array}{ll}\text { CARSMARU MARIA M DR } & \text { CRISTESCU CORNELIA G DR } \\ \text { DRAMBA C PROF } & \text { DUMITRACHE CHRISTANA } \\ \text { MARIS GEORGETA DR } & \text { MIHAILA IERONIM PROF } \\ \text { NADOLSCHI V PROF DR } & \text { OPROIU TIBERIU DR } \\ \text { POP VASILE DR } & \text { PREDEANU IRINA DR } \\ \text { RUSU L DR } & \text { STANILA GEORGE DR } \\ \text { TODORAN IOAN DR } & \text { TORO TIBOR PROF }\end{array}$


M170

Country : SIMGAPORE

WAN FOOK SUN

WILSON S J

Country : SRI LANKA

DE SILVA L N K DR

MAHESWARAN MURUGESAPILLA]

Country : SYRIA

YAO ZHENG-QIU

CountTY : THAILAND
PORNCHAI P.-TANAKUN SONGSATHAPORN RUANGSAK DR

Country : VIETMAM

HAN NGEYEN DUSH DR

TUNG NGUYEN MAU DR 
4. Alphabetical iist of members

Note: In field "COM".

"EC" means Member of the Executive Committee or Adviser to the Executive Committee

"p" means President of Commission

" $V$ " means Vice-President of Commission

"C" means Member of the Scientific Committee of the related Commission 


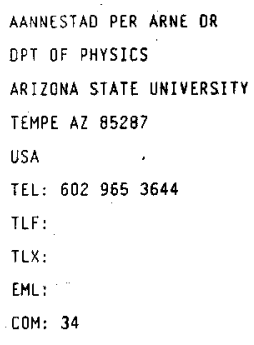

AARSETH SVERRE J DR:
INSTITUTE OF ASTRONOMY
THE OBSERVATORIES
MADINGLEY RD
CAMBRIOGE CB3 OHA
UR
TEL: 22362204
TLF:
TLX: 817297 ASTRON G
EML:

COM: 33,37

ABBOTT DAYIO'C OR

JILA

UNIVERSITY OF COLORADO BOX 440

BOULDER CO B0309 0440

USA

TEL:

TLF:

TLX:

EML:

COM: 36

ABDULLA SHAKER ABOUL AZIZ SARC

SCIENTIFIC RES COUNCIL

BOX 2441

JADIRIYAH BAGHDAD

IRAQ

TEL: 17765127

TLF:

TLX: 2187 BATHILMI IK

EML:

COM: 40

ABLES JOHM G OR
CSIRO
OIVISION OF RADIOPHYSICS
BOX 76
EPPING NSW 2121
AUSTRALIA
TEL:
TLF:
TLX:
EML:
COM: 40

\section{ABRAMI ALBERTO PROF}

OAT

BOX SUCC TRIESTE 5

VIA TIEPOLO $\mathrm{H}$

I 34131 TRIESTE

$$
\text { ITALY }
$$

TEL:

ILF:

TLX:

EML:

COM: 10,40
abAD ALBERTO J DR OPT FISICA TEORICA UNIVERSIDAD DE ZARAGOZA E 50009. ZARAGOZA. SPAIN

TEL: 76357011

TLF:

TLX: 58198

EML:

COM: 07

ABBOTT WILLIAM N OR UNIVERSITY OF ATHENS MICHALACOPOOULOU 42

\section{GR 11528 ATHENS}

GREECE

TEL: 17213352

TLF :

TLX:

EML:

COM: 22

ABELE MARIS $K$ OR ASTRONOMICAL OBSERVATORY LATVIAN STATE UNIVERSITY RAINIS BUL 19 226098 RIGA LATVIA

TEL:

TLF:

TLX:

$$
\text { EML: }
$$

COM: 31

\section{ABOUDARHAM JEAN OR} OBSERVATOIRE DE PARIS SECTION DE MEUDON F 92195 MEURON PPL COX FRANCE

TEL: $1 \quad 45 \quad 077784$

TLF: 145077469

TLX: 201571

EML: MESIOA: :ABOU

COM: 10,12

ABRAMOWICZ MAREX DR
SISSA
ST COSTIERA II
MIRAMARE
I 34014 TRIESTE
ITALY
TEL: 40224281
TLF:
TLX: 460392 ICTP
EML:
COM: 48

ABALAKIN VICTOR K $D R$ PULKOVO OBSERVATORY ACADEMY OF SCIENCES 10 KUTUZOV QUAY 196140 ST PETERSBURG RUSSIA

JEL: $298-2242$

TLF:

TLX: 12261 FENIKS

EML:

COM: $04 \mathrm{C}, 05,07,20$

ABDEL HADY, AHMED DR

EGYPT

TEL:

TLX:

TLF:

EML:

COM:

ABHYANKAR KRISHNA D PROF DPT OF ASTROMOMY UNIVERSITY OF OSMANIA HYDERABAD 500007 INDIA

TEL: $85 \quad 1672$

TLF:

TLX:

EML:

COM: $24,29,36,42$

ABOU-EL-ELLA MOHAMEO S DR HELWAN OBSERVATORY

HELWAN

EGYPT

IEL: 78 0645/2683

TLF:

TLX: 93070 HIAG UN

EML:

COM: 37

ABT HELMUT A DR KITT PEAK NTL OBS BOX 26732

950 N CHERRY AVE TUCSON AZ 857266732

USA

TEL: $602 \quad 325.9215$

TLF:

TLX: D666-484 AURA NOAO

EML:

COM: $05,26 \mathrm{P}, 29,30$ 


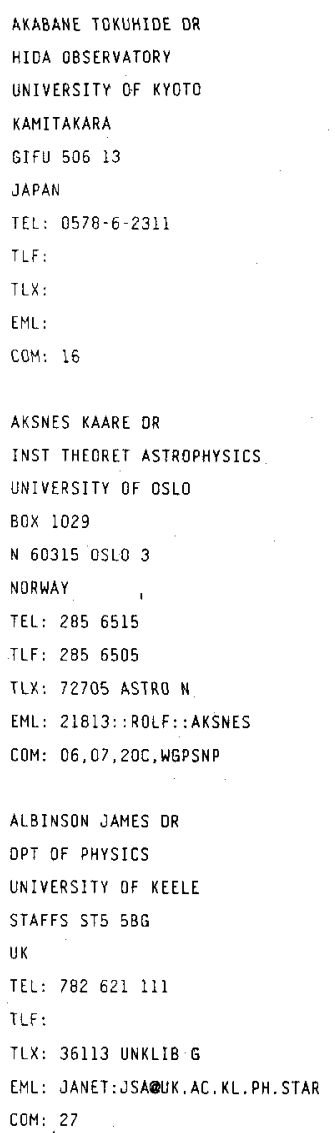

AKCAYLI MELEK MA OR

FACULTY OF SCIENCE

EGE UNIVERSITY

BOX 21

35100 BORNOVA IZMIR

TURKEY

TEL:

TLF :

TLX:

EML:

COM:

ARUJOR CHIDI E

OPT OF PHYSICS \& ASTRON

UNIVERSITY OF NIGERIA

NSUKKA ANAMBRA STATE

NIGER IA

TEL: $42.77 \quad 1532$

ILF :

TLX: 51496 U LION NG

EML:

COM: 40

ALBRECHT MIGUEL A DR

ESO

KARL-SCHWARZSCHILOSTR 2

D 8064 GARCHING MUENCHEN GERMANY

TEL: 8932006346

TLF: 8932006480

FLX: 5282820 EO O

EML: MALBRECHTOESO.ORG

COM: 05

ALDROVANDI RUBEN OR
INST OI FISICA TEORICA
RUA PAMPLONA 15
O1405 SAO PAULO SP
BRAZIL
TEL: $I 1288.5643$
TLF:
TLX:
EML:

ALEXANDER JOSEPH $K$
NASA HEADQUARTERS
CODE
OFFICE OF CHEIF SCIENTIST
WASHINGTON OC 20546
USA
TEL:
TLF:
TLX:
EML:
COM: 40.44

AKIM EFRAIM L OR

INST OF APPLIED MATHS

ACADEMY OF SCIENCES

MIUSSKAJA SQ 4

125047 MOSCOW

RUSSSIA

TEL: $251 \quad 37.39$

TLF :

FLX:

EML:

CBM: 07

AKYOL MUSTAFA UNAL PROF

FACULTY OF EDUCATION

SELCUK UNIVERSITY

42090 KONYA

TURKEY

TEL:

TEF:

TLX:

EML:

COM:

ALBRECHT RUDOLF OR

SPACE TELESCOPE EUROPEAN

COORDINATING FACILITY

KARL-SCHWARZSCHILD-STR 2

D 80.46 GARCHING MUENCHEN

GERMANY

TEL: 8932006287

TLF :

TLX: 52828222 EO 0

EML:

COM: 09,25

ALOROVANOI S M VIEgAS DR IAG

UNIVERSIDADE LE SAO PAULO

AV MIGUEL STEFANO 4200

04301 SAD PALLO SP

BRAZIL

TEL: 115778599

TLF:

ILX: 1136221 IAGM BR

EML: IAGUSP\%BRFAPESP, ANSP

COM: 34

\section{ALEXANDER MURRAY E DR}

DPT OF COMPUTER SCIENCE

UNIVERSITY OF OTAGO

$80 \times 56$

DUNEDIN

NEW' ZEALANO

TEL: $643479 \quad 8585$

TLF: 6434798577

TLX:

EML: MURRAY\%otago.AC.NZRELLAY.CS.NE EML: PA25OUK.AC.CAM. PHX

COM: 07
COM: 40

AKSENOV E P PROF DR

STERNBERG STATE ASTR INST

UNIVERSITETSKIJ PROSP 13

119899 MOSCOW

RUSSIA

TEL: $139-28-58$

TLF :

TLX:

EML :

COM: 07

ALBERS HENRY PROF

62 PROSPECT ST

FALMOUTH MA 02540

USA

TEL: $508540 \quad 0978$

TLF :

TLX:

EML:

COM: 45

ALCAINO GONZALO DR INSTITUTO ISAAC NEWTON

CASILLA 8-9

SAN\#1AGO 9

CHILE

TEL: 2472013

ILF:

TLX: C/O ESO 240853 ESOG0

EML:

COM: 28,37

ALECIAN GEORGES OR OBSERVATOIRE OE PARIS SECTION DE MEUOON DAF

F 92195 MEUDON PPL CDX FRANCE

TEL: 145347420

TLF :

T!X: 201571

EML:

COM: 29

\section{ALEXANDER PAUL OR} MULLARO RADIO ASTRON OBS CAVENDISH LABORATORY

MADINGLEY RD

CAMBaIDge CB3 GHE

UK

TEL: 26366477

TLF: 


\begin{tabular}{|c|}
\hline ALFARO EMILIO JAVIER \\
\hline INST ASTROFISICA \\
\hline DE ANDALUCIA APO 3004 \\
\hline C/SAMCHO PAHZA STN \\
\hline E 13080 GRANADA \\
\hline SPAIN \\
\hline TEL: $58 \quad 12 \quad 1311$ \\
\hline TLF: \\
\hline TLX: 78573 IAAG E \\
\hline EML: $16488:$ :EMILIO/EMIL \\
\hline $\cos : 27,37$ \\
\hline ALLAOIN SALEM MOHAMED \\
\hline DPT OF ASTRONOMY \\
\hline UNEVERSITY OF OSMANIA \\
\hline HYDERABAD $50000 \%$ \\
\hline INGIA \\
\hline TEL: 71116 \\
\hline TLF : \\
\hline$T L X:$ \\
\hline$E M L:$ \\
\hline COM: 28 \\
\hline ALLEGRE CLAUDE PROF \\
\hline INST PHYSIQUE DU GLOBE \\
\hline 4 PLACE JUSSIEU \\
\hline F 75005 PARIS \\
\hline FRANCE \\
\hline TEL: \\
\hline TLF: \\
\hline$T L X:$ \\
\hline EML: \\
\hline COM: 1 \\
\hline
\end{tabular}

\begin{tabular}{|c|c|}
\hline ALLEN RONALD J DR & ALLEN WILLIAM \\
\hline STSCI & ADAMS LANE OBSERVATORY \\
\hline HOMEWOOO CAMPUS & 46 ADAMS LANE \\
\hline $37 C 0$ SAN MARTIN DR & BLENHEIM \\
\hline BALTIMORE MO 21218 & NEW ZEALAND \\
\hline USA & TEL: $0578725 \mathrm{~g}$ \\
\hline TEL : $301338 \quad 4574$ & TLF: \\
\hline TLF: $301338 \quad 5090$ & $T L \dot{X}:$ \\
\hline$T L X:$ & EML: \\
\hline EML: VJALLENESTSCI.EDU & COM: \\
\hline COM: 28,40 & \\
\hline ALLER MARGO $F$ OR & ALLEY CARROL OOR \\
\hline DPT OF ASTRONOMY & ASTRONOMY PROGRAM \\
\hline UNIVERSITY OF MICHIGAN & UNIVERSITY OF MARYLANO \\
\hline DENMISON BLOG & COLLEGE PARK MO 20742 \\
\hline ANN ARBOR MI 481091090 & USA. \\
\hline USA & TEL: $302 \quad 4543405$ \\
\hline TEL: 3137643465 & TLF : \\
\hline TLF: & $T L X: 908787$ \\
\hline TLX: $810-223-6056$ & EML: \\
\hline EML: & COM: 31 \\
\hline
\end{tabular}

COM: 47,48

ALLAN OAVIO W MR

BUREAU DF STANDAROS

TIME \& FREQUENCY OIV

COOE 524

BOULDER CO 80302

USA

TEL: 3034975637

TLF:

J.XX: 9109405906

EML:

COM: $31 \mathrm{C}$

ALLEN ANTHONY JOHN OR

ASTRONOMY UNIT

MILE END RO

LONDON EI 4NS

UK

TEL: 19804811

TLF:

TLX: 893750 QMCUOL G

COM:

ALFVEN HANNES PROF
OPY OF PLASMA PHYSICS
ROYAL INST OF TECHNOLOGY
510044 STOCKHOLM 70
SWEOEN
TEL: B7 877000
TLF:
TLX: 10389 KTHB
EML:

QUEEN MARY/WESTFIELO COLL

EML: allenUUK. AC. QMC. MATHS

ALISSANORAKIS C PH D

DPT OF ASTROPHYSICS

NTL UNIVERSITY OF ATHENS

PANEPISTIMIOPOLIS

GR 15771 ATHENS

GREECE

TEL: 17235122

TLF:

TLX:

EML:

COM: 10,12

ALLAN PETER M:

DPT OF ASTRONOMY

UNIVERSITY OF MANCHESTER

MANCHESTER MI3 $\mathrm{gPL}$

UK

TEL: 612737121

TLF:

TLX:

EML:

COM: 47

\section{ALLEN CHRISTINE}

INSTITUTO DE ASTRONOMIA

UNAM

APDO POSTAL 70-264

04510 MEXICO OF

MEXICD

TEL:

TLF:

TLX:

EML:

COM: 25,37

ALLER HUGH O DR
OPT OF ASTRONOMY
UNIVERSITY OF MICHIGAN
OENNISON BLOG
ANN ARBOR MI 481091090
USA
TEL: 3137643466
ILF:
TLX:
EML:
COM: 40

ALLOIN DANIELLE DR observatoire de paris SECTYON DE MEUDON

DAEC

F 92195 MEUDON PPL. COX

FRANEE

TEL: 145077404

TLF: $\quad 2,45 \quad 07>7469$

TLX: 201571

EML: ALLOTN RMEUSI

COM: 28
ALKSNIS ANOREJS OR RADIOASTROPHYSICAL OBS LATVian aCad of sEIENCES TURGENEVA 19 226524 RIGA

LATVIÁ

TEL: 226796 RIGQ

TLF:

TLX:

EML:

COM: 37

ALLARO NICOLE DR OBSERVATOIRE DE PARIS SECTION DE MEUDON F 92125 MEUDON PPL CDX FRANCE

TEL: 145077449

TLF: $145 \quad 077469$

TLX: $20157 \mathrm{t}$

EML: 17670: :ALLARD

COM: 14

ALLEN DAVIO A OR

AAO

OBSERVATORY

BOX 296

EPPING NSW 2121

AUSTRALIA

TEL: 28681666

ILF:

TLX: 23999 OSYO AA

EML:

COM:

ALLER LAWRENCE HUGH OPT OF ASTRONOMY UNIVERSITY OF CALIFORNIA MATH SCIENCES BLDG LOS ANGELES CA 90024 USA

TEL: 2138253515

TLF:

TLX: $910-342-7597$

EML:

COM: $29,34,36$

ALMAR IVAN PROF KONKOLY OBSERVATORY THEGE U 13/L?

BOX 67

H 1525 BUOAPEST

HUNGARY

TEL: 175 5866/75 4122

TLF:

TLX: 227460

EML:

COM: 51 


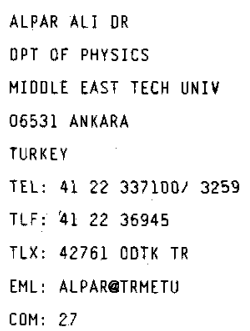

AMBARTSUMIAN V A PROF DR
BYURAKAN ASTROPHYSICAL
OBSERVATORY
378433 ARMENIA
ARMENIA
TEL: 8852284580
TLF:
TLX: 412623
EML:
COM: $28,33,51$

\author{
ALTAMORE ALDO \\ ISTITUTO ASTRONOMICO \\ UNIVERSITA OI ROMA \\ VIA G M LANCISI 29 \\ I OOLGI ROMA \\ ITALY \\ TEL: 68442977 \\ TLF: \\ TLX: 613255 INFRO \\ EML: \\ COM
}

ALTSCHULER MARTIN D PROF

OPT RAD THERAPY BOX 522

HOSP UNIV OF PENNSYLVANIA

3400 SPRUCE ST

PHILADELPHIA PA 19104

USA

TEL: 2156626472

TLF:

TLX:

EML:

COM: $\quad 10,12$

\section{ALVAREZ PEDRO OR}

INST OE ASTROFISICA

DE CANARIAS

OBS DEL TEIOE

E 38071 LA LAguna

SPAIN

TEL:

TLF:

TLX: 92640 IACE E

EML :

COM: 05,09

AL-NAIMY HAMIO M K OR

- SARC

SCIENTIFIC RES COUNCIL

BOX 2441

JADIRIYAH BAGHDAD

IRAD

TEL: $1776 \quad 5127$

TLF :

TLX: $2121 B 7$

EML:

COM: 42,5 :

AMBASTHA A K $K$ DR

UDAIPIJR SOLAR OBSERVATORY

11 VIOYA MARG

UQATPLIR $3: 3001$

INDIA

TEL : $25 \quad 626$

TLF :

TLX:

EML:

COM: 10,33

\section{altaVista CARLOS A OR \\ OBSERVATORIO ASTRONOMICO \\ PASEO DEL BOSQUE}

1900 LA PLATA (BS AS)

ARGENTINA

IEL: 21217308

TLF:

TLX: 31151 BULAP

EML:

COM: 07

ALURKAR S K OR

PHYSICAL RESEARCH LAB

NAVRANGPURA

AHMEDABAD 390009

INOIA

TEL: $27246 \quad 2129$

TLF: 272445292

TLX: 121-397 PRL IN .

EML:

COM:

ALVAREZ POMARES A 0 DR

INST GEOPHYS \& ASTRONOMY

CALLE 212 N 2906/29 Y 31

LISA

LA HABANA

CUBA

TEL: 218416

TLF :

TLX: 0511240

EML:

COM:

AL-SABTI ABDUL ADIM OR DPT OF PHYSICS

UNIVERSITY OF BAGHDAD

SCIENCE COLLEGE

JADIRIYAH BAGHDAD

IRAO

TEL: I 5552340

TLF :

TLX:

EML:

COM: 38.51

AMBROZ PAVEL DR

ASTRONOMICAL INSTITUTE CZECH ACADEMY DF SCIENCES ONDREJOV OBSERVATORY

CS 25165 ONDREJOY

CZECHOSLOVAKIA

TEL: 20485201

TLF: 20485314

TLX: 121579 ASTR C

EML:

COM: 10
ALTENHOFF WILHELM 3 DR MPI FUER RADIOASTRONOMIE

AUF DEM HUEGEL 69

D 5300 BONN 1

GERMANY

TEL: 228525293

TLF :

TLX: 886440

EML:

COM: $33,34,40$

ALVAREZ HECTOR BR

OPT DE ASTRONOMIA

UNIVERSIOAD OE CHILE

CASILLA 360

SANTIAGO

CHILE

TEL: 22294101

TLF :

TLX: 440001

EML:

COM:

ALY JEAN JACQUES OR

CEA CEN.

DAPNIA/SAP

BP 2

F 9Llgl GIF/YVETTE COX FRANCE

TEL; $1 \quad 6908 \quad 4030$

TLF: $1 \quad 6908 \quad 9266$

$T L X: 604860$

EML: JJALYSOLAR

COM: 10

AMARI TAHAR OR OBSERVATOIRE DE PARIS SECTION DE MEUDON SECTION O'ASTROPHYSIQUE F 92195 MEUDON PPL COX FRANCE

TEL: $14507 \quad 7760$ TLF: $145 \quad 07 \quad 7469$ TLX: 270912

EML: AMAR I FRMEU5] COM : 10

AMBRUSTER CAROL OR DPT OF ASTRONOMY VILLANOVA UNIVERSITY MENDEL HALL VILLANOVA PA 19085 USA

TEL:

TLF :

TLX

EML: A?BRUSTERQVUVAXCOM COM: 
ANANO $S P S D R$

APPLIEO RESEARCH CORP

8201 CORPORATE ORIVE

SUITE 920

LANDOVER MD 20785

USA

TEL: 3014598442

TLF:

TLX:

EML:

COM: 35

ANDERSEN 80 NYBORG OR NORWEGIAN SPACE CENTRE

BOX 85

SMESTAD

N. DO309 OSLO 3

NORWAY

TEL: 2523800

TLF : 472522397

ILX: 005678174 SPACEN

EML: $21813::$ BANDERSEN

COM: $10,12,44$

ANDERSON CHRISTTOPHER M DR

WASHEURN OBSERVATORY

UNIVERSITY OF WISCONSIN

$475 \mathrm{~N}$ CHARTER ST

MADISON WI 53706

USA

TEL: $608 \quad 262 \quad 0492$

TLF:

$T\llcorner X:$

EML:

COM:

ANDREANI PAOLA MICHELA DR

OPT DI ASTRONOMIA

UNIVERSITA DI PADOVA

VIC OELL OSSERVATORIO 5

I 35122 PADOVA

ITALY

TEL: $49 \quad 829 \quad 3442$

TLF: 498759840

ILX: 432071 ASTROS I

ENL: 39003: : ANOREANI SPAN

COM: 47

ANDREWS PETER J DR

ROYAL GREENWICH OBS

HERSTMONCEUX CASTLE

HAILSHAM BN27 IRP

UK

rEL: 323833171

$T \perp X: 87451$

EML:

TLF:

COM:
ANANTHAKR 1SHNAN $S$

TATA INST OF FUNDAMENTAL

RESEARCH

POONA UNIVERSITY CAMPUS -

PUNE 411007

INDIA .

TEL: 212 J3 6105

TLF: 212335760

TLX: 0145658 GMRT IN

EML: Uunet!shakti!gmrt!ananth

COM: 49

ANDERSEN JOHANNES

COPENHAGEN UNIVERSITY OBS

BRORFELDEVEJ 23

DK 4340 TOLLOSE

DENMARK

TEL: 53488195

TLF; $5348 \quad 8755$

TLX: 44155 DANAST OK

EML:

[OM: $30[, 33,42$

ANDERSON KINSEY A PROF

SPACE SLIENCES LABORATORY

UNIVERSITY OF CALIFORNIA

BERKELEY CA 94720

USA

TEL: $415 \quad 6421313$

TLF :

TLK: $910-3667945$ UC SPACE

EML:

COM: $10,21,49$

ANOREW BRYAN H DR

CHIEF PROGRAM SERVICES

NTL RESEARCH COUNCIL

100 SUSSEX DR

OTTAWA ON KLA ORE

CANADA

TEL: $613993 \quad 3731$

TLF: 6139526602

TLX: 0533145

EML:

COM: 34,40

\section{ANDRIENKO OMLTRY A OR}

ASTRONOMICAL OBSERVATORY

KIEV STATE UNIVERSITY

OBSERVATORNAYA UL 3

252022 KIEV

UKRAINE

TEL: 250775

TLF :

$T L X: 132201$

EML:

COM: 15
ANANTHARAMAIAH $K R$ RR

NRAO

VLA SITE

BOX 0

SOCORRO NM 878010387

USA

TEL: 5057724306

TLF :

TLX: 9109881710

EML:

COM: 34,40

ANDERSEN TORBEN BRENDER

LOCKHEEO PALO ALTO RES LB

OPTICAL DESIGN GR B254 E.

3251 HANOVER ST

PALD ALTO CA 94304

USA

TEL:

TLF:

TLX:

EML:

COM:

ANDERSIN KURT S

DPT OF ASTRONOMY

NEW MEXICO STATE UNIV

LAS CRUCES NM BBOOJ

USA

TEL: 5056461032

TLF:

TLX: 210-983-0549 NHSUCI

EML:

COM:

ANDREW KENNETH L PROF

1637 MAY - 1002

WICHITA 67213

USA

TEL: 3162438381

TLF: *

TLX:

EML:

COM: 14

ANDRIESSE CORNELIS D DR

POGGENBECSKSTRAAT 31

NL 6813 KD ARNHEM

NETHERLANDS

TEL:

TLF :

TLX:

EML:

COM: 34
ANDERNACH HEINZ DR

INST DE ASTROFISICA

VIA LACTEA S/N

E. 38200 LA LAGUNA

SPAIN

TEL: $22605200 / 37$

TLF: $22 \quad 605210$

TLX: 92640 IAC E

EML: HJAOIAC. ONET. NASA.GOV

COM: 05,40

\section{ANDERSON BRYAN OR}

NRAL

JODRELL BANK

MACCLESFIELO SKII 9OL

UK

TEL:

TLF :

TLX:

EML:

COM:

ANDO HIROYASU QR

TOKYO ASTRONOMICAL CBS

NAOJ

OSAWA MITAKA

TOKYO 181

JAPAN

TEL: 0422-32-5111

ILF:

ILX: 2622307 TAOMK J

EML:

COM: $12,27,51$

ANDREWS DAVIO A DR

ARMAGH OBSERVATORY

COLLEGE HILL

ARMAGH BT6L 9DG

UK

TEL:

TLF:

TLX:

EML:

COM:

ANDRILLAT HENRI L PROF

LAB ASTRONOMIE

USTL II

PLACE EUgene bataillon

F 34095 MONTPELLIER CQX 5

FRANCE

TEL: $67 \cdot 14 \quad 3415$

TLF: 67543079

TLX: 490944 USTMONT $F$

EML:

COM: $29,34,46,47$ 


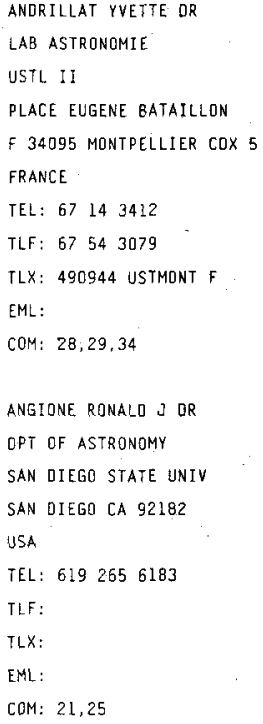

\author{
ANGEL J ROGER P PROF \\ STEWARO OBSERVATORY \\ UNIVERSITY OF ARIZONA \\ TUCSON AZ 85721 \\ USA \\ TEL: $602 \quad 6216541$ \\ TLF : \\ TLX: 467175 \\ EML:
}

COM: 25

ANGLATA GIJILLEM OR OPT ASTRON I METEOROLOG UNIVERSIDAD OE BARCELONA AVO OIAGONAL 647

E O8028 BARCELONA

SPAIN

TEL: $3402 \quad 1121$

TLF: 34110873

TLX:

EML: 16488::50179: : GUILLEM

COM: 34

ANSARI S M RAZAULLAH PROF

OPT OF PHYSICS

ALIGARH MUSLIM UNIVERSITY

ALIGARH UP 202002

INOIA

TEL: 57129001

TLF:

TLX: 564230 AMI-IN

EML:

CDM: $12,41 \vee P, 46$

ANTIOCHOS SPIRO KOSTA

NAVAL RESEARCH LABORATORY

COOE 4170 SA

4555 OVERLOOK AVE. SW

WASHINGTON' OC 203755000 USA

TEL: 2027676199

TLF :

TLX:

EML:

COM: 10

ANTONOPOULOU E OR
OPT OF ASTRONOMY
NTL UNIVERSITY OF ATHENS
PANEPISTIMIOPOLIS
GR 15771 ZOGRAFOS
GREECE
TEL:
TLF:
TLX:
EML:
COM: 42

\author{
ANGELETTI LULCIO OR \\ OAR \\ VIA DEL PARCO MELLINI B4 \\ I OOL3E ROMA \\ ITALY \\ TEL: 6347056 \\ ILF: \\ TLX: \\ EML: \\ COM:
}

$\begin{array}{ll}\text { ANGLITA CLAUOLO A OR } & \text { ANILE ANGELO M } \\ \text { DPT DE ASTRONOMIA } & \text { DPT MATEMATICA } \\ \text { UNIVERSIDAD OE CHILE } & \text { CITTA UNIVERSITARIA } \\ \text { CASILLA } 360 & \text { VIA A OORIA 6 } \\ \text { SANTIAGO } & \text { I } 95125 \text { CATANIA } \\ \text { CHILE } & \text { ITALY } \\ \text { TEL: } 22294101 & \text { TEL: } 95330533 \\ \text { TLF: } & \text { TLF: } \\ \text { TLX: } 440 \text { DOL } & \text { TLX: } \\ \text { EML: } & \text { EML: } \\ \text { COM: } 0 B & \text { COM: }\end{array}$

ANTALOVA ANNA

ASTRONOMICAL INSTITUTE

SLOVAK ACADEMY SCIENCES

CS 05960 TATRANSKA LOMNI

CZECHOSLOVAKIA

TEL: $959967866 / 7 / 8$

TLF: $96996 \quad 7656$

$T L X$ :

EML:

COM: 10

ANTIPOVA LYUDMILA DR INST OF ASTRONOMY

ACADEMY OF SCIENCES PYATNITSKAYA UL 49

109017. MOSCOW

RUSSIA

TEL: $23106 \quad 80$

TLF:

TLX: 411576 ASCON SU

EME:

COM: 27,42

ANTONOV VADIM A QR ASTRONOMICAL OBSERVATORY ST PETERSBURG UNIVERSITY BIBL.IOTECHNAJA PL 2 199178 ST PETERSBURG RUSSIA

TEL:

TLF:

TLX:

EML:

COM: 33
ANGELOV TRAJKO

INSTITUTE OF ASTRONOMY UNIVERSITY OF BELGRADE STUDENTSKI TRG 16 YU 11000 BEOGRAD YUGOSLAVIA

TEL: 11639715

TLF :

TLX:

EML:

COM: 35

COM

ANTHONY-TWAROG BARBARA J DPT PHYSICS \& ASTRONOMY UNIVERSITY OF KANSAS LAWRENCE KS 66045 USA

TEL: 9138644933

TL.F :

TLX:

EML:

COM: 25

ANTONACOPQULLOS GREg PROF DPT OF ASTRONOMY UNIVERSITY OF PATRAS GR 26110 RION GREECE

TEL: $6199 \quad 1145$

TLF:

TLX:

EML

COM: 07

ANTONUCCI ESTER DR

IST DI FISICA UNIVERSITA DI TORINO CORSO 0 AZEGLIO 46 I 10125 TORINO ITALY

TEL: 11657694

TLF :

TEX: 211041 INFNTO I

EML:

CON: $10 \mathrm{C}$ 


\begin{tabular}{|c|c|c|c|}
\hline ANZER ULRICH OR & AOKI SHINKO PROF & APARICI JUAN OR & APARICIO ANTONIO DR \\
\hline MPI FUER PHYSIK UND & TOKYO ASTRONGMICAL OBS & DPT DE ASTRONOMIA & INST DE ASTROFISICA \\
\hline ASTROPHYSIK & NABJ & UNIVERSIDAD DE CHILE & DE CANARIAS \\
\hline KARL-SCHWARZSCHILO-STR 1 & OSAWA MITAKA & CASILLA 360 & OBS DEL TEIDE \\
\hline D 8046 GARCHING MUENCHEN & TOKYO 181 & SANTIAGO & E 38Z00 LA LAGUNA \\
\hline GERMANY & JAPAN & CHILE & SPAIN \\
\hline TEL: 8932990 & TEL: 422325111 & TEL: 22294101 & TEL: $22 \quad 282211$ \\
\hline ILF: & TLF : & TLF : & TLF: 22263005 \\
\hline$T L X: 524629$ ASTRO D & TLX: 2822307 TAOMTK J & TLX: 440005 & TLX: 92640 IAC E \\
\hline EML: & EML: & EML: & EML: \\
\hline $\operatorname{CoM}: 10$ & COM: $04,07,31,33$ & COM: 09,40 & COM: 37 \\
\hline APPARAO K M $\vee D R$ & APPENZELLER IMMO PROF. & APPLEBY JOHN $F$ & APPLETON PHLLIP NDEL OR \\
\hline TIFR & LANDESSTERNWARTE & JPL/CALTECH & OPT. OF PHYSICS \\
\hline HOMI BHABHA RO & KOENIGSTUHL & MS $183 \quad 301$ & IOWA STATE UNIVERSITY \\
\hline COLABA & D 6900 HEIOELBERG I & 4800 OAK GROVE OR & AMES IA 50012 \\
\hline BOMBAY 400005 & GERMANY & PASADENA CA 91109 & USA \\
\hline INDIA & TEL: $62 \quad 215090$ & USA & TEL: $515 \quad 294 \quad 3667$ \\
\hline TEL: $22 \quad 219 \quad 11 \mathrm{~L} * 341$ & TLF: $62 \quad 21509202$ & TEL: 8183543943 & TLF : \\
\hline TLF: & TLX: 461153 LSWHO 0 & TLF: & TLX: \\
\hline TLX: 011-3009 TIFR IN & EML: CTOEHDURZI & $T L x:$ & EML: BITNET:S1, PNAISUMVS \\
\hline EML: & COM: 29,35 & EML: & COM: \\
\hline COM: 48 & & COM: 15 & \\
\hline AQUILANO ROEERTO OSCAR DR & ARABELOS DIMITRIOS OR & ARAFUNE JIRO OR & ARAI KENZO OR \\
\hline INSTITUTO DE FISICA & DPT GEODESY \& SURVEYING & INST COSMIC RAY RESEARCH & DPT OF PHYSICS \\
\hline ROSARIO CONICET UNR & UNIVERSITY THESSALONIKI & UNIVERSITY OF TOKYO & KUMAMOTO UNIVERSITY \\
\hline BV 27 OE FEBRERO 210 BIS & UNIV BOX 503 & MIDORICHO TANASHI & 2-39-I KUROKAMI \\
\hline 2000 ROSARIO & GR 54006 THESSALONIKI & TOKYO 188 & КUМАMOTO 860 \\
\hline ARGENTINA & GREECE & JAFAN & JAPAN \\
\hline TEL: $41821769 / 72$ & TEL: 31992693 & TEL: 04224614131 & TEL: $096-344-2111$ \\
\hline TLF: $41 \quad 257164$ & TLF: & TLF: $0424 \quad 68 \quad 1438$ & TLF: \\
\hline TLX: 41817 CIROS AR & TLX: 412181 AUTH GR & TLX: 2822371 ICRTUJ & TLX: \\
\hline EML: USUARIOSKIFIR, EQU. ARQUUNET, UU & EML: & EML: & EML: \\
\hline COM: 42 & COM: 19 & COM: 48 & COM: 35 \\
\hline ARAIJUO FRANCISCO $\times$ DE OR & ARDAVAN HOUSHANG OR & ARDEBERG ARNE L PROF & ARDEBILI M REZA OR \\
\hline OBSERVATORIO NACIONAL & INSTIIUTE OF ASTRONOAY & LUNO OBSERVATORY & BOX 47415341 \\
\hline RUA GL BRUCE 586 & THE OBSERVATORIES & $B 0 \times 43$ & \\
\hline SAO CRISTOVAD & MADINGLEY RD & S 22100 LUND & BABOLSAR \\
\hline 20921 RIO DE JANEIRO RJ & CAMBRIOGE CB3 OHA & SWEOEN & IRAN \\
\hline BAAZIL & uk & TEL: $46 \quad 10 \quad 7290$ & TEL: \\
\hline TEL: $21 \cdot 5800235$ & TEL: 22362204 & TLF: & TLF: \\
\hline ILF: $21 \quad 580 \quad 0332$ & TLF: & $T L X: 33199$ OBSNOT $S$ & TLX: \\
\hline$T L X: 21288$ & TLX: 817297 ASTRON G & EML: & EML: \\
\hline EML : USERFXAOLNC. BITNET & EML: & COM: $28,33,45,50 \mathrm{C}$ & COM: \\
\hline COM: 29 & COM: & & \\
\hline ARDUINI-MALINOVSKY M DR & ARELLANO FERRO ARMANOO & ARGUE A NOELL MR & ARGYLE ROBERT WILLIAM MR \\
\hline CAES & INSTITUTO DE ASTRONOMIA & INSTITUTE OF A'STRONOMY & ROYAL GREENWICH OBS \\
\hline 2 PLACE MAURICE QUENTIN. & UNAM & THE OBSERVATORIES & MADINGLEY RD. \\
\hline F 75039 PARIS COX OL & APDO POSTAL $70-264$ & MADINGLEY RD & CAMBRIDGE CB3 OEZ \\
\hline FRANCE & 04510 MEXICO OF & CAMBRIDGE CB DHA & UK \\
\hline TEL: & MEXICO & uk & TEL: 22337.4783 \\
\hline TLF: & TEL: $905-548-5305$ & TEL: 22362204 & ILF: 223374700 \\
\hline$I L X:$ & $T L F:$ & TLF: & IEX: 817235 RGOSTR $G$ \\
\hline EMLL: & TLX: 01760155 CICME & TLX: 817297 ASTRON. G & EML: RWAUK.AC. R60.SRF \\
\hline COM: 14 & EML: & EML: & COM: 08 \\
\hline & COM: 27,45 & COM: $24[, 25,26$ & \\
\hline
\end{tabular}


ARGYRAKOS JEAN PROF DR 193 PATISSON ST -GR 11253 ATHENS GREECE

TEL: 18677000

TLF :

EML:

COM: 08,41

TLX:

ARKHIPOVA $\checkmark P$ OR
STERNBERG STATE ASTR INST
UNIVERSITETSKIJ PROSP 13 .
119899 MOSCOW
RUSSIA
TEL: $139-26-57$
TLX:
EML:
TLF:
COM: $27,28.34$

ARNAL MARCELO EOMUNDO OR MPI FUER RADIOASTRONOMIE AUF DEM HUGEL GG D 5300 BONN 1 GERMANY

TEL:

TLX:

EML:

ILF:

COM: 40

ARNOLD JAMES $R$ OR

DPT OF CHEMSTRY

UCSO

B 017

LA JOLLA CA 920930216

USA

TEL: 6195342908

TLF:

TLX: 9103371271

EML: Bitnet: jarnoideucsd

COM: 15

ARONS JONATHAN
ASTRONOMY DPT
UNIVERSITY OF CALIFORNIA
601 CAMPBELL HALL
BERKELEY CA 94720
USA
TEL: 4156424730
TLF:
TLX: 820181 UCB AST RAL
EML:
COM: 48

ARIAS OE GREIFF J PROF OBSERVATORIO NACIONAL

APDO 2584

BOGOTA 1 DE

COLOMBIA

TEL:

TLF :

TLX:

EML:

COM: 04,50

ARLOT JEAN-EUDES

BUREAU DES LONGITUDES

77 AVE DENFERT ROCHEREAU

F 75014 PARIS

FRANCE

TEL: 140512267

TLF:

TLX:

EML:

COM: $04,20 \mathrm{C}$

ARNAUL JEAN PALLL

OBS MIOI PYRENEES

14 AVE E BELIN

F 31400 TOULOUSE CDK

FRANCE

TEL: $6133 \quad 2929$

ILF: 615536722

TLX: $530776 \mathrm{~F}$

EML: :

COM: $09,12,2 b$

ARNOULO MARCEL L DR
IAAG
VRIJE UNIV BRUSSELS
CP 165
B 1050 BRUSSELS
BELGIUM
TEL: 2649 0030
ILF:
TLX: 23069 UNILIB
EML:
COM: 35,48

ARP HALTON DR

MAX PLANCK INSTITUT FUER

PHYSIK UND ASTROPHYSIK

KARLSEHWARZSCHILOSTR I

D 8046 GARCHING MUENCHEN GERMANY

TEL:

TLF:

TLX:

EML

COM: 28
ARIAS. ELISA FELICITAS

UNIV NACIONAL OE LA PLATA

FCAG .

1900 LA PLATA (BS AS)

ARGENTINA

TEL: 21217308

TLF:

TLX:

EML:

COM: 19

ARMANCROFF TAFT E OR

KITT PEAK NTL OBS

BOX 26732

$950 \mathrm{~N}$ CHERRY AVE

TUCSON AZ $85726 \quad 6732$

USA

TEL: $602 \quad 3259382$

TLF: 6023259360

ILX: 0666484

EML: ARMANDONOAO.EDU

COM: 37

ARNAUD MONIQUE

CEA CEN

DAPNIA/SAP

$\mathrm{BP} 2$

F 91191 GIF/YVETTE CDX

FRANCE

TEL: 169087017

TLF:

TLX: 604860

EML: SPAN: 32779 : : ARNAUD

COM: 48

ARNQUIST WARREN N DR

8127 DELGANY AVE

PLAYA DEL REY CA 90291

USA

TEL: $213 \quad 821 \quad 2724$

TLF:

TLX:

EML:

COM:

ARPIGNY CLAUDE PROF

IHSTITUT D'ASTROPHYSIQUE

UNIVERSITE OE LIEGE

AVE COINTE 5

B 4000 COINTE-LIEGE

BELGIUM

TEL: $41529980^{*} 263$

TLF: 41527474

TLX:

EML:

COM: 15,36
ARIMOTO NOBUD OR

OBSERVATOTRE DE PARIS

SECTION OE MEUDON

LAM

F 92195 MEUDON PPL CDX

FRANCE

TEL: 145347570

TLF:

ILX: 207571

EML:

COM: 35

ARMSTRONG JOHN THOMAS DR

US NAVAL OBSERVATORY

ASTROMETRY DIV AD 5

34 \& MASSACHUSETTS AVE NW

WASHINGTON OC 203925100

USA

TEL: $202 \quad 653 \quad 1769$

TLF:

TLX:

EML: ' ATLAS! ARMSTREINTERF. COM

COM: 26

ARNETT W DAVIO PROF

ENRICO FERMI INSTITUTE

UNIVERSITY OF CHICAGO

933 E 56TH ST

CHICAGO IL 6063 ?

USA

TEL: 3129628208

ILF:

TLX: 9102215617

EML:

COM: 35 .

ARNY THOMAS T OR DPT PHYSICS \& ASTRONOMY UNIV OF MASSACHUSETTS

GRC B

AMHERST MA 01003

USA

TEL: 4135452194

TLF:

TLX:

EML:

COM: 34

ARRIBAS SANTIAGO DR INST DE ASTROFISICA DE CANARIAS

OBS DEL TEIOE

E 38200 LA LAGUNA.

SPAIN

TEL: $22 \quad 262211$

TLF :

TLX: 92640

EML: SPAN: IAC: : SAM

COM: 
ARSENIJEVIC JELISAVETA

ASTRONOMICAL OBSERVATORY VOLGINA?

YU 11050 BEOGRAD

YUGOSLAVIA

TEL:

ILF:

ILX:

EML:

COM: $25,27,50$

ASCHENEACH BERNO PH D

MPI F. PHYSIK \& ASTROPHYS

INST F. EXFRATERR PHYSIK

KARL-SCHWARZSEHILD-STR 1

D. 8046 GARCHING MUENCHEN

GERMANY

TEL:

TLF :

TLX:

EML:

COM: 44.48

\section{ASLANOV I A DR}

SHEMAKHA ASTROPHYSICAL

OBSERVATORY

AZER ACADEMY OF SCIENCES

373243 SHEMAKHA

AZERBAIDZHAN

TEL:

TLF :

$T L X:$

EML:

CON:

ASSUS PIERRE DR

OCA OBSERV DE NICE

BP 139

F 06003 NICE COX

FRANCE

TEL: $92 \quad 00 \quad 3086$

TLF: 92.003033

$T ! X$ :

EML:

COM: 09

ATHANASSOULA EVANGELIE OR OBSERVATOIRE DE MARSEILLE

2 PLACE LE VERRIER

F 13248 MARSEILLE CDX 04 FRANCE

TEL: 91959088

TLF :

TLX: $420241 \mathrm{~F}$

EML:

COM: 28,33
ARTHUR DAVID W $G$

US GEQLOGICAL SURVEY

BRANCH OF ASTROGEOLOGY

2255 N GEMINI OR

FLAGSTAFF AZ 86001

USA

TEL:

TLF:

TLX:

EML:

COM: 16

ASCHWANDEN MARKUS QR ASTRONOMY PROGRAM

UNIVERSITY OF MARYLANO

COLLEgE PARK MO 20742

USA

TEL: $301405 \quad 1525$

TLF: 3013149067

TLX:

EML: MARKUSOASTRO. UMO.EOU

COM: 10,40

ASPIN COLIN OR

JOINT ASTRONOMY CENTER

665 KOMOHANA ST

HILO HI 96720

USA

TEL: BOB 9613756

ILX:

EML: CAAOJACH. HAWAII.EOU

TLF: 8089616516

COM: 25

ASTERIAOIS GEORGIOS DR

OPT GEODESY \& SURVEYING

UNIVERSITY THESSALONIKI

UNIV BOX 503

GR 540 OE THESSALONIKI

GREECE

TEL: 31992693

TLF:

TLX: 412181 AUTH GR

EML:

COM: 27,33

ATHAY R GRANT OR

HIGH ALTITUDE OBSERVATORY

NCAR

BOX 3000

BOULOER CO 803073000

USA

TEL: 3034971556

TLF:

TLX:

EML:

COM: $10,12,36$
ARTRU MARIE-CHRISTINE DR OBSERVATOIRE DE LYON

AVE CHARLES ANORE

F 69561 5 GENIS LAVAL CDX

FRANCE

TEL: $7856 \quad 0705$

TLF: 72399791

TLX: 310926

EML:

COM: 14,29

ASHOK N M QR

PHYSICAL RESEARCH LAB

NAVRANGPURA

AHMEDABAD 380009

INDIA

TEL: $272 \quad 46 \quad 2129$

TLF : 272445292

TLX: 121397

EML:

COM: 09,25

ASSEO ESTELLE DR

CENTRE PHYSIQUE THEORIQUE

ECOLE POLYTECHNIQUE

F 9112B PALAISEAU CDX

- FRANCE

TEL: 169418200

TLF:

TLX: 691596 .

EML:

COM: 4 日

ATAC TAMER

KANDILLI DBSERVATORY

BOGAZICI UNIVERSITY

CENGELKOY

B.1220 ISTANBUL

TURKEY

TEL: 13320240

TLF:

$T E X: 26411$ BOLN TR

EML:

COM: 10,29

ATHERTON FAUL DAVID

ASTROPHYSICS GROUP

IMPERIAL COLLEGE

BLACKETT LABORATORY

LONOON SW7

UK

TEL:

FLF:

ILX:

EML:

COM: 09
ARTZNER GUY

IAS

BP 10

$F$ G1371 VERRIERES BUISSON

FRANCE

TEL: $164 \quad 474309$

TLF:

TLX: 600252

EML:

COM:

ASLAN ZEKI OR

FACULTY OF SCIENCE

INONU UNIVERSIIY

44069 MALATYA

IURKEY.

TEL:

ILF:

TLX:

EML:

COM:

ASSOUSA GEORGE ELIAS DR 545 BOYLSTON ST

SUITE 901

BOSTON MA 02116

USA

TEL:

TLF:

$T L X$ :

EML:

COM: 40

ATANASIJEVIC IVAN OR FACULTY OF SCIENCES

NL 6500 GL. NIJMEGEN

NETHERLANDS

TEL:

TLF:

TLX:

EML:

COM:

ATREYA SUSHIL $K$

DPT ATM \& OCEANIC SCIENCE

UNIVERSITY OF MICHIGAM

SPACE RESEARCH BLOG

ANN ARBOR MI 481092143

USA

TEL: 3137643335 .

TLF :

TLX: 8102236056

EML:

COM: 15 
AUBIER MONIQUE G DR observatoire de paris SECTION DE MEUDON

F 92195 MEUDON PFL COX. FRANCE

TEL: 145347755

TLF :

TLX: 270912

EML:

COM:

AUGASON GEREQN.C'DR NASA AMES RESEARCH CTR MS 2456 MOFFETT FIELO CA 94035 USA

TEL: 4156944156

TLF:

ILX:

EML:

COM:

AURASS HENRY OR

AKADEMIE DER WISSENSCHA. OER GOR ZENTRALINSTITUT FUR ASTROPHYSIK

D 1501 TREMSOORF

GERMANY

TEL: 2261

TLF:

TLX: 15420

EML:

COM: 10,40

AVCIOGLU KAMURAN PROF DR UNIVERSITY OBSERVATORY UNIVERSITY OF ISTANBUL 3445Z ISTANBUL

TURKEY

TEL: 15223597

TLF:

TLX:

EML:

COM:

AVRETT EUGENE H DR

CENTER FOR ASTROPHYSICS

HCO/SAO .

60 Garden St

CAMBRIDGE MA 02138

USA

TEL: $617495 \cdot 7423$

TLF:

TLX: 921428 SATELLITE CAM

EML:

COM: 36
aUdDUZE JEAN PROF

INSTITUT OAASTROPHYSIQUE

S8BIS BO ARAGO

F 75014 PARIS.

FRANCE

TEL: 143201425

TLF :

TLX:

EML:

COM: $35,47,48$

AULUCK FAQIR CHAND PROF OPT PHYSICS \& ASTROPHYS UNIVERSIIY OF OELHI NEW DELHI 110007

INIIA

TEL: 112918993

TLF:

TLX:

EML: .

COM: 47

\section{AURIEMMA GIULIO OR}

DPT. DI FISICA

UNIVERSITA DI ROMA

PA MORD 2

I 00187 ROMA

ITALY

TEL: 64976336

TLF:

ILX: 613255 INFNRO

EML:

COM:

AVERY LORNE W OR

HERZBERG INST ASTROPHYS

NTL RESEARCH COUNCIL

100 SUSSEX DR

OTTAWA ON KIA ORG

CANADA

TEL: 6139936060

ILF: 6139526602

ILX: 053.3715

EML:

COM: 34,40

AWAD MERVAT EL-SAID DR

DPT OF ASTRONOMY

FACULTY OF SCIENCES

CAIRO UNIVERSITY

GEZA

EGYPT

IEL:

TLF:

TLX:

EML:

COM:
AUER LAWRENCE H OR

los alamos national lab

MS F665

ESS 5

LOS ALAMOS NM 87545

USA

TEL: $505 \quad 6675824$

TLF:

TLX:

EML:

COM: 36

AUMAN JASON R PROF

OPT GEOPHYS \& ASTRONOMY

UNIV OF BRITISH COLUMBIA

2075 WESBROOK PL

VANCOUVER BC VGT IWS

CANADA

TEL: 6042282892

TLF: 6042286047

TLX:

EML:

COM: 36

AURI ERE MICHEL

OBS MIDI PYRENEES

14 AVE E BELIN

F 31400 TOULOUSE COX

FRANCE

TEL: 62951969

TLF:

$T(X:$

EML:

COM: 37

AVGOLOUPIS STAVROS DR

DPT OF ASTROPHYSICS

UNIVERSITY THESSALONIKI

GR 54006 THESSALONIKI

GREECE

TEL: $3 L 99$ L357.

TLF:

TLX: 041218। AUTH GR

EML:

COM: 27

AWADALLA NABIL SHOJKRY DR HELWAN OBSERVATORY

HELWAM

EGYPT

TEL: $78 \quad 06,45 / 2683$

TLF:

ILX: 93070 HIAG UN

EML:

COM: 42
AUGAROE RENEE DR

OBSERVATOIRE DE MARSEJLLE

2 PLACE LE VERRIER

F 13248 MARSEILLE COX 04

FRANCE

TEL: $919590 B B$

TLF:

TLX: 420241

EML:

COM:

AUNER GERHARD DR

INSTITUT FUER ASTRONOMIE

TUERKENSCHANZSTK 17

A 1180 WIEN

AUSTRIA

TEL:

TLF :

TLX:

EML:

COM:

AUVERGNE MICHEL

OCA OBSERV DE NICE

BP 139

F 06003 NIEE COX

FRANCE

TEL: 93890420

TLF :

TLX: 460004 OBSNICE $F$

EML:

COM:

\section{AVIGNON YVETTE DR}

OBSERVATOIRE DE PARIS

SECTION DE MEUDON

F $92 L 95$ MEUDON PPL CDX FRANCE

TEL: 145347771

TLF:

TLX: 200590

EML:

EOM: 10,40

AXFORD W IAN PROF

MPI FUER AERONOMIE

POSTFACH 20

O 3411 KATLENBURG LINOAU

GERMANY

TEL: $555 \quad 64 \quad 1414$

TLF :

TLX: 965527

EML :

COM: $\quad 15,34,48$ 


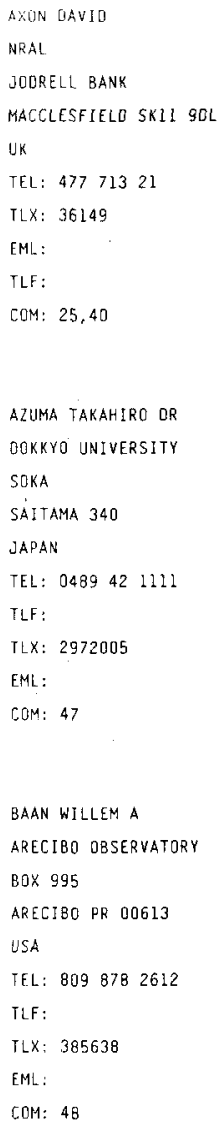

BABA NAOSHI DR OPT OF PHYSICS HOKKA]DO UNIVERSITY KITA 13 NISHI 8 SAPPORO 060 JAPAN

TEL: 0117162111
TLF: 0117264336
TLX: 932302 HOKUEN-J
EML: Al0156 JPNAC.BITNET
COM: 09

BABIN ARTHUR OR

UKRAINE
TEL:
TLF:
EML:
TLX:

COM: 10
AYDIN CEMAL PROF DR

DPT OF ASTRONOMY

UNIVERSITY OF ANKARA

FEN FAKULTESI

O6100 BESEVLER

TURKEY

TEL: $41232105 * 94$

TLF:

TLX:

EML:

COM:

AZZOPAROI MARC OR OBSERVATOIRE OE MARSEILLE

2 PLACE LE VERRIER

F 13248 MARSEILLE CDX 04

FRANCE.

TEL: 91959088

TLF :

TLX: $420241 \mathrm{~F}$

EML:

COM: 28,30

BAARS JACOB W M DR

STEWARD OBSERVATORY

UNIVERSITY OF ARIZONA

TULSON AZ 85721

USA

TEL: 6026211515

TLF: 6026211532

TLX: 467175

EML: BAARSOTUCSMT. AS. ARIZONA. EOU

COM: 34,40

BABADZHANIANC MICHAIL OR

ASTRONOMICAL OBSERVATORY

ST PETERSBURG UNIVERSITY

194904 ST PETERSBURG

RUSSIA

TEL:

TLF:

TLX:

EML:

COM:

BABIN V G OR

SIBIZMIR

ACADEMY OF SCIENCES

664697 IRKUTSK 33

RUSSIA

TEL: $602 \quad 65$

TLF :

TLX:

EML:

COM: 10
AYRES THOMAS $R$

CASA

UNIVERSITY OF COLORADO

BOX 391

BOULOER CO 803090391

USA

TEL: $303 \quad 4925320$

TLF:

TLX: 755842 JILA

EML:

COM: $12 \mathrm{C}, 44$

A'HEARN MICHAEL F OR

ASTRONOMY PROGRAM

UNIVERSITY OF MARYLAND

COLLEGE PARK MD 20742

USA

TEL: 3014056076

TLF: 3013149067

TLX: $710 \quad 826 \quad 0352$

EML: MALASTRO.UMU. EDU

COM: 05,15 VP, 20

BAART EDWARD E PRDF

DPT OF PHYSICS

RHODES UNIVERSITY

BOX 94

GRAHAMSTOWN 6140

SOUTH AFRICA

TEL: 046L-7L28

TLF:

FLX: 244226

EML:

COM: $34.40 C$.

BABADZHANOV PULAT B DR

ASTROPHYSICAL INSTITUTE

IADJIK ACAD OF SCIENCES

734670 DUSHANBE

TADZHIKISTAN

TEL:

TLF:

TLX:

EML:

COM: $15,20,22 \mathrm{C}$

BABU G S D

INDIAN INSTITUTE OF

ASTROPHYSICS

KORAMANGALA

BANGALORE 560034

INDIA

TEL: $812 \quad 569179$

TLF:

ILX: 8452763 IIAB IN

EML:

COM: 45
AZCARATE ISMAEL N OR

IAR

CC 5

1894 VILLA ELISA (BS AS)

ARGENTINA

TEL: $21 \quad 43793$

TLF :

TLX: 28052 CICYT AR

EML: AZCARATEUUMA. EDU. AR

COM: 34

BAADE OIETTRICH DR

ST /ECF

ESO

KARL - SCHWAR ZSCHILD-STR 2

D B046 GARCHING MUENCHEN

GERMANY

TEL: 8932006388

TLF:

TLX: 05282820 EO D

EML: DBAADEOOGAESO51, BITNET

COM: $27,29 C, 36$

BAATH LARS 8 OR

BOX 8481

S 439 OO ONSALA

SWEDEN

TEL:

TLF :

TLX: 2400 ONSPACE S

EML:

COM: $40 C$

BABCOCK HORACE W DR

MT WILSON \& LAS CAMPANAS

OBSERVATORIES

813 SANTA BARBARA ST

PASADENA CA 91101

USA

TEL: $818577 \quad 1122$

FLF:

TLX:

EML:

COM: 09

BAEALOV MIHAIL

PEOPLE'S ASTRONOMICAL OBS

\& PLANETARIA

$J$ BRUNO

B6. DIMITROVGRAD

BULGARIA

TEL: 3913797

TLF :

TLX:

EML:

COM: 46 
A14

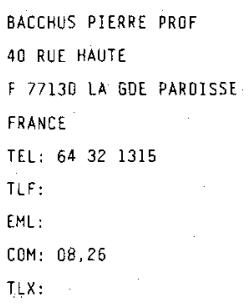

BACHILLER RAFAEL DR CTR ASTRON DE YEBES DAN

APD 148

E 19080 gUAOALAJARA

SPAIN

TEL: 11290311

TLF:

TLX: 23465 IGC E EML:

COM: 34,40 STERREXUNDIG OBSERV RIJKSUNIVERSITEIT GENT KRIJGSLAAN 281

B 9000 GENT

BELGIJM

TEL: 91644757

TLF: $9164 \quad 4995$

TEX:

EML: BAECKAASTRO, RUG AC.BE COM

BAFFA CARLO OR
OSS ASTROFISICO
OI ARCETRI
LARGO E FERMI 5
I 50125 FIRENZE
ITALY
TEL: 554378540
TLF: 55435939
TLX: 572268 ARCETR I
EML: baffodastrfi.cineca.it
COM: 09

BAGILOINSKIJ BRONISLAV K PULKOVO OBSERVATORY ACADEMY OF SCIENCES 10. KUTUZOV QUAY 196140 ST PETERSBURG RUSSIA

TEL:

TLF :

TLX:

EML:

COM: 08

BAHCALL JOHN N PROF INST FOR ADVANCEO STUDY SCHOOL OF NATURAL SCIENCE OLDEN LN BLDG E PRINCETON NJ 08540 USA

TEL: 6097348054 TLF :

TLX: 837680

EML:

COM: 28,33
BACKER DONALD CH DR RADIO ASTRONOMY LAB UNIVERSITY OF CALIFORNIA 601 CAMPBELL HALL BERKELEY CA 94720 USA TEE: $415 \quad 642 \quad 5128$ TLF :

TLX: 820181 UCB AST RAL EML:

COM: $08.40 \mathrm{C}$

BAEK CHANG RYONG

DPT DF PHYSICS

KIM IL SUNG UNIVERSITY TAESONG OISTRICT

PYONGYANG

KOREA OPR

TEL:

TLF :

TLX:

EML:

COM:

BAGARE S P' OR

INDIAN INSTITUTE OF ASTROPHYSICS

KORAMANGALA

BANGALORE 560 034

INDIA

TEL: $81256 \quad 6585 / 497$

TLF :

TLX: 845763 IIAB IN

EML:

COM: 10

BAGLIN ANNIE OR OBSERVATOIRE OE PARIS SECTION DE MEUDON

DASGAL

F 92195 MEUDON PPL CDX FRANCE

TEL: $147 \quad 057855$

TLF:

TLX:

EML:

COM: $27,35,45$

BAHNER KLALSS OR MPI FUER ASTRONOMIE ADOLF-ROLPING-STR 5 D 6903 NECKARGEMUEND GERMANY

TEL: $62 \quad 233735$

TLF :

TLX:

EML:

COM:
BAOIALI MASSIMO

IAS

CNR

VIA ENRICO FERMI 23

100044 FRASCATI

ITALY

TEL: 69425655

TLF: 69416847

TLX: 610261 CNR FRA

EML: MASSIMOIRMIAS. BITNET

COM: 44

BAERENTZEN JORN

ELMEHOJVEJ 66

DK 8270 HOJBJERG

DENMARK

TEL: $86 \quad 27 \quad 2428$

TLF :

TLX:

EML:

COM:

BAGGALEY WILLIAM J PROF DPT OF PHYSICS. UNIVERSITY OF CANTERBURY PRIVATE BAG

CHR ISTCHURCH I

NEW ZEALAND

TEL: $482 \quad 009 * 767$

TLF :

TLX: 4144 UNICANT NZ

EML:

COM: '21, 22

BAGNUOLO WILLJAM G JR OR CHARA

GEORGIA STATE UNIVERSITY ATLANTA GA 303033083 USA

TEL: 4046512932

TLF :

TLX:

EML:

COM: 26

BAHNG JOHN O R PROF DPT PHYSICS \& ASTRONOMY NORTHWESTERN UNIVERSIIY DEARBORN DBSERVATORY EVANSTON IL 60208 USA

TELL: 3124918645

TLF :

TLX:

EML:

COM: 25,45 


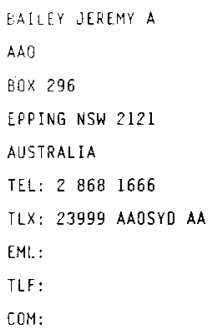

BAILEY MARK EDWARD
DPT OF ASTRONOHY
UNIVERSIYY OF MANCHESIER
MANCHESTER MI3 GPL
UK
TEL: 612737121
TLF: 612735867
TLX: 66517 UNIMAN
EML: MEBOUK, AC.MAN, AST, STAR
COM: $15,20,28$

BAKER JAMES GILBERT
14 FRENCH OR
BEBFORO NH 03102
USA
TEL: 6034725860
TLF:
EML:
COM:
TLX:

BALAZS BELA A PROF DR
OIREKTOR
COLLEGILM HUNGARICUM
HOLLANDSTR 4
A 1020 WIEN 2
AUSTRIA
TEL: 1240.581
TLF: 16296575
TLK:
EML:
COM: 37.51

BALDWIN JACK A DR

CERRO TOLOLO

INTERAMERICAN OBSERVATORY

CASILLA 603

- LA SERENA

CHILE

TEL: 51213352

TLF: $51212466 * 342$

TLX: 359620301 AURA CT

EML:

COM: 28

BALICK BRUCE PROF

OPT OF ASTRONOMY

UNIVERSITY OF WASHINGTON

FM 20

SEATTLE WA 98195

USA

TEL: 2065437683

ILF :

TLX: 4740096 UW UI

EML:

COM:
BAIRO SCOTT R

OPT PHYSICS \& ASTRONOMY

BAIZE PALL OR

BENELICTINE COLLEGE

N 14

ATCHISON KS 660021499

USA

TEL: 9133675340

TLF:

TLX:

EML:

COM: 36

BAKER NORMAN H PROF

DPT OF ASTRONOMY

COLUMBIA UNIVERSITY

PUPIN HALL 538 W L20TH ST EGYPT

NEW YORK NY 10027 TEL:

USA. TLX:

TEL: 2122803280 TLF:

TLF :

TLX: 220094 COLU UR COM:

EML:

COM: $05,27,35$

BALAZS LAJOS G OR BALBUS STEVEN A OR

KONKOLY OBSERVATORY

THEGE U $13 / 17$

BOX 67

H 1525 BUCAPEST

HUNGARY

IEL: 175 5866/75 4122

TLF :

TLX: 227460 KONOB H

EML:

COM: 33

BALOWIN JDHN E QR

MULLARI RADIO ASTRON OBS

CAVENDISH LABORATORY

MADINGLEY RD.

CAMBrioge CB3 OHE

UK

TEL: 22366477

TLF :

TLX: 81292 CAVLAB G

EML:

COM: $33,34,40,47$

BALIUNAS SALLIE L

CENTER FOR ASTROPHYSICS

HCO/SAO

60 GAROEN ST

CAMBRIOGE MA 02138

USA

TEL: 6174957415

TLF :

TLX:

EML:

COM: $12,29,35,44$

BALKLAVS A E DR
6 RUE DAUBIGNY

F 75017 PARIS

FRANCE

IEL:

TLF:

TLX:

COM: 26

BAKRY ABDEL AZIZ OR UNIVERSITY STATION UNIVERSITY OF VIRGINIA BOX 3818.

CHARLOTTESVILLE VA 22903

USA

TEL: 8049244897

TLF :

TLX:

EML: BITNET : sbovirginia COM: 33

BALOWIN RALPH B 6190 GATEHOUSE OR SE

GRANO RAPIOS MI 49506

USA

TEL: 6199495190

TLF:

TLX:

ENL:

COM: RAOIOASTROPHYSICAL OBS LATVIAN ACAD OF SCIENCES TURGENEVA 19 225524 RIGA LATVIA

IEL:

FLF:

TLX:

EML:

COM: 40 
BALL JUHN A DR HAYSTACK DBSERVATDRY WESTFORO MA 01886 USA

TEL: $617 \quad 692 \quad 4764$

TLF :

EML:

COM: 51

TLX:

BALLEREAU DOMINIQUE DR OBSERVATOIRE DE PARIS SECTION DE MEUOON SECTION D'ASTROPHYSIQUE F 92195 MEUDON PPL COX FRANCE

TEL: $145 \quad 07 \quad 7854$

TLF: $14507 \quad 7878$

TLX: 631987

EML: MES IOA: :BALLEREAU

COM: 29

BALMINO GEORGES G DR

CAES/GRGS/BGI

18 AVE E BELIN

F 31055 TOULOUSE COX

FRANCE

TEL: $61 \quad 274427$

TEF:

TLX: 531081 CNEST B F

EML:

COM: 07

BALUTEAU JEAN-PAUL DR

OBSERVATOIRE DE MARSEILLE

2 PLACE LE VERRIER

F 13248 MARSEILLE COX 04

FRANCE

TEL: 91.959088

FLF :

TLX: $420241 \mathrm{~F}$

EML: EARN: :OBSMRSOFROMRS5]

COM: 34

BANERJI SRIRANJAN OR
OPT OF PHYSICS
UNIVERSITY OF BURDWAN
GOLOPBAG
BUROWAN 713104
INOIA
TEL:
TLF:
ILX:
EML:
COM: 47

BALKOWSK!-HAUGER CH DR OBSERVATOIRE DE PARIS SECTION DE MEUDON.

DAF

F 92195 MEUDON PPL COX FRANCE

\section{TEL: 145347556}

TLF:

EML:

COM: 28

BALLARIO M C PROF

OSS ASTRONOMICO

OI ARCETRI

VIA S LEONARDO

I $50: 00$ FIRENZE

ITALY

TEL:

TLF:

TLX:

EML:

COM:

\section{BALLY JOHN OR}

AT \& BELL LABORATORIES

HOH L 245

USA

TEL: 2018887124

TLF :

TLX: 219879 BTLH UR

EML: jbohohn-2.att.com

COM: 40
TLX: 20157i

HOLMDEL NJ] 07733

BALL LEWIS DR

THEORETICAL ASTROPHYSICS

UNIVERSITY OF SYDNEY

SYENEY NSW 2006

AUSTRALIA

TEL: $2 \quad 692 \quad 3241$

TLF: 26602903

TLX:

EML: BALLPHYSICS.SU.DZ.AU

COM: 40

BALLESTER JOSE LUIS OR

DPT FISICA

UNIYERSIBAD DE LAS ISLAS

BALEARES

E 07071 PALMA DE MALLORCA

SPAIN

TEL: $71 \quad 17 \quad 3228$

TLF: $7143 \quad 8028$

TLX: 69121 UNPM E

EML： DFSULBO@PS.UJB.ES

COM: 10

BALONA LUIS ANTERO OR

SAAD

BOX 9

OBSERVATORY 79́35

SOUTH AFRICA

TEL: 470025

TLF :

TLX: 20309

EML:

COM: $25,27,30,36$

BANDERMANN $L W D R$

21131 GRENOLA QR

CUPERTINO CA 95014

USA.

TEL:

TLF :

TLX:

EML:

COM:

COM: 12

BANOYOPADHYAY A PROE
RESEARCH OIVISION
BIRLA PLANETARIUM
96 JAWAHARLAL NEHRU RO
CALCUTTA 700071
INDIA
TEL: 33281515
TLF:
TLX:
EML:
COM: 04,41

BANG YONG GOL

PYONGYANG ASTRON OBS

ACADEMY OF SCIENCES DPRK

TAESONG DISTRICT

PYONGYANG

KOREA OPR

FEL:

TLF :

TLX:

EML:

COM: 19
BALLABH G M OR

OPT OF ASTRONOMY

UNIVERSITY OF OSMANIA

HYOERABAD 500007

INOIA

TEL: $71951 * 247$

TLF:

TLX:

EML:

COM: 24,28

BALLI EOIBE PROF

UNIVERSITY RASATHANESI

ISTANBUL

TURKEY

TEL:

TLF:

ILX:

EML:

COM: 10

BALONEK THOMAS J DR DPT OF PHYSICS \& ASTRON COLGATE UNIVERSITY HAMILTON NY 13346 USA

TEL: $315 \quad 824 \quad 1000$

TLF :

TLX:

EML: BITNET: TBALONEKGCOLGATHU UJ COM: 40

BANDIERA RINO OR OSS ASTROFISTCO. DI ARCETRI

LARGO E FERMI 5

1. 50125 FIRENZE

ITALY

TEL: $55 \quad 437 \quad 8540$

TLF: 55435939

ILX: 572268

EML: BANOIERABASTRFI. INFN. IT

COM:

BANHATII DILIF GOPAL OR

SCHOOL OF PHYSICS

MADURAI KAMARAJ UNIVERS

PALKALAINAGAR

MADURAI 625021

INDIA

TEL: 85252

TLF:

TLX: 445308 MKU IN

EML:

COM: $28,33,40,47$ 


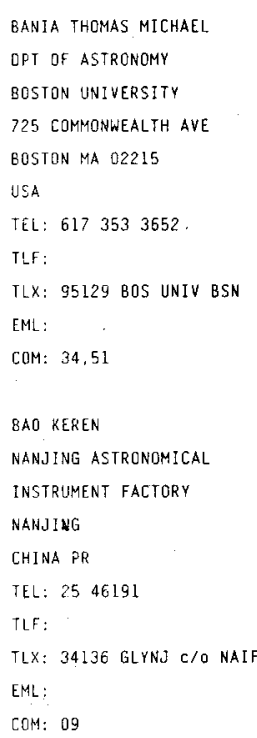

BANNI ALGO DR

STAZIONE ASTRONOMICA

VIA OSPEDALE 72

I 09124 CAGLIARI

ITALY

TEL: $70 \quad 663544$

TLX:

EML: VAXGA2: : LASER

TLF: 70657657

COM: 19

\section{BARANNE A DR}

observatoire oe marseille

2 PLACE le verrier

F 13248 Marseille COX 04 FRANCE

TEL: 91959088

TLF :

TLX:

EML:

COM: 09

\section{BARBERIS BRUND}

IST OI FISICA MATEMATICA UNIVERSITA DI TORINO

VIA C ALBERTO 10

I 10123 TORINO

ITALY

TEL: 11539214

TLF :

TLX:

EML:

COM: $07,33,47$

BARBUY BEATRIZ OR UNIVERSIDADE DE SAO PAULO DEPT DE ASTRONOMIA

CP 30627

01051 SAO PAULO SP BRAZIL

TEL: 115778599

TLF : 112763848

TLX: 1156735 IAGM BR

EML:

COM: $29 \mathrm{C}$

BARDEEN JAMES M PROF
OPT OF PHYSICS
UNIVERSITY OF WASHINGTON
FM 15
SEATTLE WA 98195
USA
TEL: 2065452394
TLF:
TLX: 4740096 LWW UI
EML:
COM: 47

BANOS COSMAS J OR ASTRONOMICAL INSTITUTE NTL OBSERVATORY OF ATHENS BOX 20048

GR 11810 ATHENS

GREECE

TEL: 13461191

TLF:

TLX: 215530 DBSA GR

EML:

COM: 21

BARATTA GIOVANNI BATTISTA OAR

VIA DEL PARCO MELLINI 84

I 00136 ROMA

ITALY

TEL: 6347056

TLF:

TLX:

EML:

COM: 29

BARBIERI CESARE PROF OPT DI ASTRONOMIA UNIVERSITA DI PADOVA VIC DELL OSSERVATORIO 5 135122 PADOVA

ITALY

TEL: 49651499

TLF:

ILX: 430176 UNPADU

EML:

COM: 51

BARCIA ALBERTO OR CTR ASTRON DE YEBES OAN

APD 148

E 19080 GUADALAJARA

SPAIN

TEL: 11290311

TLF: 11290063

TLX:

EML: BARCIA CAY.ES

COM: 09

BARKAT ZALMAN PROF RACAH INST OF PHYSICS HEBREW UNIV OF JERUSALEM JERUSALEM 91904

ISRAEL

TEL: 2584490

TLF:

TLX: 25391

EML:

COM
BANOS GEORGE J PROF DPT OF PHYSICS/DAG UNIVERSITY OF IOANNINA GR 45332 IOANNINA GREECE

TEL: $65 \quad 19 \quad 1697$

TLF :

TLX: 322160

EML:

COM:

\section{BARBANIS BASIL PROF} DPT OF ASTRONOMY JNIVERSITY THESSALONIKI GR 540 O6 THESSALONIKI GREECE TEL: $3199 \quad 1357$ TLF:

TLX: 412181

EML:

COM: 33

BARBIER-BROSSAT M DR observatOIRE DE MARSEILLE 2 PLACE LE VERRIER F 13248 MARSEILLE CDX 04 FRANCE

TEL: 91959088

ILF :

TLX: $420241 \mathrm{~F}$

EML:

COM: 30,45

BARCONS KAVIER DR DPT DE FISICA MOOERNA UNIVERSIDAD OE CANTABRIA AVO LOS CASTROS SAN E 39005 SANTANaER SPAIN

TEL: 42201461

TLF: $42 \quad 20 \quad 1402$

ILX: 35861 EQUCI E

EML: BARCONS COM: 28

BARKER EDWIN S GR ASTRONOMY DPT UNIVERSITY QF TEXAS RLM 15308 AUSTIN TX 787121083 USA TEL: 5124714461 TLF :

TLX: 910-874-1351

EML:

COM: 15 


\author{
BARKER PAUL K OR \\ DPT OF PHYSICS \\ YORK UNIVERSITY \\ 4700 KEELE ST \\ NORTH YORK ON M3J 1 P3 \\ CANADA \\ TEL: $416736 \quad 2100$ \\ TLF: 4167365386 \\ TLX: 065 '24736 \\ EML: \\ COM: \\ BARLIER FRANCOIS E DR \\ DCA CERGA \\ AVE COPERNIC \\ F O6130 GRASSE \\ FRANCE \\ TEL: $93 \quad 365849$. \\ TLF: \\ ILX: 470865 \\ EML: \\ COM: 19
}

BARNES III THOMAS G DR

ASTRONOMY DPT

UNIVERSITY OF TEXAS

MCOONALO OBSERUATORY

AUSTIN TX 787121083

USA

TEL: 5124714461

TLF :

TLX: 910-874-1351

EML:

COM: $25,27 \tau$

BARRETO LUIZ MUNIZ PROF OBSERVATORIO NACIONAL

RUA GL BRUCE 585

SAO CRISTOVAO

20921 RIO OE JANEIRO RJ

BRAZIL

TEL: 215807313

TLF : 215800332

TLX: 02121288 OBSN

EML:

COM: 50

\section{BARROW COLIN H OR}

MAX PLANCK INSTITUT

MAX PLANCK STRASSE 2

POSTFACH 20

D 34 II KATLENBURG LINDAU

GERMANY

TEL:

TLF :

TLX:

EML:

COM: $10,16,40,49$

BARKER TIMOTHY OR
OPT PHYSICS \& ASTRONOMY
WHEATON COLLEGE
NORTON MA 02766
USA
TEL: 5082857722
TLF: :
TLX:
EML:
COM:

BARLOW MICHAEL I OR DPT PHYSICS \& ASTRONOMY UNIVERSITY COLLEGE LONDON GOWER ST

LONOON WEIE $68 \mathrm{~T}$

UK

TEL: 1713877050

TLF :

TLX: 28722 LCPHYS G

EML:

COM: 34

'BARNOTHY JENO DR PRDF

833 LINCOLN ST

EVANSTON IL 60201

USA

TEL: 3123285729

ILF :

EML:

COM: 47,48

$T \perp X$ :

BARRETT ALAN H PROF

15 TREASURE RD

MARATHON FL 33050

USA

TEL:

TLF:

EML:

COM: 34,40

TLX:

BARROW JOHN OAVIO

ASTRONOMY CENTRE

UNIVERSITY OF SUSSEX

FALMER

BRIEHTON BNI $90 H$

LIK

TEL: $273 \quad 606755$

TLF :

TLX: 877159 UNISEX G

EML:

COM: 47

\author{
BARLAI KATALIN QR \\ KONKOLY OBSERVATORY \\ THEGE $\cup \quad 3 / 17$ \\ BOX 67 \\ H 1525 BUGAPEST \\ HUNGARY \\ TEL: 175 5866/75 4122 \\ TLF: \\ YLX: 227460 KONOB H \\ EML: \\ COM: .
}

BARNARO HANNES A J OR

DPT OF PHYSICS

UNIV OF BRITISH COLUMBIA

6224 AGRICULTURE RD

VANCQUVER BC V6T $2 A 6$

CANADA

TEL: $604 \quad 228 \quad 2894$

TLF: 6042285324

ILX: $045 \quad 08576$

EML:

COM: 14

BAROCAS VINICIO PROF

11 VEWLANOS AVE

FULWORD

PRESTON PR2 AQR

UK

TEL: 772719249

TLF:

TLX:

EML:

COM:

BARRETT PAUL EVERETT OR

NASA/GSFC

CODE 668.1

GREENBELT MO 20771

USA'

TEL:

TLF :

TLX:

EML:

COM: 25

8ARROW RICHARD F OR

PHYSICAL CHEMISTRY LAB

UNIVERSITY OF OXFORD

SOUTH PARKS RD

OXFORO OXI $30 Z$

UK

TEL: 86553322

TLF :

TLX:

EML:

COM: 14
BARLETTI RAFFAELE ENG

OSS ASTROFISCIO

DI ARCETRI

LARGO E FERMI 5

I 50125 FIRENZE

ITALY

TEL: $\begin{array}{lll}55 \quad 437 & 8540\end{array}$

TLF: 55435939

TLX: 572268

EML:

COM:

BARNES AARON DR

NASA AMES RESEARCH CTR

MS 2453

MOFFEtT FIELD CA 94035

USA

TEL: 4156945506.

TLF :

TLX:

EML:

COM: 34,49

BARONE FABRIZIO OR

OPT OI SCIENZE FISICHE.

UNIVERSITA OI NAPOLI

MOSTRA D' OLTREMARE PAD 19

I BOI25 NAPGLI

ITALY

TEL: 817253447

TLF: BI 61 4508

TLX: $720320^{\circ}$

EML: FBARONEONAPOLJ. INFN.II

COM: 42

BARROSO JR JAIR

OBSERVATOR IO NACIONAL

RUA GL J [RISTINA 77

SAO CRISTOVAO

20921 RIO OE JANEIRO RJ

BRAZIL

TEL: $215807313 * 273$

TLF: 215800332

TLX: $021-21280$

EHIL:

CDM: 09

BARRY, DDN T DR

DPT DF ASTRONOMY

UNIV SOUTHERN CALIFORNIA

LOS ANGELES CA 90099

USA

TEL: 2137432764

TLF :

TLX:

EML:

COM: 29,45 


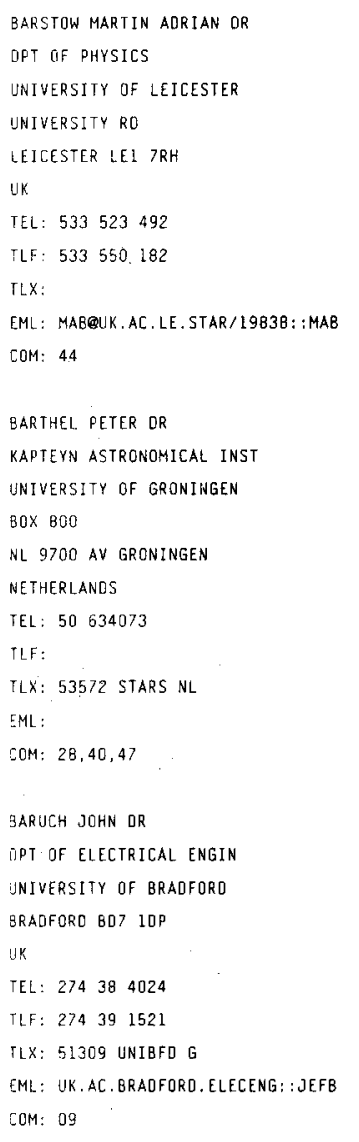

BARTAYA R A DR ABASTUMANI ASTROPHYSICAL OBSERVATORY

GEORGIAN ACAD OF SCIENCES 383762 ABASTUMANI

GEORGIA

TEL: 237 ABASTUMANI

TLF :

TEX: 327409

EML:

COM: $45 C$

BARTHOLOI PAUL OR OBSERVATOIRE OE GENEVE CHEMIN DES MAILLETTES 51 CH 1290 SAUVERNY SWITZERLAND

TEL: 227552611

TLF: 227553983

TLX: 419209 OBS CH

EML: Darthoobs.unige.ch COM:

BARVAINIS RICHARD OR HAYSTACK OBSERVATORY WESTFORD MA 01886 USA

TEL: 6176924764

TLF:

EML:

COM: 40

TLX: 948149
BASCHEK BODO PROF

INSTITUT F THEORETISCHE

ASIROPHYSIK

IM NEUENHEIMER F 561

76900 HEIOELBERG I

GERMANY

TEL: $62 \quad 215 \quad 62837$

TLF:

TLX: 461515 UNIHD D

EHL:

COM: 36

BASIIAN ULRICH
ASTRONOMISCHES-RECHEN
INSTITUT
MOENCHHIOFSTR $12-14$
D 6900 HEIOELBERG 1
GERMANY
TEL: 622149026
TLF:
TLX: 461336 ARI HD D
EML:
COM: 24

BASH FRANK N PROF
ASTRONOMY OET
UNIVERSITY OF TEXAS
RLM
AUSTIN TX 787121083
USA
TEL: 5124714461
TLF:
TLX: $910-874-1351$
EML:
COM: $33,34,40$

BASTIEN PIERRE OR

DPT DE PHYSIQUE UNIVERSITE DE MONTREAL

CP 6128 SUCC A

MONTREAL QC H3C $3 J 7$

CANADA

TEL: $514 \quad 343 \quad 7355$

TLF: 5143432071

TLX: 05562425 UDEMPHYSA

EML:

COM: 27
BARTEL NORBERT HARALD DR CENTER FOR ASTROPHYSICS HCO/SAD.

60 GAROEN ST

CAMBRIDGE MA 02138

USA

TEL: $617 \quad 495 \quad 9278$

TLF:

ILX: 921428

EML:

COM: 40

BARTOLINI CORRADO

DPT II ASTRONOMIA

LNIVERSIIA DI BOLOGNA

VIA ZAMEONI 33

I 40126 BOLOGNA

ITALY

IEL: $5122 \quad 6577$

TLF :

TLX: 211664

EML:

COM: 27,42

BARWIG HEINZ

INST F ASTRON \& ASTROPHYS

UNIVERSITAET MUNCHEN

SEHEINERSTR 1

D 8000 MUENCHEN 80

GERMANY

TEL: 89989021

TLF :

TLX: 529815 UNIVM D

EML:

COM: 09.27

BASRI GIBOR B

ASTRONOMY BPT

UNIVERSITY OF CALIFORNIA

601 CAMPBELL HALL

BERKELEY CA 94720

USA

TEL: $415 \quad 642 \quad 8198$

TLF :

TLX: 820181 UCB ASTRAL UD

EML:

COM: 29.36

BASTIN JOHN A PROF

DPT OF PHYSICS

QJEEN MARY/WESTFIELD COLL

MILE END RD

LONOON EI 4NS

UK

TEL:

TLF :

TLX:

EML:

COM:
BARTH CHARLES A PROF

LASP

UNIVERSITY OF COLORADO

BOX 392

BOULDER CO 803090392

USA

TEL: 303.4927502

FLF:

TLX:

EML:

COM: 49

BARUCCI MARIA A OR OBSERVATOIRE DE PARIS SECTION DE MEUDON

F 92195 MEUDON PPL COX FRANCE

TEL: $145 \quad 07 \quad 7539$

TLF: $145 \quad 077469$

TLX: 201571

EML: MESIOA: : BARUCCI/17670::BARUCCI

CDM: $15 C$

BASART JOHN $P$

OPT OF PHYSICS

IOWA STATE UNIVERSITY

AMES IA 50011

USA

TEL: $515294 \quad 2663$

TLF:

TLX:

EML:

COM:

BASSINO LILIA P OR

INIV NACLONAL OE LA PLATA

FCAG

1900 LA PLATA (BS AS)

ARGENTINA

TEL: 21217308

TLF: $21 \quad 211761$

TLX: 3His: BULAP

EML: LBASSINOQFCAGLP. EDU. AR

COM: 28

BASU BAIDYANATH PROF

DPT APPLIEO MATHEMATICS

UNIVERSITY OF CALCUTTA

92 A P. C RD

CALCUTTA 700.009

INOIA

TEL:

TLF :

TLX:

EML:

COM: $28,33,5]$ 
BASU OIPAX DR

OPT OF PHYSICS

UNIVERSITY OF WEST INDIES

ST AUGUSTINE

TRINIDAO WEST INOIES

INDIA

TEL:

TLF :

TLX:

EML:

COM: $40,47,48,51$

BATES OAVIO:R PROF

DPT OF APPLIED MATHS

\& THEORETICAL PHYSICS

QUEEN'S UNIVERSITY

BELFAST BT? INN

UK

TEL:

TLF :

$T L X:$

EML.

COM: 14,21

BATSON RAYMOND MILNER OR US GEOLOGICAL SURVEY BRANCH OF ASTRDGEOLOGY $2255 N$ GEMINI DR FLAGSTAFF AZ 86001 USA

TEL: 6025567260

TLF : $602 \quad 5567090$

TLX:

EML: NASAMAI LRA\%BATSON

COM: 16

BATTISTINI PIERLUIGI DR OSS ASTRONOMICO

UNIVERSITA OI BOLOGNA

VIA ZAMBONI 33

I 40126 BOLOGNA

ITALY

TEL: 51519593

TLF:

TLX:

EML:

COM

BAUER CARL A DR

OPT OF ASTORNOMY

PENNSYLVANIA STATE UNIV

506 DAVEY LAB

UNIVERSITY PARK PA 16802

USA

TEL

TLF :

TLX:

EML :

COM
BATALHA CELSO CORREA DR

OBSERVATORIO NACIONAL

RUA GL BRUCE $5 B 6$

SAD CRISTOVAO

20921 RID DE JANEIRO RJ

BRAZIL

TEL: 215807181

TLF : $21 \quad 580 \quad 0332$

TLX: 021288

EML: IAGUSP:: CELSO/CCBLLNCCUM

COM: 29

BATESON FRANK M OBE DR

ASTRONOMICAL RESEARCH LTO

BOX 3093

GREERTON TAURANGA

NEW ZEALAND

TEL: $64-075-410-216$

TLF :

TLX: 2880 CPO TG NZ

EML:

COM: 27,42

BATTANER EQUARDO OR

DPT FIS TEBRICA \& COSMOS

FAC DE CIENCIAS

Avo Fuentenueva

E 18002 GRANADA

SPAIN

TEL: 202212306

$T \succeq F$ :

TLX:

EML:

COM: 16,28

\section{BATTY MICHAEL DR}

SCHOOL OF MATHS PHYSICS

COMPUTING \& ELECTRONONICS

MACQUARIE UNIVERSITY

NSW 2109

AUSTRALIA

TEL:

TLF :

TLX:

EML:

COM: 40

BAUER WENDY HAGEN

WHITIN OBSERVATORY

WELLESLEY COLLEGE

WELLESLEY MA 02181

USA

TEL: $617 \quad 235 \quad 0320$

TLF :

TLX:

EML:

LOM: 27,29
BATCHELOR DAVID ALLEN OR

NASA/GSFC

CODE. 633

GREENBELT MD 20771

USA

TEL: 3012862988

TLF :

TLX: 892339

EML:

COM: 10

BATH GEPFFREY T DR

DPT OF ASTROPHYSICS

UNIVERSITY OF OXFORD

SOUTH PARKS RD

OXFORD OXI $3 R 0$

uk

TEL: 865511336

TLF

TLX: 83295

EML:

COM: 27,42

BATTEN ALAN H DR

HERZBERG INST ASTROPHYS

DOMINION ASTROPHYS OBS

5071 W SAANICH RD

VICTORIA BC VBX $4 M$

CANADA

TEL: 6043880009

TLF: 6043630045

TLX: 0497295

EML:

COM: $26,30,42$, WGWWOA

BAUT BOUDEWIJN OR

FOKKER BV

SPACE DIVISION

BOX 7600

NL 1117 ZJ SHIPHOL

NETHERLANDS

TEL: $2054 \quad 49111$

TLF :

TLX:

EML:

COM: 33

BAUM WILLIAM A DR

DPT OF ASTRONOMY

UNIVERSITY OF WASHINGTON

2124 NE PARK RAOD

SEATTLE WA 98195

USA

TEL :

TLF :

TEX:

EML:

COM: $09,16,28,5$
BATES ERIALI UK

DPT OF PURE \& APPL. PHYY

QUEEN'S UNIVERSITY

BELFAST BT7 INN

UK

TEL: $232 \quad 245133$

TLF :

TLX: 74487

EMI: :

COM:

BATRAKOV YU $V$ OH

INST OF THEORET ASTRONOMY

ACADEMY OF SCIENCES

N KUTUZOVA 10

191187 ST PETERSBURG

RUSSTA

TEL: 2724023

TLF:

TLX: 121578 ITA SU

EML:

COM: 07,20

BATTINELLI PADLO QR

OAR

VIA DEL PARCO MELLIINI 84

I 00136 ROMA

ITALY

TEL: 6347056

TLF : 6347802

TLX: 626326 DAROMA I

EML: $40061:$ : BATTINELLI

COM: 28

BAUDRY ALAIN OR

OBSERVATOIRE DE BORDEAUX

BP $89 \cdots$

F 33270 FLOIRAC

FRANCE

TEL: $56 \quad 86 \quad 4330$

TLF: 55404251

TLX:

EML:

COM: 34,40

BALSTIAN W MR

KITT PEAK NTL OES

BaX 26732

$950 \mathrm{~N}$ CHERRY AVE

TUCSOH AZ 857266732

USA

TEL:

TLF:

TLX:

EML:

COM: 


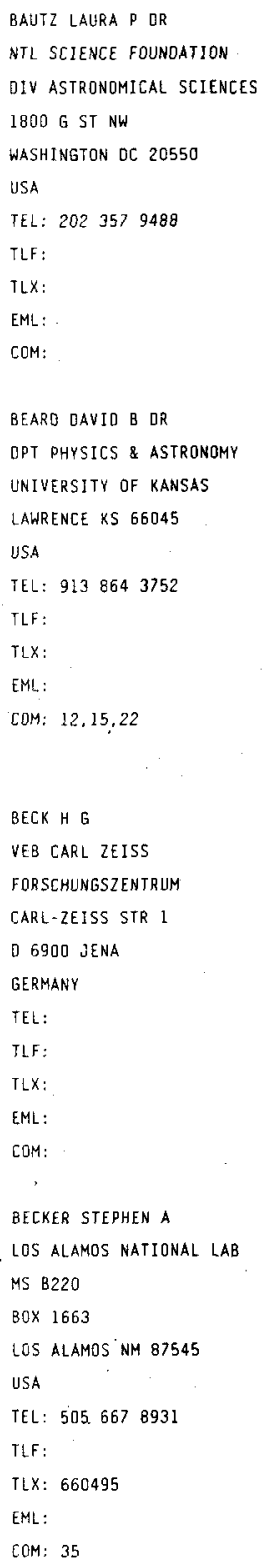

BAYM GOROON ALAN OR
DPT OF PHYSICS
UNIVERSITY OF ILLINOIS
HILO W GREEN ST
URBANA IL 61801
USA
TEL: 2173334363
TLF:
TLX: $910-830-6599$ PHYSIC
EML:
COM: 35,48

BEAUDET GILLES OR

OPT OE PHYSIQUE

UNIVERSITE OE MONTREAL

CP 6128 SUCC A

MONTREAL QC H3C 3.J

CANADA

TEL: 5143436669

TLF: 5143432071

TLX: 05562425

EML:

COM: 35,51

BECK RAINER

MPI FUER RADIOASTRONOMIE

AUF DEM HUEGEL 69

D 5300 BONN 1

GERMANY

IEL: 228525320

TLF:

JLX: 886440

EML:

COM: $25,28,40$

BECKER WILHELM PROF

ASTRONOMISCHES INSTITUT

UNIVERSITAET BASEL

VENUSSTRASSE?

CH 4102 BINNINGEN

SWITZERLAND

TEL: 6L $22771 \mathrm{~L}$

TLF:

$T \perp X:$

EML:

COM: $25,33,37$

\section{BECKWITH STEVEN V W}

MPI FUER ASTRONOMIE

KOENIGSTUHL 17

D 6900 HEIOELBERG 1

GERMANY

TEL: $6221528 \quad 210$

TLF: 6221528246

TLX:

EML: BECKWITHOMPIAHD.MPI-HD.MPG.OE

COM: 34.51
BAZILEVSKY ALEXANDR T

VERNADSKY INST GEOCHEM \&

ANALYTICAL CHEMISTRY.

KOSYGIN STR 19

117334 MOSCOW

RUSSIA

TEL:

TLF:

TLX:

EML

COM: $16 C$

BEAVERS WILLET I DR

ERWIN W FICK OBSERVATORY IOWA STATE UNIVERSITY AMES IA 50011

LSA

TEL: 5152943667

TLF :

$T L X:$

EML:

COM: 26,30

BECKER ROBERT A DR

BOX 4609

CARMEL CA 93921

USA

TEL:

TLF:

$T L X$ :

EML:

COM:

\section{BECKERS JACQUES M DR}

ESO

KARL SCHWARZSCHILDSTR 2

D 8046 GARCHING MUENCHEN

GERMANY

TEL: 89320060

TLF: 893202362

TLX: 052828222 EO D

EML:

COM: 10,12

BEC-BORSENBERGER ANNICK

BUREAU DES LONGITUDES

77 AVE DENFERT ROCHEREAU

F 75014 PARIS

FRANCE

TEL: 140512273

ILF: 146332834

$T L X:$

EML: SPAN: IAPCOA: :Borsenberger

COM: $04,07,20$
BEALE JOHN S DR

231 MARLBOROUGH RD

SWINDON SN3 I.NN

UK

TEL: $793 \quad 34725$

TLF:

TLX:

EML:

COM:

BECHTOLD JILL DR STEWARD OBSERVATORY UNIVERSITY OF ARIZONA TUCSON AZ 85721

USA

TEL: $602 \quad 6216533$ TLF: 6024282854

TLX:

EML:

COM: 47

BEÇKER ROBERT HOWARO DPT OF PHYSICS

UNIVERSITY OF CALIFORNIA

DAVIS CA 95616

USA

IEL: 9167526921

TL.F :

TLX: 910-531-0785 UC DAVS

EML:

COM: 48

BECKLIN ERIC E DR DPT OF ASTRONOMY UNIVERSITY OF CALIFORNIA LOS ANGELES CA 90024 USA

TEL: 2132060208

TLF :

TLX:

EML: BECKLINNUCLASTRO.BITNET COM: 09,34

BEDOGNI ROBERTO

DPT DI ASTRONOMIA UNIVERSITA OI BOLOGNA CP 596

I 40100 BOLOGNA

ITALY

TEL: 5 L 222956

TLF :

TLX: 211664 INFNBO I

EML:

COM: 27,34 


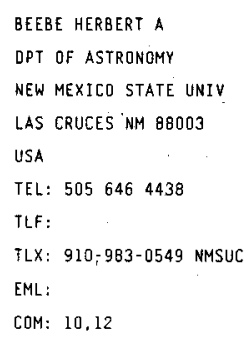

BEEBE RETA FAYE OR

DPT OF ASTRONOAY

NEW MEXICO STATE UNIV

BOX 4500

LAS CRUCES NM 88003

USA

TEL: $505 \quad 6461938$

TLF:

TLX:

EML:

COM: 16,51

BEgEMAN KLR G DR

KAPTEYN ASTRONOMICAL INST

BOX 800

NL 9700 AV GRONINGEN

NETHERLANOS

TEL: $50 \quad 63 \quad 4073$

TLX: 53572

EML: KGBQRUGFX4.RUG.NL

TLF: 50636100

COM: 28

\section{BEXENSTEIN JACOB O OR}

DPT OF PHYSICS

BEN GURION UNIVERSITY

B0X 653

BEERSHEVA 84105

ISRAEt

TEL: $57 \quad 66 \quad 4271$

TLF:

TLX: 5253 UNASI IL

EML:

COM:

\section{BELL BARBARA DR}

CENTER FOR ASTROPHYSICS

HCO/SAO

60 GARDEN ST

CAMBRIOGE MA 02138

USA

TEL: 6174952688

TLF:

TLX:

EML:

COM: 10

\section{BELL MORLEY B}

HERZBERG INST ASTROPHYS

NTL RESEARCH COUNCIL

100 SUSSEX OR

OTTAWA ON KLA ORG

\section{CANADA}

TEL: 6139936060

TLF : 6139526602

TEx: 0533715

EML:

COM:

\author{
BEER REINHARO OR \\ JPL/CALTECH \\ MS 183301 \\ 4800 DAK GROVE DR \\ PASADENA CA 91109 \\ USA \\ TEL: 8183544748 \\ TLF: \\ ILX: \\ EML: \\ BEERS TIMOTHY C OR \\ PHYSICS ASTRONDMY DPT \\ MICHIGAN STATE UNIVERSITY \\ EAST LANSING MI 48824 \\ USA \\ TEL: 517353454 ] \\ TLF: \\ $T L X:$ \\ EML: BEERSMSUPA.BITNET \\ COM: 30
}

COM: 09, 16

BEGGS DENIS W MR

INSTITUTE .OF ASTRONOMY

THE OBSERVATORIES

MADINGLEY RD.

CAMBRIDGE CB3 OHA

UK

TEL: 223.62204

TLF:

TLX: 817297 ASTRON-G

EML:

COM:

BEL NICOLE J OR

observatoire de paris

Section oe meudon

F 92195 MEUDON PPL COX

FRANCE

TEL: $145 \quad 347412$

TLF

TLX: 2015?L

EML:

COM: $12,34,47$

BEHR ALFREO PROF EMERITUS ESCHENWEG 3

D 3406 BOVENOEN

GERMANY

TEL: 5518897

TLF:

TLX:

EML:

COM: 25

BELINSKY VLADIMIR DR

LANOAU INST THEOR PHYSICS ACADEMY OF SCIÉNCES

117940 MOSCOW

RUSSIA

TEL: $137 \quad 32 \quad 44$

TLF :

TLX:

EML:

COM: 47

BELL BURNELL S JOCELYN DR DPT OF PHYSICS

THE OPEN UNIVERSITY

WALTON HALL

MILTON KEYNES MKT GAA

uK

TEL: $908 \quad 274 \quad 066$

TLF: 908653744

TIX:

EML:

COM:

BELL ROGER A DR

ASTRONOMY PROGRAM

UNIVERSITY OF MARYLAND

COLlege PARK MO 20742

USA

TEL: 3014546282

TLF:

TLX: 887294

EML:

CoM: $36.37,45$
BELL KENNETH LLOYD DR OPT OF APPLIEO MATHS \& THEORETICAL PHYSICS QUEEN'S UNIVERSITY. BELFAST BT7 INN UK

TEL: $232 \quad 245133$

TLF:

TLX: 74487 QUBAOM

EML:

COM:

BELL STEVEN OR

DPT OF PHYSICS \& ASTRON UNIVERSITY OF ST ANOREWS NORTH HAUGH ST ANDREWS FIFE KY16 9SS UK

TEE: $334761 \quad 61 * 8306$

TLF: 33474487

TLX: 9312110846 SA G

EML: ASRSB@STAR.ST-AND.AC.UK COM: 42 
BELLAS-VELIOIS IOANNIS OR ASTRONOMICAL INSTITUTE NTL OBSERVATORY OF ATHENS BOX 20048

GR 11810 ATHENS

GREECE

TEL: I 346 119]

TLF: $1346 \quad 3803$

ILX:

EML: YBELLASGgRATHUNI

COM: 29

BELVEDERE GAETANO OR

IST DI ASTRONOMIA

CITTA UNIVERSITARIA

VIA A DORIA 6

I 95125 CATANIA

ITALY

TEL: $9533 \quad 0533$

TLF:

TLX: 970359 ASTRCT I

EML:

COM: 10,27

BENACCHIO LEOPOLDO

OSS ASTRONOMICO DI PADOVA

VIC DELL OSSERVATORIO 5

I 35122 PADOVA

ITALY

TEL: $49 \quad 56 \quad 1499$

TLX: 430176 UNPAOU I

EML :

TLF :

COM: 05

BENDER RALF DR

LANDESSTERNWARTE

ROENIGSTUHL 12

O 6900 HEIDELBERG I

GERMANY

TEL: $62 \quad 215090$

TLF: $62 \quad 21509202$

TLX: 491153 LSWHD

EML: H36:: DHDURZI

COM: 28

BENEVIDES SUARES $P$ DR

INST ASTRON. E GEOFISICO

CP 30627

01051 SAO PAULO SP

BRAZIL

TEL: 112753720

TLX: 113622L IAGM BR

EML:

TLF :

COM: $08 \mathrm{C}$
BELMONTE AVILES I A OR

INST DE ASTROFISICA

DE CANARIAS

OBS DEL TEIDE

E 38200 LA LAGUNA

SPAIN

TEL: 22605200

TLF: $22 \quad 263005$

TLX: 92640 IAC E

EML: IAC: : JBA,

COM: 27,35

BELYAEV. NIKOLAJ A DR

INST OF THEORET ASTRONOMY

ACADEMY OF SCIENCES

N KUTUZOVA 10

191187 ST PETERSBURG

RUSSIA

TEL: $27906 \quad 67$

ILF :

TLX: 121578 ITA SU

EML:

COM:

\section{BENAVENTE JOSE}

URBANIZACION LAS REOES

OCEANO ALANTICO 11

E 11500 PUERTO SANTA MARI

SPAIN

TEL:

TLF:

TLX:

EML:

COM: 31

BENOINELLI ORAZIO

DPT OI ASTRONOMIA

UNIVERSITA DI BOLOGNA

VIA ZAMBONI 33

I 40126 BOLOGNA

ITALY

TEL: $5122 \quad 6677 * 956$

TLF :

TLX: 211664 INFNBO I

EML:

COM: 28

BENFORD GREGURY DR

OPT OF PHYSICS

UNIVERSITY OF CALIFORNIA

IRVINE CA 92717

USA

TEL: $714 \quad 8565147$

TLF :

TLX:

EML:

COM: 12,48
BELSERENE EMILIA P MARIA MITCHELL OBS

3 VESTAL STREET

NANTUCKET MA 02554

USA

TEL: $617228 \quad 9273$

$\mathrm{TLF}$ :

TLX:

EML:

COM: 27

BELY-QUBAL FRANCOISE OCA OBSERV DE NICE

BP 139

F 06003 NICE COXX

FRANCE

TEL: 93890420

TLF:

TLX: 460004 OBSNICE F

EML:

COM: 14

BENAYDOUN JEAN-JACQUES DR OBSERVATOIRE DE GRENOBILE

CERMO/ASTROPHYSIQUE

BP $53 \mathrm{X}$

F 38041 GRENOBLE CDX

FRANCE.

TEL: $76 \quad 51 \quad 4914$

TLF: $76 \quad 44 \quad 8821$

TLX: USMG 980134

EML: BENAYOUNGFGAG5]

COM: 34

BENEDICT GEORGE F OR

ASTRONOMY DPT

UNIVERSITY OF TEXAS

RLM 15308

AUSTIN TX $78712 \quad 1083$

USA

TEL: $512 \quad 4714461$

TLF:

TLX:

EML

COM: $24,28,44$

BENN CHRIS $R$ DR

ROYAL GREENWICH OBS

HERSTMONCEUX CASTLE

HAILSHAM

EAST SUSSEX BN27 IRP

UK

TEL: $323 \quad 833 \quad 171$

TLF :

TLX: 87451 RGOBS $6 B$

EML: JANET: CRB@JK.AC.RGO.STAR

COM: 05,40
BELTON MICHAEL J S OR SOLAR SYSTEM PROGRAM BOX 26732

950 N CHERRY AVE

TUCSON AZ $85726 \quad 6732$

USA

TEL: 6023275511

TLF :

TLX: $666 \% 484$ AURA KNPO TU

EML:

COM: 15C, 16C

BEM JERZY DR

ASTRONOMICAL INSTITUTE

WROCLAW UNIVERSITY

UL. KOPERNIKA II

PL 51 G22 WROCLAW

POLAND

TEL:

TLF:

TLX

EML:

COM: 08

BENDER PETER L OR

JILA

UNIVERSITY OF COLORADO

BOX 440

BOULDER CO 803090440

USA

TEL: $303492 \quad 6793$

TLF:

TLX: 755842 JILA

EML:

COM: $16,19,31$

BENEST DANIEL OR OCA DBSERY DE NICE

BP 139

F 06003 NICE CDX

FRANCE

TEL: $93 \quad 990420$

TLF :

TLX: 460004 OBSNICE $F$

EML:

COM: $07,20,51$

BENNETT CHARLES L DR

NASA/GSFC

CODE 685

GREENBELT MO 20771

USA

TEL: 3012863902

TLF :

ILX:

EML: CHAMP:: BENNETT

COM: $40,44,47$ 


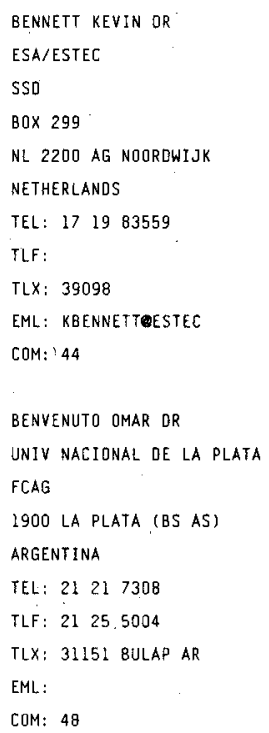

BENSAMMAR SLIMANE DR OBSERVATOIRE OE PARIS SECTION OE MEUDON

F 92195 MEUDON PPL COX FRANCE

TEL: $145 \quad 347835$

TLF:

TLX: 270912

EML:

COM: 09,50

\section{BENZ ARNOLD OR}

INSTITUT FUER ASTRONOMIE

ETH ZENTRUM

CH 8092 ZUERICH

SWITZERLAND

TEL: 12564223

TLF: 12520192

ILX: 53178 ETHBI CH

EML:

COM: $10 \mathrm{C}, 40$

\section{BERGE GLENN L DR \\ CALTECH}

MS 17025

OWENS Valley radio obs

PASABENA CA 91125

USA

TEL: $818 \quad 356 \quad 6969$

TLF :

TŁX: 675425

EML:

COM: 16,40

\section{BERGER MITCHELL DR}

OPT OF MATHEMATICAL SCI

UNIVERSITY OF ST ANOREWS

NORTH HAUGH

ST ANOREWS FIFE KY16 9SS

UK

TEL: 33476161

TLF :

TLX: 76213 SAULIB GB

EML: EARN\%SOLAR: : MBERGER

COM: 10

BERKHUIJSEN ELLY M OR
MPI FUER RADIOASTRONOMIE
AUF OEM HUEGEL 69
D 5300 BONN 1
GERMANY
TEL:
TEX: 886440
EML:
TLF:
COM: $28,33,34,40$

BENSON PRISCILLA J DR

WHITIN OBSERVATORY

WELLESLEY COLLEGE

WELLESLEY MA O21BI

USA

TEL: 617.2350320

TLF:

TLX:

EML: PBENSONGLUCY, WELLESLEY, EOU

COM: $27,40,41,46$

BENZ WILLY

CENTER FOR ASTROPHYSICS

HCO/SAO

60 GARDEN ST

CAMBRIOGE MA 02138

USA

TEL: 6174959889

TLF

TLX:

EML:

COM: 35

BERGEAT JACQUES G DR OBSERVATOIRE DE LYON AVE CHARLES ANDRE

F 69561 S GENIS LAVAL CDX FRANCE

TEL: $78 \quad 56 \quad 0705$

TLF: 72399791

TLX:

EML:

COM :

BERgERON JACQUELINE A OR INSTITUT O'ASTROPHYSIQUE 988 IS 80 ARAGO

F 75014 PARIS

FRANCE

TEL: $143 \quad 201425$

TLF : I 43298673

TLX:

EML: IAPOBS : : BERGERON

COM: $28,34,44,47,48$, GS

BERMAN MARCELO S DR RUA CANDIDO HARTMAN 575 AP 17 ED RENOIR 80430 CURITIBA PR BRAZIL

TEL: 412246426

TLF : $41226 \quad 1679$

TLX:

EML:

COM: 47
BENVENUTI PIERD OR

ST/ECF

C/O ESO

KARL-SCHWARZSCHILO-SIR 2

D 8046 GARCHING MUENCHEN GERMANY

TEL: 9932006291

TLF :

TLX: 52828222 EO D

EML :

COM:

BERENDZEN RICHARO OR PRESIOENT'S OFFICE

THE AMERICAN UNIVERSITY WASHINGTON OC 20016

USA

TEL: $282 \quad 885 \quad 2121$

TLF :

TLX:

EML :

COM: 41.51

BERGER CHRISTIANE DR OCA EERGA AVE COPERNIO

F OGIJO GRASSE FRANCE

TEE: $93 \quad 365849$

TLF:

TLX: $470865 \mathrm{~F}$

EML:

COM:

BERGSTRALH JAY T DR JPL.CLATECH.

MS 183301

4800 OAK GROVE DR PASADENA CA 91109

USA

TEL: 8183542296

TEF:

TLX:

EML:

COM: 16

BERMAN ROBERT HIRAM OR DPT OF PHYSICS

MIT

BOX 165

CAMBRIOGE MA 02139

USA

TEL: 6172531000

TLF :

TLX:

EML:

COM: 


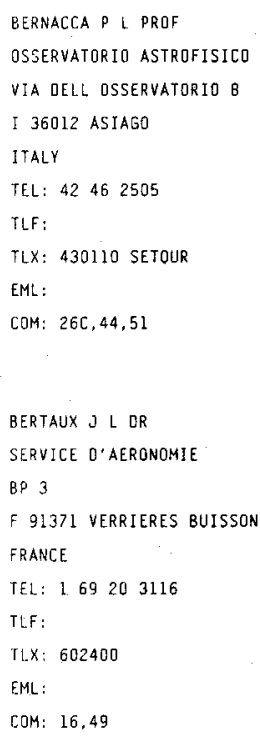

BERNAT ANDREW PLOUS DR OPT OF COMPUTER SCIENCE UNIVERSITY OF TEXAS EL PASO IX 79968 USA TEL: 9157475494 TLF:

TLX:

EML:

COM: 34,36

BERTELLI GIANPAOLO OR DPT DI ASTRONOMIA UNIVERSITA OI PADOVA VIC DELL OSSERvatorio 5 I 35122 PADOVA ITALY

TEL: $4966 \quad 1499$

TLF:

TLX: 432071 ASTROS I

EML:

COM:

BERTOLA FRANCESCO PROF
OSS ASTRONOMICO DI PADOVA
VIC DELL OSSERVATORIO 5
I 35122 PACOVA
ITALY
TEL: 49661499
TLX: 430176 UNPADU I
EML:
TLF:
COM: $28 C, 47$

\section{BETANCORT-RIJO JUAN DR} INST DE ASTROFISICA DE CANARIAS OBS DEL TEIOE E 38200 La Laguna SPAIN

TEL: $22 \quad 262211$

TLF: $22 \quad 263005$

TLX: 92540 IAC E

EML:

COM: 47

BEUTLER GERHARO PROF
ASTRONOMISCHES INSTIIUT
UNIVERSITAET BERN
SIOLERSTRASSE 5
CH 3012 BERN
SWITZERLANO
TEL: 31658591
TLF: 4131653869
TLX: 912643 PIBE CH
EML: BEUTLEREAIUB. UNIBE.CH
COM: 19.

BERRINGTON KEITH AORIAN BPT OF APPLIEO MATHS \& THEORETITCAL PHYSICS QUEEN'S UNIVERSITY BELFAST BT7 INN UK

TEL:

TLF:

TLX:

EML:

COM: 14

BERTHOMIEU' GABRIELLE DR OCA OBSERV DE NICE

BP 139

F 06003 NICE COX

FRANCE

TEL: 93.890420

TLF:

TLX: 460004 OBSNICE F

EML:

COM: 27,35

BERTOUT CLALIEE

INSTITUT D'ASTROPHYSIQUE

$98 B$ IS BO ARAGO

F 75014 PARIS

FRANCE

TEL: 143201425

TLF: 143298573

TLX:

EMt:

COM: 34,36

BETIIS DALE G PROF

TICOM

UNIVERSITY OF TEXAS AUSTIN IX 797121083

USA

TEL:

TLF:

TLX:

EML:

COM: 07

BHANDARI N DR

PHYSICAL RESEARCH LAB

NAVRANGPURA

AHMEDABAD 380009

INOIA

TEL: $272 \quad 46 \quad 2129$

TLF: 272445292

TLX: 0121397

EML:

COM: 22
BERRUYER-DESIROTTE N DR OCA OBSERV DE NICE BP 139

F 06003 NICE COX

FRANCE

TEL: 92003011

TLF :

TLX: 460004 OBSNICE F

EML:

COM:

BERTIAU FLOR C PROF

WAVERSEBAAN 220

B 3030 HEVERLEE

BELGIUM

TLF:

TEL:

TLX:

EML:

COM: 30

BERTSCHINGER EDMMUND DR DPT DF PHYSICS MIT RM 6207 BDX 165

CAMBRIDGE MA 02139

USA

TEL: 6172535083

ILF: 6172539798

TLX:

EML: EOBERT COM: 47

BETTONI DANIELA OR OSS ASTRONOMICO OI PADOVA

VIC DELL OSSERVATORIO 5 I 35122 PADOVA

ITALY

TEL: $4966 \quad 1499$

TLF :

TLX: 430176 UNPADU I

EME:

COM: 28

BHANDARI RAJENDRA OR RAMAN RESEARCH INSTITUTE SACASHIVANAGAR BANGALORE $56000^{\circ}$ INDIA

TEL: 812360122

TLF: 812340492

TLX: $845267 I$ RRI IN

EML: :

COM: 40 


\author{
BHAT CHAMAN LAL QR \\ HEAD NRL HARL C 7 \\ BHABHA ATOMIL RES CENTRE \\ SHASTRI NAGAR \\ JAMMU $180 \quad 004$ \\ INDIA \\ TEL: \\ TLF : \\ TLX: \\ EML:
}

COM:

\section{BHATIA V B DR}

DPT PHYSICS \& ASTROPHYS

UNIVERSITY .OF DELHI

NEW DELHI 110007

INDIA

TEL: 112918993

TLF:

TLX:

EML:

COM:

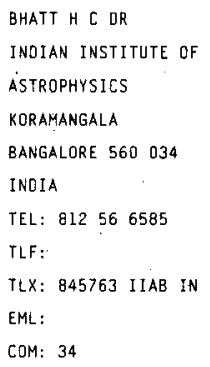

BHATT H C DR

INDIAN INSTITUTE OF

ASTROPHYSICS

KORAMANGALA

BANGALORE 560034

INOIA

TEL: 812566585

TLF:

TLX: 845763 IIAB IN

EML:

COM: 34

\section{BHAVSAR SUKETU $P$}

OFT PHYSICS \& ASTRONOMY UNIVERSITY OF KENTUCKY LEXINGTON KY 405060055 USA

TEL: $606 \quad 257 \quad 6722$

TLF

ILX:

EML:

COM: 47

BIANCHINI ANTONIO OR
OSSERVATORIO ASTROFISICO
VIA DELL OSSERVATORIO B
I 36012 ASIAGO
ITALY
TEL: 42462665
TLF:
TLX:
EML:
COM: 27

BHAT NARAYANA P OR
TIFR
HOMI BHABHA RO
CQLABA
BOMBAY 400005
INOIA
TEL: 224952311
TLF:
TLX: 0113009 TIFR IN
EML:
COM:

BHATNAGAR ARVIND DR

UDAIPUR SOLAR OBSERVATORY

11 VIDYA MARG

UDAIPUR 313001

INO1A

TEL: $25 \quad 626 / 23861$

TLF :

TLX

EML:

COM: 10,12

BHATTACHARYA DIPANKAR
RAMAN RESEARCH INSTITUTE
SADASHIVANAGAR
BANGALORE 560080
INDIA
TEL: 812340122
TLF: 812340492
TLX: $845-2671$
EML:
COM:

BHONSLE RAJARAM $\checkmark$ PROF

PHYSICAL RESEARCH LAB

NAVRANGPURA

AHMEDABAD 380009

INDIA

TEL: $27246 \quad 2129$

TLF: 272445292

ILX: 121397 PRL IN

EML:

COM: 40

BIBARSOV RAVIL'SH OR
ASTROPHYSICAL INSTITUTE
IADJIK ACAD OF SCIENCES
734670 OUSHANBE
TADZHIKISTAN
TEL:
TLF:
TLX:
EML:
COM: 22

BHATIA PREM $K$ OR
DPT OF MATHEMATICS
UNIVERSITY OF JODHPUR
JODHPUR 342001
INDIA
TEL:
TLF:
TLX:
EML:
COM:

BHATIA R K OR

DPT OF ASTRONOMY

UNIVERSITY OF OSMANIA

HYDERABAD 500007

INDIA

TEL: 71951

TLF:

TLX:

EML:

COM: 16

BHATNAGAR ASHOK KUMAR

POSITIONAL ASTR CTR

P 546 BLOCK N $1 S T$ FL

NEW ALIPORE

CALCUTTA 700053

INDIA

TEL: $33 \quad 450 \quad 321 / 493541$

TLF :

TLX:

EML:

COM: 04

BHATTACHARYYA I C PROF

INDIAN INSTITUTE OF

ASTROPHYSICS

KORAMANGALA

BANGALORE $560 \quad 034$

INDIA

TEL: B12 $56 \quad 6583 / 6585$

TLF :

ILX: 845753 IIAB IN

EML:

BHATNAGAR K B DR

ZAKIR HUSSAIN COLLEGE

UNIVERSITY OF OELHI

AJMERI GATE

NEW DELHI 110006

INOIA

TEL: $1152 \quad 2802$

ILF: 117234544

ILX: $3162442,316243 \mathrm{~L}$

EML:

COM: $07 C$

BHATTACHARYYA TARA OR

JOGAMAYA DEVI COLLLEE

92 SYAMAPRADAO MUKERJEE

CALCUTTA 700026

INOIA

TEL:

ILF :

TLX:

EML :

COM: 28

COM: O9P, 12, 50VP

BIAN YUS-LIN

BEIJING ASTRONOMICAL OBS

BIANEHI LUCIANA

OSS ASTRONOMICO DI TORINO

W SUBURB

BEIJING 100080

CHINA PR

TEL:

TLF: 12561085

TLX: 22040 BADAS CN

EML: BMABADICA. BEIJING. CANET.CN

COM:

ST OSSERVATORIO 20

I 10025 PINO TORINESE

ITALY

TEL: 11842040

TLF :

TLX: 213236 TO ASTR I

EML:

COM: 34,44

BICA EDUARDO L D DR

INSTITUTO DE FISICA

UFRGS

CP 15051

90000 PORTO ALEGRE RS

BRAZIL

TEL: $512 \quad 36 \quad 4677$

TLF:

TLK: 515730 CCUF BR

EML:

COM: 28

BICAK JIRI OR

OPT OF MATH PHYSICS

CHARLES UNIVERSITY

HOLESOVICKACH 2

CS 180 OO PRAHA 8

CZECHDSLOVAKIA

TEL: 2849951

TLF :

TLX:

EML:

COM: 


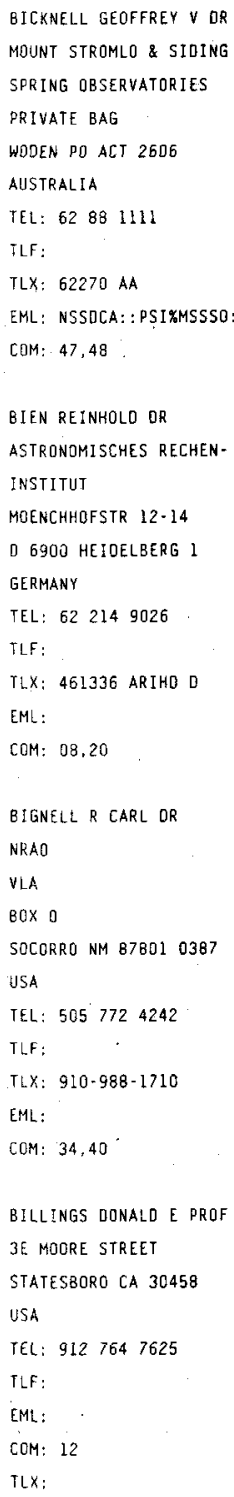

COM: 15,16

8IDELMAN WILLIAM P PROF WARNER \& SWASEY OBS

CASE WESTERN RESERVE UNIV CLEVELANO OH 44106

USA

TEL: $216 \quad 368 \quad 6699$

TLF :

TLX:

EML:

COM: 05,45

BIENAYME OLIVIER DR OBSERVATOIRE DE BESANCON BP 1615

F 25010 BESANCON COX

FRANCE

TEL: 81666900

TLF : 81666944

TLX: 361144

EML: BIENAYMEEROBES5 L

COM: 33

BIJAOUI ALBERT OR OCA OBSERV OE NICE

BP 139

FRANCE

TEL: $93 \quad 890420$

TLF:

TLX: 460004 OBSNICE F

EML

COM: 28,37

BINETTE LUC

CITA MCLENNAN LABS

UNIVERSITY OF TORONTO

60 ST GEQRGE ST

TORONTO OH MSS IAI

CANADA

TEL: $416978 \quad 8497$

TLF: $416978392 \mathrm{l}$

TLX:

EML:

COM: 28,34

BINZEL RICHARD P OR

DPT OF EARTH SCIENCE

MIT RM 54426

BOX 165

CAMBRIDGE MA 02139

USA

TEL: $6 \mathrm{~L} 72536486$

TLF :

ILX:
F 06003 NICE CDX

BIEGING JOHN HAROLD DR

\section{STEWARO OBSERVATORY}

UNIVERSITY OF ARIIZONA TULSON AZ 85721

USA

TEL:

TLF:

ILX:

EML:

COM: 34,40

BIERMANN PETER L DR MPI FUER RADIOASTRGNOMIE AUF DEM HUEGEL 69

D 5300 BONN 1

GERMANY

TEL: 228525279

TLF :

FLX: 886440

EML:

COM: $28,40,48$

BILLAUD GERARO J

DCA CERGA

AVE COPERNIC

F 06130 GRASSE

FRANCE

TEL: 93365849

TLF :

TLX: 470865

EML:

COM: $0 B$

BINGGELI BRUNO

ASTRONOMISCHES INSTITUT UNIVERSITAET BASEL

VENUSSTBASSE?

CH 4102 BINNINGEN

SWITZERLAND

TEL: 6L 227711

TLF :

TLX:

EML:

COM: 28

BIRAUD FRANCOIS OR OBSERVATOIRE OE PARIS

SECTION DE MEUDON

F 92195 MEUDON PPL CDX FRANCE

IEL: 145077602

TEF :

TLX: 270912

EML:

COM: $06,40,51$
BIEMONT EMILE OR INSTITUT D'ASTROPHYSIQUE UNIVERSITE DE LIEGE AVE COINTE 5

B 4000 COINTE-LJEGE

BELGIUM

TEL: $4152 \quad 9980$ ILF: $41 \quad 52 \quad 7474$

TLX:

EML:

COM: 14

BIGNAMI GIOVANNI F IST DI FISICA COSMICA CNR

15/A VIA BASSINI

I 20133 MILANO

ITALY

TEL: 22367587

TLF:

TLX: 313839 MUACNR I -

EML:

COM: 47,48

BILLINGHAM JOHN NASA AMES RESEAREH CTR LIFE SCIENCE DIV MOFFETT FIELD CA 94035 USA

TEL: $415694 \backslash 5181$ TLF:

TLX: $34840 B$ NASA AMES MOF EML:

COM: 51

BINGHAM RICHARO G DR ROYAL GREENWICH OBS MADINGLEY RD CAMBRIOGE CB3 OEZ UK TEL: $223^{2} 83 \quad 3171$ TLF: $T L X: 87451$ EML: COM: 09

BIRCH PETER MR PERTH OBSERVATORY

BICKLEY WA 6075 AUSTRALIA

TEL: 92938255

TLF :

ILX:

EML:

COM: 15 


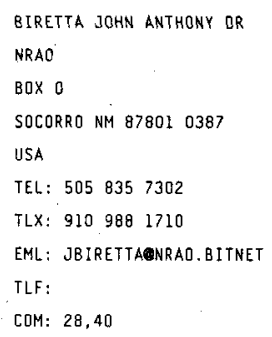

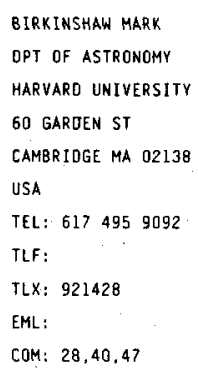

BIRKLE KURT PH D MPI FUER ASTRONOMIE KOENIGSTUHL

D 6900 HetDelberg I

\section{GERMANY}

TEL:

TLF:

TLX:

EML:

COM: 34

\author{
BISWAS SUKUMAR OR \\ TIFR/COSMIC RAY GROUP \\ HOMI BHABHA RD \\ COLABA \\ BQMBAY 400005 \\ INDIA \\ TEL: $22 \quad 219111$ \\ TLF: \\ TLX: 213009 TIFR IN \\ EML: \\ COM: 48
}

BLACKMAN CLINTON PAUL OR CARLSTON LODGE

CAMPSIE RD

TORRANCE

GLASGOW G64 4HD

uk

TEL:

TLF :

TLX:

EML:

COM:

BLAHA MILAN OR

NAVAL RESEARCH LABORATORY CODE 4720

4555 OVERLOOK AVE SW

WASHINGTON OC 203755000 USA

TEL:

TLF :

TLX:

EML:

COM: 14

\section{BLAMONT JACQUES E PROF}

CNES

2 PLACE MAURICE QUENTIN

F 75039 PARIS 'COX 01

FRANCE

TEL: 145087612

TLF:

TLX: 214674

EML:

COM: $12,15,16,21,44$
BISHOP ROY L DR

OPT OF PHYSICS

ACADIA UNIVERSITY

WOLFYILLE NS BOP $I X O$

\section{CANAOA}

TEL: 9025422201

TLF:

TLX:

EML:

COM: 41

\author{
BJORNSSON CLAES - INGVAR \\ STOCKHOLM OBSERVATORY \\ $S 13336$ SALTSJOEBADEN \\ SWEDEN \\ TEL: 87170195 \\ TLF : 87. 174719 \\ TLX: 12972 SOBSERV 5 \\ EML: BJORNSSONEASTRO.SU. \\ COM:
}

BLACKWELL ALAN TREVOR NTL RESEARCH COUNCIL 1771 BELVAL CRESCENT ORLEANS ON KIC 6J6 CANADA TEL: 6139938521 TLF :

TLX:

EML:

COM: 22

bLAIR OAYID GERALO

DPT OF PHYSICS UNIVERSITY W AUSTRALIA NEDLANDS WA 6009 AUSTRALIA TEL:

TLF :

TLX: 92992 AA

EML:

COM: 40

BLANCO CARLO DR

IST DI ASTRONOMIA

CITTA UNIVERSITARIA

VIA A DORIA 6

I 95125 CATANIA

ITALY

TEL: 95330533

TLF :

TLX: 970359 ASTRCT I

EML:

COM: $20,36,50$ 


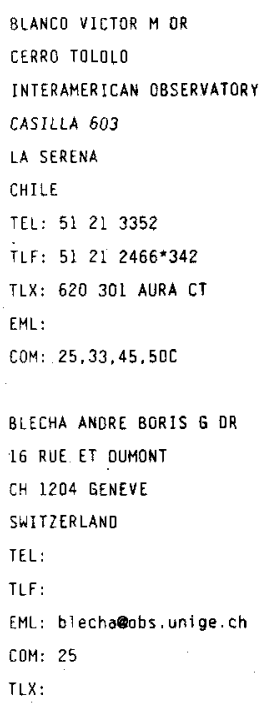

\section{BLITZ LEO DR \\ ASTRONOMY PROGRAM UNIVERSITY OF MARYLAND COLLEGE PARK MD 20742 USA \\ TEL: 3014056650 \\ TLF: 3013149067 \\ TLX: 7108260352 \\ EML: blitzeastro. umd.edu COM: $28,33 P, 34$}

BLOEMHOF ERIC E OR
CENTER FOR ASTROPHYSICS
HCO/SAO
60 GARDEN ST
CAMBRIDGE MA 02138
USA
TEL: 617.4957314
TL.F:
TLX:
EML: BLOEMHOFECFA
COM: 40
BLUDMAN SIDNEY A PROF
OPT OF PHYSICS
UNIV OF PENNSYLVANIA
PHILADELPHIA PA 19104
USA
IEL: 2158988151
TLF:
TLX: 831908
EML:
COM: $35,47,40$

BLANDFORD ROGER DAVID OR CALTECH

MS 13033

THEROETICAL ASTROPHYSICS

PASADENA CA 91125

USA

TEL: 2133564200

TLF:

TLX: 675429

EML:

COM: $40,48,49$

BLEEKER JOHAN A M OR IR SPACE RESEARCH LABORATORY SRON

SORBONNELAAN 2

NL 3584 CA UTRECHT

NETHERLANDS

TEL: 30535600

TLF: 30540860

TLX: 47224 ASTRO NL

EML:

COM: 44,48

BLITZSTEIN WILLIAM OR

DPT ASTRON \& ASTROPHYS

UNIV OF PENNSYLVANIA

DAVIO RITTENHOUSE LAB EI

PHILADELPHIA PA 19104

USA

TEL: 2158987899

TLF:

$T L X: 834621$

EML:

COM: 09,42

BLOMME RONNY DR

DBSERVATOIRE ROYAL DE

BELGIQUE

AVE CIRCULAIRE 3

B 1180 BRUSSELS

BELGIUM

TEL: 23752484

TLF: 23749822

TLX: 21565

EML: RONNYAASTRO. OMA.BE

C.0M: 36

BLUM PETER PROF

INSTITUT F. ASTROPHYSIK

UNIVERSITAET BONN

AUF DEM HUEGEL 71

D 5300 BOHN 1

GERMANY

TEL: $228 \quad 73 \quad 3665$

TLF:

TLX: 886440

EML:

COM: 49
BLASIUS KARL RICHARO DR

3839 MYRTLE

LONG BEACH CA 90807

USA

TEL:

TLF:

TLX:

EML:

COM:

BLESS ROBERT C PROF
OPT OF ASTRONOMY
UNIVERSITY OF WISCONSIN
475 N CHARTER ST
MAOISON WI 53706
USA
TEL: 6082621715
TLF:
ILX:
EML:
COM: $34,36,44.51$

BLOCK DAVIU LAZAR PROF DPT COMPUT \& APPL MATHS WITWATERSRAND UNIVERSITY PRIVATE BAG 3

WITS 2050

SOUTH AFRICA

TEL: $27 \quad 11 \quad 7163761$

,TLF: $27 \quad 117163000$

TLX: 427125 SA

EML: O76BLOKOWITSVMA.WITS.AC.ZA

COM: 28

\section{BLONOIN JOKN M OR}

DPT PHYSICS \& ASTRONOMY

UNIVERSITY NORYH CORALINA

PHILLIPS HALL CB 3255

CHAPEL HILL NC 27599

USA

TEL: 9199623018

TLF: 919962.0460

TLX:

EML: BLONDINGPHYSICS. UNC. EDU

COM: $4 \mathrm{~B}$

\section{BLUMENTHAL GEQRGE R OR}

LICK OBSERVATORY

UNIVERSITY OF CALIFORNIA

SANTA CRUZ CA 95064

USA

TEL : $408 \quad 429 \quad 2005$

TLF:

TLX:

EML: BITNET: george@ortal

COM: 28
BLAZIT ALAIN DR

OCA OBSERV DU CALERN

CAUSSOLS

F 06460 S YALLIER THIEY

FRANCE

TEL: $93 \quad 42 \quad 6270$

TLF :

TLX: 461402

EML:

COM:

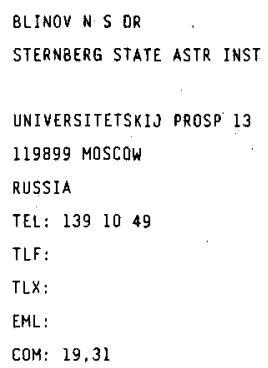

EML:

COM: 19,31

BLOEMEN JOHANNES B GM OR STERR EWACHT

B0X 9513

NL 2300 RA LEIDEN

NETHERLANDS

TEL: $7127581 B$

TLF:

TLX: 39058 ASTRO NL

EML:

COM: $33 \mathrm{C}$

BLOW GRAHAM L

CARTER OBSERVATORY

BOX 2909

WELLINGTON

NEW ZEALAND

TEL: 4728167

TLF:

TLX: NZ 30172 NATOBS

EML:

COM: 20

\section{BO SHU-REN}

INST HISTORY NAT SCIENCE

1 GONG YUAN WEST RD

BEIJING

CHINA PR

TEL: 1557180

TLF:

TLX:

EML:

COM: 41 


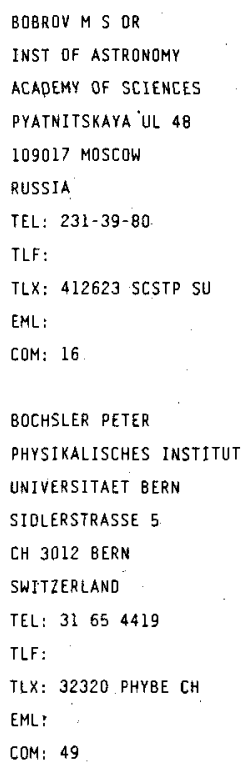




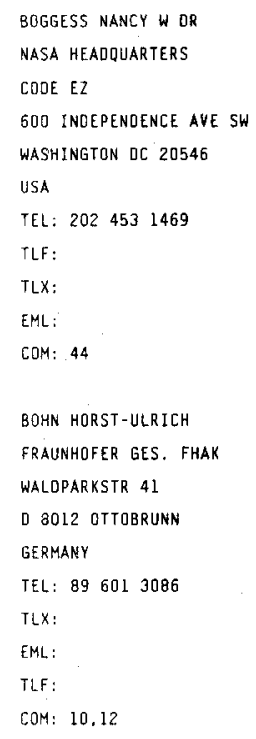

BohanNAN bruCE EDWARD SOMMERS-BAUSCH OBS UNIVERSITY OF COLORADO BOX 391 .

BOULDER CO 803090391 USA

TEL: 3034928782

TLF:

ILX:

EML:

COM:

BOHRMANN ALFREO PROF SCHAERSTR 23

0 2050 HAMBURG 80 GERMANY

TEL: 7399800

TLF:

EML:

COM:

TLX:

BOISSON CATHERINE DR observatoire de paris SECTION DE MEUDON

DAEC

F 92195 MEUDON PPL COX FRANCE

TEL: $145 \quad 07 \quad 7436$

TLF: $1 \quad 45 \quad 07 \quad 7469$

TLX: 201571

EML: 17733: :BOISSON/BOISSONEFRMEU5I COM: 28,44,47 COM: 28

BOLOT ELIHU OR
NASA/GSFC
COOE 661
GREENBELT MO 20771
USA
TEL: 301286.5853
TLF:
TLX: 89675 NASCOM-GBLT
EML:
COM:

BOLTON JOHN 6

39 PANORAMA CRESCENT BUDERIM QLD 4556 AUSTRALIA

TEL: $7 \quad 145 \quad 3374$

TLF:

EML:

COM: 40

TLX:
BOHLIN J DAVIO OR NASA HEADQUARTERS

COOE EZ

600 INDEPENDENCE AVE SW WASHINGTON OC 20546

USA

TEL: 2024531466

TLF:

ILX: 89530

EML:

COM:

BOIGEY FRANCOISE

IMTA LAB MECAN CELESTE

UNIVERSITE PARIS VI

4 PLACE JUSSIEU TOUR 66

F 75230 PARIS CDK 05

FRANCE

TEL:

TLF:

$T L X:$

EML:

COM: 07

BOKSENBERG ALEC PROF

ROYAL GREENWICH OBS

HERSTMONCEUX CASTLE

HAILSHAM BN27 IRP

uK

TEL: $323 \quad 833: 71$

rLF:

.TLX: B7451 RGOBSY G

EML:

BOLEY FORREST I

DPT OF PHYSICS \& ASTRON

darTMOUth COLLEge

WILDER LABORATORY

HANOVER NH 03755

USA

TEL: 6036462966

TLF:

TLX:

EML:

COM:

BOMMIER VERONIQUE OR

OBSERVATOIRE OE PARIS

SECTION DE MEUDON

DAMAP

F 92195 MEUDON PPL CDX

FRANCE

TEL: 145077454

TLF:

TLX: 201571

EML: 28726: BOMMIER

COM: $10,12,14$
BOHLIN RALPH C DR

STSCI

HOMEWOOO CAMPUS

3700 SAN MARTIN DR

BALTIMORE MD 21218

USA

TEL: 3013384804

TLF:

TLX: 6849101 STSCI UWI

EML:

COM: 34,44

BOISCHOT ANDRE OR OBSERVATOIRE OE PARIS

SECTION OE MEUDON

F 92195 MEUDON PPL COX FRANCE

TEL: $145 \quad 077774$

TLF:

TLX: 200590

EML:

COM: 40

BOLAND WILFRIED

NFRA

BOX 2

NL 7990 AA OWINGELDO

NETHERLANOS

TEL: 52197244

TLF:

TLX: 42043

EML:

COM: 34

BOLOIX RAFAEL DR

REAL INST Y .OBSERVATORIO

DE LA ARMADA

CÉcilio pujazon S/N

E 11110 San fernando

SPAIN

TEL:

TLF:

TLX:

EML:

COM: 31

BONACCINI DOMENICO OR OSS ASTROFISICO

DI ARCETRI

LARGO E FERMI 5

I 50125 FIRENZE

ITALY

TEL: 554378540

TLF: 55435939

TLX:

EML: $38954:$ : BONACCINI

COM: 
BONANNO GIOVANNI QR OSS ASTROFISICO

CITTA UNIVERSITARIA

VIA A DORIA 6

I 95125 . CATANIA

ITALY

TEL: 95330533

TLF: 95330592

TLX:

EML: 40297::GIOVANNI

COM: 09

BONO JOHN RICHARD

CITA MCLENNAN LABS

UNIVERSITY OF TORONTO

60 ST GEORGE ST

TORONTO ON MSS IAI

CANADA

TEL: $416 \quad 978 \quad 6874$

TLF: 4169783921

TLX:

EML: bondoutorphys.bitnet

COM: $47 \mathrm{C}$

\section{BONET JOSE A \\ INST DE ASTROFISICA \\ DE CANARIAS \\ OBS DEL TEIDE \\ E 38071 LA LAGUNA \\ SPAIN \\ TEL: \\ TLF : \\ TLX: \\ EML: \\ COM:}

\section{BONNET ROGER M DR}

ESA

8-IO RUE MARTO NIKIS

F 75738 PARIS COX 15

FRANCE

TEL: 142737197

TLF:

TLX: 202746

EML:

COM: $12.44,49$

\section{BONOLI FABRIZIO}

OSS ASTRONOMICO

UNIVERSITA OI BOLOGNA

CP 596

I 40100 BOLOGNA

ITALY

TEL: 51222956

TLF :

TLX: 211664 INFNBO I

EML:

COM:
BONANOMI JACQUES DR

SOUS LES BUIS 28

CH 2068 HAUTERIVE

SWITZERLAND

TEL:

TLF:

EML:

COM: 31

ILX:

BONDAL KRISHNA RAJ DR

UTTAR PRADESH STATE

OBSERVATORY

PO MANORA PEAK 263129

NAINITAL 263129

INOIA

TEL: $2136 / 2583$

TLF:

TLX:

EML: ASTRONOMY NAINITAL

COM: 10

\section{BONEV BONU K MR}

PEOPLE'S ASTRONOMICAL OBS

\& PLANETARIA IN BULgaria

ST AVGUSTA TRAIANA 29/B

BG 6000 STARA ZAGORA

BULGAR IA

TEL: $42 \quad 43 \quad 183$

TLF :

TLX:

EML:

COM:

BONNET-BIDAUD J M OR

CEA CEN

DAPNIA/SAP

BP 2

F 91191 GIF/YvetTe CDX

FRANCE

TEL: 169089259

TLF: 169086577

TLX: 604860

EML: 32779::BOBI BOBI AT FRSACH!

COM: 48

\section{BONOMETTO SILVIO A OR}

DPT DI FISICA G GALILEI

UNIVERSITA DI PADOVA

VIA MARZOLO 8

I 35131 PADOVA

IIALY

TEL: 49844111

TLF: 49844245

TLX:

EML:

OM: 48
BONAzZOLA SILVANO DR OBSERVATOIRE DE PARIS

SECTION DE MEUDON

F 92195 MEUDON PPL COX

FRANCE

TEL: $145 \quad 07 \quad 7429$

TLF :

TLX: 20157.1

EML:

COM: 42,48

BONOARENKO LN DR

STERNBERG STATE ASTR INST

UNIVERSITETSKIJ PROSP 13

119899 MOSCOW

RUSSIA

TEL: $139-3721$

TLF :

TIX:

EML:

COM: 16

BONIFAZI ANGELO DR

OSS ASTRONOMICO

UNIVERSITA OI BOLOGNA

CP 596

I 40100 BOLOGNA

ITALY

TEL:

TLF:

TLX:

EML:

COM:

BONNOR W $B$ PROF

1 SOUTH BANK TERRACE

SURBITON SURREY KTE GDG

uK

TEL: 13991103

ILF :

TLX:

EML:

COM: 47

BONSACK WALTER K PROF

SUITE 298

5100 IB CLAYTON ROAO

CONCORD CA 94521

USA

TEL:

TLF :

TLX:

EML:

COM: 29
BOND HOWARD E OR

STSCI

HOMEWOOD CAMPUS

3700 SAN MARTIN OR

BALTIMORE MO 21218

USA

TEL: 3013384718

TLF :

TLX: 6849101

EML:

COM: 27.29

BONOI HERMANN PROF SIR

CHURCHILL COLLEGE

CAMBRIDGE CB3 ODS

UK

TEL:

ILF :

ILX:

EML:

COM: 35.47

BONNEAU DANIEL

OCA OBSERV DU CALERN

CALSSOLS

F.06460 S VALLIER THIEY

FRANCE

TEL: $9342 \quad 6270$

ILF :

$T L X: 461402$

EML:

COM: 09.26

BONO GIUSEPPE DR

OAT.

BOX SUCC TRIESTE 5

VIA TIEPOLO II

I 34131 TRIESTE

ITALY

TEL: $40 \quad 3199233$

TLF: $40 \quad 30 \quad 9418$

TLX: 461137 OAT I

EML: ASTRTS: : BONO/38439: : BONG

COM: 35

BONTEKOE ROMKE OR

ESA/ESTEC

SSD

B0X 299

NL 2200 AG NOOROWIIJK

NETHERLANDS

TEL: $17 \quad 1985160$

TLF: $31 \quad 171984690$

TLX:

EML: ROMKE GUSPACE, RUG. NL

COM: 28 
BOOK DAVID L

NAVAL RESEARCH LABORATORY

CODE 4040

4555 OVERLOOK AVE SW

WASHINGTON OC $20375500 Q$

USA

TEL :

TLF :

$T t X$ :

EML :

COM: 12

BOOTH ROY S PROF

INSALA SPACE OBSERVATORY

GOETEBORG UNIVERSITY

S 439 OO ONSALA

SWEDEN

TEL: 30 O6 2590

TLF :

TLX: 2400 ONSPACE 5

EML:

COM: 40

BOREERIES NICOLE

JPL

MS 301150

4800 OAK GROVE OR

PASADENA CA 91109

USA

TEL: $818 \quad 354 \quad 8211$

TLF :

TLX: 675429

EML:

COM: 07

\section{BORIAKOFF VALENTIN}

NAIC

CORNELL UNIVERSITY

420 SPACE SCIENCES BLDG

ITHACA NY 148536801

USA

TEL: $607256 \quad 3734$

TLF:

TLX: 932454

EML:

COM: 40

BOS ALBERT OR
NFRA
BOX 2
NL 7990 AA DWINGELOO
NETHERLANOS
TEL: 52197244
TLX: 42043 SRZM NL
EML: PSIU்02041521004: :SYSTEM
TLF: 3152197332
COM: 40

BOOKBINOER JAY A DR

CENTER FOR ASTROPHYSICS

HCO/SAO MS 58

60 GARDEN ST

CAMBRIDGE MA 02138

USA

TEL: 617.4957058

TLF:

TLX: 921428 SATELLITE CAM

EML: BOOKBINOECFA227. HARVARD.EDU

COM:

BOPP BERNARD W QR

OPT PHYSICS \& ASTRONOMY

UNIVERSITY OF TOLEDO

2801 W BANCROFT ST

TOLEDO OH 43506

USA

TEL: $419 \quad 5372274$

TLF :

TLX:

EML:

COM: 27.29 .36 .42

BORGEEST ULF QR

HAMBURGER STERNWARTE

GOJENBERGSWEG 112

D 2050 haMbURG 80

GERMANY

TEL: 4072524121

TLX: 217884

EML: ST40OLOOOHHUNI4. BITNET

TLF: 4072524198

COM: 47

BORKOWSKI KAZIMIERZ M OR

INST OF RADIO ASTRONOMY

$N$ COPERNICUS UNIVERSITY

UL CHOPINA $12 / 18$

PL 87100 TORUN

POLAND

TEL: $48 \quad 56 \quad 783327$

TLF: $4856 \quad 11651$

TLX: 552324 TRAO PL

EML: KAZ IKOPLTUMKII

COM:

BOSCHAN PETER OR
ASTRONOMISCHES INSTITUT
UNIVERSITAT MUNSTER
WELHELM-KLEMM-STR 10
O 4400 MUENSTER
GERMANY
TEL: 251833561
TLF: 251833669
TLX: 892529
EML: PAS04 AT BMSWWU IA. BITNET
COM:

BOOKMYER BEVERLY B OR

OPT PHYSICS \& ASTRONOMY

CLEMSON UNIVERSITY

CLEMSON SC 29631

USA

TEL: 8036563417.

TLF:

TLX:

EML:

COM: 25,42

BORCHKHADZE TENGIZ M ER

ABASTLMANI ASTROPHYSICAL OBSERVATORY

GEORGIAN ACAD RF SCIENCES

383762 ABASTUMANI

GEORGIA

TEL:

TLF:

$T L X$ :

EML:

COM: 28

BORGMAN JAN QR PROF

KAPTEYN OBSERVATORY

WERKGROEP

MENSINGHEWEG 20

NL 9301 KA RODEN

NETHERLANDS

TEL:

TLF:

TLX:

EML:

COM: 25,34

BORNMANN PATRICIA L DR

NOAA ERL R/E/SE

SPACE ENVIRONMENT LAB

325 BROADWAY

BOUEDER CO 80303

USA

TEL: $303 \quad 4973532$

TLF:

TLX: 45897 SOLTERWARN BDR

EML: SPAN: SELVAX:pbornmann

COM: 10,12

BOSMA ALBERT OR

observatoire oE MARSEILle

2 PLACE LE VERRIER

F 13248 MARSEILLE. CDX 04

FRANCE

TEL: 91959088

TLF :

TLX: 420241

EML:

cam: 28
BOOTH ANDREW J

SCHOOL OF PHYSICS

UNIVERSITY OF SYDNEY

SYDNEY NSW 2006

AUSTRALIA

TEL:

TLF:

TLX:

EML:

COM:

BORD DONALO JOHN

DPT DF NATURAL SCIENCES

UNIVERSITY OF MICHIGAN

DEARBORN

DEARBORN MI $4 B 128$

USA

TEL: 3135935483

TLF:

TLX:

EML:

COH:

BORGNINO JULIEN OR

OPT ASTROPHYSIQUE

UNIVERSITE DE NIEE

PARC VALROSE

F 06034 NICE CDX

FRANCE

TEL: 93519100

TLF:

TLX:

EML:

COM: 09

BORRA ERMANNO F OR

DPT DE PHYSIQUE

UNIVERSITE DE LAVAL

STE FOY OC GIK TP4

CANADA

TEL: $418 \quad 6567405$

TLF: 4186562040

TLX: 05131621

EML:

COM: 25

BOSMA PIETER B OR

OPT PHYSICS \& ASTRONOMY

FREE UNIVERSITY

DE BOELELAAN 1081

NL LÓBI HV AMSTERDAM

NETHERLANOS

TEL: $20 \quad 5484139$

TLF : 20461459

$T L X:$

EML:

COM: 16 


BOSMAN-CRESPIN DENISE
BVD D AVROY 68
BOX 093
B 4000 COINTE-LIEGE
BELGIUM
TEL: 41237486
TLF:
TLX:
EML:
COM:

BOUCHER CLAUDE DR

INSTITUT GEOGRAPHIQUE NTL

2 AVE PASTEUR

F 94160 SAINT MANDE

FRANCE

TEL: 143989000

TLF:

YLX:

EML:

COM: 19

BOUGERET J L DR

OBSERVATOIRE DE PARIS

SECTION DE MEUDON

DESPA

F 92195 MEUDON PPL CDX

FRANCE

TEL: $145 \quad 077704$

TLF :

TLX: 204464

EML:

COM: $10,12,44$

BOULON JACQUES J DR

OBSERVATOIRE DE PARIS

61 AVE OBSERVATOIRE

F 75014 PARIS

FRANCE

TEL: $140 \quad 51.2253$

TLF:

TLX: 270776 DBS F

EML:

COM: $27,30,33$

BOWELL EDWARD L G DR

LOWELL OBSERVATORY

1400 W MARS HILL RD

BOX 1149

FLAGSTAFF AZ BG00L

USA

TEL: $602 \quad 774 \quad 3358$

TLF :

TLX:

EML:

COM: 15,20
BOSS ALAN P BR

OPT TERRESTR MAGNETISM

CARNEGIE INST WASHINGTON

5241 BROAD BRANCH RD NW

WASHINGTON DC 20015

USA

TEL: 2026864402

TLF :

TLX: 440427

EML:

COM: 16,35

BOUCHET FRANCOIS R DR

INSTITUT O'ASTROPHYSIQUE

98BIS BD ARAGO

F 75014 PARIS

FRANCE

TEL: $14320 \quad 1425$

TLF: 143.298673

TLX:

EML: BOUCHETEFRIAP5I

COM:

BOUIGUE $R$

11 RUE PELLETIER DOOISY

F 31400 TOULOUSE

FRANCE

TEL:

ILF:

EML:

COM: 24

TLK:

BOLSKA JIRI OR

DPI OF ASTRONOMY

CHARLES UNIVERSITY

SVEOSKA $B$

CS 150 00 PRAHA 5

CZECHOSLOVAKIA

TEL: 2540395

$\mathrm{T} L F:$

TLX: $121673 \mathrm{MFF}$

EML:

COM: 05,15

BOWEN EOWARO $G$ DR

1/39 CLARKE STREET

NARRABEEN NSW 2101

AUSTRALIA

TEL: 989565

TLX:

EML:

TLF:

COM:
BOTEZ ELVIRA DR

INSTITUT D'ENSEIGNEMENT

SUPERIEUR

13 RUE EM BOONARAS

R 5800 SUCEAVA

RUMANIA

TEL: 98716147

TLF:

TLX:

EML:

COM: 46

BOUCHET PATRICE DR

ESO

CASILLA 19001

SANTIAGO 19

CHILE

TEL: 26988757

TLF:

$T L X: 24088 \mathrm{l}$

EML: PBOUCHETOOGAESO51/ESBMCI : : PATO EML

COM: 28

CON: 08

BOULANGER FRANCOIS

RAOIOASTRONOMIE ENS

24 RUE LHOMONO

F 75231 PARIS CDX 05

FRANCE

TEL:

TLF:

TLX:

EML:

COM: 34

BOUVIER JEROME

CERMO

BP 68

F 38402 S MARTIN HERES CO

FRANCE

TEL: 76514790

TLF: $76 \quad 44 \quad 6821$

TLX:

EML: BOUVIERQFRGAG5L. BITNET

COM: 29,34

BOWEN GEORGE H DR

DPT OF PHYSICS

IOWA STATE UNIVERSITY

AMES IA 50011

USA

TEL: 5152947659

TLF:

TLX:

EML: BITNET:SL. GHBOISUMVS

COM: 27,36
BOTIINELLI LUCETTE OR OBSERVATOIRE DE PARIS SECTION DE MEUDON

RADIOASTRONOMIE

F 92195 MEUDON PPL COX

FRANCE

TEL: 145077604

TLF:

ILX: 270912

EML:

COM: $28,40,46$

BOUGEARD MIREILLE L OR OBSERVATOIRE DE PARIS

61 AVE OBSERVATOIRE

F 75014 PARIS

FRANCE

TEL: $1405 \mathrm{~L} 2226$

TLF: 140512232

TLX: 270775 OBS F

BOULESTEIX JACQUES

OBSERVATOIRE DE MARSEILLE

2 PLACE LE VERRIER

F 13248 MARSEIILLE CQX 04

ERANCE

TEL: 91959088

ILF:

TLX: $420241 F$

EML:

COM:

BOUVIER PIERRE PROF

OBSERVATOIRE DE GENEVE

CHEMIN DES MAILLETTES 51

CH 1290 SAUVERNY

SWITZERLAMO

TEL: 227552611

TLF: 227553983

TLX: 419209 OBS CH

EML:

COM: 29,37

BOWERS PHILLIP F

NAVAL RESEARCH LABORATORY CODE 4134 .

4555 OVERLOOK AVE SW

WASHINGTON DC 203755000

USA

TEL: 2027672495

TLF:

TLX:

EML:

COM: 40 


BOWYER C STUART PROF
ASTRONOMY OPT
UNIVERSITY OF CALIFORNIA
60L CAMPBELL. HALL
BERKELEY CA 94720
USA
TEL: 4156421648
TLF:
TLX: $910-3667945$
EML:
COM: $21 C, 44,51$
BOYO ROBERT L F PROF SIR
41 CHURCH ST
LITTLEHAMPTON BN17 SPU
UK
TEL:
TLF:
EML:
COM: 44,48
TLX:

BOYLE RICHARD P OR VATICAN OBSERVATORY I Q0120 VATICAN CITY VATICAN CITY STATE

TEL: $6 \quad 6985256$

TLF:

EML: BOYLEARIZRVAX. BITNET

COM:

ILX: 5042020 VATOBS VA

BOZKURT JUMNU Un

OBSERVATORY

EGE UNIVERSITY

BOX 21

35100 BORNOVA IZMIR

TURKEY

TEL: $51 \quad 18 \quad 0306$

TLF :

TLX:

EML:

COM:

BRAES L L E QR
STERREWACHT
BOX 9513
NL 2300 RA LEIOEN
NETHERLANOS
TEL: 71272727
TLX:
EML:
TLF:
COM: 46

BOYARCHUK A A OR

INST OF ASTRONOMY

ACADEMY OF SCIENCES :

PYATNITSKAYA UL 4B

109017 MOSCOW

RUSSIA

TEL: $7095 \quad 2312129$

TLF: 70952302081

TLX: 411576 ASCON SU

EML: IAASENODE. IAS.MSK. SU

COM: $27,29,38,44, E C$

BOYDAG-YILDIZOOGOU F $S$ OR

KING SAUD UNIVERSITY.

COLLEGE OF SCIENCE

BOX 2452

RIYADH 11495

SAUOI ARABIA

TEL:

TLF :

TLX:

EML:

COM:

BOYNTON PAUL EOWARD OR

OPT DF ASTRONOMY

UNIVERSITY OF WASHINGTON

FM 20

SEATTLE WA 98195

USA

TEL:

TLF:

ILX:

EML:

COM:

BRACCESI ALESSANLRO PROF

OPT OI ASTRONOMIA

UNIVERSITA, DI BOLOGNA

VIA ZAMBONI 33.

I 40126 BOLOGNA

ITALY

IEL: 51222956

TLF:

TLX: 211664 INFNBOI

EML:

COM: 28

\section{BRAGA JOAO OR}

INPE

CP 515

$12201 S$ JOSE DOS CAMPOS

BRAZIL

IEL: $123418977^{* 679}$

TLF: 123218743

TLX: 123353 INPE BR

EML: INPEDASQBRFAPESR. BITNET

COM: 48
BOYARCHUK MARGARITA E DR

INST OF ASTRONOMY

ACADEMY OF SCIENCES

PYATNITSKAYA UL 48

109017 MOSCOW

RUSSIA

TEL:

TLF :

TLX:

EML:

COM: 27

BOYER RENE

OBSERVATOIRE OE PARIS

SECTION DE MEUDON

DASOP

F 92195 MEUDON PPL COX

FRANCE

TEL: $1 \quad 45 \quad 077741$

TLF :

TLX: 201571

EML:

COM: 10

BOYTEL JORGE DEL PINO OR. INST GEOPHYS \& ASTRONOMY CALLE 212 N 2906/29 Y 31 LISA

LA HABANA

CUBA

TEL: $21 \quad 8416 / 0644$

ILF :

TLX: 511240 GEOAS CU

EML:

COM: 19

BRACEWELL RONALD N PROF

DURAND 329 A

STANFORD UNIVERSITY

STANFORO EA 94305

USA

TEL: $415 \quad 497 \quad 3545$

TLF :

TLX:

EML:

COM: 40,51

BRAHDE ROLF

INST THEORET ASTROPHYSICS

UNIVERSITY OF OSLO

BOX 1029

N 0315 BLINDERN OSLO 3

NDRWAY

TEL: 2456508

TLF:

TLX:

EML:

COM:
BOYCE PETER B OR AMERICAN ASTRON SOCIETY 2000 FLORIOA AVE NW SUITE 300

WASHINGTON OC 20009

USA

TEL: $202328 \quad 2010$

TLF :

ILX: 257588 AASW UR

EML:

COM: $09.16,51$

BOYLE BRIAN DR

AAO

$80 \times 296$

EPPING NSW 2121

AUSTRALIA

TEL: 2 868 1666

JLF :

ILX: 23999 AAOSYD AA

EML:

COM: 47

BOZIS GEOR'GE PROF

DPT THEORET MECHANICS

UNIVERSITY THESSALONIKI

GR 54006 THESSALONIKI

GREECE

TEL: 31992845

TLF:

TLX:

EML:

COM: 07

BRADSTREET DAVID H. OR DPT DF PHYSICAL SCIENCE EASTERN COLLEGE

ST DAVIOS PA 19087

USA

TEL: 2153415945

TLF :

TLX:

EML:

COM: 42

BRAHIC ANERE OR OBSERVATOIRE DE PARIS SECTION DE MEUDON F 92195 MEUDON PPL COX FRANCE

TEL: $145 \quad 077402$

TLF :

TLX: 201571

EML:

COM: 16C, WGPSNC 
BRANCH DAVIO R DR

DPT PHYSICS \& ASTRONOMY UNIVERSTIY OF OKLAMOMA NDRMAN OK 73019

USA

TEL: 4053253961

TLF:

TLX: 9108306521

EML:

COM:

BRANOIE GÉORGE W DR ENYIRONMENTAL ENGINEERING QUEEN'S UNIVERSITY KINGSTON ON KOL 3NG CANADA

TEL:

TLF:

TLX:

EML:

COM:

BRANHAM RICHARD L JR CENTRO REgIONAL DE INVESI CIENTIFICAS Y TECNOL.

CC L3:

5500 MENDO2A

ARGENTINA

TEL: 612411794

TLF:

TLX: 55438 CYTME AR

EML:

COM: $07,20,24$

BRAUDE SEMION YA PROF AG INSTITUTE OF RADIO ASTRON UKRAINIAN ACAD OF SCIENCE KRASNOZNAMENNAYA UL 4 310002 KHARKOV UKRAINE

TEL: 441092

TLF:

TLX:

EML:

COM: 40

BRAUNINGER HEINRICH DR MPI F PHYSIK \& ASTROPHYS INST F. EXTRATERR. PHYSIK O BO46 GARCHING MUENCHEN GERMANY

TEL: 893299566

TLF:

TLX:

EML:

COM:
BRAND PETER W J L OR .

ROYAL OBSERVATORY

BLACKFORO HILL

EDINBURGH EHS 3HJ

uk

TEL: 316673321

ILX: 72383 ROE EOIN 6

FML:

TLF:

COM: 34

BRANDT JOHN C DR

LASP

UNIVERSITY OF COLORADO

BOX 392

BOULDER CO 803090392

USA

TEL: 3034923215

ILF:

TLX: 9109403441

EML:

COM: $15 C, 44,49$

BRANSCOMB L M OR

NTL BUREAU OF STANOARDS

WASHINGTON DC 20025

USA

TEL:

TLF:

EML:

COM: 14

TLX:

BRAULT JAMES $W$ OR

NTL. SOLAR OBSERVATORY

BOX 26732

$950 \mathrm{~N}$ CHERRY AVE

TUCSON AZ 857266732

USA

TEL: 6023259363

TLF:

TLX: 666484 AURA NOAO IUC

EML:

COM: $09.12,14$

BRAUNSFURTH EOWARO PH D

IM HAARMANNSBOCH $99 \mathrm{~A}$

O 4630 BOCHUM 1

GERMANY

TEL:

TLF:

EML:

COM: 34

TLX:
BRANDENBURG AXEL DR

NORDITA

BLEgOAMSVEJ 17

OK 2100 CDPENHAGEN $O$

DENMARK

TEL: 31421616

ILF: 31389157

TLX: 15216 NBI OK

EML: BRANDENBENOROITA. DK

COM: 10

BRANOT PETER N

KIEPENHEUER INSTITUT FUER

SONNENPHYSIK

SCHONECKSTR, -

D 7800 FREIBURG BREISGAU

GERMANY

TEL: 76132864

TLF:

TLX: 7721552

EML:

COM: 10,12

BRANSON NICHOLAS J B A OR GENERAL BDARD OFFICE

THE OLO SCHODLS

CAMBRIOGE CB2 ITT

uK

TEL: 223332250

ILF: 223332332

ILX: 81240 CAMSPL G

EML:

COM:

BRAUN ARIE

RACAH INST OF PHYSICS

HEBREW UNIV OF JERUSALEM

JERUSALEM 91904

ISRAEL

TEL: 2584521

TLF:

TLX: $25391 \mathrm{HU}$ IL

EML:

COM:

BRAY RGBERT J DR

31/126 CRIMEA ROAD

MARSFIELO NSW 2122

AUSTRALIA

TEL:

TLF:

ILX:

EML:

COM: 10,12
BRANOI ELISANDE ESTELA DR OBSERVATORIO ASTRONOMICO PASEO DEL BOSQUE

1900 LA PLATA (BS AS) ARGENTINA

TEL: 21217308

ILF:

TLX:

EML:

COM: 29,42

BRANDUARDI - RAYMONT G MULLARD SPACE SCIENCE LAB UNIVERSITY COLLEGE LONDON HOLMBURY ST MARY DORKING SURREY RHS ENT uk

TEL: 30670292

TLF:

TLX: 859185

EML:

COM:

\section{BRATLJCHUK MATRONA V}

SATELITE DBSERVATORY

UZHGOROD STATE UNIVERSITY

HORKIY 46

294000 UZHGOROD

UKRAINE

TEL: 36065

TLF:

ILX: 274155 KNIGA

EML:

COM:

BRAUN ROBERT DR

NFRA

BOX 2

NL 7990 AA DWINGELOO

NETHERLANDS

TEL: 52197244

TLF: 52197332

TLX: 42043 SRZM NL

EML: RBRAUNENFRA.NL

COM: 28

BREAKIRON LEE ALLEN OR US NAVAL OBSERVATORY TIME SERVICE OPT

34 \& massachusetTs ave nh WASHINGTON OC 203925100 USA

TEL: $202653188 B$

TLF:

TLX:

EML: I: navobsymardacva.arpa

COM: 24 


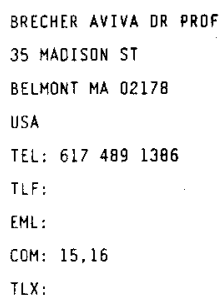

\section{BRECHER KENNETH PROF}

OPT OF ASTRONOMY

BOSTON UNIVERSITY

725 COMMONWEALTH AVE

BOSTON MA 02215

USA

TEL: 617353.3423

TLF:

TLX: 95-1289 BIS UNIV BSN

EML:

COM: $28,47,48$

GREGMAN JOEL N
OPT OF. ASTRONOMY
UNIVERSITY OF MICHIGAN
OENNISON BLOG
ANN ARBOR MI 461091090
USA
TEL: 3137643440
TLF:
TLX:
EML: JBREGMANASTRO. LSA. UMICH, EDU
COM:

\section{GRETAGNON PIERRE OR}

BUREAU DES. LONGITUDES

77 AVE DENFERT ROCHEREAU

F 75014 PARIS

FRANCË

TEL: 140512269

TLF:

ILX:

EML:

COM: 04,07

BRIOLE ALAN H PROF
NRAO
EDGEMONT RD
CHARLOTIESVILLE VA 22903
USA
TEL: 8042960375
TLX: $910-997-0174$
EML:
TLF:
COM: 40

\section{BRINKMAN BERT C OR}

SPACE RESEARCH LA'BORATORY

SRON

SORBONNELAAN 2

NL 3584 CA UTRECHT

NETHERLANDS

TEL: 30535600

ILF: 30540860

rLX: 47224 SRON NL

EML:

COM: 44
BRECKINRIDGE JAMES B OR JPL/CALTECH

MS 183301

4BOO OAK GROVE DR

PASADENA CA 91109

USA

TEL: 2133546785

TLF :

TLX: 675429

EML:

COM: 09,12

BREINHORST ROBERT A OR ASTRONOMISCHES INSTITUT STERNWARTE

AUF DEM HUEGEL 71

D 5300 BONN 1

GERMANY

TEL: $22873 \quad 3660$

TLF:

ILX:

EML:

COM: 42

BREUKERS R J L H DR

STERREWACHT

BOX 9513

NL 2300 RA LEIOÉN

NETHERLANDS

TEL:

TLF:

TLX:

EML:

COM:

BRIEVA EDUARDO PROF

OBSERVATORIO NACIONAL

\section{APARTADO 2584}

BOgOTA 1 DE

COLOMBIA

TEL: 1423786

TLF:

TLX:

EML:

COM: 07,46

BRINKMANN WOLFGANG

MP.I F PHYS \& ASTROPHYSIK INST F EXTRATERR PHYSIK KARL-SCHWARZSCHILO-STR I D 8046 GARCHING MUENCHEN GERMANY

TEL: $89329 \quad 9877$

TLF:

TLX: 05215845. XTER O

EML:

COM: 28.34
BREGER MICHEL PROF DR INSTITUT FUER ASTRONOMIE TUERKENSCHANZSTRASSE 17

A 1180 WIEN

AUSTRIA

TEL: 13453605

TLF: 134536015

TLX: 133099 VIAST A

EML: BREGER@AVIA.UNA. AC.AT

COM: $25 \mathrm{C}, 27 \mathrm{C}, 30$

BREITSCHWEROT DIETER DR MPI FÜR KERNPHYSIK

POSTFACH 1039 BO

D 6900 HEIDELBERG I

GERMANY

TEL: $62 \quad 21516471$

TLF: $62 \quad 21516324$

TLX: 461666

EML: WINOS DHOMPISV (BITNET)

COM: 34

BREYSACHER JACQUES

ESO

KARL-SCHWARZSCHILO STR. ?

o 8046 garching mUENCHEN

GERMANY

TEL: $B 932006224$

TLF: 893202362

TLX: 5282820

EML:

COM: 29

BRIHAYE CHARLES C A DR

IAAG

VRIJE UNIV BRUSSELS

CP. 165

B 1050 BRUSSELS

BELGIUM

TEL: 26876928

TLF:

TLX:

EML:

COM:

BRINKS ELIAS DR

NRAD

BOXं 0

SOCORRO NM 878010387

USA

TEL: 5058357000

TLF: $505 \quad 8357027$

TLX: 9109981710

EML: EBRINKSENRAO

COM: 28,40 


\author{
BRIOT DANIELLE OR \\ OBSERVATOIRE DE PARIS \\ 61 AVE OBSERVATOIRE \\ F. 75014 PARIS \\ FRANCE \\ TEL: 140512239 \\ TLF: \\ TLX: 270776 OBS F \\ EML: \\ COM:
}

BRODIE JEAN $P^{\prime}$

SPACE SCIENCES LABORATORY UNIVERSITY OF CALIFORNIA BERKELEY CA 94720

USA

TEL: $\mathbf{4 1 5} 642 \quad 1579$

TLF :

TLX: $910-366-7945$

EML:

COM: 28

BRONNIKGVA NINA M

PULKOVO OBSERVATORY

ACADEMY OF SCIENCES

10 KUTUZOV QUAY

196140 ST PETERSBURG

RUSSIA

TEL:

TLF:

TLX:

EML:

COM: 24

BROSCHE PETER PROF

OBSERVATORIUM HOHER LIST

UNIV STERNWARTE BONN

D 5568 DAUN

GERMANY

TEL: 65922150

TLF:

TLX:

EML:

COM: $19,24,26,28$

\section{BROUW W N OR}

CSIRO

ATNF

BOX 76

EPPING NSW 2121

AUSTRALIA

TEL:

TLF:

TLX:

EML: WBROUWATNF.CSIRO.AU

COM: 08,40

BROADFOOT A LYLE OR
LUNAR PLANETARY LAB
UNIVERSITY OF ARIZONA
901 GOULO SIMPSON BLOG
TUCSON AZ 85721
USA
IEL: 6026214301
ILF:
TLX: $910-952-1143$
EML:
COM: 16,21
BROGLIA PIETRO OR
OSS ASTRONOMICO OI MILANO
VIA E BIANCHI 46
I 22055 MERATE
ITALY
TEL: 592035
TLX:
EML:
TLF:
COM: 42

BROOKES CLIVE J DR

DPT OF MATHEMATICS

EARTH/SATELLITE RES UNIT

ASTON UNIVERSITY

BIRMINGHAM B4 7ET

UK

TEL: 213593611

TLF:

TLX: 335787

EML:

COM: 07

BROSTERHUS E B F OR
LOCKHEEO CITY
BOX 6308
JEDDAH
SAUDI ARABIA
TEL: $26562501 \approx 355$
TLX:
EML:
TLF:
COM:

BROWN ALEXANDER
JILA
UNIVERSITY OF COLORADD
BOX 440 -
BOULDER CO 803090440
USA
TEL: 3034928962
ILF:
TLX: 755842 JILA
EML:
COM: 35,44

BROCATO ENZO DR

OSS DI TERAMO

COLLURANIA

I 64100 TERAMO

ITALY

TEL: BE1 210490

TLF: $861 \quad 210493$

$T \perp X$ :

EML:

COM:

BROMAGE GORDON E DR

RUTHERFORD APPLETON LAB

SPACE \& ASTROPHYSICS DIV

BLDG R25/R68

CHILTON DIOCOT OXII DOX

UK

TEL: 23521900

TLF:

ILX: 83159

EML:

COM: 14,34

BROOKS RANDALL C DR

PHYSICS SCIENCES

NTL MUSEUM SCIENCE \& TECH

BOX 9724

OTTAWA ONT K1G $5 A 3$

CANADA

TEL: 6139902804

TLF: 613.9913636

TLX:

EML: BROOKSOHUSKYL. STMARYS.CA

COM: 41

\section{BROTEN NORMAN W}

HERZBERG INST ASTROPHYS

NTL RESEARCH COUNCIL

100 SUSSEX OR

OTTAWA ON KIA OR6

CANADA

TEL: 6139936060

TLF: 6139526602

TLX: 0533715

EML:

COM: 40

BROWN DOUGLAS NASON

DPT OF ASTRONOMY

UNIVERSITY OF WASHINGTON

FM 20

SEATTLE WA 98195

USA

TEL: $206543 \quad 4313^{\star} 2888$

TLF :

ILX:

EML:

COM: $25,27,29,36$
BRODERICK JOHN DR

PHYSICS OPT

VIRGINIA TECH

BLACKSBURG VA 24061

USA

TEL: 7032315321

TLF:

TLX: 9103331861 VPIBKS

EML:

COM: 40,51

BRONFMAN LEONAROO OR

DPT DE ASTRONOMIA

UNIVERSIOAD DE CHILE

CASILLA 360

SANTIAGO

CHILE

TEL;'2 $228 \quad 1941$

TLF:

ILX: 440001

EML:

COM: 33,40

BROSCH NOAH DR

WISE OBSERVATORY

TEL AVIV UNIVERSITY

RAMAT AVIV

TEL AVIV 69978

ISRAEL

TEL: $341 \quad 3788$

TLF:

TLX: 342171 VERSY IL

EML: BITNET: H38@TAUNOS.

COM: 28,46

BROUCKE ROGER DR

7203 RUNNING ROPE CIRCLE

AUSTIN TX 7B731

USA

TEL: 5123456435

TLF :

TLX:

EML:

COM: 07

BROWN HARRISON DR

3005 LA MANCHA DR

ALBUQUERQUE NM 87104

USA

TEL:

TLF :

TLX:

EML:

COM: 
BROWN JOHN C PROF

DPT OF PHYSCIS \& ASTRON

UNIVERSITY OF GLASGOW

GLASGOW G12 800

UK

TEL: $413 \quad 305 \quad 182$

TLF :

TLX: 777070 UNIGLA

EML:

COM: 10

\author{
BROWNE IAN W A DR \\ NRAL \\ JOORELL BANK \\ MACCLESFIELO SK11 9DL \\ UK \\ TEL: 47771321 \\ TLX: 36149 \\ EML: \\ TLF : \\ COM: 40
}

BRUCATO ROBERT J

CALTECH

MS 10524

PALOMAR DBS

PASADENA CA 91125

USA

TEL: $818 \quad 356 \quad 4035$

ILF : $818 \quad 568 \quad 1517$

TLX: 675425 OR 188192

EML:

COM:

BRUECKNER GUENTER E DR NAVAL RESEARCH LABORATORY CODE 4160

4555 OVERLOOK AVE SW

WASHINGTON OC 203755000

USA

YEL: 2027673287

TLF:

TLX:

EML:

CON: $10,12,44$

BRUNER MARILYN E DR

LOCKHEEO PALO ALTO RES LB

OPT 9120 BLDG 255

3251 HANOVER ST

PALO ALTO CA 9.4304

USA

TEL: 415 B5B 4023

TLF :

TLX: 346409 LMدL

EML:

Con: $10,12.44$

\section{BROWN ROBERT HAMILTON \\ JPL/CALTECH}

MS 183501

4800 OAK GROVE DR

PASADENa CA 91109

USA

TEL: 8183542517

TLF :

TLX:

EML:

COM: $15,16,50$

BROWNING PHILIPPA OR DPT OF PURE \& APPLIED PHY

UMIST

BOX 88

MANCHESTER M60 100

UK

TEL: 612363311

TLF :

TLX: 666094

EML: MCCPPBEUK.AC.UMRCC.CMS

COM: 10

BRUCH ALBERT

ASTRONOMISCHES INSIITUT

DER UNIVERSITAET MUENSTER

DOMAGKSTR 75

D 4400 MUENSTER

GERMANY

TEL:

TLF :

TLX:

EML:

COM:

BRUGEL EDWARO W OR

CASA

UNIVERSITY OF COLORADO

BOX 391

BOULDER CO 803090391

USA

TEL: 3034924054

TLF :

TLX:

EML: BRUGELECYGNUS. COLORADO.EQU

COM:

BRUNET JEAN-PIERRE DR

OBS MIOI PYRENEES

14 AVE E BELIN

F 31400 TOULOUSE CDX

FRANCE

TEL: 61252101

TLF: ,

TLX: 503776

EML:

COM: 41
BROWN ROBERT L DR

N'RAO

EDGEMONT RD

CHARLOTTESVILLE VA 22901

USA

TEL: 8042960232

TLF:

TLX: 910-997-0174

EML:

EOM:

BRDWNLEE DONALD E PROF

OPT OF. ASTRONOMY

UNIVERSITY OF WASHINGTON

FM 20

SEATTLE WA 98195

USA

TEL: $206543288 B$

ILF :

ILX:

EML:

COM: 15,22

BRUCK HERMANN A PROF

CRAIGOWER

PENICUIK EH2G GLA

UK

TEL: 96875918

TLF :

TLX:

EML:

COM: 25

BRIHWEILER FREO C JR

10102 GARDINER AVE

SILUER SPRING MO 20902

USA

TEL:

TLF :

TLX:

EML:

COM: $29,34 \mathrm{C}, 42,44$

BRUNING DAVID H DR

STELLAR RESEARCH \&

EDUCATION-

BOX 1223

WAUKESHA WI 53187

USA.

TEL:

TLF :

TLX:

EML: B1TNEET: dhbrun010ul kyvx

COM: 12,29
BROWN RONALD D PROF

JPT. OF CHEMISTRY

MONASH UNIVERSITY

WELLINGTON RD

CLAYTON VIC 3168

AUSTRALIA

TEL: 35654550

TLF: 35654597

TLX: AA 32691

EML: CHE265KVVAXC.CC. MONASH, EDU.AU

COM: $34,51 \mathrm{P}$

BROWNLEE ROBERT R DR

LOS ALAMOS NATIONAL LAB

MS $F 670$.

B0X 1663

LOS ALAMOS NM 87545

USA

TEL: $505 \quad 662 \quad 6427$

TLF :

TLX:

EML:

COM: 35,42

BRUCK MARY T OR

ROYAL OBSERVATTORY

BLACKFORO HILL

EDINBURGH EH9 $3 \mathrm{HJ}$

UK

TEL: $316 \quad 673 \quad 321$

T.LF :

TLX: 72383

EML:

COM: 46

BRUMBERG VICTOR A DR

INST OF APPLIEO ASTRONOMY

ACADEMY OF SCIENCES

ZOANOVSKAYA UL 8

197042 ST PETERSBURG

RUSSIA

TEL:

TLF :

TLX:

EML:

COM: $04,07,31 \mathrm{C}$

BRUNINI. ADRIAN DR

IAG

UNIVERISDADE DE SAO PAULO

AV MIGUEL STEFANO 4200

04301 SAO PAULO SP

BRAZIL

TEL:

ILF: $11276 \quad 3848$

TLX: II 56735 IAGM BR

EML: IAGUSPXFPSP. HEPNETALB. BITNET

COM: 07 
BRUNK WILLIAM E OR

NASA HEADQUARTERS

COOE SL OFF SPACE SCIENCE.

400 MARYLAND AVE $S W$

WASHINGTON DC 20546

USA

TEL: $202 \quad 453 \quad 1596$

TLF:

TLX

EML:

COM: 15,16

BRYANT JOHN DR

47 AVE FELIX FAURE.

F 75015 PARIS

FRANCE

TEL: 145577647

TLF:

EML:

COM:

TLX:

BUDDING EDWIN DR

CARTER OBSERVATORY

BOX 2909

WELLINGTON I

NEW ZEALANO

TEL: $04-728-167$

TLK: 30172 NATOBS NZ

EML:

ILF :

COM: 42,46

BUFF JAMES S DR.
DPT OF PHYSICS \& ASTRON
DARTMOUTH COLLEGE
HANOVER NH O3755
USA
TEL:
TLF:
TLX:
EML:
COM:

BUMBA VACLAV OR

ASTRONOMICAL INSTITUTE

EZECH ACADEMY OF. SCIENCES

ONOREJOV OBSERVATORY

CS 25165 ONDREJOV

CZECHOSLOVAKIA

TEL: 20485201

TEF : 20485314

TLX: 121579 ASTR C

EML:

COM: $10,12,44$
BRUSTON PAUL OR

IAS

BP 10

F 91371 VERRIERES BUISSON

FRANCE

TEL: $16920 \quad 1060$

TLF:

TLX:

EML :

COM:

BRZEZINSKI ALEKSANDER OR

SPACE RESEARCH CENTER

POLISH ACAD OF SCIENCES

UL ORDONA'21

$P L \quad 01237$ WARSAW

POLAND

TEL: 410041

TLF: $22 \quad 3765.64$

TLX: 825670 CBKPL

EML: EARN: : "CBKPAN PLEARN"

COM: 19

BUECHER ALAIN DR

OBS MIDI PYRENEES

$9 R$ PONT DE LA MOUETTE

F 65200 BAGNERES BIGORRE

FRANCE

TEL: 62951969

JLF: 62951070

TLX: 531625 OBSPIC $F$

EML:

COM: 10

BUHL DAVIO OR
NASA/GSFC
CODE 693
INFRAREO \& RADIO ASTRO BR
GREENBELT MO 20771
USA
TEL: 3012868810
TLF:
TLX:
EML:
COM:

BUNCLARK PETER STEPHEN DR INSTITUTE OF ASTRONOMY THE OBSERVATORIES

MAOINGLEY RO CAMBRIOGE CB3 OHA UK

TEL: 223337548

TLF :

TLX: 817297 ASTRON E

EML:

COM: 24
BRUZEK ANTON DR

SCHWAIGHOFSTR 7

- 7800 fREIBURG bREISGAU

GERMANY

TEL: 76178522

TLF:

TLX:

EML:

COM: 10,12

BUCCIARELLI BEATRICE OR STSCI

HOMEWOOD CAMPUS

3700 SAN MARTIN DR

BALTIMORE MD 21218

USA

TEL: 3013384570

TLF: $301338 \quad 2617$

TLX: 684910178724

EML: 6559: :BUCC/BUCCESTSCI.EDU

COM: 08

BUECHNER JORG DR

ZNTRLINST.F ASTROPHYSIK

ROSA LUXEMBURG STR 17A

D 1591 POTSOAM

GERMANY

IEL: 23.75105

TLF:

$T L X: 15,471$

EML:

COM: 10,49

BUITRAGO JESUS

INST DE ASTROFISICA

DE CANARIAS

OBS DEL TEIDE

E 38071 LA LAGUNA

SPAIN

TEL: 22262211

ILF:

ILX: 92640

EML:

COM:

\section{BUNNER ALAN N OR}

PERKIN ELMER CORP

MS 897

100 WOOSTER HEIGHTS RD

DANBURY CT O6810 7859

USA

TEL: 2037976339

TLF:

TLX:

EML:

COM: $42,44,48$
BRUZUAL GUSTAVO

CIDA

B0X 264

MERIOA 5101 A

VENEZUELA

TEL: 74639930

TLF:

TLX: 74174 CIOA VC

EML: emsca!cida!bruzualosun. CO COM: $28 \mathrm{C}$

BUCHLER I ROBERT PROF

OPT OF PHYSICS

UNIVERSITY OF FLORIDA

GAINESVILLE FL 32611

USA

TEL: 9043739942.

ILF:

ILX:

EML:

CDM: 35

BUES IRMELA D OR

REMEIS STERNWARTE

STERNWARTSTR 7

D 9600 BAMBERG

GERMANY

TEL: 95157708

TLF:

ILX:

EML: BUESOSTERNWARTE. UNI - ERLANI

COM: 29,36

BUJARRABAL VALENTIN

CTR ASTRON DE YEBES

OAN

APO 148

E 19080 GUADALAJARA

SPAIN

TEL: $11 \quad 223358$

TLF:

ILX:

EML:

COM: 34,40

BUONANNO ROBERTO

OAR

VIA DEL PARCO MELLINI 84

I OOL36 ROMA

ITALY

TEL: 63452656

TLF:

$T L X$ :

EML:

COM: 37 


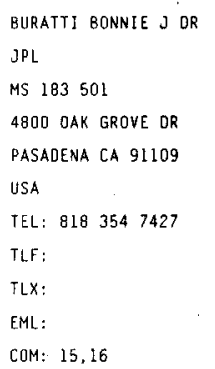

\section{BURBIOGE E MARGARET PROF}

CASS

UCSO

C 011

LA JOLLA CA 920930216

USA

TEL: $619452 \quad 4477$

TLF:

TLX:

EML:

COM: 28

BURGESS ALAN DR

DPT APPLIEO MATHS

\& THEORETICAL PHYSICS

SILVER STREET

CAMBRIOGE CB3 9EW

UK

TEL:

TLF :

TLX:

EML:

COM: 14,34

BURKHART CLAUDE OR OBSERVATOIRE DE LYON

AVE CHARLES ANDRE

F 69561 S GENIS LAVAL COX FRANCE

TEL: $78 \quad 56 \quad 0705$

ILF: $72 \quad 399791$

TLX: $310-226$

EML:

COM: 29

\section{BURNAGE RDBERT}

OHP

F 04870 S MICHEL OBS

FRANCE

TEL: 92766368

TLF :

EML:

COM: 30

ILX: 410690 OHP F

\section{BURSA MILAN OR}

ASTRONOMICAL INSIITUTE

CZECH ACADEMY SCIENCES

1 BUDECSKA 6

CS 12023 PRAHA 2

CZECHOSLOVAKIA

TEL: 2250551

TLF :

ILX: 122486

EML:

COM:
BURBIDGE GEOFFREY, R PROF

CASS

UCSD

C 011

LA JOLLA EA 920930216

USA

TEL: 6194526626

TLF :

TLX:

EML

COM: $28,35,40,47,48$

BURGESS DAVID D PRDF

BLACKETT LABORATORY

IMPERIAL COLLEGE

PRINCE CONSORT RD

LONDON SW7 2BZ

UK

TEL: I $5895111 * 6931$

TLF :

TLX:

EML:

COM:

\section{BURKHEAD MARTIN $S$}

ASTRONOMY QPT

INOIANA UNIVERSITY

SWAIN WEST 319

BLOOMINGTON IN 47405

USA

TEL: 812 3356917

$T L F$ :

TLX:

EML:

COM: 37

BURNS JACK D'NEAL JR DPT PHYSICS \& ASTRONOMY UNIVERSITY OF NEW MEXICD BOO YALE BLVO NE ALBUQUERQUE NM B7131 USA

TEL: $505 \quad 277 \quad 2705$

TLF :

TLX:

EML:

COM: 28

BURSTEIN DAVID

DPT OF PHYSICS

ARIZONA STATE UNIVERSITY TEMPE AZ 85287

USA

TEL:

TLF:

TLX:

EML:

COM: 28,50
BURGER J J DR IR ESA/ESTEC

SSD

BOX 299

NL 2200 AG NOORDWIJK NETHERLANDS

TEL: $17 \quad 1984404$

TLF :

$T L X: 39098$

EML:

COM:

BURKE BERNARD F OR DPT OF PHYSICS MIT RM $26 \quad 335$ B0X 165

CAMBRIOGE MA 02139 USA

TEL: 6172532572

TLF:

TLX: $92-1473$

EML:

COM: $33,34,40,44 C, 51$

BURKI GILBERT PROF OBSERVATOIRE OE GENEVE CHEMIN OES MAILLETTES 51 CH 1290 SAUVERNY SWITZERLAND TEL: 227552611 FLF: 227553983 rLX: 419209 OBS CH EML: burki@obs,unige,ch COM: $27,30 \mathrm{P}$

BURNS JOSEPH A PROF CORNELL UNIVERSITY THURSTON HALL ITHACA NY 14850 USA

TEL: $607 \quad 256 \quad 4875$

TLF:

TLX: 937478

EML: COM: $15,16,20$

BURTON MICHAEL G DR AAO

EPPING LABORATORY

BOX 296

EPPING NSW 2121 AUSTRALIA

TEL: 28681666

TLF: $2876 \quad 8536$

TLX: 23999 AAOSYD AA

EML: PSIXNSSOCA: : AADEPP : :MGB COM: 34 
BURTON W BUTLER DR STERREWACHT

BaX 9513

NL 2300 RA LEIOEN NETHERLANDS

TEL: 71272727

TLX: 39058 ASTRONL

EML:

TLF :

COM: $09,33,34$

BUSKO IVO C DR

INPE

CP 515

12200 S JOSE DOS CAMPOS

BRAZIL

TEL: $123 \quad 22 \quad 9977 \star 392$

TLF: $12321 \quad 8743$

TLX: 011-33530 INPE BR

EML

COM: 27

BUTCHER HARVEY $R$ PROF DR

KAPTEYN ASTRONOMICAL INST

BOX 800

NL 9700 AV GRONINGEN

NETHERLANDS

TEL: 59 08 1963]

TLX: 53767 KSWRO NL

EML:

ILF:

COM: 28

BUTLER DENNIS OR

YALE UNIVERSITY OBS

YALE STATION

BOX 2023

NEW HAVEN CT 06520

USA

TEL:

TLF:

TLX:

EML:

COM: 27,37

BYARD PALL L DR

OPT OF ASTRONOMY

OHIO STATE UNIVERSITY

174 W 18TH AVE

COLUMBUS OH $43210 \quad 1106$

USA

TEL: $614 \quad 422 \quad 1773$

TLF:

TLX:

EML:

COM:
BURTON WILLIAM M

RUTHERFORO APPLETON LAB

SPACE \& ASTROPHYSICS DIV

BLDG R25/R68

CHILTON DIOCOT OXII OQX

UK

TEL: 23521900

TLF:

TLX: 83159

EML:

COM: 44

BUSOH LUCIÒ M OR

OSS ASTRONOMICO DI PADOVA

VIC DELL OSSERVATORIO 5

135122 PADOVA

ITAL

TEL: $49 \quad 66 \quad 1499$

TLX: 432071 ASTROS I

EML: BUSONEASTRPO. INFNET

- TLF:

Con:

BUTCHINS SYDNEY ADAIR

DPT OF CIVIL AVIATION STU

FAC OF MATHS

THE MINORIES

TOWER HILL EC3N 2JY

UK

TEL: 717227344

TLF :

TLX:

EML:

COM:

BUTLER KEITH DR

INSTITUT FUR ASTRONOMIE

UNO ASTROPHYSIK

SCHEINERSTR 1

O BOOO MUENCHEN BD

GERMANY

TEL: 89989021

TLF :

TLX:

FML :

COM: 29

BYKOY MIKĹE F DR

ASTRONOMICAL INSTITUTE

UZBEK ACADEMY OF SCIENCES

700000 TASHKENT

UZBEKISTAN

TEL:

TLF :

TLX:

EML:

COM: 08
IBUSEOMBE WILLIAM PROF

OPT PHYSICS \& ASTRONOMY

NORTHWESTERN UNIVERSITY

OEARBORN OBSERVATORY

EVANSTON IL 60208

USA

TEL: 3124917527

TLF:

TlX:

EML:

COM: 45,46

BUSSO MAURIZIO

OSS ASTRONOMICO DI TORINO

ST OSSERVATORIO 20

I 10025 PINO TORINESE

ITALY

TEL: 11841067

TLF :

TLX: 213239 TO ASTR I

EML:

COM: 42

BUTI BIMLA PROF

PHYSICAL RESEARCH LAB

NAVRANGPURA

AHMEOABAD 380009

INOIA

TEL: $272 \quad 46 \quad 2129$

TLF: 272445292

TLX: 1216397 PRL IN

EML: BUTIOPRL.ERNET. IN

COM: $49 \mathrm{P}$

BUTTERWORTH PAUL

NASA/GSFC

CODE 633

GREENBELT MD 20771

USA

TEL: 3012863995

TLF:

TLX:

EML:

COM: 44

BYLEVELD WILLEM OR

OMNIVERSUM SPACE THEATRE

PRES KENNEQYLAAN 5

NL 2517 JK THE HAGUE

NETHERLANDS

TEL: 70547479

TLF: $70 \quad 52 \quad 4280$

TLX:

EML:

COM:
BUSER ROLANO DR

ASTRONOMISCHES INSTITUT

UNIVERSITAET BASEL

VENUSSTRASSE ?

CH 4102 BINNINGEN

SWITZERLAND

TEL: $6122^{\circ} 7711$

TLF:

TLX:

EML:

COM: 25,45

BUTA RONALO J DR

ASTRONOMY OPT

UNIVERSITY OF TEXAS

RLM 15308

AUSTIN TX 78712 1003

USA

TEL: 5124713466

TLF :

TLX: $910 \quad 874 \quad 1351$

EML:

COM: 28

BUTLER C JOHN DR

ARMAGH OBSERVATORY

COLLEGE HILL

ARMAGH BT6I 906

UK

TEL: 861522928

TLF :

TLX: 747937 ARMOBS 6

EML:

COM: 27,44

BUYUKLIEY GEORGI OR

NTL ASTRONOMICAL OBS

BULGARIAN ACAD SCIENCES

BOX 136

BG 4700 SMOLJAN

BULGARIA

TEL: 7341599

TLF ;

TLLX: 48446 SST RZNBG

EML:

COM: 10

BYRO GENE G OR

DPT OF PHYSICS \& ASTRON

UNIVERSITY OF ALABAMA

BOX 1921

UNIVERSITY AL 354870324

USA

TEL: 2053485050

TLF :

TLX:

EML:

COM: 28,37 


BYRNE PATRICK B OR
ARMAGH OBSERVATORY
COLLEGE HILL
ARMAGH BT61 $90 G$
UK
TEL: 861522928
TLF:
TLX: 747937 ARMOBS G
EML: PBBESTAR. ARM. GUB. AC.UK.
COM: 27

CACEIARI CARLA OR

OSS ASTRONOMICO

UNIVERSITA OI BOLOGNA

CP 596

I 40100 BOLOGNA

ITALY

TEL: $51 \quad 25 \quad 9301 * 9401$

TLF: 51259407

TLX: 520634 INFNBO I

EML: CACCUARULASTBOI. IMFMET

CDM:

CAHN JULIUS H PROF

DPT OF ASTRONOMY

UNIVERSITY OF ILLINOIS

1011 W SPRINGFIELD AVE

IRBANA IL 61801

USA

TEL: 2173333090

TLF:

$T L X:$

EML:

CaM:

\section{CALOWELL JOHN JAMES}

DPT OF PHYSICS

YORK UNIVERSITY

4700 KEELE ST

NORTH YORK ON M3J IP3

CANADA

TEL: 4167362100

TLF : 4167365386

TLX: 06524736

EML:

COM: 16

CALDI VITTORIA OR

IAS

CNR

CP 57

I DOO44 FRASCATI

ITALY

TEL: 69425654

TLF: 69416847

TLX: 610261 CNR FRA

EML:

COM: 35,37
BYSTROVA NATALIJA Y OR

PULKOVO OBSERVATORY

acAaemy OF SCIENCES

10 KUTUZOV QUAY

196140 ST PETERSBURG

RUSSIA

TEL: $297-9452$

TLF:

TLX:

EML:

COM: 34

CACCIN BRUNo

DPT DI FISICA

VIA RAIMONDO SNC

UNIVERSTTA TOR VERGATA

I 00173 ROMA

ITALY

TEL: 679792323

TLF:

TLX: 626382 FIUNTV

EML:

COM: 38

CAILlAULLT JEAN PIERRE DR

OPT PHYSICS \& ASTROHOMY

UNIVERSJTY OF GEORGIA

ATHENS GA 30602

USA

TEL: $404542 \quad 2883$

TLF:

TLX: 490999.1619

EML:

COM:

CALlanan PAUl oR

DPT OF ASTROPHYSICS

UNIVERSITY OF OXFORD

KEBLE RD

OXFORO OXI $3 R H$

uk

TEL: 865273293

TLF: 865273418

TLX: 83295 NUCLOX G

EML: UK.AC.OX.ASTRO (JANET)

COM: 42

CALVANI MASSIMO DR

DPT OI ASTRONOMJA

UNIVER'SITA DI PADOVA

VIC DELL OSSERVATORIO 5

135122 PADOVA

ITALY

TEL: $49.66 \quad 1499$

TLF :

TLX: 430176 UNPAOU 1

EML:

COM: 47
CABRITA EZEQUIEL DR

OBS. ASTRONOMICO DE LISBOA

TAPADA DA LISBDA

P 1300 LISBOA

PORTUGAL

TEL: $637351-634669$

TLF :

TLX:

EML:

COM: 26

CADEZ ANGREJ OR"

DPT OF PHYSICS

UNIVERSITY OF LJUBLJANA

JADRANSKA 19

YU 61000 LJUBLJANA

YUGOSLAVIA

TEL: 61265061

TLF: 61217281

TEX:

EML: ANDREJ .CADEZUUN - LJ AC. MAIL. YU COM: 10,12

COM:

CALAME ODILE DR

OCA CERGA

AVE COPERNIC

F 06130 GRASSE

FRANCE

TEL: 93365849

TLF:

TLX: 470865

EML:

COM: $07,16,20$

CALLEBAUT OIRK $K$ OR

DPT OF PHYSICS

UNIVERSITY OO ANTWERPEN

UNIVERSITEITSPLEIN 1

B 2610 ANTWERPEN WILRIJK

BELGIUM

TEL: · 38202457

TLF: 38202245

TLX: 33646

EML:

COM: 35,37

CALVET NURIA DR

CIDA

BOX 264

MERIDA 5101 A

VENEZUELLA

TEL: 74639930

TLF:

TLX: 74174 CIDA VC

EML:

COM: 46
CACCIANI ALESSANDRO PROF

DPT DI FISICA

UNIVERSITA DI ROMA

PA MORO 2

I 00185 ROMA

ITALY

TEL: 64976265

TLF:

TLX: 613255 INFNRO

EML:

COM:

CADEZ VLADIMIR

INSTITUTE OF PHYSICS

BOX 57

YU 11001 BEOGRAD

YugosiaVia

TEL: 11212219

TLF:

TLX: 11002 INFIZ YU

EML:

(a)

CALOWELL JOHN A R

SAAO

BOX 9

OBSERVATORY 7935

SOUTH AFRICA

TEL: 021470025

TLF:

TEX: $57-20309$

EML:

COM: 33

CALLY PAUL S OR

dPT OF MATHEMATICS

MONASH UNIVERSITY

WELLINGTON RD

CLAYTON VIC 3168

AUSTRALIA

TEL: 35654471

TLF:

TLX: MONASH AA 32691

EML: apm150 fevaxc.cc.monash.edu. au

COM: 10

CALVIN HILLIAM H DR

DPT OF PHYSICS

UNIVERSITY OF WASHINGTON

FM 15

SEATTLE WA 98195

USA

TEL: 2063281192

TLF: 2065433041

TLX:

EML: WCALVINUU. WASHINGTON. EDU

COM: 51 
CALYO MANUEL

DPT DE ASTRONOMIA

UNIVERSIDAD DE ZARAGOZA

E 50009 ZARAG0ZA

SPAIN

TEL: 357011

TLF :

TLX:

EML

COM

CAMERON ANOREW COLLIER DR

ASTRONOMY CENTRE

UNIVERSITY OF SUSSEX

FALMER

BRIGHTON BNI 9QH

UK

TEL: $273 \quad 67 \quad 8117$

TLF :

TLX: 877159 BHVTXS 6

EML:

COM: 27

CAMPBELL ALISON DR

DPT PHYSICS \& ASTRONOMY

JOHNS HOPKINS UNIVERSITY

CHARLES \& 34TH ST

BALTIMORE MD 21218

USA

TEL: 3013385186

TLF: 3013388260

TLX:

EML: AWCOSTSCI,BITNET SCIVAX: :AWC

COM: 26

CAMPBELL JAMES W
ROYAL OBSERVATORY
BLACKFORO HILL
EOINBURGH EHS $3 H \mathrm{HJ}$
UK
TEL:
TLX:
EML:
TLF:
COM:

CAMPUSANO LUIS E

DPT OE ASTRONOMIA

UNIVERSIOAD DE CHILE

CASILLA 360

SANTIAGO

CHILE

TEL: 22294101

TLF:

TLX: 440001

EML:

COM: 28,51
CAMARENA BADIA VICENTE PR OPT MATEMATICA APLICADA

UNIVERSIDAD DE ZARAGOZA

E 50009 ZARAGOZA

SPAIN

TEL:

TLF :

TLX:

EML:

COM:

CAMEROH LUZIUS MARTIN

ASTRONOMISCHES INSTITUT

UNIVERSITAET BASEL

VENUSSTRASSE ?

CH 4102 BINNINGEN

SWITZERLAND

TEL: 61227711

TLF :

TLX:

EML:

COM: 28

CAMPBELL BELVA G S OR

OPT PHYSICS \& ASTRONOMY

UNIVERSITY OF NEW MEXICD

800 YALE BLVD NE

ALBUQUERQUE NM 87131

USA

TEL: $505 \quad 277 \quad 5148$

TLF :

TLX:

EML: BITNET: BEL OUNMB

COM:

CAMPBELL MURRAY $F$

OPT PHYSICS \& ASTRONOMY

COLBY COLLEGE

WATERVILLE ME 04901

USA

TEL: $207 \quad 8723251$

TLF:

ILX:

EML:

COM: 44

CANAL RAMON M OR

DPT FISICA DE ATMOSFERA

UNIVERSIDAD DE BARCELONA

AVD DIAGONAL 645

E OBO2B BARCELONA

SPAIN

TEL:

TLF:

TLX:

EML:

COM: $35 \mathrm{C}$

\author{
CAMENZIND MAX DR \\ LANDESSTERNWARTE \\ KOENIGSTUHL \\ 0 6900 HEIOELBERG I \\ GERMANY \\ TEL: $62 \quad 21509262$ \\ TLF : $62 \quad 21509202$ \\ ILX: 461153 \\ EML: AB4AOHOURZ1. BITNET \\ COM: 48
}

CAMERON WINIFRED S MRS

LA RANEHITA DE LA LUNA

BLOG 26

200 ROJO DR

SEDONA AZ 86336

USA

TEL:

TLF :

TLX:

EML:

COM: 16

CAMPBELL BRUCE OR

4537 RITHEWOOO PLC

VICTORIA BC VX 4.39

CANADA

TEL:

TLF :

TLX:

EML:

COM: $29,30,51 \mathrm{C}$

CAMPINS HLMBERTO DR

DPT OF ASTRONOMY

UNIVERSITY OF FLORIOA

211 SSRB

GAINESVILLE FL 3261$]$

USA

TEL: 9043923066

TLF:

TLX:

EML: compinseastro.ufl.edu.

COM: 15

CANAVAGgia RENEE DR

oBservatoIRE DE PARIS

61 AVE OBSERVATOIRE

F 75014 PARIS

FRANCE

TEL:- $143 \quad 20 \quad 1210$

TLF :

TLX: 270776 OBS F

EML:

COM:
CAMERON ALASTAIR G W PROF CENTER FOR ASTROPHYSICS

HCO/SAO

6O GAROEN ST

CAMBRIDGE MA 02138

USA

TEL: 6174955374

TLF :

TLX:

EML:

COM: 35,48

CAMICHEL HENRI DR

24 AVE C FLAMMARION

F 31500 TOULOUSE

FRANCE

TEL: $61 \quad 489691$

TLF :

TLX:

EML:

COM: 16

CAMPBELL DONALD B

CORNELL UNIVERSITY

SPACE SCIENCES BLDG

ITHACA NY 14853

USA

TEL: $607255 \quad 5274$

TLF :

TLX: 932454

EML:

COM: 16

CAMPOS L M BRAGA DA COSTA INST SUPERIOR TECNICO

AVE ROVISCO PAIS

P 1096 LISBOA CODEX

PORTUGAL

TEL: 800525

TLF :

TLX: 63423 ISTUTL $P$

EML:

COM:

CANDY MICHAEL P MR

PERTH OBSERVATORY

BICKLEY WA 6076

AUSTRALIA

TEL:

TLF :

TLX:

EML:

COM: $06,07,15,20$ 


CANE HILARY VIVIEN
OPT OF PHYSICS
UNIVERSITY OF TASMANIA
GPO BOX $252 C$
HOBART TAS 7001
AUSTRALIA
TEL: 2202401
TLF: 2202186
TLX: AA58150
EML: HILARY, CANE
COM: 10,33

CANNON WAYNE H DR
DPT PHYS/EARTH \& ATM SCI
YORK UNIVERSITY
4700 KEELE ST
DOWNSVIEW ON M3J 193
CANADA
TEL: 4166676410
TLF:
TLX: O65 24736
EML:
COM:
CAD LIHONG
PURPLE MOUNTAIN OBSERV
CAS
NANJING
CHINA PR
TEL: 25 46700
TLF:
TLX:
EML:
COM:

CAPELATO HUGO VICENTE DR INPE

C.P 515

$12201 S$ JOSE DOS CAMPOS

BRAZIL

TEL: 123229977

ILF:

LLX: 1233530 INPE BR

EML:

COM:

CAPPA DE NICOLAU CRISTINA
IAR
CC 5
1894 VILLA ELISA (BS AS).
ARGENTINA
TEL: 2143793
FLX:
EML:
TLF:
COH: 34

CANFIELO RICHARO C OR
INSTITUTE FOR ASTRONOMY
UNIVERSITY OF HAWAII
2680 WOOOLAWN OR
HONOLULU HI 96822
USA
TEL:
TLF:
TLX:
EML:
COM:

CANTO JORGE DR INSTITUTO OE ASTRONOMIA UNAM

APDO POSTAL 70-264

04510 MEXICO DF

MEXICO

TEL： 5485305

TLF :

TLX: 1760155 CIC ME

EML:

COM: 34 .

CAO SHENGLIN
OPT OF ASTRONOMY
BEIJING NDRMAL UNIVERSITY
BEIJING 100875
CHINA PR
TEL: 12012255
TLF:
TLX: 222701
EML:
COM:

CAPEN CHARLES
SOLIS LACUS OBSERVATORY
RT 2
BOX 262 E
CUBA MO 65453
USA
TEL:
TLF:
TLX:
EML:
COM:

CAPPELLARO ENRICO DR

oss astronomico di padova

VIC DELL OSSERVATORIO 5

I 35122 PADOVA

ITALY

TEL: $4966 \quad 1499$

TLX: 43207I ASTROS I

EML: SPAN:ASTRPO: : CAPPELLARO

TLF:

COM:
CANIZARES CLAUDE R PROF CENTER FOR SPACE RESEARCH MIT

\section{RM 37-24L}

CAMBRIOGE MA 02139

USA

TEL: $617 \quad 2537480$

TLF: 6172530861

TLX: 921473 MITCAM

EML: brendapspace.mit.edu

COM:

CANTU ALBERTO M OR

IST CIBERNETICA/BIOFISICA

C.NR

I 16032 CAMOGLI

ITALY

TEL: 185770646

TLF:

TLX:

EML:

COM:

CAPACCIOLI MASSIMO DR DPT DI ASTRONOMIA

UNIVERSITA DI PADOVA

VIC DELL OSSERVATORIO 5

I 35122 PADOVA

ITALY

TEL: $49 \quad 66 \quad 1499$

TLF:

TLX: 430176 INDAPU [

EML:

COM: 28

CAPITAINE NICOLE

OBSERVATOIRE DE PARIS

61 AVE OBSERVATOIRE

F 75014 PARIS

FRANCE

TEL: $140 \quad 512231$

TLF:

TLX: 270776 OBS F

EML:

COM: $04,19 \mathrm{C}$

CAPRIA MARIA TERESA QR

IAS

REPARTO OI PLANETOLOGIA

VIA DELL'UNIVERSITA II

I 00185 ROMA

ITALY

TEL: 64456951

TLF: 64454969

TLX:

EML: TERESAQIRMIAS

COM: 15,16 .
CANNON RUSSELL D DR

AAO

BDX 296

EPPING NSW 2121

AUSTRALIA

TEL: $2868 \quad 1666$

TLF:

TLX: 23999 AAOSYD

EML:

COM: 28,37

CAO CHANGXIN

NANJING ASTRONOMICAL

INSTRUMENT FACTORY

NANJING

CHINA PR

TEL: 2546191

TLF :

TLX: 34136 GLYNJ $\mathrm{CN}$

EML:

COM: 09

CAPACCIONI FABRIZIO OR.

IAS

REPORTO OI PLANETOLOGIA

VIA OELL'UNIVERSITA II

I 00185 ROMA

ITALY

TEL: $6 \quad 4456951$

TLF: 64454969

TLX:

EML:

COM: 15

CAPLAN JAMES

OBSERVATOIRE DE MARSEILLE

2 PLACE LE VERRIER

F 13246 MARSEILLE COX 04

FRANCE

TEL: 91959088

TLF :

TLX:

EML:

COM: 34

CAPRIOLI GIUSEPPE PROF

DAR

VIA TRIONFALE 204

I 00136 ROMA

ITALY

TEL: 6347050

TLF:

TLX:

EML:

COM: 31 


CAPRIOTTI EUGENE R DR
OPT OF ASTRONOMY
OHIO STATE UNIVERSITY
5058 ALPHEUS SMITH LAB
COLUMBUS OH $43210 \quad 1106$
USA
TEL: 6144221773
TLF:
TEX:
EML:

CAPUTO FILIPPINA DR
IAS
CNR
CP 67
I. 00044 FRASCATI
ITALY
TEL: 69425651
TLF: 69416847
TLX: 610261 CNR FRA
EML:
COM: 35,37
CARDELLI JASON A OR
OPT OF ASTRONOMY
UNIVERSITY OF WISCONSIN
475 N CHARTER ST
MADISON WI 53706
USA
TEL: 608 262 7921
TLF:
TLX:
EML: MAORAF: : CARDELLI
COM: 34 .

CARDUS ALMEDA J o MR OBSERVATORIO DEL EBRO. E 43520 RQQuetes

SPAIN.

TEL: 77500511

TLF:

EMt:

COM:

ILX:

CARLBERG RAYMONO GARY OR
OPT OF PHYSICS
YORK UNIVERSITY
4700 KEELE ST
TORONTO ON MJJ IF3
CANADA
TEL: 4166673851
TLF:
TLK: 06524736
EML:
COM:
CARLSSON MATS OR
INST THEORET ASTROPHYSICS
UNIVERSITY OF OSLO
BOX 1029
N 0315 BLINOERN OSLO 3
NORWAY
TEL:
TLF:
TLX:
EML:
COM: 36

\section{CARESTIA REINALDO A DR} OBSERVATORIO ASTRONOMICO FELIX AGUILAR

AV BENAVIOEZ 2175 OESTE 5400 SAN JUAN

ARGENTINA

TEL: $64 \quad 23 \quad 1615$

TLF:

TLX:

EML:

COM: 08

CARLETON NATHANIEL P DR CENTER FOR ASTROPHYSICS HCO/SAO

60 GARDEN ST

CAMBRIOGE MA 02138

USA

TEL: 6174957405

ILF :

TLX: 921428 SATELLITE CAM

EML:

COM:

\section{CARNEY BRUCE WILLIAM}

OPT PHYSICS \& ASTRONOMY UNIVERSITY NORTH CAROLINA 204 PHILLIPS HALL 039A CHAPEL HILL NC 27514 USA

TEL: 9199623023

ILF:

ILX:

EML:

COM: $25.29,30,37$

CAPUZZO DOLCETTA ROBERTO
ISTITUTO ASTRONOMICO
UNIVERSITA DI ROMA
VIA G M LANCISI 29
I 00161 ROMA
ITALY
TEL: 6867525
TLF:
TLX:
EML:
COM: 34,37

CARDINI DANIELA OR

IAS

CNR

CP 67

I 00044 FRASCATI

ITALY

TEL: 69425655

TLF: 6.9416847

ILX: 610261 CNR FRA

EML: DANIELAEIRMIAS

CGM: 48

CARGILL PETER J IR

NAVAL RESEARCH LABORATORY

CODE 4790

4555 OVERLOOK AVE SW

WASHINGTON DC 203755000

USA

TEL: 2027674978

TLF: 2027670631

ILX:

EML: CARGILL®PPPI1. NRL. NAVY.MIL

COM: 10

CARLOVIST PER A OR

DPT OF PLASMA PHYSICS

ROYAL INST OF TECHNOLOGY

S 10044 STOCKHOLM 70

SWEOEN

TEL: $87 \quad 877697$

TLF ;

ILX:

EML:

COM: 10

CAROFF LAWRENCE J

NASA AMES RESEARCH CTR

MS 2456

SPACE SCIENCE OIV

MOFFETT FIELD CA 94035

USA

TEL: $415694 \quad 5523$

TLF:

TLX:

EML:

COM:
CARANICOLAS NICHOLAS DR. DPT OF ASTRONOMY UNIVERSITY THESSALONIKI GR 540 O6 THESSALONIKI GREECE

TEL: 3199 1357/59

TLF :

TLX:

EML:

COM: 07

CARDONA OCTAVIO DR INAOE

TONANTZINTLAZ

APDO POSTAL 216 y 51

72000 PUEBLA PUE

MEXICO

TEL: $22 \quad 470500$

TLF:

TLX:

EML:

COM:

CARIGNAN CLAUDE oR.

DPT DE PHYSIQUE UNIVERSTTE OE MONTREAL.

CP $612 B$ SUCC A

MONTREAL OC H3C $3 J 7$

CANADA

TEL: 5143437355

FLF: 5143432071

ILX: 05562425

EML: CARIGNAN CCC. UMONTREAL, CA COM: 28

CARLSON JOHN B

ARCHAEOASTRONOMY CENTER

$B O X X$

COLLEGE PARK MO 20740

USA

TEL: $301864 \quad 6637$

TLF :

TLX:

EML:

COM: 41,51

CAROUBALOS C A PROF

LAB ELECTRONIC PHYSICS NYL UNIVERSITY OF ATHENS KTHRIA TYPA-ILISSIA GR ATHENS 144 GREECE

TEL: $17244 \quad 096 / 11119$

ILF:

TLX: 215530 GBSA GR

EML:

COM: 40 
CARPENTER KENNETH G OR NASA/GSFC

COOE 681

GREENBELT MO 20771

USA

TEL: 3012863453

TLF

TLX: $89675 \mathrm{~K}$ CARPENTER

EML: SPAN : $6172:$ : HRSCARPENTER

COM: 29,44

\begin{abstract}
CARR BERNARO JOHN
\end{abstract}
SCHOOL OF MATHEMATICAL SC DUEEN MARY/WESTFIELO COLL

MILE ENO RO

LONDON EI 4NS

UK

TEL: 1980481

TLF

TLX:

EML:

COM: 47

CARRASCO LUIS DR

INSTITUTO OE ASTRONOMIA

UNAM

APDO POSTAL 70-264

04510 MEXICO OF

MEXICO

TEL: $905-54 \theta-5305$

TLF:

TLX:

EML:

COM: 33

CARSMARU MARIA M DR

ASTRONOMICAL OBSERVATORY CUTITUL DE ARGINT 5

$80 \times 28$

R 75212 BUCHAREST

RUMANIA

TEL: 236892

TLF:

TLX: 11882 ASTRO R

EML:

COM: 16

EARTER WILLIAM EUGENE

NES

N/CG 114

ROCKYILLE MO 20852

USA

TEL: 3014438423

TLF:

TLX:

EML:

COM: 19,31
CARPENTER LLOYD OR

13902 RESIN CT

BOWIE MD 20720

USA

TEL:

TLF :

EML:

COM:

TLX:

CARR THOMAS O PROF
DPT OF ASTRONOMY
UNIVERSITY OF FLORIOA
211 SSRB
GAINESVILLE FL 32611
USA
TEL: 9043922066
TLF:
TLX:
EML: tacarreuffsc,bitnet
COM: 40,51

CARROLLL P KEVIN PROF

OPT OF PHYSICS

UNIVERSITY COLLEGE

BELFIELO

DUBLIN 4

IRELAND

TEL: 1693244

TLF :

TLX:

EML:

COM: 14,44

CARSON T R DR

DPT OF PHYSICS \& ASTRON

UNIVERSITY OF ST ANDREWS

NORTH HAUGH

ST ANOREWS FIFE KY16 9SS

TEL: 33476161

TLF: $334^{1} 744 \quad 87$

TLX:

EML:

COM: 35.36

CARUSI ANDREA

IAS

REPARTO DI PLANETOLOGIA

VIA OELL'UNIVERSITA II

I 00185 ROMA

ITALY

TEL: 64456951

TLF: $644 \quad 54969$

TLX: 610261 CNRFRA

EML: CARUSIOIRMIAS

COM: $15,20 \mathrm{P}, 22$
CARPINO MARIO OR OSS ASTRONOMICO OI BRERA

VIA BRERA 28

I 20121 MILAND

ITALY

TEL: 2874444

TLF: $272 \quad 00 \quad 1600$

TLX:

EML: CARPINO@ASTMIB.ASTRO.IT

COM: 07

CARRANZA GUSTAVO J DR
LAPRIOA 880
5000 COROOBA
ARGENTINA
TEL: 5140613
TLF:
TLX:
EML:
COM: 28

CARRUTHERS GEORGE R DR NAVAL RESEARCH LABORATORY CODE 7123

SPACE SCIENCE DIVISION WASHINGTON OC 203755000 USA

TEL: $202 \quad 7672764$

TtF :

TLX:

EML: :

COM: 15.34

CARSWELL ROBERT $F$ OR

INSTITUTE OF ASIRONOMY

THE OBSERVATORIES

MAOINGLEY RD

CAMBRIDGE CB3 OHA

UK

TEL: 22362204

TLF:

TLX: 817297 ASTRON G

EML:

COM: 28

CARVER JOHN H PROF

AUSTRALIAN NTL UNIVERSITY

RES SCHOOL PHYS SCIENCE

BOX 4

CANBERRA aCt 2601

AUSTRALIA:

TELL: $62 \quad 492 \quad 476$

ILF :

TLX: 62615 RPHYS

EML:

COM: 14,44
EAROUILLAT JEAN-MICHEL DBS MIDI PYRENEES

14 AVE E BELIN

F 31400 TOULOUSE CDX

FRANCE

TEL: 61252101

TLF :

TLX: 530776 OBSTLSE

EML:

COM: 30

CARRASCO GUILLERMO DR DPT OE ASTRONOMIA UNIVERSIOAO OE CHILE CASILLA 360

SANT IAGO

CHILE

TEL: 22294002

TLF :

TLX: 440001

EML:

COM: 08

CARSENTY URI DR

OLR. NE-OE-PE

OBERPFAFFENHOFEN

D 8031 WESSLING

GERMANY

TEL: 8153281328

TLF : 81532476

TLX :

EML: SPAN: DOEPES: : Carsenty

COM: 15

CARTER DAVIO OR

MOUNT STROMLO \& SIOING

SPRING OBSERVATORIES

PRIVATE BAG

WODEN PO ACT 2606

AUSTRALIA

TEL: $62 \quad 8 B \quad 1111$

TLF :

TEX: $62270 \mathrm{AA}$

EML:

COM: 28

CASANOVAS JUAN OR

VATICAN OBSERVATORY

I Q0120. VATICAN CITY

VATICAN CITY STATE

TEL: $6 \quad 698 \quad 3411 / 5266$

ILF:

TLX: 2020 VATOBS VA

EML:

COM: 


CASERTANO STEFANO OR
OPT OF ASTRONOMY
UNIVERSITY OF ILLINOIS
IOLI W SPRINGFIELO AVE
URBANA IL 61801
USA
TEL: 2173339390
TLF:
TLX:
EML: STEFANDERIGEL.ASTRO.ULUC. EDU
COM: 28

CASSE MICHEL. DR

CEA CEN

DAPNIA/SAP

$B P 2$

F 91191 GIF/YVETTE COX FRANCE

TEL:

TLF:

TLX:

EML:

COM: 48

CASTELAZ MICHEAL W OR
OPT OF PHYSICS
E TENMESSEE STATE UNIV
BOX
JOHNSON CITY TN $=37614$
USA
TEL: 6154617064
TLF:
TLX:
EML: R29CASTZOETSU. BITNET
COM: 25

\section{CASTETS ALAIN DR}

OBSERVATOIRE DE GRENOBLE

CERMO/ASTROPHYSIDUE

Bp $53 x$

F 380415 MARTIN HERES CD

FRANCE

TEL: 76514786

TLF: 76448821

TLX:

EML: EARN: CASTETSEFRAG5]

COA: 40

\section{CATALA POCH M A \\ OPT FISICA DE ATMOSFERA \\ UNIVERSIDAD, DE BARCELONA \\ AVD DIAGONAL 645 \\ E 08028 BARCELONA \\ SPAIN \\ TEL: $33307311 * 244$ \\ TLF: \\ TLX: \\ EML:}

COM: 46

\author{
CASH WEBSTER C JR \\ LASP \\ UNIVERSITY OF COLORADO \\ BOX 392 \\ BOULOER CO 803090392 \\ USA \\ TEL: 3034928208 \\ TLF: \\ TLX: \\ EML: \\ COM: 48 \\ CASSINELLI JOSEPH P OR \\ DPT OF ASTRONOMY \\ UNIVERSITY DF WISCONSIN \\ $475 \mathrm{~N}$ CHARTER ST \\ MADISON WT 53706 \\ USA \\ TEL': $608 \quad 262 \quad 1752$ \\ TLF: \\ TLX: 265452 UOFWISC MES \\ EML: \\ COM: 36
}

CASTELLANI VITTORIO PROF ISTITUTO ASTRONOMICO

UNIVERSITA DI ROMA

VIA GM LANCISI 29

I 00161 ROMA

ITALY

TEL: 6867525

TLF:

TLX:

EML:

COM: 35,37

CASTOR JOHN I QR ,

LAWRENCE LIVERMORE LAB

L 23

BOX 808

LIVERMORE CA 94550

USA

TEL: 4154224664

TLF:

TLX: 910-3868339 LLNL

EML:

COM: 35,36

Catalan manuel dR

REAL INST' Y OBSERVATORIO

oE la armada

CECILIO PUJAZON S/N

E 11110 SAN FERNANDO

SPAIN

TEL: $56 \quad 88 \quad 3548$

TLF :

TLX: 76108

EML:

COM: 04.08 .31
CASOLI FABIENNE DR

RADIOASTRONOMIE ENS

24 RUE LHOMOND

F 75231 PARIS CDX 05

FRANCE

TEL: 143291225

TLF:

TLX: 202601

EML: CASOLI TRRULMII

COM:

CASTAGNINO MARIO DR
IAFE
CC 67 SUC 28
1428 BUENOS AIRES
ARGENTINA
TEL: 17816755
TLF: 18144299
TLX: 17181 VERBA
EML: CASTAGNINO@IAFE EDU.AR
COM: 47

\section{CASTELLI FIORELLA OR}

OAT

BOX SUCC TRIESTE 5

VIA TIEPOEO 11

I 34131 TRIESTE

ITALY

TEL: 40793921

TLF :

TLX: 461137 OAT I

EML:

COM: 29

CASHELL JAMES L DR

CSIRO

DIVISION OF RADIOPHYSICS

BOX 76

EPPING NSW 2121

AUSTRALIA

TEL: $2868 \quad 0222$

TLF :

ILX: 26230 ASTRO AA

EML: JCASWELLLATNF.CSIRO.AU

COM: 33.34 .40

CATALANO FRANCESCO A DR

IST OI ASTRONOMIA

CITTA UNIVERSITARIA

VIA A OORIA 6

195125 CATANIA

ITAEY

TEL: 95330533

TLF:

ILX: 970359 ASTRCT I

EML:

COM: 36

\author{
CASSATELLA ANGELO OR \\ IAS \\ CNR \\ CP 67 \\ 100044 FRASCATI \\ ITALY \\ TEL: 69425655 \\ TLF : 69416847 \\ TLX: \\ EML: \\ COM: $29 C$
}

CASTANEOA HECTOR INST DE ASTROFISICA DE CANARIAS OBS DEL TEIDE E 38200 LA LAGUNA SPAIN

TEL: 22.262211

TLF: $22 \quad 263005$

FLX: $926 \dot{6} 40$ IAC E

EML:

COM: 34

CASTELLI JOMN $P$

A F GEOPHYSICS LABORATORY

SPACE PHYSICS DIV PHP

HANSCOM AFB

BEDFORO MA 01731

USA

TEL:

TLF :

ILK:

EML:

COM:

CATALA CLAUDE DR OBSERVATOIRE DE PARIS SECTIDN DE MEUDON F 92195 MEUDON PQL CDX FRANCE

TEL: $145 \quad 07 \quad 7663$

TLF:

TLX: 204464

EML:

COM: 29.36

CATALANO SANTO OR IST OI ASTRONOMIA CITTA UNIVERSITARIA VIA A DORIA 6 I 95125 CATANIA

ITALY

TEL: $95 \quad 330533$

TLF:

TLX: 970359 ASTRCT I

EML:

COM: $16,29,36,42$ 


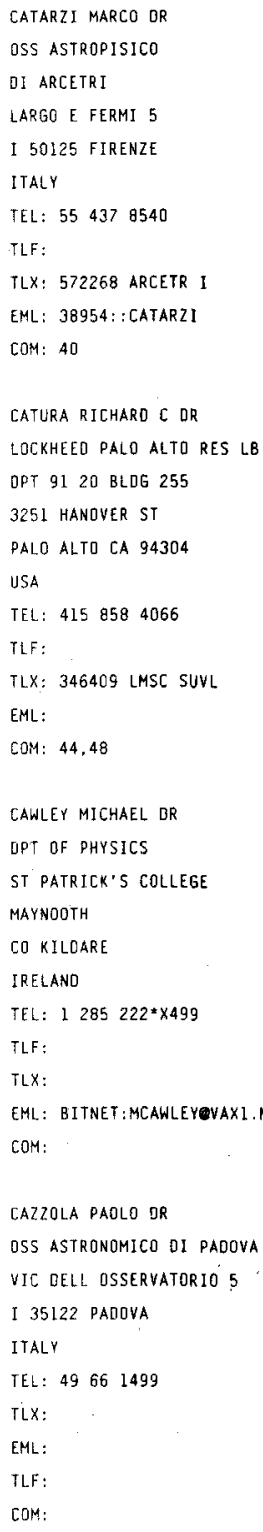

CELNIKIER LUDWIK DR observatoire de paris SECTION DE MEUDON F 92195 MELIDON PPL CDX FRANCE

TEL: $145 \quad 077410$

ILF:

TLX: 201571

EML:

COM:

CATCHPOLE ROBIN M OR
ROYAL GREENHICH OBS
MAOINGLEY RO
CAMBRIDGE CB3 OEZ
UK
TEL: 223374000
TLF: 223374700
TLX: $26541 / 265871$
EML: CATHPOLEOUK. AC. CAM.AST-STAR
COM: $27 ; 29$

CATO B TORGNY DR NORDISK TELESATELLITSTAT BOX 107

S 457 OO TANUMSHEDE SWEOEN

TEL: 52529155

TLF:

TLX: 20164 NORDSAT S

EML:

COM:

CAUGHLAN GEORGEANNE R
DPT OF PHYSICS
MONTANA STATE UNIVERSITY
BOZEMAN MT 59717
USA
TEL: 4069946170
TLF:
TLX:
EML:
COM: 35,48

CAYREL DE STROBEL GIUSA observatoire de paris SECTION OE MEUDON F 92195 MEUDON PPL CDX FRANCE.

TEL: $1 \quad \begin{array}{llll}45 & 07 & 7863\end{array}$

TLF:

TLX:

EML:

COM: 29,36

CEFOLA PAUL J DR
MAIL STATION 64
C S DRAPER LAB
555 TECHNOLOGY SO
CAMBRIOGE MA 02139
USA.
TEL: 6172581787
TLF:
TLX:
EML:
COM: 87

\section{CENTRELLA JOAN M OR} DPT OF PHYSICS OREXEL UNIVERSITY PHILADELPHIA PA 19104 USA

TEL: $215 \quad 895 \quad 2715$

TLF:

TLX:

EML:

COM:

\begin{tabular}{|c|c|}
\hline CAVALIERE ALFONSO G PROF & CAVALLINI FABIO \\
\hline OPT OI ASTROFISICA & OSS ASTROFISICO \\
\hline II UNIVERSITA DI ROMA & OI ARCETRI \\
\hline VIA ORAZIO RATMONDO & LARGO E FERMI 5 \\
\hline I 00173 ROMA & I 50125 FIRENZE \\
\hline ITALY & ITALY \\
\hline TEL.: & TEL: $55 \quad 4378540$ \\
\hline TLF: & TLF: $55 \quad 435939$ \\
\hline TLX: & TLX: 572268 \\
\hline EMt: & EML: \\
\hline COM: 47,48 . & COM: 12 \\
\hline CAYREL ROGER OR & CAZENAVE ANNY DR \\
\hline OBSERVATOIRE OE PARIS & CNES/GRGS \\
\hline 61 AVE OBSERVATOIRE & 1B AVE E BELIN \\
\hline F 75014 PARIS & F 31055 TOULOUSE COX \\
\hline FRANCE & FRANCE \\
\hline TEL: $14051 \quad 2251$ & TEL: $61 \quad 274011$ \\
\hline ThF: & TLF : \\
\hline$T L X: 270776$ OBS $F$ & TLX: 531081 \\
\hline EMt: & EML: \\
\hline COM: $29,36,50$ & COM: \\
\hline CELIS LEOPOLDO DR - & CELLINO ALBERTO DR \\
\hline DPT DE ASTRONOMIA & OSS ASTRONOMICO \\
\hline UNIVERSIDAD CATOLICA & OI TORINO \\
\hline CASILLA 6014 . & I 10025 PINO TORINESE \\
\hline SANTIAGO & ITALY \\
\hline CHILE & TEL: 11842040 \\
\hline TEL: $2 \quad 552 \quad 2375$ & TLF: \\
\hline TLF: 25525692. & FLX: 213236 TOASTR \\
\hline TLX: 440001 & EML: CELLINO@ASTTOZ. INFN. IT \\
\hline EML: & COM: 15 \\
\hline COM: 25,45 & \\
\hline CEPA. JORDI OR & CEPLECHA ZDENEK OR \\
\hline INST OE ASTROFISICA & ASTRONOMICAL INSTITUTE \\
\hline DE CANARIAS & CZECH ACADEMY OF SCIENCES \\
\hline OBS, DEL TEIDE & ONDREJOV OBSERVATORY \\
\hline E 38200 LA LAGUNA & CS 25165 ONDREJOV \\
\hline SPAIN & CZECHOSLOVAKIA \\
\hline TEL: $22 \quad 262211$ & TEL: 20485201 \\
\hline TLF: 22263005 & ILF: 20485314 \\
\hline TLX: 92640 IAC E & TLX: 121579 \\
\hline EML: IAC: : JCN & EML: \\
\hline COM: 28 & COM: $15,22 \mathrm{C}$ \\
\hline
\end{tabular}

CATON DANIEL B DR ASSISTANT PROFESSOR PHYSICS ANO ASTRONOMY APPALACHIAN 'STATE UNIV BOONE NC 28608

USA

TEL: 7042622446

TLF :

TLX: 888370 OR 62671500 EML: BI INET: CATONDB APPSTATE COM:

COM: $15,22 \mathrm{C}$ 


CEPPATELLI GUIDO DR
OSS ASTROFISICO
DI ARCETRI
LARGO E FERMI 5
I 50125 FIRENZE
ITALY
TEL: 554378540
TLF: 55435939
TLX: 572268
EML:
COM: 12
CERSOSIMO JUAN CARLOS DR
IAR
- -
L894 VILLA ELISA (8S AS)
ARGENTINA
TEL: 214 3793
TLX:
EML:
TLF:
COM: 34

\section{CEVOLANI GIOROANO}

FISBAT

CNR

VIA CASTAGNOLI 1

I 40126 BOLOGNA

ITALY

TEL: $51239593 / 94$

TLF :

TLX: 511350

EML:

COM: 22

CHAISSON ERIC J PROF

STSCI

HOMEWOOD CAMPUS

3700 SAN MARTIN OR

BALTIMORE MD $2121 B$

USA

TEL: 3013384757

TIF:

TLX: 6849101

EML:

COM: 51

\section{CHAMARAUX PIERRE DR}

OBSERVATOIRE DE PARIS

SECTION DE MEUDON

F 92195 MEUDON PPL CDX FRANCE

TEL: $1 \quad 45 \quad 07 \quad 7594$

TLF:

TLX: 270912

EML:

COM: 28

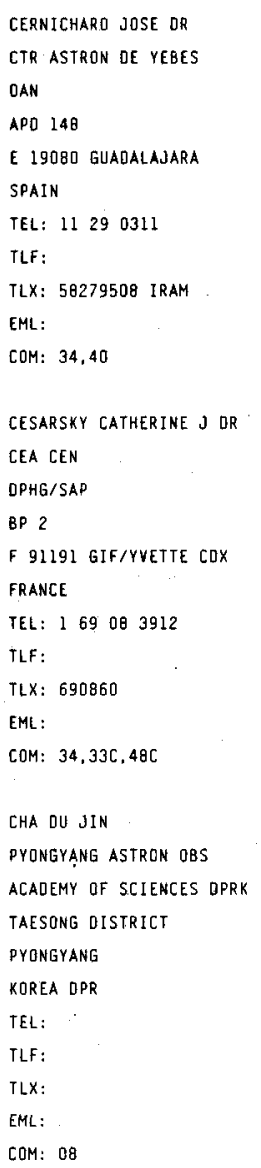

\section{CHAKRABARTI SANOIP $K$ OR} TIFR

HOMI BHABHA RO

COLABA

BOMBAY 400005

INDIA

TEL: $22 \quad 215 \quad 2971 * 305$

TLF: $22 \quad 2152110$

TLX: 01183009 TIFR IN

EML: CHAKRABAeTIIFRVAX. BITNET

COM: 48

CHAMBE GILBERT
OBSERVATOIRE OE PARIS
SECTION OE MEUDON
DASOP
F 92195 MEUDON PPL CDX
FRANCE
TEL: 145347793
FLF:
FLX:
EML:
COM: 10,12

\begin{tabular}{|c|c|}
\hline CERRONI PRISCILLA DR & CERRUTI-SOLA MONICA \\
\hline IAS & OSS ASTROFISICO \\
\hline REPARTO DI PLANETOLOGIA & DI ARCETRI \\
\hline VIA OELL'UNIVERSITA II & LARGO E FERMI 5 \\
\hline I 00185 ROMA & I 50125 FIRENZE \\
\hline ITALY & ITALY \\
\hline TEL: 64956951 & TEL: 554378540 \\
\hline TLF: 64454969 & TLF: $55 \quad 43 \quad 5939$ \\
\hline TLX: 610261 CNR FRA & TLX: 572268 ARCETR 1 \\
\hline EML: BITNET: CARUSI IRMUNISA & EML: \\
\hline COH: 15 & COM: 34 \\
\hline $\begin{array}{l}\text { CESARSKY OIEGO A DR } \\
\text { INSTITUT O ASTROPHYSIQUE }\end{array}$ & $\begin{array}{l}\text { CESTER BRUNO PROF } \\
\text { OAT }\end{array}$ \\
\hline 98BIS BD ARAGO & BOX SUCC TRIESTE 5 \\
\hline F 75014 PAR1S & VIA TIEPOLO 11 \\
\hline FRANCE & I 34131 TRIESTE \\
\hline TEL: 143201425 & ITALY \\
\hline TLF: $143 \quad 29 \quad 8673$ & TEL: $4079 \quad 3921 * 221$ \\
\hline$T L X_{i}$ & TLF : \\
\hline EML: & TLX: 461137 OAT I \\
\hline COM: 33,34 & EML: \\
\hline & COM: $26,42,45$ \\
\hline
\end{tabular}

CHA GI UNG

PYONGYANG ASTRON OBS

ACADEMY OF SCIENCES - DPRK TAESONG DISTRICT

PYONGYANG

KOREA DPR

TEL:

TLF:

TLX:

EHL:

COM:

\section{CHAKRABORTY DEO K DR}

DPT OF PHYSICS

UNIVERSITY OF RAVISHANKAR

RAIPUR 492010

INDIA

TEL: 27064

TLF :

TLX:

EML:

COM: 48

CHAMBERLAIN JOSEPH M DR

ADLER PLANETARIUM

1300 S LAKE SHORE DR

CHICAGO IL 60605

USA

TEL: 3123220325

TLF :

TLX:

EML:

COM: $08,31,46$
CHAFFEE FREOERIC $H$ OR

MULT. MIRROR TELESCOPE OBS UNIVERSITY OF ARIZONA TUCSON AZ 85721

USA

TEL:

TLF :

TLX:

EML:

COM:

CHALABAEY ALMAS OR OHP

F 04870 S MICHEL OBS FRANCE

TEL: $9276 \quad 6368$

TLF :

TLX: 410690 OHP $F$

EML: CHALABAEVOFONI51

COM: 28

CHAMBERLAIN JOSEPH W PROF OPT SPACE PHYS \& ASTRON RICE UNIVERSITY HOUSTON TX 77001 USA

TEL: 7135278101

TLF:

TLX: 556457

EML:

COM: $16,21,49$ 


CHAMBLISS CARLSON R OR
OPT PHYSICAL SCIENCES
KUTZTOWN UNIVERSITY
KUTZTOWN PA 19530
USA
TEL: 2156834439
TLF:
TLX:
EML:
COM: 42
CHANGRA SURESH DR
OPT OF PHYSICS
UNIVERSITY OF GORAKHPUR
GORAKHPUR 273 OO9
INDIA
TEL:
TLF:
TLX:
EML:
COM: 10,34

CHANMUGAM GANESAR PROF DPT PHYSICS \& ASTRONOMY LUUISIANA STATE INIV BATON ROUGE LA 708034001 USA TEL: 5043886894

TLF :

TLX:

EML:

COM: 42

CHAPMAN ROBERT D OR

LYNDON JOHNSON SPACE CTR. CODE PO3L1

HOUSTON TX 77058

USA

TEL:

TLF:

$T L X:$

EML:

COM: $15,42,44$

CHASSEFIERE ERIC

SERVICE D'AERONOMIE

$B P 3$

F 91371 VERRIERES BUISSON

FRANCE

TEL: 164474211

TLF:

$T L X: 602400$

EML:

COM: 49
CHAN KWING LAM APPLIED RESEARCH CORP 8201 CORPORATE DR SUITE 920

LANDOVER MO 20785

USA

TEL: 3014598442

TLF:

TLX:

EML:

COM: $12,35,36,40$

CHANDRASEKHAR S PROF ASTROPHYS \& SPACE RES LAB UNIVERSITY OF CHICAGO 933 E 56TH ST CHICAGO IL 60637 USA

TEL: 3129627860

TLF :

TLX:

EML:

COM: $35,4 \mathrm{~B}$

CHAPMAN CLARK R OR PLANETARY SCIENCE INST 2030 E SPEEOWAY SUITE 201

TUCSON AZ 85719

USA

TEL: $602 \quad 8810332$

TLF:

TLX:

EML:

COM: 15,16

CHAPRONT JEAN DR

BUREAU DES LONGITUDES

77 AVE DENFERT ROCHEREAU

F 75014 PARIS

FRANCE

TEL: $1405.1227 \mathrm{i}$

TLF :

TLX:

EML:

COM: $04 \mathrm{C}, 07 \mathrm{C}$

CHATTERJEE TAPAN K OR

FAC OE CIENCIAS

UNIVERISDAD A PUEBLA

APDO POSTAL 1152.

72000 PUEBLA PUE

MEXiCO

TEL: 9122330455

TLF: $9122 \quad 44 \quad 8947$

TLX:

EML:

COM: $2 B$
CHAN RDBERTO DR OBSERVATORIO NACIONAL

CHANORA SUBHASH MIS PHILIPS LABS 345 SEARBOROUGH RO BRIAR CLIFF NY 10510 SAO CRISTOVAO

20921 RTO DE JANEIRO RJ BRAZIL

USA

TEL:

TLF:

TLX:

EML:

COM:

EML: USERCHANOLNCCVM

COM: 35

CHANDRASEKHAR T OR PHYSICAL RESEARCH LAB

NAVRANGPURA

AHMEDABAD 380009

INDIA

TEL: $272 \quad 46 \quad 2129$

ILF: 272445292

TLX: $121397 \cdot$ PRL IN

EML:

COM: 15

CHAPMAN GARY A DR

DPT PHYSICS \& ASTRONOMY

SAN FERNANGO OBSERVATORY CALIFORNIA STATE UNIV. NORTHRIDGE CA 91330

USA

TEL: $818 \quad 885 \quad 2775$

ILF:

$T L X:$

EML:

COM: 10,12

CHAPRONT-TOUZE MICHELLE BUREAU DES LONGITUDES 77 AVE DENFERT ROCHEREAU F 75014 PARIS

FRANCE

TEL: 14051.2266

$T L F$ :

$T L X:$

EML:

COM: $04,07,20$

CHAU WAI Y PROF

OPT OF PHYSICS

QUEEN'S UNIVERSITY

KINGSTON ON KZL JNE

CANADA

TEL: 6135473526

TLF: 6135456463

TLX:

EML:

COM:
CHANG KYONGAE OR' OPT PHYSICS \& OPTICS CHUNGJU UNIVERSITY CHUNGJU KOREA R

TEL:

TLF :

TLX:

EML:

COM: 47 UNIVERSITY OF OXFORD
CHAPMAN JESSICA DR
CSIRO
AUSTRALIAN TELESCOPE
BOX 296
EPPING NSW 2121
AUSTRALIA
TEL: 28680222
TLF: 28680310
TLX: 26230 ASTRO AA
EML: JCHAPMANEATNF.CSIRO.AU
COM: 33

CHARLES PHILIP ALLAN DPT OF ASTROPHYSICS SOUTH PARKS RO OXFORO OXI 3RQ UK TEL: $865511 \quad 336 * 506$ TLF: TLX: 83295 NULCLOX EML: COM: 44

CHAUBEY UMA SHANKAR DR UTTAR PRADESH STATE OBSERVATORY PO MANORA PEAK 253129 NAINITAL 283129 INDIA

TEL: $2136 / 2583$

TLF:

TEX:

EML:

CQM: 42 


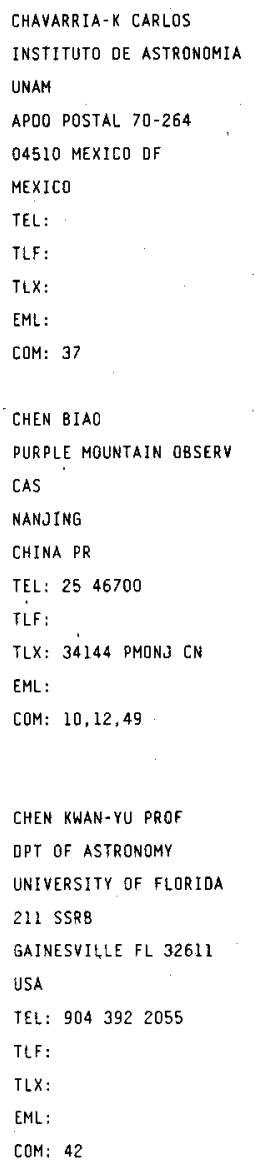

CHAVIRA ENRIQUE SR
INAOE
TONANTZINTLAZ
APDO POSTAL 216 y 51
72000 PUEBLA PUE
MEXICO
TEL: 47-05-00
TLF:
TLX:
EML:
COM: 27
CHEN DA0-HAN
PURPLE MOUNTAIN OBSER
CAS
NANJING
CHINA PR
TEL: 2531096
TLF:
TLX: 34144 PMONJ CN
EML:
COM: 15,16

\section{CHEN PEISHENG}

YUNNAN OBSERVATORY EAS

BOX 110

KUNMING 72946 YUNNAN

CHINA PR

TEL: 8712035

TLF:

TLX:

EML:

COM: 36

\section{CHEN ZUN-GUI}

BEIJING PLANETARTum

138 XI WAI ST

BEIJING

CHINA PR

TEL: 1893003

TLF:

TLX:

EML:

COM: 41

CHEREDNICHENKO V I OR

KIEV POLYTECHNICAL INST 252056 KIEV

UKRAINE

TEL:

TLF:

EML:

COM: 15

TLX:
CHECHETKIN VALERIJ M OR INST OF APPLIEO MATHS ACADEMY OF SCIENCES MIUSSKAJA SQ 4 125047 MOSCOW RUSSIA

TEL: 2513739

TLF :

TLX:

EML:

COM: 35,48

CHEN HONGSHENG

BEIJING ASTRONOMICAL OBS CAS

\section{W SUBURB}

BEIJING 100080

CHINA PR.

TEL:

TLF :

TLX: 9053

EML:

COM: 40

\section{CHEN XIAO-ZHONG}

BEIJING PLANETARIUM

130 XI WAI ST

BEIJING

CHINA PR

TEL: 1893003

TLF :

TLX:

EML:

COM:

CHENG CHUNG-CHIEM DR NAVAL RESEARCH LABORATORY COOE $4175 C C$

4555 OVERLOOK AVE SW WASHINGTON OC 203755000 USA

TEL: 2027672350

TLF:

TLX:

EML:

COM: 10,12

CHEREPASHCHUK A M PROF
STERNBERG STATE ASTR INST
UNIVERSITETSKIJ PROSP 13
11989g MOSCOW
RLSSIA
TEL: $139-38-38$
TLF:
TLX:
EML:
COM: $27,42,45$

CHELLI ALALN

INSTITUTO DE ASTRONOMIA

UNAM

APDO POSTAL 70-254

O4510 MEXICO OF

MEXICO

TEL: $548-3712 / 5306$

TLF :

TLX: 1760155

EML:

COM: 09

CHEN JIAN-SHENG

BEIJING ASTRONOMICAL OBS CAS

\section{W SUBURB}

BEIJING 100080

CHINA PR

TEL:

TLF:

TLX: 22040 BADAS CN

EML:

COM: $28 \mathrm{C}, 47$

CHEN ZHEN

PURPLE MOUNTAIN OBSERV

CAS

NANUING

CHINA PR

TEL: 2546700

TLF :

TLX: 34144 PMONJ CN

EML:

COM: $07,26,33$

CHENG FU-HUA

GENTER FOR ASTROPHYSICS

UniV SCLENCE \& TECHNOLOGY

HEFEI 230026 ANHUI

CHINA PR

TEL: $55133 \quad 1134 * 526$

TLF:

TLX: 90028 USTC CN

EML:

COM: 47

CHERNEGA N A A DR ASTRONOMICAL OBSERVATORY KIEV SYAYE UNIVERSITY observatornaya UL 3 $252053 \mathrm{kIEV}$ UKRAINE

TEL: $26 \quad 2391$

TLF:

TLX:

EML:

COM: 08 


CHERNOV GENNADIJ OR.
IZMIRAN
ACAOEMY OF SCIENCES
142092 TROITSK
RUSSIA
TEL: 3340902
TLF: 3340124
TLX: 412623 SCSTP SU
EML:
COM: 10

CHEVALIER ROGER A OR

UNIVERSITY STAIION

UNIVERSITY OF VIRGINIA

BOX 3818

CHARLOTIESVILLE VA 22903

USA

TEL: 8049244889

ILF :

$F ! X:$

EML:

[COM: 34

CHINCARINI GUIDO L DR

OSS ASTRONOMICO DI MILANO

VIA E BIANCHI 46

I 22055 MERATE

ITALY

TEL: 596412

rLX:

EML:

TLF :

COM: 28,47

CHISTYAKOV VLADIMIR E OR

USSURIISK SOLAR STATION

PRIMORSKY KRAY

692533 GORNOTAEZHNOE

RUSSIA

TEL : 91121 USSURIISK

TLX: 213954 SOLNZE

EML:

ILF :

COM: 12

CHIU LIANG-TAI GEORGE
IBM
THOMAS J WATSON RES CTR
BOX 218
YORKTOWN HEIGHTS NY 10598
USA
TEL: 9149452436
TLF:
TLX:
EML:
COM: 24

CHERNYKH N S DR

CRIMEAN ASTROPHYS OBS

UKRAINIAN ACAD OF SCIENCE

NAUCHNY

334413 CR IMEA

UKRAINE

TEL: 432945

TLF:

ILX:

EML:

COM: 15,20

CHE-BOHNENSTENGEL ANWE

SUELZBRACKRING 39A

D 2050 HAMBURG 80

GERMANY

TEL: 407238550

TLF:

EML:

COM:

TLX:

CHINI ROLF

MPI FUER RADIOASTRONDMIE

AUF DEM HUEGEL 69

D 5300 BONN 1

GERMANY

TEL:

TLX: 886440

EML:

TLF :

COM: 34,40

CHITRE DATTAKUMAR M DR

COMPUTER SCIENCES.CORP

SYSTEM SLIENCES DIV

8728 COLESVILLE RD

SILVER SPRING MD 20910

USA

TEL:

TLF :

TLX:

EML:

COM: $38 \mathrm{C}, 47,49$

CHIUOERI ELAUDIO PROF

DPT DI ASTRONOMIA

UNIVERSITA DI FIRENZE,

LARGO E FERMI 5

I 50125 FIRENZE

ITALY

TEL: 5527521

TLF: $55 \quad 220039$

TLX: 572268 ARCETA I

EML:

COM :
CHERTOPRUD V E DR

HYDROMETEOROLOGICAL CTR

123376 MOSCOW

RUSSIA

TEL:

TLF :

TLX:

EML:

COM: 10

CHIAN ABRAHAM CHIAN-LONG INPE

CP 515

$12200 S$ JOSE DOS CAMPOS

BRAZIL

TEL: $123 \quad 229977$

TLF: $123 \quad 21 \quad 8743$

TLX: $011-33530$

EML:

COM: 48

CHIO CHOL ZONG

PYONGYANG ASTRON OBS

ACADEMY OF SCIENCES OPRK

TAESONG GISTRICTं

PYONGYANG

KOREA DPR

TEL:

TLF:

TLX:

EML:

COM: 20

CHITRE SHASHIKUMAR M DR

TIFR

HOMI BHABHA RO

COLABBA

BOMBAY 400005

INDIA

TEL: $22 \quad 219111$

TLF :

TLX: 011-3009 TIFR IN

EML:

COM: $35,48,49$

CHIUDERI-DRAGO FRANCA PR OSS ASTRDFISICO

DI ARCETRI

LARGO E FERMI 5

I 50125 FIRENZE

ITALY

TEL: 554378540

TLF: 55435939

TLK: 572268 ARCETR I

EML:

COM: 10
CHEVALIER CLAUOE DR

OHP

F $04870 S$ MICHEL DBS

FRANCE

TEL: $9276 \quad 6368$

TLF:

TLX: 410690 OHP F

EML:

EOM: 35

CHIKADA YOSHIHIRO DR

NOBEYAMA RADIO OBS

NAOJ

MINAMIMAKI MURA

NAGANO $384 \quad 13$

JAPAN

TEL: $267-98-2831$

TLF :

TLX: 3329005 TAONRO

EML:

COM : 40

CHIOSI CESARE S OR

OPT BI ASTRONOMIA

UNIVERSITA DI PADOVA

VIC DELL OSSERVATORIO 5

I 35122 PADOVA

ITALY .

TEL: $49 \quad 66 \quad 1499$

TLF :

TLX: 430176 UNDAPU I

EML:

COM: $35 \vee P, 37$

CHIU HONG-YEE OR

MUDO BLOG RM 828

COLUMBIA UNIVERSITY

NEW YORK NY 10027

USA

TEL:

TIF :

TLX:

EML:

COM: 35

CHIUEH TZIHONG OR INST PHYSICS \& ASTRONOMY NTL CENTRAL UNIVERSITY

CHUNG LI

TAIWAN

CHINA R.

TEL: $3422 \quad 7151 \star 5341$

TLF: $3425 \quad 1175$

TLX:

EML: CHIUEH@PHYAST. QNET.NCU.EOU.TW

COM: 10 


\author{
CHIUMIENTO GIUSEPPE \\ OSS ASTRONOMICO DI - TOR INO \\ ST OSSERVATORIO 20 \\ I 10025 PINO TORINESE \\ ITALY \\ TEL: 11841067 \\ TLX: 213236 TOASTR I \\ EML: \\ TLF \\ COM: 08,19
}

CHO SE HYUNG QR
ISSA
YOOSUNG KOON
OAEJEON 305348
XOREA R
TEL: $042 \quad 851 \quad 1281$
TLF: $042 \quad 861 \quad 5610$
TLX:
EML:
COM: 40

CHOI WON CHOL

PYONGYANG ASTRON OBS

ACADEMY OF SCIENCES OPRK

TEASONG OISTRICT

PYONGYANG

KOREA DPR

TEL: 5-3134, 5-3239

TLF:

$T L X:$

EML:

COM:

CHOU CHIH-XANG DR

ASTRONOHY,

NTL CENTRAL UNIVERSITY

CHUNG LI

TAIWAN ROC

CHINA R

TEL: 34251175

TLF:

TLX:

EML:

COM: 28,49

\section{CHR ISTENSEN-DALSGAARD J}

INST OF PHYSICS \& ASTRON

UNIVERSITY OF AARHUS

NY MUNKEGADE

OK 8000 AARHUS $C$

DENMARK

TEL: $86 \quad 12 \quad 8899$

TLF: $86 \quad 202711$

TLX: 64767 AAUSCI DK

EML:

COM: $12,27[, 35$
CHKHIKVAOZE IAKOB N

ABASTUMANI ASTROPHYSICAL

OBSÉRVATORY

GEORGIAN ACAD OF SCIENCES

383762 ABASTUMANI

GEORGIA

TEL: $2-78$

TLF:

TLX: 327409

EML:

COM: 35

CHOCHOL ORAHOMIR

ASTRONOMICAL INSTITUTE

SLOVAK ACADEMY SCIENCES

CS 05960 TATRANSKA LOMNI

CZECHOSLOVAKIA

TEL: 969 96. 7866/7/8

ILF: 969967656

TLX: 78277

EML:

COM: 42.44

ChOLlet fernand dR

observatoire de paris

61 AVE OBSERVATOIRE

F 75014 PARIS

FRANCE

TEL: 140512205

TLF:

TLX: 270776 OBS F

EML:

COM: 04,08

CHOU DEAN-YI DR

PHYSICS DPT

NTL TSING HUA UNIVERSITY

HSIN CHU 300043

CHINA R

TEL:

TLF:

TLX:

EML: BITNET : DYCHOU®TWNCTUOI COM:

CHRISTIAN CAROL ANM

CTR FOR EUV ASTROPHYSICS

UNIVERSITY OF CALIFORNIA

2150 KITTREOGE ST

BERKELEY CA 94720

USA

TEL:

TLF :

TLX:

EML:

COM: 37
CHLISTOVSKY FRANCA DR

OSS ASTRONOMICO DI BRERA

VIA BRERA 28

I 20121 MILANO

ITALY

TEL: 2874444

ILF: 272001600

TLX:

EML:

COM: 08

CHOE SEUNG URN OR

OPT DF EARTH SCIENCE \& ED

SEOUL NTL UNIVERSIIY

SINLIM OONG GWANG GU

SEOUL 151742

KOREA R

TEL:

TLF:

TLX:

EML:

COM:

CHOLONIEWSSKI JACEK DR

ASTRONOMICAL OBSERVATORY

WARSAW UNIVERSITY

AL UJAZOOWSKIE 4

PL 00478 WARSAW

POLAND

TEL: 294011

TLF:

TLX: 817063 OAUW PL

EML: JCH PLWAUWEL.BITNET

COM:

CHOU KYONG CHOL PROF

OPT ASTRONOMY SPACE SCI

KYUNG HEE UNIVERSITY

YONG IN KUN

KYUNGGI DO 17073

KOREA R

TEL: 966-0061/5

TLF:

TLX:

EML:

COM: 51

CHRISTIANSEN WAYNE A DPT PHYSICS \& ASTRONOMY UNIVERSITY NORTH CAROLINA 204 PHILLIPS HALL O39A CHAPEL HILL NC 27514 USA

TEL: 9199623011

TLF :

TLX:

EML:

COM: 40
CHMIELEWSKI YVES OR QBSERVATOIRE OE GENEVE CHEMIN DES Maillettes 51 CH 1290 SAUVERNY SWITZERLANO

TEL: 227552611 TLF: 227553983 TLK: 419209 OBS CH EML: igorobs.unige.ch CDM:

CHOI KYU-HONG OPT ASTRON \& METEOROLOGY YONSEI UNIVERSITY SUDAEMUN

SEOUL $120 \quad 749$

KOREA $R$

TEL: 02-392-0131

TLF:

TLX:

EML:

COM: 07,42

CHOPINET MARGUERITE DR

57 RUE THIERS

F 92L00 BOULOGNE

FRANCE

TEL: $147 \quad 61 \quad 1144$

TLF:

TLX:

EML:

COM: 34

CHRISTENSEN PER R OR

NIELS BOHR INSTITUTE BLÉGOAMSVEJ 17

OK 2100 COPENHAGEN 0

DENMARK.

TEL: 31421616

TLF: 31389157

TLX: 15216 NBI DK

EML: PERREXanBIVAX.NBI.OK COM:

CHRISTIANSEN WILBUR PROF RMB 436

MAC'S REEF RD

VIA BLUNGENDORE 2621

AUSTRALIA

TLF:

TEL: $62 \quad 303287$

TLX:

EML:

COM: 40 


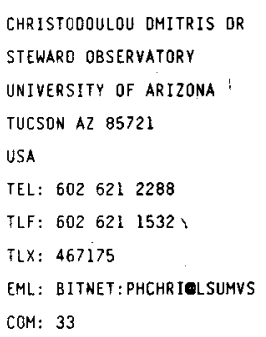

CHRISTY JAMES WALTER OR HUGHES AIRCRAFT CO

1720 W NIONA PL

IUCSON AZ 85704

USA

TEL: $602 \quad 297 \quad 1377$

TLF :

$T L X$ :

EML:

COM: $09,24,45$

CHU YOU-HUA
DPT OF ASTRONOMY
UNIVERSITY OF ILLIMOIS
LOLI W SPRINGFIELO AVE
URBANA IL 6LBOI
USA
TEL: 2173335535
TLF:
TLX:
EML:
COM: 34
CHUN MUN-SUK OR
OPT ASTRON \& METEOROLOGY
YONSEI UNIVERSITY
SUOAEMUN
SEOUL 120 749
KOREA R
TEL: 2 - $392-0131$
TLF:
TLX:
EML:
COM: 37

CHUVAEV K K DR

CRIMEAN ASTROPHYS OQBS UKRAINIAN ACAD OF SCIENCE NAUCHNY

3344 L3 CR IMEA

UKRAINE

TEL: 432945

TLF :

$T L X$ :

EML:

COM: 28

CILLIE G G PROF

4 MINSERIE ST

STELLENBOSCH 7600

SOUTH AFRICA

TEL: 02231-3515

TLF :

EML:

COM: 42

$T L X$ :
CHRISTY ROBERT F DR

CALTECH

MS 10524

patamar obs

PASADENA CA 91125

USA

TEL: 2137956811

TLF :

TLX:

EML:

COM: 27,35

CHUBB TALBOT A OR

5023 N 3BTH ST

ARLINGTON VA 22207

USA

TEL:

TLF :

TLX:

EML:

COM: 44,48

CHUPP EDWARD L DR

DPT OF PHYSICS

UNIV OF NEW HAMPSHIRE

DEMERITT HALL

DURHAM NH 03824

USA

TEL: $603 \quad 862 \quad 2750$

TLF :

TLX: 950030

EML: SOLMAX: : CHUPP

COM: 10,48

CHVOJXOVA WOYK E OR

ASTRONOMICAL INSTITUTE CZECH ACADEMY SCIENEES BUDECSKA 6

CS 12023 PRAHA 2

CZECHOSLOVAKIA

TEL:

TLF :

TLX:

EHL:

COM: 12

CIURLA TADEUSZ

ASTRONOMICAL INSTITUTE WROCLAW UNIVERSITY UL KOPERNIKA 11 PL 51622 WROCLAW POLAND

TEL:

TLF:

TLX:

EML:

COM: 33
CHU HAN-SHU PROF PURPLE MOUNTAIN OBSERV CAS

NANJING CHINA PR TEL: 25301096 TLF : TLX: 34144 PMONJ CN EML:

COM: 40

CHUGAI NIKOLAI N DR INST OF ASTRONOMY ACABEMY OF SCIENCES PYATNITSKAYA UL 48 109017 MOSCOW RUSSIA TEL: 2312129 TLF:

TLX: 411576 ASCON SU EML:

COM: 28,36

CHURCHWELL EQWARD B DR WASHBURN OBSERVATORY UNIVERSITY OF WISCONSIN 475 N CHARTER ST MADISON WI 53706 USA YEL: $608 \quad 262 \quad 7857$ TLF : TLX: 265452 UOFWISC MOS EML: COM: 33,34

CIATTI FRANEO DR OSSERVATORIO ASTROFISICO VIA OELL OSSERVATORIO 8 136012 ASIAGO ITALY TEL: 42462665 TLF : TLX: 430110 SETURIST EML: COM:

CLAIREMIOI JACQUES DR OBSERVATOIRE OE BESANCON 418IS AVE OBSERVATOIRE. F 25000 BESANCON CDX FRANCE TEL: 81666900 TLF: ILX: $361144 \mathrm{~F}$ EML: CLAIREMIOFROBES5I COM: $15,16,21$ 
CLARIA JUAN OR OBSERVATORIO ASTRONGMICO DE CORDOBA

\section{LAPRIDA 854}

5000 COROOBA

ARGENTINA

TEL: 5140613

TLF :

TLX: 51822 BUCOR

EML:

COM: $37 \mathrm{C}, 42,45,47$

CLARK FRANK OLIVER OR DPT PHYSICS \& ASTRONOMY UNIVERSITY OF KENTUCKY LEXINGTON KY 40506

USA

TEL: $606 \quad 2573376$

TLF:

$T L \dot{X}$

EML:

COM: 34,40

\section{CLARKE OAVID DR \\ DPT OF ASTRONOMY UNIVERSITY OF GIASGOW GLASGOW G12 800 UK TEL: $413398 \quad 855$ TLF : \\ TLX: 778421 \\ EML: \\ COM: 09}

\section{CLAUSSEN MARK J DR}

NAVAL RESEARCH LABORATORY

CODE 4210

4555 OVERLOOK AVE SW

WASHINGTON OC 203755000

USA

TEL: 2027670670

TLF:

TLX:

EML:

COM:

\section{CLAYTON ROBERT N DR} ENRICO FERMI INSTITUTE UNIVERSITY OF CHICAGO 56405 ELLIS AVE CHICAGO IL 60637

USA

TEL: 3127027777

TLF :

TLX:

EMt:

COM: 15
CLARK ALFRED JR PROF DPT ṀECH \& AEROSPACE SCI UNIVERSITY. OF ROCHESTER ROCHESTER NY. 14627

USA

TEL:

TEF :

TLX:

EML:

COM:

CLARK GEORGE W PROF CENTER FOR SPACE RESEARCH MIT RM 37611

BOX 165

CAMBRIDGE MA 02139

USA

TEL: $617253 \quad 5842$

TLF:

TLX:

EML:

COM: $44,48 C$

CLARKE JOHN T

NASA/GSFC

CODE 6BI

HUBBLE SPACE TELESCOPE

GREENBELT MO 20771

USA

TEL: $301286 \quad 5781$

FLF:

TLX: $710-828-9716$

EML:

COM:

CLAVEL JEAN

ESA/ESTEC

SSD

B0X 299

NL 2200 AG NOORDWIJK

NETHERLANDS

TEL:

TLF:

TLX:

EML:

COM: 28

\section{CLEGG PETER E DR}

ROYAL GREENWICH OBS

MADINGLEY RO

CAMBRIOGE CB3 OEZ

UK

TEL: 223337548

TLF:

TLX: B17297 ASTRON.G

EML: SPAN: rlvad: : cavad:: oper

COM:

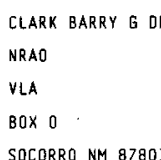

SOCORRO NM $87801038 \%$ USA

TEL: 5057724011

TLF :

ILX: 9109881710

EML:

COM: 40

CLARK THOMAS A OR

NASA/GSFC

CODE 974

GREENBELT MD 20771

USA

TEL: 3012865957

TLF :

TLX:

EML:

COM: 51

CLARKE THOMAS R DR

MCLAUGHLIN PLANETARIUM

ROYAL ONTARIO MUSEUM

100 QUEENS PARK CRESCENT

TORONTO ON MSS 2C6

CANADA

TEL: 4169988551

TLF :

TLX:

EML:

COM:

CLAYTON DONALD D PROF DPT PHYSICS \& ASTRONOMY CLEMSON UNIVERSITY CLEMSON SC 296341911 USA

TEL: 8036565299

TLF :

TLX:

EML: BITNET Claytonelemson

COM:

CLEGG ROBIN E S DR

ROYAL GREENWICH OBS

MADINGLEY RD

CAMBRIOGE CBJ OEZ

UK .

TEL:

TLF :

TLX:

EML:

COM: 34
CLARK DAVIO H DR

SCIENCE OIV

SCI \& ENGINEER RES COUNCI

NORTH STAR AVE

SWINDON SN2 IET

UK

TEL: $79,3262 \quad 22$

TLF:

TLX: 449466

EML:

COM: 40

CLARK THOMAS ALAN DR

OPT OF. P.HYSICS

UNIVERSITY OF CALGARY

2500 UNIVERSITY DR NW

CALGARY AB T2N IN4

CANADA

TEL: 4032845392

TLF: $403 \quad 2893331$

TLX:

EML:

COM: 12,44

CLAUSEN JENS VIGGO LEKTOR COPENHAGEN UNIVERSITY OBS BRORFELDEVEJ 23

DK 4340 TOLLOSE

DENMARK

TEL: $53 \quad 48 \quad 8195$

ILF: $58 \quad 48 \quad 8755$

TLX: 44155 DANAST

EML:

COM: 42

CLAYTON GEOFFREY C DR NASA HEADQUARTERS

CDOE EZ

600 INDEPENDENCE AVE SW WASHINGTON DC 20546

USA

TEL: $202 \quad 453 \quad 1469$

ILF :

TLX:

EML: SPAN: POLLUX: :GCLAYTON

COM: 15

CLEMENS DAN $P$. OR

OPT OF ASTRONOHY

BOSTON UNIVERSIIY

725 COMMONWEALTH AVE

BOSTON MA 02215

USA

TEL: $617353 \quad 6140$

TLF :

TLX:

EML:

COM: 33,40 


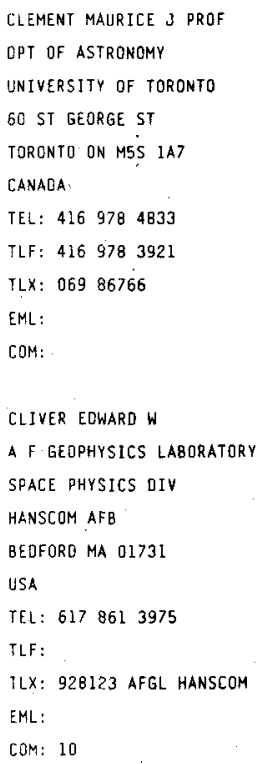

CLIFTON KENNETH ST NASA/MSFC

CODE ES 63

HUNTSVILLẸ AL 35812

USA

TEL: 2054532305

TLF:

ILX: $5944 ! 6$

EML:

COM: 22

CLUBE S V M OR

OPT OF ASTROPHYSICS UNIVERSITY OF OXFORD SOUTH PARKS RO OXFORD OXI 3RQ UK

TEL: 865511336

TLF:

ILX:

EML:

COM: $15,22,24,33$

COCKE WILLIAM JOHN PROF STEWARO OBSERVATORY UNIVERSITY OF ARIZONA TUCSON AZ 85721

USA

TEL: 6026216540

TLF:

ILX:

EML:

COM: 47

COELHO BALSA MARIO C DR RUA TRINDADE COELHO 21 20 OTO

P 3000 COIMBRA

PORTUGAL

TEL:

TLF :

ILX:

EML:

COM:

COHEN JEFFREY M OR
OPT OF PHYSICS
UNIV OF PENNSYLVANIA
PHILADELPHIA PA 19104
USA
TEL:
TLF:
TLX:
EML:
COM: $35,47,48$

CLIMENHAGA JOHN. L PROF OPT OF PHYSICS UNIVERSITY OF VICTORIA BOX 1700

VICTORIA BC VBW $2 Y 2$ CANADA

TEL: 6047217741

TLF: 6047217715

TLX:

EML:

COM: 29

CLUTTON-BROCK MARTIN DR DPT OF MATHS \& ASTRONOMY UNIVERSITY OF MANITOBA WINNIPEG MB R3T 2N2

CANADA

TEL: 2042619255

TLF:

TLX:

EML:

COM:

CODE ARTHUR D

WASHBURN OBSERVATORY

UNIVERSITY OF WISCONSIN

475 N CHARTER ST

MADISON WI 53706

USA

TEL: $608 \quad 262 \quad 9594$

ILF :

$T L X:$

EML:

COM: 34,44

COFFEEN QAVIO L QR

BOX 151

HASTINGS HUDSON NY 10706

USA

TEL: $914 \quad 478 \quad 2594$

ILF:

ILX:

EML:

COM:
CLINE THOMAS L OR NASA/GSFC

CODE 661

LHEA

GREENBELT MO 20771

USA

TEL: $301286 \quad 8375$

TLF :

TLX: 89675 NASCOM 68LT EML: SPAN: 6197: : CLINE COM:

COCHRAN ANITA L OR ASTRONOMY DPT UNIVERSITY OF TEXAS RLM 15308 AUSTIN TX 78712 1063 USA

TEL: $512 \quad 471 \quad 1471$

TLF:

TLX: $910 \quad 874 \quad 1351$

EML: BITNET: as 1j 72060 ta 3081 COM: 15,16

COOINA LANDABERRY SAYO J OBSERVATORIO NACIONAL RUA GL BRUCE 586 SAO CRISTOVAO 20921 RIO DE JANEIRO RJ BRAZIL

TEL: $215807313 * 267$

TLF: 215800332

TLX: 21288

EML:

COM: 46

COFFEY HELEN E MS NDAA

NGDC E/GC2

325 BROAOWAY

BOULDER CO 80303

USA

TEL: 3034976223

TLF:

TLX: 592811 NOAA MASC BDR EML:

COM: 10

COHEN SUDITH DR

CALTECH

MS 10524

PALOMAR DBS

PASADENA CA 91125

USA

IEL: 8183564005

TLF :

TLX:

EML: JLCODEIMOS.CALTECH,EDU

COM:
COHEN LEON PROF

DPT OF PHYSICS HUNTERS COLLEGE

695 PARK AVE

NEW YORK NY 10021

USA

TEL: 2125705696

TLF :

ILX:

EML:

COM: 


\begin{tabular}{|c|c|c|c|}
\hline COHEN MARSHALL H PROF & COHEN MARTIN OR & COHEN RAYMOND I DR & COHEN RICHARDS \\
\hline CALTECH & RADIO ASIRONOMY LAB & NRAL & INST FOR SPACE STUBIES \\
\hline MS 10524 & UNIVERSITY OF CALIFORNIA & JODRELL BANK & \\
\hline PALOMAR OBS & 601 CAMPBELL HALL & MACCLESFIELD SK11 90L & 2880 BROADWAY \\
\hline PASADENA CA 91125 & BERKELEY CA 94720 & uk & NEW YORK NY 10025 \\
\hline USA & USA & TEL: $477 \quad 71321$ & USA \\
\hline TEL: 2133564000 & TEL: $415 \quad 642 \quad 2833$ & TLF: & TEL: 2126785611 \\
\hline TLF: & TLF : & TLX: 36149 & TLF : \\
\hline TLX: 675425 & TLX: 820181 UCB AST RALUD & EML: & ILX: \\
\hline EML: & EML: & COM: 40 & EML: \\
\hline COM: 34,40 & COM: 27 & & $\operatorname{COM}: 09,33,40$ \\
\hline COHEN ROSSS O OR & COHN HALDAN N & COLBURN DAVID S OR. & COLE TREVOR WILLIAM PROF \\
\hline CASS & ASTRONOMY OPT & 1944 WAVERLEY STREET & SCHOQL OF ELECTRICAL ENG \\
\hline UCSD & INOIANA UNIVERSITY & PALO ALTO CA 94301 & UNIVERSITY OF SYDNEY \\
\hline coll & SWAIN WEST 31.9 & USA & SYONEY NSW 2006 \\
\hline LA JOLLA CA 920930216 & BLOOMINGTON IN 47405 & TEL: & AUSTRALIA \\
\hline USA & USA & TLF : & TEL: 26922682 \\
\hline IEL: 6195342664 & TEL: 8123354174 & TLX: & TLF: \\
\hline TLF: & TLF: & EML: & TLX: \\
\hline$T L X:$ & $T L X:$ & COM: & EML: \\
\hline EML: & EML: & & COM: 40 \\
\hline COM: 28,47 & COM: & & \\
\hline COLEMAN PAUL' HENRY DR & COLES PETER OR & COLGATE STIRLING A OR & COLIN JACQUES OR \\
\hline KAPTEYN ASTRONOMICAL INST & ASTRONOMY UNIT & LOS ALAMOS SCIENTIFIC LAB & OBSERVATOIRE DE BORDEAUX \\
\hline $80 \times 800$ & OUEEN MARY/WESTFIELO COLL & MS 8275 & $8 P 89$ \\
\hline NL 9700 AV GRONINGEN & MILE ENO RO & THEORETICAL DIV & F 33270 FLOIRAC \\
\hline NETHERLANOS & LONOON EI 4 AS & LOS ALAMOS NM 87545 & FRANCE \\
\hline TEL: 5063.064 & $u K^{\prime}$ & USA & TEL: $56 \quad 86 \quad 4330$ \\
\hline$T L X: 53572$ STARS NL & TEL: $1719755 \quad 481$ & TEL: $505 \quad 6672897$ & TLF: $58 \quad 404251$ \\
\hline EML: BITNET:GRUFFHGRUG5 & TLF: $1819 \quad 819587$ & TLF : & TLX: \\
\hline TLF : & TLX: 893750 & TLX: & EML: \\
\hline COM: 40 & EML: PCOLESEUK.AC. OMW.STARLINK & EML: & COM: $28,37,33$ \\
\hline & COM: 47 & COM: & \\
\hline COLLADOS MANUEL DR & COLLINS GEORGE W II PROF & COLLIN-SOUFFRIN SUZY DR & COLOMB FERNANOO R DR \\
\hline INST DE ASTROFISICA & DPT OF ASTRONOMY & INSTITUT D'ASTROPHYSIQUE & IAR. \\
\hline OE CANARIAS & OHIO STATE UNIVERSITY & $98 B^{\circ}$ IS BO ARAGO & \\
\hline OBS DEL TEIDE & 174 W LQTH AVE & F 75014 PARIS & CC 5 \\
\hline E 38200 LA LAGUNA & COLUMBUS OH 432101106 & FRANCE & 1894 VILLA ELISA (BS AS) \\
\hline SPAIN & USA & TEL: $143 \quad 20 \quad 1425$ & ARGENTINA \\
\hline YEL: $22 \quad 262211$ & TEL: 6144225467 & TLF: $1143 \quad 298673$ & TEL: 2143793 \\
\hline TLF : & TLF: & TLX: & TLF: \\
\hline$I L X: 92640$ & TLX: & EML: & TLX: 18052 CICYT AR \\
\hline EML: & EML: & COM: 34,48 & EML: \\
\hline $\operatorname{COM}: 10,12$ & COM: 42 & & COM: $34,40 C, 51 \mathrm{C}$ \\
\hline & & & \\
\hline COLOMBO G PROF DR & COLUZZI REGINA OR & COMA JUAN CARLOS & COMBES FRANCOISE OR \\
\hline IST MECCANICA APP,L & OAR & REAL INST Y OBSERVATORIO & OBSERVATOIRE DE PARIS \\
\hline UNIVERSITA OI PADOVA & VIA DEL PARCO MELLINI 84 & DE LA ARMADA & SECTION DE MEUDON \\
\hline VIA F MARZOLO 9 & I $00136 \cdot$ ROMA & CECILIO PUJAZON S/N & DEMIRM \\
\hline I 35122 PADOVA & ITALY & E 11110 SAN FERNANDO & F 92195 MEUDON PPL CDX \\
\hline ITALY & TEL: 6347056 & SPAIN & FRANCE \\
\hline TEL: $4966 \quad 1499$ & TLF: & TEL: & TEL: 145077898 \\
\hline ILF : & TLX: 626226 OA ROMA I & TLF : & TLF: \\
\hline$T L X:$ & EML: & TLX: & TLX: 270912 \\
\hline EML: & COM: $05,29,45$ & EML: & EML: \\
\hline COM: 16 & & COM: 04 & COM: \\
\hline
\end{tabular}




\author{
COMBES MICHEE \\ OBSERYATOIRE DE PARIS \\ SECTION DE MEUDON \\ F 92195 MEUDON PPL COX \\ FRANCE \\ TEL: 145077691 \\ TLF: \\ TLX: 201571 \\ EML: \\ COM:
}

COMTE GEORGES OR

observatoire de marseille

2 PLACE LE VERRIER

F I3246 MARSEILLE COX 04

FRANCE

TEL: 91959088

ILF:

TLX: .420241 F

EML: EARN: : "COMTEAROMRS51"

COM: 28

CONNES JANINE DR

CIRCE

BP 63

F 91400 ORSAY

FRANCE

TEL: L 69287675

TLF:

TLX: 692166

EML:

COM: 16

CONTI PETER S DR
JILA
UNIVERSITY OF COLORADO
BOX 440
BOULOER CO 803090440
USA
TEL: 3034927789
TLF: 3034925235
ILX: 755842 JILA.
EML: $33833:$ PCONTI/PCONTICJILA
COM: 296,36
COOK JOHN W
8O32 SLEEPY VIEW LN
SPRINGFIELO VA 22153
USA
IEL: 2027672161
ILF:
EML:
COM: 10,12
TLX:

COMBI MICHAEL R OR

SPACE PHYSICS RESEARCH LB

UNIVERSITY OF MICHIGAN

2455 HAYWARD ST

ANN ARBOR MI 481092143

USA

TEL: 3137647226

TLF: 3137473083

TIX:

EML: COMBIOSPRLC.SPL, UMICH. EDU

COM: 15,16

CONCONI PADLO DR

OSS ASTRONOMICO DI MILANO

VIA E BIANCHI 46

I 22055 MERATE

ITALY

TEL: 592035

TLX:

EML:

TLF :

COM:

CONNES PIERRE DR

SERVICE O'AERONOMIE

BP 3

F 91371 VERRIERES BUISSON

FRANCE

TEL: $1 \quad 64 \quad 474277$

TLF :

TLX: 602400

EML:

COM: 24,51

CONTOPOULOS GEORGE PROF

DPT OF ASTRONOMY

NTL UNIVERSITY OF ATHENS

PANEPISTIMIOPOLIS

GR 15771 ATHENS

GREECE

TEL: $1724 \quad 3211$

TLF :

TLX:

EML:

COM: $07,28,33$

COOK KEM HOLLANO DR

LAWRENCE LIVERMORE LAB

L 401

BOX $80 B$

LIVERMORE CA 94550

USA

TEL: $510423 \quad 4634$

TLF: 5102945512

TLX:

EML: KCOOKOIMAGER, LLNL, GOV

COM: 28
COMINS NEIL FRANCIS

OPT PHYSICS \& ASTRONOMY

UNIVERSITY OF MAINE

BENNETT HALL.

ORONO ME 04469

USA

TEL: 2075811037

TLF:

TLX:

EML:

COM: 33

CONOON JAMES D DR

NRAO

EOGEMONT RD

CHARLOTTESVILLE VA 22903

USA

TEL: 8042960211

TLF:

TLX: 910-997-0174

EML:

COM: $40,47,48$

CONNDLLY LEO PAUL

DPT OF PHYSICS

CALIFORNIA STATE UNIV

5500 UNIVERSITY PARKWAY

SAN BERNARDINO CA 92407

USA

TEL: 7148805400

TLF:

TLX:

EML:

C.OM: $25,27,35$

CONWAY ROBIN G OR

NRAL.

JOORELL BANK

MACCLESFIELO SK $1190 L$ UK

TEL: 47771321

TLF :

TLX: 36149

EML:

COM: 40

COOKE B A DR

QPंT OF PHYSICS

UNIVERSITY OF LEICESTER

$X$-RAY ASTRONOMY GROUP.

LEICESTER LEI 7RH

UK

TEL: $533 \quad 554 \quad 455^{\star} 188$

TLF :

TLX: 341664

EML:

COM:
COMORETTO GIOVANNI

OSS ASTROFISICO

DI ARCETRI

LARGO E FERMI 5

I 50125 FIRENZE

ITALY

TEL: 554378540

TLF : 55435939

TLX: 572268 ARCETR I

EML:

COM:

CONKLIN EDWARD K

FORTH INC

111 N SEPULLEDA BLVD 300

MANHATTAN BEACH CA 90266

USA

TEL:

TLF :

TLX: 275182 FORT UR

EML:

COM: 40

CONTADAKIS MICHAEL E DR DPT GEODESY \& SURVEYING UNIVERSITY THESSALONIKI UNIV BOX 503

GR 54006 . THESSALONIKI

GREECE

TEL: 31992693

TLF :

TLX: 412181 AUTH GR

EML:

COM: 27

COOK ALAN H PROF

OPT PHYS/UNIV CAMRIOGE

THE MASTER'S LOOGE

SELWYN COLLEGE

CAMBRIDGE CB3 9DQ

UK

TEL: $22362381 * 29$

TLF :

TLX: 81292 CAVLAB

EML:

COM: 07,14

CODKE JOHN ALAN

ROYAL OBSERVATORY

BLACKFORD HILL

EOINBURGH EHG 3 HJ

UK

TEL: 316.673221

TLF :

TLX: 72383 ROEOIN G

EML:

COM: 09 


\begin{tabular}{|c|c|c|c|}
\hline CORADINI ANGIOLETTA & CORBALLY CHRISTOPHER & CORBELLI EOVIGE OR & CORBIN THOMAS ELBERT DR \\
\hline IAS & STEWARD OBSERVATORY & OSS ASTROFISICO & US NAVAL OBSERVATORY \\
\hline REPARTO DI PLANETOLOGIA & UNIVERSITY OF ARIZONA & OI ARCETRI & ASTROMETRY OPT \\
\hline VIA DELL'UNIVERSITA 11 & VATICAN OBS RESEARCH GP & LARGO E FERMI 5 & $34 \&$ MASSACHUSETTS AVE NW \\
\hline I 00185 ROMA & TUCSON AZ 85721 & I 50125 FIRENZE & WASHINGTON DC·20392 5100 \\
\hline ITALY & USA & ITALY & USA \\
\hline TEL: 64956951 & TEL: 6026213225 & TEL: 554378540 & TEL: $202653 \quad 1557$ \\
\hline TLF: & TLF: & TLF: 55435939 & TLF: \\
\hline TLX: 580489 CNR FRA & TLX: 467175 & $T L X:$ & $T L X: 710-8221970$ \\
\hline EML: & EML: & EML: EDVIGESISIFO.ARCETRI. ASTRO.IT & EML: \\
\hline COM: & COM: $29.45 \mathrm{C}$ & COM: 34 & EOM: $08 \mathrm{C}, 24$ \\
\hline CORDES JAMES M & CORDOVA FRANCE A D & CORLISS CHOR & CORNEJO ALEJANORO A DR \\
\hline CORNELL UNIVERSITY & DPT OF ASTRONOMY & FOREST HILLS LABORATORY & INAOE \\
\hline SPACE SCIENCES BLDG & PENNSYLVANIA STATE UNIV & 2955 ALBEMARLE STREET NW & TONANTZ INTLAZ \\
\hline ITHACA NY 14853 & 525 DAVEY LAB & WASHINGTON DC 20008 & APDO POSTAL 216 y 51 \\
\hline USA & UNIVERSITY PARK PA 16802 & USA & 72000 PUE.BLA PUE \\
\hline TEL: $607 \quad 256 \quad 3734$ & USA & TEL: 2023626085 & MEXICO \\
\hline$T L x: 9322458$ & TEL: & TLF: & TELL: 47-05-00 \\
\hline EML: & TLF: & $T L X:$ & TLF: \\
\hline VLF: & TLX: & EML: & TLX: \\
\hline COM: 40 & EML: & COM: 14 & EML: \\
\hline & CDM: 44 & & COM: 09,41 \\
\hline & & & \\
\hline CORNIDE MANUEL & CORNILLE MARGUERITE OR & CORREIA EMILIA DR & CORWIN HAROLD $G \mathrm{JR}$ \\
\hline DPT DE ASTROFISICA & OBSERVATOIRE DE PARIS & CRAAE/ESCOLA POLITECNICA & CALTECH \\
\hline FAC DE FISICA & SECTION DE MEULON & UNIVERSIOADE DE SAO PAULO & MS 10022 \\
\hline UNIVERSIOAD COMPLUTENSE & UA 812 & CP 8174 & IPAC \\
\hline E 28040 MADRID & F 92195 MEUDON PPL COK & 05508 SAO PAULO'SP & PASADENA CA 91125 \\
\hline SPAIN & FRANCE & BRAZIL & USA \\
\hline TEL: 14495316 & TEL: $1 \quad 45 \quad 07 \quad 7455$ & TEL: 118156289 & TEL: 8185842902 \\
\hline TLF: & TLF: & TLF: $11815 \quad 4272$ & TLF: $818 \quad 584 \quad 2902$ \\
\hline$T L X: 47273$ FFUC & TLX: 201571 & TLX: 1180127 INPE BR & $T L X: 675429$ \\
\hline EML: & EML: BITNET:FRORS3I & EML: ECORREIA@BRUSP.ANSP.BR & EML: infoxipachamlet \\
\hline COM: 29 & COM: 14 & COM: 10 & $\operatorname{COM}: 28$ \\
\hline COSMOVICI BATALLI C DR & COSIA EDGARDO OR & COSTA ENRICO & COSTA JOAQUIM EDUARDO OR \\
\hline IAS & DPT DE ASTRONOMIA & IAS & CRAAE/PTE ESCOLA POLI USP \\
\hline ENR & UNIVERSIDAD DE CHILE & CNR & \\
\hline I 00044 FRACASTI & CASILLA 360 & CP 67 & CP B174 CEP \\
\hline ITALY & SANTIAGO & I 00044 FRASCATI & O1051 SAO PAULO SP \\
\hline TEL: 69423801 & CHILE & ITALY & BRAZIL \\
\hline TLF : & TEL: 22294101 & TEL: 69425655 & TEL: 118156289 \\
\hline$T L X: 610261 \mathrm{I}$ & TLF: 22712799 & TLF: 69416847 & TLF: $11 \quad 815 \div 4272$ \\
\hline EML: & ILX: 440001 & $T L X: 610261 \mathrm{I}$ & $T L K: 1180127$ INPE $B R$ \\
\hline \multirow[t]{2}{*}{ COM: 15} & EML: & EML: & EML: JERCOSTA@BRUSP.ANSP.QR \\
\hline & COM: 08.33 & COM: & COM: 10 \\
\hline COSTA VICTOR DR & COSTAIN CECIL C DR & COSTERO- RAFAEL & COTION WILLIAM D Jr \\
\hline INST ASTROFISICA & DIVISION OF PHYSICS & INSTITUTO OE ASTRONOMIA & NRAO \\
\hline DE ANDALUCIA APD 2144 & NTL RESEARCH COUNCIL & UNAM & \\
\hline C/SANCHO PANZA S/N & 100 SUSSEX OR & APDO POSTAL $70-264$ & EOGEMONT RD \\
\hline E IBOBO GRANADA & DTTAWA ON KLA ORG & 04510 MEXICO DF & CHARLOTTESVILLE VA 22901 \\
\hline SPAIN & CANADA & MEXICO & USA \\
\hline TEL: $\begin{array}{rlll}58 & 12 & 1311\end{array}$ & TEL: 6139936060 & TEL: $548-5305$ & TEL: $904 \quad 296 \quad 0319$ \\
\hline TLF: & TLF : $613952 \quad 6602$ & TLF : & $T L F:$ \\
\hline TLX: 78573 IAGG E & $T L X:$ & TLX: 1760155 CICME & TLX: 5105875482 \\
\hline EML: & EML: & EML: & EML: \\
\hline COM: & COM: & COM: $34,50 \mathrm{C}$ & COM: 40 \\
\hline
\end{tabular}


COTTRELL PETER LEDSAM

DPT OF PHYSICS

UNIVERSITY OF CANTERBURY

PRIVATE BAG

CHR ISTCHURCH 1

NEW ZEALAND

TEL: $03 \quad 482009$

TLF:

TLX: $4144 \mathrm{NZ}$

EML:

COM: 29

COUPER HEATHER MISS

COLLINS COTTAGE

LOWER RD

LOOSLEY ROW

BUCKS HP17 OPF

uK

TEL:

TLF:

TLX:

EML:

COM: 46,51

COUSINS A W J DR

SAAO

BQX 9

OBSERVATORY 7935

SOUTH AFRICA

TEL: 021-47-0025

TLF:

tLX: 5720309

EML:

COM: 25

COUTURIER PIERRE
INSU
77 AVE OENFERT ROCHEREAU
F 75014 PARIS
FRANCE
IEL: 140512004
TLF: 140512101
TLX:
EML: IAPOBS: : COUTURIER
COM: 49

COWLEY ANNE $P$ OR

DPT OF PHYSICS

aRIZONA STATE UNIVERSITY

TEMPE AZ 85287

USA

IEL: 6029652919

TLF:

TLX:

EML:

COM: 29.42 .45
COUEH WARRICK OR

$\triangle A O$

BOX 296

EPPING NSW 2121

AUSTRALIA

TEL: 28681666

ILX: 23999 AAOSYO AA

EML:

ILF:

COM: 28

COUPINOT GERARD OR

OBS MIOI PYRENEES

9 R PONT DE LA MOULEITE

F 65200 BAGNERES BIGORRE

FRANCE

IEL: $62 \quad 95,1969$

ILF: 62951969

ILX:

EML:

COM:

COUTEAU PAUL PROF

OCA OBSERY DE NICE

BP 139

F 06003 NICE CDX

FRANCE

TEL: $93 \quad 890420$

TLF :

TLX: 460004 OBSNICE F

EML:

COM: 26

COVINGTON ARTHUR E

131 COLLEGE STREET

KINGSTON ON KOL $4 L 7$

CANADA

TEL:

TLF:

EML:

COM: 10,40

TLX:

COWLEY CHARLES R PROF

OPT OF ASTRONOMY

UNIVERSITY OF MICHIGAN

DENNISON BLOG

ANN ARBOR MI 481091090

USA

TEL: 313.7643437

TLF :

TLX: $810-2236056$

EML:

COM: 29.36
COULSON IAIN M OR

JOINT ASTRONOMY CENTER

UKIRT

665 KOMOHANA ST

HILO HI 96720

USA

TEL:

TLF :

TLX:

EML:

COM: 27

COURTES G PROF

LAS

LES TROIS LUCS

TRAVERSE DU SIPHON

F 13012 MARSEILLE COX4

FRANCE

TEL: 91055900

TLF: $91 \quad 66 \quad 1855$

TLX: 410584

EML:

COM: $28,33,34,44$

COUTREZ RAYMOND A J PROF

G RUE EgIDE BOUVIER

B 1160 BRUSSELS

BELGIUM

TEL:

TLF:

TLX:

EML:

COM: 40

COWAN JOHN J DR

DPT PHYSICS \& ASTRONOMY

UNIVERSITY OF OKLAHOMA

NORMAN OK 73019

USA

TEL: 4053253961

TLF:

$T L X$ :

EML:

COM: 35

COWSIK RAMANATH

TIFR

HOHI BHABHA RO

COLABA

BOMBAY 400005

INDIA

TEL: 22219111

TLF:

I $L X:$

EML:

COM: $28 ; 48$
COUNSELMAN CHARLES C PROF DPT OF EARTH- PLANET \& SCI MIT RH 54620

BOX 165

CAMBRIDGE MA 02139

USA

TEL: $617 \quad 253 \quad 7902$

TLF:

TLX: 921473 MIT CAM

EML:

COM: $07,08,16$

COURVOISIER THIERRY J-L OBSERVATOIRE DE GENEVE CHEMIN DES MAILLETTES 51 CH 1290 SAUVERNY SWITZERLAND

TEL: 227552611

TL'F: 227553983

TLX: 419209 OBS CH

EML: COLRVOI SOOBS. UNIGE. CH

COM: 28,44

COUTTS-CLEMENT CHRISTINE BPT OF ASTRONOHY UNIVERSITY OF TORONTO 60 ST GEORGE ST TORONTO ON M5S IAT CANADA

TEL: 4169785186 TLF: 4169783921

TLX: 06986766

EML:

COM: 27

COWIE LENNOX LAUCHLAN DR INSTITUTE FOR ASTRONOMY UNIVERSITY OF HAWAII 2680 WOODLAWN DR HONOLULU HI 96822 USA

TEL:

TLF:

TLX:

EML:

COM: 34,48

COX ARTHUR N DR

LOS ALAMOS NATIONAL LAB

BOX 1663

LOS ALAMOS NM 87545

USA

TEL: 5056677648

TLF:

$T L X: 910-988-1773$

EML:

COM: 12,27 


\author{
COX DONALO P PROF \\ DPT OF ASTRONOMY \\ UNIVERSITY OF WISCONSIN \\ 475 N CHARTER ST \\ MADISON WI 53706 \\ USA \\ TEL: 6082625916 \\ TLF: \\ Tex: \\ EML: \\ COM: 34 \\ CRAig ian Jonathan O oR \\ DPT APPLIED MATHEMATICS \\ UNIVERSITY OF WAIKATO \\ HAMILTON \\ NEW ZEALAND \\ TEL: $628 B 9$ \\ TLF : \\ TLX: \\ EML: \\ COM: 12
}

CRAMPTON DAVIO OR HERZBERG INST ASTROPHYS OOMINION ASTROPHYS OBS 5071 W SAANICH RO VICTORIA BC VQX 4M6 CANADA

TEL: 6043883900

TLF: 6043630045

TLX: 0497295

EML:

COM: $30,33,45$

CRAWFORD DAVID L DR
KITT PEAK NTL OBS
BOX 26732
$950 \mathrm{~N}$ CHERRY AVE
TUCSON AZ 857266732
USA
TEL: 6023259346
TLF: 6023259360
TLX: 1561401 AURA UT
EML:
COM: 09,25, 33, $45,50 C$
CRISTALDI SALVATORE OR
OSS ASTROFISICO
CITIA UNIVERSITARIA
VIA ARTALE ALAGONA 75
I 95126 CATANIA
ITALY
TEL: 33 O734
TLF:
TLX: 970359 ASTRCT I
EML:
COM: 42

COX PIERRE DR

observatoire de marseille

2 PLACE LE VERRIER.

F 13248 MARSEILLE COX 04

FRANCE

, TEL: 91959088

TLF: $9162 \quad 1190$

TLX:

EML: COXEFROMRSSI

COM: 34

CRAINE ERIC RICHARD OR

WESTERN RESEARCH CO

5061 W CAMINO OE GIRASOL

TUCSON AZ 85745

USA

TEL: 6027437377

TLX:

EML:

TLF:

COM:

CRANE PATRICK $\mathrm{C}$

NRAD

BOX 0

SOCORRO NM 878010387 USA

TEL: 5057724011

TLX: 910-988-1710

EML:

TLF:

COM: 40,47

CRAWFORD IAN ANDREW DR OPT PHYSICS \& ASTRONOMY UNIVERSITY COLLEGE LONDON GOWER ST

'LONOON WCLE 6BT uK

TEL: $1713877 \quad 050 \star 3498$

TLF: I $713807 \quad 145$

TLX: 28722

EML: IACASTAR. UCL. AC UK

COM: 34

CRISTESCU CORNELIA G IR ASTRONOMICAL OBSERVATORY CUTITUL DE ARGINT 5

BOX 28 .

R 75212 BUCAREST

RUMANIA

TEL: 236892

TLF:

TLX:

EML:

COM: 15,20
COYNE GEORGE $\checkmark D R$

DIRETTORE DELLA SPECQLA

VATICANA

I O0120 VATICAN CITY

VATICAN CITY STATE

TEL: 6 699 3411

TLF:

TLX: 2020 VAT OBS VA

EML:

COM: $25,34.50$

CRAM LAWRENCE EDWARD PROF SCHOOL OF PHYSICS UNIVERSITY OF SYONEY SYDNEY NSW 2006 AUSTRALIA

TEL: $2 \quad 692 \quad 2537$

TLF: 26602903

TEX: AA26169 UNISYO

EML: LCOPHYSICS.SU,OZ,AU COM: $12,36 \mathrm{YP}$

CRANE PHILIPPE

ESO

KARL-SCHWARZSCHILD-STR 2

D 8046 GARCHING MUENCHEN GERMANY

TEL: 89792098

TLF :

TLX: 528-28222 EO D

EML:

COM: $28,34,47$

CREZE MICHEL DR

BBS DE STRASBDURE

11 RUE UNIVERSITE

F 67000 STRASBOURG

FRANCE

TEL: 88358216

TLF: 88250160

TLX: OBSBES $361144 \mathrm{~F}$.

EMt: crezesimbad. $u$-strasbg. $f \mathrm{r}$

COM: $05 C, 24,33$

CRISTIANI STEFANO DR

OPT DI ASTRONOMIA

UNIVERSITA DI PAgOVA

VIC OELL OSSERVATORIO 5

I 35122 PADOVA

ITALY

TEL: $49 \quad 66 \quad 1499$

TLF :

TLX: 432071

EML: SPAN: $39003:$ :CRISTIANI

COM: 47
CRABTREE DENNIS DR HERZBERG INST ASTROPHYS DOMINION ASTROPHYS OBS 5071 W SAANICH RO VICTORIA BC VBX $4 M 6$ CANADA

TEL: 6043980025

TLF : 6043630045

TLX: 0497295

EML:

COM:

\section{CRAMER NEIL OR} SCHOOL OF PHYSICS UNIVERSITY OF SYDNEY SYONEY NSW 2006 AUSTRALIA

TEL: 26923162 TLF: 26602903

TLX: AA 26169

EML:

COM: $10,12,49$

CRANNELL CAROL JO DR NASA/GSFC

CODE 682

GREENBELT MO 2077

USA

TEL: 3012865007

TLF :

TLX: 89675

EML:

COM: 10,44

CRIFO FRANCOISE DR observatoire de paris SECTION DE MEUDON. DEPEG

F 92195 MEUDON PPL CDX FRANCE

TEL: $1 \quad 45 \quad 077834$

TLF :

TLX: 201571

EML:

COM: 08,24

CRIVELLARI LUCIO

OAT

BOX SUCC TRIESTE 5

VIA TIEPOLO 1

I 34131 TRIESTE

ITALY

TEL: 40793221

TLF:

TLX: 461137 DAT I

EML:

COM: 36 


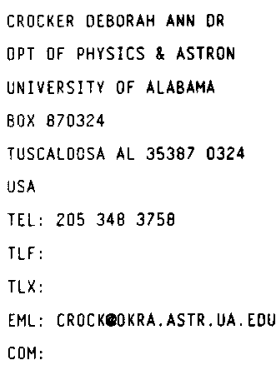

CROOH DAVID L DR RUTHERFORD APPLETON LAB SPACE \& ASTROPHYSICS DIV BLOG R25/R6B CHILTON OIOCOT OXI1 OQX UK

TEL: 23521900

TLF :

$T L X: 83159$

EML:

COM: 40

CRUTCHER RICHARD M OR DPT OF ASTRONOMY

UNIVERSITY OF ILLINOIS

1011 W SPRINGFIELO AVE. URBANA IL 61801

USA

TEL: 2173339581

TLF :

ILX:

EML:

COM:

CUBARSI RAFAEL OR

OPT MATEMATICA APLICADA

UNIV POLITEC DE CATALUNYA

BOK 30002

E 08080 BARCELONA

SPAIN

TEL: 34016799

ILF: 34016801

ILX: 5282 I UPC-E.

EML: MATRCMMMAT.UPC.ES

COM: 33

CUGIER HENRYK DR

ASTRONOMICAL INSTITUTE.

WROCLAW UNIVERSITY

UL KOPERNIKA 11

PL 51622 WROCLAW

POLAND

TEL: $48 \quad 24 \quad 34$

TLF :

TLX: 712791 UWR PL

EML:

COM: 36

\section{CUI ZHEN-HUA}

BEIJING PLANETARIUM

\section{$138 \times 1$ WAI ST}

BEIJING

CHINA PR

TEL: 1 G9 3003

TLF:

TLX:

EML :

COM: 41.46
CROVISIER JACQUES OBSERVATOIRE DE PARIS SECTION DE MELIDON

$F$ 92195 MEUDON PPL CDX FRANCE

TEL: $145 \quad 077599$

TLF :

TLX: 270912

EML:

COM: $15,34,40$

CRUVELLIER PAUL E OR

LAS

TRAVERSE OU SIPHON

LES TROIS LUCS

F 13012 MARSEILLE

FRANCE

TEL: 91055900

TLF: $9166 \quad 1855$

TLX: 420584

EML:

COM: 34

CUDABACK OAVIO D DR

RADIO ASTRONOMY LAB

UNIVERSITY OF CALIFORNIA

601 CAMPBELL HALL

BERKELEY CA 94720

USA

TEL: 4156425724

TLF:

TLK: 820181 UCB AST

EML:

CDM: 34,40

CUGNON PIERRE DR

OBSERVATOIRE ROYAL DE

BELGIQUE

AVE CIRCULAIRE 3

B 1180 BRUSSELS

BELGIUM

TEL: $2 \quad 375 \quad 2484$

TLF:

$T\lfloor X: 21565$ OBSBE

EML:

COM: 34

CULHANE LEONARO PRDF.

MULLARD SPACE SCIENCE LAB UNIVERSITY COLLEGE LONDON HOLMBURY ST MARY DORKING SURREY RHE GNT UK

TEL: 30670292

TLF:

TLX: 859185 UCMSSL G

EML:

COM: $10,44,48$
CRUIKSHANK DALE P DR NASA AMES RESEARCH CTR MS 2456 MOFFETT FIELD CA 94035 USA

TEL:

TLF:

$T L X$ :

EML:

COM: 15,160

CRUZ-GONZALEZ IRENE INSTITUTO DE ASTRONOMIA UNAM

APDO POSTAL 70-264

04510 MEXICO OF

MEXICO

TEL; 905-548-5306

TLF :

TLX:

EML:

COM:

CUOWORTH KYLE MCCABE OR YERKES OBSERVATORY UNIVERSITY OF CHICAGO BOX 258 WILLIAMS BAY WI 53191 USA

TEL: 4142455555

TLF:

TLX:

EML:

COM: $24,33,37$

CUGUSI LEONINO DR DPT OI SCIENZE FISICHE UNIVERSITA OI CAGLIARI VIA OSPEDALE 72 I 09100 CAGLIARI ITALY

TEL: 7066.4770

TLF :

TLX:

EML:

COM:

CULLUM MARTIN DR

ESO

KARL SCHWARZSCHILD STR. 2 D 8046 GARCHING MUENCHEN GERMANY

TEL: 89320060

TLF: $89320 \quad 2362$

TLX: 5282820 EO 0

EML: EARN : cullumedgaes 051

COM: O9C 
CULVER ROGER BRUCE OR

DPT OF PHYISCS

CQLORAGO STATE UNIVERSITY FORT COLLINS CO 80523

USA

TEL: 3034916206

TLF:

TLX: 9109309000 ENGRCSUFT

EML:

COM: 26

\section{CURIR ANNA}

OSS ASTRONOMTCO DI TORINO

ST OSSERVATORIO 20

I LO02S PINO TORINESE

ITALY

TEL: 11841067

TLX: 213236 TO ASTR 1

EML:

TLF:

COM: 48

\section{CZERNY BOZENA DR} COPERMICUS ASTRON CENTER POLISH ACAD OF SCIENCES

UL BARTYCKA 18

PL 00716 WARSAW

\section{POLAND}

TEL: WARSAW 41 DO 41

TLF:

TLX:

EME:

COM:

DA COSTA GARY STEWART OR

$\triangle A O$

B0X 296

EPPING NSW 2121

AUSTRALIA

TEL: 28681666

TLX: 123999 AAOSYO

EML: DACOSTA\%adicpp. oz. au uunet.uu. TLX: 1133530 INPEBR

TLF:

COM: $37 \mathrm{C}$

DA SILVA a V C S

observatorio astronomico

UNIVERSIQADE SANTA CLARA

P 3000 COIMERA

PORTUGAL

TEL:

TLF:

TLX:

EMt:

COM:

\section{CZERNY MICHAL DR}

TEL: 215807313
COM: 48

CUNTZ MANFRED DR

JILA

UMIVERSITY OF COLORADO

BOX 440

bovLder CO BO3090440

USA

TEL:

TLF :

TLX:

EML:

COM: 36

CURRIE DOUGLAS G DR

ASTRONOMY PROGRAM

UNIVERSITY OF MARYLANO

COLLEGE PARK MD 20742

USA

TEL: 3014543405

TLF:

TLX:

EML:

COM: 09,51

DPT OF ASTRONOMY

UNIVERSITY OF LEICESTER

UNIVERSITY RO

LEICESTER LEI TRH

UK

TEL: $533 \quad 522 \quad 073$

TLF:

TLX: 347250 LEICUN

EML:

com:

DA COSTA JUSE MARQUES OR

INPE

CP 515

$12200 S$ JOSE DOS CAMPOS

BRAZIL

TEL: $123 \quad 229977$

TLF: $123 \quad 21 \quad 8743$

EMt:

DA SILVA LICIO DR

GBSERVATOR IO NACIONAL

RUA GL BRUCE $586^{\circ}$

SAO CRISTOVAO

20921 RTO DE JANEIRO R.J

BRAZIL

TLF: 215800332

TLX: 21288

EML:

COM:
CUNY YVETTE J OR

observatotre de paris

SECTION DE MEUUDON

F 92195 MEUDON PPL COX

FRANCE

TEL: 145077838

TLF:

TLX:

EML:

COM: $36 \mathrm{C}$

CUTISPOTO GIUSEPPE DR

OSS ASTROFISICO

CITTA UNIVERSITARIA

VIA A DORIA 6

I 95125 CATANIA

ITALY

TEL: 95337241.

TLF: $95 \quad 330592$

TLX: 970359 ASTRCT I

EML: 40297::GIUSEPPE

COM: 27

CZYZAK STANLEY 3 DR

DPT OF PHYSICS

OHIO STATE UNIVERSITY

174 W. 18TH AVE

COLUMBUS OH 43210

USA

TEL: $614422 \quad 6543$

TLF:

TLX:

EML:

COM: 14,34

DA COSTA NICOLAI L-A OBSERVATORIO NACIONAL

RUA GL BRUCE 586

SAO CRISTOVAO

20921 RIO DE JANEJRO RJ

BRAZIL

TEL: 215807181

TLF: 215800332

TLX:

EML: LNOCOLNCCVM

COM: $28,30 \mathrm{C}$

DACHS JOACHIM PROF OR

ASTRONOMISCHES INSTITUT

RUHR-UNIVERSI TAET

POSTFACH 102148

D 4630 BOCHUM 1

GERMANY

TEL: 2347003454

TLF:

TZX: 825860

EML:

COM: 21,25
CUPERMAN SAMI PROF

DPT OF PHYSICS \& ASTRON

TEL AVIV UNIVERSITY

RAMAT AVIV

TEL AVIV 69978

ISRAEL

TEL: $342022 \tilde{L} / 425697$

TLF:

TLX: 342LYL VERSY IL

EML:

COM: 33,49

CUYPERS JAN DR

OBSERVATOIRE ROYAL DE

Belgique

aVE CIRCULAire 3

B 1180 ERUSSELS

BelgiUM

TEL: 23752484

TLF:

TLX: 21565

EML:

COM: $15,25,27$

DA COSTA ANTONIO A. DR COMPLEXO INTEROISCIPLINAR - COMPLEXO INTEROISCIPLANAR INSTITUTO SUPERIOR TECNIC

P 1096 LISBOA COOEX PORTUGAL

TEL: 35113524303

TLF: 35113524372

TLX:

EML:

COM: 48

DA ROCHA VIEIRA E DR INSTITUTO DE FISICA

UFRGS

CP 15051

90000 PORTO ALEGRE RS

BRAZIL

TEL: 512217666

TLF:

TLX: 0511055 LUFRSBR

EML:

COM:

DADAEV ALEKSANOR N DR PULKOVO OBSERVATORY ACADEMY OF SCIENCES

10 KUTUZOV QUAY 196140 ST PETERSBURG RUSSIA

TEL :

TLF :

TLX:

EML:

COM: 26,42 


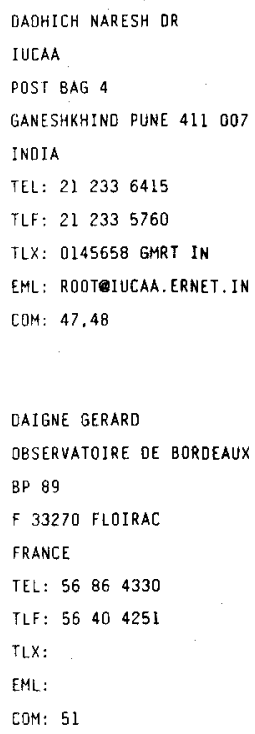

DADIC ZARKO DR zAVOD ZA POUVIJEST ZNANOSTI JAZU ANTE KOVACICA 5 YU 41000 ZAGREB YUGOSLAVIA TEL: $41 \quad 440 \quad 124$ TLF:

TLX:

EML

COM: 41

DAINTREE EDWARD J OR NRAL

JOORELL BANK

MACCLESFIELO SKLI 9OL UJK

TEL: 47771321

ILX: 36149

EML:

TLF :

COM: 40

DALL'OGLIO GIORGIO DR DPT DI FISICS UNIVERSITA DI ROMA

PA MORO 2

I 00185 ROMA

ITALY

TEL: $6 \begin{array}{lll}6 & 49 & 91427]\end{array}$

ILF: $6 \quad 495,7697$

TLX: 613255 INFN RO

EML: VAXMA:: DALLAGLIO

COM: 09

DAN XH1-XIANG

SHANGHAI OBSERVATORY

CAS

BO NANDAN RD

SHANGHAI

CHINA PR

TEL: $2138 \quad 619$ ]

TLF :

TLX: 33164 SHAO CN

EML:

COM: 09

OANFORD STEPHEN C OR
DPT OF PHYSICS \& ASTRON
UNIVERSITY OF N CAROLINA
AT GREENSBORO
GREENBORO NC 27412
USA
TEL: 9193345669
TLF:
TLX:
EML: DANFOROEUNCG.BITNET
COM: $25,27.37$

DAGKESAMANSKY RUSTAM D DR

LEBEDEV PHYSICAL INST

ACADEMY OF SCIENCES

LENINSKY PROSPEKT 53

117924 MOSCOW

RUSSIA

TELL: $135 \quad 14 \quad 29$

TLF:

TLX: 411479 NEOD SU

EML:

COM: 40

DAISHIDO TSUUNEAKI PROF OPT OF SCIENCE

WASEDA UNIVERSITY

SHINJUKU KU TOKYO 160

JAPAN

TEL: $2-203-4141$

TLF:

TLX: 2323280 WASEDA $J$

EML:

COM: 40

DALTABUIT ENRIQUE DR INSIITUTO DE ASTRONOMIA

UNAM

APDO POSTAL 70-264

04510 MEXICO DF

MEXICO

TEL:

TLF :

TLX:

EML:

COM:

JANBY J M ANTHONY DR

DPT DF MATHEMATICS

N CAROLINA STATE UNIV

RALEIGH NC 276958205

USA

TEL: 9197373210

TLF :

TLX:

EML:

COM: 07

DANILOV VLADIMIR M OR OPT OF ASTRONOMY URALSKIJ STATE UNIVERSITY 629983 SVERTLLVVSK

RUSSIA

TEL: 22.3386

TLF :

TLX:

EML:

COM:
DAHN CONARO CURTIS DR US NAVAL OBSERVATORY FLAGSTAFF STATION BOX 1149

FLAGSTAFF AZ 86002

USA

TEL: 6027795132

TLF:

TLX:

EML:

COM: $24: 25,34$

DALGARNO ALEXANDER PROF CENTER FOR ASTROPHYSICS HCO/SAO

60 GARDEN ST

CAMBRIDGE MA 02138

USA

TEL: 6174954403

TLF :

TLX: $92142 B$

EML: DALGARNDECFA7

COM: 14,34

DAMINELI NETO AUGUSTO OR IAG

UNIVERSIDADE OE SAO PAULO CP 30627

OLO5I SAO PAULO SP

BRAZIL

FEL:

TLF :

TLX:

EML:

COM:

DANESE. LUIGI DR OSS ASTRONOMICO DI PADOVA

VIC DELL OSSERVATORIO 5

I 35122 PADOVA

ITALY

TEL: $4966 \quad 1499$

TLF:

ILX: 430176 UNPADJ-I

EML:

COM: 47

DANKS ANTHONY C DR

$13: 5$ PEACHTREE CT

BOWIE MO 20721

USA

TEL: 3012498206

TLF:

TLX:

EML:

CGM: 15,$28 ; 34$ 


\begin{tabular}{|c|c|c|c|}
\hline DANLY LAURA OR & OANZIGER I JOHN OR & DAPERGOLAS A DR & DAPPEN WERNER \\
\hline STSCI & ESO & ASTRONOMICAL INSTITUTE & OBSERVATOIRE DE PARIS \\
\hline HOMEWOOD CAMPUS & KARL-SCHWARZSCHIILO-STR 2 & NTL OBSERVATORY OF ATHENS & SECTION OE MEUOON \\
\hline 3700 SAN MARTIN OR & D 8046 GARCHING MUENCHEN & $80 \times 20048$ & F 92195 MEUDON PPL COX \\
\hline BALTIMORE MD 21218 & GERMANY & GR 11810 ATHENS & FRANCE \\
\hline USA & TEL: 89320060 & GREECE & TEL: 145347530 \\
\hline TEL: $301338 \quad 4422$ & TLX: & TEL: $1346119 \mathrm{l}$ & TLF: \\
\hline TLF: 3013385090 & EML: & TLF: & TLX: \\
\hline TLX: 6839101 & . TLF: 893202362 & TLX: & EML: \\
\hline EML: SCIVAX: : DANLY/DANLY Y TSCI & COM: & EML: & COM: \\
\hline COM: 34 & & COM: 37 & \\
\hline DARA HELEN DR & OARIUS JON DR & DAS MRINAL KANII & DAS P K QR \\
\hline RES CENTER FOR ASTRONOMY & SCIENCE MUSEUM & OPT PHYSICS/DELHI UNIV & INOIAN INSTITUTE OF \\
\hline ACADEMY OF ATHENS & LONDON SW7 200 & SRI VENKATESWARA COLLEGE & ASTROPHYSICS \\
\hline 14 ANAGNOSTOPOULOU ST & UK & DHAULLA KUAN & KORAMANGALA \\
\hline GR 10673 ATHENS & TEL: Y $5893456 * 643$ & NEW OELHI $110 \quad 021$ & BANGALDRE 560034 \\
\hline GREECE & TLF: & INOIA & INOIA \\
\hline TEL: 13613589 & EML: & TEL: & TEL: 812566585 \\
\hline TLF : & COM: $41,46,51$ & TLF: & TLF: \\
\hline TLX: & TLX: 21200 SCMLIB G & $T L X:$ & $T L X: 845763$ IIAB IN \\
\hline EML: EXAKAZOEGRTHUN1 & & EML: & EML: \\
\hline COM: 12 & & COM: 35 & COM: 47 \\
\hline DATLOWE DAYTON DR & DATTA BHASKAR OR & DAUBE-KURZEMNIECE I A OR & DAUTCOURT G OR \\
\hline LOCKHEEO PALO ALTO RES LB & INDIAN INSTITUTE OF & RADIOASTROPHYSICAL OBS & ZNTRLINST, F ASTRGPHYSIK \\
\hline DPF $9120 \quad B L 06 \quad 255$ & ASTROPHYSICS & LATVIAN ACAB DF SCIENCES & STERNWARTE BABELSBERG \\
\hline 3251 HANOVER ST & KORAMANGALA & TURGENEVA 19 & ROSA-LUXEMBURG-STR $17 \mathrm{~A}$ \\
\hline PALO ALTO CA 94304 & BANGALORE 560034 & 226524 RIGA & O 1502 POTSDAM \\
\hline USA & INOIA & LATVIA & GERMANY \\
\hline TEL: 4158584074 & TEL: $812566585 / 6497$ & TEL: 226796 & TEL: \\
\hline TLF: & TLF: & TLF: & TLF : \\
\hline TLX: & ILX: 845763 IIAB IN & TLX: & $T L x:$ \\
\hline EML: & EML: & EML: & EML: \\
\hline COM: 10 & COM: 47 & COM: 37 & COM: 48 \\
\hline DAVID LAURENCE P OR & DAVIDGE TIMOTHY J DR & OAVIOSEN ARTHUR FALNES DR & OAVIDSON KRIS DR \\
\hline CENIER FOR ASTROPHYSICS & HERZBERG INST ASTROPHYY & DPT PHYSICS \& ASTRONOMY & SCHOOL OF PHYS \& ASTRON \\
\hline MS 4 & DOMINION ASTROPHYS OBS & JOHNS HOPKINS UNIVERSITY & UNIVERSITY OF MINNESOTA \\
\hline 60 GARDEN ST & $5071 \mathrm{~W}$ SAANICH RD & CHARLES \& 34 TH ST & 116 CHURCH ST SE \\
\hline CAMBRIDGE MA D213日 & VICTORIA BC VBX AME & BALTIMORE MO 21218 & MINNEAPOLIS MN 55455 \\
\hline USA & CANADA & USA & USA . \\
\hline TEL: 6174957245 & TEL: $604 \quad 363 \quad 0047$ & TEL: 3013387370 & TEL: $612 \quad 373 \quad 7795$ \\
\hline TLF: 6174957356 & TLF: & TLF: & TLF: \\
\hline$T L x:$ & TEx: & TLX: & $T L x:$ \\
\hline EML: DAVIDCFA & EML: DAVIOGECOAD.NRC.CA & EML: & EMI : KD@APS2.SPA.UMK.EOL \\
\hline COM: & COM: 28 & COM: $28,44,48$ & COM: \\
\hline DAVIOSON WILLIAM PROF & DAVIES MERTON E MR & DAVIES PAUL CHARLES $W$ & DAVIES RODNEY O PROF \\
\hline 80 WEST CLOSE & THE RANO CORPÓRATION & SCHOOL OF PHYSICS & NRAL \\
\hline FERNHURST & 1700 MAIN ST & UNIVERSITY OF NEWCASTLE & \\
\hline HASLEMERE & SANTA MONICA CA 90406 & NEWCASTLE/TYNE NEI TRU & JODRELL BANK \\
\hline SURREY GU27 3JT & USA & uk & MACCLESF IELD SK11 9DL \\
\hline UK & TEL: $213393041 \mathrm{~L}$ & TEL: & UK \\
\hline rEL: & TLF: & TLF: & TEL: 47771321 \\
\hline TLF : & TLX: & $T L x:$ & TLF: $477716 \quad 18$ \\
\hline TLX: & EML: & EML: & TLX: 36149 \\
\hline EML: & COM: $04,16 C$ & COM: 47 & EML: \\
\hline COM: 47,48 & & & COM: $28,33,34,40$ \\
\hline
\end{tabular}


OAVIES ROGER L DR

KITI PEAK NTL OBS

BOX 26732

$950 \mathrm{~N}$ CHERRY AVE

IULSON AZ $85726 \quad 6732$

USA

TEL: 6023259353

ILF:

TLX: 0666484 AURA NOAO

EML:

COM: 47

DAVIS LEVERETT JR PROF
CALTECH
MS 40547
PASADENA CA 91125
USA
TEL: $8 L B \quad 3564243$
TLF:
ILX:
EML:
COM: 48

DAVIS RICHARO J DR

NRAL

JOORELL BANK

MACCLESFIELD SKIY 9OL

UK

TEL:

TLX:

EML:

TLF :

COM: $40 C$

OANANAS DJONI N DR

OPT OF ASTRONOMY

BANDUNG INSTTTUTE OF TECH

$J L$ GANESHA 10

BANDUNG 40132

INGONESIA

TEL: $622 \quad 244 \quad 0252$

TLF:

TLX: 28324 ITB BD

EML:

COM: 29

OE BIASE GIUSEPPE A OR

OAR

VIA OEL PARCO MELLINI 84

100136 ROMA

ITALY

TEL: 6347056

TLF:

TLX:

EML:

COM:
DAVILA JOSEPH OR

NASA/GSFC

CODE 682.1

GREENBELT MO 20771

USA

TEL: 3012868366

TLF:

TLX:

EML: SOLAR: : IDAVILA

COM: 10

DAVIS MARC OR

ASTRONOMY DPT

UNIVERSITY OF CALIFORNIA

601 CAMPBELL HALL

BERKELEY CA 94720

USA

TEL: 4156425156

TLF:

TLX: 820181 UCB AST

EML:

COM: $28,30,47$

DAVIS ROBERT J OR

CENTER FOR ASTROPHYSICS

HCO/SAO MS 20

60 GARDEN ST

CAMBRIOGE MA 02138

USA

TEL: 6164957335

TLF:

TLX: 921428 SATELLITE CAM

EML:

COM: $05,30,40,44$

DAWE JOHN ALAN OR

ANU

SIDING SPRING OBSERVATORY

PRIVATE BAG

COONABARABRAN NSW 2857

AUSTRALIA

TEL: $68426 \quad 221$

TLF:

TLX: 63945 AA CANOPUS

EML:

COM: 50.51

DE BOER KLAAS SJOERDS OR ASTRONOMISCHES INSTITUT UNIVERSITAET BONN AUF OEM HUEGEL $7 \mathrm{~L}$

D 5300 BONN 1

GERMANY

TEL: 228733656

TLF:

TLX: 886440

EML:

COM: $05,28,34$
DAVIS CECIL G JR

LoS alamos national Lab

MS 0406

GROUP P-15

LOS ALAMOS NM 87545

USA

TEL: 5056675908

TLF :

TLX:

EML:

COM: 35,36

DAVIS MICHAEL M OR ARECIBO OBSERVATIRK

BOX 995

ARECIBO PR 00513

USA

TEL: 8098782612

TLF:

TLX: 385638

EML:

COM: $40,47,48,51$

DAVIS SUMNER $P$ DR

PHYSICS DPT

UNIVERSITY OF CALIFORNIA

BERKELEY EA 94720

USA

TEL: 4156424857

TLF:

TLX:

EML:

COM: 14

DE ALMEIDA AMAURY A DR

IAG.

UNIVERSIDADE DE SAO PAULO

CP 30627

01065 SAO PAULO SP

BRAZIL

TEL: $11 \quad 577 \quad 8599 * 38$

TLF: 112763848

ILX: 11 56735 IAEM BR

- EML: 47556: : AMAURY.

COM: 15

DE BRUYN A GER OR

\section{NFRA}

\section{BOX 2}

NE 7990 AA DWINGELOO

NETHERLANDS

TEL: 52197244

TLF:

TLX: 42043 SRZM NL

EML:

COM: 28
DAVIS JOHN PROF

SYDNEY UNIV STELL INTER

THE PAUL WILD OBSERVATORY

BOX 94

NARRABRI NSW 2390

AUSTRALIA

TEL:

TLF : 67959270

TLX:

EML:

COM: 09C, 50C

OAVIS MORRIS S PROF

OAI PHYSICS \& ASTRONOMY

UNIKERSITY NORTH CAROLINA

204 PHILLIPS HALL 039A

CHAPEL HILL NC 27514

USA

TEL: 9199623011

TLF:

TLX:

EML:

COM: 05,07

DAVOUSt EMMANUEL

OBS MIDI PYRENEES

14 AVE E BELIN

F. 31400 TOULOUSE COX

FRANCE

TEL: 6I $25210 \mathrm{~L}$

TLF :

TLX: 530776

EML:

EOM:

OE BERGH CATHERINE DR OBSERVATOIRE OE PARIS SECTION DE MEUOON OPT DE RECHERCHE SPATIALE F 92195 MEUDON PPL CDX FRANCE

TEL: $145 \quad 077666$

TLF:

TLX: 201571

EML: MESIOA: : OEBERGH/DEBERGH RMEUS

COM: 16VP

de carvalho reinaldo or observatorio NaCiOnal.

RUA GL BRUEE 586

SAO CRISTOVAO

2092:1 RIO DE JANEIRO RJ

BRAZIL

TEL: 215807181

TLF: 215800332

TLX: $2128 B$

EML: $47556:$ :REINALOO

COM: 28 
DE CASTRO ANGEL OR OBS ASTRONOMICO NCL

APO 12354

ALFONSO XII-3

E 28014 MAORIO

SPAIN

TEL: 12271935

TLF :

TLX: 234651 GCE

EML:

CON: 04

DE FREITAS PACHECO J A OR

IAG

UNIVERSIDADE DE SAO PAULO

CP 30627

01051 SAO PAULO SP

BRAZIL

ITEL: 217173518

TLF :

TLX

EML:

COM:

DE GREVE JEAN-PIERRE OR

IAAG

VRIJE UNIV BRUSSELS

CP 165

B 1050 BRUSSELS

BELGIUM

TEL: 26413498

TLF :

TLX: 61051 VUBCD

EML:

COM: 35,42

DE JAGER GERHARD PROF

DPT PHYSICS/ELECTRONICS

RHODES UNIVERSITY

BOX 94

GRAHAMSTOWN 6140

SOUTH AFRICA

TEL: $0461-7128$

TLF :

TLX: 244226

EML:

COM:

DE KORT JULES J DR

HOUTLAAN 4

NL 6500 GV NIJMEGEN

NETHERLANOS

TEL:

TLF:

EML:

COM: 42

ILX:
De cÁstro elisa

DPT DE ASTROFISICA

FAC DE FISICA

UNIVERSIDAD COMPLUTENSE

E 28040 MADRID

SPAIN

TEL: 14495316

TLF :

TLX: 47273 FFUC

EML:

COM: 29

DE GRAAF T DR

INSTITUUT VOOR FONETISCHE

WETENSCHAPPEN

GROTE ROZENSTRAAT 31

NL 9712 TG GRONINGEN

NETHERLANOS

TEL:

TLF :

TLX:

EML:

COM: 48

DE GROOT MART DR

ARMAGH OBSERVATORY

COLLEGE HILL

ARMAGH BT6I 9DG

UK

TEL: 861522928

TLF :

TLX: 747937 ARMOBS G

EML:

COM: $27,29.42$

QE JAGER OCKER $C$ DR

DPT OF PHYSICS

POTCHEFSTROOM UNIVERSITY

POTCHEFSTROOM 2520

SQUTH AFRICA

TEL: 148992418

TLF:

TLX: 346019

EML:

COM:

OE KORTE PIETER A J OR SPACE RESEARCH LABORATORY

SRON

SORBONNELAAN 2

NL 3584 CA UTRECHT

NETHERLANDS

TEL: 30535600

TLF: 30540860

ILX: 47224

EML:

COM: de fELICE fERnANDO DR DPT OI FISICA G GALILEI UNIVERSITA OI PADOVA

VIA MARZOLO 8

I 35131 PADOVA

ITALY

TEL: 49844278

TLF:

TLX: 430308 DFGGPOI

EML:

COM: 48

DE GRAAFF W DR

APPELGAARDE 117

NL 3992 JO HOUTEN

NETHERLANDS

TEL:

TLF:

ILX:

EML:

COM: 51

DE GROOT T OR

STERREKUNDIG INSTITUTE

BOX 80000

NL 3508 TA UTRECHT

NETHERLANDS

TEL: 30 535200

ILF :

TLX: 47224 ASTRD

EML:

COM; 40

DE JONG TEIJE OR

ASTRONOMICAL INSTITUTE

UNIVERSITY OF AMSTERDAM

KRUISLAAN 403

HL 1098 SJ AMSTERDAM

NETHERLANOS

TEL: 205257491

TLF: 205257484

TLX: 10262 HEF NL

EML:

COM: 33,34

DE LA HERRAN $\checkmark$ JOSE ENG INSTITUTO OE ASIRONOMIA APDO POSTAL 971

O4510 MEXICO DF

MEXICO

TEL:

TLF :

TLX:

EML:

COM:
DE FREES DOUGLAS J DR IBM ALMADEN RESEARCH CENT OPT $K 84 / 801$

650 HARRY ROAD

SAN JOSE CA $95120-6099$

USA

TEL: $408 \quad 9272854$

TLF : 4089272100

TLX:

EML: OEFREESOALMVMA

COM: 14

DE GRAAUW TH DR

ESA/ESTEC

SSO

BOX 800

NL 2200 AG NOOROWIJK

NETHERLANDS

TEL:

TLF :

TLX:

EML:

COM:

DE JAGER CORNELIS PROF SPACE RESEARCH. LABORATORY SRON

SORBONNELAAN 2

NL 3584 CA UTRECHT

NETHERLANDS

TEL: 30535600

TLF: 30540860

TLX: 47224 SRON NL

EML: XMKEESJEHUTRUUO. BITNET

COM: $12,35,40,44,49,51$

DE JONGE J K DR

DPT OF ASTRONOMY

UNIVERSITY OF PITTSBURGH

RIVERVIEW PARK

PITTSBURGH PA 15214

USA

TEL:

TLF :

TLX:

EML:

COM: 30,51

OE LA NOE JEROME OR OBSERVATOIRE DE BORDEAUX

BP 89

F 33270 FL.OIRAC

FRANCE

TEL: $56 \quad 86 \quad 4330$

TLF: $56 \quad 40 \quad 4251$

TLX:

EML:

COM: $28,34,40$ 


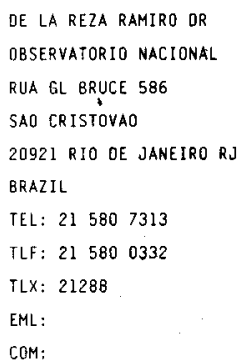

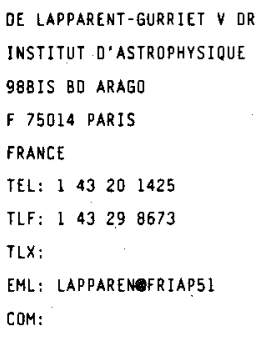

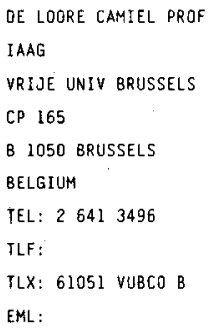

COM:

DE VRIES CORNNELLIS OR

SRL

HUYGENS LAB

B0X 9504

NL 2300 RA LEIDEN

NETHERLANDS

TEL: 71275816

TLF :

TLX:

EML: SRONEGJHLERUL2

COM:

DEARBORN DAVIO PAUL S DR LAWRENCE LIVERMORE LAB

L 23

BOX 808

LIVERMORE CA 94550

USA

TEL:

TLF :

FLX:

EML:

COM: 35 
DEBARBAT SUZANNE V DR OBSERVATOIRE DE PARIS 6.I AVE OBSERVATOIRE

F 75014 PARIS

FRANCE

TEL: 140512209

TLF :

TLX: 270776 OBS F

EML

COM: $08,19,41 P$

DEERENBERG A J M DR

SRL

HUYGENS LAB

BOX 9504

NL 2300 RA LEIDEN

NETHERLANDS

TEL: 71272727

TLF :

TLX:

EML:

COM:

DEHANT VERONIQUE DR

OBSERVATOIRE ROYAL DE

BELGIQUE

AVE CIRCULAIRE 3

B 1180 BRUSSELS

BELGIUM

IEL: 23730246

TLF: $237 \dot{4} 9822$

TLX: 21565 OBSBEL B

EML:

COM: 19

DEJAIFFE RENE J DR

OBSERVATOIRE ROYAL DE

BELGIQUE

AVE CIRCULAIRE 3

B 1180 BRUSSELS

BELGIUM

TEL: 23752484

TLF: 23749822

TLX: 21565 OBSBEL B

EML:

COM: 08,19

DEL OLMO OROZCO A DR

INST ASTROFISICA

OE ANDALUCIA APO 2144

C/SANCHO PANZA S/N

E 18080 GRANADA

SPAIN

TEL: $58 \quad 121311$

TLF: 58 81 4530

TLX: 78573 IAAG E

EML: CHONYEIAA, ES/16488: : CHONY

COM: 28
DEBEHOGNE HENRI OR SC OBSERVATOIRE ROYAL DE BELGIQUE

AVE CIRCULAIRE 3

B 1180 BRUSSELS

BELGIUM

TEL: 23743801

TLF: 23749822

TLX: 21565 B

EML:

COM: 25,20

DEgAOHKAR SS DR

PHYSICAL RESEARCH LAB

NAVRANGPURA

AHMEDABAO 380009

INDIA

TEL: $272 \quad 46 \quad 2129$

TLF: 272445292

TLX: 121397 PRL IN

EML:

COM: 40

DEHARVENG JEAN-MICHEL OR LAS

TRAVERSE OU SIPHON

LES TROIS LUCS

F 13012 MARSEILLE

- france

TEL: 91055900

TLF: $9166 \quad 1855$

TLX: $420584 \mathrm{~F}$.

EML :

COM:

DEJONGHE HERWIG BERT DR

STERREKUNDIG OBSERY

RIJKSUNIVERSITEIT GENT

KRIJGSLJAN 281

B 9000 GENT

BELGIUM

TEL: 91225715

TLF: 91240634

TLX:

EML:

COM: 28.33 .37

DEL RIO GERARDO OR OBS ASTRONOMICO NCL

ALFONSO XII-3

E 28014 MADRID

SPAIN

TEL: $12270107 / 1935$

TLX: 23465 IGCE

EML:

ILF:

COM:
DEBRUNHER HERMANN DR PHYSIKALISCHES INSTITUT UNIVERSITAET BERN

SIDLERSTRASSE 5

CH 3000 BERN

SWITZERLAND

TEL: 31654051

TLF :

TLX: $32320 \mathrm{CH}$

EML:

COM: 48

OEGEWIJ JOHAN DR MOOOERMANSTRAAT 66 NL. 2313 GS LEIDEN NETHERLANOS

TEL:

TLF :

TLX:

EML:

COM: 15,16

DEHARVENG LISE DR

OBSERVATOIRE DE MARSEILLE

2 PLACE LE VERRIER

F 1324 B MARSEILLE COX 04 FRANCE

TEL: 91959088

TLF :

TLX:

EML:

COM: 34.

DEKEL AVISHAI

DPT OF PHYSICS

HEBREW UNIV OF JERUSALEM

JERUSALEM 91904

ISRAEL

TEL: 2584605

TLF :

TLX:

EML:

COM: $28,33,47$

DEL TORO INIESTA JOSE DR INST DE ASTROFISICA

DE CANARIAS

OBS DEL TEIOE

E 38200 LA LAGUNA

SPAIN

TEL: $22 \quad 262211$

TLF: $922 \quad 263005$

TLX: 92640 IACE

EML: SPAN:IAC::JTI

COM: 10,12
DEEMING TERENCE J DR

ICARUS RESEARCH

BOX 540205

HOUSTON TX 77254

USA

TEL: $713772 \quad 8414$

TLF :

TLX:

EML:

COM: 41

DEGUCHI SHUJI DR

NOBEYAMA RAOIO OBS

NAOJ

MINAMIMAKI MURA

NAGANO $384 \quad 13$

JAPAN

TEL: $026798 \quad 2831$

TLF :

TLX: 3329005 TAONRO J

EML:

COM: 34

OEINZER W PROF OR

UNIVERSITAETS - STERNWARTE

GEISMARLANOSTR 11

3400 goettingén

GERMANY

TEL: 551395044

TLF :

TLX: 96753

EML:

COM: 35

DERKER E DR

MEIOOORNLAAN I3

NL 3461 ES LINSCHOTEN

NETHERLANDS

TEL: 348015406

TLF :

TLX:

EML:

COM: 41

DELABOUDINIERE J-P

IAS

BP 10

F 91371 VERRIERES BUISSON

FRANCE

TEL: $164 \quad 47 \quad 4305$

TLF :

TLX: 600252

EML:

COM: 


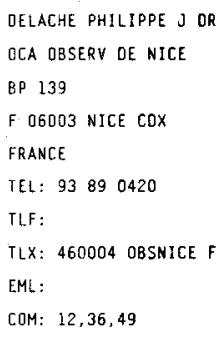

OELANNOY JEAN OR
IRAM
300 RUE OE LA PISCINE
F 38406 S MARTIN HERES CD
FRANCE
TEL: 76423383
TLF:
TLX: 980753
EML:
COM: 40

DELHAYE JEAN PROF

2 RUE DE LA PLEIADE

F 94240 L'HAY LES ROSES FRANCE

TEL: $146 \quad 645771$

TLF :

EML:

COM: 24,33

TLX:

DELSEMme ARMAND H PROF DR DPT PHYSICS \& ASTRONOMY UNIVERSITY OF TOLEDO 2801 W BANCROFT ST TOLEDO OH 43606 USA

TEL: 4195372654

TLF :

TLX:

EML:

COM: $14,15,20,51$

DEMERS SERGE OR

DPT DE PHYSIQUE UNIVERSITE OE MONTREAL

CP 6128 SUCC A

MONTREAL QC H3C 3J7 CAMAOA

TEL: 5143436718

TLF: $514 \quad 3432071$

TLX: 05562425

EML:

COM: $27,28,37$

DENG ZUGAN OR
GRAOUATE SCHOOL
DPT OF PHYSICS
BOX 3908
BEIJING 100039
CHINA PR
TEL: $1289461 * 89$
TLF:
TLX: 22040 BAOAS CN
EML:
COM:

DELBOUILLE LUC PROF INSTITUT D'ASTROPHYSIOUE UNIVERSITE DE LIEGE AVE COINTE 5 B 4000 COINTE-LIEGE BELGIUM

TEL: 41529980

TLF : 41527474

TLX: 41264 ASTRLG B

EML:

COM: 12

DELIYANNIS JEAN OR DPT OF ASTROPHYSICS NTL UNIVERSITY OF ATHENS PANEPISTIMIOPOLIS

GR 157 B3 ZOGRAFOS

GREECE

TEL: 17243414

TLF: $1722 \quad 8981$

TLX:

EML: YANNISEGRATHNI

COM: 12 .

demARCo JeAN ING

FRANCE

TEL:

TLF :

FLX:

EML':

COM:

DEMIN $\checkmark$ G PROF DR STERNBERG STATE ASTR INST UNIVERSITETSKIJ PROSP 13 119899 MOSCOW

RUSS IA

TEL: $139-36-81$

TLF :

TLX:

EML:

COM: 07.

DENIS CARLO DR INSTITUT DAASTROPHYSIQUE UNIVERSITE OE LIEGE AVE COINTE 5 B 4000 COINTE-LIEGE BELGIUM

\section{TEL: 41529980}

TLF: $41 \quad 52 \quad 7474$

TLX: 41264

EML:

COM:
DELCROIX ANDRE J S DR 19A RUE E VANDERVELDE 19A RUE E VANOERVELDE B 7230 FRAMERIES BELGIUM

TEL:

TLF :

TLX:

EML :

COM:

DELLA VALLE MASSIMO OR OPT DI ASTRONOMIA UNIVERSITA OI PADOVA VIC DELL OSSERVATORIO 5 I 35122 PADOVA

ITALY TEL: 49661499 TLF :

TLX: 432071

EML: INFNET: $39003:$ : GELLAVALLE COM:

DEMARET JACQUES DR 158/033 AVE OBSERVATOIRE

B 4000 COINTE-LIEGE

BELGIUM

TEL: 41527261

TLF :

TLX: 41264 ASTROLIEGE

EML:

COM: 47

DEMING LEO ORAKE DR NASA/GSFE

CODE 693

GREENBELT ML 20771

USA

TEL: $301286 \quad 6519$

TLF: 3012863271 .

TLX: 89675 NASCOM

EML: LEPVAX::YSLOD SPAN COM: 12

DENISSE JEAN-FRANCOIS OR 4B. RUE MR LE PRINCE

F 75006 PARIS

FRANCE

IEL: $143 \quad 294874$

ILF :

ILX:

EML:

CDM: 40 
DENISYUK EDVARD K DR ASTROPHYSICAL INSTITUTE KAZAKH ACAD OF SCIENCES 480068 ALMA ATA KAZAKHSTAN

TEL:

TLF :

TLX:

EML:

COM:

\begin{abstract}
UK
TEL:

TLF :

TLX:

EML:

COM:
\end{abstract}

DENNISON P A DR

TRINITY HALL

UNIVERSITY OF CAMBRIDGE CAMBRIDGE CB3

DERE KENNETH PAUL

NAVAL RESEARCH LABORATORY

CODE 4163

4555 OVERLOOK AVE SW

WASHINGTON DC 203755000

USA

TEL : 2027672517

TLF :

TLX:

EML:

COM: 10

DESAI JYOTINDRA N
PHYSICAL RESEARCH LAB
NAVRANGPURA
ROOM 763
AHMEOABAD 380009
INOIA
IEL: 272462129
TLF: 272445292
TLX: 121397
EML:
COM: 09

DESPAIN KEITH HOWARO OR LOS ALAMOS NATIONAL LAB MS B220 $\times 2$

BOX 1663

LOS ALAMOS NM 87545

USA

TEL: $505 \quad 6672388$

TLF :

TLX:

EML:

COM: 35
DENNEFELO MICHEL

INSTITUT D'ASTROPHYSIOUE

98BIS BD ARAGO

F 75014 PARIS

FRANCE

TEL: 143201425

TLF: 143298673

TLX:

EML: SPAN : IAPOBS: : DENNEFELO

COM:

DENDYELLE JOZEF KIC

OBSERVATOIRE ROYAL DE

BELGJQUE

AVE CIRCULAIRE 3

B 1180 BRUSSELS.

BELGIUM

TEL: 23752484

TLF: $2374^{\circ} 9822$

TLX: 21565 OBSBEL

EML:

CoM: 25,33

DERMAN I ETHEM OR

DPT OF ASTRONOMY

UNIVERSITY OF ANKARA

FEN FAKULTESI

06100' BESEVLER

TURKEY

TEL: $4123 \quad 6550 / 0109$

TLF:

TLX:

EML:

COM:

DESESQUELLES JEAN OR

UNIVERSITE LYON I

CAMPUS LA DOUA

43 BO DU 11 NOVEMBRE

F 6962l VILLEURBANNE

FRANCE

TEL: $78 \quad 89 \quad 8124$

TLF :

TLX: 380273 IPN F

EML:

CON: 14

OESPOIS OIDIER DR

OBSERVATOIRE DE BORDEAUX

BP 89

F 33270 FLOIRAC

FRANCE

TEL: $56 \quad 86 \quad 4330$

TLF : $56 \quad 40 \quad 4251$

TLX:

EML: EARN : OESPOIS ROROBD:

COM: 40
DENNIS BRIAN ROY DR

NASA/GSFC

CODE $6 B 2$

GREENBELT MO 20771

USA

TEL: 3012866604

TLF :

TLX: 89675

EML:

COM: $10,44,48$

DENT WILLIAM A PROF

DPT PHYSICS \& ASTRONOMY

UNIV OF MASSACHUSETTS

GRC B

AMHERST MA 01003

USA

TEL: $413 \quad 545 \quad 3665$

TLF :

TLX:

EML:

COM: 40

DERMENDJIEV VLADIMIR OR

DPT OF ASTRONOMY

BULGARIAN ACAO SCIENCES

72 LENIN BLVD

BG 1784 SOFIA

BULGARIA

TEL: 2758927

TLF :

TLX: 23561 ECF BAN BG

EML:

COM: 10

\section{DESHPANDE AVINASH}

RAMAN RESEARCH INSTITUTE

SADASHIVANAGAR

BANGALORE 560080

INDIA

TEL: $812 \quad 36 \quad 0122$

TLF : 812340492

TLX: 8452671 RRI IN

EML:

COM: 40

DETTMAR RALF-JURGEN OR

RAOIOASTRON INSTITUT DER

UNIVERSITAET BONN

AUF OEM HUEGEL 71

D 5300 BONN 1

GERMANY

YEL: 228735658

TLF : 228525229

TLX: 886440

COM: 28
DENNISON EDWIN W DR

985 CYNTHIA AVE

PASALENA CA 91107

USA

TEL: $818 \quad 351 \quad 8751$

TLF :

TLX:

EML:

COM:

DEPRIT ANDRE PROF

NTL BUREAU OF STANDARDS

APPLIED MATHEMATICS CTR

GAITHERSBURG MO 20899

USA

TEL: 3019212631

TLF:

TLX:

EML:

COM: $04,07 P$

DERMOTT STANLEY F

OPT OF ASTRONOMY.

UNIVERSITY OF FLORIDA

224 SSRB

GAINESVILLE FL 32611

USA

TEL: $904392 \quad 2361$

TLF: 9043929605

TLX:

EML: DERMOTTOUUPITER. ASTRO.UFL. EH

COM: $15,16,21$

DESHPANDE M R DR

PHYSICAL RESEARCH LAB

NAVRANGPURA

AHMEDABAD 380009

INOIA

TEL: $272 \quad 46 \quad 2129$

TLF : 272445292

TLX: 121397 PRL IN

EML:

COM: 25

OEUBNER FRANZ-LUDWIG DR

INST F ASTRONOMIE \&

ASTROPHYSIK

AM HUBLAND

D 8700 WUERZBURG

GERMANY

TEL: 931 BBB 5030

TLF: 931706297

TLX: 68671 UNIWBG

COM: $10,12 \mathrm{VP}$ 


DEUL ERIK DR
STERREWACHT
BOX 9513
NL 2300 RA LEIDEN
NETHERLANDS
TEL: 71275880
TLX: 39058 ASTRO NL
EML: DUEL HLEUL5!/RULHLI
TLF: 71275819
COM:
OEVORKIN OAVIO H
NTL AIR \& SPACE MUSEUM
SMITHSONIAN INSTITUTION
WASHINGTON DC 20560
USA
TEL: 2023572828
TLF:
TLX:
EML:
COM: 41

\section{DEWITT JOHN H JR}

3602 HODOS HILL RD NASHYILLE TN 37215 USA

TEL: 6153838272

$$
\text { TLF : }
$$

$$
\text { EML: }
$$$$
\text { COM: }
$$$$
\text { TLX: }
$$

DI COCCO GUIDO
IST TE S R E
CNR
VIA DE CASTAGNOLI 1
I 40126 BOLOGNA
ITALY
TEL: 5 I 519593
TLF:
TLX: 511350 CNR BO J
EML:
COM: 44
OI TULLIO GRAZIELLA DR
OSS ASTRONOMICO DI PADOVA
VIC OELL OSSERVATORIO 5
I 35I22 PABOVA
ITALY
TEL: 4966 1499
TLX:
EML:
TLF:
COM:

DEUPREE ROBERT G OR los alamos national lab MS F665 ESS 5

BOX 1663

LOS ALAMOS NM 87545

USA

TEL: $505 \quad 6678215$

TLF :

TLX:

EML:

COM: 27,35

DEWDNEY PETER E F DR DOMINION RADIO ASTROPHYS OBSERVATORY

BOX 248

PENTJCTON BC V2A 6K3

CANADA

TEL: 6044932277

TLF: 6044937767

TLX: 04888127

EML:

COM: 34,40

DEWITT-MORETIE CECILE PR

ASTRONOMY OPT

UNIVERSITY OF TEXAS

RLM 15308

AUSTIN TX 787121083

USA

TEL: 5124711052

TLF:

TLX: $910-8741305$

EML:

COM:

DI FAZIO ALBERTO

OAR

VIA Del PARCO MELLINI 84

1 00136 ROMA

ITALY

TEL: 6347056

TLF:

TLX: 613103 PPRMT I

EML:

COM: $28,34.37$

DI XIAO-HUA

PURPLE MOUNTAIN OBSERV.

CAS

NANJING

CHINA PR

TEL: 2537609

TLF:

TLX: 34144 PMaNJ EN

EML:

COM: 04
DEUTSCHMAN WILLIAM A DR OREGON LASER CONSULTANTS 455 HILLSIOE AVE

KLAMATH FALLS OR 97601

USA.

TEL: 5038823295

ILF:

TLX:

EML:

COM: 15

DEWHIRST DAVIO W OR INSTITUYE OF ASTRONOMY THE OBSERVATORIES MADINGLEY RD CAMBRIDGE CB3 OHA UK

TEL: 23362204

TLF:

TLX: 817297. ASTRON G

EML:

COM: 05,41

DEZSO LORANT PROF

HELIOPHYSICAL OBSERVATORY BOC 30

h 4010 debrecen

HUNGARY

TEL: 5211015

ILF:

TLX: 72517 DEOBS L

EML:

COM: 10,12

OI MARTINO MARIO

OSS ASTRONOMICO DI TORINO

ST OSSERVATORIO 20

I 10025 PINO TORINESE

ITALY

TEL: 1184.1067

TLF:

ILX: 213236 TO ASTR I

EML:

COM: 15

DIALETIS DIMITRIS DR

AStRonOMICAL INSTItUTE

NTL observatory of athens

BOX 20048

GR 11 110 ATHENS

GREECE

TEL: $1346 \quad 1191 / 1804 \quad 0619$

ILF:

$T L X: 215530$ OBSA GR

EML:

COM: 10
OEVINNEY EOWARO J OR

100 union ave

DELANCO NJ 08075

USA

TEL: 6097641250

TLF:

TLX:

EML:

COM:

DEWITT BRYCE $S$ DR

ASTRONOMY OPT

UNIVERSITY OF TEXAS

RLM 15308

AUSTIN IX $78712 \quad 1083$

USA

TEL: 5124715055

ILF:

TLX: 9108741305

EML:

COM: 48

DHAWAN VIVEK OR.

RAMAN RESEARCH INSTITUTE

SADASHIVANAGAR

BANGALORE 560080

INOIA

TEL: 812340122

ILF: 812340492

ILX: 8452671 RRI IN

EML: RRIOVIGYAN. ERNET. IN

COM: 40

Di SEREgo Alighieri S DR

OSS ASTROFISICO

DI ARCETRI

LARGO E FERMI 5

I 50125. FIRENZE

ITALY

TEL: $55 \quad 4378540$

TLF: 55.435939

TLX:

EML: ASTRFI: : SPERELLL

COM: 28

DIAMONO PHILIP JOHN DR

NRAO

BOX 0

SOCORRO NM 878010387

USA

TEL: 5058357900

TLF: :

ILX: 9109881710

EML: POIAMONDENRAO.EDU

COM: 
DIAZ ANGELES ISABEL DR DPT OE FISICA TEORICA C-XI UNIVERSIDAD AUTONOMA DE MAdRID CANTOBLANCO

\section{E 28049 MADRIO}

SPAIN

TEL: 13974223

TLF:

TLX: 27810

EML: BITNET: ADIAZEMDUAMIL

COM: 28
OICK STEVEN J

US NAVAL OBSERVATORY

34 \& MASSACHUSETTS AVE NW

WASHINGTON DC 203925100

USA

TEL:

TLX:

EML:

TLF :

COM: $08,41 \mathrm{C}, 51$

OICKEL JOHN R

UNIVERSITY OF ILLINOIS

349 ASTRONDMY BLDG

1002 W GREEN ST

URBANA IL 61801

USA

TEL: 2173335532

TLF:

ILX: 910-245-2434 PURCH

EML:

COM: $16,33,34,40$

DICKINSON DALE F DR

LOCKHEEO PALO ALTO RES LB

DPT 9220 BLOG 205

3251 HANOVER ST

PALO ALTO CA 94304

USA

TEL: 4154242701

TLF :

TLX:

EML:

COMM:

DIERCKSEN GEERD H F PH D

MPI F, PHYSIK \& ASTROPHYS

KARL-SCHWARZSCHILD-STR I

D 8046 GARCHING MUENCHEN

GERMANY

TEL: 8932990

TLX: 524629 ASTRO D

EML:

TLF:

COM: 14

DINERSTEIN HARRIET L
ASTRONOMY DPT
UNIVERSITY OF TEXAS
RLM 15308
AUSIIN TX 787121083
USA
TEL: 5124713449
TLF:
TLX: $910-874-1351$
EML:
COM: 34

DICK WOLFGANG OR

OBSERVATORIUM HOHER LIST

UNIV STERNWARTE BONN

- 5568 OAUN

GERMANY

TEL: $6592 \quad 2150$

TLF:

TLX:

EML:

COM: 24

DICKENS ROBERT J OR

RUTHERFORO APPLETON LAB

SPACE \& ASTROPHYSICS OIV

BLDG R25/RGB

CHILTON DIDCOT OX11 OQX UK

TEL: 23521900

TLF :

TLX: 83159

EML:

COM: $27,28,37$

DICKMAN ROBERT L DR

DPT OF RAOIO ASTRONOMY UNIV OF MASSACHUSETTS

GRC

AMHERST MA OLOO3:

USA

TEL: $413545 \quad 0925$

TLF:

TLX: 955491

EML:

COM: 33,40

DIETER NANNIELOU H OR
CLAY RD
N THERFORO VT 05054
USA
TEL: 8023334079
TLF:
TLX:
EML:
COM: 33,40

DINESCU A DR

INSTITUE OF GEOOESY

PHOTOGRAMM CARTOGRAPHIE

IA BLVO DE L'EXPOSITION

R 78334 BUCAREST

RIJMANIA

TEL:

TLF:

TLX:

EML:

COM:
OLCKE ROBERT H PROF

OPT OF PHYSICS

PRINCETON UNIVERSITY

JOSEPH HENRY LABS

PRINCETON NJ 08544

USA

TEL: $609452 \quad 4317$

TLF:

TLX:

EML:

COM: 47,48

OICKEY JEAN O'BRIEN

JPL/CALTECH

MS 138208

4800 OAK GROVE DR

PASADENA CA 91109

USA

TEL: $818 \quad 354 \quad 3235$

TLF :

TLX: 675429

EML:

COM: $04,16,19 \mathrm{C}, 31$

OICKMAN STEVEN R

DPT GEOLOGICAL SCIENCES

STATE UNIV OF NEW YORK

BINGHAMTON NY 13901

USA

TEL: $607777 \quad 4378$

TLF :

TLX:

EML:

COM: $19 \mathrm{C}$

OIKOVA SMILIANA D

OPT OF ASTRONOMY

BULGARIAN ACAD SCIENCES

72 LENIN BLVD

BG 1784 SOFIA

BULGARIA

TEL: 2758927

TLF:

TLX: 23561 ECF BAN

EML:

COM: 07

DING YOU-JI

YUNNAN OESERVATORY

CAS

BOX 110

XUNMING 72946 YUNNAN

CHINA PR

TEL: 87! 2035

TLF :

TLX: 64040 YUOBS CN

EML:

COM: 10 


\author{
DINGENS P PROF DR \\ KORTRIJKSE STEENWEG 763 \\ B 9000 GENT \\ BELGIUM \\ TEL: 91221966 \\ TEF \\ EML \\ COM: 35 \\ TLX
}

OISNEY MICHAEL I PROF

PHYSICS DPT

UNIV WALES COLLEGE

BOX 913

CARDIFF CFI 3 TH

UK

TEL: $222 \quad 874785$

TLF: 222371921

TLX: 498635

EML :

COM: 34,48

DIZER MUAMMER PROF

KANOILLI OBSERVATORY

BOGAZICI UNIVERSITY

CENGELKOV

B1220 ISTANBUL

TURKEY

TEL: 13320277

TLF :

TLX:

EML:

COM: 10

\author{
OLUZHNEVSKAYA O B OR \\ INST OF ASTRONOMY \\ ACADEMY OF SCIENCES \\ PYATNITTSKAYA UL 48 \\ 109017 MOSCOW \\ RUSSIA \\ TEL: 2315461 \\ TLF : \\ TLX: 412623 SCSTP SV \\ EML \\ COM: 05 YP. 35.37
}

DOBRZYCKI JERZY PROF

HISTORY OF SCIENCE

POLISH ACAD OF SCIENCES

GWIA2O2ISTA 27/169

PL 01814 WARSAH

POLAND

TEL: $33-22-03$

TLF :

TLX:

EML

COM: 41
DINTINJANA BOJAN OR

ASTRONOMICAL OBSERVATORY

UNIVERSITY OF E KARDELJ

JADRANSKA 19

YU 61110 LJUBLJANA

YUGOSLAVIA

TEL: 61265061

TLF: 61 217281

TLX:

EML: BOJAN DINTINJANAOUNI-LJ AC MAI EML:

COM:

\section{DIVAN LUCIENNE OR}

INSTITUT D'ASTROPHYSIQUEE

9BBIS BD ARAGO

F 75014 PARIS

FRANCE

TEL: $143 \cdot 20,1425$

ILF: 143298673

TLX:

EML:

COM: 29,45

DJORGOVSKI STANISLAV OR

CALTECH

MS 10524

PASAOENA CA 91125

USA

TEL: $818 \quad 356 \quad 4415$

TLF

TLX: 675425

EML: SPAN:GEORGEO6035

COM: 22.27 .51

\section{DOAZAN VERA DR}

OBSERVATOIRE DE PARIS

61 AVE OBSERVATOIRE

F 75014 PARIS

FRANCE

TEL: 140512235

TLF

ILX: 270776 OBS F

EML:

COM: 29.36

DOCOBO DURANTEZ JOSE A

OBSERVAFORIO ASIRONOMICO

RAMON MARIA ALLER

AVD DE LAS CIENCIAS S/N

E SANTIAGO DE COMPOSTELA

SPAIN

TEL:

TLF:

TLX:

EML:

COM: 26
DIONYSIOU DEMETRIOS PROF HELLENIC AIR-FORCE ACAD

DERELIA-ATTICA

IB AMASSIAS STR

GR 11634 ATHENS

GREECE

TEL: $17238436 / 12466366$

TLF :

TLX:

COM: 47

DIVARI N B DR

DDESSA POLYTECHNICAL INST

270044 ODESSA

UKRAINE.

IEL:

TLF :

ILX

EML:

COM: 21

DJUURASEVIC GOJKO

ASTRONOMICAL OBSERVATORY

VOLGINA $?$

YU 11050 BEQGRAD

YUGOSLAVIA

TEL: 11419553

TLF :

TLX

EML:

COM:

DOBRITSCHEV V M MR

DPT OF ASTRENOMY

BULGARIAN ACAD SCIENCES

7TH NOVEMBER ST 1

BG 1000 SOFIA

BULGARIA

TEL: 2.7341

TLF: 23561 ECFBAN BE

TLX: 23561 ECFBAN BG

EML:

COM

OODD RICHARD J DR

CARTER OBSERVATORY

BOX 2909

WELLINGTON

NEW ZEALANO

TEL: $728-16$ ?

TLF :

ILX: 30172 NATOBS NZ

EML:

COM:

M:

DIRIKIS M A DR

ASTRONOMICAL OBSERVATORY LATVIAN STATE UNIVERSITY

RAINIS BUL 19

226098 RIGA

LATVIA

TEL:

TLF :

TLX:

EML:

COM: 20

DIXON ROBERT S OR

RADIO OBSERVATORY

OHIO STATE UNIVERSITY.

2015 NEIL AVE

COLUMBUS OH 43210

USA

TEL: $614422 \quad 6789$

TLF:

ILX:

EML:

COM: $05,40,51$

OJUROVIC DRAGUTIN M OR

DPT OF ASTRONOMY

FACULTY OF SCIENCES

STLDENTSKI TRG 16

YU 11000 BEOGRAD

YUGOSLAVIA

TEL: 11420221

TLF:

TLX:

EML:

COM: 08,19

DOBRONRAVIN PETER OR CRIMEAN ASTROPHYS OBS UKRAINIAN ACAD OF SCIENCE NAUCHNY

334413 CR IMEA

UKRAINE

TEL: 432945

TLF :

TLX:

EML:

COM: 09,29

OOGAN NADIR PROF DPT OF ASTRONOMY UNIVERSITY OF ANKARA FEN FAKULTESI

06100 BESEVLER

TURKEY

TEL:

TLF:

TLX:

EML:

COM: 12 
DOGGETT LEROY E DR

US NAVAL OBSERVATORY NAUTICAL ALMANAC OFFICE

34 \&ASSACHUSETTS AVE NW WASHINGTON OE 203925100 USA

TEL: 2026531572

TLF:

TLX:

EML:

COM: $04 C .07$

OOKUCHAEVA OLGA D DR

STERNBERG STATE ASTR INST

UNIVERSITETSKIJ PROSP 13

119899 MOSCOW

RUSSIA

TEL:

TLX:

EML:

TLF :

COM: 09,34

DOLIDZE MADONA $\checkmark$ DR

ABASTUMANI ASTROPHYSICAL

OBSERVATORY

GEORGIAN ACAD OF SCIENCES

383762 ABASTUMANI

GEORGIA

TEL:

TLF

TLX

EML:

COM: 29

DOMINSKI IRENEUSZ OR

ASTRONOMICAL LATITUDE OBS

BOROWIEC

B0X 62035

PL 62035 KORNIK

POLAND

TEL: POZNAN 170187

TLF :

TLX: 4L2623 AOS PL

EML:

COM: 31

DONG IL ZUN

PYONGYANG ASTRON OBS

ACADEMY OF SCIENCES DPRK

TAESONG BISTRICT

PYONGYANG

KOREA DPR

TEL:

TLF:

TLX:

EML:

COM:
ODHERTY LORNE H OR

HERZBERG INST ASTROPHYS

NTL RESEARCH COUNCIL

100 SUSSEX OR

OITAWA ON KIA ORG

CANADA

TEL: 6139936060

TLF: 6139526602

TLX: 0533715

EML:

COM:

DOLAN JOSEPH F DR

NASA/GSFC

CODE 681

GREENBELT MD 20771

USA

TEL: 3012865920

TLF:

ILX: 89675

EML:

CON: $25,44,48$

DOLLFUS AUDOUIN PROF

OBSERVATOIRE DE PARIS

SECTION DE MEUDON

F 92195 MEUDON PPL CDX

FRANCE

TEL: $145 \quad 347530$

TLF :

TLX:

EML:

COM: 10.16 .20

DOMKE HELMUT PH D

ZNTRLINST F ASTROPHYSIK

STERNWARTE BABELSBERG

ROSA-LUXEMBURG-STR 17A

D 1502 POTSOAM

GERMANY

TEL:

TLF:

ILX:

EML:

COM: 36

DONN BERTRAM D

NASA/GSFC

COOE 69I

GREENBELT MD 20771

USA

TEL: 3012866859

ILF :

ILX: 89675

EML:

COM: 15.34
OOHERTY LOWELL R PROF

OPT OF ASTRONOMY

UNIVERSITY OF WISCONSIN

475 N CHARTER ST

MADISON WI 53706

USA

TEL: 6082621249

TLF :

ILX:

EML:

COM:

QOLEZ NOEL DR

OBS MIDI PYRENEES

14 AVE E BELIN

F 31400 TOULOUSE COX

FRANCE

TEL:

ILF :

ILX:

EMLL:

COM:

DOMINGO VICENTE DR

ESA/ESTEC

SSD

BOX 299

NL 2200 AG NOOROWIJK

NETHERLANDS

TEL: $17 \quad 1983576$

TLF: 171984698

ILX: 39098

EML: ESTCl: : VDOMINGO/VDOMINGOESTEC COM:

COM: 44

DOMMANGET J OR

OBSERUATOIRE ROYAL DE

BELGIQUE

AVE CIRCULAIRE 3

B 1180 BRUSSELS

BELGIUNA

TEL: 23752484

ILF: 23749822

ILX: 21565

EML:

COM: $24,26,50$

DONNER KARL JOHAN

OBS \& ASTROPHYSICS. LAB

UNIVERSITY OF HELSINKI

TAEHTITORNINMAK

SF OOI3O HELSINKI 13

FINLAND

TEL:

TLF :

TLX:

EML:

COM: $2 B$
DOKUCHAEV VYACHESLAV DR

INST NUCLEAR RESEARCH

ACADEMY OF SCIENCES

EOTH ANIV OCT PROSPEKT $7 \mathrm{~A}$

117312 MOSCOW

RUSSIA

TEL: 0953827678

TLF: 0952926511

TLX: 411051 INR SU

EML: dokuchaeveinucres.msk, su

COM: 34

DOLGINOV ARKADY Z PRQF OR IOFFE PHYSICAL TECH INST ACADEMY OF SCIENCES POLYTECHNICHESKAYA UL 26 194021 ST PETERSBURG RUSS.IA

TEL:

ILF :

YLX:

EML:

COM: 49

DOMINKO FRAN PROF DR SARANOVICEVA 11

YU 61000 LJUBLLJANA

YUGOSLAVIA

TEL: 61322210

TLF :

TLX:

EML:

DONAS LOSE OR

LAS

TRAVERSE DU SIPHON

LES TROIS LUCS

f 13012 MARSEILLE

FRANCE

TEL: 91055900

TLF : $91 \quad 66 \quad 1855$

ILX; $420584 \mathrm{~F}$

EML: BITNEET: DONASEFLASMS:

COM: 28

DONNISON JOHN RICHARO DR DPT OF MATH SCIENCES

GOLDSMITHS' COLLEGE

NEW CROSS

LONDON SEI4 6NW

UK

TEL: $1 \quad 716 \quad 927 \quad 171$

TLF :

TLX:

EML:

COM: 20 
DOPITA MICHAEL ANOREW DR MOJNT STROMLO \& SIOING SPRING OBSERVATORIES

PRIVATE BAG

WODEN PO ACT 2606

AUSTRALIA

TEL: $62 \quad 88 \quad 1111$

TLF:

TLX: 62270 CANOPUS AA

EML:

COM: $34 \mathrm{C}$

DOROSHKEVICH ANDREI G DR INST OF APPLIEO MATHS ACADEMY OF SCIENLES

MIUSSKAJA SO 4

125047 MOSCOW

RUSSIA

TEL:

TLF :

TLX:

EML:

COM: 47

\section{DOSSIN F DR}

INSTITUT D'ASTROPHYSIAUE

UNIVERSITE DE LIEGE

AVE COINTE 5

B 4000 COINTE-LIEGE

BELGIUM

TEL: 41529980

TLF : $4152 \quad 7474$

TLX: 41264 ASTRLG

EML:

COM: 15

OOUGHTY NOEL A OR

OPT OF PHYSICS

UNIVERSITY OF CANTERBURY

PRIVATE BAG

CHRISTCHURCH I

NEW ZEALAND

TEL:

TLF:

TLX:

EML:

COM: 42

DOUGLASS GEOFFREY $G$

US NAVAL OBSERVATDRY

34 \& MASSACHUSETTS AVE NW WASHINGTON DC 203925100

USA

TEL: $202 \quad 653 \quad 1457$

TLX: 7108221970

EML:

TLF: $202 \quad 653 \quad 1497$

COM: 24,26
DORENWENDT KLAUS OR

PHYSIKALISCH - TECHNISCHES

BUNDESANSTALT

BUNDESALLEE 100

D 3300 BRAUNSCHWEIG

\section{GERMANY}

TEL: $531592 \quad 1210$

TLF:

TLX: 952822

EML:

COM:

\section{DORSCHNER JOHANN OR UNIV STERNWARTE JENA SCHILLERGAESSCHEN 2 \\ D 6900 JENA \\ GERMANY \\ TEL: $78 \quad 822 \quad 2637$ \\ TLX: 5886134 \\ EML: \\ TLF : \\ COM: 34,51}

OOTANI TADAYASU OR
ISAS
$3-1-1$ YOSHINODAI
SAGAMIHARA
KANAGAWA 229
JAPAN
TEL: 427513911
TLF: 427.594253
TLX: J27758 ISAS ERO
EML: DOTANIQASTRO. ISAS.AC.JP
COM: 48

DOUGLAS JAMES N PRDF

ASTRONOMY OPT

UNIVERSITY OF TEXAS

RLM. 15308

AUSTIN TX $78712 \quad 1083$

USA

TEL: $512 \quad 4714461$

TLF:

TLX: $910874-1351$

EML:

COM: 40

DOURNEAU GERARD DR

OBSERVATOIRE DE BORDEAUX

BP 89

F 33270 FLOIRAC

FRANCE

TEL: $56 \quad 86 \quad 4330$

TLF: 56404251

TLX:

EML: EARN : :OOURNEAUAFROBOR5I

COM: 07,20
DORFI ERNST ANTON DR

INSTITUT FUER ASTRONOMIE

UNIVERSITAET WIEN

TUERKENSCHANZSTR 17

A 1180 WIEN

AUSTRIA

TEL: 13453600

TLF :

TLX: 133099

EML:

COM: 42

DOS REIS M PROF

OBSERVATORIO ASTRONOMICO

P. 3000 COIMBRA

PORTUGAL

TEL:

TLF :

TLX:

EML:

COM:

DOTTORI HORACIO A DR

INSTITUTO OE FISICA

IFRGS

CP 15051

90049 PORTO ALEGRE RS

BRAZIL

TEL: $512 \quad 36 \quad 4677$

TLF :

TLX: 511055 UFRS BR

EML:

COM: 28,34

DOUGLAS NIGEL DR

KAPTEYN STERRENWACHT

UNIVERSITY OF GRONINGEN

BOX 800 .

NL 9700 AV GRONINGEN

NETHERLLANOS

TEL: 590828818

TLF : $5908 \quad 28800$

TLX:

EML: NOOUGLAS HROKSW5

COM: 09

DOVAL JORGE PEREZ DR

INST GEOPHYS \& ASTRONOMY

CALLE 212 N 2906/29 Y 31

RPTO COROMELA/LA LISA

LA HABANA

CUBA.

TEL: $218435 / 0644$

TLF :

TLX: 511240 GEOAS CU

EML:

COM : 20
DORMAND JOHN RICHARD OR

DPT OF MATHEMATICS

TEESSIOE POLYTECHNIC

MIDOLESBROUGH

CLEVELANO TSL 3BA

UK

TEL: $642 \quad 218 \quad 121 \star 4365$

TLF:

TLX:

EML:

COM: 07

DOSCHEK GEORGE A DR

NAVAL RESEARCH LABORATORY

CODE 4170

4555 OVERLOOK AVE SW

WASHINGTON OC 203755000

USA

TEL: 2027676473

ILF :

TLX:

EML:

COM:

DOUBINSKIJ B A DR

INST OF RADLO ELECTRON

ACADEMY OF SCIENCES

103907 MOSCOW

RUSSIA

TEL:

TLF :

TLX:

EML:

COM: 40

DOUGLAS R J MR

PHYSICS DIVISION (M-36)

NTL RESEARCH COUNCIL

100 SUSSEX DR

OTTAWA ON KIA OSI

CANADA

TEL: 6139936060

TLF : 6139526602

TLX:

EML:

COM: 31

DOWNES ANN JULIET B

MULLARO RADIO ASTRON OBS

CAVENOISH LABORATORY

MADINGLEY RD

CAMBRIOGE CB3 OHE

UK

TEL: $233 \quad 66477$

TLF :

TLX: 81292

EML:

COM: 


OOWNES DENNIS DR
IRAM
300 RUE DE LA PISCINE
F 38406 S MARTIN HERES CD
FRANCE
TEL: 76423383
TLF:
TLX: 980753
EML:
COM: $33,34,40$

DDYle laURANCE R DR

SETI INSTITUTE 245 ? NASA AMES RESEARCH CENTER MAFFETT FIELO CA 94035 USA

TEL: $415 \quad 6043372$

TLF:

TLX:

EML: 24609: :DOYLE SPAN

COM:

ORAMBA C PROF
ASTRONOMICAL OBSERVATORY

CUTITUL DE ARGINT 5

BOX 28

R 75212 BUCAREST

RUMANIA

TEL: 753998; 193407

TLF:

TLX:

EML:

COM:

DRECHSEL HORST OR

DR-REMEIS-STERNWARTE

ASTR INST UNIV ERLANGEN-N

STERNWARTSTR 7

D Bg00 BAMBERG

GERMANY

TEL: $9515770 \mathrm{~B}$

TLF :

TLX: 629830 UNIER $D$

EML: ORECHSELOSTERNWARTE. UNI-ERLANG EML

CQM: 42

\section{DRESSLER KURT PROF}

LAB PHYSIK CHEMIE

ETH ZENTRUM

CH 8092 ZUERICH

SWITZERLAND

TEL: 12564441

TLF :

TLX: 53178 ETHBI CH

EML:

COM: 14

\author{
DOWNES RONALD A DR \\ STSCI \\ C/O USER SUPPORT BRANCH \\ 3700 SAN MARTIN OR \\ BALTIMORE MO 21218 \\ USA \\ TEL: 3013384700 \\ TLF: \\ TLX: \\ EML: SCIVAX: :DOWNES \\ COM: 27
}

DRAINE BRUCE T

PRINCETON UNIVERSITY OBS PEYTON HALL

PRINCETON NJ 08544

USA

TEL: 6094523574

TLF:

TLX:

EML:

COM: 34

DRAPATZ SIEGFRIED W DR

MPI F EXTRATERRESTRISCHE PHYSIK

KARL-SCHWARZSCHILD-STR 1

O 8046 garching MUENCHEN GERMANY

TEL: 893299880

TLF:

TLX: 05215845

EML:

COM: 34

\section{OREHER JOHN W}

OPT OF PHYSICS

MIT RM $26 \quad 315$

BOX 265

CAMBRIDGE MA 02139

USA

TEL: 6172538519

TEF :

TLX: 921473

COM: $09,34,40$

OREVER RONALO W $P$ DR

CALTECH

PASADENA CA 95064

USA

TEL:

TLF : $818 \quad 795 \quad 1547$

EML:

COM:

TLX: 675425 CALTECH PSD
DOWNS GEORGE S DR

MIT LINCOLN LABORATORY

ROOM B285

$\mathrm{BOX} 73$

LEXINGTON MA 02173

USA

TEL:

TLF :

TLX:

EML:

COM: 40,51

DRAKE FRANK O PROF

DIV OF NATURAL SCIENCES

UNIVERSITY OF CALIFORNIA

SANTA CRUZ CA 95064

USA

TEL: 4084292931

TLF:

TLX: 7607936 UCSC UC

EML:

COM: $16,40,48,51 C$

DRAVINS DAINIS PROF

LUNO OBSERVATORY

BOX 43

S 22100 LUND

SWEDEN

TEL: $46 \quad 107297$

TLF: $46 \quad 104614$

TLX: 33199 OBSNOT $S$

EML: dainisastro.lu.se (internet) COM: $09,12,29,36 \mathrm{C}$

DRESSEL LINDA L

DPT SPACE PHYS \& ASTRON .

RICE UNIVERSITY

BOX 1892

HOUSTON TX 772511892

USA

TEL: 7135278101

TLF :

TLX:

EML:

COM: 28

DREW JANET

DPT OF ASTROPHYSICS

UNIVERSITY OF OXFORO

KEBLE RD

OXFORD OXI JRH

UK

TEL:

TLF:

TLX:

EMt:

COM:
DOYLE JOHN GERARD

ARMAGH OBSERVATORY

COLLEGE hILL

ARMAGH BTEI 906

UK

TEL: 861522928

TLF:

ILX: 747937

EML:

COM: 36

DRAKE STEPHEN A

NASA/GSFC

CODE 668

GREENBELT MO 20771

USA

TEL: 3012866962

TLF:

TLX:

EML:

COM: 36.40

DRAVSKIKH A F DR

PULKOVO OBSERVATORY

ACADEMY OF SCIENCES

10 KUTUZOV QUAY

196140 ST PEJERSBURG

RUSSIA

TEL: 297-94-52

TLF:

TLX:

EML:

COM: 08,40

\section{DRESSLER ALAN}

MT WILSON \& LAS CAMPANAS OBSERVATORIES

913 SANTA BARBARA ST

PASADENA CA 911011292

USA

TEL: 8183040245

TLF:

TLX: 675425 CALTECH PSD

EML:

COM: $28,47 \mathrm{C}$

DRILLING JOHN S

DPT PHYSICS \& ASTRONOMY

LOUISIANA STATE UNIY

BATON ROUGE LA 708034001

USA

TEL: 5043886795

TLF :

TLX:

EML:

COM: 33 


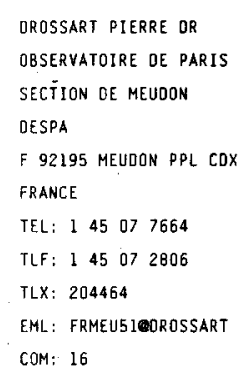

OROZYNER ANORZEJ

INSTITUTE OF ASTRONOMY

N COPERNICUS UNIVERSITY CHOPINA , 12/18

PL 87100 TORUN

' POLAND

TEL: $260 \quad 1726037$

TLF:

TLX: 0552234 ASTR PL.

EML:

COM: 07

DUBIN MAURILE DR

NASA/GSFC

COOE 616

LAB FOR ATMOSPHERES

GREENBELT MO 20771

USA

TEL: 3012865475

RLF: 3012862630

TLX:

EML:

COM: 21.22

DUBOUT RENEE

OBSERVATOIRE OE LYON

AVE CHARLES ANDRE

F 69561 S GENIS LAVAL CDX FRANCE

TEL: 78560705

TLF: 72399791

TLX: 310926

EML:

COM: 25,34

OUERST JOHANNES. DR

LANGWIES

CH 8821 SCHONENBERG

SWITZERLANO

IEL: $1788 \quad 1785$

ILF:

EML:

COM:

ILX:

QUFOUR REginalo JAMES

DPT SPACE PHYS \& ASTRON

RICE UNIVERSITY

204X SPACE SCIENCE BLDG

HOUSTON TX ?700L

USA

TEL: 7135278101

TLF:

TLX:

EML:

COM: 28.34
ORURYY LUKE O. CONNOR OR

DIAS

SCHOOL OF COSMIC PHYSCIS

5 MERRION SQ

DUBLIN 2

IRELAND

TEL: I 774321

TLF:

TLX: 31687 OIAS

EML: LO@IASCP. UUCD

COM: 48

OUBNER GLORIA DR

IAR

CC 5

1894 VILLA ELISA (BS AS)

ARGENTINA

TEL: 2143793

TLF:

TLX: 22414 CEDOC AR

EML:

COM: 34

QUCATI JORGE RICARDO DR INSTITUTO DE FISICA

UFRGS

CP 15051

90000 PORTO ALEGRE RS

BRAZIL

TEL: 512364677

TLF:

TLX: 051-1055 UFRS BR

EML:

COM: $05,25,33,38 \mathrm{C}$

DUFAY MAURICE PROF

UNIVERSITE LYON 1

CAMPUS LA DOUA

43 BO OU 11 NOVEMBRE

F 69621 VILLEURBANNE

FRANCE

TEL:

TLF:

TLX:

EML:

COM: 14,21

DUFTON PHILIP L DR

DPT OF PURE \& APPL PHYS

QUEEN'S UNIVERSITY

BELFAST BT7 INN

UK

TEL: $232 \quad 245133$

TLF:

TLX: 74487

EML:

COM: 36
DRYER MURRAY OR

NOAO ERL R/E/SE

SPACE ENVIRONMENT LAB

325 BROADWAY

BOULDER CO 80303

USA

TEL: 3034973978

TLF:

TLX: 592811 NOAA MASC BDR

EML:

COM: $10,15,49$

DUBOIS MARC A

CEA CEN

DRFC

BP 6

F 92260 fontenay aUX ROSE FRANCE

TEL: 146547881

TLF:

TLX:

EML:

COM: 10

DUCHESNE MAURICE dR oBSERVATOIRE OE PARIS

61 AVE OBSERVATOIRE

F 75014 PARIS

FRANCE

TEL: 143201210

TLF:

TLX:

EML:

COM: 09

DUFFETT-SMITH PETER JAMES MULLARD RAOIO ASTRON OBS CAVENOISH LABORATORY MADINGLEY RD CAMBRIDGE CB3 OHE UK

TEL: 22366477

TLF:

TLX: 81292 CAVLAB G.

EML:

COM: 40

DULDIG Marcus Lestie or OPT OF PHYSICS UNIVERSITY OF TASMANIA GPO BOX $252 \mathrm{C}$ HOBART TAS 7001 AUSTRALIA

TEL: 2202022 TLF: 2202410 TLX: AA 58150 COSANT EML: OULGIGPHYSVAX. PHYS. UTAS. EOU.A COM: $10 C, 49$ 
' DULEY WALTER W PROF

OPT OF PHYSICS

YORK UNIVERSITY

4700 KEELE ST

DOWNSVIEW ON M3J 1 P3

CANADA

TEL: $416 \quad 6673040$

TLF:

TLX:

EML:

COM: 34

DUMITRACHE CHRISTANA

ASTRONOMICAL OBSERVATORY

CUTITUL OE ARGINT 5

BOX 28

R 75212 BUCHAREST 28

RUMANIA

TEL: 90236892

TLF:

TLX: 11882 ASTRO R

EML:

COM: 10

DUNCAN ROBERT A PROF
CSIRO
DIVISION OF RADIOPHYSICS
BOX 76
EPPING NSW 2121
AUSTRALIA
TEL:
TLF:
TLX:
EML: RDUNCAN ATNF. CSIRO.AUI
COM: 10

\section{DUNLOP STORM}

140 STOCKS LANE

EAST WITTERING

CHICHESTER W SUS POZO $8 \mathrm{NT}$ uK

TEL: $243 \quad 670354$

TLF:

TLX: $9312-1107-40$

EML: TELECOM GOLO72:MAG100665

COM: 27

\begin{tabular}{|c|c|}
\hline DUPUY DAVID L DR & DURGAPRASAD N DR \\
\hline DPT OF PHYSICS & TIFR \\
\hline VIRGINIA MILITARY INST & HOMI BHABHA RO \\
\hline LEXINGTON VA 24450 & COLABA \\
\hline USA & BOMBAY $400 \quad 005$ \\
\hline TEL: 7034647504 & INOIA \\
\hline TLF: & TEL: $22 \quad 219 \quad 111 * 342$ \\
\hline$T L X:$ & TLF : \\
\hline EML: fpydupuyzfacultysumist.vini.e & TLX: 0113009 \\
\hline COM: 27.46 & EML: \\
\hline & COM: \\
\hline
\end{tabular}

OULK GEORGE A PROF
CASA
UNIVERSITY OF COLORADO
BOX 391
BOULDER CO 803090391
USA
TEL: 3034928788
TLF:
TLX:
EML:
COM: 10,40

DUMONT RENE OR.

OBSERVATOTRE DE BORDEAUX

BP 89

F 33270 FLOIRAC

FRANCE

TEL: $56 \quad 86 \quad 4330$

TLF: 56404251

TLX:

EML:

COM: 210

DUNCOMBE RAYNOR L OR

AEROSPACE. ENGINEERING OPT UNIVERSITY OF TEXAS

\section{WRW 414}

AUSTIN TX 78712

USA

TEL: 5124714250

TLF:

TLX: 704265 CSRUTX UD

EML:

COM: $04,05,07,08$

DUNN RICHARO B OR

AIR FORCE GEOPHYSICS LAB

NTL SOLAR OBSERVATORY

SUNSPOT NM 88349

USA

TEL: $505 \quad 4341390$

TLF:

TLX:

EML:

COM: 10,12
DULTZIN-HACYAN O OR

INST ASTROFISICA

DE ANOULUCIA APO 2144

PROFESOR ALBAREDA I

E 18080 GRANADA

SPAIN

TEL: $58 \quad 12: 1300$

TLF:

TLX: 78573 IAAGE

EML:

COM: 28.47

DUMONT SIMONE OR

INSTITUT DAASTROPHYSIQUE

98BIS BD ARAGO

F 75014 PARIS

FRANCE

TEL: 143201425

TLF: 143298673

ILX:

EML:

COM: 12,41

DUNHAM DAVID W

COMPUTER SCIENCES CORP

SYSTEM SCIENCES DIV

8728 COLESVILLE RD

'SILVER SPRING MO' 20910

USA

TEL:

TLF:

TLK:

EML:

COM: $04,20,26$

DUDRAH 'HIRA LAL DR

DPT OF PHYSICS

UNIVERSITY OF GAUHATI

GUWAHATI 781014

INDIA.

TEL: BB 531

ILF:

TLX:

EML:

COM: 48

\section{DURIEZ, LUC DR}

LABORATOIRE O'ASTRONOMIE

I IMPASSE OBSERVATOIRE

F 59000 LILLE

FRANCE

TEL: $20 \quad 524424$

TLF:

TLX:

EML: EARN: DURIEZ@FRCIIL7I

COM: 07
DUMA DMITRIJ P DR

MAIN ASTRONOMICAL OBS

UKRAINIAN ACAD OF SCIENCE

GOLQSEEVO

252127 KIEV

UKRAINE

TEL: 663120

TLF:

TLX: 131406 SKY SU

EML:

COM: 08

OUNCAN DOUGLAS KEVIN OR

STSCI

HOMEWOOD CAMPUS

3700 SAN MARTIN UR

BALTIMORE MD 21218

USA

TEL: 3013384935

TLF :

TLX:

EML:

COM: 29

OUNKELMAN LAWRENCE

LUNAR \& PLANETARY LAB

UNIVERSITY OF ARIZONA

BOX 36241

TUCSON AZ 6572L

USA

TEL: 6026216963

TLF:

TLK:

EML:

COM: $09,12,21,44,50$

DUPREE ANDREA $K$ DR

CENTER FOR ASTROPHYSICS

SOLAR \& STELLAR DIVISION

60 GARDEN ST

CAMBRIOGE MA 02138

USA

TEL: 6174957489

TLF:

TLX: 921428 SATELLITE CAM

EML:

COM: $34,36,44$

DURISEN RICHARQ H DR

ASTRONOMY OPT

INDIANA UNIVERSITY

SWAIN WEST 319

BLOOMINGTON IN 47405

USA

TEL: 8123356921

TLF:

TLK:

EML:

COM: 35.42 


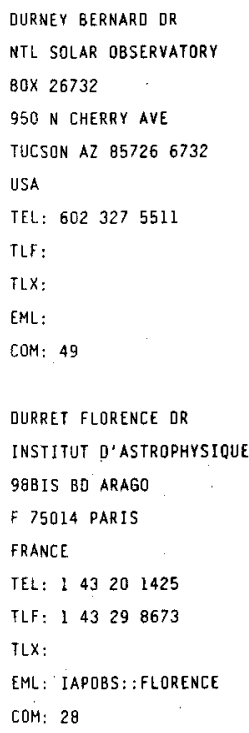

\author{
DUROUCHOUX PHILIPPE \\ CEA CEN \\ DAPNIA/SAP \\ BP 2 \\ F 91191 GIF/YVETTE CDX \\ FRANCE \\ TEL: 169083376 \\ TLF : \\ TLX: 690860 \\ EML: \\ COM: 48
}

DUSCHL WOLFGANG I DR INSTITUT F. THEORETISCHE ASTROPHYSIK

IM NEUENHEIMER F 561

D 6900 HEJOELBERG I

GERMANY

IEL: $62 \quad 21562967$

TLF :

TLX: 46515 UNIHD D

EML: BITNET: CJOOOHOURZI

COM: 42

DUVERT GILLES DR

OBSERVATOIRE DE GRENOBLE

CERMO/ASTROPHYSIQUE

BP $53 \mathrm{X}$

F 38041 S MARTIN HERES CD FRANCE

TEL: $76 \quad 51 \quad 4885$

ILF: $76 \quad 448821$

TLX:

EML: BITNET: DUVERT FRGAG5 I

COM:

OWIVEOI BHOLA NATH DR OPT OF APPLIEO PHYSICS IT UNIVERSITY BANARAS HINOU VARANASI 221005

INDIA

TEL:

TLF:

TLX: 0.545-208 TECH IN

EML:

COM: 10

OVER EOWARO R OR

3626 DAVIS ST NW WASHINGTON OC 20007

USA

TEL:

TLF :

EML:

COM:

ILX:
DURRANCE SAMUEL T DR OPT PHY'SICS \& ASTRONOMY JOHNS HOPKINS UNIVERSITY CHARLES \& 34TH ST BALTIMORE MO 21218 USA

FEL: $301338 \quad 8707$

TLF :

TLX: 9102400225 JHU CAS

EML:

COM: 16

DUTHIE JOSEPH G PROF DPT PHYSICS ASTRONOMY UNIVERSITY OF ROCHESTER ROCHESTER NY 14627

USA

TEL:

TLF:

TLX:

EML:

COM: 48

DVORAK RUDOLF DR

INSTITUT FUER ASTRONOMIE UNIVERSITAETSSTERNWARTE TUERKENSCHANZSTR 17

A 1180 WIEN

AUSTRIA

TEL: 13453600

TLF:

TLX:

EML:

COM: 07,20

DWORETSKY MICHAEL M DR OPT PHYSICS \& ASTRONOMY UNIVERSITY COLLEGE LONDON GOWER ST

LONDON WCLE GBT

UK

TEL: $1713877 \quad 050$

TLF :

$T L X: 28722$

EML:

COM: 29

OYSON F J OR

INST FOR ADVANCED STUDY

SCHOOL OF NATURAL SCIENCE.

PRINCETON NJ 08540

USA

TEL: $609734 \quad 8055$

$T L F$ :

TLX:

EML:

COM: 40,51
DURRANT CHRISTOPHER J DR OPT APPLIEO MATHEMATICS UNIVERSITY OF SYDNEY SYDNEY NSW 2006 AUSTRALIA

TEL: $2 \quad 692 \quad 3.373$

TLF:

TLX: 20056 FISHLIB AA

EML:

COM:

DUVAL MAR IE-FRANCE OBSERVATDIRE DE MARSEILLE 2 PLACE LE VERRIER F 13248 MARSEILLE COX 04 FRANCE

TEL: 91959088

TLF :

TLX:

EML:

COM: 28,46

DWARAKANATH KS

RAMAN RESEARCH INSTITUTE

SADASHIVANAGAR

BANGALORE 560080

INDIA

TEL: $812 \quad 36 \quad 0122$

TLF: 812340492

TLX: 8452671 RRI IN

EML:

COM: 40

DYCK M DR

KITT PEAK NTL OBS BOX 26732 $950 \mathrm{~N}$ CHERRY AVE TUCSON AZ $85726 \quad 6732$ USA

TEL:

TLF :

TLX:

EML:

COM:

OYSON JOHN E OR DPT OF ASTRONOMY UNIVERSITY OF MANCHESTER MANCHESTER MI3 9PL UK

TEL: 612754235

TLF:

TLX: 668932

EML:

COM: 34,49 


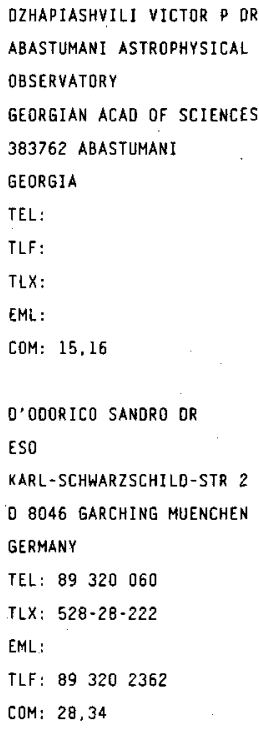

ECHEVARRIA JUAN OR OBS ASTRONOMICO NACIONAL UNAM

APDO POSTAL 677

22800 ENSENADA B CALIF MEXICO

TEL:

TLF :

TLX:

EML:

COM: 42

\section{EDELSON RICK OR}

CASA

UNIVERSITY OF COLORADO

BOX 391

BOULOER CO 803090391

USA

TEL: $303492 \quad 6784$

TLF :

TLX:

EML: ELROY: :RICK SPAN

COM: $28,40,48$

\section{EOVAROSSON BENGT OR}

ASTRONDMICAL OBSERVATORY

BOX 515

S 75120 UPPSALA

SWEDEN

TEL: 18512488

TLX: 76024 UNIVUPS S

EML: ASTBEOSEUOAC21 BITNET

TLF: $18 \quad 527583$

COM: 36
OZIEMBOWSKI WOJCIECH PROF COPERNICUS ASTRON CENTER POLISH ACAD OF SCIENCES

UL BARTYCKA 18

PL 00716 WARSAW

POLAND

TEL:

TLF:

TLX:

EML:

COM: 27,35

EATON JOEL A DR

ASTRONOMY DPT

INDIANA UNIVERSITY

SWAIN WEST 319

BLOOMINGTON IN 47405

USA

TEL: 8123354176

TLF:

$T \perp X$ :

EML:

COM: 42

ECHEVERRIA ROMAN JUAN M OBS ASTRONOMICO NACIONAL UNAM

APDO POSTAL 977

22860 ENSENADA B CALIF

MEXICO

TEL:

TLF:

TLX:

EML:

COM:

EDLEN BENGT PROF

DPT OF PHYSICS

UNIVERSITY OF LUND

SOELVEGATAN 14

S 22362 LUND

SWEDEN

TEL: $45 \quad 10 \quad 7730$

TLF :

TLX:

EML:

COM: 14

EDWARDS ALAN CH BR DPT PHYSICS \& ASTRONOMY UNIVERSITY OF GEORGIA ATHENS GA 30602

USA

TEL:

TLF :

TLX:

EML:

COM: 35
DZIGVASHVILI R M OR ABATSUMANI ASTROPHYSICAL OBSERVATORY

GEORGIAN ACAD OF SCIENCES

383762 ABASTUMANI

GEORGIA

TEL:

TLF:

$T \perp X$ :

EML:

COM: 33

EBISUZAKI TOSHIKAZU OR KOMABA MEGURO-KU

TOKYO 153

JAPAN

TEL: 81334671171 (665)

ILF: 81 334653925

TLX:

EML:

COM: 28

EOOY JOHN A OR

3460 ASH AVE

BOULOER CO BO303

USA

TEL: $303 \quad 497 \quad 1680$

TLF:

TLX: 45694

EML:

COM: 10,41

EOMONOSON FRANK K PROF

ASTRONOMY DPT

INDIANA UNIVERSITY

SWAIN WEST 319

BLOOMINGTON IN 47405

USA

TEL: 8123356918

TLF :

TLX:

EML:

COM: $20,30,33,41$

EOWARDS PAUL $J$ BR

MOUNT STROMLO \& SIDING

SPRING OBSERVATORIES

PRIVATE BAG

WODEN PO ACT 2606

AUSTRALIA

TEL: $62 \quad 881111$

TLF:

TLX: $68270 \mathrm{AA}$

EML:

COM: $25,27,48,50$
D'ANTONA FRANCESCA OR

DAR

MONTE PORZIO

I OOO40 MONTEPORZIO

ITALY

TEL: 69449019

TLF:

ILX:

EML:

COM: $35,37,42$

ECCLES MICHAEL $\mathbf{J}$ DR BALLENCRIEFF TOLL

SUNNYSIDE

BATHGATE EH48 4LD

UK

TEL: 50653989

TLF :

TLX: 727484

EML:

COM: 51

EDELLMAN COLETTE DR

BUREAY DES LONGITUOES

77 AVE DENFERT ROCHEREAL

F 75014 PARIS

FRANCE

TEL: I 40512272

TLF :

TLX:

EML:

COM: 07,20

EOMUNDS MICHAEL GEOFFREY PHYSICS OPT UNIV WALES COLLEGE BOX 913 CARDIFF CFI 3TH UK

TEL: $222 \quad 874 \quad 785$

TLF: $222 \quad 371921$

TLX:

EML:

COM: 28

EOWARDS TERRY W DPT PHYSICS \& ASTRONOMY UNIVERSITY OF MISSOURI COLUMBIA MO 65211

USA

TEL: $314 \quad 8823036$

TLF:

TLX:

EML:

COM: 35 
EOWIN ROGER $P$

UPT OF PHYSICS \& ASTRON

UNIVERSITY OF ST ANOREWS NORTH HAUGH

ST ANDREWS FIFE KY16 9SS

UK

TEL: 33476161

TLF: 33474487

ILX:

EML:

COM: 09 .

EFSTATHIOU GEORGE

INSTITUTE OF ASTRONOMY

THE OBSERVATDRIES

MADINGLEY RO

CAMBRIDGE CB3 OHA

UK

TEL: 22362204

TLF :

TLX: B17297 ASTRON G

EML:

COM: 28,47

EICHHORM HEINRICH $K$ QR

OP.T OF ASTRONOMY

UNIVERSITY OF FLORIDA

$23 L$ SSRB

EATNESVILLE FL 32611

USA

TEL: $904392 \quad 2052$

TLF:

$T L X:$

EML:

COM: $07,08,24,26$

\section{EINASTO JAAN DR}

TARTU ASTROPHYSICAL OBS

ESTONIAN ACAD OF SCIENGES

202444 TARTU

ESTONIA

TEL:

TLF:

TLX:

EML:

COM: $28,33,37,47$

\section{EKERS RONALO D DR}

\section{CSIRO}

OIVISION OF RADIOPHYSICS

$80 \times 76$

EPPING NSW 2121

AUSTRALIA

TEL: $2868 \quad 0222$

TLF: 28680310

TLX: 26230

EML: REKERS ATNF.CSIRO.AU

COM: 28,40
EELSALU HEINO OR

TARTU ASTROPHYSICAL OBS

ESTONIAN ACAD OF SCIENCES

202400 TARTU 4

ESTONIA

TEL: 143428163

TLF: $1434 \quad 10205$

TLX:

EML:

COM: $25,30,41$

EGGLETON PETER P DR

INSTITUTE OF ASTRONOMY

THE OBSERVATORIES

MADINGLEY RD

CAMBRIOGE CB3 OHA

UK

TEL: 22362204

TLF ;

TLX: 817297 ASTRON $G$

EML:

COM: $35,42 C$

EICHLER DAVIO OR

ASTRONOMY PROGRAM

UNIVERSITY OF MARYLANO

COLLEGE PARK MO 20742

USA

TEL: 3014546448

TLF:

TLX: $710-8260352$

EML:

COM: 48 .

EINAUDI GIORGIO

DPT DI ASTRONOMIA

UNIVERSITA DI FIRENZE

LARGO E FERMI 5

I 50125 FIRENZE

ITALY

TEL: 5527521

TLF: 55220039

TLX: 572268 ARLETR I

EML:

COM: 12

\section{EL BAKKALI LARBI DR}

FAC DE SCIENCES

BOX 2121

TETOUAN

MOROCCO

TEL: 974509 .

TLF: 971763

ILX:

EML:

COM: 07
EFREMOY YU I DR

INST OF APPLIEO MATHS

ACADEMY OF SCIENCES

MIUSSKAJA SQ 4

125047 MOSCOW

RUSSIA

TEL:

TLF:

TLX:

EMt:

COM:

EGRET DANIEL DR .

OBS OE STRASBOURG

11 RUE UNIVERSITE

f 67000 STRASBOURG

FRANCE

TEL: 88354300

TLF: 88 '25 0160

TLX: 890506 STAROBS

EML:

COM: $05,33,45 \mathrm{C}$

EIDELSBERG MICHELE OR

OBSERVATOIRE DE PARIS

SECTION DE MEUDOON

F 92195 MEUDON PPL COX

FRANCE

TEL: $145 \quad 077562$

TLF: 145077469

TLX:

EML: FRMEUO51

COM: 14

EINICKE OLE H QR

COPENHAGEN UNIVERSITY OBS

BRORFELDEVEJ 23

OK 4340 TOLLOSE

DENMARK

IEL: 5338 48 8195

TLF: 58488755

TLX:

EML:

COM: 08

EL EIO MOUNIB DR

UNIVERSITATS-STERNWARTE

GEISMARLANOSTR. 11

98 BIS. BQULEVARO ARAGO

D 3400 GOETIINGEN

GERMANY

TEL: 551395042

ILF:

TLX: 96753

EML:

COM:
EFREMOV YURY. N OR

STERNBERG STATE ASTR INST

UNIVERSITETSKIJ PROSP 13

119899 Moscow

RUSSIA

TEL: 1392657

TLF:

TLX:

EML:

COM: $27,33,37$

EHLERS JURGEN PROF

MPI FUER PHYSIK UND

ASTROPHYSIK

KARL-SCHWARZSCHILD-STR I

D 8046 GARCHING MUENCHEN

GERMANY

TEL: 8932999444

ILF:

TLK: 524629 ASTRO D

EML:

COM: 47

EILEK JEAN

DPT OF PHYSICS

NEW MEXICO TECH

CAMPUS STATION

SOCORRO NM 97801

USA

TEL: $505 \quad 8355433$

TLF:

TLX:

EML:

COM: 48

EIROA DE SAN FRANCISCO C OBS ASTRONOMICO NCL

ALFONSO XII-3

E 28014 MADRID

SPAIN

TEL: 12270107

TLF :

TLX: 49880 OANM

EML:

COM:

EL NAWAWAY M S DR

ACADEMY OF SCIENTIFIC

RESEARCH AND TECHNOLOGY

101 KASR EL EINI STREET

CAIRO

EGYPT

TEL:

TLF :

TLX:

EML:

COM: 
ELFORD WILLIAM GRAHAM OR OPT OF PHYSICS

UNIVERSITY OF ADELAIDE

BOX 498

ADELAIDE SA 5001

AUSTRALIA

TEL: 22285321

TLF:

ILX: B9I4I UNIVQOO AA

EML:

COM: 22

ELLDER JOEL DR

ONSALA SPACE OBSERVATORY

S 43034 ONSALA

SWEDEN

TEL:

TLF:

EML:

COM:

TLX:

ELLIS G R A PROF

DPT OF PHYSICS

UNIVERSITY. QF TASMANIA

GPO BOX $252 C$

HOBART TAS 7001

AUSTRALIA

TEL:

TLF :

TLX: 58150

EML:

COM: 40

ELMEGREEN OEBRA MELOY

DPT PHYSICS \& ASTRONOMY

VASSAR COLLEGE

POUGHKEEPSIE

NY 12601

USA

TEL: 9144377356

TLF:

TLX:

EML: ELMEGREENOVASSAR.EDU

COM: $28,33,34$

ELSTE GUNTHER H. DR

DPT OF ASTRONOMY

UNIVERSITY OF MICHIGAN

DENNISON BLDG

ANN ARBOR MI 481091090

USA

TEL: 3137643444

ILF:

TLX:

EML:

COM: $10,12,36$
ELgaroy OYSTEIN PROF

INST THEORET ASTROPHYSICS

UNIVERSITY OF OSLO

BOX 1029

N 0315 BLINOERN OSLO 3

NORWAY

TEL: $02-456-504$

TLF:

ILK:

EML:

COM: 40

ELLIOT JAMES L DR

DPT .OF EARTH \& PLANET SCI

MIT RM 54 422A

BOX 165

CAMBRIDGE MA 02139

USA

TEL: 6172536308

TLF:

ILX: 921473 MIT CAM

EML:

COM: 16.20

ELLIS GEORGE F R PROF

SISSA

ST COSIIERA II

MIRAMARE

I 34014 TRIESTE

ITALY.

JEL: $40 \quad 224118$

ILF:

TLX: 460392 ICTP

EML:

COM: 47.51

ELSAESSER HANS PROF

MPI FUR ASTRONOMIE

KOENIGSTUHL

0. 6900 HEIDELBERG I

GERMANY

TEL: $62 \quad 21528200$

TLF :

TLX:

EML:

COM: 21.33

ELSTON WOLFGANG E PROF

OPT OF GEOLOGY

UNIVERSITY OF. NEW MEXICO

800 YALE BLVD NE

ALBUQUERQUE NM 87131

USA

TEL: $505 \quad 277 \quad 5339$

TLF :

TLX: 660461

EML:

COM: 16
ELIPE SANCMEZ ANTONIO.

DPT FIS TIERRA \& COSMOS

UNIVERSIDAD DE ZARAGOZA

E 50009 ZARAGOZA

SPAIN

TEL: $76 \quad 357011$

TLF :

TLX: 58198

EML:

COM: 07

ELLIOTT IAN DR

DUNSINK OBSERVATORY

DIAS

DUBLIN 15

IRELAND

TEL: 1387959

TLF:

TLX: 31687 OIAS EI

EML:

COM: 12

ELLIS RICHARG S

DPT OF PHYSICS

UNIVERSITY OF DURHAM

SOUTH RO

DURHAM OHL 3LE

UK

TEL:

TLF :

ILX:

EML:

COM: $28 C, 47$

ELSMORE BRUCE DR

MULLARD RADIO ASTRON OBS

CAVENDISH LABORATORY

MADINGLEY RD

CAMBRIDGE CB3 OHE

UK

TEL: 22366477

TLF :

TLX: 81292

EML:

COM: $19,24,40$

ELVIS MARTIN S DR

CENTER FOR ASTROPHYSICS

HCO/SAO

GO GAROEN ST

CAMBRIOGE MA 02138

USA

TEL: $6.17 \quad 495 \quad 7442$

TLF:

$T L X: 921428$

EML:

COM: $28,44,47,48$
ELITZUR MOSHE

OPT PHYSICS \& ASTRONOMY

UNIVERSITY OF KENTUCKY

LEXINGTON KY 405060055

USA

TEL: 6062574720

TLF:

TLX:

EML:

COM: 34

ELLIOTT KENNETH H DR

OPT OF SPACE RESEARECH

UNIVERSITY OF BIRMINGHAM

BOX 363

BIRMINGHAM B15 $21 T$

UK

TEL: 214721301

TLF :

TLX: 338938

EML:

COM: 34

ELMEGREEN BRIUCE GORDON-DR

IBM

THOMAS I WATSON RES CTR

BOX 21.8

YORKTOWN HEIGHTS NY 10598

USA

TEL: $914945 \quad 2448$

ILF: 9149452141

TLX: 137456

EML: BGECYKTVMT

COM: 34,37

ELST ERIC WALTER DR OBSERVATOIRE. ROYAL DE BELGIOUE

AVE CIRCULAIRE 3

B 1180 BRUSSELS

BELGIUM

TEL:

ILF: 23749822

$T L X:$

EHL:

COM: 20

ELVIUS AINA M PROF

STOCKHOLM OBSERVATORY.

S 13336 SALTSJOEBADEN

SWEDEN

TEL: $87 \quad 170195$

TLF: 87174719

ILX: 12972 SOBBSERV $S$

EML: ELVIUSEASTRO.SU.SE

COM: 28.34 


ELVIUS IORD PROF EMERITUS
NORRLANOSGATAN $34 \mathrm{~F}$
S 75229 UPPSALA
SWEDEN
TEL: 18100857
TLF:
EML:
COM: 33.45
TLX:

EL-RAEY MOHAMEO E PROF OPT ENVIRONMENT STUDIES INST GRADUATE STUD \& RES UNIVERSITY OF ALEXANDRIA ALEXANDRIA

EGYPT

TEL:

TLF :

TLX:

EML:

COM: 44

EMERSON QAVID

ROYAL OBSERVATORY

BLACKFORD HILL

EUINBURGH EH9 $3 \mathrm{HJ}$

UK

TEL:

$T\lfloor X$ :

EML:

TLF :

COM: $2: 8940,46$

ENARD DANIEL OR

ESO

KARL-SCHARZSCHILDSIR. 2

0 8046 GARCHING MUENCHEN

GERMANY

TÉL: 8932006251

TLF: 89.3202362

TLX:

EML:

cont:

ENGELBRECHT CHRISTIAN DR

UNIVERSITY OF STELLENBOSC

PRETORIA

SOLTH AFRICA

TEL:

TLF:

EML:

COM:

TLX:
ELWERT GERHARO PROF LEHRSTUHL F THEORETISCHE ASTROPHYSIK

UNIVERSITAET TUEBINGEN

D 7400 TUEBINGEN

GERMANY

TEL: 7071296483

TLF:

ILX: 7-262714 ALT 0

EML:

COM: 10,40

EL-SHAHAWY MOHAMAD PROF

DPT ASTRON \& METEOROLOGY

FACULTY OF, SCIENCES

CAIRO UNIYERSITY

GEZA

EGYPT

TEL: 272022

TLF:

TLX: 94372 UNCAI

EML: '

COM: 19

EMERSON JAMES P

DPT OF PHYSICS

QUEEN MARY/WESTFIELO COLL

MILE ENO RO

LONDON EI 4NS

UK

TEL: 19804811

TLF:

TLX: 893750 QMCUOL G

EML:

COM: 34

ENCRENAZ PIERRE J DR

RAOIOASTRONOMIE ENS

24 RUE LHOMONO

F 75231 PARIS COX 05

FRANCE

TEL: 143291235

TLF :

TLX:

EML:

COM: 34

\section{ENGELS DIETER DR}

STERNWARTE DER UNIVERS.

HAMBURG

GOJENSBERGSWEG. 112

D 2050 HAMBURg 80

GERMANY

TEL: $40725 \quad 24136$

TLF: 4072524198

TLX: 217884

EML: ST20050 DHHUNI4 , EARN

COM: 09

\author{
EL-BASSUNY ALAWY A A OR \\ HELWAN OBSERVATORY \\ HELWAN \\ EGYPT \\ TEL: $78 \quad 0645 / 2683$ \\ TLF: \\ TLX: 9703 HIAG UN \\ EML: \\ COM: 27,37
}

EMANUELE ALESSANORO OR

IAS

CNR

CP 67

I 09044 FRASCATI

ITALY

TEL: 69425655

TLF: $6 \quad 9416847$

TLX: 610261

EML: POLIFEMOARMIAS

COM: 48

EMINZADE T A DR

SHEMAKHA ASTROPHYSICAL

OBSERVATORY

AZER ACAGEMY OF SCIENCES

373243 SHEMAKHA

AZERBAIDZHAN

TEL:

TLF :

TLX:

EML:

COM: 35

ENCRENAZ THERESE DR

OBSERVATOIRE DE PARIS

SECTION DE MEUDON

GROUPE PLANETES

F 92195 MEUDON PPL CDX

FRANCE

TEL: 145077691

TLF:

TLX: 204464

EML:

COM: $15,16 \mathrm{C}$

ENGIN SEMANUR PROF

DPT OF ASTRONOMY

UNIVERISTY OF ANKARA

FEN FAKULTESI

O6LOD BESEVLER

TURKEY

TEL:

TLF:

TLX:

EML:

COM:
EL-BAZ FAROUK OR

ITEK OPTICAL SYSTEMS

10 MAgUiRE RD

LEXINGTON MA 02173

USA

TEL: $617276 \quad 2532$

TLF:

TLX: 923456

EML:

COM: 16

EMELIANOV NIKOLAJ V OR

STERNBERG STATE ASTR INST

UNIVERSITETSKIJ PROSP 13

119899 MOSCOW

RUSSIA

TEL: $139-37-64$

TLF:

TLX:

EML:

COM: 07

EMSLIE A GORDON

DPT OF PHYSICS

UNIVERSITY OF ALABALA

HUNTSVILLE AL 35899

USA

TEL: 2058956167

TLF:

TLX:

EML:

COM: 10

ENDAL ANOREW S. OR

APPLIED RESEARCH CORP

8201 CORPORATE OR

SUITE 920

LANDOVER MD 20785

USA

TEL: 3014598442

TLF:

TLX:

EML:

COM: 35

ENGINOL TURAN B OR

INST FOR GRADUATE STUOIES

IN SCIENCE \& ENGINEERING

BOGAZICI UNIVERSITY

TURKEY

TEL: $1163 \quad 1500$

TLF: $11658480^{\circ}$

TLX: 26411 BOUN TR

EML: ENGINOL F TR BOUN

COM: 47 


\begin{tabular}{|c|c|c|c|}
\hline ENGVOLD ODDBJOERN DR & ENOME SHINZZO PROF & ENSLIN HEINZ DR & $\begin{array}{l}\text { EPPS HARLAND, WARREN PROF } \\
\text { OPT OF ASTRONOMY }\end{array}$ \\
\hline $\begin{array}{l}\text { INST THEORET ASTROPHYSICS } \\
\text { UNIVERSITY OF OSLO }\end{array}$ & $\begin{array}{l}\text { NOBEYAMA RADIO OBS } \\
\text { NAOJ }\end{array}$ & $\begin{array}{l}\text { YIERLAENDER WEG } 5 \\
\text { D } 2057 \text { REINBECK }\end{array}$ & $\begin{array}{l}\text { OPT OF ASTRONOMY } \\
\text { UNIVERSITY OF CALIFORNIA }\end{array}$ \\
\hline$B 0 \times 1029$ & MINAMIMAKI MURA & GERMANY & MATH SCI RM 8983 \\
\hline N 0315 BLIMDERN OSLO 3. & NAGANO $384 \quad 13$ & TEL: $407223616 * 194$ & LOS ANGELES CA 90024 \\
\hline NORWAY & JAPAN & TLF:" & USA \\
\hline TEL: 472856521 & TEL: $81 \quad 267 \quad 98 \quad 2831$ & TLX: & TEL: 2138253025 \\
\hline TLF: 472856505 & TLF: $81 \quad 267 \quad 98 \quad 2884$ & EML: & TLF: \\
\hline TLX: 2456515 & TLX: 3329005 NAONRD J & COM: 19.31 & $T L X: 910-3427597$ \\
\hline EML: $21813:: 55418:: 000 B J O R N$ & EML: & & EML: \\
\hline COM: 09.10VP & COM: 10.40 & & COM: \\
\hline EPSTEIN EUGENE E DR & EPSTEIN GABRIEL LEO DR. & EPSTEIN ISADORE PROF & EPSTEIN RICHARO I DR \\
\hline AEROSPACE CORPORATION & NASA/GSFC & DPT OF ASTRONOMY & LOS ALAMOS NATIONAL LAB \\
\hline 211B PATRICIA AVE & CODE 682 & COLUMBIA UNIVERSITY & MS 436 \\
\hline LOS ANGELES CA 90009 & GREENBELT MO 20771 & PUPIN HALL 53B W $120 T H$ ST & BOX 1663 \\
\hline USA & USA & NEW YORK NY 10027 & LOS ALAMOS NM 37545 \\
\hline TEL: $213 \quad 648 \quad 6798$ & TEL: & USA & USA \\
\hline$T L X: 664460$ & TLF: & TEL: $212 \quad 2803280$ & TEL: 5056879595 \\
\hline EML: & $T L X:$ & TLF : & TLF : \\
\hline TLF : & EML: & TLX: 125953 COLUMBIA & TLX: \\
\hline \multirow[t]{2}{*}{ COM: 40.51} & COM: 12.14 & EML: & EML: \\
\hline & & COM: 35 & COM: \\
\hline ERCAN E NIHAL & EROI B OR & ERGMA E V OR & ERICKSON WILLIAM C DR \\
\hline KANOILLI OBSERVATORY & ASTRONOMY OPT & DPT THEORET PHYS \& ASTOPH & DPT OF PHYSICS \\
\hline BOGAZICI UNIVERSITY & EOTVOS UNIVERSITY & TARTU UNIVERSITY & UNIVERSITY OF TASMANIA \\
\hline CENGELKOY & KUN BELA TER 2 & ULIKOOLI IB & GPO BOX $252 \mathrm{C}$ \\
\hline 81220 ISTANBUL & H 1083 BUDAPEST & 202400 TARTU : & HOBART TAS 7001 \\
\hline TURKEY & HUNGARY & ESTONIA & AUStRALiA \\
\hline TEL: $1332 \cdot 0240 / 41$ & TEL: 141019 & TEL: 73775 & TEL: 2202401 \\
\hline TLF: & TLF: & TLF : & TLF: $2202 \quad 186$ \\
\hline TLX: 26411 BOUN TR & TLX: & TLX: 412623 SCSTP SU & $T L X: A A 58150$ \\
\hline EML: & EML: & EML: & EML: \\
\hline COM: & COM: 07 & CDM: 35,49 & COM: 40 \\
\hline ER1GUCHI YOSHIHARU OR & ERIKSEN GUNNAR PROF & ERIKSSON KJELLL DR & ERPYLEV N P OR \\
\hline OPT EARTH SCI \& ASTRONOMY & INST THEORET ASTROPHYSICS & ASTRONOMICAL OBSERVATORY & INST OF ASTRANOMY \\
\hline UNIVERSITY OF TOKYO & UNIVERSITY OF OSLO & $80 \times 515$ & ACADEMY OF SCIENCES \\
\hline MEGURO KU & $80 \times 1029$ & S 75120 UPPSALA & PYATNITSKAYA UL 48 \\
\hline TOKYO 153 & N 0315 BLINDERN OSLO 3 & SWEOEN & 109017 MOSCOW \\
\hline JAPAN & NORWAY & TEL: : IB 112488 & RUSSIA \\
\hline TEL: $03-467-1171 \times 439$ & TEL: $02-45-65-15$ & TLF: & TEL: $231-54-61$ \\
\hline TLF : & TLF: & ILX: 76024 UNIVUPS $S$ & TLF: \\
\hline TLX: 25510 UNITOKYO & TLX: & EML: & TLX: 412623 SLSTP SU \\
\hline EML: & EML: & COM: $36^{\prime}$ & EML: \\
\hline COM: 35 & COM: 40 & & COM: 41 \\
\hline ERSHKOVICH ALEXANDER PROF & ERTAN A YENER OR & ESCALANTE VLADIMIR DR & ESHLEMAN VON R PROF \\
\hline OPT GEOPHYS \& PLANET SCT & FACULTYY OF SCIENCE & INSTITUTO OE ASTRONOMIA & DURANO 221 \\
\hline TEL AVIV UNIVERSITY & EGE UNIVERSITY & UNAM & STANFORD UNIVERSITY \\
\hline TEL AVIV & $B D \times 21$ & APDO.POSTAL $70-264$ & STANFORD CA 94305 \\
\hline ISRAEL. & 35100 BORNOVA IZMIR & O4510 MEXICO DF & USA \\
\hline TEL: 3413505 & TURKEY. & MEXICO & TEL: 4154973531 \\
\hline TLF: & TEL: & TEL: 5255485305 & TLF: \\
\hline TLX: 342171 ' VERSY IL & TLF: & TLF: $525 \quad 5 \quad 483712$ & TLX: \\
\hline EML: & TLX: & TLX: 01760155 CICME & EML: \\
\hline COM: 15 & EML: & EML: VLAOIMIR ALFA.ASTROS. UNAM.MK & CDM: $16,40,49$ \\
\hline & COM: & COM: 34 & \\
\hline
\end{tabular}




ESIPOV VALENTIN F OR
STERNBERG STATE ASTR INST
117234 MOSCOW
RUSSIA
TEL:
TLF:
EML:
COM: 34
TLX:

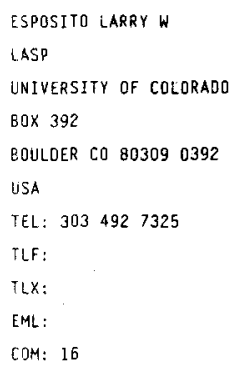

ESKIOGLU A NIHAT
DEVLET MUHENDISLIK
MIMARLIK AKADEMISI
ADAPAZARI SAKARYA
TURKEY
TEL:
TLF:
TLX:
EML:
COM: 27

ESSER RUTH OR

INST MATHS \& PHYSICAL SCI UNIVERSITY OF TROMSO BOX 953

N 9000 TROMSO

NORWAY

TEL: $4783 \quad 45164$

TLF: $47 \quad 83 \quad 89852$

TLX: 64124 SUROB $\mathrm{N}$

EML: RUTH-ESSEREESUIT.UIT.NO

COM: 12

EVANGELIOIS E OR

PLASMA PHYSICS DIVISION

NUCOR / PELINDABA

PRIVATE BAG X256

PRETORIA 0001

SOUTH AFRICA

TEL: $27-12-21-3311$

TLF:

TLX: 30253 SA

EML:

COM: 33,36

EVANS KENTON DOWER DR

DPT OF PHYSICS

UNIVERSITY OF LEICESTER

UNIVERSITY RD

LEICESTER LEL TRN

UK

T.EL: 533554455

TLF :

TLX: 341664

EML:

COM: 40

EVANS ROGER G OR
RUTHERFORO APPLETON LAB
SPACE \& ASTROPHYSICS OIV
BLOG R25/R68
CHILTON DIOCOT OXLI OQX
UK
TEL: 23521900
TLF:
TLX: 83159 RUTHLB 6
EML:
COM: 28

ESKRIDGE PAUL B DR CENTER FOR ASTROPHYSICS

MS $B 1$

60 GARDEN ST

CAMBRIDGE MA 02138

USA

TEL: 6174967585

TLF:

TLX:

EML: ESKRIDGEECFA. HARVARD. EOU COM:

ESTALELLA ROBERT

DPT FISICA DE ATMDSFERA

UNIVERSIDAD DE BARCELONA AVD DIAGONAL 645

E 08028 BARCELONA

SPAIN

TEL: $33307311 / 298$

TLF:

TLX:

EML:

COM:

\section{EVANS ANEURIN}

DPT DF PHYSICS

UNIVERSITY OF KEELE

KEELE STS 5BG

UK

TEL: 732621111

TLF ;

TLX: 36113 UNKLIB G

EML:

COM: 27,34

EVANS NANCY REMAGE DR

SAL/ISTS

YORK UNIVERSITY

4700 KEELE ST

NORTH YORK ON LAK 3C8

CANADA

TEL:

TLF : $416 \quad 736 \quad 5386$

TLX:

EML:

COM: 27

\section{EVANS W DOYLE}

390 EL CONEJO

LOS ALAMOS NM 87544

USA

TEL: $505 \quad 667 \quad 3644$

TLF :

TLX:

EML:

COH: 48
ESPOSITO F PAUL PRDF

OPT OF PHYSICS

UNIVERSITY OF CINCINNATI

210 BRAUNSTEIN ML 11

EINCINNAII OH 452210111

USA

TEL: $513 \quad 4752233$

TLF:

TLX:

EML:

COM:

ETZEL PAUL B DR

DPT OF ASTRONOMY

SAN DIEGO STATE UNIV

SAN DIEGO CA 92182

USA

TEL: 6194956169

TLF: 6195945485

$T L X$ :

EML: ETZELOHINTAKA, SOSU, EO

COM: 42

EVANS I $\checkmark$ DR

COMSAT LABORATORIES

22300 COMSAT OR

CLARKSBURG MD 20871

USA

TEL: 3014284422

TLF:

ILX: 908753

EML:

COM: 12

EVANS NEAL J II ASS PRDF ASTRONOMY OPT UNIVERSITY OF TEXAS

RLM 15308

AUSTIN TX 787121083 USA

TEL: 5124714461

TLF:

TLX:

EML:

COM: 34,51

EVOOKIMOV YU V OR ENGELHAROT ASTRONOMICAL OBSERVATORY oBSERVATORIA STATION 422526 KAZAN RUSSIA

TEL:

TLF:

$T L X$ :

EML:

CDM: 20 


\author{
EVIATAR AHARON PROF \\ OPT GEOPHYS \& PLANET SCI \\ TEL. AVIV UNIVERSITY \\ IEL AVIV \\ ISRAEL \\ TEL: 3420620 \\ ILF: \\ TLX: 342171 VERSY IL \\ EML: \\ COM: 15,49 \\ EZER-ERYURT DILHAN PROF \\ DPT OF PHYSICS \\ MIODLE EAST TECH UNIV \\ O6531 ANKARA \\ TURKEY \\ TEL: $41 \quad 237100 * 3255$ \\ ILF: \\ TL.X: 42761 ODTK TR \\ EML: \\ CaM: 35
}

\section{FABREGAT JUAN QR}

DPT MATEMATICA Y ASTRON

UNIVERSIDAD OE VALENCIA

BURJASOT

E 46100 VALENCIA

SPAIN

TEL: $6386 \mathbf{4 5 7 3}$

TLF: 63864735

TLX:

EML: 16444 : : FABREGAT

COM: 25

\section{FADEYEV YURI A}

INST OF ASTRONOMY

ACADEMY OF SCIENCES

PYATNITSKAYA UL 48

109017 MOSCOW

RUSSIA

TEL: 231-54-61

TLF:

TLX: 412623 SESTP SU

EML:

COM: 27,35

FAHY EOHARD F PROF
DPT OF PHYSICS
UNIVERSITY COLLEGE CORK
CORK
IRELAND
TEL: $1212 \cdot 6871$
TLF:
TLX: 26050
EML:
COM:

$\begin{array}{ll}\text { EVREN SERDAR DR } & \text { EWEN HAROLD I QR } \\ \text { FACULTY OF SCIENCE } & \text { HILLCREST DRIVE } 60 \\ \text { EGE UNIVERSITY } & \text { BEAVER } \\ \text { BOX } 21 & \text { SOUTH OEERFIELO MA 01373 } \\ 35100 \text { BORNOVA IZMIR } & \text { USA } \\ \text { TURKEY } & \text { TEL: } \\ \text { TEL: } 51.180110 * 2322 & \text { TLF: } \\ \text { TLF: } & \text { ILX: } \\ \text { TLX: } & \text { EML: } \\ \text { EML: EFEASTOIOTREARN.BITNET } & \text { COM: }\end{array}$

FABER SANDRA M PROF

LICK OBSERVATORY

UNIVERSITY OF CALIFORNIA

SANTA CRUZ CA 95064

USA

TEL: $408 \quad 4292944$

TLF:

TLX:

EML:

COM: $28,33,47$

EML:

COM: 28

\section{FABRICANT DANIEL $G$}

CENTER FOR ASTROPHYSICS

HCO/SAO

60 GARDEN ST

CAMBRIDGE MA 02138

USA

TEL: 6174957398

TLF :

tLX: 921428 SATELLITE CAM

EML:

COM: $09,28,44$

FAELTHAMMAR CARL GUNNE PR
OPT OF PLASMA PHYSICS
ROYAL INST OF TECHNOLOGY
S 10044 STOCKHOLM 70
SWEOEN
TEL: 86877685
TLF:
TLX: 10389 KTHB
EML:
COM:

\section{FAIRALL ANTHONY P PROF}

DPT OF ASTRONOMY

UNIVERSITY OF CAPE TOWN

RONDEBOSCH 7700

SOUTH AFRICA

TEL: $21698531 \times 629$

TLF:

TLX: 5721439

EML:

COM: $28,30 \mathrm{C}, 46$

FABRICIUS CLAUS V

COPENHAGEN UNIVERSITY OBS

BRORFELDEVEJ 23

OK 4340 TOLLOSE

DENMARK

TEL: $53 \quad 488195$

TLF: 58.488755

TLX: 44155 DANAST DK

EML:

COM: $0 B$

FAHLMAN GREGORY G DR

OPT GEOPHYS \& ASTRONOMY

UNAIV OF BRITISH COLUMBIA

2075 WESBROOK PL

VANCOUVER BC VGT IW5

CANADA

TEL: 6042284891

TLF: 6042286047

TLX:

EML:

COM:

\section{FALCHI AMBRETTA}

OSS ASTROFISICO

DI ARCETRI

LARGO E FERMI 5

I 50125 FIRENZE

ITALY

IEL: $55 \quad 275 \quad 2236$

TLF :

TLX: 572268 ARCETR I

EML:
EWING MARTIN S

YALE UNIVERSITY SECF

10 HILLHOUSE AVE $2330 \mathrm{~L}$

NEW HAVEN CT 06520

USA

TEL: 2034324342

TLF: 2034322797

ILX: 9102508365 YALE

EML: EWINGGYLEVM. YCE, YALE. EDU

COM: 40

FABIAN ANDREW $C$ OR

INSTITUTE OF ASTRONOMY

THE OBSERVATORIES

MADINGLEY RD

CAMBRIDGE CB3 DHA

UK

TEL:

TLF:

TLX:

EML:

COM: 48

FACONOI SILVIA ROSA DR IST OI RADIOASTRONOMIA CNR

VIA IRNERIO 46

I. 40126 BOLOGNA

ITALY

TEL: $5128 \quad 7835$

TLF: $5124 \quad 3130$

TLX: $520634^{\circ}$ INFN. BO.

EML: SFACONDI ASTBOI.INFN. IT

COM: 40

FAHR HANS JOERG PROF OR INSTI IUT FUR ASTROPHYSIK DER UNIVERSITAET BONN AUF DEM HUEGEL 71 D 5300 BONN 1 GERMANY

TEL: 228733677

TLF:

TLX: 886440

EML:

COM: 49

COM: 10
FALCIANI ROBERTO OR OPT OI ASTRONOMIA UNIVERSITA DI FIRENZE LARGO E FERMI 5

I 50125 FIRENZE

ITALY

TLF: 55220039

TLX: 572268 ARCETR

EML:

COM: 10,12
TEL: 5527521 


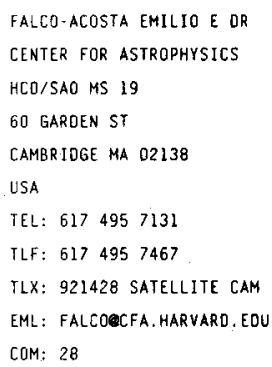

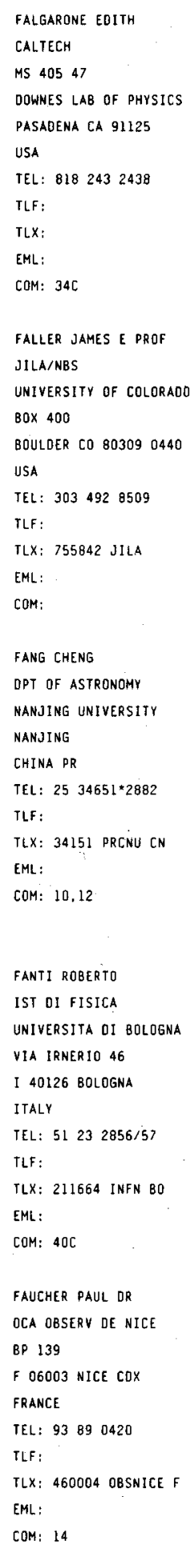

FALK SYONEY W JR OR ASTRONOMY DPT UNIVERSITY OF TEXAS RLM 15308 AUSTIN TX 787121083 USA

TEL:

TLF :

ILX:

EML:

COM: 34,47

FALLON FREDERICK W OR NGS

N/CG 114 NOAA 6010 EXECUTIVE BLVD ROCKVILLE MD 20852 USA TEL: 3014438424 TLF:

FLX:

EML:

COM: 24,31

FANG LI-ZHI

DPT OF PHYSICS UNIVERSITY OF ARIZONA TUCSON AZ 85721

USA

TEL:

TLF:

TLX:

EML:

COM: 47,48

FARAGGIANA ROSANNA PROF OAT BOX SUCC TRIESTE 5 VIA -TIEPOLO II I 34131 TRIESTE ITALY. TEL: $40 \quad 793921$ TLF: TLX: 461137 OAT I EML: COM: $29,36,44,45$

FAULKNER DONALD J DR MOUNT STROMLO \& SIOING SPRING OBSERVATORIES PRIVATE BAG WODEN PO ACT 2606 AUSTRALIA TEL: $62 \quad 88 \quad 1111$. TLF : TLX: 62270 EML: COM: 34,35
FALL S MICHAEL DR

STSCI

HOMEWOOD CAMPUS

3700 SAN MARTIN OR

BALTIMORE MD 21218

USA

TEL:

ILF:

FLX:

EML:

COM: $28,33,37,47$

FALOMO RENATO DR OSS ASTRONOMICO OI PADOVA

VIC DELL OSSERVATORIO 5 I 35122 PADOVA

ITALY

TEL: $49 \quad 56 \quad 1499$

FLF:

TLX: 432071 ASTROS I

EML: ASTRPD : : FALOMO COM:

FANSELOW JOHN LYMAN

JPL

MS 264748

4800 OAX GROVE DR PASADENA CA 91109 USA

TEL: 2133546323

TLF:

TLX: 675429

EML:

COM: 24

FARINELLA PAOLO OR IST DI MATEMATICA UNIVERSITA DI PISA VIA BUONARROTI 2

I 55127 PISA ITALY TEL: $50 \quad 599524$ TLF: $50 \quad 599524$ TLX: 500371 CNUCE I EML: TWIN2 ICNUCEVM.BITNE.T COM: $07,15,16$

FAULKNER JOHN PROF LICK. OBSERVATORY UNIVERSITY OF CALIFIRNIA SANTA CRUZZ CA 95064 USA TEL: $408 \quad 4292815$ TLF:

TLX:

EML:

COM: 35,42 


\author{
FAUNDEZ-ABANS M DR \\ DPT OE FISICA \\ UNIVERSIOA OE SANTIAGO \\ CASILLA 307.2 \\ SANIIAGO \\ CHILE \\ TEL: \\ TLF: \\ ILX: \\ EML: \\ COM:
}

FAZIO GIDVANNI G OR

CENTER FOR ASTROPHYSICS

HCO/SAO

60 GAROEN ST

CAMBRIOGE MA 02138

USA

TEL: 6174957458

TLF: 6174957490

TLX: 921428 SATELLITE. CAM

EML: FAZIDECFA,BITNET

COM: $44 \vee P, 48,51$

FEDERICI LUCIANA

DPT OI ASTRONOMIA

UNIVERSITA OI BOLOGNA

CP 596

I 40100 BOLOGNA

ITALY

TEL: 51222956

TLF:

TLX: 211664 INFN BO I

EML:

COM: 14

FEHRENBACH CHARLES PROF

LES MAENANARELLES

LOURMARIN

F 84160 CADENET

FRANCE

TEL: 90680028

TLF:

TLX:

EML:

COM: $09,30,33,45$

FEISSEL MARTINE OR

observatoire dE PARIS

61 AVE OBSERVATOIRE

F 75014 PARIS

FRANCE

TEL: $14051 \cdot 2226$

TLF: 140512232

TLX: 270776 OBS F

EML: IAPOBS: : FEISSEL/FEISSELCRIAPS TLX: 825860

COM: $08,19 C 31$
COM: $28,33,34$

FAUROBERT-SCHOLL M DR

OCA OBSERV DE NICE

BP 139

F 06003 NICE CDX

FRANCE

TEL: 92003011

TLF: 92003033

TLX: 460004 OBSNICE F

EML: FALROBEFRONI51. BITNET

COM: 36

FEAST MICHAEL W PROF
SAAO
BOX 9
OBSERVATORY 7935
SOUTH AFRICA
IEL: 2721470025
TLF: 2721473639
TLX: 520309
EML:
COM: $27,28,29,33,37,45$

FEDERMAN STEVEN ROBERT

OPI PHYSICS ASTRONOMY

UNIVERSITY OF TOLEOO

2801 W BANCROFT ST

TOLEDO OH. 43606

USA

TEL: 4195372652

TLF:

TLX:

EML:

COM: 14.34

FEIBELMAN WALTER A OR

NASA/GSFE

CODE 685

GREENBELT MD 20771

USA

TEL: 3012865272

TLF:

TLX:

EML:

COM: $10,27,34$

FEITZINGER JOHANNES PROF

ASTRONOMISCHES INSTITUT

RUHR UNIVERSITAET BOCHUM

POSTFACH 102148

D 4630 BOCHUM 1

\section{GERMANY}

TEL: 2347003450

TLF:
FAWELL DEREK R DR

UNIVERSITY OF LONOON OBS

MILL HILL PARK

LONDON NW7 2OS

ux

TEL:

TLF:

TLX:

EML:

COM:

FEAUTRIER NICOLE OR

obserVATOIRE DE PARIS

SECTION DE MEUDON

F 92195 MEUDON PPL CDX

FRANCE

TEL: 145077552

TLF:

TLX: 201571

EML:

COM: 14

FEDOROVA RIMMA T OR

NIKOLAEY OBSERVATORY

UKRAINIAN ACAD OF SCIENCE

327000 NIKOLAEV

UKRAINE

TEL: 375714

ILF:

TLX:

EML:

COM: 08

FEIGELSON ERIC D DR

DPT OF ASTRONOMY

PENHSYLVANIA STATE UnIV

525 DAVEY LAB

UNIVERSITY PARK PA 16802

USA

TEL: 8148650162

TLF:

TLX: 842510

EML: INTERNET: EDF ASTRO, PSU, EOU

COM: 40

FEIX GERHARD DR

RUHR UNIVERSITAET: BOCHUM

DEPT XII

POSTFACH 102148

D 4630 BOCHUM I

GERMANY

TEL: 2347002051

TLF:

ILX: 0825860

EML:

COM: 40
FAY THEODORE O DR TELEOYNE BROWN ENG CUMMINGS RES PARK

MS 19

HUNTSVILLE AL 35807

USA

TEL:

TLF:

TLX:

EML:

COM:

FECHTIG HUGO DR

SANSERWEG 3

D 6906 LEIMEN

GERMANY

TEL:

TLF :

TLX:

EML:

COM: $15,21,22$

FEGAN DAVID J OR

DPT OF PHYSICS

UNIVERSITY COLLEGE

BELFIELD

DUBLIN 4

IRELAND

TEL: 1693244

TLF:

TLX: 32693 UCD EI

EML:

COM:

FEINSTEIN ALEJANDRO DR OBSERVATORIO ASTRONOMILO paseo oel bosque 1900 LA PLATA (BS AS) ARGENTINA

TEL: $2 \downarrow 21 \quad 6357$

TLF: $21 \quad 21 \quad 1761$

TLX: 31216 CESLA AR

EML: AFEINSTEINAFCAGLP. EOU.AR

COM: $25,37 \mathrm{VP}$

FEJES ISTVAN OR

FOMI SATELLITE

GEOOETIC OBSERVATORY

BOX 546

H 1373 BUDAPEST

HUNGARY

TEL:

TL'F:

TLX:

EML:

COM: 51 
FEKEL FRANCIS C

DYER OBSERVATORY

VANDERBILT UNIVERSITY

NASHVILLE TN 37235

USA

TEL: $615322 \quad 2804$

TLF :

TLX:

EML:

COM: $26 \mathrm{C}, 30,42$

FELDMAN URI DR

NAVAL RESEARCH LABORATORY

COOE. 4174

4555 OVERLOOX AVE SW

WASHINGTON OC 203755000

USA

TEL:

TLF:

TLX:

EME:

COM

\section{FENG HESHENG}

YUNNAN OBSERVATORY

CAS

BOX 110

KUNMING 72946 YUNAAN

CHINA PR

TEL: 8712035

TLF:

TLX:

EML:

COM:

FERETTI LUIGINA

IST OI RADIOASTRONOMIA

CAR

VIA IRNERIO 46

I 40126 BOLOGNA

ITALY

TEL: 51.232856

TLF:

TLX: 520634 INFNBO I

EML:

COM: 40

FERNANOEZ JEAN-CLAUDE DR OCA OBSERY DE NICE

BP 139

F 06003 NICE COX

FRANCE

TEL: 93890420

TLF:

TLX: 460004 OBSNICE $F$

EML:

COM: 15
fELdMAN PAUL A DR

HERZBERG INST ASTROPHYS

NTL RESEARCH COUNCIL

100 SUSSEX OR

OTTAWA ON KIA DRG

\section{CANADA}

TEL: 6139936060

TLF: 6139526602

TLX: 0533715

EML:

COM: 40,51

\section{FELENBOK PAUL DR}

OBSERVATOIRE DE PARIS

SECTION DE MEUDON

F 92195 MEUDON PPL CDX

FRANCE

TEL: $145 \quad 07 \quad 7523$

TLF :

TLX: 201571

EML :

COM: 09.29

FENG KE-JIA

DPT OF ASTRONOMY

BEIJING NORMAL UNIVERSITY

BEIJING 100875

CHINA PR

TEL: I $653531 * 6967$

TLF :

TLX:

EML:

COM: 10,46

FERLANO GARY JOSEPH

OPT OF ASTRONOMY

OHIO STATE UHIVERSITY

174 W 18TH AVE

COLUMBUS OH 432101106

USA

TEL: $\begin{array}{lll}614 & 422 \quad 1773\end{array}$

TLF:

TLX: 8104821715

EML:

COM: 27,28

FERNANOEZ JULIO A DR
OPT DE ASTRONOMIA
FACUT HUMANIDADES Y CIENC
TRISTAN NARVAJA 1674
MONTEVIDEO
URUGUAY.
TEL: $598 \quad 24911044$
TLF:
TLX: UDELAR UY 26692
EML:
COM: $15,20,46$

FELOMAN PAUL DONALD DR DPT PHYSICS \& ASTRONOMY JOHNS HOPKINS UNIVERSITY CHARLES \& $34 \mathrm{TH}$ ST BALTIMORE MO 21218 USA

TEL: 3013387339

TLF:

TLX: 710-234-1090

EML:

COM: $15,21,44$.

FELLI MARCELLO DR

OSS ASTROFISICO

DI ARCETRI

LARGO E FERMI 5

I 50125 FIRENZE

ITALY

TEL: 552752240

TLF :

TtX: 572268

EML:

COM: 34,40

FENKART ROLF P PROF DR

ASTRONOMISCHES INSTITUT UNIVERSITAET BASEL

VENUSSTRASSE 7

CH 4102 BINNINGEN

SWITZERLAND

TEL: 61227711

TLF:

TLX:

EML:

COM: 33

FERLET ROGER OR INSTITUT DAASTROPHYSIQUE 98BIS BO ARAGO

F 75014 PARIS

FRANCE

TEL: 143201425

ILF: 143298673

TLX:

EML: EARN: FERLETORRIAP5I

[DM: 34

FERNANDEZ SILVIA M OR OBSERVATORIO ASTRONOMICO

DE CORDOBA

LAPRIDA 854

5OOD CORDOBA

ARGENTINA .

TEL: 5140613

TLF :

ILX: 51822 BUCOR

EML:

EOM: 07
FELOMAN UR I

NAVAL RESEARCH LABORATORY

HOLBURT C.TR FOR SPACES RE 4555 OVERLOOK AVE SW

WASHINGTON OC 203755000

USA

TEL: 2027673286

TLF :

TLX:

EML:

CDM: 12

FELTEN JAMES E DR

NASA/GSFC

CODE 685

GREENBELT MO 20771

USA

TEL: 3015521526

TLF :

TLX:

EML:

COM: $34,40,47.48$

FENTON $K B$ B

DPT OF PHYSICS

UNIVERSITY OF TASMANIA

GPO BOX 252C

HOBART TAS 7001

AUSTRALIA

JEL: 2202411

TLF:

TLX: 58150 AA

EML:

COM: 48

FERLUGA STENO OR

OPT OI ASTRONOMIA

UNIVERSITA OI TRIESTE

VIA TIEPOLO 11

I 34131 TRIESTE

ITALY

TEL: $40 \quad 76 \quad 3912$

TLF: $40 \quad 309418$

TLX: 461137 OAT I

EML: ASTRONET : ASTRTS : : FERLUGA

COM: 42

FERNANDEZ - FIGUEROA M J DR

DPT DE ASTROFISICA

FAC DE FISICA

UNIVERSIDAD COMPLUTENSE

E 28040 MADRID

SPAIN

TEL: 14495316

TLF:

TLX: 47273 FF UC

EML:

COM: 29,46 


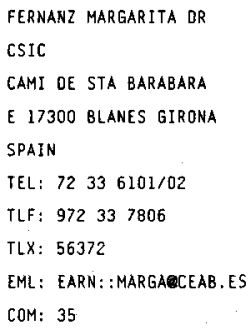

FERNIE J DONALD PROF DAVID DUNLAP OBSERVATORY UNIVERSITY OF TORONTO

BoX 360

RICHMOND HILL ON LAC 4 YG CANADA

TEL: 4168849562

TLF: 4169783921

TLX: 06986756 TELEXPERTS

EML: ferniecentaur. astro.utoronto. EM

COM: 25.27 .41

FERRARI D'OCCHIEPPO $*$ OR OESTERREICHISCHE AKADEMIE DER WISSENSCHAFTEN

DR IGNAZ-SEIPEL-PLATZ 2

A 1010 WIEN

AUSTRIA

TEL: 522281991

TLF :

TLX: $01-12628$

EML:

COM: 41,42

FERRER OSVALDO EDUARDO OR UNIV NACIONAL OE LA PLATA FCAG

1900 LA PLATA (BS ASS) ARGENTINA

TEL: 21217308

TLF:

$T L X$ :

EML:

COM: 25,42

FESTOU MICHEL C DR

OBS MIOI PYRENEES

14 AVE E BELIN

F 3L400 TOULOUSE COX

FRANCE

TEL: 61252101

TLF :

TLX: 530776

EML:

COM: 15

FICH MICHEL OR

DPT OF PHYSICS UNIVERSITY OF WATERLOO WATERLOO ON N2L 361 CANADA

TEL: $519 \quad 865 \quad 1572$

TLF: $519746 \quad 8115$

$T L X: 06955259$

EML: BITNET:FICHENATSCI COM
FERRANOIZ JOSE MANUEL DR

ETS

INGENIEROS INDUSTRIALES

PASEO DEL CAUCE S/N

E 47011 VALLADOLID

SPAIN

TEL: : $83 \quad 30 \quad 4700$

TLF: 83392026

TLX:

EML:

FERRARI TONIOLO MARCO

IAS

CNR

[P 67

I 00044 FRASCATI

ITALY

TEL: 69425651

TLF: 69416847

TLX: 610261 CNR-FRA I

EML:

COM: 44 .

FERRERI WALTER

OSS ASTRONOMICO DI TORINO

ST OSSERVATORIO 20

I 10025 PINO TORINESE

ITALY

TEL: $11 \quad 842040$

TLF :

TLX: 213236 ASTR I

EML:

COM: 20

FEYNMAN JOAN DR

JPL

MS 144218

\$BOD OAK GROVE DR PASADENA CA 91109 USA

TEL: $818 \quad 354 \quad 3454$

TLF:

TLX: 675429

EML:

COM: 49

FICHTEL CARL E OR

NASA/GSFC

COOE 560

GREENBELT MO 20771

USA

TEL: $301286 \quad 6281$

TLF :

TLX: 89675

EML:

COM: 44,48
FERRANDO PHILIPPE DR

\section{CEA CEN}

DPHG/SAP

BP 2

F 91191 GIF/YVETTE COX

FRANCE

TEL: $169 \quad 08 \quad 2020$

TLF :

TLX: 604860

EML: BITHET:FERRANDDO32779

COM:

FERRAZ-MELLO S PROF OR

IAG

UNIVERSIOADE OE SAD PAULO

CP 30627

01065 SAO PAULO SP

BRAZIL

TEL: 11577 B599*27

TLF : II 2763848

TLX: 1156735 IAGM BR

EML: SYLVIOOIAG.USP.ANSP.BR

COM: OTVP, 20,46

FERRIN IGKACIO

UNIVERSIOAO DE LOS ANDES

FACULTAD DE CIENCIAS

DPT DE FISICA

MERIOA 5101 A

VENEZUELA

TEL: $74 \quad 639930$

TLF :

TLX:

EML:

COM: 15

\section{FIALA ALAN D OR}

US NAVAL OBSERVATORY

NAUTICAL ALMANAC OFFICE

34 \& MASSACHUSETTS AVE NW WASHINGTON DC 203925100 USA

TEL: $202 \quad 653 \quad 1274$

TLF:

$T L X: 710 \cdot 822 \cdot 1970$

EML:

COM: $04,07,12$

FIEDLER RALPH L DR NAVAL RESEARCH LABORATORY CODE 4210

4555 OVERLOOK AVE SW WASHINGTON OC 203755000 USA

TEL: 2027670644

TLF :

$T L X$;

EML: FIEDLERERIRA.NRL. NAVY.MIL COM: 
FIELL DAVIO

SCHOOL OF CHEMISTRY

CANTOCKS CLOSE

BRISTOL BSB ITS

UK

TEL: $272 \quad 24 \quad 161$ *505

TLF:

TLX: 444174 BUPHYS

EML:

COM: 34

FIERRO JULIETA

INSTITUTO OE ASTRONOMIA

UNAM

APGO POSTAL 70-264

04510 MEXICO OF

MEXICO

TEL:

TLF:

TLX:

EML:

COM: $34,46 \mathrm{C}$

filloY emilio manUel E E

IAR

CC 5

1894 VILLA ELISA (BS AS)

ARGENIINA

TEL: 2143793

TLX:

EML:

TLF:

COM:

FINN $G$ OR
USA
TEL:
TLF:
EML:
TLX:
COM: 36
FIRNEIS MARIA G OR
INSTITUT FUER ASTRONOMIE
TUERKENSCHANZSTR 17
A 1180 WIEN
AUSTRIA
TEL: 1345 3600
TLF:
ILX:
EML:
COM: $24,41 C, 51$

FIELO GEORGE B PROF CENTER FOR ASTROPHYSICS HCO/SAO

60 GARDEN ST

CAMBRIDGE MA O2L138

USA

IEL: $617495472 \mathrm{~L}$

ILF:

ILX: 921428 SATELLITE CAM

EML:

COM: $28,34,40,47,48,49,51$

FIGUERAS FRANCESCA OR

DPT ASTRONOMIA

UNIVERSIOAD DE BARCELONA

AVO DIAGONAL 64?

E 08028 BARCELONA

SPAIN

TEL: 33307311

TLF:

TLX:

EML: DJFAFFSOEEBOUBOLL

COM: 33

FINOLAY JOHN W OR

NRAD

EDGEMONT RO

CHARLOTTESVILLE VA $2290 \mathrm{~L}$

USA

TEL:

TLX: 910-997-0174

EML:

TLF:

COM: 40

FINZI ARRIGO QR

OPT OF MATMEMATICS

III

TECHNION CITY

HAIFA 32000

ISAAEL

TEL:

TLF:

TLK: 46406 TECON IT

EML:

COM:

FIROR JOHN W DR

HIGH ALTITUOE OBSERVATORY

NCAR

BOX 3000

BOULOER CO 803073000

USA

TEL: $303 \quad 497 \quad 600$

TLF:

$T L X: 45694$

EML:

COM:
FIELDER GILBERT DR

WILLOW TREE

ELDROTH

AUSTWICK

LANCASTER LA2 BAH

UK

TEL:

TLF:

YLX:

EML:

COM: 16

FILIPOV LATCHEZAR

CTR LAB FOR SPACE RES

BULGARIAN ACAD SCIENCES

MOSKOVA ST 6

BG 1000 SOFIA

BULGAR IA

TEL: 870978 '

TLF:

TLX: 23351 CLSR BE

EML:

COM:

\section{FINK UWE DR}

LUNAR \& PLANETARY LAB

UNIVERSITY OF ARIZONA

TUCSON AZ 85721

USA

TEL: 6026212736

TLF:

TLX: 9109521143

EML:

COM: 14,16

FIRMANI CLAUDIO A PRDF

INSTITUTO DE ASTRONOMIA

UNAM

APDO POSTAL 70-264

04510 MEXICO DF

MEXICO

TEL: 905-548-3712

TLF:

TLX: 1760155 CICME

EML:

COM: 42

FISCHEL OAYIO OR

EARTH OBSERVATION

SATELLITE COMPANY

4300 FORBES BLVB

LANHAM MD 20706

USA

TEL: 3015520500

TLF:

TLX: 277685

EML:

COM:
FIENBERG RICHARO T DR

SKY \& TELESCOPE

49 BAY STATE ROAD

CAMBRIDGE MA 0213B

USA

TEL: 6179647360

TLF:

TLX:

EML: SPAN: CFA: : FIENBERG

COM: 46

FILIPPENKO ALEXEI V DR

ASTRONOMY OPT

UNIVERSITY OF CALIFORNIA

6BI CAMPBELL HALL

BERKELEY CA 94720

USA

TEL: $415 \quad 642 \quad 1813$

TLF:

TLX: 820181

EML: BITNET: ALEXERYAST

COM: $06,28,47$

FINKELSTEIN ANDREJ M OR

INST OF APPLIED ASTRONOMY ACADEMY OF SCIENCES

ZDANOVSKAYA UL 8

197042 ST PETERSBURG

RUSSIA

TEL:

TLF:

TLX:

EML:

COM: 40

FIRNEIS FRIEDRICH J DR INST INFO PROC/OEAW

SONNENFELSGASSE $19 / 2$

A 1010 WIEN

AUSTRIA

TEL:

TLF:

TLX:

EML:

COM: 24,51

FISCHER JACQUELINE

NAVAL RESEARCH LABORATORY

COOE $4138 \mathrm{~F}$

4555 OVERLOOK AVE SW

WASHINGTON OC 203755000

USA

TEL: 2027673058

TLF:

ILX:

EML:

COM: 34 
FISCHER. STANISLAV OR ASTRONOMICAL INSTITUTE CZECH ACADEMY SCIENCES BUDECSKA 6

CS 12023 PRAHA 2

CZECHOSLOVYKIA

TEL: 2252438

TLF:

TLX: 122486 ASTRC

EML:

COM:

FISHER RICHARD R OR

HIGH ALTITUDE OBSERVATORY

NCAR

BOX 3000

BOULDER CO 803073000

USA

TEL: 3034945151

TLF:

ILX:

EML:

COM:

FITTON BRIAN QR

ESA/ESTEC

ASTROPHYSICS OIV

BOK 299

NL 2200 AG NOOROWIJK

NETHERLANDS

TEL: $25 \quad 24 \quad 4635$

TLF:

ILX:

EML:

COM: 44

FLANNERY BRIAN PAUL OR

EXXON RES \& ENGINEERING

RQUTE 22 EAST

ANNANDALE NJ 08801

USA

TEL: 2017302540

ILF:

ILX: 136140 EXKONRES

EML:

COM: $34,35,42$

\section{FLETT \#LISTAIR M}

OPT OF PHYSICS

UNIVERSITY OF ABERDEEN

FRASER NOBLE BLOG

ABEROEEN ABg $2 U E$

UK

TEL: $224 \quad 40241$

TLF:

TLX: 73458 UNIABN $G$

EML:

COM: 40
FISHER GEORGE HEWITT OR

INSTITUTE FOR ASTRONOMY

UNIVERSITY OF HAWAII

2680 WOODLAWN OR

HONOLULU HI 96822

USA

TEL: 8089566665

TLF: $808 \quad 9882790$

TLX:

EML: FISHERQUHIFA, IFA. HAWAII EOU

COM: 10.12

FISHKOVA LUISA M PROF

ABASTUMANI ASTROPHYSICAL

OBSERVATORY

georgian aCAD .0F SCIENCES

383762 ABASTUMANI

GEORGIA

IEL:

TLF:

TLX:

EML:

COM: 21

FITZGERALO M PIM PROF

DPT OF PHYSICS

UNIVERSITY OF WATERLOO

WATERLOO ON N2L 361

CANADA

IEL: $519885 \quad 1572$

TLF: 5197468115

TLX: 06955259

EML:

COM: 33,37

FLECK ROBERT CHARLES OR OPT OF MATH \& PHYSICAL SC EMBRY-RIDOLE AERON UNIVER DAYTONA FL 32114

USA

TEL: $904 \quad 2266612$

TLF: 9042266713

ILX:

EML:

COM: 34.46

FLIEGEL HENRY F

3730 EL MORENO AVE

BOX 8682 ,

LA CRESCENTA CA 91214

USA

TEL: 2136487452

TLF: 2133365076

TLX:

EML:

COM: 19,3 !VP
FISHER J RICHARD

NOAD

BOX 2

GREEN BANK WV 24944

USA

TEL: 3044562011

TLF:

TLX: 7109381530

EML:

COM:

fishman geralo J

NASA/MSFC

CODE ES 62

ASTROPHYSICS BRANCH

HUNTSVILLE AL 35012

USA

TEL: 2054530117

TLF:

TLX:

EML:

COM: 44

FITZPATRICK EOWARD L DR

PRINCETON UNIVERSITY OBS

PEYTON HALL

PRINCETON NJ 08544

USA

TEL: 6094523702

TLF: 6092437333

$T \perp X:$

EML: FITZASTROVAX. PRINCETON, EDU

COM: $29,36,45$

\section{FLEISCHER ROBERT QR}

18712 KEEDYSVILLE RD

KEEDYSVILLE MO 217561112

USA

TEL:

TLF :

TLX:

EML:

COM: 40

FLIN PIOTR

ASTRONOMICAL OBSERVATORY

JAGIELLONIAN UNIVERSITY

BL ORLA 171

PL 30244 KRAKOW

POLANO

TEL:

TLF :

TLX: 0322297 UJ PL

EML:

COM: 28
FISHER PHILIP C

RUFFNER ASSOCIATES

BOX 7070

MENLO PARK CA 94026

USA

TEL:

TLF :

TLX:

EML:

COM: $44,48,51$

FITCH WALTER S OR

BOX 100

ORACLE AZ 85623

USA

TEL: 6028962911

TLF :

TLX: 467175

EML:

[OM: 27

FIX JOHN D OR

OPT PHYSICS \& ASIRONOMY

UNIVERSITY OF IOWA

IOWA CITY IA 52240

USA

TEL: 3193537064

TLF:

TLX: $910-525-1398$

EML:

COM:

FLETCHER I MURRAY

HERZBERG INST ASTROPHYS

DOMINION ASTROPHYS OBS

5071 W SAANICH RD

VICTORIA BC VBX $4 M 6$

CANADA

TEL: 6043883905

TLF: 6043630045

TLX: $049 \cdot 7295$

EML:

COM: $09,26,30$

FLOQUET MICHELE DR

OBSERVATOIRE DE PARIS

SECTION OE MEUDON

DEPEG

F 92195 MEUOON PPL COX

FRANCE

TEL: $1 \quad 4507 \quad 7851$

TLF :

TLX:

EML:

COH: 29 


\section{FLORENTIN-NIELSEN RALPH COPENHAGEN UNIVERSITY OBS BRORFELDEVEJ 23 \\ DK 4340 TOLLOSE DENMARK \\ TEL: 53 4B B195 \\ TLF: $58 \quad 48 \quad 8755$ \\ ILX: 44155 DANAST \\ EML: \\ COM:}

FLOWER DAVIO R OR
DPT OF PHYSICS
UNIVERSITY OF OURHAM
SOUTH RD
OURHAM OHI $3 L E$
UK
TEL: 913742145
TLF: 913743749
TLX: 537351
EML: DAV10. FLOWEREOURHAM. AC. UK
COM:

FOCARDI PAOLA DR

OSS ASTRONOMICO

UNIVERSITA OI BOLOGNA

CP 596

I 40100 BOLOGNA

ITALY

TEL: 51259301

TLF:

TLX: 520634 INFNBO I

EML: SPAN:ASTBO3: : PAOLA

COM: 47

\section{FOGARTY WILLIAM G OR \\ IBM \\ NCMB \\ - 411 east hisconsin ave MILWAUKEE WI 53202 \\ USA \\ TEL: \\ TLF: \\ TLX: \\ EML: \\ COM:}

FOMALONT EOWARO B OR
NRAO
BOX O
SOCORRO NM 878010387
USA
IEL:
TLX: $910-988-1710$
EML:
TLF:
COM: 40

FOMINOV ALEXANOR M DR

INST OF THEORET ASTROMOMY

ACADEMY OF SCIENCES

N KUTUZOVA 10

191187 ST PETERSBURG

RUSSIA

TEL: 278-8B-98

ILF:

TLY: 121578 ITA SU

EML:

COM: 04

\author{
FOING BERNARO H OR \\ IAS \\ BP 10 \\ F 91371 VERRIERES BUISSON \\ FRANCE \\ TEL: 164474328 \\ TLF: \\ TLX: 600252 \\ EML: SPAN : IAPOBS: : FOING \\ COM: $10.29,44$
}

\section{FOMENKO ALEXANOR $F$ OR}

SPECIAL ASTROPHYSICAL OBS

ACADEMY OF SCIENCES

NIZHNIJ ARKHYZ

357147 STAVROPOLSKIJ

RUSSIA

TEL:

TLF:

TLX:

EML:

COM: 09

\section{FONG CHU-GANG}

SHANGHAI OBSERYATORY

CAS

80 NANDAN RO
SHANGHA1
CHINA PR
TEL: 21386191
TLF:
TLK: 33164 SHAO CN
EML:
COM: 07,19

FLORKOWSKI OAVIO R DR

US NAVAL OBSERVATORY

34 \& MASSACHUSETTS. AVE NW

WASHINGTON DC 203925100

USA

TEL: $202 \quad 653 \quad 1509$

TLF: 202.6530587

TLX:

EML: ORFPHOBOS.USNO.NAVY.MIL

COM: 40

FODERA SERIIO GIORGIA OR

OSS ASTRONOMICO

UNIUERSITA II PALERNO

PALAZZO DEI NORMANNI

I 90134 PALERMO

ITALY

TEL: 916570451

TLF: $9148 \quad 8900$

ILX: 910402 ASTROP I

EML: ASTROPA PACUC. BItNet

COM: 41

FOLEY ANTHONY DR

NFRA

BOX 2.

NL 7990 AA OWINGELOO

NETHERLANOS

TEL: 52197244

TLF : 52197332

ILX: 42043 SRZM NL

EML: FOLREDQNFRA.NL

COM: 40

FOMICHEV VALERI V DR

IZMIRAN

ACADEMY OF SCIENCES

142092 TROITSK

RUSSIA

TEL:

TLF:

TLX:

EML:

COM: 12

\section{FONG RICHARD}

DPT OF PHYSICS

UNIVERSITY OF OURHAM

SOUTH RO

DURHAM OHI 3LE

UK

TEL: 9138564971

ILF:

ILX: 537351

EML:

COM: 47
FLORSCH ALPHONSE OR OBS DE STRASBOURG

11 RUE UNIVERSITE

F 67000 STRASBOURG

FRANCE

TEL: 88354300

ILF: 88250160

TLX: 890506 STAROBS $F$

EML:

COM: $28,30,38$

FOFI MASSIMO OR

OAR

VIA DEL PARCO MELLINI 84

I 00136 ROMA

ITALY

TEL: 6347056

TLF:

TLX: 626326 OAROMA I

EML: 40061: : FOFI

COM: 12

FOLTZ CRAIG B

MULT MIRROR TELESCOPE OBS

UNIVERSITY OF ARIZONA

TUCSON AZ 85721

USA

TEL: $602621 \quad 1269$

TLF:

$T\lfloor X: 467175$

EML:

COM: 28,30

FOMIN VALERY A DR

PULKOVO OBSERVATORY

ACADEMY OF SCIENCES

10 KUTUZOV QUAY

196140 ST PETERSBURG

RUSSIA

TEL:

ILF:

TiX:

EML:

COM: 08

FONTAINE GILLES OR

DPT DE PHYSIQUE

- UNIVERSITY OF MONTREAL

CP 6128 SUCC A

MONTREAL OC H3C 3JJ

CANADA

TEL: 5143436680

ILF: 5143432071

TLX: 05562425

EML:

COM: 35,36 


FONTENLA UUAN MANUEL OR
NASA/MSFC
COOE ES 52
HUNTSVILLE AL 35812
USA
TEL:
TLF:
TLX:
EML:
COM: 12,36

FORD HOLLAND C RES PROF STSCI

HOMEWOOD CAMPUS

3700 SAN MARTIN DR

BALTIMORE MD 21218

USA

TEL: 3013384803

TLF :

TLX:

EML:

COM: $28,34,47$

FORSTER JAMES RICHARD DR HAT CREEK RADIO OBSERV

RT 2

B0X 500

CASSEL CA 96016

USA

TEL: $916 \quad 3352364$

TLF: 9163353968

TLX:

EML:

COM: 34

\section{FORTI GIUSEPPE DR}

OSS ASTROFISICO

DI ARCETRI

LARGO E FERMI 5

I 50125 FIRENZE

ITALY

TEL: $55 \quad 2752236$

TLF:

TLX: 572268 ARCETR

EML: FORTI ARCETRI.ASTRO.IT

COM: $15,20,22$

\section{FOSSAT ERIC DR}

OCA OBSERV OE NICE

BP 139

F 06003 NIEE CDX

FRANCE

TEL: $93 \quad 89 \quad 0420$

TLF:

TLX: 460004 OBSNICE F

EML:

COM: $10,12,35$
FORBES DOUGLAS DR

SIR WILFRED GRENFELL COLL

MEMORIAL UNIVERSITY

NEWFOUNOLANO

CORNER BROOK NF A2H $6 \mathrm{Pg}$

CANADA

TEL: 7096376295

TLF: 7096376390

TLX: 01644190

EML: OFORBESEKEAN. UCS. MUN. CA

COM: 37

FORD W KENT JR DR

OPT TERRESTR MAGNETISM

CARNEGIE INST WASHINGTON

5241 BROAD BRANCH RD NW

WASHINGTEN OC $20015^{\circ}$

USA

TEL: 2029660863

TLF:

TLX: 440427 MAGN UI

EML:

COM: 09,28

FORT BERNARD P OR

OBS MIOI PYRENEES

14 AVE E BELIN

F 31400 TOULOUSE COX

FRANCE

TEL: 61252101

TLF :

TLX: $530776 \mathrm{~F}$

EML :

COM: 09

FORTINI TERESA DR

VIA F D GUERRAZZI 19

I 00152 ROMA

ITALY.

TEL:

TLF :

EMI:

COM: 10

TLX:

FOUKAL PETER $V$ DR
CAMBRIOGE RESEARCH
INSTRUMENTATIONM INC
21 ERIE ST
CAMBRIDGE MA 02139
USA
TEL: 6174912627
TLF:
TLX:
EML:
COM: 12.

FORBES O E DR

BOX B8120

INOLANAPOLIS IN 46208

USA

TEL:

TLF:

TLX:

EML:

COM: 35

FORMAN WILLIAM RICHARD DR SMITHSONIAN ASTROPHYS OBS 60 GARDEN ST

CAMBRIDGE MA 02138

USA

TEL: 6174957210

TLF :

TLX: 921428

EML:

COM: 47,48

FORT DAVID NORMAN OR

JPL

4BOO OAK GROVE DR

PASADENa CA 91109

ISA

TEL: $818 \quad 354 \quad 9132$

TLF :

TLX:

EML:

COM: 40

FORVEILLE THIERRY DR OBSERVATOIRE OE GRENOBLE CERMO/ASTROPHYSIQUE

BP $53 \mathrm{X}$

F 3B04.1 S MARTIN HERES CO

FRANCE

TEL: $76 \quad 514567$

TLF: $76 \quad 448821$

TLX:

EML: FORVEILLFFRAG5I. BITNET

COM: 40

FOUQUE PASCAL DR

GRUPO ASTROFIS PONTIFICA

UNIVERSIDAD CATOLICA

CASILLA 104

SANTIAGO 22

CHILE

TEL:

TLF:

TEX:

EML: PFOUQUEASTRQUC. PUE.CL

COM: $28,40,47$
FORBES TERRY G DR

SPACE SCIENCE CENTER/EOS

UNIV OF NEW HAMPSHIRE

SC ENGIN RES BLOG

DURHAM NH 03857

USA

TEL: $603 \quad 862 \quad 3872$

TLF :

TLX: 950030 UNH PHYS

EML: BITNETX"T_FORBES@UNH"

COM: $10 \mathrm{C}$

FORREST WILLIAM JOHN

DPT PHYSICS \& ASTRONOMY

UNIVERSITY OF ROCHESTER

ROCHESTER NY 14627

USA

TEL: $716 \quad 275 \quad 4343$

TLF:

TLX:

EML: FORRESTOBORIS.PAS.ROCHESTEI

COM:

FORTE JUAN CARLOS DR

UNIY NACIONAL OE LA PLATA

FCAG

1900 LA PLATA (BS AS)

ARGENTINA

TEL: 21217308 .

TLF :

TLX:

EML:

COM: $25,28,37$

FOSBURY ROBERT A E. OR

ST/ECF

C/O ESO

KARL-SCHWARZSCHILD-STR 2

D 9046 GARCHING MUENCHEN

GERMANY

TEL: 8932006235

TLF:

TLX: 52828222 EO D

EMt:

COM:

FOWLER WILLIAM' A PROF

CALTECH

MS 10638

PASADENA CA 91125

USA

TEL: $B 18 \quad 356 \quad 4272$

TLF :

TLX:

EML:

COM: 35,48 


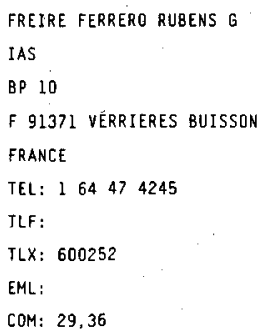

FREITAS MOURAO $R R$ OR

MUSEUM ASTR E CIENCIAS

RUA GL BRUCE 586

SAN CRISTOVAO

20921 RIO DE JANEIRO RJ

BRAZIL

TEL: $21580 \quad 7154 / 7204$

TLF: 215800332

TLX: 22653

EML:

COM: $20,26,41$

FRIBERG PER

JOINT ASTRONOMY CENTER

UKIRT

665 KOMOHANA ST

HILO HI 96720

USA

TEL:

TLF :

TLX:

EML: FRIBERGQJACH, HAWAII, EOU

COM: 40

FRIED JOSEF WILHELM DR

MPI FUER ASTRONOMIE

KOENIGSTUHL

D 6900 HEIDELBERG I

GERMANY

TEL: $62 \quad 215281$

TLX: 461789 MPIA 0

EML:

TLF :

COM: 28

FRIEDMAN HERBERT DR NAVAL RESEARCH LABORATORY CODE 7100

4555 OVERLOOK AVE SW WASHINGTON OC 203755000 USA

TEL:

TLF:

TLX:

EML:

COM: $10.12 .40,44,48$

FRINGANT ANNE-MARIE DR OBSERVATOIRE DE PARIS 61 AVE OBSERVATOIRE

F 75014 PARIS

FRANCE

TEL: 140512248

TLF :

TLX: 270776 OBS F

EML:

COM:
FRENCEH RICHARO G

OPT OF EARTH \& PLANET SCI MIT RM 54422

BOX 165

CAMBRIOGE MA 02139 USA

TEL: $617 \quad 2533392$

TLF :

TLX:

EML:

COM:

\section{FRICKE KLAUS DR} UNIVERSITAETSSTERNWAR TE UNI VERSI TAET GOETT INGEN GEISMARLANDSTR 11 D 3400 GOETTINGEN GERMANY

TEL: 551395051

TLF:

TLX: 96753

EML:

COM: 28

FRIEDEMANN CHRISTIAN DR UNIV STERNWARTE JENA SCHILLERGAESSCHEN - 2

D 6900 JENA GERMANY

TEL: $78 \quad 822 \quad 2637 / 27122$ TLF :

TLX: 05886134

EML:

CON: 34

FRIEDMAN SCOTT OAVID DR DPT PHYSICS \& ASTRONOMY JOHNS HOPKINS UNIVERSITY CHARLES \& 34TH ST BALTIMORE MD 21218 USA

TEL: $301338 \quad 5317$

TLF: 3013385494

ILX:

EML: SCIVAX: : CASA: :SCOTT

COM:

FRISCH HELENE OR
OCA OBSERV DE NICE
BP 139
F 06003 NICE COX
FRANCE
TEL: 93890420
TLF:
TLX: 460004 OBSNICE F
EML:
COM: 36

FRENK CARLOS $S$

DPT OF PHYSICS

UNIVERSITY OF DURHAM

SOUTH RO

DURHAM OHI 3LE

UK

TEL: $913 \quad 8564971$

TLF :

TLX: 537351 DURLIB G

EML:

COM: 47

\section{FRIOLUND MALCOLM OR}

ESA/ESTEC

ASTROPHYSICS DIV

BOX 299

NL 2200 AG NOORDWIJK

NETHERLANOS

TEL: $17 \quad 19 \quad 84768$

TLF: $17 \quad 1984690$

TLX: 39098

EML: ESTCS51::MFRIOLUN

COM: 34

FRIEDJUNG MICHAEL OR INSTITUT D'ASTROPHYSIQUE 988IS BD ARAGD

F 75014 PARIS

FRANCE

TEL: $14320 \quad 1425$

TLF: 143298673

TLX:

EML: FRIEDJUNGEFRIAP5I

COM: $27,29,42$

FRIEL EILEEN D DR HERZBERG INST ASTROPHYS DOMINION ASTROPHYS DBS 5071 W SAANICH RD VICTORIA BC V8X $4 M 6$ CANADA

TEL: 6043880062 TLF: 6043630045 TLX: 0497295

EML:

COM: 29

FRISCH PRISCILLA ASTRONOMY \& ASTROPHYS CTR UNIVERSITY OF CHICAGO . 5640 S ELLIS AVE CHICAGO IL 60637 USA

TEL: $312962 \quad 8211$ TLF :

TLX: $910 \quad 2215617$

EMt: FRISCH@OODJOB.UCHICAGO. EOU COM: 34 


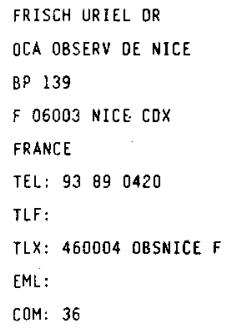

COM:
FRISK URBAN DR

SPACE SCIENCE SECTION

SWEOISH SPACE CORPORATION

$80 \times 4207$

S 17104 SOLNA

SWEOEN

TEL: $86 \quad 276200$

ILF: 8987069

TLX:

EML:

COM: 40,44

FROESCHLE CLALUE OR
OCA OBSERV OE NICE
BP 139
F 06003 NICE COX
FRANCE
TEL: 93890420
TLF:
TLX: 460004 OBSNICE F
EML:
COM: $07 C, 20$

FROST KENNETH J OR
NASA/GSFC
CODE 600.2 BLOG 16
SPACE STATION OFFICE
GREENBELT MD 20771
USA
TEL: 3012868824
TLF:
TLX:
EML:
COM:

FU DELIAN

BEIJING ASTRONOMICAL OBS CAS

\section{W SUBURB}

BEIJING 100080

CHINA PR

TEL: 1282070

TLF:

ILX: 22040 BAOAS CN

EML:

COM: 09

FUENSALIOA JIMENEZ J OR INST OE ASTROFISICA

DE CANARIAS

OBS DEL TEIDE

E 38200 LA LAGUNA

SPAIN

TEL: 22262211

TLF:

ILX: 92640

EML:

COM:
FRITZOVA-SVESTKA L OR

DOPPERSTRAAT 147

NL 3752. JC BUNSCHOTEN NETHERLANDS

TEL: 349984403

TLF :

TLX:

EML:

COM:

FROESCHLE MICHEL, OR

\section{OCA CERGA}

AVE COPERNIC

F 06130 GRASSE

FRANCE

TEL: $93 \quad 355849$

TLF:

TLX:

EML:

COM: 08

FRVE GLENN M PROF

OPT OF PHYSICS

CASE WESTERN RESERVE UNIV ROCK BDG

CLEVELAND OH 44106

USA

IEL: 2163682997

TLF :

TLX:

EML:

COM:

FU QI JUN

BEIJING ASTRONOMICAL OBS CAS

\section{W SUBURB}

BEIJING 100080

CHINA PR

IEL: $1275580^{\circ}$

TLF:

ILX: 22040 BAOAS CN

EML:

COM: 10

FUERST ERNST DR MPI FUER RADIOASTRONOMIE AUF DEM HUEGEL 69

D 5300 BONN 1

GERMANY

TEL:

TLF :

TLX: 886440

EML:

COM: 40
FROEHLICH CLAUSS

WORLO RADIATION CENTER

PHYSIKALISCH-METEOROL OBS

POSFACH 173

CH 7260 OAVOS-DORF

SHITZERLAND

TEL: 8352131

TLF : 83.35058

TLX: 853232 PMOO CH

EML:

COM: 12

FROGEL JAY ALBERT DR DPT OF ASTRONOMY OHIO STATE UNIVERSITY 174 W 18TH AVE. COLUMBUS OH 432101106 USA

TEL: 6142925651

ILF :

ILX:

EML: FROGELOHSTPY.BITNET COM: 28

FTACLAS CHRIST

PERKIN ELEMR CORP

MS 897

100 WOOSTER HEIGHTS RD DANBURY CT 068107859

USA

TEL: 2037976448

TLF:

TLX:

EML:

COM: 28

FUCHS BURKHARD DR ASTRONOMISCHES-RECHEN INSTITUT

MOENCHHOFSTR. 12-14

D. 6900 heiduelberg 1

GERMANY

TEL: $62 \quad 2149026$

TLF:

TLX: 461336 ARIHD D

EML:

COM: 28,33

FUHR JEFFREY ROBERT DR NTL BUREAU OF STANDARDS PHYSICS BLOG RM A267 GAITHERSBURG MO 20899 USA.

TEL: 3019753204 TLF : 3019753038 TLX: 197674 NIST UT EML: FUHRONISTCS2 COH: 14 
FUJIMOTO MASAYUKI DR

FAC OF EDUCATION

NIIGATA UNIVERSITY

6050 IXARASHI 2

NIIGATA 95021

JAPAN

TEL:

TLF :

TLX:

EML:

COM: 28,35

\section{FUJITA YOSHID PRGF}

DPT OF ASTRONOMY

UNIVERSITY OF TOKYO

BUNKYO KU.

TOKYO 113

JAPAN

TEL: 423-74-4186

TLF :

TLX:

EML:

COM: 29

FUKUE JUN DR

ASTRONOMICAL INSTITUTE

OSAKA KYOIKU UNIVERSITY

488 MINAMI KAWAHOR ICHO

OSAKA 543

JAPAN

TEL:

TLF :

TLX:

EML:

COM:

FUXUNAGA MASATAKA OR

ASTRONOMICAL INSTITUTE

TOHOKU UNIVERSITY

SENDAI AOBA

MIYAGI 980

JAPAN

TEL: $22 \quad 2221800$

TLF:

ILX:

EML:

COM: 33

FURNISS IAN

DPT PHYSICS \& ASTRONOMY

UNIVERSITY COLLEGE LONDON

GOWER ST

LONDON WCIE 6BT

UK

TEL: I 713877050

TLF:

ILX: 28722 UCPHYS

EML:

COM: 34,44
FUJ IMOTO MASA-KATSU OR

TOKYO ASTRONOMICAL OBS

NAOJ

OSAWA MITAKA

TOKYO 181

JAPAN

TEL: 422325111

TLF:

TLX: 2822307 TAOMTK J

EML:

COM: $31 \mathrm{C}, 33,5 \mathrm{I}$

FUJIWARA AKIRA OR
OPT OF PHYSICS
KYOTO UNIVERSITY
SAKYO KU
KYOTO 606
JAPAN
TEL: $075-751-2111$
TLF:
TLX: 5422693 LIBKYU J
EML:
COM: $15: 16.21$

FUKUGITA MASATAKA DR

RES INST FUNDAMENTAL PHYS

KYOTO UNIVERSITY

KITASHIRAKAWA SAKYO KU

KYOTO 606

JAPAN

TEL: 075 $711 \quad 1381$

TLF:

TLX: 5423179 RIFPK

EML:

COM: 28,47

FUKUSHIMA TOSHIO OR

HYOROGRAPHIC DPT

GEOOESY \& GEOPHYSICS DIV

TSUKIJI 5 CHUD $\mathrm{KU}$

TOKYO 104

JAPAN

TEL: 35413811

TLF :

TLX: 2522452 HOJOOC J

EML: GE MarkIII NOOE-8C28 G60HDJ

COH: 04

FURSENKO M A OR.

INST OF THEORET ASTRONOMY

ACADEMY OF SCIENCES

N KUTUZOVA 10

191187 ST PETERSBURG

RUSSIA

TEL: $27 \mathrm{~B}-88-98$

TLF :

TLX: 121578 ITA SU

EML:

COM: 04

FUJIMOTO MITSUAKI OR
DPT OF PHYSICS
NAGOYA UNIVERSITY
FUROCHO CHIKUSA KU
NAGOYA 464
JAPAN
TEL:
TLF:
TLX:
EML:
COM: 47,51

\section{FUJIWARA TAKAO BR}

KYOTO CITY IJNIV OF ARTS

NISHIKYO KU

KYOTO 61011

JAPAN

TEL: $75 \quad 3320701$

TLF: $75 \quad 3320709$

TLX:

EML: C53926

COM: 33

FUKUI TAKAO DR

OPT OF LIBERAL ARTS

DOKKYO UNIVERSITY

SAKAE-MACHI 600

SOKA SAITAMA

JAPAN

TEL: $0489-42-1111$

TLF :

TLX:

EML:

COM: 47

FULLCHGMONI MARCELLO PROF

IAS

CNR

CP 67

I 00044 FRASCATI

ITALY

TEL: 64956951

TLF:

TLX: 680489 CNR FRA

EML:

COM: 15, WGPSNC

FURUKAWA KIICHIRO OR,

TOKYO ASTRONOMICAL OBS

NADJ

OSAWA MITAKA

TOKYO 181

JAPAN

TEL:

TLF :

TLX:

EML:

COM: 20
FUJISHITA MITSUMI DR

DIV OF EARTH ROTATION

NTL ASTRONO OBSERVATORY

2-12 HOSHIGAOKA

IWATE 023

JAPAN

TEL: 197247111

TLF :

TLX: 837628 1LSMIZ J

EML: TELEMAIL IO:MIZUSAWA

COM: 08,19

FUKUOA ICHIRO

KANAZAWA TECHNOLOGY INST

7-1 OGIGAOKA

NONOICHINACHI

ISHIKAWA 921

JAPAN

TEL: $0762-48-1100$

TLF :

TLX: 5L22456 KIT LC J

EML:

COM: 45

FUKUI YASUO DR

DPT OF PHYSICS.

NAGOYA UNIVERSITY

FUROCHO CHIKUSA KU

NAGOYA 464

JAPAN

TEL: $052-781-5111$

TLF:

TLX: 4477323 SCUNAG J

EML:

COM: 34,40

FURENLID INGEMAR $K$ DR

DPT PHYSICS \& ASTRONOMY

GEORGIA STATE UNIVERSITY

ATLANTA GA 303033083

USA

TEL: $404 \quad 6582932$

TLF :

TLX:

EML:

COM: 26,29

FUSCQ-FEMIANO ROBERTO

IAS

CNR

CP 67

I 00044 FRASCATI

ITALY

TEL: 69425655

TLF :

TLX: 610261

EML :

COM: 


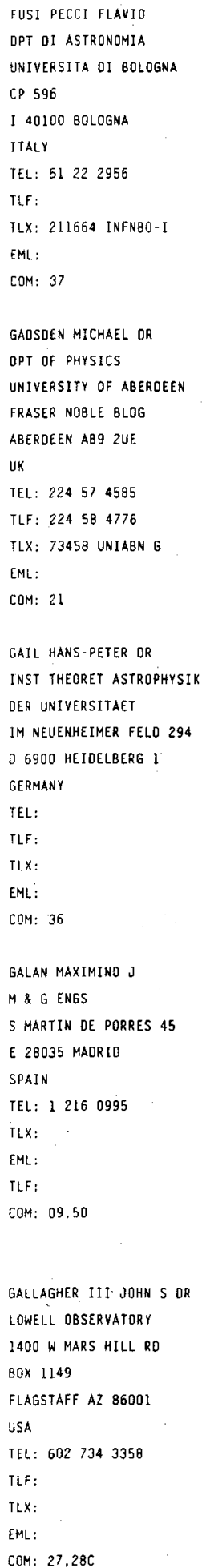

FU-SHONG KUD
OPT OF PHYSICS

NTL CENTRAL UNIVERSITY

CHUNG LI

CHINA R

TEL:

TLF:

TLX:

EML:

COM:

GAETZ TERRANCE J DR

DPT OF. ASTRONOMY

UNIV OF WESTERN ONTARIO

LONDON ON NEA 3K7

CANADA

TEL: 5196613183

TLF: 5196613486

FLX: 0647134

EML: BITNET:GAETZOUWOVAX

COM:

GAISSER THOMAS $K$ BARTOL RESEARCH INSTITUTE UNIVERSITY OF OELAWARE NEWARK OE 19716

USA

TEL: $302 \quad 451 \quad 0111$

TLF:

TLX: 510-665-0805 BARTOL

EML:

COM: 48

GALEOTTI PIERO PROF

IST OI COSMO GEOFISICA

CNR

CORSO FIUME 4

I 10133 TORINO

ITALY

TEL: $11 \quad 658979$

TLF:

TLX: 224379 COSMOT I

EML:

COM: 48

GALLAGHER JEAN W OR

NIST

PHYSICS BUILOINO 221

ROOM A 323

GAITHERSBURG MO 20899

USA

TEL: 3019752204

TLF: 3019260416

TLX: 197674 NISTUT

EML: JWG̣ENISTCS2,BITNET

COM: 14
GABRIEL ALAN H

IAS

BP 10

F 91371 VERRIERES BUISSON

FRANCE

TEL: $164 \quad 47 \quad 4315$

TLF :

TLK: 600252

EML:

COM: $10,12,14[, 44$

GAHM GOESTA F DR

STOCKHOLM OBSERVATORY

513336 SALTSJOEBADEN SWEDEN

TEL: $87 \quad 170637$

ILF: $87 \quad 17 \quad 4719$

ILX: 12972

EML: GAHMASTRO.SU.SE

COM: 27

GAIZAUSKAS VICTOR OR HERZBERG INST ASTROPHYS NTL RESEARCH COUNCIL

100 SUSSEX OR

OTTAWA ON KLA ORG

CANADA

TEL: 6139937395

TLF: $613952 \quad 0974$

TLX: 0533715 NRCOTT

EML: 18584::VICGZ

COM: $10 P, 12$

GALIBINA I $\checkmark D R$

INST OF THEORET ASTRONOMY

ACADEMY OF SCIENCES

N KUTUZOVA 10

191187 ST PETERSBURG

RUSSIA

TEL: $186 \quad 1974$

TLF :

TLX: 121578 ITA SU

EML:

COM: 07

GALLET ROGER $M$

964 JTH ST

BOULOER CO 80302

USA

TEL:

TLF:

TLX:

EML:

COM:
GABRIEL MAURICE R DR

INSTITUT D.ASTROPHYSIQUE

UNIVERSITE OE LIEGE

AVE COINTE 5

B 4000 COINTE-LIEGE

BELGIUM

TEL: 41.529980

TLF: 41527474

ILX: 41264 ASTRLG

EML:

COM: 35

GAIGNEBET JEAN OR

OCA CERGA

AVE COPERNIC

F OEL3O GRASSE

FRANCE.

TEL: 93365849

TLF:

TLX: $470865 \mathrm{~F}$

EML:

COM: 31

GALAL A A PROF

HELWAN OBSERVATORY

HELWAN

EGYPT

TEL: $78 \quad 0645 / 2683$

TLF :

TLX:

EML:

COM: $10 \mathrm{C}$

GALINDO TREJO JESUS DR INSTITUTO DE ASTRONOMIA UNAM

APDO POSTAL 70-264

04510 MEXICO DF

MEXICO

TEL: $905 \mathbf{5 4 8 5 3 0 5}$

TLF:

TLX: 1760155 CICME

EML: BITNET: JGALOUNAMYMI

COM:

GALLETTA GIUSEPPE PROF DPT DI ASTRONOMIA

UNIVERSITA DI PADQVA

VIC DELL OSSERVATORIO 5

I 35122 PADOVA

ITALY

TEL: $4966 \quad 1499$

TLF:

TLX: 430176 UNPADU 1

EML:

COM: 28 
GALLETTO OIONIGI

IST OI FISICA MATEMATICA UNIVERSITA DI TORINO

VIA C ALBERTO 10

I LOL23 TORINO

ITALY

TEL: 11539214

TLF:

TLX:

EML:

COM: $07,33,47$

GALLOUET LOUIS DR

OBSERVATOIRE OE PARIS

61 AVE OBSERVATOIRE

F 75014 PARIS

FRANCE

TEL: 140512207

TLF:

TLX: 270776 OBS F

EML:

COM: 24,25

GAMALELOIN ABOULLA I OR HELWAN OBSERVATORY

HELWAN

EGYPT

TEL: $78 \quad 0645 / 2683$

TLF:

EML:

COM: 28

TEX:

GAO BUXI

INSTITUTE OF GEODESY \&

GEOPHYSICS

XU DONG LU

WUCHANG HUBEI

CHINA PR

TEL: B1 3805

TLF :

TLX:

EML:

COM: 08,19

GARCIA DE MARIA J M DR

DPT FISICA APLICADA

RONDA DE VALENCIA 3

E 28012 MAORID

SPAIN

TEL: 13367686

TLF: 15309244

TLX:

EML:

COM: 42 galliano pier giorgio

IST ELETTRONICO NAZ1ONALE

ST DELLA CACCE 91

I 10135 TORINO

ITALY

TEL: 3488933

TLF:

TLX: 211553 IENGF

EML:

COM: 31

GallowaY DAVIO DR

DPI OF APPLIEO MATHS

UNIVERSITY OF SYONEY

SYONEY NSW 2006

AUSTRALIA

TEL: 26922222

TLF :

TLX: 26169 UNISYO AA

EML:

COM: 10

gambis DaNiEL oR observatoIre de PARIS 61. AVE OBSERVATOIRE

F 75014 PARIS

FRANCE

TEL: 140512233

TLF:

TLX: 270776 OBS F

EML: GAMBISEFRIAP5]

COM: 19,31

GAPOSCHKIN EOWARD M OR

55 FARMCREST AVE

LEXINGTON MA 02173

USA

TEL: 6178622538

TLF:

EML:

COM: 07,19

TLX:

GARCIA EOUARDO DEL POZO

INST GEOPHYS \& ASTRONOMY

CALLE 212 N 290629 Y $3 \mathrm{k}$

LISA

LA HABANA

CUBA

TEL:

TLF:

TLX:

EML:

COM:
GALLIAND PIER gIOAEIO

IST ELETTRONICO NAZIONALE

ST DELLA CACCE 91

I 10135 TORINO

ITALY

TEL: 3488933

TLF :

TLX: 2ป1553 IENGF I

EML:

COM: 31

GALPERIN YU I PROF

SPACE RESEARCH INSTITUTE

ACADEMY OF SCIENCES

PROFSOJUSNAYA UL $84 / 32$

117810 MOSCOW

RUSSIA

TEL: $333-31-22$

ILF:

TLX: 411498 STAR SU

EML:

COM: $21 \mathrm{C}$

gAMMELGAard PETER Mag SCI INST OF PHYSICS \& ASTRON UNIVERSITY OF AARHUS

NY MUNKEGADE

DK 8000 AARHUS $C$

denMark

TEL: $86 \quad 128999$

TLF: $86 \quad 202711$

TLX: 64767 AAUSCI DK

EML:

COM: 15

GARAY GUIDO DR

OPT DE ASTRONOMIA

UNIVERSIDAD DE CHILE

CASILLA 36 D

SAMTIAGO

CHILE

TEL:

TLF:

$T L X:$

EML:

COM: 40

GARCIA LAMBAS OIEGO DR UNIV NACIONAL DE LA PLATA

FCAG

1900 LA PLATA (BS AS)

ARGENT INA

TEL: 21217308

TLF:

TLX:

EML:

COM: 28
GALLINO ROBERTD

IST DI FISICA GENERALE

CORSO D AZEGLIO 46

I 10125 TORINO

ITALY

TEL: 11655103

TLF:

TLX: 21104 INFN TO

EML:

COM: $35,36,46$

GALT JOHN A OR

DOMINION RADIO ASTROPHYS

OBSERVATORY

BOX 248

PENTICTON BC Y2A 6K3

CANADA

TEL: $604 \quad 493 \quad 2277$

TLF: $604 \quad 493 \quad 7767$

TLX: 04888127

EML:

COM: 40

GAO BILIE

NANJING ASTRONOMICAL

INSTRUMENT FACTORY

NANJINE

CHINA PR

TEL: $25 \quad 46191$

TLF:

ILX:

EML:

COM: 09

GARCIA DE \LA ROSA JOSE

INST DE ASTROFISICA

oE CANARIAS

OBS OEL TEIDE

E 38071 LA LAGUNA

SPAIN

TEL: $22 \quad 262211$

TLF:

TLX: 92640

EML:

COM: 10

GARCIA MICHAEL $R$ OR

CENTER FOR ASTROPHYSICS

HCO/SAO

60 GARDEN ST

CAMBRIDGE MA 02138

USA

TEL: 6174957169

TLF: 6174957356

TLX:

EML: GARCLAGCFA200. HARYARD. EOU

COM: 
GARCIA-BARRETO JOSE A

OBS ASTRONOMICO NACIONAL

UNAM

APDO POSTAL 877

22860 ENSENADA B CALIF

MEXICO

TEL: $667-830-93$

TLF :

TLX:

EML:

COM:

\section{GARFINKEL BDRIS OR}

YALE UNIVERSITY OBS

YALE STATION

BOX 2023

NEW HAVEN CT 06520

USA

TEL: $203 \quad 4363460$

TLF :

TIX:

EML:

COM: 07,20

GARMIRE GOROON P PROF

DPT OF ASTRONOMY

PENNSYLVANIA STATE UNVI

525 DAVEY LAB

UNIVERSITY PARK PA 16802

USA

TEL: $814 \quad 8650418$

TLF :

TLX: 842510 PENNSTBSTR SC

EML :

COM: 48

GARRISON RQBERT F PROF

DAVIO OUNLAP OBSERVATORY

UNIVERSITY OF TORONTO

BOX 360

RICHMONO HILL ON LAC $4 Y G$

CANADA

TEL: $416 \cdot 8849562$

TLF : 416,9783921

TLX: 06986766

EML:

COM: $29,45,47$

GARY GILMER ALLEN OR

NASA/MSFC

CODE ES 52

SPACE SCIENCE LAB

HUNTSVILLE AL 35812

USA

TEL: 2055447609

ILF: 2055445862

ILX: 62026079

EML: SPAN SSL: :GARY

COM: 10
GARCIA-BERRO ENRIQUE OR

DPT FISICA APLICADA

UNIV POLITEC DE CATALUNYA

E. 08032 BARCELONA

SPAIN

TEL: 34016898

TLF: 34016801

TLX: 52821 UPC E

EML: GARCIAEETSECCPB.UPC.ES

COM: 12

GARGAUD MURIEL DR

OBSERVATOIRE DE BORDEAUX

BP 89

F 33270 FLOIRAC

FRANCE

IEL: $56 \quad 86 \quad 4330$

TLF: $56 \quad 40 \quad 4251$

ILX:

EML: MURIELEFROBOR51

COM: 14

\section{GARNIER ROBERT ING \\ OBSERVATOIRE DE LYON \\ AVE CHARLES ANORE \\ FRANCE \\ TEL: 7\& 560705 \\ ILF: 72399791 \\ TLX: \\ EML: \\ COM:}

F 69561 S GENIS LAVAL COX

\section{GARSTANG ROY H PROF \\ JILA \\ UNIVERSITY OF COLORADO \\ BOX 440}

BOULDER CO 803090440

USA

TEL: 3034927795

TLF :

TLX: 755842 JILA

EML:

COM: 05,14

GARZON FRANCISCO DR

INST DE ASTROFISICA

DE CANARIAS

OBS DEL TEIDE

E 38200 LA LAGUNA

SPAIN

TEL: 22262211

TLF:

TLX: 92640

EML: SPAN: IAC: ; FGL

COM: 33
GARCIA-PELAYO JOSE DR INST ASTROFISICA

OE ANDULUCIA APD 2144

PROFESOR ALBAREOA 1

E 18080 GRANADA

SPAIN

TEL: $58 \quad 25 \quad 6103$

TLF:

FLX:

EML:

COM:

GARLICK GEORGE F DR

267 SOUTH BELOIT AVE

LOS ANGELES CA 90049

USA

TEL: 2134723512

TLF:

TLX:

EML:

COM:

GARRIDO RAFAEL

INST ASTROFISICA

DE ANDULUCIA APD 2144

PROFESOR ALBAREDA 1

E 18080 GRANAOA

SPAIN

TEL: $5812 \quad 1311$

TLF :

Ti.X: 78753

EML:

COM: 27

BARTON W R S PROF

BLACKETT LABORATORY

IMPERIAL COLLEGE

PRINCE CONSORT RO

LONDON SW7 2BZ

UK

TEL: '1 23321657

TLF:

TLX: 261503

EML:

COM: 14

GASCOIGNE 5 C B DR

MOUNT STROMLO \& SIOING

SPRING OBSERIORIES

PRIVATE BAG

WODEN PO ACT 2606

AUSTRALIA

TEL:

TLF:

TLX:

EML:

COM: $27,28,37$
GARDNER FRANCIS, F DR

CSIRO

OIVISION OF RADIOPHYSICS

B0X 76

EPPING NSW 2121

AUSTRALIA

TEL: 28680222

TLF :

TLX: $26230 \mathrm{AA}$

EML:

COM: 34,40

GARMANY CATHERINE D DR

IILA

UNIVERSITY OF COLORADO

BOX 440

BOULOER CO BO309 0440

USA

TEL: 3034927836

TLF :

TLX:

EML: :

COM: $29,42,45$

GARRINGTON SIMON DR

NRAL.

JODRELL BANK

MACCLESFIELD SK11 9DL

UK

TEL: 47771231

YLF: 447771618

ILX: 36149

EML: JANET STGOUK, AC.MAN.JB, STAR

COM: 40

GARY OALE E

CALTECH

MS 26433

$S A$

PASADENA CA 91125

USA

TEL: 8183563863

TLF :

TLX: 675425 CALTECH PSD

EML:

COM:

GASKA STANISLAW DR

INSTITUTE OF ASTRONOMY

N COPERNICUS UNIVERSITY

UL CHOPINA $12 / 18$

PL 87100 TORUN

POLANO

TEL:

FLF :

TLX:

EML:

COM: 07 
GATEWOOD GEORGE DIRECTOR ALLEGHENY OBSERVATORY OBSERVATORY STATION PITTSBURGH PA 15214 USA

TEL: 4123212400

TLF:

TLX:

EML:

COM: $24,26,51$

GAUR V $\mathrm{P}$

UTTAR PRADESH STATE

OBSERVATORY

PO MANORA PEAK 263129

NAINITAL 263129

INDIA

TEL: $5942 \quad 2136$

TLF:

ILX:

EML:

COM: 12

\section{GAVAZZI GIUSEPPE DR}

OSS ASTRONOMICO DI BRERA

VIA BRERA 28

I 20121 MILANO

ITALY

TEL: 2874444

TLX:

EML: 39216: : GAVAZZ1

TLF: 272001600

COM: $2 \theta$

geballe thomas a dr JOINT ASTRONOMY CENTER

UKIRT

665 KOMOHANA ST

HILO HI 96720

USA

TEL: 8089613756

TLF:

TLX: 633135

EML: NSSOCA: : PSIXUKTH: : TOM

COM: 34

GEHRELS TOM PROF

LUNAR \& PLANETARY LAB

UNIVERSITY OF ARIZONA

TUCSON AZ 85721

USA

TEL: 6026216970

TLF:

TLX:

EML:

COM: $15,16,20,25,34,51$
GATLEY IAN

NOAO

BOX 26732

$950 \mathrm{~N}$ CHERRY AVE

TUCSON AZ 857266732

USA

TEL: 6023275511

TLF:

TLX: 0666484 AUR

EML:

COM:

GAUSS F STEPHEN

US NAVAL OBSERVATORY

34 \& MASSACHUSETTS AVE NW

WASHINGTON OC 203925100

USA

TEL: 2026531510

TLX:

EML: FSGESICON.USNO.NAVY.MIL

TLF : $202 \quad 6531497$

COM: 08,09

GAY JEAN DR
OCA CERGA
AVE COPERNIC
F O6130 GRASSE
FRANCE
TEL: 93365849
TLF:
TLX: 470865
EML:
COM: 09.34

GebBIE KATHARINE B OR

JILA

UNIVERSITY OF, COLORAOO

BOX 440

BOULDER CO 803090440

USA

TEL: 3034927825

TLF:

TLX: 755842 JILA

EML:

COM: 36

GEHREN THOMAS PH D

INST ASTRON \& ASTROPHYSIK UNIVERSITAETS STERNWARTE

SCHEINERSTR 1

O 8000 MUENCHEN 80

GERMANY

TEL: 89989021

TLF:

TLX: 529815 UNIVERS D

EML:

COM: 29
GAUDENZI SILVIA DR

istituto Astronomico

UNIVERSITA OI ROMA

VIA G M LANCISI 29

I 00161 ROMA

ITALY

TEL: $644 \quad 03734$

TLF: 64403673

TLX: 613255 INFNRO

EML: 40058 : :GAUDENZI

COM: 26

gAUSTAD JOHN E PROF

DPT OF ASTRONOMY

SWARTHMORE COLLEGE

SWARTHMORE PA 19081

USA

TEL: $215328827 !$

TLF: 2153288673

TLX:

EML: JGAUSTAI SCC. SWARTHMORE. EDU

COM: 34

GAYLARD MICHAEL JOHN

HARTEBEESTHOEK RADIOASTRO

OBSERVATORY

BOX 443

KRUGERSDORP $1740 \hat{\mathrm{O}}$

SOUTH AFRICA

TEL: $27 \quad 116424692$

TEF: $27 \quad 11 \quad 642 \quad 2446$

TLX: 321006

EML:

COM: 40

GEBLER KARL-HEINZ OR

RADIDASTRONOMISCHES INST

DER UNIVERSITAET BONN

AUF DEM HUEGEL 71

O 5300 BONN 1

GERMANY

TEL: 228733662

TLF :

TLX:

EML:

COM: 40

GEHRZ ROBERT DOUGLAS DR DPT PHYSICS \& ASTRONOMY UNIVERSITY OF WYOMING

BOX 3905

LARAMIE WY B2071

USA

TEL: 3077666176

TLF :

TLX:

EML:

COM: 25
GAUME RALPH A DR

NAVAL RESEARCH LABORATORY

CODE 4210

4555 OVERLOOK AVE SW

WASHINGTON OC 203755000

USA

TEL: 2027670670

TLF:

TLX:

EML: GAUMEER IRA1. NRL. NAYY.MIL

COM:

GAUTIER DANIEL

OBSERVATOIRE DE PARIS

SECTION DE MEUDON

F 92195 MEUDON PPL COK

FRANCE

TEL: $14507 \quad 7707$

TLF:

TLX: 201571

EML:

COM: $16 \mathrm{C}$

GEAKE JOHN E DR

DPT Of PHYSICS

UMIST

BOX 88

MANCHESTER M60 100

UK

TEL: 612363311

TLF:

TLX: 666094

EML:

COM: 16

GEFFERT MICHAEL DR OBSERVATORIUM HOHER LIST

D 5568 DAUN

GERMANY

TEL: $6592 \quad 2150$

TLF:

TLX:

EML:

COM:

GEISLER DOUGLAS P DR

CERRO TOLOLO

INTERAMERICAN OBSERVATORY CASILLA 603

LA SERENA

CHILE

TEL: $5122 \quad 5415 * 208$

TLF: $5122 \quad 5415^{*} 342$

TLX: 620301 AURA CT

EML: DGEISLERENOAO.EDU

COM: 37 


GEISS JOHANNES PROF
PHYSIKALISCHES INSTITUT
UNIVERSITAET BERN
SIOLERSTRASSE 5
CH 3012 BERN
SWITZERLAND
YEL: 31654402
TLF:
TLX: 32320
EML:
COM: $: 5,16$

GEMMO ALESSANDRA OR

DPT OL ASTRONOMIA.

UNIVERSITA di padova

VIC DELL OSSERVATORIO 5

I 35122 padova

ITALY

TEL: 49661499

ILF: 4938919

TLX: 432071 ASTROS I

EML: 39003 : GEMMO

COM: 33

GENT HUBERT MR
PROSPECT HOUSE
SHERWODD LANE
WORCESTER WR2 $4 \mathrm{NX}$
UK
TEL: 422186
TLF:
TLX:
EML:
COM: 40

GEORGIEV TSVETAN DR

OPT OF ASTRONOMY

BULGAR IAN ACAD SCIENCES

72 LENIN BLYO

BG 1784 SOFIA

BULLaria

TEL: 2883503

TLF:

TLX: 23351 IKI BAN BG

EML:

COM: 28

GERBALDI MICHELE DR INSTITUT O'ASTROPHYSIQUE gaBIS BO ARAGO

F 75014 PARIS

FRANCE

TEL: $14320 \quad 1425$

TLF: 143298673

TLX:

EML:

COM: $25,29 \mathrm{C}, 45,46 \mathrm{C}$ gelozahler bermaro J MAVAL RESEARCH LABORATORY CODE 4121.6

4555 OVERLOOK AVE SW WASHINGTON OC 203755000 USA

TEL:

TLF :

TLX:

EML:

COM: 40

GENET R M DR

FAIRBORN OBSERVATORY

3435 E EOGEWOOD ABE

MESA AZ 85204

USA

TEL: $602988 \quad 6561 * 223$

TLF:

TLX:

EML:

COM: $25 \mathrm{C} .27$.

GENZEL REINHARD OR

MAX PLANCK INSTITUT FLER PHYSIK UND ASTROPHYSIK KARLSCHARZSCHILO STR I

D 8046 GARCHING MUENCHEN GERMANY

TEL: 8932990

TLF:

ILX: 524629

EML:

COM: $34 \mathrm{C}, 40$

GERARD ERIE OR OBSERVATOIRE DE PARIS SECTION DE MEUDON F 92195 MEUBON PPL CDX FRANCE

TEL: $1 \quad 45 \quad 07 \quad 7607$

TLF:

TLX: 270912

EML:

COM: $15,34,400$

GERGELY TOMAS ESTEBAN DR NTL SCIENCE FOUNDATION DIV ASTRONOMICAL SCIENCES 1800 G ST NW WASHINGTON OC 20550 USA

TEL: 2023579696

TLF:

ILX:

EML:

COM: $10,40,500$
GELFREIKH GEORGIJ B OR PULKOVO OBSERVATORY ACAOEMY OF SCIENCES 10 KUTUZOV QUAY 196140 ST PETERSBURG RUSSIA

TEL:

TLF:

TLX:

EML:

COM: 10,40

GENKIN IGOR L PROF DR

FAC OF PHYSICS

KAZAKH STATE UNIVERSITY KOMSOMOLSKAYA 96

480012 ALMA ATA

KAZAKHSTAN

TEL: $67-70 \cdot 18$

TLF:

TLX:

EML:

COM: 33

GEORGELIN YVON P. DR

OBSERVATOIRE OE MARSEILLE

2 PLACE LE VERRIER

F 13248 MARSEILLE COX 04 FRANCE

TEL: 91959088

TLF :

TLX: 42024I F

EML:

COM: $30,33,34$

GERARD JEAN-CLAUDE M C DR INSTITUT O.ASTROPHYSIQUE UNIVERSITE OE LIEGE AVE COINTE 5

B 4000 COINTE-LIEGE BELGTUM

TEL: 41529980

TLF : $41 \quad 527474$

TLX: 41264

EML: U2163JC AT BLIULG 11

COM: 16

GERHARD ORTWIN
LANDESSTERNWARTE
KONIGSSTUHL
O 6900 HEIDELBERG I
GERMANY
TEL: 6221509222
TLF:
TLX:
EML:
COM: 28

GELLER MARGARET 'JOAN CENTER FOR ASTROPHYSICS HCO/SAO

60 GAROEN ST

CAMBRIDGE MA 02138

USA

TEL: $617 \quad 4957409$

TLF:

TLX: $92142 B$ SATELLITE CAM EML :

COM: $28,47 \mathrm{C}$

GENOVA FRANCOISE DR CNES/SU/AS -

2 PLACE maurice Quentin

F 75039 PARIS COX 01

FRANCE

TEL: $145 \quad 087741$

TLF: $145 \quad 087557$

rLX: 214674 .

EML:

COM:

GEORGELIN YVONNE M DR OBSERVATOIRE OE MARSEILLE 2 PLACE LE VERRIER

F 13248 MARSEILLE COX 04 FRANCE

TEL: 91959088

ILF:

TLX: $420241 \mathrm{~F}$

EML:

LOM: 33

GERBAL DANIEL DR OBSERVATOIRE DE PARIS SECTION de MEUDON F 92195 MEUDON PPL COX FRANCE

TEL: 145077419

TLF:

TLX: 201571

EML:

COM:

GERIN MARYVONNE OR RAOIOASTRONOMIE ENS 24 RUE LHOMONO F 75231 PARIS COX O5 FRANCE

TEL: 143291225

TLF:

ILX:

EML: GERIN@FRULMLI

COM: 


\begin{tabular}{|c|c|c|c|}
\hline GERLEI OTTO & GEROLA HUMBERTO DR & GEROYANNIS VASSILIS S DR & GERSHBERG R E OR \\
\hline HELIOPHYSICAL OBSERVATORY & 20390 KNOLLWOOD DRIVE & OPT OF ASTRONOMY & ERIMEAN ASTROPHYS OBS \\
\hline $80 \mathrm{C} 30$ & SARAT0GA CA 95070 & UNIVERSITY OF PATRAS & UKRAINIAN ACAD OF SCIENCE \\
\hline H 4010 DEBRECEN & USA & GR 261 10 RION & NAUCHNY \\
\hline HUNGARY & TEL: & GREECE & 334413 CRIMEA \\
\hline TEL: $52 \quad 11 \quad 015$ & TLF: & TEL: & UKRAINE \\
\hline TLX: & EML: & TLF : & TEL: 432945 \\
\hline EML: & COM: 34 & TLX: & TLF: \\
\hline TLF : & TLX: & EML: & TLX: \\
\hline \multirow[t]{2}{*}{ COM: } & & COM: 35 & EML: \\
\hline & & & COM: $27 \dot{C} .29$ \\
\hline GEYER EDWARD H PROF OR & GEZARI DANIEL YSA DR & GHEIDARI S NASSIRI OR & GHIGO FRANCIS D DR \\
\hline OBSERVATORIUM HOHER LIST & NASA/GSFC & DPT Of PHYSICS & NRAO \\
\hline UNIVERSITAET BONN & CODE 693 & LINIVERSITY OF SHIRAZ & \\
\hline O 5568 OAUN/EIFEL & GREENBELT MO 20771 & BIRUNI OBSERVATORY & BOX 2 \\
\hline GERMANY & USA & SHIRAZ 71459 & GREEN BANK WV 24944 \\
\hline TEL: $6592 \quad 2150$ & TEL: 3012863432 & IRAN & USA \\
\hline TLF : & TLF: & TEL: & TEL: \\
\hline TLX: & TLX: & TLF: & TLF : \\
\hline EML: & EML: & $T L x:$ & TLX: \\
\hline \multirow[t]{2}{*}{ COM: $26,27,42,45$} & COM: 34,44 & EML: & EML: \\
\hline & & COM: 35 & COM: $28,40,51$ \\
\hline GHOBROS ROSHDY AZER PROF & GHOSH KAJAL KUMAR DR & GHOSH P DR & GHOSH S K DR \\
\hline HELWAN OBSERVATORY & INDIAN INST OF ASTROPHYS & TIFR & TIFR \\
\hline HELWAN & VAINU BAPPU OBSERVATQRY & HOMI BHABHA RD & HOMI BHABHA RD \\
\hline EGYPT & KAVALUR & COLABA & COLABA \\
\hline TEL: 780645 & ALANGAYAM NAA TN 635701 & BOMMBAY 400005 & BOMBAY 400005 \\
\hline TLF : & INDIA & INOIA & INDIA \\
\hline EML: & TEL: 530676 & TEL: $22 \quad 219 \quad 111 * 260$ & TEL: $22 \quad 215 \quad 2311$ \\
\hline COM: & TLF: & TLF : & TLF: $22 \quad 215 \quad 2110$ \\
\hline \multirow[t]{3}{*}{ TLX: } & TLX: B452763 IIAB IN & $T L X: 011-3009$ & TLX: 0113009 TIFR IN \\
\hline & EML: SHAKTI!VIGYAN!IIAP!GCA & EML: & EML: SWARNATIFRVAX, BETNET \\
\hline & COM: 29 & COM: 28 & COM: 25 \\
\hline GIACAGLIA GIORGIO E PROF & GIACANI ELSA BEATRIZ DR & GIACCONI RICCARDO PROF & GIACHETTI RICCARDO PROF \\
\hline CRAAE/ESCOLA POLITECNICA & IAR & STSCI & OPT OI FISICA \\
\hline UNIVERSIDADE DE SAO PAULO & cc 5 & HOMEWOOD CAMPUS & UNIVERSITA DI FIRENZE \\
\hline CP 8174 & 1894 VILLA ELISA (BS AS) & 37OO SAN MARTIN DR & LARGO E FERMI 2 \\
\hline 05508 SAO PAULO SP & ARGENTINA & BALTIMORE MD 21218 & I. 50100 FIRENZE \\
\hline BRAZIL & TEL: 2143793 & USA & ITALY \\
\hline TEL: 118159322 & TLX: 19052 CICYT AR & TEL: 3013384711 & TEL: 552298141 \\
\hline TLF: & EML: & TLF: & TLF: \\
\hline$T L X:$ & TLF : & TLX: 6849101 ST SCI & TLX: 572570 \\
\hline EML: & COM: 28.34 & EML: & EML: \\
\hline COM: 07 & & COM: $44,48 C$ & COM: \\
\hline GIALLONGO EMANUELE OR & GIAMPAPA MARK $S$ & GIANNONE PIETRO PROF & GIANNUZZI MARIA A OR \\
\hline OAR & NTL SOLAR OBSERVATORY & OAR & DPT OI MATEMATICA \\
\hline I OOO40 MONTEPORZIO & B0X 26732 & VIA DEL PARCO MELLINI 84 & UNIV OI ROMA LA SAPIENZA \\
\hline ITALY & $950^{\circ} \mathrm{N}$ CHERRY AVE & 100136 ROMA & PIAZZA GRAMSCI 5 \\
\hline TEL: 69449019 & TUCSON AZ $85726 \quad 6732$ & ITALY & I 00041 ALBANO/LAZIALE \\
\hline TLF: & USA & TEL: $634 \quad 52794$ & ITALY \\
\hline EML: OECNET: $17468:$ : GIALLIONGO & TEL: 6023275511 & TLF: & TEL: 69321101 \\
\hline COM: 47 & TLF: & TLX: & TLF: \\
\hline \multirow[t]{3}{*}{ TLX: } & TLX: 0666484 AURA NOAOTUC & EML: & TLX: \\
\hline & EML: & COM: 35,42 & EML: \\
\hline & COM: 29,36 & & COM: \\
\hline
\end{tabular}


GIBSON DAVIO MICHAEL DR SIIE MAN GEODSS FIELO ST MIT LINEOLN LABORATORY BOX 1707

SOCORRO NM B7801 1707 USA

TEL: 5056794244

flF:

TLX:

EML:

COM: $09,10,50$

gieren WOLFGang P OR GRUPO ASTROFIS PONTIFICA UNIVERSIDAO CATOLICA

CASILLA 104

SANTIAGO 22

CHILE

TEL:

TLF:

ILX:

EML: WGieRen astrouc. PUC.CL. COM: 27

GIGAS DETLEF OR
NASA GSFC
COOE 9102
GREENBELT MO 20771
USA
TEL:
TLF:
TLX:
EML:
COM: 36

\section{GILLINGHAM PETER MR}

AAO

PRIVATE bag

COONABARABRAN NSW 2357

AUSTRALIA

TEL: 68421122

TLX: 63945AA

EML:

TLF:

COM: 09

GILMOZZI ROBERTO
ESA IUE OBSERVATORY
APD 54065
E 28080 MADRID
SPAIN
TEL: 14019661
TLX: 42555
EML:
TLF:
COM:

GIBSON JAMES

JPL/CALTECH ITT \& FEC

MS 264781

4800 OAK GROVE OR

PASADENA CA 91109

USA

TEL: 8183542900

TLF:

TLX: 675429

EML:

COM: 15,20

GIES DOUGLAS R DR

OPT PHYSICS \& ASTRONOMY

georgia STATE UNIVERSITY

ATLANTA GA 303033083

USA

TEL: 4046512932

TLF:

TLX:

EML: BI TNET: PHYODGeGSUYMI

COM: 27

GIL JANUSZ A PROF

THE ASTRONOMICAL CENTER

LUBUSKA 2

PL 65001 ZIELONA GORA

POLAND

TEL: $48 \quad 68 \quad 66385$

TLF: $4868663 \quad 85$

TLX: 043346? WSPPL

EML:

COM: 40

GILMAN PETER A DR
HIGH ALTITUOE OBSERVATORY
NCAR
BOX 3000
BOULDER CO 803073000
USA
TEL: 3034971560
TLF:
TLX:
EML:
COM: 10
GILRA OAYA P OR
SM SYSTEMS \& RESEARCH CO
840L CORPORATE OR
SUITE 450
LANDOVER MO 20785
USA
TEL: 3017634483
TLF:
TLX:
EML:
COM: $29,34,44$

GICLAS HENRY L MR

120 E. ELM AVE

FLAGSTAFF AZ 86001

USA

TEL: 6027744769

TLF:

TLX:

EML:

COM: $16,20,24$

GIESEKING FRANK OR

OBSERYATORIUM HOHER LIST

UNIVERSITAET BONN

D 5568 DAUN/EIFEL

GERMANY

TEL:

TLF:

TLX:

EML:

COM: 30

GILLET D DR

OHP

F $04870 S$ MICHEL OBS

FRANCE

TEL: 92766368

TLF:

TLX: 410690 OHP F

EML: BITNET:GILLETERONISI

COM:

GILMORE ALAN C MR

MT JOHN OBSERVATORY

BOX 57

LAKE TEKAPO

NEW ZEALANO

TEL: 645056813

TLF:

TLX:

EML:

COM: 06,20

GIMENEZ ALVARO
INST ASTROFISICA
DE ANDALUCIA APD 2144
PROFESOR ALBAREDA 1
E 18080 GRANAOA
SPAIN
TEL: 58121311
TLF:
TLX: 785731 AAE E
EML:
COM: $35,42 C$

GIERASCH PETER J DR

DPT OF ASTRONOMY.

CORNELL UNIVERSFTY

SPACE SCIENCES BLDG

ITHACA NY 14853

USA

IEL: 6072563507

TLF:

TLX:

EML:

COM: 16

GIEJZEN JOSEPH W

ROYAL GREENWICH OBS

HERSTMONCEUX CASTLE

HAILSHAM BN27 IRP

UK

TEL: 323813171

TLF:

TLX:

EML:

COM:

GILLILAND RONALO LYNN

STSCI

HOMEWOOD CAMPUS

3700 SAN MARTIN OR

BALTIMORE MO 21218

USA

TEL:

ILF :

TLX:

EML:

COM: 10

GILMORE GERARD FRANCIS INSIITUTE OF ASTRONOMY THE OBSERVATORIES

MADINGLEY RD

CAMBRIOGE CBЗ OHA

UK

TEL: $223 \quad 622 \quad 04$

TLF:

TLX: 81797 ASTRON G

EML:

COM: $30.33 \mathrm{C}$

GINGERICH OWEN PROF

CENTER FOR ASTROPHYSICS

HCO/SAO

60 GARDEN ST

CAMBRIOGE MA 02138

USA

TEL: 6174957216

ILF: $617 \quad 4967564$

TLX: 921428 SATELLITE CAM

EML: GINGER@CFA/CFA:; GINGER

COM: 41,46 
GINGOLD ROBERT ARTHUR OR AUSTRALIAN NTL UNIVERSITY ANU SUPERCPTR FACILITY

BOX 4

CANBERRA ACT 2600

AUSTRALIA

TEL: $62 \quad 493 \quad 437$

TLF :

$T L X:$

EML:

COM: 35

GIOVANE FRANK

NASA HEABQUARTERS.

COOE $S Z$

600 INDEPENDENCE AVE SW

WASHINGTON OC 20546

USA

TEL: $202453 \quad 1472$

TLF:

TLX:

EML: 6646: : FGIOVANE

COM: 15,21

giraud edMONO

CENTRE PHYSIQUE THEORIQUE

CNRS

LUMINY CASE 07

f 13288 MARSEILLE COK

FRANCE

TEL: 91269519

TLF :

TLX: 430838 CNRSLUM $F$

EML: EARN: : GIRAUDOFRCPTM5I

COM:

GLASER HAROLD OR

1346 BONITA ST

BERKELEY CA 94709

USA

TEL: 4155271860

ILF:

EML:

COM: 44

TLX:

GLASS IAN STEWART OR

SAAO

$B 0 \times 9$

OBSERVATORY 7935

SOUTH AFRICA

TEL: $21 \quad 470025$

TLF :

TLX: 5720309 SA

EML: ISGQSAAD.AC. ZA

COM: $09,25,28$
GINZBURG VITALY L. PROF

LEBEDEV PHYSICAL INST

ACADEMY OF SCIENCES

LENINSKY PROSPEKT 53

117924 MOSCOW

RUSSIA

TEL:

TLF:

TLX:

EML:

COM: $40,48,51$

GIOVANELLI RICCAROO OR

OPT OF ASTRONOMY

CORNELL UNIVERSITY

SPACE SCIENCES BLOG

ITHACA NY 14853

USA

TEL:

TLF:

$T L X:$

EML:

COM: $28,30,34$

GIRIDHAR SUNETRA OR

INDIAN INSTITUTE OF

ASTROPHYSICS

KORAMANGALA

BANGALORE 560034

INDIA

TEL: $81256 \quad 6585$

TLF:

TLX: 845763 IIAB IN

EML:

COM: 35

GLASNER SHIMON AMI

RACAH INST OF PHYSICS

HEBREW UNIV OF JERUSALEM

JERUSALEM 91904

ISRAEL

TEL: 2584521

TLF :

TLX: 25391 HUIL

EML:

COM:

GLASSGOLD ALFRED E PROF

DPT OF PHYSICS

NEW YORK UNIVERSITY

4 WASHINGTON PLACE

NEW YORK NY 10003

USA

TEL: 2125982020

TLF:

TLX: 235128 NYU UR

EML:

COM:

\author{
GIOIA ISABELLA M OR \\ INSTITUTE FOR ASTRONOMY \\ UNIVERSITY OF HAWAII \\ 2680 WOODLAWN DR \\ HONOLULU HI 96822 \\ USA \\ TEL: \\ TLF: \\ TLX: 7238459 UHAST HR \\ EML: \\ COM : 40
}

GIOVANNELLI FRANCO OR

IAS

CNR

CP 67

I 00044 FRASCATI

ITALY

TEL: 69425655

TLF:

TLX: 610261 CNRFRAI

EML:

COM: $29,42,51$

GIURICIN GIULIANO

OPT OI ASTRONOMIA

UNIVERSITA DI TRIESTE

VIA TIEPOLO 11

I 34131 TRIESTE.

ITALY

TEL: 40768005

TLF:

TLX: 461137 OAT I

EML:

COM: 42,47

GLASPEY JOHN W OR

CANAOA FRANCE HAWAII

TELESCOPE CORPORATION

BOX 1597

KAMUELA HI 96743

USA

TEL:

TLF:

TLX:

EML: GLASPEYUUHCFHT.BITNET

COM:

GLATZEL WOLFGANG DR

MAX PLANCK INSTITUT FUER

PHYSIK UND ASTROPHYSIK

KARL-SCHWARZSCHILOSTR , I

D 6046 GARCHING MUENCHEN

GERMANY

TEL: 6932990

TLF:

TLX: 524629

EML: BITNET:WOG OGAIPPIS

COM:
GIOVANAROI CARLO

OSS ASTROFISICO

DI ARCETRI

LARGO E FERMI 5

I 50125 FIRENZE

ITALY

TEL: $55 \quad 275 \quad 2239$

TLF :

TLX: 572268 ARCETR I

EML:

COM: 28

GIOVANNINI GABRIELE

IST DI RADIOASTRONOMIA

CNR

VIA IRNERIO 46

I 40126 BOLOGNA

ITALY

TEL: 51232856

TLF :

TLX: 211664 INFN BO I

EML:

COM: 40

GLAGOLEVSKIJ JU $\checkmark$ OR

SPECIAL ASTROPHYSICAL OBS

ACADEMY OF SCIENCES

NIZHNIJ ARKHYZ

357147 STAVROPOLSKIJ

RUSSIA

TEL: 93.577

TLF :

TLX:

EML:

COM: $\quad 14,27,29,45$

GLASS BILLY PRICE DR

OPT OF GEOLOGY

UNIVERSITY DF OELAWARE

NEWARK OE 19716

USA

TEL: 3024518458

TLF:

TLX:

EML:

COM: 22

GLATZMAIER GARY A

LOS ALAMOS NATIONAL LAB MS F665

ESS 5

LOS ALAMOS NM 87545

USA

TEL: 5056677647

TLF:

TLX:

EML:

COM: $10,12,35$ 
GLEBOCKI ROBERT PROF INST THEORETICAL PHYSICS UNIVERSITY OF GOANSK

UL WITA STWOSZA 57 PL 80952 GDANSK POLAND

TEL: 4l-87-00

TLF :

TLX: 0512706 IFAS

EML:

[014: 36

GLIESE WIHELM PROF

ASTRONMISCHES-RECHEN

INSTITUT HEIOELBERG

MOENCHHOFSTR 12-14

06900 HEIDELBERG 1

GERMANY

TEL: $62 \quad 214050$

TLF: $62 \quad 21 \quad 4052 \quad 97$

TEX: 461336 ARIHD D

EML:

COM: $24,26,33$

GODART ODON PROF

9 AVE DES CERISERS

B 1488 BOUSVAL

BELGIUM

TEL: 10613817

$T ! F$ :

EML:

COM: 47

TLX:

\section{GOEBEL ERNST OR}

INSTITUT FUER ASTRONOMIE

UNIVERSITAET WIEN

TUERKENSCHANZSTR 17

A 1180 WIEN

AUSTRIA

TEL: 1345360186

TLF :

TLX:

EML:

COM: 50

\section{GOETZ WOLOEMAR OR}

STERNWARTESTRASSE 23A

PSF $5527 / 28$

06400 SONNEBERG

GERMANY

TEL: $96 \quad 74 \quad 3793$

$T L X: 627180$

EML:

TLF :

COM: 27
GLEBOVA NINA I DR

INST OF THEORET ASTRONOMY

ACADEMY OF SCIENCES

N KUTUZOVA 10

191187 ST PETERSBURG

RUSSIA

TEL: $278-88-98$

TLF :

TLX: 121578 ITA SU

EML:

COM: 04

GLUSHNEVA I N DR

STERNBERG STATE ASTR INST

UNIVERSITETSKIJ PROSP 13

119899 MOSCOW

RUSSIA

TEL: $139-20-46$

TLX:

ENL:

TLF :

COM: 29

GODFREY PETER DOUGLAS DR

DPT OF CHEMISTRY

MONASH UNIVERSITY

WELLINGTON RD

CLAYTON VIC 3168

AUSTRALIA

TEL: 35410811

ILF :

TLX: $32691 \mathrm{AA}$

EML:

COM: 34

GOEBEL JOHN H OR

NASA AMES RESEARCH CTR

MS 2447

SPACE SCIENCE DIV

MOFFETT FIELD CA 94035

USA

TEL: 4156946525

TLF:

TLX:

EML:

COM: 29.34

GOKOOGAN NUZHET PROF

UNIVERSITY OBSERVATORY

UNIVERSITY OF ISTANBUL

34452 ISTANBUL

TURKEY

TEL: 15223597

TLF:

TEX:

EML:

COM: 12,36
GLEISSBERG WOLFGANG PROF

BUCHENWEG 12

D 6374 DBERURSEL

GERMANY

TEL:

TLF:

TLX:

EML:

COM: 10

GNEOIN YURIJ N OR

IOFFE PHYSICAL IECH INST

ACADEMY OF SCIENCES

POLYTECHNICHESKAYA UL 26

194021 ST PETERSBURG

RUSSIA

TEL:

TLF :

ILX:

EML:

COM:

GQOQLI GIOVANNI PROF

DPT DI ASTRONOMIA

UNIVERSITA OI FIRENZE

LARGO E FERMI 5

I 50125 FIRENZE

ITALY

TEL: 5527521

ILF: 55220039

TLX: 572268 ARCETR I

EML:

COM: $10,12,27,51$

GOEOBLOEO JOHAN P PROF DR

INST VOOR PLASMAFYSICA

BOX 1207

NL 3430 BE NIEUWEGEIN

NETHERLANGS

TEL: $34 \quad 02 \quad 31224$

ILF: $34 \quad 02 \quad 31204$

TLX:

EML: GOEDBLOEDSARA.NL

COM: 10

GOKHALE MORESHWAR HARI PR

INDIAN INSTITUTE OF

ASTROPHYSICS

KORAMANGALA

BANGALORE $560 \quad 034$

INDIA

TEL: $81256 \quad 6585$

TLF:

TLX: 845763 IIAB IN

EML:

COM: 10
GLENCROSS WILLIAM M DR DPT PHYSICS \& ASTRONOMY UNIVERSITY COLLEGE LONOON GOWER ST

LONDON WCIE GBT

TEL: 1713877050

ILF :

TLX: 28722 UCPHYS G

EML:

COM:

GNEVYSHEVA RAISA S OR PIJLKOVO OBSERVATORY ACADEMY OF SCIENCES 10 KUTUZOV QUAY 196140 ST PETERSBURG RUSSIA

TEL:

TLF :

TLX:

EML:

COM: 10

GODWIN JON GUNNAR DR UNIVERSITY OF OXFORO B5 CHERWELL DRIVE OXFORD OX3 OND

TEL: $865 \quad 722 \quad 313$

TLF: 865721000

TLX:

EML: UK.AC.RL. STAR: : JG COM:

GOELBASI ORHAN OR FACUTLY OF SCIENCE INONU UNIVERSITY 44069 MALATYA TURKEY

TEL: 82121871

TLF: 82118133

TLX: 66140

EML:

COH:

GOLAY MARCEL PROF OBSERVATOIRE DE GENEVE CHEMIN DES MAILLETTES 51 CH 1290 SAUVERNY SWITZERLAND TEL: $22755 \quad 2611$ TLF: 227553983 TLX: 419209 OBS CH EML: gol ayoobs, unige.ch COM: $25,37,45 \mathrm{C}$ 


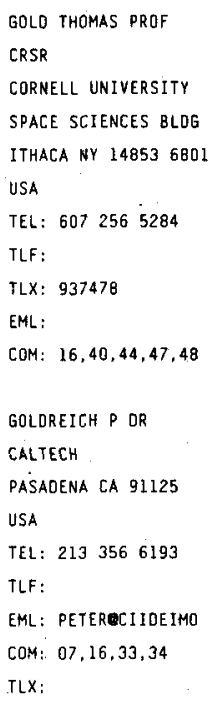

$\begin{array}{ll}\text { GOLDBACH CLAUOINE MME } & \text { GOLDMAN ITZHAK OR } \\ \text { INSTITUT D'ASTROPHYSIQUE } & \text { DPT OF PHYSICS ASTRON } \\ 98 B I S \text { BD ARAGO } & \text { TEL AVIV UNIVERSITY } \\ \text { F } 750.14 \text { PARIS } & \text { RAMAT AVIV } \\ \text { FRANCE } & \text { TEL AVIV } 69978 \\ \text { TEL: } 143201425 & \text { ISRAEL } \\ \text { TLF: } 143298673 & \text { TEL: } 35450303 \\ \text { TLX: } & \text { TLF: } \\ \text { EML: } & \text { TLX: } 342171 \text { VERSY IL } \\ \text { COM: } 14 & \text { EML: BITNET: E170TAUNOS } \\ & \text { COM: } 42\end{array}$

GOLDSMITH PAUL F DR BPT PHYSICS \& ASTRONOMY UNIV OF MASSACHUSETTS

GRC B 626

AHFERST MA 01003

USA

TEL:

TLF :

TLX:

EML:

COM: 34,40

gOLDSTEIN SAMUEL J PROF UNIVERSITY STATION UNIVERSITY OF VIRGINIA BOX 3818 CHARLOTTESVILLE VA 22903 USA TEL:

TLF :

TLX:

EML:

COM: 34,40

GOLLNOW H DR

MOUNT STROMLO DBSERVATORY PRIVATE BAE

WDDEN PO

CANBERRA ACT 2606

AUSTRALIA

TEL:

TLF :

TLX:

EML:

COM:

GOMEZ GONZALEZ JESUS DR PASEO IMPERIAL $296 \mathrm{GH}$ E MADRID 5

SPAIN

TEL:

TLF :

TLX:

EML:

COM: $34,40 \mathrm{C}$
GOLDMAN MARTIN $V$

CASA

UNIVERSITY OF COLORADO BOX 391

BOULDER CO 803090391

USA

TEL: 3034928896

TLF:

TLX:

EML:

COM: 12,49

GOLOSMITH PAUL F DR LEADMINE HILL ROAD 5

AMHERST MA 01002

USA

TEL: 4135450925

TLF:

TLX: 955491

EML:

COM:

GOLOSWORTHY FREDERICK A DPT OF APPL MATHEMATICS UNIVERSITY OF LEEOS LEEOS LS2 9JT UK

TEL: $532 \quad 431 \quad 751$

TLF:

TLX:

EML:

COM: 34

GOLUB LEON DR CENTER FOR ASTROPHYSICS HCO/SAO 60 GAROEN ST CAMBRIDGE MA 02138 USA TEL: $617 \quad 4957177$ TLF :

TLX:

EML:

COM:

GOMEZ MARIA THERESA OR OSS ASTRONOMICO

I 60131 NAPOLI
ITALY
TEL:
TLF:
TLX:
EML:
COM: 12




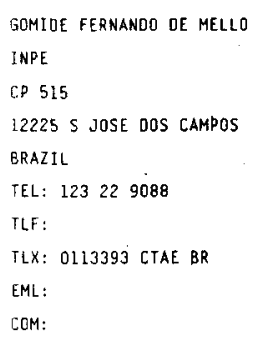

GONCZI GEORGES

\section{OCA OBSERV DE NICE}

BP 139

F 06003 NICE CDX

FRAMCE

TEL: 93890420

TLF :

TLX: 460004 OBSNICE F

EML:

COM:

GOHG SHU-MO
PURPLE MOUNTAIN OBS
CAS
NANJING
CHINA PR
TEL: 2546700
TLF:
TLX: 34144 PMO CN
EML:
COM: 35.47

GONZALEZ SERRANO J I OR DPT DE FISICA MODERNA UNIVERSIOAD DE CANTABRIA AVO LOS CASTROS S/N E 39005 SANTANOER SPAIN

TEL: $42 \quad 20 \quad 1450$

ILF: $42 \quad 20 \quad 1402$

TLX: 35851 EQUCI E

EML: GSERRANDECCUCVX. UNICAN.ES

COM: 28

GOODRICH ROBERT W OR

CALTECH

MS 10524

PASADENA CA 91125

USA

TEL: 8183563693

TLF :

TLX:

EML: RWGEDEIMOS, CALTECH, EDU

COM: 28

GOPALSWAMY N OR
ASTRONOMY PROGRAM
UNIVERSITY OF MARYLANO
COLLEGE PARK MO 20742
USA
TEL: 3014546649
TLF:
TLX: 62891478
EML:
COM: 12,40

GONDHALEKAR PRABHAKAR DR RUTHERFORD APPLETON LAB SPACE \& ASTROPHYSICS DIV BLDG R25/R68 CHILTON DIDCOT OXI1 OQX UK

TEL: 23521900

TLF :

TLX: 83159

EML:

COM: 44

GONZALES-A WALTER D DR INPE

CP 515

$12200 S$ JOSE DOS CAMPOS BRAZIL

TEL: $123 \quad 229977$

TLF: $123 \quad 21 \quad 8743$

TLX: $011-33530$ INPE BR

EML:

COM: 48

GONZALEZ-RIESTRA R DR

ESA IUE OBSEERVATORY

VILSPA

APD 54065

E 28080 MADRID

SPAIN

TEL: 14019661

TL.F :

TLX: 42555 VILSE

EML: EARN: IUEHOTODOAESA10

CDM:

G000Y R M

CEPP

PIERCE HALL

29 OXFORD ST

CAMBRIBGE MA 02138

USA

TEL: 6174954517

TLF :

TEX:

EML:

COM: 16

GOPASYUK S I DR.

CRIMEAN ASTRDPHYS OBS

UKRAINIAN ACAD. OF SCIENCE

NAUCHNY

334413 CR IMEA

UKRAINE

TEL: 432945

TLF :

TLX:

EML:

COM: 10,12
GONDOLATSCH FRIEDRICH PRF ASTRONOMISCHES RECHEN

INSTITUT

MOENCHHOFSTR 12-14

D 6900 HEIOELBERG 1 GERMANY

TEL: $62 \quad 2149026$

TLF:

TLX:

EML:

COM: 04

GONZALEZ CAMACHO ANTONIO INST DE ASTRON \& GEODESIA FAC DE CIENCIAS MATEMAT UNIVERSIDAD COMPLUTENSE E 28040 MADRID

SPAIN

TEL: 12442501

TLF :

TLX:

EML :

COM: 07

GONZE ROGER $F$ J IR OBSERVATOIRE ROYAL OE BELGIQLEE AVE CIRCULAIRE 3 B 1180 BRUSSELS BELGIUM

TEL: 23752484

TLF: 23749822

TLX: 21565

EML:

COM: 40

GOOSSENS MARCEL DR ASTRONOMISCH INSTITUUT KATHOLIEKE UNIV LEUVEN CELESTIJNENLAAN 200 B B 3030 HEVERLEE BELGIUM

TEL: -

TLF :

TLX:

EML:

COM: 10

GORBATSKY VITALIJ G PROF ASTRONOMICAL OBSERVATORY ST PETERSBURG UNIVERSITY BIBLIOTECHNAJA PL 2 198904 ST PETERSBURG RUSSIA

TEL: 257-94-91

TLF:

TLX:

EML:

COM: 27 


GORDON CHARLOTTE PROF
11 RUE TOURNEFORT
F 75005 PARIS
FRANCE
TEL:
TLF:
EML:
COM: 12.36
TLX:

GORDON MARK A OR
NRAO
CAMPUS BLDG 65
949 N CHERRY AVE
TUCSON AZ 857210655
USA
TEL: 6028828250
TLF:
TLX:
EML:
COM: $33,34,40$
GORGAS GARCIA JAVIER OR
DPT OE ASTROFISICA
FAC OE FISICA
UNIVERSIOAD COMPLUTENSE
E 28040 MADRID
SPAIN
TEL: I 549 5316
TLF:
FLX: 47273
EML: EARN/BITNET: WO620EEMDUCMI1
COM: 28

GOSS W MILLER PROF

NRAO

VLA

BOX 0

SOCORRO NM $87801 \quad 0387$

USA

TEL: 5057724011

TLF :

TLX: $910-988-1710$

EML:

COM: $28,34,40$
GOTTESMAN STEPHEN T OR BPT OF ASTRONOMY UNIVERSITY OF FLORIDA

211 SSRB

GAINESVILLE FL 32611

USA

TEL: 904392 2050/2052

TLF :

TLX: 8108252308

EML:

COM: $28,33,40$
GORDON COURTNEY P PROF OPT OF ASTRONOMY

HAMPSHIRE COLLEGE

AMHERST MA 01002

USA

TEL:

TLF:

TLX:

EML:

COM: 34

GORENSTEIN MARC $V_{i}$

CENTER FOR ASTROPHYSICS

MS 42

60 GAROEN ST

CAMBRIDGE MA 02138

USA

TEL: 6174959296

JLF :

TLX:

EML:

COM:

GORGOLEWSKI STANISLAW PR INST OF RAOIO ASTRONOMY N COPERNICUS UNIVERSITY UL. CHOPINA 12/18

PL 87 IOO TORUN POLAND

TEL: 20651 TORUN

TLF :

TLX: 0552324 TRAO PL

EML:

COM: 40

GOSSET ERIC DR

INSTITUT D'ASTROPHYSIQUE

UNIVERSITE DE LIEGE

AVE COINTE 5

B 4000 COINIE-LIEGE

BELGIUM

TEL: 41529980

TLF: 41527474

FLX: 41264 ASTRLG

EML: U2I4IEGEBLIULGIL

COM: 47

GOTTLIEB CARL A OR
INST FOR SPACE STUDIES
2880 BROAOWAY
NEW YORK NY 10025
USA
TEL: 2126785566
TLX:
EML:
TLF:
COM:

GOTTLIEB CARL A DR

SPACE STUDIES

-

GORDON ISAAC M DR INST RADIOPHYS \& ELECTRON $3100 B 5$ KHARKOV UKRAINE

TEL:

TLF :

TLX:

EML:

COM:

GORENSTEIN PAUL OR CENTER FOR ASTROPHYSICS HCO/SAO

GD GARDEN ST

CAMBRIDGE MA 02138 USA

TEL: 6174957250

TLF:

TLX: 921428 SATELLITE CAM

EML:

COM: 16

GOSACHINSKIJ I V DR

PULKOVO OBSERVATORY ACADEMY OF SCIENCES 10 KUTUZOV QUAY 196140 ST PETERSBURG RUSSIA

TEL: 2979452

TLF:

TLX: 321262

EML:

COM: $34,40 C$

GOSWAMI J N DR

PHYSICAL RESEARCH LAB

NAVRANGPURA

AHMEOABAD 380009

INOIA

TEL: $272 \quad 46 \quad 2129$

TLF: 272445292

ILX: OL2L397 PRL IN

EML:

COM: 22

GOTTLIEB KURT

46 JENNINGS STREET

CURTIN ACT 2605

AUSTRALIA

TEL: 62814166

TLF:

TLX:

EML:

COM:
GORDON KURTISS J PROF OPT OF ASTRONOMY HAMPSHIRE COLLEGE AMHERST MA 01002 USA

TEL: 4135494600

TLF:

TLK:

EML:

COM:

GORET PHILIPPE OR

EEA CEN

DAPNIA/SAP

BP 2

F 91191 GIF/YVETTE CDX FRANCE

TEL: 169084463

ILF :

TLX: 690860

EML:

COM: 47

GOSLING JOHN T DR

LOS ALAMOS NATIONAL LAB MS 0438

ESS 8

LOS ALAMOS NM 87545

USA

TEL: 5056675389

TLF:

ILX: 660495

EML:

COM: 49

GOTT III J RICHARO DPT ASTROPHYSICAL SCI PRINCETON UNIVERSITY PRINCETON NJ $08544 \quad 1001$ USA

TEL: 6094523813

TLF:

TLX:

EML:

COM: 51

GOUDA NADTERU OR DPT OF PHYSICS KYOTO UNIVERSITY SAKYO XU

KYOTO 696 JAPAN

TEL: $8175753 \quad 3844$

TLF: $8175753 \quad 3886$

TLX: 5422829 KUNSOP J

EML: GQUDAOJPNRIFP. BITNET

COM: 47 
gOUDAS CONSTANTINE L PROF OPT OF MATHEMATICS UNIVERSITY DF PATRAS GR 261 10 RION GREECE

TEL: $61 \quad 991889$

TLF:

TLX: 312239 EFAP GR

EMI:

COM: 07,16

GOULO ROBERT J PROF

OPT OF PHYSICS

UCSO

B 01

LA JOLLA CA 920930216

USA

TEL: 6194523649

TLF :

Tix:

EML:

COM:

GQWER J F R. DR

1615 MCTAVISH ROAD RR.2

SIDNEY BC VOL 3SI

CANADA

TEL: 6046565457

TLF :

EML:

COM: 40

TEx:

GRABOWSKI BOLESLAW DR INSTITUTE OF PHYSICS PEDAGOGICAL UNIVERSITY UL OLESKA 48

PL 45951 OPOLE

POLANO

TEL: $358-41$

TLF :

TLX: 0732230 WSP PL

EML:

COM

GRAHAM OAVIO A

MPI FUER RADIOASTRONOMIE

AUF DEM HLEGEL 69

D 5300 BONN 1

GERMANY

TEL: 228525282

$T(x: 886440$

EML:

TLF :

COM: 34,40
GOUDIS CHRISTOS O PROF OPT OF PHYSICS

UNIVERSITY OF PATRAS

GR 261 I0 PATRAS

GREECE

TEL:

TLF

TLX:

EML:

COM: 51

GOUPIL MARIE JOSE

OBSERVATOIRE DE. PARIS

SECTION DE MEUDON

DASGAL

F 92195 MEUDON PPL COX

FRANCE

TEL: $145 \quad 07 \quad 7880$

TLF :

TLX: 270912

EML: EARN : GOUPILFRMEU5 I

COM: 27,35

GOY GERALD PROF

OBSERVATOIRE OE GENEVE

CHEMIN DES MAILLETTES 51

CH 1290 SAUUERNY

SWITZERLAND

TEL: 227552611

TLF : 227553983

TLX: 419209 OBS CH

EML: goyoobs.unige.ch

COM: 25

GRADIE JONATHAN CAREY

INSTITUTE OF GEOPHYSICS

UNIVERSITY OF HAWAII

OIV PLANETARY GEOSCIENCES

HONOLULU HI 96822

USA

TEL: $808948 \quad 6488$

TLF:

TLX:

EML:

COM: 15

GRAHAM ERIC OR

BOC 9056

WOOOLAND PARK

COLORAOO 80866

USA

TEL:

TLF :

TLX:

EML:

COM: 35
GOLGH DOUGLAS O OR

INSTITUTE OF ASTRONOMY

THE OBSERVATORIES

MADINGLEY RD

CAMBRIDGE CB3 OHA

UK

TEL: 22362204

TLF:

TLX: 817297

EML:

COM: $27,35,36$

GOUTTEBROZE PIERRE DR

IAS

BP 10

F 91371 VERRIERES BUISSON

FRANCE

TEL: $1 \quad 64 \quad 47 \quad 4204$

TLF :

TLX: 600252

EML:

COM

GOYAL A N OR

DPT OF MATHEMATICS

UNIVERSITY OF RAJASTHAN

JAIPUR 302004

INDIA

TEL: $74 \quad 060$

TLF :

TLX:

EML:

COM: 24

GRADSZTAJN ELI DR

DPI OF PHYSICS ASTRON

TEL AVIV UNIVERSITY

RAMAT AVIV

TEL AVIV 69978

ISRAEL

TEL:

ILF:

TLX:

EML:

CON:

GRAHAM JOHN A DR

DPT TERRESTR MAGNETISH

CARNEGIE INST WASHINGTON.

5241 BROAO BRANCH RO NW

WASHINGTON DC 20015

USA

TEL: $202966 \quad 0863$

TLF:

TLK: 440427 MAGN UI

EML:

COM: $25,27,28$
GOUGUENHE IM LUCIENNE

OBSERVATOIRE DE PARIS

SECTION DE MEUDON

RADIOASTRONOMIE

F 92195 MEUDON PPL COX

FRANCE

TEL: $145 \quad 07 \quad 7604$

TLF: $145 \quad 07 \quad 7939$

TLX:

EML: GOUGUENHEIMGFRMUS

COM: $28,30,46 \mathrm{P}$

GOWER ANN C DR

DPT DF PHYSICS

UNIVERSITY OF VICTORIA

BOX 1700

VICTORIA BC V8W $2 Y 2$

CANADA

TEL: 6047217700

TLF : 6047217715

TLX:

EML:

COM:

GRABDSKE HAROLD [ JR LAWRENCE LIVERMORE LAB L 23

$80 \times 808$

LIVERMORE CA 94550

USA

TEL: 4154227262

TLF:

TLX:

EML:

COM:

GRADY CAROL ANNE OR

OPT OF PHYSICS

THE CATHOLIC UNIVERSITY

OF AMERICA

WASHINGTON DC 20064

USA

TEL: 2023195315

TLF :

TLX:

EML: IUE: : GRAGY ON SPAN

COM: 29

GRAHL BERNO H DR

MPI FUER RAOIOASTRONOMIE

AUF DEM HUEGEL 69

D 5300 BONN 1

GERMANY

TEL: 22573112

ILF :

TLX: 886440

EML:

COM: 
GRAINGER JOHN F OR

OPT OF PHYSICS

UMIST

BOX 88

MANCHESTER MGO $1 Q 0$

UK

TEL: $612 \quad 363 \quad 311$

TLF :

TLX: 666094

EML:

COM:

GRANVEAUT MICHEL

observatoIRE DE PARIS

61 AVE OBSERVATOIRE

LPIF

F 75014 PARIS

FRANCE

TEL: 143201210

TLF:

TLX: $270776085 \mathrm{~F}$

EML:

COM: $31 \mathrm{C}$

GRAY DAVIO F PROF

DPT OF ASTRONOMY

UNIV OF WESTERN ONTARIO

LONOON ON NGA $3 K 7$

CANADA

TEL: $519 \quad 6793184$

TLF: 5196613486

JLX: 0647134

EML: DFGRAYUWO.BITNET

COM: $29,36 \mathrm{C}$
GRANDI STEVEN ALORIDGE DR

NOAO

BOX 26732

$950 \mathrm{~N}$ CHERRY AVE

TUCSON AZ $85726 \quad 6732$

USA

TEL: 6023275511

TLF :

TLX:

EML:

COM: $2 \theta$

GRASDALEN GARY L DR

DPT PHYSICS \& ASTRONOMY

UNIVERSITY OF WYOMING

BOX 3905 UN STA

LARAMIE WY B207I

USA

TEL: $307 \quad 766 \quad 4385$

TLF:

TLX:

EML:

COM: $27,28.34$

GRAY PETER MURRAY
AAO.
BOX 296
EPPING NSW 2121
AUSTRALIA
IEL: 28681666
TLX: 23999 AAOSYO AA
EML:
TLF:
COM: 09

GREEN DAVIO DR

MULLARD RADIO. ASTRON OBS CAVENDISH LABORATORY

MADINGLEY RO

CAMBRIOGE CB 3 OHE

$U K$

TEL: 223337310

TLF: $223 \quad 354599$

TLX: 81292 CAVLAB 6

EML: DAGg@UK.AC.CAM. PHX

COM: 40

\section{GREEN RICHARO F OR}

KITT PEAK NIL OBS

BOX 26732

$950 \mathrm{~N}$ CHERRY AVE

TUCSON AZ $85726 \quad 6732$

USA.

TEL: 6023259299

TLF:

TLX: 0666484 AURA NOAO

EML:

COM:
GRANDPIERRE ATTILA DR

KONKOLY OBSERVATORY

THEGE U 13/17

$B 0 \times 67$

H 1525 BUDAPEST

HUNGARY

TEL: $175 \quad 5866 / 754122$

TLF: 11569640

TLX: 277460 KONOB H

EML: H697 KNO E ELLA.UUCP

COM: 10

GRATTON R G DR

OAR

VIA OELL' OSSERVATORIO

I 00040 MONTEPORZIO

ITALY

TEL: 69449019

TLF :

TLX:

EML: SPAN: 17468: :RAFFAELE

COM: 29.37

GRAYZECK EOWIN I OR

DPT OF PHYSICS

UNIVERSITY OF NEVADA

45055 MARYLAND PARKWAY

LAS VEGAS NV 89154

USA

TEL: 7027393507

TLF:

TLX:

EML:

EOM: 33

GREEN ELIZABETH M OR

MOUNT STROMLO \& SIDING

SPRING DBSERVATORIES

PRIVATE BAG

WOOEN PO ACT 2606

AUSTRALIA

TEL: $6288 \quad 1111$

TLF :

ILX: $62270 \mathrm{AA}$

EML:

COM: 37

\section{GREEN ROBIN $M$ DR}

DPT OF ASTRONOMY

UNIVERSITY OF. GLASGOW

GLASGOW GL2 800

UK

TEL: 413398855

ILF :

ILX: 778421

EML:

COM:
GRANT IAN $P$ DR

PEMBROKE COLLEGE

OXFORD OXI LOW

UK

TEL: $865 \quad 242 \quad 271$

TLF :

TLX:

EML:

COM: 14.36

GRAUER ALBERT O

OPT PHYSICS ASTRONOMY

UALR

33RD \& UNIVERSITY

LITTLE ROCK AR 72204

USA

TEL: 5015693275

TLF:

ILX:

EML:

COM: 25

GREBENIKOV E A PROF OR LOMONOSOV STATE UNIVER

117234 MOSCOW

RUSSIA

TEL:

TLF:

ILX:

EML:

COM: 07

GREEN JACK PROF

DPT OF GEOLOGY

CALIF STATE UNIVERSITY

LONG BEACH CA 90840

USA

TEL: $213 \quad 498 \quad 4809$

TLF :

$T L X$ :

EML:

COM: 16

GREEN SIMON $F$

PHYSICS LABORATORY

UNIVERSITY OF KENT CANTERBURY CT2 7NR

UK

TEL: $227764000 * 3780$

TLF: 227762616

ILX: 965449

EML:

COM: 15 


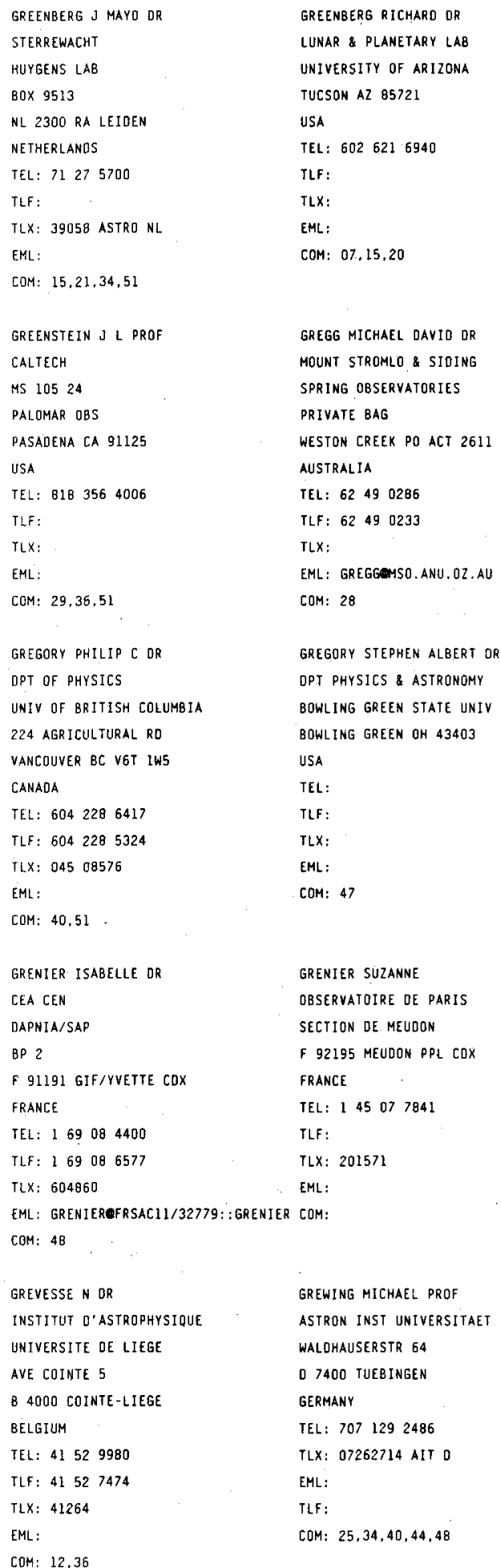

GREENHILL JOHN DR

OPT OF PHYSICS

UNIVERSITY OF TASMANIA

GPO BOX $252 C$

HOBART TAS 700.1

AUSTRALIA

TEL: 2202429

TLF: 2202410

TLX: AA 58150

COM: 48

GREGGIO LAURA DR

OPT OI ASTRONOMIA

UNIVERSITA OI BOLOGNO

CP 596

I 40125 BOLOGNA

ITALY

TEL: 51259413

TLF:

TLX: 520634 INFNBO 1

EML: SPAN/OECNET: 37928: : LAURA

COM: 35

GREGUL A YA DR

ASTRONOMICAL OBSERVATORY

KIEV STATE UNIVERSITY

OBSERVATORNAYA UL 3

$252053 \mathrm{KIEV}$

UKRAINE

TEL: 262391

TLF:

TLX:

EML:

COM:

GRENON MICHEL DR

OBSERVATOIRE DE GENEVE

CHEMIN DES MAILLETTES $5 \mathrm{I}$

CH 1290 SAUVERNY

SWITZERLAND

TEL: 227552611

TLF: 227553983

TLX: 419209 OBS CH

EML: grenonøobs, unige.ch

COM: $25,33,45 \mathrm{C}$

GREYBER HOWARD 0 OR

10123 FALLS RD

POTOMAC MO 20854

USA

TEL:

TLF:

TLX:

EML:

COM: $44,47,48$
GREENSTEIN GEORGE PROF

DPT OF ASTRONOMY

amHerst COLlege

AMHERST MA 01002

USA

TEL: 4135422075

TLF:

TLX:

EML:

GREGORINI LORETTA.

IST OI RADIOASTRONOMIA

CNR

VIA IRNERIO 46

1. 40126 BOLOGNA

ITALY

TEL: $51 \quad 23 \quad 2856$

TLF:

TLX: 211664 INFN BOI

EML:

COM: 40

GREISEN KENMETH I PROF

336 - FOREST HOME DR

ITHACA NY 14850

USA

TEL: 6072571650

TLF:

TLX:

EML:

COM: 48

GREVE ALBERT DR

IRAM

300 RUE DE LA PISCINE

F 38406 S MARTIN HERES CO

FRANCE

TEL: $76 \quad 824931$

TLF:

TLX:

EML:

COM:

GRIFFIN MATTHEW J DR

DPT OF PHYSICS

QUEEN MARY/WESTFIELD COLL

MILE END RO

LONDON EI. 4NS

UK

TEL: 19804811

TLF:

$T L X: 893750$

EML: MJG@UK. AC, QMC. STAR

COM: 
GRIFFIN RITA E M OR

INSTITUTE OF ASTRONOMY

THE OBSERVATORIES

MADINGLEY RD

CAMBRIOGE CB3 OHA

UK

TEL: 22362204

TLF :

TLX: 817297 ASTRON G

EML:

COM: 29

GRIFFITHS WILLIAM $K$

DPT OF PHYSICS

UNIVERSITY OF LEEDS

LEEOS LS2 9JT

UK

TEL:

TLF:

TLX:

EML:

COM: 37

GRINDLAY JONATHAN E DR

CENTER FOR ASTROPHYSICS

HCÓ/SAO .

60 GAROEN ST

CAMBRIBGE MA 02138

USA

TEL: 6174957204

TLF : 6174957356

TLX: 921428 SATELLITE CAM

EML: CFA: : "JOSHECFA255"/6699: : J0SH

COM: $06 P, 37,48$

GROSBOL PREBEN JOHNSON DR

ESO

KARL SCHWARZSCHILO STR 2

O B046 GARCHING MUENCHEN

GERMANY

TEL: $89320 \quad 06237$

TLX: 52828222 EOD

EML:

TLF: 893202362

COM: $05 \mathrm{C}, 09$

GROSSMANN-DOERTH U DR

KIEPENHEUER INSTITUT

FUER SONNENPHYSIK

SCHOENECKSTR 6

D 7800 FREIBURG BREISGAU

GERMANY

TEL: 76132864

TLF :

TLX: 7721552 KIS 0

EML:

COM:
GRIFFIN ROGER F OR

INSTITUTE OF ASTRONOMY

THE OBSERVATORIES

MADINGLEY RO

CAMBRIDGE CB3 OHA

UK

TEL: 22362204

TLF :

TLX: 617297 ASTRON $G$

EML: RFFIDUK, AC. CAM. PHX

COM: $05,29,30$

GRIGORJEV VICTOR M DR

SIBIZMIR

ACADEMY OF SCIENCES

664697 IRKUTSK 33

RUSSIA

TEL:

TLF:

TLX:

EML:

COM: 09

GRININ VLADIMIR P OR

CRIMEAN ASTROPHYS OBS UKRAINIAN ACAD OF STIENCE NAUCHNY

334413 CRIMEA

UKRAINE

TEL: 432945

TLF :

TLX:

EML:

COM: 27,36

GROSS PETER G PROF

714 OXFORD RO

BALA CYNWYO PA 19004

USA

TEL:

TLF :

EML:

COM:

TLX:

GROTEN ERWIN PROF

INST/PHYSIKALISCHE GEOO

PETERSENSTR 13

D 6100 DARMSTADT

GERMANY

TEL: $615 \quad 116 \quad 3109$

TLX: 419579

EML:

TLF :

COM: 19
GRIFFITH JOHN S PROF

DPT OF MATH SCIENCE

LAKEHEAD UNIVERSITY

THUNDER BAY ON P7B 5EI

CANADA

TEL: $B 073452121$

TLF :

TLX:

EML:

COM:

GRIJO DE OLIVEIRA A K OR OBSERVATORIO NACIONAL

RUA GL BRUCE 586

SAN CRISTOVAO

2092 I RIO OE JANEIRO RJ

BRAZIL

TEL: 215807181

TLF: $21580 \quad 0332$

TLX:

EML:

COM:

GRISHCHUK L P DR

STERNBERG STATE ASTR INST

UNIVERSITETSKIJ PROSP 13

119899 MOSCOW

RUSSIA

TEL: $139-50-06$

TLF :

TLX:

EML:

COM: 47

GROSSMAN ALLEN S PROF

ERWIN W FICK OBSERVATORY

IOWA STATE UNIVERSITY

AMES IA 50011

USA

TEL: 5152943666

TLF:

TLX:

EML:

COM:

GROTH EDWARD I 111

DPT OF PHYSICS

PRINCETON UNIVERSITY

JADWIN HALL

PRINCETON Nu 08544

USA

TEL: 6094524361

TLF :

TLX:

EML:

COM:
GRIFFITHS RICHARD E DR

STSCI

HOMEWOOD CAMPUS

3700 SAN MARTIN DR

BALTIMORE MD $2121 B$

USA

TEL: 3013384824

TLF:

TLX:

EML: scivax: griffiths

CDM: $09,28,44,48$

GRIMLEY PETER DR

OPT OF PHYSICS

ST PATRICK'S COLLEGE

MAYNOOTH

CO KILDARE

IRELAND

TEL: 1285222

TLF:

$T L X: 31493$

EML:

COM:

GRDOTE OETLEF

HAMBURGER STERNWARTE

GOJENSBERGSWEG 112

D 2050 HaMburg 80

GERMANY

TEL: 4072524112

TLF:

TLX:

EML:

COM:

gROSSMAN LAWRENCE PROF DPT GEOPHYSICAL SCIENEES UNIVERSITY OF CHICAGO

5734 S ELLIS AVE

CHICAGO IL 60637

USA

TEL: $312962 \quad 8153$

TLF :

ILX:

EML:

COM: 15,16

GROTH HANS G PROF OR

INST ASTRON \& ASTROPHYS

UNIVERSITAT MUENCHEN

SCHEINERSTR 1

D BOOO MUENCHEN 80

GERMANY

TEL: 89989021

TLF :

TLX:

EML:

COM: 29,36 


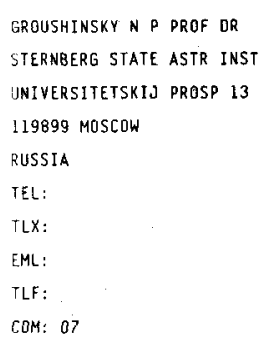

GRUBISSICH C PROF OR
VI AOSTA $34 / 5$
I 35142 PADOVA
ITALY
TEL: 4938301
ILF:
EML:
COM: 37
TLX:

GRUEN EBERHARO DR

MPI FUER KERNPHYSIK POSTFACH 103980

D 6900 HEIDELbERG I

GERMANY

TEL: $62 \quad 21516478$

ILX: 461666 MPIHO D

EML:

TLF :

COM: . 15C, 21,22

\section{GRYGAR JIRI OR}

INSTITUTE OF PHYSICS

CZECH ACADEMY OF SCIENCES

NA SLOVANCE 2

CS 18040 PRAHA 8

CZECHOSLOVAKIA

TEL:

TLF :

TLX:

EML: GRYGARECSPGASII

COM: 27,42

Gllbandy VADIM $S$ OR PULKOVO OBSERVATORY ACADEMY OF SCIENCES 10 KUTUZOV QUAY 196140 ST PETERSBURG RUSSTA

TEL: $297-94-81$

$$
\text { TLF : }
$$$$
\text { TLX: }
$$$$
\text { EML: }
$$$$
\text { COM: } 08
$$

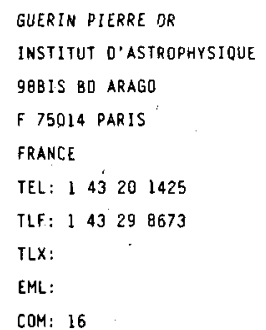

GRUDLER PIERRE

OCA CERGA

AVE COPERNIC

F O6LI3O GRASSE

FRANCE

TEL: 93365849

TLF:

ILX:

EML:

COM: 08,31

GRUENWALD RUTH DR

IAG

UNIVERSIDADE DE SAO PAULO

CP 30627

01051 SAO PALLO SP

BRAZIL

TEL: 115778599

TLF:

TLX: 36221 IAGM BR

EML:

COM:

GRZEOZIELSKI STANISLAW PR

SPACE RESEARCH CENTER

POLISH ACAD OF SCIENCES

UL ORDONA 21

PL OI $237^{\circ}$ WARSAW

POLAND

TEL:

TLF:

TLX:

EML:

COM: 49

GURMUNDSSON EINAR $K$

RAUNVISINDASTOFNUN

HASKOLANS

DUNHAGA 3

IS 107 REYKJAVIK

ICELAND

TEL: 121340

ILF:

TLX: 2307 ISINFO

E़ML:

COM: 47

GUERRERO GIANANTONIO DR

OSS ASTRONOMICO DI MILAND

VIA E BIANCHI 46

122055 MERATE

ITALY

TEL: 592035

TLF:

TLX:

EML:

COM: 27
GRUDZINSKA STEFANIA OR INSTITUTE OF ASTROMOMY N COPERNICUS UNIVERSITY UL CHOPINA $12 / 18$

PL 87 100 TORUN

POLAND

TELL: 20655

TLF :

TLX: 0552234 ASTR PI

EML:

COM: 15

GRUNDMANN WALTER

HERZBERG INST ASTROPHYS DOMINION ASTROPHYS OBS 5071 W SAANICH RD VICTORIA BC VBX $4 M 6$ CANADA

TEL: 6043883157

TLF: 6043630045

ILX: 0497295

EML:

COM: 09

GU XIAO-MA

YUNNAN OBSERVATORY

CAS

BOX 110

KUNMING 72946 YUNNAN

CHINA PR

TEL: 8712035

TLF:

TLX: 64040 YUOBS CN

EML:

COM: 10,12

GUDUR N OR

OBSERVATORY

EGE UNIVERSITY

BOK 21

35100 BORNOVA IZMIR

TURKEY

TEL: $5118 \quad 0110 * 2326$

TLF:

TLX:

EML:

COM:

GUERTLER JOACHIN OR UNIVERSITÁTS-STERNWARTE

SCHILLERGAESSCHEN 2

- 6900 JENA

GERMANY

IEL: 788222637

ILF: $78 \quad 822 \quad 2345$

TLX: 588634 UNI DO

EML:

COM: 34 
A118

GUEST JOHN E DR

UNIVERSITY OF LONDON OBS

MILL HILL PARK

LONDON NW7 2QS

uk

TEL: 19597367

ILX: 28722 UCPHYS

EML: ,

ILF:

COM: 16

GUIDICE DONALO A DR

A F GEOPHYSICS LABORATORY

SPAÇE PHYSICS OIV

HANSCOM AFB

BEDFORO MA 01731

USA

TEL: 6178613989

TLF:

TLX:

EML:

COM: 40

GULL STEPHEN F OR
MULLARD RADIO ASTRON OBS
CAVENOISH LABORATORY
MADINGLEY RO
CAMBRIOGE CB3 OHE
UK
TEL: 22366477
TLF:
TLX: 81292
EML:
COM: 40

\section{GULYAEY A P DR}

STERNBERG STATE ASTR INST UNIVERSITETSKIJ PROSP 13

119899 MOSCOW

RUSSIA

TEL: $139-19-70$.

TLX:

EML:

TLF:

COM: 08

GUD QUAN SHI

PURPLE MOUNTAIN OBSERV

CAS

NANJING

CHINA PR

TEL: 2546700

TLF:

ILX:

EML:

COM:
GUESTEN ROLF

MPI FUER RADIOASTRONOMIE

AUF DEM HUEGEL 69

O. 5300 BONN 1

GERMANY

TEL: $228 \quad 525379$

ILX:

EML:

TLF:

COM: $34.40 \mathrm{C}$

GUINAN EOWARD FRANCIS DR

DPT OF ASTRONOMY

VILLANOVA UNIVERSITY

VILLANOVA PA 19085

USA

TEL: $2155272100^{\circ}$

TLF:

TLX:

EML:

COM: $27,42 \mathrm{C}$

GULL THEODORE R DR
NASA/GSFC
CODE 680
LASP
GREENBELT MO 20771
USA
TEL: $301286 \quad 8060$
TLF:
TLX: $710-8289716$
EML:
COM: 34,44

COM: 34,44

GULYAEY RUDOLF A OR
IZMIRAN
ACADEMY OF SCIENCES
142092 TROITSK
RUSSIA
TEL:
TLF:
TLX:
EML:
COM:

GUPTA SUNIL $K$ DR
TIFR
HOMI BHABHA RO
COLABA
BOMBAY 400005
INOIA
TEL: $22 \quad 2152971 * 545$
TLF: $22 \quad 2152110$
TLX:
EML: GUPTASKOIIFRVAX. BITNET
COM: 33

COM: 33
GUETTER HARRY HENORIK US NAVAL OBSERVATORY

FLAGSTAFF STATION

BOX 1149

FLAGSTAFF AZ 86002

USA

TEL: $602 \quad 7795132$

TLF :

$T L X:$

EML:

COM: $25,37,45$

GUINOT BERNAFD R PROF

2 RUE DES SOUPIRS

F 77590 CHARTRETTES

FRANCE

TEL:

TLF :

TLX:

EML:

COM: $19,31 \mathrm{C}$

GULLIVER AUSTIN FRASER OR

DPT OF PHYSICS

UNIVERSITY OF BRANDON

2500 UNIVERSITY OR NW

BRANDON MB RTA GA9

CANALA

TEL: 2047289520

TLF :

TLX: 07502721

EML:

COM: 42

GUNN JAMES E PROF

OPT ASTROPHYSICAL SCI

PRINCETON UNIVERSITY

PEYTON HALL

PRINCETON NJ 085441001

USA.

TEL: 6094523802

TLF :

TLX:

EML:

COM: $28,47,48.51$

GURM KARDEV S PROF

OPT ASTRONOMY \& SPACE SCI

UNIVERSITY OF PANJABI

PATIALA 147002

INOIA

IEL: $73262 * 96$

TLF :

TLX:

EML:

COM: $27,35,46,51$
GUIBERT JEAN DR

OBSERVATOIRE OE PARIS

61 AVE OBSERVATOIRE

F 75014 PARIS

FRANCE

TEL: 140512098

TLF :

TLX: 270776 OBS F

EML:

COM: $05,09,24$

GULKIS SAMUEL OR

JPL

MS 169506

4800 DAK GROVE DR

PASADENA CA 91109

USA

TEL: 8183545708

TLF : $818 \quad 354.2946$

TLX:

EML:

COM: $16,40,51$

GULMEN OMUR OR

OBSERVATORY

EGE UNIVERSITY

BOX 21

35100 BORNOVA IZMIR

TURKEY

TEL: 51180110

TLF:

TLX:

EML:

COM:

GUO NEI-SHU DR

NANJING ASTRONOMICAL INSTRUMENT FACTORY

BOX 846

NANJING

CHINA PR

TEL: 25646191

ILF:

ILX: 34136

EML:

COM:

GURMAN JOSEPH, B DR

NASA/GSFC

CODE 602.6

GREENBELT MO 20771

USA

TEL: 301 2867599

TLF :

TLX: 89675

EML: SPAN: SOLMAX: : GURMAN

COM: 10 
GURSHTEIN ALEXANDER A DR INST HIST DF SCI \& TECHN ACADEMY OF SCIENCES STAROPANSKY $1 / 5$ 103012 MOSCOW RUSSIA

TEL:

TLF :

TLX:

EML:

COM: 16,41

GUSEINOV O H PROF INSTITUTE OF PHYSICS NARIMANOV UL 33 370143 BAKU AZERBAIDZHAN TEL: 39 3951

ILX:

EML:

ILF :

COM: $34,42,44,48$

GUSTAFSSON BENGT DR ASTRONOMICAL OBSERVATORY BOX 515

575120 UPPSALA

SWEOEN

TEL:

$T L X:$

EML:

ILF:

COM: $29 C, 3 E$

GWINN CARL R OR

OPT OF PHYSICS

UNIVERSITY OF CALIFORNIA

SANTA BARBARA CA 93106

USA

TEL: 8059612814

ILF: $805961 \quad 4170$

ILX:

EML: cgwinnevoodoo.ucsb.edu

COM: 40

HABIBULLIN SH T PROF OR

OPT OF ASTRONOMY

KAZAN STATE UNIVERSITY

LENIN UL 18

$42000 B$ KAZAN

RUSSIA

TEL: 323641

TLF :

TLX:

EML:

COM: 16
GURSKY HERBERT OR

NAVAL RESEARCH LABORATORY

COOE 4100 .

4555 OVERLOOK AVE SW

WASHINGTON DC 203755000

USA

TEL: 2027676343

TLF :

TLX

EML

COM: $27,42,44,48$

GUSEJNOV RAGIH EH DR

SHEMAKHA ASTROPHYSICAL

OBSERVATORY

AZER ACAOEMY OF SCIENCES

373243 SHEMAKHA

AZERBAIDZHAN

TEL:

TLF :

TLX:

EML:

COM:

GUTCKE DIETRICH

CARL-ZEISS STR 1

O 6900 JENA

GERMANY

TEL:

TLF :

EML:

COM: 09

TLX:

GYLDENKERNE KJELD DR

COPENHAGEN UNIVERSITY OBS

BRORFELOEVEJ 23

OK 4340 TOLLOSE

DENMARK

TEL: $53 \quad 48 \quad 8195$

TLF: $58 \quad 48 \quad 8755$

TLX: 44155

EML:

COM: 33,42

HABING H J DR

STERREWACHT

B0X 9513

NL 2300 RA LEIDEN

NETHERLANDS

TEL: 71272727

TLX: 39058

EML:

TLF:

COM: $33,34 \mathrm{P}$
GURTOVENKO E A OR

MAIN ASTRONOMICAL OBS

UKRAINIAN ACAO OF SEIENCE

GOLOSEEVO

$252127 \mathrm{KIEV}$

UKRAINE

TEL: $66 \quad 1065$

TLF :

TLX: 131406

EML:

COM: 10,12

GUSSMANN E A DF

ZNTRLINST F ASTROPHYSIK

STERNWARTE BABALSBERG

ROSA-LUXEMBURG-STR 17A

D 1502 POTSDAM

GERMANY

TEL:

TLF :

TLX:

EML:

COM: 36

GUTHRIE BRUCE N G DR

ROYAL OBSERVATORY

BLACKFORD HILL

EOINBURGH EHS 3 HJ

TEL: $316 \quad 673 \quad 321$

TLF :

TLX: 72383 ROEOIN G

EML:

COM: 29

HABBAL SHADIA RIFAI

CENTER FOR ASTROPHYSICS

$\mathrm{HCO} / \mathrm{SAO}$

60 GARDEN ST

CAMBRIDGE MA 02138

USA

TEL: $617495 \quad 7348$

TLF :

TLX: 921428

EML:

COM: 49

HACHENBERG OTTO PROF OR RADIOASTRONOMISCHES INST UNIVERSITAET BONN

AUF DEM HUEGEL 71

D 5300 BONN 1

GERMANY

TEL:

TLF :

TLX:

EML:

COM:
GURZAOIAN G A PROF OR BVURAKAN ASTROPHYSICAL OBSERVATORY

378433 ARMENIA

ARMENIA

TEL: $8 B \quad 52 \quad 28 \quad 3453$

ILF :

TLX:

EML:

COM: $28,34,45$

GUSTAFSON BO A S

DPT OF ASTRONOMY

UNIVERSITY OF FLORIDA

211 SSRB

GAINESVILLE FL 32611

USA

TEL: 9043927677

TLF: 9043925089

TEX:

EML: gustafeastro.ufi.edu

COM: $15,21,22$

GUTIERREZ-MORENO A OR MRS DPT DE ASTRONOMIA

UNIVERSIDAD DE CHILE

CASILLA 36 D

SANTIAGO

CHILE

TEL: $2 \quad 229 \quad 4101 / 4002$

TLF :

TLX: 440001

EML:

COM: 25

HABE ASAO

DPT OF PHYSICS

HOKKAIDO UNIVERSITY

KITA 10 NISHI 8

SAPPORO 060

JAPAN

TEL: $11-711-2111$

TLF:

TLX:

EML:

[OM: 33

HACHISU IZUMI OR DPT AERONAUTICAL ENGINEER KYOTO UNIVERSITY

KITASHIRAKAWA SAKYO KU KYOTO 606

JAPAN

TEL: 075-751-2111

TLF:

TLX: 05422693 LIBKYU J

EML:

COM: 35 


\section{HACK MARGHER ITA PROF}

OAT

BOX SUCC TRIESTE. 5

VIA TIEPOLO 11

I 34131 TRIESTE

ITALY

TEL: 40793921

TLF:

TLX: 461137 DAT I

EML:

COM: $29,36,44,45$.

HADJIDEMETRIOU JOHN O

DPT THEORET MECHANICS

UNIVERSITY THESSALONIKI

GR 54006 THESSALONIKI

GREECE

TEL: 31991410

TLF :

ILX:

EML:

COM: 07

HAEMEEN ANTTILA KAARLE A

DPT OF ASTRONOMY

UNIVERSITY OF OULU

SF 90570 OULU 57

FINLAND

TEL:

TLF : $81 \quad 56 \quad \overline{1278}$

TLX:

EML:

COM:

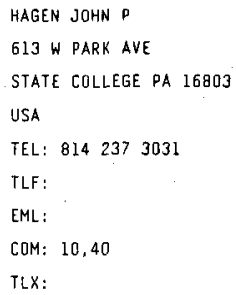

HACKWELL JOHN A DR AEROSPACE CORPORATION BDX 92957

LOS ANGELES CA 90009

USA

TEL: $307766 \quad 6296$

TLX:

EML:

TLF:

COM: 27,34

\section{HADLEY BRIAN W \\ ROYAL OBSERVATORY \\ BLACKFORO HILL \\ EDINBURGH EH9 $3 \mathrm{HJ}$ \\ U.K \\ TEL: $316 \quad 688 \quad 296$ \\ TLX: 72383 ROEDIN G \\ EML: BWHUK.AC.ROE.STAR, \\ TLF: \\ COM: 09}

HAENSEL PAWEL GR

COPERNICUS ASTRON CENTER

POLISH ACAD OF SCIENCES

UL BARJYCKA 18

PL 00716 WARSAW.

POLANO

TEL: 410828

TLF :

ILX: 113970 ZAPLAN PL

EML:

$\mathrm{COM}$

HAGEN-THORN VLAOIMIR A OR ASTRONOMICAL OBSERVATORY ST PETERSBURG UNIVERSITY BIBLIOTECHNAJA PL 2 198904 ST PETERSBURG RUSSIA

TEL: 257.94 .91

ILF:

TLX:

EML:

COM: 28

HAHN GERHARD J DR
ASTRONOMICAL OBSERVATORY
BOX 515
$S 75120$ UPPSALA
SWEDEN
TEL: 18525724
TLX: 76024 UNIVUPS S
EML: GERHARDLABAN. SUNET.SE
TLF: 18527583
COM: 20

HACYAN SHAHEN DR

HADOOCK FREO T DR

INSTITUTO OE ASTRONOMIA

UNAM

APOO POSTAL 70-264

04510 MEXICO DF

MEXICO

TEL: $905-548-5305$

TLF :

TLX: 1760155 CICME

EML:

COM: 47

\section{HAORAVA PETR}

ASTRONOMICAL INSTITUTE

CZECH ACADEMY OF SCIENCES

ONDREJOV OBSERVATORY

CS 25165 ONDREJOV

CZECHOSLOVAKIA

TEL: 20485201

TLF: 20485314

TLX:

EML:

COM: 42

HAERENDEL G OR

MPI F. PHYSIK \& ASTROPHYS

INST F. EXTRATERR. PHYSIK

0 8046 GARCHING MUENCHEN

GERMANY

TEL: 8932993516

TLF: 8932993569

TLX: 05215845 XTER D

EML:

COM:

HAGFORS T OR
NAIC
CORNELL UNIVERSITY
SPACE SCIENCES BLDG
ITHACA NY 148536801
USA
TEL: 6072563734
TLF:
TLX: 932454
EML:
COM: 16

HAIKALA LAURI $K$

OBS \& ASTROPHYSICS LAB UNIVERSITY BF HELSINKI

TAEHTITORNINMAKI

SF 00130 HELSINKI 13

FINLAND

TEL: 1912948

TLF :

TLX: 124690 UNIH SF

EML:

COM

TEL: $415 \quad 858 \quad 4073$
DPT OF ASTRONOMY

UNIVERSITY OF MICHIGAN

OENNISON BLOG

ANN ARBOR MI 48109 1090

USA

TEL: 3137643430

TLF :

TLX:

EML:

COM: $40,44,51$

HAEFNER REINHOLD DR

UNIVERSITAETS STERNWARTE

SCHEINERSTR 1

D BOOO MUENCHEN BO

GERMANY

TEL: 89989021

TLF :

TLX:

EML:

COM: 27

HAGEN HANS-JUERGEN DR

HAMBURGER STERNWARTE

GOJENSBERGSWEG 112

(1) 2050 HAMBURG BO

GERMANY

TEL: 4072524136

TLF: $407252 \quad 4198$

TLX: 217884

EML:

COM: 47

HAGIO FUMIHIKO DR XUMAMOTO INST TECHNOLOGY

IKEOA 4

RUMAMOTO

JAPAN

TEL: 963263111

TLF: 963263000

TLX:

EML:

COM: 28

HAISCH BERNHARD MICHAEL LOCKHEEO PALO ALTO RES LB DPT 9120 BLOG 255

3251 HANOVER ST

PALO ALTO CA 94304

USA

TLF :

TLX: 346409

EML:

COM: $27,36,51$ 


HAJDUK ANTON OR
ASTRONOMICAL INSTITUTE
SLOVAK ACADEMY SCIENCES
CS CS 842 28 BRATISLAVA
CZECHOSLOVAKIA
TEL: 7375157
TLF:
TLX: 93373 SEIS
EML:
COM: $15,22,51$

HALL AMOREW NORMAN

OPT OF ASTROPHYSICS

UNIVERSITY OF OXFORD

SOUTH PARKS RD

OXF0RO OX1 3RO

UK

TEL:

TLF:

TLX:

EML:

COM: $4 B$

HALL R GLENN DR

3612 SPRING ST

CHEVY CHASE MD 20815

USA

TEL: 3016527221

TLF:

EML:

COM: 19.31

TLX:

HAMADA TETSUO PROF

DPT OF PHYSICS

IBARAKI UNIVERSITY

BUNKYO

MIT0 310

JAPAN

TEL: $0292-252-35-24$

TLF :

TLX:

EML:

COM:

HAMILTON P A DR
OPT OF PHYSICS
UNIVERSITY OF TASMANIA
GPO BOX $252 C$
HOBART TAS 7001
AUSTRALIA
TEL: 2202419
TLF:
TLX: 58150
EML:
COM: 40

HAJDUKOVA MARIA

DPT OF ASTRONOMY

COMENIUS UNIVERSITY

MLYNSKA DOLINA

CS B42 15 BRATISLAVA

CZECHOSLOVAKIA

TEL: 7320003

TLF :

TLX:

EML:

COM: 22

HALL DONALD N OR

INSTITUTE FOR ASTRONOMY

UNIVERSITY OF HAWAII

2680 WOODLAWN OR

HONOLULU HI 96822

USA

TEL: 8089488312

ILF:

TLX: 723-8459

EML:

COM:

HALLAM KENNETH L OR

701 E CAPITOL ST SE WASHINGTON DC 20003

USA

TEL:

TLF:

EML:

COM: 09,44,45

IŁX:

HAMAJIMA KIYOTOSHI DR

KISO OBSERVATORY

UNIVERSITY OF TOKYO

MITAKEMURA KISOGUN

NAGANO 39701

JAPAN

TEL:

ILF:

TLX:

EML:

COM: 33

HAMMAN WOLF-RAINER
INST THEOR PHYS \& STERHW
UNIVERSITAET KIEL
OLSHAUSENSTR
O $2300 \mathrm{KIEL} 1$
GERMANY
TEL: 4318804110
TLF:
TLX: 292706
EML:
COM: 36

HAKKILA JON ERIC OR

DPT MATHS ASTRONOMY \& STA

MANKATO STATE UNIVERSITY

BOX 41

MANKATO MN 56002 B400

USA

TEL: $507389 \quad 1840$

TLF :

TLX:

EML: JHAKKMYSUSI

COM: 33

HALL DOUGLAS S DR

DYER OBSERVATORY

VANDERBILT UNIVERSITY

NASHVILLE TN 37235

USA

TEL: : 6153734897

TLF:

TLX: 554323

EML:

COM: $25,27,36,42$

HALLIDAY IAN DR

HERZBERG INST ASTRGPHYS

NTL RESEARCH COUNCIL

100 SUSSEK OR

OTTAWA ON KLA ORG

CANADA

IEL: 6139909704

TLF: 6139526602

rLX: 0533715

EML:

COM: $15,16,21,22$

HAMOY M A M PROF

HELWAN OBSERVATORY

HELWAN

EG.YPT

TEL: 78 0645/2683

TLF:

TLX: 93070 HIAG UN

EML:

COM: 27

HAMMER FRANCOIS OR

OBSERVATOIRE DE PARIS

SECTION DE MEUDON

DAEC

F 92195 MEUDON PPL CDX

FRANCE

TEL: 145077408

TLF: 145072806

TLX:

EML: HAMMER ERMEUSI

COM: 28
HALBWACHS JEAN LOUIS DR

OBS DE STRASBQURG

11 RUE UNIVERSITE

F 67000 STRASBOURG

FRANCE

TEL: 88358200

ILF: $88 \quad 250160$

TLX: 890506

EML: EARN: UD1103बFRCCSC21

COM: 26,30

HALL PETER J DR

CSIRO AUSTRALIA TELESCOPE

NATIONAL FACULTY

BOK 94 ;

NARRABRI NSW 2390

AUSTRALIA

TEL: 67 959205

ILF: 67959255

$T \perp X$ :

EML: PHALLOATNF.CSIRO.AU

COM: 40

HAMABE MASARU OR

KISO OBSERVATORY

UNIVERSITY OF TOKYO

MITAKEMURA KISOGUN

NAGAND 39701

JAPAN

TEL: $26452-3360$

TLF :

TLX: 3347577 KSOOBS J

EML:

COM: 28

HAMIOS EL DIN PROF

DPT OF ASTRONOMY

FACULTY OF SCIENCES

CATRO UNTYERSITY

GEZA.

EGYPT

TEL:

TLF:

TLX:

EML:

COM: 07

HAMMER REINER

KIEPENHEUER INSTITUT FUER SONNENPHYSIK

SCHOENECKSTR 6

D 7800 FREIBURG BREISGAU GERMANY

TEL: 76132864

TLF :

TLX: 7721552 KIS D

EMt:

-COM: 10,12 
HAMMERSCHLAG ROBERT H DR STERREKUNDIG INSTITUTE BOX 80000

NL 3508 TA UTRECHT NETHERLANDS

TEL: 30535218

TEX: 40048 FYLUT, NL

EML: BITNET: WNMMAILOHUTRUUD

TLF

COM: 09

HAN FU
PURPLE MOUNTAIN OBSERV
CAS
NANJING
CHINA PR
TEL: 2533738
TLF:
TLX: 34144 PMONJ CN
EML:
COM: 40

HANAMI HITOSHI OR

CQLL HUMANITIES SOCIAL SC IWATE UNIVERSITY

UEDA 3

MORIOKA 020

JAPAN

TEL: $0196 \quad 235171 * 2284$

TLF: 0196542289

TLX:

EML: 012697@PNKUDPC

COM: $28,33,34$

HANBURY BROWN ROBERT PROF

WHITE COTTAGE

PENTON MEWSEY

HANTS SPII ORQ

UK

IEL: $264772 \quad 334$

TLF :

TLX:

EML:

COM: 40

HANG HENG-RONG

PURPLE MOUNTAIN OBSERV

CAS

NANJING

CHINA PR

TEL: 2533583

$T L F$ :

TLX: 34144 PMONJ CN

EML:

COM: 44,48
HAMMERSCHLAG-HENSBERGE G ASTRONOMICAL INSTITUTE UNIVERSITY OF AMSTERDAM

KRUISLAAN 403

NL 1098 SJ AMSTERDAM

NETHERLANDS

TEL: $205257491 / 7492$

TLF: 205257484

TLX: 10262 HEF NL

EML: A4IOINSTEHASARALI.BITNET

COM: 42

HAN İIANQI

INSTITUTE OF GEODESY \&

GEOPHYSICS

XU DONG LU

WUCHANG HUBEI

CHINA PR

TEL: $81 \quad 3712 \star 570$

TLF :

TLX:

EML:

COM: 19.31

HANAOKA YOICHIRO OR

NOBEYAMA RADIO OBS

NADJ

MINAMIMAKI MURA

NAGANO 38413

JAPAN

TEL: : $81 \quad 267 \quad 63 \quad 4381$

TLF: 81267.982506

TLX: 3329005 NADNRO J

EML: E52863)JPNKUDPC.BITNET

COM: 10

HANDA TOSHIHIRO QR

INST ASTRO FAC SCIENCE

UNIVERSITY OF TOKYO

OSAWA MITAKA

TOKYO 181

JAPAN

TEL: $42241 \quad 3735$

TLF: $42241 \quad 3749$

TLX: 2822307 TAOMTK J

EML:

COM: 28,40

HANISCH ROBERT J OR

STSCI

HDMEWOOD CAMPUS

3700 SAN MARTIN DP

BALTIMORE ML 21218

USA

TEL: $301 \quad 338 \quad 4910$

TLF :

TLX: 6849101

EML: SPAN: SCIVAX: :HANISCH

COM: $05,09,40$
HAMMOND GOROON L OR

OPT OF MATHEMATICS

UNIVERSITY OF S FLORIDA

ASTRONOMY PROGRAM

TAMPA FL 33620

USA

IEL: 8137831226

TLF:

TLX:

EML: OKGAWAAECFRVM.BITNET

COM: 35

HAN WENJUN

BEIJING ASTRONOMICAL OBS

CAS

W SUBURB

BEIJING 100080

CHINA PR

TEL: 1281698

TLF :

TLX:

EML:

COM: $40^{\circ}$

HANAS2 JAN DR

INSTITUTE OF ASTRONOMY

$N$ COPERNICUS UNIVERSITY

UL CHOPINA $12 / 18$

PL 87 IOO TORUN

POLANO

TEL: $260-37$

TLF:

TLX: 0552234 ASTR PL

EMt:

COM: 10,40

HANOLIROVA DAGMAR DR

$N$ COPERNICUS OBSERVATORY

\& PLANETARIUM

KRAVI HORA

CS 616 DO BRNO

CZECHOSLOVAKIA

TEL: $574 \quad 4374$

TLF:

TLX:

EML:

COM:

HANKINS TIMOTHY HAMILTON OPT OF PHYSICS \& ASTRON NEW MEXICO TECH

CAMPUS STATION

SOCORRO NM B B 7801

USA

TEL: 5054768011

TLF:

TLX:

EML:

COM: 40
HAMZAOGLU ESAT E H OR

KING SAUO UNIVERSITY

COLLEGE OF SCIENCE

BOX 2455

RIYAOH 11453

SAUDI ARABIA

IEL:

TLF:

TLX:

EML:

COM:

HAN ZHENG-ZHONG

PURPLE MOUNTAIN OBSERV

CAS

NANJING

CHINA PR

TEL: 2533583

TLF :

TLX: 34144

EML:

COM: 44

HANAWA TOMOYUKI OR

DPT OF ASTROPHYSICS

NAGOYA UNIVERSITY

FUROCHO CHIKUSA KU

NAGOYA 464 Ol

JAPAN

TEL: 527816769

TLF:

TLX: 4477323

EML: BITNET: B42287@JPNXUDPC

COM: 42

HANES OAVID A DR

OPT OF PHYSICS

QUEEN'S UNIVERSITY

KINGSYON ON K7L 3NG

CANADA

TEL: 6135475750

TLF : 6135456463

TLX:

EML:

COM: 37

HANNER MARTHA S DR

JPL

MS 183601

4800 DAK GROVE DR

PASADENA CA 91109

USA

TEL: 8:B $354 \quad 4100$

TLF:

TLX: 675429

EML: SPAN:JPLSCB::MSH

COM: $15,21 \mathrm{P}, 22$ 


HANSEN CARL J PROF
JILA
UNIVERSITY OF COLORADO
BOX 440
BOULDER CO 803090440
USA
TEL: 3034927811
TLF:
TLX: 755842 JILA
EML:
COM: 27
HANSON ROBERT B OR
LICK OBSERVATORY
UNIVERSITY OF CALIFORNIA
SANTA CRUZ CA 95064
USA
TEL: 408 429 2755
TLF:
TLX:
EML:
COM: 24

HAO YUN-XIANG
OPT OF ASTRONOMY
BEIJING NORMAL UNIVERSITY
BEIJING 100875
CHINA PR
TEL: $1656531 * 6285$
TLF:
TLX:
EML:
COM: 09

HARA TETSUYA OR
OPT OF PHYSICS
KYOTO SANGYO UNIVERSITY
KAMIGAMO
KYOTO 603
JAPAN
TEL: $075-701-2151$
TLF:
TLX: 5422661 KSU J
EML:
COM: 28
HARMANEC PETR OR
ASTRONOMICAL INSTITUTE
CZECH ACADEMY OF SCIENCES
OHOREJOY O8SERVATORY
CS 251 65 ONDREJOV
CZECHOSLOVAKIA
TEL: 204 B5201
ILF: 204 85314
TLX: 121579
EML:
COM: 27,42

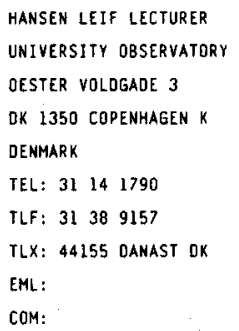

HANSSON NILS OR
LUND OBSERVATORY
BOX 43
S 221 OO LUND
SWEOEN
TEL: 46107000
TLX: 33533 LUNIVER S
EML:
TLF:
COM:

HAPKE BRUCE W OR DPT GEOL \& PLANETARY SCI UNIVERSITY OF PITTSBURGH 321 OLD ENGINEERING HALL PITTSBURGH PA 15235

USA

TEL: $412 \quad 624 \quad 4719$

TLF:

TLX:

EML:

COM: 15

HARDEBECK ELLEN G OR
3106 TUMBLEWEEO RD
BISHOP CA 93514
USA
TEL:
TLF:
EML:
COM: 34
TLX:-

HARMER CHARLES F W MR
NOAD
BOX 26732
950 W CHERRY AVE
TUCSON AZ 857266732
USA
TEL: 6023275511
TLF:
TLX: $0666-484$ AURA NOAO
EML:
COM: 09,29

HANSEN RICHARD T MR

ENGINEERING 138

VAMC

150 S HUNTINGTON AVE

BOSTON MA 02130

USA

TEL: $617 \quad 734 \quad 2534$

TLF :

TLX:

EML:

COM: 10

HANTZIOS PANAYIOTIS DR ASTRONOMICAL INSTITUTE NTL OBSERVATORY OF ATHENS BOX 20048

GR 118 ID ATHENS

GREECE

TEL: 13461191

TLF :

TLX:

EML:

COM: 42

HARA KEN NOSUKE DR SECOND TECHNICAL HIGH SCH MAYAGINO KU SENDAI 983

JAPAN

TEL:

TLF :

TLX:

EML:

COM: 31,47

HARDEE PHILIP

DPT OF PHYSICS \& ASTRON

UNIVERSITY OF ALABAMA

BOX 1921

UNIVERSITY AL $35487 \quad 0324$

USA

TEL: $205348 \quad 5050$

TLF :

TLX:

EML:

COM: 40

HARMER OIANNE L MRS

NOAO

BOX 26732

950 N CHERRY AVE

TUCSON AZ 857266732

USA

TEL: $602 \quad 3259218$

TLF :

TLX:

EML: DIHARMERONOAD.EQU

COM: 09.29
HANSLMEJER ARNOLD

INSTITUT FUER ASTRONOMIE

KARL-FRANZENS-UNIVERSITAT

UNIVERSITAETSPLATZ 5

A 8010 GRAZ

AUSTRIA

TEL: $316 \quad 3805275$

TLF :

TLX:

EML:

COM: 07,10

HANUSCHIK REINHARO OR ASTRONOMISCHES INSTITUT RUHR UNIVERSITAT BOCHUM POSTFACH 102148

o 4630 BOCHUM 1

\section{GERMANY}

TEL: 2347003450

TLF :

TLX: 0825860

EML:

COM: 29 .

HARA TADAYOSHI DR

NTL ASTRONOMICAL OBS

DIV OF EARTH ROTATION

MIZUSAWA SHI

IWATE 023

JAPAN .

TEL: $197 \quad 247111$

TEF :

TLX: 837628 ILSMIZ J

EML:

COM:

HAROY EQUARDO

DPT DE PHYSIQUE

UNIVERSITE LAVAL

FAC SCIENCES \& GENIE

LAVAL QC GIK 794

CANADA

TEL: 4186562960

TLF: $418 \quad 6562040$

YLX: 051. 31621

EML:

COM: 28,47

HARMS RICHARD JAMES DR APPLIEO RESEARCH CORP

8201 CORPORATE DR

SUITE 920

LANDOVER MD 20785

USA

TEL: 3014598442

TLF :

ILX:

EML:

COM: $28,44,47$ 
HARNDEN FRANK R JT CENTER FOR ASTROPHYSICS HCO/SAO

GO GARDEN ST

CAMBRIOGE MA 02138

USA

TEL: $617495 \quad 7143$

TLF

TLX: 921428 SATELLITE CAM EML:

COM:

HARRINGTON 3 PATRICK OR ASTRONOMY PROGRAM UNIVERSITY OF MARYLANO COLLEGE PARK MD 20742 USA

TEL: 3014545944

TLF :

TLX: 7108260352

EML:

COM: 34

HARRIS DANLEL E DR

CENTER FOR ASTROPHYSICS

HCO/SAO

60 GARDEN ST

CAMBRIOGE MA 02138

USA

TEL: 6174957148

TLF :

TLX: 921428

EML:

COM: 40

HARRIS WILLIAM E DR

DPT OF PHYSICS

MCMASTER UNIVERSITY

HAMILTON ON LES $4 M I$

CANABA

TEL: $416 \quad 525 \quad 9140$

TLF : $416 \quad 546 \quad 1252$

TLX: $061 \quad 8347$

EML:

COM: 37

HART MICHAEL H OR

7301 MASONVILLE OR

ANNANDALE VA 22003

USA

TEL:

TLF :

EML:

COM: : 51

TLX:
HARNETT JULIEINE OR

SCHOOL OF PHYSICS

UNIVERSITY OF SYDNEY

SYDNEY NSW 2006

AUSIRALIA

TEL: $2692 \quad 2727$

TLF: 26602903

TLX: AA 26169

EML: JHATNETTEROBIN.RP.CSIRO, AU

COM: 28,40

HARRINGTON ROBERT S OR

US NAYAL OBSERVATORY

34 \& MASSACHUSETTS AVE NW

WASHINGTON OC 203925100

USA

TEL: $202 \quad 653 \quad 1533$

TLX:

EML:

TLF :

COM: $20,24,26,51$

HARRIS GRETCHEN L H OR

OPT OF PHYSICS

UNIVERSITY OF WATERLDO

WATERLOO ON N2L $3 G 1$

CANADA

TEL: 5198851211

TLF: $519 \quad 746 \quad 8115$

TLX: 06955259

EML: glharriseastro.waterloo. edu COM: $37 \mathrm{C}$

\section{HARRISON EOWARD R PROF}

DPT PHYSICS \& ASTRONOMY

UNIV OF MASSACHUSETTS

GRC

AMHERST MA 01003

USA

TEL: 4135452194

TLF :

TLX:

EML:

COM: 47,5 !

HARTEN RONALO H OR

RCA ASTRO ELECTRONIC

TB-1

BOX 800

PRINCETON NJ 08540

USA

TEL: 6094263551

TLF :

TLX:

EML :

COM: 34,40
HARPAZ AMOS DR

DPT OF PHYISICS/SPACE RES

IIT

TECHNION CITY

HAIFA 32000

ISRAEL

TEL : $4293 \quad 521$

TLF

TLX:

EML: BITNET: PHRB9AH@TECHNION

COM:

HARRIS ALAN WILLIAM

MPI FUER PHYS H ASTROPHY

INST FUER EXTRATER PHYSIK

D 8046 GARCHING MUENCHEN

GERMANY

TEL: 8932990

TLF: 8932993235

TLX: 05215845 XTERR D

EML: SPAN: $19457:$ : AWH

COM: 16,34

HARRIS HUGH C

US NAVAL OBSERVATORY

FLAGSTAFF STATION

POX 1149

FLAGSTAFF AZ 86002

USA

TEL: 6027795132

TLF :

TLX:

EML:

COM: 37

HARR ISON RICHARD A DR RUTHERFORO APPLETON LAB SPACE \& ASTROPHYSICS DIV BLOG R25/R68

CHILTON DIOCOT OXI1 OOX UK

TEL: 235446497

TLF :

TLX: 83159 RUTHLB G

EML: SPAN: $19457:$ : RAH

COM:

HARTKOPF WILLIAM I OR

CHARA

GEORGIA STATE UNIVERSITY

ATLANTA GA 303033083

USA

TEL: $404 \quad 6512932$

TLF :

TLX:

EML:

COM: $24,26,33$
HARPER DAVID DR

ASTRONOMY UNIT

QUEEN MARY/WESIFIELD COLL MILE END RD

LONDON EI ANS

TEL: 1719755492

TLF : 1819819587

TLX: 893750

EML: ADHOSTAR, QMW, AC,UK

COM: 20

HARRIS ALAN WILLIAM DR

JPL

MS 183501

4800 OAK GROVE DR

PASADENA CA 91109

USA

TEL: $818 \quad 354 \quad 6741$

TLF: $818 \quad 3540966$

TLX: 675429/91058B3294/69

EML: AWHARRISUPLGP.JPL. NASA.GO

COM: $15 P, 20$

HARRIS STELLA

OPT OF PHYSICS

QUEEN MARY/WESTFIELO COLL

MILE END RD

LONDON EI 4NS

UK.

TEL: $19804811 * 4050$

TLF:

TLX: 893750

EML:

COM: 34

HARROWER GEORGE A OR 2041033 BELMONT AVENUE

VICTORIA BC VBS $3 T 4$

CANADA

TEL:

TLF :

TLX:

EML:

COM:

HARTL HERBERT DR

INSTITUT FUER ASTRONOMIE

TECHNIKERSTR 15

A 6020 INNSBRUCK

AUSTRIA

TEL: 5222.7485263

ILF: 52227485252

TLX: 533808 UNITE A

EML: [706059AINLNIOI

COM: 34 
HARTLEY KENNETH F DR RUTHERFORD APPLETON LAB SPACE \& ASTROPHYSICS DIV BLDG R25/R6B

CHILTON OIOCOT OXII OQX UK

TEL: $235 \quad 21900$

TLF:

TLX: 83159

EML:

COM:

HARTQUIST THOMAS WILBUR MPI FUR PHYS \& ASTROPHYS KARL-SCHWARZSCHILO-STR I D 8046 GARCHING MUENCHEN GERMANY

TEL: $B 93299838$

TLX: 05215845 XTERR O

EML:

TLF:

COM: 34

HARVEL CHRISTOPHER ALVIN 6161 STEVEN'S FOREST RD COI.UMB IA MO 21045

USA

TEL: 3019640211

TLF

EML:

COM: 05.37

TLX:

HARVEY PAUL MICHAEL OR ASTRONOMY OPT UNIVERSITY OF TEXAS

RLM 15308

AUSTIN TX $78712 \quad 1093$

USA

TEL: 512,4714461

$T L F$ :

ILX: 910-874-1351

EML:

COM: 34,44

HASAN SAIYID STRAJUL

INOIAN INSTITUTE OF

ASTROPHYSICS

KORAMANGALA

BANGALORE 560034

INDIA

TEL: $8 L 256 \quad 6585$

TLF:

$I \perp X$ :

EML:

COM: 10
HARTMANN LEE WILLIAM CENTER FOR ASTROPHYSICS

HCO/SAO

60 GARDEN ST

CAMBRIDGE MA 02138

USA

TEL: 6174957487

TLF:

TLX:

EML:

COM: 29,36

HARTWICK F DAVID A OR

DPT OF PHYSICS

UNIVERSITY OF VICTORIA

BOX 1700 .

VICTORIA BC VOW 2 Y2

CANADA

TEL: 6047217742

TLF: 6047217715

TLX:

EML:

COM:

HARVEY CHRISTOPHER C DR OBSERVATOIRE DE PARIS

SECTION DE MEUDON

F 92195 MEUOON PPL CDK

FRANCE

TEL: $145 \quad 077669$

TLF :

ILX: 204464

EML: MEUDON: : HARVEY

COM: 44.49

HARWIT MARTIN PROF

OPT OF ASTRONOMY

CORNELL UNIVERSITY

SPACE SCIENCES BLOG

ITHACA NY 14853

USA

TEL: 6072564805

TLF :

TLX:

EML:

COM: $15,21,48$

HASCHICK AUBREY
HAYSTACK OBSERVATORY
WESTFORO MA 01886
USA
TEL: 6176924764
TLF:
EML:
COM: 40
TLX:

HARTMANN WILLIAM $K$ PLANETARY SCIENCE INST 2030 E SPEEDWAY

SUITE 201

TUCSON AZ 85719

USA

TEL: 6028810332

TLF:

TLX:

EML:

COM: 15

HARTZ THEOOORE R DR 915 MOUNTAINVIEW AVENUE OTTAWA ON K2B $5 G 3$ CANADA

TEL: $613 \quad 596 \quad 1211$

TLF:

TLX:

EML:

COM: 40,44

HARVEY GALE A OR INST F GESCHICHTE D NATURWISSENSCHAFTEN GOETHE UNIVERSITAET D 6000 FRANKFURT GERMANY

TEL:

$T L F$ :

$T L X$ :

EML:

COM: 22

HARWOOD DENNIS MR

PERTH OBSERVATORY

BICKLEY WA 6076

AUSTRALIA

TEL: 92938255

TLF :

ILX:

EML:

COM: 24.25

HASEGAWA ICHIRO OR

4-18-5 FUJIWARADAI KITA KIYA KU

KOBE 651-13

JAPAN

TEL: $8178 \quad 982 \quad 5255$

ILF :

ILX:

EML:

COM: $15,20,22$ C
HARTOOG MARK RICHARD OR LICK OBSERVATORY UNIVERSITY OF CALIFORNIA SANTA CRUZ CA 95064 USA

TEL:

TLF:

$T L X$ :

EML:

COM:

HARUTVUNIAN HAIK A DR BYURAKAN ASTROPHYSICAL OBSERVATORY

378433 BYURAKAN

ARMENIA

TEL: $89 \quad 52 \cdot 28 \quad 3453 / 4142$ TLF:

TLX: 411576 AS CON SU

EML:

COM: 36

HARVEY JOHN W OR NTL SOLAR OBSERVATORY BOX 26732 $950 \mathrm{~N}$ CHERRY AVE TUCSON AZ $85726 \quad 6732$ USA

TEL: 6023275511

TLF: $602 \quad 3259278$

ILX: L56140I AURA UT EML: JHARVEYNOAO. EDU COM: $10,12 \mathrm{C}$

HASÁN HASHIMA OR STSCI

HOMEWODD CAMPUS 3700 SAN MARTIN DR BALTIMORE MD 21218 USA.

TEL: 3013384519 TLF: 3013384767 TLX: 3016849101 STSCI EML: HASANESTSCI

COM: 28

HASEgANA TATSUHIKO DR DPT OF PHYSICS OHIO STATE UNIVERSITY 174 W I8TH AVE COLUMBUS OH 43210 USA

TEL:

TLF: 6142927557

TLX:

EML:

COM: 
HASEGAWA TETSUO DR

UNIVERSITY OF TOKYO

DSAWA MITAKA

TOKYO 181

JAPAN

TEL: 422.413737

TLF :

TLX: 2822307

EML:

COM: 40

HASSALL BARBARA J M DR

ROYAL GREENWICH OBS

MAOINGLEY RO

CAMBRIDGE CB3 OEZ

UK

TEL: $223 \quad 337548$

TLF :

TLX: 817297 ASTRON G

EML: JANET:BJMHOUK. AC.CAM. AST-STAR

COM: 42
INST OF ASTRONOMY

HAUSER MICHAEL $G$ DR

NASA/GSFC

CODE 680

LASP

GREENBELT MD 20771

USA

TEL: $30 \mathrm{~L} 2868701$

TLF :

$T L X$ :

EML: STARS: : HAUSER

COM: 2IVP, 44
HASER LEO N $K$ DR

MPI F. EXTRATERR. PHYSIK

D 8046 GARCHING MUENCHEN

GERMANY

TEL: 893299803

TLF :

EML:

COM: 15

TLX: 5215845 XTER O

HASSAN S M PROF

HELWAN OBSERVATORY

HELWAN

EGYPT,

TEL: $78 \quad 0645 / 2683$

TLF :

EML:

COM: 37

TLX: $93070^{\circ}$

HAUBOLD HANS JOACHIM

ZNTRLINST F ASTROPHYSIK

STERNWARTE BABELSBRG

ROSA LUXEMBURG STR I7A

O 1591 POSTOAM.

GERMANY

TEL: 7620

TLF:

TLX: 15305

EML:

COM: 48

HAUGE OIVIND DR

INST THEORET ASTRQPHYSICS

UNIVERSIIY OF OSLO

BOX 1029

N 0315 BLINDERN OSLO 3

NORWAY

TEL: 245-65-06

TLF :

ILX:

EML:

COM:

HASHIMOTO MASA-AKI DR

OPT OF PHYSICS

COLLEGE OF GENERAL EOU

KYUSHU UNIV ROPPONMATSU

FUKUOKA B10

JAPAN

TEL: 0927714161 EXT 360

ILF: 0927318745

ILX:

EML: E76051AJPNCCKU

COM: 35

HATHAWAY DAVIO H DR

NASA/MSFC

CODE ES 52

HUNTSVILLE AL 35812

USA

TEL: 2055447610

TLF:

TLX:

EML:

COM: 10

HAUCK BERNARO PROF INSTITUT O'ASTRONOMIE UNIVERSITE DE LAUSANNE

CH 1290 CHAVANNES-D-BOIS SWI TZERLAND

IEL: 227552611

TLF: 227553983

TLX: 27720 OBSG CH

EML: hauckobs.unige.ch

COM: $05 P, 25,45$

HALPT HERMANN F PROF INSTITUT FUER ASTRONOMIE

DER UNIVERSITAET

UNIVERSITAETSPLATZ 5

A BOIO GRAZ

AUSTRIA

TEL: $316380 \quad 5271$

TLF:

TLX: 31078A

EML:

COM: $15,20,38,46$

HAVLEN ROBERT O OR

NRAD

BOX O. ;

SOCORRO NM 878010387

USA

TEL: $505 \quad 835$ 7330

TLX: 9109881710

EML: BITNET: RHAVLENONRAO

ILF: 5058357027

COM:
HAVNES OVE QR

AURORAL OBSERVATORY

UNIVERSITY OF TROMSO

BOX 953

N 9001 TROMSO

NQRWAY

TEL: $83-86060$

TLF :

TLX: 64124 AUROB N

EML:

COM:
HASLAM C GLYN T BR

MPI FUER RADIOASTRONOMIE

AUF DEM HUEGEL 69

D 5300 BONN 1

GERMANY

TEL:

TLF:

TLX: 886440

EML:

COM: 40

HATZES ARTIE P OR

ASTRONOMY DPT

UNIVERSITY OF TEXAS

RLM 15308

AUSTIN TK $78712 \quad 1083$

USA

TEL: $512471 \quad 1473$

ILF:

ILX:

EML: ARTIEOASTRO, AS, UTEXAS. EQU

COM:

HAUG EBERHARD DR

MOZARTSTR 20

O 7430 METZINGEN

GERMANY

IEL: $707 \quad 1296483$

TLF:

TLX:

EML:

COM: 10

HAUPT WOLFGANG DR

GIRONDELLE 105

D 4630 BOCHUM 1

GERMANY

TEL:

TLF:

TLX:

EML:'

COM:

HAWAROEN TIMOTHY G OR

ROYAL OBSERVATORY

BLACKFORD HILL

EDINBURGH EH9 $3 \mathrm{HJ}$

UK.

TEL: 316.673321

TLF:

TLX: 72383 ROEOIN G

EML:

COM: 37 


HAWKES ROBERT LEWIS DR
OPT OF PHYSICS
MOUNT ALLISON UNIVERSITY
SACKVILLE NB EOA 3 CO
CANADA
TEL: 5063642580
TLF: 5063642216
TLX:
EML: BITNET:A014EMTAM
COM: 22

HAWKINS MICHAEL R S

ROYAL OBSERVATORY

BLACKFORO HILL

EDINBURGH EH9 $3 \mathrm{HJ}$

UK

TEL:

TLX:

EML:

TLF:

COM: 33

HAYES DONALO S OR

B0X 1907

SCOTTSOALE AZ 85252

USA

TEL: 6029473572

ILF :

EML:

COM: 25,45

$T\llcorner X:$

HAYNES RAYMONO F PROF
CSIRO
OIVISION OF RADIOPHYSICS
BOX 76
EPPING NSW 2121
AUSTRALIA
TEL: 2.868 0276
TLF: 2 B6B 0457
TLX: ASTRO 26230
EML: RHAYNESERPEPPING.0Z.AU
COM: 34,40
HAZEN MARTHA L OR
CENTER FOR ASTROPHYSICS
HCO/SAO
60 GAROEN ST
CAMBRIDGE MA O213B
USA
TEL: 617 4953362
TLF:
TLX:
EML:
COA: 37

HAWKING STEPHEN W PROF DPT APPLIEO MATHS

\& THEORETICAL PHYSICS

SILVÉR STREET

CAMBRIDGE CB3 9EW.

UK

TEL: 223351645

TLF:

TLX: BI240 CAMSPL G

EML:

COM: 47,48

HAYASHI CHUSHIRO PROF

MOMOYAMA YOGORO-CHO I

FUSHIMI KU

KYOTO 612

JAPAN

TEL: 075-6L1L-1062

TLF :

TLX

EML:

COM: 35,47

HAYLI AVRAM PROF

OBSERVATOIRE DE LYON

AVE CHARLES ANDRE

F 695615 GENIS LAVAL CDX

FRANCE

TEL: 78560705

TLF: $7239979 \mathrm{l}$

TLX: 310916

EML:

COM: 33,41

\section{HAYWARD JOHN}

DPT MATHS \& COMPUTING

POLYTECHNIC OF WALES

PONTYPRIOD

MIO GLAMORGAN [F3g $2 \mathrm{PJ}$

UK

TEL:

TLF :

TLX:

EML:

COM: 10

HAZER'S OR

FACULTY OF SCIENCE.

EGE UNIVERSITY

BOX 21

35100 BORNOVA IZMIR

TURKEY

TEL:

TLF:

TLX:

EML:

COM:
HAWKINS GERALO S OR

CONSUL 906

2400 VIRGINIA AVE NW WASHINGTON DC 20037

USA.

TEL: 2024852050

TLF:

TLX:

EML:

COM: 22,41

HAYASHI MASAHIKO DR

OPT OF ASTRONOMY

UNIVERSITY OF TOKYO

BUNKYO KU

TOKYO 113

JAPAN

TEL: 38.122111

TLF:

TLX: 2722126 UTGAB J

EML:

COM: 40

HAYMES ROBERT C PROF OPT SPACE PHYS \& ASTRON

RICE UNIVERSITY

HOUSTON TX 77001

USA

TEL: 7135274045

TLF:

TLX: 556457

EML:

COM: 48

HAYWOOD J

DPT OF TEACHER EDUCATION

TRINITY COLLEGE

OUBLIN 2

IRELAND

TEL: 1772941

TLF :

$T L X$ :

EML:

COM: 46

HAZLEHURST JOHN OR

HAMBURGER STERNWARTE

GOJENSBERGSWEG 112

2050 HAMBURG 80

GERMANY

TEL:

TLF :

TLX:

EML:

COM: 42
HAWKINS ISABEL DR -

CIR FOR EUV ASTROPHYSICS

UNIVERSITY OF CALIFORNIA

2150 KITTREOGE ST

BERKELEY CA 94720

USA

TEL: $415 \quad 6420816$

TLF: 4156437629

ILX:

EML:

COM: 44

HAYASHI SAEKO S DR

TOKYO ASTRONOMICAL OBS

NAOJ

OSAWA MITAKA

TOKYO 181

JAPAN

TEL: 0422413609

TLF: 0422 4l 3608

TLX:

EML: SAEKDOOPTIK.MMTK, NAO,AC.JP

COM: 34

HAYNES MARTHA $P$

DPT OF ASTRONOMY

CORNELL UNIVERSITY

SPACE SCIENCES BLDG

ITHACA NY 14853

USA

TEL: 6072563734

TLF:

ILX: 932454

EML:

COM: 40

HAZARD CYRIL DR

INSTITUTE OF ASTRONOMY

THE OBSERVATORIES

MADINGLEY RD

CAMBRIDGE CB3 OHA

UK

TEL: 22362204

TLF :

TLX:

EML:

COM: 40

HE MIAO-FU

SHANGHAI OBSERVATORY

CAS

BO NANDAN RD

SHANGHAI

CHINA PR

TEL: 21386191

TLF :

TLX: 33164 SHAO CN

EML:

COM: $04,07 C, 20$ 


\section{A128}

HE XIANG-TAD

DPT OF ASTRONOMY

BEIJING NORMAL UNIVERSITY

BEIJING 100875

CHINA PR

TEL: $165 \quad 6531 * 6285$

TLF :

ILX:

EML:

COM: 28,47

HEASLEY JAMES NORTON

INSTITUTE FOR ASTRONOMY

UNIVERSITY OF HAWAII

2680 WOODLAWN OR

HONOLULU HI, 96822

USA

TEL: $808 \quad 948 \quad 6826$

TLF

ILX: 7238459 UHAST HR

EML:

COM: 36

\section{HECK ANDRE OR}

DBS DE STRASBOURG

11 RUE UNIVERSITE

F 67000 STRASBOURG

FRANCE

TEL: $88 \quad 358216$

TLF: 88250160

TLX: 890506 STAROBS $F$

EML: heckfrccsc2l.bitnet

COM: $05 \mathrm{C}, 25,5 \mathrm{I}$

HEDOLE DOUGLAS W O PROF

DPT OF PHYSICS

ROYAL HOLLOWAY COLLEGE

UNIVERSITY OF LONDON

EGHAM SURREY TWZO OEX

UK

TEL: $784 \quad 35351$

TLF :

TLX: 935504

EML:

COM: 14

HEFFERLIN RAY A PROF

DPT OF PHYSICS

SOUTHERN COLLEGE

DRAWER $H$

COLLEGEOALE TN 373150370

USA

TEL: $615 \quad 238 \quad 2869$

TLF :

TLX:

EML:

COM: 14

HEAP SARA R DR
NASA/GSFC
CODE 672
GREENBELT MO 20771
USA
TEL:
TLF:
ILX:
EML:
COM:

HEAVENS ALAN DR

ROYAL OBSERVATORY

BLACKFORD HILL

EOINBURGH EHS 3HJ

UK

TEL: $316 \quad 688352$

ILX: 72383 ROEOIN G

EML: AFHOUK.AC, ROE. STAR

ILF :

COM: 47

HECKATHORN HARRY M

NAVAL RESEARCH LABORATORY

COOE 41432

4555 OVERLOOK AVE SW

WASHINGTON DC 203755000

USA

TEL: $202767 \quad 2764$

TLF :

$T \perp X$ :

EML:

COM: 09,44

HEOEMAN E RUTH MISS

3440 ST JEFFERSON ST

FALLS CHURCH VA 22041

USA

TEL:

TLF:

EML:

COM:

TLX:

HEFTY JAN DR

OBSERVATORY OF THE SLOVAK

TECHNICAL UNIVERSITY

RADLINSKEHO 11

CS 813 68 BRATISLAVA

CZECHOSLOVAKIA

TEL: 7498047

TLF: 752027

TLX: 92720 ELFAKC

EML:

COM: 19
HEARN ANTHONY G DR

STERREKUNOIG INSTITUTE

$80 \times 80000$

NL $350 B$ TA UTRECHT

NETHERLANDS

TEL: 30535200

TLF :

TLX: 40048 FYLUT NI

EML: BITNET: WNMTONYHUTRUUO

COM: 36,44

HEBER ULRICH

INST THEOR PHYS \& STERNW

UNIVERSITAET KIEL

LEIBNIZSTR

D 2300 KIEL 1

GERMANY

TEL: 431 B80 4103

TLF:

TLX: 292706

EML:

COM: 29,36

HECKMAN TIMOTHY M

ASTRONOMY PROGRAM

UNIVERSITY OF MARYLAND

COLLEGE PARK MD 20742

USA

TEL: 3014543001

TLF

TLX: 7108260352 ASTR CORP

EML:

COM: 28

HEESCHEN DAVID S DR

NRAO

EOGEMONT RD

CHARLOTTESVILLE VA 22901

USA

TEL:

TLF;

TtX: $910-997-0174$

EML :

COM: $28,40,51$

HEGGIE DOUGLAS C OR

DPT OF MATHEMATICS

UNIVERSITY OF EDINBURGH

KING'S BUILDINGS

EDINBURGH EH9 3JZ

UK

TEL: $316 \quad 67108$

TLF:

TLX: 727442 UNIVEOG

EML: O.C.HEGGIE D.AC.UK

COM: 07,37
HEARNSHAW JOHN B OR

OPT OF PHYSICS

UNIVERSITY OF CANTERBURY

PRIVATETBAG

CHR ISTCHURCH I

NEW ZEALAND

TEL: $348 \quad 2009 * 771$

TLF :

TLX: 4144 UNICANT NZ

EML:

COM: $29.30 \mathrm{C}$

HECHT JAMES H DR

AEROSPACE CORPORATION

MS M2 255

BOX 92957

LOS ANGELES CA 90009

USA

TEL: 213.3367017

TLF:

TLX:

EML:

COM: 34

HECQUET. JOSETTE DR

OBS MIOI PYRENEES

14 AVE E BELIN

F 31400 TOULOUSE COX

FRANCE

TEL: $6125 \quad 2101$

TLF:

TLX: $530776 \mathrm{~F}$

EML:

COM:

HEFELE HERBERT PH D

MPI FUER ASTRONOMIE

KONIGSTUHL

D 6900 heidelberg a

GERMANY

TEL:

TLF :

TLX :

EML:

COM: 05

HEGYI DENNIS I ASSOC PROF

RANDALL LABORATORY

UNIVERSITY OF MICHIGAN

ANN ARBOR MI 4B109 1090

USA

TEL: 3137645448

TLF :

TLX: B10-223605

EML:

COM: 
HEIDMANN JEAN DR OBSERVATOIRE DE PARIS SECTION DE MEUIOON

F 92195 MEUOON PPL COX FRANCE

TEL: $145 \quad 07 \quad 7598$

ILF:

TLX: 270912

EML:

COM: $28,40,47,51 \mathrm{C}$

HEINTZE J R W OR

STERREKUNOIG INSTITUTE

$80 \times 80000$

NL 3508 TA UTRECHT

NETHERLANOS

TEL: 30535235

ILX: 40048 FYLUT NI

EML: BITNET: WNMMAILEHUTRUUD

TLF:

COM: 29,30

HEJWA LADISLAV OR

FAC OF MATHS \& PHYSIC

CHARLES UNIVERSITYY

MALOSTRANSKE NAM $2 / 25$

CS 110 OO PRAHA 1

CZECHOSLOVAKIA

TEL: 2532132

ILF: 2847688

ILX: L2L673 MFF C

EML: LHEJNAOCSPGUKII

COM; 12

HELFER h LaWRence PROF

DPT PHYSICS \& ASTRONOMY

UNIVERSITY OF ROCHESTER

ROCHESTER NY 14627

USA

TEL: $716 \quad 275 \quad 4377$

ILF :

iLX:

EML:

COM: 34

HELMER LEIF

COPENHAGEN UNIVERSITY OBS

BRORFELDEVEJ 23

DK 4340 TOLLOSE

DENMARK

TEL: $53 \quad 4 B \quad B 195$

TLF: $58 \quad 48 \quad 8758$

TLX: 44155

EML:

COM: $0 B C, 50$
HEILES CARL PROF

ASTRONOMY OPT

UNIVERSITY OF CALIFORNIA

601 CAMPBELL HALL

BERKĘLEY CA 94720

USA

TEL: $415 \quad 642 \quad 4510$

TLF :

TLX: B2018L UCB AST RAL

EML:

COM: $33,34,40$

HEINZEL PETR DR

ASTRONOMICAL INSTITUTE

CZECH ACADEMY OF SCIENCES

ONDREJOY OBSERVATORY

CS 25165 ONDREJOV

CZECHOSLOVAKIA

TEL: 20485201

TLF : 20485314

TLX: 121579 ASTR :

EML:

COM: 10,12

HEKELA JAN OR

ASTRONOMICAL INSTITUTE

CZECH ACADEMY OF SCIENCES

ONOREJOV OBSERVATORY

CS 25165 ONDREJOY

CZECHOSLOVAKIA

TEL: 204 B5201

TLF : $204 \quad 85314$

TLX:

EML:

COM: 36

HELIN ELEANOR FRANCIS

JPL

MS 183501

4800 OAK GROVE DR

PASADENA CA 91109

USA

TEL: B1B 3544606

TLF:

TLX: 675429

EML:

COM: $15,20,22$

HELMKEN HENRY $F$ DR

CENTER FOR ASTROPHYSICS

HCO/SAO

60 GARDEN ST

CAMBRIOGE MA 02138

USA

TEL:

TLF :

TLX:

EML:

COM: 44
HEINRICH INGE

ASTRONOMISCHES RECHEN-

INSTITUT

MOENCHHOFSTR $\quad 12 \cdot 14$

D 6900 HEIDELBERG 1

GERMANY

TEL: $62 \quad 2149025$

TLF :

TLX:

EML:

COM: 05

HEISE JOHN DR

SPACE RESEARCH LABORATORY

SRON

SORBONNELAAN 2

NL 3584 CA UTRECHT

NETHERLANDS

TEL: 30535600

ILF: 30540860

TLX: 47224 ASTRO NL

EML:

COM: 44,48

HELALI YHYAE ER

HELWAN OBSERVATORY

HELWAN

EGYPT

TEL: $78 \quad 0645 / 2683$

TLF :

TLX:

EML:

COM: 07

HELLER MICHAEL PROF

POWSTANCOW WARSAWY $13 / 94$

PL 33110 TARNOW

POLAND

TEL:

TLF :

TLX:

EML:

COM: 47

HELOU GEORGE OR

CALTECH

MS 10022

IPAC

PASADENA CA 91125

USA

TEL: 8185842928

TLF:

ILX: 5849945

EML: BITNEI : HELOU* I PACOHAMLET.

COM: $05,28,34,40,44$
HEINTZ WULFF $D$ DR OPT OF ASTRONOMY

SWARTHMORE COLLEGE

SWARTHMORE PA 19081

USA

IEL: 2154477265

ILF:

TLX:

EML:

COM: $05,08,24,42$

HEISER ARNOLO M OR

OYER OBSERVATORY

VANDERBILT UNIVERSITY

BOX 1803 STA B

NASHVILLE TN 37235

USA

TEL: $615 \quad 3734897$

TLF:

TLX:

EML:

COM: 27

HELFAND DAVIO JOHN

COLUMBIA ASTROPHYSICS LAB

COLUMBIA UNIVERSITY

538 WEST 120TH ST

NEW YORK NY 1002 ?

USA

FEL: 2128542150

TLF: 212. 3169504

TLX: 220094 COLU UR

EML: BIINET: DJHTECUPHYO

COM: 48

HELLWIG HELMUT WILHELM OR FREQUENCY \& TIME SYSTEMS

34 TOZER RD

BEVERLY MA D1915

USA

TEL: 6179278220

TLF:

TLX: 940518

EML:

COM: 31

HELT BODIL E

UNIVERSITY OBSERVATORY

OESTER VOLOGADE 3

CK 1350 COPENHAGEN $K$

DENMARK

TEL: $31 \quad 14 \quad 1790$

TLF: 31389157

TLX: 44155 DANAST DK

EML:

COM: 42 
HEMENWAY MARY KAY M DR

ASTRONOMY OPT

UNIVERSITY OF TEXAS

RLM 15308

AUSTIN TX $78712 \cdot 1083$

USA

TEL: 5124711309

TLF: 5124716016

TLX: 9108741351

EML: MARYKAYYASTRO.AS. UTEXAS. EDU

COM: 46

HENDECOURT D' LOUIS OR

GROUPE PHYSIQUE SOLIOES

UNIVERSITE PARIS VII

4 PLACE JUSSIEU TOUR 23

F 75251 PARIŚ COX 05

FRANCE

TEL:

TLF:

TLX:

EML:

COM: $14,21,34$

HENON MICHEL C OR

OCA OBSERV DE NICE

BP 139

F 06003 NICE COX

FRANCE

TEL: $93 \quad 890420$

TLF:

TLX: 460004 OBSNICE $F$

EML:

COM: $07,33,37$

HENRI KSEN RICHARD N
OPT OF PHYSICS
QUEEN'S UNIVERSITY
KINGSTON ON K7L 3N6
CANADA
TEL: 6135475536
TLF: 6135456463
TLX:
EML:

HENSLER GERHARD PROF

INST THEORICAL PHYS \& OBS

UNIVERSITY OF KIEL

OLSHAUSENSTR 40

D 2300 KIEL I

GERMANY

TEL: 4318804125

TLF : 4318804432

TLX: 292706

EML: PASJOERZ.UNI-KIEL. OBP. DE

COM: 42

\author{
HEMENWAY PALL D DR \\ ASTRONOMY DPT \\ UNIVERSITY OF TEXAS \\ RLM 15308 \\ AUSTIN TX 78712 1083 \\ USA \\ TEL: $512 \quad 4714461$ \\ TLF: \\ TLX: \\ EML: \\ COM: $08,20,24 \mathrm{C}$
}

HENIZE KARL G ASTRONAUT

NASA/JOHASON SPACE CENTER

COOE CB

HOUSTON TX 77058

USA

TEL: 7134832411

TLF:

TLX:

EML:

COM: $28,29,34,44,45$

HENDUX JEAN-CLAUDE DR

observatotre oE paris

SECTION DE MEUDON

F 92195 MEUDON PPL COX

FRANCE

TEL: $145 \quad 077803$

TLF:

TIX:

EML:

COM: 10,44

\section{HENRY RICHARD B C DR}

OPT PHYSICS \& ASTRONOMY

UNIVERSITY OF OKLAHOMA

NORMAN OK 73019

USA

TEL: $405325396 \mathrm{~L}$

TLF:

TLX:

EML:

COM: 28,35

HERAS ANA M OR
ESA/ESTEC
SSO
BOX 299
NL: 2200 AG NOOROWIJK
NETHERLANOS
TEL: 171985016
TLF: 171984698
TLX:
EML: ESTCSI: :AHERAS/AHERASEESTEC
COM: 49

HEMMLEB GERHARO OR

ZENTRALINSTITUT FUR

PHYSIK DER ERDE

TELEGRAFENBERG A 17

D 1500 POTSOAM

GERMANY

TEL: 4551

TLF :

TLX: 15305

EML:

COM: 19,31

HENKEL CHRISTIAN

MPI FUER RADIOASTRONOMIE

AUF DEM HUEGEL 69

D 5300 BONN 1

GERMANY

TEL:

TLF:

TLX: 886440

EML:

COM: 34,40

HENRARD JACQUES PROF

DPT DE PHTSIQUE

FACULTES UNIVERSITAIRES

RUE DE BRUXELLES 6 !

B 5000 NAMUR

BELGIUM.

TEL: B1 229061

TLF: $81 \quad 230.391$.

TLX: 59222

EML: JHENRARD@BNANDP5 1

COM: $04,07 \mathrm{C}, 20$

HENRY RICHARO C PRQF

DPT PHYSICS \&I ASTRONOMY

JOHNS HOPKINS UNIVERSITY

CHARLES \& 34TH ST

BALTIMORE MD 21218

USA

TEL: 3013387350

TLF :

TLX:

EML:

COM: 21,48

HERBIG GEORGE H DR

INSTITUTE FOR ASTRONOMY

UHIVERSITY OF HAWAII

2680 WOODLAWN DR

HONOLULU HI 96022

USA

TEL: $808 \quad 94 B \quad 8312$

TLF :

TLX: 723-B459

EML:

COM: 27,29
HEMPE KLAUS

GRENZWEG 24 B

D 2057 REINBEK

GERMANY

TEL: 407105628

TLF :

TLX:

EML:

COM:

HENNING THOMAS OR

UNIVERSITY OBS JENA

SCHILLERGAESSCHEN 2

D 6900 JENA

GERMANY

TEL: 7827122

TLF: $78 \quad 425039$

TLX: 05886134

EML: PHYSFAKOPHYSIK, UNI-JENA, OBP

COM: 34

HENRICHS HUBERTUS F OR

ASTRONOMICAL INSTITUTE

UNIVERSITY OF AMSTERDAM

KRUISLANN 403

NL 1098 SJ AMSTERDAM

NETHERLANDS

TEL: 205257491

TLF: $31205 \quad 257484$

TLX: 10262 HEF NL

EML:

COM: 29

HENSBERGE MERMAN

KONINKLIJJKE STERRENWACHT

RINGLAAN 3

B 1180 BRUSSELS

BELGIUM

TEL: 223730284

TLF: 223749822

TLX: 21565 OBSBEL

EML: HERMANOASTRO. OMO.BE

COM: 25,44

HERBST ERIC DR

OPT OF PHYSICS

OHIO STATE UNIVERSITY

174 W 18TH AVE

COLUMBUS OH 43210.

USA

IEL: $614292 \quad 2653$

TLF:

$T L X:$

EML:

COM: 
HERBST WILLIAM OR

DPT OF ASTRONOMY

VAN. VLECK OBSERVATORY

WESLEYAN UNIVERSITY

MIOOLETOWN CT 06457

USA

$\mathrm{TEL}: 2033479411$

TLF:

TLX:

EML:

COM: 33,37

HERMSEEN WILLEM OR

SRL

HUYGENS LAB

BOX 9504

NL 2300 RA LEIOEN

NETHERLANOS

TEL: 71275810

TLF:

TLX: 39058 ASTRO NL

EML:

COM:

\section{HERR RICHARO B DR}

DPT OF PHYSICS

UNIVERSITY OF DELAWARE

NEWARK DE 19716

USA

TEL: $302 \quad 4512673$

TLF:

TLX:

EML:

COM: 27

HERS JAN MR
BOX 48
SEOGEFIELD 6573
SOUTH AFRICA
TEL: 4455736
TLF:
EML:
COM: $06,20,27,31$
TLX:

HESKE ASTRID DR

ESA/ESTEC

ASTROPHYSICS OIV

BOX 299

NL 2200 AG NOOROWIJK

NETHERLANDS

TEL: $17 \quad 19 \quad 83173$

ILF: $17 \quad 1984690$

TLX: 39098

EML: ESTCSL: : AHESKE / AHESKEEESTEC

COM: $29,40,44$
HERCZEG TIBOR J PROF DR

OPT PHYSICS \& ASTRONOMY

UNIVERSITY OF OKLAHOMA

NORMAN OK 73019

USA

TEL: 4053253961

TLF:

TLX:

EML:

COM: 42,51

HERNANDEZ CARLOS ALBERTO

OBSERVATORIO ASTRONOMICO

PASED DEL BOSQUE

1900 LA PLATA (BS AS)

ARGENTINA

TEL: 21217308

TLF :

TLX:

EML:

COM:

HERRERA MIGUEL ANGEL DR

INSTITUTO ASTRONOMIA

UNAM

APDO POSTAL 70.264

04510 MEXICO DF

MEXico

TEL: 5485305

TLF :

TLX: 1760155 CICME

EML: BITNET:MANHEReumamVmI

COM:

HERSHEY JOHN L DR

US NAVAL OBSERVATORY

34 \& MASSACHUSETTS AVE NW

WASHINGTON DC 203925100

USA

TEL: $202 \quad 653 \quad 1554$.

$T L X: 710-822-1970$

EML:

TLF:

COM: $24 ; 26,51$

HESSER JAMES E DR

HERZBERG INST ASTROPHYS

DOMINION ASTROPHYS OBS

5071 W SAANICH RO

VICTORIA BC V8X $4 M 6$

CANADA

TEL: $604 \quad 388 \quad 3974$

TLF: 6043630045

TLX: 0497295

EML:

COM: $14,27,37$
HERMAN JACOBUS DR

MAX PLANCK. INSTITUT FUR

EXTRATERRESTRISCE PHYSIK

D 8046 GARCHING MUENCHEN

GERMANY

TEL:

TLF :

TLX:

EML:

COM: 33

HERNQUIST LARS ERIC OR

LICK OBSERVATORY

UNIVERSITY OF CALIFORNIA

NTL SCIENCES II

SANTA CRUZ CA 95064

USA

TEL: $408 \quad 425 \quad 4733$

TLF: $408 \quad 4263115$

TLX: 7607936

EML: LARS SHELIOS. UCSU. EDU

COM: 28

HERRERO DAVO ARTEMIO OR INST DE ASTROFISICA

DE EANARIAS

OBS DEL TEIDE

E 38200 LA LAGUNA

SPAIN

TEL: $22 \quad 262211$

TLF:

TLX: 92640

EML:

COM :

HERTZ PAUL L OR

NAVAL RESEARCH LABORATORY

CODE 41215

4555 OVERLOOK AVE SW

WASHINGTON OC 203755000

USA

TEL: 2027672438

TLF:

$T E X$ :

EML:

COM:

HEUDIER JEAN-LOUIS OR

OCA OBSERV DE NICE

BP 139

F 06003 NICE CDX

FRANCE

TEL: 92003011

TLF: 92 00 3033

TLX: 460004 OBSNICE $F$

EML:

COM: $09,20,24,37,46,51$
HERMANS DIRK DR

SEHOOL MATHS/STATISTICS

UNIVERSITY OF BIRMINGHAM

EDGBASTON

BIRMINGHAM BI5 2TT

UK

TEL: $214 \quad 143961$

TLF : $214 \quad 143907$

TLX: 337262 UOBHAM G

EML: D.F.H.HERMANSEUK. AC. BHAM

COM: 10

HEROLO HEINZ

THEORETISCHE ASTROPHYSIK

UNIVERSITY TUEBINGEN

AUF. DER MORGENSTELLE $12, \mathrm{C}$

D 7400 TUEBINGEN

GERMANY

TEL: $707 \quad 1292043$

TLF :

TLX:

EML:

COM: 14,36

HERRMANN DIETER PROF DR ARCHENHOLD STERNWARTE

ALT TREPTOW 1

D 1193 BERLIN

\section{GERMANY}

TEL: $272 \quad 887 i \div 494$

TLF :

TEX:

EML:

COM: 41

HER ZBERG GERHARD OR HERZBERG INST ASTROPHYS NTL RESEARCH COUNCIL

100 SUSSEX OR

OTTAWA ON KIA ORG

CANAOA

TEL: $613 \quad 9900917$

TLF: 6139526602

TLX: $053 \quad 3715$

EML:

COM: $14,15,16,34$

HEWETT PAUL

INSTITUTE OF ASTRONOMY

THE OBSERVATORIES

MADINGLEY RO

CAMBRIDGE EB3 OHA

UK

TEL: $223 \quad 622 \quad 04$

TLF :

FLX: 817297 ASTRON G

EML:

COM: 30,47 


HEWISH ANTONY PROF
MULLARD RADIO ASTRON OBS
CAVENOISH LABORATORY
MADINGLEY RO
CAMBRIDGE CB3 OHE
UK
TEL: 223.66477
TLF:
TLX: 81292
EML:
COM: 40
HEYVAERTS JEAN OR
OBSERVATOIRE OE PARIS
SECTION DE MEUDON
F 92195 MEUDON PPL COX
FRANCE
TEL: 145 O7 7405
TLF:
TLX: 201571
EML:
COM: 49
TL
TLX:
EML:
COM:
HIDALGO MIGUEL A DR
FAC OE CIENCIAS FISICAS
UNIVERSIDAD DE ZARA60ZA
E 50009 ZARAG0ZA
SPAIN
TEL:
TLF:

HILDEBRAND ROGER H

ENR ICO FERMI INSTITUTE UNIVERSITY OF CHICAGO 5640 S ELLIS AVE CHICAGO IL 60637 USA TEL: $312 \cdot 9627581$ TLF :

TLX:

EML:

COM: 34

\section{HILL FRANK DR}

NTL SOLAR OBSERVATORY BOX 26732

$950 \mathrm{~N}$ CHERRY AVE TUCSON AZ B5726 6732 USA

TEL: $602323 \quad 4138$

TLF: 6023259278

TLX: 1561401 AURA UT

EML: FHILLONOAO,EOU

COM: 12

HEWIIT ADELAIDE
CASS
UCSD
C OII
LA JOLLA CA 92093

LA JOLLA CA 920930216

USA

TEL: $619.534 \quad 6627$

ILF:

TLX:

EML: INT: hewittycass.spaneucsd. edu

COM: 28,47

HIBBS ALBERT R MGR PLANS

781 PROSPECT BL

PASADENA CA 91103

USA

TEL:

TLF:

EML:

COM:

TLX:

$$
\begin{aligned}
& \text { HIDE RAYMOND PROF } \\
& \text { GEOPHYSICAL FLUID } \\
& \text { OYNAMICS LABORATORY } \\
& \text { METEOROLOGICAL OFF } \\
& \text { BRACKNELL BERKS RG } \\
& \text { UK } \\
& \text { TEL: } 34442242 \\
& \text { TLF: } \\
& \text { TLX: } 849801 \\
& \text { EML: } \\
& \text { COM: } 16,19
\end{aligned}
$$

oYNamics Laboratory

METEOROLOGICAL OFFICE

BRACKNELL BERKS RGI2 2SZ

HILOLTCH RONALD W OR
OPT OF PHYSICS \& ASTRON
UNIVERSITY OF ST ANOREWS
NORTH HAUGH
ST ANOREWS FIFE KYI6 955
UK
TEL: 33476161
TLF: 33474487
TLX: 9312110846 SA 6
EML: ASSRH@UK. AC.ST-AND. STAR
COM: $25,30,42 C$

HILL GRAHAM DR

HERZBERG INST ASTROPHYS DOMINION ASTROPHYS OBS

5071 W SAANICH RD

VICTORIA BC VBX $4 M 6$

CANADA

TEL: 6023883935

ILF: 6043630045

TLX: 0497295

EML:

COM: $24,26,30,42 \mathrm{C}$
HEWITT ANTHONY $V$ OR

GE MEDICAL SYSTEMS.

PEt ENGINEERING

BOX 206

PEWAUKEE WI 53072

USA

TEL: 4143205170

TLF:

TLX:

EML:

COM: 09,28

HICKSON PAUL DR

OPT. GEOPHYS \& ASTRONOMY

UNIV OF BRITISH COLUMBIA

2219 MAIN MALL

VANCOUVER BC VGT IWS

CANADA

TEL: $604 \quad 2282267$

TLF: 6042286047

TLX:

EML:

COM: 28

HIEI EIJIRO OR

TOKYO ASTRONOMICAL OBS

NAOJ

OSAWA MITAKA

TOKYO 18:

JAPAN

TEL: 0422-32-5111

TLF:

TLX: 2822307

EML:

COM: 10,12

HILONER ERNEST OR

DIRECTOR R/S/SE

SPACE ENVIRONMENT LAB

325 BROADWAY

BOULDER CO 803033328

USA

TEL:

TLF :

TLX:

EML:

COM: $10 C, 12$

HILL HENRY ALLEN OR

DPT OF PHYSICS

UNIVERSITY OF ARIZONA

BLDG BL

TUCSON AZ 85721

USA

TEL: $602 \quad 621 \quad 6784$

ILF:

TLX: $910-9521143$

EML:

COM: 27 hey James Stanley DR

4 SHORTLANOS CLOSE

EASTBOURNE BN22 OJE

UK

TEL:

TLF:

TLX:

EML:

COM: 22,40

hidAJAT bambang PROF. OR BOSSCHA OBSERVATORY

LEMBANG 40391

INDONESIA.

TEL: 2296001

TLF:

ILX: 28234 BD IIB

EML:

com: $26,34,46,50$

HIGGS LLOYO A DR

DOMINION RADIO ASTROPHYS OBSERVATORY

BOX 248

PENTICTON BC V2A $5 \mathrm{KK}$

CANADA

TEL: 6044932277

ILF: 6044937767

TLX: 04888127

EML:

COM: 34,40

HILF EBERHARD R H PH D

PESTRUPSWEG 30

D 2900 OLDENBURG

GeRMANY

TEL:

TLF:

TLX:

EML:

COM: 35,40

HILL PHILIP W OR

DPT OF PHYSICS ASTRON

UNIVERSITY OF ST ANOREWS

NORTH HAUGH

ST ANDREWS FIFE KYI6 gSS

uK

TEL: 33476161

ILF: 33474487

TLX: 76213 SALLIB G

EML: P.W.HILLUK, AC, ST-ANDREWS

COM: 25,27 
HILLEBRANOT WOLFGANG PH D

MPI F. PHYSIK \& ASTROPHYS

KARL-SCHWARZSCHILD-STR I

D 8046 GARCHING MUENCHEN

GERMANY

TEL: 8932999409

$T L X:$

EML:

TLF:

COM:

HILTON JOHN OR
DPT OF MATH SCIENCES
GOLOSMITHS ' COLLEGE
NEW CROSS
LONDON SE14 $6 \mathrm{NW}$
UK
TEL:
TLF:
TLX:
EML:
COM:

HIOTELIS NICOLAOS OR

OPT OF ASTRONOMY

NTL UNIVERSITY QF ATHENS

PANEPISTIMIOPOLIS

GR 15783 ZOGRAFOS

GREECE

TEL: 1724.3414

TLF:

TLX:

EML: NHIOTOGRATHUNI

COM: 28

HIRATA RYUKO
OPT OF ASTRONOMY
XYOTO UNIVERSITY
RITASHIRAKAWA SAKYO KL
KYOTO 606
JAPAN
TEL:
TLF:
TLX:
EML:
COM: 29
HIRTH WOLFGANG ERNST PH O
THEODOR-HEUS STR 18
O 5354 WEILERSWIST
GERMANY
TEL:
TLF:
EML:
COM:
TLX:

HILLIARD R DR

USA

TEL:

TLF :

EML:

EOM: 09

ILX:

HINKLE KENNETH H
KITI PEAK NIL OBS
BOX 26732
950 N CHERRY AVE
TUCSON A2 857266732
USA
TEL: 6023275511
TLF:
TLX: 0666-484 AURA NOA
EML:
COM: 29
HIPPELEIN HANS H DR
MPI FUER ASTRONOMIE
KOENIGSTUHL
O 6900 HEIOELBERG I
GERMANY
TEL:
TLF:
TLX:
EML:
COM: 34

HIRAYAMA TADASHI PROF

TOKYO ASTRONOMICAL OBS

NAOJ

DSAWA MITAKA

TOKYO 181

JAPAN

TEL: 0422-32-5111

TLF :

TLX: 2822307 TAOMTK -J

EML:

COM: 10,12

HITOTSUYANAGI JUICHI PROF

KATAHIRA 1-4-6-401

SENDAI 980

JAPAN

TEL: $0222-27-9351$

TLF:

EML:

COM: 35,36

TLX:
HILLS JACK G OR

LOS ALAMOS NATIONAL. LAB M5 B228

THEORETICAL OIV T6

LOS ALAMOS NM 87545

USA

TEL: 5056679152

ILF:

TLX:

EML:

COM: 37,42

HINTEREGGER HANS E' DR

HAYSTACK OBSERVATORY

WESTFORD MA 01886

USA

TEL:

TLF:

TLX:

EML:

COM: 44

HIRABAYASHI HISASHI DR NOBEYAMA RADIO DBS

NADJ

MINAMIMAKI MURA

NAGANO $384 \quad 13$

JAPAN

TEL: $2679-8-2831$

ILF:

$T L X: 3329005$.

EML:

COM: $40.51 \mathrm{C}$

HIROMOTO NORIHISA QR

COMMUNICATIONS RES LAB

4-2-1 NUKUIKITAMACHI

KOGANEI

TOKYO 184

JAPAN

TEL: 0423277548

TLF: $04232766 \overline{67}$

TLX: 2832611 DEMPA J

EML: HIROMOTOQBC.CRL.GO.JP

COM: 34

HJALMARSON AKE G OR ONSALA SPACE OBSERVATORY

GOETEBORG UNIVERSITY

S 439 OO ONSALA

SWEDEN

TEL: $30 \quad 060653$

TLF:

TLX: 2400 ONSPACE

EML:

COM: $28,34,40$
HILLS RICHARD E DR

MULLARD RADIO ASTRON OBS

CAVENOISH LABORATORY

MADINGLEY RD

CAMBRIDGE CB3 OHE

UK

TEL: 22366477

TLF:

ILX: 81282

EML:

COM: 40

HINTZEN PAUL MICHAEL N DR

NASA GSFE

CODE 681

GREENBELT MD 20771

USA

TEL: 3012865101

TLF:

TLX:

EML:

COM: 28

HIRAI MASANORI OR

DPT EARTH SCI \& ASTRONOMY

FUKUOKA UNIVERSITY OF EQU

729 MUNAKATA

FUKUOKA B11-41

JAPAN

TEL: 0940322381

ILF:

$T L X:$

EML:

COM: 29

HIRST WILLIAM $P$

1 CLIFFORD CRESENT

BERGVLIET 7945

SOUTH AFRICA

TEL:

ILF :

TLX:

EML:

COM:

HJELLMING ROBERT M OR
NRAO
BOX O
SOCORRO NM 878010387
USA
TEL: 5058357273
TLF:
TLX: $910-988-1710$
EML: BITNET:RHJELLMIENRAO.edU
COM: $34,40,42$


HO PAUL T P

SMITHSONIAN ASTROPHYS OBS

60 GARDEN ST

CAMBRIDGE MA 02138

USA

TEL: 6174953627

ILX: 921428

EML:

TLF :

COM: 40

HOBBS LEWIS M DR

YERKES OBSERVATORY

UNIVERSITY OF CHICAGO

BOX 258

WILLIAMS BAY WI 53191

USA

TEL: $414 \quad 245 \quad 5555$

TLF :

TLX:

EML:

COM: 34

\section{HOEG ERIK DR}

UNIVERSITY OBSERVATORY

OESTER VOLDGADE 3

OK 1350 COPENHAGEN $K$

DENMARK

TEL: $31 \quad 14 \quad 1790$

TLF: 31389157

TLX: 44155 DANAST

EML:

LOM: 08

HOESSEL JOHN GREG WASHBURN OBSERVATORY UNIVERSITY OF WISCONSIN 475 N CHARTER ST MADISON WI 53706 USA

TEL: $60 \mathrm{~B} 2621752$

TLF :

TEX:

EML :

COM:

HOFFMAN JEFFREY ALAN OR NASA/JOHNSON SPACE CENTER CODE CB 4

HOUSTON TX 77058

USA

TEL: $713 \quad 483 \quad 2411$

TLF :

TLX:

EML:

COM: 44,48
HOAG ARTHUR A OR

4410 E 14TH ST

TUCSON AZ 85711

USA

TEL: $602795 \quad 8644$

TLF:

EML:

COM: 50

TLX:

HOBBS ROBERT W OR

COMPUTER TECHN ASSOCIATES

6116 EXECUTIVE BOULEVARD

APT 800

ROCKVILLE MO 20952

USA

TEL:

TLF:

TLX:

EML:

COM: 33.40

\section{HOEGBOM JAN A OR}

STOCKHOLM OBSERVATORY

513336 SALTSJOEBADEN

SWEDEN

TEL: 87170195

TLF: 8717.4719

EML: HOGBOMEASTRO.SU,SE

COM: 40

TLX: 12972 SOBSERV $S$

HOEY MICHAEL J OR

OPT OF PHYSICS

UNIVERSITY COLLEGE

BELFIELD

DUBLIN 4

IRELAND

TEL: $1693 \quad 244$

TLF:

TLX:

EML:

COM:

HOFFMANN MARTIN OR'

OBSERVATORIUM HOHER LIST

STERNWARTE DER

UNIVERSITAET BONN

- 5568 DAUN

GERMANY

TEL:

TLF :

TLX:

EML:

COM: 42
HOANG BINH DY DR

OBSERVATOIRE DE PARIS

SECTION DE MEUDON

LAM

F 92195 MEUDON PPL COX

FRANCE

TEL: $1 \quad 45 \quad 07 \quad 7445$

TLF:

TLX: 201571

EML:

COM: $12,14,40,51$

HODGE PAUL W PROF

OPT OF ASTRONOMY

UNIVERSITY OF WASHINGTON

FM 20

SEATTLE WA 98195

USA

TEL: 2065432888

TLF :

TLX: 9104740096

EML:

COM: 22,28

HOEGLUND BERTIL PROF

ONSALA SPACE OBSERVATORY

GOETEBORG UNIVERSITY

S 439 OO ONSALA

SWEDEN

TEL: $30 \quad 06 \quad 0652$

TLF :

TLX: 2400

EML:

COM: 34,40

HOFF DARREL BARTON

CENTER FOR ASTROPHYSICS

PROJECT STTAR

60 GARDEN ST

CAMBRIOGE MA 02138

USA

TEL: 6174959798

TLF :

TLX:

EML:

COM: 46

HOFMANN WILFRIEO OR

ASTRONOMISCHES RECHEN-

INSTITUT

MOENCHHOFSTR 12-14

D 6900 HEIOELBERG 1

GERMANY

TEL: 62,2149026

TLF :

TLX: 46L336 ARIHO D

EML:

COM: 21
HOARE MELVIN OR

DPT OF ASTROPHYSICS

UNIVERSITY OF OXFORD

KEBLE RO

OXFORO OXI 3RH

UK

TEL: $865 \quad 273292$

TLF : $865 \quad 273418$

TLX: 83295 NUCLOX G

EML: MGHEUK.AC.OX.ASTRO

COM: 36

HOEFLICH PETER OR

MAX PLANCK INSTITUT FUER

ASTROPHYSIK

KARL-SCHWARZSCHILOSTR I

0 8046 GARCHING MUENCHEN GERMANY

TEL: 89329.93249

TLF: 8932993235

TLX: 524629 ASTRO D

EML: PAHEOGAIPPIS

COM: 29.36

HOEKSTRA ROEL DR

TPD/INO/TH

BOX 155

NL 2600 AD DELFT

NETHERLANOS

TEL:

TLF:

$T L X:$

EML:

COM:

HOFFLEIT E DORRIT OR

DPT OF ASTRONOMY

YALE UNIVERSITY.

BOX 6666

NEW HAVEN CT 06520

USA

TEL:

ILF :

TLX:

EML:

COM: 24,27

HOGAN CRAIG J DR

OPT OF ASTRONOMY

UNIVERSITY OF WASHINGTON

FM 20

SEATTLE WA 98195

USA

TEL:

ILF:

TLX:

EML:

CON: 


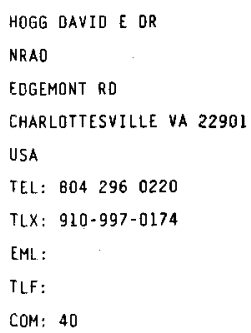

COM: 10
HOLBERG JAY B

LUNAR \& PLANETaR'Y LaB

UNIVERSITY OF ARIZONA

901 GOULO SIMPSON BLDG TUCSON AZ 85721

USA

TEL: 6026214301

TLF:

TLX: 9109521143

EML:

COM: 16,44

HOLLOWAY NIGEL J DR

SAFETY \& RELIABILITY DIR

WIGSHAW LANE

CULCHETH

WARR INGTON WA3 4NE

uK

TEL: 9531244

TLF:

TLX: $62930 \mathrm{i}$

EML:

COM: $4 B$

\section{MOLMBERG ERIK B PROF}

ENELIDEN 2

S 433 DO PARTILLE

SWEDEN

TEL: 31265842

TLF :

EML:

COM: 25,28

TLX:

HONEYCUTT R KENT PROF

ASTRONOMY OPT

INOIANA UNIVERSTTY

SWAIN WEST 319

BLOOMINGTON IN 47405

USA

TEL: 8123356916

TLF :

TLX:

EML:

COM: 09,42

\section{HOOD ALAN DR}

DPT MATHEMATICAL SCIENCE UNIVERSITY OF ST ANDREWS FIFE KY16 9SS

UK

TEL: $334762 \quad 13$

TLF:

TLX: 932110846 SA O

EML: AMSAHOSAVB. ST-AND. AC. UK. ILX:

COM:
HOLOEN FRANK

2 COLWICH CRESENT

KINGSTON HILL

STAFFORD ST16 3XP

UK

TEL: 78553120

TLF:

$T L X:$

EML:

COM: 26

HOLLOWELL DAVID EARL DR

LOS ALAMOS NATIONAL LAB

MS $8220 \times 2$

LOS ALAMOS NM 87545

USA

TEL: 5056674812

TLF

TLX:

EMt: U095874MLANL. GOY

COM: 35

HOLT STEPHEN S
NASA/GSFC
COOE 660
GREENBELT MD 20771
USA.
TEL: 3012868801
TLF:
TLX:
EML:
COM: 42.44

HONG HYON IK OPT OF PHYSICS

KIM IL SUNG UNIVERSITY TAESONG OISTRICT

PYONGYANG

KOREA DPR

TEL:

TLF:

TLX:

EML

COM: 10

HOOGHOUDT B G IR
NAIC
CORNELL UNIVERSITY
SPACE SCIENCES BLDG
ITHACA NY 149536801
USA
TEL:
TLF:
TLX:
EML:
CGM: 09,40

HOLLENBACH DAVID JOHN OR NASA AMES RESEARCH CTR MS 2456 MOFFETT FIELD CA 94035 USA

TEL: 4159976426

TLF:

TLX:

EML:

COM: 34

HOLLWEG JOSEPH V

DPT OF PHYSICS

UNIV OF NEW HAMPSHIRE DEMERITT HALL

DURHAM NH 03824

USA

TEL: 6038623869

ILF:

ILX:

EML:

COM: 10,49

HOLWEGER HARTMUT PROF INST THEOR PHYS \& STERNW UNIVERSITAET KIEL

OLSHAUSENSTR

O 2300 KIEL 1

GERMANY

TEL: $4318804 \quad 107$

TLF:

TLX: 292706

EML:

COM: 12,36

HONG SEUNG SOO OR OPI OF ASTRONDMY SEOUL NTL UNIVERSITY KWANAK KU

SEOUL 151

KOREA R

TEL: $877-2131$

TLF:

TLX: 29664

EML:

COM: 2lC, 22,34

HOPP ULRICH DR MAX PLANCK INSTITUT FUR AStronomie

KOENIGSTUHL 17

O 6900 HEIDELBERG I GERMANY

TEL: 6221528224 TLF: 6221528246

TLX: 461789' MPIA O EML: HDPPODDMPISV, BITNET COM: 28 


HORAK TOMAS B OR
VIRK
RIECNA 1
CS 81558 BRATISLAVA
CZECHOSLOVAKIA
TEL: 7338451
TLF:
TLX:
EML:
COM: 42
HORIUCHI RITOKU DR
NTL INSTITUTE FOR
FUSION SCIENCE
NAGOYA 46401
JAPAN
TEL: $52 \quad 781511$
TLF: 0527827106
TLX: $0447-3691$ NIFS
EML:
COM: 42

HORVATH ANDRAS DR TIT PLANETARIUM \& URANIA OBSERVATORY BOX 46

H. 1476 BUDAPEST HUNGARY

TEL: 133425

TLF:

ILX:

EML:

COM:

HOSKING ROGER J PROF JAMES COOK UNIVERSITY OF NGRTH QUEENSLAND TONNSUILLE QLO 4BII AUSTRALIA

TEL: 778141113

TLF:

TLX: AA47009

EML:

COM:

HOUCK JAMES $R$

DPT OF ASTRONOMY

CORNELL UNIVERSITY

220 SPACE SCIENCE BLOG ITHACA NY 14853

USA

TEL: 6072564806

TLF: 6072552365

ILX: 937478 IICA

EML:

COM: $21 \mathrm{C}$
HORAK ZOENEK PROF DR VIETNAMSKA 2

CS 16000 PRAHA 6

CZECHOSLOVAKIA

TEL:

TLF :

EML:

COM:

TLX:

HOROWITZ PAUL PROF

DPT OF PHYSICS

HARVARO UNIVERSITY

60 GARDEN ST

CAMBRIOGE MA $02138^{\circ}$

USA

TEL: 6174953265

TLF :

TLX: 499211$\}$

EML:

COM: 51

HORWITZ GERALQ PRQF

RACAH INST OF PHYSICS

HEBREW UNIV OF JERUSALEM

JERUSALEM 91904

ISRAEL

TEL: 2584592

TLF:

TLX:

EML:

COM:

HOSOKAWA YOSHIMASA H PROF

SAKIGAOKA 3-4-9

FUNABASHI CITY

CHIBA PREFECTURE 274

JAPAN

TEL: $0474 \quad 48 \quad 6679$

TLF:

TLX:

EML:

COM:

HOUGH JAMES OR

DPT OF PHYSICS \& ASTRON

UNIVERSITY OF GLASGOW

GLASGOW G12 $8 Q 0$

UK

TEL: 413304706

TLF: 413349029

TLX: 777070 UNIGLA

EML: GWO4XGLA; PH.11 UUK. AC.RL.BITNET TLX:

COM: OS
EML:

COM: $27,29,45$

HOREOT GEORG PAUL DR

\section{OFVLR}

D 8031 WESSLING

GERMANY

TEL:

TLF :

TLX:

EML:

COM: 16

MORSKY JAN PROF

OPT OF THEORETICAL PHYS

PURRYME UNIVERSITY

KOTLARSKA 2

CS 61137 BRND

CZECHOSLOVAKIA.

TEL: 51112

TLF:

TLX:

EML:

COM:

HOSHI REIUN DR

OPT OF PHYSICS

RIKKYO UNIVERSITY

NISHI I I KEBUKURO

TOSHIMA KU TOKYO 171

JAPAN

TEL: 03-985-2414

TLF:

TLX:

EML:

COM: 35

HOSOYAMA KENNOSHUKE DR

INTL LATITUDE OBSERVATORY

NAOJ

HOSHIGAOKA MIZUSAWA SHI

IWATE 023

JAPAN

TEL:

TLF:

TLX:

EML:

COM:

\section{HOUK NANCY DR}

OPT OF ASTRONOMY

UNIVERSITY OF MICHIGAN

1045 PHYS-ASTRO BLDG

ANN ARBOR MI $48109 \quad 1090$

USA

TEL: 3137643436

TLF :

LX:
HORI GENICHIRO PROF

DPT OF ASTRONOMY

UNIVERSITY OF TOKYO

BUnKYo KU

TOKYO 113

JAPAN

TEL: 03-8122111×4251

TLF:

TLX: 33659 UTYOSCI J

EML:

COM: 07,33

HORTON BRIAN H OR

JACABRI ENT

B0X 309

GOOLWA SA 5214

AUSTRALIA

TEL: 85553376

TLF:

TLX:

EML:

COM: 12

HOSKIN MICHAEL A DR

CHURCHILL COLLEGE

CAMBRIDGE CB3 ODS

UK

TEL: $223 \quad 35$ 83B1

TLF :

TLX:

EML:

COM: 41

HOTINLI METIN OR

UNIVERSITY RASATHANESI

ISTANBUL

TURKEY

TEL:

TLF:

TLX:

EML:

COM: 12,36

HOUSE FRANKLIN C OR

HEIDENREICHSTR 42

O 6100 DARMSTADT

GERMANY

TEL: $615 \quad 142 \quad 2412$

TLF:

TLX:

EML:

COM: 
HOUSE LEWIS L OR

HIGH ALTITUDE OGSERVATORY

NCAR

$B O \times 3000$

BOULOER CO 803073000

USA

IEL: 303494515 I

ILF:

TLX:

EML :

COM: $12,14,36$

HOWARO W MICHAEL DR

LAWRENCE LIVERMORE LAB

L 297

BOK 808

LIVERMORE CA 94550

USA

IEL: 4154224138

TLF :

I!X:

EML:

COM:

HOWSE H DEREK

12 BARNFIELD RO

RIVERHEAD

SEVENOAKS TN13 2AY

UK

TEL: 732454366

TLF :

ILX:

EML:

COM: 41

HRIVNAK BRUCE J

OPT OF PHYSICS

VALPARAISO UNIVERSITY

VALPARAISO IN 46383

USA

TEL: 2194645379

TLF :

TLX:

EML:

COM: 30,42

\section{HU ESTHER M OR}

INSTITUTE FOR ASTRONOMY

UNIVERSITY OF HAWAII

2680 WOODLAWN DR

HONOLULU HI 96822

USA

TEL: $808 \quad 948 \quad 7190$

TLF

TLX: 723 8459 UHAST HA

EML: INT: huouhifa,ifa.hawail.edu

COM: 47
HOUZIAUX L PROF

INSTITUT DE MATHEMATIQUES

15 AV TILLEULS

B 4000 LIEGE

BELGIUM

TEL: $41 \quad 66 \quad 9494$

ILF: $41 \quad 669493$

TLX:

EML: U2141LH@BLIULGLI

COM: $29,34,44,46 \mathrm{C}$

HOWARO WILLIAM E III DR

1653 QUAIL HOLLOW CT

MCLEAN

VA 221013234

USA

TEL: 7036951447

TLF: 7036976956

TLX:

EML: howardxpeo-mis-emhl.army.mfl.

COM: 40

HOYLE FRED SIR

102 AOMIRALS WALK

WEST. CLIFF RD

BOUREMOUTH

DORSET 8 H2 $5 \mathrm{HF}$

TEL

ILF :

TLX:

EML:

COM: $28,35,47,48$

HRON JOSEF OR

INSTITUT FUER ASTRONOMIE

UNIVERSITAET WIEN

TUERKENSCHANZSTR 17

A 1180 WIEN

AUSTRIA

TEL: I 3453600

TLF :

TLX: 133099 viast a

EML: EARN: AWIUNIIILAB2OIDAH

COM: 29.33

HU FU-XING

PURPLE MOUNTAIN OBSERV

CAS

NANJING

CHINA PR

TEL: 2546700

TLF:

ILX:

EML:

COM: 28
HOVENIER J W DR

DPT PHYSICS \& ASTRONOMY

FREE UNIVERSITY

DE BOELELAAN 1081

NL 1081 HV AMSTERDAM

NETHERLANOS

TEL: 205402414

TLF :

ILX

EML:

COM: 16

HOWARTH IAN DONALD

DPT PHYSICS \& ASTRONOMY

UNIVERSITY COLLEGE LONDON

GOWER ST

LONOON WCIE 6BT

UK

TEL: $1713 \quad 877 \quad 0.50$

TLF :

TLX: 28722

EML:

COM: 44

HOYNG PETER OR

SPACE RESEARCH LABORATORY

SRON

SORBONNELAAN 2

NL 3584 CA UTRECHT

NETHERLANDS

TEL: 30535600

ILF: 30540860

TLX: 47224 SRON NI

EML:

COM: $10,12,44$

HSIANG YAN-YU

BEIJING ASTRONOMICAL OBS

CAS

W SUBURB

BEIJING 100080

CHINA PR

TEL: 1281698

$T L F$

TLX: 22040 BAOAS CN

EML:

COM:

HU JING-YAO

BEIJING ASTRONOMICAL OBS

CAS

W SUBURB

BEIJING 100080

CHINA PR

TEL: 1281698

TLF:

$T X_{i} 22040$ BAOAS CN

EML:

COK: 09,25
HOWARQ ROBERT F DR

NTL SOLAR OBSERVATORY

BOX 26732

$950 \mathrm{~N}$ CHERRY AVE

TUCSON AZ $85726 \quad 6732$

USA

TEL: $602-327-5511$

TLF :

TLX: 0666484 AURA NOAOTUC

EML:

$\mathrm{COH}: 10,12$

HOWELL STEVE BRUCE DR PLANETARY SCIENCE INST 2030 E SPEEOWAY

SUITE 201

TUCSON AZ 85719

USA

TEL: $602 \quad 8 B 1 \quad 0332$

TLF : 6028810335

TLX:

EML: 5470::HOWELL

COM: 27

HRIC LADISLAV OR

ASTRONOMICAL INSTITUTE

SLOVAK ACADEMY SCIENCES

CS 05960 TATRANSKA LOMNI

CZECHOSLOVAKIA

TEL: $96996 \quad 7866 / 7 / 8$

ILF: 969967656

TLX: 7827?

EML:

COM: 42

HSIANG-KUANG TSENG

OPT OF PHYSICS

NTL CENTRAL UNIVERSITY

CHUNG LI

CHINA R

TEL:

TLF:

TLX:

EML:

COM:

HU NING - SHENG

NANJING ASTRONOMICAL

INSTRUMENT FACTORY

BOX 846

NANJING

CHINA PR

TEL: 2546191

TLF:

TLX: 34136 GLYNJ CN : MAIF

EML:

COM: $08 \mathrm{C}, 09$ 
HU WEN-RUI

INSTITUTE OF MECHANICS

CAS

\section{W SUBURB}

BEIJING 100080

CHINA PR

TEL: 1284185

TLF:

TLX: 22474 ASCHI CN

EML :

COM: 44

HUAN NGEYEN DUSH DR

UNIVERSITY OF VINH

PROVINCE NGH^E AN

VIETNAM

TEL:

TLF :

EML:

COM:

TLX:

HUANG JIE-HAO

ASTROPHYSICS INSTITUTE

NANJING UNIVERSITY

NANJING

CHINA PR

TEL:

TLF:

TLX: 34151 PRCNU CN

EML:

COM: 28

\section{HUANG RUN-OIAN}

YUNNAN OBSERVATORY

CAS

BOX 110

KUNMING 72946 YUNNAN

CHINA PR

TEL: $B 712035$

TLF:

TLX: 64040 YUOBS CN

EML: :

COM: 35,42

HUANG YINN-NIEN 'DR

DPF OF POSTS \& TELECOMMUN

MIN OF TRANSPORT \& COMMUN

2 CHANGSHA ST SEC 11

TAIPEI 100

CHINA R

TEL:

TLF:

TLX:

EML:

COM:

HU ZHONG-WE:
DEPI OF ASTRONOMY
NANJING UNIVERSITY
NANJING
CHINA PR
TEL: $253765 \mathrm{I}$
TLF:
TLX: 0909
EML:
COM: 15,16

HUANG BI-KUN

PURPLE MOUNTAIN OBSERY

LIBRARY

CAS

NANJING

CHINA PR

TEL: 25307521

TLF :

TLX: 34144 PMONJ CN

EML:

COM: 05

HUANG KE-LIANG

DPT OF ASTRONOMY

NANJING UNIVERSITY

NANJING

CHINA PR

TEL: $2534651 * 2882$

TLF :

TLX: 34151 PRCNU CN

EML:

COM: 28,48

HUANG SONG-NIAN DR

ICTP CP 586

ST COSTIERA 11

MIRAMARE

I 34100 TRIESTE

ITALY

TEL:

TLF:

TLX:

EML:

COM: 28,33

HUANG YIN-LIANG

TIANJIN INST OF TECH

HONG QI NAN RD

TIANJIN 300191

CHINA PR

TEL: 368787256

TLF:

TLX:

EML:

COM: 50
HUA CHON TRUNG OR

LAS

TRAVERSE OU SIPHON

LES TROIS LUCS

F 13012 MARSEILLEE

FRANCE

IEL: 91055932

TLF: $9156 \quad 1855$

TLX: $420584 F$

EML:

COM: 28,34

hUANG CHANG-CHUN

PURPLE MOUNTAIN OBSERV

CAS

NANJING

CHINA PR

TEL: 25 46700/42817

TLF :

TLX; 34144 PMONJ CN

EML:

COM: 29,30

HUANG KUN-YI

PURPLE MOUNTAIN OBSERV

CAS

NANJING

CHINA PR

TEL: 2532893

TLF:

TLX: 34144 PMONJ CN

EML:

COM:

MUANG TIANYI

DEPT OF ASTRONOMY

NANJING UNIVERSITY

NANJING

CHINA PR

TEL:

TLF :

TLX: 34151 PRCNU CN

EML:

COM: 07

HUANG YI-LONG OR

INST OF HISTORY

NTL TSING HUA LNIVERSITY

HSIN CHU 300043

CHINA $R$

TEL: 35716780

TLF :

TLX:

EML:

COM:
HUA YING-MIN

SHANGHAI OBSERVATORY

CAS

80 NANDAN RD

SHANGHAI 200030

CHINA PR

TEL: $21 \quad 38 \quad 8191$

TLF: $2138 \quad 4618$

TLX: 33164 SHAO CN

EML:

COM: 08,19

HUANG CHENG DR

SHANGHAI OBSERVATORY

CAS

80 NANDAN RD

SHANGHAI

CHINA PR

TEL: $21 \quad 386191$

TLF :

TLX:

EML

COM: 07

HUANG LIN

BEIJING ASTRONOMICAL' OBS CAS

W SUBURB

BEIJING 100000

CHINA PR

TEL: 1281698

$T L F:$

TLX: 22040 BAOAS CN

EML:

COM: 25.45

HUANG TIE-QIN

NANJING ASTRONOMICAL INSTRUMENT FACTORY

NANJING

CHINA PR

TEL: 2546191

TLF

TLX: 34136 GLYNJ CN

EML:

COM: 09

HUANG YONGWEI

BEIJING ASTRONGMICAL OBS

CAS

W SUBURB

BEIJING 100080

CHINA PR

TEL: $128 \quad 1698$

TLF :

TLX: 9053

EML:

COM: 28 


HUANG. YOU-RAN
OPT OF ASTRONOMY
NANJING UNIVERSITY
NANJING
CHINA PR
TEL:
TLF:
TLX: 34151
EML:
COM: 10

HUBENY IVAN
JILA
UNIVERSITY OF COLORADO
BOX 440
BOULOER CO 80309.0440
USA
TEL: 3034927838
ILF:
ILX: 755842 JILA
EML:
COM: 29,36

HUCHRA JOHN PETER DR CENTER FOR ASTROPHYSICS
HUBbaro WILLIAM B PROF LUNAR \& PLANETARY LAB UNIVERSITY OF ARIZONA TUCSON AZ 85721

USA

TEL: $602 \quad 6216942$

ILF:

TLX: 9109521143

EML:

COM: 16

HUBER MARTIN CE DR

ESA/ESTEC

SSD

. $80 \times 299$

NL 2200 AG NOOROWIJK

NETHERLANDS

TEL: 171983552

TLF:

TLX: 39098

EML:

COM: 14,44

HUCHTMEIER WALTER K OR

MPI FUER RADIOASTRONOMIE

AUF DEM HUEGEL 69

O 5300 BONN 1

GERMANY

TEL: 228525215

Tix: 886440

EML:

TLF:

COM: 28,40

HUENEMOERDER DAVID P. DR

OPT OF ASTRONOMY

pennsylVania state uniV

525 daver LAB

UNIYERSITY PARK PA 16802

USA

TEL: $814865660 \mathrm{~L}$

TLF:

TLX:

EML: INTERNET: dpheastro.psu. edu

COM: 27,29

\section{HUGHES SHAUN}

CALTECH

DPT OF AStronamy

PASADENA CA 91125

USA

TEL:

TLF:

TLX:

EML:

COM:
HUBE DQUGLAS P. OR

OPT OF PHYSICS

UNIVERSITY OF ALBERTA

EDMONTON AB TGG 2JI

CANADA

TEL: 4034325410

TLF: 4034324256

TLK: 0372979

EML:

COM: 30,42

HUBERT HENRI QR

OBSERVATOIRE DE PARIS

SECTION DE MEUDON

DASGAL

F 92195 MEUDON PPL CDX. FRANCE

TEL: $145 \quad 077850$

ILF:

TLX: 270912

EML: HUBERT TRMEUSI

COM: 29

HUDEC RENE DR

ASTRONOMICAL INSTITUTE

CZECH ACADEMY OF SCIENCES

ONDREJOV OBSERVATORY

CS 25165 ONOREJOV

CZECHOSLOVAKIA

TEL: 20485201

TLF: 20485314

TLX: : 121579

EML:

COM:

\section{HUGHES DAVID W DR}

OPT OF PHYSICS

THE UNIVERSITY

SHEFFIELD S3 7RH

UK

TEL: 74278555

TLF:

$T L X: 54348$ ULSHEF 6

EML:

COM: $15 C, 22$

HUGHES VICTOR A PROF

OPT OF PHYSICS

QUEEN'S UNIVERSITY

KINGSTON ON K7L 3N6.

CAMADA

TEL: 6135476633

TLF: 6135456463

TLX:

EML: HUGHESV YUCON BITANET

COM: $33,34,40$
HUBENET HENRI OR

STERREKLNGIG INSTITUTE

B0X 80000

NL $350 B$ TA UTRECHT

NETHERLANOS

TEL: 30535200

TLF:

ILX: 40048 FYLUT NL

EML:

COM:

HUBERT-DELPLACE A-M DR OBSERVATOIRE OE PARIS SECTION OE MEUDON F 92195 MEUDON PPL COX FRANCE

TEL: 1 45. 347856

TLF:

ILX: 270912

EML:

COM: 29

HUOSON HUGH S DR

OPT OF PHYSICS

UesD

C 011

LA JOLLA CA 920930216

USA

TEL: $619452 \mathbf{4 4 7 6}$

TLF:

TLX:

EML:

COM: 10

HUGHES JOHN P DR CENTER FOR ASTROPHYSICS HCO/SAO

60 GAROEN ST

CAMBRIOGE MA 02138

USA

TEL: 6174957142

TLF:

ILX: 921428 SATELLITE CAM

EML: BITNET: HUGHES SFA.

COM:

HUGUENIN G RICHARD MULTITECH CORPORATION BOX 109 SOUTH DEERFIELL RES PARK SOUTH DEERFIELD MA 01373 IJSA

TEL: 4136658551

TLF:

TLX: 3719862 TRUB

EML:

EOM: 
HULSBOSCH A N M OR

STERRENKUNDIG INSTITUTE

KATHOLIEKE UNIVERSITEIT

TIERNOOIVELO

NL 6525 ED NIJMEGEN

NETHERLANOS

TEL: $B 055 \quad 8833$

TLF:

TLX: 48228

EML :

COM: $33,34,40$

HUMPHREYS ROBERTA M PROF

OPT OF ASTRONOMY

UNIVERSITY OF MINNESOTA

116 CHURCH ST SE

MINNEAPOLIS MN 55455

USA

IEL: 6123739747

ILF:

TLX:

EML:

COM: $28,33,35,45$

HUNSTEAD RICHARD W DR

SCHOOL OF PHYSICS

UNIVERSITY OF SYONEY

SYONEY NSW 2006

AUSTRALIA

TEL: 26923871

TLF :

TEX: 26169 UNISYO AA

EML:

COM: 28.40

HUNTER CHRISTOPHER PROF

DPT OF MATHEMATICS

FLORIDA STATE UNIVERSITY

TALLAHASSEE FL 32306

USA

TEL: $904 \quad 644 \quad 2488$

TLF

ILX:

EML:

COM: 28.33

HUOVELIN JUHANI OR

OBS \& ASTROPHYSICS LAB

UNIVERSITY OF HELSINKI

TAEHT I TORN I NMAK I

SF OOI30 HELSINKI 13

FINLAND

TEL: : 1912948

ILF: 1912952

TLX: 124690 UNIH SF

EML: HUOVELIN AT CC.HELSINKI.FI

COM: 25
HUMBLE JOHN EDMUND 'OR

DPT OF PHYSICS

UNIVERSITY OF TASMANIA

GPO BOX $252 C$

HOBART IAS 7001

AUSTRALIA

TEL: 02202401

TLF; 02202410

TLX: AA 58150

EML: JOHN. HUMBLEPHYS.UTAS. EDU.AU

COM: 49

HUMPHRIES COLIN M DR

ROYAL OBSERVATORY

BLACKFORO HILL

EDINBURGH EHS $3 \mathrm{HJ}$

UK

TEL: $316 \quad 673 \quad 321$

ILX: 72383

EML:

TLF :

COM: 095

HUNT GE OR

ELBURY

37 BLENHEIM ROAD

RAYNES PARK

LONDON SW20 9BA

UK

TEL: i 5422374

TLF:

ILX:

EML:

COM: 16

\section{HUNTER OEIORE ANN}

OPT TERRESTR MAGNETISM

CARNEGIE INST WASHINGTON

5241 BROAO BRANCH RO NW

WASHINGTON OC 20015

USA

TEL: 2029660863

TLF :

TLK: 440427 MAGN UI

EML:

COH:

HURFORO GOROON JAMES

CALTECH

MS 26433

PASADENA CA 91125

USA

TEL: $818 \quad 356 \quad 3866$

TLF:

TLX: 67542

EML:

COM: 10
HUMMEL EOSHO

NRAL

JOORELL BANK

MACCLESFIELO SKLL 90L

UK

TEL:

TLF :

TLX:

EML:

COM: 28

HUNDHAUSEN ARTHUR DR

HIGH ALTITUDE OBSERVATORY

NCAR

BOX 3000

BOULDER CO B0307 3000

USA

TEL:

TLF:

TLX:

EML:

COM:

HUNT LESLIE DR

OSS ASTROFISICO

DI ARCRETI CAISMI CNR

LARGO E FERMI 5

I 50125 FIRENZE.

ITALY

TEL: 552752296

TLF: 55220039

TLX: 572268 ARCETR

EML: HUNTOSISIFO, ARCETRI, ASTRO.IT

COM: 48

HUNTER JAMES H PROF

DPT OF ASTRONOMY

UNIVERSITY OF FLORIDA

211 SSRB

GAINESVILLE FL 32611

USA

TEL: $904392 \quad 1078$

TLF :

TLX:

EML:

COM: 28,51

HJRNIK HIERONIM PROF

ASTRONOMICAL OBSERVATORY

A MICKIEWICZ UNIVERSITY

UL SLONECZNA 36

PL 60286 POZNAN

POLAND

TEL: 679670

TLF :

TLX:

EML:

COM: 20
HUMMER DAVIO G DR

JILA

UNIVERSITY OF COLORADO

BOX 440

BDULOER CO 803090440

USA

TEL: 3034927837

TLF:

TLX: 755842 JILA

EML:

COM: 34,36

HUNGER KURT PROF

INST THEOR PHYS \& STERNW

MEUE UNIV PHYSIK ZENTRUM OLSHAUSENST 40 NGIC

D 2300 KIEL 1

GERMANY

TEL: 4318804110

TLF :

TLX: 292706

EML :

COM: 29,36

HUNTEN DONALO M PROF

LUNAR \& PLANETARY LAB

UNIVERSITY OF ARIZONA

TUCSON AZ 85721

USA

TEL: 6026214002

TLF :

TLX:

EML:

COM: 16,51

HUNTRESS WESLEY T OR

NASA HEADQUARTERS

COOE SL

600 INDEPENDENCE AVE SW

WASHINGTON DC 20546

USA

TEL: $202 \quad 453 \quad 1588$

TLF : $202 \quad 426 \quad 1023$

TLX:

EML:

COM: 15

HUSFELD DIRK DR

INSTITUT FÖR ASTRONOMIE

UNO ASTROPHYSIK

SCHEINERSTR 1

D 8000 MUENCHEN 80

GERMANY

TEL: 8992209440

TLF: $89922 \quad 09427$

TLX:

EML: UHIOIBS DMOLRZOL.EARN

COM: 36 
HUT PIET

INST FOR ADVANCED STUDY SCHOOL OF NATURAL SCIENCE PRINCETON NJ 08540 USA

TEL: 6097348075

TLF:

TLX: 229734 IAS UR

EML:

COM: 37

HWANG WOEI-YANN P PROF

DPT OF PHYSICS

NTL TAIWAN UNIVERSITY

TAIPEI

TAIWAN 10764 ROC

CHINA $R$

IEL: $23630231 * 3159$

TLF: $2363 \quad 7204 / 9984$

ILX:

EML:

COM:

HYUN JONG-JUNE PROF

DPT OF ASTRONOMY

SEOUL NTL UNIVERSITY

KWANAK KU

SEOUL 151

KOREA $R$

TEL: $877-3010 / 2542$

TLF:

YLX:

EML:

COM:

IBANEZ S MIGUEL H DR

UNIVERSIDAD OE LOS ANDES

FACULDAD DE CIENCIAS

DPT OE FISICA

MERIDA 5101 A

VENEZUELA

TEL: $7463 \cdot 9930 / 7477$

TLF:

TLX: 74174 CIOA

EML:

COM:

IBRAHIM JURGA

DPT OF ASTRONOMY

BANDUNG INSTITUTE OF TECH

JL. TAMANSARI 64

BANDUNG

INOONESIA

TEL:

T.LF:

$T L X$ :

EML:

COM:
HUTCHEON RICHARD J OR

OPT OF PHYSICS

UNIVERSITY OF LEICESTER

$X$-RAY ASTRONOMY GROUP

LEICESTER LEI TRH

UK

TEL:

TLF:

TLX:

EML:

COM:

HYOER C \& OR

HIGH ALTITUDE OBSERVATORY.

NCAR

BOX 3000

BOULOER CO 603073000

USA

TEL:

TLF:

TLX:

EML:

COM: 10

IANNA PHILIP A

UNIVERSITY STATION

UNIVERSITY OF VIRGINIA

BOX 3818

CHARLOTIESVILLE VA 22903

USA

TEL: 8049244898

TLF :

TLX:

EML:

COM: $20,24,26$

IBANOGLU C OR

FACULTYY OF SCIENCE

EGE UNIVERSITY

BOX 21

35100 BORNOVA IZMIR

TURKEY

TEL: $51 \quad 18 \quad 0110 * 2332$

TLF:

$T\lfloor X$ :

EML:

COM: 42

\section{ICHIKAWA SHIN-ICHI DR}

TOKYO ASTRONOMICAL OBS

NAOJ

OSAWA MITAKA

TOKYO 181

JAPAN

TEL: 422413604

TLF: 422413608

TLX: 02822307 TAOMTK

EML: ICKIKAWAOCI.MTK. NAO.AC.JP.

COM: 28
HUTCHINGS JOHN B DR HERZBERG INST ASTROPHYS DOMINION ASTROPHYS OBS

5071 W SAANICH RD

VICTORIA BC VBK AMG

CANADA

TEL: 60433803909

TLF: $604363 \quad 0045$

TLX: 0497295

EML:

COM: $27,34,36,42,44 \mathrm{C}$

HYLAND A R HARRY DR

OPT PHYSICS UNIV COLLEGE

UNIVER OF NEW SOUTH WALES

NORTHCOTT QR

CAMPBELL ACT 2600

AUSTRALIA

TEL: $62 \quad 68 \quad 8787$

TLF: 62688786

TLX: 62030 ADFAOM AA

EML:

COM: 25,29

IANNINI GUALBERTO OR

DBSERVATORIO ASTRONOMICO

DE CORDOBA

LAPRIOA 854

5000 CORDOBA

ARGENTINA

TEL: 5140613

TLF: ,

TLX:

EML:

COM:

IBBETSON PETER AARON DR

WISE OBSERVATORY

TEL AVIV UNIVERSITY

RAMAT AVIV

TEL AVIV 69978

ISRAEL

TEL: 3 41 3788

TLF:

TLX: 342171 VERSY IL

EML:

COM:

IEHIKAWA TAKASHI

OPT OF ASTRONOMY

KYOTO UNIVERSITY

KITASHIRAKAWA SAKYO KU

KYOTO 606

JAPAN

TEL: 757513890

TLF :

$T \backslash X:$

EML:

COM: 28
HUTSEMEKERS DAMIEN DR INSTITUT O'ASTROPHYSIQUE UNIVERSITE DE LIEGE

AVE COINTE 5

B 4000 COINTE-LIEGE

BELGIUM

IEL: 4152.9980

TLF: 41527474

ILX: 41264 ASTRLG B

EML: U214LMGEBLIULGII

COM: 34

HVSOM EOMUND J

8 EAST DRIVE

CALDECOTE

CAMBRIDGE CB3 7NZ

UK

TEL: $223954211 * 137$

TLF:

TLX:

EML:

COM: $09,41,51$

IBADINOV KHURSANDKUL OR ASTROPHYSICAL INSTITUTE TADJIK ACAO OF SCIENCES 734670 DUSHANBE

TAOZHIKISTAN

TEL:

TLF :

$T L X$ :

EML:

COM: 15

IBEN ICKO JR PROF

OPT OF ASTRONOMY

UNIVERSITY OF ILLINOIS IOIL W SPRINGFIELO AVE URBANA IL .6180I

USA

TEL: 2173333090

TLF:

TLX: 9102452434 AST

EML:

COM: $27,35 \mathrm{C}, 37$

ICHIMARU SETSUO OR

DPT OF PHYSICS

UNIVERSITY OF TOKYO

BUNKYO KU

TOKYO 113

JAPAN

TEL: $03-812-2111$

TLF :

TLX: UTPHYSIC J23472

EML:

COM: $4 B$ 
ICKE VINCENT OR

STERREWACHT

B0X 9513

NL 2300 RA LEIDEN

NETHERLANDS

TEL: 71272727

TLK: 39058 ASTRO NL

EML:

TLF:

COM: 47

\section{IKEUCHI SATORU DR}

TOKYO ASTRONOMICAL OBS

\section{NAOS}

OSAWA MITAKA

TOKYO 181

JAPAN

TEL: $0422-32-5111$

TLF:

TLX: 2822307

EML:

COM: 33,47

\section{ILL MARTON J OR}

KONKOLY OBSERVATORY

TOTH Kalman U 19

H 6501 BAJA

HUNGARY

TEL: 7912110

TLK: 281303

EML:

TLF:

COM:

\section{ILYAS MOHAMMAD DR}

\section{SCHOOL OF PHYSICS}

UNIVERSIII SAINS MALAYSIA

11800 USM

PENANG

\section{MALAYSIA}

TEL: 883822

TLF:

ILX: 40254 MA

$$
\text { EML: }
$$

COM: 04.09 .46

IMPEY CHRISTOPHER D DR

\section{STEWARD OBSERVATORY}

UNIVERSITY OF ARIZONA TUCSON A2 85721

$$
\text { USA }
$$$$
\text { TEL: } 602 \quad 6216522
$$

TLF:

$I L x: 467175$

EML: impeyesolpl,as.arizona.edu

[DM: $28,46,47$

IOLIS G M OR
INST HIST OF SCI \& TECH
ACACEAY OF SCIENCES
STAROPANSKY $1 / 5$
103012 MOSCOW
RUSSIA
TEL: 2281969
TLF:
ILX:
EML:
COM: 41.51

IKHSANOV ROBERT N DR PULKOVO OBSERVATORY ACADEMY OF SCIENCES 10 KUTUzOV QUAY I96140 ST PETERSBURG RUSSIA

TEL:

TLF :

TLX:

EML:

COM: 40

ILLES ALMAR ERZSEBET OR KONKOLY OBSERVATORY THEGE $\cup$ 13/17

BOX 67

H 1525 BUDAPEST

HUNGARY

TEL: 1.75 5866/75 4122

TLF :

TLX: 227460

EML:

COM:

IMAMURA JAMES OR

OPT OF PHYSICS

UNIVERSITY OF OREGON

EUGENE OR 97403

USA

TEL: $503 \quad 6865212$

TLF:

TLX:

EML: Imamurahost ro. UOREGON. EOU COM: 42

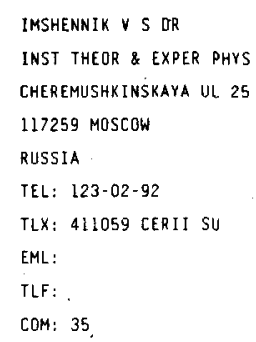

IIJIMA SHIGETAKA PROF

MUSASHI INSTITUTE OF

TECHNOLOGY

TAMAZUTSUMI, SETAGAYA-KU

TOKYO 158

JAPAN

TEL: 337033111

TLF:

ILX:

EML:

COM: 19,31

IKHSANOVA VERA N OR
PULKOVO OBSERVATORY
ACADEMY OF SCIENCES
I0 KUTUZOV QUAY
196140 ST PETERSBURG
RUSSIA
TEL:
TLF:
TLX:
EML:
COM: 40

ILLING RAINER M E

BALL AEROSPACE SYSTEMS

DIVISION

BOX 1062

BOULDER CO 80306

USA

TEL: $303939588 B$

TLF :

TLX:

EML:

COM: 12

IMBERT MAURICE OR

observatoire de MARSEILLE

2 PLACE LE VERRIER

F 13248 Marseille COX 04

FRANCE

TEL: 91959088

TLF:

TLX: 420241

EML:

COM: 30,42

INAGAKI SHOGO DR
OPT OF ASTRONOMY
KYOTO UNIVERSITY
KITASHIRAKAWA SAKYO KU
RYOTO 606
JAPAN
TEL: $075-75 Y-2111$
TLF:
TLX: 5422693 LIBKYU J
EML:
COM: $33,37,49$

IIJIMA TAKASHI DR OSSERVATORIO ASTROFISTCO VIA DELL OSSERVATORIO B I 36012 ASIAGO

ITALYY

TEL: $42 \quad 462505$

TLF: 424462884

TLX:

EML: IIJIMA STRAS. CINECA,IT

COM: 27

ILIEV ILIAN

NTL ASTRONOMICAL OBS

BULGARIAN ACAD SCIENCES

BOX 136

BG 4700 SMOLJAN

BULGARIA

TEL: 7341559

TLF:

TLX: 23561

EML:

COM: 14,35

ILLINGWORTH GARTH O DR LICK OBSERVATORY UNIVERSITY OF CALIFORNIA SANTA CRUZ CA 95064

USA

TEL:

TLF :

TLX:

EML:

COM: 28,37

IMHOFF CATHERIME L DR

NASA/GSFC

COOE 684.9

IUE OBS

GREENBELT MO 20771

USA

TEL: 3012865749

TLF:

TLX: 89675

EML:

COM: 44

INATANI JUNJI

NOBEYAMA RADIO OBS

NAOJ

MINAMIMAKI MURA

NAGANO 38413

JAPAN

TEL: $267-98-2831$

TLF:

TLX: 3329005 TAO NRO J

EML:

COM: 40 


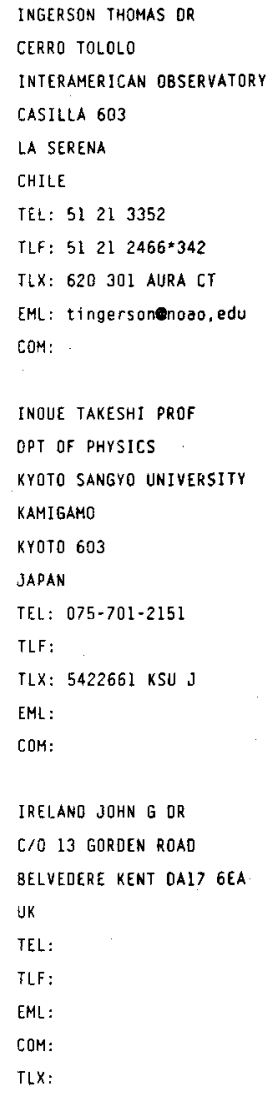

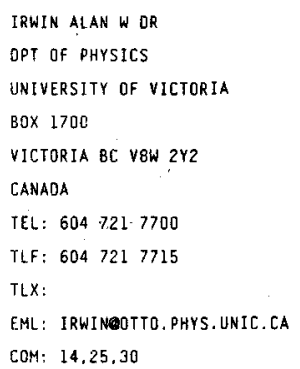

INNANEN KIMMO A PROF
OPT OF PHYSICS
YORK UNIVERSITY
4700 KEELE ST
NORTH YORK ON M3J 1P3
CANADA
TEL: 4166673937
TLF: 4167365396
TLX: 06524736
EML:
COM: 33
10SHPA B A OR
IZMIRAN
ACADEMY OF SCIENCES
142092 TROITSK
RUSSIA
TEL: 2321921
TLF:
TLX: 412623 SCP
EML:
COM: 10

\section{IRIGOYEN MAYLIS}

UNIVERSITE OE PARIS II

12 PLACE dU PANTHEON

\section{$F 75005$ PARIS}

FRANCE

TEL:

TLF:

TLX:

EML:

COM:

IRWIN JOHN B PROF

2744 N TYNDALL AVE

TUCSON AZ 85719

USA

TEL: $602 \quad 6237423$

TLF :

EML:

COM: 33,42

TLX:

ISERN JORGE OR
C/SEPULVEDA $83-6-3 A$
E 08015 BARCELONA
SPAIN
TEL:
TLF:
EML:
COM: 35
TLX:

INOUE HAJIME OR

INST SPACE \& ASTRON SCI

UNIVERSITY OF TOKYO

MEGURO KU

TOKYO 153

JAPAN

TEL: 03-467-1111

TLF:

TLX:. $24550 \mathrm{~J}$

EML:

COM: 44,48

IP WING-HUEN

MPI FUER ASTRONOMIE

D 3411 KATLENBURG LINDAU GERMANY

TEL: 0049-555-6416

TLF:

TLX: 0965527

EML:

COM: 15

IRIYAMA JUN DR

FAC OF ENGINEERING

CHUBU UNIVERSITY

1200 MATSUMOTO

KASUGAI - SHI, AICHI 487

JAPAN

TEL: $56851 \quad 1111$

ThF:

TLX:

EML:

COM: 17

IRWIN JUDITH OR

DPT OF ASTRONOMY

UNIVERSITY OF TORONTO

60 ST GEORGE ST

TORONTO ON MSS IAI

CANADA

TEL: 4169785558

TLF: 4169783921

TLX: 06218915

EML:

COM: 28

ISHIDA GORO DR

BROADCAST UNIVERSITY

23-11 AKABANE NISHI

I CHOME KITA KU

TOKYO 115

JAPAN

TEL: 03-909-3871

TLF:

TLX:

EML:

COM: 26
INOUE MAKOTO DR

NOBEYAMA RADIO OBS

NAOJ

MINAMIMAKI MURA

NAGANO $384 \quad 13$

JAPAN

TEL: $0267-98-2831$

TLF :

TLX: 3329005 TAONKKRO IJ

EML:

COM: 40

IPSER JAMES $R$ PROF

OPT OF PHYSICS

UNIVERSITY OF FLORIOA

WILLIAMSON HALL

GAINESVILLE FL 32611

USA

TEL: 904392.0521

TLF:

TLX:

EML:

CON: 48

IRVINE WILLIAM M PROF

FIVE COLLEGE

RADIO ASTRONOMY OBSERV

B619 LEOERLE GRAO RES TWR

AMHERST MA 01003.

USA

TEL: 4135450733

ILF :

TLX: 955491 UNIV MASS AMS

EML:

COM: $15,16,34,51$

IRWIN MICHAEL JOHN DR INSTITUTE OF ASTRONOMY THE OBSERVATORIES MAOINGLEY RD CAMBRIDGE CB3 OHA UK

TELL: 223337548

TLF:

TLX:

EML: MIKEUK.AC. CAM.AST-STAR

COM: 24

ISHIDA KEIICHI PROF

TOKYO ASTRONOMICAL OBS

NAOJ

OSANA MITAKA

TOKYO 181

JAPAN

TEL: $04-22-32-5211$

TLF:

TLX: 2822307 TAOMTK J

EML:

COM: 37 
A144

\begin{tabular}{|c|c|c|c|}
\hline ISHIGURO MASATO PROF & ISHIHARA HIDEKI OR & ISHIZAWA TOSHIAKI A PROF & ISHIZUKA TOSHIHISA DR \\
\hline NOBEYAMA RADIO OBS & QPT OF PHYSICS & DPT OF ASTRONOMY & DPT OF PHYSICS \\
\hline NAOJ & KYOTO UNIVERSITY & KYOTO UNIVRESITY & IBARAKI UNIVERSITY \\
\hline MINAMIMAKI MURA & SAKYO KU & KITASHIRAKAWA SAKYO KU & BUNKYO \\
\hline NAGANO $384 \quad 13$ & KYoт0 606 & KYOTO 606 & MITO 310 \\
\hline JAPAN & JAPAN. & JAPAN & JAPAN \\
\hline TEL: 0267982831 & TEL: 0757533850 & TEL: $075-751-2111$ & TEL: $0292-26-1621$ \\
\hline ILF: & TLF: & TLF: & TLF : \\
\hline TLX: 3329005 NAONRO J & $T \perp X:$ & TLX: 5422693 LIBKYU J & $T L X_{i}$ \\
\hline EML: & EML: ISHIHARAENWS841. SCPHYS,'KYOTOUA & EML: & EML: \\
\hline COM: 40 & COM: 47 & COM: & COM: 35 \\
\hline ISOBE. SYUZZO OR & ISRAEL FRANK P DR & ISRAEL GUY MARCEL DR & ISRAEL WERNER PROF \\
\hline TOKYO ASTRONOMICAL OBS & STERREWACHT & SERVICE O'AERONOMIE & DPT OF PHYSICS \\
\hline NAOJ & BOX 9513 & BP 3 & UNIVERSITY OF ALBERTA \\
\hline OSAWA MITAKA & NL 2300 RA LEIDEN & F 91371 VERRIERES BUISSON & EOMONTON AB T6G $2 J 1$ \\
\hline TOKYO 181 & NETHERLANOS & FRANCE & CANADA \\
\hline JAPAN & TEL: & TEL: $1 \quad 64 \quad 47 \quad 4289$ & TEL: 4034323552 . \\
\hline TEL: 422413645 & TLX: & TLF: & TLF: $403 \quad 432 \quad 4256$ \\
\hline TLF: 422413608 & EML: & TLX: & TLX: 0372979 \\
\hline TLX: 2822307 TAQMTK J & TLF: & EML: & EML: \\
\hline EML: OISOBEX $\triangle$ LL.MTK.NAO.AC.JP & COM: $28 C, 33,34,51$ & COM: & COM: 48 \\
\hline COM: $06 C, 15,20,33,34,46 C$ & & & \\
\hline ISSA ALI DR & ISSERSTEOT JOERG DR. & ITO KENSAI A PROF & ITOH HIROSHI DR \\
\hline HELWAN OBSERVATORY & INSTITUT FUER ASTRONOMIE & DPT OF PHYSICS & OPT -OF ASTRONOMY \\
\hline HELWAN & UND ASTROPHYSIK & RIKKYO UNIVERSITY & KYDTO UMIVERSITY \\
\hline EGYPT & AM HUBLAND & NISHI - IKEBUKURO & KITASHIRAKAWA SAKYO KU \\
\hline TEL: $780645 / 2683$ & O 8700 WUERZBURG & TOKYO 171 & KYOTO 606 \\
\hline TLF: & GERMANY & JAPAN & JAPAN \\
\hline EML: & IEL: & TEL: $03-985-2384$ & TEL: $075-751-2111$ \\
\hline COM: 28.34 & TLF: & TLF : & TLF : \\
\hline TLX: 93070 & TLX: & $T L X:$ & TLX: \\
\hline & EML: & EML: & EML: \\
\hline & COM: & COM: 48 & COM: 34 \\
\hline ITOH MASAYUKI OR & ITOH NAOKI DR & IVANCHUK VICTOR I OR & IVANOV GEORGI $R$ OR \\
\hline ISAS & DPT OF PHYSICS & ASTRONOMICAL OBSERVATORY & DPT OF ASTRONOMY \\
\hline 3-1-1 YOSHINODAI & SOPHIA UNIVERSITY & KIEV STATE UNIVERSITY & UNIVERSITY OF SOFIA \\
\hline SAGAMIHARA & 7-1 XIOI-CHO CHIYOOA KU & OBSERVATORNAYA UL 3 & ANTON IVANOV ST 5 \\
\hline KANAGAWA 229 & TOKYO 102 & $252053 \mathrm{KIEV}$ & BG 1126 SOFIA \\
\hline JAPAN & JAPAN & UKRAINE & BULGARIA" \\
\hline TEL: 427513911 & TEL: $03-238-3431$ & TEL: & TEL: 2544852 \\
\hline TLF: $42759 \quad 4253$ & TLF: & TLF: & TLF : \\
\hline TLX: J2775B ISAS ERO & TLX: & TLX: & TLX: \\
\hline EML: & EML: & EML: & EML: \\
\hline COM: 48 & COM: 35 & COM: 10 & COM: \\
\hline IVANOV VSEVOLOD $\vee$ DR PROF & IVANOVA VIOLETA OR. & IVANOV-KHOLOONY G S DR & IVES JOHN CHRISTOPHER MR \\
\hline ASTRONOMICAL OBSERVATORY & ASTRONOMICAL OBSERVATORY & IZMIRAN & ESA/ESTEC \\
\hline ST PETERSBURG UNIVERSITY & BULGARIAN ACAD SCIENCES & ACADEMY OF SCIENCES & SSO \\
\hline BIBLIOTECHNAJA PL 2 & 72 LENIN BLVO & $142092 \mathrm{MOSCOW}$ & $80 \times 299$ \\
\hline 198904 ST PETERSBURG & BG 1784 SOFIA & RUSSIA & NL 2200 AG NOORDWIJK \\
\hline RUSSIA & BULGARIA & TEL: & NETHERLANDS \\
\hline TEL: $257-94-91$ & TEL: 2758927 & TLF : & TEL: $17 \quad 1983629$ \\
\hline TLF : & TLF: & TLX: & TLF: \\
\hline TLX: & TLX: 23561 ECF BAN BG & EML: & TLX: 39098 \\
\hline EML: & EML: & COM: 21 & EML: \\
\hline $\mathrm{COM}: 36$ & COM: $07,15,20$ & & COM: \\
\hline
\end{tabular}




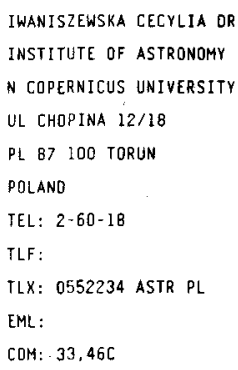

IWANOWSKA WILHELMINA PROF INSTITUTE OF ASTRONOMY $N$ COPERNICUS UNIVERSITY UL CHOPINA $12 / 18$ PL 87100 TORUN POLAND

TEL: $260-18$

TLF:

TLX: $06412 \mathrm{PL}$

EMI:

COM: 33

IYER B R OR

RAMAN RESEARCH INSTITUTE SADASHIVANAGAR BANGALORE 560080 INDIA

TEL: 812360122

TLF: 812340492

TLX: 8452671 RRI IN

EML:

COM: 47

JABbAR SABEH RHAMAN

SARC

SCIENTIFIC RES COUNCIL

BOX 2441

JABIRIYAH BAGHDAD

IRAO

TEL: $1776 \quad 5127$

TLF :

TLX: 213976 SRC IK

EML:

COM: 12,42

JACKISCH GERHARD DR ZNTRLINST,..F, ASTROPHYSIK STERNWARTE BABELSBERG

D 6400 SONNEBERG

GERMANY

TEL:

TLF:

TLX:

EML:

COM: 41

JACKSON PETER DOUGLAS OR JG-CHESAPEAKE ASSOCIATES BOX 1667

ANNAPOLIS MD 21404

USA .

TEL: 3017573613

TLF :

TLX:

EML:

COM: 33
IWASAKI KYOSUKE DR KYOTO GAKUEN UNIVERSITY

NANJO

SOGABECHO KAMEOKA

KYOTO 621

JAPAN

TEL: 771222001

TLF: $77124 \quad 8150$

TLX:

EML:

COM: 16

IZOTOV YURI OR

MAIN ASTRONOMICAL OBS

UKRAINIAN ACAD OF SCIENCE

GOLOSEEVO

$252127 \mathrm{KIEV}$

UKRAINE

TEL: $66 \quad 3110$

TLF:

TLX: 131406 SKY SU

EML:

COM: 28

jABIR NIAMA LAFTA

SARC

SCIENTIFIC RES COUNCIL

BOX 2441

JADIRIYAH BAGHOAO

IRAO

TEL: 17765127

TLF :

TLX: 213976 SRC IK

EML:

EOM: 34

JACKSON BERNARO V OR

CASS

UCSO

C 011

LA JOLLA CA 920930216

USA

TEL: 6195343358

TLF :

TLX:

EML: SOLAR::BJACKSON/CASSO1::BVJCME CDM: 4

COM: 12,21

$\begin{array}{ll}\text { JACKSON WILLIAM M OR } & \text { JACOBS KENNETH C OR } \\ \text { OPT OF CHEMISTRY } & \text { OPT OF PHYSICS } \\ \text { UNIVERSITY OF CALIFORNIA } & \text { HOLLINS COLLEGE } \\ \text { ROOM 214 } & \text { BOX } 9661 \\ \text { OAVIS CA } 95616 & \text { ROANOKE YA } 24020 \\ \text { USA } & \text { USA } \\ \text { TEL: } 9167520503 & \text { TEL: } 7033626478 \\ \text { TLF: } & \text { TLF: } \\ \text { TLX: } & \text { TLX: } \\ \text { EML: } & \text { EML: } \\ \text { COM: } 15 & \text { COM: }\end{array}$

IVE MASANORI DR

TOKYO ASTRONOMICAL OBS NAOJ

OSAWA MITAKA

TOKYO 181

JAPAN

TELL: $0422-32-511 \times 313$

TLF :

TLX: 2822307 TAOMTK J

EML:

COM: 33

IZVEKOV $\checkmark A D R$

INST OF THEORET ASTRONOMY ACADEMY OF SCIENCES

N KetTuzova 10

191187 ST PETERSBURG

RUSSIA

TEL: 2724023

TLF :

TLX: 121578 ITA SU

EML:

COM: 07,20

JABLONSKI FRANCISCO DR INPE

CP 515

12201 S JOSE DOS CAMPOS BRAZIL

TEL: $\begin{array}{lll}123 & 41 & 8977\end{array}$

ILF: $123 \quad 218743$

TLX:

EML:

COM: 27

JACKSON JOHN CHARLES OR 16 THE PARK

NEWARK NG24 150

UK

EL:

TLF:

TLX:

EML:

COM 


JACOBSEN THEOOOR S PROF
6205 17TH PARK RD
SEATTLE WA 98115
USA
TEL: 2065235245
TLF:
EML:
COM:
TLX:
JAEGER FRIEDRICH W PROF
TELEGRAFENBERG A 33
O 1500 POTSDAM
GERMANY
TEL: 4551
TLF:
EML:
COM:
TLX:
TLI
TEL: 25 626/27 457
TLF:
TLX:
EML:
COM: 10
JAIN RAJMAL DR
UDAIPUR SOLAR OBSERVATORY
I1 VIOYA MARG
UDAIPUR 313 001
INDIA

JAKS WALDEMAR OR ASTRONOMICAL LATITUOE OBS BOROWIEC

BoX 62035

PL 62 035 KORNIK

POLAND

TEL: POZNAN 170187.

TLF:

TLX: 0412623 AOS PL

EML:

COM: 19

JAMESON RICHARD $F$ OR

OPT OF ASTRONOMY

UNIVERSITY OF LEICESTER

UNIVERSITY RO

LEICESTER LEI TRH UK

TEL: $533554 \quad 455$

TLF:

TLX: 341198

EML:

COM:
JACOBY GEORGE $\cdot$ H

KITT PEAK NTL OBS

BOX 26732

$950 \mathrm{~N}$ CHERRY AVE

TUCSON AZ 857266732

USA

TEL: 6023259292

TLF:

TLX:

EML:

COM: 34

JAFFE DANIEL T

SPACE SCIENCES LABORATORY

UNIVERSITY OF CALIFORNIA

BERKELEY CA 94720

USA

TEL: $415642 \quad 1930$

TLF :

TLX:

EML: DTJEASTRO.AS. UTEXAS. EDU

COM: 34

JAIN SURENDRA DR
INOIAN INSTITUTE OF
ASTROPHYSICS
KORAMANGALA
BANGALORE 560034
INDIA
TEL: 812569702
TLF;
TLX: 8452763 IIAB IN
EML:

COM:

JAMAR CLA

IAL SPACE

UNIVERSITE OE LIEGE

AVE DU PRE AILY

B 4900 ANGLEUR-LIEGE

BELGIUM

TEL: 41676760

TLF:

TLX: 41320 IAL SP

EML:

COM: 44

\section{JANES KENNETH A DR}

OPT. OF ASTRONGMY

BOSTON UNIVERSITY

725 COMMONWEALTH AVE

BOSTON MA 02215

USA.

TEL: 617.353 2627

TLF :

TLX: 95-1289 BOS UNIV BSN

EML:

COM: 376
JACQ THIERRY

OBSERVATOIRE DE BORDEAUX

BP 89

F 33270 FLOLRAC

FRANCE

TEL: $5686 \quad 4330$

TLF: $56 \quad 404251$

TLX:

EML: JACOEFROBOR5

COM: 34,40

JAFFE WALTER JOSEPH DR

STSCI

HOMEWOOD CAMPUS

3700 SAN MARTIN OR

BALTIMORE MO 21218

USA

TEL: 3013384762

TLF:

TLX: 6849101 STSCI

EML:

COM: $28,40,48$

JAKIMIEC JERZY PROF

ASTRONOMICAL INSTITUTE

WROCLAW UNIVERSITY

UL KOPERNIKA IL

PL 51622 WROCLAW

POLAND

TEL: 482434

TLF:

TLX: 0712791 UWRPL

EML:

COM: $10 \mathrm{C}$

JAMES JOHN F MR

SCHUSTER LABORATORY

UNIVERSITY OF MANCHESTER MANCHESTER MI3 $\mathrm{gPL}$

UK

TEL: $612737 \quad 121$

TLF:

FLX:

EML:

COM: 21

Janiczek PaUl M dR

US NAVAL OBSERVATORY

34 \& MASSACHUSETTS AVE NW WASHINGTON OC 203925100 USA

TEL: 2026531569

ILF:

TLX: $710-822 \cdot 1970$

EML:

COM: 04,07
JACQUINOT PIERRE DR LABORATOIRE AIME COTTON UNIVERSITE PARIS XI

BT 505

F 91405 ORSAY COX

FRANCE

TEL:

TLF :

TLX:

EML:

COM: 14 .

JAHREISS HARTMUT OR ASTRONOMISCHES RECHENINSTITUT

MOENCHHOFSTR 12-14

O 6900 HEIOELBERG 1

GERMANY

TEL: $62 \quad 2149026$

TLF :

TLX: 461336 ARIHO D

EML:

COM: $24 C, 33$

JAKOBSEN PETER

ESA/ESTEC

SSD.

BOX 299

NL 2200 AG NOOROWIJK

NETHERLANDS

TEL: $17 \quad 19833614$

TLF :

TLX: 39098

EML:

COM:

JAMES RICHARD A OR

OPT OF ASTRONOMY

UNIVERSITY OF MANCHESTER MANCHESTER MI3 9PL

UK

TEL:

TLF :

TLX:

EML:

COM: 35

JANKOVICS ISTVAN DR KONKOLY OBSERVATORY THEGE U 13/17

80X 67

H 1525 BUDAPEST

HUNGARY

TEL: $175 \quad 5866 / 75 \quad 4 ! 22$

TLF:

$T L X: 227460$

EML:

COM: 
JANOT-PACHECO EDUARDO DR

IAG

UNIVERSIOADE DE SAO PAULO

CP 30627

01051 SAO PAULO.SP

BRAZIL

TEL: 115778599

ILF

TLX: 36221 IAGM BR

EML:

COM:

JARRETT ALAN H PROF
BOYOEN OBSERVATORY
BOX 334
BLOEMFONTEIN 9300
SOUTH AFRICA
TEL: $051-37605$
TLF:
TLX: 267666 SA
EML:
COM: 21,46
JASNIEWICZ GERARD OR
OBSERVATOIRE ASTROMOMIOUE
II RUE UNIVERSITE
F 67000 STRASBOURG
FRANCE
TEL: 88 35 9200
TLF: 88 25 0160
TLX: 890 506 STAROBS
EML: EARN: UDLLO9OFCCSC2L
COM: 33,42

JAYARAJAN A P MR

INOLAN INSTITUTE OF

ASTROPHYSICS

KORAMANGALA

GANGALORE $560 \quad 034$

INOIA

TEL: 812566585

TLF :

TLX: 845763 IIAB IN

EML:

COM: 09

JEFFERY CHRISTOPHER S QR UNIVERSITY OBSERVATORY

UNIVERSITY OF ST ANOREWS BUCHANAN GARDEN

ST ANDREWS FIFE KYIG 9LZ

UK

TEL:

ILF:

TLX:

EML:

COM: 27

\author{
JANSSEN MICHAEL ALLEN \\ JPL \\ MS 183301 \\ 4800 OAK GROVE DR \\ PASADENA CA 91109 \\ USA \\ TEL: 2133547247 \\ TLF : \\ TLX: \\ EML: \\ CDM: 40
}

JARZEBOWSKI TADEUSZ DR ASTRONOMICAL INSTITUTE WROCLAW UNIVERSITY

UL KOPERNIKA II

PL 51622 WROCLAW

POLAND

TEL :

TLF :

TLX:

EML:

CDM: 27

JASTROW ROBERT

INST FOR SPACE STUDIES

2880 BROADWAY

NEW YORK NY 10025

USA

TEL:

TLX:

EML:

TLF :

COM: 51

JEFFERIES JOHN T OR
NOAO
BOX 26732
$950 N$ CHERRY AVE
TUCSON AZ 857266732
USA
TEL: 602881 1960
TLF:
TLX: 0666484 AURANOAOTUC
EML:
COM: 12,36

JEFFERYS WILLIAM H OR

ASTRONOMY DPT

UNIVERSITY OF TEXAS

RLM 15308

AUSTIN TX 78712 1083

USA

TEL: $512 \quad 471 \quad 4461$

TLF :

TLX:

EML:

COM: 07,24
JAROINE MOIRA MARY DR

ASTRONOMY CENTRE

UNIVERSITY OF SUSSEX

FALMER

BRIGHTON BNI 9QH

UK

TEL: $273606755 * 3085$

TLF: $27367 \quad 8335$

TLX: 877159.8HV TKS G

EML: MMJOUK.AC.SUSSEX.STARLINK

COM: 10

JASCHEK CARLOS O R PROF

OBS OE STRASBOURG

11 RUE UNIVERSITE

F G7000 STRASBOURG

FRANCE

TEL: 88358200

TLF: $88 \quad 250160$

TLX: 890506 STAROBS

EML:

COM: $05,26,33,42,45$

JAUNCEY DAVIO L DR

CSIRO

OIVISION OF RADIOPHYSICS

BOX 76

EPPING NSW 2121

AUSTRALIA

TEL:

TLF :

TLX: 26230 ASTRO

EML: DJAUNEE YEATNF. CSIRO. AU

COM: $40,47 C$

JEFFERIES STUART DR

NTL SOLAR OBSERVATORY

BARTOL RESEARCH INST

$950 \mathrm{~N}$ CHERRY AYE

TUCSON AZ B5726 6732

USA

TEL: 6023234182

TLF :

TLX: 0666484 AURANOADTUC

EML: STUARTJENOAO EOU/5355 : STUARTJ EML:

COM: 12

JENKINS CHARLES R

ROYAL GREENWICH OBS

HERSTMONCEUX CASTLE

HAILSHAM BN27 IRP

UK

TEL: $323 \quad 833 \quad 17$

TLF:

TLX: 87451

EML:

COM: 40
JAROSZYNSKI MICHAL

ASTRONOMICAL OBSERVATORY

WARSAW UNIVERSITY

AL UJAZDOWSKIE 4

PL OO 478 WARSAW

POLAND

TEL: 294011

TLF:

TLK: 813978 ZAPAN PL

EML:

COM: 47

JASCHEK MERCEDES DR

OBS DE STRASBOURG

11 RUE UNIVERSITE

F 67000 . STRASBOURG

FRANCE

TEL: $88 \quad 358200$

LF: $88 \quad 250160$

TLX: 890506 STAROBS $F$

EML:

COM: 45

JAYANTHI UDAYA B DR

INPE

CP 515

12201 S JOSE DOS CAMPOS

BRAZIL

TEL: 123 41 $8977 * 392$

TtF: $123 \quad 21 \quad 8743$

FLX: 1233530 INPE BR

EML:

COM:

JEFFERS STANLEY DR

CRESS PHYSICS DPT

YORK UNIVERSITY

4700 KEELE ST

OOWNSVIEW ON M3J IP3

CANADA

TEL: $416 \quad 6673851$

TLF:

'TLX:

COM: 09,51

JENKINS EGWARD B DR

PRINCETON UNIVERSITY OBS

PEYTON HALL

PRINCETON NJ 08544

USA

TEL: 6094523826

TLF: 6092581020

TLX: 322409 ASTRO PRIN

EML: ebjeastro.princeton.edu

COM: $34,44 \mathrm{C}$ 


\section{A148}

JENKINS L F MS

YALE UNIVERSITY OBS

VALE STATION

BOX 2023

NEW HAVEN CT 06520

บSA

TEL:

TLF:

ILX

EML:

COM: 34

JENHISON ROGER C PROF ELECTRONICS LABORATORY UNIVERSITY OF KENT CANTERBURY CT2 7NT UK

TEL:

TLF:

TLX: 965449

EMt:

COM: $22,40,51$

JEWELL PHILIP R DR

NRAO

CAMPUS BLDG 65

$949 \mathrm{~N}$ CHERRY AVE

TUCSON AZ 857210655

USA

TEL: $602882 \quad 0250$

TLF

TLX: 9102409524 NRAO TUC

EML: BITNET: PJEWELLENRAO

COM: 27,40

JIANG DONG-RONG

SHANGHAI OBSERVATORY

CAS

80 NANOAN RD

SHANGHAI

CHINA PR

TEL: 21386191

TLF :

TLX: 33164 SHAO CN

EML:

COM: 33

JIANG YAO-TIAO

OPT OF ASTRONOMY

NANJING UNIVERSITY

NANJING

CHINA PR

TEL: 2534151

TLF :

TLX:

EML:

COM: 10
JENKNER HELMUT OR

STSCI

HOMEWOOD CAMPUS

3700 SAN MARTIN DR

BALIIMORE MD 21218

USA

TEL: 3013384842

TLF:

TLX: 6849101 STSCI

EML:

COM: 05,09

JENSCH A

PESTALOZZISTR 9

D 6900 JENA

GERMANY

TEL:

TLF :

EML:

COM

ILX:

JI HONG-QING

INTL LATITUTOE STATION

TIANJIN

CHINA PR

TEL:

TLF :

EML:

COM

TLX:

JIANG SHI-YANG

BEIJING ASTRONOMICAL OBS

CAS

W SUBURB

BEIJING 100080

CHINA PR

TEL: $128 \quad 1698$

TLF :

TLX: 22040 BADAS CN

EML:

COM: $09,29,27,50$

JIANG ZHAOJI

BEIJING ASTRONDMICAL OBS

CAS

W $S$ S B URB

BEIJING 100080

CHINA PR

TEL:

ILF:

ILX: 22040 BAOAS CN

EML:

COM:
JENMER GAVIO C OR

OPT OF ASTRONOMY

UNIVERSITY OF WASHINGTON

FM 20

SEATTLE WA 98195

USA

TEL: $206543 \quad 6182$

TLF :

TLX:

EML:

COM:

JENSEN EBERHART PROF

INST THEORET ASTROPHYSICS

UNIVERSITY OF OSLO

BOX 1029

N 0315 BLINOERN OSLO 3

NORWAY

TEL: 02-456502

TLF:

TLX: 72425N UNIOS

EML:

COM: 10

JI SHUCHEN DR

BOX 110 KUNMING

YUNNAN PROVINCE

YUNNAN

CHINA PR

TEL: 72946

TLF :

TLX: 64040 YUOBS CM

EM: :

COM: 40

JIANG SHUDING
GRADUATE SCHOOL
UNIV SCIENCE \& TECHNOLOGY
BOX 3908
BEIJING 100039
CHINA PR
TEL: $1817031 \times 253$
TLF:
TLX:
EML:
COM: 47

JIN BIAOREN DR

OPT OF GEOPHYSICS

WUHAN TECHNICAL UNIVER

39 LO-YU RD

WUHAN 430070

CHINA PR

TEL: $87557 !$

TLF :

TLX: 40210 WTUSM CN

EML:

COM:
JENNINGS R E PROF

OPT PHYSICS ASTRONOMY

UNIVERSITY COLLEGE LONDON

GOWER ST

LONDON WCIE $6 B T$

TEL: $1713 \quad 877050$

TLF:

TLX: 28722

EML:

COM: 34

JERZYKIEWICZ MIKOLAJ OR ASTRONOMICAL INSTITUTE WROCLAW UNIVERSITY

UL KOPERNIKA II

PL 51622 WROCLAW

POLAND

TEL: $48-24-34$

TLF:

ILX: 0712791 UWR PL

EML:

COM: $25,27 \mathrm{VP}$

JIANG CHONG-GUO YUNNAN OBSERVATORY

CAS

BOX 110

KUNMING 72946 YUNNAN

CHINA PR

TEL: 8712035

TLF :

TLX:

EML:

COM: 08

JIANG XIAO-YUAN DR SHANGHAI OBSERVATORY CAS

80 NANOAN RD

SHANGHAI 200030

CHINA PR

TEL: $2138 \quad 6191 * 49$

TLF:

TLX: 33164 SHAO CN

EML:

COM: 41

JIN SHEN-ZENG

BEIJING ASTRONOMICAL OBS CAS

W SUBURB

BEIJING 100080

CHINA PR

TEL: $128 \quad 1698$

TLF :

TLX: 22040 BAOBS CN

EML:

COM: 40 
JIN WEN-JING

SHANGHAI OBSERVATORY

CAS

BO NANOAN RO

SHANGHAI

CHINA PR

TEL: 21386191

TLF :

TLX: 33164 SHAO CN

EML:

COM: $19 C, 31$
JOCKERS KLAUS OR

MPI FUER AERONOMIE

POSTFACH 20

D 3411 KATLENBURG LINDAU

GERMANY

TEL: 5556411

TLX: 965527

EML:

TLF :

COM: 10,15

JOHANSSON LARS ERIK B OR

LADAEMNESGATAN 7

S 41679 GOETEBORG

SWEOEN

TEL:

ILF :

EML:

COM: 40

TLX:

JOHNSON FRED M PROF OR PHYSICS \& ASTRONOMY DPT CALIFORNIA STATE UNIV FULLERTON CA 92634 USA

IEL: $714 \quad 773 \quad 3366$

TLF:

ILX:

EML:

COM: 14,34

JOHNSTON KENNETH J

NAVAL RESEARCH LABORATORY

CODE 7134

4555 OVERLOOK AVE SW

WASHINGTON OC 203755000

USA

TEL: $202 \cdot 7672351$

TLF:

ILX:

EML:

COM: $04.08,24.34 .40$

JONAS JUSTIN LEONARD

DPT PHYSICS/ELECTRONICS

RHODES UNIVERSITY

BOX 94

GRAKAMSTOWN 6140

SOUTH AFRICA

TEL: (461) 22023

TLF:

ILX: 244226 RUANT SA

EML :

COM: 33,40
JOERSAETER STEVEN DR

STOCKHOLM DBSERVATORY

S 13336 SALTSJDEBADEN SWEDEN

TEL: $81 \quad 64 \quad 463$

TLF: $87 \quad 174719$

ILX:

EML: STEVENOASTRO.SU. SE

COM:

JOHANSSON LENNART DR

ASTRONOMICAL OBSERVATORY

BOX 515

S 75120 UPPSALA

SWEDEN

IEL: $18 \quad 51 \quad 1274$

TLF: $18 \quad 52 \quad 7583$

ILX:

EML: LABAN: : LJ

COM:

JOHNSON HOLLIS R PROF
ASTRONOMY DPT
INDIANA UNIVERSITY
SWAIN WEST 319
BLDOMINGTON IN 47405
USA
TEL: 8123354172
TLF:
TLX: 272279
EML:

COH: 29,36

JOKIPII J R PROF

LUNAR \& PLANETARY LAB

UNIVERSITY OF ARIZONA

TUCSON AZ B5T21

USA

TEL: $602 \quad 6214256$

TLF :

TLX:

EML:

COM: 48,49

JONCAS GILLES DR

DPT DE PHYSIQUE

UNIVERSITE OE LAVAL,

STE FOY QQC GIK 7 P4

CANADA

TEL: $418 \quad 656 \quad 2652$

TLF: $418 \quad 656 \quad 2040$

TLX: 05131621

EML: BITNET: 1150041 LAVALVXI

COM:
JOG CHANDA J OR

OPT OF PHYSICS

INDIAN INSTITUTE SCIENCES

BANGALORE 560012

INOIA

TEL: 812344411

TLF:

TLX: 8458349 IISC IN

EML:

COM: 28,33

JOHANSSON SVENERIC DR

DPT OF PHYSICS

UNIVERSITY OF LUNO

SOELVEGATAN 14

S 22362 LUND

SWEOEN

TEL: $46 \quad 12 \quad 6097$

TLF: $46 \quad 10 \quad 4709$

TLX: 33533 LUNIVER $S$

EML: ATOMSEJPSELDC52

COM: 14

JOHNSON HUGH M OR

2017 NEWELL RD

PALO ALTO CA 94303

USA

TEL: 4153267223

TLF :

TLX:

EML:

COM: 33,34

JOLY FRANCOIS OR

UNIVERSITE OE BORDEAUX

123 RUE LAMARTINE

F 33405 TALENCE

FRANCE

TEL: $56 \quad 86 \quad 4330$

TLF: $56 \quad 40 \quad 4251$

TLX:

EML:

COM: 14,40

JONES ALBERT F MR

31 RANUI RD

STOKE:

NELSON

NEW IEALANO

TEL: 054-73-905

TLF:

TLX:

EML:

COM: 27 


\author{
JONES BARBARA \\ CASS \\ UCSD \\ c 011 \\ LA JOLLA CA 920930216 \\ USA \\ TEL: 7144524474 \\ TLF : \\ TLX: \\ EML: \\ COM: 09
}

JONES DAYTON L

$\mathrm{JPL}$

MS 238700

4BOD DAK GROVE OR

PASADENA CA 91109

USA

TEL: $818 \quad 354 \quad 7774$

TLF :

TLX: 675429

EML:

COM: 40

JONES HARRISON PRICE DR KITT PEAK NTL OBS

SOLAR STATION

$900 \mathrm{~N}$ CHERRY AVE TUCSON AZ $85726 \quad 6732$

USA ,

TEL: $602 \quad 3259354$

TLF:

ILX:

EML:

COM: 10.12

JOROAN CAROLE DR
OPT THEORETICAL PHYSICS
UNIVERSITY OF OXFORO
I KEEBLE RD
OXFORO OKI $3 \mathrm{NP}$
UK
TEL: 86553281
TLF:
TLX: 83295 NUCLOX
EML:
COM: $12,14,29,44$

JOROI NEBOT CARME DR

DPT ASTRONOMIA

UNIVERSIDAD OE BARCELONA

AVD DIAGONAL 647

E OBO28 BARCELONA

SPAIN

TEL: 33307311

TLF

TLX

EML: D3FACJNOEEBOUBOII

COM: 25
JONES BARRIE W DR

DPT OF PHYSICS

THE OPEN UNIVERSITY

WALTON HALL

MILTON KEYNES MK7 GAA

uk

TEL: $908 \quad 653378$

TLF: 908653744

ILX: 825061

EML: BW_JONESEUK.AC.OPEN.ACS.VAX

COM: 46

JONES DEREK H P.DR

ROYAL GREENWICH OBS

MADINGLEY RD

CAMBRIDGE CB3 OEZ

UK

TEL: $223 \quad 374000$

ILF: 223374700

TLX: 265451

EML:

COM: $24,33,37$

JONES JAMES DR

DPT OF PHYSICS

UNIV OF WESTERN ONTARIO

LONDON ON NGA $5 B 9$

CANADA

TEL: 5196613183

TLF: 5196613486

TLX: 0.547134

EML:

COM: $22 C$

JOROAN H L OR DIREKTOR

INSIITUT F. PLASMAPHYSIK

KERNFORSCHUNGSANLAGE

JUELICH GMBH PF 365

D 5170 JUELICH

GERMANY

TEL:

TLF :

TLX:

EML:

COM: 14

JORGENSEN HENNING E PROF UNIVERSITY OBSERVATORY oEster VOLDGADE 3

DK 1350 COPENHAGEN $K$ DENMARK

TEL: $3 i \quad 14 \quad 1790$

TĹF: $31 \quad 38 \quad 9157$

TLX: 44155

EML: HENNINGEASTRO . KU. OK

COM: 38VP
JONES BERNARD J T DR

NORDITA

BLEgDAMSVEJ 17

DK 2100 COPENHAGEN 0

DENMARK

TEL: $3142 \quad 1616$

TLF: 31389157

TLX: 15216 NBI DK

EML:

COM: 47

JONES ERIC $M$

LOS ALAMOS NATIONAL LAB

MS F665

BOX 1663

LOS ALAMOS NM 87545

USA

IEL: $505.667 \quad \dot{6386}$

TLF :

TLX:

EML:

COM: 51

JONES JANET E DR

NOROITA.

BLEGOAMSVEJ 17

OK 2100 COPENHAGEN O

DENMARK

TEL: $3142 \quad 1616$

TLF: 31399157

TLX: 15216 NBI DK

EML:

COM:

JORDAN STUART OO DR

NASA/GSFC

CODE 682

LASP

GREENBELT MD 20771

USA

TEL: 3012868811

TLF:

TLK: 89675

EML:

COM: $10,12,44 \mathrm{C}$

JORGENSEN UFFE GRAE DR NIELS BOHR INSTITUTE BLEgDAMSVEJ 17

DK 2100 COPENHAGEN 0

DENMARK

TEL: 31421616

TLF: $31 \quad 389157$

T.LX: 15216

EML:

COM:
JONES BURTON OR LICK OBSERVATORY UNIVERSITY OF CALIFORNIA SANTA CRUZ CA 95064 USA

TEL: $408 \quad 4292384$

TLF:

TLX:

EML:

COM: 24

JONES FRANK CULVER DR NASAFGSFC

CDOE 665

GREENBELT MO 20771

USA

TEL: 3012865506

TLF:

TLX: $710-828-9716$

EML:

COM: 34,48

JONES THOMAS WALTER DR OPT OF ASTRONOMY

UNIVERSITY OF MINHESOTA

116 CHURCH ST SE

MINNEAPOLIS MN 55455

USA

TEL: 6123733307

TLF:

TLX:

EML:

COM: 28,48

JOROEN PAUL RICHARO

ROYAL GREENWICH OBS

MADINGLEY RD

CAMBRIDGE CB3 OEZ

UK

TEL: 223374000

TLF: $223 \quad 374700$

TLX: 265451/265871MONREFG

EML: AC.RO-GREENWICH.STARLINK: ;

COM:

JOSELYN JO ANN C DR

NOAA ERL R/E/SE2

SPACE ENVIRONMENT LAB

325 BROADWAY

BOULOER CO BO303

USA

TEL: 3034975147

TLF:

TLX: $8 B B 776$ NOAA BLDR

EML: SPAN: SELVAX: : JJOSELYN

COM: 10,49 
JOSEPH CHARLES LYNN OR OPT ASTROPHYSCIAL, SCI PRINCETON UNIVERSITY PRINCETON NJ 085441001

USA

TEL: 6092583808

TLF: 6092581020

TLX:

EML: CLJ eastro.princeton. eOU COM: 09

JOSHI MOHAN N PROF

TIFR/RADIO ASTRONOMY CTR

\section{$80 \times 8$}

JUHAGAMANDALAM 643001

INDIA

TEL: 4232032

TLF :

TLX: 8458488 TIFR IN

EME:

COM: $28,40,47$

JOUBERT MARIINE

LAS

TRAVERSE DU SIPHON

LES TROIS LUCS

F 13012 MARSEILLE

FRANCE

TEL: 91055900

TLF: $91 \quad 66 \quad 1855$

TLX: 420584

EML:

COM: 21

\begin{tabular}{|c|c|}
\hline JOY MARSHALL J OR & JUDGE PHILIP OR \\
\hline NASA/MSFC & HIGH ALTITUDE OBSERVATOR \\
\hline CODE ES 65 & NCAR \\
\hline SPACE SCIENCE LAB & $B 0 \times 3000$ \\
\hline HUNTSVILLE AL 35812 & BOULDER CO 803073000 \\
\hline USA & USA \\
\hline TEL: 20,5443423 & TEL: \\
\hline TLF: $205 \quad 5447754$ & TLF : \\
\hline TLX: & TLX: \\
\hline EML: SSL: : JOY/JOY $巴 S S L$, MSFC. NASA, GOV & EML: \\
\hline COM: 28 & CON: 36 \\
\hline JUNG JEAN DR & JUNKKARINEN VESA T DR \\
\hline THOMSON & CASS \\
\hline 173 BD HAUSSMANN & UCSO \\
\hline F 75379 PARIS CDX 08 & C 011 \\
\hline FRANCE & LA JOLLA CA 920930216 \\
\hline TEL: $\quad 1.45619600$ & USA. \\
\hline TLF : & TEL: 6195340735 \\
\hline TLX: 204780 & TLF: \\
\hline EML: & TLX: \\
\hline COM: & EML: SPAN:27783::VESA \\
\hline & COM: 28,47 \\
\hline
\end{tabular}

JOSEPH ROBERT D DR

ASTROPHYSICS GROUP

IMPERIAL COLLEGE

BLACKETT LABORATORY

LONDON SW7 $2 B Z$

UK

TEL: 15895111 *6660

TLF:

TLX: 261503

EML:

COM:

JOSHI U C DR

PHYSICAL RESEARCH LAB

NAVRANGPURA

AHMEDABAD 380009

INDIA

TEL: 27246.2129

TLF: 272445292

TLX: 121397

EML:

COM: $25,28,37$

JOURNET ALAIN

DCA CERGA

AVE COPERNIC

F 06130 GRASSE

FRANCE

TEL: 93365849

ILF:

TLX: 470865

EMLL:

COM: 07,08

JUGAKU JUN DR

TOKYO ASTRONOMICAL OBS

NADJ

OSAWA MITAKA

TOKYO 181

JAPAN

TEL:

TLF:

ILX: 2822307 TAOMTKJ

EML:

COM: $28,29,51$

JUPP ALAN H OR

DPT APPL MATHS THEOR PHYS

UNIVERSITY OF LIVERPOOL

BOX 147

LIVERPOOL L69 $3 B X$

UK

TEL: 517096022

ILF :

ILX: 627095

EML:

COM: 07
JOSHI G C OR

UTTAR PRADESH STATE

OBSERVATORY

PO MANORA PEAK 263129

NAINITAL 263129

INDIA

TEL: 594212136

TLF:

TLX:

EML:

COM: 12

JOSS PAUL CHRISTOPHER DR

DPT OF PHYSICS

MIT RM 6203

BOX 165

CAMBRIDGE MA 02139

USA

TEL: $617243 \quad 4845$

TLF :

TLX:

EML:

COM: 42,48

JOVANOVIC BOZIDAR

FAC OF AGRICULTURE

INST WATERRANGING

VELJKA VLAHOVICA 2

YU 21000 NOVI SAD

YUGOSLAVIA

TEL: 215366

TLF:

TLX:

EML:

COM: 07,10

JULIUSSON EINAR OR

UNIVERSITY IF ICELAND

V/SUDURGÖTU

IS 101 REYKJAVIK

ICELAND

TEL: 179979

TLF:

TLX: 05012307 ISINFO

EML:

COM: 48

JURA MICHAEL DR

OPT OF ASTRONOMY

UNIVERSITY OF CALIFORNIA

MATH SCIENCES BLDG

LOS ANGELES CA 90024

USA

TEL: $213 \quad 8254302$

TLF:

TLX:

EML:

CON: 34 


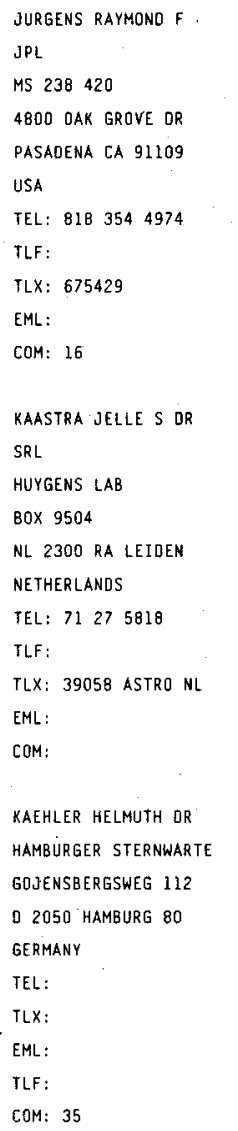

KAASTRA JELLE S OR
SRL
HUYGENS LAB
BOX 9504
NL 2300 RA LEIDEN
NETHERLANDS
TEL: 71275818
TLF:
TLX: 39058 ASTRO NL
EML:
COM:
KAEHLER HELMUTH DR
HAMBURGER STERNWARTE
GOJENSBERGSWEG 112
D 2050 HAMBURG 80
GERMANY
TEL:
TLX:
EML:
ILF:
COM: 35

kahane claudine oR OBSERVATOIRE DE GRENDBLE CERMO/ASTROPHYSIQUE BP $53 X$

F $38041 \mathrm{~S}$ MARTIN HERES CD FRANCE

IEL: 76514600

TLF: 76448821

TLX: 980134 USMG

EML: KAHANEARGAG5

COM:

\section{KAI KEIZO OR}

TOKYO ASTRONOMICAL OBS

NAOJ

OSAWA MITAKA

TOKYO 181

JAPAN

TEL: $0422-32-5111$

TLF :

TLX: 3329005 TAONRO J

EML:

COM: 10,40
JURKEVICH IGOR DR

3130 PORT WAY

ANNAPOLIS MD 21403

USA

TEL: 2027672003

TLF :

EML:

CON: 42

ILX:

KABURAKI MASAKI PROF
4-24-9 KICHIJYOJI
MINAMI MUSASHINO
TOKYO 180
JAPAN
TEL:
TLF:
TLX:
EML:
COM: 33

KAFATOS MINAS DR

DPT OF PHYSICS

GEORGE MASON UNIVERSITY

FAIRFAX VA 22030

USA

TEL: $703 \quad 3232303$

TLF:

TLK:

EML:

COM: $34,44,51$

KAHLER STEPHEN W OR
A F GEOPHYSICS LABORATORY
SPACE PHYSICS OIV PHP
HANSCOM AFB
BEOFORD MA 01731
USA
TEL: 6178613975
TLF:
TLX:
EML:
COM: 10

\section{KAIFU NORIO OR} NOBEYAMA RADIO OBS

NAOJ

MINAMIMAKI MURA

NAGANO $394 \quad 13$

JAPAN

TEL:

TLF :

TLX:

EML:

COM: $34 \mathrm{C}, 40$
JUST ANDREAS OR

INST FUER THEORETISCHE

PHYSIK DER UNIVERSITAEI

ROBERT-MAYER-STR 10

D 6000 FRANKFURT MAIN 1

GERMANY

TEL: 697982636

TLF : 697988350

TLX:

EML:

COM: 34

\section{KAOLA ZOENKA I OR}

PULKOVO OBSERVATORY

ACADEMY OF SCIENCES

10 KUTUZOV QUAY

196140 ST PETERSBURG

RUSSIA

TEL:

TLF :

TLX:

EML:

COM: 05,37

KAFKA PETER

MPI F. PHYSIK \& ASTROPHYS

INSTITUT FUR ASTROPHYSIK

KARL-SCHWARZSCHILD-STR I

O B046 GARCHING MUENCHEN

GERMANY

TEL: 8932990

TLF :

TLX: 524629 ASTRO D

EML

COM: 48,51

KAHL.MANN HANS CORNELIS DR RAOIO OBS WESTERBORK

SCHATTENBERG 1

NL 9433 TA ZWIGGELTE

NETHERLANDS

TEL: 59392421

TLF: 59392486

TLX:

EML: KAHLMANN_HANSENFRA.NL

COM: 40,50

KAISER THOMAS R PROF

DPT OF PHYSICS

THE UNIVERSITY

SHEFFIELD S3 7RH

UK

TEL: $742 \quad 785 \quad 55^{\star 4} 4277$

TLF :

TLX: 547216 UGSHEF 6

EML:

COM: 22
JUSZKIEWICZ ROMAN

COPERNICUS ASTRON CENTER

POLISH ACAD OF SCIENCES

UL BARTYCKA 18

PL OO 716 WARSAL

POLAND

TEL:

TLF

TLX:

EML:

COM: 47

KADOURI TALIB HADI

SARC

SCIENTIFIC RES COUNCIL

BOX 2441

JADIRIYAH BAGHOAD

IRAD

TEL: 17765127

TLF :

TLX: 213976 SRC IX

EML:

COM: $27,30,36,42$

XAFTAN MAY A OR

NRAD

BOX 2

GREEN BANK WV 24944

USA

TEL:

TLF :

TLX: 710-938-1530

EML:

COM: 34,40

KAHN FRANZ O PROF OPT OF ASTRONOMY UNIVERSITY OF MANCHESTER MANCHESTER M13 $9 P L$

UK

TEL: 612737121

TLF:

TLX: 668932 MCHRUL G

EML:

COM: $34,40,48$

KAITCHUCK RONALD H

DPT OF PHYSICS \& ASTRON BALL STATE UNIVERSITY

MUNCIE IN 47306

USA

TEL:

TLF :

TLX:

EML:

COM: 42 


KAKINUMA TAKAKIYO T PROF
INSI FOR ATMOSPHERIC RES
NAGOYA UNIVERSITY.
3-13 HONOHARA
TOYOKAWA AICHI 442
JAPAN
TEL: $05338-6-3154$
TLF:
TLX:
EML:
COM: 40,49
KALBERLA PETER
RADIOASTRONOMISCHES INST
OER UNIVERSITAET BONN
AUF DEM HUEGEL 71
O 5300 BONN 1
GERMANY
TEL: 228733645
TLF:
TLX: B86440
EML:
COM: 05,40

KALLOGLIAN ARSEN T DR BYURAKAN ASTROPHYSICAL OBSERVATORY

378433 BYURAKAN

ARMENIA

TEL: $88 \quad 52 \quad 283453$

TLF :

TLX:

EML:

COM: 28

XALUZNY JANUSZ OR
ASTRONOMICAL OBSERVATORY
WARSAW UNIVERSITY
AL UJAZOOWSKIE 4
PL 00478 WARSAW
POLAND
TEL: 294011
TLF:
TLX: 817063
EML:
COM: 42

KAMINISHI KEISUKE PROF OPT OF PHYSICS KUMAMOTO UNIVERSITY 2-39-1 KUROKAMI KUMAMOTO 860 JAPAN

TEL: $096-344-2111$

TLF:

TLX:

EML :

COM: 35
KAKUTA CHUICHI OR ASTROgEODYNAMICS OBS HOSHIGAOKA 2-12

MIZUSAWA SHI

IWATE 023

JAPAN

TEL: 197247111

ILF:

TLX: 837628 ILSMIZ J

EML:

COM: 19.31

KALER JAMES B PRQF

UNIVERSITY OF ILLINOIS

103 ASTRONOMY BLDG

1002 W GREEN ST

URBANA IL 51801

USA

TEL: $217 \quad 33 \dot{3} 9382$

ILF:

TLX: $910-2452434$ AST

EML:

COM: 34

KALMAN BELA DR

HELIOPHYSICAL OBSERVATORY

BOK 30

H 4010 debrecen

HUNGARY

TEL: 5211015

TLX: 72517 DEOBS H

EML:

TLF:

COM: 10.12

KAMEL OSMAN M PROF

DPT OF ASTRONOMY

FACULTY OF'SCIENCES

CAIRO UNIVERSITY

GEZA

EGYPT

TEL:

TLF:

TLX:

EML:

COM:

KAMMEYER PETER C DR

US NAVAL OBSERVATORY 34 \& MASSACHUSETTS AVE NW WASHINGTON OC 203925100 USA

TEL: $202 \quad 653 \quad 1563$

ILX:

EML;

ILF:

COM: 07
KALAFI MANOUCHER

CTR FOR ASTRON RESEARCH

UNIVERISTY OF TABRIZ

TABRIZ 51664

IRAN.

TEL: 4132564

TLF:

TLX:

EML:

COM: 28

KALINKOV MARIN P OR

OPT OF ASTRONOMY

BULGARIAN ACAD SCIENCES

72 LENIN BLVO

BG 1784 SOFIA

BULGARIA

TEL: 2758927

TLF:

TLX: 22774 ELANP BE

EML:

COM: 28

KALMYKOV A M DR

ASTRONOMICAL INSTITUTE

UZBEK ACADEMY OF SCIENCES

700000 TASHKENT

UZBEKISTAN

TFL:

ILF:

TLX:

EML:

COM:

KAMEYA OSAMU DR NOBEYAMA RAOIO OBS

NAOJ

MINAMIMAKI MURA

NAGANO $384: 13$

JAPAN

TEL: $26798 \quad 2 B 31$

TLF:

TLX: 3329005 NADNRO

EML:

COM: 19,40

KAMP LUCAS WILLEM DR.

DPT OF ASTRONOMY

BOSTON UNIVERSITY

725 COMMONWEALTH AVE

BOSTON MA 02215

USA

TEL:

TLF:

TLK:

EML:

COM: 36.37
KALANDADZE N B DR

ABASTUMANI ASTROPHYSICAL OBSERVATORY

GEORGIAN ACAD OF SCIENCES

383762 ABASTUMANI

GEORGIA

TEL: 227

TLF:

TLX: 327409

EML:

COM: 33

KALKOFEN WOLFGANG BR CENTER FOR ASTROPHYSICS HCO/SAO

60 GARDEN ST

CAMBRIDGE MA 02138

USA

TEL: 6174957285

TLF:

TLX:

EML:

COM: $12,36 \mathrm{P}$

KALNAJS AGRIS J OR

MOUNT STROMLO \&IOING SPRING OBSERVATORIES

PRIVATE Bag

WODEN PO ACT 2606

AUSTRALIA

TEL: 62 88 1111*248

TLF:

TLX: $62270 \mathrm{AA}$

EML: agrismso.anu.oz

COM: $33 \mathrm{C}$

KAMIJO FUMIO PROF OR OPT OF ASTRONOMY UNIVERSITY OF TOKYO BUNKYo KU TOKYO 113 JAPAN

TEL:

TLF:

TLX:

EML:

COM: 34

KAMPER KARL W DR DAVID DUNLAP OBSERVATORY UNIVERSITY OF TORONTO BOX 360 RICHMOND HILL ON L4C $4 Y 6$ CANADA

TEL: 4168849562

TLF: 4169783921

TLX: 06986766

EML:

COM: 
KANAEV IVAN I OR

PULKOVO OBSERVATORY

ACADEMY OF SCIENCES

10 KUTUZOV QUAY

196140 ST PETERSBURG

RUSSIA

TEL:

TLF :

TLX:

EML:

COM: 24

XaNDPAL CHANORA D

UtTAR pradesh State

OBSERYATORY

PO MANORA PEAK 263129

NAINITAL 263129

INDIA

TEL: $5942 \quad 2136,2325$

TLF:

TLX:

EMH :

COM: 42

KANG GON IK

PYONGYANG ASTRON OBS

ACADEMY OF SCIENCES DPRK

TAESONG DISTRICT

PYONGYANG

KOREA OPR

TEL:

TLF:

TLX:

EML:

COM: 40

\section{RAPAHI VIJAY $K$ DR}

TATA INST OF FUNDAMENTAL

RESEARCH

POONA UNIVERSITY CAMPUS

PUNE $41100 \%$

INDIA

TEL: $21233 \quad 6105$

TLF: 212335760

TLX: 0145658 GMRT IN

EML: uunet! shakt ! gmrt!vijay

COM: 28,40

\section{KAPLAN LEWIS O OR}

ATMOSPH \& ENVIRONMENTAL

RESEARCH INC

840 MEMORLAL DR

CAMBRIOGE MA 02139

USA

TEL: 6175476207

TLF:

TLX: 951417 AERC

EML:

COM:
RANBACH GOTTFRIEO OR

MAX PLANCK INSTITUT FUR

EXTRATERR, PHYSIK

D 8046 garChING MUENCHEN

germany

TEL: 893299544

TLF:

TLX: 5215845 XTER D

EML: BITNET:GOKEOGAIPPIS

COM:

KANDRUP HENRY EMIL DR

DPT OF ASTRONOMY

UNIVERSITY OF FLORIDA

211 SSRB

GAINESVILLE FL 32611

USA

TEL: 9043922681

TLF: 9043929741

TLX:

EML: KANORUP QASTRO. UFL. EDU

COM: $28,37,47$

KANG JIN SOK

PYONGYANG ASTRON OBS

ACADEMY OF SCIENCES DPRK

TAESONG OISTRICT

PYONGYANG

KOREA DPR

TEL:

ILF:

TLK:

EML:

COM: 10

KAPISINSKY IGOR

ASTRONOMICAL INSTITUTE

SLOVAK ACAOEMY SCIENCES

DUBRAVSKA 9

CS B42 28 BRATISLAVA

CZECHOSLOVAKIA

TEL: $>375157$

ILF:

ILX: 093355

EML:

COM: 22

\section{KAPOOR RAMESH CHANDER}

INDIAN INSTITUTE OF

ASTROPHYSICS

KORAMANGALA

BANGALORE 560034

INOTA

TEL: $81256 \quad 6585$

TLF:

rLX: 845763 IIAB IN

EML:

COM: 47,48
KANDEL ROBERT S DR

LMO

ECOLE POLYTECHNIQUE

F 91128 palaISEAU COX

FRANCE

TEL: $1 \quad 69418200$

TLF:

TLX: 691596

EML:

COM: 36

KANE SHARAD R DR

SPACE SCIENCES LABORATORY

UNIVERSITY OF CALIFORNIA

BERKELEY CA 94720

USA

TEL: $415 \quad 642 \quad 1719$

TLF:

TLX: $910-366-7945$

EML:

COM: 10

KANG YOUNG WOON OR

DPT OF EARTH SCIENCE

KING SEJONG UNIVERSITY

g9 KOONJA-DONG

SEOUL SUNGDONG 133747

KOREA R

TEL: 4675121

TLF:

TLX:

EML:

COM: 42

RAPLAN GEORGE H OR

US NAVAL OBSERVATORY

34 \& MASSACHUSETTS AVE NW

WASHINGTON OC 203925100

USA

TEL: $202 \quad 653 \quad 0722$

TLF: 2026530179

TLX:

EML: GHKRHOBOS.USNO.NAYY.MIL

COM: $04,05,08$

KARAALI SALIH. OR *

FACULTY OF SCIENCE

UNIVERSITY OF ISTANBUL

34452 UNIVERSITY

TURKEY

IEL: $15224200 * 610$

TLF:

TLX:

EML:

COM:
KANDEMIR GUELCIN

ISTANBUL TECHNICAL UNIV

FEN FAKULTESI, FIZIK B

MASLAK

34452 ISTANBUL

TURKEY

TEL: 1609109

TLF:

TLX:

EML:

COM:

KANEKO NOBORU OR

DPT OF PHYSICS

HOKKALOO UNIVERSITY

KITA 10 NISHI $B$

SAPPORO 060

JAPAN

TEL: 11-716-2111

TLF :

TLX: 9325LO HOKUSC $\mathrm{J}$

EML:

COM: 28

KANYO SANDOR OR

KONKOLY OBSERVATORY

THEGE $\cup 13 / 17$

BOX 67

H 1525 BUOAPEST

HUNGARY

TEL: 175 5866/75 4122

TLF:

ILX:

EML:

COM: 27

KAPLAN $J$ BR

OPT OF PHYSICS

UNIVERSITY OF CALIFORNIA

LOS ANGELES CA 90024

USA

TEL:

TLF :

ILX:

EML:

COM: 21

KARACHENTSEV I D DR

SPECIAL ASTROPHYSICAL OBS

ACADEMY OF SCIENCES

ZELENCHUKSKAJA

357147 STAVROPOLSKIJ

RUSSIA

TEL:

TLF:

TLX:

EML:

COM: $09,28,30,47$ 


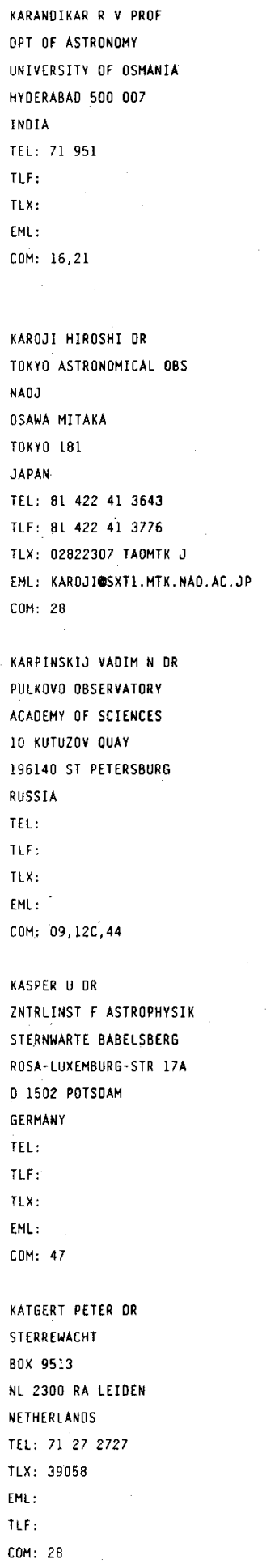

KARDASHEY N S OR
SPACE RESEARCH INSTITUTE
ACADEMY OF SCIENCES
PROFSOYUZNAYA UL $84 / 32$
IL781O MOSCOW
RUSSIA
TEL:
ILF:
TLX:
EML:
COM: 40.51

$\begin{array}{ll}\text { KAROUSKA MARGARITA OR } & \text { KARP ALAN HERSH OR } \\ \text { CENTER FOR ASTROPHYSICS } & \text { SCIENTIFIC CENTER IBM } \\ \text { HCO/SAO } & \text { I530 PAGE MILL RD } \\ 60 \text { GAROEN ST } & \text { PALO ALTO CA } 94304 \\ \text { CAMBRIDGE MA 02138 } & \text { USA } \\ \text { USA } & \text { TEL: } 4158553127 \\ \text { TEL: } 6174957347 & \text { TLF: } \\ \text { TLF: } & \text { TLX: } \\ \text { TLX: } & \text { EML: } \\ \text { EML: KAROVSKAOCFA } & \text { COM: } 27,36\end{array}$

COM: 27

KARTTUNEN HANNU DR CENTRE FOR SCIENTIFIC COMPUTING

$B 0 \times 40$

SF 0210L ESPOO

FINLAND

TEL: 4572709

TLF: 4572302

TLX:

EML: HKARTTUNEN AT CSC.FI

COM: 15

KASTURIRANGAN K OR
ISRO SATELLITE CTR
AIRPORT RO
VIMANAPURA POST
BANGALORE 5600.17
INDIA
TEL: 81254779
TLF:
TLX: $845325 \& 769$
EML:
COM: 44
KATGERT-MERKELIJN J K OR
STERREWACHT
HUYGENS LAB
BOX 9513
NL 2300 RA LEIOEN
NETHERLANDS
TEL:
TLF:
TLX:
EML:
COM:

KARETNI KOV VALENTIN 6 R

ASTRONOMICAL OBSERVATORY ODESSA STATE UNIVERSITY SHEVCHENKO PARK

270014 ODESSA

UKRAINE

TEL: 250356

TLF:

TLX:

EML:

COM: 42

XARYGINA ZOYA V DR ASTROPHYSICAL INSTITUTE KAZAKH ACAD OF SCIENCES 48006B ALMA ATA KAZAKHSTAN

TEL:

TLF:

TLX:

EML:

COM: 21

KASUGA TAKASHI
NOBEYAMA RAOIO OBS
NAOJ
MLNAMIMAKI MURA
NAGANO 38413
JAPAN
TEL: $267-98-2831$
TLF:
TLX: 3329005 TAO NRO J
EML:
COM: 40
KATO MARIKO
OPT OF ASTRONOMY
KEIO UNIVERSITY
HIYOSHI KOULOKU-KU
YOKOHAMA SHI 223
JAPAN
TEL: $44-63-1111$
TLF:
TLX:
EML:
COM: 35

KARLICKY MARIAN ASTRONOMICAL INSTITUTE CZECH ACADEMY OF SCIENCES ONOREJOY OBSERVATORY CS 25165 ONOREJOY CZECHOSLOVAKIA

TEL: 20485201

TLF : 20485314

TLX: 121579 ASTR $~ C$

EML:

COM: $10 C, 12$

KARPEN JUOITH T NAVAL RESEARCH LABORATORY CODE $4175 \mathrm{~K}$

4555 OVERLOOK AVE SW WASHINGTON OC 203755000 USA

TEL: 2027673441

TLF:

TLX:

EML:

COM: 10,12

KASHSCHEEV B L. PROF OR KHARKOV INSTITUTE FOR RADIOELECTRONICS 310059 KHARKOY UKRAINE

TEL:

TLF:

TLX:

EML:

COM: 22

KASUMOV FIKRET K O OR PHYSICS INSTITUTE NARIMANQVA PR 33 AKADEMGORODOC 370122 BAKU AZERBAIDZHAN TEL: 396784

TLF:

TLX:

EML:

COM: 33

KATO SHOJI PROF OPT OF ASTRONOMY KYOTO UNIVERSITY KITASHIRAKAWA SAKYO KU KYOTO 606

JAPAN

TEL: 075-751-2111

TLF:

TLX: 5422693 LIBKYU J

EML:

COM: $12,33,47$ 
KATO TAKAKO DR

INST OF PLASMA PHYSICS

NAGOYA UNIVERSITY

FUROCHO CHIKUSA KU

NAGOYA 464

JAPAN

TEL: 052-781-5111

ILF:

TLX: 0447-3691 1PPJNU J

EML:

COM: 14 C
KATSIS DEMETRIUS OR

12 RUE VARNIS

GR 17124 NEA SMYRNE

GREECE

JEL: 9336014

TLF :

EML:

COM: 07

TLX:

KAUFMANN JENS PETER DR INSTITUT FUR ASTRONOMIE TECHNISCHE UNIVERSITAT HARDENBERGSTR. 36

D 1000 BERLIN 12

GERMANY

TEL: 303145462

TLF :

TLX: 184262

EML:

COM:

\section{KAWABATA KIYOSHI}

DPT OF PHYSICS COLL SCI

SCIENCE UNIVERSITY TOKYO

1-3 KAGURAZAKA SHINJUKU

TOKYO

JAPAN

TEL: $3-260-4271$

TLF :

TLX:

EML:

COH:

KAWARA KIMIAKI

TOKYO ASTRONOMICAL OBS

NAOJ

DSAWA MITAKA

TOKYO 181

JAPAN

TEL:

TLF :

TLX:

EML:

COM: 25

KAZES ILYA DR OBSERVATOIRE DE PARIS SECTION DE MEUDON SECTION ASTROPHYSIQUE F 92195 MEUDON PPL CDX FRANCE

TEL: $145 \quad 077606$

TLF:

Tix:

EML:

COM: 34.40
KATZ JONATHAN I

DPT OF PHYSICS

WASHINGTON UNIVERSITY

ST LOUIS MO 63130

USA

TEL: 3148896202

TLF :

ILX:

EML:

COM: 48

KAUTMANN PIERRE PROF

CRAAE/ESCOLA POLITECNICA

UNIVERSIDADE OE SAO PAULO

CP 8174 .

05508 SAO PAULO SP

BRAZIL

TEL: 118159322

TLF :

TLX:

EML:

COM: $10,12,40,51$

KAWABATA SHUSAKU PROF

KYOTO GAKUEN UNIVERSITY

NANJO

SOGABECHO KAMEOKA

KYOTO 621

JAPAN

TEL: $07712-2-2001$

TLF:

TLX:

EML:

COM: 42

KAWATA YOSHIYUKI OR

KANAZAWA TECHNOLOGY INST

7-I OGIGAOKA

NONOICHOMACH I

ISHIKAWA 921

JAPAN

TEL:

TLF:

TLX:

EML:

COM:

KEAY COLIN S L PROF

OPT OF PHYSICS

NEWCASTLE UNIVERSITY

NEWCASTLE NSW 2308

AUSTRALIA

TEL: $49215451 / 5440$

TLF: 49216907

TLX: 28194 NEWUN AA

EML: PHCSLKECC . NEWCASTLE, EDU.AU

COM: $15,22 C$
KATZ JOSEPH DR

RACAH INST OF PHYSICS

HEBREW UNIY OF JERUSALEM JERUSALEM 91904

ISRAEL

$T E L: 2584604$

TLF:

TLX: 25391 HUIL

EML:

COM:

KAULA WILLIAM M PROF DPT OF EARTH \& SPACE SCI UNIVERSITY OF CALIFORNIA LOS ANGELES CA 90024

USA

TEL:

TLF :

TLX:

EMLL:

COM: 07,16

KAWAGUCHI ICHIRO PROF OPT OF ASTRONOMY KYOTO UNIVERSITY KITASHIRAKAWA SAKYO KU KYOTO 606

JAPAN

TEL:

TLF :

TLX:

EML:

COM: 12

KAYSER RAINER OR HAMBURGER STERNWARTE

GOJENSBERGSWEG 112

D 2050 hamBURG Bo GERMANY

TEL: $40 \quad 7252 \quad 4126$

'TLF: 4072524198

TLX: 217884

EML: ST40010\%DHHUNI4.BITNET COM: 47

KEEL WILLIAM C DPT OF PHYSICS \& ASTRON UNIVERSITY OF ALABAMA BOX 1921

TUSCALOOSA AL $35487 \quad 0324$ USA

TEL: 2053485050

TEF :

TLX:

EML:

COM: 28 
KEENAN PHILIP C PROF EMER PERKINS OBSERVATORY OHIO STATE UNIVERSITY BOX $449^{\circ}$

DELAWARE OH 43015

USA

IEL: $\quad 614 \quad 363 \quad 1257$

TLF :

$T L X: B 10-482-1715$

EML:

COM: 29,45

RELEMEN JANDS

KONKOLYY OBSERVATORY

THEGE U 13/1?

BOX 67

H 1525 BUDAPEST

HUNGARY

TEL: $175 \quad 5866 / 75 \quad 4122$

TLF :

TLX: 227460 KONOB H

EML:

COM:

KELLER HUWE ULRICH OR OBSERVATORY \& PLANETARIUM NECKARSTRASSE 47

D 7000 STUTTGART 1 GERMANY

TEL: 711291004

TLX: 721855 STBST D

EML:

TLF:

COM: 46,51

KENDEROINE SIDNEY OR

MULLARD RADIO ASTRON OBS CAVENDISH LABORATORY

MADINGLEY RD

CAMBRIDGE CB3 OHE

UK

TEL: 22366477

TLF:

TLX: 81292

EML:

COM: 40

\section{KENNICUTT ROBERT [ JR}

OPT OF ASTRONOMY

UNIVERSITY OF MINNESOTA

116 CHURCH ST SE

MINNEAPOLIS MN 55455

USA

TEL: 6123765224

TLF;

ILX:

EML:

COM: 28,34
KEGEL WILHELM H PROF INST THEORETISCHE PHYSIK UNIVERSITAT FRANKFURT

ROBERT-MAYER-STR 8-10

D 6000 FRANKFURT MAIN I

GERMANY

TEL: $69798 \quad 2357$

TLF:

ILX: 413932

EML:

COM: 34

KELLER CHARLES $F$

LOS ALAMOS NATIONAL LAB

MS F665

B0X 1663

LOS ALAMOS NM 87545

USA

TEL: 5056675648

TLF:

$T L X$ :

EML:

COM:

KELLERMANM KENNETH I OR

NRAD

EDGEMONT RD

CHARLOTIESVILLE VA 22903

USA

TEL: 8042960240

TLX: 9109970174

EML: NRAO: : KKELLERM

TLF: 8042960278

COM: $28,40,47,48,51$

KENDZIORRA ECKHARD DR

ASTRONOMISCHES INSTITUT DER UNIV TUEBINGEN

WALOHAEUSERSTR 64

D 7400 TUEBINGEN

GERMANY

TEL: $707 \quad 129 \quad 6127$

TLF : $707 \quad 129 \quad 3458$

TLX: 7262714 AIT 0

EML: 29382: : KENDZIORRA

COM

\begin{tabular}{|c|c|}
\hline KENT STEPHEN M & KENYON SCOTT J DR \\
\hline FERMILAB & CENTER FOR ASTROPHYSICS \\
\hline MS 127 & $\mathrm{HCO} / 5 \mathrm{AO}$ \\
\hline $80 \times 500$ & 60 GARDEN ST \\
\hline BATAVIA IL 60510 & EAMBRIDGE MA 02138 \\
\hline USA & USA \\
\hline TEL: & TEL: 6174957235 \\
\hline TLF: & TLF : \\
\hline$T L X:$ & $T L X: 921428$ \\
\hline EML: & EML: BITNET: kenyonefa \\
\hline COM: & COM: 42 \\
\hline
\end{tabular}

KEIL STEPHEN L

AIR FDRCE GEQPHYSICS LAB

NTL SOLAR OBSERVATORY

SACRAMENTO PEAK OBS

SUNSPOT NM 88349

USA

TEL: 5054341390

TLF :

TLX:

EML:

COM: 12

KELLER HORST UWE OR

MPI FUER AERONOMIE

POSTFACH 20

D 3411 KATLENBURG LINDAL GERMANY

TEL: 555641419

TLF :

TLX: 965527

EML:

COM: $15 C, 49 C$

KEMBHAVI A.JIT $K$

TIFR

HOMI BHABHA RD

COLABA

BOMBAY 400005

INDIA

TEL:

TLF:

TLX:

EML:

COM: 47,48

KENNEDY JOHN E PROF

323 LAKE CRESENT

SASKATOON SK STH 3AL

CANADA

IEL: $306 \quad 3744614$

ILF :

TLX:

EML:

COM: 41.46

KEPLER S D

INSTITUTO DE FISICA

UFRGS

CP 15051

90049 PORTO ALEgRE RS

BRAZIL

TEL: $512 \quad 36 \quad 4677$

TLF :

ILX: 051-1055 UFRS BR

EML:

COM: 25,27 


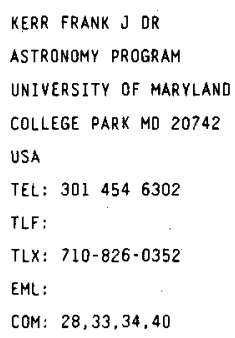

KERR ROY P PROF
OPT OF PHYSICS
UNIVERSITY OF CANTERBURY
PRIVATE BAG
CHRISTCHURCH 1
NEW ZEALAND
TEL: 348 2009
TLF:
TLX: NZ 4144
EML:
COM:

\section{KHACHIKIAN E YE PROF} BYURAKAN ASTROPHYSICAL OBSERVATORY

378433 BYURAKAN
ARMENIA
TEL: $8852 \quad 284142 / 283453$
TLF: 8852523640
TLX: 243344 ORION SU
EML:
COM: $28 P$

KHARIN A S DR

MAIN ASTRONOMICAL DBS UKRAINIAN ACAD OF SCIENCE GOLOSEEVO

$252127 \mathrm{KIEV}$

UKRAINE

TEL: $66 \$ \mathbf{4 7 6 5}$

ILF:

TLX: 132517 NEBO

EML:

COM: 05,08

KHOKHLOVA V L DR

INST OF ASTRONOMY

ACADEMY OF SCIENCES

PYATNITSKAYA UL 48

109017 MOSCOW

RUSSIA

TEL: $231-54-61$

TLF

TLX: 412623 SCSTP SU

EML:

COM: 29.36

KIANG TAO PROF
DUNSINK OBSERVATORY
DIAS
OUBLIN 15
IRELAND
TEL: 1387911
TLF:
TLX: 31687 OIAS EI
EML:
COM: 20,41

KESSLER KARL G DR NTL BUREAU OF STANOARDS

A 505 ADMIN

GAITHERSBURG MO 20899

USA

TEL: 3019213643

TLF:

TLX: 197674 TRT

EML:

COM: 14,31

KHALESSEH BAHRAM DR OPT OF PHYSICS 91735654 UNIVERSITY OF FERDOWSI SCHOOL OF SCIENCES MASHHAD

IRAN

TEL: $5132021 * 64$

TLF : 5187079

TLX: 512271 FUON IR

EMt:

COM: 42

KHARI TONOV ANOREJ V DR ASTROPHYSICAL INSTITUTE KAZAKH ACAO OF SCIENCES 480068 ALMA ATA KAZAKHSTAN

TEL:

TLF:

TEX:

EML:

COM:

KHOLSHEVNI KOV K $\mathrm{K}$ DR

ASTRONOMICAL OBSERVATORY ST PETERSBIRRG UNIVERSITY BIBLIOTECHNAJA PL 2 198904 ST PETERSBURG RUSSIA

TEL: 257-94-88

TLF:

TLX:

EML:

COM: $07 C$

KIASATPOOR AHMAO PROF OPT OF PHYSICS UNIVERSITY OF ESFAHAN DANESHGAH E

ESFAHAN

IRAN

TEL: 3144321

TLF:

TLX: 31-2295 IRE U

EML:

COM:
KESSLER MARTIN $F$ QR

ESA/ESTEC

ASTROPHYSICS OIV

BOX 299

NL 2200 AG NOOROWIJK

NETHERLANDS

TEL: 171983623

TLF: $17 \quad 1985434$

ILX: 39098

EML: ESTCSI: :MKESSLER

COM: 44

KHARADZE E K PROF

ABASTUMANI ASTROPHYSICAL OBSERVATORY

GEORGIAN ACAD OF SCIENCES 383762 ABASTUMANI

GEORGIA

TEL: 998891/225460

ILF:

TLX: 327409

EML:

COM: $33,34,45$

KHATISASHVILI ALFEZ SH OR ABASTUMANI ASTROPHYSICAL. OBSERVATORY

GEORGIAN ACAD OF SCIENCES 363762 ABASTUMANI

GEORGIA

TEL:

TLF:

TLX:

EML:

COM: 20

KHOZOV GENNADIJ $V$

ASTRONOMICAL OBSERVATORY ST PETERSBURG UNIVERSITY BIBLIOTECHNAJA PL 2 198904 ST PETERSBURG RUSSIA

TEL: 2-57-94-84

TLF:

TLX: 12168 PHOBOS

EML:

COM: 35

KIBBLEWHITE EDWARO J DR INSTITUTE OF ASTRONOMY THE OBSERVATORIES MADINGLEY RD CAMBRIDGE CBЗ OHA UK TEL:

TLF:

TLX:

EML:

COM: 


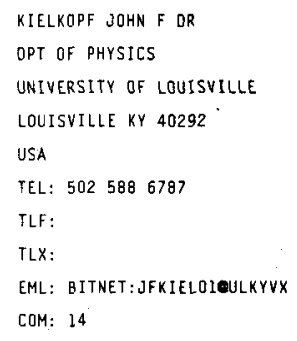

KIGUCHI MASAYOSHI DR
RES INST SCIENCE \& IECH
KINKI UNIVERSITY
HIGASHI
OSAKA 577
JAPAN
TEL: $06-721-2332$
TLF:
TLX:
EML:
COM: 35

KILAMBI G C OR

DPT OF ASTRDNOMY

UNIVERSITY OF OSMANIA

HYOERABAO 500007

INDIA

TEL: $71951 * 247$

TLF :

TLX:

EML:

COM: 37

KIM CHULHEE DR

DPI OF EARTH SCI EDU

CHONBUK NTL UNIVERSITY

CHONJU. 560756

KOREA R

TEL:

TLF:

TLX:

EML:

COM: 27

\section{KIM KWANG-TAE DR}

OPT ASTRON \& SPACE RES CHUNGNAM NTL UNIVERSITY DAEJOEN 304764

\section{KOREA R}

TEL: 0428215463

TLF :

TLX:

EML:

COM: 40

\section{KIM YUL}

PYONGYANG ASTRON OBS ACADEMY OF SCIENCES DPRK TAESONG DISTRICT

\section{PYONGYANG}

KOREA DPR

TEL:

TLF:

TLX:

EML:

COM:
KII TSUNEO OR

ISAS

3-1-I YOSHINODAI

SAGAMIHARA

KANAGAWA 229

JAPAN

TEL: 427513911 EX 2624

TLF: 0427594253

TLX: J27758IASA ERO

EML:

COM: 48

KILAR BOGDAN DR

FAC DF GEODESY

UNIVERSITY OF LJUBLJANA

JAMOVA?

YU 61000 LJUBLJANA

YUGOSLAVIA

TEL:

TLF:

$T L X_{i}$

EML:

COM:

KIM IRADTA S

STERNBERG STATE ASTR INST

117234 MOSCOW

RUSSIA

TEL:

TLF:

TLX:

EML:

COM: $L O C$

KIM TU HWAN

KOREA ASTR/SPACE SCI INST

36-1 WHAAM-DONG JUNG-GU

TAEJEON CHUNGCHUNGNAM-DO

TAEJEON 30031

KOREA R

TEL: $042-823-1497$

TLF:

TLX: $45532 \mathrm{k}$

EME:

COM: 27

KIM ZONG DOK

PYONGYANG ASTRON OBS

ACADEMY OF SCIENCES DPRK

TAESONG DISTRICT

PYONGYANG

KOREA DPR

TEL:

TLF:

TLX:

EML:

COM: 14
KIKUCHI SADAEMON PROF ASTRONOMICAL INSTItUTE TOHOKU UNIVERSITY

SENDAI AOBA

MIYAGI 980

JAPAN

TEL:

TLF :

TLX:

EML:

COM:

KILKENNY DAVIO OR

SAAO

BOX 9

OBSERVATORY 7935

SOUTH AFRICA

TEL: 021-47-0025

TLF:

TLX: 57-20309 SA

EML:

COM: 25

KIM JIK SU

PYONGYANǴ ASTRON OBS ACADEMY OF SCIENCES DPRK TAESONG OISTRICT

PYONGYANG

KOREA DPR

TEL:

TLF:

TLX:

EML:

COM: 47

KIM YONG HYOK DR

PYONGYANG ASTRON OBS ACADEMY OF SCIENCES OPRK TAESONG OISTRICT

PYONGYANG

KOREA DPR

TEL: $5-3134,53239$

ILF:

TLX:

EML:

COM:

KIMBLE RANDY A DR. DPT PHYSICS \& ASTRONOMY JOHNS HOPKINS UNIVERSITY CHARLES \& $34 \mathrm{TH}$ ST BALTIMORE MO 21218 USA

TEL: 3013388738

TLF :

TLX: 9102400225 JHU CASMD EML: SPAN:SCIVAX: : CASA: : RAK COM: 44 
A 160

KIMURA HIROSHI OR PURPLE MOUNTAIN ÓBSEV

CAS

NANJING

CHINA PR

TEL: 2533921

TLF :

TLX: 34144 PMONJ CN

EML:

COM: 34

KING IVAN R PROF

ASTRONOMY DPT

UNIVERSITY OF CALIFORNIA

60L CAMPBELL HALL

BERKELEY CA 94720

USA

TEL: 4156422206

TLF:

TLX: 820181 UCB ASTRAL

EML:

COM: $25,28,33,37$

KING-HELE DESMOND G DR ROYAL AIRCRAFT ESTABL

FARNBORQUGH HANTS

UK

IEL: $252 \quad 24461$

TLF :

EML:

COM: 07

TLX:

KIPLINGER ALAN L DR

APAS QPR

UNIVERSITY OF COLORADO

B0X 391

BOULOER CO 803090391

USA

TEL: 3034975892

TLF:

TLX:

EML: SPAN 9555: : AKIPLINGER

COM: 10,27

\section{XIRBIYIK HALIL DR}

DPT OF PHYSICS

MIDOLE EAST TECH UNIV

06531 ANKARA

TURKEY

TEL: $4122 \quad 37100 / 3528$

TLF:

TLX: 42761 OOTK TR

EML:

COM:

KING ANDREW R DR
OPT OF ASTRONOMY
UNIVERSITY OF LEICESTER
UNIVERSITY RD
LEICESTER LEI $7 R H$
UK
TEL: 533555455
TLF:
TLX: 341198
EML:
COM:
KING R B OR
BOX 725
MEOOCINO CA 95460
USA
TEL:
TLF:
EML:
COM: 14,29
TLX:

KINMAN THOMAS D OR KITT PEAK NTL OBS

BOX 26732

950 N CHERRY AVE

TUCSON AZ $85726 \quad 6732$

USA

TEL: 6023275511

TLF :

TLX: D6EG-484 AURA NOAO

EML:

COM: 28,33

KIPPENHAHN RUDOLF PROF

RAUTENBREITE 2

D 3400 GOETTINGEN

GERMANY

TEL: 55124714

TLF : 55122902

EML:

COM: 27,35

TLX:

KIRBY KATE P OR
CENTER FOR ASTROPHYSICS
HCO/SAO
GO GAROEN ST
CAMBRIDGE MA 02138
USA
TEL: 6174957237
TLF:
TLX:
EML:
COM: 14

\author{
KING DAVIOS PROF \\ DPT PHYSICS \& ASTRONOMY \\ UNIVERSITY OF NEW MEXICO \\ 800 YALE BLVO NE \\ ALBUQUERDUE NM 87131 \\ USA \\ TEL: \\ TLF: \\ TLX: \\ EML: \\ COM: 35,41
}

KING ROBERT WILSON JR DR OPT OF EARTH \& PLANET SCI

MIT RM 54620

BOX 165

CAMBRIUGE MA 02139

USA

TEL: 6172537064

TLF:

ILX: 921473 MIT CAM

EML:

COM: 04

KINNEY ANAE L DR

STSCI

HOMEWOOD CAMPUS

3700 SAN MARTIN DR

BALTIMORE MO 21218

USA

TEL: 3013384831

TLF :

TLX: 6849102 STSEI

EML: 6549: : KINNEY

COM:

KIPPER TONU DR

TARTU ASTROPHYSICAL OBS

ESTONIAN ACAD OF SCIENCES 202444 TARTU

ESTONIA

TEL:

TLF:

TLX:

EML:

COM: $09,14,29$

KIRK JOHN DR

MAX PLANCK INSTITUT

F. PHYSIK \& ASTROPHYSIK

KARLSCHWARZ SCHILO STR. 1

D 8046 GARCHING MUENCHEN

GERMANY

TEL: 8932990

TLF :

$T L X:$

EML:

COM: 48
KING HENRY C OR

TRILLIUM

206 WHITE LION RDAO

LITTLE CHALFONT

BUCKS HP7 $9 N U$

UK

TEL:

TLF:

TLX:

EML

COM: 41

RINGSTON ARTHUR E PROF OPT OF APPLIED MATHS

\& THEORETICAL PHYSICS QUEEN'S UNIVERSITY

BELFAST BT7 INN

UK

TEL: $232 \quad 245133$

ILF :

ILX: 7448? QUB AMD 6

EML:

COM: 14

KINOSHITA HIROSHI DR

TOKYO ASTRONOMICAL OBS

NADJ

OSAWA MITAKA

TOKYO 181

JAPAN

TEL: 422413615

TLF : $422 \quad 413793$

ILX: 02822307 TAOMTK

EML: KINOSHITAOC I. MTK, NAD, AC.JP

COM: 04VP,07C, 20

KIRAL ADNAN PROF

UNIVERSITY OBSERVATORY UNIYERSITY OF ISTANBUL 34452 ISTANBUL

TURKEY

TEL:

TLF:

TIX:

EML:

COM:

KIRKPATRICK RONALD C OR LOS ALAMOS NATIONAL LAB MS 220

BOX 1663

LOS ALAMOS MM B7545

USA

TEL: $505 \quad 6674812$

TLF:

TLX:

EML:

COM: 34 
KIRSHNER ROBERT PAUL OR DPT OF ASTRONGMY HARVARD UNIVERSITY 60 GARDEN ST CAMBRIOGE MA 02138 USA TEL: 6174957390

TLF:

TLX:

EML:

COM: 28.34

KISLYYK VITALIJ S DR

MAIN ASTRONOMICAL OBS UKRAINIAN ACAD OF SCIENCE GOLOSEEVO

252127 KIEV

UKRAINE

TEL:

TLF :

TLX: 131406 SKY US

EML:

COM: 16,24

\section{KITAMURA M PROF}

TOKYO ASTRONOMICAL OBS

NAOU

OSAWA MITAKA

TOKYO 181

JAPAN

TEL: 0422-32-5111

TLF:

TLX: 2822307 TAOMTKK J

EML:

COM: 42

\section{KIZILOGLU UEMIT DR}

DPT OF PHYSICS

MIOOLE EAST TECH UNIV

06531 ANKARA

TURKEY

TEL: $41 \quad 2237100 * 3275$

TLF: 412868638

TLX: 42751 ODTU TR

EML: UMKETRMETU.BITNET

COM:

KLAPP JAIME OR
OPT DE FISICA
UNAM UNIDAD
APOO POSTAL $55-534$
09340 IZTAPALAPAO DF
MEXICO
TEL: 5156442
TLF:
TLX: 1764186 KEBEME
EML:
COM:

XISELEV NIKOLAI N OR ASTROPHYSICAL INSTITUTE TADJIK ACAD OF SCIENCES SVIRIDENKO UL 22 734042 DUSHANBE TADZHIKISTAN

TEL:

TLF:

ILX:

EML:

COM: $15 \mathrm{C}$

KISSELEVA taMara $P$ PULKOVO OBSERVATORY ACADEMY OF SCIENCES 10 KUTUZOV QUAY 196140 ST PETERSBURG RUSSIA

TEL:

TLF :

TLX:

EML:

COM: 20

KITAMURA SEIICHI DR

DPT OF EARTH SCIENCE SHIGA UNIVERSITY

2-5-1 HIRATSU

OTSU 520

JAPAN

TEL: 775-37-0081

TLF:

TLX:

EML:

COM:

\section{KJAERgAaRo PER OR}

UNIVERSITY OBSERVATORY OESTER VOLOGADE 3

OK 1350 COPENHAGEN K OENMARK

TEL: $31 \quad 14 \quad 1790$

TLF: 31389157

TLX: $44 ! 55$ DANAST OK

EML:

COM:

KLARE GERHARD OR
LANDESSTERNHARTE
KOENIGSTUHL
O 6900 HEIOELBERG I
GERMANY
TEL: 622110036
TLF:
TLX:
EML:

XISELYOV ALEXEJ A OR PULKOVO OBSERVATORY ACADEMY OF SCIENCES 10 KUTUZOV QUAY 196140 ST PETERSBURG RUSSIA

TEL:

TLF :

TLX:

EML:

COM: 26

KISSELL KENNETH E DR CHIEF SCIENTIST

RPS MAUL BOX 758 AMOS/MOTIF OBSERVATORIES PAUNENE HI 96784 USA

TEL:

ILF:

TLX:

EML:

COM: 09

RITCHIN CHRISTOPHER R DR HATFIELD POLYTECHNIC OBSERVATORY BAYFORDBURY HERTFORD HERTS SGI3 BLO uK

TEL: $992 \quad 558451$

TLF :

TLX: 262413

EML:

COM: 29,46

KJELOSETH-MOE OLAV DR INST THEORET ASTROPHYSICS UNIVERSITY OF OSLO B0X 1029

N 0315 BLINDERN OSLO 3 NORWAY

TEL: 47-2-456510

TLF:

TLX: 72425 UNIOS N

EML:

COM: 10

KLARMANN JOSEPH PROF DPT OF PHYSICS WASHINGTON UNIVERSITY ST LOUIS MO 63130 USA

TEL: 3148896299

TLF:

TLX: 650-2557719 MCI

EML:

COM:
KISLYAKOY ALBERT G DR INST OF APPLIEO PHYSICS ACADEMY OF SCIENCES ULYANOV UL 46 603600 N NOVGOROO RUSSLA

TEL:

TLF:

TLX:

EML:

COM: 40

KITAMOTO SHUNJI OR FAC OF SCIENCES OSAKA UNIVERSITY machi KaneYama TOYONAKA OSAKA 560 JAPAN

TEL: $6844 \quad 1151$

TLF:

ILX:

EML: BITNET: KITAMOTOUPNOSKFM COM:

KIZILOGLU NILGUEN OR GPT OF PHYSICS MIDOLE EAST TECH UNIV 06531 ANKARA TURKEY TEL: : $412237100^{*} 3268$ TLF: 412868638 TLX: 42761 ODTU TR EML: NLK RRMETU.BITNET COM: 35

KUURKCHIEVA DIANA DR DPT OF PHYSICS HIGHER PEDAGOGICAL INST BG 9700 SHOUMEN BULGARIA

TEL: 63151216

TLF:

TIX:

EML:

COM: 27,42

KLECZEK JOSIP OR ASTRONOMICAL INSTITUTE CZECH ACADEMY OF SCIENCES ONOREJOY OBSERVATORY CS 251 65 ONDREJOY CZECHOSLOVAKIA TEL: 20485201 TLF: 20485314 TLX: 121579 ASTR C EML: COM: 05,10 
KLEIN KARL LUOWIG OR OBSERVATOIRE DE PARIS SECTION DE MEULON DASOP

F 92195 MEUDON PPL COX FRANCE

TEL: 145347761

TLF :

TLX:

EML: SPAN:MEUDON: : KLEIN

COM: $10,12,40$

KLEINMANN DOUGLAS E DR

HONEYWELL ELECTRO OPTICS OPERATION

2 FORBES RD

LEXINGTON MA 02173

USA

TEL: $617863 \quad 3841$

TLF :

TLX: $92-3477$

EML:

COM:

KLIMCHUK JAMES A DR

CTR FOR SPACE SCIENCES \&

ASTROPHYSICS

STANFORD UNIV ERL

STANFORO CA 943054055

USA

TEL: $415723 \quad 1765$

TLF: $415 \quad 725 \cdot 2333$

TIX:

EML: KLIMCHUKOFLARE. STANFORD. EOU

COM: 10

KLIORE ARVYDAS JOSEPH DR

JPL

4800 OAK GROVE DR

PASADENA CA 91109

USA

TEL: $818354 \quad 6164$

TLX: 675429

EML:

TLF:

COM:

KLVANA MIROSLAV
ASTRONOMICAL INSTITUTE
CZECH ACAOEMY OF SCIENCES
ONDREJOV OBSERVATORY
CS 25165 ONOREJOV
CZECHOSLOVAKIA
TEL: 20485201
ILF: 20485314
TLX:
EML:
COM: 10

\author{
KLEIN MICHAEL J OR \\ JPL/SSD \\ MS 303401 \\ 4800 OAK GROVE DR \\ PASADENA CA 91109 \\ USA \\ TEL: $818 \quad 354 \quad 7132$ \\ TLF : \\ TLX: \\ EML: \\ COM: $51 \mathrm{C}$
}

\section{KLEMOLA ARNOLD R DR}

LICK OBSERVATORY

UNIVERSITY OF CALIFORNIA

UCSC

SANTA CRUZ CA 95064

USA

TEL: $408 \quad 4292907$

TLF:

TLX:

EML:

COM: 20,24

\section{KLIMISHIN I A PROF}

PEDAGOGIC INSTITUTE

PUSHKIN UL 96 APT 66

284000 IVANOFRANKOVSK

UKRAINE

TEL:

$T L X$ :

EML:

TLF :

COM:

KLOCK B L OR

6601 S HOMESIAKE DR

BOWIE MO 20715

USA

TEL: $301262 \quad 1506$

TLF :

EML:

COM: $08,09,24$

TLX:

KNACKE ROGER F OR
OPT OF EARTH \& SPACE SC1
ASTRONOMY PROGRAM
SUNY AT STONY BROOK
STONY BROOK NY 117942100
USA
TEL: 516246.7673
TLF:
TLX: 5102287767
EML:
COM: 15,34

KLEIN RICHARD I OR
LAWRENCE LIVERMORE LAB
L 23
BOX $80 B$
LIVERMORE CA 94550
USA
TEL: 4154223548
TLF:
TLX:
EML:
COM: 36

KLEMPERER W $K$ DR

NBS

ELECTROMAGNETIC FIELDS D

325 BROADWAY

BÖULDER CO 80303

USA

TEL: 3034973757

TLF ;

TLX: 592811 NOAA MASC BOR

EML :

COM:

KLINGLESMITH DANIEL A OR

NASA/GSFC

CODE $6 B 4$

GREENBELT MO 20771

USA

TEL: 3012866541

TLF:

TLX:

EML:

COM:

KLOCOK LUBOMIR OR

ASTRONOMICAL INSTITUTE

SLOVAK ACADEMY SLIENCES

CS 05960 TATRANSKA LOMNI

CZECHOSLOVAKIA

TEL: $96996 \quad 7866 / 7 / 8$

ILF: 969967656

ILX: 78277

EML:

COM: 09

KNAPP GILLIAN R DR

DPT ASTROPHYSICAL SCI

PRINCETON UNIVERSITY

PRINCETON NJ 085441001

USA

TEL: $609452 \quad 3824$

TLF:

TLX:

EML:

COM: $28,33,34$
KLEIN ULRICH

RADIOASTRONOMISCHES INST

DER UNIVERSITAET BONN

AUF OEM HUEGEL 71

O 5300 BONN 1

GERMANY

TEL: $22873 \quad 3644$

ILF:

TLX:

EML:

COM: 28,40

KLEPCZYNSKI WILLIAH J DR

US NAVAL OBSERVATORY

34 \& MASSACHUSETS AVE NW WASHINGTON OC 203925100 USA

TEL: $202 \quad 653 \quad 1521$

TLF :

TLX: 710-822-1970

EML:

COM: $04,19,31 \mathrm{C}$

KLINKHAMER FRANS DR NIKHEF H CHEAF

BOX 41882

NL 1009 OB AMSTERDAM

NETHERLANDS

TEL: 205929444

TLF : $20 \quad 5925155$

TLX:

EML： KLINKHAMERNI KHEFH. NIK'HEF. WE

COM: 48

KLOKOCNIK JAROSLAV OR

ASTRONOMICAL INSTITUTE

CZECH ACAOEMY OF SCIENCES

ONOREJOV OBSERVATORY

CS 25165 DNOREJOV

CZECHOSLOVAKIA

TEL: 204.65201

TLF: 20485314

TLX: 121579 ASTR C

EML:

COM: 07

KNEER FRANZ OR

UNIVERSITATS STERNWARTE

GE ISMARLANDSTRASSE II

D 3400 GOETTINGEN

GERMANY

TEL: 551395042

TLF :

TLX: 96753

EML:

COM: 12 
KNEZEVIC ZORAN

ASTRONOMICAL OBSERVATORY

VOLGINA 7

YU 11050 BEOGRAD

YUGOSLAVIA

TEL: $11 \quad 419357 / 421875$

TLF :

TLX: 72610 AOB YU

EML: EAOPOOZ YUBGSS21.BITNET

COM: $07,15 C, 20$

KNOWLES STEPHEN H OR

CODE 02

NAVSPASUR

DAHLGREN VA 224485180

USA

TEL:

TLF :

TLX:

EML:

COM: 19,51

KOBAYASHI YUKISAYU

TOKYO ASTRONOMICAL OBS

NADJ

OSAWA MITAKA

TOKYO 181

JAPAN

TEL: $422 \quad 325111$

ILF:

ILX: 2822307 TAOMTK

EML :

COM: 31

KDCHAROV GRANT E PROF IOFFE PHYSICAL TECH INST ACADEMY OF SCIENCES POLYTECHNICHESKAYA UL 26 194021 ST PETERSBURG RUSSIA

TEL: $247-91-67$

TLF :

TLX:

EML:

COM: 48

KODAMA HIDEO

COLL LIB ARTS KYOTO UNIV YOSHIDA NIHONMATSU-CHO

SAKYO KU

KYOTO 606

JAPAN

TEL: 075-7512111

TLF:

TLX:

EML:

COM: 47
KNIFFEN DONALO A OR

OPT OF PHYSICS

BOX 862

HAMPOEN SYONEY COLLEGE

VA 23943

USA

TEL:

TLF :

TLX:

EML

COM:

KNUDE JENS KIRKESKOY DR UNIVERSITY OBSERVATORY oester voldgade 3

DK 1350 COPENHAGEN $K$ DENMARK

TEL: $31 \quad 141790$

TLF: 31389157

TLX: 44155 DANAST DK

EML:

COM: $25 \mathrm{C}, 34$

KOCER OURCUN DR

DPT OF ASTRONOMY

ISTANBUL UNIVERSITY

34452 ISTANBUL

TURKEY

TEL: 15223597

TLF: 15226123

TLX:

EML:

COM: 51

KOCHHAR R K OR

INDIAN INSTITUTE OF

ASTROPHYSICS

KORAMANGALA

BANGALORE 560034

INDIA

TEL: $81256 \quad 6585$

TLF :

TLX: 845763 IIAB IN

EML:

COM: 28,35

KOEBERL CHRISTIAN OR

INSTITUTE OF GEOCHEMISTRY

UNIVERSITY OF VIENNA

OR KARL-LUEGER - RING I

A 1010 VIENNA

AUSTRIA

TEL: 122243002360

TLF :

TLX:

EML:

COM: $15,22[, 5]$
KNOLKER MICHAEL DR UNIV STERNWARTE

GOTTINGEN

GEISMARLANDSTRASSE 11

D 3400 GOETTINGEN

GERMANY

TEL: 551395046

TLF :

TLX:

EML:

COM: 12.35

KO HSIEN C PROF

DPT. OF ELECT ENGINEERING

OHIO STATE UNIVERSITY

1958 NEIL AVE

COLLUMBUS OH 43210

USA

TEL: 6144222571

TLF: 1

TLX: $24-5334$

EML:

COM: 40 .

KOCH DAVID $G$

NASA AMES RESEARCH CTR

MS 2456

MOFFETT FIELD CA 94035

USA

TEL:

TLF :

TLX:

EML:

COM:

KOCH-MIRAMOND LYOIE DR

CEA CEN

IRF/OPHG/SAP

BP 2

F 91191 GIF/YVETTE CDX

FRANCE

TEL: $1 \quad 6908 \quad 4329$

$T L F$ :

ILX: 690860

EML:

COM: 44,48

KOECKELENBERGH ANDRE DR

OBSERVATOIRE ROYAL DE

BELGIQUE

AVE CIRCULAIRE 3

B $\$ 180$ BRUSSELS

BELGIUM

TEL: 23730311

TLF: 23749822

TLX: 2.1565 OBSBEL

EML:

COM: 10
KNOSKA STEFAN

ASTRONOMICAL INSTITUTE

SLOVAK ACADEMY SCIENCES

CS 05960 TATRANSKA LOMNI CZECHOSLOVAKIA

TEL: $96996 \quad 7866 / 7 / 8$

TLF : 969967656

TLX:

EML:

COM: 10

KOBAYASHI EISUKE BR SCIENCE INST OF OSAKA 459 SUGIMOTO CHO SUMIYOSHI KU OSAKA 558

JAPAN

TEL: $06-692-18 B 2$

TLF :

TLX:

EML:

COM :

KOCH ROBERT H OR

OPT ASTRON \& ASTROPHYS

UNIV OF PENNSYLVANIA

DAVID RITTENHOUSE LAB

PHILADELPHIA PA 19104

USA

TEL: $215 \quad 898 \quad 7882$

TLF: $215 \quad 898 \quad 9336$

TLX: 834621

EML: RKOCHOPENNDRLS

COM: $25,42 C, 51$

KODAIRA KEIICHI PROF

TOKYO ASTRONOMICAL OBS

NADJ

DSAWA MITAKA

TOKYO 181

JAPAN

TEL: 0422-32-5111

TLF:

TLX: 2822307 TAOMTK J

EML:

COM: $28,29,36$

KOEHLER H PROF OR

SAUERBRUCHSTR 6

D 7920 HEIDENHEIM

GERMANY

TEL: 732144560

TLF:

$T L X:$

EML: .

COM: 09 
KOEHLER JAMES A PROF DPT OF. PHYSICS UNIV OF SASKATCHEWAN SASKATOON SK STN OWO CANADA

TEL: $306966 \quad 6442$

TLF:

ILX:

EML:

COM:

KOEPPEN JOACHIM DR INST F. THEOR ASTROPHYSIK DER UNIV HEIDELBERG IIM NEUENHEIMER FELO 561 D 6900 HEIDELBERG 1 GeRMANY

TEL: $62 \quad 21562988$

TLF:

TLX: 461515 UNIHO D

EML:

COM: 34

KOHL JOHN L DR

CENTER FOR ASTROPHYSICS

HCO/SAO

60 GARDEN ST

CAMBRIDGE MA 0213B

USA

TEL: 6174957377

TLF:

TLX: 921428

EML:

COM: 14

KOKKOTAS KONSTANTINOS OR

DPT ASTROPHY ASTRON MECH UNIVERSITY THESSALONIKI GR 540 O6 THESSALONIKI GREECE

TEL: 31991357

TLF: 31992777

TLX: 412181 AUTH GR

EML: CAAZO104@RTHEUN1. EARN

COM: 47

KOLCHINSKIJ I $G$ DR

MaIN ASTRONOMICAL OBS

UKRAINIAN ACAD OF SCIENCE

GOLOSEEVO

252127 KIEV

UKRAINE

TEL:

TLF:

$T L X:$

EML:

COM: 24
KOEHLER PETER

CARL ZEISS JENA GMBH

ASTRON INSTRUMENTS OIV

PO BOX 125

D 6900 JENA

GERMANY

TEL: 785882575

TLF: $78 \quad 580 \quad 2083$

TLX: 587452 CZG DD

EML:

COM: 09

KOESTER DETLEV DR

OPT PHYSICS \& ASTRONOMY

LOUISIANA STATE UNIV

RATON ROUGE LA 708034001

USA

TEL: $504388226 \mathrm{~L}$

TLF:

TLX: 559184

EML:

COM: 35,36

KOHOUTEK LUBOS OR

HAMBURGER STERNWARTE

GOJENSBERGSWEG 112

O 2050 HAMBURG 80

GERMANY

TEL: 4072524112

TLX: 217894

EML:

TLF:

COM: $15,20,34$

KOKURIN YURIJ L DR

LEBEDEV PHYSICAL INST

ACADEMY OF SCIENCES

LENINSKY PROSPEKT 53

117924 MOSCOW

RUSSIA

TEL: $135-03-60$

FLF:

TLX: 411479 NEOO SU

EML:

COM: 08

KOLESNIK IGOR GG OR

MAIN ASTRONOMICAL OBS

UKRAINIAN ACAD OF SCIENCE

goloseevo

$252127 \mathrm{kIEV}$

UKRAINE

TEL: 663110

TLF:

TLK: 131406 SKY SU

EML:

COM: 33,34

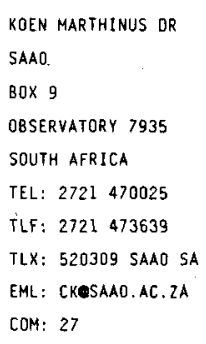

KOGOSHVILI NATELA G

ASTROPHYSICAL OBSERVATORY

MOUNT KANOBILI

383762 ABASTUMANT

GEORGIA

TEL: 283

TLF :

TLX:

EML:

COM: 28

KOJIMA MASAYOSHI OR

SOLAR TERRESTRIAL LAB

NAGOYA UNIVERSITY

3-13 HONOHARA TOYOKAWA

AICHI 442

JAPAN

TEL: $8 L 5338 \quad 6 \quad 3154$

TLF: 81533860811

TLX: 432231L STELAB J

EML: KOJIMAQSTELAB. NAGOYA-U. AC.JP

COM: 40

KOLACZEK BARBARA OR

PLANETARY GEODESY DEPT

POLISH ACAD OF SCIENCES

UL BARIYCKA 18

PL DO 716 WARSAW

POLAND

TEL: 4l-00-41

TLF:

TLX: 815670 CBK PL

EML:

COM: $04,19 P, 31$

KOLESNIK L N DR

MAIN ASIRONOMICAL DBS

UKRAINIAN ACAD OF SCIENCE

GOLOSEEVO

$252127 \mathrm{KIEV}$

UKRAINE

TEL: 660869

TLF:

TLX: 131406

EML:

COM: 33
KOENIGSBERGER GLORIA

INSTIJUTO OE ASTRONOMIA

UNAM

APDO POSTAL 70-264

O4510 MEXICO DF

MEXICO

TEL: 905-548-5305/05

TLF :

TLX:

EML:

COM:

KOGURE TOMOKAZU OR

TOGANO-0 1.10

HASHIMOTO

YAWATA

KYOTO 614

JAPAN

TEL: $075-983-2984$

TLF :

TLX:

EAL:

COM: 29

KOJOIAN GABRIEL DR

OPT OF PHYSICS

UNEVERSITY OF WISCONSIN

EAU CLAIRE WI 54701

USA

TEL: $715 \quad 836 \quad 3148$

TLF :

TLX:

EML:

COM: 28,40

KOLB EDWARD W OR

FERMILAB

MS 209

THEORETICAL ASTROPHYSICS

BATAVIA IL 60510

USA,

TEL: 3128404695

TLF :

TLX: 720481

EML:

COM: 47,48

\section{KOLESOV A K DR}

ASTRONDMICAL OBSERVATORY

ST PETERSBURG UNIVERSITY BIBLIOTECHNAJA PL 2

199178 ST PETERSBURG

RUSSIA

TEL:

TLF :

TLX:

EML:

COM: 36 
KOLEV OIMITAR ZORAVKOV NTL ASTRONOMICAL OBS BULGARIAN ACAD SCIENCES BOX 136

BG 47,00 SMOLJAN

BULGARIA

TEL: 7341559

TLF:

TLX:

EML:

COM:

KOMAROV NS DR

ASTRONOMICAL OBSERVATORY

ODESSA STATE. UNIVERSITY

SHEVCHENKO PARK

270014 ODESSA

UKRAINE

TEL: 220396

ILF :

TLX:

EML:

COM:

KONIGL ARIEH DR

ASTRONOMY \& ASTROPHYS CTR

UNIVERSITY OF CHICAGO

5640 S ELLIS AVE

CHICAGO IL 60637

USA

TEL: 3127027968

TLF :

TLX: $2 B 2132$

EML: SPAN: lasr: :oddjob::arieh

COM:

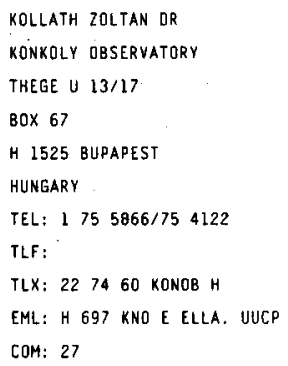

KOLLATSCHNY WOLFRAM DR UNIVERSITAETS STERNWARTE GEISMARLANDSTRASSE 11

D 3400 GOETTINGEN

GERMANY

TEL: 551395067

TLF :

TLX: 96753

EML: BITNET:WKOLLAT OOGOGWOGI

COM: $2 B$

\author{
KONBO MASAYUKI OR \\ TOKYO ASTRONOMICAL OBS \\ NAOJ \\ OSAWA MITAKA \\ TOKYO 181 \\ JAPAN \\ TEL: $0422-32-5111$ \\ TLF : \\ TLX: 2822307 TAOMTK 3 \\ EML:
}

COM:

KONONOVICH EOWARD V OR

SIÉRNBERG STATE ASTR INST UNIVERSITETSKIJ PROSP 13

119899 MOSCOW

RUSSIA

TEL:

TLF:

ILX:

EML:

COM: 12,46

KOO DAVIO L-Y DR

LICK OBSERVATORY

UNIVERSITY OF CALIFORNIA

NTL SCIENCES II

SANTA CRUZ CA 95064

USA

TEL: $408 \quad 429 \quad 2130$

TLF : $408 \quad 426 \quad 3115$

TLX: 9109971741 UNICAL

EML: KOOHELIOS.UCSC. EOU

COM: 28,47

\section{KOPP ROGER A DR}

LOS ALAMOS NATIONAL LAB

MS E531.

80X 1663

LOS ALAMOS NM 87545

USA

TEL: $5015 \quad 6674398$

TLF :

TLX: 660495 LOS ALAMOS

EML:

COM:
KOLLBERG ERIK L PROF

OPT OF PHYSICS

CHALMERS TECHNICAL UNIV

S 41296 GOETEBORG

SWEDEN

TEL: 31810100

TLF:

TLX: 2400 ONSPACE

EML:

COM:

KONDO YOJI DR

NASA/GSFC

CODE 684

GREENBELT MD 20771

USA

TEL: $301286 \quad 6247$

TLF : 302867642

$T 1 X$ :

EML: JUE: : KONDO

COM: $34,36,42 P, 44$

KONOPLEVA VARVARA P OR

MAIN ASTRONOMICAL OBS

UKRAINIAN ACAD OF SCIENCE

GOLOSEEVO

252127 KIEV

UKRAINE

IEL: 663110

TLF:

TLX: 131406 SKY SU

EML:

COM: 15

KOORNNEEF JAN OR

STSCI

, HOMEWOOD CAMPUS

3700 SAN MARTIN OR

BALTIMORE MD 21218

USA

TEL: 3013384802

TLF:

TLX: 6849101

EML:

COM: 34

KOPYLOV I M OR PULKOVO OBSERVATORY ACADEMY OF SCIENEES 10 KUTUZOV QUAY 196140 ST PETERSBURG RUSSIA

TEL: $928 \quad 22 \quad 42$

TLF:

TLX:

EML:

COM: 09,27 


\section{A166}

KORCHAK A A OR
IZMIRAN
ACADEMY OF SCIENCES
142092 TROITSK
RUSSIA
IEL:
TLF:
TLX:
EML:
COM:

KOSHIBA MASA-TOSHI DR TOKAI UNIVERSITY

2-28 TOMIGAYA

SHIBUYA

TOKYO 151

JAPAN

TEL: 0334672211 EXT 483

TLF: 034854958

TLX:

EML:

COM: 48
KORMENOY JOHN DR INSTITUTE FOR ASTRONOMY UNIVERSITY OF HAHAII 2680 WOODLAUN OR HONOLULU H1 96822 USA

TEL: 8089566680

TLF: 8089882790

TLX: 7238459

EML: KORMENT+DYUHIFA, IFA, HAWAII, EO EML COM: $28,33,47$

KOSIN GENNADIJ S OR PULKOVO OBSERVATORY ACADEMY OF SCIENCES 10 KUTUZOV QUAY 196140 ST PETERSBURG RUSSIA

TEL:

TLF:

TLX:

EML:

COM: 08

KOSTYAKOVA ELENA B DR STERNBERG STATE ASTR INST 117234 MOSCOW RUSSIA.

TEL:

TLF: ,

EML:

COM: 34

ILX:

EML:

COM:

KOTANYI CHRISTOPHE OR

NEPAE

UFSM

CIDADO UNIVERSITARIA

97100 SANTA MARIA RS

BRAZIL

TEL: $55226 \quad 1616$

TLF

TLX: 0552230 UFSM BR

EML:

COM:

KOTRC PAVEL

ASTRONOMICAL INSTITUTE

CZECH ACADEMY OF SCIENCES

ONOREJOY OBSERVATORY

CS 25A 65 ONOREJOV

CZECHOSLOVAKIA

TEL: 20485201

TLF: 20485314

TLX: 121579

EML:

COM: 10,12
KOROVYAKOVSKIJ YURIJ P DR SPECIAL ASTROPHYSICAL OBS ACADEMY OF SCIENCES

NIZHNIU ARKHYZ

357247 STAVROPOLSKIJ

RUSSIA

TEL:

TLF:

TLX:

COM: 09

KOSOVICHEV ALEXANOER

INSTITUTE OF ASTRONOMY

THE OBSERVATORIES

MADINGLEY RO

CAMBRIDGE CB3 OHA

UK

TEL: $223337548^{\star} 7516$

TLF: 223337523

TLX: 817297 ASTRON G

EML: agkuk, ac, cam,ast-star

COM: 35

KOSTYLEV K VR

ENGERHAROT ASTRONOMICAL

DBSERVATORY

OBSERVATORIA STATION

422526 KAZAN

RUSSIA

TEL:

ILF:

TLX:

EML:

COM: 22

KOTHARI D S DR

DPT OF PHYSICS UNIVERSITY OF DELHI NEW OELLI 110007 INDIA

TEL: 112918993

ILF:

ILX:

EML:

COM: 35

KOUPELIS THEODOROS OR

DPT PHYSICS \& ASTRON UNIVERSITY OF ROCHESTER ROCHESTER NY 14627

USA

TEL: $716 \quad 2754389$

TLF: $716 \quad 2758527$

TLX:

EML: TKOUEUOROBV, BITNET

COM: 48
KOSAI HIROKI

TOKYO ASTRDNOMICAL OBS NAOJ

OSAWA MITAKA

TOKYO IBI

JAPAN

TEL: 0422413638

TLF: 0422413698

TLX:

EML:

COM: 20

KOSTIK ROMAN. I

MAIN ASTRONOMICAL OBS UKRAINIAN ACAD OF SCIENCE GOLOSEEVO

$252127 \mathrm{KIEV}$

UKRAINE

TEL: 664762

ILF:

TLK: 131406 SKY SU

EML:

COM: 10,12

KOSUGI TAKEO

TQKYO ASTRONOMICAL OBS

NAOJ

OSAWA MITAKA

TOKYO 181

JAPAN

TEL:

TLF :

TLX:

EML:

COM: io

KOTOV VALERY OR

CRIMEAN ASTROPHYS OBS UKRAINIAN ACAD OF SCIENCE NAUCHNY

334413 CR IMEA

UKRAINE

TEL: 432945

TLF:

TLX:

EML:

COM: 12

KOURGANOFF YLADIMIR PROF 20 AVE PAUL APELL

F 75014 PARIS

FRANCE

TEL: 145405053

TLF:

TLX:

EML:

COM: $\cdot 46$ 
KQUTCHMY SERGE DR INSTITUT D'ASTROPHYSIQUE $98 B 1 S$ BD ARAGO

F 75014 PARIS

FRANCE

TEL: $1 \quad 43 \quad 201425$

TLF: 143298673

TLX:

EML

COM: $10,12,21$

KOVACS GEZA DR KONKOLY OBSERVATORY THEGE U 13/17

$80 \times 67$

H 1525 BUDAPEST

HUNGARY

TEL: $175 \quad 5866 / 754122$

TLF :

TLX: 227460

EML:

COM:

KDVAR ROBERT P OR

966G E ORCHARO OR

ENGLEWODO CO 80111

USA.

TEL: 3033944494

TL.F :

EML:

EOM:

TLX:

KOYAMA KO-U-ICH I OR

RES INST SCIENCE \& TECH

KINKI UNIVERSITY

HIGASHI

DSAKA 577

JAPAN

TEL: $06 \quad 7212332$ EXT 4711

TLF: 067212353

TLX

EML:

COM: 35

KOZIEL KAROL PROF DR

ASTRON OBSERVATORY KRAKOW

UL 22 LIPCA 16

PL 43460 WISLA

POLAND

TEL: $32-42$

TLX:

EML:

TLF :

COM:
KOUVELIOTOU CHRYSSA OR

NASA/MSFT

COOE ES 62

HUNTSVILLE AL 35812

USA

TEL: 2055447711

TLF : 2055445800

TLX:

EML: BATSE::KOUVELIOTOU SSL::KOUVEL TLX: 23561 ECF BAN BG COM:

KOVAL I K OR

MAIN ASTRONOMICAL OBS

UKRAINIAN ACAD OE SCIENCE

GOLOSEEVO

252127 KIEV

UKRAINE

TEL: 660869

TLF:

$T L X:$

EML:

COM:

KOVETZ ATTAY PROF

DPT OF PHYSICS \& ASTRON

TEL AVIV UNIVERSITY

RAMAT AVIV

TEL AVIV 69978

ISRAEL

TEL: 3420234

ILF

TLX: $342-171$ VERSY IL

EML:

COM: 35,47

KOYAMA SHIN PROF DR
FAC OF EOUCATION
KAGAWA UNIVERSITY
SAIWAI CHO TAKAMATSUSHI
KAGAWA 760
JAPAN
TEL: $878-61-4141$
TLF:
TLX:
EML:
COM: 12

KOZLOVSKY B Z OR

DPT OF PHYSICS \& ASTRON

TEL AVIV UNIVERSITY

RAMAT AVIV

TEL AVIV 69978

ISRAEL

TEL:

TLF:

TLX:

EML:

COM: A7
KOVACHEV B J OR

DPT OF ASTRONOMY

BULGARIAN ACAD SCIENCES

72 LENIN BLVD

BG 1784 SDFIA

BULGARIA

TEL: 2758827

TEL:

EML

COM: 09,29

KOVALEVSKY JEAN QR

OCA CERGA

AVE COPERNIC

F 06130 GRASSE

FRANCE

TEL: $93 \quad 36 \quad 5849$

TLF:

FLX: 470865 CERGA F

EML:

COM: $07,08 C, 24,31 C, 50 C$

KOWAL CHARLES THOMAS

STSCI

HOMEWOOD CAMPUS

3700 SAN MARTIN OR

BALTIMORE MO 21218

USA

TEL:

TLF :

TLX:

EML:

COM: $15,26,20$

KOZAI YOSHIHIDE PROF

TOKYO ASTRONOMICAL OBS

NAOJ

osawa MITAKa

TOKYO 181

JAPAN

TEL: 422413650

TLF: $422 \quad 413690$

TLX: 2822307 TAOMTK

EML:

COM: $06,07,20,38,50$, EC

KOZLOWSKI MACIEJ OR

COPERNICUS ASTRON CENTER

POLISH ACAO OF SCIENCES

UL BARTYCKA 18

PL 00716 WARSAW

POLAND

TEL: $41 \quad 1086$

$T L F$

$T L X: 813978$ 2APAN PL

EML:

COM: 35,48
ROVACS AGNES DR

HELIOPHYSICAL OBSERVATORY

BOX 30

H 4010 DEBRECEN

HUNGARY

TEL: $52 \quad 11015$

ILF :

TLX: 72517 DEOBS H

EML:

COM: 10

KOVAR N S DR

OPT OF PHYSICS

UNIVERSITY OF HOUSTON

HOUSTON TX 77004

USA

TEL:

TLF:

TLX:

EML:

COM:

KOYAMA KATSUJI

INST SPACE \& ASTRON SCT UNIVERSITY OF TOKYO

MEGURO KU

TOKYO 153

JAPAN

TEL: $3-467-1111$

TLF:

TLX: 34757 ISASTRO J

EML:

COM: 48

KOZASA TAKASHI

MAX PLANCK INSTITUT FOR

KERNPHYSIK

PO BOX 103980

D 6900 HEIOELBERG L

GERMANY

TEL: $62 \quad 215 \quad 16271$

TLF: $52 \quad 215 \quad 16540$

TLX:

EML: KOZASAEOHOMPI5

COM: 34

KRAAN-KORTEWEG RENEE C DR

KAPTEYN ASTRONOMICAL INST

$B 0 \times 800$

NL 9700 AV GRONINGEN

NETHERLANDS

TEL: $50 \quad 634045$

ILF: 50636100

TLX:

EML: KRAAN@RUG.NL

COM: 28 


KRAEMER GERHARD OR'
ASTRONOMISCHES INSTITUT
OER UNIVERSITAET
PHILOSOPHENWEG 37
D 7400 TUEBINGEN
GERMANY
TEL:
TLF:
TLX:
EML:
COM: 12,44
KRANUC ALOO DR
OSS ASTRONOMICO DI BRERA
VIA BRERA 26
I 20121 MILANO
ITALY
TEL: 2874444
TLX:
EML:
TLF: 272001600
COM:

KRAUS JOHN D PROF

RADIO OBSERVATORY DHIO STATE UNIVERSITY 2015 NEIL AVE COLUMBUS OH 43210 USA

TEL: 6145487895

TLF :

TLX:

EML:

COM: 40,51
KREMPEC-KRYGIER JANINA DR INSTITUTE OF ASTRONOMY N COPERNICUS UNIVERSITY UL CHOPINA $12 / 18$

PL B7 100 TORUW PGLANO

TEL: $260-18$

TLF:

TLX: 0552234 ASTR PL

EML:

COM: 29

\author{
KRAFT ROBERT P PROF \\ LICK OBSERVATORY \\ UNIVERSITY OF CALIFORNIA \\ SANTA CRUZ CA 95064 \\ USA \\ TEL: 408429299 ] \\ TLF: \\ TLX: 9109971741 \\ EML: \\ COM: $27,29,30,37,42$
}

KRASINSKI ANORZEJ PROF COPERNICUS ASTRON CENTER POLISH ACAD OF SCIENCES UL BARTYCKA 18

PL DO 716 WARSAW

POLANO

TEL: 410828

TLF:

TLX: 813978

EML:

COM: 47

KRAUSE F OR.

ZNTRLINST F ASTROPHYSIK

ASTROPHYSIKALISCHES OBS

TELEGRAFENBERG

D 1500 potSDAM

GERMANY

TEL:

- TLF:

TLK:

EML: ELSTNERQVAX. HMI. CBP. DE

COM: 10

KREINER JERZY MAREK OR
INSTITUTE OF PHYSICS
PEDAGOGICAL UNIVERSITY
UL POOCHORAZYCH 2
PL 30 O84 KRAKOW
POLANO
TEL: 12378286
TLF: 123722243
TLX: 322444 WSP PL
EML:
COM: $27,42,46$
KRESAK LUBOR DR
ASTRONOMICAL INSTITUTE,
SLOVAK ACADEMY SCIENCES
DUBRAVSKA 9
CS 942 28 8RATISLAVA
CZECHOSLOVAKIA
TEL: 737 5157
TLF:
TLX: 93373 SEIS
EML:
COM: $15 C, 20 C, 22$

\author{
RRAICHEVA ZORAVSKA OR \\ DPT OF ASTRONOMY \\ bulgarian acad sciences \\ TTH NOVEMBER ST 1 \\ BG 1000 SOFIA \\ bulgar ia \\ TEL: 27341 \\ TLF: 23561 ECFBAN B6 \\ TLX: \\ EML: \\ COM: 42
}

KRASINSKY GEORGE A OR INST OF APPLIED ASTRONOMY ACADEMY OF SCIENCES ZOANOVSKAYA UL $B$ 197042 ST PETERSBURG RUSSIA

TEL:

$T L F$ :

TLX:

EML:

COM: 04,07

KRAUSHAAR WILLIAM L PROF DPT OF PHYSICS UNIVERSITY OF WISCONSIN 1150 UNIVERSITY AVE MAOISON WI 53706 USA TEL: 6082625916

ILF:

TL.X: 265452

EML:

COM: 44

\section{KREISEL E PROF}

EINSTEIN LABORATORIUM ROSA-LUXEMBURG-STR $17 \mathrm{~A}$ O 1502 POISOAM

GERMANY

TEL: 762225

TLF:

TLX: 15471

EML:

COM: 48

KRESAKOVA MARGITA DR

ASTRONOMICAL INSTITUTE. SLOVAK ACADEMY SCIENCES DUBRAVSKA 9

CS 84228 BRATISLAVA

CZECHOSLOVAKIA

TEL: 7375157

TLF:

TLX: 93373 SEIS

EML:

COM: 15,22
KRAMER KH N OR ASTRONOMICAL OBSERVATORY ODESSA STATE UNIVERSITY SHEVCHENKO PARK 270014 ODESSA UKRAINE TEL: 220396 TLF: TLX:

EML:

COM: 22

KRASSOUSKY V I OR INST PHYSICS OF ATMOSPH ACADEMY OF SCIENCES PYSHEVSKY PER 3 109017 MOSCOW RUSSIA

TEL: 231-88-62 ILF:

TLX:

EML:

COM:

KRALTTER JOACHIM OR LANDESSTERNWARTE KOENIGSTUHL

D G900 HEIDELBERG I GERMANY

TEL: 622110036 TLF:

ILX: 461789 MPIA D

EML:

COM: $27,34,42$

KRELOWSKI JACEK OR INSTITUTE OF ASTRONDMY N COPERNICUS UNIVERSITY UL CHOPINA $12 / 18$ PL 87100 TORUN POLAND TEL: $856-206-55$

TLF :

TLX: 055-2234 ASTR PL

EML:

COM:

KREYSA ERNST

MPI FUER RADIOASTRONOMIE

AUF DEM HUEGEL 69

D 5300 BOWN:

GERMANY

TEL: 228525269

TLF;

TLX: 886440

E.ML:

COM: 34,40 


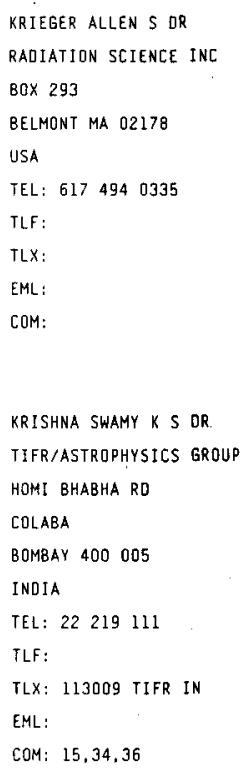

KRIKORIAN RALPH DR

INSTITUT 'D'ASTROPHYSIQUE

98BIS BD ARAGO

F 75014 PARIS

FRANCE

TEL: $143 \quad 201425$

TLF: 143298673

TLX:

EML:

COM: 36
KRISCIUNAS KEVIN OR JOINT ASTRONOMY CENTER 665 KOMOHANA ST

HILO HI 96720

USA

TEL: $808 \quad 9354332$

TLF:

TLX:

EML: KEVINOJACH. HAWAII I EOU COM: 27

KRISHNAN THIRUVENKATA MR HELIOS ANTENNAS/ELECTRON 234 AVVAI SHANMUGHAM RO GOPALAPURAM

MADRAS 600086

INDIA

TEL: $44 \quad 472 \quad 680$

TLF :

TLX:

EML:

COM: 40

KRISTIAN JEROME OR MT WILSON \& LAS CAMPANAS OBSERVATORIES 813 SANTA BARBARA ST PASADENA CA 91101 USA

TEL: 8185771122

TLF:

TLX:

EML:

COM:

KROGDAHL W S DR DPT PHYSICS \& ASTRONOMY UNIVERSITY OF KENTUCXY LEXINGTON KY 40506 USA

TEL: $606 \quad 2722659$

TLF :

TLX:

EML:

COM:

KRON RICHARD G YERKES OBSERVATORY UNIVERSITY OF CHICAGO BOX 258 WILLIAMS BAY WI 53191 USA

TEL: $312 \quad 2365468$

TLF:

TLX:

ENL:

COM: 28
KRISHNA GOPAL

TATA INST OF FUNDAMENTAL RESEARCH

POONA UNIVERSITY CAMPUS PUNE 411007

INDIA

TEL: 212337107

TLF: 212335760

TLX: 0145658 GMRT IN

EML: unnet ! shakt i !gmrt!gk

COM: $28,38 \mathrm{C}, 40$

KRISS GERARD A OR

OPT PHYSICS \& ASTRONOMY

JOHNS HOPKINS UNIVERSITY

CHARLES \& $34 T H$ ST

BALTIMORE MD 21218

USA

TEL: 3013387679

TLF:

TLX: 9101300225 JHU CASMO EML:

COM: 47

KRISTIANSSON KRISTER PROF DPT OF PHYSICS UNIVERSITY OF LUNO SOELVEGATAN 14 S 22362 LUNO SWEDEN

TEL: $46 \quad 107726$

TLF :

TLX:

EML:

COM: 48

KROLIK JULIAN H OPT PHYSICS \& ASTRONOMY JOHNS HOPKINS UNIVERSITY CHARLES \& 34TH ST BALTIMORE MD 21218 USA

TEL: 3013387926 TLF:

$T L X:$

EML:

COM:

KRONBERG PHILIPP OR DPT OF ASTRONOMY UNIVERSITY OF TORONTO 60 ST GEORGE ST TORONTO ON MSS IAT CANADA

TEL: $416 \quad 9784971$ ILF: 4169783921 TLX: 06986766 EML:

COM: 40 


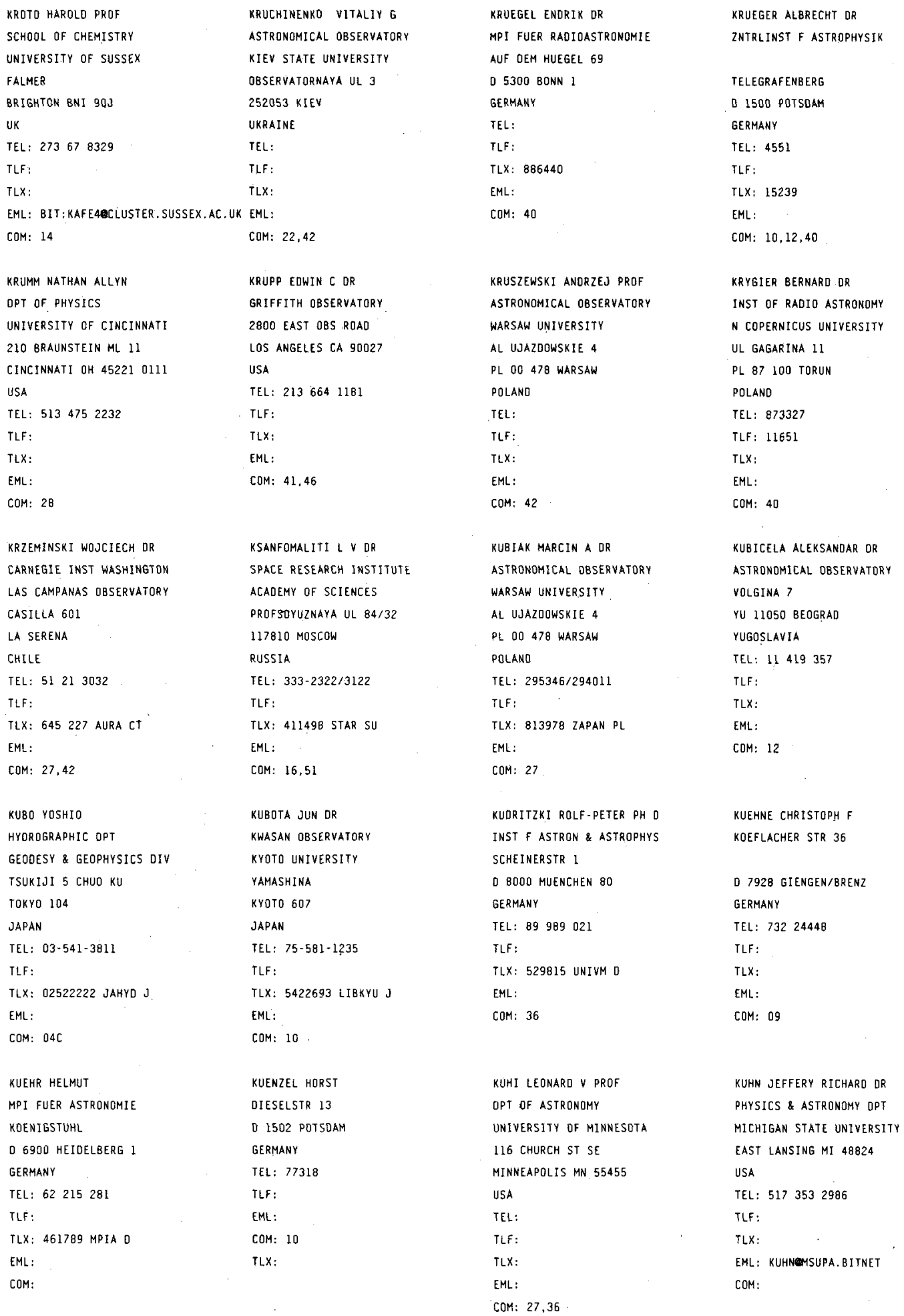


KUIJPERS H JAN ME OR STERREKUNDIG INSTITUTE BOX 80000

NL 3508 TA UTRECHT NETHERLANDS

TEL: 30535209

TLX: 40048 FYLUT NL

EML: KUIJPERS

TLF: 30531601

COM: 40

KULKARNI PRABHAKAR V PRÓ PHYSICAL RESEARCH LAB NAVRANGPURA

AHMEOABAD 380009

INDIA

TEL: $27246 \quad 2129$

TLF: 272445292

TLX: 8458488 TIFR IN

ENL:

COM: $09,21,25,40$

KULTIMA JOHANNES

GEOPHYSICAL OBSERVATORY

EISCAT

SF ggG00 SOKANKYLAE

FINLAND

TEL:

TLF:

TLX:

EML:

COM:

KUMAR SHIV S PROF

UNZVERSITY STATION

UNIVERSITY OF VIRGINIA

BOX 3818

CHARLOTTESVILLE VA 22903

USA

TEL: $804924 \quad 4896$

TLF

TLX:

EML

COM: $16,35,36$

KUN MARIA DR

KONKOLY OBSERVATORY

THEGE U $13 / 17$

BOX 67

H 1525 BUDAPEST

HUNGARY

TEL: $175 \quad 5866 / 754122$

TLF

TLX: 227460 KONOB H

EML:

COM: 37
KUIPER THOMAS B H DR

SPL

MS 1695065

4800 OAK GROVE OR

PASADENA CA 91109

USA

TEL: 8183545479

TLF :

TLX: 675429

EML:

COM: $34,40,51$

KULKARNI SHRINIVAS R OR

CALTECH

MS 10524

PASADENA CA 91125

USA

TEL: 8183564010

TLF:

$T L X: 188192675425$

EML

COM: 40

KUMAJGORODSKAYA RAISA OR

SPECIAL ASTROPHYSICAL OBS

ACADEMY OF SCIENCES

NIZHNIJ ARKHYZ

357147 STAVROPOLSKIJ

RUSSIA

TEL: 93-515

TLF:

TLX:

EML:

COM:

KUMKOVA IRINA I QR

INST OF APPLIED ASTRONOMY

ACADEMY OF SCIENCES

ZDANOVSKAYA UL 8

197042 ST PETERSBURG

RUSSIA

TEL:

TLF :

TLX:

EML:

COM: 24

\section{KUNCHEY PETER DR}

OPT OF ASTRONOMY

UNIVERSITY OF SOFIA

ANTON IVANOV ST 5

BG 1126 SOFIA

BULGARIA

TEL: 2544852

TLF:

TLX:

EML:

COM: 28
KUKLIN G V OR

SIBIZMIR

ACADEMY OF SCIENCES

664697 IRKU'SK 33

RUSSIA

TEL: $6-02-65$

TLF:

TLX:

EML:

COM: 10,12

KULKARNI VASANT $K$ OR

TATA INST OF FUNDAMENTAL

RESEARCH

POONA UNIVERSITY CAMPUS

PUNE 411007

INDIA

TEL: $212 \quad 337107$

TLF: 212335760

TLX: 0145658 GMRT IN

EML: uunet!shokt)!gmrt!vasant

COM: 40

KUMAR C KRISHNA QR

OPT PHYSICS \& ASTRONOMY

HOWARD UNIVERSITY

WASHINGTON OC 20059

USA

TEL: : $202 \quad 636 \quad 6245$

TLF:

$T L X:$

EML:

COM: 34

KUMSIASHVILY MZIA I DR

ABASTUMANI ASTROPHYSICAL

OBSERVATORY

GEORGIAN ACAD OF SCIENCES

383762 ABASTUMANI

GEORGIA

TEL: 252

TLF :

TLX: 327409

EML:

COM: 42

KUNDT WOLFGANG PROF OR

INSTITUT F ASTROPHYSIK

\& extraterr forschung

AUF DEM HUEGEL 71

D 5300 BONN I

GERMANY

TEL: $228 \quad 26 \quad 7400$

TLF:

$T L X: 888440$

EML:

COM: 40,48
KULCAR LADISLAV DR

PEDAGOGICKA FAKULTA

KATEORA FYZIXY

TAJOVSKEHO 40

CS 97549 BANSKA BYSTRICE

CZECHOSLOVAKIA

TEL: 8834553

TLF: $88 \quad 3 \quad 3132$

$T L X$ :

EL:

COM: 12

KULSRUD RUSSELL M DR

OPT ASTROPHYSICAL SEI

PRINCETON UNIVERSITY

PRINCETON NJ OB544 1001

USA

TEL: 6096832613

TLF:

TLX:

EML:

COM: 33,48

KUMAR SHAILENORA

4 CLOVERLEAF ORIVE

MARLBORO NJ 07746

USA

TEL:

TLF :

TLX:

EML:

COM:

KUMSISHVILI J I OR

ABASTUMANI ASTROPHYSICAL. OBSERVATORY

GEORGIAN ACAD OF SCIENCES

383762 ABASTUMANI

GEORGIA

TEL: '279

TLF:

TLX: 327409

EML:

COM: 26,27

KUNDU MUKUL R DR

ASTRONOMY PROGRAM.

UNIVERSITY OF MARYLAND

COLLEGE PARK MO 20742

USA

TEL: 3014543005

TLF :

TLX: 710-826-0352

EML:

COM: $10,12,34,40$ 
KUNIEOA HIOEYO DR

OPT OF ASTROPHYSICS

NAGOYA UNIVERSITY

FUROCHO CHIKUSA KU

NAGOYA 464

JAPAN

TEL: 527815111

TLF: 52781354

TLX: 4477323 SCUNAG $J$

EML:

COM:

KUNZE RUEOIGER DR

INST THEOR PHYSIK STERN

DER UNIVERSITAET KIEL

OLSHAUSENSTR 40

D $2300 \mathrm{KIEL} 1$

GERMANY

TEL: 4318801575

TLF: 4318804432

TLX: 292706

EML: PAS29@RZ.UNI-KIEL. OBP.DE

COM: 34

KUROCHKA L N OR
ASTRONOMICAL OBSERVATORY
KIEV STATE UNIVERSITY
OBSERVATORNAYA UL 3
252053 KIEV
UKRAINE
TEL: 26 2691
TLF:
TLX:
EML:
COM: 10,12
KLSTZ, OONALD WAYNE OR
DPT OF ASTRONOMY
UNIVERSIYY DF CAPE TOWN
RONDEBOSCH 7700
SOUTH AFRICA
TEL: 69 853L
TLF:
TLX: 521439
EML:
COM: $25,27 C$ 45

KUSHWAHA R S PROF

DPT OF MATHEMATICS

UNIVERSITY OF JOOHFUR

JOOHPUR

INDIA

TEL:

TLF :

TLX:

EML

COM: 35,36
KUNITZSCH PAUL PROF

INST. FOR SEMITIC STUDIES

UNIVERSITY OF MUNICH

VETERINAERSTRASSE ।

D 8000 MUENCHEN 22

GERMANY

TEL: $892180 * 2352$

TLF :

TLX: 916280

EML:

COM: 41

KUPERUS MAX PROF OR

STERREKUNDIG INSTITUTE

B0X 80000

NL 3508 TA UTRECHT

NETHERLANOS

TEL: 30535212

TLX: 40048 FYLUT NL

EML: BITNET: WNMMAI LOHUTRUUD

TLF :

COM: 10,12

KUROKAWA HIROKI OR

HIQA OBSERVATORY

UNIVERSITY OF KYOTO

KAMI FAKARA

GIFU $506 \quad 13$

JAPAN

TEL: $0578-6-2628$

TLF:

TLX:

EML:

COM: 10

KURUCZ ROBERT L. OR

CENTER FOR ASTROPHYSICS

HCD/SAO

60 GARDEN ST

CAMBRIDGE MA 02138

USA

TEL: 6174957429

TLF:

ILX: 921428

EML: Kuruczecta.harvard.edu

COM: 36

KUSTAANHEIMO PAUL E FROF

OANMARKS TEKN HOJSKOLE

LUNDTOFTEVEJ ?

OK 2800 LYNGBY

DENMARK

TEL: $42 \quad 88 \quad 3022$

TLF :

$T L X:$

EML:

COM: $07,28.47$
KUNKEL WILLIAM E DR

CARNEGIE INST WASHINGTON

LAS CAMPANAS OBSERVATORY

CASILLA 601

LA SERENA

CHILE

TEL: 51213032

TLF :

TLX: 645227 AURA CT

EML:

COM: 25,27

RURFESS JAMES O

NAYAL RESEARCH LABORATORY

CODE 4150

4555 OVERLOOK AVE SW

WASHINGTON OC 203755000

USA

TEL: 2027673182

TLF:

TLX:

EML:

COM:

KURPINSKA-WINIARSKA M DR

ASTRONOMICAL OBSERVATORY

JAGIELLONIAN UNIVERSITY

UL ORLA $17 L$

PL 30244 KRAKOW

POLAND

TEL:

TLF:

TLX: 0322297 UJ PL

EML:

COM: 42

KURZYNSKA KRYSTYNA OR

ASTRONOMICAL OBSERVATORY

A MICKIEWICZ UNIVERSITY

UL SLONECZNA 36

PL 60286 POZNAN

POLAND

TEL: 61679670

TLF: 61535535

ILX: 413260 UAMPL

EML: KURZASTR@PLPUAMM

COM: 08

KUSUNOSE MASAAKI OR

COPERNICUS ASTRON CENTER

POLISH ACAD OF SCIENCES

UL BARTYCKA 19

PL DO 716 WARSAW

POLAND

TEL: 22411086

ILF: $22 \quad 410046$

TLX: 813978 ZAPLAN

EML: KUSUNOSECAMK.EDU.PI

COM: 48
KUNTH OANIEL

INSTITUT D'ASTROPHYSIQUE

9BBIS BD ARAGD

F 75014 PARIS

FRANCE

TEL: 143201425

ILF: 143298673

TLX:

EML :

COM: $28,34,47$

KURIL-CHIK V N DR

STERNBERG STATE ASTR INST

UNIVERSITETSKIJ PROSP 13

119899 MOSCOW

RUSSIA

TEL: $139-10-30$

TLF :

TLX:

EML:

COM: 40

KURT V G OR

SPACE RESEARCH INSTITUTE

ACADEMY OF SCIENCES

PRDFSOYUZNAYA UL 84/32

117810 MOSCOW

RUSSIA

TEL: $333-31-22$

TLF :

TLX: 411498 STAR SU

EML:

COM: $16,44,48$

KUS ANDRZEJ JAN DR

INST OF RADIOASTRONOMY

$N$ COPERNICUS UNIVERSITY

UL CHOPINA L2/1B

PL 87 100 TORUN

POLANO

TEL: 04856-2065L

TLF:

TLX: 0552324 TRAO PL

EML:

COM: 40

KUTNER MARC LESLIE DR

DPT OF PHYSICS

RENSSELAER POLYTECHN INST

TROY NY 121803590

USA

YEL: $518 \quad 265 \quad 6417$

ILF :

TLX:

EML:

COM: 34,40 


KUTTER G SIEGFRIEO OR
NASA/GSFC
COBE 681
GREENBELT MO 20771
USA
TEL:
TLF:
TLX:
EML:
COM:

KVIZ ZOENEK OR

SCHOOL OF PHYSICS

UNIVERSITY OF SOUTH WALES

BOX 1

KENSINGTON NSW 2033

AUSTRALIA

TEL: 26974578

TLF:

TLX: 26054 AA

EML:

COM: $22,25,42$

KYLAFIS NIKOLAOS O OR

DPT OF PHYSICS

UNIVERSITY OF. CRETE

BOX 1527

GR 71111 IRAKLION

GREECE

TEL: 81239757

TLF:

TLX:

EML:

COM: 34

LABAY JAVIER
OPT FISICA OE ATMOSFERA
UNIVERSIDAD DE BARCELONA
AVD DIAGONAL 645
E O8028 BARCELONA
SPAIN
TEL: 33307311
TLF:
TLX:
EML:
COM: 35

LABS DIETRICH PROF
LANOESSTERNWARTE
XOENIGSTUHL
O 6900 HEIDELBERG 1
GERMANY
TEL: 622110036
TLF:
TLX: 461153 LSWHO O
EML:
COM: 12

KUTUZOV S A DR

ASTRONOMICAL OBSERVATORY

ST PETERSBLRG UNIVERSITY

BIBLIOTECHNAJA PL 2

199164 ST PETERSBURG

RUSSIA

TEL:

TLF :

TLX:

EML:

COM: 33

\section{KWEE K K OR \\ STERREHACHT \\ BOX 9513 \\ NL 2300 RA LEIDEN \\ NETHERLANOS}

TEL: 71272727

TLX: 39058 ASTRO NL

EML:

TLF:

COM: 27,42

LA BONTE BARRY JAMES

INSTITUTE FOR ASTRONOMY

UNIVERSITY OF HAWAII

2680 WOOBLAWN OR

HONOLULU HI 96822

USA

TEL: 8089486531

ILF:

TLX: 723-8459 UHAST HR

EML:

COM: 12

LABEYRIE ANTOINE DR
OCA CERGA
AVE COPERNIC
F 06130 GRASSE
FRANCE
IEL: 93,365849
ILF:
TLX: 461402
EML:
COM: 09

LACEY CEDRIC OR
OPT OF ASTROPHYSICS
UNIVERSITY OF OXFORO
KEBLE RD
OXFORD OXI JRH
UK
TEL: 865273352
TLF: 865273418
TLX: 83295 NUCLOX G
EML: $19464:$ : CGL (SPAN)
COM: 47

KUZMANOSKI MIKE INSTITUTE OF ASTRONOMY UNIVERSITY OF BELGRADE STUDENTSKI TRG 16 YU 11000 BEOGRAD YUGOSLAVIA

YEL: 11638715

TLF:

TLX:

EML:

COM:

KWITTER KAREN BETH DR DPT OF PHYS \& ASTRONOMY THOMPSON PHYSICS LAB WILLIAMS COLLEGE WILLIAMSTOWN MA 01267 USA

TEL: 4135972272

TLF:

TLX:

EML:

COM: 34

LA DOUS CONSTANzE A DR. NASA/GSFC

CODE 630.2

GREENBELT MO 20771

USA

TEL: 3012869793

TLF:

TLX: 817297 ASTRON $G$

EML: NSSDCA: : LADOUS

COM: 42

LABEYRIE JACQUES OR

CEA CEN

CFR

BP 2

F 91191. GIF/YVETTE CDX FRANCE

TEL: $1 \quad 69677828$

TLF:

TLX: 691137

EML:

COM:

LACHIEZE-REY MARC

CEA CEN

DAPNIA/SAP

$B P 2$

F 91191 GIF/YVETTE CDX FRANCE

TEL: 169086292

TLF:

TLX: 690860

EML:

COM: 47
KUZMIN ARKADII D PROF DR LEBEOEY PHYSICAL INST ACADEMY OF SCIENCES LENINSKY PRDSPERT 53 117924 MOSCOW RUSSIA

TEL:

TLF:

TLX: 411479 NEOD SU

EML:

COM: $16,40,51$

KWOK SUN OR

DPT OF PHYSICS

UNIVERSITY OF CALGARY

2500 UNIVERSITY DR NW

CALGARY AB T2N IN4

CANADA

TEL: 4032845414

TLF: $403 \quad 2893331$

TLX: 03821545

EME:

COM: 29.34 .35 .40

LA PAQULA CESARE

IAS

CNR

CP 67

I 00044 FRASCATI

ITALY

TEL: 69425655

TLF:

TLX:

EML:

COM:

LABHARDT LUKAS

ASTRONOMISCHES INSTITUT UNIVERSITAET BASEL VENUSSTRASSE 7

CH 4102 BINNINGEN SWITZERLANO TEL: 61227711 TLF:

TLX:

$$
\text { EML: }
$$$$
\text { COM: } 25,45
$$

\section{LACLARE F MR}

\section{OCA CERGA}

AVE COPERNIC

F 06130 GRASSE

FRANCE

TEL: 93365849

TLF:

TLX:

EML:

COM: 08 


LACROUTE PIERRE A PROF
2 RUE O'ALISE
F 21000 OIJON
FRANCE.
TEL: 80661154
TLF:
EML:
COM: 08,24
TLK:

LAFFINEUR MARIUS MR

21 BD BRUNE

F 74014 PARIS

FRANCE

TEL

TLF :

EML:

COM

TLX:

LAGO MARIA TERESA V
GRUPO DE MATEM APLICAOA
UNIVERSIDADE DO PORTO
RUA DAS TAIPAS I35
P 4000 PORTO
PORTUGAL
TEL: 380313
TLF:
TLX: 28109
EML:
COM: $27,29,46$
LAI SEBASTIANA
ISTITUTO OI ASTRONOMIA
VIA OSPEDALE 72
I 09I00 CAGLIARI
ITALY
TEL: 7066 3544
TLX:
EML:
TLF:
COM: 46,49

$\begin{array}{ll}\text { LAL DEVENDRA } & \text { LALA PETR OR } \\ \text { PHYSICAL RESEARCH LAB } & \text { OUTER SPACE AFFAIRS DIV } \\ \text { NAVRANGPURA } & \text { UNITEO NATIONS SECRETARIA } \\ \text { AHMEOABAD } 380009 & \text { NEW YORK NY } 10017 \\ \text { INDIA } & \text { USA } \\ \text { TEL: } 272.462129 & \text { TEL: } \\ \text { TLF: } 272445292 & \text { TLF: } \\ \text { TLX: } & \text { TLX: } \\ \text { EML: } & \text { EML: } \\ \text { COM: } & \text { COM: } 07\end{array}$

LAFON JEAN-PIERRE J DR OBSERVATOIRE DE PARIS SECTION DE MEUDON

F 92195 MEUDON PPL CDX FRANEE

TEL: 145077858

TLF :

TLX: 204464

EML:

COM: $28,33,34,49,51$

LAHAV OFER UR

INSTITUTE OF ASTRONOMY

MAOINGLEY RD

CAMBRIDGE CB3 OHA

UK.

TEL: 223337548

TLF: 223337523

TLX: 817297 ASTRON G

COM: 47

LAING ROBERT

ROYAL GREENWICH OBS

HERSTMONCEUX CASTLE

HAILSHAM BN2? IRP

$u K$

TEL: $323833 \quad 171$

ILX: 87451 RGOBS G

EML:

ILF:

COM: 40

$\begin{array}{ll}\text { LACY JOHN H DR } & \text { LADA CHARLES JOSEPH OR } \\ \text { ASTRONOMY OPT } & \text { STEWARO OBSERVATORY } \\ \text { UNIVERSITY OF TEXAS } & \text { UNIVERSITY OF ARIZONA } \\ \text { RLM } 15308 & \text { TUCSON AZ } 85721 \\ \text { AUSTIN TX } 787121083 & \text { USA } \\ \text { USA } & \text { TEL: } 6026214878 \\ \text { TEL: } 5124711469 & \text { TLF: } \\ \text { TLF: } & \text { TLX: } \\ \text { TLX: } & \text { EML: } \\ \text { EML: } & \text { COM: } 34,37,40\end{array}$

THE OBSERVATORIES

EML: OLLEAST-STAR.CAM.AC.UK

COM:

LAGERKYIST CLAES-INGVAR
ASTRONOMICAL OBSERVATORY
BOX 515
S 75120 UPPSALA
SWEDEN
TEL: 18113522
TLF:
TLX: 76024 UNIV UPS S
EML:
COM: 15,20

LAHIRI N C

INDIAN METEOROLOGIC OPT

P 546 BLOCK N

NEW ALIPORE

CALCUTTA 700053

INOIA

TEL:

TLF:

$T L X:$

EML:

COM: 04

AIRO JOHN B OR

DPT PHYSICS ASTRONOMY

BOWLING GREEN STATE UNIV

BOWLING GREEN OH 43403

USA

TEL: 4193727244

TLF:

ILX:

EML: LAIRDOANDY.BGSU.EOU

EOM: 29

LALLEMENT ROSINE OR

SERVICE D'AERONOMIE

BP 3

F 91371 -VERRIERES BUISSON

FRANCE

TEL: $1 \quad 64 \quad 47 \cdot 4235$

TLF:

TLX: 602400

EML: BITNET:ROSINEOFRIAPSI

COM:

\author{
LAGERQVIST ALBIN PROF \\ INST OF THEORETICAL PHYS \\ VANADISVAEGEN $g$ \\ S 11346 STDCKHOLM \\ SWEOEN \\ TEL: 8164500 \\ TLF : \\ TLX: 1.5433 FYSTOS \\ EML: \\ COM: 14 . \\ LAHULLA J FORNIES OR \\ OBS ASTRONOMICO NCL, \\ ALFONSO XII-3 \\ E 28014 MADRID \\ SPAEN \\ TEL: 12270107 \\ TLF: \\ TLX: 22465 IGC E \\ EML: \\ COM: \\ LAKE KAYLL WILLIAM OR \\ OPT OF PHYSICS \\ QUEEN'S UNIVERSITY \\ KINGSTON ON K $7 L$ 3NG \\ CANADA \\ TEL: 6135473020 \\ TLF: 6135456463 \\ TLX: \\ EML: \\ CON: 47
}

LAMB OONALO QUINCY JR DR UNIV OF CHICAGO PRESS UNIVERSITY OF CHICAGO 58015 ELLIS AVE CHICAGO IL 60637 USA

TEL: 312962 B203

TLF:

TLX: 269266

EML:

COM: $35,42,48$ 


LAMB FREQERICK K PROF
OPT OF PHYSICS
UNIVERSITY OF ILLINOIS
111O WREEN $5 T$
URBANA IL 61801
USA
TEL: 2173336363
TLF:
TLX: 6502272050 MCI
EML:
COM: 48
LAMBERT QAV10 L PROF
ASTRONOMY DPT
UNIVERSITY OF TEXAS
RLM 15308
AUSTIN TX 78712 1083
USA
TEL: 5124717438
TLF: 5124716016
TLX: 9108741351
EML: $0 L L A S T R O$. AS. UTEXAS. EDU
COM: $14,29 P, 36$

\section{LAMPENS PATRICIA OR}

OBSERVATOIRE ROYAL OE

BELgIQUe

AVE CIRCULAIRE 3

B IIBO BRUSSELS

BELGIUM

TEL: 23730263

TLF ; 23749822

TLX: 21565 OBSBEL

EML: PATRICIAEASTRO, OMA, BE

COM: 26

LANDE KENNETH PROF
GPT OF PHYSICS
UNIV OF PENNSYLVANIA
PHILADELPHIA PA 19104
USA
TEL: 2158988177
TLF:
TLX:
EML:
COM:

LANOI DEGL'INNOCENTI $M$

OSS ASTROFISICO

DI ARCETRI

LARGO E FERMI 5

I 50125 FIRENZE

ITALY

TEL: $55 \quad 2752256$

TLF:

TLX: 572268 ARCETR

EML:

cont 12
LAMB RICHARD C DR

DPT OF PHYSICS

IOWA STATE UNIVERSITY

AMES IA 50011

USA

TEL: $515 \quad 294 \quad 3873$

TLF:

TLX:

EML: BITNET: LAMBEALISUVAX

COM:

\section{LAMERS HENNY I G L M DR \\ SPACE RESEARCH LABORATORY \\ SRON}

SORBONNELAAN 2

NL 3584 CA UTRECHT

NETHERLANOS

TEL: 30535720

TLF: 30540860

TLX: 47224 SRON NL

EML: HENNYLE SRON.RUU.NL

COM: $29,36,44$

\section{LAMPTON MICHAEL}

SPACE SCIENCES LABORATORY

UNIVERSITY OF CALIFORNIA

BERKELEY CA 94720

USA

TEL: $415 \quad 642 \quad 3576$

$T L F$;

TLX: 9103667945

EML:

COM: 48

LANOECKER PETER BRUCE OR
HUGHES AIRCRAFT CO
SPACE \& COMM GR BLOG S41
MS B322 BOX 92919
LOS ANGELES CA 90009
USA
TEL: 2136480815
TLF:
TLX: 664480
EML:
COM:

LANOINI MASSIMO PROF

OSS ASTROFISICO

OI ARCETRI

LARGO E FERMI 5

I 50125 FIRENZE

ITALY

TEL: 552752247

TLF :

TLX:

EML:

COM:
LAMB SUSAN ANN DR

DPT OF PHYSICS

UNIVERSITY OF MISSOURI

8001 NATURAL BRIOGE RO

ST LOUIS MO 63121

USA

TEL:

TLF:

TLX:

EML:

COM: 35,48

\section{LAMLA ERICH E OR}

BRUESSELERSTR 9

D 5300 BONN 1

GERMANY

TEL:

ILF:

TLK:

EML:

COM:

LAMY PHILIPPE OR

LAS

TRAVERSE DU SIPHON

LES TROIS LUES

F 13012 MARSEILLE

FRANCE

TEL: 91055932

TLF: $9166 \quad 1855$

TLX: $420584 \mathrm{~F}$

EML:

COM: $15,21 C, 22$

LANDECKER THOMAS L:DR DOMINION RADIO ASTROPHYS OBSERVATORY

$B 0 \times 248$

PENTICTON BC V2A $6 K 3$

CANADA

TEL: 6044932277

TLF : 6044937767

TLX: 04888127

EML:

COM:

LANDI-DESSY J DR

OBSERVATORIO ASTRONOMICO

DE COROOBA

LAPRIDA 854

5000 CORDOBA

ARGENT INA

TEL: 5140613

TLF :

TLX:

EML:

COM:
LAMBECK KURT PROF

AUSTRALIAN NTL UNIVERSITY RES SCHOOL EARTH SCIENCE

BOX 4

CANBERRA ACT 2600

AUSTRALIA

IEL: $62 \quad 492 \quad 487$

TLF :

$T L X: 62693$

EML:

COM:

LAMONTAGNE ROBERT OR

DPT DE PHYSIQUEE

UNIVERSITY DE MONTREAL

CP 6128 SUCC A

MONTREAL QC H3C $3 J 7$

CANADA

TEL: $514 \quad 342 \quad 7273$

TLF: 5143432071

TLX: 05562425

EML: 5007@CC. UMONTREAL.CA

COM: 29

LANCASTER BROWN PETER

IOA ST PETER'S ROAD

ALOEBURGH SUFFOLK

UK

TEL:

TLF :

TLX:

EML:

COM: 15

LANDI DEGL'INNDCENTI E PR OPT DI ASTRONOMIA UNIVERSITA DI FIRENZE LARGO E FERMI 5

I 50125 FIRENZE

ITALY

TEL: $552752 \mathrm{i}$

ILF: 55220039

TLX: 572268 ARCETR I

EML:

COM: $12 C$

LANDMAN DONALD ALAN 525 CAMINO LAGUNA VISTA

GOLETA CA 93117

USA

TEL:

$T L F$ :

TLX:

EML:

COM: $10,12,14$ 
LANCOLFI MARCO

DSS ASTROFISICD

OI ARCETRI

LARGO E FERMI 5

150125 FIRENZE

ITALY

TEL: 552752256

ILF :

TLX: 572268 ARCETR

EML:

LOM: 12

LANE ARTHUR LONNE DR

JPL

4800 OAK GROVE DR

PASADENA CA 91109

USA

TEL: 818 3452725

TLX:

EML:

TLF :

COM: 15,26

LANGER GEORGE EOWARD DR

PHYSICS OPT

COLORADO COLLEgE

COLORADO SPRINGS CO 80903

USA

TEL: $303473 \quad 2233 * 578$

TLF :

TLX:

EML:

COM: 29

\section{LANTOS PIERRE OR}

GBSERVATOIRE DE PARIS

SECTION OE MEUDON

DASOP

F 92195 MEUDON PPL CDK

FRANCE

IEL: $145 \quad 077767$

TLF :

YLX:

EML:

COM: $05,10,12,40$

\section{LAPUSHKA K K OR}

ASTRONOMICAL DBSERVATORY LATVIAN STATE UNIVERSITY

RAINIS BUL 19

226098 RIGA

LATVIA

TEL: $223149 / 611984$

TLF :

TLX:

EML:

COM: 24
LANDOLT ARLO U PRQF

DPT PHYSICS \& ASTRONOMY

LOUISIANA STATE UNIV

BATON ROUGE LA 708034001

USA

TEL: $504 \quad 388 \quad 8276$

TLF:

TLX: 559184

EML:

COM: $25 \mathrm{C}, 27,37,42$

LANEY CLIFTON D DR
SAAO
BOX 9
OBSERVATORY 7935
SOUTH AFRICA
TEL: $470-025$
TLF:
TLX:
EML:
COM: 27

LANGER NORBERT DR

UNIVERSITAET STERNWARTE

GOETTINGEN

GEISHARLANDSTR LI

D 3400 gOETTINGEN

GERMANY

TEL: 551395054

TLF: 551395043

ILX: 96753

EML: NLANGERQDGOGWDGL. BITNET

COM: 35

\section{LANZ THIERRY OR}

NASA/GSFC

CODE 681

LASP

GREENBELT MO 2077 !

USA

TEL :

TLF :

TLX:

EML: LANZOSTARS.GSFC.NASA.GOV

COM: 29

\section{LAQUES PIERRE DR}

QBS MIDI PYRENEES

9 R PONT DE LA MOULETTE

F 65200 BAGNERES BIGQRRE

FRANCE

TEL: $62 \quad 95 \quad 1969$

TLF :

TLX: 531625

EML :

COM: 09.51
LANOSTREET JOHN O PRDF

DPT OF ASTRONOMY

UNIV OF WESTERN ONTARIO

LONOON ON NEA 3K7

CANADA

TEL: $519 \quad 679 \quad 3184$

TLF : 5196613486

TLX: 0647134

EML: QLANDSTRUNOVAX.BITNET

COM: $25 \mathrm{VP}, 29,36$

LANG JAMES DR

RUTHERFORO APPLETON LAB

SPACE \& ASTROPHYSICS DIV

BLDG R25/R68

CHILTON DIDCOT OX12 OQX

UK

TEL: 23521900

TLF :

TLX: 83159

EML:

COM: 14

LANGER WILLIAM DAVID DR

JPL

MS 169506

4800 OAK GROVE DR

PASADENA CA 91109

USA

TEL: $818 \quad 3545823$

TLF :

TLX:

EML:

COM: 34,40

LAPASSET EMILIO OR

OBSERVATORIO ASTRONOMICO

DE CORDOBA

LAPRIDA 854

5000 CORDOBA

ARGENTINA

TEL: 5136876

TLF :

TLX: 51822 BUCOR

EML:

COM: 37,42

LARGE MICHAEL I QR

SCHOOL OF PHYSICS

UNIVERSITY OF SYDNEY

SYONEY NSW 2006

AUSTRALIA

TEL: 26922222

ILF:

TLX: 26169 UNISYD AA

EML:

COM: 40
LANE ADAIR P

DPT OF ASTRONOMY

BOSTON UNIVERSITY

725 COMMONWEALTH AVE

BOSTON MA O2215

USA

TEL: 6173532633

TLF :

TLX:

EML:

COM:

LANG KENNETH R ASST PRDF

OPT OF PHYSICS

TUFTS UNIVERSITY

ROBINSON HALL

MEDFORD MA 02155

USA

TEL: 6173813390

TLF :

TLX:

EML:

COM: $10,40,41$

LANNES ANORE DR

OBS MIDI PYRENEES

14 AVE E BELIN

F 31400 TOULOUSE COX

FRANCE

TEL: $61 \quad 252101$

TLF :

TLX: $530776 \mathrm{~F}$

EML:

COM:

\section{APOINTE SM OR}

UNIVERSITE DU QUEBEC

2875 BLO LALIRIER

STE FOY OC GIV $2 M 3$

CANADA

TEL: $418 \quad 6573551$

TLF :

TLX: 05131623

EML:

COM:

LARI CARLO DR

IST DI RADIOASTRONOMIA

ENR

VIA IRNERIO 46

I 40126 BOLOGNA

ITALY

TEL: 51232856

ILF :

TLX:

ENL:

COM: 


LARSON HAROLD P DR
LUNAR \& PLANETARY LAB
UNIVERSITY OF ARIZONA
TUCSON AZ 85721
USA
TEL: 6026216943
TLF:
TLY:
EML:
COM: 15,16
LARSSON-LEANDER G PROF
LUNO OBSERVATORY
BOX 43
S 221 OO LUND
SWEOEN
TEL: 46 LO 7000
TLX: $331990 B S N O T S$
EML:
TLF:
COM: 37,42

LASKAR JACQUES DR

BUREAU OES LONGITUDES

77 AVE OENFERT ROCHEREAU

F 75014 PARIS

FRANCE

TEL: 140512274

TLF :

TLX:

EML:

COM: 04,07

LATHAM DAVID W DR

CENTER FOR ASTROPHYSICS

HCO/SAO

60 GAROEN ST

CAMBRIOGE MA 02138

USA

TEL: 6174957215

TLF :

TLX: 921428 SATELLITE CAM

EML: LATHAMECFA3

COM: $26,30 \mathrm{C}, 33,37$

\section{LATYPOV A A OR}

ASTRONOMICAL INSTITUTE

UZBEK ACADEMY OF SCIENCES

700052 TASHKENT

UZBEKISTAN

TEL: 358102

TLF :

TLX: 116012 VREMJA

EML:

COM: 24
LARSON RICHARO B PROF

DPT OF ASTRONOMY

YALE UNIVERSITY

BOX 6666

NEW HAVEN CT 06520

USA

TEL: 2034363015

TLF:

TLX:

EML :

COM: $28,33,35$

LASALA GERALO J DR

DPT OF PHYSICS

UNIV OF SOUTHERN MAINE

96 FALMOUTH ST

PORTLAND ME 04103

USA

TEL: 2077804557

TLF: 2077804933

TLX: *

EML: LASALAGPORTLAND

COM: 45

LASKARIOES PAUL 6 ASSPROF

DPT OF ASTRONOMY

NTL UNIVERSITY OF ATHENS

PANEPISTIMIOPOLIS

GR 15771 ZOGRAFOS

GREECE

TEL: 17243211

TLF:

TLX:

EML:

COM: $25,27,35$

LATQUR JEAN J

OBS MIDI PYRENEES

14 AVE E BELIN

F 31400 TOULOUSE COX

FRANCE

TEL: 61252101

TLF :

TLX:

EML:

COM: 35

LAUBERTS ANDRIS DR.

FOA 3

BOX 1165

$S 58111$ LINKOPING

SWEDEN

TEL: $13 \quad 11 \quad 8235$

TLF: $13 \quad 131665$

TLX: 50073 foatre $S$

EML:

COM: 28
LARSON STEPHEN $M$

LUNAR \& PLANETARY LAB

UNIVERSITY OF ARIZONA

TUCSON AZ 85721

USA

TEL: $602 \quad 6214973$

TLF:

TLX: $910-952-1143$

EML:

COM: 15,16

LASENBY ANTHONY

MULLARO RADIO ASTRON OBS

CAVENOISH LABORATORY

MAOINGLEY RD

CAMBRIOGE CB3 DHE

UK

TEL: $223 \quad 66477$

TLF :

TLX: B1292 CAVLAB G

EML:

COM: 40

LASKER BARRY M DR

STSCI

HOMEWOOD CAMPUS

3700 SAN MARTIN DR

BALTIMORE MD 21218

USA

TEL: 3013384840

TLF:

TLX: 684919L STSI

EML:

COM: $09,25,28,34$

LATTANZI MARIO G

OSS ASTRONOMICO OI TORINO

ST OSSERVATORIO 20

I 10025 PINO TORINESE

ITALY

TEL: $1184 \quad 1057$

ILF:

TLX: 213236 TO ASTR 1

EML: SPAN:391B1:: LATTANZI

COM: 08,26

LAUNAY JEAN-MICHEL DR

OBSERVATOIRE OE PARIS

SECTION DE MEUDON

F 92195 MEUDON PPL CDX

FRANCE

TEL: $145 \quad 07 \quad 7554$

TLF :

TLX: 201571

EML:

COM: 14
LARSSON STEFAN DR

STOCKHOLM OBSERVATORY

S 13336 SALTSJOEBADEN

SWEDEN

TEL: $81 \quad 64 \quad 464$

TLF: $87 \quad 174719$

TEX:

EML: LARSSONOASTRO.SU.SE

COM: 42

LASHER GOROON JEWETT OR

IBM

THOMAS J WATSON RES CTR

BOX 218

YORKTOWN HEIGHTS NY10598

USA

TEL:

TLF :

TLX:

EML:

COM: 48

LASOTA JEAN-PIERRE OR OBSERVATOLRE DE PARIS SECTION OE MEUDON

F 92195 MEUDON PPL COX

FRANCE.

TEL: $145 \quad 07 \quad 7416$

TLF:

TLX: 201571

EML:

COM: 35,47

LATTIMER JAMES $M$ DR

OPT OF EARTH \& SPACE SCI

ASTRONOMY PROGRAM

SUNY AT STONY BROOK

STONY BROOK NY 117942100

USA

TEL: $516246 \quad 8223$

TLF:

TLX:

EML:

COM: 48

LAURENT BERTEL E PROF

INST OF THEORETICAL PHYS

VANAJISVAEGEN 9

S 11346 STOCKHOLM

SWEDEN

TEL: 8164500

TLF:

TLX: 15433 FYSTO S

EML:

COM: 


\author{
LAURENT CLAUDJNE DR \\ INSU \\ 77 AVE DENFERT ROCHEREAU \\ F 75014 PARIS \\ FRANCE \\ TEL: 140512118
TLF:
TLX:
EML:
COM: 34
}

LAVROV M I PROF
ENGELHARDT ASTRONOMICAL
OBSERVATORY
OBSERVATORIA STATION
422526 RAZAN
RUSSIA
TEL:
TLF:
TLX:
EML:
COM: 42

LAWRENCE GM OR
LASP
UNIVERSITY OF COLORAOO
BOX 392
BOULOER CO 803090392
USA
TEL:
TLF:
TLX:
EML:
COM: 14

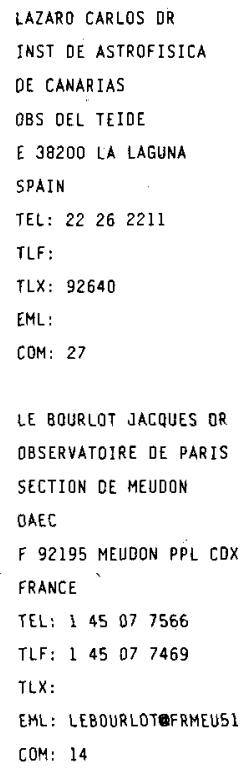

LAUSBERG ANORE DR
INSTITUT O.ASTROPHYSIQUE
UNIVERSITE DE LIEGE
AVE COINTE 5
B 4000 COINTE-LIEGE
BELGIUM
TEL: 41529980
TLF: 41527474
TLX:
EML:
COM: 28.47

LAVRUKHINA A K PROF DR INST OF GEOCHEMISTRY ANALYTICAL CHEMISTRY USSR ACADEMY OF SCIENCES 117334 MOSCOW RUSSIA

TEL: $137-75-38$

ILF:

TLX:

EML:

com:

\section{LAWRIE DAVIO 6}

AEROSPACE CORPORATION

MS M4 041

BOX 9295?

LOS ANGELES CA 90009

USA

TEL: $213 \quad 6486142$

TLF:

TLX:

EML:

COM:

LAZOVIC JOVAN P PROF
DPT OF ASTRONOMY
FACULTY OF SCIENCES
STUDENTSKI TRG 16
YU 11000 BEOGRAD
YUGOSLAVIA
TEL: 11638715
TLF:
TLX:
EML:
COM: 07
LE CONTEL JEAN-MLCHEL
OCA OBSERV OE NICE
BP 139
F OGO03 NICE COX
FRANCE
TEL: 9389 O420
TLF:
TLX: 460004 OBSNICE F
EML:
COM: 29

LAUTMAN D A DR

CENTER FOR ASTROPHYSICS HCO/SAO

60 GARDEN ST

CAMBRIDGE MA 02138

USA

TEL:

TLF:

TLX:

EML:

COM:

\section{LAWRENCE ANDREW OR}

ASTRONOMY UNIT

QUEEN MARY/WESTFIELO COLL

MILE ENO RO

LONDON EI ANS

uK

TEL: 19755481

TLF:

$T \perp X: 893750$

EML: ALUUK. AC. QMC. STAR

COM: 28

LAYZER DAVID PROF

CENTER FOR ASTROPHYSICS

HCO/SAD MS 31

60 GARDEN ST

CAMBR IDGE MA 02138

USA

TEL:

TLF:

TLX:

EML:

COM: $14,28,47$

\section{LAZZARO DANIELA DR} OBSERVATOR TO NACIONAL RUA GL BRUCE 586

SAN CRISTOVAO 20921 RIO DE JANETRO RJ BRAZIL

TEL: 215807181

TLF: 215800332

$T L X: 2121288$ OBSN BR

EML: DAZAELNCCVM

COM: 20

LE gourneuf marYvonne observatoire oe paris SECTION DE MEUDON

F 92195 MEUDON PPL COK FRANCE

TEL: $145 \quad 077555$

TLF:

TLX: 201571

EML:

COM: 14
Laval, anNie or

observatoire de marseiLle

2 PLACE LE VERRIER

F 13248 MARSEILLE COX 04 FRANCE

TEL: 91959088

TLF:

$T L X: 420241 \mathrm{~F}$

EML:

COM: 37

LAWRENCE CHARLES R DR

CALTECH

MS 10524

PASADENA CA 91125

USA

TEL: 8183564976

TLF :

TLX: 675429

EML: BITNET:CRLOCITQEIMO

COM: 40

LAZAREFF BERNARD DR

OBSERVATOIRE DE GRENOBLE CERMO/ASTROPHYSIQUE

BP 53X.

f 38041 S MARTIN HERES CO FRANCE

TEL: $76 \quad 514600$

TLF: 76448821

ILX: $980134 \mathrm{~F}$

EML:

COM:

LE Borgne Jean francoIs OBS MIDI PYRENEES

24 AVE E BELIN

F 31400 TOULOUSE COX

FRANCE

TEL: 61252101

TLF:

TLX: $530776 \mathrm{~F}$

EML: BITNET: LEBORGNEGFROMP5I COM:

LE FEVRE OLIVIER DR
OBSERVATOIRE DE PARIS
SECTION OE MEUDON
OAECC
F 92195 MEUDON PPL COX
FRANCE
TEL:
TLF:
TLX:
EML:
COM: 28




LE POOLE RUDOLF S OR
STERREWACHT
BOX 9513
NL 2300 RA LEIOEN
NETHERLANDS
TEL: 71272727
TLX: 39058 ASTRO NL
EML:
TLF:
COM: 24

LEACOCK ROBERT JAY

DPT OF ASTRONOMY UNIVERSITY OF FLORIDA

211 SSRB

GAINESVILLE FL 32611

USA

TEL: 9043922052

TLF:

TLX:

EML:

COM: 28

LEBLANC YOLANDE IR observatoire de parts SECTION OE MEUDON

F 92195 MEUDON PPL CDX FRANCE

TEL: 145077759

TLF:

TLX:

EML:

COM: 40

LECAR MYRON OR

CENTER FOR ASTROPHYSICS

HCO/SAO

60 GARDEN ST

CAMBRIDGE MA 02138

USA

TEL: 6174957251

ILF:

ILX: 921428 SATELLITE CAM

EML:

COM: 33

LEE PAUL O OR
OPT PHYSICS \& ASTRONOMY
LOUISIANA STATE UNIV
BATON ROUGE LA 708034001
USA
TEL:
TLF:
TLX:
EML:

LE SQUEREN ANNE-MARIE OR ogservatoire de paris SECTION de MELUOON F 92195 MEUDON PPL CDX fRANCE

TEL: 145077595

TLF:

TLX:

EML:

COM: 34.40

LEAHY J PATRICK OR
NRAL
JOORELL BANK
MACCLESFIELO SK11 $90 L$
UK
TEL: 47771321
TLX: 36149
EML: JPLEUK. AC. MAN.JB.STAR
TLF: 477716 18
COM: 40

LEBOFSKY LARRY ALLE
LUNAR \& PLANETARY LAB
UNIVERSITY OF ARIZOMA
TUCSON AZ 85721
USA
TEL: 6026216947
TLF:
TLX:
EML:
COM: 15

LECKRONE OAVIO S DR

NASA/GSFC

CODOE $68 \mathrm{~L}$

GREENBELT MD 20771

USA

TEL: 3012868904

TLF:

ILX:

EML:

COM: 29.44

LEE SANG GAK
OPT OF ASTRONOMY
SEOUL NTL UNIVERSITY
KWANAK KU
SEOUL 151
KOREA R
TEL: $877-2131 / 2139$
TLF:
TLX:
EML:
COM: $33,45,51$

LEA SUSAN MAUREEN DR OPT PHYSICS \& ASTRONOMY SAN FRANSISCO STATE UNIV 1600 HOLLOWAY AVE SAN FRANCISCO CA 94332 USA TEL: 405469 L880 Tik:

TLX:

EML:

COM: 48

LEAHY DENIS A DR

OPT OF PHYSICS

UNIVERSITY OF CALGARY

2500 UNIVERSITY DR' NWW

CALGARY AB T2N IN4

CANADA

TEL: 4032207192

TLF : 4032893331

TLX:

EML: BJTNET: LEAHYUNCAMULT

COM:

LEBOVITZ NORMAN R PROF

OPT OF MATHEMATICS

UNIVERSITY OF CHICAGO

5734 S UNIVERSITY AVE

CHICAGO Il 60637

USA

TEL: 3127538074

TLF:

TLX:

EML:

COM: 35

LEDERLE TRUDPERT DR

ASTRONOMISCHES-RECHEN

INSTITUT

MOENCHHDFSTR 12-14

D 6900 HEIDELBERG i

GERMANY

TEL: 622149026

TLF:

TLX: 461336 ARIHO O

EML:

COM: $04,05,08$

LEE SEE-HDO DR

DPT OF ASTRONOMY

SEOUL NTL UNIVERSITY

SEOUL CITY

KOREA R

TEL: $877-2131 \cdot 9 \times 3308$

TLF:

TLX:

EML:

COM:
LEACH SYONEY DR observatoire de PARIS SECTION DE MEUDON DAMAP

F 92195 MEUDON PPL CDX FRANCE

TEL; 145077561

TLF: $4707 \quad 7469$

TLX: 201571

EML: LEACH巴FrMEU5!

COM: 14

LEBEDINETS VLADIMIR N DR INST OF ASTRONOMY

ACADEMY OF SCIENCES PYATNITSKaYA UL 48 109017 MOSCOW

RUSSTA

TEL:

TEF:

TLX:

EML:

COM: 22

LEBRETON YVELINE DR OBSERVATOIRE DE PARIS SECTION DE MEUOON F 92195 MEUDON PPL COX FRANCE

TEL: 145077859

TLF:

TLX: $20157 t$

EML: LEBRETON@RMEUS1

COM: 35

LEE HYUNG MOK DR

DPT EARTH SCIENCES PUSAN NTL UNIVERSITY KUM JONG KU

PUSAN

KOREA R

TEL: 515102702

TLF: 515137495

ILX:

EML:

COM: 33

LEE TERENLE J OR ROYAL OBSERVATORY HEAD OF TECHNOLOGY BLACKFORO HILL EOINBURGH EH9 3 HJ UK. TEL: $316 \quad 673321$ TLF : ILX: 72383 ROEOING UK EML:

COM: 34 


LEE THYPHOON
INST EARTH SCIENCES
ACADEMIA SINICA
BOX 2359
TAIPEI 107
CHINA R
TEL: 23963211
TLF:
TLX:
EML:
COM: 15,35

LEFEBVRE. MICHEL OR
CNES/GRGS
18 AVE E BELIN
F 31055 TOULOUSE COX
FRANCE.
TEL:
TLF:
TEX:
EML:
COM:

\section{LEHMANN MAREK OR}

astronomical latituoe obs BOROWIEC

$80 \times 62035$

PL 62035 KORNIK

POLAND

TEL: (61)170187

TLF:

TLX: 412623 ADS PL

EML:

COM: $04,08.19$

\section{LEIGHTON R \& PRDF}

CALTECH

MS 10524

PALOMAR OBS

PASADENA CA 91125

USA

TEL: 8183564286

TLF:

TLX:

EML:

COM: 12

Leite schejo paulo or OBSERVATORIO NACIONAL RUA GL BRUCE 586 SAO CRISTOVAO 20921 RIO DE JANEIRO RJ BRAZIL

TEL: 215807313

TLF: 215800332

TLX:

EML:

COM: 27

\author{
LEE WOO-BAIK DR \\ ISSA \\ 36-1 WHAAM DONG \\ YUSEONG GU \\ DAEJEON 305348 \\ NOREA $R$ \\ TEL: 0428615611 \\ TLF: 0428615610 \\ TLX: \\ EML: \\ COM: 42
}

LEFEVRE JEAN OR
OCA OBSERV DE NICE
BP 139
F O6003 NICE COX
FRANCE
TEL: 93 B9 0420
TLF:
TLX: 460004 OBSNICE F
EML:
COM:

LEHNERT B P PROF

DPT Of PLASMa PHYSICS

ROYAL INST OF TELHNOLOGY

S 10044 STOCKKOLM 70

SWEDEN

TEL: $78 \gg 763$

TLF:

TLX: 10389 KTHB'S

EML:

COM:

LEIKIN G A OR
INST OF ASTRONOMY
ACADEMY OF SCIENCES
PYATNITSKAYA UL 48
109017 MOSCOW
RUSSIA
TEL: $231-54-61$
TLF:
TLX: 412623 SCSTP SU
EML:
COM: 16.

LELIEVRE GERARO OR observatotre oE PaRIS

61 AVE OBSERVATOIRE

F 75014 PARIS

France

TEL: 140512255

TLF: 140512232

TLX: 270776 OBS F

EML: LELIEVRE TRIAP51

COM: 09vP, 28
LEE YONG-SAM OR

OPT ASTRONOMY \& SPACE SCI

CHUNGBUK NIL UNIVERSITY

CHEONGJU $360>63$

KOREA R

TEL: $431 \quad 612314$

TLF: 431674232

ILX:

EML: YSLEECBUCC.CBNU.AC. KR

COM: 42

LEGER ALAIN OR
GROUPE PHYSIQUE SOLIDES
UNIVERSITE PARIS VII
4 PLACE JUSSIEU TOUR 23
F 75251 PARIS COX 05
FRANCE
TEL: $143362525 * 4673$
TLF:
TLX:
EML:
COM: $14,21,34$

LEIBACHER JOHN OR

NTL SOLAR BBSERVATORY

BOX 26732

$950 \mathrm{~N}$ CHERRY AVE

TUCSON AZ 857266732

USA

TEL: 6023259302

TLF: 6023259305

TLX: 066484 AURA NOAO TU

EML: JLEIBACHERENOAO AR IZONA. EDU

COM: $10,12,36$

LEINERT CHRISTOPH OR

MPI FUER ASTRONOMIE

KOENIGSTUHL

D 6900 HEIOELBERG L

GERMANY

TEL: $62 \quad 21528264$

ILF:

TLX: 461789 MPIAD

EML:

COM: $21 \mathrm{C}, 26$

LEMAIRE JEAN-LOUIS OR OBSERVATOIRE DE PARIS

SECTION DE MEUDON

F 92195 MEUDON PPL COX

FRANCE

TEL: 145077563

TLF: $145 \quad 077469$

TLX:

EML:

COM: 14
LEER EGIL PROF

INST THEORET ASTRDPHYSICS

UNIVERSITY OF OSLO

BOX 1029

N O315 BLINDERN OSLO 3

NORWAY

TEL: $2-456503$

TLF: $2-456505$

TLX: 64124 AUROB N

EML:

COM:

LEGG THOMAS H OR

HERZBERG INST ASTROPHYS

NTL RESEARCH COUNCIL

100 SUSSEX DR

OITAWA ON KLA ORG

CANADA

TEL: 6139936060

TLF: 6139526602

TLX: 0533715

EML:

COM: 40

LEIBOWITZ ELIA M OR

OPT OF PHYSICS \& ASTRON

TEL AVIV UNIVERSITY

RAMAT AVIV

TEL AVIV 69978

ISRAEL

TEL: 3413788

TLF:

TLX: 343171 VERSY IL

EML: :

COM: 50

LEISAWITZ DAVID OR

NASA/GSFC

CDDE 685

GREENBELT MD 20771

USA

TEL: 3012862150

YLF: 3012868709

TLX:

EML: 6168: :LEISAWITZ

COM: $33,34,37$

LEMAIRE PHILIPPE DR
IAS
BP 10
F 91371 VERRIERES BUISSON
FRANCE
TEL: 164474312
TLF:
TLX: 600252
EML:
COM: 44


LEMAITRE ANNE DR

DPT DE MATHEMATIQUES

FACULTES UNIVERSITAIRES

REMPART DE LA VIERGE 8

B 5000 NAMUR

BELEIUM

TEL: $8122906 \mathrm{~L}$

TLF. 81. 23039

TLX: 59222 FACNAM $B$

EML:

COM: 07

LENZEN RAINER DR

MPI FUER ASTRONOMIE

KOENIGSTUHL

D 6900 HEIDELBERG I

GERMANY

TEL:

TLF :

TLX:

EML:

COM: 25

LEQUEUX JAMES DR

RADIOASTRONOMIE ENS

24 RUE LHOMOND

F 75231 PARIS CDX 05

FRANCE

TEL: $143 \quad 291215$

TLF :

TLX: 202601

EML:

COM: $05,28,34,40,47$

LESCH HAROLO DR

MPI FUR RADIOASTRONOMIE

AUF DEN HUGEL 69

D 5360 BONN 1

GERMANY

TEL: 2285251

TLF :

TLX:

EML:

COM: 40

LESTRADE JEAN FRANCOIS OR

BUREAU OES LONGITUDES

77 AVE OENFERT ROCHEREAL

F 75014 PARIS

FRANCE

TEL: 140512265

TLF:

TLX: 270070

EML:

COM : 40
LEMALTRE GERARD R DR

obSERVATOIRE ge marSEILLE

2 PLACE LE VERRIER

F 13248 MARSEILLE COX 04

FRANCE

TEL: 91959088

TLF:

$T L X$ :

EML:

CDM: 09

LEORAT JACQUES DR

OBSERVATOIRE DE PARIS

SECTION DE MEUDON

F 92195 MEUDON PPL COX

FRANCE

TEL: 145077421

TLF:

TLX: 201571

EML:

COM:

LEROY BERNARD DR

OBSERVATOIRE DE PARIS

SECTION OE MEUDON

DASOP

F 92195 MEUDON PPL CDX

FRANCE

TEL: $1 \quad 45 \quad 07 \quad 7812$

TLF;

TLX:

EML:

COM: 10

LESCHIUTTA S PROF
DPT ELECTRONICA
POLITECNICO
CORSO DUCA O ABRUZZI 24
I 10129 TURINO
ITALY
TEL: 115567235
TLF:
TLX: 220646 POLITO
EML: BITNET:LESCHIUTTAOITOPOLI
COM: 31

LETFUS VOJTECH DR

ASTRONOMICAL INSTITUTE

CZECH ACADEMY OF SCIENCES

ONOREJOV OBSERVATORY

CS 25165 ONDREJOY

CZECHOSLOVAKIA

TEL: 20485201

TLF: 204.85314

TLX:

EML:

COM:
LEMKE DIETRICH DR

MPI FUER ASTRONOMIE

KOENIGSTUHL

D 6900 HEIDELBERG 1

GERMANY

TEL: $62 \quad 21528259$

TLF :

rLX: 461789 IMPIA-D

EML:

COM:

LEPINE JACQUES R D DR

IAG

UNIVERSIDADE DE SAO PAULO

AV MIGUEL STEFANO 4200

04301 SAO PAULO SP

BRAZIL

TEL: 112753720

$T L F$

ILX: 1136221 .

EML:

COM: $34,35,40$

LEROY JEAN-LOUIS

OBS MIDI PYRENEES

14 AVE E BELIN

F 31400 TOULOUSE CDX

FRANCE

TEL: 61332929

TLF :

TLX: $530776 \mathrm{~F}$

EML: EARN : LEROYOFMEU5I

COM: 10,12

LESTER DANIEL F OR

ASTRONOMY DPT

UNIVERSITY OF TEXAS

RLM 15308

AUSTIN TX $78712 \quad 1083$

USA

TEL: $512 \quad 471 \quad 3442$

TLF:

TLX: 9108741351

EML: ARPA:df1Bastro.as.utexas.edu

COM:

LEUNG CHUN MING DR

DPT OF PHYSICS

RENSSELAER PQLYTECHN INST

IROY NY 121803590

USA

TEL: $518 \quad 266 \quad 6318$

TLF:

TLX:

EML:

COM: 34,40
LENA PIERRE J PROF

ÓBSERVATOIRE OE PARIS

SECTION DE MEUDON

F 92195 MEUDON PPL CDX

FRANCE

TEL: $145 \quad 07 \quad 7719$

TLF:

ILK: 201571

EML

COM:

LEPP STEPHEN H DR

CENTER FOR ASTROPHYSICS

HCO/SAO

60 GARDEN ST

CAMBRIDGE MA 02138

USA

TEL: 6174954086

TLF :

TLX:

EML:

COM:

LESAGE ALAIN DR

OBSERVATOIRE DE PARIS

SECTION DE MEUDON

DASGAL

F 92195 MEUDON PPL COX

FRANCE

TEL: $1.45 \quad 07 \quad 7829$

TLF: $145 \quad 077878$

TLX:

EML: LESAGEFRMEU51/MESIOA: :LESAGE

COM: 14

LESTER JOHN B DR

ERINOALE COLLEGE

UNIVERSITY OF TORONTO

DPT OF ASRONOMY

MISSISSAUGA L5L ICE

CANADA

TEL: $416 \quad 8285356$

TLF: 4168285328

ILX:

EML:

COM: 29

LEUNG KAM CHING PROF

DPT OF PHYSICS \& ASTRON

UNIVERSITY OF NEBRASKA

BEHLEN OBSERVATORY

LINCOLN NE 68588

USA

TEL: $402472 \quad 2770$

TLF

TLX: 484340 UNL

EML:

COM: $27 ; 38 \mathrm{C}, 42$ 
LEYASSEUR-REgOURR A $C$ PR SERVICE D'AERONOMIE

BP 3

F 91371 VERRIERES BUISSON

FRANCE

TEL: 164474293

TLF: $1 \quad 43 \quad 298673$

TLX: 602400

EML

COM: $15,21 \mathrm{C}, 22$

LEVREAULT RUSSELL M DR

DPT OF ASTRONOMY

VAN VLECK OBSERVATORY

WESLEYAN UNIVERSITY

MIOOLETOWN CT 06457

USA

TEL: $203 \quad 3479411$

TLF

TIX

EML: BITNET: rlevreaulawesieyan

COM: 40

\section{LEWIS BRIAN MLRRAY OR}

AREC IBO OBSERVATORY

BOX 995

ARECIBO PR 00612

USA

TEL: $809 \quad 878 \quad 2612$

TLF :

ILX:

EML

COM: 30

LI DONG-MING

PURPLE MOUNTAIN OBSERV

CAS

NANJING

CHINA PR

TEL: 2546700

TLF:

$T L X$

EML

COM: 08

LI HYOK HO

PYONGYANG ASTRON OBS

ACABEMY OF SCIENCES OPRK

TAESONG OISTRICT

PYONGYANG

KOREA OPR

TEL:

TLF :

TLX:

EML:

COM: 04
LEVATO ORLANDO hUGO DR COMPLEJO ASTRONOMICO

EL LEONCITO

CC 467

5400 SAN JUAN

ARGENTINA

TEL: 64225720

TLF: $64 \quad 21 \quad 1475$

TLX: 59134 ENTOP AR

EML: MSSDCA: : PSI\%: : IAFE: : LEVATO

COM: $29,30,45 \vee P$

\section{LEVY EUGENE $H$ OR}

LUNAR \& PLANETARY LAB

UNIVERSITY OF ARIZONA

TUCSON AZ 85721

USA

TEL: 6026216962

TLF:

TLX: 9109521143

EML:

COM: 41,49

LEWIS $J S$

LUNAR \& PLANETARY LAB

UNIVERSITY OF ARIZONA

TUCSOH A2.85721

USA

TEL: $602 \quad 6214972$

ILF:

TLX:

EML:

COM: 16

LI GI MAN

PYONGYANG ASTRON OBS

ACADEMY OF SCIENCES OPRK

TAESONG OISTRICT

PYONGYANG

XOREA DPR

TEL :

ILF :

TLX:

EML:

COM: 04

LI I Y

PYONGYANG ASTRON OBS

ACADEMY OF SCIENCES QPRK

TAESONG DISTRICT

PYONGYANG

KOREA OPR

TEL:

TLF:

TLX:

EML:

COM:
LEVINE RANOOLPH H QR

50 CARVER RD

NEWTON MA 02161

USA

TEL: 6179655953

TLF :

TLX

EML:

COM:

LEVY JACQUES $R$ DR

OBSERVATOIRE OE PARIS

61 AVE OBSERVATOIRE

F 75014 PARIS

FRANCE

TEL: $143 \quad 20 \quad 1210$

TLF :

TLX: 270776 OBS F

EML:

COM: 41

LI CHUN-SHENG

DPT OF ASTRONOMY

NANJING UNIVERSITY

NANJING $21000 B$

CHENA PR

TEL: $253465 ! * 2882$

TLF:

TLK: 34151 PRCNLI CN

EML:

COM: 10,40

LI GYONG WON

PYONGYANG ASTRON OBS

ACADEMY OF SCIENCES DPRK

TAESONG OISTRICY

PYONGYANG

KOREA DPR

IEL:

TLF :

TLX:

EML:

COM: 40

LI JING

BEIJING ASTRONOMICAL OBS

CAS

W SUBURB

BEIJING 100080

CHINA PR

TEL: 122040

TEF:

TLX:

EML:

COM: $2 B .33$
LEVISON HAROLD F OR

US NAVAL OBSERVATORY

FLAGSTAFF STATION

BOX 1149

FLAGSTAFF AZ 86002

USA

TEL: 6027795132

TLF :

TLX:

EML: PERCY::LEVISON

CON:

LEWIN WALTER H G PROF

OPT OF PHYSICS

MIT RM 37627

B0X 165 .

CAMBRIDGE MA 02139

USA

TEL: $617 \quad 2534282$

TLF :

TLX:

EML:

COM: 44

LI DEPEI

MANJING ASTRONOMICAL

INSTRUMENT FACTORY

NANJING

CHINA PR

TEL: 2546191

TLF :

TLX: 1131

EML:

COM: 09

LI HONG-WE!

OPT OF ASTRONOMY

NANJING UNIVERSITY

NANJING

CHINA PR

TEL: 25 3465L/34751

TLF :

ILX: 34151 PRCNU CN

EML:

COM: 40

LI NED C DR

CALIFORNIA UNIVERSITY

6531 WITHWORTH RO

LOS ANGELES CA 90035

USA

TEL:

TLF :

TLX:

EML

COM 


\begin{tabular}{|c|c|c|c|}
\hline LI NENG-YAD & LI OI-BIN & LI SIN HYONG & LI SON JAE \\
\hline PURPLE MOUNTAIN OBSERV & BEIJING ASTRONOMICAL OBS & PYONGYANG ASTRON OBS & PYONGYANG ASTRON OBS \\
\hline CAS & CAS & ACADEMY OF SCIENCES OPRK & ACADEMY OF SCIENCES OPRK \\
\hline NANJING & W SUBURB & TAESONG DISTRICT & TAESONG OISTRICT \\
\hline CHINA PR & BEIJING 100080 & PYONGYANG & PYONGYANG \\
\hline TEL: 2537609 & CHINA PR & KOREA OPR & KOREA DPR \\
\hline TLF : & TEL: $\quad$ I $28 \quad 1968$ & TEL: & TEL: \\
\hline TLX: 34144 PMONJ CN & TLF : & TLF : & TLF: \\
\hline EML: & TLX: 22040 BADAS CN & TLX: & TLX: \\
\hline \multirow[t]{2}{*}{ COM: 04,08} & EML: & EML: & EML: \\
\hline & COM: $05 C, 28,46,48$ & COM: 25 & COM: 10 \\
\hline LI TING & LI TIPEI & LI WEI BAD & LI XIAO-QING \\
\hline NANJING ASTRONOMICAL & INSTITUTE OF HIGH ENERGY & YUNNAN OBSERVATORY & PURPLE MOUNTAIN OBSERV \\
\hline INSTRUMENT FACTORY & PHYSICS & CAS & CAS \\
\hline NANJING & $80 \times 9183$ & BOX 110 & NANJING \\
\hline CHINA PR & BEIJING & KUNMING 72946 YUNNAN & CHINA PR \\
\hline TEL: 2546191 & CHINA PR & CHINA PR & TEL: 2531096 \\
\hline TLF : & TEL: 1 日1 $2971 * 464$ & TEL: 8712035 & TLF : \\
\hline TLX: & TLF: & TLF : & TLX: 34144 PMO NJ CN \\
\hline EML: & TLX: 22082 IHEP CN & TLX: & EML: \\
\hline \multirow[t]{2}{*}{ COM: 09} & EMt: & EML: & COM: 28,49 \\
\hline & COM: 44,48 & COM: 10 & \\
\hline LI YUAN-JIE & LI ZHENG-XIN OR & LI ZHIAN OR & LI ZHIGANG \\
\hline OPT OF PHYSICS & SHANGHAI OBSERVATORY & DPI OF ASTRONOMY & SHAANXI OBSERVATORY \\
\hline HUAZHONG UNIVERSITY OF & CAS & BEIJING NORMAL UNIVERSITY & CAS \\
\hline SCIENCE AND TECHNOLOGY & 80 NANDAN RD & BEIJING 100875 & LINTONG XIAN \\
\hline WUHAN & SHANGHAI & CHINA PR & SHAANXI \\
\hline CHINA PR & CHINA PR & TEL: $12012255 * 2618$ & CHINA PR \\
\hline TEL: 870541 & TEL: $2138 \quad 6191$ & TLF: & TEL: 332255 \\
\hline TLF: & TLF : & TLX: $222701 \mathrm{BNU} \mathrm{CH}$ & TLF: \\
\hline TLX: 7122 & $r L x:$ & EML: & $\mathrm{TLX}: 70121$ CSAOCN \\
\hline EML: & EML: & COM: 19 & EML: \\
\hline COM: 48 & COM: 19 & & COM: 08,09 \\
\hline LI ZHI-FANG & LI ZHI-SEN & LI ZHONGYUAN & LI ZONG-WEI \\
\hline SHANGHAI OBSERVATORY & BEIJING ASTRONOMICAL OBS & DPT EARTH \& SPACE SCI & OPT OF ASTRONOMY \\
\hline CAS & CAS & UNIV SCIENCE \& TECHNOLOGY & BEIJING NORMAL UNIVERSITY \\
\hline 80 NANDAN RD & W SUBURB & HEFEI 230026 ANHUI & BEIJING 100875 \\
\hline SHANGHAI & BEIJING 100080 & CHINA PR & CHINA PR \\
\hline CHINA PR & CHINA PR & TEL: 551331134 & TEL: $1656531 * 683$ \\
\hline TEL : 21386191 & TEL: 1281698 & $T L F:$ & TLF: \\
\hline TLF : & TLF: & TLX: 90028 USTC CN & TLX: \\
\hline TLX: 33164 SHAD CN & $T L X: 9053$ & EML: & EML: \\
\hline EML: & EML: & COM: 42,44 & COM: 35,48 \\
\hline COM: 08,47 & COM: 41 & & \\
\hline LI ZONG-YUN & LIANG EDISON P DR & LIANG SHI-GISANG & LIANG ZHONG-HUAN \\
\hline DPT OF ASTRONOMY & DPT SPACE PHYY \& ASTRON & SHANGHAI OBSERVATORY & $80 \times 18$ \\
\hline NANJING UNIVERSITY & RICE UNVIERSITY & CAS & LINTONG \\
\hline NANJING & $80 \times 1892$ & BO NANDAN RO & XIAN \\
\hline EHINA PR & HOUSTON TX $77251 \quad 1892$ & SHANGHAI & CHINA PR \\
\hline TEL: & USA & CHINA PR & TEL: 332255 \\
\hline TLF: & TEL: $7135278101 * 3524$ & TEL: $2138 \quad 6191$ & TLF: \\
\hline TLX: 34151 PRCNU CN & TLF: $713 \quad 285 \quad 5143$ & TLF: & TLX: 70121 CSAD CN. \\
\hline EML: & TLX: 556457 & TLX: 33164 SHAO CN & EML: \\
\hline COM: & EML: LIANGQVEGA, RICE, EOU & EML: & COM: 31 \\
\hline & COM: 48 & $\cos 40$ & \\
\hline
\end{tabular}




\author{
LIAO XINHAO DR \\ DPT OF ASTRONOMY \\ NANJING UNIVERSITY \\ NANJING 210008 \\ CHINA PR \\ TEL: $25 \quad 63 \quad 7651 * 2884$ \\ TLF: \\ TLX: 34151 PRCNU CN \\ EML: 210008 \\ COM: 07
}

\section{LIEBSCHER DIERCK-E OR \\ ZNTRLINST F ASTROPHYSIK \\ STERNWARTE BABELSBERG \\ ROSA-LUXEMBURG-SIR 17A \\ O 1502 POTSOAM \\ GERMANY \\ TEL: \\ TLF : \\ TLX: \\ EML:}

COM: 47

\author{
LILLEY EOWARD A PROF \\ CENTER FOR ASTROPHYSICS \\ HCO/SAO \\ 60 GARDEN ST \\ CAMBRIDGE MA 02130 \\ USA \\ TEL: 6174953971 \\ TLF : \\ TLX: 921428 SATELLITE CAM \\ EML: \\ COM: 40,51

LIN DOUGLAS N C OR
LICK OBSERVATORY
UNIVERSITY OF CALIFORNIA
SANTA CRUZ CA 95064
USA
TEL: 4084292732
TLF:
TLX:
EML:
COM:

LINDBLAD PER OLOF PROF
STOCKHOLM OBSERVATORY
S 13336 SALTSJOEBADEN
SWEOEN
TEL: 87170380
TLF: 87174719
EML: LINOBLADOASTRO.SU.SE
COM: 28,33, EC
TLX: 12972 SOBSERV S

LIBBRECHT K. G OR
CALTECH
MS 26433
BIG BEAR SOLAR OBS
PASAOENA CA 91125
USA
TEL: 8183563722
TLF:
TLX: 675425 CALTECH PSO
EML: KGLESUNDOG.CALTECH. EOU
COM:

LIDDELL U MR
NASA HEADQUARTERS
SPACE SCI \& APPLICATIONS
600 INOEPENDENCE AVE SW
WASHINGTON OC 20546
USA
TEL:
TLF:
TLX:
EML:
COM:

LIESKE JAYH DR LILJE PER VIDAR BARTH OR

JPL/CALTECH

MS 264664

4BOO OAK GROVE DR

PASADENA CA 91109

USA

TEL: $818 \quad 354 \quad 3642$

TLF: $818 \quad 3543437$

ILX: 675429

EML: NAIF : : JHL

COM: 04.07C.19.20

\section{LILLIE CHARLES F OR}

TRW SPACE \& TECHNOLOGY

1 SPACE PARK

REDONDO BEACH CA 90278

USA

TEL: $213 \quad 8122248$

TLX: 910-325-6511

EML:

TLF :

COM: 15.21

\section{LIN YUANZHANG}

BEIJING ASTRONOMICAL QBS

CAS

W SUBURB

BEIJING 100080

CHINA PR

TEL: 1281698

ILF :

TLX: 22040 BADAS CN

EML:

COM: 10,12

LINDE PETER DR
LUND OBSERVATORY
BOX 43
S. 22100 LUND
SWEDEN
TEL: 46104701
TLX: 33199 OBSNOT
EML: PETER SSTRO.LU.SE
TLF:
COM: 05,25

\section{NOROITA}

BLEgDAMSVEJ 1 ?

OK 2100 COPENHAGEN $O$

DENMARK

TEL: $3142 \quad 1616$

ILF: $31 \quad 389157$

TLX: 15216 NBI DK

EML: LILJEONORDITA.DK

COM: 47

\author{
LILLY SIMON J DR \\ DPT OF ASTRONOMY \\ UNIVERSITY OF TORONTO \\ 60 ST GEORGE ST \\ TORONTO ON M5S IAL \\ CANADA \\ TEL: $416 \quad 978 \quad 3150$ \\ TLF: 4169783921 \\ ILX: 06286766 . \\ EML: \\ COM: 28,47
}

LINCOLN J VIRGINIA MISS

2005 ALPINE OR

BOULOER CO 80304

USA

TEL: $303 \quad 4426757$

TLF :

TLX:

EHL:

COM:

LINOEGREN LENNART OR
LUND OBSERVATORY
BOX 43
S 22100 LUND
SWEDEN
TEL: 46107000
TLF:
TLX: 33199 OBSNDT S
EML:
COM: OBC

LIEBERT JAMES W DR

STEWARD OBSERVATORY

UNIVERSITY OF ARIZONA

TUCSON AZ 85721

USA

TEL: $602 \quad 6214513$

TLF:

TLX: 62141410

EML: LIEBERT

COM: $29,33,35,36$

LILLER WILLIAM OR

INSTITUTO ISAAC NEWTON

CASILLA 8-9

VINA DEL MAR

CHILE

TEL: 32970864

TLF:

TLX:

EML:

COM: $15,28,34$

LIN CHIA C PROF

OPT OF MATHEMATICS

MIT

77 MASSACHUSETTS AVE

CAMBRIDGE MA 02139

USA

TEL: $617 \quad 2531796$

TLF:

ILX: 921473 MIT CAM

EML:

COM: $28,33,34$

LINDBLAO BERTIL A DR

LUND OBSERVATORY

$80 \times 43$

S 221 OO LUNO

SWEOEN

TEL: $46 \quad 107000$

TLF :

TLK: 33199 OBSNOT $S$

EML:

COM: $20,22,44$

LINDGREN HARRI

GRUPO NOROICO DEL OBS DEL

ROQUE DE LOS MUCHACHOS

APD 474

E 39700 SANTA CRUZ

SPAIN

TEL:

TLF:

ILX:

EML:

COM: 30 


LINDSEY CHARLES ALLAN
INSTITUTE FOR ASTRONOMY
UNIVERSITY OF HAWAII
2680 WOODLAWN DR
HONOLULU HI 96822
USA
TEL: $80 B \quad 9486526$
TIF:
TLX:
EML:
COM: 15
LINKE RICHARO ALAN DR
8 ANDERSON LANE
PRINCETON NJ O8540
USA
TEL:
TLF:
EML:
COM: 34,40
TLX:

LIPOVETSKY $\checkmark$ A

SPEEIAL ASTROPHYSICAL OBS ACADEMY OF SCIENCES NIZHNIH ARKHYZ

357147 STAVROPOLSKIJ RUSSIA

TEL: 93-2-42

ILF:

TLX:

EML:

COM: 28

LISEAU RENE DR

IAS

CNR

VIA G GALILEI CP 27

I 00044 FRASCATI

ITALY

TEL: 69423801

TLF:

TLX: 610261 CNR FRA I

EML:

COM:

IITTLE LESLIE T OR
ELECTRONICS LABORATORY
UNIVERSITY OF KENT
CANTERBURY CT2 7NT
UK
TEL: 22766822
TLF:
TLX: 965449 UKCLIB
EML:
COM: 40

LING CHIH-BING DR INSTITUTE OF MATHEMATICS ACADEMIA SINICA BOX NO 143

TAIPEI

CHINA R

TEL:

TLF:

TLX:

EML:

COM:

LINNELL ALBERT P PROF PHYSICS \& ASTRONOMY DPT MICHIGAN STATE UNIVERSITY EAST LANSING MI 48824 USA

TEL: 5173536670

TLF:

TLX:

EML:

COM: $35,36,42$

LIPPINCOTT SARAH LEE DR OPT OF ASTRONOMY SharthMORE COLLEGE SPOUL OBSERVATORY SWARTHMORE PA 19081 USA

TEL: 2155439058

TLF:

TLX:

EML:

COM: $24,26,51$

LISI FRANCO OR

OSS ASTROFISICO

DI ARCETRI

LARGO E FERMI 5

I 50125 FIRENZE

ITALY

TEL: 552752289

TLF: 55220039

TLX: 572268 I

EML: SPAN:38954::LISI

COM:

LITTLETON JOHN E

DPT OF PHYSICS

WEST VIRGINIA UNIVERSITY

BOX 6023

MORGANTOWN WV 265066023

USA

TEL: 3042933498

TLF:

TLX: $710-921-0309$

EML:

COM: 35
LING J OR

OBSERVATORIA ASTRONOMICO

RAMON MARIA ALLER

AVD DE LAS CIENCIAS S/N

E SANTIAGO DE COMPOSTELA

SPAIN

TEL:

TLF:

TLX:

EML:

COM: 26

LINSKY JEFFREY L DR

JI LA

UNIVERSITY OF COLORADO

BOX 440

BOULDER CO 803090440

USA

TEL: 3034927838

TLF :

TLX: 755842 JILA

EML:

COM: $12,36 \mathrm{C}, 44$

LIPSCHUTZ MICHAEL E DR WETHERILL CHEMISTRY BLOG PURDUE UNIVERSITY

W LAFAYETTE IN 47907

USA

TEL: 3174945326

TLF:

TLX: 272396

EML: BITNET: RNAAPUMLPURCCVM

COM: 15

LISSAUER JACK J DR

OPT OF EARTH \& SPACE SCI

ASTRONOMY PROGRAM

SUNY AT STONY BROOK

STONY BROOK NY 117942100

USA

TEL: $516 \quad 6328225$

TLF :

ILX:

EML:

COM: $07,15,16$

LITTLE-MARENIN IRENE R DR WHITIN OBSERVATORY

WELLESLEY COLLEGE

WELLESLEY MA 02181

USA

TEL: 6172355303

TLF :

TLX:

EML; jlittle@ucy, wel les]ey, edu COM: $27,29,46$
LINGENFELTER RICHARO E

CASS

UCSO

C. 011

LA JOLLA CA 920930216

USA

TEL: 6194522464

TLF:

TLX: 9103371271 SIOCCAN

EML:

COM:

LINSLEY JOHN

OPT PHYSICS \& ASTRONOMY UNIVERSITY OF NEW MEXICO 800 YALE BLVD NE ALBUQUERQUE NM 87131 USA

TEL: 5052431.924

TLF :

TLX: 910989

EML:

COM: 44.48

LIRITZIS IOANNIS DR RES CENTER FOR ASTRONOMY ACADEMY OF ATHENS

14 ANAGNOSTOPOULOU ST GR 10673 ATHENS GREECE

TEL: 13613589 TLF: 13631606 TLX:

EML:

COM: 10

LISZT HARVEY STEVEN NRAO

EDGEMOMT RO CHARLOTTESUILLE VA 22901 USA

TEL: $804 \quad 296 \quad 0344$

TLF:

$T L K: 910-997-0714$

EML:

COM: 34

LITVAK MARVIN M DR TRW INC $01 / 1260$

I SPACE PARK

REDONDO BEACH CA 90278

USA

TEL:

TLF :

TLX:

EML: :

COM : 
LITVINENKO LEONID N DR INSTITUTE OF RADIO ASTRON UKRAINIAN ACAD OF SCIENCE KRASNOZNAMENNAYA UL 4 310002 KHARKOV

UKRAINE

TEL: 451009

TLF: 320273

TLX: 115144 METIL SU

EML:

COM: 40

LIU JINMING

SHANGHAI OBBSERVATORY

CAS

80 NANDAN RO

SHANGHAI

CHINA PR

TEL: 21386191

ILF:

ILX: 33164 SHAO CN

EML:

COM: $05,06,31$

LIU OINGYAO DR

YUNNAN OBSERVATORY

CAS

BOX 110

KUNMING 72946 YUNNAN

CHINA PR

TEL: 8712035

TLF :

TLX: 64040 YUOBS CN

EML:

COM: 42

\section{LIU XUEFU}

DPT BF ASTRONOMY

BEIJING NORMAL UNIVERSITY

BEIJING 100875

CHINA PR

TEL: $1656531 * 6285$

TLF:

TLX: 8511

EML:

COM: 42

LIVIO MARIO PROF

DPT OF PHYSICS

I IT

TECHNION CITY

HAIFA 32000

ISRAEL

TEL: 4293549

TLF:

TLX: 46550 TECLI IL

EML: PHROIMLIECHNION

COM: 35
LIU BAD-LIN

PURPLE MOUNTAIN OBSERV

CAS

NANUING

CHINA PR

TEL: $25 \quad 42817 / 46700$

TLF :

TLX: 34144 PMONJ CN

EML:

COM: 04

LIU LIAO

DPT OF PHYSICS

BEIJING NORMAL UNIVERSITY

BEIJING 100071

CHINA PR

TEL:

TLF:

TLX:

EML:

COM: 47

LIU RU-LIANG

PURPLE MOUNTAIN OBSERV

CAS

NANJ IHG

CHINA PR

TEL: $25 \quad 42817 / 46700$

TLF:

TLX: 34144 PMONJ CN

EML:

COM: 28,48 .

LIU YONG-ZHEN

GRADUATE SCHOOL

UNIV SCIENCE \& TECHNOLOGY

BOX 3908

BEIJING 100039

CHINA PR

TEL: 1 8L 7031

ILF:

ILX:

EML:

COM: 28,47

LIVSHITS MA OR
IZMIRAN
ACADEMY OF SEIENCES
142092 TROITSK
RUSSIA
TEL:
TLF:
TLX: 412623 SCSTP SU
EML:
COM: 10

LIU CAIPIN

PURPLE MOUNTAIN OBSERV

CAS

NANJING

CHINA PR

TEL: 2542817

TLF :

TLX: 34144 PMONJ CN

EML:

COM: 36

LIU LIN

OPT OF ASTRONOMY

NANJING UNIVERSITY

NANJING.

CHINA PR

TEL: $25,34651 * 2882$

TLF :

TLX: 34151 PRCNU CN

EML:

COM:

LIU SOU-YANG OR

COMPUTER SCIENCES CORP

SYSTEM SCIENCES DIV

8728 COLESVILLE RD

SILVER SPRING MO 20910

USA

TEL: 3015891545

TLF:

TLX:

EML:

COM:

LIU ZONGLI

BEIJING ASTRONOMICAL OBS

CAS

W SUBURB

BEIJING 100080

CHINA PR

TEL:

ILF :

TLX: 22040

EML:

COM: 15,27

LIZAND-SOBERON SUSANA OR

INSTITUTO DE ASTRONOMIA

UNAM

APDO POSTAL 70-264

04510 MEXICO DF

MEXICO

TEL: $011525 \quad 548 \quad 53 \quad 05$

TLF: $011525 \quad 548 \quad 3712$.

TLX: 017 60155 CICME

EML: LIZANO\%ALFA. ASTROSCU, UNAM, MX

COM: 34
LIU CI - YUAN DR SHAANXI OBSERVATORY CAS

LINTONG XIAN

SHAANXI

CHINA PR

TEL: 332255

TLF: 92373496

TLX: 70121 CSAO

EML:

COM: 19,41

LIU LIN-ZHONG

PURPLE MOUNTAIN OBSERV

CAS

NANJ ING

CHINA PR

TEL: 2546700

TLF :

TLX: 34144 PMONT CN

EML:

COM: 15

LIU XINPING PROF

INSTITUTE OF MECHANICS

CAS

BEIJING L00080

CHINA PR

TEL: $128 \quad \$ 185$

TLF: 12561284

TLX: 222554 MEHAS CN

EML:

COM: 10

LIVINGSTON WILLIAM C

NOAO/NSO

BOK 26732

$950 \mathrm{~N}$ CHERRY AVE

TUCSON AZ $85726 \quad 6732$

USA

TEL: $602 \quad 327 \quad 5511$

TLF :

rLX: 0666484 AURA NOAO TU

EML:

COM: 09.12

LLOYO EVANS THOMAS OR

SAAO

BOX 9

OBSERVATORY 7935

SOUTH AFRICA

TEL: $021-47-0026$

TLF :

TLX: $5720309 \mathrm{SA}$

EML:

COM: $37,45 C$ 


LO KWOK-YUNG OR
CALTECH
MS 10524
OWENS VALLEY RADIO OBS
PASADENA CA 91125
USA
TEL: 8183564415
ILF:
TLX: 675425 CALTECH PSO
EML:
COM: $28,34,40$
LOCKMAN FELIX J
NRAO
EOGEMONT RO
CHARLOTTESVILLE VA 22903
USA
IEL: 804296 O211
TLX: $910-997-0174$
EML:
TLF:
COM: $33,34,40$

LOISEAU NORA DR INST DE ASTROFISICA DE CANARIAS OBS DEL TEIOE E 38200 LA LAGUNA SPAIN

TEL: 22605237

TLF: 22605210

TLX: 92640 IACE E

EML: NLLOIAC. DNET, NASA.GOV COM: 40

LOCANTHI DOROTHY DAVIS OR
2180 PINECREST OR
ALTADENA CA 91001
USA
TEL: 2137970629
TLF:
EML:
COM: 29
TLX:

LOCKWOOD G WESLEY OR
LOWELL OBSERVATORY
1400 W MARS HILL RO
BOX 1149
FLAGSTAFF AZ 86001
USA
TEL: 6077743358
TLF:
TLX:
EML:

COM: $16,25,27$

LOMB NICHOLAS RALPH DR
SYONEY OBSERVATORY
MUSEUM APPLIED ARTS \& SCI
BOX K 346
HAYMARKET NSW 2000
AUSTRALIA
TEL:
TLF:
TLX:
EML:
COM: 20,46

$\begin{array}{ll}\text { LONSDALE CAROL J OR } & \text { LOOSE HANS-HERMANN DR } \\ \text { CALTECH. } & \text { UNIVERSTAETS STERNWARTE } \\ \text { MS } 10022 & \text { GEAISMARLANDSTRASSE } 11 \\ \text { IPAC } & \text { O 3400 GEOTTINGEN } \\ \text { PASADENA CA } 91125 & \text { GERMANY } \\ \text { USA } & \text { TEL: } 551395056 * 953 \\ \text { TEL: } 0185842929 & \text { TLF: } \\ \text { TLF: } & \text { TLX: } \\ \text { TLX: } 675429 & \text { EML: } \\ \text { EML: INTERNET:CJLOIPAC.CALTECH.EDU COM: } 28 \\ \text { COM: } 05.28,47\end{array}$

\section{LOPEZ CARLOS LIC}

OBSERVATORIO ASTRONOMICO

FELIX AGUILAR

AV BENAVIDEZ BI75 OESTE

5407 SAN JUAN

ARGENTINA

TEL: $64 \quad 231494$

TLF :

TLX:

EML:

COM: 24
LOCHMAN JAN

ASTRONOMICAL INSTITUTE

CZECH ACADEMY OF SCIENCES

DVORAKOVA 298

CS 511 O1 TURNOV

CZECHOSLOVAKIA

TEL: 43622622

TLF:

TLX:

EML:

COM: 09

LODEN KERSTIN R DR STOCKHOLM OBSERVATORY

S 13336 SALTSJDEBADEN SWEDEN

TEL: 87170195

TLF : $87 \$ 74719$

TLX: 12972 SOBSERV $S$

EML: LOOEN_KOASTRO.SU.SE

COM: $26,33,45$

LONG KNOX S DR

DPT PHYSICS \& ASTRONOHY

JOHNS HOPKINS UNIVERSITY

CHARLES \& 34TH ST

BALTIMORE MD 21218

USA

TEL: 3013387391

TLF:

TLX: 9102400225 JHUCASMD

EML:

COM: 44 .

LOOSE HANS-HERMANN DR

STAETS SIERNWARTE

- 3400 GEOTTINGEN

TEL: $551395 \quad 056 * 953$

TLF :

COM: 28

LOPEZ DE COCA M OP DR

INST ASTROFISICA

DE ANDALUCIA APJ 2144

PROFESOR ALBAREDA 1

E 18080 GRANADA

SPAIN

TEL: $58 \quad 12 \quad 1311$

TLF: $58 \quad 114530$

ILX: 78573 IAAGE

EML: PILAR@IAA,ES

COM: 27
LOCKE JACK L DR

250 BRAESIDE AVENUE

OTTAWA ON KIH 7J5

CANADA

TEL: 6135230812

TLF:

TLX:

EML:

COM: 12,40

LODEN LARS DLOF PROF ASTRONOMICAL OBSERVATORY

B0X 515

S 75120 UPPSALA

SWEDEN

TEL: 18 II 4490

TLF:

TLX: 76024

EML:

COM: $26,33,37,51$

LONGAIR MS PROF

MULLARD RADIO ASTRON OBS

CAVENOISH LABORATORY

MADINGLEY RD

CAMBRIDGE CB 3 OHE

UK

TEL: $223 \quad 337083$

TLF: $223 \quad 354599$

TLX: 81292 CAVLAB

EML: ms luk, ac.cam.phy.ras

COM: $40,47,48$

LOPES DALTON DE FARIA DR OBSERVATORIO NACIONAL RUA GL JOSE CRISTINO 77 SAO CRISTOVAO 20921 RIO DE JANEIRO TJ BRAZIL

TEL: $21 \quad 5807181$

TLF: $21 \quad 580 \quad 0332$

TLX: 021288

EML: DFLOLLCCVB.BITNET

COM: 29

LOPEZ GAREIA ZULEMA L. DR OBSERVATORIO ASTRONOMICO FELIX AGUILAR

AV BENAVIOEZ 8175 OESTE 5407 MARQUESAOO (S.J.) ARGENIINA

TEL:

TLF:

ILX:

EML:

COM: 
LOPEZ JOSE A ING

OBSERVATORIO ASTRONOMICO

FELIX AGUILAR

AV BeNAVIOEZ 8175 OESTE

5407 MARquesado (S.J.)

ARGENTINA

TEL: $64 \quad 231494$

ILF:

TLX: 59100 UNSJA AR

EML:

COM: $\triangle B C$

LOPEZ-GARCIA FRANCISCO DR OBSERVATORIO ASTRONOMICO

\section{FELIK AGUILAR}

AV BENAVIDEZ 8175 OESTE

5407 MaRqueSATO (5.J.)

ARGENTINA

TEL: $64 \quad 231494$

TLF:

TLX:

EML:

COM:

LOPEZ-VALVERDE M A OR INST ASTROFISICA

OE ANDALUCIA APD 3004

PROFESOR aLBAREOA I

E 18080 GRANADA

SPAIN

TEL: 58121311

TLF: $58 \quad 814530$

TLX: 78573 IAAGE

EML: 16488 : : VALVERDE

COM: 16

\section{LORTET MARIE CLAIRE}

observatoire oE PARIS

SECTION DE MEUDON

DAPHE

F 92195 MEUDON PPL CDX

FRANCE

TEL: $145 \quad 077426$

TLF:

TLX: 201571

EML:

COM: $05,28,34$

\section{LOUISE RAYMOND PROF}

FACULTE DES SCIENCES

DEPT DE PHYSIQUE

33 RUE ST-LEU

F 80039 AMIENS

FRANCE

TEL:

ILF:

TLX:

EML:

COM: 34

\author{
LOPEZ JOSE ALBERTO OR \\ INSTITUTO DE ASTRONOMIA \\ UNAM \\ APDO POSTAL 877 \\ 22800 ENSENADA B CALIF \\ MEXICO \\ TEL: $667-44580$ \\ ILF: \\ TLX: 56539 CICEME \\ EML: \\ COM:
}

LOPEZ-GONZALEZ MARIA J DR

INST ASTROFISICA

DE ANOALUCIA APO 3004

PROFESOR ALBAREDA I

E 18080. GRANADA

SPAIN

TEL: 58121311

TLF: 58814530

TLX: 78573 IAAG E

EML: 16488 : :MARIAJOSE

COM: 21

LORO STEVEN OONALO OR

NASA AMES RESEARCH CTR

MS 2456

MOFEEFT FIELD CA 94035

USA

TEL: $415 \quad 6043571$

TLF :

TLX:

EML: LORD GAL ARC. NASA. GOV

COM: 28

LOSCO LUCETTE OR

FACULTE oES SCIENCES

F 25030 BESANCON COX

FRANCE

$$
\text { TEL: }
$$

TLF:

EML:

COM:

TLX:

\section{LOULERGUE MICHELLE OR} observatoire de paris SECtion dE MEULOON

F 92195 MEUDON PPL CDX

$$
\text { FRANCE }
$$

TEL: 145077455

TLF:

TLX: 270912

EML:

COM: 14
LOPEZ ROSARIO DR

OPT FISICA

UnIVERSADAD DE barCELONA

AVD DIAGONAL 647

E 0802B BarCELONA

SPAIN

TEL: 33307311

ILF:

TLX:

EML: BITNET: DJFARLHOEEBOUBOLL

COM: 28

LOPEZ-MORENO JOSE JUAN

INST ASTROFISICA

DE ANDALUCSA APO 2144

PROFESOR ALBAREOA 1

E 18080 granada

SPAIN

IEL: 58121300

TLF:

ILX: 78573 IAAG E

EML:

COM: 16,21

LOREN ROBERT BRUCE DR

BOX 2915

SILVER CITY NM 88062

USA

TEL:

TLF:

TLX:

EML:

COM: 34,40

LOTOVA N A BR

IZMIRAN

ACADEMY OF SCIENCES

142092 TROLTSK.

RUSSIA

TEL:

TLF:

TLX:

EML:

COM: 49

LOVAS FRANCIS JOHN OR

NTL BUREAU OF STANDARDS

OIV 545

MOLECULAR SPECTROSCOPIC

WASHINGTON OC 20234

USA

TEL: 3019212023

ILF:

TLX: 898993

EML:

COM: 14,34
LOPEZ-ARROYO M

OBS ASTRONOMICO NCL

ALFONSO XII- 5

E 28014 MAORIO

SPAIN

TEL:

TLF :

TLX:

EML:

COM: 12

LOPEZ - PUERTAS MANUEL INST ASTROFISICA

DE ANOALUCTA APB 2144 PROFESOR ALBAREDA I

E 18080 GRANADA

SPAIN

TEL: $58 \quad 12 \quad 1300$

TLF:

ILX: 78573 IAAG E

EML:

COM: 16,21

LORENZ HILMAR

ZNTRLINST F ASTROPHYSIK

AKAD WISSENSCHAFTEN ODR

ROSA-LUXEMBURG-STR ITA

D 1502 POTSDAM

GERMANY

TEL:

TLF:

TLX:

EML:

COM: 28,40

LOUGHHEAD RALPH E OR

CSIRO

DIV OF APPLIED PHYSICS

BOX 218

LINDFIELLO NSW 2070

Australia

TEL: 24676355

TLF:

TLX: 26296

EML:

COM: 10,12

LOVAS MIKLOS

KONKOLY OBSERVATORY

THEGE U 13/1?

BOX 67

H 1525 BUDAPEST

HUNGARY

TEL: 175 5866/75 4122

TLF:

TLX: 227460 KONOB

EML:

COM: 20 
LOVELACE RICHARD Y E OR CORNELL UNIVERSITY SPACE SCIENCES BLDG ITHACA NY 14853 USA

TEL: $607 \quad 256 \quad 3968$

ILX:

EML:

ILF:

COM: 48

LOWE ROBERT $P$ OR

OPT OF PHYSICS

UNIV OF WESTERN ONTARIO

LONDON ON NGA $3 K 7$

CANADA

TEL: 5196792917

TLF : 5196613486

TLX: 0647134

EML:

COM:

LU BEN-KUI

PURPLE MOUNTAIN OBSERV

CAS

\section{NANJING}

CHINA PR

TEL: 2532893

TLF :

TLX: 34144 PMONJ CN

EML:

COM: 07,31

LU RUWEI DR

YUNNAN OBERVATORY

CAS

$80 \times 110$

KUNMING 650011 YUNNAN

CHINA PR

TEL: 8712035

TLF: 87171845

TEX: 64040 YUOBS

EML:

COM: 09

LUCAS ROBERT OR,
OBSERVATOIRE OE GRENOBLE
CERMO/ASTROPHYSIQUE
BP 68
F 3804 I S MARTIN HERES CO
FRANCE
TEL: 76514600
TLF:
TLX:
EML:
COM: 34

LOVELL SIR BERNARD PROF

\section{NRAL}

JODRELL BANK

MACCLESFIELD SKIL 90L

UK

TEL: 47771321

ILX: 36149

EML:

TLF :

COM: $22,40,44,51$

LOYOLA PATRICIO DR

DPT DE ASTRONOMIA

UNIVERSIDAD DE CHILE

CASILLA 360

SANTIAGO

CHILE

TEL: 22294101

TLF :

TLX: 440001

EML:

COM: 08

LU CHUN-LIN

PURPLE MOUNTAIN OBSERV

CAS

NANJING

CHINA PR

TEL: $25 \quad 42700$

TLF :

TLX: 34144 PMONJ CN

EML:

COM: $O B$

LU TAN

DPT OF ASTRONOMY

NANJ ING UNIVERSITY

NANJING

CHINA PR

TEL: $253465 \mathrm{~L} * 2882$

TLF:

TLX: 34151 PRCNU CN

EML:

COM: 47.48

LUCCHIN FRANCESCO

OPT DI FISICA G GALILEI

UNIVERSITA OI PADOVA

VIA MARZOLO 8

135131 PAQOVA

ITALY

TEL: $49 \quad 84 \quad 4333$

TLF :

TLX: 430308 DF GGPOI

EML:

COM: 47
LOW BOON CHYE

HIGH ALTITUDE OBSERVATORY

NCAR.

BOX 3000

BOULDER CO $8030 \% 3000$

USA

TEL: 3034971553

TEF :

TLX: 45694

EML:

COM: 10

LOZINSKAYA TAT' YANA A DR

STERNBERG STATE ASTR INST

UNIVERSITETSKIJ PROSP 13

119899 MOSCOW

RUSSIA

TEL: $139-10-30$

TLF :

TLX:

EML:

COM: $34 \mathrm{C}, 40$

LU JU FU OR

ASTROPHYSICS DIVISION

UNIV SCIENCE \& TECHNOLOGY

HEIFI 230026 ANHUI

CHINA PR

TEL: $551 \quad 33 \quad 1134 * 527$

TLF: 551331760

TLX: 90028 USTC CN

EML:

COM: 48

LU YANG

DPT OF ASTRONOMY

NANJING UNIVERSITY

NANJING

CHINA PR

TEL: $2534651 * 2882$

TLF :

TLX: 34151 PRCNU CN

EML:

COM: 40

LUCEY JOHN DR

OPT OF PHYSICS

UNIVERSITY OF OURHAM

SOUTH RD

DURHAM DHI 3LE

UK

IEL:

TLF :

TLX:

EML:

COM: 28
LOW FRANK 」 DR

4940 CALLE BARRIL

TUCSON AZ 85718

USA

TEL: $602 \quad 621 \quad 2779$

TLF :

TLX:

EML

COM: $28 ; 34,45$

LOZINSKIJ A M DR

INST OF ASTRONOMY

ACADEMY OF SCIENCES

PYATNITSKAYA UL 48

109017 MOSCOW

RUSSIA

TEL: 231-54-61

TLF:

TLX: 412623 SCSTP SU

EML:

COH: 24

LU PHILLIP $K$ OR

OPT PHYSICS \& ASTRONOMY

W CONNECTICUT STATE UNIV

181 WHITE $S T$

DANBURY CT O6B1D 7859

USA

TEL: 2037974218

TLF :

TLX:

EML:

COM: $24,33,37$

LUB JAN OR

STERREWACHT

HUYGENS LAB

BOX 9513

NL 2300 RA LEIDEN

NETHERLANDS

TEL: $71 \quad 27 \quad 2727$

TLF :

TLX: 39068 ASTRO NL.

EML:

COM: $25[, 27$

LUCK J DR

DIVISION OF NTL MAPPING

BOX 31

BELCOMMEN ACT 2626

AUSTRALIA

TEL: $62 \quad 525172$

TLF:

TLX: 62622 LUNAR AA

EML:

COM: $31 \mathrm{C}$ 
LUCK R EARLE OR

WARNER \& SWASEY OBS

CASE WESTERN RESERVE UNIV

CLEVELAND OH 44106

USA

IEL: 2163686697

TLF :

ILX:

EML:

COM: 29.36

\section{LUEST RHEA OR}

MPI FUER PHYSIK UND

ASTROPHYSIK

K-SCHWARZSCHILDSTR 1

D BO46 GARCHING MUENCHEN

GERMANY

TEL: 8932032990

TLF :

TLX: 524629 ASTROD

EML:

COM: 15

LUMME KARI A OR

OBS \& ASTROPHYSICS LAB

UNIVERSITY OF HELSINKI

TAEHT I TORN INMAK I

SF 00130 HELSINKI 13

FINLAND

TEL: 1912910

TLF :

TLX

EML:

COM: $15,16,21$

LUNOSTEDT HENRIK DR
LUND OBSERVATORY
BOX 43
S 221 OO LUNO
SWEOEN
TEL: 46107294
TLX: 33199 OBSNOT S
EML: henrikEastro.1u.se
TLF: 46104614
COM: 10,49

\section{LUO BAO-RONG}

YUNNAN OBSERVATORY

CAS

BOX 110

KUNMING 72946 VUNNAN

CHINA PR

TEL: 8712035

TLF:

$r: X:$

EML:

CON: 10
LUCKE PETER B DR

DPT PHYSICS \& ASTRONOMY

MOUNT UNION COLLEGE

ALLIANEE OH $\mathbf{4 4 6 0 1}$

USA

TEL: $216 \quad 8215320$

TLF :

$T L X:$

EML:

COM:

\begin{tabular}{|c|c|}
\hline LUGGER PHYLLIS M & LUKACEVIC ILIJA S OR \\
\hline ASTRONOMY DPT & DPI OF MECHANICS \\
\hline INDIANA UNIVERSITY & FACULTY OF SCIENCES \\
\hline SWAIN WEST 319 & STUDENTSKI TRG 16 \\
\hline BLOOMINGTON IN 47405 & YU 11000 BEOGRAD \\
\hline USA & YUGOSLAVIA \\
\hline TEL: 8123356929 & TEL: 11638715 \\
\hline TLF : & TLF : \\
\hline TLX: & TLX: \\
\hline EML: & EML: \\
\hline COM: 28 & COM: \\
\hline LUNA HOMERO G OR & LUND NIELS \\
\hline IAR & DANISH SPACE RESEARCH INS \\
\hline CC 5 & LUNDTOFTEVEJ 7 \\
\hline 1894 FILLA ELISA (BS AS) & OK 2800 LYNGBY \\
\hline ARGENTINA & DENMARK \\
\hline TEL: 2143793 & TEL: $42 \quad 8 B \quad 2277$ \\
\hline$T L X:$ & TLF : \\
\hline EML: & TLX: 37198 \\
\hline TLF: & EML: \\
\hline COM: 25 & COM: . \\
\hline
\end{tabular}

LUNDSTROM INGEMAR OR

LUND OBSERVATORY

$B 0 \times 43$

S 22100 LUNO

SWEDEN

TEL: $46 \quad 10 \quad 7300$

TLX: $33199^{\circ}$

EML:

TLF:

COM: 29

LUD DINGCHANG

BEIJING ASTRONOMICAL DBS

CAS

\section{W SUBURB}

BEIJING 100080

CHINA PR

TEL: 1275580

ILF:

$T \perp X: 2204 \Omega$

EML:

COM: 31
LUCY LEON B PROF

ESO

KARL-SCHWARZSCHILD-STR 2

D B046 GARCHING MUENCHEN

GERMANY

TEL: 8932006249

ILF: $89320 \quad 2362$

ILX: 0528282-0

EML:

COM: 42

LUNEL MADELEINE OP

OBSERVATOIRE OE LYON

AVE CHARLES ANORE

F 69561 S GENIS LAYAL COX

FRANCE

TEL: $78 \quad 560705$

TLF: 72399791

TLX: $310-926$

EML:

COM: 33

LUO DING-JIANG

BEIJING ASTRONOMICAL OBS

CAS

\section{W SUBURB}

BEIJING 100080

CHINA PR

TEL: $128 \quad 1698$

ILF:

TLX: 22040 BAO ASCN

EML:

COM: 08.19
LUEST REIMAR PROF

MAX PLANCK INSTITUTE FOR

METEOROLOGY

BUNDESSTRASSE 55

D 2000 haMBURG 13

GERMANY

TEL: 40411730

TLF: $4041 \quad 173298$

ILX: 211092

EML:

COM: $12,44,48,49$

LUMINET JEAN-PIERRE

OBSERVATOIRE DE PARIS

SECIION OE MEUDON

F 92195 MEUDON PPL COX

FRANCE

TEL: $145 \quad 07 \quad 7423$

TLF :

ILX: 201571

EML:

COM: $28,47,48$

LUNDQUIST CHARLES A OR RESEARCH INSTITUTE

UNIVERSITY OF ALABAMA

BOX 209

HUNTSVILLE AL 35899

USA

TEL: $205 \quad 895 \quad 6100$

TLF :

TLX:

EML:

COM: 07

LUNGU NICOLAIE DR INSTITUTE POLITEHNIC CATEDRa dE matematica STR EMIL ISAC 15

R 3400 CLUJ NAPOCA

RUMANIA

TEL: $951-17229$

TLF:

TLX:

EML:

COM:

LUO SHI - FANG

SHANGHAI OBSERVATORY

CAS

BO NANDAN RD

SHANGHAI

CHINA PR

TEL: 21386191

ILF :

TLX: 33164 SHAO EN

EML:

COM: 19.31 


LUO XIANHAN,
OPT OF GEOPHYSICS
BEIJING UNIVERSITY
BEIJING 100071
CHINA PR
TEL: 122239
TLF:
TLX:
EML:
COM: 10,40

LUTZ BARRY L OR LOWELL OBSERVATORY 1400 W MARS HILL RO BOX 1149

FLAGSTAFF AZ 86001 USA

TEL: $602774335 \mathrm{~B}$

ILF:

TLX: 6502358958 MCI EML:

COM: $14,15,16$

LYNAS-GRAY ANTHONY E

OPT PHYSICS ASTRONOMY

UNIVERSITY COLLEGE LONOON

GOWER ST

LONOON WCIE 6BT

UK

TEL:

TLF:

TLX:

EML:

COM:

LYNDS ROGER C OR
KITT PEAK NTL OBS
BOX 26732
950 N CHERRY AVE
TUCSON AZ 857266732
USA
TEL: 6023275511
TLF:
TLX:
EML:
COM: 28
LYTTLETON RAYMOND A PROF
INSTITUTE OF ASTRONOMY
THE OBSERVATORIES
MADINGLEY RD
CAMBRIDGE CB3 OHA
UK
TEL: 223 622 04
TLF:
TLX: 817297 ASTRON G
EML:
COM: 15

LUPISHKO DMITRIJ F ASTRONOMICAL OBSERVATORY KHARKOY UNIVERSITY

SUMSKAYA UL 35 310022 KHARKOY UKRAINE

TEL: 432428

TLF:

TLX: 12553L ICAR

EML: .

COM: $15 \mathrm{C}$

LUTZ JULIE H DR

PROGRAM IN ASTRONOMY

WASHINETON STATE UNIV

PULLMAN WA 991642930

USA

TEL: 5093353136

TLF:

TLX: 5107741091 WSUOIPPMA

EML:

COM: 45

LYNCH DAVID K

AEROSPACE CORPORATION

HS M2 226

BOX 92957

LOS ANGELES CA 90009

USA

TEL: 2136486686

TLF:

TLX: 664460

EML:

COM: 09

LYNE ANOREW G DR

NRAL

JOORELL BANK

MACCLESFIELO SKII 906

uk

TEL: 47771321

TLX: 36149

EML:

TLF:

COM: 40

LYUBIMKOY LEONIDS OR

CRIMEAN ASTROPHYS OBS UKRAINIAN ACAD OF SCIENCE NAUCHNY

334413 CR IMEA

UKRAINE

TEL: 432945

TLF:

TLX:

EML:

COM: 05,36
LUSTIG GUENTER DR

INSTITUT FUER ASTRONOMIE

KARL-FRANZENS-UNIVERSITAT

UNIVERSITATSPLATZ 5

A 8010 GRAZ

AUSTRIA

TEL: 3163805272

TLF:

TLX: 0311662 UBGRZ

EML:

COM: 10,12

LUTZ THOMAS E DR

PROGRAM IN ASTRONOMY

WASHINGTON STATE UNIV

PULLMAN WA 991642930

USA

TEL: : 5093353141

TLF:

TLX: 5107741091 WSUOIPPMA

EML:

COM: 24

LYNDEN-BELL OQNALO PROF

INSTITUTE OF ASTRONOMY

THE OBSERVATORIES

MADINGLEY RO

CAMBRIOGE CB3 OHA

UK $\therefore$

TEL: 22362204

TLF :

TLX: 817297 ASTRON $G$

EML:

COM: $28,33,37,47,48$

LYNGA GOSTA DR

MOUNT STROMLO \& SIDING

SPRING OBSERVATORIES

PRIVATE BAG

WODEN PO ACT 2606

AUSTRALIA

TEL:

TLF:

TLX:

EML: GOSTAOMSO .ANU. EDU. AU

COM: $05,33,37,45$

LYUTY VICTOR M OR

CRIMEAN STATION OF

STERNBERG INSTITUTE

NAUCHNY

334413 CRIMEA

UKRAINE

TEL: 432945

TLF:

TLX:

EML:

COM: 42
LUTTERMOSER DONALO OR

DPT OF PHYSICS

IOWA STATE UNIVERSITY

AMES IA 50010

USA

TEL

FLF :

TLX:

EML:

COM: 36

LUYTEN WILLEM J PROF SPACE SCIENCE CENTER UNIVERSITY OF MINNESOTA 100 UNION ST SE MINNEAPOLIS MN 55455

USA

TEL: 6123733366

TLF:

TLX:

EML:

COM: 24.26 .33

LYNOS BEVERLY T OR

KITT PEAK NTL OBS

BOX 26732

950 N CHERRY AVE

TUCSON AZ $85726 \quad 6732$

USA

TEL: $602 \quad 3259396$

TLF:

TLX: 0666-484 ALRA NDAO

EML:

[OM: 28,34

LYTTKENS EJNAR DR

SKOLGATAN 33B

$\$ 75221$ UPPSALA

SWEDEN

TEL:

TLF:

TLX:

EML:

COM:

MA ER

BEIJING ASTRONOMICAL OBS CAS

W SUBURB

BEIJING 100080

CHINA PR

TEL: $128 \quad 698$

TLF :

TLX: 22040 BAOAS CN

EML:

COM: 28 
MA XING-YUAN

OPT OF GEQGRAPHY

BEIJING TEACHERS COLLEGE

BALI ZHUANG

BEIJING

CHINA PR

TEL:

TLF :

TLX:

EML:

COM: 46

\section{MACCAGNI DARIO}

IST DI FISICA COSMICA

CNR

VIA BASSINI 15

120133 MILANO

ITALY

TEL: $2 \quad 298 \quad 237$

TLF :

ILX: 313839 MUACNR I

EML:

COM: 48

MACDONALD GEOFFREY H DR ELECTRONICS LABORATORY UNIVERSITY OF KENT CANTERBURY CT2 7NT UK

TEL: $227 \quad 668 \quad 22 \star 258$

TLF :

TLX: 965449 UKCLIB

EML:

COM: 40

MACHADO LUIZ E OA SILYA
UNIV FED RIO DE JANEIRO
AV SERNAMBETIBA 3300 BL7
22630 BARRA DA TIJUCA
20080 RIO DE JANEIRO RJ
BRAZIL
TEL: 213992589
TLF:
TLX:
EML:
COM: 20,24
MACIEL WALTER J OR
IAG
UNIVERSIDADE OE SAO PAULO
CP 30627
OLO5I SAO PAULO SP
BRAZIL
TEL:
TLF:
TLX:
EML:
COM: 34,46

MA YU-QIAN

INSTITUTE OF HIGH ENERGY

PHYSICS

$80 \times 9183$

BEIJING

CHINA PR

TEL: 1 B1 2971*464

TLF:

TLX: 22082 IHEP CN

EML:

COM: 44,48

MACCALLUM MALCOLM A H

SCHOOL OF MATHEMATICAL SC QUEEN MARY/WESTFIELO COLL

MILE ENO RD

LONDON EI 4NS

UK

TEL: 19804811

TLF :

TLX: 893750 QMCUOL

EML:

COM: 47

macoONALD JAMES

DPT OF PHYSICS

UNIVERSITY OF DELAWARE

NEWAR $\vec{K}$ OE 19716

USA

TEL: 302451266 ]

TLF :

TLX:

EMt:

COM: 40,42

MACHAOO MARCOS
CNIE
AVENIOA MITRE 3100
1663 SAN MIGUEL (BS AS)
ARGENTINA
TEL: 16648371
TLF:
TLX: 17511 LANBA AR
EML:
COM: $10 C$

MACK PETER DR
MDM OBSERVATORY
HC 04
BOX 7520
TUCSON AZ 85735
USA
TEL: 6026205360
TLF:
TLX: 9109521116
EML:
COM: 09

MACALPINE GORDON M

DPT OF ASTRONOMY

UNIVERSITY OF MICHIGAN

DENNISON BLDG

ANN ARBOR MI 481091090

USA

TEL: 3137643433

TLF :

TLX: $810-223-6056$

EML:

COM: 28

MACCHETTO FERDINANDO OR

STSCI

HOMEWDOD CAMPUS

3700 SAN MARTIN DR

BALTIMORE MO 21218

USA

TEL: 3013384790

TLF :

TLX； 6849101

EML:

COM: $28,40.44,48$

MALERONI CARLA

OAR

VIA DEL PARCO MELLINI 84

I O0136 ROMA

ITALY

TEL:

TLF :

TLX:

EML:

COM: 42

MACHALSKI JERZY OR ASTRONOMICAL OBSERVATORY JAGIELLONIAN UNIVERSITY UL. MAZOWIECKA $36 / 33$

PL 30019 KRAKOW

POLAND

TEL:

TLF:

TLX: 0322297 UJ PL

EML:

COM: 40

MACKAY CRAIG O DR INSTITUTE OF ASTRONOMY THE OBSERVATORIES MABINGLEY RB CAMBRIOGE CB3 OHA UK

TEL: $223 \quad 622 \quad 04$

TLF :

TLX: B17297 ASTRON G

EML:

COM
MACCACARO TOMMASO OR CENTER FOR ASTROPHYSICS

HCO/SAO

60 GAROEN ST

CAMBRIDGE MA 02138

USA

TEL: 6174957253

TLF:

TLX: 921428 SATELLITE EAM

EML:

COM: 48

MACCONNELL DARRELL J OR

STSCI/CSC

HOMEWOOD CAMPUS

3700 SAN MARTIN OR

baLTIMORE MO 21218

USA

TEL: $301338 \quad 4800$

TLF: 3013384746

TLX:

EML: SCIVAX: :MACCONKELL

COM: $24,33,45 \mathrm{P}$

MACGILLIVRAY HARVEY T OR ROYAL OBSERVATORY

BLACKFORO HILL

EOINBURGH EHS 3HJ

UK

TEL: $316 \quad 673321$

TLF:

ILX: 72383 ROEDIN G

EML:

COM:

MACIEJEWSKI ANORZEJ J BR INSIITUTE OF ASTRONOMY N COPERNICUS UNIVERSITY UL CHOPINA $12 / 18$

PL 87100 TORUN

POLAND

TEL: $26018^{*} 53$

TLF :

TLX: 552234 ASTR PL

EML: MACIEJKAEPLTUMKLI

COM: 07

MACKINNON ALEXANOER L DPT OF ASTRONOMY UNIVERSITY OF GLASGOW GLASGOW G12 BOW

UK

TEL: $413398 \quad 855$

TLF :

TLX: 777070 UNIGLA

EML:

COM: 10 


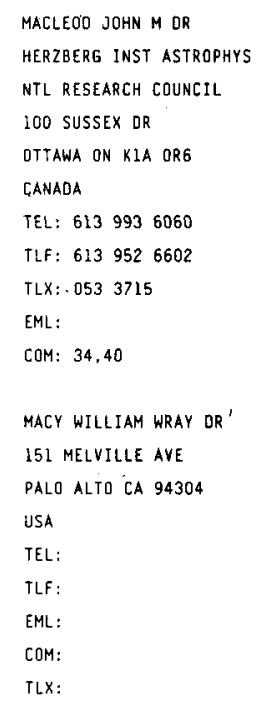

$$
\text { EML: }
$$

COM: 34,40

MACY WILLIAM WRAY OR' 151 metville aVe PALO ALTO CA 94304 USA$$
\text { TEL: }
$$$$
\text { TLF : }
$$$$
\text { EML: }
$$$$
\text { COM: }
$$$$
\text { TLX: }
$$

MAOORE BARRY FRANCIS OR DAVIO DUNLAP OBSERVATORY UNIVERSITY OF TORONTO $80 \times 360$

RICHMOND HILL ON L4C 4 YG CANADA

TEL: 4168849562

TLF: 4169783921

TLX: 06986766

EML:

COM: 27,28

\section{MAEDER ANORE PROF}

observatoire oE genEVE CHEMIN oEs maillettes $5 \mathrm{I}$ CH 1290 SAUVERNY SWITZERLAND

TEL: 227552611

YLF: 227553983

TLX: 419209 OBS CH

EML: MAEDER @CGEUGE 11

COM: $27,35 C, 37$

\section{MAGAIN PIERRE DR}

INSTITUT D'ASTROPHYSIQUE UNIVERSITE OE LIEGE

AVE COINTE 5

B 4000 COINTE-LIEGE

BELGIUM

TEL: 41529980

TLF: 41527474

TLX:

EML:

COM: 29
MACQUEEN ROBERT M DR

DPT OF PHYSICS

RHOOES COLLEGE

2000 N PARKWAY

MEMPHIS TENNESSEE 38112

USA

TEL: 9017263000

TLF:

TLX:

EML:

COM: 10,49

MADDISON RONALD CH DR

UNIVERSITY OF KEELE

2 CHURCH PLANTATION

KEELE PARK

KEELE STAFFS

UK

TEL: $782621 \quad 111$

TLF :

TLX:

EML:

COM: 46

\section{MADSEN JES}

INST OF PHYSICS \& ASTRON

UNIVERSITY OF AARHUS

NY MUNKEGAOE

DK 8000 AARHUS C

DENMARK

TEL: $86 \quad 128999$

TLF: 86202711

TLX: 64767 AAUSCI DK

EML:

COM:

MAEHARA HIOEO OR
OKAYAMA ASTROPHYSICAL OBS
NAOJ
KAMOGATA ASAKUCHI
OKAYAMA 71902
JAPAN
TEL: 865442155
TLF: $86544-2360$
TLX:
EML: MAEHARAGKIBI. OAO, NAO, AC,JP
COM: $45 C$

MAgAKIAN TIGRAN Y DR

gyuRakan astrophysical

\section{oBSERVATORY}

\section{BYURAKAN}

\section{ARMENIA}

TEL: $88 \quad 52 \quad 28 \quad 28 \quad 4142$

TLF:

TLX: 411576 ASCON SU

EML:

COM:
MACRAE DOMALD a PROF DAVIO OUNLAP OBSERVATORY UNIVERSITY OF TORONTO

BOX 360

RICHMOND HILL ON L4C $4 Y 6$ CANADA

TEL: 4168949562

TLF: 4169783921

TLX: 06986766

EML:

COM: $33,38,40$

\section{MADDOX STEPHEN DR}

DPT, OF ASTROPHYSICS

UNIVERSITY OF OXFORD

KEBLE RO

OXFORO OXI 3RH

UK

TEL: 865273310

TLF: 865273418

TLX: 83295 NUCLOX G

EML: SJMUK, AC, OX. ASTRO/19464: : SJM EML:

COM: 47

MAEDA KEI-ICHI OR

OPT OF PHYSICS

WASEDA UNIVERSITY

OKUBO 3-4-1 SHINJUKU-KU

TOKYO 160

JAPAN

TEL: $03 \quad 2034141$

TLF:

TLX: 2323280 WASEDA J

EML: BITNET: Taeda jonwasoo

COM: 47

MAETZLER CHRISTIAN DR

PHYSIKALISCHES INSTITUT

UNIVERSITAET BERN

SIOLERSTRASSE 5

CH 3012 BERN

SWITZERLAND

TEL: 31658911

TLF:

TLX: 32320 PHYBE CH

EML:

COM:

magalhaes antonio as eng OBSERVATORIO ASTRONOMICO UNIVERSIDADE DO PORTO

MONTE DA VIRGEM

P 4400 VILA NOVA GAIA

PORTUGAL

TEL: 782-0404

TLF:

TLX:

EML:

COM:
MACRIS CONSTANTIN J PROF RES CENTER FOR ASTRONOMY ACADEMY OF ATHENS

14 ANAGNOSTOPOULOU ST

GR 10673 ATHENS

GREECE

TEL: 13613589

TLF:

TLX:

EML:

COM: 10

\section{MADEJ JERZY}

ASTRONOMICAL OBSERVATORY WARSAW UNIVERSITY

AL UJAZOOWSKIE 4

PL. 00478 WARSAW

POLANO

TEL: $4822-29-40-1 \mathrm{~L}$

TLF:

TLX:

COM: 36

MAEDA KOITIRO

DPT OF PHYSICS

hYOGO COLLEGE OF MEDICINE

NISHINOMIYA

HYOGO 663

JAPAN

TEL: $798-45-6111$

ILF:

TLX:

EML:

COM:

\section{MAFFEI PAOLO PROF} CATTEDRA DI ASTROFISCA UNIVERSITA OI PERUGIA VIA DELL'ELCE DI SOTTO I 06100 PERUGIA

ITALY

TEL: 7545647

TLF :

TLX:

EML:

COM: 27,51

MagalmaEs antonio Mario OPT OF ASTRONOMY UNIVERSITY OF WISCONSIN 475 N CHARTER ST MADISON HI 53706 USA

TEL: $508 \quad 263 \quad 4680$

TLF :

TLX: 265 452 UW MAD

EMI:

COM: 


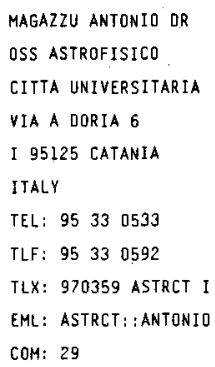

MAGGIO ANTONIO DR
OSS ASTRONOMICO
UNIVERSITA OI PALERNO
PALAZZO DEI NORMANNI
I 90134 PALERMO
ITALY
TEL: 91657 045I
TLF: 9148 8900
TLX: 910402 ASTROP I
EML: ASTROPAEIPACUC.BITNE
COM: 48
MAGNI GIANFRANCO
IAS
VIA DELL'UNIVERSITA 11
I OOI85 ROMA
ITALY
IEL:
ILX:
EML:
TLF:
COM:

MAHMOUD FAROUK M A B DR HELWAN OBSERVATORY

HELWAN

EGYPT -

TEL: $78 \quad 0645 / 2683$

TLF:

EML:

COM: 27

TLX:

\section{MAITZEN HANS $M$ DR}

INSTITUT FUER ASTRONOMIE TUERKENSCHANZSTR 17

\section{A 1180 WIEN}

AUSTRIA

TEL: 134536094

TLX: 116222 PHYSI A

EML:

TLF:

COM: 25,29

\section{MAKAROV VALENTINE I}

XISLOVOOSK STATION OF THE PULKOVO OBSERVATORY

357741 KISLOVOOSK

RUSSIA

TEL:

TLF:

TLX:

EML:

COM: 10,12
MAGNAN CHRISTIAN DR

GRAAL CC 72

USTL II

PLACE EUGENE BATAILLON

F 34095 MONTRELLIER COX 5

FRANCE

TEL: $67 \quad 143902$

TLF:

TLX:

EML:

COM:

MAGUN ANDREAS DR

PHYSIKALISCHES INSTITUT

UNIVERSITAET BERN

SIDLERSTRASSE 5

CH 3012 BERN

SWITZERLAND

TEL: 31658923

TLF:

TLX:

EML:

COM:

MAHRA HS QR

UTTAR PRADESH STATE

OBSERVATORY

PO MANORA PEAK 263129

NAINITAL 263129

INDIA

TEL: 5942 2136/2583

TLF :

TLX:

EML:

COM: $09,16,20,27,50$

MAJIO ABDUL BIN A H OR JABATAN UKUR DAN PEMETAAN JALAN SEMARAK

50578 KUALA LUMPUR

MALAYSIA

TEL: 032926327

TLF:

TLX:

EML:

COM: 04

MAKAROVA ELENA A OR

STERNBERG STATE ASTR INST

117234 MOSCOW

RUSSIA

TEL: 139-1973

TLF :

TLX: 113037 JAPET

EML:

COM: 12
MAGNANI LORIS ALBERTO DR DPT PHYSICS \& ASTRONOMY UNIVERSITY OF GEORGIA ATHENS GA 30602 USA

TEL: 4045422876 TLF: 4045422492

TLX: 414190

EML: LORISAJOVE. PHYSAST. UGA. EDU COM:

MAHAT ROSLI H DR DPT OF ASTRONOMY UNIVERSITY OF MALAYA 59100 KUALA LUMPIJR MALAYSIA

TEL:

TLF:

TLX:

EML:

COM:

MAIHARA TOSHINORI OR OPT OF PHYSICS KYOTO UNIVERSITY SAKYO KU KYOTO 606 JAPAN TEL: 075-751-2111 TLF : TLX: 5422693 LIBKYU O EML: COM: 21,34

MAJOR JOHN DR DPT OF PHYSICS UNIVERSITY OF DURHAM SOUTH RD DURHAM DHI 3LE UK TER: 913742111 TLF :

TLX:

EML: COM: 09

MAKINO FUMIYOSHI QR ISAS 3-1-1 YOSHINODAI SAGAMIHARA KANAGAWA 229 JAPAN TEL: $81-427 \quad 513911$ TLF: $81-427594253$ TLX: 34757 ISASTRO J EML: COM: 
MAKISHIMA KAZUD

INST SPACE \& ASTRON SCI

UNIVERSITY OF TOKYO

MEGURO KU

TOKYO 253

JAPAN

TEL: $03 \cdot 467-1111 \times 303$

TLF:

TLX: 34757 ISASTRO J

EML:

COM:

\section{MALAISE DANIEL J DR}

INSTITUT O'ASTROPHYSIQUE UNIVERSITE DE LIEGE

AVE COINTE 5

B 4000 COINTE-LIEgE

BELGIUM

TEL: 41529980

TLF: $41 \quad 527474$

TLX:

EML:

C.OM: 15,44

MALIN DAVID F MR

AAO

BOX 296

EPPING NSW 2121

AUSTRALIA

TEL: 2 86B 1666

ILX: 23999 AA

EML:

TLF:

COM: 09

MALKAMAEKI LAURI J DR ELSON RESEARCH INC BOX 167

SALEM NH 030799998

USA

TEL: 6038930766

TLF: 6038932131

TLX:

EML:

COM: 09

MALTBY PER PROF -

INST THEORET ASTROPHYSICS

UNIVERSITY OF OSLO

BOX 1029

N 0315 BLINDERN OSLO 3

NORWAY

TEL: $2-456509$

TLF :

TLX:

EML:

COM: 10
MAKITA MITSUGU DR

KWASAN \& HIDA OBS

KYOTO UNIVERSITY

YAMASHINA

XYOTO 607

JAPAN

TEL: $075-581-1235$

TLF:

TLX:

EML:

COM: 10,12

\section{MALAKPUR IRADJ OR}

INSTITUTE OF GEOPHYSICS

UNIVERSITY OF TEHRAN

KARGAR SHOMALI

TEHRAN 14394

IRAN

TEL: $2163 \quad 1081 / 3$

TLF:

TLX: 215319 UTIG

EML:

COM:

\section{MALIN STUART}

national maritime museum

GREENWICH

LONDON SELO 9NF

UK

TEL: $1858 \quad 1167$

TLX:

EML:

TLF:

COM: 41

MALKAN MATTHEW ARNOLD DR OPT OF ASTRONOMY

UNIVERSITY OF CALIFORNIA

LOS ANGELES. CA 90024

USA

TEL: $213 \quad 825 \quad 3404$

ILF :

TLX:

EML: MALKANGASTRO. UCLA. EOU

COM: 44

MALVILLE J MCKIM PROF
CASA
UNIVERSITY OF COLORADO
BOX 391
BOULDER CO 803090391
USA
TEL: 3034928788
TLF:
TLX:
EML:
COM: 10

MALACARA DANIEL

CENTRO DE INVESIIGACIONES

EN OPTICA

APDO POSTAL 948

37000 LEON GTD

MEXICO

TEL: $758-23$

TLF:

TLX:

EML:

COM:

MALARODA STELLA M DR

COMPLEJO ASTRONOMICO

EL LEONCITO

CC 467

5400 SAN JUAN

ARGENTINA

TEL: $64 \quad 225718$

TLF :

TLX: 59134 ENTOP AR

EML:

COM: 29,45

MALINA ROGER FRANK DR

CTR FOR EUV ASTROPHYSIES UNIVERSITY OF CALIFORNIA

2150 KITTREDGE ST

BERKELEY CA 94720

USA

TEL: 5106435636

TLF: 5106435660

TLX:

EML: RMALINAGSSL, BERKELEY, EOU

COM:

MALLIA EOWARO A DR

OPT OF ASTROPHYSICS

UNIVERSITY OF OXFORD

SOUTH PARKS RO

OXFORO OXi $3 R O$

UK

TEL:

TLF:

ILX:

EML:

COM:

MAMMANO AUGUSTO OR

OSSERVATORIO ASTROFISICO

VIA DELL OSSERVATORIO 8

I 36012 ASIAGO

ITALY

TEL: $42 \quad 46 \quad 2665$

TLF :

TLX:

EML:

COM: 42
MALAGNINI MARIA LUCIA

OAT

BOX SUCC TRIESTE 5

VIA TIEPOLO 11

I 34:3I TRIESTE

ITALY

TEL: $40 \quad 793921$

TLF :

TLX: 461137 OAT I

EML:

COM:

MALHERBE JEAN MARIE DR OBSERVATOIRE DE PARIS

SECTION DE MEUDON

DASOP

F 92195 MEUDON PPL COX

FRANCE

TEL: $145 \quad 07 \quad 7796$

TLF :

TLX: 201571

EML: BITNET: MALHERBEERMEU5I

COM: 10

MALITSON HARRIET H MS

13315 MAGELLAN AVE

ROCKVILLE MO 20853

USA

TEL: $301946 \quad 0496$

TLF:

TLX:

EML:

COM: 10,44

MALLIK OC V OR

INDIAN INSTITUTE OF

ASTROPHYSICS

KORAMANGALA

BANGALORE 560034

INDIA

TEL: $81256 \quad 6585 / 6497$

TLF:

TLX: 845763 IIAB IN

EML:

COM: 34,35

MANABE SEIJI QR

INTL LATITUDE OBSERVATORY

NAOJ

HOSHIGAOKA MIZUSAWA SHI

IWATE D23

JAPAN

TEL:

TLF :

TLX:

EML:

COM: 19 
MANARA ALESSANDRO A DR

OSS ASTRONDMICO OI BRERA

VIA BRERA 28

I 20121 MILANO

ITALY

TEL: 2874444

ILX:

EML:

TLF: 272001600

COM: 20.44

MANCUSO SANTI PROF
OSS ASTRONOMICO
OI CAPODIMONTE
VIA MOIARIELLO 16
I 80131 NAPOLI
ITALY
TEL: 44 0101
TLF:
TLX:
EML:
COM:
MANGENEY ANORE' DR
OBSERVATOIRE OE PARIS
SECTION OE MEUDON
F 92195 MEUDON PDL COX
FRANCE
TEL: 1 45 O7 7661
TLF:
TLX:
EML:
COM: 49

MANRIQUE WALTER T PROF OBSERVATORTO ASTRONOMICO FELIX AGUILAR

AV BENAVIDEZ 8175 OESTE 5407 MARQUESADO (S.J.) ARGENTINA

TEL: 64231494

TLF :

TLX:

EML:

COM: 08

MAO WEI
YUNNAN O8SERVATORY
CAS
BOX 110
KUNMING 72946 YUNNAN
CHINA PR
TEL: 8712035
TLF:
TLX:
EML:
COM: 08

MANCHAOO ARTURO OR

INST OE ASTROFISICA

DE CANARIAS

OBS DEL TEIDE

E 38200 LA LAGUNA

SPAIN

TEL: $22 \quad 26 \quad 2211$

TLF: $22 \quad 263005$

TLX: 92640 IAC E

EML:

COM: 34

MANDOLESI NAZZARENO

IST TE S RE

CNR

VIA DE CASTAGNOLI 1

I 40126 BOLDGNA

ITALY

TEL: 51238022

TLF :

FLX: 511350

EML:

COM: $40,44,47$

MANN GOTTFRIED OR

ZNTRLINST F ASTROPHYSIK

OBSERVATORIUM FUER SOLARE

RADIO ASTRONOMIE

D 1501 TREMSDORF

GERMANY

TEL:

TLF :

TLX: 15420

EML:

COM: 10

MANSFIELD VICTOR N PROF DPT OF PHYSICS \& ASTRON COLGATE UNIVERSITY HAMILTON NY 13346 USA

TEL: $315824 \quad 1000$

TLF :

TLX:

EML:

COM:

MARABINI RODOLFO JOSE
UNIV NACIONAL OE LA PLATA
FCAG
1900 LA PLATA (BS.AS.)
ARGENTINA
TEL: 21217308
TLF:
TLX:
EML:
COM:

\section{MANCHANDA $R \quad K$ DR}

TIFR

HOMI BHABHA RD

COLABA

BOMBAY 400005

INDIA

IEL: $22 \quad 219 \quad 111 * 336$

TLF:

TLX: 113009 TIFR IN

EML:

COM:

MANDZHOS ANDREJ $V$ 'DR

SPECIAL ASTROPHYSICAL OBS

ACADEMY OF SCIENCES

NIZHNIJ ARKHYZ

357147 STAVROPOLSKIJ

RUSSIA

TEL: $86 \quad 578 \quad 92501$

TLF :

TLX: 123244 ZENIT

EML: AMANDESAO.STAVROPOL.SU

COM: 47

MANN PATRICK J OR

DPT OF ASTRONOMY

UNIV OF WESTERN ONTARIO

LONDON ON NEA 3K7

CANADA

TEL: 5196613183

TLF: 5196613486

TLX: 0647134

EML: BITNET: 2014-562@UWOVAX

COM:

MANTEgAZZA LUUCIANO

OSS ASTRONOMICO DI MILANO

VIA E BIANCHI 46

I 22055 MERATE

ITALY

TEL: 592035

TLF :

TLX:

EML:

COM: 27

MARAN STEPHEN P QR

NASA/GSFC

COOE 680

GREENBELT MO 20771

USA

TEL: 3012868607

TLF:

ILX: 89575

EML:

COM: $15,40,44$
MANCHESTER RICHARD N DR

CSIRO

DIVISION DF RADIOPHYSICS

BDX 76

EPPING NSW 2121

AUSTRALIA

TEL: 2 B6B 0225

TLF :

TLX: 26320 ASTRO

EML: RMANCHES @ATNF.CSIRO.AU

COM: $33,34,40$

MANFROIO JEAN DR

INSTITUT D'ASTROPHYSIQUE

UNIVERSITE OE LIEGE

AVE COINTE 5

B 4000 COINTE-LIEgE

BELGIUM

TEL: 41529980

TLF: $4152 \quad 7474$

ILX: 41264 ASTRLG

EML:

COM: 25.34

MANNINO GIUSEPPE PROF IST MATEMATICO

VIA CAMPI 181

I 41100 MODENA

ITALY

TEL:

TLF :

TLX:

EML:

COM: 27

MANTOVANI FRANCO

IST OI RAOIOASTRONOMIA

CNR

VIA IRNERIO 46

I 40126 BOLOGNA

ITALY

TEL: 51232856

TLF :

TLX: 211664 INFNBO I

EML:

COM:

MARAND BRUND

OPT OI ASTRONOMIA

UNIVERSITA DI BOLOGNA

CP 596

I 40100 BOLOGNA

ITALY

TEL: 51222956

ILF:

TLX: 211664 INFNBD I

EML:

COM: 47 


MARAR T M K
ISRO SATELLITE CTR
AIRPORT RO
VIMANAPURA POST
BANGALORE 560017
INDIA
TEL: $81256 \quad 6251-$
TLF:
TLX:
EML:
COM: 44
MARCHAL CHRISTIAN OR
OPT ETUDES DE SYHTHESE
ONERA
F 92320 CHATILLON
FRANCE
TEL: 14657 1160
TLF:
TLX: 260907
EML:
COM: 07

MARGONI RINO

OSSERVATORIO ASTROFISICO

VIA DELL OSSERYATORIO 8

I 36012 ASTAGO

ITALY

TEL: $42 \quad 462665$

FLF:

TLX: 430110 SETOUR

EML:

COM:

MARILLI ETTORE OR
OSS ASTROFISICO
CITTA UNIVERSITARIA
VIA A DORIA 6
I 95125 CATANIA
ITALY
TEL: 95330533
TLF:
TLX: 970359 ASTRCT I
EML:
COM: $12,29,42$
MARISKA JOHN THOMAS
NAVAL RESEARCH LABORATORY
CODE $4175 M$
4555 OVERLOOK AVE SW
WASHINGTON DC 203755000
USA
TEL: 2027672605
TLF:
TLX:
EML:
COM: 10,12

MARASCHI LAURA OR
IST DI FISICA
UNIVERSITA DI MILANO
VIA CELORIA 16
I 20133 MILANO
ITALY
TEL: 22392275
TLF: 22366583
TLX:
EML:
COM:

\section{MARDIROSSIAN FABIO}

OPT DI ASTRONOMIA

UNIVERSITA OI TRIESTE

VIA TIEPOLO 11

I 34131 TRIESTE

ITALY

TEL: $40793921 * 221$

TLF:

TLX: 461137 DAT I

EML:

COM: 42.47

MARGRAVE THOMAS EWING JR
400 JOHNSON ST
VIENNA VA 22180
USA
TEL:
TLF:
EML:
COM: $27.5 \mathrm{I}$
TLX:

MARINO BRIAN F ENG
AUCKLAND OBSERVATORY
BOX 72009
NORTHCOTE
AUCKLAND 9
NEW ZEALAND
TEL: 649486951
TLF:
TLX:
EML:
COM: 42

$\begin{array}{ll}\text { MARK IAMES WAI-KEE OR } & \text { MARKELLOS VASSILIS } \vee \text { OR } \\ \text { LAWRENCE LIVERMORE LAB } & \text { OPT ENFINEERING SCIENCE } \\ \text { L } 477 & \text { UNIVERSITY OF PATRAS } \\ \text { BOX } 808 & \text { GR } 260 \text { OO RION } \\ \text { LIVERMORE CA } 94550 & \text { GREECE } \\ \text { USA } & \text { TEL: } 61991465 \\ \text { TEL: } 4154225931 & \text { TLF: } \\ \text { TLF: } & \text { TLX: } \\ \text { TLX: } 910-386-8339 \text { UCCLLL } & \text { EML: } \\ \text { EML: } & \text { COM: } 07\end{array}$

MARCAIDE JUAN-MARIA DR. INST ASTROFISICA DE ANOALUCIA APO 2144 PROFESOR ALBAREDA 1

E 18080 GRANADA SPAIN

TEL: $58 \quad 12 \quad 1311$

ILF:

TLX: 78573 IAAG E

EML:

COM: 40

MAREK JOHN

44 PERCY RD

WREXHAM CLWYO

UK

TEL:

TLF :

TLX:

EML:

COM: 47

MARIE M A OR

DPT OF ASTRONOMY

FACULTY OF SCIENCES

CAIRO UNIVERSITY

GEZA

EGYPT'

TEL:

TLF :

TLX:

EML:

COM:

MARIOTTI JEAN MARIE DR OBSERVATOIRE DE PARIS SECTION DE MEUDON

F. 92195 MEUOON PPL CDX FRANCE

TEL: 145077570

TLF :

TLX: 204464

EML: BITNET: MAR IOTTI FRMEU5I

COM:

COM: 33
MARCELIN MICHEL

OBSERVATOIRE DE MARSEILLE

2 PLACE LE VERRIER

F 13248 MARSEILLE COX 04

FRANCE

TEL: 91959088

TLF :

TLX: $420241 F$

EML:

COM: 28

MARGON BRUCE H PROF

OPT OF ASTRONOMY

UNIVERSITY OF WASHINGTON

FM 20

SEAITLE WA 98195

USA

TEL: 2065430089

TLF :

TLX: 4740096

EML: MARGON JANUS. ASTRO, WASHINGTON.

COM:

MARIK MIKLOS DR

ASTRONOMY OPT

EOTVOS UNIVERSITY

KUN BELA TER 2

H 1083 BUDAPEST

HUNGARY

TEL: 141019

TLF :

TLX:

EML:

COM: 12,38

MARIS GEORGETA OR ASTRONOMICAL OBSERVATORY CUTITUL DE ARGINT 5

BOX 28

R 75212 BUCURESTI 28 RUMANIA

TEL: 90236010

TLF:

TLX: 11882 ASTRO $R$

EML:

COM: 10

MARKERT THOMAS H DR CENTER FOR SPACE RESEARCH MIT RM 37621

BOX 165

CAMBRIDGE MA 02139

USA

TEL: 6172535169

TLF :

TLX:

EML: THHOSPACE.MIT,EDU

COM: 
MARKKANEN TAPIO DR OBS \& ASTROPHYSICS LAB UNIVERSITY OF HELSINKI TAEHT I TORNI IMAKI

SF 00130 HELSINKI 13

FINLANO

TEL: 0908391

TLF:

TLX:

EML:

COM: $25,37,50$

MAROCHNIK LS PROF OR SPACE RESEARCH INSTITUTE ACADEMY OF SCIENCES PROFSOJOSNAYA UL 84/32 117810 MOSCOW RUSSIA

TEL: $333-31-22$

TLF:

TLX: $41149 B$ STAR SU

EML:

COM: 33

MARRACO HUGO G DR.

UnIV NaCIONAL OE LA PLATA

FACULTAD OE CIENCIAS

ASTRONOMICAS Y GEOFISICAS

1900 LA PLATA (BS.AS.)

ARGENTINA

TEL: 21217308

TLF:

TLX: MI BULAP AR

EML:

COM: 25,37

MARSDEN PHILIP L PROF

OPT OF PHYSICS

University OF LeEDS

LEEOS LS? פנT

$\begin{array}{llll}\text { UK } & \cdot \\ \text { TEL: } & 532 \quad 431751\end{array}$

ILF:

TLX: 556473 UNIOS

EML:

COM:

MARSHALL KEVIN P

INSTITUTE OF ASTRONOMY

THE OBSERVATORIES

MADINGLEY RO

CAMBRIDGE CB3 OHA

UK

TEL: 223337548

TLF:

TLX: 817297 ASTRON G

EML: K.PMOUK. AC. CAM. AST-STAR

COM: 37
MARKOWITZ WILLIAM DR

APT 15 B

2800 E SUNRISE BLVD'

FORT LAUDERDALE FL 33304

USA

TEL: 3055632859

TLF:

TLX:

EML:

COM: 19,31

MAROY MIKHAIL YA PROF

INST OF APPLIEO MATHS

ACADEMY OF SCIENCES

MIUSSKAYA SQ 4

125047 MOSCOW

RUSSLA

TEL: 0952500485

TLF: 0959720737

TLX:

EML:

COM: 16VP, 44,51, HGPSNC "

MARSCHALL LAURENCE A

DPT OF PHYSICS

GETTYYSBURG COLLEGE

GETIYSB̈URG PA 17325

USA

TEL: 7173371865

TLF:

TLX:

EML:

COM: 24,30

MARSH JUL JAN C D

hatFiELO POLYTECHAIC

OBSERVATORY

BAYFORDBURY

HERTFORO HERTS SG13 8LD

uK

TEL: 992558451

TLF :

TLX: 262413

EME:

COM: 46

MARSOGLU A OR

UNIVERSITY OBSERVATORY

UNIVERSITY OF ISTANBUL.

34452 ISTANBUL

TURKEY

TEL: 15223597

TLF:

TLX:

EML:

COM:
MARLBOROUGH J M PROF

OPT OF ASTRONOMY

UNIV OF WESTERN ONTARIO

LONOON ON MEA 3KT

CANADA

TEL: 5196793184

TLF: 5196613486

TLX: 0647134

EML:

COM: 36

MARQUES DOS SANTOS P PRBF IAG

universidade oE sao paulo

CP 30627

Dio5l say paulo sp

BRAZIL

TEL: 112763941

TLF:

TLX: 36221 IAGM BR

EML:

COM: 28,40

MARSCHER ALAN PATRICK

OPT OF ASTRONOMY

BOSTON UNIVERSitY

725 COMMONWEALTH AVE

BOSTON MA O2215

USA

TEL: $617 \quad 3535029$

TLF:

YLX: 951289 BOS UNIV BSN

EML:

COM: 40

MARSH THOMAS OR

OPT OF ASTROPHYSICS

UNIVERSITY OF OXFORD

KEBLE RO

OXFORO OXI $3 R H$

uK

TEL: 865273303

TLF: 865273418

TLX: 83295 NUCLOX G

EML: 19464 : :TRM

COM: 42

MARSTON ANTHONY PHILIP OR DPT PHYSICS \& ASTRONOMY DRAKE UNIVERSITY

DES MOINES IA 503 LI

USA

TEL: 5152713034

TLF:

TLX:

EML: TMgggIR@RAKE

COM: 34
MARMOLINO CIRO

OPT DI FISICA

UNIVERSTIA DI NAPOLI

MOSTRA D OLTREMARE PAD 19

I 80125 NAPOLI

ITALY

TEL: 817253428

TLF :

TLX: 720320 INFNNA I

EML:

COM: 12

MARQUES MANUEL N DR

OBSERVATORIO ASTRONOMICO

TAPADA DA AJUDA

P I300 LISBOA 3

PQRTUGAL

IEL:

TLF :

TLX:

EML:

COM:

MARSDEN BRIAN G DR

CENTER FOR ASTROPHYSCIS

HCO/SAO

60 GARDEN ST

CAMBRIOGE MA 02138

USA

TEL: 6174957244

TLF :

TLX: 7103206842 ASTROGRAM

EML: CFAPS1: : BRIAN

COM: 06C, 07, 15,20C

MARSHALL HERMAN LEE DR

CTR FOR EUV ASTROPHYSICS UNIVERSITY OF CALIFORNIA

2150 KITTREOGE ST

BERKELEY CA 94720

USA

TEL: $415 \quad 643 \quad 5671$

TLF: 4156435660

TLX: 910414 061 UCBSAG

EML: HERMANMASSL, BERELEY,EQU

COM:

MARTENS PETRUS C OR

LOCKHEED PALO ALTO RES LB

DPT 9130 BLOG 252

3251 HANOVER ST

PALO ALTO CA 94304

USA

TEL: $415 \quad 3545819$

TLF: $415 \quad 4243994$

TLX: .

EML: 24707: : MARTENS

COM: 10 
MARTIN ANTHONY $R$ OR

UK CULHAM LABORATORY

\section{RM F4/135}

ABINGOON OXI4 $30 B$

TEL: $235 \quad 21840$

TLF:

TLX: 83189

EML:

COM: 51

MARIIN MARIA CRISIINA OR

IAR

CC 5

1894 VILLA ELISA (BS AS)

ARGENTINA

TEL: 2143793

TLX: 31216 CESLA AR

EML:

TLF:

COM: 28,51

MARTIN WILLIAM C DR

NTL BUREAU OF STANDARDS

PHYSICS BLOG ALE7

GAITHERSBURG MD 20899

USA

TEL: 3019212011

TLF :

TLX:

EML:

COM: 14

MARTINEZ ROGER CARLOS DR INST OE ASTROFISICA

OE CANARIAS

OBS OEL TEIDE

E 38200 LA LAGUNA

SPAIN

TEL: $22 \quad 26 \quad 2211$

FLF:

TLX: 92640

EML:

COM:

MARTIN-DIAZ CARLOS OR

INST DE ASTROFISICA

DE CANARIAS

OBS DEL TEIDE

E 38200 LA LAGUNA

SPAIN

TEL: $22 \quad 26221 \mathrm{~L}$

TLF: $22 \quad 263005$

TLX: 92640 IACE

EML: SPAN: IAC : CMD

COM:
MARTIN DEREK. H PROF

OPT OF PHYSICS

QUEEN MARY/WESTFIELD COLL

MILE END RD

LONDON EI 4NS

UK

TEL:

TLF :

TLX:

EML:

COM:

MARTIN NICOLE OR OBSERVATOIRE DE MARSEILLE

2 PLACE LE VERRIER

f 13248 marSeILLE COX 04 FRANCE

TEL: $9195908 B$

TLF:

TLX: 420241

EML:

COM: 30

MARTIN WILLIAM L OR
ROYAL GREENWICH OBS
HERSTMONCEUX CASTLE
HAILSHAM BN27 IRP
UK
TEL: 323833171
TLX: 87451 RGOBSY G
EML:
TLF:
COM: 27

MARTINEZ-GONZALEZ E OR

OPT DE FISICA MODERNA

UNIVRESIOAD DE CANTABRIA

AVD LOS CASTROS S/N

E 39005 SANTANDER

SPAIN

TEL: $42 \quad 201468$

TLF : $42 \quad 201402$

TLX: 35861 EOUCI E

EML: 16438: : CCUCVX::E_MARTINEZ

COM: 47

MARTIN-LORON M OR

HERMANOS MIRALLES I4

E MADRID 1

SPAIN

TEL:

TLF :

EML:

COM:

TLX:
MARFIN FRANCDIS DR

OPT ASIROPHYSIQUE

UNIVERSITE OE NICE

PARC VALROSE

F 06034 NICE CÓX

FRANCE

TEL: 93519100

TLF :

TLX: 970281

EML:

COM:

MARTIN PETER G PROF

CITA MCIENNAN LABS

UNIVERSITY OF TORONTO

60 ST GEORGE ST

TORONTO ON M5S IAT

CANADA

TEL: 4169786840

TLF: $416 \quad 9783921$

TIX:

EML:

EOM: $34 \mathrm{C}$

MARTINET LOUIS PROF

observatoIRE DE gENEVE

CHEMIN DES MAILLETTES 51

$\mathrm{CH} 1290$ SAJVERNY

SWITZERLAND

TEL: 227552611

TLF: 227553983

TLX: 419209 OBS CH

EML: martinetoobs.unige.ch

COM: $28,33,46$

MARTINI ALOO OR

IAS

CNR

CP 67

I 00044 fRASCATI

ITALY

IEL: 69425655

TLF :

TLX:

EML:

COM:

MARTIN-PINTADO JESUS

CTR ASTRON DE YEBES

OAN

APO 148

E 19080 gUAdALAJARA

SPAIN

TEL: 11223358

TLF :

TLX:

EML

COM: 34,40
MARTIN INACIO MALMONGE OR INSTITUTO OE FISICA UNIVERSIDADE EST CAMPINAS CP 1170

13100 CAMPINAS SP

BRAZIL

TEL: 192391301

TLF:

TLX: $019-1150$

EML:

COM: 48

MARTIN ROBERT N DR STEWARD OBSERVATORY UNIVERSITY OF ARIZONA TUCSON AZ 85721

USA

TEL: 6026211539

TLF:

TLX: 467175

EML:

COM: 34,40

MARTINEZ MARIO DR OPT DE GEOFISICA

CIESE

APDO POSTAL 2732

22860 ENSENADA B CALIF

MEXICO

TEL:

TLF:

TLX:

EML:

COM :

MARTINS DONALD HENRY DR DPT PHYSICS \& ASTRONOMY UNIVERSITY OF ALASKA 3221 UAA' DRIVE ANCHORAGE AK 99508 USA

TEL: $907786 \quad 1238$

TLF :

TLX:

EML:

COM: 09,37

MARTRES MARIE-JOSEPHE OBSERVATOIRE DE PARIS SECTION DE MEUDON

F 92195 MEUDON PPL CDX FRANCE

IEL: $145 \quad 347530$

TLF :

TLX:

EML:

COH: 10 
MARVIN USRSULA B DR CENTER FOR ASTROPHYSICS HCO/SAD

60 GARDEN ST CAMBRIDGE MA 02138 USA

TEL: 6174957270

TLF :

TLX: 921428 SATELLITE CAM EML:

COM: 22

MASEgOSA GALLEGO J DR INST ASTROFISICA OE ANDALUCIA APQ 2144 C/SANCHO PANZA S/N E 18080 GRANADA SPAIN

TEL: $58 \quad 12 \quad 1311$

TLF: 58 B1 4530

TLX: 78573 IAAG E EML:

COM: 28

MASON GLENN M

ASTRONOMY PROGRAM

UNIVERSITY OF MARYLAND COLLEGE PARK MD 20742 USA

TEL: 3014542616

TLF :

TLX: $71-8261125$

EML:

COM: 10.48 .49

MASSAGLIA SILVANO

IST OI FISICA GENERALE

CORSO D AZEGLIO 46

I 10125 TORINO

ITALY

TEL: 11657694

TLX: 211041

EML :

TLF:

COM: 36

MASSON COLIN R

CENTER FOR ASTROPHYSICS

HCO/SAD

60 GARDEN ST

CAMBRIDGE MA 02138

USA

TEL: 6174957000

TLF :

TLX:

EML:

COM: 34,40
MARX GYORGY PROF

EOTVOS UNIVERSITY

PUSHKIN U 5-7

H 1088 BUDAPEST

HUNGARY

TEL: 1187902

TLF: $118 \quad 0206$

TLX: 225459 ATOMF H

EML: KUERT IEAWIRAF

COM: $35.51 \mathrm{C}$

MASLOWSKI JOZEF OR

ASTRONOMICAL OBSERVATORY

JAGIELLONIAN UNIVERSITY

UL ORLA 171

PL 30244 KRAKOW

POLAND

TEL: $34-10-41$

TLF :

TLX: 0322297 UJ PL

EML:

COM: 40

MASON HELEN E DR

DPT APPLIED MATHS

* theoretical physics

SILVEK STREET

CAMBRIOGE CB3 9EW

UK

TEL: $223 \quad 351645$

TLF:

rLX: 81240

EML:

COM: 14

MASSAGUER JOSEP PROF

DPT FISICA APLICADA

UNIV POLITEC DE CATALUNYA

E 08034 BARCELONA

SPAIN

TEL: 34016827

TLF: 34016090

TiX:

EML: MASSAGUER FA.UPC.ES

COM:

MATAS VLADIMIR R DR

ASTRONOMISCHES-RECHEN

INSTITUT

MOENCHHOFSTR $\quad 12 \cdot 14$

06900 HEIDELBERG I

GERMANY

TEL: 62.2149026

TLF:

TLX:

EML:

COM: 07
MARX SIEGFRIED DR

ZNTRLINST, F, ASTROPHYSIK

KARL-SCHWARZSCHILO OBS

D 6901 TAUTENBURG

GERMANY

TEL: 7823530

TLF:

TLX: $58 B 6284$ KSOT DD

EML:

COM: 50

MASNOU FRANCOISE DR

28 ALLEE GAMBAUBERIE

F 91190 GIF/YYETTE

FRANCE

TEL:

TLF:

TLX:

EML :

COM:

MASON JOHN WILLIAM OR

51 ORCHARO WAY

WEST BARNHAM

BOGNOR REGIS

WEST SUSSEX PO2Z DHX UK

TEL: $243 \quad 553244$

TLF; 243554272

TLX:

EML:

COM: 22

MASSEVICH ALLA G QR

INST OF ASTRONOMY

ACADEMY OF SLIENCES

PYATNITSKAYA UL 48

109017 MOSCOW

RUSSIA

TEL: $231-54-61$

TLF :

TLX: 412623 SCSTP SU

EML:

COM: 35

MATERNE JUERGEN OR

ARETINSTR 27

D 8000 MUENCHEN 90

GERMANY

TEL:

TLF :

TLX:

EML

COM: 47
MASANI A PROF

OSS ASTRONOMICO DI BRERA

VIA BRERA 28

I 20100 MILANO

ITALY

TEL:

TLF:

TLX:

EML:

COM: $25,27,35$

MASNOU J L DR

OBSERVATOIRE OE PARIS

SECTION DE MEUDON

ER 176 DARC

F 92195 MEUDON PPL COX

FRANCE

TEL: $1 \quad 45 \quad 347570$

JLF :

ILX: 201571

EML:

COM:

MASON KEITH OWEN

MULLARO SPACE SCIENCE LAB

UNIVERSITY COLLEGE LONOON

HOLMBURY ST MARY

DORKING SURREY RH5 GNT

JEL: 30670292

TLF:

ILX: 859185

EML:

COM: 48

MASSEY PHILIP L

KITT PEAK NTL OBS

BOX 26732

$950 \mathrm{~N}$ CHERRY AVE

TUCSON AZ $85726 \quad 6732$

USA

TEL: 6023275511

TLF :

TLX:

EML:

COM: 29

MATHER JOHN CROMWELL

NASA/GSFC

CODE 685

LASP

GREENBELT MD 20771

USA

TEL: $301 \quad 286 \quad 8720$

TLF :

TLX: 89675

EML:

COM: $21,34,44,47$ 


MATTEUCCI FRANCESCA OR
IAS
CNR
CP 67
I 00044 FRASCATI
ITALY
IEL: 69425655
TLF:
TLX:
EML:
COM: $33 C, 35,37$

MATTHEWS THOMAS A DR

ASTRONOMY PROGRAM UNIVERSITY OF MARYLAND COLLEGE PARK MO 20742 USA

TEL:

TLF :

TLX:

EML:

COM:

MATZ STEVEN MICHEAL OR OPT PHYSICS \& ASTRONOMY NORTHWESTERN UNIVERSITY DEARBORN OBSERVATORY EVANSTON IL 60208 USA TEL: 7084918643 TLF :

TLX:

EML: MATZOOSSENU. ASTRO. NWU. EOU COM: $05,09,4 \mathrm{~B}$

\section{MAUERSBERGER RAINER DR} MPI FÖR RADIOASTRONOMIE AUF OEM HUGEL 69 D 5300 BONN 1 GERMANY

TEL: 2285251

TLX: 886440

EML: P373MAUUPIRBN.MPIFR-BONN.MPG. TLX: $420241 \mathrm{~F}$

TLF: 49228525229 EML:

COM: 40

EML:
COM: 28,30
MATTHEWS CLIFFORO PROF OPT OF CHEMESTRY M/CLII UNIVERSITY OF ILLINOIS BOX 4338 CHICAGO IL 60680 USA

JEL: 3129963161 TLF: 3129960431 TLX: .

EML:

COM: 51

MATTIG W PROF DR KIEPENHEUER INSTITUT FUER SONNENPHYSIK SCHOENECKSTRASSE 6 D 7800. FREIBURG BREISGAU GERMANY

TEL: 76132864

TLF:

TLX: 7721552 K.1S 0

EML:

COM: $10,12,50$

MATZNER RICHARD A PROF
ASTRONOMY DPT
UNIVERSITY OF TEXAS
RLM 15308
AUSTIN TX $78712 \cdot 1083$
USA
TEL: 5124715062
TLF:
TLX:
EML:
COM: 47

MAURICE ERIC N

observatoire de marseille

2 PLACE LE VERRIER

F 13248 MARSEILLE EDX O4 FRANCE

TEL: 91959088

TLF:

MAVRIOIS L N PROF OPT GEODETIC ASTRONOMY UNIVERSITY THESSALONIKI UNIV BOX 503 GR 54006 THESSALONIKI GREECE

TEL: 31992693

TLF:

TLX:

EML:

COM: $08,27,33,46,51$
MATTHEWS HENRY E DR

JOINT ASTRONOMY CENTER

JCMT

665 KOMOHANA ST

HILO HI 96720

USA

TEL: 8089613756

TLF:

TLX: 633135

EML:

TOM: 40

MATIILA KALEVI OR

OBS \& ASTROPHYSICS LAB UNIVERSITY OF HELSINKI TAEHTI TOBNI MMAKI SF 00130 HELSINKI 13 FINLAND

TEL: 1912947

TLF:

TLX: 124690 UNIH SF

EML:

COM: $21,34,40$

MAUCHERAT J DR

LAS

TRAVERSE DU SIPHON

LES TROIS LUCS

f 13012 MARSEILLE

FRANCE

TEL: 91055900

TLF: $91 \quad 66 \quad 1855$

TLX:

EML:

COM: 21

MAURON NICOLAS DR OBS MIDI PYRENEES

14 AVE E BELIN

F 31400 TOULOUSE COX FRANCE

TEL: 61 252101

TLF:

ILX: $530776 \mathrm{~F}$

EML:

COM:

MAVROMICHALAKI HELEN OR UNIVERSITY, PHYSICS DEPT NUCLEAR pHYSICS SECTION 104 SOLONOS ST

GR 106 BO ATHENS

GREECE

TEL: 13639439

TLF:

$T C X:$

EML:

COM: 49
MATTHEWS JAYMIE

DPT OF PHYSICS

UNIVERSITY OF MONTREAL

CP 612 SUCC A

MONTREAL QC H3C $3 J 7$

CANADA

TEL: $514 \quad 343 \quad 6111 * 3214$

TLF: 5143432071

TLX: 05562425

EML: MATTHEWSEASTRO. UMONTREAL.CA

COM: 27

MATVEYENKO L I DR

SPACE RESEARCH INSTITUTE

ACADEMY OF SCIENCES

PROFSOJOSNAYA UL 84/32

117810 MOSCOW

RUSSIA

TEL: $333-31-22$

TLF:

TLX: 411498 STAR SU

EML:

COM: 40

MAUOER HORST PROF DR ASTRONOMISCHES INSTITUT

WALÓHAUSER-STR 64

D 7400 TUEBINGEN

GERMANY

TEL:

TLF :

TLX:

EML:

COM: 42

MAVRAganis A G PROF DPT OF ENG SECT OF MECH NTL TECHN UNIV/5 HEROES POLYTECH AVE GR 15773 ZOGRAFOS GREECE

TEL: 18433170

TLF:

TLX:

EML:

COM: 07

MAX CLAIRE E OR LAHRENCE LIVERMORE LAB L 413

BOX BOB

LIVERMORE CA 94550

USA

TEL: 4154225442

TLF:

TLX: 9103869339 UCLLLLUMR

EML:

COM: 
MAXWELL ALAN QR

CENTER FOR ASTROPHYSICS

HCO/SAO

60 GARDEN ST

CAMBRTOGE MA 02138

USA

TEL: 6174959059

TLF

TLX:

EML:

COM: 10,40

MAYER CORNELL H

1209 VILLAMAY BLVD

ALEXANDRA

VIRGINIA 22307

USA

TEL:

TLF:

TLX:

EML:

COM: 16,40

MAZA JOSE

OPT DE ASTRONOMIA

UNIVERSIDAO OE CHILE

CASILLA 360

SANTIAGO

CHILE

TEL: 22294101

TLF:

TLX: 440001

EML: MASAOUCHCECVM

COM: 46

MAZZITELLI ITALO OR

IAS

CNR

CP 67

I. 00044 FRASCATI

ITALY

TEL: $6942 \quad 1483$

TLF:

TLX: 610261 CNRFRA

EML:

COM: 35

MCALISTER HAROLO A OR DPT PHYSICS ASTRONOMY GEORGIA STATE UNIVERSITY ATLANTA GA 303033083 USA

TEL: $404 \quad 6582932$

TLF: 4046512013

$T L X$ :

EML: HAL OCHARA.GSU.EDU

COM: $24,26[.51$
MAY J

OBS RADIDASTR DE MAIPU

UNIVERST'DAD OE CHILE

\section{CASILLA 68}

MAIPU

CHILE

TEL: 22294101

TLF:

TLX: 440001

EML:

COM: 40

MAYER PAVEL DR

DPT OF ASTRONOMY

CHARLES UNIVERSITY

SVETSKA 8

CS 150 OD PRAHA 5

CZECHOSLOVAKIA

TEL: 2540395

TLF:

TLX:

EML:

COM: 25,42

MAZEH TSEVI DR

WISE OBSERVATORY

TEL AVIV UNIVERSITY

RAMAT AVIV

TEL AVIV 69978

ISRAEL

TEL: 35450729

TLF :

TLX: 342171 VERSY IL

EML: BITNET:K23@TAUNOS

COM: 30,42

MAZZONI MASSIMO OR

DP DI ASTRONOMIA

UNIVERSTIA OI FIRENZE

LARGO E FERMI 5

I 50125 FIRENZE

ITALY

TEL: $55 \quad 27521$

TLF: $55 \quad 220039$

TLX: 572268 ARCETR I

EML:

COM:

MCBREEN BRIAN PHILIP DR

DPT OF PHYSICS

UNIVERSITY COLLEGE

BELFIELD

DUBLIN 4

IRELAND

TEL: 1693244

TLF :

TLX: 36293

EML:

COM: 28,48
MAYALL Margaret W

5 SPARKS ST

CAMBRIDGE. MA 0213B

USA

TEL: $617876 \quad 1563$

TLF :

$T L X:$

EML:

COM: 27

MAYFIELO EARLE 8 DR

CALIFORNIAN POLYTECHNIC

STATE UNIVERSITY

1427 BAYVIEW HEIGHTS QR

LOS OSOS CA 93403

USA

TEL: $805 \quad 5285231$

TLF:

TLX:

EML:

COM:

MAZURE ALAIN DR

LAB ASTRONOMIE.

USTL II

PLACE EUGERE BATAILLON

F 34095 MONTPELLIER CDX 5

FRANCE

TEL: $67 \quad 52 \quad 3548$

TLF:

TLX: 490944

EML:

COM:

MAZZUCCONI FABRIZIO OR

OSS ASTROFISICO

OI ARCETRI

LARGO E FERMI 5

I 50125 FIRENZE

ITALY

TEL: $55 \quad 2752250$

TLF :

TLX:

EML:

COM:

MCCABE MARIE K MS

1617 S BERETANIA ST \#801

HONOLULU HI 96826

USA

TEL: 8089560923

TLF:

TLX:

EML:

COM: 10
MAYALL NICHOLAS U ASTRON

7206 E CAMINO VECINO

TUCSON AZ 85715

USA

TEL: 6028862423

TLF:

$T L X:$

EML:

COM:

MAYOR MICHEL PROF

observatorre de geneve

CHEMIN DES MAILLETTES 51

CH 1290 SAUVERNY

SWITZERLAND

TEL: 227552611

TLF : 227553983

ILX: 419209 OBS CH

EML: mayoreobs.unige.ch

EOM: $30,33 C, 37$

MAZUREK THADDEUS JOHN DR 4580 NUECES DR

SANTA BARBARA CA 93110

USA

TEL:

TLF:

ILX:

EML:

COM: $35,4 B$

MCADAM W BRUCE OR

SCHOOL OF PHYSICS

UNIVERSITY OF SYONEY

SYONEY NSW 2006

AUSTRALIA

TEL: 26922222

TLF :

TLX: 26169 UNISYD

EML:

COM: 40

MCCALL MARSHALL LESTER OR

DPT OF PHYSICS

YORK UNIVERSITY

4700 KEELE ST

NORTH YORK ON M3J IP3

CANADA

TEL: 4167362100

TLF: 416736.5386

ILX: 06524736

EML: BITNET:FS300050@YUSOL

COM: 34 
MCCAMMON DAN

OPT OF PHYSICS

UNIVERSITY OF WISCONSIN

1150 UNIVERSITY AVE

MAOISON WI 53706

USA

TEL: 6082625916

TLF :

TLX: 265452 UOFWISC MDS

EML:

COM:

MCCLAIN EOWARD $F$

4133 MAPLE RD

MORNINGSIDE MO 20746

USA

TEL: $301736 \quad 8933$

TLF :

EML:

COM

TLX:

\section{MCCONNELL DAVID DR}

AUSTRALIA TELESCOPE

NATIONAL FACILITY

BOX 94

NARRABRI NSW 2390

AUSTRALIA

TEL: 67959205

TLF: 67959255

TLX:

EML: OMLCONNERPEPPING. OZ.AU

COM: 40

MCCREA J. OERMOIT
DPT OF MATHS/PHYSICS
UNIVERSITY COLLEGE
BELFIELO
OUBLIN 4
IRELAND
TEL: 1693244
TLF:
TLX:
EML:
COM: $34,35,47$

MCCUTCHEON WILLIAM h PROF

OPT OF PHYSICS

UNIV OF BRITISH COLUMBIA

2075 WESBROOK MALL

VANCOUVER BC V6T $2 A 6$

CANADA

TEL: $504 \quad 228 \quad 3853$

TLF: $604 \quad 2285324$

TLX: 04508576 UBCPHYSICS

EML:

CQQM:
MCCARROLL RONALD PROF

UNIVERSITE DE BORDEAUX

LAB ASTROPHYSIQUE

123 RUE LAMARTINE

F 33405 TALENCE

FRANCE

TEL: $56 \quad 86 \quad 4330$

TLF; $56 \quad 404251$

TLX:

EML:

COM:

MCCLINTOCK JEFFREY E DR

CENTER FOR ASTROPHYSICS

HCO/SAO

60 GARDEN ST

CAMBRIDGE MA 02138

USA

TEL: 6174957136

$T L F$ :

$T L X$ :

EML:

COM:

MCCORD THOMAS B DR

PLANETARY GEOSLIENCES DIV

HAWAII INST OF GEOPHYSICS

2525 CORREA RD

HONOLULU HI 96822

USA

TEL: $808 \quad 948 \quad 6488$

TLF :

ILX:

EML:

COM: 15.16

MCCREA WILLIAM SIR

ASTRONOMY CENTRE

UNIVERSITY OF SUSSEX

FALMER

BRIGHTON BN1 $90 \mathrm{H}$

UK

TEL: 273606755

TLF :

TLX: 877259 UNISEX G

EML:

COM: 28.47

MCOONALO FRANK B DR

NASA/GSFC

CODE 660

GREENBELT MO 20771

USA

TEL:

TLF:

ILX:

EML:

COM:
MCCARTHY DENNIS O DR

US NAVAL OBSERVATORY

34 \& MASSACHUSETIS AVE NW

WASHINGTON OC 203925100

USA

TEL : $202 \quad 6530066$

TLF :

TLX: 7108221970

EML:

COM: 196.31

MCCLURE ROBERT D PROF

HERZBERG INST ASTROPHYS

DOMINION ASTROPHYS OBS

5071 W SAANICH RD

VICTORIA BC VBX $4 M G$

CANADA

TEL: $604 \quad 388 \quad 0230$

TLF: 6043630045

TLX: 0497295

EML:

COM: 30,45

MCCRACXEN XENNETH G DR

CSIRO COSSA

LIMESTONE AVE

BOX 225

DICKSON ACT 2602

AUSTRALIA

TEL: 62484595

TLF:

TLX: 62003 AA

EML:

COM: 44

MCCROSKY RICHARD E DR

CENTER FOR ASTROPHYSICS

HCQ/SAO

60 GARDEN ST

CAMBRIDGE MA 02138

USA

TEL: 6174957212

TLF:

TLX:

EML:

COM: $15,20,22$

MCOONALD J K PETRIE OR

768 RICHMOND AVE

VICTORIA BC VOS $3 Z 1$

CANADA

TEL:

TLF :

TIX:

EML:

COM:
MCCARTHY MARTIN F OR

SPECOLA VATICANA

I O0120 VATICAN CITY

VATICAN CITY STATE

TEL: $6 \quad 698.3411$

TLF :

TLX: 5042020 VAT DBS VA

EML:

COM: $25,33,45,46,50$

MCCLUSKEY GEORGE E JR OR ASTRONOMY DIV/MATHS DPT

LEIGH UNIVERSITY

BETHLEHEM PA 18015

USA

TEL: $215 \quad 8613721$

TLF :

TLX:

EML:

COM: 42,44

MCCRAY RICHARD OR

JILA

UNEVERSITY OF COLORADO

BOX 440

BOULDER CO B́30309 0440

USA

TEL: 3034927835

TLF:

TLX:

EML: DICKSJILA

COM: 34,48

MCCULLOCH PETER M DR

DPT OF PHYSICS

UNIVERSITY OF TASMANIA

GPO BOX 252C

HOBART IAS 7001

AUSTRALIA

TEL: 2202420

TLF :

TLX: 58150

EML:

COM: 40

MCDONNELL J A M PROF UNIT FOR SPACE SCIENCES UNIVERSITY OF KENT CANTERBURY CT2 7NR UK

TEL: 227459616

TLF :

TLX: 965449 UKCLIB G

EML

COM: $15,21,22$ 


MCOONOUGH THOMAS R DR
CALTECH
$500 S$ OAK KNOLL NO 46
PASAOENA CA 91101
USA
TEL: 8187950147
TLX:
EML:
TLF:
COM: 51

MCGIMSEY BEN Q JR DR DPT PHYSICS A ASTRONOMY gEORGIA STATE UNIVERSITY ATLANTA GA 303033083 USA TEL: $404 \quad 6582279$ TLF:

TLX:

EML:

COM:

MCINTOSH BRUCE A OR
HERZBERG INST ASTROPHYS
NTL RESEARCH COUNCIL
100 SUSSEX OR
DTTAWA ON K1A OR6
CANADA
TEL: 6139936060
TLF: 6139526602
TLX: 0533715
EML:
COM: 22

MCKEITH NIALL ENDA DR DPT OF PHYSICS ST PATRICK'S COLLEGE MAYNOTTH CO KILDARE IRELAND

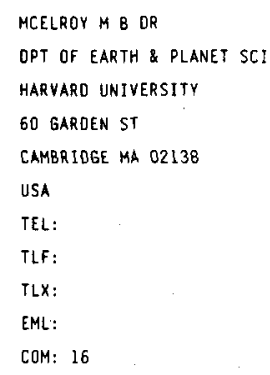

$$
\text { TLF: }
$$$$
\text { TLX: } 467175
$$$$
\text { EML: }
$$$$
\text { COM: } 27
$$

\section{MCINTOSH PATRICK S}

NOAA ERL R/E/SE3

SPACE ENVIRONMENT LAB

325 BROADWAY

BOULDER CO 80303

$$
\text { USA }
$$$$
\text { IEL: } 3034973795
$$$$
\text { TLF : }
$$$$
\text { TLX: }
$$

EML: SPAN: 9555 : : PMCINTOSH

COM: 10

\section{MCKENNA LAWIOR SUSAN \\ DPT OF EXPERIMENTAL PHYS ST PATRICK'S COLLEGE MaYNOOTH \\ CO KILOARE \\ IRELANO \\ TEL: I 285222 \\ TLF: \\ TLX: 31493 SPCM EJ \\ EML: \\ COM: $10,12,15,40,41$}

MCLEAN IAN S DR

OPT OF ASTRONOMY UNIVERSITY OF CALIFGRNIA 405 HILGARD AVENUE LOS ANGELES CA 90024 USA

TEL: $213 \quad 825 \quad 1140$

TLF: 2132062096

TLX:

EML:

COM: $09 \mathrm{C}, 25 \mathrm{C}$
MCFADDEN LUCY ANN DR CALIFORNIA SPACE INST UCSO

\section{A 016}

LA JOLLA CA 920930216

USA

TEL: 6195343915

TLF:

TLX:

EML: LMCFADDENEUCSO.EDU

COM: 15

MCGREGOR PETER JOHN DR MOUNT SIROMLO \& SIOING SPRING OBSERVATORIES PRIVATE BAG

WOOEN PO ACT 2611

AUSTRALIA

TEL: 62881111

TLF:

TLK: 62270 CANQPUS AA

EML:

COM: $09,29,33,34$

MEKEE CHRISTOPHER F PROF PHYSICS DPT

UNIVERSITY OF CALIFORNIA BERKELEY CA 94720

USA

TEL: $415 \quad 5420805$

TLF:

TLX: 820181 ULB AST RALUD

EML:

COM: 34

MCLAREN ROBERT A DR

INSTITUTE FOR ASTRONOMY UNIVERSITY OF HAWAII

2680 WOODLAWN OR

HONOLULU HI 96822

USA

TEL: 808948 8312/8566

TLF :

TLX: 8459 UHAST HR

EML:

COM:

MCMAHAN ROBERT KENNETH OR DPT PHYSICS \& ASTRONOMY UNIVERSITY NORTH CAROLINA PHILLIPS HALL CB 3255 CHAPEL HILL NC 275993255 USA

TEL: 9199627168

TLF: 9199620480

TLX:

EML:

COM:
MCGEE RICHARD $X$ OR

CSIRO

OIVISION OF RADIOPHYSICS

BOX 76

EPPING WSW 2121

AUSTRALIA

TEL: 2 B6B 0222

TLF:

TLX: 26230 ASTRO

EML:

COM: 34

MCHARDY IAN MICHAEL DR OPT OF PHYSICS

SOUTHAMPTON UNIVERSITY ASTRO \& SPACE PHYSICS GP SOUTHAMPTON SOS $5 \mathrm{NH}$ UK

TEL:

ILF:

TLX:

EML:

LOM:

MCKEITH CONAL O OR DPT OF PURE \& APPL PHYS QUEEN'S UNIVERSITY BELFAST BI7 INN

uK

TEL: 232245133

TLF:

TLX: 74487 QUB ADM

EML:

COM: 34

MCLEAN BRIAN JOHN

STSCI

HOMEHOOD CAMPUS

3700 SAN MARTIN DR

BALTIMORE MO 21218

USA

TEL: 3013339101

TLF:

TLX: 6849101 STSCI

EML:

COM: 05,24

\section{MCMILLAN ROBERT S OR} LUNAR \& PLANETARY LAB INIVERSITY OF ARIZONA SPACE SCIENCES BLDG TUCSON AZ 85721 USA

TEL: 6026216968 TLF:

TLX:

EML: NASAMAIL: RSMMILLAN

COM: 30 
MCMULLAN DENNIS DR MULLARD RADIO ASTRON OBS CAVENDISH LABORATORY MADINGLEY RD

CAMBRIDGE CB3 OHE UK

TEL:

TLF:

TLX:

EML:

COM: 09

MCWHIRTER R W PETER OR RUTHERFORD APPLETON LAB

SPACE \& ASIROPHYSICS DIV BLOG R25/R68

CHILTON DIDCOT OXII OOX UK

TEL: $235 \quad 446.424$

TLF :

TLX: B3159 RUTHLB G

EML:

COM: 14,44

\section{MEATHERINGHAM STEPHEN DR}

MOUNT STROMLO SIOING

SPRING OBSERVATORIES

PRIVATE BAG

WOOEN PO ACT 2606

AUSTRALIA

TEL: 62 B日 ILL

TLF: $62 \quad 490233$

TLX: 62270 CANOPOUS AA

EML: SJMMMOGGIE, ANU, EOU, AU

COM: 33

\section{SHEVCHENKO PARK \\ 270014 ODESSA \\ UKRAINE \\ TEL: 228442 \\ TLF : \\ TLX: \\ EML: \\ COM:}

MEDVEDEV YURI A DR

ASTRONOMICAL OBSERVATORY ODESSA STATE UNIVERSITY

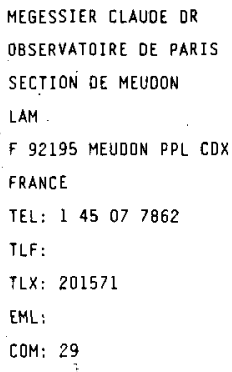

MCNALLY DEREK DR

UNIVERSITY OF LONDON OBS

MILL HILL PARK

LONDON NW? $20 S$

uk

TEL: 1819590421

ILX: 28722 ULPHYS G

EML: RLVAD::ZUVAD::DMN

TLF: 18807145

COM: $0,5,34,46,50 \mathrm{C}, \mathrm{EC}$

MEABURN J OR

DPT OF ASTRONOMY

UNIVERSITY OF MANCHESTER

MANCHESTER MI3 SPL

UK

TEL:

TLF:

TEX:

EML:

COM: 34

MEBOLO ULRICH DR PROF

RADIOASTRONOMISCHES INST

DER UNIVERSITAT BONN

AUF OEM HUEGEL 71

D 5300 BONN 1

GERMANY

TEL:

TLF :

ILX:

EML:

COM: 34,40

\section{MEECH KAREN OR}

INSTITUTE FOR ASTRONOMY

UNIVERSITY OF HAWAII

2680 WOODLAWN DR

HONOLULU HI 96822

USA

TEL: BOB 9566828

TLF: $808 \quad 9882790$

ILX: 8459 UHAST HR

EML: MEECHUHIFA. IFA. HAWAII . EOU

COM: 15

\section{MEGEVAND DENIS OR}

OBSERVATOIRE DE GENEVE

CHEMIN DES. MAILLETTES 51

CH 1290 SAUVERNY

SWITZERLAND

TEL: 227552611

TLF : 227553983

TLX: 45419209 OBSO CH

EML: MEgEVAND@SCSUn. UNIGE, CH

COM: 09
MCNAMARA DELBERT H OR

DPT PHYSICS \& ASTRONOMY

BRIGHAM YOUNG UNIVERSITY

PROVO UT B4602

USA

TEL: 8013782298

TLF:

$T L X:$

EML:

COM: $05,27,29,45$

MEAD JAYLEE MONTAGUE OR

NASA/GSFC

CODE 680

GREENBELT MD 20771

USA

TEL: $301286 \quad 8543$

TLF ;

TLX:

EML:

COM: $05 C, 44,45$

MEOIAVILLA EVENCIO OR

INST DE ASTROFISICA

DE CANARIAS

OBS DEL TEIDE

E 38200 LA LAguna

SPAIN

TEL: $22 \quad 26 \quad 2211$

TLF :

TLX: 92640

EML:

COM: 28

MEEKS M LITILETON OR

MEEKS ASSOCIATES INC

BOK 643

LINCOLN MA 01773

USA

TEL: 6172590093

TLF:

$T L X:$

EML:

COM: 40

MEIDAV MEJR DR

SCHOOL OF EDUCATION

TEL AVIV UNIVERSITY

TEL AVIV 69978

ISRAEL

TEL: 35450840

TLE: $364 \quad 13944$

TLX:

EML:

COM: 46
MCNAUGHT ROBERT H

SIOING SPRING OBSERVATORY

PRIVATE BAG

COONABARABRAN NSW 2357

AUSTRALIA

TLF: 68842298

TEL: 68426269

TLX: CANOPUS AA 163945

EML: NSSDCA: : PSI\%AAOCBN : : UKESRU

COM: 20

MEADOWS A JACK PRGF

DPT ASTRONOMY HISTORY

UNIVERSITY OF LEICESTER

UNIVERSITY RD.

LEICESTER LEI 7RH

UK

TEL:

TLF:

TLX:

EML:

COM: 05,16

MEDINA JOSE DR

DPT FISICA

UNIYERSIDAD DE ALCALA

APD 20

E 28871 ALCALA DE HENARES

SPAIN

TEL: I 8854940

TLF: 18854953

TLX:

EML:

COM: 48

MEERSON BARUCH DR

RACAH INST OF PHYSICS

HEBREW UNIVERSITY

BOX 4040

JERUSALEM 91904

ISRAEL

TEL: $2 \quad 584 \quad 470$

TLF: $2 \quad 584 \quad 437$

ILX: 25391 HUIL

EML: MEERSONEHUJIVMS

COM: 10

MEIER DAVIO L

JPL

MS 264700

4800 OAK GROVE OR

PASADENA CA 91109

USA

TEL: 2133545062

TLF :

TLX: 675429

EML: DLMOCENA.JPL. NASA, GOV/CENA:

COM: · $28,48,40$ 
MEIER ROBERT R

WAVAL RESEARCH LABORATORY

CODE 4140

4555 OVERLOOK AVE SW

WASHINGTON OC 203755000

USA

TEL: $202767 \quad 2773$

TLF:

TLX

EML

COM: 34

MEIN PIERRE

OBSERVATOIRE DE PARIS

SECTION DE MEUDON

F 92195 MEUDON PPL CDX

FRANCE

TEL: $1 \quad 45 \quad 07 \quad 7801$

TLF :

TLX: 270912

EML:

COM: $05,10,12$

MEISEL DAVIO D OR

DPT PHYSICS \& ASTRONOMY

STATE UNIVERSITY COLLEGE

SUNY

GENESEO NY 14454

USA

TEL: $716 \quad 245 \quad 5280$

TLF:

ILX:

EML:

COM: 15.22

MELBOURNE WILLIAM G OR

JPL

MS 238540

4800 OAK GROVE OR

PASADENA CA 91109

USA

TEL: $818 \quad 354 \quad 5071$

TLF

TLX:

EML:

COM: $07,19 \mathrm{C}, 31$

MELLIER YANNICK OR

OBS MIOI PYRENEES

14 AVE E BELIN

F 31400 TOULOUSE CDX

FRANCE

TEL: 61252101

TLF:

TLX: 530776

EML:

COM:
MEIKLE WILLIAM P S ASTROPHYSICS GROUP IMPERIAL COLLEGE BLACKETT LABORATORY LONDON SW7 2BZ UK

TEL: I 5895111

TLF:

TLX: 261503

EML:

COM: 28

MEINEL ADEN B PROF

JPL

MS $186 \quad 134$

4800 OAK GROVE DR

PASADENA CA 91109

USA

TEL: $818 \quad 354 \quad 6827$

ILF:

TLX:

EML:

COM: 09,24

MEISENHEIMER KLAUS DR MPI FUER ASTRONOMIE

KUNIGSTUHL 17

D 6900 HEIOELBERG I

GERMANY

TEL: 6221528206

TLF: 6221528246

TLX: 461789 MPI D

EML: MEISEODHOMPI5V

COM: 28

MELCHIOR PAUL J PROF DIR

OBSERVATOIRE ROYAL DE

BELGIQUE

AVE CIRCULAIRE 3

B 1180 BRUSSELS

BELGIUM

TEL: $2375 \quad 2484$

TLF: 2374 BB22

TLX: 21565 OBSBEL

EML:

COM: $08,19,31$

MELNICK GARY J

CENTER FOR ASTROPHYSICS

HCO/SAD.

60 GAROEN ST

CAMBRIDGE MA 02138

USA

TEL: 6174957388

TLF :

TLX:

EML:

COM: $30,34,44$
MEIKSIN AVERY ABRAHAM BR CITA MCLENNAN LABS UNIVERSITY OF TORONTO

60 ST GEORGE ST

TORONTO ON MES IAT

CANADA

TEL: $416978 \quad 8494$

TLF : $416 \quad 978 \quad 3921$

TLX:

EML: MEIKSINUTORPHYS

COM: 48

MEINIG MANFRED OR

ZNTRLINST F PHYSIK ERDE

TELEGRAFENBERG A17

D 1500 POTSDAM

GERMANY

TEL: 4551

TLF:

ILX: 15305

EML:

COM: 19,31

MEKARNIA DJAMEL DR

OCA OBSERV OE NICE

BP 139

F 06003 NICE CDK

FRANCE

TEL: $92 \quad 003011$

TLF : $92 \quad 00 \quad 3033$

TLX: 460004

EML: MEKARNIAOFRONI5I

COM:

MELIA FULVIO OR

OPT PHYSICS \& ASTRONOMY

NORTHWESTERN UNIVERSITY

DEARBORN OBSERVATORY

EVANSTON IL 60208

USA

TEL: $31249 ! 4568$

TLF

TLX

EML: SPAN: nssdCa: : 11340::me if

COM: 42,44

MELNICK JORGE

DPT DE ASTRONOMIA

UNLVERSLDAD OE CHILE

CASILLA 36"D

SANTIAGO

CHILE

FEL: 22294101

TLF:

$T L X: 440001$

EML:

COM:
MEIM NICOLE QR OBSERVATOIRE DE PARIS SECTION DE MEUDON DASOP

F 92195 MEUDON PPL COX FRANCE

TEL: $145 \quad 07 \quad 7801$

TLF:

TLX

EML

COM:

MEIRE RAPHAEL

ASTRON STERRENWACHE

RIJKSUNIVERSITEIT GENT

WEIOESTR IL

B 9050 EVERGEM

BELGIUM

TEL: 91538755

TLF :

TLX:

EML:

COM: 07

MEKLER YURI PROF

DPT GEOPHYS \& PLANET SCI

TEL AVIV UNIVERSITY

TEL AVIV

ISRAEL

TEL: 3413505

TLF :

TLX:

EML:

COM:

MELIK-ALAVERDIAN YU DR BYURAKAN ASTROPHYSICAL OBSERVATQRY

378433 ARMENIA

ARMENIA

TEL:

TLF :

TLX:

EML:

COM: 35

MELOTT ADRIAN L PROF DPT PHYSICS \& ASTRONOMY UNIVERSITY OF KANSAS LAWRENCE KS 66045 USA -

TEL: 9138644626

TLF:

ILX

EML: BITNET: melot toukanvax COM: 47 
MELROSE DONALO B PROF DPT THEORETICAL PHYSICS UNIVERSITY OF SYONEY SYONEY NSW 2006 AUSTRALIA

TEL:

TLF:

TLX:

EML:

COM: $10,12,48,49$

MENDEZ ROBERTO H DR

IAFE

[C. 67 SUC 28

- 1428 BUENOS AIRES

ARGENTINA

TEL: 17816755

TLX: 22414 CEDOC AR

EML:

TLF:

COM: 34

MENEGUZZI MAURICE M OR

CEA CEN

DAPNIA/SAP

BP 2

F 91191 GIF/YVETTE COX

\section{FRANCE}

TEL: 169084438

ILF:

ILX: 690860

EML:

COM:

MENTESE HUSEVIN DR UNIVERSITY OBSERVATORY UNIVERSITY OF ISTANBUL 34452 ISTANBUL TURKEY

TEL: ] 5223597

ILF:

ILX:

EML:

COM:

MERAT PARVIZ OR
INSTITUT D'ASTROPHYSIQUE
98BIS BD ARAGO
F 75014 PARIS
FRANCE
TEL: 143201420
TLF: 143298673
TLX:
EML:
COM:

MENDES DA COSTA ARACY OR CRAAE/PIR ESCOLA POLI USP CP 8174 CEP 05508 01051 SAO PAULO SP BRAZIL

TEL: 118155936

ILX: 1180127 INPE BR

EML: AMDCOSTA BRUSP. BITNET

TLF: 118156289

COM: 10

MENDIS DEVAMITTA ASOKA QR

EECS

UCSD

LA JOLLA CA 920930216

USA

TEL: $619452 \quad 2719$

TLF:

TLX:

EML:

COM: 15,49

MENG XINMIN

YUNNAN OBSERVATORY

CAS

BOX 110

KUNMING 72946 YUUNAN

CHINA PR

TEL: 8712035

TLF:

TLX: 64040 YUOBS CN

EML:

COM: 09

\section{MENZIES JOHN W DR}

SAAO

BOX 9

OBSERVATORY 7935

SOUTH AFRICA

TEL: 47-0025

TLF:

TLX: 5720309

EML:

COM: $25 C, 34,37,50$

\section{mercier claUde DR} observatoire de paris SECTION DE MEUDON

DASOP

F 92195 MEUDON PPL CDX

\section{FRANGE}

TEL: $145 \quad 077815$

TLF:

TLX: 201571

EML:

COM:
MENOEZ $z$ MANUEL OR

INSTITUTO DE ASTRONOMIA

UNAM

APOO POSTAL 70-264

O4510 MEXICO OF

MEXICO

JEL:

TLF:

ILX:

EML:

COM:

mendoza claudio

IBM VENEZUELA SCIENT CTR

BOX 388

CARACAS $1010 A$

VENEZUELA

IEL: 29088697

ILF:

TLX: 23283 IBMVE VC

EML:

-COM:

MENNESSIER MARIE-ODILE DR

LAB ASTRONOMIE

USTL II

PLACE EUGENE BATAILLON

F 34095 MONTPELLIER CDX 5

FRANCE

TEL: $67 \quad 639144$

TLF:

TLX: 490944 USTMONT $F$

EML:

COM: $24,27,33$

MEN' A V OR

INST RADIOPHYS \& ELECTRON

UKRAINIAN ACAO OF SCIENCE

310085 KHARKOV

UKRAINE

TEL:

TLF:

TLX:

EML:

COM:

MERGENTALER JAN PROF

ASTRONOMICAL INSTITUTE

UL KOPERNIKA 19

PL 51617 WROCLAW

POLANO

TEL: $48-23-29$

TLF:

TLX:

EML:

COM: 10,12
MENDEZ MARIANO DR

DESERVATORIO ASTRONOMICO

paseo del bosque

1900 LA PLATA (BS.AS.)

ARGENTINA

TEL: 2121 6357

TLF: $2121 \quad 1761$

TLX: 31151 BULAP AR

EML: MMENDEZ ZOFCAGLP, EDU AR

COM: 37

MENDOZA V EUGENIO E OR

INSTITUTO DE ASTRONOMIA

UNAM

APDO POSTAL 20.528

01000 MEXICO DF

MEXICO

TEL: 683-3094

TLF:

TLX:

EML:

COM: $25,45,51$

MENON T. K PROF

OPT GEOPHYS \& ASTRONOMY

UNIV OF BRITISH COLLMBIA

2075 WESBROOK PL

VANCOUVER BC V6T JWS

CANADA

TEL: 6042282082

TLF: 6042286047

TLX:

EML:

COM: $28,34,37,40$

MERAT PARVIZ

INSTITUT O'ASTROPHYSIQUEE 98BIS BD ARAGO

F 75014 PARIS

FRANCE

TEL: 143201425

TLF: 143298673

TLX:

EML:

COM: 47

MERIGHI ROBERTO DR

DSS ASTRONOMICO

UNIVERSITA OI BOLOGNA

CP 596

I 40100 BOLOGNA

ITALY

TEL: 51259401

TLF:

TLX: 520634 INFNBO I

EML: SPAN: $37929:$ : MERIGHI

COM: 47 
MERKLE FRIT? OR

ESO

RARLSCHWARZSCHILD-STR 2

D 8046 GARCHING MUENCHEN

GERMANY

TEL: 89320060

TLF: 893202362

TLX: 5828222

EML:

COM: $O 9 \mathrm{C}$

MERMILLIOD JEAN-CLAUDE DR INSTITUT D. ASTRONOMIE

UNIVERSITE OE LAUSANNE CH 1290 CHAVANNES-D-BOIS SWITZERLAND

TEL: 227552611

TLF:

TLX: 27720 OBSG CH

EML: mermi ooobs. unige. ch

COM: $05,30,37 \mathrm{P}$

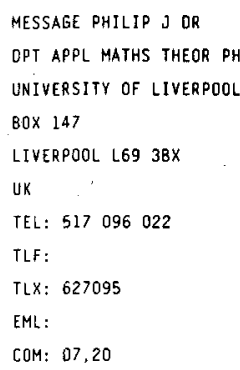

MERLEAU-PONTY J PROF

5 RUE GL DE CASTELNAU

F 75015 PARIS

FRANCE

TEL:

TLF:

EML:

COM: 41

ILL:

MERRIAM JAMES B

DPT GEOLOGICAL SCIENCES

UNIV OF SASKATCHEWAN

SASKATOON SK STN OWO

CANADA

TEL: 3069665716

TLF:

TLX:

EML:

COM: 19

MESSEROTTI MAURO OR

SOLAR ASTROPHYSICS GROUP ASTRONOMICAL OBSERVATORY BASOVIZZA 302

I 34012 TRIESTE

ITALY

TEL: 40226176

TLF: 40226630

TLX: 461137 OAT I

EML: ASTRTS: : MESSEROTTI

COM: 10

MESZAROS PETER OR
OPT OF ASTRONOMY
PENNSYLVANIA STATE
525 OAVEY LAB
UNIVERSITY PARK PA
USA
TEL: 8148650418
TLF:
TLX: 842510
EML:
COM: $34,47,48$

MEWE R DR

SPACE RESEARCH LABORATORY

SRON

SORBONNELAAN 2

NL 3584 CA UTRECHT

- NETHERLANOS

TEL: 30535600

TLF: 30540860

TLX: 47224

EML:

COM: $12,14,44$
MERMAN G A DR

MERMAN NATALIA V DR PULKOVO OBSERVATORY ACADEMY OF SCIENCES 10 KUTUZOV QUAY 196140 ST PETERSBURG RUSSIA

TEL:

TLF :

TLX:

EML:

COM:

MERzANIDES CONSTANTINOS ZALOGOU 15

GR 65403 KAVALA

GREECE

TEL: 5122840

TLF:

$T L X:$

EML:

COM:

MESSINA ANTONIO

OPT OI ASTRONOMIA

UNIVERSTIA OI BOLOGNA

CP 596

I 40100 BOLOGNA

ITALY

TEL: 51222956

ILF:

TLX:

EML:

COM

METCALFE LEO OR

ESA/ESTEC

SSD

BOX 299

NL 2200. AG NOOROWIJK NETHERLANDS

TEL: 171983616

TLF:

TLX: 39098

EML: EARN : LMETCALF@ESTEC

COM:

MESTEL LEON PROF

ASTRONOMY CENTRE

UNIVERSITY OF SUSSEX

FALMER

BRIGHTON BNI 9 QH

UK

TEL: 273606755

TLF:

TLX: 877159 UNISEX G

EML:

COM: $35,48,49$

MET2 KLAUS OR INSTITUT F ASTRONOMIE

\& ASTROPHYSIK

SCHEINERSTR 1

D 8000 MUENCHEN 80

GERMANY

TEL: 89989021

TĹF:

TLX:

EML:

COM: 27

MEYER CLAUDE OR

OCA CERGA

MEYER DAVIO M OR

OPT PHYSICS \& ASTRENOMY NORTHWESTERN UNIVERSITY OEARBORN OBSERVATORY EVANSTON IL 60208 USA

TEL: 3124914516

TLF :

TLX:

EML:

COM: 47 


\section{MEYER FRIEORICH OR}

MPI FUER PHYSIK UND

ASTROPHYSTK

KARL-SCHWARZSCHILO-ST I

D 8046 GARCMING MUENCHEN

GERMANY

TEL: 8932990

TLF:

ILX: 524629 ASTRD D

EML:

LOM: 12,48

MEYLAN GEORGES OR

STSCI

HOMEWOOD CAMPUS

3700 SAN MARTIN DR

BALTIMORE MD 21218

USA

TEL: $301338 \quad 4700$

TLF: 3013384788

TLX: 05282820 ES D

EML: BI TNET: mey lanestc 1

COM: $30,37 \mathrm{C}, 47$

MIANES PIERRE OR

OBS MIOI PYRENEES

14 AVE E BELIN

F 31400 TOULOUSE CDX

FRANCE

TEL: 61252101

TLF:

TLX:

EML:

COM: 25

MICHALEC AOAM

ASTRONOMICAL OBSERVATORY

JAGIELLONIAN UNIVERSITY

UL. ORLA $17 \mathrm{~L}$

PL 30244 KRAKOW

POLAND

TEL: 221817/3856

TLF :

TLX: 0322297 ปJ PL

EML:

COM: 40

MICHAUD GEORGES I DR

250 DU FINISTERE

ST LAMBERT J4S $1 P 5$

CANAOA

TEL: 5143436672

TLF:

EML:

COM: $35 \mathrm{C}, 36$

TLX: 05562425 UDEMPHYSAS
HEYER JEAN-PAUL OR

CEA CEN

DAPNIA/SAP

BP 2

F 9119 GIF/YVETTE COX

FRANCE

TEL: 169085025

TLF :

TLX: 690860

EML:

COM: 48

MEYNET GEORGES DR OBSERVATOIRE DE GENEVE CHEMIN DES MAILLETTES 51

CH 1290 SAUVERNY SWITZERLAND

TEL: 227552611

TLF: 227553983

TLX: 419209 OBSG CH

EML: MEYNET OCGEUGEII

COM: 35

MIAO YONG-KUAN
DPT OF ASTRONOMY
NANJING UNIVERSITY
NANJIMG 210008
CHINA PR
TEL:
TLF:
TLX:
EML:
COH:

MICHALITSIANOS ANDREW

\section{NASA/GSFC}

CODE 684.1

GREENBELT MO 20771

USA

TEL: $301286 \quad 6177$

TLF:

TLX:

EML:

COM: $10.34,44$

MICHEL F CURTIS PROF OPT PHYISCS \& ASTRONOMY

RICE UNIVERSITY

BOX 1892

HOUSTON TX 772511892

USA

TEL: 7135274925

TLF :

TLX: 556457

EML:

COM: 48,49
MEYERS KARIE ANN

NOAO

BOX 26732

950 N CHERRY AVE

TUCSON A2 $85726 \quad 6732$

USA

TEL: 6023259202

TLF: 6023259360

TLX:

EML: KMEYERS@NOAO. EOU

CDM:

MEZGER PETER g PRDF MPI FUER RADIOASTRONOMIE

AUF OEM HUEGEL 69

D 5300 BONN 1

GERMANY

TEL: 228525297

TLF: 228525229

TLX: 886440

EML:

COM: $33,34,40 C$

MIAO YONG-RUI

SHAANXI OBSERVATORY

CAS

LINTONG XIAN

SHAANXI

CHINA PR

TEL: 332255

TLF :

TLX: 70121 CSAO CN

EML:

Com: 31

MICHALOWSKI TADEUSZ DR

ASTRONOMICAL OBSERVATORY

A MICXIEWICZ UNIVERSITY

UL SLONECZNA 36

PL 60286 POZNAN

POLAND

TEL: 61679670

TLF: 61 535535

TLX: 413260 UAM PL

EML: MICHASTROPLPUAMII

COM: 15

MICKELSON MICHAEL E OR

DPT OF PHYSICS \& ASTRON

DENISON UNIVERSITY

GRANVILLE OH 43023

USA

TEL: $614587 \quad 6467$

TLF:

TLX:

EML: MICKELSONODENISON

COM: 14.26
MEYER-HOFMEISTER E DR

MPI F PHYSIK \& ASTROPHYS

KARL - SCHWARZSCHILO-STR I

O 8046 GARCHING MUENCHEN

GERMANY

TEL: 8932990

TLF:

TLX: 524629 ASTRO O

EML:

COM: 35,42

\section{MEZZETII MARINO}

OAT

BOX SUCC TRIESTE 5

VIA TIEPOLO 11

I 34131 TRIESTE

ITALY

TEL: 40793221

TLF :

TLX: 461137 OAT I

EML:

COM: 42,47

MICELA GIUSEPPINA OR

OSS ASTRONOMICO

UNIVERSITA OI PALERNO

PALAZZO DEI NORMANNI

I 90134 PALERMO

ITALY

TEL: 916570451

TLF: $9148 \quad 8900$

TLX: 910402 ASTROP 1

EML: ASTROPAOIPACUC

COM: 48

MICHARO RAYMONO DR OCA OBSERV DE NICE

BP 139

F 06003 NICE COX

FRANCE

TEL: 93890420

TLF:

TLX: 460004 OBSNICE F

EML:

COM: 12

MIOOLEHURST BARBARA M MS

16567 EL CAMINO REAL

HOUSTON TX 77062

USA

TEL:

TLF :

TLX:

EML:

COM: 16 
MIETELSKI JAN S OR ASTRONOMICAL OBSERVATORY JAGIELLONIAN UNIVERSITY

UL ORLA 171

PL 30244 KRAKOW

POLAND

TEL: $48-12-22-38-56$

TLF :

TLX: 0322297 UJ PL

EML:

COM:

MIHALAS DIMITRI DR

DPT OF ASTRONOKY

UNIVERSITY OF ILLINOIS

1011 W SPRINGF IELD AVE

URBANA IL 6180 I

USA

TEL: 2173333090

TLF :

TLX

EML:

COM: 12,36

MIXHAL JOSEPH SIOKY PROF

HELWAN OBSERVATORY

HELWAN

EGYPT

TEL: $78 \quad 0645 / 2683$

FLF:

EML:

COM: 16

ILX: 93070 HIAG UN

MIKULASEK ZOENEK DR

$N$ COPERNICUS OBSERVATORY

\& PLANETARIUM

KRAVI HORA

CS 61600 BRNO 16.

CZECHOSLOVAKIA

TEL: $5 \quad 74 \quad 4347$

TLF:

TLX:

EML:

COM: 29,42

MILET BERNARD L DR

OCA OBSERV DE NICE

BP 139

F 06003 NICE CDX

FRANCE

TEL: 93890420

TLF:

TLX: 4600Q4 OBSNICE F

EME:

COM: $15,20,28,51$
MIGNART FRANCOIS OR

OCA LERGA

AVE COPERNIC

F OS130 GRASSE

FRANCE

TEL: $93 \quad 365849$

TLF :

ILX: 470865

EML:

COM: 07

MIKAMI TAKAO DR

OSAKA GAKUIN UNIVERSITY

2-36-1 KISHIBE MINAMI

SUITA SHI

OSAKA 564

JAPAN

TEL: $06-381-8434$

TLF :

TLX:

EML:

COM:

MIKHELSON NIKOLAJ N DR

PULKOVO OBSERVATORY

ACADEMY OF SCIENCES

10 KUTUZOY OUAY

196140 ST PETERSBURG

RUSSIA

TEL: 2-979-465

TLF :

TLX:

EML:

COM: 09

MILANI ANDREA

IST DI MATEMATICA

UNIVERSITA OI PISA

VIA BUONARROTI 2

I 56127 PISA

ITALY

TEL:

TLF:

TLX:

EML:

COM: $07 C, 15,20$

MILEY G K OR

STERREWACHT

NIELS BOHRHEG 2

BOX 9513

NL 2333 RA LEIDEN

NETHERLANDS

TEL: 71275849

TLF:

TLX: $3905 B$ ASTRO NL

EML:

COM: 28,40
MIHAILA IERONIM PROF BUCHAREST UNIVERSITY ACAOEMIEI 14

R 70109 BUCAREST RUMANIA

TEL: 230819

TLF:

TLX:

EML:

COM:

MIKESELL ALFRED H MR

8316 WALDNUT RD NE

OLYMPIA WA 9850669550

USA

TEL: $206 \quad 493 \quad 1457$

TLF:

TLX:

EML:

COM:

MIKKOLA SEPPO DR

TURKU UNIVERSITY OBS

THORLA

SF 21500 PIIKKIO

FINLAND

TEL: 21435822

TLF: 21433767

TLX: 62638 TYF SF

EML:

COM: $07,26,33,37$

MILANO LEOPOLDO OR

DPT DI SCIENZE FISICHE

UNIVERSITA OI NAPOLI

MOSTRA O OLTREMARE PAD 19

I 80125 NAPOLI

ITALY

TEL: 817253447

TLF:

TLX: 720320

EML:

COM: 42

MILKEY ROBERT W DR

STSCI

HOMEWOOD CAMPUS

3700 SAN MARTIN DR

BALTIMORE MD 21218

USA

TEL: 3013384720

TLF :

TLX: 6849101

EML:

COM: 12
MIHALAS BARBARA R WEIBEL

HIGH ALTITUDE OBSERVATORY

NCAR

$80 \times 3000$

BOULDER CO 803073000

USA

TEL: 3044945151

TLF:

TLX:

EML: :

COM:

MIKHAIL FAHMY I PROF DR AIN SHAMS UNIVERSITY

FACULTY OF SCIENCES

CAIRO UNIVERSITY

CAIRO

EGYPT

TEL: 575887

TLF :

TLX: 94070 USHMS UN

EML:

COM:

MIKOLAJEWSKA JOANNA DR INSTITUTE OF ASTRONOMY N COPERNICUS UNIVERSITY

UL CHOPINA $12 / 18$

PL 87100 TORUN

POLANO

TEL: 562601726018

TLF :

TLX: 552234 ASTR PL

EML:

COM: 42

MILES HOWARD G MR

LANE PARK

PTIYNE

ST MINVER

WAOEBRIDGE PL27 6PN

UK

TEL: $208 \quad 863 \quad 153$

TLF :

TLX:

EML:

COM: 22

MILLAR THOMAS J DR

OPT DF MATHEMATICS

UMIST

BOX 88

MANCHESTER M6O IQD UK

TEL: $612 \quad 363 \quad 311$

TLF:

$T L X: 666094$

EML:

COM: 34 
MILLER FREEMAN D PROF OPT OF ASTRONOMY UNIVERSITY OF MICHIGAN OENNISON BLDG

ANN ARBOR MI 461091090 USA

TEL: $313764 \quad 3447$

TLF:

TLX:

EML:

Col4: 15

MILLER RICHARD H OR

ASTRONOMY \& ASTROPHYS CTR UNIVERSITY OF CHICAGO

5640 S ELLIS AVE

CHICAGO IL 60637

USA

TEL: 312962.8201

TLF:

TLX: 6871133

EML:

COM: 28,33

MILLIKAN ALLAN G MR

RESEARCH LAB Q-59

EASTMAN KODAK CO

343 STATE ST

ROCHESTER NY $\$ 4650$

USA

TEL:

TLF:

TLX:

EML:

COM: 09

MILNE DOUGLAS K DR

CSIRO

DIVISION OF RADIOPHYSICS

BOX 76

EPPING NSW $212 ?$

AUSTRALIA

IEL: 2 86̈ 0222

TLF:

TLX: 26230 ASTRO

EML: DMILNEATNF. CSIRO.AU

COM: 34,4 [

MILOVANOVIC VLADETA OR

INSTITUT ZA GEODEZIJU

BULEVAR REVOLUCIJE 73

YU 11000 BEOGRAD

YUGOSLAVIA

TEL:

TLX:

EML:

TLF :

COM:
MILLER HUGH R PROF

OPT PHYSICS \& ASTRONOMY

GEORGIA STATE UNIVERSITY

ATLANTA GA 303033083

USA

TEL: $404 \quad 658 \quad 2279$

TLF:

TLX:

EML:

COM: 28

MILLET JEAN OR

LAS

TRAVERSE OU SIPHON

LES TROIS LUCS

F 13012 MARSEILLE

FRANCE

TEL: 91055900

TLF : $91 \quad 66 \quad 1855$

TLX: 420584 ASTROSP F

EML:

COM:

MILLIS ROBERT L DR

LOWELL OBSERYATORY

1400 W MARS HILL RD

BOX $\mathrm{H} 49$

FLAGSTAFF AZ 86001

USA

TEL: 6027743358

TLF:

TLX:

EML:

COM: $15,16,20$

MILOGRADOV-TURIN JELENA

INSTIJUTE OF ASTRONOMY

UNIVERSITY OF BELGRADE

STUDENTSKI TRG 16

YU 11000 BEOGRAD

YUGOSLAVIA

TEL: 11638715

TLF: '

TLX:

EML:

COM: 40

MINAROVJECH MILAN.

ASTRONOMICAL INSTITUTE

SLOVAK ACADEMY SCIENCES

CS 05960 TATRANSKA LOMNI

CZECHOSLOVAKIA

TEL: $969967866 / 7 / 8$

TLF : $96996 \quad 7656$

TLX: 78277

EML:

COM: 09
MILLER JOHN C DR

DPT OF ASTROPHYSICS

UNIVERSITY OF OXFORO

SOUTH PARKS RO

OXFORO OXI $3 R Q$

UK

TEL: 865511336

TLF:

TLX: 83295 NUCLOX G

EML:

COM: 48

MILLIARD BRUNO

LAS

TRAVERSE DU. SIPHON

LES TROIS LUUCS

F 13012 MARSEILLE

FRANCE

TEL: 91055900

TLF: $91 \quad 66 \quad 1855$

TLX: 420584 ASTROSP, F

EML:

COM:

MILLS ALLAN A DR

DPT OF ASTRONOMY

UNIVERSITY OF LEICESTER

UNIVERSITY RD

LEICESTER LEI TRH

UK

TEL: $533 \quad 554 \quad 455$

TLF :

TLX:

EML:

COM:

MILONE EUGENE F PROF

OPT OF PHYSICS

UNIVERSITY OF CALGARY

2500 UNIVERSITY OR NW

CALGARY AB T2N IN4

CANADA

IEL: 4032205412

TLF: 4032893331

TLX: $038 \quad 21545$

EHL:

COM: $25,27,37,42$

MINESHIGE SHIN OR

DPT OF PHYSICS

IBARAKI UNIVERSITY

BUNKYO

MITO 310

JAPAN

TEL:

TLF :

TLX:

EML:

COM: 42
MILLER JOSEPH S PROF

LICK OBSERVATORY

UNIVERSITY OF CALIFORNIA

SANTA CRUZ CA 95064

USA

TEL: 4084292135

TLF:

TLX:

EML:

COM: $25,28,34$

MILLIGAN J $\varepsilon$

NASA/GSFC

INFRARED ASTROPHYSICS BR

GREENBELT MD 20771

USA

TEL:

TLF:

TLX:

EML:

COM:

MILLS BERNARO $Y$ PROF

SCHOOL OF PHYSICS

UNIVERSITY DF SYONEY

SYONEY NSW 2006

AUSTRALIA

TEL: 26922544

TLF :

TLX: 26169 UNISYD

EML:

COM: 28,40

MILONE LUIS A DR

OBSERVATORIO ASTRONOMICO

DE CORDOBA

LAPRIOA B54

5000 CORDOBA

ARGENTINA

TEL: $36876 / 40629$

TLF :

TLX: 51822 BUCOR

EML:

COM: 27

MINEVA VENETA DR

DPT DF ASTRONOMY

BULGARIAN ACAD SCIENCES

72 LENIN BLVD

BG 1784 SOFIA

BULGARIA

TEL: 2758827

TLF :

TLX: 23561

EML:

COM: 28 
MININ I N PROF

ASTRONOMICAL OBSERVATORY

ST PETERSBURG UNIVERSITY

BIBLIOTECHNAJA PL 2

198904 ST PETERSBURG

RUSSIA

TEL: 257-94-89

ILF .

ILX:

EML:

COM: 34

MIOC VASILE DR

ASTRONOMICAL OBSERVATORY

STR CIRESILOR 19

R 3400 CLUJ NAPOCA

RUMANIA

TEL: 95111592

TLF:

TLX:

EML:

COM: 07

MISCONI NEBIL YOUSIF OR

FIT

328 W HIBISCUS

MELBOURNE 32901

USA

TEL: 4077686000

ILF :

$\mathrm{I} \perp \mathrm{X}$

EML:

COM: 21,22

MITALAS ROMAS ASSOC PROF

OPT OF ASTRONOMY

UNIV OF WESTERN ONTARIO

LONOON ON NGA $5 B 9$

CANADA

TEL: 5196793184

TLF: 5196613486

TLX: 0647134

EML:

CON: 35

MITCHELL WALTER E JR

OPT OF ASTRONOMY

OHIO STATE UNIVERSITY

174 W 18TH AVE

COLUMBUS OH $43210 \quad 1106$

USA

TEL: 6144225554

TLF :

TLX:

EML:

COM:
MINN YOUNG KEY OR

DPT ASTRONOMY SPACE SCI

KYUNG HEE UNIVERSITY

YONG IN KUN

KYUNGGI DO $170 \quad 73$

KOREA A

TEL; $02-764-6131$

ILF:

LX:

EML:

COM: 34,51

MIRABEL IGOR FELIX OR

CEA EEN

DAPNIA/SAP

BP 2

F 91191 GIF/YVETTE COX FRANCE

TEL: 169 O8 3492

TLF: $1 \quad 6908 \quad 6577$

TLX: 604860

EML: 32773: :MIRABEL

COM: $28,33,40,51$

\section{MISNER CHARLES W PROF}

ASTRONOMY PROGRAM

UNIVERSITY OF MARYLAND

COLLEGE PARK MO 20742

USA

TEL:

TLF :

TLX:

EML:

COM: 47

MITCHELL GEORGE F OR

BPT OF ASTRONOMY

ST MARY'S UNIVERSITY

HALIFAX NS B3H 3 C 3

CANADA

TEL: $902 \quad 4299780$

TLF : 9024205551

ILX:

EML:

COM: 34

MITIC LJUBISA A OR

ASTRONOMICAL DBSERVATORY

VOLGINA 7

YU 11050 BEOGRAD

YUGOSLAVIA

TEL: $11419357 / 421875$

TLF :

TLX:

EML:

COM: OB
MINNET HARRY C MR

CSIRO

DIVISION OF RADIOPHYSICS

B0X 76

EPPING NSW 2121

ALSTRALIA

TEL:

TLF :

TLX:

EML:

COM:

MIRONOV NIKOLAY T

MAIN ASTRONOMICAL OBS

UKRAINIAN ACAO OF SCIENCE

GOLOSEEVO

252127 KIEV.

UKRAINE

TEL: $66 \quad 4759$

TLF:

TLX: 131406 SKY

EML:

COM: 190

MISSANA MARCO OR

OSS ASTRONOMICO DI BRERA

VIA CREMAGNANI $13 / 11$

I 20059 VIMERCATE

ITALY

TEL:

TLF :

TLX:

EML :

COM:

MITCHELL KENNETH J OR

APPLIEO RESEARCH CORP

8201 CORPORATE OR

SUITE 920

LANDOVER MD 20785

USA

TEL: 3014598442

TLF :

TLX:

EML:

COM: 40

MITRA A P OR

NTL PHYSICAL LABORATORY

HILLSIOE RO

NEW OELHI 110012

INOIA

TEL: $11.585 \quad 298 / 440$

TLF :

ILX: 3162454 RSD IN

EML:

COM:
MINTZ BLANCO BETTY MRS

CERRO TOLOLO

INTERAMERICAN OBSERVATORY

CASILLA 603

LA SERENA

CHILE

TEL： $5 ! 213352$

ILF: $5121 \quad 2466 * 342$

TLX: 620301 AURA CT

EML:

COM: 20,25

MIRZOYAN L V DR PROF

BYURAKAN ASTROPHYSICAL

OBSERVATORY

378433 ARMENIA

ARMENIA

TEL:

TLF :

TLX:

EML:

COM: 27,33

MISSANA NATALE PROF

VIA PUCCINI 2

I 10025 PINO TORINESE

ITALY

TEL:

TLF :

TLX:

EML:

COM:

MITCHELL RICHARD MR

C $/ O$ OR-SCHUSTER WILLIAMS

BOX 73027

SAN YSIDRO CA 92073-9027

USA

TEL:

TLF :

TLX:

EML: :

COM:

MITROFANOVA LYUDMILA A OR PULKOVO OBSERVATORY ACAOEMY OF SCIENCES

10 KUTUZOV QUAY 196140 ST PETERSBURG RUSSIA

TEL:

TL.F :

ILX:

EML:

COM: 


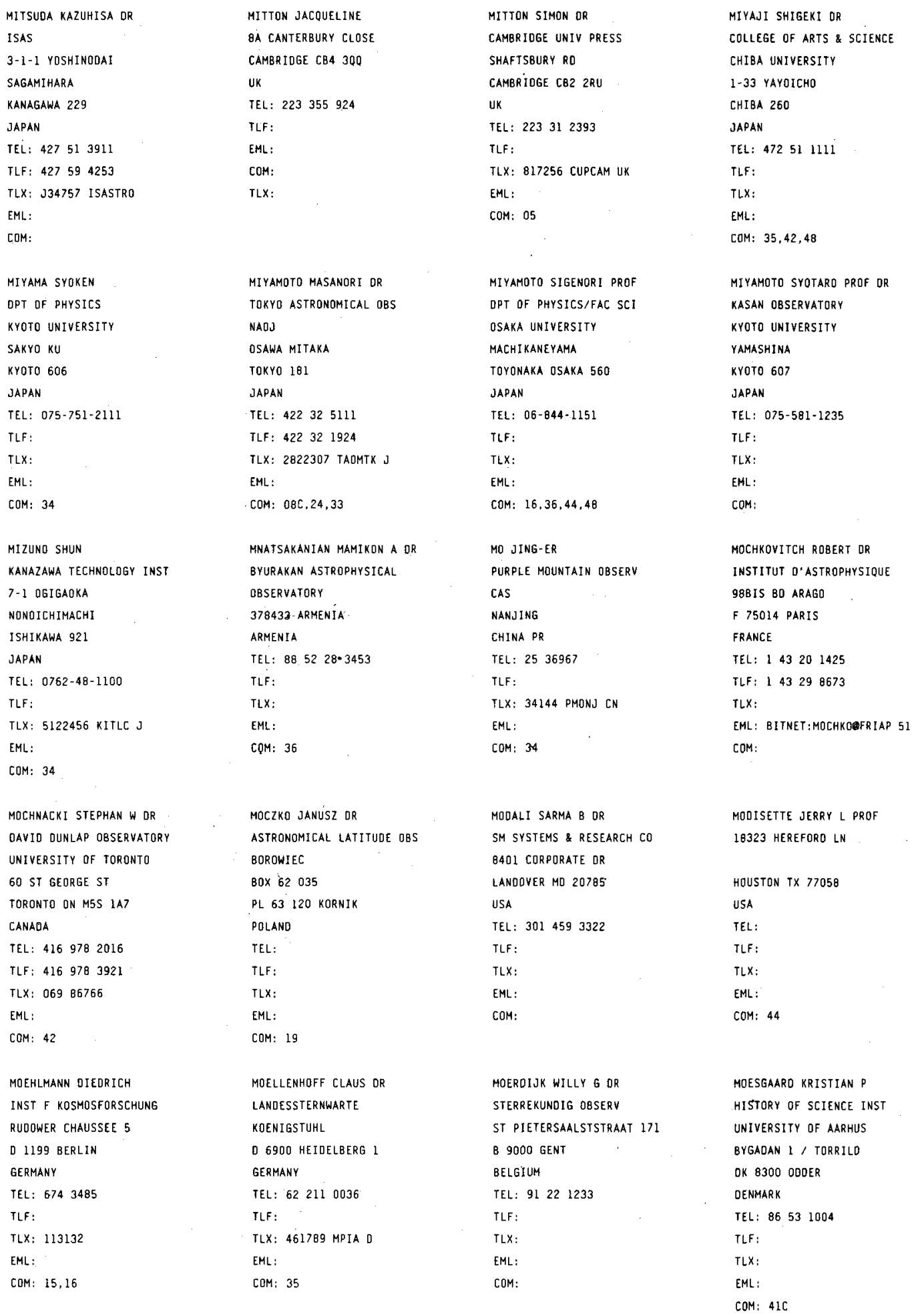


MOFFAT ANTHONY \& J DR

OPT DE PHYSIQUE

UNIVERSITE OE MONTREAL

CP 6128 SUCC A

MONTREAL QC H3C 337

CANADA

TEL: 5143436682

TLF: 5143432071

TLX: 05562425 UDEMPHYSAS

EML:

COM: $29,33,37$

MDGILEVSKIJ EH I OR

IZMIRAN

ACADEMY OF SCIENCES

142092 TROITSK

RUSSIA

TEL: $232-19-31$

TLF:

TLX: 412623 SCSTP SU

EML:

COM: 10

MOISEEV I G OR

CRIMEAN ASTROPHYS OBS

UKRAINIAN ACAD OF SCIENCE

NAUCHNY

334413 CRIMEA

UKRAINE

TEL: 432945

TLF :

TLX:

EML:

COM: 10.40

MOLINA ANTONIO
INST ASTROFISICA
OE ANDALUCIA APO 2144
PROFESOR ALBAREDA 1
E IBOBO GRANADA
SPAIN
TEL: 58121300
TLF:
TLX: 78573 IAAG E
EML:
COM: 16,21
MONET ALICE K B DR
US NAVAL OBSERVATORY
FLAGSTAFF STATION
BOX LI49
FLAGSTAFF AZ B6002
USA
TEL:
ILF:
TLX:
EML:
COM: 19

MOFFAT JOHN W DR

DPT OF PHYSICS

UNIVERSITY OF TORONTO

60 ST GEORGE ST

TORONTO ON MSS IAT

CANADA

TEL: $416 \quad 9782949$

TLF : 4169783921

TLX: 06986766

EML:

COM:

MOHAN CHANDER DR

OPT OF MATHEMATICS

UNIVERSITY OF ROORKEE

ROQRKEE 247667

INDIA

TEL: 26

TLF :

TLX:

EML:

COM: $26,27,35$

\section{MOLARO PAOLO DR}

OAT

BOX SUCC TRIESTE 5

VIA TIFPOLO 11

I 34131 TRIESTE

ITALY

TEL: 40309342

TLF:

TLX: 461137 OAT I

EML:

COM: 29

MOLNAR MICHAEL R PROF

MOLNAR TECHNOLOGIES

3 STONINGHAM DR

WARREN NJ 07060

USA

TEL: 2015801404

TLF :

TLX:

EML :

COM:

\section{MONET DAVID G}

US NAVAL DBSERVATORY

FLAGSTAFF STATION

BOX 1149

FLAGSTAFF AZ 86002

USA

TEL: 6927795132

TLF :

$T L X:$

EML: percy: :dmonet

COM: $24,33,44$
MOFFATT HENRY KEITH PROF

DPT APPLIEO MATHS

\& theORETICAL PHYSICS

SILVER STREET

CAMBRIOGE CB3 9EW

UK

TEL: $223 \quad 351645$

ILF :

TLX: 81249 CAMSPL G

EML:

COM :

MOHAN VIJAY OR

UTTAR PRAOESH STATE

OBSERVATORY

PO MANORA PEAK 263129

NAINITAL 263129

INDIA

TEL: $5942: 2136$

TLF:

TLX:

EML:

COM: 37

MDLCHANOV A P PROF

ASTRONOMICAL OBSERVATORY

ST PETERSBURG UNIVERSITY

BIBLIOTECHNAJA PL 2

199178 ST PETERSBURG

RUSSIA

TEL:

TLF

ILX:

EML:

COM: 40

MOMCHEV GOSPODIN

ASTRONOMICAL OBSERVATORY

$B O X 7$

BG 8800 SLIVEN

BULGARIA

TEL: 4427204

TLF :

TLX:

EML:

COM: 46

MONFILS ANDRE G PROF

IAL SPACE

UNIVERSITE DE LIEGE

AVE QU PRE AILY

B 4900 ANGLEUR-LIEGE

BELGIUM

TEL: 41676668

TLF :

TLX: 41320 IAL SP B

EML:

COM: 44
MOFFETT THOMAS J PROF

OPT OF PHYSICS

PURDUE UNIVERSITY

WEST LAFAYETTE IN 47907

USA

TEL: 3174945508

TLF:

TLX:

EML:

COM: $25 C .27$

MOHD ZAMBRI ZAINUDOIN OR

CENTRE FOR FOUNDATION

UNIVERSITY OF MALAYA

59100 KUALA LUMPUR

MALAYSIA

TEL: 037552744

TLF : 6037573661

TLX: MA39845

EML:

COM:

MOLES MARIANO J DR

INST ASTROFISICA

DE ANOALUCIA APO 2144

PROFESOR ALBAREDA 1

E IODSO GRANADA

SPAIN

TEL: $58 \quad 12 \quad 1311$

TLF:

TLX: 78573 IAAG E

EML:

COM: 28

MONAGHAN JOSEPH J DR

OPT OF MATHEMATICS

MONASH UNIVERSITY

WELLINGTON RD

CLAYTON VIC 3168

AUSTRALIA

TEL: 35412563

TLF:

ILX: MONASH AA 32691

EML:

COM: 35

MONIN JEAN-LOUIS OR

OBSERVATOIRE DE GRENOBLE

CERMO/ASTROPHYSIQUE

BP $53 \mathrm{X}$

F 38041 S MARTIN HERES CD

FRANCE

TEL: $76 \quad 514786$

TLF: $7644 \quad 8821$

TLX: $980134 \mathrm{~F}$

EML: MONINEFRGAG51/GAGVX3:: MONIN

COM: 34 


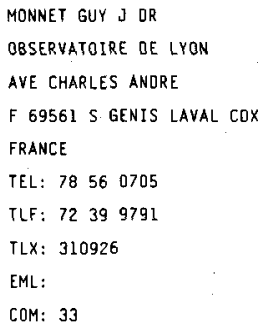

MONSIGNORI FOSSI BRUNA OR
OSS ASTROFISICO
OI ARCETRI
LARGO E FERMI 5
I 50125 FIRENZE
ITALY:
TEL: 552752239
TLF:
TLX:
EML:

COM: 10,12

MOOK OELO E PROF
DPT OF PHYSICS \& ASTRON
DARTMOUTH. COLLGE
HANOVER NH 03755
USA
TEL: 6036462972
TLF:
TLX:
EML:
COM:

MOORE ELLIOTT P PROF

JOINT OBSERVATORY

NEW MEXICO TECH

CAMPUS STATION

SOCORRO NM 87801

USA

TEL: 5058355431

TLF:

TLX:

EML:

COM: 15

MOORWDOD ALAN F M

ESO

KARL-SCHWARZSCHILO-STR 2

D 8046 GARCHING MUENCHEN

GERMANY

TEL: 8932006294

TLX: 052828224 EO D

EML:

TLF: 893202362

COM: 28

MORANOO BRUNO L DR

BUREAU. OES LONGITUDES

77 AVE OENFERT ROCHEREAU

F 75014 PARIS

FRANCE

TEL: 140512276

TLF: $146 \quad 33 \quad 2834$

TLX:

EML: OBLMCA: :MORANDO

CDM: 04C.07.20
MONTEIRO TANIA S DR DPT OF MATHEMATICS ROYAL HOLLOWAY COLLEGE UNIVERSITY OF LONDON EgHAM SURREY TW2O OEX UK

TEL: $78434455 * 3106$

ILF :

TLX:

EML: ASLOT03eULCC

COM:

MOON SHIN HAENG OR
ISSA
$36-1$ WHAAM DONG
YUSEONG GU
OAEJEON 305348
KOREA R
TEL: $042 \quad 861 \quad 1497$
TLF: 0428815610
TLX:
EML:
COM: 48

MOORE PATRICK OR

FARTHINGS

39 WESI ST

SELSEY SUSSEX

UK

TEL: 243603668

TLF :

TLX:

EML

COM: 16

MOOS HENRY WARREN OR OPT PHYSICS \& ASTRONOMY JOHNS HOPKINS UNIVERSITY CHARLES \& 34TH ST BALTIMORE MO 21218

USA

TEL: 3013387337

TLF :

TLX: 7102341090

EML:

COM: 29,44

MORBEY CHRISTOPHER L HERZBERG INST ASTROPHYS DOMINION ASTROPHYS OBS 5071 W SAANICH RO VICTORIA BC VBX $4 M 6$ CANADA

TEL: 6043880220

TLF: 6043630045

TLX: 0497295

EML:

COM: 26,30
MONTES CARLOS OR OCA OBSERY DE NICE

BP 139

F 06003 NICE CDX

FRANCE

TEL: 93890420

TLF:

TLX: 460004 OBSNICE F EML:

COM:

MOONS MICHELE B MM DPT DE MATHEMATIQUE FACULTES UNIVERSITAIRES REMPART DE LA VIERGE 8 B 5000 Namur BELGIUM

TEL: $81229061 * 2438$ TLF: $81 \quad 230391$ TLX: 59222 FACNAM $B$ EML:

COM: 07

MOORE RONALD L DR NASA/MSFC COOE ES 52

SPACE SCIENCE LAB HUNTSVILLE AL 35812 USA TEL: 2054530118 TLF: TLX: 59-4416 NASA MSFC HT EML: COM: 12

MORALES-OURAN CARMEN INTA OPT DE PROGR ESPACIALES E 28850 TORREJON DE AROOZ SPAIN.

TEL: 1.6750700

TLF :

TLX: 22026 INTA E

EML:

COM:

MORBIDELLI ROBERTO DR OSS ASTRONOMICO OI TORINO

ST ISSERVATORIO 20 I 10025 PINO TORINESE ITALY

TEL: $11 \quad 84 \quad 1067$

TLF: $11 \quad 84 \quad 1281$

TLX: 213236 TOASTR I

EML: 39181: :MORBIDELLI

COM: 26 
MOREELS GUY DR OBSERVATOIRE DE BESANCON 41BIS AVE OBSERVATOIRE F 25000 BESANCON COX

\section{FRANCE}

TEL: 81502266

TLF:

TIX: $361144 \mathrm{~F}$

EML:

COM: 46

MORENO FERNANDO DR

INST ASTROFISICA

DE ANDALUCIA APO $3004^{\circ}$ PROFESOR ALBAREDA I

E 18080 GRANADA

\section{SPAIN}

TEL: $5812 \quad 1311$

TLF: 58 81 4530

TLX: 78573 IAAG E

EML: 16488: ; FERNANDO

$$
\text { COM: } 16
$$

\section{MORGAN BRIAN LEALAN}

BLACKETT LABORATORY IMPERIAL COLLEGE PRINCE CONSORT RO LONDON SW7 $2 B Z$

$$
\text { UK }
$$$$
\text { TEL: } 15895111
$$

TLF:

TLX: 261503

$$
\text { EML: }
$$$$
\text { COM: } 09
$$

\section{MORGAN THOMAS. H DR} NASA/JOHNSON SPACE CENTER CODE SN3 HOUSTON TX 77058

$$
\text { USA }
$$$$
\text { TEL: } 7134835039
$$$$
\text { TLF: }
$$$$
\text { TLX: } 762931
$$

$$
\text { EML: }
$$$$
\text { COM: } 42,44
$$

MORISON IAN MR
NRAL
JOORELL BANK
MACLLESFIELD SK11 9DL
UK
TEL: 47771321
TLX: 36149
EML:
TLF:
COM: 40

MOREL PIERRE JACQUES QR OCA OBSERV OE NICE

BP 139

F 06003 NICE COX

FRANCE

TEL: 93890420

TLF:

TLX: 460004 OBSNICE $F$

EML:

COM: 26

MORENO HUGO PROF

DPT OE ASTRONOMIA

UNIVERSIDAD DE CHILE

CASILLA 360

SANTIAGO

CHILE

TEL: 2229 4101/4002

TLF:

ILX: 440001

EML:

COM: 25

MORGAN DAVID H DR

ROYAL OBSERVATORY

BLACKFORO HILL

EDINBURGH EHS 3HJ

UK

TEL: 316673321

ILX: 72383 ROEOIN G

EML:

TLF :

COM: 21,34

MORGAN WILLIAM W PROF

YERKES OBSERVATORY

UNIVERSITY OF CHICAGO

BOX 258

WILLIAMS BAY WI 53191

USA

TEL: $414245 \quad 5555$

TLF:

TLX:

EML:

COM: 28,45

MORITA KAZUHIKO
OPT OF PHYSICS
HOKKAIDO UNIVERSITY
KITA 10 NISHI 8
SAPPORO 060
JAPAN
TEL: $11-711-2111$
TLF:
TLX:
EML:
COM: 40

MORENO CORRAL MARCO A OR OBS ASTRONOMICO NACIONAL UNAM

APDO POSTAL 977

22830 ENSENADA B CALIF

MEXICO

TEL: 66744580

TLF: 66744607

TLX: 56539 CICEME

EML:

COM: 34

MORENO-INSERTIS FERNANDO

INST DE ASTROFISICA

DE CANARIAS

OBS DEL TEIDE

E 38071 LA LAGUNA

SPAIN

TEL: 22262211

ILF:

TLX: 92640

EML:

COM: 10,12

MORGAN JOHN ADRIAN

AEROSPACE CORPORATION

MS M4 041

BOX 92957

LOS ANGELES CA 90009

USA

YEL:

XLF:

TLX:

EML:

COM: 35

MORIARTY-SCHIEVEN G H DR JPL

MS 169506

4800 OAK GROVE OR

PASADENA CA 91109

USA

TEL: 8183547894

TLF:

ILX: 675429

EML: JPLRAG: : SCHIEVEN

COM:

MORIYAMA FUMTO PROF OSAKA GAKUIN UNIVERSITY 2-36-1 KISHIBE MINAMI

SUITA SHI

OSAKA 564

JAPAN

TEL: $06-381-8434$

TLF:

TLX:

EML:

COM: $10,12,40$
MORENO EDMUNDO DR INSTITUTO OE ASTRONOMIA UNAM

APDO POSTAL 70-264

04510 MEXICO DF

MEXICO

TEL: $52554853 \quad 06$

TLF : $9011525 \quad 548 \quad 3712$

TLX:

EML:

COM: 28

MORETON $G$ E

155 THE ESPLANADE

BALMORAL BEACH NSW $208 B$

AUSTRALIA

TEL:

TLF :

TL,X:

EML:

COM: 10

MORGAN PETER DR CANBERRA COLL ADY EDUC SCHOOL OF APPLIEO SCIENCE Box 1

BELCONNEN ACT 2616

AUSTRALIA

TEL: 62522557

TLF:

TLX: 62267 CANCOL AA

EML:

COM: 19.31

MORIMOTO MASAKI DR NOBEYAMA RADIO OBS

NAOU

MINAMIMAKI MURA

NAGANO $384 \quad 13$

JAPAN

TEL: $267 \quad 634372$

TLF: 267982884

ILX: 7203329005 NAONRO J

EML: MORIMOTOENRO.NAO.AC.JP

COM: $34,38 \mathrm{C}, 40 \mathrm{P}, 51$

MOROSSI CARLO

OAT

BOX SUCC TRIESTE 5

VIA TIEPOLIO 11

I 34131 TRIESTE

ITALY

TEL: 40768506

TLF:

TLX: 461137 OAT I

EML:

COM: $2.9,45$ 


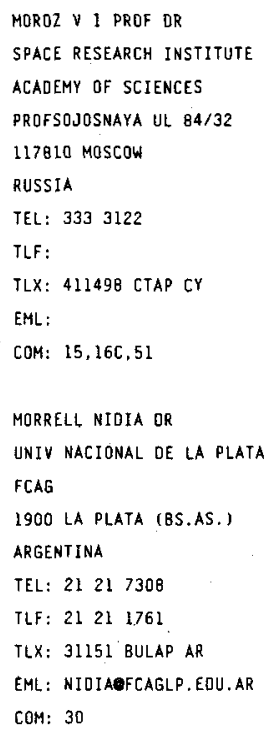

MOROZHENKO A V OR

MAIN ASIRONOMICAL OBS UKRAINIAN ACAD OF SCIENCE

GOLOSEEVO

252127 KIEV

UKRAINE

TEL: 663110

TLF:

ILX: 131406 SKY

EML:

COM: 16

MORRIS CHARLES $S$

JPL

MS 300319

4800 OAK GROVE DR

PASADENA CA 91109

USA

TEL: 8183548074

TLF:

TLX: 675429 JPL USA

EML: SPAN: STANS: : CSM

COM:

\section{MORRIS STEPHEN C DR \\ HERZBERG INST ASTROPHYS \\ DOMINION ASTROPHYS OBS \\ 5071 W SAANICH RO \\ VICTORIA BC V8X $4 M 6$ \\ CANADA \\ TEL: 6043883976 \\ TLF: 6043630045 \\ TLX: 0497295 \\ EML: \\ COM: 25,35}

MORRISON NANCY DUNLAP DR DPT PHYSICS \& ASTRONGMY UNIVERSITY OF TOLEOO 2801 W BANCROFT ST TOLEOO OH 43606 USA

TEL: 4195372659 .

TLF:

TLX:

EML:

COM: 27,29

MOSKALIK PAWEL OR

COPERNICUS ASTRON CENTER POLISH ACAD OF SCIENCES UL BARTYCKA 18

PL 00716 WARSAW

POLAND

TEL:

ILF:

ILX:

EML:

COM: 35

MOROZHENRO N N DR
MAIN ASTRONOMICAL OBS
UKRAINIAN ACAO OF SCIENCE
GOLOSEEVO
252127 KIEY
UKRAINE
TEL: 663110
TLF:
TLX: 131406 SKY
EML:
COM: 10

COM: 10

MORRIS DAVIO QR

IRAM

300 RUE DE LA PISCINE

F 38406 S MARTIN HERES CD FRANCE

TEL: 76423383

ILF:

TLX: 950753

EML:

COM: 40

\section{MORRIS STEVEN OR \\ APT 2 \\ 2860 W 235TH ST \\ TORRANCE CA 90505 \\ USA \\ TEL: 2135308708 \\ TLF: \\ TLX: \\ EML: \\ COM:}

MORRISON PHILIP PROF
OPT OF PHYSICS
MIT RM 6205
BOX 165
CAMBRIOGE MA 02139
USA
TEL: 6172535086
TLF:
TLX:
EML:
COM: $47,48,51$

MOSS CHRISTOPHER OR INSIITUTE OF ASTRONOMY THE QBSERVATORIES MADINGLEY RD CAMBRIDGE CB3 OHA UK TEL: 223333548

TLF:

TLK: 817297 ASTRON G

EML:

COM: 28
MORRAS RICARDO DR

IAR

CC 5

1894 VILLA ELISA (BS AS) ARGENT INA

TEL: $21 \quad 4 \quad 3793$

TLF :

TLX: 18052 CICYR-AR

EML:

COM: 40

MORRIS MARK ROOT DR OPT OF ASTRONOMY UNIVERSITY OF CALIFORNIA MATH SCIENCES BLDG LOS ANGELES CA 90024 USA TEL: $213 \quad 8253320$ TLF :

TLX:

EML:

COM: $33 C, 34,40,51$

MORRISON DAVID PROF NASA AMES RESEARCH CTR MS 2451 SPACE SCIENCE OIV MOFFETT FIELD CA 94035 USA

TEL: $415 \quad 6045028$

TLF : 4156046779

TLX:

EML: DMORRISONenasamail COM: $15,16 P$, WGPSNC

MORTON DONALO C DR HERZBERG INST ASTROPHYS NTL RESEARCH COUNCIL 100 SUSSEX OR OTTAWA ON KIA ORE CANADA

TEL: 6139936060 TLF: 6139526602 TLX: 0533715 EML:

COM: $09,34,44$

MOSS DAVID L OR OPT OF MATHEMATICS UNIVERSITY OF MANCHESTER MANCHESTER ML3 9PL UK

TEL:

TLF:

$T L X:$

EML:

COM: 35 


MOTTA SANTO OR
OPT OI MATIMATICA
CIUIA UNIVERSIIARIA
VIA A DORIA 6
I 95125 CATANIA
IJALY
TEL: 95 33 0533*668
TLF:
TLX: 970359 ASTRCT-1
EML:
COM: 10
MOURADIAN ZADIG M OR
OBSERVATOIRE DE PARIS
SECTION OE MEUDON
DASOP
F 92195 MEUDON PPL COX
FRANCE
TEL: 1 45 07 7800
TLF:
TLX:
EML:
COM: 12

MOZURKEWICH DAVIO OR US NAVAL OBSERVATORY AD 5 BLDG 22 RM 220 34 \& MASSACHUSETTS AVE NW WASHINGTON DC 203925100 USA

TEL: 2026530948

TLF:

ILX:

EML:

COM:

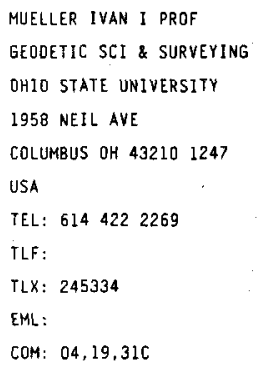

MOTZ LLOYO PROF

DPT OF ASTRONOMY

COLUMEIA UNIVERSITY

PUPIN HALL 538 W 120TH ST

NEW YORK NY 10027

USA

TEL: $212 \quad 2803279$

TLF:

TLX:

EML:

COM:

MOUSCHOVIAS TELEMACHOS CH OPT OF ASTRONOMY UNIVERSITY OF ILLINOIS 1011 W SPRINGFIELO AVE URBAANA IL 61801 USA

TEL: 2173333090

TLF:

TLX:

EML:

COM: 34

\section{MRKOS ANTONIN DR}

DPT OF ASTRONOMY

CHARLES UNIVERSITY

SVEOSKA \&

CS 150 OO PRAHA 5

CZECHOSLOVAKIA

TEL: 2540395

TLF:

TLX: 144307 KLET CZ

EML:

COM: $06,15,20$

\section{MUENCH GUIDO PROF}

MPI FUER ASTRONOMIE

KOENIGSTUHL

D G900 heidelberg 1

GERMANY'

TEL: $62 \quad 21528210$

TLF :

TLX: 461789 MPIA D

EML:

COM: $33.34,36$

MUKAI SONOYO DR

KANAZAWA TECHNOLOGY INST

7-1 OGIGAOKA

NONOICHIMACHI

ISHIKAWA 921

JAPAN

TEL: $0762-48-1100$

TLF:

TLX: $5122456 \mathrm{KITLCJ}$

EML:

COM: 21,36
MOUCHET MARTINE OR

observatoire DE PARIS

MOULO JEREMY $R$

SECTION DE MEUDON

DAEC

F 92195 MEUDON PPL COX

CALTECH

MS 10524

FRANCE

TEL: $1 \quad 4507 \quad 7522$

PASADENA CA 91125

USA

TEL: $818 \quad 3564168$

TLF:

ILX:

EML:

TLX: 201571

EML: MELAMA: :MOUCHET/MOUCHET RMEU5 COM: 28,37

COM: 42

MOUSSAS XENOPHON PH D

OPT OF ASTROPHYSICS

NTL UNIVERSITY OF ATHENS

PANEPISTIMIOPOLIS

GR 157 B3 ATHENS

GREECE

TEL: $1723 \quad 5122 / 18843877$

TLF :

TLX:

EML: MOUSSAS

COM: 49

MOUTSOULAS MICHAEL PROF DPT OF EARTH SCIENCES NTL UNIVERSITY OF ATHENS PANEPISTIMIOPOLIS

GR 157 B4 ATHENS

GREECE

TEL: 17247569

TLF:

TLX: 215255 GR

EML:

COM: 16,51

\section{MUELLER EDITH A PROF}

RENNWEG 15

CH 4052 BASEL

SWITZERLANO

TEL: 61423168

TLF:

TLX:

EML:

COM: $12,38,44$

MUELLER EwALD

MPI F PHYS \& ASTROPHYSIK

INSTITUT F. ASTROPHYSIK

KARL-SCHWARZSCHILQ-STR, I

D 8046 GARCHING MUENCHEN

GERMANY

TEL: 8932990

TLF:

TLX: 524629 ASTRO D

EML:

COM: 35

MUFSON STUART LEE DR

ASTRONOMY OPT

INDIANA UNIVERSITY

SWAIN WEST 319

BLOOMINGTON IN 47405

USA

TEL: 8123356927

TLF:

TLX:

EML:

COM: 34

MUINONEN KARRI OR

LOWELL OBSERVATORY

1400. W MARS HILL RD

BOX 1149

FLAGSTAFF A2 86001

USA

TEL: 6027743358

TLF: 6027746296

ILX:

EML: S:PERCY: :KOM

COM: 21

MUKAI TADASHI DR

DPT OF EARTH SCIENCES

KOBE WOMEN'S UNIVERSITY

SUMA KU

KOBE 657

JAPAN

TEL: $78881 \quad 1212$ EXT 4475

MUKHERJEE KRISHNA

OPT PHYSICS \& ASTRONOMY

UNIVERSITY OF KANSAS

LAWRENCE KS 66045

USA

TEL: 9138644030

TLF: 788821549

TLF :

TLX:

EML:

EML: E01142 PNAC.BITNET COM:

COM: $15,21 \mathrm{C}$ 


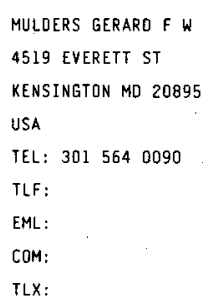

MULHOLLANO J OERRAL OR
OCA CERGA
AVE COPERNIC
F O6130 GRASSE
FRANCE
TEL: 93365849
TLF:
TEX:
EML:
COM: $07,16,20$

MULLER C A PROF JR

ODINKSVELL 8

NL 7491 HO DELOEN

NETHERLANOS

TEL: 54 O7 2428

TLF:

EML:

COM:

ILX:

MUMFORD GEORGE S PROF

DPT OF EOUCATION

TUFTS UNIVERSITY

FILENP - CENTER

MEDFORD MA 02155

USA

TEL: $617 \quad 6538923$

ILF :

TLX:

EML:

COM: $25,27,42$

MUNOZ-TUNON CASIANA.

INST DE ASTROFISICA

DE CANARIAS

OBS DEL TEIOE

E 38200 LA LAGUNA

SPAIN

TEL: $22 \quad 262211$

TLF:

TLX: 92640

EML:

COM: 28

MURDOCH HUGH S DR

OPT OF ASTROPHYSICS

UNIVERSITY OF SYONEY

SYONEY NSW 2006

AUSTRALIA

TEL: 26922222

TLF:

TLX: 26169 UNISYD

EML:

COM: 40
MULLALY RICHARO F DR SCHOOL DF ELECTRICAL ENG UNIVERSITY OF SYONEY SYDNEY NSW 2006 AUSTRALIA

TEL:

TLF:

TLX:

EML:

COM:

MULLER PAUL

3 RUE Chauvain

F 06000 NICE

FRANCE

TEL:

TLF:

TLX:

EML:

COM: 26

MUMMA MICHAEL JON NASA/GSFC

CODE 693

GREENBELI MO 20771

USA

TEL: 301286.6994

ILF:

TLX:

EML:

COM: $14,15,16$

MUNRO RICHARD H DR 2378 OENNISON LANE BOULDER CO 80302

USA

TEL:

TLF:

TLX:

EML:

COM: 12

MURDOCK THOMAS LEE GENERAL RESEARCH CORP OPT OF TECHNOLOGY

5 CHERRY HILL DANVERS MA 01923 USA

TEL: $617777 \quad 6323$

TLF:

TLX:

EML:

COM: 44
MULLAN DERMOTT J OR BARTOL RESEARCH INSIITUTE UNIVERSITY OF DELAWARE NEWARK OE 19716 USA TEL: 3013983368 TLF: TLX: 5106660805 EML:

COM

MULLER RICHARO A LAWRENCE BERKELEY LAB BLDG 50 RM 238 BERKELEY CA 94720 USA TEL: 4154865235 TLF:

TLX:

EML:

COM: $47,5 \mathrm{~L}$

MUNOT REINHARD OR MPI FUR ASTRONOMIE KOENIGSTUHL D 6900 HeIDELBERG 1 GERMANY

TEL: $62 \quad 215 \cdot 28227$ TLF :

TLX: 461789

EML:

COM:

MURAKAMI TOSHIO INST SPACE \& ASTRON SCI UNIVERSITY OF TOKYO MEGURU KU TOKYO 153 JAPAN

TEL: $03-467-111 ! \times 303$ TLF:

TLX: 34757 ISASTRO J EML:

COM: 48

MUREOOU LEONARDO DR STAZIONE ASTRONDMICA

VIA OSPEDALE 72 I 09100 CAGLIARI ITALY

TEL: $70 \quad 66 \quad 3544$

TLF : 70657657

TLX:

EML:

COM: 


MURPHY BRIAN WILLIAM OR
STERREKUNDIG INSTITUTE
BOX 80000
NL 3508 TA ULTRECHT
NETHERLANDS
TEL: 30535203
TLX: 40048 FYLUT
EML: MURPHYORUUNSC.FYS.RUU
TLF:
COM:
MURRAY JOHN B OR
UNIVERSITY OF LONDON OBS
MILL HTLL PARK
LONOON NW7 2OS
UK
TEL:
TLX:
EML:
TLF:
COM:

\section{MUSMAN STEVEN DR}

NGS

N/CG 112

ROCKVILLE MO 20852

USA

TEL:

TLF:

ILX:

EML:

LOM:
MURPHY ROBERT E DR NASA HEADQUARTERS

CODE EEL

600 INDEPENDENCE AVE SW

WASHINGTON OC 20546

USA

TEL: $202453 \quad 1720$

TLF :

TLX: 89530 NASA WSH

EML:

COM: 16

MURRAY STEPHEN S DR
CENTER FOR ASTROPHYSICS
HCO/SAO
60 GARDEN ST
CAMBRIDGE MA 02138
USA
TEL: 6174957205
TLF:
TLX: 921428 SATELLITE CAM
EML:
COM: 09, 28
MUTEL ROBERT LUCIEN
OPT PHYSICS \& ASTRONOMY
UNIVERSITY OF IOWA
IOWA CITY IA 52242
USA
TEL: 3193537205
TLF:
TLX:
EML:
COM: $40,42 C$

MYACHIN VLADIMIR F OR INST OF THEORET ASTRONOMY ACADEMY OF SCIENCES N KUTUZOVA 10 192187 ST PETERSBURG RUSSIA

TEL:

ILF:

TLX: 31216 CESLA AR

EML: jemuzzioxfcaglp.edu. oreuccpnet TLX:

COM: $28,33,37,46$

EML:

COM: 07

NADAL ROBERT
OBS MIDI PYRENEES
14 AVE E BELIN
F 31400 TOULLOUSE COX
FRANCE
TEL: 61252101
TLF:
TLX: 530776 F
EML:
COM: 41

NADEAU DANIEL DR

OPT DE PHYSIQUE UNIVERSITE OE MONTREAL

\section{CP 6128 SUCC $A$}

MONTREAL QC H3C $3 J 7$

CANADA

TEL: 5143436676

TLF: 5143432071

TLX: 055.62425

EML:

COM: 40
MURRAY C ANDREW DERWENT COTTAGE 12 OERWENT RO

MEADS

EASTBOURNE ON2O $7 \mathrm{PH}$$$
\text { UK }
$$

TEL:

TLF:

ILX:

EML:

COM: $08,22,24,33,37$

MUSEN PETER DR

BBO4 ORBIT LANE

LANHAM MD 20801

USA

TEL: 3015523848

TLF :

TLX:

EML:

COM: 07

MUTSCHLECNER J PAULL DR ASTRONOMY OPT

INDIANA UNIVERSITY

SWAIM WEST 319

BLOOMINGTON IN 47405

USA

TEL:

TLF:

TEX:

EML:

COM: 36

MYERS PHILIP C

CENTER FOR ASTROPHYSICS

HCO/SAO MS 42

60 GARDEN ST

CAMBRIDGE MA 02138

USA

TEL:

TLF:

TLX:

EML:

COM: $34 \mathrm{C}, 40$

NADOLSCHI $\checkmark$ PRDF OR

COM AROEOANI OF TESCANI

JUO

R BACAU

RUMANIA

TEL:

TLF:

TLX:

EML:

COM:
MURRAY CARL O OR

SCHOOL OF MATHEMATICAL SC QUEEN MARY/WESTFIELD COLL MILE END RD

LONDON EI ANS

UK

TEL: 1719804811

ILF:

ILX: 893750

EML:

COM: 20

MUSIELAK ZOZISLAW E OR NASA/MSFC

CODE ES 52

SPACE SCIENCE LAB

HUNTSVILLE AL 35812

USA

TEL: $205 \quad 5447619$

TLF :

TLX: 594416

EML: SPAN:SSL: : MUSIELAK

COM: 10,36

MUXLOW THOMAS

NRAL

JOORELL BANK

MACCLESFIELL SKII $90 L$

uK

TEL: 47771321

TLF:

TLX: 36149

EML:

COM: 40

NACOZY PAUL E OR

FEOEREAL SPACE SYSTEMS

BOX 26712

AUSTIN TX 78755

USA

TEL: $512467 \quad 6659$

TLF:

TLX:

EML:

COM: 07,20

NADYOZHIN O K OR

INST THEOR \& EXPER PHYS

CHEREMUSHKINSKAJA UL 25

117259 MOSCOW

RUSSIA

TEL: $123-02-92$

ILF:

TLX: 411059 CERII SU

EML:

COM: 35 


NAGASAWA SHINGO PROF
$3-20-9$ HIGASHI MACHI
KICHIJOJI
MUSASHINO SHI
TOKYO 180
JAPAN
TEL:
TLF:
TLX:
EML:
COM: 10

NAHON FERNAND PROF 25 AVE DE L'EUROPE F 92310 SEVRES FRANCE

TEL: $14534 \quad 1805$

TLF

EML :

COM: 07,33

TLX:

NAKAGAWA TAKAO OR
ISAS
$3-1-1$ YOSHINGDAI
SAGAMIHARA
KANAGAWA 229
JAPAN
TEL: 427513911
TLF: 427594253
TLX: J 34757 ISASTRU
EML: NAKAGAWA ASTRO. ISAS.AC.JP
COM: 34

NAKAI YOSHIHIRO
KWASAN \& HIDA OBS
KYOTO UNIVRSITY
YAMASHINA
KYOTO 607
JAPAN
TEL: 075-581- 1235
TLF:
TLX:
EML:
COM: O9
NAKAMURA TSUKO DR
TOKYO ASTRONOMICAL DBS
NAOJ
OSAWA MITAKA
TOKYO 181
JAPAN
TEL: 3 8122111
ILF:
TLX:
EML:
COM: 15,20

\author{
NAGASE FUMIAKI OR \\ OPT OF ASTROPHYSICS \\ NAGOYA UNIVERSITY \\ FUROCHO CHIKUSA KU \\ NAGOYA 464 \\ JAPAN \\ TEL: 052-781-5111 \\ TLF: \\ TLX: 4477323 SCUNAG J \\ EML: \\ COM:

NAIOENOV VICTOR O
IOFFE PHYSICAL TECH INST
ACADEMY OF SCIENCES
POLYIECHNICHESKAYA UL 26
194021 ST PETERSBURG
RUSSIA
TEL:
TLF:
TLX:
EML:
COM: 48

NAKAGAWA YISHINARI OR CHIBA INST OF TECHNOLOGY NARASHINO 275

JAPAN -

TEL: 0474752111

TLF:

EML:

COM: $10,49 C, 51$

ILX:

NAKAJIMA HIROSHI
NOBEYAMA RADIO OBS
NAQJ
MINAMIMAKI MURA
NAGANO 38413
JAPAN
TEL: $267-98-2034$
TLF:
TLX: 3329005 TAONRO J
EML:
COM: 10

NAKAMURA YASUHISA

OP.T OF EARTH SCINECE

FUKUSHIMA UNIVERSITY

MATSUKAWA MACHI

FUKUSHIMA $960-12$

JAP.AN

TEL: $0245485151 / 421$

TLF:

TLX:

EML:

COM: 42

\section{NAGIRNER DMITRIJ I OR \\ ASTRONOMICAL OBSERVATORY ST PETERSBURg UNIVERSITY BIBLIOTECHNAJA PL 2 198904 ST PETERSBURG} RUSSIA

TEL: 257-94-89

TLF:

TLX:

EML:

COM: 36

NAKADA YOSHIKAZU OR
OPT OF ASTRONOMY
UNIVERSTTY OF TOKYO
BUNKYO KU
TOKYO 113
JAPAN
TEL: $03-812-2111$
TLF:
TLX:
EML:
COM: 34

NAKAGAWA YOSHITSUGU QR GEOPHYSICAL INSTITUTE UNIVERSITY OF TOKYO BUNKYO KU TOKYO 113 JAPAN

TEL: $3-812-2111 \times 4304$

TLF: $3-818-3247$

TLX: 2722126 UTGAB J

EML: a81938\%tansei.cc.u-tokyo.ac.jo EML: NRONAKA

COM: 16

NAKAJIMA KOICHI OR

FAC OF ECONOMICS

HITOTSUBASHI UNIVERSITY

NAKA 2-I KUNITACHI

TOKYO 186

JAPAN

TEL: 435721101

TLF:

TLX: 2842107

EML:

COM: 08

NAKANO SABURO DR

KOBENATO 1-21-7

BUNKYO KU

TOKYO 112

JAPAN.

TEL:

TLF:

TLX:

EML:

COM:
COM: 28

NAGNIBEDA VALERY G DR ASTRONOMICAL OBSERVATORY ST PETERSBURG UNIVERSITY BIBLIDTECHMAJA PL 2 298904 ST PETERSBURG RUSSIA

TEL: 257-94-91

TLF:

TLX:

EML:

COM: 40

NAKAGAWA NAOYA OR

UNIV OF ELECTRO-COMMUNICA CHOFU-SHI

TOKYO 182

JAPAN.

TEL: 0424-83-2161

ILF:

TLX: 2822446 UEC J

EML:

COM:

NAKAI NAOMASA OR NOBEYAMA RADIO OBS NAOJ

MINAMIMAKI MURA NAGANO 38413

JAPAN

TEL: 267634367

TLF: 267634387

TLX: 3329005 NAONRO J

NAKAMURA TAKASHI DR OPT OF PHYSICS KYOTO UNIVERSITY SAKYO KU KYOTO 606 JAPAN

TEL:

TLF :

TLX:

EML:

COM: 35

NAKANO SYUICHI

3-19 I СHOMO

TAKENOKUCHI

SUMOTO

HYOGO KEN 656

JAPAN

TEL:

TLF:

TLX:

EML:

COM: 06,20 
NAKANO TAKENORI DR

OPT OF PHYSICS

KYOTO UNIVERSITY

SAKYO KU

KYOTO 606

JAPAN

TEL: $075-751-2111$

TLF :

TLX:

EML:

COM: 35

NAMBU YASUSADA DR

OPT OF PHYSICS

KYOTO UNIVERSITY

SAKYO KL

KYOTO 606

JAPAN

TEL: $\theta 175753 \quad 3844$

TLF: B1 757533866

ILX:

EML: NAMBUU.JPNRIFP

COM: 47

NAQVI $S I$ H PROF

DPT PHYSICS \& ASTRONOMY

UNIVERSITY OF REGINA

REGINA SK \$4S OA2

CANADA

TEL: $306584 \quad 4262$

TLF: 3065869862

TLX: $0712683 \cup R$ REG

EML:

COM:

NARAYANA J V

REGIONAL METEOROLOGICAL

DFFICE

4 COLlege RD

MAORAS $600 \quad 006$

INDIA

TEL:

TLF:

TLX:

EML:

COM:

NATALI GIULIANO DR

IAS

CNR

CP 67

I 00044 FRASCA.TI

ITALY

TEL: 69425655

TLF :

TLX:

ENL:

COM:
NAKAYAMA SHIGERU OR

FACULTY OF GENERAL EDU

UNIVERSITY OF TOKYO

MEGURO KU

TOKYO 153

JAPAN

TEL: $03-467-1171$

TLF :

TLX:

EML:

COM: 41

NAN REN-DONG

BEIJING ASTRONOMICAL OBS

CAS

W SUBURB

BEIJING 100080

CHINA PR.

TEL: 1281698

ILF :

TLX: 22040 BAOBS CN

EML:

COM: 40

NARANAN S PROF
TIFR
HOMI BHABHA RO
COLABA
BOMBAY 400005
INOIA
TEL: 22219111
TLF:
TLX: OL13009
EML:
COM:

NARIAI KYOJI PROF

TOKYO ASTRONOMICAL OBS

NAOJ

OSAWA MITAKA

TOKYO 181

JAPAN

TEL: $0422-32-5111$

TLF:

ILX:

EML:

COM: 36,42

NATHER R EOWARD

ASTRONOMY OPT

UNIVERSITY OF TEXAS

RLM 15308

AUSTIN TX $78712 \quad 1083$

USA

TEL:

TLF :

TLX:

EML:

COM: 27,42
NAKAZAWA KIYOSHI DR

TOKYO INST OF TECH

OHOKAYAMA 2-12-1

MEGUROKU TOKYO 152

JAPAN

TEL:

TLF:

$T L X:$

EML:

COM: 22,35

NANQY KASHINATH DR

ROYAL OBSERYATORY

BLACKFORO HILL

EOINBURGH EH9 3HJ

UK

TEL: $316 \quad 673321$

TLF::

TLX: 72383 ROEOING

EML:

COM: 34,45

NARASIMHA DELAMPADY OR

TIFR

HOMI BHABHA RO

COLABA

BOMBAY 400005

INDIA

TEL: $22495 \quad 2311$

TLF:

TLX: 3009 IN

EML:

COM: $35,36,47$

NARITA SHINJI DR DOSHISHA UNIVERSITY KYOTO 602

JAPAN

TEL:

TLF :

TLX:

EML:

COM: 35

NATTA ANTONELLA OR CENTRO PER ASTRONOMIA IR LARGO E FERMI 5

I 50125 FIRENZE

ITALY

TEL: 55.2752239

TLF :

TLX: 572268 ARCETRI

EML:

COM:
NAMBA OSAMU DR

MARCO POLOLAan 319

NL 3526 GE UTRECHT NETHERLANDS

TEL:

TLF:

TLX:

EML:

COM: 10,12

NAPIER WILLIAM M OR ROYAL OBSERVATORY

BLACKFORD HILL

EDINBURGH EHg ЗHJ

UK

TEL: $316 \quad 673 \quad 321$

TLF :

TLX: 72383 ROEDIN $G$

EML:

COM: 15,22

NARAYAN RAMESH DR STEWARD OBSERVATORY UNIVERSITY OF ARIZONA TUCSON AZ B572L

USA

TEL: 6026212560

TLF :

TLX: 467175

EML:

COM:

NARLIKAR JAYANT V PROF

IUCAA

BOST BAG 4

GANESHKHIND

PUNE 411007

INDIA

TEL: 212336415

TLF: 212335760

TLX: 0145658 GMRT IN

EML: jayant iucad.ernet in

COM: $28,46,47 \mathrm{VP}$

NAUMOY VITALIJ A DR

PULKOVO OBSERVATORY

ACADEMY OF SCIENCES

10 KUTUZOV QUAY

196140 ST PETERSBURG

RUSSIA

TEL: 2982242

TLF :

TLX:

EML:

COM: 19,31 


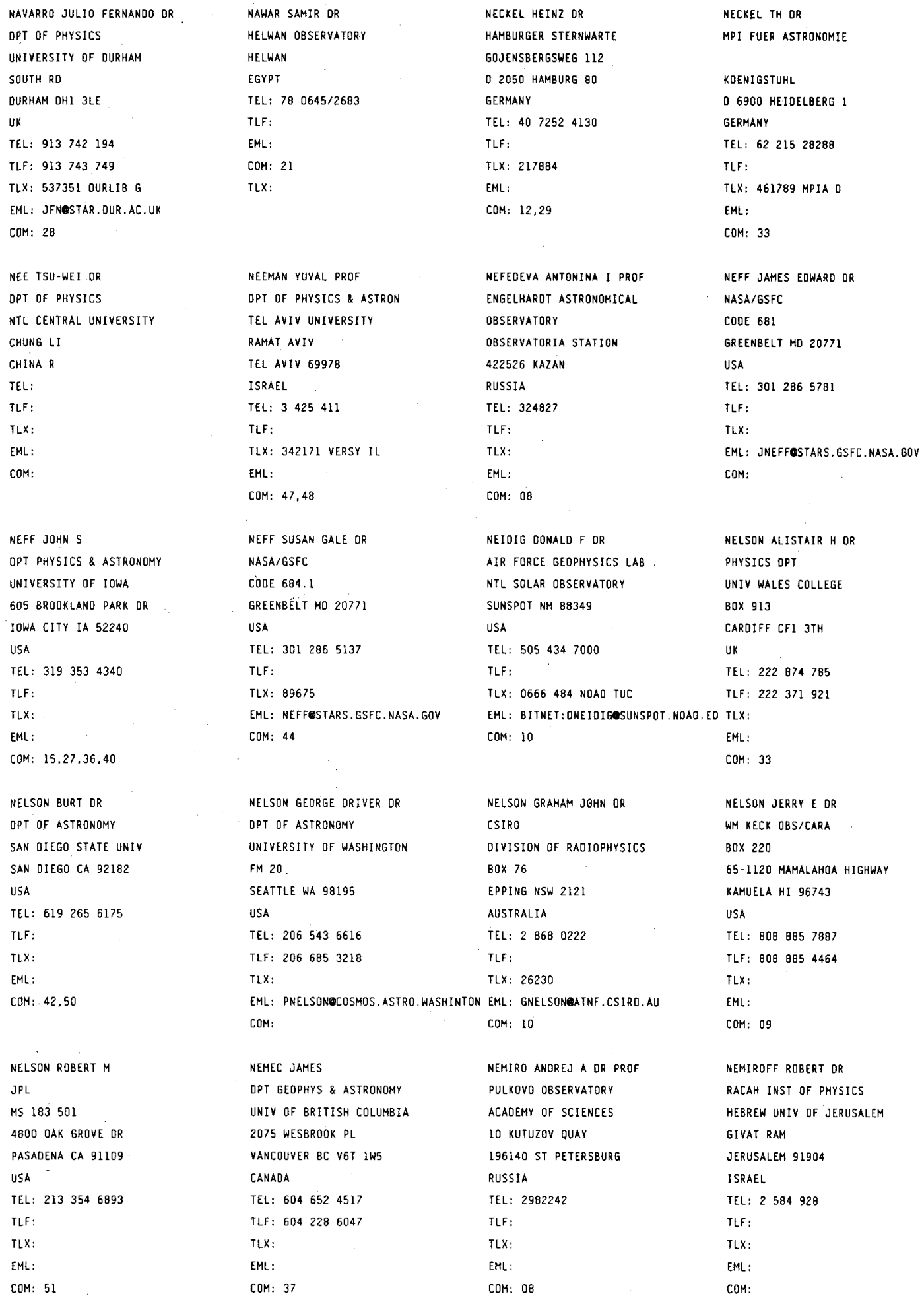




NESCI ROBERTO
ISTITUTO ASTRONOMICO
UNIVERSITA DI ROMA
VIA LANCISI 29
I OOI6I ROMA
ITALY
TEL: 6867525
ILF:
TLX: 613255 INFNRO
EML:
COM: 37

NEWSOM GERALD H PROF

DPT OF ASTRONOMY OHIO STATE UNIVERSITY 174 W $18 T$ H AVE COLUMBUS OH 432101106 USA

TEL: 6144227082

TLF :

TEX:

EML:

COM: 14,42

NG KIN-WANG

INSTITUTE OF PHYSICS ACADEMIA SINICA

TAIPE I

TAIWAN 11529

CHINA R

TEL: 27823075

TLF: 27834187

$T L X$ :

EML: PHKWNGeTWMAS886

COM:
NESIS ANASTASIOS OR

KEIPENHEUER INSTITUT FUR SONNENPHYSIK

SCHONECKSTRASSE 6

D 7800 freiburg breisgau GERMANY

TEL: 761382067

TLF: 76132280

TLX: 7721552 KISO

EML:

COM: 12

NEUKUM G DR

D F $V$ LR

NE-DE-PE

D g03I WESSLING

GERMANY

TEL: $815 \quad 328731$

TLF :

TLX: 0526419 OVLOP D

EML:

COM: 15,16

NEWELL EDWARO B DR

MOUNT STROMLO \& SIDING

SPRING OBSERVATORIES

PRIVATE BAG

WODEN PO ACT 2606

AUSTRALIA

TEL: 62 B日 ILLL

TLF:

TLX: AA 62270 CANOPUS

EML:

COM: 37

NEWTON GAVIN DR

OPT OF PHYSICS \& ASTRON

UNIVERSITY OF GLASGOW

GLASGOW G12 QQQ

UK

TEL: $413398 \quad 855 \star 4196$

ILF : $413 \quad 349029$

TLX: 777070 UNIGLA

EML:

COM: 09

NGUYEN-QUANG RIEU DR

OBSERVATOIRE DE PARIS

SECTION DE MEUDON

F 92195 MEUDON PPL CDX

FRANCE

TEL: 145347530

TLF :

TLX: 270912

EML:

COM: 34,40
NESS NORMAN F DR

BARTOL RESEARCH INSIITUTE

UNIVERSITY OF DELAWARE

NEWARK DE 19716

USA

TEL: $302 \quad 4518116$

TLF :

TLX: 510-6665

EML:

COM: 16,44

NEUPERT WERNER M DR

NASA/GSFC

CODE 680

GREENBELT MD 20771

USA

TEL: $301286 \quad 8169$

TLF :

TLX:

EME:

COM: 10,44

NEWHALL $X \times$ OR

$J P L$

MS $238 \quad 332$

4800 OAK GROVE DR

PASADENA CA 91109

USA

TEL: 8183540000

TLF:

$T L X: 192961003$

EML: SPAN: LOGOS: XXN

COM: $04,19,31$

NEWTON ROBERT R OR

APPLIEO PHYSICS LAB

JOHNS HOPKINS UNIVERSITY

JOHNS HOPKINS RD

LAUREL MO 20707

USA

TEL: 3019537100

TLF:

TLX:

EML:

COM:

NHA IL-SEONG DR

YONSEI UNIVERSITY OBS

134 SINCHON-DONG

SUDAEMUN

SEOUK 120

KOREA $R$

TEL: $392-0131$

TLF :

TLX:

EMt:

COM: $38,42,46$
NETZER HAGAI PROF

SCHOOL OF PHY \& ASTRON

TEL AVIV UNIVERSITY

RAMAT AVIV

TEL AVIV 69978

ISRAEL

TEL: 35450208

TLF:

TLX: 342171 VERSY IL

EML: BITNET: H3IETAUNOS

COM:

NEUZIL LUDEK DR

ASTRONOMICAL INSTITUTE

CZECH ACADEMY OF SCIENCES

ONDREJOV OBSERVATORY

CS 25165 ONDREJOV

CZECHOSLOVAKIA

TEL: 20485201

TLF: 20485314

TLX:

EML:

COM: 21

NEWMAN MICHAEL JOHN DR LOS ALAMOS NATIONAL LAB MS $8220 \times 2$

BOX 1663

LOS ALAMOS NM 87545

USA

TEL: $505 \quad 6677698$

TLF :

TLX:

EML:

COM: 35

NEY EDWARD P PROF

DPT OF ASTRONOMY

UNIVERSITY OF MINNESOTA

116 CHURCH ST SE

MINNEAPOLIS MN 55455

USA

TEL: $612 \quad 373 \quad 4687$

ILF :

$\mathrm{TLX}$ :

EMLL:

COM: 21

NIARCHOS PANAYIOIIS PH D OPT OF ASTRONOMY

NTL UNIVERSITY OF ATHENS PANEPISTIMIOPOLIS

GR 15.771 ZOGRAFOS

GREECE

TEL: 17243414

TLF:

TLX:

EML:

COM: $27,42,51$ 


NLAZY ADNAN MOHAMMAD OR
KACST
BOX 6086
RIYADH 11442
SAUDI ARABIA
TEL: 14883751
TLX:
EML:
TLF:
COM:

NICOLACI DA COSTA L-A OBSERVATORIO NACIONAL RUA GL BRUCE 586 SAO CRISTOVAO 20921 RIO DE JANEIRO RJ BRAZIL

TEL: 21580.7313

TLF: $21580 \quad 0332$

TLX: 021-21288

EML:

COM:

NICOLET MARCEL PROF

IASB

AVE DEN DOORN 30

B 1180 BRUSSELS

BELGIUM

TEL: 23742948

TLX: 21563 ESPACE B

EML:

TLF:

COM: 12,21

NIEONER MALCOLM DR

NASA/GSFC

GREENBELT MO 20771

USA

TEL: 3012862000

ILF:

EML:

COM:

TLX:

NIEUWENHUIJZEN HANS DR STERREKUNDIG INSTITUTE BOX 80000

NL 3508. TA UTRECHT

NETHERLANOS

TEL: $30 \quad 535237$

TLX: 40048 FYLUT NL

EML: BITNET: XMHANSEN@HUTRUUO

TLF:

COM:
NICHOLLS RALPH W PROF DPT OF PHYSICS

YORK UNIVERSITY

4700 KEELE ST

NORTH YORK ON MOJ IP3

CANADA

TEL: 4167365247

TLF: 4167365386

TLX: 06524736

EML: FS3000030YUSOL

COM: $14 \mathrm{C}, 29$

NICOLAIOIS EFTHYMIOS OR NTL RESEARCH FOUNOATION 48 VAS CONSTANTINOU AVE GR 11635 ATHENS

GREECE

TEL: 17210554

TLF : 17212729

TLX: 224064

EML:

COM: 41

NICOLOV NIKOLAIS SOR

DPT OF ASTRONOMY

UNIVERSITY OF SOFIA

aNTON fYaNOV ST 5

BG 1126 SOFIA

BULGarIa

TEL: 2544852

TLF:

TLX:

EML:

COM: 27,46

NIELL ARTHUR E DR

HAYSTACK OBSERVATORY

WESTFORD MA O1886

USA

TEL: 6176924764

TLF:

EML:

COM:

TLX: 948149

MIIMI YUK10

TOKYO ASTRONOMICAL OBS

NAOJ

osahA MITAKA

TOKYO 181

JAPAN

TEL: 422325111

TLF :

TLX: 2822307 TAOMTK J

EML:

COM: 31
NICHOLSON WILLIAM

ROYAL GREENWICH OBS

HERSTMONCEUX CASTLE

HAILSHAM BN27 IRP

UK

TEL:

TLF:

ILX:

EML:

COM: 24

NICOLAS KENNETH ROBERT NAVAL RESEARCH LABORATORY CODE 4163

4555 DVERLOOK AVE SW WASHINGTON OC 203755000 USA

TEL: 2027672517

TLF:

TLX:

EML:

COM: 12

NICOLSON GEORGE D DR

HARTEBEESTHOEK RADIOASTRO

OBSERVATORY

BOX 443

KRUGERSDORP 1740

SOUTH AFRICA

TEL: $27 \quad 116424692$

TLF: $27 \quad 11642 \quad 2446$

TLX: 321006 HART SA

EML:

COM: 40

NIEMELA VIRPI S DR

CALLE 51 ESO 11

1894 VILLA ELISA (BS AS)

ARGENT INA

IEL: 2143793

TLF:

$T L K$ :

EML:

COM: 29.42

\section{NIKITIN A A OR}

ASTRONOMICAL OBSERVATORY

ST PEJERSBURG UNIVERSITY

B1BLIOTECHNAJA PL 2

198904 ST PETERSBURG

RUSSIA

TEL: 293-22-62

TLF :

TLX:

EML:

Con:
NICHOLS-BOHLIN JOY OR COMPUTOR SCIENCES CORP

10000 A AEROSPACE RO LANHAM SEABROOK MD 20706 USA

TEL: 3017941410

TLF:

TLX:

EML: 6890 : : NICHOLS

COM: 44

NICOLET BERMARD

OBSERVATOLRE DE GENEVE

CHEMIN DES MAILLETTES 51

CH 1290 SAUVERNY

SWITZERLAND

TEL: 227552611

ILF: 227553983

TLX: 419209 OBS CH

EML: nicoletoobs,unige.ch

COM: 25,45

NIEONER MALCOLM B DR

NASA/GSFC

LASP

GREENBELT MO 20771

USA

TEL:

TLF:

TLX:

EML:

COM: 15

NIEMI AIMO

TURKU UNIVERSITY OBS

TUORLA

SF 21500 PIIKKIO

FINLAND

TEL: $21 \quad \mathbf{4 3} 5822$

TLF: 21433767

TLX: 62638 TYF SF

EML:

COM: 09,19

NIKOGHOSSIAN ARTHUR G DR BYURAKAN ASTROPHYSICAL OBSERVATORY

378433 BVURAKAN

ARMENIA

TEL: $88 \quad 52 \quad 28 \quad 3453$

TLF:

TLX: 411576 ASCON SU

EML:

COM: 36 


\author{
NIKOLOFF IVAN OR \\ PERTH OBSERVATORY \\ BICKLEY WA 6076 \\ AUSTRALIA \\ TEL: $9293 \quad 1865$ \\ TLF: \\ EML: \\ COM: 08 \\ TLX:
}

NINKOVIC SLOBODAN

ASTRONOMICAL OBSERVATORY

VOLGINA 7

YU 11050 BEOGRAD

YUGOSLAVIA

TEL: $11419357 / 421 \quad 075$

TLF :

TLX:

EML:

COM: 33,38

NISHIMURA JUN OR

INST SPACE \& AERON SCI

UNIVERSITY OF TOKYO

MEGURO KU

TOKYO 153

JAPAN

TEL: $03-467-1111 \times 388$

TLF :

ILX: J 24550 SPACETKY

EML:

COM:

NISHIO MASANORI DR NOBEYAMA RADIO OBS

NAOJ

MINAMIMAKI MURA

NAGANO 38413

JAPAN

IEL: 81267634381

ILF: $81 \quad 267982506$

ILX: 3329005

EML:

COM: 40

NOBILI ANNA M

IST DI MATEMATICA

UNIVERSITA OI PISA

VIA BUONARROTI 2

I 56127 PISA

ITALY

TEL:

TLF :

TLX:

EML:

COM: 07,20
NIKDLOV ANOREJ OR

DPT OF ASTRONOMY

UNIVERSITY OF SOFIA

ANTON IVANOV ST 5

BG 1126 SOFIA

BULGARIA

TEL: 2544852

ILF:

TLX: 23296 SUKOR BG

EML:

COM: 27

\section{NISHI KEIZO DR}

TOKYO ASTRONOMICAL OBS

NAOS

OSAWA MI TAKA

TOKYO 181

JAPAN

TEL: 0422-32-5111

TLF:

TLX: 02822307 TAOMTK J

EML:

COM: 10,12

NISHIMURA MASAKI
DPT OF PHYSICS
HOKKAIDO UNIVERSITY
KITA 1O. NISHI 8
SAPPORO 060
JAPAN
TEL: $11-71-2111$
TLF:
TLX:
EML:
COM:

NISSEN POUL E PROF

INST OF PHYSICS \& ASTRON

UNIVERSITY OF AARHUS

NY MUNKEGADE

OK 8000 AARHUS $C$

OENMARK

TEL: $86 \quad 128899$

TLF: 86202711

TLX: 64767 AAUSCI DK

EML:

COM: 29,37

NOBILI L DR

OPT DI FISICA G GALILEI

UNIVERSITA DI PADOVA

VIA MARZOLO 8

I 35131 PADOVA

ITALY

TEL: $49844205 * 111$

TLF:

TLK: 430308 DFGGPDI

EML:

COM:
NILSON PETER DR

ASTRONOMICAL OBSERVATORY

BOX 515

S 75120 UPPSALA

SWEDEN

TEL:

TLF:

TLX:

EML:

COM:

NISHIDA MINORU PROF

DPY OF PHYSICS

KYOTO UNIVERSITY

SAKYO KU

KYOTO 606

JAPAN

TEL:

TLF:

TLX: 5422693 LIBKYU J

EML:

COM: $33,35,47$

NISHIMURA SHIRD DR TOKYO ASTRONOMICAL OBS

NAOJ

OSAWA MITAKA

TOKYO 181

JAPAN

TEL: 422-32-5111

TLF:

TLX: 2822307 TAOMTK -0

EML:

COM: $05,09,29$

NITTMAN JOHANN
ETUDES ET FABRICATION
DOWELL SCHLUMBERGER
BP 90
F 42003 ST ETIENME
FRANCE
TEL: 77326423
TLF:
TLX:
EML:
COM:
NOCERA LUIGI DR
IST DI FISICA
ATOMICA E MOLECOLARE
VIA GIARDINO 7
I 56127 PISA
ITALY
TEL: 5050 1384
TLF: 5025175
TLX:
EML: BISTAB
CGM: 10

NILSSON CARL OR

CENTER FOR ASTROPHYSICS

HCO/SAO

60 GARDEN ST

CAMBRIDGE MA 02138

USA

TEL:

TLF:

TLX:

EML:

COM:

NİSHIDA MITSUGU

DPT OF LITERATURE

KOBE WOMEN'S UNIVERSITY

SUMA KU

KOBE 654

JAPAN

TEL: 078-731-4416

ILF:

TLX:

EML:

COM: 33

NISHIMURA TETSUO DR STEWARO OBSERVATORY UNIVERSITY OF ARIZONA TUCSON AZ 85721

USA

TEL: $602 \quad 6212054$

TLF :

TLX: 467175

EML:

COM: 21

NITYANANDA $R$ DR RAMAN RESEARCH INSTITUTE SADASHIVÁNAGAR BANGALORE 560080 INOIA

TEL: 812360122

TLF: B12 340492

TLX: B452671 RRI IN

EML:

COM: 28,48

NOCI GIANCARLO PROF

DPT OI ASTRONOMIA

UNIVERSITA OI FIRENZE

LARGO E FERMI 5

I 50125 FIRENZE

ITALY

TEL: 5527521

TLF : $55 \quad 220039$

TLX: 572268 ARCETR I

EML:

COM: 


\author{
NOEL FERNANDO \\ DPT DE ASTRONOMIA \\ UNIVERSIDAD DE CHILE \\ CASILLA 36 D \\ SANTIAGO \\ CHILE \\ TEL: 22294101 \\ TLF: \\ TLX: 40853 \\ EML: \\ COM: 08C, 31 \\ NOGUCHI MASAFUMI OR \\ TOKYO ASTRONOMICAL OBS \\ NAOJ \\ OSAWA MITAKA \\ TOKYO 181 \\ JAPAN \\ TEL: 0422325111 \\ TLF: \\ ILX: 2822307 TAOMTK J \\ EML: \\ COM: 28 \\ NOONAN THOMAS W PROF \\ SUNY AT BROCKPORT \\ DPT OF PHYSICS \\ BROCKPORT NY 14420 \\ USA \\ TEL: $716 \quad 395 \quad 5581$ \\ TLF: \\ TLX: \\ EML: \\ COM: 28,47
}

NOELS ARLETTE OR

50 AVE DE LA PAIX

BOX 063

B 4030. GRIVEGNEE

BELGIUM

TEL: $41529980 * 7517$

ILF:

TLX:

EML:

COM: 35,46

NOLL KEITH STEPHEN OR

STSCI

HOMEWOOO CAMPUS

3700 SAN MARTIN DR

BALTIMORE MD $2121 \mathrm{~B}$

USA

TEL: $41033 B 5080$

TLF :

TLX:

EML: NOLLOSTSCI.EDU/STSCIC: : NOLL

COM: 15,16

NOROH H LENNART OR

STOCKHOLM OBSERVATORY

S 13336 SALTSJOEBADEN

SWEDEN

TEL: 87170195

TLF: $87 \quad 174719$

EML: NOROHAASTRO,SU,SE

CDM: 34,44

TLX: 12972

NORGAARD-NIELSEN HANS U

UNIVERSITY OBSERVATORY

OESTER VOLOGADE 3

DK 1350 COPENHAGEN K

DEMMARK

TEL: $31141790^{\circ}$

TLF: 31389157

$T \perp X:$

EML:

COM:

NORRIS RAYMOND PAULL

CSIRO

OIVISION OF RAOIOPHYSICS

BDX 76

EPPING NSW 2121

AUSTRALIA

TEL: $2 \quad 8680222$

TLF:

TLX: 26230 ASTRO

EML: RNORRISATNF.CSIRO.AU

COM:
NOENS JACQUES-CLAIR DR

OBS MIOI PYRENEES

9 R PONT DE LA MOUETTE

F 65200 BAGNERES BIGORRE

FRANCE

TEL: $6295 \quad 1969$

TLF: $6295 \quad 1070$

TLX: 531625

EML:

COM: 10

NOLLEZ GERARD DR

INSTITUT D'ASTROPHYSIOUE

9EBIS BD ARAGO

f 75014 PARIS

FRANCE

TEL: 143201425

TLF: 143298673

TLX:

EML:

COM: 14

'.

NORDLUND AKE DR

UNIVERSITY OBSERVATORY

OESTER VOLOGADE 3

DK 1350 COPENHAGEN $K$

DENMARK

TEL: $31 \quad 141790$

TLF: 31389157

TLX: 44155 DANAST

EML:

COM: $12 C, 36$

NORIEGA-CRESPO ALBERTO DR

DPT OF ASTRONOMY

UNIVERSITY OF WASHINGTON

FM 20

SEATTLE WA 98195

USA

TEL: $206 \quad 6852155$

TLF:

TLX:

EML: AIPSACPHAST. PHYS. WASHINTON. ED EML: STSCIC: : NORMAN

COM:

NORTH JOHN DAVIO PROF

FILOSOFISCH INST

RIJKSUNIVERSITEIT

NL 9718 CW GRONINGEN

NETHERLANDS

TEL: 59071846

TLF: 50636160

TLX:

EML:

COM: $41 \mathrm{C}$
COM: $28,33,34,44,47,48$

NOERDLINGER PETER O PROF APPLIEO RESEARCH CORP

8201 CORPORATE DR

SUITE 1120

LANDOVER MD 20785

USA

TEL: 3014598442

TLF:

TLX:

EML:

COM: 47

NOMOTO KEN' ICHI OR

DPT OF ASTRONOMY

UNIVERSITY OF TOKYO

BunkYo KU

TOKYO 113

JAPAN

TEL: $33 \quad 812 \quad 2111 * 4255$

ILF: 338139439

ILX: 2722126 UTGABJ

EML: nomotomopsunl astron, s, u. -toky

COM: $35 C, 48$

NORDSTROEM BIRGITTA DR

COPENHAGEN UNIVERSITY OBS

BRORFELOEVEJ 23

OK 4340 TIKKISE

DENMARK

TEL: $53 \quad 48 \quad 8195$

TLF: 53488755

TLX: 44155 DANAST DK

EML: BIRGITTAEASTRO.KU.OK

COM: $30,33,42$

NORMAN COLIN A PROF

STSCI

HOMEWOOD CAMPUS

3700 SAN MARTIN DR

BALTIMORE MD 21218

USA

TEL: 3013384895

TLF:

NORTH PIERRE

INSTITUT O.ASTRONOMIE

UNIVERSITE OE LAUSANNE

CH 1290 Chavannes-D-80IS

SWITZERLAND

TEL: 227552611

TLF :

TLX: 27720 OBSG CH

EML: northeobs.unige.ch

COM: 45 
NOSKOV BORIS N OR

STERNBERG STATE ASTR INST

UNIVERSITETSKIJ PROSP 13

119899 MOSCOW

RUSSIA

TEL:

TLX:

EML:

TLF:

COM: 07

NOVICK ROBERT

DPT OF PHYSICS

COLUMBIA UNIVERSITY

538 W 120TH ST

NEW YORK NY 10027

$$
\text { USA }
$$

TEL: $212 \quad 2803293$

TLF:

TLX: 22094 COLU UR

EML:

COM: 44,48

NOVOTNY JAN OR
FAC OF SCIENCE
MSARYK UNIVERSITY
XOTLARSKA 2
CS 611 37 BRNO
CZECHOSLOVAKIA
TEL:
TLF:
TLX:
EML:
COM: 47

\section{NULSEN PAULL DR}

MOUNT STROMLO \& SIOING

SPRING OBSERVATORIES

pRIVATE bag

WOOEN PO ACT 2606

AUSTRALIA

TEL: $6280 \quad 111 \mathrm{~L}$

TLF:

TLX: 62270 AA

EML:

COM: $28,34,48$

NUTH JOSEPH A III

NASA/GSFE

CODE 691

LEP

GREENBELT MO 20771

USA

TEL: 3012866364

TLF:

TLX:

EML:

COM: 22,34
NOTNI $P$ OR

2NTRLINST F ASTROPHYSIK

STERNWARTE BABELSBERG

ROSA- LUXEMBURG-STR $17 \mathrm{~A}$

D 1502 potsdaM

GERMANY

TEL:

TLF:

TLX:

EML:

COM: 25,45

NOVIKOV I O DR

SPACE RESEARCH INSTITUTE

ACADEMY OF SCIENCES

PROFSOJOSNAYA UL B4/3Z

117810 MOSCOW

RUSSIA

TEL:

TLF:

TLX:

EML:

COM: 47

\section{NOVOTNY VACLAY}

ASTRONOMICAL INSTITUTE

CZECH ACADEMY OF SCIENCES ONDREJOV OBSERVATORY

CS 25165 ONOREJOV

CZECHOSLOVAKIA

TEL: 20485201

TLF: 20485314

TLX: 121579

EML:

COM: 44

NUNES ROGERIOS DE SOUSA

GRUPO DE MATEM APLICAOA

UNIVERSIOADE DO PORTO

RUA DAS TAIPAS 135

P 4000 PORTO

PORTUGAL

TEL: 380313/769

TLF:

TLK:

EML:

COM: 09

\section{NYMAN LARS-AKE DR}

ONSALA SPACE OBSERVATORY GOETEBORG UNIVERSITY

S 439 DO ONSALA

$$
\text { SWEDEN }
$$

TEL: 30060651

TLF :

TLX: 2400

EML:

COM:
NOTTALE LAURENT

OBSERVATOIRE OE PARIS

SECTION DE MEUDON

DAF.

F 92195 MEUDON PPL CQX

FRANCE

TEL: $145 \quad 077403$

TLF:

TLX: 201571

EML:

COM: 47

NOVIKOV SERGEJ B DR

STERNBERG STATE ASTR INST

117234 MOSCOW

RUSSIA

TEL:

ILF :

TLX:

EML:

COM:

NOYES ROBERT W PROF

CENTER FOR ASTROPHYSICS

HCO/SAO

60 GARDEN ST

CAMBRIDGE MA 02138

USA

TEL: 6174957424

TLF:

TLX: 921428 SATELLITE CAM

EML:

COM: $10,12,44$

NUNEZ JORGE DR

OBSERVATORIO FABRA

TIBIDADO

E 08022 BARCELONA

SPAIN

TEL: 32475736

TLF:

TLX:

EML:

COM: 24

OBASHEV SAKEN O OR

ASTROPHYSICAL INSTITUTE

KAZAKH ACAD OF SCIENCES

46006B ALMA ATA

KAZAKHSTAN

TEL:

TLF:

ILX: 275

EML:

COM:
NOVELLO MARIO OR

CTR BRAS PESQUISAS FISIC RUA OR XAVIER SIGAUD

150 URCA

22290 RIO DE JANEIRO RJ

BRAZIL

TEL: $21 \quad 5410337$

TLF :

TLX: 2122563

EML:

COM: 47

NOVOSELOV V S PROF DR ASTRONOMICAL OBSERVATORY ST PETERSBURG UNIVERSITY BIBLIOTECHNAJA PL 2

198904 ST PETERSBURG

RUSSIA

TEL: 257-94-91

TLF:

TLX:

EML:

COM: 07

NUGIS TIIT

TARTU ASTROPHYSICAL OBS

ESTONIAN ACAD OF SCIENCES 202444 TARTU

ESTONIA

TEL:

TLF:

ILX:

EML:

COM: $27,29 \mathrm{C}$

NUSSBAUMER HARRY PROF INSTITUT FUER ASTRONOMIE ETH ZENTRUM

CH 9092 ZUERICH

SWITZERLAND

TEL: 12563631

TLF:

TLX: 53178 ETHBI CH

EML:

COM: $10,14 \mathrm{C}, 34$

OBI SHINYA PROF

FACULTY OF GENERAL EOU

UNIVERSITY OF TOKYO

MEGURO KU

TOKYO 153

JAPAN.

TEL:

TLF:

ILX:

EML:

COM: 14 


\section{OBLAK EDOUARD}

OBSERVATOIRE OE BESANCON

41 IS AVE OBSERVATOIRE

F 25000 BESANCON CDX

FRANCE!

TEL: 81503088

TLF:

TLX: 361144

EML:

COM: $25,26,33$

OCHSENBEIN FRANCOIS DR

OBSERVATOLRE ASTRONOMLQUE

11 RUE UNIVERSITE

F 67000 STRASBDURG

FRANCE

TEL: $88 \quad 358218$

TLF: $88 \quad 25 \quad 0160$

TLX: 890506 STAROBSF

EML:

COM: 05

ODENWALO STEN F DR

NAVAL RESEARCH LABORATORY

CODE 41380

4555 OVERLOOK AVE SW

WASHINGTON OC 203755000

USA

TEL: 2027673010

TLF:

TLX:

EML:

COM:

\section{OEHMAN YNGVE PROF}

JHULELEM 53

S 22367 LUND

SWEOEN

TEL: $46 \quad 143362$

TLF:

EML:

COM:

TLX:

OESTERWINTER CLAUS'
BOX 1270
OAHLGREN VA 224485180
USA
TEL: 7036632555
TLF:
EML:
COM: 04,07
TLX:

OBREGON OIAZ OCTAVIO J DR

DPT DE FISICA

UNAM UNIOAO

APDO POSTAL 55-534

09340 MEXICO OF

MEXICO

TEL: 6860322

TLF :

TLX: 1764296 UAM ME

EML:

COM:

ODA MINORU PROF

WAKD SHI

SALTAMA 35101

JAPAN

TEL: 0484621111

TLF: 0484675942

EML:

COM: $44,48,51$

ILX: 02962818 RIKEN J

ODGERS GRAHAM J DR

HERZBERG INST ASTROPHYS

DOMINION ASTROPHYS OBS

5071 W" SAANICH RO

VIETORIA BC VOX 4 MG

CANADA

TEL: 6043683977

ILF: 6043630045

TLX: 0497295

EML:

COM: 09,27

OEKTEN ADNAN DR

UNIVERSITY OBSERVATORY

ISTAMBUL UNIVERSITY

34452 ISTANBUL

TURKEY

TEL: 15223597

TLF : I 5226123

TLX:

EML: JKO17@TRIUVM11

COM: 10

OESTGAARD ERLENO

OPT OF PHYSICS

UNIVERSITY OF TRONDHEIM

AVH

N 7055 DRAGVOLLM

NORWAY

TEL: $07-920411 \times 117$

TLF:

TLX:

EML:

COM:
OBRIOKO VLADIMIR N OR

IZMIRAN

ACADEMY OF SCIENCES

242092 TROITSK

RUSSIA

TEL: $232-1921$

TLF:

TLX: 412623 SCSTP

EML:

COM: 10

ODA NAOKI

MICRO-ELECTRONICS RES LAB

NIPPON ELECTRIC COMPANY

4-1-1 MIYAZAKI MIYAMAE KU

KAWASAKI KANAGAWA 213

JAPAN

TEL:

TLF:

ILX:

EML:

COM: 44

OEGELMAN HAKKI B DR

MAX PLANCK INSTITUT FÜ

EXTRATERESTRISHE PHYSICS

KARL-SCHWARZSCHILOSTR

D GO4E GARCHING MUENCHEN

GERMANY

TEL: 8932993833

ILF: 8932993569

TLX:

EML: HBO AT DGAIPPIS

COM:

OEMLER AUGUSTUS JR OR

YALE UNIVERSITY OBS

BOX 6666

NEW HAVEN CT 06511

USA

TEL: 2034363460

TLF:

TLX:

EMt:

COM: $2 B, 47$

OESTREICHER ROLAND

LANDESSTERNWARTE

KOENIGSTUHL

D 6900 HEIDELBERG

GERMANY

TEL: 622110036

TLF

TLX:

EML:

COM: 25
OCCHIONERO FRANCO PROF

OAR

VIA DEL PARCO MELLINI 84

100136 ROMA

ITALY

TEL: 63452556

TLF :

TLX:

EML:

cOM:

ODELL ANOREW $P$

ASTROPHYSICAL DBSERVATORY

N ARIZONA UNIVERSITY

FLAGSTAFF AZ 86011

USA

TEL:

TLF :

TLX:

EML:

COM: 35

OEGERLE WILLIAM R

5924 BERWYN RD

COLLEGE PARK MO 20740

USA

TEL:

TLF:

TLK:

EML:

COM:

OERTEL GOETZ K DR

AURA INC

1625 MASSACHUSETTS AVE NW

SUITE 701

WASHINGTON OC 20036

USA

TEL: 2024832102

TLF

TLX:

EML: TELEMAIL: G, OERTEL

COM: 44

OETKEN L DR

ZNTRLINST F ASTROPHYSIK ASTROPHYSIK OBSERVATORIUM

TELEGRAFENBERG

D 1500 POTSDAM

gerMANY

TEL:

TLF:

TLX:

EML:

COM: 14,30 


OEZEL MEHMET EMIN OR
OPT OF PHYICS
CURUROVA UNIVERSITY
O133O ADANA
TURKEY
TEL: $711333394 \times 2480$
TLF: 71121945
TLX: 62934
EML:
COM: $40,48,50$
OGIR MAYA OR
CRIMEAN OBSERVATORY
NAUCHNY
CRIMEA
UXRAINE
TEL: 432945
TLF:
TLX:
EML:
COM: 10

DHISHI MASATOSHI OR NOBEYAMA RADIO OBS NAOJ

MINAMIMAKI MURA

NAGANO 38413

JAPAN

TEL: $81 \quad 267 \quad 634373$

TLF: $81 \quad 26798 \quad 2884$

ILX: 3329005 NADNRO J

EML: NROOIST IPNNRO.BITNET

COM: 40

OHTSUBO JUNJI DR
FACULIY OF ENGINEERING
SHIZUOKA UNIVERSITY
3 CHOME JYOHOKU
HAMAMATSU 432
JAPAN
TEL: 534711171 EXT 5
TLF: 534751764
TLX:
EML:
COM: 09
OKA TAKESHI OR
OPT OF CHEMISTRY
UNIVERSITY OF CHICAGO
5735 S ELLIS AVE
CHICAGO IL 60637
USA
TEL: 3129627070
TLF:
TLX:
EML:
COM: 14

OEZKAN MUSTAFA TUERKER DR UNIVERSITY OBSERVATORY ISTANBUL UNIVERSITY 34452 ISTANBULL TURKEY

TEL: I 5283847

TLF: 15226123

TLX:

EML: JKOL7 TETRIUYMII

COM: 42

\section{OgURA KATSUO DR \\ COL OF Literature \\ KOKUGAKUIN UNIYERSITY \\ HIGASHI 4-10-28 \\ SHIBUYAKU TOKYO 150 \\ JAPAN \\ TEL: 298-42-6913 \\ TLF : \\ TLX: 28899 SIBINBTH J \\ EML: \\ COM: 37}

OHKI KENICHIRO OR
TOKYO ASTRONOMICAL OBS
NAOJ
OSAWA MITAKA
TOKYO 181
JAPAN
TEL: $0422-32-5111$
TLF:
ILX: 2822307
EML:
COM: 10

OHYAMA NOBORU PROF
FACULTY OF ENGINEERING
SHIZUOKA UNIVERSITY
3 CHOME JYOHOKU
HAMAMATSU 432
JAPAN
TEL:
TLF:
TLX:
EML:
COM: 35
OKAMOTO ISAO DR
INTL LATITUDE OBSERVATORY
NAOJ
HOSHIGAOKA MIZUSAWA SHI
IWATE 023
JAPAN
TEL: 0197-24-7111
TLF:
TLX: 8376-28 ILSMIZ J
EML:
COM: 35

OGAWARA YOSHIAKI

INST SPACE \& ASTRON SCI UNIVERSITY OF TOKYO

MEGURO KU

TOKYO 153

JAPAN

TEL: 3-467-1111

TLF:

TLX: 34757 ISASTRO $J$

EML:

COM: 44,48

OH KYU DONG DR

DPT EARTH SCIENCE

CHONNAM NTL UNIVERSITY

KWANGJU CHONNAN

KOREA R

TEL:

ILF:

TLX:

EML:

COM: 42

OHRING GEORGE PROF

DPT GEOPHYS \& PLANET SCI

TEL AVIV UNIVERSITY

IEL AVIV

ISRAEL

IEL:

ILF:

TLX:

EML:

COM:

OJA HEIKKI OR

OBS \& ASTROPHYSICS LAB

UNIVERSITY OF HELSINKI

TAERTI TORNIMMAKI

SF 00130 HELSINKI 13

FINLAND

TEL: 1912942

ILF:

TLX:

EML:

COM: 46

OKAMURA SADANORI DR
KISO OBSERVATORY
UNIVERSITY OF. TOKYO
MITAKEMURA KISOGUN
NAGANO 39701
JAPAN
TEL: $0264-52-3360$
TLF:
TLX: 3347577 KS00BS
EML:
COM: $28 C$

OGELMAN HAKKI B DR

OPT OF PHYSICS UNIVERSITY OF WISCONSIN 1150 UNIVERSITY AVE MADISON WI 53706 USA

TEL: 6082652052

TLF; 6082630800 TLX: 265452 UOFWISC MOS EML: 47452: :0GELMAN

COM:

OHASHI TAKAYA DR

OPT OF PHYSICS

UNIVERSITY OF TOKYO

BUNKYO KU

rokYO 113

JAPAN

TEL: 38122111

TLF: 38126938

TLX: UTPHYSIC $23472 \mathrm{~J}$

EML:

COM:

OHTANI HIROSHI OR

OPT OF ASTRONOMY

KYOTO UNIVERSITY

KITASHIRAKAWA SAKYO KU KYOTO 606

JAPAN

TEL: 075-751-2111

TLF:

TLX: 5422693 LIBKYU J

EML:

COM: 34

OJA TARMO PROF KVISTABERG OBSERVATORY

S 19700 BRO

SWEOEN

TEL: $7584 \quad 0157$

TLF:

TLX:

EML:

COM: $24,33,45$

OKAZAKI AKIRA DR DPT SCIENCE EOUCATION GUNMA UNIVERSITY MAEBASHI GUNMA 371 JAPAN

TEL: $0272 \quad 321611$ TLF: 0272339231

TLX:

EML:

COM: 42 


OKAZAKI ATSUO T DR
COL OF GEN EDUCATION
HOKKAI GAKUEN UNIVERSITY
TOYOHIRA-KU
SAPPORO 062
JAPAN
TEL: $0118411161 * 284$
TLF:
TLX:
EML: ALO935EJPNKUDPC. BITNE
COM: 29

OKOYE SAMUEL E PROF

DPT OF PHYSICS ASTRON

UNIVERSITY OF NIGERIA

NSUKKA ANAMBRA STATE

NIGER IA

TEL: $042-770752$

TLF:

TLX:

EML:

COM: $38,40,46,47,4 \theta$

OLAH KATALIN OR

KONKOLY OBSERVATORY

THEGE U 13/17

BOX 67

H 1525 BUDAPEST

HUNGARY

TEL: 175 5866/75 4122

TLF :

ILX: 227460 KONOB H

EML:

COM: 27,42

OLIVA ERNESTO OR
OSS ASTROFISICO
OI ARCETRI
LARGO E FERMI 5
I 50125 FIRENZE
ITALY
TEL: 552752310
TLF: 15220039
TLX: 572268
EML: SPAN: $38954:$ : OLIVA
COM:

OLLONGREN A PROF DR STERREWACHT

DPT MATHS \& COMPUTER SCI

B0X 9512

NL 2300 RA LEIOEN

NETHERLANDS

TEL: $71272727 * 5006$

TLF:

TLX: 39058 ASTRO NL

EML:

COM: 33,51

OKAZAKI SEICHI DR
$2-4-4$ OSAWA.
MITAKA
TOKYO 181
JAPAN
TEL: 0422316770
TLF:
TLX:
EML:
COM:

OKUDA HARUYUKI OR PROF
INST SPACE \& ASTRON SCI
UNIVERSITY OF TOKYO
MEGURO KU
TOKYO 153
JAPAN
TEL: 03-467-1111
TLF:
TLX: 24550 SPACETKY J
EML:
COM: 33,34

OLANO CARLOS ALBERTO OR IAR.

CC. 5

1894 VILLA ELISA (BS AS) ARGENTINA

TEL: 2143793

TLX:

EML:

TLF:

COM: 33

OLIVER BERNARD M OR
NASA AMES RESEARCH CTR
MS 229 B
MOFFETT FIELO CA 94035
USA
TEL: 4156945165
TLF:
TLX: 348408 NASA AMES
EML:
COM: 51

OLNON FRISO

NFRA

BOX 2

NL 7990 AA DWINGELOO

NETHERLANDS

TEL: $52 \quad 197244$

TLX: 42043 SRZM NL

EML:

TLF:

COM:
OKE J BEVERLEY PROF

CALTECH

MS 10524

PASADENA CA 91125

USA

TEL: 8183564007

TLF:

TLX:

EML:

COM: 28

OKUDA TORU

INST OF EARTH SCIENCE

HOKKAIDO UNIV OF EOUCAT

1.2 HACHIMAN CHO

HAKODATE 040

JAPAN

TEL: $0139-41-1121$

TLF:

TLX:

EML:

COM: 44

OLBERG MLCHAEL DR

ONSALA SPACE OBSERVATORY

GOETEBORG UNIVERSITY

S 43900 ONSALA

SWEDEN

TEL: 30060650

ILF: 30062621

TLX: 2400 ONSPACE $S$

EML: OLBERGOSO.CHALMERS. SE

COM: 40

OLIVER BERNARD M PROF

NASA AMES RESEARCH CTR CHEIF SETI PROGRAM OFFICE MOFFETT FIELO CA 94035

USA

TEL: 4156945166

TLF:

TLX:

EML:

COM: 51

OLOFSSON HANS

ONSALA SPACE OBSERVATORY

GOETEBORG UNIVERSITY

$S 43900$ ONSALA

SWEOEN

TEL: 30060650

TLF:

TLX: 8542400 ONSPACE

EML:

COM: 34
OKEKE PIUS N DR

DPT OF PHYSICS \& ASTRON

UNIVERSITY OF NIGERIA

NSUKKa ANAMBRA StaTE

NIGERIA

TEL: 42771911

TLF:

TLX:

EML:

COM: 48

OKUMURA SACHIKO DR

OPT EARTH SCI \& ASTRON

UNIVERSITY OF TOKYO

MEGURO KU

TOKYO 153

JAPAN

TEL: 81334671171

TLF: 81334852904

TLX: 2426728 TODAIK J

EML: OKUMURAGKYOHOU.C.U-TOKYO.AC.

COM: 40

OLEAK H OR

ZNTRLINST F ASTROPHYSIK

STERNWARTE BABELSBERG

ROSA-LUXEMBURG-STR I7A

D 1502 POTSOAM

GERMANY

TEL:

TLF:

ILX:

EML:

COM: 28

OLIVER JOHN PARKER IR DPT OF ASTRONOMY

UNIVERSITY OF FLORIDA

211 SSRB

GAINESVILLE FL 32611

USA

TEL:

ILF:

TLX:

EML:

COM: 42

DLOFSSON S GOERAN DR STOCKHOLM OBSERVATORY

S 133 J6 SALTSJOEBADEN

SWEDEN

TEL: 87172639

TLF: 87174719

TLX: 12972

EML: OLOFSSONASTRO.SU.SE

COM: 


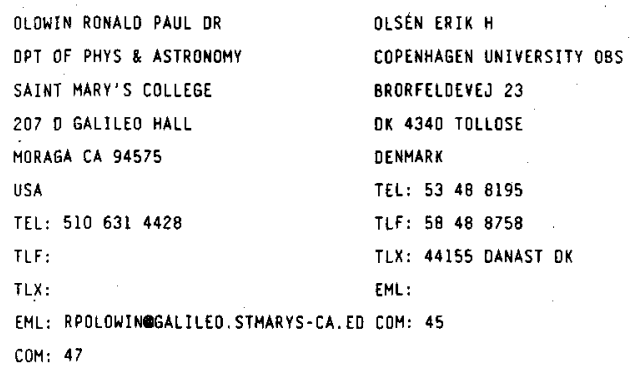

EML: RPOLOWINEGALILEO. STMARYS-CA. ED COM: 45

OLSEN ERIK H

COPENHAGEN UNIVERSITY OBS

BRORFELDEVEJ 23

DK 4340 TOLLOSE

DENMARK

TEL: 53488195

TLF: 59488758

TLX: 44155 DANAST OK

EML:

OLTHOF HINOERICUS OR
ESA/ESTEC
SSD
BOX 299
NL 2200 AG NOOROWIJK
NETHERLANDS
TEL: 171986555
TLF:
TLX: 39098 ESTC NL
EML: SPAN:ESTEC $1:$ : HOLTHOF
COM: 44

\section{ONAKA TAKASH!}

DPT OF ASTRONOMY

UNIVERSITY OF TOKYO

BUNKYO KU

TOKYO 113

JAPAN

TEL: 3-812-2111

TLF:

TLX: 33659 UTYOSCI $]$

EML:

COM: 34

ONUORA LESLEY IRENE DR DPT OF PHYSICS \& ASTRON UNIVERSITY OF NIGERIA NSUKKA ANAMBRA STATE NIGERIA

TEL:

TLF:

TLX: 51496 ULIONS

EML:

COM: $40,46,47$

OPOLSKI ANTONI PROF

ASTRONOMICAL INSTITUTE WROCLAW UNIVERSITY UL KOPERNIKA 11

PL 51622 WROCLAW POLANO

TEL:

TLF:

TLX:

EML:

COM: 27
OLSEN FOGH H J

COPENHAGEN UNIVERSITY OBS BRORFELOEVEJ 23

OK 4340 TOLLOSE

DENMARK

TEL: 53488195

ILF: 58488758

TLX: 44155 OQNOST

EML:

COM: $08 ; 46$

OMAROV TUKEN B PROF

ASTROPHYSICAL INSTITUTE

KAZAKH ACAD OF SEIENCES

480068 ALMA ATA

KAZAKHSTAN

TEL: $64 \cdot 40 \cdot 40$

TLF:

TLX:

EML:

COM: 07

ONDERLICKA BEDRICH OR

OPT OF ASTRONOMY

PURKYNE UNIVERSITY

KOTLARSKA 2

CS 61137 BRNO

CZECHOSLOVAKIA

TEL: 51112

TLF:

TIX:

EML:

COM:

DOE MASATSUGU DR

INTL LATITUDE OBSERVATORY

NAOJ

HOSHIGAOKA MIZUSAWA SHI

IWATE 023

JAPAN

TEL: 0197-24-7111

TLF:

TLX: 837628 MIZ J

EML:

COM: 19

OPROIU TIBERIU DR

ASTRONOMICAL OBSERVATORY

BLOC R-5 SC I \& II AP 10

STR BUCIUM 25

R 3400 CLUJ NAPOCA

RUMANIA

TEL: $951-62616$

TLF:

TLX:

EML:

COM:
OLSEN KENNETH H OR

GCS

BOX 1273

LYNNWOOD WA $98046 \quad 1273$

USA

TEL:

ILF:

TLX:

EML:

COM:

OMNES ROLANO PROF

LPTHE

UNIVERSITE PARIS XI

BT 211

F 91405 ORSAY CDX

FRANCE

TEL: 169417744

TLF :

TLX: 692166

EML:

COM: 47

ONEGINA A B DR

MAIN ASTRONOMICAL OBS

UKRAINIAN ACAD OF SCIENCE

GOLOSEEYO

252127 KIEV

UKRAINE

TEL: 663744

TLF:

TLX:

EML:

COM: 24

OORT JAN H PROF

PRES KENNEOYLAAN 169

NL 2343 GZ DEGSTGEEST

NETHERLANDS

TEL:

TLF:

TLX:

EML:

COM: $28,33,40,47$

ORCHISTON WAYAE OR

27 MARIAN DRIVE

GISBORNE

NEW ZEALAND

TEL: 83 332

TLF:

TLX:

EML:

COM: 41 
ORELLANA ROSA BEATRIZ OR OBSERVATORIO ASTROMOMICO paseo OEL bosque 1900 LA PLAT́a (BS.AS.) ARGENTINA

TEL: 21217308

TLF:

TLX:

EML: RORELLANAXFCAGLP. EOU. AR. COM: 07

ORRALL FRANK O PROF

INSTITUTE FOR ASTRONOMY UNIVERSITY OF HAWAII 2680 WOODLAWN $D R$ HONOLULU HI 96822 USA TEL: 8089488667 TLF: TLX: 723-8459 EML: COM: $10,12,36$

ORTON GLENN S DR

JPL

MS 183301

4800 OAK GROVE DR

PASADENA CA 91109

USA

TEL: 8183542460

TLF:

TLX:

EML:

COM: 14

OSAWA KIYOTERU DR
TOKYO ASTRONOMICAL OBS
NAOJ
OSAWA MITAKA
TOKYO 181
JAPAN
TEL: $0422-32-5111$
TLF:
TLX:
EML:
COM:
OSMER PATRICK S OR
HOAO
BOX 26732
95O N CHERRY AVE
TUCSON AZ 857266732
USA
TEL: 6023275511
TLF:
TLX: 666484
EML:
COM:

ORLIN HYMAN DR

NTL ACADEMY OF SCIENCES

NTL RESEARCH COUNCIL

2101 CONSTITUTION AVE NW

WASHINGTON OC 20418

USA

IEL:

TLF:

TLX:

EML:

COM:

ORSATTI ANA M DR

UNIV NACIONAL DE LA PLATA

FCAG

1900 LA PLATA (BS.AS.)

ARGENTINA

TEL: 21217308

TLF:

TLX: 31151 BULAP

EML: AMDECAGLP.EDU.AR

COM: 25

ORUS JUAN 3 PRBF

DPT FISICA OE ATMOSFERA

UNIVERSIDAD DE BARCELONA

AVD DIAGONAL 645

E 08028 BARCELONA

SPAIN

TEL:

TLF:

TLX:

EML:

COM: 07

OSBORN WAYNE DR

OPT OF PHYSICS

CENTRAL MICHIGAN UNIV

MT PLEASANT MI 48859

USA

TEL: $517 \quad 7743321$

TLF:

TLX:

EML: 3Y2LW5GACMUVM. BITNET

COM: $24,45,46$

OSORLO JOSE J S P PROF observatorio astronomico. UNIVERSIDADE DO PORTO MONTE. OA VIRGEM

P 4400 VILA NDVA DE GAIA PORTUGAL

TEL: 7820404

TLF:

TLX: 22367

EML:

COM: $07,08,46,50$
ORLOV MIKHAIL OR

MAIN ASTRONOMICAL OBS

UKRAINIAN ACAO OF SCIENCE

GOLOSEEVO

252127 KIEV

UKRAINE

TEL: 663110

TLF:

TLX: $131406 \mathrm{SKY}$

EML:

COM: 29

\section{ORTE ALBERTO}

CECILIO PUJAZON 22-3 A

E 11100 SAN FERnando

SPAIN

TEL: $56 \quad 89 \quad 5441$

TLF :

TLX:

EML:

COM:

OSAKI TORU OR

RYUKOKU UNIVERSITY

FUKAKUSA TSUKAMOTO

FUSHIMI KU

KYOTO 612

JAPAN

TEL: $075-64 \dot{z}-1111$

TLF:

TLX:

EML:

COM: 34

OSBORNE JOHN L OR

DPT OF PHYSICS

UNIVERSITY OF OURHAM

SOUTH RD

OURHAM OHI $3 L E$

UK

TEL: 9138564971

TLF:

TLX: 537351 QURLIB G

EML:

COM: 34

OSTER LUOWIG F PRDF DR NTL SCIENCE FOUNDATION div AStRONOMICAL SCIENCES 1800 G ST NW

WASHINGTON OC 20550

USA

TEL: $202 \quad 3579857$

TLF:

YLX:

EML:

COM: 12
ORMES JONATHAN $F$ DR

NASA/GSFC

COOE 660

GREENBELT MO 20771

USA

TEL: 3012868801

TLF:

TLX: 89675

EML: SPAN LHEAVX: : ORMES

COM:

ORTOLANI SERGIO

OSS ASTRONOMICO DI PADOVA

VIC DELL OSSERVATORIO 5

I 35122 PADOVA

ITALY

TEL: $4966 \quad 1499$

TLF:

TLX: 432071 ASTROS I

EML:

COM: 37

OSAKI YOJI OR

DPT OF ASTRONOMY

UNIVERSITY OF TOKYO

BUNKYO KU

TOKYO 113

JAPAN

TEL: $03-812-2111$

TLF :

TLX: 33659 UTYOSCI J

EML:

COM: 35,42

osman anAS MOHAMED PROF HELWAN OBSERVATORY

HELWAN

EGYPT

TEL: $780645 / 2683$

TLF:

TLX:

EML:

COM: 28,37

OSTERBROCK DONALO E PROF LICK OBSERVATORY UNIVERSITY OF CALIFORNIA SANTA CRUZ CA 95064 USA

TEL: $408 \quad 4292605$

TLF:

TLX:

EMt:

COM: $28,34,40,41$ 


OSTRIKER JEREMIAH P PROF
PRINCETON UNIVERSITY OBS
PEYTON HALL
PRINCETON NJ 08544
USA
TEL: 60925838900
TLF:
TI.X: 322409
EML: jpOestro.princeton.edu
COM: $33,35.48 P .51$

OTTELET I J DR

INSTITUT Q.ASTROPHYSIQUE

UNIVERSITE DE LIEGE

AVE COINTE 5

B 4000 COINTE-LIEGE

BELGIUM

TEL: 41529980

TLF: 41527474

TLX: 41264

EML

COM: 16

OWEN FRAZER NELSON DR

NRAD

VLA

BOX 0

SOCORRO NM $87801 \quad 0387$

USA -

TEL: 5057724011

TLF:

TLX: $910-988-1710$

EML:

COM: $28,40,50$

OZERNOY LEONIO M PROF

NASA/GSFC

CODE 665

LAB HIGH ENERGY ASTROPHYYS

GREENBELT MD 20771

USA

TEL: 3012868801

TLF:

TLX:

EML: OZERNOVELHEAVX,GSFC.NASA,GOV

COM: $34,47,48$

T'CONNELL ROBERT F PROF

OPT PHYSICS \& ASTRONOMY

LOUISIANA STATE UNIV

BATON ROUGE LA 708034001

USA

TEL: 5043886848

TIF :

TLX:

EML:

COM: 48
OSWALT TERRY 0 DR

OPT OF PHYS \& SPACE SCI

FLORIOA INST TECHNOLOGY

150 W UNIVERSITY BLVO

MELBOURNE FL 32901

USA

TEL: 3057688098

TLF :

TLX:

EML:

COM: $26,27,35$

OUHRABKA MIROSLAV DR

COLLEGE OF EOUCATION IN

HRADEC KRALOVE

NAM SVOBOOY 301

CS 50191 HRAQEL KRALQVE

CZECHOSLOVAKIA

TEL: 4925226

TLF: 4925785

TLX:

EML:

COM:

OWEN TOBIAS C PRDF

INSTITUTE FOR ASTRONOMY

UNIVERSITY OF HAWAII

2680 WOODLAWN OR

HONOLULU HI 96822

USA

TEL: 8089568007

TLF: $B 08 \quad 988 \quad 2790$

TLX:

EML:

COM: $16 C, 44,51$, WGPSNC

OZGUT ATILA

KANDILLI OBSERVATORY

BUGAZICI UNIVERSITY

CENGELKOY

81220 ISTANBUL

TURKEY

TEL: $13320240 / 41$

TLF:

TLX:

EML:

COH: 10

O'CONNELL ROBERT WEST DR

UNIVERSITY STATION

UNIVERSITY OF VIRGINIA

BDX 3818

CHARLOTTESVILLE VA 22903

USA

TEL: 8049247494 .

TLF :

TLX: $510-587-5453$

EML:

COM: 28,47
OTERMA LIISI PROF

SIRKKALANKATU 31

SF 20700 TURKU

FINLAND

TEL: ' 21332081

TLF:

TLX:

EML:

COM: 20

OVERBEEK MICHIEL DANIEL

BOX 212

EDENVALE 1610

SOUTH AFRICA

TEL: 11535447

TLF :

TLX:

EML:

COM: 20

OWOCKI STANLEY PETER OR BARTOL RESEARCH INSTITUTE UNIVERSITY OF OELAWARE NEWARK DE 1.9716

USA

TEL: 3024518357

TLF: $302 \quad 451 \quad 1843$

TLX: $510 \quad 666 \quad 0805$

EML: BARTOL: : OWOCKI

COM: $12,29,36$

OZSVATH I PROF

UNIVERSITY OF TEXAS

PROGRAMS IN MATHEMAT SCI

B0X 830688

RICHARDSON TX 750830688

USA

TEL: $214 \quad 6902174$

TLF :

TLX:

EML:

COM: 47

D'CONNOR SEAMUS L OR

DPT OF PHYSICS

UNIVERSITY COLLEGE

BELFIELD

DUBLIN 4

IRELAND

TEL: I 693244

TLF:

TLX: 32693 ULC EI

EML:

COM:
OTHMAN MAZLAN

DPT OF PHYSICS

UNIVERSITI KEBANGSAAN

MALAYSIA

43600 BANGI SELANGOR

MALAYSIA

TEL: 8250001

TLF :

TLX: 31496 UNIKEB MA

EML:

COM: 46

OWAKI NAOAKI OR

OPT ASTRON \& EARTH SCI

TOKYO GAKUGEI UNIVERSITY

KDGANEI

TOKYO 184

JAPAN

TEL:

TLF:

TLX:

EML:

COM: 46

OXENIUS JOACHIM DR

IAAG

VRIJE UNIV BRUSSELS

CP 231

81050 BRUSSELS

BELGIUM

TEL: 26400015

TLF :

TLX: 23069 UNILIB B

EML:

COM: 36

O'BRIEN PAUL THOMAS OR DPT PHYSICS, \& ASTRONOMY UNIVERSITY COLLEGE LONDON GOWER ST

LONDON WCIE 6BT

UK

TEL: $1713 \quad 877050$

TLF: $1713 \quad 807 \quad 145$

TLX: 28722

EML: PTOEUK.AC.UCL. STAR

CON: 44

O'DEA CHRISTOPHER P DR

STSCI

HOMEWOOD CAMPUS

3700 SAN MARTIN DR

BALTIMORE MD 21218

USA

TEL: 3013382590

TLF: 3013385085

TLX: 684910!

EML:

COM: 28,40 
O'OELL CHARLES R DR

DPT SPACE.PHYS \& ASTRON

RICE UNIVERSITY,

BOX 1892

HOUSTON TK 772511892

USA

TEL: 713527 8101

TLF :

TLX: 556457

EML:

COM: $09,15,34$

O'KEEFE JOHN A DR

NASA/GSFE

CODE 681

GREENBELT MO 20771

USA

TEL: $301286 \cdot 8445$

TLF:

TLX: 89675

ENL:

COM: $15,16,22$

D'sUllivan DENIS F

OIAS

SCHOOL OF COSMIC PHYSICS

5 MERRION SO

DUBLIN 2

IRELANO

TEL: 1774321

TLF :

TLX: 31687 DIAS EI

EML:

COM: 48

PACIESAS WILLIAM S DR

OPT OF PHYSICS

UWIVERSITY OF ALABAMA

HUNTSVILLE AL 35899

USA

TEL: 2055447712

FLF:

TLX: 594416 ES62

EML:

COM: 44

PADEVET VLADIMIR DR

ASTRONOMICAL INSTITUTE

CZECH ACADEMY OF SCIENCES

ONDREJOV OBSERVATORY

CS 25165 ONDREJOV

CZECHOSLOVAKIA

TEL: 20485201

TLF: 20485314

ILX: 121579

EML:

COM: 22
O'DELL STEPHEN L

NASA/MSFC

CODE ES 65

SPACE SCIENCE LAB

HUNTSVILLE AL 35812

USA

TEL: 2055447708

TLF :

TLX:

EML:

COM: 34

D'LEARY BRIAN T

FUTURE FOCAS

5136 E KAREN DR

SCOTTSOALE AZ 85254

USA

TEL:

TLF :

TLX:

EML:

COM:

O'SULLIVAN JOHN DAVIO DR

CSIRO

DIVISION OF RADIOPHYSICS

BOX 76

EPPING NSW 2121

AUSTRALIA

TEL:

TLF :

TLX: 26230 ASTRO

EML:

COM: 40 .

PACINI FRANCD PROF

OPT OI ASTRONOMIA

UNIVERSITA DI FIRENZE

LARGO E FERMI 5

I 50125 FIRENZE

ITALY

TEL: 5527521

TLF: 55220039

TLX: 572258 ARCETR-I

EML:

COM: $44,48 C, 51$

PADMAN RACHAEL

MULLARD RADIO ASTRON OBS

CAVENOISH LABORATORY

MADINGLEY RO

CAMBRIDGE CB3 OHE

UK

TEL: $223 \quad 664 \quad 77$

TLF :

TLX: 81292

EML: .

COM: 40
O'DONOGHUE DARRAGH OR

OPT OF ASTRONOMY

UNIVERSITY OF CAPE TOWN

RONOEBOSCH 7700

SOUTH AFRICA

TEL:

TLF:

TLX:

EML:

COM: 27

Q.MARA BERNARD J PROF

DPT OF PHYSICS

UNIVERSITY OF QUEENSLAND

ST LUCIA

BRISBANE QLO 4067

AUSTRALIA

TEL:

TLF:

TLX:

EML:

COM: 36

pachner jaroslav prof

60655 WYNFORD HTS CR

TORONTO ON M3C 1L4

CANADA

TEL: $416 \quad 447 \quad 2015$

TLF :

TLX:

EML:

COM: 47

PACZYNSKI BOHDAN PROF COPERNICUS ASTRON CENTER POLISH ACAD OF SCIENCES

UL BARTYCKA 18

PL 00716 WARSAW

POLANO

TEL:

TLF :

TLX:

EML:

COM: 35,42

PADMANABHAN T DR

TIFR/PHYSICS GROUP

HOHI BHABHA RD

COLABA

BOMBAY 400005

INDIA

TEL: $22 \quad 4952311$

TLF:

TLX: 011-3009 TIFR IN

EML:

COM: 47
O'HANOLEY DOUGLAS A DR NASA HEADQUARTERS

COOE $Z$

600 INDEPENDENCE AVE SW

WASHINGTON DC 20546

USA

TEL: 2024538932

ILF: 2024260408

TLX:

EML:

COM: 04,07

O'MONGAIN EON

OPT OF PHYSICS

UNIVERSITY COLLEGE

BELFIELO

OUBLIN 4

IRELAND

TEL: 1693244

TLF :

TLX: 32693 UCD

EML:

COM: 44

PACHOLCZYK ANDRZEJ G PROF

STEWARO OBSERVATORY

UNIVERSITY OF ARIZONA

TUCSON AZ 85721

USA

TEL: 6026216928

TLF :

TEX: 467175 .

EML:

COM: $28,40,48$

PADALIA T D OR

UTTAR PRADESH STATE

OBSERVATORY

PO MANORA PEAK 263129

NAINITAL 263129

INOIA

TEL: $5942 \quad 2136$

TLF :

TLX:

EML:

COM: 42

PADRielli lucia

IST DI RAOIOASTRONOMIA

CNR

VIA IRNERIO 46

I 40126 BOLOGNA

ITALY

TEL: 51232856

TLF:

TLX: 211664 INF BO I

EML:

COM: 40.47 
PAGE ARTHUR MR

MT TAMBORINE OBSERVATORY

BOX 44

ASPLEY QLO 4034

AUSTRALIA

TEL: ? 2634813

$T L F$ :

$T$ I. $X$ :

EML

COM: 25

PAGEL BERNARD E J PROF

NDROITA

BLEGDAMSVEJ 1 ?

OK 2100 COPENHAGEN O

DENMARK

TEL: $3142 \quad 1616$

TLF: 31389157

TLX: 15216 NBI

EML: norditaenbivax,nbt,dk

COM: $29,34,36$

PALLA FRANCESCO

OSS ASTROFISICO

DI ARCETRI

LARGO E FERMI 5

I 50125 FIRENZE

ITALY

TEL: $55 \quad 2752242$

TLF :

TLX: 572268 ARCETR I

EML

COM: 34

PALMER PATRICK E PROF

ASTRONOMY \& ASTROPHYS CTR

UNIVERSITY OF CHICAGO

5640 S ELLIS AVE

CHICAGO IL 60637

USA

TEL: 3129627972

TLF :

TLX: 6871133

EML:

COM: $33,3.4,40$

PALUS PAVEL DR

FAC OF MATH \& PHYSICS

DPT ASTRONOMY \& ASTROPHYS

MLYMSKA DOLINA

CS 94215 BRATISLAVA

CZECHOSLOVAKIA

TEL: 7723611

TLF: 7325882

TLX:

EML:

COM: 10,12
PAGE CLIVE G DR

DPT OF PHYSICS

UNIVERSITY OF LEICESTER

UNIVERSITY RD

LEICESTER LEI 7RH

TEL: $533554 \quad 455 \star 23$

TLF

TLX: 341664 LUXRAY G

EML

COM: 48

\section{pakvor IVAN}

ASTRONOMICAL OBSERVATQRY

VOLGINA 7

YU 11050 BEOGRAD

YUGOSLAVIA

TEL: $11419 \quad 357 / 421 \quad 875$

TLF

ILX: 72610 A0B YU

EML

COM: $O B$

PALLAVICINI ROBERTO DR
OSS ASTROFISICO
OI ARCETRI
LARGO E FERMI 5
I 50125 FIRENZE
ITALY
IEL: 552752252
TLF:
TLX: 572268 ARCETR I
EML:
COM: $10,36 C$

PALMER PHILIP DR

ASTRONOMY UNIT

QUEEN MARY/WESTFIELO COLL

MILE END RO

LONOON EI 4NS

UK

TEL: 19755462

TLF

TLX: 893750

EML: PHILIP QMC.MATHS.

COM: 28

PAMYATNIKH A OR

INST OF ASTRONOMY

ACADEMY OF SCIENCES

PYATNITSKAYA UL 48

109017 MOSCOW

RUSSIA

TEL: $231-54-61$

TLF:

TLX: 412623 SCSIP SU

EML:

COM: 05.35
PAGE DON NELSON

DPT OF ASTRONOMY

PENNSYLVANIA STATE UNIV

104 DAVEY LAB

UNIVERSITY PARK PA 16802

USA

TEL: $81.4863 \quad 0163$

TLF

TLX: 842510

EML:

COM: 47

PAL ARPAD PROF DR

FAE OF MATHEMATICS

UNIVERSITY OF [LUJ-NAPOCA

STR RAKOCZI 72

R 3400 CLUJ NAPOCA

RUMANIA

TEL: $951-16101 / 11592$

TLF :

TLX:

EML:

COM: 07

palle pere-lluis do

INST DE ASTROFISICA

DE CANARIAS

OBS DEL TEIDE

E 38200 LA LAGUNA

SPAIN

TEL: $22 \quad 26 \quad 2211$

TLF: $22 \quad 263005$

TLX: 92640

EML: SPAN:IAC: :PLP

COM: 10,12

PALOUS JAN BR

ASTRONOMICAL INSTITUTE

CZECH ACADEMY SCIENCES

BUDECSKA 6

CS 12023 PRAHA 2

CZECHOSLOVAKIA

TEL: 225 8757

TLF:

TLX: 122486

EML:

COM: 33

PAN JUN- HUA

NANJING ASTRONOMICAL

INSTRUMENTS FACTORY

BOX 846

NANJING

CHINA PR

TEL: 2546191

TLF

TLX: 34136 GLYNJ CN

EML:

COM:
PAGE THORNTON L DR NASA/JOHNSON SPACE CENTER

18639 POINT LOOKOUT OR HOUSTON TX 77058

USA

TEL: $713 \quad 483 \quad 3728$

TLF :

ILX:

EML

COM: 28,51

PALAGI FRANCESCO

OSS ASTROFISICO

DI ARCETRI

LARGO E FERMI 5

I 50125 FIRENZE

ITALY

TEL: 5527521

TLF :

TLX: 572268 ARCETR I

EML:

COM:

palmeira ricardo a R dR

INPE

CP 515

12200 S JOSE DOS CAMPOS

BRAZIL

TEL: $123 \quad 229977$

TLF: $123 \quad 218743$

TEX:

EML:

COM

PALUMBO giorgio G C DR LABORATORIO TESRE

VIA CASTAGNDLI

I 40100 BOLOGNA

ITALY

TEL: 51519593

TLF

TLX: 511350 CNR-80

EML:

COM: 28,48

PAN LIANDE

SHAANXI OBSERVATORY

CAS

LINTONG XIAN

SHAANXI

CHINA PR

TEL: 332255

TLF :

TLX: 70121 CSAO CN

EML:

COM: 10 
PAN NING-BAO

BEIJING ASTRONOMICAL OBS

CAS

W SUBURB

BEIJING 100000

CHINA PR

TEL: $128 \quad 1698$

TLF:

TLX:

EML:

COM:

PANDE GIRISH CHANDRA PROF

126 ARYANAGAR

LUCKNOW 226004

INDIA

TEL:

TLF:

EML :

COM: 35

TLX:

PANDEY UMA SHANKAR OR

DPT OF PHYSICS

UNIVERSITY OF GORAKHPUR

GORAKHPUR 273009

INDIA

TEL: 336601

TLF:

TLX:

EML:

COM: $42,46,48$

PANNUNZIO RENATO

OSS ASTRONOMICO DI TORIND

ST OSSERVATORIO 20

I 10025 PINO TORINESE

ITALIY

TEL: 11841067

TLX: 213236 TOASTR I

EML:

TLF: $1184 \quad 1281$

COM: 26

PAPAELIAS PHILIP OR

OPT OF PHYSICS

NTL UNIVERSITY OF ATHENS

PANEPISTIMIOPOLIC

GR 15771 ZOGRAFOS

GREECE

TEL: 17235122

TLF:

TLX: 223815 UNIVGR

EML:

COM:
PAN RONG-SHI

SHANGHAI OBSERVATORY

CAS

GO NANDAN RD

SHANGHAI

CHINA PR

TEL: 21386191

TLF:

TLX: 33164 SHAO CN

EML:

COM: $24,28,47$

PANDE MAHESH CHANORA DR

UTTAR PRADESH STATE

OBSERVATORY

PO MANORA PEAK $263 \quad 129$

NAINITAL 263129

INDIA

TEL: $5942 \quad 2136$

TLF :

TLX:

EML:

COM: 12

PANEK ROBERT J DR

DPT OF ASTRONOMY

PENNSY LVANIA STATE UNIV

525 DAVEY LAB

UNIVERSITY PARK PA 16802

USA

TEL:

TLF:

TLX:

EML:

COM: 36

PANOV KIRIL DR

OPT OF ASTRONOMY

BULGARIAN ACAD SCIENCES

7TH NOYEMBER ST 1

BG 1000 SOFIA

BULGARIA

TEL: 27341

TLF: 23561 ECFBAN BG

TLX: 23561 ECF BAN BG

EML:

COM:

Papagiannis michael D pro

DPT OF ASTRONOMY

BOSTON UNIVERSITY

725 COMMONWEALTH AVE

BOSTON MA 02215

USA

TEL: 6173532626

TLF:

TLX:

EML:

COM: $40,44,51 \mathrm{C}$
PAN XIAO-PEI

CALTECH

MS 10524

PASADENA CA 91125

USA

TEL: B18 3564015

TLF:

TLX: 67425 CALTECH PSD

EML: XPPEOEIMOS. CALTECH. EDU

COM:

PANOEY A $K$

UTTAR PRADESH STATE

OBSERVATORY

PO MANORA PEAK 263129

NAINITAL 263129

INDIA

TEL: $59 \quad 42 \quad 2136$

TLF :

TLX:

EML:

COM: $20,33,37$

PANG KEVIN

JPL

MS Tll 823

4800 OAK GROVE OR

PASADENA CA 91109

USA

IEL: $818 \quad 354 \quad 5392$

ILF :

TLX: 675429

EML:

COM: 16

PAOLICCHI PAOLO DR

IST OI ASTRONOMTA

UNIVERSITA DI PISA

PIAZZA TORRICELLI 2

I 56100 PISA

ITALY

TEL: 5043343

TLF :

TLX:

EML:

COM: 15,16

PAPALIOLIOS COSTAS DR

CENTER FOR ASTROPHYSICS

HCO/SAO

60 GARDEN ST

CAMBRIDGE MA 02138

USA

TEL:

TLF:

TLX:

EML:

COM:
PANAGIA NINO OR

STSCI

HOMEWOOD CAMPUS

3700 SAN MARTIN DR

BALTIMORE MO 21218

USA

TEL: 3013384916

TLF :

TLX: 6849101 ST SCI

EML:

COM: 34

PANDEY S $K$

DPT OF PHYSICS

UNIVERSITY OF RAVISHANKAR

RAIPUR 492010

INOIA

TEL: 27064

TLF :

TLX:

EML:

COM:

PANKONIN VERNON LEE DR

NTL SCIENCE FOUNDATION

DIV ASTRONOMICAL SCIENCES

1800 G ST NW

WASHINGTON OC 20550

USA

TEL: $202 \quad 3579696$

TLF :

TLX:

EML:

COM: $34,40,50$

PAP JUDIT

JPL/CALTECH

MS 171400

4800 OAK GROVE OR

PASADENA CA 91109

USA

TEL: $818 \quad 3542662$

TLF: $818 \quad 3544707$

TLX:

EML: jpapesolar.stanford.edu

COM: 10

PAPALOIZOU JOHN C B DR

SCHOOL OF MATHEMATICAL SC

QUEEN MARY/WESTFIELD COLL

MILE END RO

LONDON EL 4NS

UK

TEL:

TLF :

TLX:

EML:

COM: 27,35 


\author{
PAPARB MARGIT DR \\ KONKOLY OBSERVATORY \\ THEGE U 13/1? \\ BOX 67 \\ H 1525 BUDAPEST \\ HUNGARY \\ TEL: $17,55866 / 75 \quad 4122$ \\ ILF: \\ TLX: 227460 \\ EML:
}

COM: 27

PAQUet PAUl eg DR OBSERVATOIRE ROYAL DE BELGIQUE

AVE CIRCULAIRE 3

B 1180 BRUSSELS

BELGIUM

TEL: 23730211

TLF: 23749822

TLX: 21565 OBSBEL

EML:

COM: $19,31 \mathrm{C}$
TLF: 539570431

PARKER EUGene $N$

ASTROPHYS \& SPACE RES LAB UNIVERSITY OF CHICAGO

933 E 56 TH ST

CHICAGO IL 60637

USA

TEL: 3129627847

TLF :

TLX: 910-221-5617

EML:

COM: $34,48,49$ papathanasoglou o dR

OPT OF ASTRONOMY

NTL UNIVERSITY OF ATHENS

PANEPISTIMIOPOLIS

GR 15771 ZOGRAFOS

GREECE

TEL: 17243414

TLF :

TLX:

EML:

COM: 12

PARCELIER PIERRE DR

OBSERVATOIRE OE PARIS

61 AVE OBSERVATOIRE

F 75014 PARIS

FRANCE

TEL: 143201210

TLF :

TLX: 270776 OBS F

EML:

COM:

PARIJSKIJ YU N DR

PULKOVO OBSERVATORY

ACAOEMY OF SCIENCES

10 KUTUZOV QUAY

196140 ST PETERSBURG

RUSSIA

TEL: 2979452

TLF

TLX:

EMr:

COM: 40.51

PARK MYEONG-GU OR

DPT ASTRON \& METEOROLOGY

COLLEGE OF NTL SCIENCES

KYUNGPOOK NTL UNIVERSITY

TAEGUS 702701

KOREA $R$

TEL: 539506364

TLX:

EML:

COM: 48

papayannopoulos TH OR

DPT OF ASTRONOMY

NTL UNIVERSITY OF ATHENS

PANEPISTIMIOPOLIS

GR 15783 ZOGRAFOS

GREECE

TEL: 17243414

ILF :

TLX: 223815 UNIV GR

EML: SPM25EGRATHUWI

COM: 28,33

PAREOES JOSE MARIA DR

DPT ASTRONOMIA

UNIVERSIOAD OE BARCELONA

AVD DIAGONAL 647

E 08028 BARCELONA

SPAIN

TEL: 33307311

TLF:

TLX:

EML: D3FAJPPO EBOUBOHI

COM: 40

PARISE RONALO A OR

NASA/GSFC

CODE 684.9

GREENBELT MO 20771

USA

TEL: 3012863896

TLF:

rLX:

EML: SPAN:UIT::PARISE

COM:

PARK SEOK JAE DR

1444-3 SHIN-LIM DONG

KWANAK KU

SEOUL 151742

KOREA $R$

TEL:

ILF:

TLX:

EML:

COM:

PARKER ROBERT A R

PARKER QUENTIN DR

ROYAL OBSERVATORY

BLACKFORD HILL

EDINBURGH EHS JHJ

UK

TEL: $316 \quad 688 \quad 379$

TLX: 72383 ROEDIN $G$

EML:

TLF:

COM: 28
NASA/JOHNSON SPACE CENTER

CODE CB

HOUSTON TX $7705 B$

USA -

TEL: 7134832221

TLF

TLX:

EML:

COM:
PAPOUSEK JIRI

DPT OF ASTROPHYSICS

PURKYNE UNIVERSITY

KOTLARSKA 2

CS 61137 BRNO

CZECHOSLOVAKIA

TEL: 51112

TLF:

TLX:

EML:

COM: 27

PARESCE FRANCESCO DR

STSCI

HOMEWOOO CAMPUS

3700 SAN MARTIN OR

BALTIMORE MO 21218

USA

TEL:

TLF:

TLX:

EML:

COM: 21,49

PARISOT JEAN-PAUL

DBSERVATOIRE DE BORDEAUX

BP 89

F 33270 FLOIRAC

FRANCE

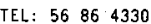

TLF: $56 \quad 404251$

TLX:

EML: EARN : $<$ USerid $>$ FROBOR51

COM: 15,46

PARKER EDWARD A OR

ELECTRONICS LABORATORY UNIVERSITY OF KENT CANTERBURY CT2 TNT

TEL: $227 \quad 66822$

TLF :

TLX: 965449

EML:

COM: 40

PARKINSON JOHN H OR MULLARD SPACE SCIENCE LAB UNIVERSITY COLLEGE LONDON HOLMBURY ST MARY DORKING SURREY RH5 ENT UK

TEL: 483274111

TLF: 483287312

TLX: 859185 UCMSSL G

EML:

COM: $10,44,48$ 


\author{
PARKINSON TRUMAN DR \\ KITT PEAK NTL OBS \\ BOX 26732 \\ 950 N CHERRY AVE \\ TUCSON AZ $85726 \quad 6732$ \\ USA \\ TEL: \\ ILF : \\ TLX: \\ EML: \\ COM:
}

PARSAMYAN ELMA S DR BYURAKAN ASTROPHYSICAL OBSERVATORY

378433 ARMENIA

ARMENIA

TEL: $88 \quad 52 \quad 28 \quad 3453$

TLF :

TLX:

EML

COM: 27.37

PASACHOFF JAY M PROF HOPKINS OBSERVATORY

WILLIAMS COLLEGE WILLIAMSTOWN MA 01267 USA

TEL: 413.5972105

ILF :

TLX:

EML: BITNET:PASACHOFFWILLIAMS COM: $10,12 \mathrm{C}, 40,46 \mathrm{C}$

PASINETTI LAURA E PROF

DPT DI FISICA

UNIVERSITA DI MILANO

VIA CELORIA 16

I 20133 MILANO

ITALY

TEL: $22392275^{\star 2} 272$

TLF: 22366583

TLX: 334687 INFN MI

EML: PASINETTI MILANO. INFN. IT

COM: $05,29,36,45,51$

\section{PATERSON-BEECKMANS $F$}

VINCENT VAN GOGHLAAN 19

NL. 2343 RH OEGSTGEES

NETHERLANDS

TEL: $71 \quad 170829$

TLF :

EML:

COM: 29

TLX:
PARKINSON WILLIAM H DR CENTER FOR ASTROPHYSICS HCOO/SAO MS 50

60 GARDEN ST

CAMBRIOGE MA 02138

USA

TEL: 6174954865

TLF: 6174957052

TLX: $92142 B$

EML: PARKINSONECFA

COM: $10,12,14 V P, 44$

PARSONS SIONEY B DR

STSCI

HOMEWOOO CAMPUS

3700 SAN MARTIN DR

BALTIMORE MO 21218

USA

TEL: 3013384807

TLF :

TLX:

EML:

COM: 29.45

PASCOAL ANTONIO J B SCI

OBSERVATORIO ASTRONOMICO

UNIVERSIOADE BO PORTO

MONTE-BA VIRGEM

P 4400 VILA NOVA DE GAIA

PORTUGAL

TEL: 7820404

TLF

TLX:

EML:

COM:

PASTORI LIVIO

OSS ASTRONOMICO DI MILANO

VIA E BIANCHI 46

I 22055 MERATE

ITALY

TEL: 592035

TLX:

EML

TLF :

COM:

PATHRIA RAJ K PROF

OPT OF PHYSICS

UNIVERSITY OF WATERLOO

WATERLOD ON N2L $3 G I$

CAMAOA

TEL: $519 \quad 885 \quad 1211$

TLF: $519746 \quad 8115$

TLX: 06955259

EML:

COM:
PARMA PAOLA.

IST DI RADIOASTRONOMIA

CNR

VIA IRNERIO 46

I 40126 BOLOGNA

ITALY

TEL: $51 \quad 23 \quad 2856$

TLF:

TLX:

EML:

COM: 40

PARTHASARATHY M OR

INDIAN INSTITUTE OF

ASTROPHYSICS

KORAMANGALA

BANGALORE 560034

INDIA

TEL: $81256 \quad 6585 / 6497$

TLF :

TLK: 845763 IIAB IN

EML:

COM: $27,29,42$

PASCU DAN OR

US NAVAL OBSERVATORY

34 \& MASSACHUSETtS AVE NW

WASHINGTON OC 203925100

USA

TEL: $202 \quad 653 \quad 1178$

TLF:

TLX:

EML:

COM: 20.24

PASTORIZA MIRIANI G DR

INSTITUTO DE FISICA

UFRGS

CP 15051

90000 PORTO ALEGRE RS

BRAZIL

TEL: $\begin{array}{lll}512 & 36 & 4677\end{array}$

TLF :

TLX: 0511055 UFRS BR

EML:

COM: 28

PATI A K

INOIAN INSTITUTE OF

ASTROPHYSICS

KORAMANGALA

BANGALQRE $560 \quad 034$

INDIA

TEL: 812569702

TLF :

TLX: 08452763

EML:

COM:
PARRISH ALLAN DR

STATE UNIV OF NEW YORK UNIV OF MASSACHUSETTS

GRC 6191

AMHERST MA 01003

USA

TEL:

TLF :

TLX:

EML:

COM: 40

PARTRIOGE ROBERT B PROF HAVERFORO COLLEGE

HAVERFORD PA 1904]

USA

TEL: $215 \quad 896 \quad 1144$

TLF:

FLX:

EML

COM: $47 \mathrm{P}$

PASIAN FABIO

OAT

BOX SUCC TRIESTE 5

VIA TIEPOLO II

I $3413 L$ TRIESTE

ITALY

TEL: 40768005

ILF

TLX: 461137 OAT I

EML:

COM: 09

PATERNO LUEIO PROF

OSS ASTRDFISICO

CITTA UNIVERSITARIA

VIA A DORIA 6

I 95125 CATANIA

ITALY

TEL: 95330533

TLF :

TLX: 970359 ASTRCT I

EML:

COM: 10,27

PATKOS LASZLO DR

KONKOLY OBSERVATORY

THEGE U 13/17

BOX 67

H 1525 BUDAPEST

HUNGARY

TEL: $175 \quad 5866 / 75 \quad 4122$

TLF :

TLX: 227460

EML:

COM: 42 


PATNAIK ALOK DR
NRAL
JORELL BANK
MACCLESFIELO SK1L $90 L$
UK
TEL: 47771321
TLX:
EML: ALOKOUK.AC. MAN. JB. STAR
TLF: 477716 18
COM:
PAULORACH ADALBERT W A DR
UNIVERSITAETS STERNWARTE
SCHEINERSTR 1
D 8OOO MUENCHEN 80
GERMANY
TEL: 89922 09436
TLF:
TLX:
EML:
COM:

\section{PAVLOVSKAYA E D DR}

STERMBERG STATE ASTR INST

117234 MOSCOW

RUSSIA

TEL:

TLF :

EML

COM: 33

TLX:

PEACH GILLIAN DR

DPT PHYSICS \& ASTRONOMY

UNIVERSITY COLLEGE LONDON

GOWER ST

LONOON WCLE GBT

UK

TEL: $1>13877050$

TLF:

TLX: 28722

EML:

COM: 14

PEALE STANTON J PROF

DPT DF PHYSICS

UNIVERSITY OF CALIFORNIA

SANTA BARBARA CA 93106

USA

TEL: 8059612977

ILF:

TLX:

EML:

COM: $07 C$
PATRIARCHI PATRIZIO DR

OSS ASTROFISICO

DI ARCETRI

LARGO E FERMI 5

I 50125 FIRENZE

ITALY

TEL: 552752282

TLF :

ILX: 572268 ARCETR I

EML:

COM:

PAULINY TOTH IVAN $K K$ DR MPI FUER RAOIOASTRONOMIE AUF DEM HUEGEL 69

D 5300 BONN 1

GERMANY

TE.L: $22852 \quad 5243$

TLX: 886440

EML:

TLF:

COM: 40.48

\section{PAVLOVSKI KRESIMIR}

HVAR OBSERVATORY

FACULTY OF GEODESY

KACICEVA 26

YU 41000 ZAGREB

YUGOSLAVIA

TEL: 41442600

TLF :

TLX:

EML:

COM: 27,42

PEACH JOHN $\checkmark$ V OR

DPT OF ASTROPHYSICS

UNIVERSITY OF OXFORO

SOUTH PARKS RO

OXFORO OXI JRO

UK

TEL: 865511336

TLF:

TLX:

EML:

COM:

PEARCE GILLIAN OR

DPT OF ASTROPHYSICS

UNIVERSITY OF OXFORD

KEBLE RD

OXFORD OXI 3RH

UK

TEL: $865 \quad 273 \quad 297$

TLF:

TLX: 83295 NUCLOX G

EML:

COM: 35
PATUREL geORges

observatoIRE de LyON

AVE CHARLES ANDRE

F 69561 S GENIS LAVAL COX

FRANCE.

TEL: $78 \quad 56 \quad 0705$

TLF: $72 \quad 399791$

TLX: 310926

EML:

COM: 05,28

PAULS THOMAS ALBERT DR NAVAL RESEARCH LABORATORY COOE 4130

4555 OVERLOOK AVE SW WASHINGTON DC 203755000 USA

TEL:

TLF :

TLX:

EML:

COM: $33,34,40$

PAXTON HAROLO'J B R

ROYAL GREENWICH OBS

HERSTMONCEUX CASTLE

HAILSHAM BN27 IRP

UK

TEL:

TLF:

TLX:

EML:

COM:

PEACOCK ANTHONY DR

ESA/ESTEC

SSD

B0X 299

NL 2200 AG NDORDWIJK

NETHERLANDS

TEL: $17 \quad 1983563$

TLF: 171917400

TLX: 39098

EML:

COM: 44

PEARSON TIMOTHY J

CALTECH

MS 1.0524

OWENS VALLEY RADIO OBS

PASADENA CA 91125

USA

TEL: $818 \quad 356 \quad 4980$

TLF:

TLX: 675425

EML:

COM: 40
PAUL JACOUES DR

CEA CEN

OAPNIA/SAP

BP 2

F 91191 GIF/YVETTE CDX

FRANCE

TEL: 169084462

TLF :

TLX:

EML:

COM:

PAUWELS T OR

OBSERVATOIRE ROYAL DE

BELGIQUE

AVE CIRCULAIRE 3

B 1180 BRUSSELS

BELGIUM

TEL: $2 \quad 375 \cdot 2484$

TLF: 23749822

TLX: 21565 OBS BEL

EML: THIERRYASTRO. OMA.BE

COM: 07,20

PAYNE DAYID G

JPL

MS 264748

4800 OAK GROVE DR

PASAOENA CA 91109

USA

TEL:

TLF:

TLX:

EML:

COM: 40

PEACOCK JOHN ANOREW

ROYAL OBSERVATORY

BLACKFORO HILL

EDINBURGH EH9 $3 \mathrm{HJ}$

UK

TEL: $316 \quad 673 \quad 32$ !

TLF :

TLX: 72383 ROEDIN G

EML:

COM: 47

PECINA PETR

ASTRONOMICAL INSTITUTE

CZECH ACADEMY OF SCIENCES

ONDREJOV OBSERVATORY

CS 25165 ONOREJOV

CZECHOSLOVAKIA

TEL: 20495201

TLF : 20485314

TLX: 122486

EML:

COM: 22 
PECKER JEAN-CLAUDE PROF CQLLEGE DE FRANCE

3 RUE D'ULM

F 75331 PARIS CDX 05 FRANCE

TEL: I $4427 \quad 1695$

TLF:

TLX:

EML:

COM: $05,12,34,36,47$

PEOLAR ALAN DR

NRAL

JDORELL BANK

MACCLESFIELO SKII 9OL

UK

TEL:

TLX:

EML:

TLF :

COM: 40

PEERY BENJAMIN $F$ PRDF

DPT PHYSICS \& ASTRONOMY HOWARO UNIVERSITY

WASHINGTON OC 20059

USA

TEL: $202 \quad 636 \quad 6267$

TLF :

TLX:

EML:

COM: 29

PEL JAN WILLEM OR

KAPTEYN STERREWACHT

WERKGROEP

MENSINGHEWEG 20

NL. 9301 KA RODEN

NETHERLANDS

TEL:

TLF:

TLX: $53767 \mathrm{kSW} R O \mathrm{NL}$

EML:

COM: 25

\section{PELLET ANDRE}

OBSERVATOIRE DE MARSEILLE

2 PLACE LE VERRIER

F 13248 MARSEILLE COX 04

FRANCE

TEL: $9195908 \mathrm{~B}$

TLF:

TLX: $420241 \mathrm{~F}$

EML:

COM:
PEDERSEN BENT M OR

OBSERVATOIRE DE PARIS

SECTION DE MEUDON

F 92195 MEUDON PPL COX

FRANCE

TEL: $145 \quad 077809$

TLF:

ILX:

EML:

COM: 10

PEDOUSSAUT ANORE

OBS MIDI PYRENEES

14 AVE E BELIN

F 31400 TOULOUSE COX

FRANCE.

TEL: $6125 \quad 2101$

TLF:

TLX: 530776 OBSTLSE

EML:

COM: 29,30

PEIMBERT MANUEL DR

INSTITUTO DE ASTRONOMIA

UNAM

APDO POSTAL 70-264

04510 MEXICO DF

MEXICO

JEL: 905-548-5306

ILF :

ILX: 01760155 CIMCE

EML:

COM: $28,33,34$

PELLAS PAUL OR

LABORATOIRE MINERALOGIE

61 RUE BUFFON

F 75005 PARIS

FRANCE

TEL: $1 \quad 47 \quad 072824$

TLF:

FLX:

EML:

COM: 15

PELLETIER GUY DR PR OBSERVATOIRE DE GRENOBLE CERMO/ASTROPHYSIQUUE

BP $53 \mathrm{X}$

F 3804l $S$ MARTIN HERES CO

FRANCE

TEL: 76514570

TLF: $76 \quad 448821$

TLX:

EML:

COM:
PEOERSEM HOLGER DR

UNIVERSITY OBSERVATORY

OESTER VOLDGADE 3

DK 1350 COPENHAGEN $K$

DENMARK

TEL: $31 \quad 141790$

TLF: 3136915 ?

TLX:

EML:

CON:

PEQREROS MARIO OR

DPT OF PHYSICS

ST MARY'S UNIVERSITY

HALIFAX NS B3H 3C3

CANADA

TEL: $902 \quad 4205640$

TLF : 9024205561

TLX:

EML:

COM: 25,37

PEKERIS CHAIM LEIB PROF

DPT OF APPLIED MATHS

WEIZMANN INSTITUTE OF SCI

BOX 26

REHOVOT 76100

ISRAEL

TEL: 8483292

'TLF :

TLX: 361900

EML:

COM:

PELLEGRINI PAULO S S OR OBSERVATORIO NACIONAL

RUA GL JOSE CRISTINO 7

SAO CRISTOVAO

20921 RIO DE JANEIRO RJ

BRAZIL

TEL: 215803683

TLF: $21580 \quad 0332$

ILX: 21288

EML:

COM: 30

PENA JOSE

INSTITUTO DE ASTRONOMIA

UNAM

APDO POSTAL 70-264

04510 MEXICO DF

MEXICO

TEL: $905-5484537$

TLF : (905)5463712

TLX:

EML: BITNET: PENASEUNAMYMI

COM:
PEOERSEN OLAF PROF

HISTORY OF SCIENCE INST

UNIVERSITY OF AARHUS

NY MUNKEgADE

OK 8000 AARHUS $C$

DENMARK

TEL: $86 \quad 12 \quad 7188$

TLF: $86 \quad 202711$

$T \perp X$ :

EML:

COM: 41

PEEBLES P JAMES E

DPT OF PHYSICS

PRINCETON UNIVERSITY

JADWIN HALL

PRINCETON NJ 08544

USA

TEL: 6094524386

TLF :

TLX: 499-3512

EML:

COM: 47

pekuenlue e rennan or

FACULTY OF SCIENCE

EGE UNIVERSITY

BoX 21

35100 BORNOVA IZMIR.

TURKEY

TEL: 222295

TLF :

TLX:

EML:

COM:

PELLERIN JR CHARLES J OR NASA HEADQUARTERS

COOE E?

600 INDEPENDENCE AYE SW

WASHINGTON OC 20546

USA

TEL: 2024531437

JLF :

TLX:

EML:

COM:

PENA MIRIAM DR

INSTITUTO DE ASTRONOMIA

UNAM

APDO POSTAL $70-264$

04510 MEXICO OF

MEXICO

TEL: $525 \quad 548 \quad 5305$

TLF : $525 \quad 548 \quad 3712$

TLX:

EML: MIRIAMEALFA.ASTROSCU. UNAM,

COM: 34 


PENG OIU-HE
OPT OF ASTRONOMY
NANJING UNIVERSITY
NANIING
CHINA PR
TEL: 25 34651 *2882
TLF:
TLX: 34151 PRCNU CN
EML:
COM: 28,48
PENSADO JOSE OR
OBS ASTRONOMICO NCL
ALFONSO XI1-5
E 28014 MADRID
SPAIN
TEL: 1227 O107
TLX:
EML:
TLF:
COM:

PEREZ ENRIQUE DR INST DE ASTROFISICA DE CANARIAS OBS DEL TEIDE E 38200 LA LAGUNA SPAIN

TEL: 22262211

TLF: $22 \quad 263005$

TLX: 92640 IAC E

EML: IAC: : EPS/EPJUIAC. ES

COM: 28
PENG YUN-LOU OPT OF ASTRONOMY NANUING UNIVERSITY NANJING

CHINA PR

TEL: $2537551 * 2882$

TLF:

ILX: 34151 PRCNU CN

EML:

COM: 40

\section{PENSTON MARGARET}

ROYAL GREENWICH OBS

MADINGLEY RD

CAMBRIDGE EB3 OEZ UK

TEL: 223374000

TLF: $223 \quad 374700$

ILX:

EML:

COM:

PERAULT MICHEL

RADIOASTRONOMIE ENS

24 RUE LHOMOND

F 75231 PARIS COX 05

FRANCE

TEL: $1 \quad 4329 \quad 1225$

TLF: 145873489

TLX: 202601

EML: DECNET: IAPOBS: : PERAULT

COM: 34

Perea-duarte Jatme o dR

INST ASTROFISICA

DE ANDALUCIA APO 2144

PROFESOR ALBAREDA 1

E $180 B 0$ granada

SPAIN

TEL: 58121311

TLF:

ILX: 78573 IAAG E

EML: JAIMEIAA: ES

COM: 28

PEREZ FourNon ISMAEL oR

INST DE ASTROFISICA

OE CANARIAS

OBS DEL TEIOE

E 38200 LA LAGUNA

SPAIN

TEL: $22 \quad 262211$

TLF: $22 \quad 263005$

TLX: 92640 IAC E

EML:

COM: 28,40
PENICHE ROSARIO DR

INSTITUTO DE ASTRONOMIA

UNAM

APDO POSTAL 70-264

04510 MEXICO DF

MEXICO

TEL: $905-5484537$

ILF: (905)5483712

TLX:

EML: BITNET:PENAS UNAMUML

COM:

PENZIAS ARNO A OR

at\&T BELL LABORATORIES

RM 6A-409

600 mountain avenue

MURRAY HILL NJ 07974

USA

TEL: 2015823361

TLF :

TLX: 13-8650 OR 219348

EML:

COM: $34,40,47$

PERCY JOHN R PROF

ERINDALE COLLEGE

UNIVERSITY OF TORONTO

DIVISION OF SCIENCE MISSISSAUGA ON L5L IC6

CANADA

TEL: 4168285343

TLF: 4168285328

TLX:

EML: PERLYUUTORPHYS

COM: $27 P, 46 \mathrm{YP}$

PEREK LUBOS DR

ASTRONOMICAL INSTITUTE

CZECH ACADEMY SCIENCES

BUOECSKA 6

CS 12023 PRAHA 2

CZECHOSLOYAKIA

TEL: 2254234

TLF:

$T L X: 122486$

EML:

COM: 33,51

PEREZ-DE-TEJADA H A DR INSTITUTO DE GEOFISICA UNAM

APDO POSTAL 877

22860 ENSENADA B CALIF

MEXICO

TEL: 706-674-060L

ILF:

$i L x_{:}$

EML:

COM: 15
PENNY ALAN JOHN DR RUTHERFORD APPLETON LAB SPACE \& ASTROPHYSICS DIV BLDG R25/R68 CHILTON OIOCOT OXII OOX uK

TEL: 23521900

TLF:

TLX: 83159 RUTHBL G

EML:

COM: $09,25,37$

PEQUIGNOT DANIEL

OBSERVATOIRE DE PARIS

SECTION DE MEUDON

DAF

F 92195 MEUOON PPL COX

FRANCE

IEL: $145 \quad 077438$

TLF :

TLX: 201571

EML:

COM: 34

PERDANG JEAN M OR INSTITUT O'ASTROPHYSIQUE UNIVERSITE DE LIEGE AVE COINTE 5

B 4000 COINTE-LIEGE BELGIUM

TEL: $41 \quad 529980$

TLF: 41527474

TLX: 41264 ASTRLG B

EML :

COM:

PERES gIOVANHI OR

OSS ASTROFISICO

CITTA UNIVERSITARIA

VIA A DORIA 6

I 95125 CATANIA

ITALY

TEL: 91330533

TLF : 91330592

TLX: 970359 ASTRCT I

EML: GIANNI PEASTRCT

COM: 10

PEREZ-PERAZA JORGE OR

INA0E

TONANT.ZINTLAZ

APDO POSTAL 216 y 51

72000 PUEBLA PUE

MEXICO

TEL: $47-04-19$

TLF :

$T L X:$

EML:

COM: 
PERINOTTO MARIO PROF

DPT OI ASTRONOMIA

UNIVERSITA OI FIRENZE

LARGO E FERMI 5

I 50125 FIRENZE

ITALY

TEL: $55 \quad 27521$

TLF: 55220039

TLX: 572268 ARCETR

EML:

COM: 34

PERRIER CHRISTIAN DR OBSERVATOIRE DE LYON

AVE CHARLES ANDRE

F 69561 S GENIS LAVAL CDX FRANCE

TEL: 78580705

TLF: 72 39 979

TLX: 310926 OBSLYON $F$

EML: EARN:perfierefrgag51

COM:

\section{PERRY JUOITH J OR \\ INSTITUTE OF ASTRONOMY \\ THE OBSERVATORIES \\ MAOINGLEY RD \\ CAMBRIOGE CB3 OHA \\ UK \\ TEL: $223 \quad 62204$ \\ TLF: \\ TLX: 817297 \\ EML: \\ COM}

PERSIOES SOTIRIOS L

DPT OF ASTRONOMY

UNIVERSITY THESSALONIK I

GR 540 O6 THESSALONIKI

GREECE

TEL: 31991357

TLF :

TL:

EML:

COM: 47

PETERS WILLIAM L III DR

ASTRONOMY OPT

UNIVERSITY OF TEXAS

RLM 15308

AUSTIN TX $78712 \quad 1083$

USA

TEL

TLF :

ILX:

EML:

COM: $28,34,40$
PERKINS FRANCIS W OR

PLASMA PHYSICS LAB

PRINCETON UNIVERSITY

$B O \times 451$

PRINCETON NJ 08544

USA

TEL: 6096832603

TLF :

TLX: 5106852399

EML:

COM: $49 C$

PERRIN JEAN MARIE DR

LAS

TRAVERSE DU SIPHON

LES, TROIS LUCS

F 13012 MARSEILLE

FRANCE

TEL: 91055900

TLF: $9166 \quad 1855$

TLX: 420584 E ASTROSP

EML: BITNET: PERRIN@FRLASM5]

COM: 21

\section{PERRY PETER M OR}

COMPUTER SCIENCES CORP

SYSTEM SCIENCES OIV

8728-COLESVILLE RO

SILVER SPRING MD 20910

USA

TEL: $301589 \quad 1545$

TLF:

TLX:

EML:

COM: 44

\section{PESCH PETER OR}

NTL SCIENCE FOUNDATION

OIV ASTRONOMICAL SCIENCES

$1 B 00$ G ST NW

WASHINGTON DC 20550

USA

TEL: 2023577622

TLF:

TIX:

EML:

COM: 33 .

PETERSEN J O OR

UMIVERSITY OBSERVATORY

OESTER VOLDGADE 3

DK 1350 COPENHAGEN $K$

DENMARK

TEL: 31141790

TLF: $31 \quad 38 \quad 9157$

TLX:

EML:

COM: 27
PERLEY RICHARD ALAN

NRAO

BOX 0

SOCORRO NM 87801 0387

USA

TEL: 5057724011

TLF:

TLX: $910-988-1710$

EML

COM: 40

PERRIN MARIE-NOEL DR

OBSERVATOIRE DE PARIS

61 AVE OBSERVATOIRE

F 75014 PARIS

FRANCE

TEL: 140512245

TLF :

TLX: 270776 OBS F

EML!

COM: 29

PERRYMAN MICHAEL A C OR

ESA/ESTEC

SSD

BOX 299

NL 2200 AG NOOROWIJK

NETHERLANDS

TEL: $17 \quad 1983615$

ILF: 17.1984690

TLX: 39098

EML: ESTCS1: :MPERRRMA

COM: 06.09 .24 .47

PESEK RUDOLPH PROF

ASTRONOMICAL INSTITUTE

CZECH ACADEMY SCIENCES

BUDECSKA 6

CS 12023 PRAHA 2

CZECHOSLOVAKIA

TEL:

TLF :

TLX:

EML:

COM: 51

PETERSON BRAOLEY MICHAEL

OPT OF ASTRONOMY

GHIO STATE UNIVERSITY

174 W 18TH AVE

COLUMBUS OH $43210 \quad 1106$

USA

TEL: 6144227886

TLF :

TLX:

EML:

COM; 28
PEROLA GIUSEPPE C DR ISTITUTO ASTRONOMICO UNIVERSITA OI ROMA

VIA G M LANCISI 29

100161 ROMA

ITALY

TEL: $6 \quad 86 \quad 7525$

TLF:

TLX: 613255 INFNRD

EML:

COM: 48

PERRY CHARLES L DR OPT PHYSICS \& ASTRONOMY LOUISIANA STATE UNIV BATON ROUGE LA 708034001 USA

TEL: 5043888287

TLF:

TLX: 559184

EML:

COM: $25,30,33,45$

PERSI PAOLO

IAS

CNR

CP 67

I 00044 FRASCATI

ITALY

TEL: 69425655

TLF:

YLX: 610261 CNR FRA

EML:

COM: 34

PETERS gERALOINE JgAN OR SPAEE SCIENCES CENTER UNIV SOUTHERN CALIFORNIA UNIVERSITY PARK LOS ANGELES CA 900891341 USA

TEL: 2137436962

TLF :

ILX: 4720490 USC LSA

EML:

COM: $29,36,42,44$

PETERSON BRUCE A DR MOUNT STROMLO SIOING SPRING OBSERVATORIES

PRIVATE BAG

WODEN PO ACT 2606

AUSTRALIA

TEL: $6288 \quad 1111$

TLF :

TLX: 62270

EML:

COM: 47,48 
PETERSON CHARLES JOHN DR DPT PHYSICS \& ASTRONOMY UNIVERSITY DF MISSOURI 223 PHYSICS BLDG COLUMBIA MO 652II USA

TEL: $314 \quad 8823217$

TLF :

TLX:

EML:

COM: $28,37,41$

PEJFORD A DAVID OR DPT OF ASTROPHYSICS UNIVERSITY OF OXFORO SOUTH PARKS RD OXFORD OXI 3RQ UK

TEL: $865 \quad 511336$

TLF:

TLX

EML:

COM: 09

PETON ALAIN OR
OBSERVATOIRE DE MARSEILLE
2 PLACE LE VERRIER
F 13248 MARSETLLE COX 04
FRANCE
TEL: 91959088
TLF:
TLX:
EML:
COM:

PETROPQULOS BASIL CH DR

RES CENTER FOR ASTRONOMY ACADEMY OF ATHENS

14 ANAGNOSTOPOULOUS ST GR 10673 ATHENS

GREELE

TEL: 13613589

TLF:

TLX:

EML:

COM: 14,16

PETROV GENNADIJ M
INST OF RADIO \& ELECIRON
ACADEMY OF SCIENCES
MARKS AVENJU 18
103907 MOSCOW
RUSSIA
TEL:
TLF:
TLX:
EML:
COM:

PETERSON DEANE M DR

OPT OF EARTH SPACE SCI

ASTRONOMY PROGRAM

SUNY AT STONY BROOK

STONY BROOK NY 117942100

USA

TEL: $526 \quad 6328223$

TLF: $516 \quad 6326240$

TLX:

EML: OPETERSONEASTRO. SUNYSB. EOU

COM: 26

PETHICK CHRISTOPHER J DR

NOROITA

BLEgDAMSVEJ 17

OK 2100 COPENHAGEN $O$

DENMARK

TEL: $3142 \quad 1616$

TLF: 31389157

TLX: $15216 \mathrm{NBIOK}$

EML: PETHICKENBIVAX. NBI.DK

COM: 48

PETRI WINFRIED PROF OR

UNTERLEITEN 2

POSTFACH 106

D 8162 SCHLIERSEE

GERMANY

TEL: $80 \quad 266428$

TLF:

TLX:

EML:

COM: 41

\section{PETROSIAN ARTASHES R DR}

BYURAKAN ASTROPHYSICAL

OBSERVATORY

378433 BYURAKAN

ARMENIA

TEL: $\begin{array}{llll}88 & 52 & 28 & 3453\end{array}$

ILF: $88 \quad 52 \quad 28 \quad 4142$

TLX: 243344 ORION SU

EML:

COM: 28

PETROV GEORGIJ I PROF OR

SPACE RESEARCH INSTITUTE

ACADEMY OF SCIENCES

-PROFSOJOSNAYA UL 84/32

117810 MOSCOW

RUSSIA

TEL:

TLF :

TLX:

EML:

COM:
PETERSON LAURENCE E PROF

CASS

UCSO

C O11.

LA JOLLA CA 920930216

USA

TEL: 6194523461

ILF:

TLX: 910-337-1271 SIOCEAN

EML:

COM: 44,48

Petit gerard or

BIPM

PAVILLON DE BRETEUTL

F 92312 SEVRES COX

FRANCE

TEL: $145 \quad 077067$

ILF: 145342021

TLX: 631351

EML: BIPMARMEU51

COM: 31

PETRINI DANIEL DR

OCA OBSERV DE NICE

BP 139

F 06003 NICE CDX

FRANCE

TEL: 93 89 0420

TLF:

TLX: 460004 OBSNICE F

EML:

COM: 14

PETROSIAN VAHE PROF

CTR FOR SPACE SCIENCES \& ASTROPHYSICS

STANFORD UNIV ERL 304

STANFORD CA 943054055

USA

TEL: $415497 \quad 1435$

TLF:

TLX:

EML:

COM: $10,34,47,48$

PETROV GEORGY TRENDAFILOV

OPT OF ASTRONOMYY

BULGARIAN ACAD SCIENCES

72 LENIN BLVD

$8 G 1784$ SOFIA

BULGARIA

TEL: 2758927

TLF:

TLX: 23561 ECF BAN BG

EML:

COM: 28
PETERSON RUTH CAROL DR 607 MARION PL

PALO ALTO CA 94301

USA

TEL: $415 \quad 321 \quad 1281$

TLF :

TLX:

EML:

COM: 29.30

PETIT JEAN-MARC OR OCA OBSERV DE NICE BP 139

F 06003 NICE COK

FRANCE

TEL: 92003009

TLF : $92 \quad 003033$

TLX:

EML: PETITEFRONI5I

COM: 07,16

PETRO LARRY DAVID

STSCI

HONEWOOD CAMPUS

3700 SAN MARTIN DR

BALTIMORE MD 21218

USA

TEL: 3013384501

TLF:

TLX: 684910]

EML:

COM:

\section{PETROV G M DR}

NI KOLAEV OBSERVATORY

UKRAINIAN ACAD OF SCIENCE 327000 NIKOLAEV

UKRAINE

TEL: $36 \quad 1824$

TLF :

ILX:

EML:

COM: $O B$

PETROY NIKOLAI

ASTRONOMICAL OBSERVATORY

BOX 120

Bg 9000 VARNA

BULGARIA

TEL: $52 \quad 222890$

TLF :

ILX:

EML:

COM: 


PETROY PETER P OR
CRIMEAN ASTROPHYS OBS
UKRAINIAN ACAD OF SCIENCE
NAUCHNY
334413 CRIMEA
UKRAINE
TEL: 432945
TLF:
TLX:
EML:
COM: 09,27

\section{PETTINI MARCO}

OSS ASTROFISICO

OI ARCETRI

LARGO E FERMI 5

I 50125 FIRENZE

ITALY

TEL: 552752282

TLF:

TLX: 572268 ARCETR

EML:

COM: 14

PFEIFFER RAYMOND J
8 BARBARA LANE
TITUSVILLE NJ 08560
USA
TEL: 6098834612
TLF:
EML:
COM: 25
TLX:

PFLUG KLAUS OR
ZNTRLINST F ASTROPHYSIK
SONMENOBSERVATORIUM
EINSTEINTURM
D 1500 POTSOAM
GERMANY
TEL:
TLF:
TLX:
EML:
COM: $10,12,49$

$\begin{array}{ll}\text { PHILLIPS JOHN PETER } & \text { PHILLIPS KENNETH J H } \\ \text { OPT OF PHYSICS } & \text { RUTHERFORO APPLETON LAB } \\ \text { QUEEN MARY/WESTFIELO COLL } & \text { SPACE \& ASTROPHYSICS OIV } \\ \text { MILE END RD } & \text { BLDG R25/R68 } \\ \text { LONDON EL 4NS } & \text { CHILTON DIDCOT OXII OQX } \\ \text { UK } & \text { UK } \\ \text { TEL: } 19804814 & \text { TEL: } 23521900 \\ \text { TLF: } & \text { TLF: } \\ \text { TLX: } 893750 \text { QMEUOL G } & \text { TLX: } \\ \text { EML: } & \text { EML: } \\ \text { COM: } 34 & \text { COM: } 10,12,44\end{array}$

PETROYSKAYA MS OR
INST OF THEORET ASTRONOMY
ACADEMY OF SCIENCES
N KUTUZOVA 10
191187 ST PETERSBURG
RUSSIA
TEL: 121578 ITA SU
ILF:
TLX:
EML:
COM: 07,37

PETTINI MAX

ROYAL GREENWICH OBS

HERSTMONCEUX CASTLE

HAILSHAM BN27 IRP

uK

TEL: 323833171

ILX: 87451 RGOBSY G

EML:

ILF:

COM:

\author{
PFENNIG HANS H OR \\ MPI. F PHYSIK \& ASTROPHYS \\ KARL-SCHWARZSCHILO-STR I \\ D BO46. GARCHING MUENCHEN \\ GERMANY \\ TEL: 8932999435 \\ TLX: 524629 ASTRO D \\ EML: \\ TLF : \\ COM: 14
}

PHAM-VAN. JACQUELINE MME

OCA CERGA

AVE COPERNIC

F 06130 GRASSE

FRANCE,

TEL: 93365849

TLF:

TLX: 470865

EML:

COM: $0 B$

COM: $10,12,44$
PETTENGILL GORDON H PROF

CENTER FOR SPACE RESEARCH MIT 37241

B0X 165

CAMBRIDGE MA 02139

USA

TEL: 6172537501

TLF:

TLX: $92-1473$

EML:

COM: 16,40

PEYTURAUX ROGER H PROF

INSTITUT D'ASTROPHYSIQUE

gGBIS BO ARAGO

F 75014 PARIS

FRANCE

TEL: $14320 \quad 1425$

TLF: 143298673

TLX:

EML:

COM: 12

PFENNIGER DANIEL DR

observatotre de geneVE

CHEMIN DES MAILLETTES 51

CH 1290 SAUVERNY

SWIIZERLANO

TEL: 227552611

TLF: 227553983

TLX: 419209 OBS CH

EML: BITNET: PFENNIGECGEUGE54

COM: 28

PHILIP A G DAVIS

1225 OXFORD PL

SCHENECTADY NYY 12308

USA

TEL: 5183745636

TLF:

TLX:

EML: AGOPUNION

COM: $05,25,30,33,37,45$

PHILLIPS MARK $M$ OR

CERRO TOLOLO

INTERAMERICAN OBSERVATORY

CASILLA 603

LA SERENA

CHILE

TEL: 51213352

ILF: $51212465 * 342$

ILX: 620301 AURA CT

EML:

COM: 28,35
PETTERSEN BJOERN RAGNYALO INST THEORET ASTROPHYSICS UNIVERSITY OF OSLO BOX 1029

N 0315 BLINDERN OSLO 3

NORWAY

TEL: 02-45-65-01

TLF :

TLX: 72705 ASTRO N

EML:

COM: 27

PFAU WERNER

UNIVERSITY OBSERVATORY

SCHILLERGAESSCHEN 2

D 6900 JENA

GERMANY

TEL: $78 \quad 58861347$

TLF:

TLX:

EML:

COM: 25

PFLEIDERER JORG PROF

institut fUER ASTRONOMIE

TECHNIKERSTR 15

A 6020 INNSBRUCK

AUSTRIA

TEL: 52227485251

TLF:

TLX:

EML:

COM: 21

PHILLIPS JOHN G PROF

ASTRONOMY OPT UNIVERSITY OF CALIFORNIA 601 CAMPBELL HALL BERKELEY CA 94720 USA

TEL: $415 \quad 642 \quad 5275$

TLF:

TLX:

EML:

COM: 14,36

PHILLIPS THOMAS GOULD OR CALTECH

MS 3204 ?

PASADENA CA 91125

USA

TEL: 9183564278

TLF:

TLK:

EML:

COM: 34,40 
PIACENTINI RUBEN OR

OBSERVATORIO ASTRONOMICO

DE ROSARIO

CC 606

2000 ROSARIO

ARGENTINA

TEL: 4163084

TIF:

TLX: 41817 CIROS AR

EML: RUBENXIFIR. EDU. AREUUNET. UU. NET EML:

COM: 14

\section{PICCIONJ ADALBERTO}

OSS ASTRONOMICO

UNIVERSITA DI BOLOGNA

CP 596

I 40100 BOLOGNA

ITALY

JEL: 51222956

TLF:

TLX: 211664 INFNBO I

EML:

COM: 42

PIER JEFFREY R UR

US NAVAL OBSERVATORY

FLAGSTAFF STATION

BOX 1149

FLAGSTAFF AZ 86002

USA

TEL: 6027795132

TLF:

ILX:

EML:

COM: 33

PIGATTO LUISA DR

OSS ASTRONOMICO DI PADOVA

VIC DELL OSSERVATORIO 5

I 35122 PADOVA

ITALY

TEL: 49661499

TLX: 432071

EML:

ILF :

COM: 41

\section{PILCHER CARL BERNARD DR}

4316 ELLICOTT STREET NW

WASHINGTON DC 20016

USA

TEL:

TLF ;

EML:

COM: 15

TLX:
PIAZZA LILIANA RIZZO

CRAAE/ESCOLA POLJTECNICA

UNIVERSIOADE DE SAO PAULO

CP 8174

05508 SAO PAULO SP

BRAZIL

TEL: $11 \quad 0133222$

TLF: 118154272

TLX: 1180127 INPE BR

COM:

PICK MONIQUE OR

observatotre oE paris

SECTION DE MEUDON

DASOP

f 92195 MEUDON PPL COX

FRANCE

TEL: $145 \quad 077811$

TLF :

TLX: 200590

EML:

COM: 10,40

PIERCE \& KEITH DR

NTL SOLAR OBSERVATORY

BOX 26732

950 N CHERRY AVE

TUCSON AZ 857266732

USA

TEL: 6023275511

TLF:

TLX:

EML:

COM: 07.12

PIIROLA VILPPU E OR

OBS \& ASTROPHYSICS LAB

UNIVERSITY OF HELSINKI

TAEHTITORNINMAKI

SF 00130 HELSINKI 13

FINLAND

TEL: 1912801

TLF:

TLX: 124690 UNIH SF

EML:

COM: $25,27,42$

PILKINGTON JOHN O H DR

ROYAL GREENWICH OBS

HERSTMONCEUX CASTLE

HAILSHAM BN27 IRP

UK

TEL: 323841139

TLX: 87451 RGOBSY G

EML:

TLF:

COM: 19,31
PICAT JEAN-PIERRE OR OBS MIDI PYRENEES

14 AVE E BELIN

F 31400 TOULDUSE COX

FRANCE

TEL: 61252101

TLF:

TLX:

EML:

COM: 09

PICKLES ANDREW JOHN DR.

INSTITUTE FOR ASTRONOMY

UNIVERSITY OF HAWAII

2680 WOODLAWN OR

HONOLULU HI 96822

USA

TEL: 8089486756

TLF: $B 08 \quad 98 B \quad 2790$

TLX: 7238459 UHAST HR

EML: picklescuhifa, i fa hawa i i .edu

COM: 28

PIERCE dAYIO ALLEN OR

7706 WASTLAWN AVE

LOS ANGELES CA 90045

USA

TEL:

TLF:

TLX:

EML:

COM: 20

PIKE CHR ISTOPHER DAVID

RUTHERFORD APPLETON LAB

SPACE \& ASTROPHYSICS DIV

BLDG R25/R6B

CHILTON OIOCOT OXI1 ORK

uk

TEL: 23521900

TLF:

TLX:

EML:

COM:

PILLINGER COLIN OR

OPT OF EARTH SCIENCES

THE OPEN UNIVERSITY

WALTON HALL

MILTON KEYNES NK7 GAA

UK

TEL: $900 \quad 652119$

TLF: 908655910

TLX:

EML:

COM: $15,16,22$
PICCA DOMENICO DR

DPT DI FISICA

UNIVERSITA DI BARI

VIA 6 AMENDOLA 173

I 70123 BARI

ITALY

TEL: $8024 \quad 3215$

TLF : 80242434

TLX:

EML:

COM: 19

PIDOINGTON JACK H RES FEL

CSIRO NML

LINOFIELD

SYDNEY NSW 2070

AUSTRALIA

TEL: 24676211

TLF :

TLX: $26296 \mathrm{AA}$

EML:

COM: 10,48

PIERRE MARGUERITE OR

ESO

KARL-SCHWARZSCHILD STR.2

D 8046 GARCHING MUENCHEN

GERMANY

TEL: 8932006293

TLF: 893202362

TLX: 5282820 EO D

EML: BITNET: PIERREESOMCI LOM:

PILACHOWSKI CATHERINE DR KIIT PEAK NTL OBS

BOX 26732

950 N CHERRY AVE

TUCSON A2 857266732

USA

TEL: 6023275511

TLF:

TLX: 0666484 AURA NOAOTUC

EML: EARN: : "CATYPONOAO. EDU"

COM: $29 \mathrm{C}, 37$

PILOWSKI K PROF OR GEODAETISCHES INSTITUT TECHNISCHE UNIVERSITAET NIENBURGER STR 1

D 3000 HANNOVER

GERMANY

TEL:

TLF:

TLX:

EML

COM: 08,33 


\begin{tabular}{|c|c|}
\hline $\begin{array}{l}\text { PINEAU OES FORETS G OR } \\
\text { OBSERVATOIRE OE PARIS }\end{array}$ & $\begin{array}{l}\text { PINEAULT SERGE OR } \\
\text { DPT OE PHYSIQUEE }\end{array}$ \\
\hline $\begin{array}{l}\text { SECTION DE MEUDON } \\
\text { DAEC }\end{array}$ & $\begin{array}{l}\text { UNIVERSITE DE LAVAL } \\
\text { SAINTE-FOY OC GLK } 7 P 4\end{array}$ \\
\hline F 92195 MEUDON PPL CDX & CANADA \\
\hline FRANCE & TEL: $416 \quad 6563901$ \\
\hline TEL: $1 \quad \begin{array}{llll}45 & 07 & 7454\end{array}$ & TLF: $418 \quad 6562040$ \\
\hline TLF: $1 \quad 45 \quad 07 \quad 7469$ & TLK: \\
\hline ILX: 631987 & EML: \\
\hline EML: MESIDA: : FOREIS/FORETS@FRHEUS? & COM: \\
\hline
\end{tabular}

COM: 34

\section{PINIGIN GENNADIJ I DR}

NIKOLAEV OBSERVATORY

UKRAINIAN ACAD OF SCIENCE 327001 NIKOLAEV

\section{UKRAINE}

TEL:

ILF:

TLX:

EML:

COM: 08

\section{PINTO PHILIP ALFREO DR}

CENTER FOR ASTROPHYSICS

$$
\text { HCO/SAO }
$$

60 GARDEN ST

CAMBR IOGE MA 02138

USA

TEL: 6174957174

TLF :

TLX:

EML: PINTOCCFA. HARVARO. EDU

COM: 36,48

\section{PIRRONELlo VALERIo \\ OSS ASTROFISICO \\ CITTA UNIVERSITARIA \\ VIA A DORIA 6 \\ I 95125 CATANIA \\ ITALY \\ TEL: $95 \quad 330533$ \\ TLF : \\ TLX: 970359 ASTRCT I \\ EML: \\ COM:}

PITTICH EDUARD M DR

ASTRONOMICAL INSTITUTE

SLOVAK ACABEMY SCIENCES

DUBRAVSKA 9

CS 842 2B BRATISLAVA

CZECHOSLOVAKIA

TEL: 7375157

ThF:

TLX: 93373 SEIS

EML:

COM: 15,20

\section{PINKAU K PROF \\ MPI FUER PLASMAPHYSIK \\ D 8046 GARCHING MUENCHEN GERMANY \\ TEL: 893299342 \\ TLF: \\ EML: \\ COM: 44,48 \\ TLX: $05-215-808$}

\section{PJOTTO GIAMPADLLO}

OPT OI ASTRONOMIA

UNIVERSITA DI PADOVA

VIC DELL OSSERVATORIO 5

I 35122 PADOVA

ITALY

JEL: 49661499

TLF: 49 38 919

TLX: 432071 ASTROS I

EML:

COM:

PISKUNOV anatoly E

INST OF ASTRONOMY

ALADEMY OF SCIENCES

PYATNITSKAYA UL 48

109017 MOSCOW

RUSSIA

TEL: 231-54-61

ILF:

TLX: 412623 SESTP SU

EML:

COM: 37

\section{PITZ ECKHART DR \\ MPI FUER ASTRONOMIE. \\ KOENIGSTUHL \\ D g900 hejoelberg 1 \\ GERMANY \\ TEL: 62215281 \\ TLF: \\ TLX: 461789 MPIA D \\ EML: \\ COM: 21}

PINES DAVIO PROF

DPT OF PHYSICS

UNIVERSITY OF ILLINOIS

$1110 W$ GREEN ST

URBANA IL 61801

USA

TEL: 2173330115

TLF:

TLX: 9103806599 PHYSICS S

EML:

COM: 35

PINOTSIS ANTONIS O OR

DPT OF ASTRONOMY

NTL UNIVERSITY OF ATHENS

PANEPISTIMIOPOLIS

GR 15771 ZOGRAFOS

GREECE

TEL: 17243414

TLF:

TLX:

EML:

COM: 35

PIPHER JUDITH L

OPT PHYSICS \& ASTRONOMY

UNIVERSITY OF ROCHESTER

ROCHESTER NY 14627

USA

TEL: 7162754402

TLF:

TLX:

EML:

COM: 44

PISKUNOV NIKOLAI E DR

INST OF ASTRONOMY

ACADEMY DF SCIENCES

PYATNITSKAYA UL 4B

109017 MOSCOW

RUSSIA

TEL:

TLF:

TLX:

EML:

COM: 36

\section{PIZZELLA G OR}

OPT DI FISICA

UNIVERSITA OI ROMA

PA MORO 2

I 00185 ROMA

ITALY

TEL: 64940156

ILF:

TLX: 613255 INFNRO

EML:

COM:
PINGREE DAVID PROF

BROWN UNIVERSITY

PO BOX 1900

PROVIOENCE RI 02912

USA

TEL: 4018632101

TLF:

TLX:

EML:

COM: 41

PINTO GIROLAMO PROF

oss astronomico oI padova

VIC DELL OSSERVATORIO 5

I 35122 PADOVA

ITALY

TEL: 49661499

TLF:

TLX:

EML:

COM:

- PIRO LUIGI OR

IAS

CNR

[P 67

I 00044 FRASCATI

ITALY

TEL: 69424589

TLF: 69416847

TLX: 610261 CNR FRA

EML: 40607: : PIRO

COM: 48

PISMIS OE RECILLAS PARIS INSTITUTO DE ASTRONOMIA UNAM

APDO POSTAL 70-264

04510 MEXICO OF

MEXICO

TEL: $905-548-5306$

TLF:

TLX: 1760155 CICME

EML:

COM: $28,33,34$

\section{PIZZICHINI GRAZIELLA}

IST TESRE

CNR

via oe Castagmoli 1

I 40126 BOLOGNA

ITALY

TEL: 51519593

TLF:

TLX: 511350 CNR BO

EML:

COM: $05,24,45$ 
PLANESAS PERE

CTR ASTRON DE YEBES

OAN

APO 148

E 19080 GUADALAJARA

SPAIN

TEL: 1$] 223358$

TLF:

$T 2 X$

EML:

COM: 40

PLAVEC ZDENKA DR

DPT OF ASTRONOMY

UNIVERSITY OF CALIFORNIA

405 HILGARO AVE

LOS ANGELES CA 90024

USA

TEL: 2132068596

ILF:

TLX:

EML:

COM: 22

PQGO ALEXANOER OR

MT WILSON \& LAS CAMPANAS

OBSERVATORIES

O13 SANTA BARBARA ST

PASADENA CA 91101

USA

IEL: $213577 \quad 1122$

TLF

TIX:

EML:

CON: 41

POLCARO $\checkmark F$

IAS

CNR

CP 67

I 00044 FRASCATI

ITALY

TEL: 69425651

TLF:

TLX: 610261

EML

COM: 29

POLLACK JAMES B DR
NASA MAES RESEARCH CTR
MS 2453
SPACE SCIENCE OIV
MOFFETT FIELD CA 94035
USA
TEL: 4156945530
TLF:
TLX:
EML:
COM: 16,51

PLASSARO J OR

KSARA DBSERVATORY

KSARA

LEBANON

TEL:

TLF :

EML:

COM:

TLX:

PNEUMAN GERALO W

HIGH ALTITUDE OBSERVATQRY

NCAR

BOX 3000

BOULDER CO 803073000

USA

TEL: 3034971000

TLF :

TLX: 45694

EML:

COM: 10,49

POHL ECKHARO DR

STERNWARTE NUERNBERG

REGIOMONTANUSWEG I

D 8500 nuernberg 20

GEAMANY

TEL: 911593540

TLX:

EML

TLF :

COM:

POLECHOVA PAVLA DR

OBS AND PLANETARIUM

PETRIN 205

CS 11846 PRAHA I

CZECHOSLOVAKIA

TEL: 25353513

TLF:

$I L \dot{X}$

EML:

COM: 05

POLNITZKY GERHARD OR

INSTITUT 'FUER ASTRONOMIE

UNIVERSITAET WIEN

TUERKENSCHANZSTR 17

A 1180 WIEN

AUSTRIA

TEL: 134536090

TLF :

TLX: 116222 PHYSI A

EML:

COM: 08,22 platais Imant $k$ DR

RAOIOASTROPHYSICAL OBS

LATVIAN ACAO OF SCIENCES

TURGENEVA 19

226524 RIGA

LATVIA

TEL: 932088

TLF:

$T L X$ :

EML:

COM: 24.37

POECKERT ROLAND H OR

DEFENCE RESEARCH

ESTABLISHMENT PACIFIC

FMO CFB ESQUIMALT

VICTORIA BC VOS 180

CANAOA

TEL :

TLF

TLX:

EML:

COM:

POKORNY ZOENEK OR

N COPERNICUS OBSERVATORY

\& PLANETARIUM

KRAVI HORA

CS 616 OO BANO 16

CZECHOSLOVAKIA

TEL: 5744374

TLF :

TLX:

EML:

COM: 16,46

PQLETTO GIANNINA PROF

OSS ASTROFISICO

OI ARCETRI

LARGO E FERMI 5

I 50125 FIRENZE

ITALY

TEL: 552752252

TLF:

TLX: 572268 ARCETR I

EML:

COM: 10

POLOSUKHINA-CHUVAEVA N OR

CRIMEAN ASTROPHYS OBS

UKRAINIAN ACAD OF SCIENCE

NAUCHNY

334413 CRIMEA

UKRAINE

TEL: 432945

TLF:

TLX:

EML:

COM:
PLAVEC MIREK J PROF

DPT OF ASTRONOMY

UNIVERSITY OF CALIFORNIA

MS 8979

LOS ANGELES CA 90024

USA

TEL: $213 \quad 825 \quad 1672$

TLF:

TLX:

EML:

COM: $29,35,42$

POEPPEL WOLFGANG G L DR

IAR

Cᄃ 5

1894 VILLA ELISA (BS AS)

ARGENTINA

TEL: 2143793

ILF :

TLX: 18052 CICYT-AR

EML:

COM: 34

POLAND ARTHUR I DR

NASA/GSFC

CODE 682

GREENBELT MD 20771

USA

TEL: 3012867334

TLF :

TLX: 89675

EML :

COM: 10

POLIOAN RONALO S

NASA/GSFC

CODE 681.0

LASP

GREENBELT MO 20771

USA

TEL: 3012865039

TLF: $301286 \quad 8709$

TLX: 89675

EML: (SPAN)STARS: : POLIOAN

COM: $29,42 \mathrm{C}, 44$

POLOZHENTSEV DIMITRIJ OR PULXOVO OBSERVATORY ACADEMY' DF SCIENCES

10 KUTUZOV QUAY

196140 ST PETERSBURG

RUSSIA

TEL: $298-22-42$

TLF:

TLX:

EML:

COM: OSC, 24C 


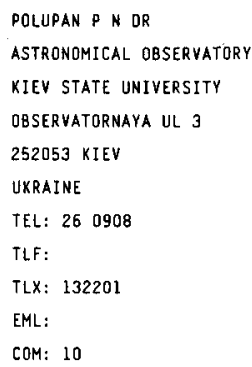

POLYACHENKO VALERIJ L DR

INST OF ASTRONOMY

ACADEMY OF SCIENCES

PYATNITSKAYA UL 48

109017 MOSCOW

RUSSIA

TEL: $95 \quad 2313980$

TLF: 952302081

TLX: 411576 ASCON SU

EML: IAASONODE. IAS.MSK.SU

COM: 33

PONMAMPERUMA CYRIL PROF DPT OF CHEMISTRY UNIVERSITY OF MARYLAND COLLEGE PARK MD 20472 USA

TEL:

TLF :

TLX:

EML:

COM: 51

POPELAR JOSEF DR

GEODETIC SURVEY DIVISION CANADA CENTRE FOR SURVEY 615 BODTH ST

OTTAWA ON KIA OEg

CANAOA

TEL:

TLF :

ILX:

EML:

COM: 19.31

POPOVIC BOZIDAR PROF DR BUEV INA $152 / 32$

YU 11000 BEOGRAD

YUGOSLAVIA

TEL: 11692352

TLF :

EML:

COM: 07,20

TLX:

PORCAS RICHARD OR
MPI FUER RADIOASTRONOMIE
AUF DEM HUEGEL 69
D 5300 BONN 1
GERMANY
TEL: 228525282
TLX: 886440
EML:
TLF:
COM: 40

POLYMILIS CHRONIS DR

DPT OF ASTRONOMY

NTL UNIVERSITY OF ATHENS

PANEPISTIMIOPOLIS

GR 15783 ZOGRAFIG

GREECE

TEL: $1724 \quad 3414$

TLF:

TLX:

EML: SPMSOEGRATHUN

COM: 33

PONSONBY JOHN E B OR

NRAL

JODRELL BANK

MACCLESFIELO SKII 9OL

UK

TEL: 47771321

TLF:

TLX: 36149

EML:

COM: 40,51

POPOV VASIL NIKOLOV

DPT OF ASTRONOMY

BULGARIAN ACAD SCIENCES

72 LENIN BLVD

BG 1784 SOFIA

BULGARIA

TEL: 2758927

$T L F$ :

TLX:

EML:

COM: 28

POPOVIC GEORGIJE OR ASTRONOMICAL OBSERVATORY VOLGINA ?

YU 11050 BEOGRAO

YUGOSLAVIA

TEL: $11 \quad 419 \quad 357$

TLF :

TLX:

EML:

COM: 26

PORCEOOU IGNAZIO E P OR ISTITUTO OI ASTRONOMIA VIA OSPEOALE 72

I 09124 CAGLIARI

ITALY

TEL: $70 \quad 66 \quad 3544$

TLF: 70657657

TLX:

EML: 4058B : : PORCEDOU

COM: 34
POMA ANGELO OR

ISTITUTO DI ASTRONOMIA

VIA OSPEDALE 72

I 09100 CAGLIARI

ITALY

IEL: $70 \quad 66 \quad 3544$

TLF :

TLX: 790326 OSSAST

EML :

COM: 08,19

POOLEY GUY DR

MULLARD RAOIO ASTRON OBS

CAVENDISH LABORATORY

MADINGLEY RD

CAMBRIDGE CBO OHE

UK

TEL: 22366477

TLF :

TLX: 81292

EML:

COM: 40

POPOV VICTOR S OR

PULKOVO OBSERVATORY

ACADEMY OF SCIENCES

10 KUTUZOV QUAY

196140 ST PETERSBURG

RUSSIA

TEL:

TLF :

TLX:

EML:

COM: 30

POPPER DANIEL M PROF OPT OF ASTRONOMY UNIVERSITY OF CALIFORNIA LOS ANGELES CA 90024 USA

TEL: 2138253622

TLF:

TLX: 9103427597

EML:

COM: 42

PORETTI ENNIO

OSS ASTRONOMICO OI MILANO

VIA E BIANCHI 46

I 22055 MERATE

ITALY

TEL: 596412

TLF :

TLX:

EML: PORETTI IOASTMIB:INFN, IT COM: 


PORFIR'EV $\checkmark \checkmark$ DR
PEOAGOGOC INSTITUTE
MINISTRY OF EOUCATION
107B46 MOSCOW
RUSSIA
TEL:
TLF:
TLX:
EML:
COM: 35

PORUBCAN VLAOIMIR OR ASTROMOMICAL INSTITUTE SLOVAK ACADEHY SCIENCES OUBRAVSKA 9

CS 842 28 BRATISLAVA CZECHOSLOVAKIA

TEL: 7375157

ILF:

TLX: 93373 SEIS

EML:

COM: 22

POULAKOS CONSTANTINE DR RES CENTER FOR ASTRONOMY ACADEMY OF ATHENS

14 ANAGNOSTOPOULOU ST GR 10673 ATHENS

GREECE

TEL:

TLF :

TLX:

EML:

COM:

POVEDA ARCADIO OR
INSTITUTO OE ASTRONOMIA
UNAM
APOO POSTAL $70-264$
O451O MEXICO OF
MEXICO
TEL: $550-5905$
TLF:
TLX: 1760155 CICME
EML:
COM: $26,28,35,37$

PRADERIE FRANCOISE OR OBSERVATOIRE DE PARIS SECTION DE MEUDON DPI RECHERCHE SPATIALE F 92195 MEUDON PPL COX FRANCE

TEL: 145077651

TLF :

TLX: 204464

EML:

COM: 29,36 pornchai P. - tanakun

DPT OF PHYSICS

CHULALONGKORN UNIVERSITY

20330 BANGKOK

THAILAND

TEL: 22527985

TLF :

TLX: 20217 UNICHUL TH

EML:

COM: 36

POTTASCH STUART R PROF

KAPTEYN ASTRONOMICAL INST

BOX 800

NL 9700 AV GRONINGEN

NETHERLANOS

TEL: 50116641

TLX: 53572 STARS NL

EML:

TLF:

COM: $34 C, 36$

poulle emmanuel prof ecole natle des chartes

19 RUE DE LA SORBONNE

F 75005 PARIS

FRANCE

TEL: $145 \quad 894857$

ILF :

TLX:

EML:

COM: 41

POYET JEAN-PIERRE DR OBS MIOI PYRENEES

14 AVE E BELIN

F 31400 TOULOUSE CDX FRANCE

TEL: 61252101

TLF

$T L X:$

EML:

COM:

PRADHAN ANIL OR
DPT OF ASTRONOMY
OHIO STATE UNIVERSITY
174 W LBTH AVE
COLUMBUS OH 432101106
USA
TEL: 6142925850
TLF: 6142922928
TLX: 755842
EML: PRADHANEOHSTPY. BITNET
COM:

PORTER JASON G OR

NASA/MSFC

COOE E5 52

SPACE SCIENCE LAB

HUNTSVILLE AL 35812

USA

TEL: 2055447607

TLF : 2055445862

TLX: 62026079 ESL

EML: SSL : : PORTERJ

COM: 10

POTTER ANOREW E DR

NASA/JOHNSON SPACE CENTER COOE SN3

HOUSTON TX 77058

USA

TEL: 7134835276

TLF: 7134835347

TLX:

EML: SN: : POTTER

COM: 16

PQUNDS KENNETH A PROF

DPT OF PHYSICS

UNIVERSITY OF LEICESTER

UNIVERSITY RO

LEICESTER LEI > $_{\text {RH }}$

UK

TEL: $533554 \quad 455 * 151$

TLF:

ILX: 341664 LUXRAYE

EML:

COM: 06.44 .48

PRABHAKARAN NAYAR 5 R DR DPT OF PHYSICS

UNIVERSITY OF KERALA

KARTYAVATTOM

TRIVANORUM 695581

INOIA

TEL: 471418920

TLF:

TLX:

EML:

COM: :46

PRANTZOS NIKOS OR

INSTITUT D'ASTROPHYSIQUE

98BIS BD ARAGO

F 75014 PARIS

FRANCE

TEL: 143201425

TLF: 1.43298673

TLX:

EML: NIKOSEFIAP51

COM:
PORIER NEIL A PROF

OPT OF PHYSICS

UNIVERSITY COLLEGE

BELFIELD

DUBLIN 4

IRELAND

TEL: $1693 \quad 244 * 211$

TLF:

TLX: 32693 UCDEI

EML:

COM: 41,48

POTTER HEINO I DR

PULKOVO OBSERVATORY ACADEMY OF SCIENCES 10 KUTUZOV QUAY 196140 ST PETERSBURG RUSSIA

TEL: $298-22-42$

TLF:

TLX:

EML:

COM: 24

POUQUET ANNICK OR OCA OBSERV DE NICE BP 139

F 06003 NICE CDX FRANCE

TEL: $93 \quad 890420$

TLF:

TLX: 460004 OBSNICE $F$ EML:

COM:

PRABHU TUSHAR P INUIAN INSTITUTE OF ASTROPHYSICS

KORAMANGALA

BANGALORE 560034

INDIA

TEL: $81256 \quad 6585$

TLF:

ILX: 845763 IIAB IN

EML:

COM: 28

PRASAD SHEO $S$

LOCKHEED PALO ALTO RES LB OPT 9120 BLDG 255 3251 HANOVER ST PALO ALTO CA 94304 USA TEL: $415424 \quad 2659$ TLF : 4154243333 TLX:

EML: PRASADXLPARL1

COM: 34 


PRASANNA A R DR
PHYSICAL RESEARCH LAB
NAVRANGPURA
AHMEOABAO 380009
INDIA
TEL: 272462129
TLF: 272445292
TLX: $021-397$ PRL IN
EML:
COM: 48
PREITE-MARTINEZ ANDREA OR
IAS
CNR
CP $6 ?$
I ODO44 FRASCATI
ITALY
TEL: 69425655
TLF:
TLX: 610261
EML:
COM: 34

PRESS WILLIAM H OR CENTER FOR ASTROPHYSICS HCO/SAO

60 GAROEN ST

CAMBrigge MA 02138 USA

TEL: 6174954900

TLF :

ILX: 921428 SATELLITE CAM EML:

COM: 28.47

PREVOT LOUIS DR

OBSERVATOIRE DE MARSEILLE

2 PLACE LE VERrier

F 13248 MARSEILLE CDX 04 FRANCE

TEL: 91959088

TLF :

TLX: 420241

EML:

COM: 30

PRICE R MARCUS DR

DPT PHYSICS \& ASTRONOMY

UNIVERSITY OF NEW MEXICO

800 YALE BLVD NE

ALBUQUERQUE NM 87131

USA

TEL: 5052772616

TLF

TLX:

EML:

COM: $33,34,40$
PRATAP R DR

INSTITUTE OF APPLIEO

SCIENCES

COCHIN 682317

INDIA

TEL:

TLF:

TLX:

EML:

COM:

PREKA-PAPADEMA P DR

LAB OF ASTROPHYSICS

NTL UNIVERSITY OF ATHENS

PANEPISTIMIOPOLIS

GR 157 B3 ATHENS

GREECE

TEL: 17235122

ILF:

TLX:

EML: SPM75 ERATHUNI

COM: 20

PRESTON GEORGE W DR

MT WILSON \& LAS CAMPANAS

OBSERVATORIES

B13 SANTA BARBARA ST

PASADENA CA 91101

USA

TEL: $818577 \quad 1122$

TLF :

TLX:

EML:

COM: 30,45

PREVIT-BURNICHON ML OR OBSERVATOIRE DE MARSEILLE 2 PLACE LE VERRIER

f 13248 MARSEILLE COX 04 FRANCE

TEL: 91959088

TLF ;

TLX: 420241

EML:

COM: 28

PRICE STEPHAN OONALD
2 POLLEY RD
WESTFORD MA 01886
USA
TEL: 6178614552
TLF:
EML:
COM: 44
TLX:

PRAVDO STEVEN H
JPL
MS 168222
4800 OAK GROVE BR
PASADENA CA 91109
USA
TEL: 8183544134
TLF:
TLX: $910-588-3294$
EML:

COM:

PRENDERGAST KEVIN H PROF

DPT OF ASTRONOMY

COLUMBIA UNIVERSITY

PUPIN HALL 538 W L20TH ST

NEW YORK NY 10027

USA

TEL: $212 \quad 2803280$

TLF :

TLX:

EML:

COM: 28

PRESTON ROBERT ARTHUR

JPL

MS $13 B \quad 307$

4800 OAK GROVE DR

PASADENA CA 91109

USA

TEL: 2133546895

TLF:

TLX: 675429

EML:

COM: 40

PRIALNIK-KOVETZ DINA OR

DPT OF GEOPHYSICS

TEL AVIV UNIVERSITY

TEL AVIV

ISRAEL

TEL: 35450633

TLF :

TLX: 342171 VERSY IL

EML: BITNET: BIJETAUNOS

COM: 15,35

PRIEST ERIC R PROF

OPT OF APPLIED MATHS

UNIVERSITY OF ST ANDREWS

NORTH HAUGH

ST ANDREWS FIFE KYIG 9SS

UK

TEL: $33476 L \quad 61 * 8156$

ILF: $334744 \quad 87$

TLX: 76213 SAULIB

EML: ERICACS.ST-ANOREWS.AC, UK

COM: $10 \mathrm{C} .12$
PREDEANU IRINA DR ASTRONOMICAL. OBSERVATORY CUTITUL OE ARGINT 5

BOX 28

R 75212 BUCHAREST

RUMANIA

TEL: 236892

TLF :

TLX: 11882 ASTRO R

EML:

COM: 16

PRENTICE ANDREW J R OR DPT DF MATHEMATICS

MONASH UNIVERSITY

WELLINGTON RO

CLAYTON VIC 3168

AUSTRALIA

TEL:

ILF:

TLX:

EML:

COM: 35

PREUSS EUGEN DR

MPI FUER RADIOASTRONGMIE

AUF DEM HUEGEL 69

D 5300 BONN 1

GERMANY

TEL: 2285251

TLF :

TLX: $\quad$ BB6440

EML:

COM: 40.48

PRICE MICHAEL J DR

SCIENCE APPLICATIONS

5151 E BROADWAY

SUITE 1100

TUCSON AZ 65711

USA

TEL: 6027487400

TLF :

TLX:

EML:

COM:

PRIESTER WOLFGANG PROF INSTITUT F ASTROPHYSIK AUF OEM HUEGEL 71

D 5300 BONN 1

GERMANY

TEL: $228 \quad 73 \quad 3671$

TLF:

TLX: 886440

EML:

COM: 33,40 


\author{
PRIETO MERCEOES \\ INST DE ASTROFISICA \\ DE CANARIAS \\ OBS DEL TEIDE \\ E 38071 LA LAGUNA \\ SPAIN \\ TEL: 22262211 \\ TLF: \\ TLX: 92640 \\ EMi : \\ COM:
}

\section{PRINJA RAMAN OR}

DPT PHYSICS \& ASTRONOMY

UNIVERSITY COLLEGE LONOON

GOWER ST

LONDON WCLE GBT

UK

TEL: 1713877050

TLF:

ILX: 28722

EML: BITNET:RKP UK. AC.UCL.STARLINK

COM: 29

PRODAN Y I QR

STERNBERG STATE ASTR INST

UNIVERSITETSKIJ PRDSP 13

119899 MosCOW

RUSSIA

TEL : 139-55-43

ILX:

EML:

TLF:

COM:

PROKOF'EV VLADIMIR K PROF

CRIMEAN ASTROPHYS OBS

UKRAINTAN ACAO OF SCIENCE

NAUCHNY

334413 CRIMEA

UKRAINE

TEL: 432945

ILF:

TLX:

EML:

COM: 14,44

PRONIK V I OR

CRIMEAN ASTROPHYS OBS

UKRAINIAN ACAD OF SCIENCE

NAUC.HNY

334413 CRIMEA

UKRAINE

TEL: 432945

ILF:

TLX:

EML:

COM: 28
PRIEUR JEAN-LOUIS DR OBS MIOI PYRENEES

14 AVE E BELIN

F 31400 TOULOUSE COX

FRANCE

TEL: 61332929

TLF: 61536722

TLX: 530776

EML: PRIEUR AT FROMP5I BITNET

COM: 28

PRITCHET CHRISTOPHER J DR

DPT OF PHYSICS

UNIVERSITY OF VICTORIA

BOX 1700

VICTORIA BC VBW $2 Y 2$

CANADA

TEL: 6047217704

TLF: 6047217715

TLK:

EML:

COM: $09,28,37$

PROFFITT CHARLES R OR

STSCI

HOMEWODO CAMPUS

3700 SAN MARTIN OR

BALTIMORE MO 21218

USA

TEL: 3013384572

TLF: 3013385090

TLX:

EML: PROFFITTESTSCI.EDU

COM: 35

PROKOF'EVA IRINA A OR

PULKOVO OBSERVATORY

aCAOEMY OF SEIENCES

10 KUTUZOV QUAY

196140 ST PETERSBURG

RUSSIA

TEL:

TLF:

TLX:

EML:

COM:

PROSZYNSKI MIECZYSLAW

COPERNICUS ASTRON CENTER

POLISH ACAD OF SCIENCES

UL BARTYCKA 18

PL 00716 WARSAW

POLAND

TEL:

ILF:

TLX:

EML:

COM: 44
PRINCE HELEN DODSON PROF 4800 FILLMORE, AVE

ALEXANDRIA VA 22311

USA

TEL: 7035781000

TLF:

TLX:

EML:

COM :

PROBSTEIN R F OR

DPT MECHANICAL ENGINEERG

MIT

BOX 165

CAMBRIOGE MA 02139

USA

TEL: 6172532240

TLF :

TLX: 921473 MIT CAM

EML:

COM:

PROISY PAUL E DR

OBSERVATOIRE OE LYON

AVE CHARLES ANDRE

F 69561 S GENIS LAVAL COX

FR.ANCE

IEL: $78 \quad 56 \quad 0705$

TLF: $72 \quad 399791$

ILX:

EML :

COM: 15

PROKOF'EVA VALENTINA $V$ OR CRIMEAN ASTROPHYS OBS UKRAINIAM ACAD OF SCIENCE NAUCHWY

334413 CRIMEA

UKRAINE

TEL: 432945

TLF:

TLX:

EML:

COM: 09

PROTHEROE RAYMONO J OR

DPT OF PHYSICS

UNIVERSITY OF AOELAIOE

BOX 498

ADELAIDE SA 5001

AUSTRALIA

TEL: B 2285996

TLF :

ILX: 89141 UNIVAD AA

EML:

CON: 48
PRINGLE JAMES E DR INSTITUTE OF ASTRONOMY

THE OBSERVATORIES

MADINGLEY RO

CAMBRIOGE $\mathrm{CB} 3 \mathrm{OHA}$

UK

TEL: $223 \quad 622 \quad 04$

TLF:

TLX: 817297 ASTRON G

EML:

COM: 27,42

PROCHAZKA FRANZ V OR INSTITUT FUER FERSTUDIEN UNIVERSITAET

KLAGENFURT

A 9020 KLAGENFURT

AUSTRIA

TEL: 4225317

TLF:

YLX:

EML:

COM: 24,51

PROKAKIS THEODORE I DR ASTRONOMICAL INSTITUTE NTL OBSERVATORY OF ATHENS BOX 20048

GR $11 B$ 10 ATHENS

GREECE

TEL: $1346 \quad 1191 / 18040619$

TLF :

TLX: 215530

EML:

COM: $10,12,41$

PRONIK I I DR

CRIMEAN ASTROPHYS OBS UKRAINIAN ACAO OF SCIENCE NAUCHNY

334413 CRIMEA

UKRAINE

TEL: 432945

TLF:

TLX:

EML:

COM: 28,34

PROTHEROE WILLIAM M PROF DPT OF ASTRONOMY OHIO STATE UNIVERSITY 174 W 18TH AVE COLUMBUS OH 432101106 USA

TEL: 6144227891

TLF :

ILX:

EML:

COM: 


PROTICH MILORAD B
ASTRONOMICAL OBSERVATORY
VOLGINA 7
YU 11050 BEOGRAD
YUGOSLAVIA
TEL: 11402.365
TLF:
TLX:
EML:
COM: 20

PRYCE MAURICE H $L$ DR

DPT OF PHYSICS

UNIV OF BRITISH COLUMBIA

2075 WESBROOK PL

VANCOUVER BC VET IW5

CANADA

TEL: 6042286417

TLF: 6042285324

TLX: 04508576

EML:

COM:

PUETTER RICHARD C OR

CASS

UCSD

C 011

LA JOLLA CA 920930216

USA

TEL: 6195344995

TLF:

YLX:

EML:

COM:

PULS JOAHIM DR

INSITUT FOR ASTRONOMIE

UND ASTROPHYSIK

SCHEINERSTR 1

O 8000 MUENCHEN 80

GERMANY

TEL: 8992209436

TLF: 8992209427

TLX:

EML: UH10LAWEOMOLRZO1/EARN

COM: 36

PUSCHELL JEFFERY JOHN
MARTIN MARIETTA
I03 CHESAPEAKE PARK PLAZA
E460
BALTIMORE MD 21220
USA
TEL: 3016820885
TLF:
TLX: 908225
EML:
COM: 40

PROUST DOMINIQUE observatoire de paris

SECTION DE MEUDON

DAPHE

F 92195 MEUBON PPL COX

FRANCE,

TEL: $145 \cdot 077411$

ILF:

TLX: 201571

EML:

COM: 28

PRYOR CARLTON PHILIP DR OPT PHYSICS \& ASTRONOMY RUTGERS UNIVERSITY

BOX 849

PISCATANAY NJ 088540849

USA

IEL: 9089325462

TLF: 9089324343

TLX:

EML: PRYOR@PRYOR.RUTGERS.EDU

COM:

PUGaCH ALEXANDER F DR

MAIN ASTRONOMICAL OBS

UKRAINIAN ACAD OF ŚLIENCE

GOLOSEEVO

252127 KIEV

UKRAINE

TEL: 664771

TLF :

TLX: 131406 SKY SU

EML:

COM: 27

PUNETHA LALIT MOHAN BR

uttar pradesh state

OBSERYATORY

PO MANORA PEAK 263129

NAINITAL 263129

INDIA

TEL: 59422136

TLF:

ILX:

EML:

COM:

PUSHKIN SERGEY B DR

TIME \& FREQUENCY SERVICE

GOSSTANDARO USSR

117049 MOSCOW

RUSSIA

TEL:

TLF:

TLX:

EML:

COM: 31
PROVERBio EDOARDO PROF

ISTITUTO DI ASTRONOMIA

VIA OSPEOALE 72

I 09100 CAGLIARI

ITALY

TEL: $7066 \quad 3544$

TLF: $70 \quad 657657$

TLX:

EML: PROVERBIDEASTRCA. ASTRO, IT

COM: $08,19,31 P, 46,41$

PSKOUSKIJ JU P OR

STERNBERG STATE ASTR INST

UN[VERSITETSKI] PROSP 13

119899 MOSCOW

RUSSIA

TEL; $139-37-21$

ILF:

TLX:

EML:

COM: 27.34

PUGET JEAN-LOUP DR

RADIOASTRONOMIE ENS

24 RUE LHOMOND.

f 75231 PARIS COX 05

FRANCE

TEL: 143291225

TLF :

TLX: 270912

EML:

COM: 34,47

PURCELL EOWARO M PROF

OPT OF PHYSICS

HARVARD UNIVERSITY

60 GARDEN ST

CAMBRIDGE MA 02L38

USA

TEL: 6174952860

TLF:

ILX:

EML:

COM: 51

PUSTYL'NIK IZOLD B OR

TARTU ASTROPHYSICAL OBS

ESTONIAN ACAD OF SCLENCES

202444 TARTU

ESTONIA

TEL: 33439

TLF:

ILX:

EML:

COM: 42
PROVOST JANINE DR

OCA OBSERV DE NICE

BP 139

F 06003 NICE COX

FRANCE

TEL: 93890420

TLF :

TLX: 460004 OBSNICE F

EML:

COM: 27,35

PUCILLO MAURO DR

OAT

BOX SUCC TRIESTE 5

VIA IIEPOLO 11

I 34131 TRIESTE

ITALY

TEL: 40793921

TLF:

ILX: 461137 OAT I

EML:

COM: 05,09

PUGLIANO ANTONIO PROF

IST UNIVERSITARIO NAVALE

VIA ACTON 38

I B0133 NAPOLI

ITALY

TEL: 815512330

TLF: $81552 \quad 1485$

TLX: 710417

EML:

COM: 00

PURTON CHRISTOPHER R OR DOMINION RADIO ASTROPHYS OBSERVATORY

BOX 248

PENTICTON BC V2A $6 K 3$

CANADA

TEL: 6044932277

TLF: 6044937767

TLX: 04888127

EML:

COM:

PYE JOHN P DR

DPT OF PHYSICS

UNIVERSITY OF LEICESTER

UNIVERSITY RD

LEICESTER LEL TRH

uk

TEL: $533554 \quad 455 * 23$

TLF

TLX: 341664 LUXRAY G

EML:

COM: 


\begin{tabular}{|c|c|c|c|}
\hline PYPER SMITH DIANE M OR & OI GUAN RONG & DIAN BO- - HEN & QIAN SHAN-JIE \\
\hline OPT OF PHYSICS & SHANNKI OBSERVATORY & SHANGHAI OBSERVATORYY & BEIJING ASTRONOMICAL OBS \\
\hline UNIVERSITY OF NEVADA & CAS & CAS & CAS \\
\hline 4505 S MARYLAND PARKWAY & LINTONG KIAN & 80 NANDAN RD & W SUBURB \\
\hline LAS VEGAS NV 89154 & SHAANKI & SHANGHAI & BEIJING 100080 \\
\hline USA & CHINA PR & CHINA PR & CHINA PR \\
\hline TEL: & TEL: 332255 & TEL: $2138 \quad 6191$ & TEL: $128 \quad 2194$ \\
\hline TLF: & TLF : & TLF: : & TLF: \\
\hline$T L X:$ & $\mathrm{ILX}: 70121 \mathrm{CSAOCN}$ & TLX: 33164 SHAO CN & TLX: 22040 BAOAS CN \\
\hline EML: & EML: & EML: & EML: \\
\hline COM: & COM: 08,31 & COM: 37 & COM: 40 \\
\hline QIAN ZHI-HAN OR & QIAN ZHONG-YU & QIAO GUOJUN & QIN DAO \\
\hline SHANGHAI OBSERVATORY & BEJJING ASTRONOMICAL OBS & OPT OF GEOPHYSICS & PURPLE MOUNTAIN OBSERV \\
\hline CAS & CAS & BEIJING UNIVERSITY & CAS \\
\hline 80 NANDAN RD & W SUBURB & BEIJING 100871 & NANUING \\
\hline SHANGHAI & BEIUING 100000 & CHINA PR & CHINA PR \\
\hline CHINA PR & CHINA PR & TEL: & TEL: 2546700 \\
\hline TEL: 21386191 & TEL: & TLF: $125 \quad 64095$ & TLF: \\
\hline TLF : & TLF : & TLX: 22239 PKUNI CN & TLX: 34144 PMONJ CN \\
\hline TLX: 33164 SHAO CN & TLX: 22040 BAOAS CN & EML: & EML: \\
\hline & EML: & COM: 42 & COM: 24 \\
\hline COM: 08 & COM: 33 & & \\
\hline QIN SONG-NIAN & QIN ZHI-HAI & QIU PUZHANG ASS PROF & QIU YU-HAI \\
\hline YUNNAN OBSERVATORY & ORT OF ASTRONOMY & YUNWAN OBSERYATORY & BEIJING ASTRONOMICAL OBS \\
\hline CAS & NANJING UNIVERSITY & CAS & CAS \\
\hline $80 \times 110$ & NANUING & $80 \times 110$ & W SUBURB \\
\hline KUNMING 72946 YUNNAN & CHINA PR & KUNMING 72946 YUNNAN & BEIJING 100080 \\
\hline CHINA PR & TEL: $2534651 * 2882$ & CHINA PR & CHINA $P R$ \\
\hline TEL: $871 \quad 2035$ & TLF: & TEL: 8712035 & TEL: \\
\hline TLF: & $T L X: 0909$ & TLF: & TLF: \\
\hline TLX: & EML: & TLX: 64040 YU0BS CN & TLX: 22040 BADAS CN \\
\hline EML: & COM: 34 & EML: & EML: \\
\hline стоM: & & COM: 09,51 & COM: 40 \\
\hline QU QIN-YUE & QUAMAR JAWALO & QUAN HEJUN & QUARTA MARTA LUCTA \\
\hline OPT OF ASTRONOMY & D 19 STAFF TOWN & SHANGHAL OBSERVATORP & IAG \\
\hline NANJING UNIVERSITY & UNIVERSITY OF KARACHI & CAS & UNIVERSIOAOE OE SAO PAULO \\
\hline NANJING & KARACHI 3201 & 80 NANDAN RD & CP 30627 \\
\hline CHINA PR & PAKISTAN & SHANGHAI & OLO51 SAO PAULO SP \\
\hline TEL: $25 \quad 37551 * 2741$ & TEL: 465491 & CHINA PR & BRAZIL \\
\hline TLF: & TLF: & TEL: 21386191 & TEL: 115778599 \\
\hline TLX: 34151 PRCNU CN & $T L X:$ & TLF: & TLF: $\quad 118153848$ \\
\hline EML: & EML: & $\mathrm{TLX}: 33164$ SHAO CN & ILX: 11.36221 IAGM BR \\
\hline COM: $35.47 .48 C$ & COM: & EML: & EML: \\
\hline & & COM: 41 & COM: \\
\hline DUAST GERMANO ROORIGO & QUENBY JOHN J OR & QUERCI FRANCOIS R OR & QUERCI MONIQUE OR \\
\hline OBSERVATORIO NACIONAL & BLACKETT LABORATORY & OBS MIOI PYRENEES & OBS MIDI PYRENEES \\
\hline RUA COLONEL RENNO 07 & IMPERIAL COLLEGE & 14 AVE E BELIN & 14 AVE E BELIN \\
\hline CP 21 & PRINCE CONSORT RD & F 31400 TOULOUSE CDX & F 31400 TOULOUSE CDX \\
\hline 37500 ITAJUBA MG & LONDON SW7 $2 B 2$ & FRANCE & FRANCE \\
\hline BRAZIL & uK & TEL: 61332929 & TEL: 61332929 \\
\hline TEL: $35 \quad 622 \quad 0788$ & TEL: $15895111 * 6661$ & TLF: $515367 z 2$ & TLF: 61536722 \\
\hline TLF: & TLF: & TLX: $530776 \mathrm{~F}$ OBSTLSE & TLX: $530776 \mathrm{~F}$ OBSTLSE \\
\hline$T L X: 0312603$ & TLX: 261503 & EML: FROMP51 & EML: FROMP51 \\
\hline EML: & EML: & COM: $14,29.36$ & COM: 29,36 \\
\hline COM: & COM: & & \\
\hline
\end{tabular}




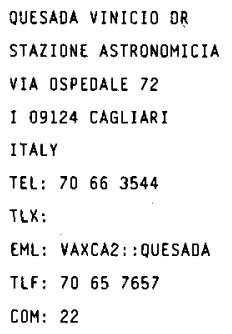

RADOSKI HENRY R DR
AFOSR/NP
BUILOING 410
BOLLING AIR FORCE BASE
WASHINGTON DC 20332
USA
TEL: 2027674906
TLF:
TLX:
EML:
COM: 21

QUIJANO LUIS

REAL INST Y OBSERVATORIO

DE LA ARMADA

CECILIO PUJAZON S/N

E 11110 SAN FERNANDO

SPAIN

TEL: $56 \quad 88 \quad 3548$

TLF :

TLX: 76108 IOM E

EML

COM: $08,20,24$

QUIRK WILLIAM J OR
LAWRENCE LIVERMORE LAB
L 35
BOX 808
LIVERMORE CA 94550
USA
TEL: 4154221852
TLF:
TLX:
EML:
COM:

RABOLLI MONICA DR

UNIV NACIONAL DE LA PLATA

FCAG

1900 LÁ PLATA (BS. AS.)

ARGENTINA

TEL: 21217308

TLF: $1786 \quad 8114$

TLX:

EML: MRABOLLI\%PSI IAFEYSSL. SPAN

COM: 33

RADHAKRISHNAN V PROF

RAMAN RESEARCH INSTITUTE

SADASHIVANAGAR

BANGALORE $560 \quad 0 B 0$

INOIA

TEL: 812340522

TLF: 812.340492

TLX: 8452671

EML:

COM: $34,40,48, E C$

RADOSLAVOVA TSVETANKA
OPT OF ASTRONOMY.
BULGARIAN ACAD SCIENCES
72. LENIN BLVD
BG 1784 SOFIA
BULGARIA
TEL: 2758927
TLF:
TEK:
EML:
COM:

QUINN PETER OR

MOUNT STROMLO \& SIOING

SPRING OBSERVATORIES

PRIVATE BAG

WESTON CREEK PO ACT 2611

AUSTRALIA

TEL: 62490272

TLF: $62 \quad 49 \quad 0233$

TLX:

EML: PJQOMINUET.ANU.0Z.AU COM: 28

RAADU MICHAEL A OR DPT OF PLASMA PHYSICS ROYAL INST OF TECHNOLOGY S 10044 STOCKHOLM 70 SWEDEN

TEL: 87877000

TLF :

TLX: 10389 KTHB STOCKHOLM

EML:

COM: $10,49 \mathrm{C}$

RACHKOVSKY D N OR

CRIMEAN ASTROPHYS OBS UKRAINIAN ACAD OF SCIENCE NAUCHNÝ

334413 CRIMEA

UKRAINE

TEL: 432945

TLF:

TLX: 192

EML:

COM: 36

RADICK RICHARO R DR

AIR FORCE GEOPHYSICS LAB

NTL SOLAR OBSERVATORY

SUNSPOT NM B8349

USA

TEL: 5054341390

TLF:

TLX:

EML:

COM: 12

RAEOLER $K$ H OR

ZNIRLINST F ASTROPHYSIK ROSA-LUXEMBURG-STR 17A

\section{1502 POTSDAM}

GERMANY

TEL:

TLF:

$I L X:$

EML:

COM: 35
QUINTANA HERNAN DR GRUPO ASTROFIS PONTIFICA UNIVERSIDAD CATOLICA CASILLA 104 SANTIAGO 22 CHILE TEL: 2775474 TLF: 25525692 TLX: 240395 EML: HQUINTANAEASTROUC. PUC.CL COM: $05,28,30,48,51$

RABBIA YVES OR OCA CERGA AVE COPERNIC F O6130 GRASSE FRANCE

TEL: 93365849 TLF:

TLX: 470865 CERGA EML: EARN: : YVRABB@FRONI5I COM:

RACINE RENE OR DPT DE PHYSIQUE UNIVERSITE DE MONTREAL CP $612 B$ SUCC A MONTREAL OC H3C $3 J 7$ CANAD́A

TEL: 5143436718 TLF : 5143432071 TLX: 05561359 RZLPNUM ML EML:

COM: 09

RADIMAN IRATIUS BOSSCHA OBSERVATORY

LEMBANG 40391

INDONESIA

TEL: 2296001

TLF:

TLX:

EML:

COM:

RAFANELLI PIERO OR OSS ASTRONOMICO DI PADOVA

VIC OELL OSSERVATORIO 5 I 35122 PADOVA

ITALY

TEL: $4966 \quad 1499$

ILF :

$T L X:$

EML:

COM: 28 


RAFERT JAMES BRUCE
DPT PHYSICS \& SPACE SCI
FLORIDA INST TECHNOLOGY
150 W UNIVERSITY BLVO
MELBOURNE FL 32901
USA
TEL:
TLF:
TLX:
EME:
COM: 42
RAHUNEN TIMO
TAMPERE SAERKAENNIEMI OY
SAERKAENNIEMI
SF 33410 TAMPERE
FINLANO
TEL: 3131333
TLF:
TLX:
EML:
COM: 42

RAITALA JOUKO T
OPT OF ASTRONOMY
UNIVERSITY OF OULU
SF 90570 OULU 57
FINLANB
TEL: 81352106
TLF: $8156 \quad 1278$
TLX: 32375
EML:
COM:

RAKAVY GIOEON PROF EINSTEIN INST OF PHYSICS HEBREW UNIV OF JERUSALEM JERUSALEM 91904 ISRAEL

TEL:

TLF :

ILX:

EML:

COM:

$\begin{array}{ll}\text { RAMADURAI SOURIRAJA OR } & \text { RAMAMURTHY SWAMINATHAN } \\ \text { TIFR } & \text { CASA } \\ \text { HOMI BHABHA RD } & \text { UNIVERSITY OF OSMANIA } \\ \text { COLABA } & \text { HYOERABAO } 500007 \\ \text { BOMBAY } 400005 & \text { INO1A } \\ \text { INOIA } & \text { TEL: } 851672 \\ \text { TEL: } 224952311 & \text { TLF: } \\ \text { TLF: } & \text { TLX: } \\ \text { TLX: } 0113009 \text { TIFR IN } & \text { EML: } \\ \text { EML: shakt }+ \text { ! } 1 \text { fr!taplseunnet.uu, net COM: } \\ \text { COM: } 35,46,48\end{array}$

RAHARTO MOEOJI

OPT OF ASTRONOMY

BANOUNG INSTITUTE OF TECH

JL GANESHA 10

BANDUNG 40132

INDONESIA

TEL: 6222440252

TLF: $622 \quad 2438388$

ILX: $28324 \mathrm{BD}$

EML:

COM: 33

RAIMOND ERNST DR

NFRA

BOX 2

NL 7990 AA DWINGELOD

NETHERLANDS

TEL:-52 197244

TLF: 52197332

TLX: 42043 SRZM NL

EML: EXREFRA.NL

COM: $05,08,34,40$

RAJCHL JAROSLAV DR

ASTRONOMICAL INSTITUTE

CZECH ACADEMY OF SCIENCES ONDREJOY OBSERVATORY

CS 25165 ONOREJOV CZECHOSLOVAKIA

TEL: 20485201

TLF: 20485314

$T \succeq X: 121579$

EML:

COM: 22

\section{RAKSHIT H PROF}

BENGAL ENGINEERG COLLEGE SIBPORE

HEWRAH

INDIA

TEL:

TLF:

TLX:

EML:

COM:

RAMANA MURTHY $P \vee O R$
TIFR
HOMI BHABHA RO
COLABA
BOMBAY 400005
INOIA
TEL: 224952979
TLF:
TLX: 0113009 TIFR IN
EML:
COM.

RAHE JURGEN PROF NASA HEADQUARTERS CODE EL 600 INDEPENDENCE AVE SW WASHINGTON OC 20546

USA

TEL: 2024531590

TLF: $202 \quad 426 \quad 1023$

TLX: 4974843

EML:

COM: $\quad 15 \mathrm{C}, 16 \mathrm{C}, 42,44$

RAINE OEREK J OR

DPT OF ASTRONOMY UNIVERSITY OF LEICESTER UNIVERSITY RO LEICESTER LEI 7RH UK

TEL: 533554455

TLF :

TLX: 341198 LEICUL

EML:

COM:

RAJU P K OR

INDIAN INSTITUTE OF ASTROPHYSICS

KORAMANGALA

BANGALORE 560034

INDIA

TEL: $81256 \quad 6585$

TLF :

TLX: 845763 IIAB IN

EML:

COM:

RAM SAGAR DR

INOIAN INSTITUTE OF

ASTROPHYSICS

KORAMANGALA

BANGALORE $560 \quad 034$

INDIA

TEL: B12 56 6585/6497

YLF:

ILX: $845763^{\circ} 11 \mathrm{AB}$ IN

EML:

COM: 37

RAMATY REUVEN DR

NASA/GSFC

CODE 665

LAB HIGH ENERGY ASTROPHYS

GREENBELT MD 20771

USA

TEL: $301286 \quad 8715$

TLF:

TLX:

EML:

COM: 40 


RAMELLA MASSIMO
OAT
BOX SUCC TRIESTE 5
VIA TIEPOLO 11
I 34131 TRIESTE
ITALY
TEL: 4076 B506
TLF:
TLX: 461137 DAT I
EML:
COM: 47

RAMSEY LAWRENCE W DR

DPT OF ASTRONOMY

PENNSYLVANIA STATE UNIV

525 DAVEY LAB

UNIVERSITY PARK PA 16802

USA

TEL: $814 \quad 6650418$

TLF:

TLX:

EML:

COM: 09,36

RAO K RAMANUJA OR
C/O OR K SURENDRA
RUA CEL JOAO CURSINO 210
APT 92 VILA ADYANA
$12200 S$ JOSE DOS CAMPOS
BRAZIL
TEL:
TLF:
TLX:
EML:
COM:
RAO RAMACHANORA $\vee$ PROF
ISRO SATELLITE CENTER
PEENYA
BANGALORE 560 O5O
INDIA
TEL:
TLF:
TLX:
EML:
COM: 44

RAMOS ISABEL FERRO OR INST GEOPHYS \& ASTRONOMY CALLE 212 N 2906/29 Y 31 LISA

\section{LA HABANA}

CUBA

TEL: $21 \quad 8416 / 0644$

TLF :

TLX: 511240 GEOAS CU

EML:

COM:

RANA NARAYAN CHANDRA OR
IIFR/THEOR ASTRO GROUP
HOMI BHABHA RD
COLABA
BOMBAY 400005
INOIA
TEL: 224952972
ILF:
TLX: 0113009
EHL:
COM: 28

RANKIN JOANHA M OR

PHYSICS OPT

UNIVERSITY OF VERMONT

A405 COOK BUILOING

BURLINGTON VT 05405

USA

TEL: 8026562644

ILF:

TLX: 5102990021

EML: RANKINZMERLIN. UVM-GEN. UVM. EOU COM:

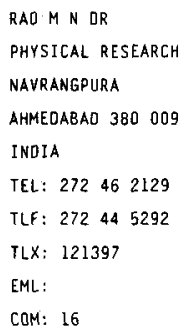

EML:

COM: $10,12,40$
RAMPAZZO ROBERTO DR OSS ASTRONOHICO DI BRERA VIA BRERA 28

I 20121 MILANO

ITALY

TEL: 2874444

TLF : 39598492

TLX:

EML: RAMPAZZOOASTMIB.ASTRO.IT COM: 28

RANDIC LEO PROF DR
GEODETICAL FACULTY
GUNDULICEVA 54
YU 41000 ZAGREB
YUGOSLAVIA
TEL: 41446675
TLF:
TLX:
EML:
COM: 19,31

RAO A PRAMESH OR

TATA INST OF FUNDAMENTAL

RESEARCH

POONA UNIVERSITY CAMPUS

PUNE 411007

INOIA

TEL: $21233710 ?$

TLF : 212335760

TLX: 0145658 GMRT IN

EML: uunet! shakt1!gmrt!pramesh COM: 10,40

RAO N KAMESWARA
INDIAN INSTITUTE OF
ASTROPHYSICS
KORAMANGALA
BANGALORE 560034
INDIA
TEL: 812566585
TLF:
TLX:
EML:
COM: 27,29

RAPAPORT MICHEL OR OBSERVATOIRE DE BORDEAUX BP $B 9$

F 33270 FLOIRAC

FRANCE

TEL: $\begin{array}{lll}56 & 86 & 4330\end{array}$

TLF: $56 \quad 40 \quad 4251$

TLX:

EML

COM: 20,21
RAMSAY DONALO A DR HERZBERG INST ASTROPHYS NTL RESEARCH COUNCIL

100 SUSSEX DR

OTTAWA ON KLA ORG

CANADA

TEL: 6139900919

TLF: $613 \quad 952 \quad 0974$

TLX: 0533715

EML: BITNET: DARENRCYMOI

COM: 20

RANIERI MARCELLO

IAS

CNR

CP 67

I 00044 FRASCATI

ITALY

TEL: 69425655

TLF :

TLX:

EML:

COM:

\section{RAO K NARAHARI}

DPT OF PHYSICS

OHIO STATE UNIVERSITY

174 W 18TH AVE

COLUMBUS OH 43210

USA

TEL: 6144226505

TLF:

TLX:

EML:

COM: 14

RAO $P$ VIVEKANANDA DR

DPT OF ASTRONOMY

UNIVERSITY OF OSMANIA

HYDERABAD 500007

INOIA

TEL: 71951

ILF:

TLX:

EML:

COM: 25

RAPLEY CHRISTOPHER G DR MULLARD SPACE SCIENCE LAB UNIVERSITY COLLEGE LONDON HOLMBURY ST MARY OORKING SURREY RH5 6NT UK

TEL: 30670292

TLF :

TLX: 859185

EML:

COM: 


\author{
ratnatunga kavan U \\ NASA/GSFC \\ CODE 630 \\ SOCD \\ GREENBELT MD 20771 \\ USA \\ TEL: 3012866276 \\ ILF: 3012863221 \\ TLX: 089675 NASCOM GBLT \\ COM: $05,30,33$ \\ RAWLINGS STEVEN DR \\ DPT OF ASTROPHYSICS \\ UNIVERSITY OF OXFORD \\ KEBLE RO \\ OXFORO OXI $3 R H$ \\ uk \\ TEL: $865 \quad 273303$ \\ TLF : 865273418 \\ TLX: 83295 NUCLOX $G$ \\ EML: SR@K.AC.OX.ASTRO
}

EML: KAVANEOFTBIT, SOCCL: : KAVAN

COM: 47

RAYCHAUDHURI AMALKUMAR OR

PRESIOENCY CQLLEGE

COLLEGE ST

CALCUTTA 73

INEIA

TEL:

TLF :

TLX:

EML:

COM: 47

RAZIN V A OR

RADIOPHYSICAL RESEARCH

INSTITUTE

LYADOV UL 25/14

603600 N NOVGOROD

RUSSIA

TEL: $36-72-94$

TLF:

TLX:

EML:

COM: 40

REAY NEWAICK K OR
ASTROPHYSICS GROUP
IMPERIAL COLLEGE
BLACKETI LABORATROY
LONDON SW7 2BZ
UK
TEL: $15895111 * 6669$
TLF:
TLX: 261503 IMPCOL
EML:
COM: $09,49,51$

RAUBENHEIMER BAREND C PR COSMIC RAY RESEARCH UNIT POTCHEFSTROOM UNIVERSITY POTCHEFSTROOM 2520

SOUTH AFRICA

TEL: 01481-27511

TLF:

TLX: 421363

EML:

COM: 48

RAY ALAK OR
IIFR
HOMI BHABHA RO
COLABA
BOMBAY 400005
INDIA
TEL: 222152971
TLF: 222152110
TLX: 1183009 TIFR IN
EML: AKR TIFRVAX
COM: 35

RAYMOND JOHN CHARLES

CENTER FOR ASTROPHYSICS HCO/SAO

60 GARDEN ST

CAMBRIOGE MA 02138

USA

TEL:

TLF:

TLX:

EML:

COM: 34

READHEAD ANTHONY $\complement, S$ DR

CALTECH

ROBINSON BLDG

PASAOENA CA 91125

USA

TEL: 2133564972

TLF:

TLX: 675425 CALTECH PSD

EML:

COM: 40,49

REBEIROT EDTTH OR

OBSERVATOIRE DE MARSEILLE

2 PLACE LE VERRIER

F 13248 MARSEILLE COX O4 FRANCE

TEL: $9195908 B$

TLF:

TLX: 420241

EML:

COM: $28,30,33$
RAUTELA B S DR

UTTAR PRADESH STATE

OBSERVATORY

PO MANORA PEAK 263129

NAINITAL 263129

INDIA

TEL: 59422136

TLF:

TLX:

EML:

COM: 29,45

RAY CHOUDHURI ARNAB OR OPT OF PHYSICS

INOIAN INSTITUTE OF SCI BANGALORE 560012

INDIA

TEL: $812 \quad 344411$

TLF :

TLX:

EML:

COM: 10

RAYROLE JEAN R DR

OBSERVATOIRE OE PARIS

SECTION OE MEUOON

F 92195 MEUDON PPL CDX

FRANCE

TEL: $145 \quad 077789$

TLF :

TLX:

EML:

COM: 10

REASENBERG ROBERT D DR CENTER FOR ASTROPHYSICS HCO/SAO RM B 217

60 GARDEN ST

CAMBRIDGE MA 02138

USA

TEL: 6174957108

TLF:

tLX: 921428 Satelite cam

EML:

COM: 04

REBER GROTE DR

C/O POST OFFICE

BOTHWELL TAS 7030

AUSTRALIA

TEL: 2. 23, 7371

TLF:

ILX:

EML:

COM: 40
RAWLINGS JONATHAN DR DPT OF ASTROPHYSICS UNIVERSITY OF OXFORD KEBLE RO OXFORO OXI $3 R H$ UK

TEL: $338 \quad 65273292$

TLF: 865273418

TLX: 83295 NUCLOX G

EML: JR@UK, AC. OX.ASTRO

COM: 34

RAY THOMAS $P$

DIAS

SCHOOL OF COSMIC PHYSICS

5 MERRION SO

DUBLIN 2

IRELAND

TEL: 1774321

TLF:

TLX: 31687 DIAS EI

EML:

COM: 40

RAZDAN HIRALAL

BHABHA ATOMIC RES CTR

ZAKURA SRINIGAR

KASHMIR 190006

INDIA

TEL:

TLF:

TLX:

EML:

COM: 48

REAVES GIBSON PROF

DEPT OF ASTRONOMY

UNIV SOUTHERN CALIFORNIA

LOS ANGELES CA 900891342

USA

TEL: 2137432039

FLF:

TLX:

EML:

COM: 28

REBOLLO RAFAEL DR INST OE ASTROFISICA DE CANARIAS obs DEL teIDE E 38200 LA LAGUNA SPAIN

TEL: 22262211

ILF:

ILX: 92640

EML: SPAN:IAC::RRL

COM: 29 
RECILLAS-CRUZ ELSA DR INSTITUTO DE ASTRONOMIA UNAM

APDO POSTAL 70-264 04510 MEXICO DF MEXICO

TEL:

TLF :

TLX:

EML:

COM:

REES MARTIN J PROF INSTITUTE OF ASTRONOMY THE OBSERVATORIES MAOINGLEY RD CAMBRIDGE CB3 OHA UK

TEL : $223 \quad 622 \quad 04$

TLF :

TLX: 817297 ASTRON G

EML:

COM: $44,47 C, 48,51$

REGEV ODEO DR

DPT OF PHYSICS

IIT

TECHNION EITY

HAIFA 32000

ISRAEL

TEL: 4293992

TLF: $4221 \quad 514$

TLX:

EML: PHR9IORQTECHNION

COM:

REICH WOLFGANG

MPI FUER RADIOASTRONOMIE

AUF DEM HUEGEL 69

D 5300 BONN I

GERMANY

TEL:

TLX: 886440

EML

TLF

COM: 40

\section{REIF KLAUS OR}

RADIOASTRONOMISCHES INST

DER UNIVERSITAET BONN

AUF DEM HUEGEL $? 1$

O 5300 BONN 1

GERMANY

TEL: 228733657

TLF :

TLX:

EML: U. 145ref\%mpi ehu\%unido, uucp

COM: 33,40
REDFERN MICHAEL R DR

DPT OF PHYSICS

UNIVERSITY COLLEGE

GALWAY

IRELANO

TEL: 4124411

TLF:

TLX : 50023

EML:

COM: 09

REEVES EOMONO $M$ OR

NASA HEADQUARTERS

CODE EM

600 INDEPENOENCE AVE SW

WASHINGTON OC 20546

USA

TEL: $202 \quad 453 \quad 1571$

TLF:

TLX: 89530

EML:

COM: $10 ; 12,44$

REGLERO-VELASCO VICTOR OR

DPT MATEMATICA Y ASTRON

UNIVERSIDAD DE VALENCIA

BURJASOT

E 46100 VALENCIA

SPAIN

TEL: $6386 \quad 4326$

ILF: 63864302

TLX: 61071

EML:

COM: 25,42

REICHERT GAIL ANNE DR

NASA/GSFC

CODE 666

GREENBELT MD 20771

USA

TEL: 3012865307

TLF: 3012863391

TLX:

EML: IUE: :REICHERT/ZBGARQSCFVM

COM: 28,48

REIMERS OIETER. PROF
HAMBURGER STERNWARTE
UNIVERS ITAET HAMBURG
GOJENSBERGSWEG 112
D 2050 HAMBURG 80
GERMANY
TEL: 4072524112
ILF:
TLX: 217884
EML:
COM: 29,36

REEO B CAMERON DR

DPT OF PHYSICS

ST MARY'S UNIVERSITY

HALIFAX NS 83 H 3 C3

CANADA

TEL: 9024205830

TLF: 9024205561

ILX:

EML: BITNET: REEDESTMARYS

COM:

REEVES HUBERT PROF

CEA CEN

SEP-SES BAT $2 B$

BP 2

F 91191 GIF/YVETTE COX

FRANCE

TEL: $16908 \quad 5159$

TLF:

TLX:

EML:

COM: $10,35,47 C, 48$

REGO FERNANDEZ $M$ DR

OPT DE ASTROFISICA

FAC DE FISICA

UNIVERSIDAO COMPLUTENSE

E $2 B 040$ MADRID

SPAIN

TEL: 14495316

TLF :

TLX: 47273 FF UC

EML:

COM: 29

REIO MARK JONATHAN DR

CENTER FOR ASTROPHYSICS

HCO/SAO

60 GARDEN ST

CAMBRIDGE MA 0213B

USA

TEL: 6174957470

TLF :

TLX: 921428 SATELLITE CAM

EML:

COM: 40

REINISCH GILBERT OR

OCA OBSERV DE NICE

BP 139

F 06003 NICE CQX

FRANCE

TEL: 93890420

TLF:

TLX:

EML:

COM:
REES DAVIO ELWYN DR

CSIRO

DIVISION OF RADIOPHYSICS

BOX 76

EPPING NSW 2121

AUSTRALIA

TEL: 28680493

TLF: 28680411

ILX:

EML: dreeserp.csiro.au

COM: 10.12

REFSDAL S PROF OR

HAMBURGER STERNWARTE

GOJENSBERGSWEG $\mathrm{LL}$

D 2050 HAMBURG 80

GERMANY

IEL: $40 \quad 7252 \quad 4124$

TLF :

TLX: 217884

EML:

COM: 42,47

REGULO CLARA DR

INST DE ASTROFISICA

DE CANARIAS

OBS DEL TEIDE

E 30200 LA LAGUNA

SPAIN

TEL: $22 \quad 26 \quad 2211$

TLF:

TLX: 92640

EML: SPAN: IAC::CRR

COM: 10,12

REIO NEILL

CALTECH

PALOMAR OBS MS 10524

PASADENA CA 9l101

USA

TEL: 8183566586

TLF :

TLX:

EML:

COM: 33

REIPURTH BO

ESO

CASILLA 19001

SANTIAGO 19

CHILE

TEL: $2 \quad 698 \quad 8757$

TLF :

TLX: 240881

EML:

COM: 34 


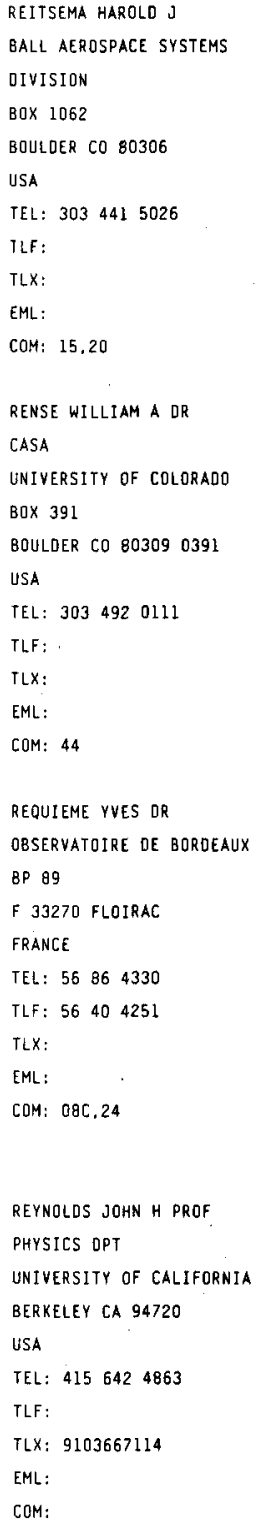

RHODES EDWARO J JR

11801 KILLIMORE AVE NORTHRIOGE CA 91326

USA

TEL:

TLF:

EML:

COM:

rLX:
REIZ ANOERS PROF LOVSPRINGSVEJ 3 B DK 2920 CHARLOTTENLUNO DEMMARK

TEL: 31632536

TLF:

EML:

COM: 08,35

TLX:

RENSON P F M OR
INSTITUT O'ASTROPHYSIQUE
UNIVERSITE DE LIEGE
AVE COINTE 5
B 4000 COINTE-LIEGE
BELGIUM
TEL: 41529980
TLF: 41527474
TLX:
EML: U2148AP@BLIULGI1
COM: 05,27

REVELLE DOUGLAS ORSON DR METEOROLOGY PROGRAM GEOGRAPHY OPT 8)5 DAVIS HALL DEKALB IL 6OLL5 USA

TEL: 8157530631

TLF :

$T \in X$ :

EML:

COM: 15,22

REYNOLDS RONALO I OR OPT OF PHYSICS UNIVERSITY OF WISCONSIN 1150 UNIVERSITY AVE MAOISON WI 53706 USA

TEL: 60.82625916

TLF :

TLX

EML:

COM: 34

RIBES ELIZABETH DR observatoIRE OE PARIS SECIION DE MEUDON F 92195 MEUDON PPL CDX FRANCE

TEL: $145 \quad 077786$

TLF :

TLX:

EML:

COM:
REMY BATTIAU LILIANE G A CONSEIL OE LA RECHERCHE UNIVERSITE OE LIEGE 7 PLACE OU XX AOUT

B 4000 COINTE-LIEGE BELGIUM

TEL: $41 \quad 420080$

TLF :

TLX: 41397 UNIV ULG

EML:

COM: 05,15

RENZINI ALVIO PROF DPT OI ASTRONOMIA VIA ZAMBONI 33

I 40126 BOLOGNA ITALY

TEL: 51222956 TLF :

TLX: 211664 INFNBD EML: ASTB03: :ALVIO COM: $35 \mathrm{C}, 37$

REYES FRANCISCO OR DPT OF ASTRONOMY UNIVERSITY OF FLORIDA

211 SSRB

GAINESVILLE FL 32611 USA

TEL: 9043922049

TLF :

TLX:

EML: BITNET FREYESQUFPINE COM: 40

REYNOLOS STEPHEN $P$

OPT OF PHYSICS

N CAROLINA STATE UNIV BOX 8202

RALEIGH NC 276958202 USA

TEL: 9197377751

TLLF:

TLX:

EML:

COM:

RIBES JEAN-CLAUUE DR

INSU

77 AVE OENFERT ROCHEREAU

F 75014 PARIS

FRANCE

IEL: 143201330

TLF:

TLX:

EML:

COM: 40
RENGARAJAN T N DR TIFR/IR ASTRONOMY HOMI BHABHA RO

COLABA

BOMBAY 400005

INOIA

IEL: 22219111

TLF :

TLX: 0LI-3009 TIFR IN

EML:

COM: 34,48

REPHAELI YOEL DR SCHOOL OF PHYSICS TEL AVIV UNIVERSITY RAMAT AVIV

TEL AVIV. 69978 ISRAEL

TEL:

TLF :

TLX: 342171 VERSY IL

EML:

COM: 28

REYNOLOS JOHN OR

AAD

ATNF

BOX 76

EPPING NSW 2121

AUSTRALIA

TEL: 28680222

TLF: 28680400

TLX: 26230 ASTRO AA

EML: JREYNOLUQATNF.CSIRO.AU

COM: 08,40

REZENDE COSTA JOAQUIM DR CRAAE/ESCOLA POLITECNICA UNIVERSIDADE DE SAO PAULO CP 8174

05508 SAO PAULO SP

BRAZIL

TEL: 118156289

TLF: 118154272

TLX: 1180127 INPE BR

EML:

COM: 10

RICE JOHN B DR OPT PHYSICS \& ASTRONOMY UNIVERSITY OF BRANDON 2500 UNIVERSITY OR NW BRANOON MB RTA $6 A 9$ CANADA

TEL: 2047279693

TLF :

ILX: 07502721

EML:

COM: 
RICHAROS MERCEDES I DR UNIVERSITY STATION UNIVERSITY OF VIRGINIA BOX $38 \mathrm{LB}$

CHARLOTIESVILLE VA 22903

USA

TEL: 8049244895

TLF: 8049243104

TLX:

EML: MTREREVIRGINIA.EDU

COM: 42

RICHER HARVEY $B$ DR

OPT GEOPHYS \& ASTRONOMY

UNIV OF BRITISH COLUMBIA

2075 WESBROOK PL

VANCOUVER BC VGT IW5

CANADA

TEL: $604 \quad 2284134$

TLF: $604 \quad 228 \quad 6047$

TLX:

EML:

COM: $2 \theta, 37$

RICHTLER TOM OR

STERNWARTE DER UNIV. BGNN AUF DEM HÜGEL 71

D 5300 BONN 1

GERMANY

TEL: $228 \quad 73 \quad 3669$

TLX: 886440

EML: $45228090071:$ : RICHTLER

TLF :

COM: 37

RICKETT BARNABY JAMES DR OPT OF ELECTRICAL ENGIN \& COMPUTER SCIENCE

UCSO

LA JOLLA CA 920930216

USA

TEL: 6194522731

TLF :

TLX:

EML:

COM: 40,49

RIEGEL KURT W OR

NTL SCIENCE FOUNDATION

3019 N OAKLAND ST

ARLINGTON VA 22207

USA

TEL: 2023579450

TLF:

TLX:

EML: kriegelenote.nsf.gov

COM: 33
RICHARDSON E HARVEY DR

HERZBERG INST ASTROPHYS

DOMINION ASTROPHYS OBS

5071 W SAANICH RO

VICTORIA BC VQX $4 M 6$

CANAOA

TEL: 6043880001

TLF: 6043630045

TLX: 0497295

EML:

COM: 09

RICHSTDNE DOUGLAS O DR

DPT OF ASTRONOMY

UNIVERSITY OF MICHIGAN

DENNISON BLDG

ANN ARBOR MI 481091090

USA

TEL: $313764 \quad 3441$

TLF :

TLX:

EML: D_Richstoneaub.cc.umich.edu

COM: 28

RICKARD JAMES JOSEPH DR

$80 \times 777$

BORREGO SPRINGS LO 92004

USA

TEL: 7147675462

TLF:

EML:

COM:

TLX:

RICKMAN HANS DR

ASTRONOMICAL OBSERVATORY

BOX 515

$S 75120$ UPPSุALA

SWEOEN

TEL: $18 \quad 123522$

TLX: 76024 UNIVUPS

EML:

TLF :

COM: $15 \mathrm{C}, 20 \mathrm{C}$

RIEGER ERICH OR

MAX PLANCK INSTITUT FUER EXTRATERRESTRISCHE PHYSIK KARL SCHWARWSCHILOSTR I

D 8046 GARCHIMG MUENCHEN

\section{GERMANY}

TEL: 8932993511

TLF : 8932993539

TLX:

EML: EIRQOGAIPPIS

COM: 10
RICHARDSON KEVIN J

DPT OF PHYSICS

OUEEN MARY/WESTFIELD COLL

MILE END RO

LONDON EL 4NS

uK

TEL:

TLF :

TLX

EML:

COM:

RICHTER G A DR

ZNTRLINST, F. ASTROPHYSIK

STERNWARTE BABELSBERG

ROSA-LUXEMBURG-STR ITA

D 6400 SONNEBERG

GERMANY

TEL: $96 \quad 74 \quad 2287$

TLF:

TLX: 6288180

EML:

COM: 27

RICKARO LEE J OR

NAVAL RESEARCH LABORATORY

CODE 4138 RRO

4555 OVERLOOK AVE SW

WASHINGTON DC 203755000

USA

IEL: 2027672495

ILF:

TLX:

EML:

COM: 34,40

RICORT GILBERT OR

DPT ASTROPHYSIQUE

UNIVERSITE DE NICE

PARC VALROSE

F 06034 NICE CDX

FRANCE

TEL: 93519100

TLF :

TLX:

EML:

COM:

RIEGLER GUENTER R OR

NASA HEADQUARTERS

CODE SZE

600 INDEPENDENCE AVE SW

WASHINGTON OC 20546

USA

TEL: $202 \quad 453 \quad 1435$

TLF :

$T L X: 89530$

EML: AMES: : "GRIEGLERONASAMAIL"

COM: 05,44
RICHAROSON R S

GRIFFITH OBSERVATORY

BOX 27787

LOS FELIX STATION

LOS ANGELES CA 90027

USA

TEL:

TLF :

TLX:

EML:

COM:

RICHTER JOHANNES PROF

INST F EXPERIMENT. PHYSIK

PHYSIKZENTRUM

OLSHAUSENSTRASSE

D 2300 KIEL 1

GERMANY.

TEL: $431880 \quad 3835$

TLF :

TLX: 292706

EML:

COM: 14

RICKER GEORGE $R$ OR

CENTER FOR SPACE RESEARCH

MIT RM 37527

BOX 165

CAMBRIDGE MA 02139

USA

TEL: 6172537532

TLF

TLX: 92-14-73

EML:

COM:

RIDDLE ANTHONY C DR

700 GRANT PL

BOULDER CO 80302

USA

TEL: $303 \quad 4478127$

TLF :

TLX:

EML:

COM: 49

RIGHINI ALBERTO PROF

OPT OI ASTRONOMIA

UNIVERSITA OI FIRENZE

LARGO E FERMI 5

I 50125 FIRENZE

ITALY

TEL: 5527521

TLF: 55220039

TLX: 572268 ARCETRI I

EML:

COM: 


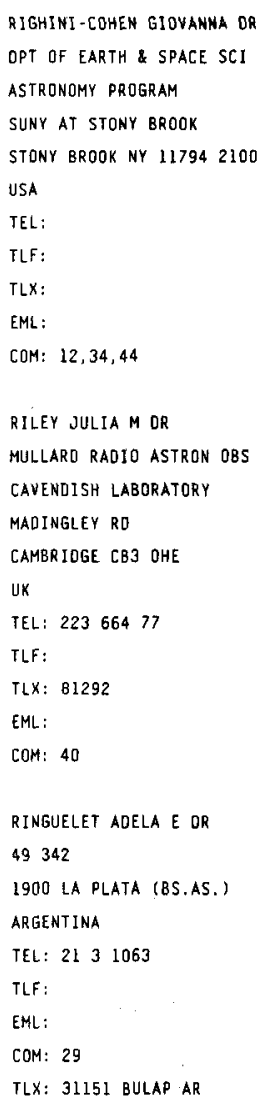

RIZVANON NAUFAL G OR ENGELHARDT ASTRONOMICAL OBSERVATORY

OBSERVATORIA STATION

422526 KAZAN

RUSSIA

TEL: 324827

TLF:

TLX:

EML:

COM: 24

ROBBINS R ROBERT PROF

ASTRONOMY DPT

UNIVERSITY OF TEXAS

RLM 15308

AUSTIN TX $78712 \quad 1083$

USA

TEL: 5124717312

TLF:

ILX:

EML:

COM: $34,46 \mathrm{C}$
RIGUTTI MARIO PROF OSS ASTRONOMICO

DI CAPODIMONTE

VIA MOTARIELLO 16

I 80131 MAPOLI

ITALY

TEL: 440101

TLF:

TLX:

EML:

COM: 12

RINDLER WOLFGANG PROF UNIVERSITY OF TEXAS

UTD

BOX 830688

RICHAROSON TX 750830688 USA

TEL: 2146902885

ILF:

TLX: $791-880$

EML:

COM: 47

RIPKEN HARTMUT W DR oARA german space agenCy KOENI GWINTERERSTRASSE

O 5300 BONN 3

GERMANY

TEL: 228447313

TLX:

EML:

TL়F :

COM: $21,22,49 \mathrm{VP}$

\section{ROACH FRANKLIN E}

1241 SADDLEBACK

COTTONWO0D AZ 86326

USA

TEL:

TLF:

EML:

COM: 21,49

TLX

ROBE H A G OR
INSTITUT D ASTROPHYSIOUE
UNIVERSITE OE LIEGE
AVE COINTE 5
B 4000 COINTE-LIEGE
BELGIUM
TEL: 41529980
TLF: 41527474
TLX: 41264 ASTRLG
EML:
COM:

RIIHIMAA JORMA J OR OPT OF ASTRONOMY UNIVERSITY OF BULU SF 90570 OULU 57 FINLAND

TEL:

TLF: 81561278

TLX:

EML:

COM: 40,51

RING JAMES PROF

BLACKETT LABORATORY

IMPERIAL COLLEGE

PRINCE CONSORT RD

LONDON SW? $2 B Z$

UK

TEL: 15895111

TLF:

TLX: 261503

EML:

COM: 09

RITTER HANS DR MAX PLANCK INSTITUT FUER PHYSIK UND ASTROPHYSIK KARL SCHARZSCHILO STR I D 8046 GARCHING MUENCHEN GERMANY

TEL:

TLF:

TLX:

EML:

COM: 35,42

ROARK TERRY P PROF

UNIYERSITY OF WYOMING

BOX 3434E

LARAMIE

WYOMING 82071

USA

TEL: 3077664121

TLF :

TLX:

EML:

COM:

ROBERGE WAYNE G DR

OPT OF PHYSICS

RENSSELAER POLYTECHN INST

TROY NY 121803590

USA

TEL: $518 \quad 276 \quad 6454$

TLF:

TLX:

EML: ROBERGEORION. PHYS. RPI, EOU

COM: 34
RIJNBEEK RICHARD OR DPT MATHEMATICAL SCIENCE UNIVERSITY OF ST ANDREWS FIFE KYI6 9SS

UK

TEL: $33476161 * 8186$

TLF: 334744 ?

ILX:

EML: RIJNBEEKGCS. ST_ANOREWS. AC. UK COM: 10

RINGNES TRULS S DR INST THEORET ASTROPHYSICS UNIVERSITY OF OSLO

BOX 1029

N 0315 BLINDERN OSLO 3

NORWAY

TEL: 472-456-503

TLF :

TLX: 72425 UNIOS $\mathrm{N}$

EML:

COM:

RIVOLO ARTHUR REX DPT ASTRON \& ASTROPHYS UNIV DF PENNSYLVANIA PHILADELPHIA PA 19104 USA

TEL: 2158986250 TLF :

TLX: 358300

EML:

COM: 47

RQBB RUSSEL $M$ DPT OF PHYSIES \& ASTRON UNIVERSITY OF VICTORIA BOX 1700 VICTORIA BC VEW $2 Y 2$ CAMADA

TEL: 6047217750

TLF: 6047217715

TLX:

EML: BITNET: ROBBQUVPHYS

COM: 42

ROBERTI GIUSEPPE DR IST DI FISICA UNIVERSITA OI NAPOLI MISTRA D OLtREMARE PAD 19 I 80125 NAPOLI

ITALY

TEL:

TLF:

ILX:

EML:

COM: 12 
ROBERTS DAVIO HALL DR

DPT OF PHYSICS

BRANDEIS UNIVERSITY

WALTHAM MA 02254

USA

TEL: 6176472846

TLF:

TLX: 703013

EML:

COM: 40,47

ROBERTSON JAMES GORDON OR

DPT OF ASTROPHYSICS

SCHOOL OF PHYSICS

UNIVERSITY OF SYONEY

SYONEY NSW 2006

AUSTRALIA

TEL:

TLF:

TLX:

EML:

COM: 28,40

\section{ROBIN ANNIE C DR}

OBSERVATOIRE DE BESANCON

BP 1615

F 25010 BESANCON CDX

FRANGE

TEL: $81 \quad 666941$

TLF: $8166 \quad 6944$

TLX: 361144

EML: ROBINAFROBES5 L/OBSBEA: : ANNIE

COM: 33

ROBINSON I PROF

UNIVERSITY OF TEXAS

MS BE 32

$80 \times 68$ 8

RICHARDSON TX.75080

USA

TEL: 2146902176

TLF:

TLX:

EML:

COM: 47

\section{ROBINSON WILLIAM J QR}

OPT OF MATHEMATICS

UNIVERSITY OF BRADFORD

BRADFORD BD7 IDP

UK

TEL: 274733466

TLF:,

TLX:

EML:

COM: 07
ROBERTS MORTON S OR

NRAO

EOGEMONT RO

CHARLOTTESYILLE YA 22903

USA

TEL:

TLX:

EML: NRAO: :MROBERTS

TLF:

COM: $28,33,38 \mathrm{C}, 40, \mathrm{EC}$

ROBERTSON JOHN ALISTAIR

DPT OF APPLIED MATHS

UNIVERSITY OF ST ANDREWS

NORTH HAUGH

ST ANDREWS FIFE KY16 9SS

uK

TEL: 33476161

ILF:

TLX: 76213

EML:

CDM: 42

\section{ROBINSON BRIAN J OR}

CSIRO

DIVISION OF RADIOPHYSICS

BOX 76

EPPING NSW 2121

AUSTRALIA

IEL: 28.580222

TLF:

ILX: 26230 ASTRO AA

EML:

COM: $33.34,40$

ROBINSON JR RICHARD D DR

AAO

BOX 296

EPPING NSW 2121

AUSTRALIA

TEL: 2 86B 1666

TLX: 23999

EML:

TLF :

COM: 10,40

ROBLEY R DR

9 ALLEE F VERDIER

F 31000 TOULOUSE

FRANCE

TEL: 61522273

TLF:

EML:

COM: 21

TLX:
ROBERTS WILLIAM W JR PRDF

OPT OF APPLIED MATHS

UNIVERSITY OF VIRGINIA

THORNTON HALL

CHARLOTTESVILLE VA 22901

USA

TEL: 8049241038

TLF:

TLX: '

EML:

COM: $28,33,34$

RDBERTSON NORNA DR

DPT OF PHYSICS \& ASTRON

UNIVERSITY OF GLASGOW

GLASGOW G12 GQQ

UK

TEL: $413398 \quad 855$

TLF : $413 \quad 349029$

TLX: 777070 UNIGLA

EML: GWO5DUK.AC.GLA.PH. II

COM: 09

ROBINSON EOWARD LEWIS OR

ASTRONOMY OPT

UNIVERSITY OF TEXAS

RLM 15308

AUSTIN TX $78712 \quad 1083$

USA

TEL: $512 \quad 4713401$

TLF:

TLX:

EML:

COM: $25,27,42$

ROBINSON LEIF J

SKY \& TELESCOPE

49 BAY STATE RD

CAMBRIUGE MA 02238

USA

TEL: $617 \quad 8647360$

TLF :

TLX:

EML:

COM: 46.51

ROBSON IAN E DR

SCHOOL OF PHYSICS \& ASTRO

LANCASHIRE POLYTECHNIC

CORPORATION ST

PRESTON PRI 2 TO

UK

TEL: $77222141 * 2188$

TLF :

TLX: 677409 LANPOL

EML:

COM:
ROBERTSON DOUGLASS

NGS

N/CG 114 NOAA

11400 ROCKVILLE PIKE

ROCKVILLE MO 20852

USA

TEL: 3014438423

TLF :

TLX:

EML:

COM: $19 C, 31,40$

ROBILLOT JEAN-MAURICE DR OBSERVATOIRE DE BOROEAUX

BP 89

F 33270 FLO1RAC

FRANCE

TEL: $56 \quad 86 \quad 4330$

TLF : $56 \quad 404251$

TLX:

EML:

CON:

ROBINSON GARRY OR

OPT PHYSICS UNIV COLLEGE

UNIVER OF NEW SOUTH WALES

NORTHCOTT OR

CAMPBELL ACT 2600

AUSTRALIA

TEL: 62686800

TLF: $6 \quad 268 \quad 0786$

TLX:

EML: GARRYPHADFA.PH.ADFA. OZ.AU

COM: 34

ROBINSON LLOYO B DR

LICK OBSERVATORY

UNIVERSITY OF CALIFORNIA

SANTA CRUZ CA 95064

USA

TEL: 4084292437

TLF :

$T L X$ :

EML:

COM: 09

ROCA CORTES TEODORO

INST DE ASTROFISICA

DE CANARIAS

DBS OEL TEIDE

E 38071 LA LAGUNA

SPAIN

TEL: $22 \quad 262211$

TLF :

TLX: 92640

EML:

COM: 10,12 
ROCCA-VOLMERANGE BRIGITTE INSTITUT D'ASTROPHYSIQUE 98BIS BD ARAGO

F 75014 PARIS

FRANCE

TEL: 143201425

TLF : 143298673

JLX:

EML

COM

RODDIER FRANCOIS PROF

NOAO/ADP

$B 0 \times 26732$

$950 \mathrm{~N}$ CHERRY AVE

TUCSON AZ $85726 \quad 6732$

USA

TEL: 6023259220

TLF :

TLX: 0666484 AURA NOAD TU

EML

COM: 09,12

RODRIGO RAFAEL

INST ASTROFISICA

DE ANDALUCIA APD 2144

PROFESOR ALBAREOA 1

E I8080 GRANADA

SPAIN

IEL: 58121300

TLF :

TLX: 79573 IAAE E

EML:

COM: 16.21

ROORIGUEZ-VILLAMIL R OR

REAL INST $\checkmark$ OBSERVATORIO

DE LA ARMAOA

CECILIO PUJAZON S/N

E 11110 SAN FERNANDO

SPAIN

TEL : $56 \quad 88 \quad 3548$

TLF: $\begin{array}{ll}56 \quad 89 & 9302\end{array}$

TLX: 76108 IOM E

EML:

COM: 07

ROEMER MAX PROF

INST F ASTROPHYSIK \&

EXTRATERR FORSCHUNG

AUF DEM HUEGEL 71

O 5300 BONN

GERMANY

TEL: $228 \quad 733670$

TLF :

Tix:

EML:

COM: 10
ROCHE PATRICK F OR

OPT OF ASTROPHYSICS

UNIVERSITY OF OXFORD

KEBLE RD

OXFORD OX1 3RH

UK

TEL: $865 \quad 273338$

TLF : $865 \quad 273 \quad 418$

TLX: 83295 NUCLOX G

EML: PERQUK,AC.OXFORO. ASTRPHYSICS

COM: 34

RODGERS ALEX W OR

MOUNT STROMLO \& SIDING

SPRING OBSERVATORIES

PRIVATE BAG

WODEN PO ACT 2606

AUSTRALIA

TEL: $62 \quad 88 \quad 1111$

TLF :

TLX: 62270 CANOPUS AA

EML:

COM: $27,29,46$

RODRIGUEZ ELOY DR

INST ASTROFISICA

DE ANDALUCIA APD 3004

PROFESOR ALBAREDA I

E IBOBO GRANADA

SPAIN

TEL: $58 \quad 12 \quad 1311$

TLF: 58 B1 4530

TLX: 78573 IAAE E

EML: 16488:: ELOY

COM: 27

ROEDER ROBERT C PROF

DPT OF PHYSICS

SOUTHWESTERN UNIVERSITY

UNIVERSITY AVENUE

GEORGETOWN TX 78626

USA

TEL: $512 \quad 863 \quad 1633$

TLF

ILX: $910-350-1677$

EMLL:

COM: 40,47

ROENNAENG BERNT O DR

ONSALA SPACE OBSERVATORY

GOETEBORG UNIVERSITY

S 439 DO ONSALA

SWEDEN

TEL: $3006 \quad 2637$

TLF :

TLX: 2400

EML:

COM: 40
ROCHESTER MICHAEL G PROF

OPT OF EARTH SCIENCES

MEMORIAL UNIVERSITY OF

NEWFOUNDLAND

ST JOHNS NF AlB $3 \times 7$

CANADA

TEL: 7097377565

TLF:

TLX: 0164101

EML:

COM: 19

ROOMAN RICHARO B OR

65 LOCUST AVE

LEXINGTON MA 02173

USA

TEL: 617 B61 8149

TLF :

ILX:

EML:

COM:

RODRIGUEZ LUIS $F$

INSTITUTO DE ASTRONOMIA

UNAM

APDO POSTAL 70-264

04510 MEXICO DF

MEXICO

TEL: $905-548-5306$

TLF:

TLX: 1760155 CICME

EML:

COM: $34 \mathrm{C}, 40$

ROELFSEMA PETER OR

KAPTEYN ASTRONOMICAL INST

$80 \times 800$

NL 9700 AV GRONINGEN

NETHERLANOS

TEL: 50634043

TLF: $50 \quad 636100$

TLX

EML: PJUTREHGRRUG

COM: 34,40

ROESER HANS-PETER

MPI FUER RAOIOASTRONOMIE

AUF DEM HUEGEL 69

D 5300 BONN :

GERMANY

IEL: 228525265

TLF:

TLX: 886440

EML:

COM: 34,40
RODOIER CLAUOE OR

NOAO /ADP

BOX 26732

$950 \mathrm{~N}$ CHERRY AVE

TUCSON AZ $85726 \quad 6732$

USA

TEL:

TLF :

TLX: 1561401

EML:

COM: 09

RODONO MARCELLO OR

IST DI ASTRONOMIA

CITTA UNIVERSIIARIA

VIA A DORIA 6

I 95125 CATANIA

ITALY

TEL: $95 \quad 337308$

TLF : 95330533

JLX: 970359 ASTRCT I

EML: ASTRCT::MARCELLO

COM: $27 C, 36,42 \mathrm{VP}$

RODRIGUEZ-ESPINOSA JOSE

INST OE ASTROFISICA

DE CANARIAS

OBS DEL TEIOE

E 38200 LA LAGUNA

SPAIN

JEL: $22 \quad 26 \quad 2211 * 449$

TLF : $22 \quad 263005$

TLX: 92640 IACE

EML: SPAN 28844::IRE

COM: 28

ROEMER ELIZABETH PROF

LUNAR \& PLANETARY LAB UNIVERSITY OF ARIZONA

TUCSON AZ 85721

USA

TEL: $602 \quad 6212897$

TLF :

TLX: 467175

EML:

COM: $06 C, 15,20,24$

ROESER HERMANN-JOSEF OR MPI FUER ASTRONOMIE KOENIGSTUHL

D 6900 HEIDELBERG I

GERMANY

TEL: $62 \quad 21528206$

TLF:

$T L X: 461789$ MPIA $O$

EML:

COM: 28 


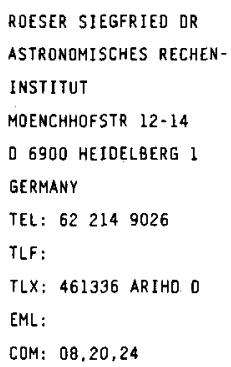

ROESSIGER SIEGFRIED DR ZENTRALINST FUER ASTROPHY STERNWARTE SONNEBERG

D 6400 SONNEBERG

GERTIANY

TEL: $9674 \quad 2287$

TLF: 96742836

TLX: 627180

EML:

COM: 05

ROGERSON JOHN B PROF

DPT ASTROPHYSICAL SCI

PRINEETON UNIVERSITY

PEYTON HALL

PRINCETON NJ OS544 1001 USA

TEL: 6094523806

TLF:

TLX: 322409

EML:

COM:

ROLLAND ANGEL DR

INST ASTROFISICA

DE ANDALUEIA APD 2144

PREFESDR ALBAREDA 1

E lo080 GRANADA

SPAIN

TEL: $58 \quad 121300$

TLF:

TLX: 78573

EML:

COM:

\section{ROMANO GIULIAND PRDF}

$\checkmark S$ ANTONIO DA PAOOVA 7

I 31100 TREVISO

ITALY

TEL:

TLF:

EML:

COM: 27

TLX:

ROMPOLT BOGDAN DR

- Astronomical institute

WROCLAW UNIVERSITY

UL KOPERNIKA 11

PL 5I 622 WROCLAW

POLAND

TEL: $071-48-24-34$

TLF:

TLX: 0712791 UWR PL

EML:

COM: 10

\section{ROGER ROBERT S DR DOMINION RADIO ASTROPHYS OBSERVATORY}

BOX 248

PENTICTON BC VZA $6 K 3$

CANADA

TEL: 6044932277

ILF: 6044937767

TLX: 04888127

EML:

COM: 34,40

ROGSTAO DAVID H OR

JPL

MS 264748

4800 DAK GROVE DR

PASADENA CA 91109

USA

TEL:

TLF:

TLX:

EML:

COM: 40

\section{ROMAN NANCY G OR}

4260 N PARK AVE

APT 306W

CHEVY CHASE MO 20815

USA

TEL: 3016566092

TLF:

TLX:

EML:

COM: $05,44,45$

RDMANOV YURI S DR

ASTRONOMICAL OBSERVATORY OOESSA STATE UNIVERSITY SHEVCHENKO PARK

270014 ODESSA

UKRAINE

TEL: 220396

TLF:

FLX:

EML:

COM: 27,30

RONAN COLIN A

FLAT 6, BOURNE CT

THE BOURNE

HASTINGS

EAST SUSSEX TN34 $3 U Z$

UK

TEL: $424 \quad 446362$

TLF:

TLX:

EML:

COM: 41
ROGERS ALAN E E OR HAYSTACK OBSERVATORY

WESTFORO MA 01BBG

USA

TEL: 6176924764

TLF:

ILX: 948149 HAYSTACK WFRD

EML:

COM: 34.40

ROHLFS K PROF DR RUHR UNIVERSITAET BOCHUM INSTITUT FUR ASTROPHYSIK POSTFACH 102148 D 4630 BOCHUM 1 GERMANY

TEL: 2347005802

TLF:

TLX: 0825860

EML:

COM: $33,34,40$

ROMANCHUK PAVEL $R$ oR ASTRONOMICAL OBSERVATORY KIEV STATE UNIVERSITY OBSERVATORNAYA UL 3 $252053 \mathrm{KIEV}$ UKRAINE TEL:

TLF :

TLX:

EML:

COM: 10

ROMERO PEREZ M PILAR INST DE ASTRON \& GEODESIA FAC DE CIENCIAS MATEMAT UNIVEASIOAD COMPLUTENSE E 28040 MADRIO SPAIN TEL: 12442501 TLF:

TLX:

EML:

COM: 04

RONG JIAN-XIANG OPT OF ASTRONOMY NANJING UNIVERSITY NANJING CHINA PR

TEL: $2534651 * 2882$

TLF:

TLX: 34151 PRCNU CN

EML:

COM; 33 
ROOD HERBERT J

INST FOR AOVANCED STUQY SCHOOL OF NATURAL SCIENCE PRINCETON NJ 08540

USA

TEL:

TLF :

TLX:

EML:

COM: 28

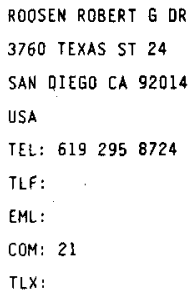

ROOD ROBERT T DR

UNIVERSITY STATION UNIVERSITY OF VIRGINIA

BOX 3818

CHARLOTTESVILLE VA 22903

USA

TEL: 8049244904

TLF :

TLX:

EML:

COM: 35,51

\section{ROQUES FRANCOISE DR}

\section{OBSERVATOIRE DE PARIS}

SECTION DE MEUOON

LAM

F 92195 MEUDON PPL COX

$$
\text { FRANCE }
$$

TEL: 145077409

TLF: 145077469

TLX: 201571

EML: MESIOA: :ROQUES/ROQUESAFRMEU51 COM

COM: 16

\section{ROSADO MARGARITA DR \\ INSTITUTO DE ASTRONOMIA \\ UNAM \\ APOO POSTAL 70-264 \\ 04510 MEXICO DF \\ MEXICO \\ TEL: $905-548-5306$ \\ TLF: \\ TLX: \\ EML: \\ COM: 28,34}

\section{ROSEN EOWARO OR}

DPT OF HISTORY

CITY COLLEGE OF NY

NEW YORK NY 10031

USA

TEL:

TLF:

TLX:

EML:

COM:

ROSLUND CURT OR
OPT OF ASTRONOMY
CHALMERS TECHNICAL UNIV
S 41296 GOETEBORG
SWEDEN
TEL: 31810100
TLF:
TLX:
EML:
COM: 25,46

ROOS NICOLAAS OR

STERREWACHT

BOX 9513

NL 2300 RA LEIDEN

NETHERLANOS

TEL: 71275864

ILF:

TLX:

EML: ROOSOHLERUL151.BITNET

CDM: 28

ROQUES SYLVIE DR

OBS MIDI PYRENEES

14 AVE E BELIN

F 31400 TOULOUSE COX

FRANCE

TEL: 61252101

TLF :

TLX: 530776 OBSTLSE

EML:

COM:

ROSCH JEAN PROF

OBS MIDI PYRENEES

14 AVE E BELIN

F 31400 TOULOUSE CDX

FRANCE .

TEL: 62951969

TLF:

TLX: $531625 \mathrm{~F}$

EML:

COM: $09,10,16$

ROSENBERG J DR

STATE UNIV OF UTRECHT

HEIOELBERLAAN $B$

NL 3584 CA UTRECHT

NETHERLANDS

TEL: $30 \quad 535124$

TLF:

ILX:

EML:

COM:

ROSNER ROBERT

ASTRONOMY \& ASTROPHYS CTR

UNIVERSITY OF CHICAGO

$5640 S$ ELLIS AVE

CHICAGO IL 60637

USA

TEL:

TLF :

TLX:

EML:

COM: 48,49
ROOSEN ROBERT G DR

RAINBOW OBSERVATORY

RR1

BOX 5068

PAHIA HI 96778

USA

TEL:

TLF :

TLX:

EML:

COM: 21,22

ROSA DOROTHEA DR

EMIL-KURZ STR 4

D 8045 ISMANING

GERMANY

TEL: 89964299

TLF :

TLX:

EML:

COM:

ROSE JAMES ANTHONY

DPT PHYSICS \& ASTRONOMY UNIVERSITY NORTH CAROLINA 204 PHILLIPS HALL 039A CHAPEL HILL NC 27514

USA

TEL: 9199827170

TLF:

TLX:

EML:

COM: 28,29

ROSENDHAL JEFFREY D DR NASA HEADQUARTERS

CODE $Z$

ASS DIRECTOR SPACE EXPLO WASHINGTON OC 20546

USA

TEL: 2024539181

ILF: 2024260408

TLK:

EML:

COM: 44

ROSQUIST KJELL

INST OF THEORETICAL PHYS

VANADISVAEGEN 9

S 11346 STOCKHOLM

SWEDEN

TEL: $8228160 * 225$

TLF :

TLX: 15433 FYSTOS

EML:

COM: 47 


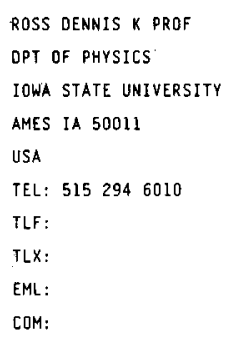

ROSS JOHN E R DR

BPT OF PHYSICS

UNIVERSITY OF QUEENSLAND

ST LUCIA

BRISBANE QLD 4067

AUSTRALIA

TEL: 73773429

TLF:

TLX: 40315 UNIVOLD AA

EML:

COM: 14,36

ROSSI LUCIO
IAS
CNR
EP 67
I 00044 FRASCATI
ITALY
TEL: $6942 \quad 5651 / 2 / 3$
TLF:
TLK: $610261 \quad$ CNR FRA
EML:

ROTH-HOPPNER MARIA LUISE HAMBURGER STERNWARTE

GOJENSBERGSWEG 112

D 2050 HAMBURG 80

GERMANY

TEL: 4072524112

ILX: 217884

EML:

TLF :

COM: 37

ROUDIER THIERRY DR

OBS MIOI PYRENEES

14 AVE E BELIN

F 31400 TOULOUSE COX

FRANCE

TEL: 61252101

TLF:

TLX:

EML:

COM: 10,12

\section{ROUSSEAU JEANINE DR}

OBSERVATOIRE DE LYON aVE CHARLES ANORE

F 69561 S GENIS LAVAL COX FRANCE

TEL: $78 \quad 56 \quad 0705$

TLF: 72399791

TLX: 310926

EML:

COM:
ROSSELLL GASPAR

OPT FISICA DE ATMOSFERA

UNIVERSIOAD DE BARCELONA

AVO DIAGONAL 645

E. OBO2B BARCELONA

SPAIN

TEL:

TLF:

TLX:

EML:

COM: 04

ROSTAS FRANCOIS DR OBSERVATOIRE OE PARIS SECTION DE MEUDON

F 92195 MEUDON PPL COX FRANCE

TEL: 145077565

ILF:

ILX: 201571

EML:

COM: 14

ROTS ARNOLD H OR
NRAO
BOK O
SOCORRO NM B7BOI 0387
USA
TEL:
TLF:
TLX:
EML:
COM: 28

ROUEFF EVELYNE. M A DR OBSERVATOIRE OE PARIS SECTION OE MEUDON

DAF

F 92195 MEUDON PPL CDX FRANCE

TEL: 145077435

TLF:

TLX: 201571

EML:

COM: 14

ROUSSEAU JEAN-MICHEL MR OBSERVATOIRE DE BOROEAUX

BP 99

F 33270 FLOIRAC

FRANCE

TEL: $56 \quad 86 \quad 4330$

TLF: 56404251

ILX:

EML:

COM: 08
ROSSI BRUNO 8 PROF

CENTER FOR SPACE RESEARCH MIT RM 37667

BOX 165

CAMBRIDGE MA 02139

USA

TEL: 6172534283

TLF:

TLX: $92-1473$

EML:

COM: 48

ROTH MIGUELL R OR CARNEGIE INST WASHINGTON LAS CAMPANAS OBSERVATORY

CASILLA 601

LA SERENA

CHILE

TEL: 51213032

TLF:

TLX: 645227 AURA CT

EML: MROTHCHCEVM

COM:

ROTTENBERG J A OR

291 BAYVIEW AVE

SUITE $110 \mathrm{C}$

WILLOWDALE ON M2K IEB

CANADA

TEL:

TLF:

TLX:

EML:

COM:

ROUNTREE JANET DR 6001 WYNNWOOD RD

BETHESDA MO 20816

USA

TEL:

TLF:

TLX

EML: ROUNTREERNSSOCA.GSFC. NASA. G COM: $09,27,37,45$

ROUTLEOGE DAVIO OR OT OF ELECTRICAL ENGEENRG UNIVERSITY OF ALBERTA EOMONTON AB T6G 2J7 CANADA

TEL: 4034325668

TLF:

ILX: 0372979

EML:

COM: 
ROUTLY PAUL M OR

US NAVAL OBSERVATORY

34 \& MASSACHUSETTS AVE NW

WASHINGTON OC 203925100

USA

TEL: $202 \quad 6531532$

TLX:

EML:

TLF

COM: 38

ROWAN-ROBINSON MICHAEL OR SCHOOL OF MATHEMATICAL SC QUEEN MARY/WESTFIELD COLL MILE END RD LONDON EI 4NS

uk.

TEL:

TLF

TLX:

EML

COM: 47,51

ROY JEAN-RENE

OPT DE PHYSIQUE

UNIVERSITE LAVAL

FAC SCIENCES 2 GENIE

LAVAL OC GIK $7 P 4$

CANADA

TEL: 4186565816

TLF: $418 \quad 656 \quad 2040$

TLX: 05131621 UNILAVAL

EML:

COM:

RUBASHEV BORIS M DR

PULKOVO OBSERVATORY

ACADEMY OF SCIENCES

LO KUTUZOV QUAY

196140 ST PETERSBURG

RUSSIA

TEL:

TLF:

ILX:

EML:

COM: 10

RUBIO MONICA OR

OPT DE ASTRONOMIA

UNIVERSIDAD OE CHILE

CASILLA 36 D

SANTIAGO

CHILE

TEL: 22294101

TLF: 22712799

TLX: 440001

EML:

COM: 40
ROVIRA MARTA GRACIELA

IAFE

CC 67 SUC 28

1428 BUENOS AIRES

ARGENTINA

TEL: 17816755

TLX:

EML:

TLF :

COM: 12,36

ROWSON BARRIE OR

NRAL

JODRELL BANK

MACCLESFIELD SK11 9DL

UK

TEL: 47771321

ILX: 36149

EML:

TLF :

COM: 40

ROZELOT JEAN $P$

OCA CERGA

AVE COPERNIC

F 06130 GRASSE

FRANCE

TEL: $93 \quad 365849$

TLF :

TLX: 470865

EML:

COM: 10

RUBEN G PROF DR

ZNTRLINST F ASTROPHYSIK

ROSA-LUXEMBURG-STR I7A

D 1502 POTSDAM

GERMANY

TEL:

TLX:

EML:

TLF :

COM: 35,44

RUCINSKI SLAWOMIR M OR

SPACE ASTROPHYSICAL LAB

YORK UNIVERSITY

4700 KEELE ST

TORONTO ON M3J IP3

CANADA

TEL: $416 \quad 6653311$

ILF:

TLX: 06524736

EML: BITNET:FS300516EYUSOL

COM: 36,42
ROVITHIS PETER DR

ASTRONOMICAL INSTITUTE

NTL OBSERVATORY OF ATHENS

BOX 20048

GR 11810 ATHENS 306

GREECE

TEL: 13463803

TLF:

$T L X: 215530$ OBSA GR

EML: -

COM: 42

ROXBURGH IAN W PROF

SCHOOL QF MATHEMATICAL SO

QUEEN MARY/WESTFIELD COLL

MILE END RD

LONDON E1 4NS

UK

TEL: 19804811

TLF :

YLX:

EML:

COM: $10,34,35,42,47,49$

ROZHKOVSKIJ OIMITRIJ A

ASTROPHYSICAL INSTITUTE

KAZAKH ACAD OF SCIENCES

480068 ALMA ATA

KAZAKHSTAN

TEL: $62-40-40$

ILF :

TLX:

EML

COM: 21,34

RUBIN ROBERT HOWARD

NASA AMES RESEARCH CTR

MS 2456

MOFFETT FIELD CA 94035

USA

TEL: 4159655528

TLF :

TLX: 348408

EML:

COM: 40,5$]$

RUDAK BRONISLAW

INSTITUTE OF ASTRONOMY

N COPERNICUS UNIVERSITY

UL CHOPINA $12 / 18$

PL 87 100 TORUN

POLANO

TEL: $26037 * 10$

ILF :

TLX: 813978 ZAPAN PL

EML :

COM:
ROVITHIS-LIVANIOU HELEN

OPT OF ASTROPHYSICS

NTL UNIVERSITY OF ATHENS

PANEPISTIMIOPOLIS

GR 15771 ZOGRAFOS

GREECE

TEL: I $724 \quad 3414$

ILF:

TLX:

EML:

COM: 42

ROY ARCHIE E PROF

DPT OF ASTRONOMY

UNIVERSITY OF GLASGOW

GLASGOW GI2 890

UK

TEL: $413398855 * 502$

TLF:

TLX: 778421 GLASUL

EML:

COM: $07 C, 46$

ROZYCZKA MICHAL

ASTRONOMICAL OBSERVATORY

WARSAW UNIVERSITY

AL UJAZZOWSKIE 4

PL 00478 WARSAW

POLAND

TEL:

TLF :

TLX: B13978 ZAPAN PL

EML:

COM: 34

RUBIN VERA C DR

DPT TERRESTR MAGNETISM

CARNEGIE INST WASHINGTON

5241 BROAO BRANCH RD NW

WASHINGTON DC 20015

USA

TEL: 2029660863

TLF:

TLX: 440427 MAGN UI

EML:

COM: $28,30,33,47$

RUDDY VINCENT $P$ OR

REGIONAL TECHNICAL COLL

ROSSA AVE

CORK

IRELANO

TEL:

TLF:

TLX:

EML:

COM: 47 


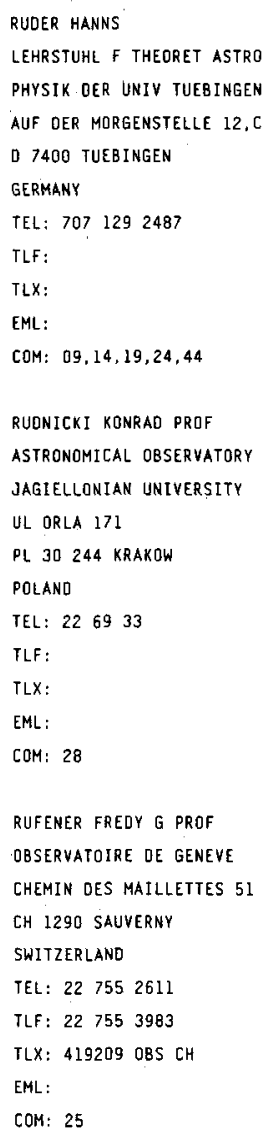

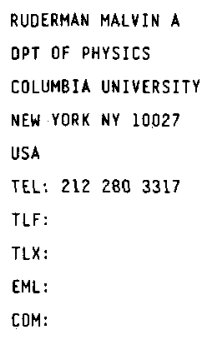

RUDZIKAS ZENONAS B INST OF THEORET PHYS/ASTR LithuENian ACADEMY OF SCI POZELOS 54

232600 YILNIUS

LithuANIA

TEL: 122620939

JLF:

ILX: 261141 LMA SU

EML:

COM: $14 \mathrm{C}$

RUFFINI REMO

OPT DI FISICA

UNIVERSITA DI ROMA

PA. MORO 2

I 00185 ROMA

ITALY

TEL: 64976304

TLF:

TLX: 613255 INFNRO I

EML:

COM: 47,48

RUMSEY NORMAN J

21 MALONE RD

LOWER HUTT

NEW ZEALAND

TEL: 4696787

TLF:

EML:

COM:

TLX:

\section{RUSCONI LUIGIA OR}

OPT OI ASTRONOMIA

UNIVERSITA DI TRIESTE

VIA TIEPOLO 11

I 34131 TRIESTE

ITALY

TEL: 40794863

ILF:

TLX: 461137 OAOTI

EML:

COM: 09

\author{
RUDKJOBING MOGENS PROF \\ INST OF PHYSICS \& ASTRON \\ UNIVERSITY OF AARHUS \\ NY MUNKEGAOE \\ DK 8000 AARUUS C \\ DERMARK \\ TEL: 86128899 \\ TLF: $86 \quad 202711$ \\ TLX: 64767 AAUSCI OK \\ EML: \\ COM: 45
}

RUEDIGER GÜNTHER OR ZNTRLINST F ASTROPHYSIK ROSA LUXEMBURG STR $17 A$ D 1591 POTSDAM GERMANY

TEL: 373377138

TLF: 06915471

TLX:

EML:

COM: 10

RUGGE HUGO R DR
AEROSPACE CORPORATION
MS M2 226
BOX 92957
LOS ANGELES CA 90009
USA
TEL: 2136487086
TLF:
TLX:
EML:

RUNCORN S K PRDF

SCHOOL OF PHYSICS UNIVERSITY OF NEWCASTLE NEWCASTLE/TYNE NEI TRU UK

TEL: 63232511

TLF:

TLX: 53654 UNINEW $G$

EML:

COM: 16,19

RUSIN VOJTECH

ASTRONOMICAL INSTITUTE

SLOVAK ACADEMY SCIENCES

CS 05960 TATRANSKA LOMNI CZECHOSLOVAKIA

TEL: $969967866 / 7 / 8$

TLF: 969967656

TLX: 8078277 AUSSAV C

EML:

COM: 10,12
RUDNICK LAWRENCE DR

OPT OF ASTRONOMY

UNIVERSITY OF MINNESOTA

116 CHURCH ST SE

MINNEAPOLIS MN 55455

USA

TEL: 6123735457

TLF:

TLX:

EML:

COM: 40,47

RUELAS-MAYORGA $R$ A DR INSTI IUTO DE ASTRONOMIA UNAM

APDO POSTAL 70-264 04510 MEXICO OF

MEXICO

TEL: 5255485306

TLF : 5255483712

TLX:

EML: RARMALFA. ASTROSCU. UNAM.MX COM: 33

RUIZ MARIA TERESA OR DPT OE ASTRONOMIA UNIVERSIDAO DE CHILE CASILLA 360

SANTIAGO

CHILE

TEL: 22294101

ILF:

TLX: 440001

EML:

COM: 33

RUPPRECHT GERO DR

ESO

KARL SCHWARZSCHILOSTR 2

D 8046 GARCHING MUENCHEN

GERMANY

TEL: 8932006355

TLF : 893202362

ILX: 5282820 EO D

EML: GRUPPREC $₫ 5 O$. ORG

COM: 09

RUSKOL EUGENIA L DR INST PHYSICS OF THE EARTH ACADEMY OF SCIENCES GRUZINSKAYA 10 123342 MOSCOW. RUSSIA

TEL: 2520726

TLF:

TLX: 411196 IFZAN SU

EML:

COM: 16 
RUSSELL CHRISTOPHER T INST OF GEOPHYS \& PLANET UNIVERSITY OF CALIFORNIA LOS ANGELES CA 90024 USA TEL: 2138253188 TLF :

TLX: 910-342-6981

EML

COM: 49

RUSSELL STEPHEN OR

DIAS

SCHOOL OF COSMIC PHYSICS

5 MERRION SO

BUBLIN 2

IRELAND

TEL: 1774321

TLF: 1682003

TLX: 31687 DIAS EI

EML: 29536: : SR/SREOIAS. IE

COM: 34

RUST OAVIO M DR

APPLIEO PHYSICS LAB

JOHNS HOPXINS UNIVERSITY

JOHNS HOPKINS RD

LAUREL MD 20707

USA

TEL: 3019535414

TLF:

TLX: 89-548 APL JHU LAUR

EML:

COM: 10

\section{RUZOJAK VLADIMIR DR}

INSTIUUTE OF PHYSICS

UNIVERSITY OF ZAGREB

BOX 304

YU 41001 ZAGREB

YUGOSLAVIA

TEL:

TLF:

TEX:

EML:

COM: 10

RYBANSKY MILAN
ASTRONGMICAL INSTITUTE
SLOVAK ACADEMY SCIENCES
CS 059 60 TATRANSKA LOHW
CZECHOSLOVAKIA
TEL: $969967866 / 7 / 8$
TLF: 969967656
TLX: 80.78277 AUSAV C
EML:
COM: 10,12

RUSSELL JANE L OR MAVAL RESEARCH LABORATORY CODE 4130R

4555 OVERLOOK AVE SW

WASHINGTON OC 203755000

USA

TEL: 2027670171

TLF:

TLX:

EML:

COM: $08,24,26,40,51$

RUSSEY RUSCHO OR

DPT OF ASTRONOMY

UNIVERSITY OF SOFIA

ANTON IVANOV ST 5

BG 1126 SOFIA

BULGARIA

TEL: 2544852

TLF:

TLX:

EML:

COM: 27

RUSU I OR

ASTRONOMICAL OBSERVATORY

CUTITUL DE ARGINT 5

$80 \times 28$

R 75212 BUCAREST 28

RUMANIA

TEL: 2363 OI

ILF:

TLX:

EML:

COM: 08,19

RUZICKOVA-TOPOLOVA B OR
ASTRONOMICAL INSTITUTE
CZECH ACAOEMY OF SCIENCES
ONDREJOV OBSERVATORY
CS 25165 ONOREJOV
CZECHOSLOVAKIA
TEL: 20485201
TLF: 20485314
TLX: 121579
EML:
COM: 10

\section{RYBICKI GEORGE B DR}

CENTER FOR ASTROPHYSICS

HCO/SAO

60 GARDEN ST

CAMBRIDGE MA 02138

USA

TEL: 6174957452

TLF:

TLX: $92-1428$

EML:

COM: 33.36
RUSSELL JOHN A PROF

DPT OF ASTRONOMY

UNIV SOUTHERN CALIFORNIA

UNIVERSITY PARK

LOS ANGELES CA 90089

USA

TEL: 2137430231

TLF:

TLX:

EML:

COM: 22

RUSSEVA TATJAMA

DPT OF ASTRONOMY

BULGARIAN ACAD SCIENEES

72 LENIN BLVO

BG 1784 SOFIA

BULGARIA

TEL: 2758927

TLF:

ILX: 23561 ECF BAN BG

EML:

COM: 37

RUSU L OR

STR MITROPOLITUL IOSIF 47

R. 75217 BUCAREST 28

RUMANIA

TEL:

ILF :

TLX:

EML:

COM:

RYABCHIKOVA TANYA DR

INST OF ASTRONOMY

ACADEMY OF SCIENCES

PYATUITSKAYA UL 48

109017 MOSCOW

RUSSIA

TEL:

TLF:

TLX:

EML:

COM: 36

\section{RYBXA PRZEMYSLAW DR}

INST HISTORY OF SCIENCES

POLISH ACAD OF SCIENCES

NOWY SWIAT 72

PL 00330 WARSAW

POLAND

TEL:

TLF:

ILX:

EML:

COM: 41
RUSSELL KENNETH S OR

UK SCHMIDT TELESCOPE

AAD

PRIVATE BAg -

COONABARABRAN NSW 2357

AUSTRALIA

TEL: 68426311

ILF: 68846298

TLX: CANOPUS AA 163945

EML: NSSOCA: ; PSIKAAOCBN ; KSR

COM: 20

RUSSO GUIDO OR

DPT DI SCIENZE FISICHE

UNIVERSITA OI NAPOLI

MOSTRA D OLTREMARE PAD 19

I 80125 NAPOLI

ITALY

TEL: $81 \quad 725 \quad 3447$

TLF:

TLX: 720320

EML:

COM: 05,42

RUTTEN ROBERT $J$ DR STERREKUNDIG INSTITUTE

BOX 80000

NL $350 B$ TA UTRECHT

NETHERLANDS

TEL: 30535200

TLF :

TLX: 40048 FYLUT NL

EML: BITNET: WMMMAILEHUTRUUO

COM: $12,29,36$

RYABOV YU A PROF OR OPT QF MATHEMATICS

MAOI

LENINGRADSKY PROSP 64

125319 MOSCOW

RUSSIA

TEL: 1550326

TLF :

ILX:

EML:

COM: 07

RYDBECK GUSTAF H B OR

ONSALA SPACE OBSERVATORY GOETEBORG UNIVERSITY

543900 ONSALA

SWEDEN

TEL: $30 \quad 062081$

TLF:

TLX:

EML:

COM: 28,40 
RYDBECK OLOF E H PROF ONSALA SPACE OBSERVATORY GOETEBORG UNIVERSITY

S 439 OO ONSALA

SWEDEN

TEL: $3006 \quad 2081$

TLF :

TLX: 8542400 ONSPACE

EML:

COM: 40

RYTER CHARLES E DR

CEA CEN

DAPNIA/SAP

BP 2

F gligl GIF/YVETTE COX

FRANCE

TEL: 169083912

ILF:

TLX:

EML:

COM:

\section{SAAR ENN DR}

TARTU ASTROPHYSICAL OBS

ESTONIAN ACAO OF SCIENCES

202444 TARTU

ESTONIA

TEL:

TLF:

TLX:

EML: sarooai.tartu.ew.su

COM: 33,47

SACKMANN I JULIANA OR

CALIECH

KELLOGG RADIATION LAB

PASADENA CA 91125

USA

TEL: $818 \quad 356 \quad 4256$

TLF:

TLX:

EML:

COM: 35

SAOIK AZIZ R OR

SARC

SCIENTIFIC RES COUNCIL

\section{BOX 2441}

JADIRIYAH BAGHDAD

IRAD

TEL: 17765127

TLF :

TLX: 213976 SRC

EML:

COM: 27,42
RYOGREN ALFRED ERIC JR OR

BOEING AEROSPACE CO

MS 8708

BOX 3999

SEATTLE WA 981242499

USA

TEL: 2067732155

TLF

TLX:

EML:

COM: 25

RYUTOVA MARGARITA $P$ DR

INSTITUTE OF NUCLEAR PHYS

ACADEMY OF SCIENCES

SIBERIAN DIV

630090 NOVOSIBIRSK.

RUSSIA

IEL: 3832359943

TLF: 3832352163

TLX: 133116 ATOM SU

EML: RYUTOVAOUXINPB.INP.NSK.SU

COM: 10

SABANO YUTAKA DR

ASTRONOMICAL INSTITUTE

TOHOKU UNIVERSITY

SENDAI AOBA

MIYAGI 980

JAPAN

TEL: $222 \quad 221800$

TLF :

TLX:

EML:

COM: 34

SADAKANE KOZO DR

ASTRONOMICAL INSTITUTE

OSAKA KYOIKU UNIVERSITY

488 MINAMIKAWAHORICHO

OSAKA 543

JAPAN

TEL:

TLF :

TLX:

EML:

COM: $29 \mathrm{C}$

SADLER ELAINE MARGARET

AAO

B0X 296

EPPING NSW 2121

AUSTRALIA

TEL: 2868 1666

TLX: 123999 AAOSYO AA

EML:

TLF:

COM: 28
RYKHLOVA LIOIJA V OR

INST OF ASTRONOMY

ACADEMY OF SCIENCES

PYATNITSKAYA UL 48

109017 MOSCOW

RUSSIA

TEL: $231-54-61$

TLF :

TLX: 412623 SCSTP SU

EML:

COM: 19

RYZHKOV NIKOLAI F DR

PULKOVO OBSERVATORY

ACADEMY OF SCIENCES

10 KUTUZOV QUAY

196140 ST PETERSBURG

RUSSIA

TEL:

TLF:

ILX:

EML :

COM: 40

SABBADIN FRANCO OR OSSERVATORIO ASTROFISICO VIA DELL OSSERVATORIO B

I 36012 ASIAGO

ITALY

TEL: $42 \quad 46 \quad 2665$

TLF :

TLX: SETUR 430110

EML:

COM: 34

SADAT RACHIDA DR
CRAAG
BP 63
BOUZAREAH
ALGER
ALGERIA
TEL: 2791443
TLF: 2132799862
TLX: $614470 Z$
EML:
COM: 47

SADUN ALBERTO CARLO DR BRADLEY OBSERVATORY

AGNES SCOTT COLLEGE

DECATUR ga 30030

USA

TEL: 4043716265

TLF :

TLX:

EML: PHYAASEGSUVMI BITNET

COM: 28
RYLOV VALERIJ S DR

SPECIAL ASTROPHYSICAL OBS ACADEMY OF SCIENCES

NIZHNIJ ARKHYZ

357147 STAVROPOLSKIJ

RUSSIA

TEL

TLF:

TLX:

EML:

COM: 09

RZHIGA OLEG N OR

INST OF RADIO \& ELECTRON

ACADEMY OF SCIENCES

103907 MOSCOW

RUSSIA

TEL:

TLF:

$T L X:$

EML:

COM:

SACK NDAM OR

DPT OF THEORETICAL PHYS HEBREW UNIV OF JERUSALEM JERUSALEM 91904

ISRAEL

TEL:

ILF:

TLX:

EML:

COM:

SADEH O DR OPT OF PHYSICS \& ASTRON TEL AVIV UNIVERSITY RAMAT AVIV

TEL AVIV 69978

ISRAEL

TEL: 3420553

TLF :

TLX: 34271 VERSY

EML:

COM:

SAOZAKOV SOFIJA DR ASTRONOMICAL OBSERVATORY VOLGINA 7

YU 11050 BEOGRAD

YUGOSLAVIA

TEL: $11 \quad 419 \quad 357 / 421875$

TLF :

TLX:

EML:

COM: 08,19 


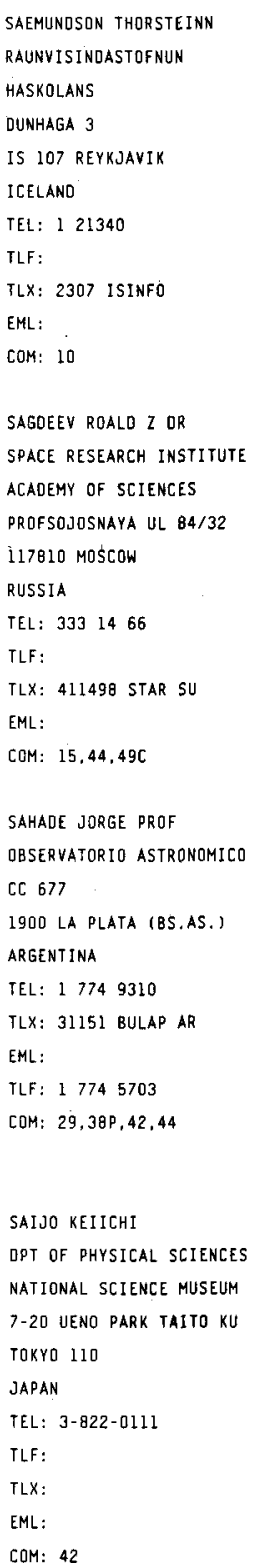

COM: 42

SAITO KUNIJI PROF

TOKYO ASTRONOMICAL OBS

NAOJ

OSAWA MITAKA

TOKYO 181

JAPAN

TEL:

TLF:

TLX:

EML:

COM: 10,36 .
SAFKO JOHN L

OPT PHYSICS ASTRONOMY

UNIVERSITY S CAROLINA

COLUMBIA SC 29208

USA

TEL: 8037776466

TLF:

TLX: UNIVSCAROL CLB

EML:

COM: 46

SAGGION ANTONIO PROF

OPT OI FISICA G GALILEI

UNIVERSITA DI PADOVA

VIA MARZOLO 8

I 35131 PADOVA

ITALY

TEL: 49844254

TLF:

TLX: 430308 OFGGPOI

EML:

COM:

SAHAI RAGHVENORA OR

OPT OF PHYSICS

CHALMERS TECHNICAL UNIV

UNIV OF GOTEBORG

S 41296 GOETEBORG

SWEDEN

TEL: 31723139

TLF:

TLX: 2369 CHALBIB S

EML: BITNET: TFARS SECTHF5I

COM:

SAIKIA DHRUBA JYOTI DR

TATA INST OF FUNOAMENTAL

RESEARCH

POONA UNIVERSITY CAMPUS

PUNE 411007

INOIA

TEL: 212337107

TLF: 212335760

TLX: 0145658 GMRT IN

EML: uunet! shakt ! gmrt!djs

COM: 40

SAITO MAMORU OR
OPT OF ASTRONOAY
KYOTO UNIVERSITY
KLTASHIRAKAWA SAKYO KU
KYOTO 606
JAPAN
TEL: $0757512111 \times 3904$
TLF:
TLX: 5422693 LIBKYU J
EML:
COM:

SAITO MAMORU OR

KYOTO UNIYERSITY

KITASHIRAKAWA SAKYO KU

TEL: $0757512111 \times 3904$
SAFRONOV VICTOR S DR

INST PHYSICS OF THE EARTH

ACADEMY OF SCIENCES

GRUZINSKAYA 10

123242 MOSCOW

RUSSIA

TEL: $252-07-26$

TLF:

TLX: 411196 IFZAN SU

EML:

COM: 16

SAGNIER JEAN-LOUIS DR

bUREAU DES LONGITUDES

77 AVE DENFERT ROCHEREAU

F 75014 PARIS

FRANCE

TEL: 140512261

ILF:

ILX:

EML:

COM: 07,20

SAHAL-BRECHOT SYLVIE DR OBSERVATOIRE OE PARIS

SECTION DE MEUDON

DAMAP

F 92195 MEUDON PPL CDK

FRANCE

TEL: 145077442

TLF: $14507 \quad 7469$

TLX: 201571

EML: MESIOA: : SAHAL/SAHALERMEUSI

COM: $10,14 C$

SAIO HIOEYUKI DR

ASTRONOMICAL INSTITUTE

TOHOKU UNIVERSITY

SENDAI AOBA

MIYAGI 980

JAPAN

TEL:

TLF:

TLX:

EML:

COM: 35

SAITO SUMISABURO DR

KHASAN OBSERVATORY

KYOTO UNIVERSITY

YAMASHINA

KYOTO 607

JAPAN

TEL: $075-5 B 1-1235$

TLF:

TLX: 5422693 LIBKYU J

EML:

COM:
SAGAN CARL DR

CORNELL UNIVERSITY

302 SPACE SCIENCE BLDG

ITHACA NY 14853

USA

TEL: 6072564971

TLF:

ILX: 937478

EML:

COM: 16,51

SAHA SWAPAN RUMAR OR INDIAN INSTITUTE OF ASTROPHYSICS

KORAMANGALA

BANGALIRE 560034

INDIA

TEL: 812569902

ILF:

TLX: 845-2763 IIAB IN

EML:

COM:

SAHU KAILASH C DR

RAPTEYN ASTRONOMICAL INST

BOX 800

NL 9700 aV GRONINGEN

NETHERLANDS

TEL: $5063 \quad 4073$

TLF: $5063 \quad 6100$

TLX: 53572 STARS NL

EML: KAILASH GRRAGG

COM: 34

SAISSAC JOSEPH DR

OBS MIDI PYRENEES

14 AVE E BELIN

F 65200 bagneres bigORRE

FRANCE

TEL: 62951969

TLF :

TLX: $531625 \mathrm{~S}$

EME:

COM: 16

SAITO TAKAO PROF

GEOPHYS INST SCIENCE FAC TOHOKU UNIVERSITY

AOBA AOBA-KU

SENOAI 980

JAPAN

TEL: 222684508

TLF: 222684500

TLX: MTA1S5COPNTOHOK

EML:

COM: 15 


\begin{tabular}{|c|c|}
\hline SAKAI JUNICHI & SAKASHITA SHIRO PROF \\
\hline FAC OF ENGINEERING & OPT OF PHYSICS \\
\hline TOYAMA UNIVERSITY & HOKKAIDO UNIVERSITY \\
\hline TOYAMA 930 & KITA 10 NISHI $\theta$ \\
\hline JAPAN & SAPPORO 060 \\
\hline TEL: $0764-41-1271$ & JAPAN \\
\hline TLF: & TEL: $011-716-2111$ \\
\hline TLX: & TLF : \\
\hline EML: & $T L X:$ \\
\hline \multirow[t]{2}{*}{ COM: 12} & EML: \\
\hline & COM: 35 \\
\hline SAKURAI TAKASHI DR & SAKURAI TAKEO T PROF \\
\hline TOKYO ASTRONOMICAL OBS & OPT AERONAITIC ENGINEER \\
\hline NADJ & KYOTO UNIVERSITY \\
\hline OSAWA MITAKA & KITASHIRAKAWA SAKYO KU \\
\hline TOKYO 181 & КYOTO 606 \\
\hline JAPAN & JAPAN \\
\hline TEL: $422 \quad 325111$ & TEL: $75-7512111 \times 5792$ \\
\hline TLF : & TLF : \\
\hline TLX: 2822307 TAOMTK $\mathrm{J}$ & TLX: 05422693 LIBKYUJ \\
\hline EML: & EML: \\
\hline COM: $10 C, 12$ & COM: \\
\hline SALETIC OUSAN & SALINARI PIERO \\
\hline ASTRONOMICAL OBSERVATORY & OSS ASTROFISICO \\
\hline VOLGINA $?$ & DI ARCETRI \\
\hline YU 11050 BEOGRAD & LARGO E FERMI 5 \\
\hline YUGOSLAVIA & I 50125 FIRENZZE \\
\hline TEL: $11 \quad 157 \quad 022$ & ITALY \\
\hline TLF: & TEL: $55 \quad 2752231$ \\
\hline TLX: & TLF: \\
\hline EML : & $T L X: 572268$ \\
\hline \multirow[t]{2}{*}{ COM: 08} & EML: \\
\hline & COM: 34 \\
\hline SALPETER EOWIN E PROF & SALUKVADZE G N DR \\
\hline CORNELL UNIVERSITY & ABASTUMANY ASTRDPHYSICAL \\
\hline NEWMAN LAB OF NUCLEAR STU & OBSERVATORY \\
\hline ITHACA NY 14853 & GEORGIAN ACAD OF SCIENCES \\
\hline USA & 383762 ABASTUMANI \\
\hline TEL: 6072563302 & GEORGIA \\
\hline TLF: & TEL: \\
\hline$T L X: 937478$ & TLF : \\
\hline EML: & TLX: \\
\hline \multirow[t]{2}{*}{ COM: $34,35,40,48 C$} & EML: \\
\hline & COM: 26,37 \\
\hline SALZER JOHN JOSEPH OR & SAMAIN DENYS OR \\
\hline DPT OF ASTRONOMY & IAS \\
\hline VAN VLECK OBSERVATORY & BP 10 \\
\hline WESLEYAN UNIVERSITY & F 91371 VERRIERES BUISSON \\
\hline MIODLETOWN CT 06457 & FRANCE \\
\hline USA & TEL: $1 \quad 64 \quad 47 \quad 4304$ \\
\hline TEL: $2033479411 * 2827$ & TLF: \\
\hline TLF : 2033447981 & $T L X: 600252$ \\
\hline TLX: & EML: \\
\hline EML: SLAZ PARCHA. ASTRO. WESL & COM: 12 \\
\hline
\end{tabular}

$\begin{array}{ll}\text { SAKHIBULLIN NAIL A OR } & \text { SAKURAI KUNITOMO PROF } \\ \text { OPT OF ASTRONOMY } & \text { OPT OF PHYSICS } \\ \text { KAZAN STATE UNIVERSITY } & \text { KANAGAWA UNIVERSITY } \\ \text { LENIN UL 18 } & \text { KANAGAWAKU } \\ \text { 420008 KAZAN } & \text { YOKOHAMA 22L } \\ \text { RUSSIA } & \text { JAPAN } \\ \text { IEL: } 32-36-41 & \text { TEL: } 454815661 \\ \text { TLF: } & \text { TLF: } \\ \text { TLX: } & \text { TLX: } \\ \text { EML: } & \text { EML: } \\ \text { COM: } 36 & \text { COM: } 10,51\end{array}$

SALA FERRAN OR SALAZAR ANTONIO DR OPT FISICA

UNIVERSIDAD DE BARCELONA

AVD DIAGONAL 647

E OBO2B BARCELONA

SPAIN

TEL: 33307311

TLF:

TLX:

EML:

COM: 28,33

SALISBURY J W DR

US GEOLOGICAL SURYEY

927 NATIONAL CENTER

RESTON VA 22092

USA

TEL: 7038606668

TLF:

ILX: 92178

EML:

COM:

SALVADOR-SOLE EOUAROO

DPT FISICA OE ATMOSFERA

UNIVERSIDAD DE BARCELONA

AVD DLAGONAL 645

E 08028 BaRCELONA

SPAIN

TEL: 33307311

TLF :

$T L X:$

EML:

COM: 28,47

SAMEC RONALD G DR

JI HOLCOMB OBSERVATORY

BUTLER UNIVERSITY

4600 SUNSET AVE

INOLANAPOLIS IN 46208

USA

TEL: 3172839282

TLF: 3172839519

TLX:

EML: SAMECEBUTLERU.BITNET

COM: 42
REAL INST $\checkmark$ OBSERVATORIO

OE LA ARMADA

CEEILIO PUJAZON S/N

E 11110 San fERnando

SPAIN

TEL: $56 \quad 88 \quad 3548$

TLF: 56899302

TLX: 76108 IOM E

EML:

COM: 04

SALO HEIKKI

JPL

MS 183501

4800 OAK GROVE DR

PASADENA CA 91109

USA

TEL: 8183543833

TLF: 35881561278

TLX:

EML: SALOOFINFUN. BITNET COM:

salvati marco

OSS ASTROF ISICO

DI ARCETRI

LARGO E FERMI 5

I 50125 FIRENZE

ITALY

TEL: 552752268

TLF:

TLX: 572230 ARCETR I

EML:

COM: 48

SAMPSON DOUGLAS H PROF OPT OF ASTRONOMY PENNSYLVANIA STATE UNIY 525 DAVEY LAB UNIVERSITY PARK PA 16802 USA TEL: 814865026 t

TLF:

TLK: 842510

EML:

COM: 


\begin{tabular}{|c|c|}
\hline SAMIIS NIKOLAI N OR & SAMAHUJA BLAS \\
\hline INST OF ASTRONOMY & DPT FISICA DE ATMOSFERA \\
\hline ACADEMY OF SCIENCES & UNIVERSIOAD DE BARCELONA \\
\hline PYATNITSKAYA UL 48 & AVO DIAGONAL 645 \\
\hline 109017 MOSCOW & E 08028 BARCELONA \\
\hline RUSSIA & SPAIN \\
\hline TEL: $231-54-61$ & TEL: $33307311 * 298$ \\
\hline TLF: & TLF: \\
\hline$T L X: 412623$ SCSTP SU & $T L x:$ \\
\hline EML: & EML: \\
\hline COM: $27[.30 .37$ & COM: 28,46 \\
\hline SANCHEZ FRANCISCO PROF & SANCHEZ MANUEL \\
\hline INST DE ASTROFISICA & REAL INST Y OBSERVATORIO \\
\hline DE CANARIAS & DE LA ARMADA \\
\hline OBS DEL TEIOE & CECILIO PUJAZON S/N \\
\hline E 38071 LA LAGUNA & E 11110 SAN,FERNANDO \\
\hline SPAIN & SPAIN \\
\hline TEL: $22 \quad 262211$ & TEL: $56 \quad 88 \quad 3548$ \\
\hline TLF : & TLF: \\
\hline TLX: 92640 IAC E & TLX: 76108 \\
\hline EML: & EML: \\
\hline COM: 21,50 & COM: 08.19 \\
\hline SANCISI RENZO DR & SANDAGE ALLAN \\
\hline KAPTEYN ASTRONOMICAL INST & MT WILSON \& LAS CAMPANAS \\
\hline$B 0 \times 800$ & OBSERVATORIES \\
\hline NL 9700 AV GRONINGEN & 813 SANTA BARBARA ST \\
\hline NETHERLANDS & PASADENA CA 91101 \\
\hline TEL: 50116695 & USA \\
\hline TLX: 53572 STARS NL & TEL: $818 \quad 577 \quad 1122$ \\
\hline EML: & TLF : \\
\hline TLF : & TLX: \\
\hline COM: $28,34,51$ & EML: \\
\hline & COM: \\
\hline SANDERS DAVID B DR & SANDERS ROBERT DR \\
\hline INSTITUTE FOR ASTRONOMY & KAPTEYN ASTRONOMICAL INST \\
\hline UNIVERSITY OF HAWAII & $80 \times 800$ \\
\hline 2680 WOOOLAWN DR & NL 9700 AV GRONINGEN \\
\hline HONOLULU HI 96822 & NETHERLANDS \\
\hline USA & TEL: 50 LL 6695 \\
\hline TEL: 8089487399 & TLX: 53572 STARS NL \\
\hline TLF: 8089882790 & EML: \\
\hline TLX: 7238459 UHAST HR & TLF: \\
\hline EML: SANDERSEUHIFA.IFA, HAWAII EDU & COM: 28 \\
\hline CEM: $2 \theta, 40$ & \\
\hline SANDFORD MAXWELL I II & SANOMANN WILLIAM HENRY \\
\hline LOS ALAMOS SCIENTIFIC LAB & OPT OF PHYSICS \\
\hline LOS ALAMOS NM 87545 & HARVEY MUDO COLLEGE \\
\hline USA & CLAREMONT CA 91711 \\
\hline TEL: $505 \quad 667 \quad 6384$ & USA \\
\hline TLF : & TEL: $714 \quad 621 \quad 8024$ \\
\hline EML: & TLF: \\
\hline COM: & ILX: \\
\hline$T L X:$ & EML: \\
\hline
\end{tabular}

SANAMIAN $V$ A DR
BYURAKAN ASTROPHYSICAL
OBSERVATORY
378433 ARMENIA
ARMENIA

TEL: $88 \quad 52 \quad 28 \quad 3453$

TLF:

TLX:

EML:

COM: 40

SANCHE Z-LAVEGA AGUSTIN OR OPT FISICA APLICADA

ETS INGENIEROS INO Y TEL AVO URQUIJO S/N

E 18013 BILBAO

SPAIN

TEL: $44416400 * 353$

TLF: 44414041

TLX:

EML:

COM: 16

SANDAKOVA E $V$ DR

KIEV STATE UNIVERSITY

ASTRONOMICAL OBSERVATQRY 252053 KIEV

UKRAINE

TEL:

TLF :

TLX:

EML:

COH:

SANDERS W L PROF

DPT OF ASTRONOMY NEW MEXICO STATE UNIV BOX 4500

LAS CRUCES NM 89003 USA

TEL: $505 \quad 646 \quad 4914$

TLF :

TLX:

ENL:

COM: 24,37

SANDQVIST AAGE OR STOCKHOLM OBSERVATORY S 13336 SALTSJOEBADEN SWEDEN

TEL: $87 \quad 17 \quad 0380$

TLF: $87 \quad 174719$

TLX: 12972 SOBSERY $S$

EML: SANDOVISOASTRO.SU.SE

COM: $33,34,46 C$
SANCHEZ ALMEIDA JORGE DR INST DE ASTROFÍSICA DE CANARIAS OBS DEL TEIOE E 38200 LA LAGUNA SPAIN TEL: 22262211 TLF: $22 \quad 263005$ TLX: 92640 IAC E EML: IAC: : JOS/JOSEIAS.ES COM: $10,12,29$

SANCHEZ-SAAVEDRA M LUISA FAC DE CIENCIAS UNIVERSIOAO DE GRANADA E 18080 GRANADA SPAIN TEL: $58 \quad 20 \quad 2212$ TLF:

TLX:

EML:

COM: $21,33,34$

SANOELL GORAN HANS \& DR 655 KOMOHAWA ST

HILO HI 96720

USA

TEL:

TLF:

TLX:

EML:

COM: 34,40

SANDERS WILTON TURNER III DPT DF PHYSICS UNIVERSITY OF WISCONSIN 1150 UNIVERSITY AVE MADISON WI 53706 USA TEL: $608 \quad 2625916$ TLF:

TLX:

EML: COM: 44,48

SANFORD PETER WILLIAM MR DPT PHYSICS \& ASTRONOMY UNIVERSITY COLLEGE LONDOH GOWER ST LONOON WCIE 6BT UK TEL: TLF :

TLX:

EML: COM: 


\section{SAMROMA MANUEL DR DPT FISICA \\ UNIVERSIOAD DE BARCELONA \\ AVD DIAGONAL 647 \\ E 08028 BARCELONA \\ SPAIN \\ TEL: 3 ' 3307311 \\ TLF: \\ $\operatorname{TLX}$ \\ EML:}

COM: 28

SANTOS FILIPE D OR

CENTRO DE FISICA NUCLEAR

UNIVERSIDADE DE LISBOA

AV PROF GAMMA PINTO N²

D 1699 LISBOA CODEX

PORTUGAL

TEL: 35117950790

TLF: 3511765622

TLX: $62593 \mathrm{P}$

EML: SANTOSAPTIFM

COM: 35

SANYAL ASHIT OR

7505 RIOGEWELL CT

BELTSVILLE MD 20705

USA

TEL :

TLF:

EML:

COM: 27.42

TLX:

SAPRE A K OR

DPT OF PHYSICS

UNIVERISTY OF RAVISHANKAR

RAIPUR 429010

INOIA

TEL: 27064

TLF:

TLX:

EML :

COM: 28

SARGENT ANNEILA I

CALTECH

MS 32047

DOWNES LAB OF PHYSICS

PASADENA CA 91125

USA

TEL: B18 3566622

TLF :

$T L X: 675425$

EML:

COM: $33,34,40$

SANSATUR IO MARIA E OR
ETS
INGENIEROS INOUSTRIALES
PASEO DEL CAUCE S/N
E 47011 VALLAOOLID
SPAIN
TEL: 83304899
TLF: 83392026
TLX:
EML:
COM: 07

SANTOS NILTON OSCAR DR OBSERVATORIO NACIONAL RUA GL BRUCE $5 B 6$ SAO CRISTOVAO 20921 RIO DE JANEIRO RJ BRAZIL

TEL: $21580 \quad 0235$

TLF : $21 \quad 580 \quad 0332$

TLX:

EML:

COM: 48

SANZ I SUBIRANA JAUME DR OPT MATEMATICA APLICAOA

UNIV POLITEC OE CATALUNYA BOX 30002

E DBOBO BARCELONA SPAIN

TEL: 34016799

TLF: 3401680 ]

TLX: 281 UPC E

EML: MATJSS MAT.UPC.ES

COM: 33

SARASSO MARIA DR

OSS ASTRONOMICO DI TORIND

ST OSSERVATORIO 20

I 10025 PINO TORINESE

ITALY

TEL: 11841067

TLX: 213236 TOASTR I

EML: 39181: :SARASSO

TLF: $1184 \quad 1281$

COM: 05,08

SARGENT WALLACE L W DR

CALTECH

MS 10524

PASADENA CA 91125

USA

TEL: $818 \quad 356 \quad 4055$

TLF :

TLX: 675425 CALTECH PSO

EML:

COM: 28,47
SANTAMARIA RAFFAELE DR

IST UNIVERSITARIO NAVALE

VIA ACTON 38

I 80133 NAPOLI

ITALY

TEL: $B 15475135$

TLF: $81552 \quad 1485$

TLX: 710417

EML:

COM:

SANWAL BASANT BALLABH OR UTTAR PRADESH STATE OBSERVATORY

PO MANORA PEAK 263129

NAINITAL 263129

IMOIA

TEL: $59 \quad 42 \quad 2136 / 2583$

TLF:

TLX:

EML:

COM: 29

SANZ JOSE L DR

OPT DE FISICA MODERNA

UNIVERSIDAD DE CANTABRIA AVO LOS CASTROS S/N

E 39005 SANTANDER

SPAIN

TEL: $42 \quad 20 \quad 1452$

TLF :

$T L X: 35681$ EOUCI E

EML:

COM: 47

SARAZIN CRAIG L DR

UNIVERSITY STATION.

UNIVERSITY OF VIRGINIA

BOX 3818

CHARLOTTESVILLE VA 22903

USA

TEL: 8049244903

TLF: 8049243104

TLX:

EML: CLSTI@VIRGINIA.EDU

COM: 28,34

SARMA M B K PROF

OPT OF ASTRONOMY

UNIVERSITY OF OSMANIA

HYOERABAD 500007

INDIA

TEL: $6522 B$

TLF:

TLX:

EML :

COM: 25,27 .
SANTIN PAOLO DR

OAT

BDX SUCC TRIESTE 5

VIA TIEPOLO II

I 34131 TRIESTE

ITALY

TEL: 40793921

ILF:

TLX: 461137 OAT I

EML:

COM:

SANWAL N B DR

DPT OF ASTRONOMY

UNIVERSITY OF OSMANIA

HYOERABAD 500007

INDIA

TEL: $71 \quad 951 * 247$

TLF :

TLX:

EML:

COM: $30,42,45$

SAPAR ARVEQ DR

TARTU ASTROPHYSICAL OBS

ESTONIAN ACAD OF SCIENCES

202444 TARTU

ESTONIA

TEL:

TLF

TLX:

EML:

COM: $36 C, 47$

SAREYAN JEAN-PIERRE OR OCA OBSERV DE NICE

BP 139

F 06003 NICE CDX

FRANCE

TEL: $93 \quad 890420$

TLF:

TLX: 460004 OBSNICE F

EML:

COM: 27.29

SARMA N $\vee G$ PROF

RAMAN RESEARCH INSTITUTE

SADASHIVANAGAR

SANGALORE 560 OBO

INOIA

TEL: 812360122

TLF : 812340492

TLX: 8452671 RRI IN

EML:

COM: 34,40 
SARMIENTO-GALAN A F DR INST DE ASTRONOMIA UNAM UNAM

APDO POSTAL 70-264

O451O MEXICO DF

MEXICO

TEL: 5485305

TLF :

TLX: 1760155 CICME

EML: ANSAR@UNAMVMI bit thet

COM:

SASAKI MISAO

RES INST FOR THEORET PHYS

HIROSHIMA UNIVERSITY

RIRONKEN

TAKEHARA 725

JAPAN

TEL: $08462-2-2362$

TLF :

TLX:

EML:

COM: 47

SASLAW WILLIAM C PROF

UNIVERSITY STATION

UNIVERSITY OF VIRGINIA

BOX 3818

CHARLOTTESVILLE VA 22903

USA

TEL: 8049244892

TLF:

$T\lfloor X$ :

EML:

COM: 28,48

SATO FUMIO OR

DPT ASTRON \& EARTH SCI

TOKYO GAKUGEI UNIVERSITY

KOGANEI

TOKYO 184

JAPAN

TEL: $0423-25-2111$

TLF :

TLX:

EML:

COM: 34,40

SATO MASSAE OR

IAG

UNIVERSIDADE DE SAO PAULO

AV MIGUEL STEFANO 4200

04301 SAO PAULO SP

BRAZIL

TEL: 115778599

TLF: $11 \quad 276 \quad 3848$

TLX: 36224 IAGM BR

EML: 47556: : MASSAE

COM: 20
SARRIS ELEFTHERIOS PH O ASTRONOMICAL INSTITUTE NTL OBSERVATORY OF ATHENS

BOX 20048

GR 11810 ATHENS

GREECE

TEL: $1346 \quad 1191$

TLF :

TLX: 215530 OBSA GR

EML:

COM:

SASAKI TOSHIYUKI OR

OKAYAMA ASTROPHYSICAL OBS

HAOJ

KAMOGATA ASAKUCHI

OKAYAMA 71902

JAPAN

TEL: 865442155

TLF : 865442360

TLX:

EML:

COM: 28

SASTRI HANUMATH I DR

INOIAN INSTITUTE OF

ASTROPHYSICS

KORAMANGALA

BANGALORE 560034

INOIA

TEL: $\theta 1256 \quad 6585$

TLF :

TLX: 845763 IIAB IN

EML:

COH: 49

SATO HUMITAKA PROF

DPT OF PHYSICS

UNIVERSITY OF KYOTO

SAKYO KU

KYOTO 606

JAPAN

TEL:

TLF:

TLX:

EML:

COM: 47

SATO NAONOBU PROF

AKITIA UNIVERSITY

1-1 TEgATA GAKUENCHO

AKITA, 010

JAPAN

TEL: 01BB-33-526]

TLX:

EML:

TLF :

COM: 27,41
SARRIS EMMANUEL T PH D

OPT OF ELECT ENGINEERING

DEMOCRITOS UNIV OF THRACE

GR 671 OO XANTHI

GREECE

TEL: $54 \quad 12 \quad 6948$

TLF:

TLX: 452312 POL.X GR

EML:

COM: 49

SASALOV DIMITAR D OR

CENTER FOR ASTROPHYSICS

HCO/SAO

60 GARDEN ST

CAMBRIDGE MA D213日

USA

TEL: $617495745 \mathrm{I}^{\circ}$

ILF: 6174957049

TLX:

EML: SASSELOVECFA. HARVARD.EOU

COM:

SASTRY CH $V$

INDIAN INSTITUTE OF

ASTROPHYSICS

KORAMANGALA

BANGALORE 560034

INOIA

TEL: $91256 \quad 6585$

TLF :

TLX:

EML:

COM: 40

SATO KATSUHIKO PROF

OPT OF PHYSICS

UNIVERSITY OF TOKYO

BUNKYO KU

TOKYO 113

JAPAN

TEL: $38122111 * 4207$

TLF: 356890465

TLX: 23472 UTPHYSIC

EML: satortkyvax. hepnetolbl.bitnet

COM: $35,44,47 \mathrm{C}$

SATO SHUJI OR

TOKYO ASTRONOMICAL OBS

NAOJ

OSAWA MITAKA

IOKYO 181

JAPAN

TEL: 0422413643

ILF: $0422 \quad 413776$

TLX:

EML:

COM: 34
SARTORI LEO PROF.

OPT OF PHYSICS \& ASTRON UNIVERSITY OF NEBRASKA

BEHLEN OBSERVATORY

LINCOLN NE 68588

USA

TEL:

TLF:

TLX:

EML:

COM: 48

SASAO TETSUD DR

INIL LATITUDE OBSERVATORY

NAOJ

HDSHIGAOKA MIZUSAWA SHI

IWATE 023

JAPAN

TEL: $197-24-7111$

TLF :

TLX: B37628 ILSMIZJ

EML:

COM: 19[

SASTRY SHANKARA $K$

DPT OF ASTRONOMY

UNIVERSITY OF OSMANIA

HYDERABAD 500007

INOIA

TEL: 71951

TLF :

TLX:

EML:

COM: $2 B$

SATO KOICHI DR

INTL LATITUDE OBSERVATORY

NAOJ

HOSHIGAOKA MIZUSAWA SHI

IWATE 023

JAPAN

TEL: 0197-24-7111

TLF :

TLX: 837628 [LSMIZJ]

EML:

COM: 08, 19

SATO YUZO DR

4-8-19 OSAWA

MITAKA

TOKYO 181

JAPAN

TEL:

ILF:

TLX:

EML:

COM: 


\begin{tabular}{|c|c|c|c|}
\hline SAULT ROBERT OR & SAUNOERS RICHARD D E & SAUVAL A JACQUES OR & SAVAGE ANN DR \\
\hline AAO & MULLARD RADIO ASTRON OBS & OBSERVATOIRE ROYAL DE & UK SCHMIOT TELESCOPE \\
\hline ATNF & CAVENDISH LABORATORY & BELGIOUE & AAO \\
\hline $\mathrm{BOX} 76$ & MADINGLEY RD & AVE CIRCULAIRE 3 & PRIVATE BAG \\
\hline EPPING NSW 2121 & CAMBRIDGE CB3 OHE & B 1180 BRUSSELS & COONABARABRAN NSW 2357 . \\
\hline AUSTRALIA & UK & BELGIUM & AUSTRALIA \\
\hline TEL: $2 \quad 868 \quad 0222$ & TEL: 22366477 & TEL: 23752484 & TEL: \\
\hline TLF : $2868 \quad 0310$ & TLF: & TLF : 23749822 & TLF : \\
\hline TLX: 26230 & TLX: 81292 & TLX: 21565 OBSBEL B & TLX: \\
\hline EML: RSAULTEATNF.CSIRO.AUU & EML: & EML: & EML: \\
\hline COM: 09 & COM: 40 & COM: 12 & COM: $28,40,47$ \\
\hline SAVAGE BLAIR O OR & SAVEDOFF MALCOLM P PROF & SAVONIJE GERRIT JAN DR & SAWANT HANUMANT S OR \\
\hline DPT OF ASTRONOMY & OPT.PHYSICS \& ASTRONOMY & ASTRONOMICAL INSTITUTE & INPE \\
\hline UNIVERSITY OF WISCONSIN & UNIVERSITY OF ROCHESTER & UNIVERSITY OF AMSTEROAM & CP 515 \\
\hline $475 N$ CHARTER ST & BAUSCH ANO LOMB BLDG & KRUISLAAN 403 & 12200 S JOSE DOS CAMPOS \\
\hline MADISON WI 53706 & ROCHESTER NY 14627 & NL 1098 SJ AMSTERDAM & BRAZIL \\
\hline USA & USA & NETHERLANDS & TEL: $123 \quad 229977$ \\
\hline TEL: $608 \quad 2623072$ & TEL: $716 \quad 275 \quad 4357$ & TEL: 205257491 & TLF: $123 \quad 21 \quad 8743$ \\
\hline TLF: & TLF: & TLF: 205257484 & ILX: $1233530^{\circ}$ \\
\hline TLX: 265452 UDFWISC-MOS & TLX: 978374 UNIBOOK ROC & TLX: 10262 HEF NL & EML: , \\
\hline EML: & EML: & EML: & COM: 40 \\
\hline COM: $34,44 C$ & COM: $34,35,48$ & COM: 35,42 & \\
\hline SAWYER CONSTANCE B DR & SAWYER-HOGG HELEN B DR & SAXENA A K OR & SAXENA P P DR \\
\hline $85020 T H 5 T * 705$ & DAVIO DUNLAP OBSERVATORY & INOIAN INSTITUTE OF & OPT OF MATHS \& ASTRONOMY \\
\hline 325 BRDADWAY & UNIVERSITY OF TORONTO & ASTROPHYSICS & UNIVERSITY OF LUCKNOW \\
\hline BOULDER CD 80302 & $B 0 \times 360$ & KORAMANGALA & LUCKNOW \\
\hline USA & RICHMOND HILL ON L4C $4 Y 6$ & BANGALORE 560034 & INDIA \\
\hline TEL: & CANADA & INDIA & TEL: \\
\hline ILF: & TEL: $416 \quad 8849562$ & TEL: $812 \quad 56 \quad 6585 / 6497$ & TLF : \\
\hline TLX: & TLF : $416978 \quad 3921$ & TLF: & TLX: \\
\hline EML: & $T L X: 06986766$ & TLX: 845763 IIAB IN & EML: \\
\hline COM: 10,49 & EML: & EML: & $\operatorname{COM}: 21,46$ \\
\hline & COM: 27.37 & COM: 09 & \\
\hline SAZHIN MICHAIL DR . & SBIRKOVA-NATCHEVA T & SCALISE JR EUGENIO OR & SCALO JOHN MICHAEL \\
\hline STERNBERG STATE ASTR INST & PLANETARIUM AND PUBLIC & INPE & ASTRONOMY OPT \\
\hline UNIVERSITETSKIJ PROSP 13 & ASTRONOMICAL OBSERVATORY & CP 515 & UNIVERSIIY OF TEXAS \\
\hline 119899 MOSCOW & Box 136 & $12200 \mathrm{~S}$ JOSE DOS CAMPOS & RLM 15308 \\
\hline RUSSIA & BG 4700 SMOLJAN & BRAZIL & AUSTIN TX 787121083 \\
\hline TEL: 959395006 & BULGARIA &. TEL: 123229977 & USA \\
\hline TLX: & TEL: 3022953 & TLF: $123 \quad 218743$ & TEL: 5124714461 \\
\hline EML: SNNOSAI.MSK.SU & TLF : & TLX: 34061 INPE BR & TLF: \\
\hline TLF : & TLX: & EML: & TLX: \\
\hline COM: 47 & EML: & COM: 40 & EML: \\
\hline & COM: 41.46 & & COM: 34,35 \\
\hline 'SCALTRITI FRANCO OR & SCARDIA MARCO & SCARFE COLIN O DR & SCARGLE JEFFREY D DR \\
\hline OSS ASTRONOMICO DI TORINO & OSS ASTRONOMICO DI MILANO & DPT OF PHYSICS & NASA AMES RESEARCH CTR \\
\hline ST OSSERVATORIO 20 & VIA E BIANCHI 46 & UNIVERSITY OF VICTORIA & MS 2453 \\
\hline I 10025 PINO TORINESE & I 22055 MERATE & BOX 3055 & MOFFETT FIELD CA 94035 \\
\hline ITALY & ITALY & VICTORIA BC VBW $3 P 6$ & USA \\
\hline TEL: II 841067 & TEL: 592035 & CANADA & TEL: 4156946330 \\
\hline TLX: 213236 TOASTR I & TLX: & TEL: $604 \quad 721 \quad 7740$ & TLF : \\
\hline EML: & EML: & TLF: 6047217715 & $T \perp X:$ \\
\hline TLF: $1184 \quad 1281$ & TLF: & $T L x:$ & EML: \\
\hline COM: 15,42 & COM: 26 & EML: SCARFEUYPHYS.UVIC.CA & COM: 48,51 \\
\hline & & COM: $26 C, 304 P, 42$ & \\
\hline
\end{tabular}




SCARIA K $K$ OR
INDIAN INSTITUTE OF
ASTRDPHYSICS
KORAMANGALA
BANGALORE 560034
INDIA
TEL: 812566585
TLF:
TLX: 845763 IIAB IN
EML:
COM: 37
SCHAEFER BRADLEY E OR
NASA/GSFC
CODE 661
GREENBELT MD 20771
USA
TEL: 301286 G955
TLF:
TLX:
EML:
COM: 27.41

SCHALEN CARL PROF
LUNO OBSERVATORY
BOX 43
S 22100 LUND
SWEDEN
TEL: 46107000
TLX: 33199 OBSNOT $S$
EML:
TLF:
COM: 34

SCHATZMAN EVRY PROF OBSERVATOIRE DE PARIS SECTION DE MEUDON

DASGAL

F 92195 MEUDON PPL CBX FRANCE

IEL: 145077873

TLF:

TLX: 201571

EML:

COM: $34,35,47,48,49,51$

SCHEIDECKER JEAN-PAUL DR OCA OBSERV DE NICE

BP 139

F 06003 NICE COX

FRANCE

TEL: 93890420

TLF:

TLX: 460004 OBSNICE $F$

EML:

COM:

SCARROTT STANLEY M OR
OPI OF PHYSICS
UNIVERSITY OF OURHAM
SOUTH. RO
DURHAM OHI $3 L E$
UK
TEL:
TLF:
TLX:
EML:
COM: 34

SCHAEFER GERHARO DR MAX PLANEK INSTITUT FUER. ASIROPHYSIK

KARL-SCHWARZSCHILOSTR 1

D 8046 GARCHING MUENCHEN GERMANY

TEL: 8932993247

TLF : 8932993235

TLX: 524629 ASTRO D

EML: GOS@DGIPPIS.BITNET

COM: 48

SCHANOA ERWIN PROF

PHYSIKALISCHES INSTITUT

INIVERSITAET BERN

SIOLERSTRASSE 5

CH' 3012 BERN

SWITZERLANO

TEL: 31658910

TLF:

TLX: 32320

EML:

COM:

SCHECHTER PAUL L DR
OPT OF PHYSICS
MIT RM 6206
BOX 165
CAMBRIOGE MA 02139
USA
TEL: 6172530690
TLF:
TLX:
EML: SCHECHEACHERNAR.MIT.E
COM: $28,33,47$
SCHERB FRANK PROF
OPT OF PHYSICS
UNIVERSITY OF WISCONSIN
II5O UNIVERSIIY AVE
MAOISON WI 53706
USA
TEL: 608 2626879
TLF:
TLX:
EML:
COM: 34,49

SCHAAL RICARDO E OR

CRAAE/ESCOLA POLITECNICA

UNIVERSIOADE DE SAO PAULO

CP 8174

.05508 SAO PAULO SP

BRAZIL

TEL: 118156289

TLF: $11 \quad 815 \quad 4272$

TLX: 1180127 INPE BR

EML: ECORRÉTAQBRUSP.BITNET

COM: 40

SCHAEFFER RICHARO QR

CEA CEN

PHYSIQUE THEORIQUE

BP 2

F 91191 GIF/YVETTE COX

FRANCE

TEL: 169087376

ILF :

TLX: 690641

EML:

COM:

SCHARMER GOERAN BJARNE

STOCKHOLM OBSERVATORY

S 13336 SALTSUOEBAOEN

SWEDEN

TEL: $87 \quad 170195$

TLF: $87 \quad 174719$

TLX: 12972

EML: SCHARMEREASTRO.SU.SE

COM: 36

SCHEEPMAKER ANTON DR

COSMIC RAY WORKING GROUP

HUYGENS LAB

WASSENAARSEWEG 78

NL 2300 RA LEIDEN

NETHERLANDS

TEL:

TLF :

TLX:

EML:

COM:

SCHERRER PHILIIP H OR

CTR FOR SPACE SCIENCES \&

ASTROPHYSICS

STANFORD UNIV ERL

STANFORD CA $94305 \mathbf{4 0 5 5}$

USA

TEL: $415497 \quad 1505$

TLF:

TLX: 348402 STANFRD STNU

EML:

COM:
SCHADEE AERT OR

STERREKUNDIG INSTITUTE

$80 \times 80000$

NL 3508 TA UTRECHT

NETHERLANDS

TEL: 30535200

TLF :

TLX: 40048 FYLUT NL

EML: BITNET/WNMAAERSQHUTRUUO

COM: 14

SCHAIFERS KARL DR

STEINBACHWEG 37

D 6900 HEIOELBERG 1

GERMANY

TEL: $62 \quad 218 \quad 01511$

TLF:

TeX:

EML:

COM:

SCHATTEN KENNETH H OR

OPT OF PHYSICS

VICTORIA UNIVERSITY

PRIVATE BAG

WELLINGTON

NEW ZEALANO

TEL:

TLF:

TLX:

EML:

COM: $10,35,48$

SCHEFFLER HELMUT PROF

CARL-ORFF-WEG 16

D 6906 LEIMEN 3

gERMANY

TEL:

TLF:

TLX:

EML:

COM:

SCHEUER PETER A G DR

MULLARO RAOTO ASTRON OBS

CAVENOISH. LABORATORY

MADINGLEY RD

CAMBRIOGE CB3 OHE

UK

IEL: $223 \quad 66477 * 344$

ILF :

TLX: 81292

EML:

COM: $34,40,47,48 C$ 


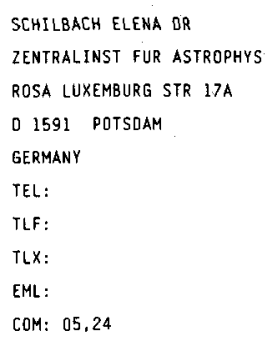

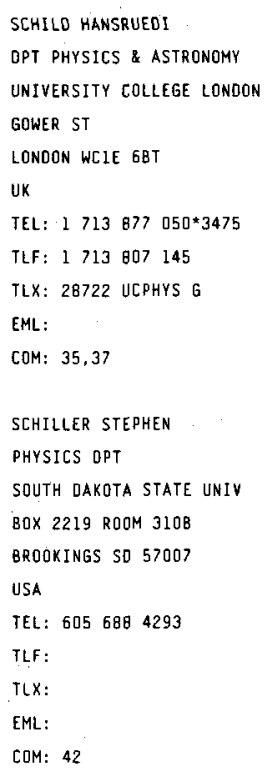

SCHLESINGER BARRY M DR ST SYSTEMS CORP [STX) 4400 FORBES BLVO LANHAMM MO 20706

USA

TEL:

TLX:

EML:

TLF:

COM:

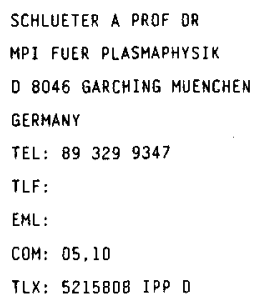

COM:

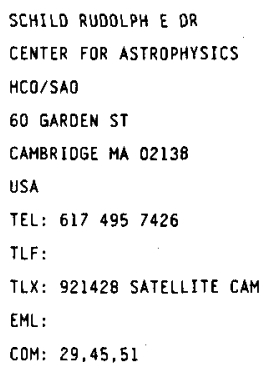

SCHLICKEISER REINHARD DR MPI FUER RADIOASTRONOMIE AUF DEM HUEGEL 69 D 5300 BONN 1

GERMANY

TEL: 2285251

ILF :

TLX: 886440

EML:

COM: 40

SCHLUETER OIETER PROF INST THEOR PHYS \& STERNW NEUE UNIV PHYSIK ZENTRUM OLSHAUSENST GEB N $61 \mathrm{C}$ D 2300 KIEL 1 GERMANY

\section{TEL: 4318804109}

TLF:

TLX:

EML:

COM:

SCHMALBERGER DONALD C OR THE ALBANY ACADEMY

\section{ACADEMY RD}

ALBANY NY 12208
USA
TEL: $518465 \quad 1461$
TLF:
TLX:
EML:
COM: 36

SCHILIZZI RICHLRD I OR

NFRA

BOX 2

NL 7990 AA DWINGELDO NETHERLAHOS

TEL: $52 \quad 197244$

TLF :

TLX: 42043 SRZM NL

EML:

COM: $40,48,50$

SCHLEGEL ERIC MATTHEW OR NASA/GSFC

COOE 66日

LAB HIGH ENERGY ASTTROPHYS GREENBELT MO 20771

USA

TEL: $301286 \quad 6636$

TLF: 3012863391

TLX:

EML: ERICEHEASFS.GSFC.NASA. GOY

COM: 27

SCHLOERB F PETER DPT PHYSICS * ASTRONOMY UNIV OF MASSACHUSETTS GRC

AMHERST MA 01003 USA TEL: 4135454303 TLF :

TLX: 955491

EML:

COM: 15,16

SCHMADEL LUTZ D DR ASTRONOMISCHES RECHEN INSTITUT MOENCHHOFSTR 12-14 O 6900 HE TOELBERG I GERMANY TEL: $62 \quad 2149026$ TLF: TLX: 461336 ARIHD D EML: COM: $05 C, 20$

SCHMEIOLER F PROF DR MAUERKIRCHERSTR. 17

D BO0O MUENCHEN 80 GERMANY

TEL:

TLF:

TLX:

EML:

COM: 08 


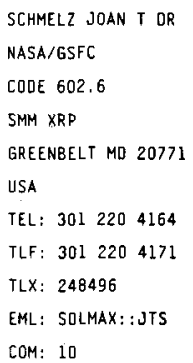

SCHMIO HANS MARTIN DR INSTITUT FUER ASTRONOMIE ETH ZENTRUM

CH 8092 ZUERICH

\section{SWITZERLAND}

TEL: 12563633

TLF: 4112620003

TLX: $817379 \mathrm{EHHG} \mathrm{CH}$

EML: SCHMTOEIFA.ETHZ.CH

COM: 42

SCHMIDT $K H$ OR
ZNTRLINST F ASTROPHYSIK
STERNWARTE BABELSBERG
ROSA-LUXEMBURG-STR 17A
O 1502 POTSDAM
GERMANY
TEL:
TLF:
TLX:
EML:
COM: $05,28,33,44$

SCHMIOTKE PAUL C OR

DPT OF PHYSICS

ARIZONA STATE UNIVERSITY

TEMPE AZ 85287

USA

TEL: 6029652918

TLF:

TLX:

EML: BITNET: SCHMIDTKEASUCPS

COM: 26,42

SCHMITT OIETER DR
UNIV STERNWARTE.
GEISMARLANOSTRASSE I1
O 3400 GOETIINGEN
GERMANY
TEL: 551395046
TLF:
TLX: 96753
EML: BIINET: dSChmi todgogwdg
COM: 12

SCHNEIDER HARTMUT DR

UNIVERSITY STERNWARTE

GEISMARLANOSTR II

D 3400 GOETIINGEN

GERMANY

TEL: 551395042

TLX: 96753

EML: BITNET: HSCHNEI@GOGWDGI

TLF: 551395043

COM: 27
SCHMIDT EOWARD G

OPT OF PHYSICS \& ASTRON UNIVERSITY OF NEBRASKA

LINCOLN NE 685880111

USA .

TEL: 4024722788

TLF:

TLX:

EML:

COM: 25,27

SCHMIDT MAARTEN PROF

CALTECH

MS 10524

ASTRONOMY OPT

PASADENA CA 91125

USA

TEL: 8183564204

TLF :

TLX: 675425

EML:

COM: $15,28,33,40,47$

SCHMIOT-KALER TH PROF ASTRONISCHES INSTITUT RUHR-UNIVERSITAET BOCHUM STEINHUEGEL 105

D. 5810 WITTEN

GERMANY

TEL: $23470 \quad 03454$

TLF:

TLX: 0825860

EML:

COM: $33,34,45$

SCHMITTER EGWARD F DR

DPT OF PHYSICS

UNIVERSITY OF LAGOS

AKOKA

LAGOS

NIGERIA

TEL: 1837864

TLF:

TLX:

EML:

COM: 46

\section{SCHNE IOER JEAN}

OBSERVATOIRE OE PARIS

SECTION DE MEUDON

F 92195, MEUDON PPL CDX FRANCE

TEL: $145 \quad 077430$

TLF:

TLX: 201517

EML: EAR: : "SCHNEIDER RMEUSI"

COM: 47.51
SCHMIOT H U DR

MPI FUER PHYSIK UND

ASTROPHYSIK

KARL-SCHWARZSCHILO-STR I

D 8046 GARCHING MUENCHEN

GERMANY

TEL: $B 932999413 / 4$

TLF:

TLX: 524629 ASTRO D

EML:

COM: $10,15,49$

SCHMIOT THOMAS OR

RUDOLF-STEINER-SCHULE

AN DER STIFTSKIRCHE 13

D 4800 BIELEFELD 1

GERMANY

TEL: $521 \quad 88 \quad 0407$

TLF:

TLX:

EML:

CDM: 34,46

SCHMIO-BURGK J OR PROF MPI FUER RADIOASTRONOMIE

AUF DEM HUEGEL 69 D 5300 BONN 1 GERMANY

TEL: 228525271

TLF:

TLX: 886440

EME :

COM: 34,36

SCHMUTZ WERNER INSTITUT FUER ASTRONOMIE ETH ZENTRUM CH BOg2 ZUERTCH SWITZERLAND

TEL:

TLF:

TLX:

EML:

COM: 36

SCHNEIOER PETER DR MAX PLANCK INSTITUT FUR PHYSIK/ASTROPHYSIK KARL-SCHARZSCHILD-STR. I D B046 GARCHING MUENCHEN GERMANY

TEL: 8932990

TLF:

TLX: 524629

EML:

COM: 47 


\author{
SCHMELL ANNELIIESE BR \\ INSTITUT FUER ASTRONOMIE \\ UNIVERSITAT WIEN \\ TUERKENSCHANZSTR 17 \\ A 1180 WIEN \\ AUSTRIA \\ TEL: 134536093 \\ TLF: \\ TLX: \\ EML: \\ COM:
}

SCHOBER HANS J OR INSTITUT FUER ASTRONOMLE UNIVERSITAETSPLATZ 5

A 9010 GRAZ

AUSTRIA

TEL: $316 \quad 380 \quad 5273$

TLX: 31078 OBSLGZ

EML:

TLF:

CoM: $10,12,15,20,42,51$

SCHOENEICH W OR

ZNTRLINST F ASTROPHYSIK

ROSA-LUXEMBURG-STR 17A

D 1502 potsoam

GERMANY

TEL:

TLX:

EML:

TLF:

COM: 25,44

SCHOLZ M PROF

INST F THEORETISCHE

ASTROPHYS DER UNIVERSITAT

IM NEUENHEIMER FELO 561

D 6900 HEIOELBERG 1

GERMANY

TEL:

TLF:

TLX:

EML: B15 OHOURZ2.BITNET

COM: 36

SCHREIER ETHAN J DR

STSCI

HOMEWOOD CAMPUS

3700 SAN MARTIN DR

BALTIMORE MD 21218

USA

TEL: 3013384740

TLF:

TLX;

EML:

COM: 48

\author{
SCHNEPS MATTHEW H \\ CENTER FOR ASTROPHYSICS \\ HCO/SAO \\ GO GARDEN ST \\ CAMBRIDGE MA 02138 \\ USA \\ TEL: 6174957472 \\ TLF : \\ TLX: 921428 SATELLITE CAM \\ EML: \\ COM:
}

SCHOEFFEL EBERHARO F OR

MERIANERSIR 42

D 8600 BAMBERG

GERMANY

TEL!

TLF :

EML:

COM: 42

TLX:

SCHOENFELOER VOLKER OR

MPI F EXIRATERRESTRISCHE

PHYSIK

D 8046 GARCHING MUENCHEN

GERMANY

TEL: 893299578

ILF :

TLX: 5215845 XTER D

EML:

COM:

SCHOOLMAN STEPHEN A DR

LOCKHEED PALO ALTO RES LB

3251 HANDVER ST

PALO ALTO CA 94304

USA

IEL:

TLX:

EML:

TLF:

COM:

\section{SCHR IJVER C J DR}

STERREKUNOIG INSTITUTE

BOX 80000

NL 3509 TA UTRECHT

NETHERLANOS

TEL: 30535224

TLX:

EML: KSCHR TJVERESOLAR

TLF: 30531601

COM: 10,36
SCHNOPPER HERBERT W OR DANISH SPACE RESEARCH INS LUNDTOFTEVEJ 7

DK 2800 LYNGBY

DENMARK

TEL: $42 \quad 882277$

TLF :

TLX: 37190 DANRU

EML:

COM: 48

SCHOEMBS ROLF OR

INSTITUT FUER ASTRONOMIE

UND ASTROPHYSIK

SCHEINERSTR 1

O 8000 MUENCHEN 80

GERMANY

TEL: 89989021

TLF :

TLX:

EML:

COM: 27

SCHOLL HANS OR

OCA OBSERY DE NICE

BP 139

F 06003 NICE CDX

FRANCE

TEL: 93890420

TLF:

TLX:

EML:

COM: $07,15,20$

SCHRAMM DAVIO N PROF

ASTRONOMY \& ASTROPHYS CTR

UNIVERSITY OF CHICAGO

5640 S ELLIS AVE

CHICAGO IL 60637.

USA

TEL: 3129628202

TLF :

TLX: 6871133 UNCGO UW

EML:

COM: $35,47,48 \mathrm{C}$

SCHRIJVER JOHANNES DR

SPACE RESEARCH LABORATORY

SRON

SORBONNELAAN 2

NL 3584 CA UTRECHT

NETHERLANDS

TEL: 30535600

TLF: 30540860

TLX: 47224 ASTRO NL

EML:

COM: 14
SCHNUR GERHARD F 0

ASTRONOMISCHES INSTITUT

RUHR UNIVERSITAET

POSTFACH 102148

D 4630 BOCHUM 1

GERMANY

TEL:

TLF :

TLX:

EML:

COM:

SCHOENBERNER DETLEF PRDF

INST THEOR PHYS \& STERNW.

OLSHAUSENSTRASSE

D 2300 KIEL 1

GERMANY

TEL: 4318804100

TLF :

TLX: 292706

EML:

COM: 35,36

SCHOLZ GERHARD DR

ZNTRLINST F ASTROPHYSIK

AKAD WISSENSCHAFTEN DDR

ROSA-LUXEMBURG-STR $17 A$

- 1502 potsDam

GERMANY

TEL:

TLF:

TLX:

EML:

COM: 29

SCHREIBER ROMAN

INSTITUTE OF ASTRONOMY

N COPERNICUS UNIVERSITY

UL CHOPINA $12 / 18$

PL 97 10D TORUN

POLAND

TEL: $48 \quad 5626017$

TLF:

TLX: 0552234 ASTR PL

EML: SCHREIBEPLLTUMKII

COM: 49

SCHROEDER OANIEL J PROF OPT PHYSICS \& ASTRONOMY BELOIT COLLEGE

BELOIT WI 53511

USA

TEL: $608 \quad 365 \quad 3391$

TLF :

TLX:

EML:

COM: 09,46 


SCHROEOER KLAUS PETER OR
HAMBURGER STERNWARTE
GOJENSBERGSWEG 112
D 2050 HAMBURG 80
GERMANY
TEL: 4072524141
TLX: 217884
EML:
TLF: 4072524198
COM: 29

SCHRUEFER EBERHARD OR
INSTITUT FUER ASTROPHYSIK
UNIVERSITAET BONN
AUF DEM HUEGEL 71
D 5300 BONN 1
GERMANY
TEL: 228733390
TLF:
SLX: 886440
EML:
COM:

SCHUECKING E $L$ OR
DPT OF PHYSICS
NEW YORK UNIVERSITY
NEW YORK NY 10003
USA
TEL:
TLF:
TLX:
EML:
COM: 28.47

SCHULTZ ALFRED BERNARD DR STSCI/CSC

HOMEWOOD CAMPUS

3700 SAN MARTIN OR BALTIMORE MD 21218

USA

TEL: 3013385044

TLF:

TLX: 6849101 STSCI

EML: SCIVAX: :SCHULTZ

COM: 09

SCHUMACHER GERARD OR

OCA OBSERV DU CALERN

CAUSSOLS

F 06460 S VALLIER THIEY

FRANCE

TEL: 93426270

TLF:

TLX: 460004

EML:

COM:

SCHROEOER ROLF OR
MOEOERKENWEG 37
D 2050 HAMBURG 80
GERMANY
TEL:
TLF:
EML:
COM:
TLX:
SCHRUTKA-RECHTENSTAMH PR
WILLERGASSE $27 / 4 / 7$
A 123B WIEN
AUSTRIA
TEL: 18848132
TLF:
TLX:
EML:
COM: 20

\section{SCHUESSLER MANFRED DR} KIEPENHEUER - INSTITUT

FUER SONNENPHYSIK

SCHOENECKSTR 6

D 7800 FREIBURG BREISGAU

GERMANY

TEL: 76132864

TLF :

TLX: 7721552 RIS D

EML:

COM: $10 \mathrm{C} .12$

SCHULTZ G $\vee$ QR
MPI FUER RADIOASTRONOMIE
AUF DEM HUEGEL 69
O 5300 BONN 1
GERMANY
TEL: 228525291
TLX: 886440
EML:
TLF:
COM: $09,28,34,40,44,47$

SCHUMANN JOERG OIETER OR OBSERVATORIUM HOHER LIST UNIV STERNWARTE BONN

\section{5568 DAUN}

GERMANY

TEL: $6592 \quad 2937$

TLF :

TLX:

EML:

COM: Og
SCHROETER EGON H PROF KIEPENHEUER INSTITUT

FUER SONNENPHYSIK

SCHDENECKSTRASSE 6

D 7800 FREIBURG BREISGAU GERMANY

TEL: 76132864

TLF :

ILX: 7721552 KIS O

EML:

COM: 10

SCHUBART JOACHIM DR ASTRONOMISCHES , RECHEN-

INSTITUT

MOENCHHOFSTR 12-14

0 6900 HEIDELBERG 1

GERMANY

TEL: $62 \quad 2149026$

TLF:

TLX: 461336 ARIHO D

EML:

COM: $07,15,20$

SCHULER WALTER DR

SONNENRAIN 15

CH 4533 RIEOHOLZ

SWITZERLAND

TEL: $65 \quad 23 \quad 2055$

TLF:

TLX:

EML:

대: 31

SCHULZ HARTMUT OR

ASTRONOMISCHES INSTITUT UNIVERSITAT BOCHUM

POSTFACH 102148

D 4630 BOCHUM 1

GERMANY

TEL: $23470 \quad 03454$

TLF :

TLX: 0825860

EML:

COM: 28

SCHUSTER WILLIAM JOHN OR INSTITUTO DE ASTRONOMIA

UNAM

APDO POSTAL 877

22860 ENSÉNADA B CALIF

MEXICO

TEL: $706 \quad 6783093$

TLF:

TLX: 56539 CICE ME

EML:

COM: 20.25
SCHROLL ALFRED DR SONNENOBSERVATOR IUM KANZELHOEHE

A 9521 TREFFEN

AUSTRIA

TEL: 42482717

TLF :

TLX: 45699 SOLOBS A

EML:

COM:

SCHUCH NELSON JORGE OBSERVATORIO NACIONAL UFSM/CTRO TECNOLOGIA CIDAOE UNIVERSITARIA 97100 SANTA MARIA RS BRAZIL

TEL: 552261616 TLF :

TLX: 0552230 UFSM

EML:

LOM: $40,47,51$

SCHULTE O H OR

ITEK CORPORATION

10 MAGUIRE RD LEXINGTON MA 02173

USA

TEL:

TLF :

TLX:

EML:

COM:

SCHULZ ROLF ANDREAS MPI FUER RADIOASTRONOMIE

AUF DEM HUEGEL 69

D 5300 BONN 1

GERMANY

TEL: 228525232

TLF :

TLX: 886440

EML:

COM: 34,40

SCHUTZ BERNARD F PROF

PHYSICS DPT

UNIV WALES COLLEGE

BOX 913

CARDIFF CFI $3 T H$ UK

TEL: $222 \quad 874 \quad 785$

TLF: 222371921

TLK: 498635

EML:

COM: 35 
SCHUTZ BOB EWALD

CENTER FOR SPACE RESEARCH

UNIVERSITY OF TEXAS

AUSTIN TX 787121083

USA

TEL: $512 \quad 471 \quad 1356$

TLF

ILX: 704265 CSRUTX UD

EML:

COM: 19

\author{
SCHWARTZ RICHARD D \\ OPT OF PHYSICS \\ UNIVERSITY OF MISSOURI \\ B001 NATURAL BRIOGE RD \\ ST LOUIS MO 63121 \\ USA \\ TEL: 3145535025 \\ TLF : \\ TLX: 447658 UMSL BOOKSTOR \\ EML : \\ COM: 34 \\ SCHWARZ ULRICH J. OR \\ KAPTEYN ASTRONOMICAL INST \\ BOX 800 \\ NL 9700 AV GRONINGEN \\ NETHERLANOS \\ TEL: 50116695 \\ TLX: 53572 STARS NL \\ EML: \\ TLF : \\ COM: $28,34,40$
}

SCHWEIZER FRANCOIS DR

OPT TERRESTR MAGNETISM

CARNEGIE INST WASHINGTON

5241 BROAD BRANCH RD NW

WASHINGTON OC 20015

IJSA

TEL: 2029660863

TLF :

TLX: 440427 MAGN UI

EML:

COM: 28

SCOTT ELIZABETH L PROF

STATISTICS DPT

UNIVERSITY OF CALIFORNIA

367 EVANS HALL

BERKELEY CA 94720

USA

TEL: $415 \quad 642 \quad 2777$

TLF :

TLX: 910-366-7114 UC BERK

EMI:

COM: 47
SCHWAN MEINER DR

ASTRONOMISCHES RECHEN-

INSTITUT

MOENCHHOFSTR $12-14$

D 6900 hejdelberg

GERMANY

TEL: $62 \quad 2149026$

TLF:

TLX: 461336 ARIHO O

EML

COM: $04 \mathrm{C}, 08 \mathrm{C}$

SCHWARTZ ROLF PH 0

MPI FUER RADIOASTRONOMIE

AUF DEM HUEGEL 69

D 5300 BONN 1

GERMANY

TEL: 228525303

TIX:

EML:

TLF :

COM

SCHWAR ZENBERG-CZERNY A

ASTRONOMICAL OBSERVATORY

WARSAW UNIVERSITY

AL UJAZOOWSKIE 4

PL 00478 WARSAW

POLAND

TEL: 294011

TLF :

TLX: 813978 ZAPAN PL

EML:

COM: 27

SCIAMA OENNIS W DR

SISSA

ST COSIIERA $1 L$

MIRAMARE

I 34014 TRIESTE

IIALY

TEL: $40 \quad 224118$

TLF:

FLX: 460392

EML:

COM: $28,47,48$

SCOTT EUGENE HOWARD

NASA/GSFC

CODE 684.9

GREENBELT ML 20771

USA

TEL: 3012868746

TLF:

$T L X:$

EML:

COM: 34
SCHWARTZ OANIEL A DR

CENTER FOR ASTROPHYSICS

HCO /SAO

60 GARDEN ST

CAMBRIDGE MA 02138

USA

TEL: 6174957232

TLF:

TLX:

EML:

COM: 44,48

SCHWARTZ STEVEN JAY

ASTRONOMY UNIT

QUEEN MARY/WESTFIELO COLL

MILE END RD

LONDON EI 4NS

UK

TEL: $1980 \quad 4811 * 3849$

TLF:

TLX:

EML:

COM: $12,44,49$

SCHWARZSCHILO MARTIN PROF PRINCETON UNIVERSITY OBS

PEYTON HALL

PRINCETON NJ 08544

USA

TEL: 6094523812

TLF:

TLX:

EML:

COM: 35

SCIORTINO SALVATORE DR

OSS ASTRONOMICO

UNIVERSITA DI PALERNO

PALAZZO DEE NORMANNI

I 90134 PALERMO

ITALY

TEL: 916570451

TLF: 91488900

TLX: $9 L 0402$ ASTROP I

EML: ASTRGPAOIPACUC.

COM: 48

SCOTT JOHN S. DR

STEWARO OBSERVATORY

UNIVERSITY OF ARIZONA

TUCSON AZ 85721

USA

TEL:

TLF:

$T L X$

EML:

COM: 40,48
SCHWARTZ PHILIP R OR

NAVAL RESEARCH LABORATORY

CODE 4138

4555 OVERLOOK AVE SW

WASHINGTON DC 203755000

USA

TEL: 2027673391

TLF :

ILX:

EML:

COM: $27,34,40$

SCHWARZ HUGO E

ESO

CASILLA 19001

SANTIAGO 19

CHILE

TEL: 21213249

TLF ;

TLX: 240853

EML:

COM:

SCHWEHM GERHARD DR

ESA/ESTEC

SSO

BOX 299

NL 2200 AG NOOROWIJK

NETHERLANDS

TEL: $1719 \quad 865555$

TLF: $17 \quad 1917400$

TLX: 39098

EML:

COM: 21,44

SCONZO PASQUALE DR

29 OLD MYSTIC ST

ARLINGTON MA 02174

USA

TEL: $617 \quad 6469315$

TLF:

TLX:

EML

COM: 07

SCOTT PAUL F OR MULLARO RADIO ASTRON OBS CAVENDISH LABORATORY MADINGLEY RD

CAMBRTBGE CB3 OHE

UK

TEL: 22366477

TLF:

TLX: 81292

EML:

COM: 40 
SCOVILLE NICHOLAS $Z$ OPT PHYSICS \& ASTRONOMY UNIV OF MASSACHUSETTS

GRC

AMHERST MA 01003

USA

TEL: 4135450789

TI.F:

TLX:

EML:

COM: 28,34

SEARLE LEONARD DR

HALE OBSERVATORIES

B13 SANTA BARBARA ST

PASADENA CA 91101

USA

TEL: 818304 U220

TLK:

EML:

TLF:

COM: 28

SEQLMAYER ERWIN OR

INST FUER ASTRONOMIE \&

ASTROPHYSIK DER TECHN UNI ERNST-REUTER-PLATZ 7

O 1000 BERLIN 10

GERMANY

TEL:

TLF :

YLX:

EML:

COM: 36

SEEGER PHILIP A OR
LOS ALAMOS NATIONAL LAB
MS H805
BOX 1663
LOS ALAMOS NM 87545
USA
TEL: 5056678843
TLF:
TL: :
EML:
COM:

SEGGEWISS WILHELM PROF

OBSERVATORIUM HOHER LIST UNIVERSITAETS-STERNWARTE

O 556B DAUN EIFEL

GERMANY

TEL: 65922150

TLF:

ILX:

EML:

COM: $29,33,42$
SCRIMGER J NORMAN DR OPT OF ASTRONOMY ST MARY'S UNIVERSITY HALIFAX NS B3H 3 C3 CANADA

TEL: $902 \quad 4205633$

TLF: 9024205561

TLX:

EML:

COM:

SEARS RICHARD LANGLEY OR DPT OF ASTRONOMY UNIVERSITY OF MICHIGAN DENNISON BLOG ANN ARBOR MI $48109 \quad 1090$ USA

TEL: 3137633295

TLF:

TLX:

EML:

COM: 35

SEDMAK GIORG TO PROF

DPT DI ASTRONOMIA

UNIVERSITA OI TRIESTE

VIA TIEPOLO $\mathrm{H}$

I 34131 TRIESTE

ITALY

TEL: 40794863

TLF:

TLX: 461137

EML:

COM: 05,09

SEGAL IRVING E OR

DPT OF MATHEMATICS

MIT RM 2224

BOX 165

CAMBRIDGE MA 02139

USA

TEL: 6172534985

TLF :

ILX:

EML:

COM: 47

SEHNAL LADISLAV OR

ASTRONOMICAL INSTITUTE

CZECH ACADEMY OF SCIENCES

ONDREJOV OBSERVATORY

CS 25165 ONOREJOV

CZECHOSLOVAKTA

TEL: 20485201

TLF: 20485314

TLX: 121579

EML:

COM: 07
SCUFLAIRE RICHARD OR INSTITUT D.ASTROPHYSIQUE UNIVERSITE OE LIEGE AVE COINTE 5

B 4000 COINTE-LIEGE

BELGIUH

TEL: 41529980

TLF: 41527474

TLX: 41264 ASTRLG B

EML:

COM: 27,35

SEATON MICHAEL J PROF

DPT PHYSICS \& ASTRONOMY

UNIVERSITY COLLEGE LONDON

GOWER ST

LONDON WC1E 6BT

uK

TEL: 1713877050

TLF :

TLX: 28722

EML:

COM: $12,14,34,36 \mathrm{C}$

SEeOS MICHAEL auguST OR

ASTRONOMY PROGRAM

FRANKLIN/MARSHALL COLLEGE

LANCASER PA 176043003

USA

TEL: 7172913800

TLF: 7172914143

TLX:

EML: BITNET\%"M_SEEOSGFANDM"

COM: 27,46

SEgaluvitz ALEXANGER DR

BOX 659

KEFAR SAVA

ISRAEL

TEL:

TLF:

TLX:

EML:

LDM:

SEIDELMANN P KENNETH DR

US NAVAL DBSERVATORY.

34 \& MASSACHUSETTS AVE NW

WASHINGTON DC 203925100

USA

TEL: $202 \quad 653 \quad 1545$

TLF: $202 \quad 653 \quad 1744$

TLX: 7108221970

EME: USNADPHOBOS.USNO.NAVY,MIL COM: $04 C .07 .20$
SEAQUIST ERNEST R PROF DPT OF ASTRONOMY UNIVERSITY OF TORONTO

60 ST GEORGE ST TORONTO ON MES 1A?

CANADA

TEL: $416 \quad 9783146$

TLF: 4169783921

TLK: 06986766

EML:

COM: 40

SECCO LUIGI OR

DPT DI ASTRONOMIA

UNIVERSITA DI PADOVA

VIC DELL OSSERVATORIO 5

I 35122 PADOVA

ITALY

TEL: 49661499

TLF:

TLX: 430176 UNPADU I

EML:

COM:

SEEger CHARLES LOUIS III SAM FRANCISCO STATE UNIV 473 JAMES RD

PALO ALTO CA 94306

USA

TEL: 4154936005

TLF:

TLX:

EML:

COM: 51

SEgAN STEVD.

INSTITUTE OF ASTRONOMY

UNIVERSITY OF BELGRADE

STUDENTSKI TRG 16

YU 11000 BEOGRAD

YUGOSLAVIA

TEL:

TLF:

TLX:

EML:

COM: 07

SEIOEN PHILIP E

IBM

THOMAS J WATSON RES CTR

BOX 218

YORKTOWN HEIGHTS NY 10598

USA

TEL: 9149451424

ILF:

ILX: 137456

EML:

COM: 28.47 
SEIDOV ZAKIR F DR SHEMAKHA ASTROPHYSICAL OBSERVATORY

AZER ACADEMY OF SCIENCES 373243 SHEMAKHA

AZERBAIDZHAN

TEL:

TLF:

TLX:

EML:

COM: 35

SEIRADAKIS JOHN HUGH OR OPT OF ASTRONOMY

UNIVERSITY THESSALONIKI GR 540 O6 THESSALONIKI GREECE

TEL: $3199 \quad 1357$

TLF:

TLX:

EML:

COM: 40,51

SEKIGUCHI KAZUHIRO OR

DPT OF ASTRONOMY

new MEXICO STATE uniV

BOX 4500

LAS CRUCES NM 88003

USA

TEL: $505 \quad 6462613$

TLF:

TLX:

EML:

COM:

\section{SEMEL MEIR OR}

OBSERVATOIRE DE PARIS

SECTION DE MEUDON

F 92195 MEUDON PPL CDX FRANCE

TEL: $145 \quad 077790$

TLF:

TLX:

EML:

COM: 10,12

SENGBUSCH KURT V OR

MPI FUER PHYSIK UND

ASTROPHYSIK

KARL-SCHWARZSCHILD-STR I

O 8046 GARCHING MUENCHEN

\section{GERMANY}

TEL:

TLF:

TLX:

EME:

COM: 35

SEIELSTAD GEORGE
NRAO
BOX 2
GREEN BANK WV 24944
USA
TEL: 3044562301
TLX: $710-938-1530$
EML:
TLF:
COM: $40,47,48,51$

SEITTER WALTRAUT C PROF ASTRONOMISCHES INSTITUT DOMAGKSTR 75

D 4400 MUENSTER

GERMANY

TEL: 251833561

$T L X: 892529$

EML:

TLF:

COM: 45

SEKIGUCHI NAOSUKE PROF
MUSASHIDAI $3-16-8$
FUCHU
TOKYO' 183
JAPAN
TEL:
TLX:
EML:
TLF:
COM: 19

SEMENIUK IRENA BR

ASTRONGMICAL OBSERVATORY WARSAW UNIVERSITY

AL UJAZOOWSKIE 4

PL 00478 WARSAW

POLAND

TEL: $29-40-11 / 12$

TLF:

TLX: B15548 DAUW

EML:

COM: 42

SEQUEIROS JUAN DR

OPT FISICA

UNIVERSIDAD DE ALCALA OE

HENARES APD 20

E 28801 aLCALA OE HENARES

SPAIN

TEL: 18894940

ILF: 18894953

TLX:

EML:

COM: 44
SEIMENIS JOHN OR

DPT OF MATHEMATICS

UNIVERSITY OF THE AEGEAN

GR 832 OO SAMOS

GREECE

TEL: 27333896

TLF: 27333896

TLX: 294268 VASM GR

EML: JSEIMEGRATHUN I

COM: 33

SEKANINA ZOENEK DR

JPL

EARTH \& SPACE SCI DIV

4800 OAK GROVE DR

PASADENÁ CA 91109

USA

TEL: 8183547589

TLF:

TLX:

EML:

COM: $15,20,22$

SELLWOOD JERRY A

OPT PHYSICS \& ASTRONOMY

RUTGERS UNVIERSITY

BOX 849

PISCATAWAY NJ 088540849

USA

TEL:

TLF: 9089324343

TLX: 703528

EML:

COM: 28,33

\section{SEMENZATO ROBERTO}

OPT DI FISICA G GALILEI UNIVERSITA OI PAODVA

VIA MARZOLO 8

I 35131 PADOVA

ITALY

TEL: 49844247

TLF:

TLX: 430300 DF GGPO I

EML:

COM:

\section{SERAFIMOV KIRIL B ACAD}

DPT OF ASTRONOMY

bulgarian aCaO SCIEnCES

72 LENIN BLVO

BG 1784 SOFIA

\section{BULGaRIA}

TEL: $275 \quad 8927$

TLF:

ILX: 23561 ECF BAN

EML:

COM:
SEIN-ECHALUCE M LUISA DR OPT DE MATEMATICA APLIC UNIVERSIDAD OE ZARAGOZA AVD MARIA ZAMBRANO 50

E 50009 ZARAGOZA

SPAIN

TEL: $7651 \quad 8143$

TLF:. 76565852

TLX: 581.98

EML:

COM: 07

SEKI MUNEZZ DR

DPT OF EARTH SCIENCES

TOHOKU UNIVERSITY

KAWAUCHI

SENDAI 980

JAPAN

TEL: $22212 \quad 1800$

TLF:

TLX:

EML:

COM: 34

SELVELLI PIERLUIGI OR OAT

BOX SUCC TRIESTE 5

VIA TIEPOLO II

I 34131 TRIESTE

ITALY

TEL: 40793221

TLF:

TLX: 461137 OQT I

EML:

COM: 44

SEN S N DR

INOLAN ASSOCIATION FOR THE CULTIVATION OF SCI JADAVPUR

INDIA

TEL:

TLF:

TLX:

EML:

COM:

SERAFIN RICHARD AUGUST THERESENSTRASSE 39a

O 4200 oberhausen 1 GERMANY

TEL:

TLF:

TLX:

EML:

COM: 
SERIO SALVATORE OR OSS ASTRONOMICO UNIVERSITA OI PALERNO PALAZZO OEI NORMANNI I 90134 PALERMO

ITALY

TEL: 91592451

TLF :

TLX: 910402 ASTROP I

EML: BITNET: ASTROPAQIPACUC

COM:

SESSIN WAGNER OR

INPE

CP 515

DPTO DE ASTRQNOMIA

12200 S JOSE DOS CAMPOS

BRAZIL

TEL: 12322 908日

TLF :

TLX: 01173437 2W0-24-73

EML:

COM: 07

SEYILLA MIGUEL 3 OR INST OE ASTRON Y GEODESIA FAC OE CIENCIAS MATEMAT UNTYERSIDAO COMPLUTENSE E 29040 MAORIO SPAIN

TEL: I 244250 ?

TLF :

TLX:

EML:

COM: 19

SHAFFER OAVID B DR

NASA/GSFC

CODE 621.9

GREENBELT MO 20771

USA

TEL: $301286 \quad 6434$

TLF :

TLX:

EML:

COM: 40

SHAHBAZIAN ROMELJA $K$ DR BYURAKAN ASTROPHYSICAL OBSERVATORY

378433 BYURAKAN

ARMENIA

TEL; $88 \quad 52 \quad 28 \quad 3453$

TLF:

TLX:

E.ML:

COM: 28
SERRANO ALFONSO DR INSTITUTO QE ASTRONOMIA UNAM

APDO POSTAL $70-264$

D4510 MEXICO OF

MEXICO

TEL:

TLF :

TLX:

EML:

COM: 05

\section{SETTI GIANCARLO PROF}

ESO

KARL-SCHWARZSCHILD-STR 2

D 8046 GARCHING MUENCHEN GERMANY

TEL: 89320060

TLF: 893202362

TLX: 52828222 EO D

EML :

COM: $28,40,47,48,49$

SEWARD FREOERICK O

CENTER FOR ASTROPHYSICS

HCO/SAO

60 GARDEN ST

CAMBR IOGE MA 02138

USA

TEL: 6174957282

TLF :

TLX

EML:

COM: 48

SHAFTER ALLEN W DR

OPT DF ASTRONOMY

SAN DIEGO STATE UNIV

SAN DIEGO CA 92182

USA

TEL: $617594 \quad 6170$

TLF:

TLX:

EML: SHAFTERQPROTEUS. SOSU. EDU

COM: 42

SHAKESHAFT JOHN R OR

MULLARO RADIO ASTRON GBS

CAVENOISH LABORATORY

MADINGLEY RD

CAMBRIOGE CB3 OHE

UK

TEL: $223 \quad 66477$

TLF:

TLX: 81292 CAVLAB G

EML:

COM: $05,28,40$
SERSIC $J$ L DR

OBSERVATORIO ASTRONOMICO

DE CORDOBA

LAPRIDA BS4

5000 COROOBA

ARGENTINA

TEL: 5125072

TLF :

TLX: $51-822$ BULOR OBSASTR

EML:

COM: 28,47

SEVARLIC BRANISLAV M PROF ASTRONOMICAL OBSERVATORY VOLGINA?

YU 11050 BEOGRAD

YUgOSLAVIA

TEL: 11419.357

TLF:

TLX:

EML:

COM: 08,19

SEYMOUR P A H

57 HERMITAGE RD

PLYMOUTH DEVON

UK

TEL :

TLF :

TLX:

EML:

COM:

SHAH GHANSHYAM A DR INOIAN INSTITUTE OF

ASTROPHYSICS

KORAMANGALA

BANGALORE 560034

INOIA

TEL: $81256 \quad 6585 / 6497$

TLF :

TLX: 845-763 IIAB IN

EML:

COM: 34

SHAKHBAZYAN YURIJ L OR BYURAKAN ASTROPHYSICAL OBSERVATORY

\section{BYURAKAK}

ARMENIA

TEL: $88 \quad 52 \quad 28 \quad 3435$

TLF:

TLX:

EML:

COM: 09,
SERVAN BERNARD

OBSERVATOIRE DE PARIS

61 AVE OBSERVATOIRE

F 75014 PARIS

FRANCE

TEL: 140512236

TLF :

TLX: 270776 OBS F

EML:

COM: 09

SEVERINO GIUSEPPE

OSS ASTRONOMICO

DI CAPOOIMONTE

VIA MOIARIELLO 16

I 80131 MAPOLI

ITALY

IEL: 81440201

TLF :

TLX:

EML:

COM: 12

SEZER CENGIZ BR

FACULTY OF SCIENCE

EGE UNIVERSITY

BoX 21

35100 BORNOVA IZMIR

TURKEY

TEL: 51280210

TLF :

TLX:

EML:

COM:

SHAHAM JACOB PROF

OPT OF PHYSICS

COLUMBIA UNIVERSITY

NEW YORK NY 10027

USA

TEL: $212 \quad 280 \quad 3349$

TLF:

TLX: 220094 COLU UR

EML:

COH: $4 B$

SHAKHOVSKOJ NIKOLAY M OR

CRIMEAN ASTROPHYS OBS

UKRAINIAN ACAO OF SCIENCE

NAUCHNY

334413 CRIMEA

UKRAINE

TEL: 432945

TLF:

ILX:

EML:

COM: 25 


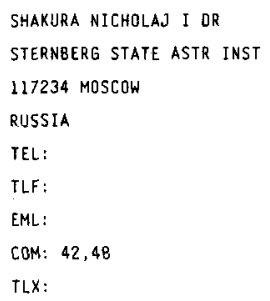

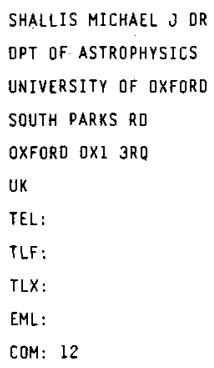

SHALTOUT MESALAM A M PROF
HELWAN OBSERVATORY
HELWAN
EGYPT
TEL: $78 \quad 0645 / 2683$
TLF:
TLX:
EML:
COM:

SHAPERO DONALD C OR NTL ACADEMY OF SCIENCES NTL RESEARCH COUNCIL 2101 CONSTITUTION AVE NW WASHINGTON OC 20418 USA

IEL: 2023343520

TLF :

TLX: 248664

EML: BITNET: DSHAPERONAS

COM:

\section{SHAPLEY ALAN H}

NOAA

\section{BROADWAY}

BOULOER CO BOJOJ

USA

TEL:

TEF:

TLX:

EML:

COM: 10

SHARMA DHARMA PAL OR

DPT OF PHYSICS

UNIVERSITY OF TASMANIA

GPO BOX 252C

HOBART TAS 7001

AUSTRALIA

TEL: 2202428

TLF: 22022410

TLX: AA 58150

EML: SHARMAGPHYSVAX. PHYS. UTAS. EQU. A EML

COM: 27

SHARPLESS STEWART PROF OPT PHYSICS \& ASTRONOMY UNIVERSITY OF ROCHESTER ROCHESTER NY 14627

USA

TEL: 7162754389

TLF:

TLX:

EML:

COM: 34,45

TLF: 893202362
COM: $06,21,33,37$

SHANDARIN SERGEI F DR INST FOR PHYSICS PROBLEMS

KOSYGIN 2

117334 MOSCOW

RUSSIA

TEL: 1373248

TLF:

TLX: 113451 MAGNIT

EML:

COM: $47 C$

SHAPIRO IRWIN I PROF CENTER FOR ASTROPHYSICS HCO/SAO RM P 209 60 GARDEN ST CAMBRIDGE MA 02138 USA

TEL: 6174957100

TLF :

TLX: 921428 SATELLITE CAM EML:

COM: $04,07,16,19$

SHARA MICHAEL DR

STSCI

HOMEWOOO CAMPUS

3700 SAN MARTIN OR BALTIMORE MO 21218

USA

TEL: 3013384743

TLF:

TLX: 6849101 STSCI UW

EML:

COM: 27

SHAROV A S OR

STERNBERG STATE ASTR INST

UNIVERSITETSKIJ PROSP 13 119899 MOSCOW

RUSSIA

TEL: 139-26-57

TLF:

TLX:

SHAVER PETER A DR

ESO

KARL-SCHWARTZSCHILD-STR 2 D 8046 GARCHING MUENCHEN GERMANY

TEL: 89320060

TLX: 52828222 EOD

EML:

COM: $28,34,40,47 C, 48$ 


SHAVIV GIORA PROF
OPT OF PHYSICS
IIT
TECHNION CITY
HAIFA 32000
ISRAEL
TEL:
TLF:
TLX:
EML:
COM: $35,42,47,48$
SHAWHAN STANLEY O OR
OPT PHYSICS \& ASTRONOMY
UNIYERSITY OF 10 OWA
IOWA CITY IA 52242
USA
TEL: 3193533294
TLF:
TLX:
EML:
COM: 49

SHCHEGOLEV DIMITRIJ E DR PULKOVO OBSERVATORY ACAOEMY OF SCIENCES 10 KUTUZOV QUAY 196140 ST PETERSBURG RUSSIA

TEL:

TLF:

TLX:

EML:

COM:

SHEFFER EUGENE $K$ DR STERNBERG STATE ASTR INST UNIVERSITETSKIJ PROSP 13 119899 MOSCOW RUSSIA

TEL: 1392046

Tix:

EML:

TLF:

COM:

SHEN BENLAMIN S P PROF

DPT OF ASTRONOMY EI

univ of PENNSYLVANIA.

PHILADELPHIA PA 19104

USA

TEL: 2158988176

ILF:

TLX:

EML:

COM:
SHAW JAMES SCOTT DR DPT PHYSICS \& ASTRONOMY UNIVERSITY OF GEORGIA ATHENS GA 30602

USA

TEL: $404542 \quad 2485$

TLF:

ILX:

EML:

COM:

SHAWL STEPHEN J DR DPT PHYSICS \& ASTRONOMY UNIVERSITY OF KANSAS LAWRENCE KS 66045 USA

TEL:

TLF:

TLX:

EML:

CGM: $25,34,37$

SHCHERBINA-SAMOJLOVA I DR INST OF SCIENCE \& TECH 125219 MOSCOW RUSSIA

TEL: 1554237

TLF :

EML:

COM: 05

TLX:

SHEFFIELO CHARLES OR EARTH SATELLITE CORP 7222 47TH STREET (CHEVY CHASE) WASHINGTON OC 20815 USA

TEL: 3019510104

TLF:

TLX: 248618 ESCO UR

EML:

COM: 44

SHEN CHANGJUN

PURPLE MOUNTAIN OBSERV

CAS

NANJING

CHINA PR

TEL: 2546700

TLF:

TLX: 34144 PMONJ CN

EML:

COM: 09
SHAW JOHN H PROF

OPT OF ASTRONOMY OHIO STATE UNIVERSITY 174 W 18TH AVE

COLUMBUS OH 432101106

USA

TEL: 6144227968

TLF;

TLX:

EML:

COM:

SHAYA EOWARD J OR
ASTRONOMY PROGRAM
UNIVERSITY OF MARYLAND
COLLEGE PARK MO 20742
USA
TEL:
TLF:
TLX:
EML:
COM: 28,47

SHEA MARGARET A DR A F GEOPHYSICS LÁBORATORY SPACE PHYSICS OIV PHC " HANSCOM AFB BEDFORO MA 01732 USA

TEL:

TLF:

TLX:

EML:

COM: 10.49

SHEFOV NICOLAI N INST PHYSICS OF ATMOSPH ACADEMY OF SCIENCES PYZHEVSKY 3

109017 MOSCOW RUSSIA

TEL:

TtF :

ILX:

EML:

COM: 21

SHEN CHUN-SHAN

ASTRONOMICAL STY OF CHINA NTL TSING HUA UNIVERSITY HSIN CHU 300043

CHINA. R

TEL: 35719039

TLF:

TLX:

EML:

COM: 46,51
SHAW R WILLIAM PROF 105 HALCYON HILL

ITHACA NY 14850

USA

TEL: 6072571948

TLF :

TLX:

EML:

COM:

SHCHEGLOV $P$ Y DR

STERNBERG STATE ASTR INST

UNIVERSITETSKIJ PROSP 13

119899 MOSCOW

RUSSIA

TEL: $139-19-73$

TLF:

TLX:

EML:

COM: $09,34,50 \mathrm{C}$

SHEELEY NEIL R DR

NAVAL RESEARCH LABORATORY CODE 4172

4555 OVERLOOK AVE SW WASHINGTON DC 203755000 USA

TEL: 2027672777

TLF:

TLX:

EML:

COM: 10,12

SHELUS PETER J DR

ASTRONOMY OPT

UNIVERSITY OF TEXAS

RLM $15 \quad 316$

AUSTIIN TX 787121083

USA

TEL: 5124713339

TLF :

TLX: 910-874-1351

EML:

COM: 20,24

SHEN KAIXIAN

SHAANXI OBSERVATORY

CAS

LINTONG XIAN

SHAANXI

CHINA PR

TEL: 332255

TLF:

TLX: 20121 CSAO CN

EML :

COM: 08 


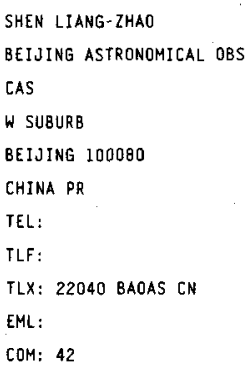

SHEN LONG-XIANG
BEIJING ASTRONOMICAL OBS
CAS
W SUBURB
BEIJING 100080
CHINA PR
TEL: 1281698
TLF:
TLX: 22040 BAOAS CN
EML:
COM: 12

SHERWODD WILLIAM A DR MPI FUER RADIOASTRONOMIE AUF DEM HUEGEL 69 D 5300 BONN 1

GERMANY

TEL: 228525362

TLX: 886440

EML:

TLF:

COM: $27,28,34$

SHI GUANG-CHEN

PURPLE MOUNTAIN OBSERV

CAS

NANJING

CHINA PR

TEL: 2533921

TLF:

ILX: 34144 PMOAS CN

EML:

COM: 08,24

SHIBATA KAZUNARI OR DPT OF EARTH SCIENCES AICHI UNIV OF EUUCATION 1 HIROSAWA

KARIYA 448

JAPAN

TEL: 566363111,596

TLF:

TLX:

EML:

COM:

\section{SHIM WOON-TAIK PROF} 236-53 SINDAN-DONG JOONG-KU

SEOUL 100

KOREA R

TEL:

TLF:

TLX:

EML:

COM:
SHEN PARN-AN

NANJING ASTRONOMICAL

INSTRUMENT FACTORY

BOX 846

NANJ ING

CHINA PR

TEL: 2546191

TLF:

TLX: 34136 GLYNJ C/O NAIF

EML:

COM: 09

SHESTAKA IVAN S DR

ASTRONOMICAL OBSERVATORY

ODESSA STATE UNIVERSITY

SHEVCHENKO PARK

270014 ODESSA

UKRAINE

TEL:

TLF :

TLX:

EML

COM: 22

SHI ZHONG-XIAN

BEIJING ASTRONOMICAL OBS

CAS

W SUBUR8

BEIJING 100080

CHINA PR

TEL: $128 \quad 1698$

TLF :

TLX: 9053

EML:

COM: 10

SHIBATA SHINPEI OR

OPT OF PHYSICS

YAMAGATA UNIVERSITY

KOJIRAKAWA

YAMAGATA 990

JAPAN

IEL: $236 \quad 311421$

TLF

TLX:

EML: BITNET:B264160JPNKUOPC

COM:

SHIMIZU MIKIO PROF

ISAS

3-1-1 YOSHINODAI

SAGAMIHARA

KANAGAWA 229

JAPAN

TEL: 042751391

TLF :

TLX:

EML:

COM: $15,16,51$
SHER DAVIO DR

BOX 9624

CINCINNATI OH 452098

USA

TEL: 5138718850

TLF :

YLX:

EML:

COM: 33,37

SHEVCHENKO VLADISLAV V DR SIERNBERG STATE ASTR INST UNIVERSITETSKIJ PROSP 13 119899 MOSCOW RUSSIA

TEL:

TLF :

TLX:

EML:

COM: 16 , WGPSNC

SHIBAHASHI HIROMOTO DR OPT OF ASTRONOMY UNIVERSITY OF TOKYO BuNKYO Ku

TOKYO 113

JAPAN

TEL: 03-812-2111

TLF:

TLX: 33659 UTYOSCI J

EML:

COM: 35

SHIBATA YUKIO OR RES INST SCIENTIFIC MEAST TOHOKU UNIVERSITY

ARAMAKI

SENDAI 980

JAPAN

TEL:

TLF:

ILX:

EML:

COM: 35

SHIMIZU TSUTOMU PROF EMER TERADA OOTANTI 26-16 JOYO SHI KYOTO FU 61001 JAPAN

TEL:

TLF :

TLX

EML:

COM: 16,33 
SHIMMINS ALBERT JOHN

18 PAGE ST

ALBERT PARK VIC 3206

AUSTRALIA

TEL: 36903803

TLF:

EML:

COM: 40

TLX:

SHISHOV VLADIMIR I DR LEBEOEV PHYSICAL INST ACADEMY OF SCIENCES LENINSKY PROSPEKT 53 117924 MOSCOW

RUSSIA

TEL:

TLF:

TEX:

EML:

COM:

SHOEMAKER EUGENE $M$

US GEOLOGICAL SURVEY

BRANCH OF ASTROGEOLOGY

2255 N GEMINI DR

FLAGSTAFF AZ 86001

USA

TEL: 6025277181

TLF :

TLX:

EML:

COM: $15,16,20$

SHORE STEVEN N

ASTROPHYSICS RESEARCH CTR

NEW MEXICO TECH

CAMPUS STATION

SOCORRO NM 87801

USA

TEL: $505 \quad 8355792$

ILF:

ILX:

ENL:

COM: $28,29,45$

SHUKRE C S OR

RAMAN RESEARCH INSTITUTE

SADASHIVANAGAR

BANGALORE 560080

INOIA

TEL: 812360122

TLF: $812,34 \quad 0492$

ILX: 8425671 RRI IN

EML:

COM: 48
SHINE RICHARD A DR

LOCKHEE PALO ALTO RES LB

DPT 9130 BLDG 256

3170 PORTER OR

PALO ALTO CA 943041211

USA

TEL: $415 \quad 858 \quad 4135$

TLF:

TLX:

EML:

COM: $10,12.36$

SHIVANANDAN KANDIAH OR

NAVAL RESEARCH LABORATORY

CODE $413 B S$

4555 OVERLOOK AVE SW

WASHINGTON OC 203755000

USA

TEL: $202 \quad 767 \quad 2749$

TLF:

TLX: 202-767-6473

EML:

COM: $09,44,47$

SHOLOMITSKY G B DR

SPACE RESEARCH INSTITUTE

ACADEMY OF SCIENCES

PROFSOJOSNAYA UL 84/32

117810 MOSCOW

RUSSIA

TEL: 333-31-22

TLF:

TLX: 411498 STAR SU

EML:

COM: 40,44

\section{SHOSTAK G SETH DR}

1372 CUERNAVACA CIRC

MOUNTAIN VIEW CA 94040

USA

TEL: 4159678193

TLF:

EML:

COM: 28,51

TLX:

SHULL JOHN MICHAEL

JILA

UNIVERSITY OF COLORADO

BOX 440

BOULDER CO 803090440

USA

TEL: 3034927827

TLF:

TLX:

EML:

COM: 34
SHIPMAN HENRY: L DR

DPT OF PHYSICS

UNIVERSITY OF DELAWARE

NEWARK DE 19716

USA

TEL: 3024512986

ILF:

TLX:

EML:

COM: 36,46

SHKODROV V G OR

DPT OF ASTRONOMY

BULGARIAN ACAD SCIENCES

72 LENIN BLVD

BG 1784 SOFTA

BULGARIA

TEL: $275 \quad 8927$

TLF:

TLX: 23761 ECF BAN BG

EML:

COM: 15,20

SHOR VIKTOR A OR

INST OF THEORET ASTRONOMY

ACADEMY OF SCIENCES

N Kutuzova 10

191187 ST PETERSBURG

RUSSIA

TEL:

TLF:

TLX: 121578

EML:

COM: $15,20 \mathrm{C}$

SHU FRANK H PROF

ASTRONOMY OPT

UNIVERSITY OF CALIFORNIA

601 CAMPBELL HALL

BERKELEY' CA 94720

USA

TEL: $415 \quad 642 \quad 2529$

TLF:

TL:X:

EML:

COM: $33,34,42$

SHULL PETER OTTO OR

OPT OF PHYSICS

OKLAHOMA STATE UNIVERSITY

STILLHATER OK 740780444

USA

TEL: 4057445705

TLF:

TLX:

EML: PHYSPOSOSUCC. BITNET

COM: 34
SHIRYAEV ALEXANDER A BR INST OF THEORET ASTRONOMY ACADEMY OF SCIENCES

N RUTUZOVA 10

191187 ST PEJERSBURG

RUSSIA

TEL: 7-812-272 40 23

TLF :

TLX: 12578 ITA SU

EML:

COM: 04

SHOBBROOK ROBERT R DR

OPT OF ASTRONOMY

UNIVERSITY OF SYONEY

SYDNEY NSW 2006

AUSTRALIA

TEL: 26923604

TLF :

TLX: 26169 UNISYD AA

EML:

COM: 27,37

SHORE BRUCE W

LAWRENCE LIVERMORE LAB

$\mathrm{B} 0 \times 808$

LIVERMORE CA 94550

USA

TEL: $415 \quad 447 \quad 1100$

TLF:

TLX:

EML:

COM: 14

\section{SHUKLA K}

OPT MATHS \& ASTRONOMY

UNIVERSITY OF LUEKNOW

LUCKNOW UP.

INDIA

TEL:

TLF :

TLX:

EML:

COM: 41

SHULOV OLEG S OR

ASTRONOMICAL OBSERVATORY

ST PETERSBURG UNIVERSITY

BIBLIOTECHNAJA PL 2

19917B ST PETERSBURG

RUSSIA

TEL:

TLF:

TLX:

EML:

COM: 


SHUL' BERG A M OR
ASTRONOMICAL OBSERVATORY
ODESSA STATE UNIVERSITY
SHEVCHENKO PARK
270014 ODESSA
UKRAINE
TEL: 250356
TLF:
TLX:
EML:
COM: 26,42

\section{SIBILLE FRANCOIS}

OBSERVATOIRE DE LYON

AVE CHARLES ANDRE

F 6956L S GENIS LAVAL COX FRANCE .

TEL: $7856 \quad 0705$

TLF: 72399791

TLX: 310926

EML:

COM:

SIEBER WDLFGANG PH D

FACHHOCHSCHULE NIEDERRHEI

FACHBEREICH ELECTR

REINARZSTR. 4969

D 4150 KREFELD 1

GERMANY

TEL: $215 \quad 18 \quad 220$

TLF :

TLK:

EML:

COM: 40

\section{SIKORSKI JERZY DR}

INST THEORETICAL PHYSICS

UNIVERSITY OF GDANSK

UL WITA STWOSZA 57

$\mathrm{PL} 80952$ GOANSK

POLAND

TEL:

ILF :

TLX: 0512706 IFAS PL.

EML:

COM:

SILVERBERG ERIC C DR

MCOONALO OBSERVATORY UNIVERSITY' OF TEXAS

BOX 1337

FORT DAVIS TX 79734

USA

TEL.

TLF :

TLX:

EML:

COM:
SHUL' MAN L M OR

MAIN ASTRONOMICAL OBS

SHUSTOV BORIS M DR

INST OF ASTRONOMY

ACADEMY OF SCIENCES

PYATNITSXAYA UL 48

109017 MOSCOW

RUSSIA

TEL: $231-54-61$

TLF :

TLX: 412623 SCSTP SU

EML:

COM: 34,35

COM: 15

SICAROY BRUNO DR

observatoIre de paris

SECTION OE MEUDON

EUROPA

F 92195 MEULON PPL CDK

FRANCE

TEL: 145077409

TLF: 145077469

ILX: 201571

EML: SICARDY YRMEU5I/MESIOA: SICARD EML:

COM: 16

COM: 07

SIENKIEWICZ RYSZARO DR

COPERNICUS ASTRON CENTER

POLISH ACAD OF SCIENCES

UL. BARTYCKKA 18

PL OO 716 WARSAW

POLAND

TEL: 411086

ILF :

ILX: 813878 ZAPAN PL

EML:

COM: 35

\section{SILBERBERG REIN OR}

NAVAL RESEARCH LABORATORY

CODE 4154

4555 OVERLOOK AVE SW

WASHINGTON OC 203755000

USA

TEL: 2027672803

TLF :

TLX:

EML:

COM: $10,34,48$

\section{SILVESTRO GIOVANNI}

IST DI FISICA

UNIVERSITA DI TORINO

CORSO D. AZEGLIO 46

I 10125 TORINO

ITALY

TEL: $11 \quad 65 \quad 8623$

TLF :

TLX: 211041 INFNTO

EML:

COM: $34,35,44$

BUDECSKA 6

CS 12023 PRAHA?

CZECHOSLOVAXIA

TEL: 225 B75?

TLF: 2255010

$T L X: 122486$

SIGNORE MONIQUE QR

RAOIOASTRONOMIE ENS

24 RUE LHOMOND

FRANCE

IEL: I $45 \quad 291225$

TLF:

TLX:

EML:

COM: $35,41,47,48$

SILK JOSEPH 1 PROF

ASTRONOMY DPT

601 CAMPBELL HALL

BERKELEY CA 94720

USA

TEL: $415 \quad 642 \quad 2113$

TLF :

TLX: 820181 UCB AST

EML:

COM: 34,47

SIM MARY E MISS

ROYAL OBSERVATORY

BLACKFORD HILL

EDINBURGH EH9 $3 \mathrm{HJ}$

JK

TEL: $316 \quad 673 \quad 321$

TLF :

TLX: 72383 ROEOIN G

EML:

COM: 09
SHUTER WILLIAM L H OR

DPT OF PHYSICS

UNIV OF BRITISH COLUMBIA

6224 AGRICULTURE RO

VANCOUVER BC V'TT $2 A G$

CANAOA

TEL: $604 \quad 228 \quad 4269$

TLF ; 6042285324

TLX: 04508576

EML:

COM: $33,34,40,51$

SIDLICHOVSKY MILOS OR

ASTRONOMICAL INSTITUTE

CZECH ACADEMY SCIENCES

F 75231 PARIS COX 05

UNIVERSITY OF CALIFORNIA

SIOORENKOV NIKOLAYS

HYOROMETEOROLDGICAL CIR

\section{MOSCOW}

RUSSIA

TEL:

TLF :

TLX:

EML:

COM: 19

COPERNICUS ASTRON CENTER

POLISH ACAD OF SCIENCES

UL BARTYCKA 18

PL OO 716 WARSAW

POLAND

TEL:

TLF:

TLX:

EML:

COM: $4 B$

SILLANPAA AIMO KALEVI DR TURKU UNIVERSITY OBS

TUORLA

SF 21500 PIIKKIO

FINLAND

TEL: 21435822

TLF: $21 \quad 43 \quad 3767$

TEX:

EML: AIMOSILLEONTL.UTU.FI

COM: $2 B$

SIMA ZOISLAV DR

ASTRONOMICAL INSTITUTE

CZECH ACAOEMYY SCIENCES

BUDECSKA 6

CS 12023 PRAHA 2

CZECHOSLOVAKIA

TEL: 2258757

TLF: 2255010

TLX: $66-122486$

EML:

COM: 07,42

\section{SIKORA MAREK}


SIMEK MILOS DR

ASTRONOMICAL INSTITUTE

CZECH ACADEMY OF SCIENCES

ONDREJOV OBSERVATORY

CS 25165 ONOREJOV

CZECHOSLOVAKIA

TEL: 20485201

TiF: 20485314

TLX: 121579

EML:

COM: 22

SIMNETT GEORGE M

DPT OF SPACE RESEARCH

UNIVERSITY OF BIRMINGHAM

BOC 363

BIRMINGHAM B15 2TT

UK

TEL:

TLF

$T L X$

EML:

COM: 20

SIMON GUY

OBSERVATOIRE DE PARIS

SECTION DE MEUDON

F 92195 MEUDON PPL EOX

FRANCE

TEL: 145077787

TLF :

TLX:

EML:

COM: 10,12

SIMON NORMAN R PROF

OPT OF PHYSICS \& ASTRON

UNIVERSITY OF NEBRASKA

BEHLEN OBSERVATORY

LINCOLN NE 685880111

USA

TEL: 4024722788

TLF:

TLX:

EML:

COM:

\section{SIMON THEODORE}

INSTITUTE FOR ASTRONOMY

UNIVERSITY OF HAWAII

268D WOODLAWN DR

HONOLULU HI 96822

USA

TEL: 8089488968

ILF:

ILX: 723-8459 UHAST HR

EML:

COM: 29,36
SIMIEN FRANCOIS OR

OBSERVATOIRE OE LYON

AVE CHARLES ANORE

F 69561 S geNIS LAVAL COX

FRANCE

TEL: $78 \quad 56 \quad 0705$

TLF: 72399791

TLX: 310926

EML:

COM: 28

SIMO CHARLES DR

FAC DE MATEMATICAS

UNIVERSIDAD DE BARCELONA

AV JOSE ANTONIO 585

E 08028 BARCELONA

SPAIN

TEL:

TLF:

TLX:

EML:

COM:

SIMON JEAN-LOUIS MR

BUREAU DES LONGITUDES

77 AVE DENFERT ROCHEREAU

F 75014 PARIS

FRANCE

TEL: 143201210

TLF :

TLX:

EML:

COM: 04,07

SIMON PAUL $A D R$

1 RUE MORTE BOUTEILLE

F 78140 VELIZY

FRANCE

TEL:

TLF:

EML:

COM: 40,44

TLX:

SIMONNEAU EOUARDO OR

INSTITUT D'ASTROPHYSIQUE

$98 B I S$ BO ARAGO

F 75014 PARIS

FRANCE

TEL: 143201425

TLF: 143298673

TLX:

EML:

COM: 36
SIMXIN SUSAN M DR

PHYSICS \& ASTRUNOMY DPT

MICHIGAN STATE UNIVERSITY

EAST LANSING MI 48824

USA

TEL: 5173534540

TLF:

TLX:

EML:

COM: 28

SIMOOA MAHIRb PROF

DEPT ASTRON/EARTH SCI

TOKYO GAKUGE I UNIVERSITY

KOGANEI

TOKYO 184

JAPAN

TEL: 0423-25-2111

TLF:

TLX:

EML:

COM: 37

SIMON KLAUS PETER

INST F ASTRON \& ASTROPHYS

DER UNIVERSITAET MUENCHEN

SCHEINERSTR 1

D BOOO MUENCHEN 80

GERMANY

TEL: 89989021

TLF:

TLX: 529815 UNIVM. Q

EML:

COM: 36

SIMON PAUL C DR

IASB

AVE CIRCULAIRE 3

B 1180 BRUSSELS

BELGIUM

TEL: 23751579

TLF :

TLX: 21563

EML:

COM: 44

SIMONS STLART DR

SCHOOL OF MATHEMATICAL SC QUEEN MARY/WESTFIELO COLL

MILE END RD

LONDON EI 4NS

UK

TEL: 1719804 81

TLF:

TtX:

EML:

COM: 34
SIMMONS JOHN FRANCIS L

31 HAVELOCK STREET

GLASGOW GIL 5HA

UK

TEL:

TLF:

TLX:

EML:

COM: 42

SIMON GEORGE W DR

AIR FORCE GEOPHYSICS LAB

NTL SOLAR OBSERVATORY

SUNSPOT NM BB349

USA

TEL: $505 \quad 4341390$

TLF:

TLX:

EML:

COM: 12

SIMON MICHAL PROF OPT OF EARTH \& SPACE SCI ASTRONOMY PROGRAM

SUNY AT STONY BROOK

STONY BROOK NY 117942100

USA.

TEL: $516 \quad 2467672$

TLF:

TLX: $510-228-7767$

EML:

COM:

SIMON RENE L E PROF INSTITUT O'ASTROPHYSIQUE UNIVERSITE OE LIEGE AVE COINTE 5

B 4000 COINTE-LIEGE BELGIUM

TEL: 41529980

TLF : $41 \quad 527474$

TLX: 41264 ASTRLG B

EML:

COM: 47

SIMONSON 5 CHRISTIAN DR 1061 RUSSELL AVE

LOS ALTOS CA 94022

USA

TEL: $415 \cdot 968 \quad 0473$

TLF:

$T L X$ :

EML:

COM: 33 


\begin{tabular}{|c|c|c|c|}
\hline SIMOVLJEVITCH JOVAN L OR & SIMS KENNETH P DR & SINACHOPQULOS D OR & SINCLAIR ANOREW T DR \\
\hline OPT OF ASTRONOMY & SYDNEY OBSERVATORY & OBSERVATOIRE ROYAL OE & ROYAL GREENWICH OBS \\
\hline FACULTY OF SCIENCES & OBSERVATORY PARK & BELGIOUE & . \\
\hline STUDENTSKI TRG 16 & SYONEY NSW 2000 & AVE IIRCULAIRE 3 & HERSTMONCEUX CASTLE \\
\hline YU 11000 BEOGRAD & AUSTRALIA & B 1180 BRUSSELS & HAILSHAM BN27 IRP \\
\hline YUGOSLAVIA & TEL: & BELGIUM & uk \\
\hline TEL: $11638>15$ & TLF: & TEL: 23730291 & TEL: $323 \quad 833 \quad 171$ \\
\hline TLF: & TLX: & TLF: 23749822 & TLF: \\
\hline TLX: & EML: & TLX: 21565 OBSBEL & TLX: 87451 RGOBSY G \\
\hline EML: & COM: 08.24 & EML: OIMITRISEASTRO.OMA.BE & EML: \\
\hline COM: & & COM: 26 & COM: 07,20 \\
\hline SINGH H P & SINGH JAGDEV DR & SINGH KULINDER PAL OR & SINGH PATAN DEEN OR \\
\hline INST. F. REINE ANGEWANOTE & INDIAN INSTITUTE OF & TIFR & IAG \\
\hline KERNPHYSIK UNVIERSTAET & ASTROPHYSICS & HOMI BHABHA RD & UNIVERSIDADE DE SAO PAULO \\
\hline OLSHAUSENSTRASSE 40 & KORAMANGALA & COLABA & CP 30627 \\
\hline O 2300 KIEL 1 & BANGALORE 560034 & BOMBAY 400005 & OIO51 SAO PAULO SP \\
\hline GERMANY & INDIA & INDIA & BRAZIL. \\
\hline TEL: & TEL: $81256 \quad 6585 / 6497$ & TEL: 224952971 & TEL: $11275 \quad 3720$ \\
\hline TLF: & TLF: & TLF : & TLF: \\
\hline TLX: & TLX: 845763 IIAB IN & $T L X: 0113009$ & TLX: 01136221 IAGM BR \\
\hline EML: PKEOGERZ. UNI-KIEL. DBP.OE & EML: & EML: BITNET: uunet!shakt 1 !tffr! root & EML: IAGUSPERFAPESP \\
\hline COM: 51 & COM: 12 & COM: & COM: 34 \\
\hline SINHA K OR & SINHA RAMESHWAR P & SINNERSTAD ULF E PROF & SINTON WILLIAM M \\
\hline UTTAR PRADESH STATE & TATA INST OF FUNDAMENTAL & STOCKHOLM OBSERVATORY & INSTITUTE FOR ASTRONOMY \\
\hline OBSERVATORY & RESEARCH & S 13336 SALTSJOEBADEN & UNIVERSITY OF HAWAII \\
\hline PO MANORA PEAK 263129 & POONA ÜNIVERSITY CAMPUS & SWEDEN & 2680 WQODLAWN DR \\
\hline NAINITAL 283129 & PUNE 411007 & TEL: 87170195 & HONOLULU HI 98822 \\
\hline INDIA & INDIA & TLF: $87 \quad 174719$ & USA \\
\hline TEL: $59 \quad 42 \quad 2136$ & TEL: 212337107 & TLX: & TEL: $808948 \quad 8007$ \\
\hline TLF : & TLF: 212335760 & EML: & TLF: \\
\hline TLX: CABLE : ASTRONOMY & TLX: 0145658 GMRT IN & COM: 29,45 & TLX: \\
\hline EML: & EML: SINHAEGMRT,ERNET.IN & & EML: \\
\hline COM: $10,12,14$ & COM: 40 & & COM: 16 \\
\hline SINVHAL SHAMBHU DAYAL DR & SINZI AKIRA M DR & SION EDWARD MICHAEL & SIREGAR SURYADI DR \\
\hline 4/3 SNEHALATAGANG & HYDROGRAPHIC DPT & DPT OF ASTRONOMY & DPT OF ASTRONOMY \\
\hline INDORE $452 \quad 003$ & GEODESY \& GEOPHYSICS DIV & VILLANOVA UNIVERSITY & BANDUNG INSTITUTE OF TECH \\
\hline INOIA & TSUKIJI 5 CHUO KU & VILLANOVA PA 19085 & JL GANESHA 10 \\
\hline TEL: & TOKYO 104 & USA & BANDUNG 40132 \\
\hline TLF : & JAPAN & TEL: $215 \quad 645 \quad 4822$ & INOONESIA \\
\hline EML: & TEL: & TLF: & TEL: $84254 \div 476$ \\
\hline COM: 27,42 & TLF : & TLX: & TLF: \\
\hline \multirow[t]{3}{*}{ TLX: } & TLX: & EML: Scionescivax.stsci.edu & TLX: 28324 ITB BANOUNG \\
\hline & EML: & COM: $35,42,45$ & EML: \\
\hline & COM: 04 & & COM: \\
\hline SIROKY JAROMIR OR & SIRY JOSEPH $W$ & SISSON GEORGE M MR & SISTERO ROBERTO F OR \\
\hline PALACKYY UNIVERSITY & 4438 42ND ST NW & PLANETREES & OBSERVATORIO ASTRONOMICO \\
\hline OPT PHYSICS \& ASTRONOMY & WASHINGTON OC 20016 & WALL & DE CORODBA \\
\hline LENIN ST 26 & USA & HEXHAM NE46 AEO & LAPRIOA 854 \\
\hline CS 77146 OLOMOUC & TEL: & UK & 5000 CORDOBA \\
\hline CZECHOSEOVAKIA & TLF: & TEL: $434 \quad 814 \quad 34$ & ARGENTINA \\
\hline TEL: 6822451 & EML: & $I L F:$ & TEL: $40613 / 36876$ \\
\hline TLF : & COM: 07 & $T\llcorner X:$ & TLF: \\
\hline TLX: & $\mathrm{TLX}$ : & EML: & TLX: 51822 BUCOR \\
\hline EML: & & COM: & EML: \\
\hline COM: 46 & & & COM: 42.47 \\
\hline
\end{tabular}


SItARSKI GRzEGORZ PROF SPACE RESEARCH CENTER POLISH ACAD OF SCIENCES UL ORDONA 21

PL 01237 WARSAW

POLAND

TEL: 41004

TLF:

TLX: $815670 \mathrm{CBK}$ PL

EML:

COM: 20

SIVAN JEAN-PIERRE DR

LAS

TRAVERSE OU SIPHON

LES TROIS LUCS

F 13012 MARSEILLE

FRANCE

TEL: 91055900

TLF: $9166 \quad 1855$

$T L X: 420584$ ASTROSP

EML:

COM: 34

SKALAFURIS ANGELO J

NAVAL RESEARCH LABORATORY

COOE 5307

4555 OVERLOOK AVE SW

WASHINGTON OC 203755000

USA

TEL: 3027673227

TLF:

TLX:

EML:

COM:

\section{SKINNER GERALD OR}

SCHOOL PHYSICS/RESEARCH

UNIVERSITY OF BIRMINGHAM

BOX 363

BIRMINGHAM B15 2 TT

UK

TEL: 214146450

TLF:

TLX: 338938

EML: SPAN 19457: :BHYAB: :GKS

COM:

SLEE O B DR
CSIRO
OIVISION OF RAOIOPHYSICS
BOX 76
EPPING NSW 2121
AUSTRALIA
TEL: 2868 O222
TLF:
TLX: 26230 ASTRO
EML:
COM: 40

SITKO MICHAEL L

DPT OF PHYSICS

UNIYERSITY OF CINCINMATI

210 BRAUNSTEIN ML 11

CINCINNATI OH 452210111

USA

TEL:

TLF:

TLX:

EML:

COM: 28,34

SIVARAM C DR

INDIAN INSTITUTE OF

ASTROPHYSICS

KORAMANGALA

BANGALORE 560034

INOIA

TEL: $81256 \quad 6585 / 6497$

TLF:

ILX: 845763 IIAB IN

EML:

COM: 51

SKILLEN IAN OR

DPT OF ASTRONOMY

UNIVERSITY OF LEICESTER

UNIVERSITY RQ

LEICESTER LEI TRH

UK

TEL:

TLF:

ILX:

EML:

COM:

SKRIPNICHENKO VLADIMIR DR INST OF APPLIED ASTRONOMY ACADEMY OF SCIENCES

ZOANOVSKAYA UL 8

197042 ST PETERSBURG

RUSSIA

TEL:

TLF:

TLX:

EML:

COM: 07

\section{SLETTEBAK ARNE PROF}

PERKINS OBSERVATORY

OHIO STATE UNIVERSITY

BOX 449

OELAWARE QH 43015

USA

TEL: $614363 \quad 125$ ?

TLF:

TLX:

EML:

COM: $29,33,45$
SITNIK G F PROF

STERNBERG STATE ASTR INST

UNIVERSITETSKIJ PROSP 13

119899 MOSCOW

RUSSIA

TEL: $139-19-73$

TLF :

TLX:

EML:

COM: $10,12,36$

SIVARAMAN K R OR
INDIAN INSTITUTE OF
ASTROPHYSICS
KORAMANGALA
BANGALORE 560034
INOIA
TEL: 812566585
TLF:
TLX: 845763 IIAB IN
EML:
COM: $12 C .15$

SKILLING JOHN OR

DPT APPLIED MATHS

\& THEORETICAL PHYSICS

SILVER Street

CAMBRIOGE CB3 9 EW

UK

TEL:

TLF :

TLX:

EML:

COM: 34,48

SKUMANICH ANDRE PROF

HIGH ALTITUDE OBSERVATORY

NCAR

BOX 3000 .

BOULOER CO 803073000

USA

TEL: $303497 \quad 1528$

TLF:

TLX: 45694

EML:

COM: 12,36

\section{SLEZAK ERIC DR}

OCA OBSERV DE NICE

BP 139

F 06003 NICE CDX

FRANCE

TEL: $9200 \quad 3124$

TLF: $92 \quad 003033$

TLX: 470865

EML: SLEZAKARONI51/17499: :SLEZAK.

COM: 28
SITTERLY CHARLOTTE M DR

3711 BRANDYWJNE ST NW

WASHINGTON OC 20016

USA

TEL: 2029669044

TLF:

TLX:

EML:

COM: 12,14

SJOGREN WILLIAM L MR

JPL/CALTECH

MS 264664

4800 DAK GROVE DR

PASADENA CA 91109

USA

TEL: 8183544868

TLF:

TLX: 675421

EML:

COM: 16

SKILLMAN EVAN D OR

DPT OF ASTRONOMY

UNIVERSITY OF MINNESOTA

116 CHURCH ST SE

MINNEAPOLIS MN 55455

USA

TEL: 6126244523

TLF: 6126262029

TLX:

EML: SKILLMAMEASTI.SPA. UMN. EOU

COM: 29,40

SLAOE MARTIN A III DR

JPL

MS 264737

4800 OAK GROVE OR

PASADENA CA 91109

USA

TEL: B18. 3546538

TLF :

TLX:

EML:

COM: 40

\section{SLONIM E M DR}

ASTRONOMICAL INSTITUTE

UZBEK ACADEMY OF SCIENCES

700000 TASHKENT

UZBEKISTAM

TEL:

TLF:

TLX:

EML:

COM: 10 


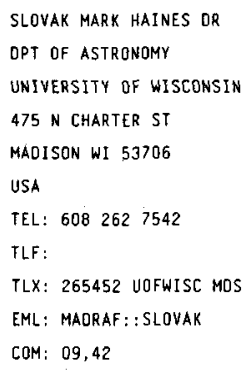

SLYSH VJACHOSLAV I DR SPACE RESEARCH INSTITUTE ACADEMY OF SCIENCES PROFSOJOSNAYA UL 84/32 117810 MOSCOW RUSSIA

TEL:

ILF:

ILX:

EML:

COM: $40,51 \mathrm{C}$

SMIT J A PROF

STERREKUNOIG INSTITUTE BOX 80000

NL 3508 TA UTRECHT NETHERLANDS TEL: 30535200

TLX:

EML:

TLF:

COM:

SMITH BRADFORD A PROF LUNAR \& PLANETARY LAB UNIVERSITY OF ARIZONA TUCSON AZ 85721 USA TEL: 6026216930 TLF:

TLX: 910-952-1143

EML:

COM: $15,16 \mathrm{C}, 44$, WGPSNC

\section{SMITH CRAIG H DR}

OPT PHYSICS UNIV COLLEGE UNIVER OF NEW SOUTH WALES NORTHCOTT OR CAMPBELL ACT 2600 AUSTRALIA

TEL: $62 \quad 68 \quad 8790$

TLF: 62688786

TLX: 62030 ADFADM AA

EML: CRAIGEPHAGFA, PH, ADFA, OZ, AU COM: 34

\section{SMITH F GRAHAM PROF}

NRAL

JOORELL BANK

MACCLESFIELD SKII 9DL. UK

TEL: 47771321

TLX: 36149

EML:

ILF: 47771618

COM: $38 \mathrm{C}, 40,50$
SMAK JOSEPH I PROF COPERNICUS ASTRON CENTER POLISH ACAD OF SCIENCES

UL BARTYCKA IB

PL 00 716 WARSAW

POLAND

TEL: 410041

TLF: $\begin{array}{llll}22 & 41 & 08 & 28\end{array}$

TLX: 813978 ZAPAN PL

EML: camklwauw61,bitnet

COM: $26,27,42$

SMITH ALEX G PROF

OPT OF ASTRONOMY UNIVERSITY OF FLORIOA

211 SSRB

GAINESVILLE FL 32611 USA

TEL: $904392 \quad 6135$

TLF :

TLX:

EML:

COM: 40

SMIJH BRUCE F DR NASA AMES RESREACH ETR MS 2453

IHEORETICAL STUDIES BR MOFFETT FIELD CA 94035 USA

TEL: $415 \quad 6945515$

TLF :

TLX:

EML:

COM: 28

SMITH DEAN F DR

BERKELEY RESEARCH ASS 290 GREEN ROCK DRIVE BOULDER CO 80302 USA

TEL: 3034441922

TLF :

TLX:

EML:

COM: $10,40,49$

SMITH GEOFFREY OR DPT OF ASTROPHYSICS UNIVERSITY OF OXFORO SOUTH PARKS RD OXFORD OX1 $3 R O$ UK

TEL: 865511336

TLF :

TEX:

EML:

COM: 14
SMALDONE LUIGI ANTONIO

DPT DI FISICA

UNIVERSITA DI NAPOLI

MOSTRA D OLTREMARE PAD 19

I 80125 NAPOLI

ITALY

TEL: 017253428

TLF :

TLX: 720320 INFNNA I

EML:

COM: 10

SMITH ANOREW M DR NASA/GSFC

CODE $6 B 1$

GREENBELT MO 20771

USA

TEL: 3012868648

TLF :

TLX:

EML:

COM:

SMITH CHARLES DITTO NATIONAL SOARING MUSEUM R D* 3 HARRIS HILL ELMIRA NY 14903 USA TEL: $607 \quad 734 \quad 3 L 28$

TLF :

TLX:

EML:

COM: 09.25

SMITH ELSKE $\checkmark P$ DR COLL HUMANITIES/SCIENCES VIRGINIA COMMON UNIV 900 PARK AVENUE RICHMOND VA 23284 USA TEL: $804 \quad 257 \quad 1674$ TLF :

TLX:

EML: COM:

SMITH GRAEME H OR LICK OBSERVATORY UNIVERSITY OF CALIFORNIA. SANTA CRUZ CA 95064 USA

TEL:

TLF :

TLX:

EML:

COM: 29,37 


\author{
SMITH HARDING E JR DR \\ CASS \\ UESD \\ c 011 \\ LA JOLLA CA 920930216 \\ USA \\ TEL: 4195344558 \\ ILF : 4195342294 \\ TEX: \\ EML: 27778::HAROING (SPAN) \\ COM: 28,47

SMITH HUMPHRY M
23 NORMANDALE
BEXHILL ON SEA TN39 3LU
UK
TEL: 424214288
TLF:
EML:
COM: 19,31
TLX:

SMITH MYRON A ASST PROF

NTL SCIENCE FOUNDATION

OIV ASTRONOMICAL SCIENCES

1800 G ST NW

WASHINGTON OC 20550

USA

TEL:

TLF:

TLX:

EML:

COH: $27 \mathrm{C}, 29,30$

SMITH RODNEY M OR

DPT OF PHYSICS

UNIVERSITY OF DURHAM

SOLTH RD

OURHAM OHI 3 LE

UK

TEL:

TLF:

TLX:

EML:

COM: 47

SMOLUCHOWSKI ROMAN PROF

ASTRONOMY DPT

UNIVERSITY OF TEXAS

RLM 25314

AUSTIN IX $78712 \quad 1083$

USA

TEL: $512471 \quad 1305$

TLF :

TLX: $910-874-1351$

EML:

COM: 15,16
SMITH HAYWOOD C OR

DPT OF ASTRONOMY

UNIVERSITY OF FLORIDA

211 SSRB

GAINESVILLE FL 32611

USA

TEL: 9043921079

ILF:

TLX:

EML:

COM: 28

SMITH LINDA J

OPT PHYSICS \& ASTRONOMY

UNIVERSITY COLLEGE LONDON

GOWER ST

LONOON WCLE 6BT

UK

TEL: $1713877050 * 788$

FLF :

ILX: 28722 UCPHYS G

EML :

COM: 44

SMITH PETER L DR

CENTER FOR ASTROPHYSICS

HCO/SAO MS 50

60 GAROEN ST

CAMBRIDGE MA 02138

USA

TEL: $617 \quad 4954984$

TLF:

TLX: 921428 SATELLITE CAM

EML:

COM: $12,14 C, 34,44$

SMITH VERNE $\checkmark$ OR

ASTRONOMY DPT

UNIVERSITY OF TEXAS

RLM 15308

AUSTIN TX $78712 \quad 1083$

USA

TEL: $512 \quad 4713351$

TLF:

TLX

EML:

COM: 29

SMOL' KOV GENNADIJ YA OR

SIBIZMIR

ACADEMY OF SCIENCES

664697 IRKUTSK 33

RUSSIA.

TEL:

TLF:

ILX:

EML:

COM: 10,40
SMITH HORACE A

PHYSICS \& ASTRONOMY DPT MICHIGAN STATE UNIVERSITY EAST LANSING MI 48824

USA

TEL: $517353 \quad 6784$

FLF :

TLX:

EML:

COM:

SMITH LINOSEY $F$ DR

1 KENNEDY RD

AUSTINMER NSW 2515

AUSTRALIA

TEL: $4 \quad 2675366$

TLF :

TLX:

EML:

COM:

SMITH ROBERT CONNON DR ASIRONOHY CENTRE

UNIVERSITY OF SUSSEX

FALMER

BRIGHTON BNI $9 Q H$

uk

TEL: $273 \quad 60 \quad 6755 * 3101$

TLF :

TLX: 877159 BHVTKS G

EML:

COM: 35,42

SMITH WM HAYDEN PRQF

DPT OF PHYSICS

WASHINGTON UNIVERSITY

MCDONNEL CTR SPACE SCI

ST LOUIS MO 63130

USA

TEL: $314 \quad 8896574$

TEF:

TLX:

EML

COM: 14

SMOOT III GEORGE F.

LAWRENCE BERKELEY LAB

1 CYCLOTRON RE

BLOG 50230

BERKELEY CA 94720

USA

TEL: $415 \quad 4865237$

TLF

TIX:

EML:

COM: 47
SMITH HOWARD ALAN

NTL AIR \& SPACE MUSEUM SMITHSONIAN INSTITUTION WASHINGTON OC 20560

USA

TEL:

TLF: 2027862262

TLX: 264729

EML:

COM: $34,44,51$

SMITH MALCOLM G DR JOINT ASTRONOMY CENTER UKIRT

665 KOMOHANA ST

HILO HI 96720

USA

TEL: 8089613756

TLF :

TLX: 708-633-135

EML:

COM: 28

SMITH ROBERT G DR

DPT PHYSICS UNIV COLLEGE

UNIVER OF NEW SOUTH WALES

NORTHCOTT OR

CAMPBELL ACT 2600

AUSTRALIA

TEL: $62 \quad 688746$

TLF: 62688786

ILX: ADFADM AA 62030

EML: RGSEPHADFA. PH, ADFA. OZ. AU

COM: 34

SMOLINSKI JAN OR

INSTITUTE OF ASTRONOMY

N COPERNICUS UNIVERSITY

UL CHOPINA $12 / 18$

PL 87100 TORUN

POLAND

TEL:

TLF :

TLX:

EML:

COM: 29

SMRIGLIO FILIPPO PROF ISTITUTO ASTRONOMICO

UNIVERSITA OI ROMA

VIA G M LANCISI 29

I 00161 ROMA

ITALY

TEL: $\delta 84 \quad 42977$

TLF :

TLX:

EML:

COM: 
SMYLIE DOUGLAS E DR

OPT EARTH ATMOSPH SCI

YORK UNIVERSITY

4700 KEELE ST

DOWNSYIEW DN M3J LP3

CANADA

TEL: 4167365245

TLF :

TLX:

EML:

COM: 31

SNEZHKO LEONIO I

SPECIAL ASTROPHYSICAL OBS

ACADEMY OF SCIENCES

NIZHNIJ ARKHYZ

357147 STAVROPOLSKIJ

RUSSIA

TEL: 93513

ILF :

ILX: 297140 ZENIT

EML:

COM: 09,36

\section{SOBERMAN ROBERT $K$ OR}

DPT ASTRON \& ASTROPHYS

UNIV OF PENNSYLVANIA

DAVID RITTENHOUSE LAB

PHILADELPHIA PA 19104

USA

TEL: 2158988176

TLF :

TLX:

EML:

COM: $21,22 C$

SOBOLEVA N S DR

PULKOVO OBSERVATORY

ACADEMY OF SCIENCES

10 KUTUZOV QUAY

196140 ST PETERSBURG

RUSSIA

TEL:

TLF:

TLX:

EML:

COM: 40

SOOERBLDM DAVIOR

SISCI

HOMEWOOD CAMPUS

3700 SAN MARTIN OR

BALTIMORE MD 21218

USA

TEL: 3013384830

TLF :

TLX: 6849101 STSCI

EML:

COM: 29, WGPSNC
SMYTH MICHAEL J OR

ROYAL OBSERVATORY

BLACKFORD HILL

EDINBURGH EHS 3HJ

UK

TEL: $316 \quad 673 \quad 321$

TLX: 72383

EML:

TLF :

COM: 09,25

SNIJDERS MATTHEUS A J DR

ASTRONOMISCHES INSTITUT

WALDHAEUSER STRASSE 64

D 7400 TUEBINGEN 1

GERMANY

TEL: $707 \quad 129 \quad 2486$

TLF :

TLX: 07262714 AIT D.

EML:

COM: 36

SOBIESKI STANLEY OR

NASA/GSFC

CODE 673

GREENBELT MD 20771

USA

TEL:

TLF:

$T \perp X$ :

EML:

COM: 42

SOBOTKA MICHAL DR

ASTRONOMICAL INSTITUTE

CZECH ACAQEMY OF SCIENCES

DNDREJOV OBSERVATORY

CS 25165 ONDREJOV

CZECHOSLOVAKIA

TEL: 20485201

TLF : 20485314

TLX:

EML:

COM: 10

\section{SODERBLOM LARRY DR}

US GEOLOGICAL SURVEY

\section{BRANHC OF ASTROGEOLOGY}

2255 N GEMINI DR

FLAGSTAFF AZ B600I

USA

TEL:

TLF:

TLX:

EML:

COM: 16

\author{
SNEDEN CHRISTOPHER A \\ ASTRONOMY DPT \\ UNIVERSITY OF TEXAS \\ RLM 15308 \\ AUSTIN TX 787121083 \\ USA \\ TEL: $512 \quad 4714461$ \\ TLF: \\ ILX: \\ EML: \\ COM: 29
}

SNOW THEODORE P PROF

CASA CB 391

UNIVERSITY OF COLORADO

BOX 391

BOULDER CO 803090391

USA

TEL: 3034926857

TLF :

TLX

EML

COM: $29,34,44$

SOBOLEV V V OR

ASTRONOMICAL OBSERVATORY.

ST PETERSBURG UNIVERSITY

BIBLIOTECHNAJA PL 2

199178 ST PETERSBURG

RUSSIA

TEL:

TLF:

TLX:

EML:

COM: 34,36

SOBOUTI YOUSEF PROF

OPT OF PHYSICS

UNIVERSITY OF SHIRAZ

BIRUNI OBSERVATORY

SHIRAZ 71459

IRAN

TEL: 7157339

TLF :

TLX:

EML:

COM: $28,33,35$

SOEDERHJELM STAFFAN OR

LUND OBSERVATORY

BOX 43

522100 LUND

SWEDEN

TEL: $46 \quad 10 \quad 7303$

TLF:

TLX: 33199 OBSNOT $S$

EML:

COM: 08,42
SNELL RONALD L

FIVE COLLEGE

RADIO ASTRONOMY OBSERY

B619 LEDERLE DRAD RES TWR

AMHERST MA 01003

USA

TEL: $413 \quad 545 \quad 1949$

TLF :

TLX: 955491

EML:

COM: 34

SNYDER LEWIS E

DPT OF ASTRONOMY

UNIVERSITY OF ILLINOIS

1012 W SPRINGFIELS AVE

URBANA IL 61801

USA

TEL: 2173335530

TLF :

TLX: 910-245-2434

EML:

COM: 15,51

SOBOLEV VLADISLAV M OR

PULKOVO OBSERVATORY

ACAOEMY OF SCIENCES

10 KUTUZOV QUAY

196140 ST PETERSBURG

RUSSIA

TEL: $298-22-42$.

ILF :

ILX

EML:

COM: 12

SOCHILINA ALLA S DR

INST TO THEORET ASTRONOMY

ACADEMY OF SCIENCES

N KUTUZOVA 10

$1911 B 7$ ST PETERSBURH

RUSSIA

TEL: $278-88-98$

TLF

TLX: 121578 ITA SU

EML:

COM: 04

SOFFEL MICHAEL OR

LEHR \& FORSCHUNSBEREICH

THEOR ASTROPHYSIK UNIV

AUF DER MORGNSTELLE IOC

D 7400 TUEB̈INGEN

GERMANY

TEL: 70741292043

TLF: $707 \quad 1295400$

TLX: 726 2867 UTNA D

EML: SOFFELETAT, PHYSIK, UNI-TUEBI

COM: 07,19 


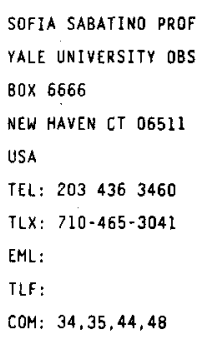

SOFUE YOSHIAKI PROF

INST OF ASTRONONOMY

SOIFER BARUCH T OR

CALTECH

MS 32047

UNIVERSITY OF TOKYO

OSAWA MITAKA

TOKYO 181

JAPAN

TEL: $81422 \quad 413734$

TLF: 81422413749

TEX: 2822307

EML: y.sifyixtansei.cc.u-tokyo.ac. EML:

COM: $34,40,5 \mathrm{~J}$

SOL HELENE OR

OBSERVATOIRE DE PARIS

SECTION DE MEUDON

TAEC

F 92195 MEUDON PPL CDX

FRANCE

TEL: $1 \quad 45 \quad 077428$

TLF:

TLX: 20157

EML: BITHET: SOLERMEUSI

COM:

SOLC MARTIN
DPT ASTRONOMY/ASTROPHYS
CHARLES UNIVERSITY
SVEDSKA 8
CS 150 OO PRAHA 5
CZECHOSLOVAKIA
TEL: 2540395
TLF:
TLX:
EML:
COM: $15,34,41$

\section{SOLIVELLA GLAOYS R LIC}

UNIV NACIONAL DE LA PLATA

FCAG

1900 LA PLATA (BS.AS.)

ARGENTINA

TEL: 21217308

TLF: 2138810

TLX: 31151 BULAP AR

EML: GLADYS\%FCAGLP, EQU AREUUNET . UU

COM: 30

SOMA MITSURU OR
TOKYO ASTRONOMICAL OBS
NAOJ
OSAWA MITAKA
TOKYO 181
JAPAN
TEL: 0422413788
TLF:
TLX: 02822307
EML:
COM: $04,08,20$

DOWNES LAB OF PHYSICS

PASADENA CA 91225

USA

TEL: $818356 \quad 6626$

TLF:

ILX: 675425

EML:

COM:

SOLANKI SAMI K DR

INSTITUT FUER ASTRONOMIE

ETH ZENTRUM

CH 8092 ZUERTCH

SWITZERLAND

TEL: 12563810

TLF: $41 \quad 12512172$

TLX:

EML: SOLANKIUIFA. ETHZ. CH

COM: 10.12

SOLF JOSEF OR

MPI FUER ASTRONOMIE

KOENIGSTUHL

D 6900 HETDELBERg 1

GERMANY

TEL: $62 \quad 21528226$

ILF:

TLX: 461799 MPIA O

EML:

COM:

\section{SOLlazzo CLAUdio}

EUROPEAN SPACE OPERATIONS

CENTER

ROBERT-BOSCH-STR 5

D GlO0 DARMSTADT

gerMANY

TEL: $615 \quad 18861$

TLF:

$T L X: 419453$

EML:

COM:

SOMERVILLE WILLIAM B OR DPT PHYSICS \& ASTRONOMY UNIVERSITY COLLEGE LONDON GOWER ST LONOON WCLE 6BT

uk

TEL: $1713 \quad B 27050$

TLF:

TLX: 28722

EML:

COM: 14,34
SOKOLOWSKI LECH

ASTRONOMICAL OBSERVATORY JAGIELLONIAN UNIVERSITY

UL ORLA 171

PL 30244 KRAKOW

POLAND

TEL: 012223856

TLF:

TLX: 0322723 UJ PL

EML:

COM: 47

SOLARIC NIKOLA

HVAR OBSERVATORY

FACULTY OF GEODESY

KACICEVA 26

YU 41000 ZAGREB

YUGOSLAVIA

TEL: 41521548

TLF :

ILX:

EML:

COM: 08

SOLHEIM JAN ERIK

INST MRTHS \& PHYSICAL SCI UNIVERSITY OF TROMSO

BOX 953

N 9001 TROMSO

NORWAY

TEL: $083-86060$

TLF:

TLX: 64124

EML:

COM: $42,46 \mathrm{NR}$

SOLOMON PHILIP M OR DPT OF EARTH \& SPACE SCI ASTRONOMY PROGRAM

SUNY AT STONY BROOK STONY BROOK NY 117942100 USA

TEL: $516 \quad 246 \quad 8383$

TLF

$T L X: 510-228-7767$

EML:

COM: 33,34

SOMMER-LARSEN JESPER DR NIELS BOHR INSTITUTE BLEgDAMSVEJ 17 DK 2100 COPENHAGEN O DENMARK

TEL: $3142 \quad 1616$

TLF: 31389157

$T L X$

EML:

COM: 
SOMOV BORIS $\checkmark$ OR

STERNBERG SIATE ASTR INST UNIVERSITETSKIJ PROSP 13

119899 MOSCOW

RUSSIA

TEL: $79593916 \quad 44$

TLX: 411483 MGU SU

EML: SNNESAI.MSK.SU

TLF: 795939 Ol 26

COM: 10

SONG JIN-AN

SHAANXI OBSERVATORY

CAS

LINTING XIAN

SHAANXI

CHINA PR

TEL: 332255

TLF :

TEX: 70121 CSAO CN

EMI:

COM: 31

SORENSEN GUNNAR OR

INST OF PHYSICS \& ASTRON

UNIVERSITY OF AARHUS

NY MUNKEGADE

OK 8000 AARHUS C

DENMARK

TEL: $86 \quad 12 \quad 8899$

ILF: $86 \quad 20 \quad 2711$

TLX:

EML:

COM: 14

SOTIROVSKI PASCAL DR

OBSERVATOIRE OE PARIS

SECTION DE MEUDON

F 92195 MEUDON PPL CDX

FRANCE

TEL: $145 \quad 07 \quad 7802$

TLF :

TLX: 270912

EML:

COM: 10,12

SPADA GIANFRANCO DR

TESRE

CNR

VIA DE CASTAGNOLI 1

I. 40126 BOLOGNA

ITALY

TEL: 51519593

TLF:

TLX: 511350 CNR BO

EML:

COM: 44,48
SONETT CHARLES P PROF

LUNAR \& PLANETARY LAB

UNIVERSITY OF ARIZONA

TULSON AZ 85721

USA

TEL: 6026216935

ILF :

TLX: 9109521143

EML:

COM: $16,49 C$

SONG MU-TAO

PURPLE MOUNTAIN OBSERV

CAS

NANUING

CHINA PR

IEL: 2546700

TLF :

TLX:

EML:

COM: 12

SDRENSEN SOREN-AKSEL DR

DPT COMPUTER SCIENCE

UNIVERSITY COLLEGE LONDON

LONDON WCIE GBT

UK

TEL:

TLF:

TLX:

EML:

COM

SOUFFRIN PIERRE B OR

OCA OBSERY DE NICE

BP 139

F 06003 NICE COX

FRANCE

TEL: $93 \quad 890420$

TLF:

TLX: 460004 OBSNICE F

EML:

COM: $12,35,36$

SPADARO DANIELE OR

OSS ASTROFISICO

CITTA UNIVERSITARIA

VIA A DORIA 6

I 95125 CATANIA

ITALY

TEL: 95330533

TLF: 95330592

TLX: 970359 ASTRCT I

EML: 40297: : DANIELE

COM: 10

\begin{tabular}{|c|c|}
\hline SONG DOO JONG OR & SONG GUO-XUAAN \\
\hline ISSA & SHANGHAI OBSERVATORY \\
\hline 36-1 WHAAM DONG & CAS \\
\hline YUSEONG GU & BO NANDAN RD \\
\hline DAEJEON 305348 & SHANGHAI \\
\hline KOREA R & CHINA PR \\
\hline TEL: $42861 \quad 1502$ & TEL: 21386191 \\
\hline TLF: 428615610 & TLF : \\
\hline TLX: 45532 TOTOROK $K$ & $T L X: 33164$ SHAO CN \\
\hline EML: DJSONG\%APISS , ISSA,RE, KReGARAM & EML: \\
\hline COM: 47 & COM: 28,33 \\
\hline
\end{tabular}

SONGSATHAPORN RUANGSAK OR

SONNEBORN GEORGE DR

DPT OF PHYSILS

CHIANG MAI UNIVERSITY

CHIANG MAI 50002

NASA/GSFC

CODE 681

LASP

GREENBELT MD 20771

USA

TEL: 3012863665

TLF:

ILX: 89675

EML: SPAN:647L: : SONNEBORN

COM: 29.44

SORU-ESCAUT IRINA MRS

OBSERVATOIRE DE PARIS

SECTION DE MEUDON

F 92195 MEUDON PPL CDX

FRANCE

TEL: $1 \quad 45 \quad 347530$

TLF :

TLX:

EML:

COM:

EML:

CON: 40

SOULIE GUY

OBSERVATOIRE DE BORDEAUX

BP 89

F 33270 FLOIRAC

FRANCE

TEL: $56 \quad 86 \quad 4330$

TLF: $56 \quad 40 \quad 425$ !

TLX:

EML

COM:

SOWELL JAMES ROBERT OR GEORGIA INSTITUTE

OF TECHNOLOGY

SCHOOL OF PHYSICS

ATLANTA GA 30332

USA

TEL: $404 \quad 8943628$

TLF :

TLX:

EML: JS5BEHYORA.GATECH,EDU

COM: 26

SPARKE LINDA

WASHBURN OBSERVATORY

UNIVERSITY OF WISCONSIN

$475 \mathrm{~N}$ CHARTER ST

MADISON WI 53706

USA

TEL: $608 \quad 262 \quad 3071$

ILF:

ILX:

EML: SPARKEOWISCMAC3 BITNET

COM: 33 
SPARKS WARREN M DR

LoS alamos national LaB

MS F669

BOX 1663

LOS ALAMOS NM 87545

USA

TEL: 5056674922

TiF:

TLX:

EML:

COM: 35,42

SPEER R J DR

OPT OF PHYSICS

IMPERIAL COLLEGE

PRINCE CONSORT RD

LONDON SW7 2BZ

UK

TEL: 15895111

TLF:

TLX: 261503 IMPCOL

EML:

COM: 44

SPICER DANIEL SHIELOS DR

NASA/GSFC

CODE 682

GREENBELT MD 20771

USA

TEL: 3012867334

TLF :

TLX:

EML:

COM: 10,12

SPITE FRANCOIS M DR

OBSERVATOIRE DE PARIS

SECTION DE MEUDON

F 92195 MEUOON PPL COX

FRANCE

TEL: 145077840

TLF:

TLX: 270912

EML:

COM: $05.29,36$

SPOELSTRA T A TH OR
NFRA
BOX 2
NL 7991 PO OWINGELOO
NETHERLANDS
TEL: 52197244
TLX: 42043 SRZM NL
EML:
TLF:
COM: $08,40,50$

SPARKS WILLIAM BRIAN

ROYAL GREENWICH OBS

HERSTMONCEUX CASTLE

HAILSHAM BN27 IRP

UK

TEL: 323 B33. 171

TLX: 87451

EML:

TLF:

COM: 28

SPENCER JOHN HOWARO

NAVAL RESEARCH LABORATORY

CODE 4134

4555 OVERLOOK AVE SW

WASHINGTON DC 203755000

USA

TEL: 2027673050

TLF :

TLX:

EML:

COM: 40

\section{SPIEGEL E DR}

DPT OF ASTRONOMY

COLUMBIA UNIVERSITY

PUPIN HALL 538 W 120TH ST

NEW YORK NY 10027

USA

TEL:

TLF:

TLX:

EML:

COM: $33,35,36$

\section{SPITE MONIQUE OR}

OBSERVATOIRE OE PARIS

SECTION DE MEUDON

F 92195 MEUDON PPL COX

FRANCE

TEL: 145077839

TLF:

TLX: 270912

EML:

COM: 29,36

SPRUIT HENK C OR

\section{MAX PLANCK INSIITUT}

KARLSCHWARSCHILD. STR. I

O 8046 GARCHING MUENCHEN

GERMANY

TEL: 8932990

TLF:

TLX: 524629 ASTRO D

EML:

COM: 10,36
SPARROW JAMES $G$ DR

AERONAUTICAL RESEARCH

LABORATQRIES

4331

MELBOURNe VIC 3001

AUSTRALIA

TEL: 36477623

TLF:

TLX: 39391 ARL AA

EML:

COM: 21

SPENCER RALPH E OR

NRAL

JOORELL BANK

MACCLESFIELO SKII $90 \mathrm{~L}$

uK

TEL: 47771321

ILF :

TLX: 36149 JODREL $G$

EML:

COM: 40

SPIELFIEDEL ARNIE DR

OBSERVATOIRE PARIS

SECTION DE MEUOON

DAMAP

F 92195 MEUDON PPL COX

FRANCE

TEL: $145 \quad 07 \quad 7453$

TLF: $14507 \quad 7469$

TLX: 201571

EML: SPILFILDQFRMEU5:

COM: 14

SPITHAS ELEFTERIOS N DR

DPT OF ASTRONOMY

NTL UNIVERSITY OF ATHENS

PANEPISTIMIOPOLIS

GR 15771 Z0GRAFOS

GREECE

TEL: $1724 \quad 3414$

TLF:

TLX:

EML:

COM:

\section{SPURZEM RAINER DR}

INSTITUT FÜR THEORETISCHE

PHYSIK UND STERNWARTE

OLSHAUSENSTR 40

D 2300 KIEL 1

GERMANY

TEL: 4318801574

TLF: 4318804432

TLX: 292706

EML: PAS2B@RZ, UNI-KIEL. OBP. OE

COM: 37
SPASOVA NEDKA MARINOVA

DPT OF ASTRONOMY

BULGARIAN ACAO SCIENCES

72 LENIN BLVO

BG 1784 SOFIA

BULGARIA

TEL: 2758927

ILF:

ILX:

EML:

COM:

SPERGEL DAVID N DR

PRINCETON UNIVERSITY OBS

PEYTON HALL

PRINCETON NJ 08544

USA

TEL: 6092583589

TLF :

TLX:

EML: DNSEASTRO.PRINCETON. EOU

COM: 33

SPINRAD HYRON PROF

ASTRONOMY DPT

UNIVERSITY OF CALIFORNIA

601 CAMPBELL HALL

BERKELEY CA 94720

USA

TEL: $415 \quad 642 \quad 2078$

TLF:

TLX:

EML:

COM: 15,28

SPITZER LYMAN JR DR

PRINCETON UNIVERSITY OBS

PEYTON HALL

PRINCETON NJ 08544

USA

TEL: 6094523809

TLF:

TLX: 322409

EML:

COM: 34,44

SPYROU NICOLAOS PROF

DPT OF ASTRONOMY.

UNIVERSITY THESSALONIXI

GR 540 OG THESSALONIKI

GREECE

TEL: 31992658

TLF:

ILX: 412181

EML:

COM: 47 


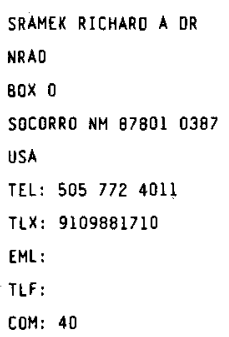

\author{
SREEKANTAN B V'OR \\ TIFR \\ HOMI BHABHA RD \\ COLABA \\ BOMBAY 400005 \\ INDIA \\ TEL: $22 \quad 219111$ \\ TLF: \\ TLK: $011-3009$ \\ EML: \\ COM:
}

SRIVASTAVA RAM KUMAR DR UTTAR PRADESH STATE OBSERVATORY

PO MANORA PEAK 263129 NAINITAL 263129 INDIA

TEL: $5942 \quad 2136$

TLF

TLX:

EML:

COM: 27,42

STAG'G CHRISTOPHER DR DPT OF PHYSICS \& ASTRON UNIVERSITY OF CALGARY 2500 UNIVERSITY OR NW CALGARY AB T2N IN4 CANADA

TEL: $403 \quad 2207423$

TLF: 4032893331

TLX: 03821545

EML: CRSTAGGUNCAMULT

COM: 42

STAHR-CARPENTER M DR

1101 HILL TOP RD CHARLOTTESVILLE VA 22903 USA

TEL: 8042937063

TLF :

EML:

COM: 40

TLX:

STANGA RUGGERO
DPT OI ASTRONOMIA
UNIVERSITA OI FIRENZE
LARGO E FERMI 5
I 50125 FIRENZE.
ITALY
TEL: 5527521
TLF: 55220039
TLX: 572269 ARCETR I
EML:
COM: 34

SREENIVASAN S RANGA PROF OPT OF PHYSICS UNIVERSITY OF CALGARY 2500 UNIVERSITY DR NW CALGARY AB T2N IN4 CANADA

TEL: 4032845385 TLF : $403 \quad 2893331$

TLX:

EML:

COM: 35

STABELL ROLF DR INST THEORET ASTROPHYSICS UNIVERSITY OF OSLO BOX 1029

N O3L5 BLINOERN OSLO 3 NORWAY

TEL: 2-456-530

T.LF:

TLK: 72705 ASTRO N

EML:

COM:

STAGNI RUGGERO

OSSERVATORIO ASTROFISICO

VIA DELL OSSERVATORIO 8

I 36012 ASIAGO

ITALY

TEL: $42 \quad 46 \quad 2665$

TLF:

TLX: 430110 SETOUR I

EML:

COM:

STALIO ROBERTO OR
OPT OI ASTRONOMIA
UNIVERSITA DI TRIESTE
VIA TIEPOLO 11
I 34131 TRIESTE
ITALY
TEL: $40793921 * 221$
TLF:
TLX: 461137 OAT I
EML:
COM: $29,36,51$
STANGE L0THAR
TECHNICAL UNIVERSITY
ORESOEN
MOMMSENSTR 13
D B027 ORESDEN
GERMANY
TEL: 514634652
TLF:
TLX: 02278
EML:
COM: 08

SRINIVASAN G

RAMAN RESEARCH INSTITUTE

SADASHIVANAGAR

BANGALORE 560080

INDIA

TEL: $01236 \quad 0122$

TLF: $812 \quad 340492$

TLX: 8452671 RRI IN

EML:

COM: 48VP

STACEY GOROON J OR

DPT OF ASTRONOMY

CORNELL UNIVERSITY

SPACE SCIEMCES BLDG

ITHACA NY 14853

USA

TEL: 6072555900

TLF: 6072555875

TIX:

EML: STACEYEASTROSUN: TN, EDU COM:

STAHL OTMAR RICHARO OR LANDESSTERNWARTE

KOENIGSTUHL

D 6900 HEIDELBERG 1

GERMANY

TEL: $62 \quad 21509232$

TLF :

ILX: 461153

EML: BITNET:BP2ODHOURZI

COM:

STANDISH E MYLES DR

JPL

MS $264 \quad 664$

4 BOO OAK GROVE OR

PASADENA CA 91109

USA

TEL: 8183543959

TLF

TLX:

EML:

COM: $04 C, 07,20$

STANILA GEORGE DR

MITROPOLIT GRIGORE $2 B$

BOX 28

R 75218 BUCAREST 28

RUMANIA

TLF :

TEL: $23 \quad 6892$

TLX:

EML:

COM: 19,31 


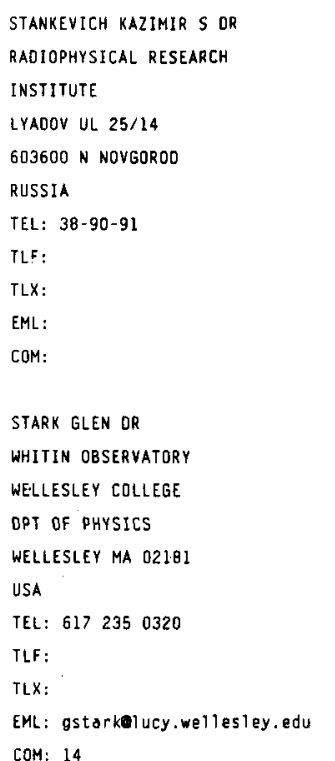

COM: 14
STANLEY G J

COM: 44.48

STAWIKOWSKI ANTONI DR

INSTITUTE OF ASTRONOMY

$N$ COPERNICUS UNIVERSITY

UL CHOPINA $12 / 18$

PL 87100 TORUN

POLAND

TEL:

TLF :

TLX:

EML:

COM: 29

STEEL DUNCAN I OR

UK SCHMIDT TELESCOPE

AAO

PRIVATE BAG

COONABARABRAN NSW 2357

AUSTRALIA

TEL: $68842 \quad 298$

ILF: $68 \quad 426 \quad 282$

$T \perp X$ :

EML: OISEAAOCBN.OZ.AU

COM: $15 \mathrm{C}, 20,22 \mathrm{C}, 42$
BOX 1348

CARMEL VALLEY CA 93924

USA

TEL: 4086592940

TLF :

EML:

COM: 40

TLX:

STARRFIELD SUMNER PROF

OPT OF PHYSICS

ARIZONA STATE UNIVERSITY TEMPE AZ 85287

USA

TEL: 6029653561

TLF :

TLX: 667391 ARIZ ST U TMP

EML:

COM: $27,35,42$

STAUDE HANS JAKOB PH D

MPI FUER ASTRONOMIE

KOENIGSTUHL

D 6900 hetoELBERG 1

GERMANY

TEL: $62 \quad 21528229$

TLF :

ILX: 461789 MPIA D

EML:

COM: 21

STANNARO DAVIO DR

NRAL

JOORELL BANK

MACCLESFIELD SKII 90L

UK

TEL: 47771321

TLF :

TLX: 36149 JOOREL $G$

EML:

COM: 40

STASINSKA GRAZYNA DR OBSERVATOLRE DE PARIS SECTION DE MEUDON

F 92195 MEUOON PPL COX FRANCE

IEL: $145 \quad 07 \quad 7422$

TLF :

TLX: 201571

EML:

COM:

STAUDE JUERGEN OR

ZNTRLINST F ASTROPHYSIK

SONNENOBSERVATORIUM

EINSTEINTURM

GERMANY

TEL:

TLF :

TLX:

EML:

COM: $12 \mathrm{C}$

STECHER THEODORE $P$

NASA/GSFC

CODE 680

GREENBELT MD 20771

USA

TEL: $301286 \quad 8718$

TLF:

TeX:

EML:

COM: $29,44,34$

EML:

COM: 12

STEENMAN-CLARK LOIS DR

OCA OBSERV DE NICE

BP. 139

F 06003 NICE COX

FRANCE

IEL: 93890420

TLF:

TLX: 460004 OBSNICE $F$

EML

COM: 14

STEFANIK ROBERT DR

OAK RIDGE OBSERVATORY

HARVARO SMITHSONIAN CTR

PINNACLE ROAD

HARVARO MA 01451

USA

TEL: 6174957070

TLF :

TLX:

EML: SPAN:cfaz: :stefanik
D 1500 POTSDAM

STARK ANTONY A

AT \& BELL LABORATORIES

HOH L 23!

HOLMOEL NJ 07733

USA

TEL: 2019494842

T!tF:

TLX:

EML:

COM:

STATHOPOULOU MARIA DR

DPT OF ASTROPHYSICS

NTL UNIVERSITY DF ATHENS

PANEPISTIMIOPOLIS

GR 15783 ZOGRAFOS

GREECE

TEL: 17243414

TLF: 17228981

$T L X$ :

EML: MARSTATHEGRATHUN1

COM: 12

STAVELEY-SMITH LISTER DR

CSIRO

PO BOX 76

EPPING NSW 2121

AUSTRALIA

TEL: 28680222

TLF: 28680310

TLX: 26230 ASTRO AA

EML: LSTAVELEEATNF. CSIRO.AU

COM: 28

STECKER FLOYD W DR

NASA/GSFC

CODE 660

LAB HIGH ENERGY ASTROPHYS GREENBELT MO 20771

USA

TEL: 3012866057

TLF :

TLX:

EML:

COM: $33,47,48$

COM: 30
STEFANOVITCH-GOMEZ A E OR OBSERVATOIRE DE PARIS

SECTION DE MEUDON

F 92195 MEUDON PPL CDX

FRANCE

TEL: $1 \quad 45 \quad 07 \quad 7843$

TLF:

TLX: 201571

EML:

COM: 33 
STEFFEN MATTHIAS OR INST FUR THEOR PHYSIK OLSHAUSENSTRASSE 40 O 2300 KIEL 1 germanY

TEL: 4318804101

TLF:

TLX: 292706

EML:

COM: $12,29,36,40$

STEIGER W R PROF

CALTECH SUBMILL OBS

BOX 4339

HILO HI 96720

USA

TEL:

TLF:

TLX:

EML:

COM:

STEIN ROBERT F ASSOC PROF PHYSICS \& ASTRONOMY DPT MICHIGAN STATE UNIVERSITY EAST LANSING MI 48824 USA

TEL: 5173538661

TLF :

TLX:

EML:

COM: 36

STEINERT RLAUS GUENTER OR LORHMANN OBSERVATORY DRESDEN UNIVERSITY OF TEC MOMMSENSTR 13

D 8027 DRESDEN

\section{GERMANY}

TEL: 514634097

TLF: 514637106

TLX: 227日 TEUNI 00

EML:

COM: 24

STEINOLFSON RICHARD S OR SOUTHWEST RESEARCH INST 6220 CULEBRA RO

SAN ANTONIO TX 78228 2510 USA

TEL: 512.5222822

TLF: 5126474325

TLX: 244046

EML: SWRI: RICH/RICHO129.162.150.30 TL:

COM:

EML:

COM: 27,35
STEFL STANISLAV DR

ESO

KARL-SCHWARZSCHILO STR 2

O 8046 gARCHING MUENCHEN

GERMANY

TEL: 8932006243

TLF: 893202362

TLX: 05282820 EO D

EML: ESO: :SSTEFL/SSTEFLOESO.ORG

COM: 29

STEIGMAN GARY PRDF

DPT OF PHYSICS

OHIO STATE UNIVERSITY

174 W IBTH AVE

COLUMBUS OH 43210

USA

TEL: $614292 \quad 1999$

TLF :

TLX: 8104821715

EML:

COM: 47,48

STEIN WAYNE A PROF

SCHOOL OF PHYS \& ASTRON

UNIVERSITY OF MINNESOTA

116 CHURCH ST SE

MINNEAPOLIS MN 55455

USA

TEL: $612 \quad 3739963$

TLF:

TLX:

EML:

COM:

STEINITZ RAPHAEL PROF

DPT OF PHYSICS

BEN GURION UNIVERSITY

BOX 653

BEERSHEVA B4105

ISRAEL

TEL: $57>0985$

TLF:

TLX:

EML:

COM:

STELLINGWERF ROBERT F QR

LOS ALAMOS NATIONAL LAB

MS. F645

$\times$ DIV

LOS ALAMOS NM B7545

USA

TEL: $505 \quad 667 \quad 4370$

TLF:

EML:
STEFL VLADIMIR

OPT THEORET PHYS ASTRO

PURKYNE UNIVERSITY

KOTLARSKA 2

CS 61137 BRNO

CZECHOSLOVAKIA

TEL: 51112

TLF:

TLX:

EML:

COM: 46

STEIMAN-CAMERON THOMAS DR

NASA AMES RESEARCH CTR

MS 2453

THEORETICAL STUDIES BR

MOFFETT FIELD CA 94035

USA

TEL: $415 \quad 6943120$

TLF:

TLX:

EML: SPAN:GAL: : TOMSC

COM: 42

STEINBERG JEAN-LOUIS OR OBSERVATOIRE OE PARIS

SECTION DE MEUDON

F 92195 MEUDON PPL COX

FRANCE

TEL: $145 \quad 077696$

TLF:

TLX: 204464

EML:

COM: 40,44

STEINLE HELMUT OR

MAX PLANCK INSTITUT

EXTRATERRESTRISCHE PHYSIK

D B046 GARCHING MUENCHEN GERMANY

TEL: 8932999470

TLF: 893299569

TLX: 215845 EXTER D

EML: BITNET:HCSODGIPPIS

COM:

STELLMACHER GOETZ

INSTITUT D'ASTROPHYSIQUE

98BIS BD ARAGO

F 75014 PARIS

FRANCE

TEL: $143 \quad 20 \cdot 1425$

ILF: 143298673

TEX:

EML:

COM: 10
STEHLE CHANTAL DR OBSERVATOIRE DE PARIS SECTION DE MEUDON DPT ATOMES \& MOLECULES F 92195 MEUDON PPL CDX FRANCE

TEL: $1 \quad 45 \quad 077453$

TLF :

TLX: 201571

EML:

COM: 14

STEIN JOHN WILLIAM

555 HILL ST

SEHICKLEY PA 15143

USA

TEL: 4127414182

TLF :

TLX:

EML:

COM: $24,26,51$

STEINER JOAO E DR

INPE

CP 515

AV DOS ASTRONAUTAS 1758 $12200 S$ JOSE DOS CAMPOS BRAZIL

TEL: $123 \quad 229977$

TLF:

TLX:

EML:

COM: $42,44,48$

STEINLIN ULI PROF

ASTRONOMISCHES INSTITUT UNIVERSITAET BASEL

VENUSSTRASSE ?

CH 4102 BINNINGEN

SWITZERLAND

TEL: 61227711

TLF :

TLX:

EML:

COM: $25,33,45$

STELLMACHER IRENE OR BUREAL DES LONGITUDES 77 AVE DENFERT ROCHEREAU

F 75014 PARIS

FRANCE

TEL: 143201210

TLF :

$T L X:$

EML:

COM: 07,20 
STENCEL ROBERT EOWARD

CASA

UNIVERSITY OF COLORADO

BOX 391

BOULOER. CO BO309 039?

USA

TEL: 3034927178

ILF :

TLK:

EML:

COM: $29,42,44$

STEPANIAN A A OR

CRIMEAN ASTROPHYS OBS

UKRAINIAN ACAD OF SCIENCE

NAUCHNY

334413 CRIMEA

UKRAINE

TEL: $43 \quad 2945$

ThF :

TLX:

EML:

COM: 28,48

STEPHENS S A DR

TIFR

HOMI BHABHA RD

COLABA

BOMBAY 400005

INOIA

†EL: $22 \quad 219111$

TLF:

TLX: 011-3009 TIFR IN

EML:

COM: 48

\section{STEPINSKI TOMASZ DR}

LUNAR \& PLANETARY INST

3303 NASA RO

HOUSTON TX 77058

USA

TEL: $713486 \quad 2170$

TLX:

EML: LPI : : STEPINSKI

TLF:

COM:

STESHENKO N $\checkmark$ DR
CRIMEAN ASTROPHYS OBS
UKRAINIAN ACAD OF SCIENCE
NAUCHNY
334413 CRIMEA
UKRAINE
TEL: 432945
TLF:
TLX:
EML:
COM: 09,10

STENFLO JAN 0 OR

INSTITUT FUER ASTRONOMIE

ETH ZENTRUM

CH 8092 ZUERICH

SWITZERLAND

TEL: 12563804

TLF: 4112620003

TLX: 817379 EHHG CH

EML: STENFLDIFA.ETHZ.CH

COM: $10,12 \mathrm{P}$

STEPANIAN JIVAN A DR

BYURAKAN ASTROPHYSICAL

OBSERVATORY

378433 BYURAKAN

ARMENIA

TEL: $8852 \quad 283435$

TLF:

TLX:

EML:

COM: 28

STEPHENSON C BRUCE PROF

WARNER \& SWASEY OBS

CASE WESTERN RESERVE UNIV

CLEVELAND OH 44106

USA

TEL: $216 \quad 368 \quad 3728$

TLF :

TLX:

EML

COM: 33,45

\section{STEPPE HANS DR}

IRAM

AVD DIVINA PASTORA 7

BLOQUE 6/2B

E 18012 GRANADA

SPAIN

TEL:

TLF :

TLX:

EML:

COM :

\section{STESHENKO N Y DR}

CRIMEAN ASTROPHYS OBS UKRAINIAN ACAD OF SCIENCE

NAUCHNY

334413 CRIMEA

UKRAINE

TEL: 432945

TLF:

$f(x:$

EML:

COM: 09,10
STENHOLM BJOERN OR

LUHD OBSERVATORY

$B 0 \times 43$

$S 22100$ LUND

SWEQCEN

TEL: $46 \quad 10 \quad 7306$

TLF: $46 \quad 10 \quad 4614$

TLX: 33199

EML: bjorneastro.1u.se

COM: 34,46

STEPANIAN N N DR

CRIMEAN ASTROPHYS OBS

UKRAINIAN ACAD OF SCIENCE

NAUCHNY

334413 LRIMEA

UKRAINE

TEL: 432945

TLF:

TLX:

EML:

COM: 10

STEPHENSON F RICHARD DR

OPT OF PHYSICS

UNIVERSITY OF DURHAM

SOUTH RD

DURHAM OHI $3 L E$

UK

TEL: $9138564971 * 208$

TLF:

TLX: 537351 DURLIB G

EMI:

COM: 19,41

STERKEN CHRISTIAAN LEO DR

IAAG

VRIJE UNIV BRUSSELS

CP 165

B 1050 BRUSSELS

BELGIUM

TEL: 26413469

TLF :

TLX: 61051 VUBCD

EML:

COM: $25 C .27$

STETSON PETER B. DR

HERZBERG INST ASTROPHYS

DOMINION ASTROPHYS OBS

5071 W SAANICH RO

VICTORIA BC V8X $4 M 6$

CAMADA

TEL: 6043390001

TLF : 604363.0045

TLX: 0497295

EML:

COM: 37
STENHOLM LARS

ASTRONOMICAL OBSERVATORY

B0X 515

S 75120 UPPSALA

SWEDEN

TEL: 18112488

TLF: $18 \quad 111853$

TLX: 76024 UNIVUPS 5

EML: stenholmolaban.uv. se

con:

STEPANOV ALEXANDER V DR

CRIMEAN ASTROPHYS OBS

UKRAINIAN. ACAD OF SCIENCE NAUCHNY

334247 KATZIVELY RT 22

UKRAINE

TEL: 727906

TLF :

TLX: 222192 VOSHOD

EML:

COM: IOC

STEPIEN KAZIMIERZ OR

ASTRONOMICAL OBSERVATORY

WARSAW UNIVERSITY

AL UJAZDOWSKIE 4

PL 00478 WARSAW

POLANO

TEL: $29-40-11$

TLF:

TLX:

EML:

COM: 27,36

STERN ROBERT ALLAN LOCKHEED PALO ALTO RES LB OPT 9120 BLOG 255

3251 HANOVER ST

PALO ALTO CA 94304

USA

TEL: $415 \quad 8584072$

TLF

TLX:

EML:

COM: 44

STEVENS GERARD A OR

SPACE RESEARCH LABORATORY

SRON

SORBONNELAAN 2

NL 3584 CA UTRECHT

NETHERLANDS

TEL: 30535600

TLF : $30 \quad 540860$

TLX:

EML:

COM 


\begin{tabular}{|c|c|}
\hline STEWART JOHN MALCOLM DR & STEWART PAUL DR \\
\hline DPT APPLIED MATHS & OPT OF MATHEMATICS \\
\hline \& THEORETICAL PHYSICS & UNIVERSITY OF MANCHESTER \\
\hline SILVER STREET & MANCHESTER M13 9PL \\
\hline CAMBRIDGE CB3 GEW & uk \\
\hline uk & TEL: \\
\hline TEL: 2233351645 & TLF: \\
\hline TLF : & TLX: \\
\hline TLX: & EML: \\
\hline EML: & COM: 40 \\
\hline COM: 47 & . \\
\hline STIAVELLI MASSIMO OR. & STIBBS DOUGLAS W N PROF \\
\hline VIA F CORRIDONI 25 & MOUNT STROMLO \& SIDING \\
\hline I 56100 PISA & SPRING OBSERVATORIES \\
\hline ITALY & PRIVATE BAG \\
\hline TEL: 5048806 & WODEN PO ACT 2606 \\
\hline TLF: & AUSTRALIA \\
\hline EML: STIAVELLEDGAES051/39196: STIAV & TEL: 62881111 \\
\hline COM: 28 & TLF: 62490233 \\
\hline TLX: & TLX: 62270 CANOPUS AA \\
\hline & EML: DWNSEMO.ANU, EOU.AU \\
\hline
\end{tabular}

STIFT MARTIN JOHANNES OR INSTITUT FUER ASTRONOMIE TUERKENSCHANZSTR 17

A 1180 WIEN

AUSTRIA

TEL: 134536096

TLF:

TLX:

EML:

COM:

STOBIE ROBERT S OR

ROYAL OBSERVATORY

BLACKFORO HILL

EOINBURGH EHG 3 HJ

UK

TELL: $316 \quad 673321$

TLX: 72383 ROEOIN G

EML:

TLF:

COM: 27

\begin{tabular}{|c|c|}
\hline STOEGER WILLIAM R OR & STOEV ALEXEI \\
\hline SPECOLA VATICANA & PEOPLE'S ASTRONOMICAL OBS \\
\hline I OOLI20 VATICAN CITY & \& PLANETARIA IN BULGARIA \\
\hline VATICAN CITY STATE & YURI GAGARIN \\
\hline TEL: $6 \quad 698 \quad 3411$ & BG 6000 STARA ZAGORA \\
\hline TLF: & BULGARIA \\
\hline EML: & TEL: $42 \quad 43 \quad 183$ \\
\hline COM: 47 & TLF: \\
\hline TLX: 504-2020 VATOBS VA & TLX: \\
\hline & EML: \\
\hline
\end{tabular}

STINEBRING DANIEL R
OPT OF PHYSICS
PRINCETON UNIVERSITY
JADWIN HALL
PRINCETON NJ 08544
USA
TEL: 6094525578
TLF:
TLX:
EML:
COM:

STOCK JURGEN O
CIOA
BOX 264
MERIOA 5101 A
VENEZUELA
TEL: 74639930
TLX: 74174
EML:
TLF:
COM: $24[, 25,30,45$

\author{
STEWART RONALD T MR \\ CSIRO \\ DIVISION OF RADIOPHYSICS \\ BOX 76 \\ EPPING NSW 2121 \\ AUSTRALIA \\ TEL: 28680222 \\ TLF: \\ TLX: 26230 \\ EML: RSTEWART TEATNF. CSIRO.AU \\ COM: 10,40 \\ STICKLAND DAVIO J DR \\ RUTHERFORD APPLETON LAB \\ SPACE \& ASTROPHYSICS OIV \\ BLOG R25/R68 \\ CHILTON OIOCOT OXII DOX \\ UK \\ TEL: $235 \quad 21900$ \\ TLF : \\ TLX: 83159 \\ EML: \\ COM: \\ STEYAERT HERMAN PROF DR \\ STERREKUNDIG OBSERV \\ RIJKSUNIVERSITEIT GENT \\ KRIJGSLAAN 28! \\ B 9000 GENT \\ BELGIUM \\ TEL: $91 \quad 225715 \star 2572$ \\ TLF: 91240634 \\ TLX: \\ EML: \\ COM: \\ STIER MARK $T$ \\ PERKIN ELMER CORP \\ MS 897 \\ 100 WOOSTER HEIGHTS RD \\ DANBURY CT 068107859 \\ USA \\ TEL: 2037975708 \\ TLF: \\ TLX: 965954 \\ EML: \\ COM: 44
}

STIRPE GIOVANNA M OR

OSS ASTRONOMICO

UNIVERSITA OI BOLOGNA

VIA ZAMBONI 33

I 40126 BOLOGNA

ITALY

TEL: 51259301

TLF: 51259407

TLX:

EML: STIRPEAASTBO3.CINECA. IT

COM: 28

STOCKMAN HERVEY S JR DR

STSCI

HOMEWOOO CAMPUS

3700 SAN MARTIN DR

BALTIMORE MO 21218

USA

TEL: 3013384820

TLF:

TLX: 6849L0। STSCI UW

EML:

COM: $25,44,48$

STOHL JAN OR

ASTRONOMICAL INSTITUTE

SLOVAK ACADEMY SCIENCES

OUBRAVSKA' 9

CS 84228 BRATISLAVA

CZECHOSLOVAKIA

TEL: 7375157

TLF:

TLX: 09จ355

EML:

COM: $15.22 \mathrm{P}$
STIX MIEHAEL OR

KIEPENHEUER-INSTITUT

FUER SONNENPHYSIK

SCHOENECKSTR 6

O 7800 FREIBURG BREISGAU

GERMANY

TEL:

TLF:

TLX: 7721552 KIS D

EML:

COM: 10,12

STOCKTON ALAN N OR

INSTITUTE FOR ASTRONOMY

UNIVERSITY OF HAWAII

2680 WOODLAWN DR

HONOLULU HI 96822

USA

TEL:

TLF :

TLX:

EML:

COM:

\section{STOKER PIETER H}

COSMIC RAY RESEARCH UNIT POTCHEFSTROOM UNIVERSITY POTCHEFSTROOM 2520

SOUTH AFRICA

TEL: 27-1481-25360

TLF:

TLX: 421363 I

EML:

COM: 10 


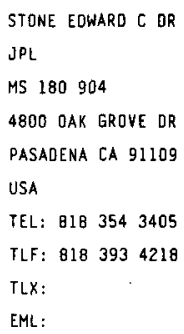

STONE R G DR

NASA/GSFC

CODE 690

LEP

GREENBELT MD 20771

USA

TELL: $301286 \quad 8631$

ILF:

ILX: 710-82089716

EML:

COM: 40.44 .49

STOREY JOHN W $V$ OR

DPT OF PHYSICS

UNIV NEW SOUTH WALES

BOX 1

KENSINGTON NSW 2033

AUSTRALIA

TEL: 26974591

TLF :

TLX: $26054 A A^{\circ}$

EML:

COM: 09

\section{STRAND KAJ AA DR 3200 ROWLAND PL NW WASHINGTON DC 20008 USA \\ TEL : $202966 \quad 0495$ \\ TLF : \\ EML \\ COM: 24,26 \\ TLX:}

STREL'NITSKIJ VLAOIMIA OR INST OF ASTRONOMY ACABEMY OF SCIENCES PYATNITSKAYA UL 48 109017 MOSCOW

\section{RUSSIA}

TEL: $231-54-61$

TLF:

TLX: 412623 SESTP SU

EML:

COM: 14

STROHMEIER WOLFGANG PROF VOLKFELLSTR 5

O B600 8AMBERG GERMANY

TEL: 95155394

TLF:

EML:

COM: $25,27,42$

TLX:
STONE REMINGTON $P S$ OR

LICK OBSERVATORY

MOUNT HAMILTON CA 95140

USA

TEL: $408 \quad 274 \quad 1809$

ILF:

ILX:

EML:

COM: 25,28

STOREY MICHELLE OR

DEPT THEORETICAL PHYSICS UNIVERSITY OF SYONEY SYDNEY NSW 2006 AUSTRALIA

TEL: 26922538

TLF: 26602903

TLX:

EML:

COM: 40

STRASSL HANS L PROF

ASTRON INST UNIV MUENSTER DOMAGKSTR 75

- 4400 muENSTER

GERMANY

TEL: 251 B6 2463

TLF :

TLX:

EML:

COM:

STRITTMATTER PETER A PROF STEWARD OBSERVATORY UNIVERSITY OF ARIZONA TUCSON AZ 85721 USA

TEL: $602 \quad 6216532$

TLF :

TLX:

EML:

COM: 35

STROM KAREN M
OPT OF ASTRONOMY
UNIV OF MASSACHUSETTS
GRC 518 B 6732
AMHERST MA 01003
USA
TEL: 4135452290
TLF:
TLX:
EML:
COM: 27

STONE RONALQ CECIL US NAVAL OBSERVATORY FLAGSTAFF STATION $B O \times 1149$ FLAGSTAFF AZ $86002^{\circ}$ USA TEL: 6027795132 TLF :

TLX:

EML:

COM: 08,24

STRAFELLA FRANCESCD DPT DI FISICA UNIVERSITA DI LECCE CP 193

I 73100 LECCE

ITALY

TEL: $832627 / 247$

TLF :

TLX: $860 B 30$ UNSTLE I

EML:

COM

STRASSMEIER KLAUS G OR

INSTITUT FUER ASTRONOMIE UNIVERSITY OF VIENNA TUERKENSCHANZSTR 17

A 1180 WIEN

AUSTRIA

TEL: I 34031695

TLF: 134031615

TLX: 133099

EML: STRASSMEIEREAVIA. UNA.AC.AT

COM: 27

STROBEL ANDRZEJ OR INSTITUTE OF ASTRONOMY N COPERNICUS UNIVERSITY UL CHOPINA $12 / 18$ PL 87 100 TORUN POLAND

TEL: 250-18

TLF :

TLX: 0552234 ASTR PL

EML:

COM: 33,45

SIROM RICHARD G QR

NFRA

BOX 2

NL 7990 AA OWINGELOD NETHERLANDS

TEL: 52197244

TLF :

TLX: 42043 SRZM NL

EML:

COM: $28,34,40$ 
STROM ROBERT G PROF

LUNAR \& PLANETARY LAB

UNIVERSITY OF ARIZONA

TUCSON AZ 85721

USA

TEL: $602 \quad 6212720$

TLF :

TLX: 9109521143

EML:

COM: 16,28

\section{STRONG XEITH T DR}

LOCKHEEO PALO ALTO RES LB

DPT 9130 BLOG 255

3251 HANOVER ST

PALO ALTO CA 94304

USA

TEL: $415 \quad 3545136$

TLF: $415 \quad 424 \quad 3333$

TLX: 346409 LMSC

EML:

COM: 10

\section{STRYKER LINOA L}

ASTRONOMY OPT

ARIZONA STATE UNIVERSITY

$B O X 37100$

PHOENIX AZ 85069

USA

TEL: 6025436000

TLF :

$T L X$ :

EML: STRYKEROASUCPS, BITNET

COM:

STUTZI JUERGEN DR
I PHYSIKALISCHE INSTITUT
UNIVERSTAET KOELN
ZUELPICHERSTR 77
D 5000 KOELN 41
GERMANY
TEL: $2214703494 / 3567$
TLF: 2214705162
TLX:
EML: STUTZKIOPHI, UNI-KOELN. DE
COM:

SU QING-RUI. DR

PURPLE MOUNTAIN OBSERV

CAS

NANJING

CHINA PR

TEL: $25 \quad 64 \quad 2817$

TLF: $25 \quad 30 \quad 1459$

TLX:

EML:

COM: 10
STROM STEPHEN E

DPT PHYSICS \& ASTRONOMY

UNIV OF MASSACHUSETTS

GRC 518 B

AMHERST MA 01003

USA

TEL: $418 \quad 545 \quad 2290$

TLF :

TLX:

EML:

COM: 27,36

STRUBLE MITCHELL $F$ OPT ASTRON \& ASTROPHYS UNIV OF PENNSYLIVANIA DAVIO RITTENHOUSE LAB PHILADELPHIA PA 19104 USA .

TEL: $215 \quad 2438176$

TLF:

ILX:

EML:

COM: 47

STUMPFF PETER PROF DR

MPI FUER RADIOASTRONOMIE

AUF DEM HUEGEL 69

D 5300 BONN 1

GERMANY

TEL: 228525360

TLX: 886440

EML:

TLF :

COM:

SU BUMEI

YUNNAN OBSERVATORY

CAS

BoX 110

KUNMING 72946 YUNNAN

CHINA PR

TEL: 8712035

TLF :

TLX: 64040 YUOBS CN

EML:

COM: 34

SU WAN-ZHEN

PIJRPLE MOUNTAIN OBSERV

CAS

NANUING

CHINA PR

TEL: 2533583

TLF:

TLX: 34144 PMONJ CN

EML:

COM: 44
STRONG IAN B OR

STRÓNG JOHN D PROF

LOS ALAMOS NATIONAL LAB

MS 436

BOX 1663

LOS ALAMOS 'NM 87545

USA

TEL: $505 \quad 667 \quad 4823$

TLF :

TLX:

EML:

COM: 48

STRUCK-MARCELL CURTIS J

OPT OF PHYSICS

IOWA STATE UNIVERSITY

AMES IA 50011

USA

TEL: 5152945440

TLF :

TLX:

EML:

COM:

STURCH CONRAD R DR

STSCI/ CSC

HOMEWOOD CAMPUS

3700 SAN MARTIN OR

BALIIMORE MO 21218

USA

TEL: 3013384856

TLF:

TLX:

EML:

COM: 33

SU DING-QIANG

NANJING ASTRONOMICAL

INSTRUMENT FACTORY

JIANGSU PROVINCE

CHINA PR

TEL: 41191

TLF :

TLX:

EML:

COM: 09

SUBRAHMANYA C R

TATA INST OF FUNDAMENTAL

RESEARCH

POONA UNIVERSITY CAMPUS

PUNE 411007

INDIA

TEL: 212337107

TLF: 212335760

TLX: 0145658 GMRT IN

EML: uunet!shakt 1 ! gmrt !crs

COM: 47

STRUKOV IGOR A OR
ASTRON RESEARCH FACILITY

UNIV OF MASSACHUSETTS

AMHERST MA 01003

USA

IEL:

ILF:

$T L X:$

EML:

COM: 16

SPACE RESEARCH INSTITUTE

ACADEMY OF SCIENCES

PROFSOJOSNAYA UL $84 / 32$

117810 MOSCOW

RUSSIA

TEL: 3331466

TLF :

TLX: 411498 STAR SU

EML:

COM: 40,47

STURROCK PETER A PROF

CTR FOR SPACE SCIENCES \&

ASTROPHYSICS

STANFORD UNIV ERL

STANFORO CA 943054055

USA

TEL: $415723 \quad 1438$

TLF :

TLX: 3484 STANFRB STNU

EML:

COM: $10,48,49,5]$

SU HONG-JUN

PURPLE MOUNTAIN OBSERV

CAS

NANUING

CHINA PR

TEL: 2536967

TLF:

TLX: 34144 PMONT CN

EML:

CON: 28

SUBRAHMANYAM $P \vee D R$

DPT OF ASTRONOMY

UNIVERSIIY OF OSMANIA

HYDERABAO 50000 ?

INDIA

TEL: $71951 * 247$

TLF :

TLX:

EML:

COM: 28 
SUBRAMANIAN KANDASWAMY DR

TATA INST OF FUNDAMENTAL

RESEARCH

\section{POONA UNIVERSITY CAMPUS}

PUNE 411007

INDIA

TEL: $212 \quad 33 \quad 7107$

TLF: 212335760

TLX: 0145658 GMRT IN

EML: uunet!shakti!gmrt!kandu

COM:

SUGAWA CHIKARA OR
HANANOI $1586-25$
KASHIWA SHI
CHIBA KEN 277
JAPAN
TEL: 471333825
TLF:
TLX:
EML:
COM: 19

SUH KYUNG-WON DR
DPT ASTRONOMY \& SPACE SCI
CHUNGBUK NTL UNIVERSITY
CHEONGJU 360763
KOREA R
TEL: 431612315
TLF: 431674232
TLX:
EML: KWSUHФCBUCC.CBNU,AC, KR
COM: 34

SULLIVAN DENIS JOHN DR DPT OF PHYSICS

VICTORIA UNIVERSITY

PRIVATE BAG

WELLINGTON

NEW ZEALAND

TEL: 721000

TLF :

$T\lfloor X:$

EML:

COM: 25

SUN JIN

OPT OF ASTRONOMY

BEIJING NORMAL UNIVERSITY

BEIJING 100875

CHINA PR

TEL: $1656531 * 6285$

TLF:

TLX:

ENL:

COM: 34
SUDA KAZUO PROF

ASTRONOMICAL INSTITUTE

TOHOKU UNIVERSITY

SENDAI AOBA

MIYAGI 980

JAPAN

TEL: $0222-22-1800$

TLF:

TLX:

EML:

COM: 35

SUGIMOTO DAIICHIRO PROF

OPT EARTH SCI \& ASTRON

UNIVERSITY OF TOKYO

KOMABA

MEGUROKU TOKYO 153

JAPAN

TEL: $03467 \quad 1171$

ILF: 34653925

TLX: 2426728 TGOAIK J

EML: B89761\%TANSEI.CCU-TOKYO.JUNET EML: 04300003PNKUDPC.BITNET

COM: $35,37,42$

SUKARTADIREDJA DARSA
PLANETARIUM ORI
JL CIKINI RAYA 73
JAKARTA 10330
INDONESIA
TEL: 21377.530
ILF:
TLX:
EML:
COM: 10.46

SULLIVAN WOODRUFF T III

DPT OF ASTRONOMY

UNIVERSITY OF WASHINGION

FM 20

SEATTLE WA 98195

USA

TEL:

TLF :

TLX:

EML:

COM: $28,40,41,51$

\section{SUN KAI}

DPT OF GEOPHYSICS

BEIJING UNIVERSITY

BEIJING 100071

CHINA PR

TEL:

TLF:

TLX: 22239 PKUNI CN

EML:

COM: 10
SUEMATSU YOSHINORI OR

TOKYO ASTRONOMICAL OBS

NAOJ

OSAWA MITAKA

TOKYO 181

JAPAN

TEL: $81422 \quad 413705$

TLF: 81422413700

EML: SUEMATSUESPOT.MTK.NAO.AC.JP

COH: 12

SUGITANI KOJI DR

COLL OF GENERAL EDUCAT

NAGOYA CITY UNIVERSITY

MIZUHO KU

NAGOYA 467

JAPAN

TEL: $81 \quad 52 \quad 872 \quad 5846$

TLF: $81 \quad 52882 \quad 3075$

TLX:

COM: 40

SUKUMAR SUNDARAJAN OR

DOMINION RADIO ASTROPHYS

OBSERVATORY

BOX 248

PENTICTON BC V2A $6 K 3$

CANADA

IEL: 6044932277

ILF: 6044937767

YLX: 04888127

EML:

COM: 40

SULTANDV G F ACAD

SHEMAKHA ASTROPHYSICAL

OBSERVATORY

AZER ACADEMY OF SCIENCES

373243 SHEMAKHA

AZERBAIOZHAN

TEL:

TLF :

TLX:

EML:

COM: 07.20

SUN WEI-HSIN DR

INST PHYSICS \& ASTRONOMY

NTL CENTRAL UNIVERSITY

CHUNG LI

TAIWAN

CHINA R

TEL: $3422 \quad 7151: 5302$

TLE: 34251175

TLX:

EML: SUN@PHYAST.DNET.NCU.EDU.TW

COM: 44
TLX: 02822307 TAOMTK J
SUESS STEVEN T DR

NASA/MSFC

CODE ES 52

SPACE SCIENCE LAB

HUNTSVILLE AL 35812

USA

TEL: 2054532824

TLF:

TLX:

EML:

COM: $49 \mathrm{C}$

SUGIYAMA NAOSHI DR

DPT OF PHYSICS

UNIVERSITY OF TOKYO

HONGO 7-3-1 BUNKYO-KU

KYOTO 113

JAPAN

TEL: $8133812 \quad 2111 * 4191$

TLF: 81356890465

TLX: 23472 UTPHYSIC J

EML: 41610 : : NSUGIYAMA

COM: 47

SULENTIC JACK $W$ OR

DPT OF PHYSICS \& ASTRON

UNIVERSITY OF ALABAMA

BOX 1921

TUSCALOOSA AL 354870324

USA

TEL: 2053485050

TLF:

TLX: 8107295845

EML: JSULENTICEUAIVM

COM: 28

SUMMERS HUGH $P$ DR

JET JOINT UNDERTAKING

CULHAM LABORATORY

ABINGOON OXI4 3EA

UK

TEL: $23528 \quad 822$

TLF:

TLX: 837505 JETEUR $G$

EML:

COM: 14

SUN YI-SUI

DPT OF ASTRONOMY

NANJING UNIVERSITY

NANJING

CHINA PR

TEL: 2537551

TLF :

TLX: 34151 PRCNU CN

EML:

COM: 07 


SUN YONGXIANG
INSTITUTE OF GEOOESY \&
GEOPHYSICS
XU DONG LU
WUHAN
CHINA PR
TEL:
TLF:
TLX:
EML:
COM: 19

SUNYAEY RASHID A DR SPACE RESEARCH INSTITUTE ACADEMY OF SCIENCES PROFSOJUSNAYA UL 84/32 117010 MOSCOW RUSS.IA

TEL: 70952302585 TLF: 7095310,7023 FL.X: 411498 STAR SU EML:

COM: $44,47,48 C$

SUTHERLAND WILLIAM OR
DPT OF ASTROPHYSICS
UNIVERSITY OF OXFORD
KE8LE RD
OXFORD OXI 3RH
UK
TEL: 865273310
TLF: 865273418
TLX: 83295 NUCLOX G
EML: WUS UK. AC. OX.ASTRO/1946
COM: 47
SVECHNIKOVA MARIA A OR
OPT OF ASTRONOMY
URALSKIJ STATE UNIVERSITY
620O83 SVEROLOVSK
RUSSIA
TEL:
TLF:
TLX:
EML:
COM: 42

SVOLOPOULOS SOTIRIOS PROF DPT OF ASTROPHYSICS NTL UNIVERSITY OF ATHENS PANEPISTIMIOPOLIS GR 15771 2OGRAFOS GREECE

TEL: 17243414

TLF :

TLX:

EML:

COM: $29,33,41$

\author{
SUNDELIUS BJOERN OR \\ DPT OF ASTRONOMY \\ CHALMERS TECHNICAL UNIV \\ 541296 GOETEBORG \\ SWEDEN \\ TEL: $31 \quad 72 \quad 3141$ \\ TLF: \\ TLX: \\ EML: BJORN@OSO. CHALMERS. SE \\ COM: 28
}

SURDEJ JEAN M G
INSTITUT D'ASTROPHYSIQUE
UNIVERSITE DE LIEGE
AVE COINTE 5
B 4000 COINTE-LIEGE
BELGIUM
TEL: 41529980
TLF: 41527474
TLX: 41264 ASTRLG B
EML:
COM: 15,47

SUTO YASUSHI OR

UJI RESEARCH CENTER

YUKAWA INST THEORICAL PHY KYOTO UNIVERSITY

UJI 611

JAPAN

TEL: $81 \quad 774 \quad 20 \quad 7432$

TLF: $81774 \quad 33 \quad 6226$

TLX:

EML: SUTO@PNKEKVX

COM: 47

SVENSSON ROLANO
STOCKHOLM OBSERVATORY
S $133 \quad 36$ SALTSJOEBAOEN.
SWEDEN
TEL: 8164472
TLF: 87174719
EML: SVENSSONOASTRO.SU.SE
COM: 48
TLX:

\section{SVOREN JAN}

ASTRONOMICAL INSTITUTE

SLOVAK ACADEMY SCIENCES

CS 05960 TATRANSKA LOMNI

CZECHOSLOVAKIA

TEL: $969967866 / 7 / 8$

Tif: 969967656

TLX: 78277 AU SAV CS

EML:

COM: 15,20
SUNOMAN ANITA OR

STOCKHOLM OBSERVATORY

S 13336 SALTSJOEBADEN SWEDEN

TEL: $87 \quad 17 \quad 0634$

TLF: $B 7174719$

TLX: 12972 SWEDEN

EML:

COM: 41,42

SUTANTYO WINAROI

BOSSCHA OBSERVATORY

LEMBANG 4039!

INDONESIA

TEL: 2296001

TLF :

TLX:

EML:

COM:

SUZUKI YOSHIMASA PROF

23-I NAKAJIMA

HIRONOMACH I

UJI 611

JAPAN

TEL:

ILF:

ILX:

EML:

COM: 34

SVESTKA JIRI OR

OBS AND PLANETARIUM

PETRIN 205

CS 11846 PRAHA 1

CZECHOSLOVAKIA

TEL: 25353513

ILF:

TLK:

EML:

COM: 22.46

SWANENBURG B N DR

SRL

HUYGENS LAB

$\mathrm{BO} \times 9504$

NL. 2300 RA LEIDEN

NETHERLANDS

TEL: 71272727

TLF :

TLX: 39058 ASTRO NE

EML:

COM:
SUNTZEFF NICHOLAS B

CERRO TOLOLO

INTERAMERICAN OBSERVATORY

CASILLA 603

LA SERENA

CHILE.

TEL: $51 \quad 213352$

TLF: $51212466 * 342$

TLX: 620301 AURA CT

EML:

COM: 29

SUTHERLAND PETER G OR

DPT OF PHYSICS

MCMASTER UNIVERSITY

HAMILTON ON LBS $4 M 1$

CANADA

TEL: 4165259140

TLF: 4165461252

TLX: 0618347

EML:

COM:

SVALGAARO LEIF DR

HERTOGENLAAN 31

83202 LUBBEEK

BELGIUM

TEL:

TLF:

TLX:

EML:

COM:

\section{SVESTKA ZOENEK OR}

SPACE RESEARCH LABORATORY

SRON

SORBONNELAAN 2

NL 3584 CA UTRECHT

NETHERLANDS

TEL : $30 \quad 535600$

TLF: 30540860

TLX: 47224 ASTRO NL

EML:

COM: 10,12

SWANK JEAN HEBB

NASA/GSFC

CODE 661

GREENBELT MD 20771

USA

TEL: $301285 \quad 6188$

TLF :

TLX: 89675 NASCOM GBLT

EML:

COM: 48 
SWARUP GOVIND PROF

TATA INST OF FUNDAMENTAL

RESEARCH

POONA UNIVERSITY CAMPUS

PUNE 411007

INDIA

TEL: 212336111

TLF : 212335760

TLX: 0145658 GMRT IN

EML: uunet ! shakt 1 !gmrt!gswarup

COM: 38,40

SWENSON GEORGE W JR PROF

OPT ELECT \& COMPUTER ENG

UNIVERSITY OF ILLINOIS

\section{W GREEN ST}

URBANA IL 61801

USA

TEL: $217333 \quad 4498$

TLF:

TLX:

EML:

COM: 40

SWINGS JEAN-PIERRE DR

INSTITUT D'ASTROPHYSIQUE

UNIVERSITE DE LIEGE

AVE COINTE 5

B 4000 COINTE-LIEGE

BELGIUM,

TEL: 41529980

TLF: $4152 \quad 7474$

TLX: 41264 ASTRLG B

EML:

COM: $09,14,29, \mathrm{EC}$

\section{SYKORA JULIUS OR}

ASTRONOMICAL INSTITUTE

SLOVAK ACADEMY SCIENCES

CS 059 60 TATRANSKA LOMN

CZECHOSLOVAKIA

TEL: $969967866 / 7 / 8$

TLF : 969967656

TLX: 78277 AUSAV CZ

EML:

COM: 10

SZABADOS LASZLO PH D

KONKOLY OBSERVATORY

THEGE U 13/17

B0X 67

H 1525 BUDAPEST

HUNGARY

TEL: $175 \quad 5866 / 75 \quad 4122$

TLF

TLX: 227460 KONOBH

EML:

COM: $26,27,42$
SWEET PETER A PROF

OPT OF ASTRONOMY

UNIVERSITY OF GLASGOW

GLASGOW G12 BQW

UK

IEL: $413398 \quad 855$

TLF:

ILX:

EML :

COM: 35

SWENSSON JOHN W DR

OPT OF THEORETICAL PHYS

UNIVERSITY OF LUND

SOELVEGATAN IA A

S 22362 LUND

SWEDEN

TEL: 10969686

TLF:

TLX:

EML:

COM: 12,29

SYGNET JEAN FRANCOIS DR

INSTITUT O'ASTROPHYSIQUE

98BIS BO ARAGO

F 75014 PARIS

FRANCE

TEL: 143201425

TLF; 143298673

TLX:

EML: SYGNETEFRIAP5I

COM: 33

SYLWESTER BARBARA OR

ASTRONOMICAL INSTITUTE

WROCLAW UNIVERSITY

UL KOPERNIKA 11

PL 51622 WROCLAW

POLANO

TEL: 483238

TLF :

TLX: 0712791 UWRPL

EML:

COM: 10

SZAFRANIEC ROZALIA DR

UL KOPERNIKA 27

PL 31501 KRAKOW

POLAND

TEL:

TLF:

EML:

COM: 42

TLX:
SWEIGART ALLEN V OR

NASA/GSFC

CODE 681

GREENBELT MD 20771

USA

TEL: $301286 \quad 6274$

TLF :

TLX: 89675

EML:

COM: 35

SWERDLOW NOEL PROF

ASTRONOMY \& ASTROPHYS CTR

UNIVERSITY OF CHICAGO

5640 S ELLIS AVE

CHICAGO IL 60637

USA

TEL: 3129627969

TLF:

TLX:

EML:

COM: 41

SYKES MARK VINCENT OR

STEWARO OBSERVATORY

UNIVERSITY OF ARIZONA

TUCSON AZ 85721

USA

TEL: $602 \quad 6212054$

TLF:

$T L X: \mathbf{4 6 7 1 7 5}$

EML: MSYKESEAS. ARIZONA. EDU

COM: 15

SYLWESTER JANUSZ

ASTRONOMICAL INSTLITUTE

WROCLAW UNIVERSITYY

UL KOPERNIKA 11

PL 51622 WROCLAW

POLAND

TEL: 481801

TLF :

TLX:

EML:

CDM: 10

SZALAY ALEX DR

DPT ATOMIC PHYSICS

EOTVOS UNIVERSITY

PUSHKIN U 5-7

H 1088 BUDAPEST

HUNGARY

JEL:

FLF :

TLX:

EML:

COM: 470
SWEITZER JAMES STUART OR

ASTRONOMY \& ASTROPHYS CTR

UNIVERSITY OF CHICAGO

5640 S ELLIS AVE

CHICAGO IL 60637

USA

TEL: 3127027783

TLF: 3127028212

TLX:

EML: JSS®ODDJOB. UCHICAGO, EDU

COM:

SWIHART THOMAS L DR

STEWARO OBSERVATORY

UNIVERSITY OF ARIZONA

TUCSON AZ 85721

USA

TEL: 6026216525

TLF :

TLX:

EML:

COM: 36

SYKES-HART AVRIL B OR DPT OF ASTROPHYSICS

UNIVERSITY OF OXFORD

SOUTH PARKS RO

OXFORO DXI 3RO

UK

TEL:

TLF:

TLX:

EML:

COM:

SYNNOTT STEPHEN P

JPL

MS 264686

4800 OAK GROVE OR

PASADENA CA g1109

USA.

TEL: B1B 3546933

TLF :

TLX:

EML:

COM: 16.20

SZATMARY KAROLY DR

JATE UNIVERSITY

DPT OF PHYSICS

DOM TER 9

H 6720 SZEGED

HUNGARY

TEL: $36 \quad 62 \quad 11622$

TLF: $36 \quad 62 \quad 12921$

TLX:

EML: H2674SZAEELLA.HU

COM: 27 


\author{
SZEBEHELY VICTOR G PROF \\ AEROSPACE ENGINEERING OPT \\ UNJVERSITY OF TEXAS \\ WRW 414 \\ AUSTIN TX 78712 \\ USA \\ TEL: 5124714239 \\ TLF: \\ $T L X: 9108741305$ \\ EML: \\ COM: 07,33 \\ SZKODY PAULA OR \\ OPT OF ASTRONOMY \\ UNIVERSITY OF WASHINGTON \\ FM 20 \\ SEATTLE WA 98195 \\ USA \\ TEL: 2065431988 \\ TLF: \\ TLX: \\ EML: \\ COM: $25,27,42 \mathrm{C}$
}

TABOADA RAMON RODRIGUEZ INST GEOPHYS \& ASTRONOMY CALLE 212 N 290629 Y 31

LISA

LA HABANA

CUBA

TEL:

TLF:

TLX:

EML:

COM:

TAFF LAURENCE G DR

STSCI

HOMEWOOD CAMPUS

3700 SAN MARTIN DR

BALTIMORE MD 21218

USA.

TEL: $301338 \quad 4799$

TLF: 3013384767

TLX: 6849101 STSCI

EML: LGTAFF

COM: 08

TAKADA-HIDAI MASAHIDE DR RES INST OF CIVILIZATION TOKAI UNIVERSITY

1117 KItAKaname

KANAGAWA 259-12

JAPAN

TEL: 0463-58-1211

TLF:

TLX: 2423402 UNITOK J

EML:

COM: 29.51

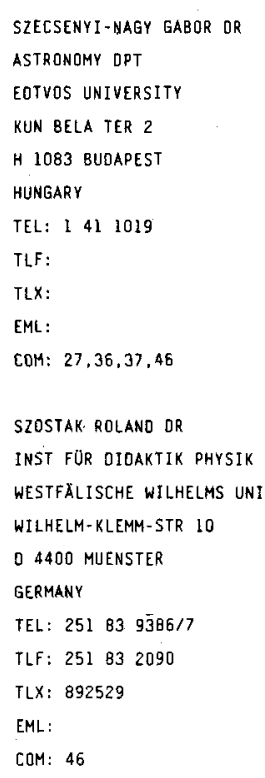

TABORDA JOSE ROSA DR FACULTY OF SCIENCES ASTRONOMICAL OBSERVATORY

$R$ ESCOLA POLITECNICA 58

P 1200 LISBOA

PORTUGAL

TEL:

TLF :

TLX:

EML:

COM: 07,46

TAFFARA SALVATORE PROF
VIA CALZA 5BIS
I $3512 B$ PADOVA
ITALY
TEL: 498071624
TLF:
EML:
COM:
TLX:

TAKAGI KOJIRO PROF OPT OF PHYSICS TOYAMA UNIVERSITY 3190 GOFUKU

TOYAMA 930

JAPAN

TEL: 0764-234716

TLF:

ILX:

EML:

COM: 40
SZEGO KAROLY OR CENTRAL RESEARCH INST FOR PHYSICS

BOX 49

H 1525 BUDAPEST

HUNGARY

TEL: 1551682

ILF: 1696567

TLX: 224722

EML:

COM: 15

TAAM RONALD EVERETT DR OPT PHYSICS \& ASTRONOMY NORTHWESTERN UNIVERSITY OEARBORN OBSERVATORY EVANSTON IL 60208

USA

TEL: 3124917528

TLF:

TLX:

EML:

COM: 35,42

TACCONI LINDA J DR

MPI FUER EXTRATERRESTRISC PHYSIK

KARLSCHWARZSCHILOSTR 2

D 8046 GARCHING MUENCHEN GERMANY

TEL: 8932993289

TLF: 8932993569

TLX: 5215845 XTER D

EML: LINDAMOGAMPE. BITNAT

COM: 28

TAGLIAFERRI GIUSEPPE PROF OSS ASTROFISICO

D. I ARCETRI

LARGO E FERMI 5

I 50125 FIRENZE

ITALY

TEL:

TLF:

ILX:

EML:

COM:

TAKAGI SHIGETSUGU OR

DPT DE FISICA-CCE-UFRN

CAMPUS UNIVERSITARIO

59000 NATAL RN

BRAZIL

TEL:

TLF:

ILX:

EML:

COM:
SZEIOL BELA DR KOHKOLY OBSERVATORY THEGE U 13/17

BOX 67

H 1525 BUDAPEST HUNGARY

TEL: 175 5866/75 4122 TLF:

TLX: 227460 KONOB

EML:

COM: $27 \mathrm{C}$

TABARA HIROTO OR

FAC OF EDUCATION

UTSUNOMIYA UNIVERSITY

MINEMACHI

UTSUNOMIYA 321

JAPAN

TEL: 0286-36-1515

TLF:

Tix:

EML:

COM: 40

TADEMARU EUGENE OR DPT OF ASTRONOMY UNIV OF MASSACHUSETIS AMHERST MA 01002

USA

TEL:

TLF:

TLX:

EML:

COM:

TAKABA HIROSHI OR KASHIMA SPACE RES CENTER CRL

893-1 HIRAI

IBARAKI 314

JAPAN

TEL: $81 \quad 29982 \quad 1211$

TLF: 81299835728

TLX: 7604038 CRL UC

EML: KASHIMAaAtTAIR.GSFC. NASA. GI

COM: 40

TAKAHARA FUMIO $D R$

DPT OF PHYSICS

TOKYO METROPOLITAN UNIVER

MINAMIOHSAWA 1-1

HACHIOJI TOKYO 192-03

JAPAN

TEL: 426771111

TLF: 426772483

ILX:

EML:

COM: 47,48 


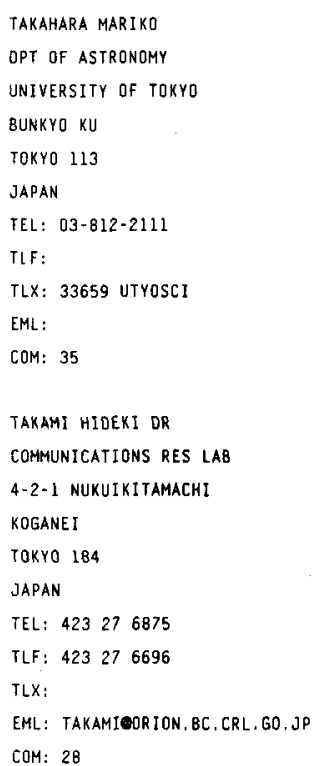

takayanagi kazuo prof

ISAS

3-1-1 YOSHINOOAI

SAGAMIHARA

KANAGAWA 229

JAPAN

TEL: 427513911

TLF:

TLX: 24550 SPACETKY J

EML:

COM: 14

TAKENS ROELF JAN OR

ASTRONOMICAL INSTITUTE

UNIVERSITY OF AMSTERDAM

KRUISLAAN 403

NL 1098 SJ AMSTERDAM

NETHERLANOS

TEL: 205257491

TLF: $20 \quad 52 \quad 57484$

ILX: 10262 HEF NL

EML:

COM:

TALON RAOUL OR
CESR
BP 4346
F 31029 TOULOUSE COX
FRANCE
TEL: 61556666
TLF: 61556701
TLX: 531329 UNSPAT F
EML:
CON: 10

TAKAKUBO KEIYA PROF

ASTRONOMICAL INSTITUTE

TOHOKU UNIVERSITY

SENDAI AOBA

MIYAGI 980

JAPAN

TEL: $222-22-1800$

TLF :

TLX: 852246 THUCOM J

EML:

COM: 34,40

TAKANO TOSHIAKI DR

NOBEYAMA RAOID OBS

NAOJ

MINAMIMAKI MURA

MAGANO $384 \quad 13$

JAPAN

TEL: $267 \quad 63 \quad 4487$

TLF: $267 \quad 634444$

TLX:

EML: TAKANOONRO,NAO,AC,JP

COM: $10,34,40$

TAKEOA HIDENORI OR
OPT AERONAUTIC ENGINEER
KYOTO UNIVERSITY
SAKYO KU
KYOTO 606
JAPAN
TEL:
TLF:
TLX:
EML:
COM: 15

TAKEUTI MINE OR

ASTRONOMICAL INSTITUTE

TOHOKU UNIVERSITY

SENDAI AOBA

MIYAGI 980

JAPAN

TEL: $22 \quad 222 \quad 1800$

TLF: $22 \quad 2612806$

TLX: 852246 THUCOM J

EML: I4AOS4OWJPNTOHOK

COM: $27 \mathrm{C}$

TALWAR SATYA P OR
DPT OF PHYSICS
UNIVERSITY OF OELHI
NEW OELHI 110007
INDIA
TEL: 112918993
TLF:
TLX:
EML:
COM:

TAKAKURA TATSUO PROF EMER

DPT OF ASTRONOMY

UNIVERSITY, OF TOKYO

BUNKYO KU

TOKYO 113

JAPAN

TEL: $03-812-2111$

TLF :

TLX: 33659 UTYOSCI J

EML:

COM: $10,40,44$

TAKARADA KATSUO OR

KYOTO INST OF TECHNOLOGY

MATSUGASAK!

SAKYO KU

KYOTO 606

JAPAN

TEL: 757913211

ILF:

$\mathrm{T} L \mathrm{X}$ :

EML:

COM:

TAKEDA YOICHI DR

INST OF ASTRONOMY

UNIVERSITY OF TOKYO

OSAWA MITAKA

TOKYO 181

JAPAN

TEL: $81422 \quad 413739$

TLF: $81422 \quad 413749$

TLX: 2822307 TAOMTK J

EML:

COM: 36

TALAVERA A DR

ESA IUE OBSERVATORY

VILSPA

APO 50727

E 28080 MAORID

SPAIN

TEL: 18131100

TLF: 18131139

TLX: 42555 VILSE

EML:

COM: 29

TAMENAGA TATSUO OR

FAC OF EOUCATION

MIE UNIVERSITY

TSU-SHI

MIE 514

JAPAN

TEL:

TLF:

TLX:

EML:

COM: 10
TAKALO LEO 0 DR

TURKU UNIVERSITY OBS

TUORLA

SF 21500 PIIKKIO

FINLAND

TEL: $21 \quad 435822$

TLF: $21 \quad 43 \quad 3767$

TLX:

EML: TAKALOKONTU.UTU.FI

CDM: 28

TAKASE BUNSHIRO PROF

TOKYO ASTRONOMICAL OBS

NAOS

OSAWA MITAKA

TOKYO 181

JAPAN

TEL: $422 \quad 325111$

TLF :

TLX: 2822307 TAOMTK

EML:

COM: 28

TAKENOUCHI TADAO OR

1-28-30 KICHIJYOJI.

KITA MACHI

MUSASHIND SHI

TOKYO 180

JAPAN

TEL:

TLF :

TLX:

EML:

COM:

TALBOT RAYMOND J JR OR THE AEROSPACE CORPORATION

1927 CURTIS AVENUE

REDONDO BEACH CA 9027B

USA

TEL: $213 \quad 379 \quad 9927$

TLF :

TLX:

EML:

COM: 28

TAMMANN G ANDREAS PROF DR ASTRONOMISCHES INSTITUT UNIVERSITAET BASEL

VENUSSTRASSE 7

CH 4102 BINNINGEN

SWITZERLANO

TEL: $6122771 \mathrm{~L}$

TLF: 612717810

TLX:

EML:

COM: $27,28 C, 33,47$ 
TAMURA SHINICHI OR

DPT OF ASTRONOMY

TOHOKU UNIVERSITY

ARAMAKI

SENDAI 980

JAPAN

TEL: $222-22-1800$

TLF:

TLX: 852246 THUCOM J

EML:

COM: 34

TANABE TOSHIHIKO DR

TOKYO ASTRONOMICAL OBS

NAOJ

DSAWA MITAKA

TOKYO 191

JAPAN

TEL: 422325111

TLF:

TLX:

EML:

COM:

TANAKA YASUO DR

DPT OF PHYSICS

IBARAKI UNIVERSITY

BUNKYO

MITO 310

JAPAN

TEL: $292-26-1621 \times 372$

TLF:

TLX:

EML:

COM: 48

TANDON JAGDISH NARAIN DR DPT PHYSICS \& ASTROPHYS

UNIVERSITY OF DELHI

NEW DELHI 110007

INBIA

TEL: $11 \quad 252 \quad 1521$

TLF:

TLX:

EML:

COM: 10

TANIGUCHI YOSHIAKI DR

KISO OBSERVATORY

UNIVERSITY OF TOKYO

MITAKEMURA KISOGUN

NAGANO 39701

JAPAN

TEL: 264523360

TLF:

TLX: 3347577 KSOOBS J

EML:

COM: 28
TAN HUISONG

VUNNAN OBSERVATORY

CAS

BOX 110

KUNMING 72946 YUNNAN

CHINA PR

TEL: .8712035

TLF:

TLX: 64040 VUOBS CN

EML:

COM: 42

TANAKA MASUO OR

INST OF ASTRONOMY

UNIVERSITY OF TOYKO

OSAWA MITAKA

TOKYO IOl

JAPAN

TEL: 81422413743

TLF: $81422 \quad 41 \quad 3749$

ILX:

EML:

COM: 34

TANAKA YASUO PROF

INST SPACE \& ASTRON SCI

UNIVERSITY DF TOKYO

MEGURO.KU

TOKYO 153

JAPAN

TEL: $03-467-1111$

ILF :

TLX: J24550 SPACE TKY

EMLL:

COM: 44

TANOON S N PROF

IUCAA

PD BOX 4

GANESHKHINO

PUNE 411007

INDIA

TEL: 212336105

TLF : $212 \quad 335760$

TLX:

EML:

COM: 25

TANZELLA-NITTI GIUSEPPE

ROMAN ATHENAEUM OF THE

HOLY CROSS

$S$ GIROLAMO DELA CARITA 64

I 00186 ROME

ITALY

TEL:

TLF :

TLX:

EML:

COM:
TANABE HIROYOSHI DR TOKYO ASTRONOMICAL OBS

NAOJ

OSAWA MITAKA

TOKYO 181

JAPAN

TEL: 0422-32-5111

TLF:

TLX: 02822307 TAOMTK J

EML:

COM: 15.21

TANAKA RIICHIRO PROF

FAC OF EDUCATION

NIIGATA UNIVERSITY

g050 IKARASHI 2

NIIGATA 95021

JAPAN

TEL:

TLF:

TLX:

EML:

COM: 40

TANAKA YUTAKA D DR

KOBE YAMATE WOMEN'S

JUNIOR COLLEGE

SUWAYAMA CHUO-KU

KOBE 650

JAPAN

TEL: 783416060

TLF:

TLX:

EML:

COM: 28

TANG YU-HUA

DPT OF ASTRONOMY

NANJING UNIVERSITY

NANJING

CHINA PR

TEL: 2537651

TLF:

TLX: 0909

EML:

COM: 10

TANZI ENRICO G

IST DI FISICA COSMICA

CNR

VIA BASSANI 15

I 20133 MILANO

ITALY

TEL: $2 \quad 298 \quad 237$

TLF:

TLX:

EML:

COM:
TANABE KENJI OR

DKAYAMA UNIV OF SCIENCE

1-I RIOAI-CHO

OKAYAMA 700

JAPAN

TEL: 0862523161

TLF: $0862 \quad 553847$

TLX:

EML:

COM: 47

TANAKA WATARU DR

DPT OF ASTRONOMY

UNIVERSITY OF TOKYO

BUNKYO KU

TOKYO 113

JAPAN

TEL: $03-812-2111$

TLF :

TLX:

EML:

COM:

TANDBERG-HANSSEN EINAR A

NASA/MSFC

CODE ES OI

HUNTSVILLE AL 35812

USA

TEL: 2055447578

TLF :

TLX:

EML:

COM: $10 \mathrm{C}, 12$

TANGO WILLIAM J DR

SCHOOL OF PHYSICS

UNIVERSITY OF SYONEY

SYONEY NSW 2006

AUSTRALIA

TEL: 26923953

TLF:

TLX: 26169 UNISYO AA

EML:

COM: $09 \mathrm{C}$

TAPIA MAURICIO DR

INSTITUTO DE ASTRONOMIA

UNAM

APDO POSTAL 877

22860 ENSENADA B CALIF

MEXICO

TEL: $4-08-80 / 8-30-93$

TLF:

TLX: 56739 CICEME

EML:

COM: 


TAPIA-PEREZ SANTIAGO
MIT LINCOLN LABORATORY
4 MAGUIRE ROAD
LEXINGTON MA 02173
USA
TEL: 6179810832
TLF: 6179810991
TLX:
EML: LOONEY: :TAPIA (SPAN)
COM: 25

TARAFOAR SHANKAR P DR

TIFR

HOMI BHABHA RD

COLABA

BOMBAY 400005

INOIA

TEL: $22 \quad 219111$

TLF :

TLX: 0113009 TIFR IN

EML: TARAFOAROTIFRVAX

COM: 34,36
TAPLEY BYRON D OR AEROSPACE ENGINEERING.DPT UNIVERSITY OF TEXAS

WRW 402

AUSTIN TX 78712

USA

TEL: $512471 \quad 1356$

TLF:

TLX:

EML:

COM: 19

TARENGHI MASSIMO DR

ESO

KARL-SCHWARZSCHILO-STR 2

D 8046 GARCHING MUENCHEN GERMANY

TEL: 8932006236

ILX: 52828223 EO D

EML:

TLF: 893202362

COM:

TARTER JILL C OR

NASA AMES RESEARCH CTR

SETI DFFICE

MOFFETT FIELO CA 94035

USA

TEL: 4156045727

TLF : 4159685830

TLX: 348408 NASA AMES MOF

EML: TARTEREBKYAST, BERKELEY, EQU

COM: $40,47,5$ IVP

TATEVYAN S K DR

INST OF ASTRONOMY

ACADEMY OF SCIENCES

PYATNITSKAYA UL 48

109017 MOSCOW

RUSSIA

TEL: $231-54-61$

TLF:

TLX: 412623 SCSTP SU

EML:

COM: 07

\section{TAVARES $J T L Q R$}

AVE OIAS DA SILVA

173 R/C ESO

P 3000 COIMBRA

PORTUGAL

TEL:

TLF :

TLX:

EML:

COM:
TAPPING KENNETH F HERZBERG INST ASTROPHYS NTL RESEARCH COUNCIL

100. SUSSEX DR

OTTAWA ON KIA ORG

CANADA

TEL: 6139915842

TLF: 6139526602

TLX: 0533715

EML:

COM:: 10

TARNSTROM GUY OR

MIT LINCOLN LABORATORY

BOX 73

LEXINGTON MA 02173

USA

TEL: $617 \quad B 635500$

TLF :

TLX: 923355

EML:

COM:

TASSOUL JEAN-LDUIS PROF DPT DE PHYSIQUE UNIVERSITE OE MONTREAL CP 6128 SUCC A MONTREAL QC H3C $3 J 7$ CANADA

TEL: $514 \quad 343 \quad 7274$

TLF : 5143432071

TLX: 05562425

EML:

COM:

TATON RENE PROF

CENTRE ALEXANDRE KOYRE

12 RUE COLBERT

F. 75002 PARIS

FRANCE

TEL: $1 \quad 42 \quad 97 \quad 5245$

TLF :

TLX:

EML:

COM: 41

TAWADROS MAHER. JACOUB OR HELWAN OBSERVATORY

HELWAN

EGYPT

TEL: $780645 / 2683$

TLF:

TLX: 93070 HIAG

EML:

COM: 07
TARAOY VLADIMIR K DR

MAIN ASTRONOMICAL OBS UKRAINIAN ACAD OF SCIENCE GOLOSEEVO

252127 KIEV

UKRAINE

TEL: 662286

TLF:

TLX: 131406 SKY SU

EML:

COM: 19

TARRAB IRENE

OBSERVATOIRE DE PARIS

61 AVE OBSERVATOIRE

F 75014 PARIS

FRANCE

TEL: 140512237.

TLF:

TLX: 270776 OBS $p$

EML:

COM:

TASSOUL MONIQUE OR

DPT DE PHYSIQUE

UNIVERSITE OE MONTREAL

CP 6128 SUCC A

MONTREAL QC H3C $3 J 7$

CANADA

TEL: $514 \quad 343 \quad 7274$

TLF: 5143432071

TLX: 05562425

EML:

CDM: 35

TATUM JEREMY B DR

CLIMENHOGA OBSERVATORY UNIVERSITY OF VICTORIA

BOX 1700

VICTORTA BC VBW $2 Y 2$

CANADA

TEL: 6047217750

TLF: 6047217715

TLX:

EML:

COM: $14,15,20$

TAWARA YUZURU OR DPT OF ASTROPHYSICS NAGOYA UNIVERSITY FUROCHO' CHIKUSA KU NAGOYA 464

JAPAN

TEL: 527815111

TLF: 527813541

TLX: 4477323 SCUNGY J

EML:

COM: 


\begin{tabular}{|c|c|c|c|}
\hline TAYLER ROGER I PROF & TAYLOR A R OR & TAYLOR DOMALO BOGGIA DR & TAYLOR DONALO J DR \\
\hline ASTRONOMY CENTRE & DPT OF PHYSICS & ROYAL GREENWICH OBS & DPT OF PHYSICS \& ASTRON \\
\hline UNIVERSITY OF SUSSEX & UNIVERSITY OF CALGARY & HERSTMONCEUX CASTLE & UNIVERSITY OF NEBRASKA \\
\hline FALMER & 2500 UNTVERSITY OR NW & HAILSHAM BN27 LRP & LINCOLN NE 68589 \\
\hline BRIGHTON BN1 9QH & CALGARY AB T2N IN4 & UK & USA \\
\hline UK & CANABA & TEL: 323833272 & TEL: 4024723686 \\
\hline TEL: 273606755 & TEL: $403 \quad 220 \quad 5385$ & TLF: & TLF: \\
\hline ILF: & TLF : $403 \quad 2893331$ & TLX: 87451 RGOBSY G. & $T L X:$ \\
\hline TLX: 877159 UNISEX $G$ & $T L X: 082 \quad 1545$ & EML: & EML: \\
\hline EML: & EML: BITNET: ARTAYLOR@UNCAMULT & COM: 07.20 & COM: \\
\hline COM: $35,47,48$ & COM: 40 & & \\
\hline TAYLOR JOSEPH H PROF & TAYLOR KEITH OR & TAYLOR KENNETH N R PROF & TCHANG-BRILLET LYOIA DR \\
\hline DPT OF PHYSICS & AAO & 105A COPELAND RD & OBSERVATOIRE DE PARIS \\
\hline PRINCETON UNIVERSITY & PO BOX 296 & BEECROFT NSW 2119 & SECTION DE MEUDON \\
\hline JADWIN HALL & EPPING NSW 2121 & AUSTRALIA & DAMAP \\
\hline PRINCETON NJ 08544 & AUSTRALIA & TEL: & F 92195 MEUDON PPL CDX \\
\hline USA & TEL: & TLF: & FRANCE \\
\hline TEL: 6094524368 & TLF: & TLX: & TEL: $1145 \quad 077576$ \\
\hline TLF: & $T\llcorner X:$ & EML: & YLF: $145 \quad 077469$ \\
\hline TLX: 4993512 & EML: & COM: 34 & $T L X: 631987$ \\
\hline EML: & COM: & & EML: BRILLETEFREUSI \\
\hline COM: & & & COM: 14 \\
\hline TEAYS TERRY J OR & TEDESCO EOWARO $F$ & TEERIKORPI VELI PEKKA DR & TEHERANY D \\
\hline NASA/GSFC & $J P L$ & TURKU UNIVERSITY OBS & 83 AVENUE REY \\
\hline COOE 684.9 & MS 183501 . & TUORLA & \\
\hline IUE OBS & 4800 OAK GROVE OR. & SF 21500 PIIKKIO & TEHERAN \\
\hline GREENBELT MD 20771 & PASADENA CA 91109 & FINLANO & IRAN \\
\hline USA & USA & TEL: 21435822 & TEL: \\
\hline TEL: $301286 \quad 5749$ & TEL: $818 \quad 354 \quad 4739$ & TLF: 21433767 & TLF: \\
\hline TLF: $301286 \quad 7642$ & TLF: & TLX: 62638 IYF SF & $T L X:$ \\
\hline TLX: & TLK: & EML: & EML: \\
\hline EML: IUESOC: : TEAYS & EML: & COM: & COM: \\
\hline COM: 42 & COM: $15 C .22 C, 51$ & & \\
\hline TEJFEL VIKTOR G DR & TEKTUNALI H GOKMEN DR & TELESCO CHARLES M DR & TEMPESII PIERD PROF \\
\hline ASTROPHYSICAL INSTITUTE & LINIVERSITY OBSERVATORY & NASA/MSFC & ISTITUTO ASTRONOMICO \\
\hline KAZAKH ACAD OF SCIENCES & UNIVERSITY OF ISTANBUL & COOE ES 63 & UNIVERSITA DI ROMA \\
\hline 480068 ALMA ATA & 34452 ISTANBUL & SPACE SCIENCE LAB & VIA G M LANCISI 29 \\
\hline KAZAKHSTAN & TURKEY & HUNTSVILLE AL 35812 & 100161 ROMA \\
\hline TEL: $68-30-53$ & TEL: 15223597 & USA & ITALY \\
\hline TLF: & TLF: & TEL: 2055447723 & TEL: 68442977 \\
\hline TLX: & TLX: & TLF: & TLF : \\
\hline EML: & EML: & ILX: 594416 & $T L X:$ \\
\hline COM: 16,51 & COM: & EML: & EML; \\
\hline & & COM: 28 & COM: 27 \\
\hline TENORIO-TAGLE G DR & TEPLITSKAYA R B DR & TER HAAR DIRK & TERASHITA YOICHI PRDF \\
\hline MPI F PHYSIK \& ASTROPHYS & SIBIZMIR & $80 \times 10$ & KANAZAWA TECHNOLOGY INST \\
\hline KARL-SCHWARZSCHILD-SIR I & ACADEMY OF SCIENCES & 349 MIDOLE ST & 7.1 OGIGAOKA. \\
\hline O 8046 GARCHING MUENCHEN & $664697 \cdot 1$ RKUTSK 33 & PETWORTH GU28 ORY & NONOICHIMACHI \\
\hline GERMANY & RUSSIA & UK & ISHIKAWA 921 \\
\hline TEL: 8932090 & TEL: $6-23-65$ & TEL: & JAPAN \\
\hline TLX: 524629 ASTRO D & TLF: & TLF : & TEL: \\
\hline EML: & TLX: & TLX: & TLF : \\
\hline TLF: & EML: & EML: & $T L X:$ \\
\hline COM: 34 & COM: 12 & COM: & EML: \\
\hline
\end{tabular}




\author{
TEREBIZH VALERY YU DR \\ CRIMEAN STATIION OF \\ STERNBERG INSTITUTE \\ NAUCHNY \\ 334413 CRIMEA \\ UKRAINE \\ TEL: 432945 \\ TIE: \\ TLX: 222192 VOSHOD \\ EML: \\ COM: \\ TERNULLL MAURIZIO OR \\ DSS ASTROFISICO \\ CIITA UNIVERSITARIA \\ VIA A DORIA 6 \\ I 95125 CATANIA \\ ITALY \\ TEL: 95330533 \\ TLF: 95330592 \\ TLX: 970359 ASTRCT I \\ EML: ASTRCT: : MAURIZIO \\ COM: 10
}

TERZAN AGOP DR

OBSERVATOIRE DE LYON

aVE CHARLES ANDRE

F 69561 S GENIS LAVAL COK

FRANCE

TEL: 78560705

TLF: 72399791

TLX: 310926

EML:

COM: 27,37

TEUBEN PETER J DR

ASTRONOMY PROGRAM

UNIVERSITY OF MARYLAND

COLLEGE PARK MO 20742

USA

TEL: 3014053001

TLF: 3013149067

TLX: 7108260352

EML:

COM:

THE PIK-SIN PROF

ASTRONOMLCAL INSTITUTE

UNIVERSITY OF AMSTERDAM

KRUISLAAN 403

NL 1098 SJ AMSTERDAM

NETHERLANOS

TEL: 202527491

ILF: $20 \quad 5257484$

TLX: 10262 HEF NL

EML:

COM: $33,34,37$

\author{
TERENTJEYA ALEXANDRA $K$ DR \\ INST OF ASTRONOMY \\ ACADEMY OF SCIENCES \\ pyatnitskaya ul 48 \\ 109017 MOSCOW \\ RUSSIA \\ TEL: 231-54-61 \\ TLF: \\ TLX: 412623 SCSTP SU \\ EML: \\ COM: 15,22
}

TERRELL NELSON JAMES JR LOS ALAMOS NaTIONAL LAB MS 0436 ESS 9

BOX 1663

LOS ALAMOS NM 87545

USA.

TEL: $505 \quad 6672044$

ILF:

TLX:

EML:

COM: 48

TERZIAN YERVANT PROF

CORNELL UNIVERSITY

SPACE SCIENCES blog

ITHACA NY 14853

USA

TEL: 607.2564935

TLX: 932454

EML:

TLF:

COM: $28,34,40,51$

TEXEREAU JEAN M
OCA CERGA
AVE COPERNIC
F 06130 GRASSE
FRANCE
TEL: 93365849
ILF:
TLX:
EML:
COM:

THEJLL PETER ANDREAS DR

\section{NORDITA}

BLEgDAMSVEJ 17

DK 2100 COPENHAGEN 0

\section{DENMARK}

TEL: $31 \quad 42 \quad 1616 * 339$

TLF: 31389157

TLX:

EML: THEJLLONORDITA.DK

COM: 36

\begin{tabular}{|c|c|}
\hline TERLEVICH ELENA OR & TERLEVICH ROBERTO JUA \\
\hline ROYAL GREENWICH OBS & ROYAL GREENWICH OBS \\
\hline \multicolumn{2}{|l|}{ HERSTMONCEUX CASTLE } \\
\hline HAILSHAM BN27 IRP & HERSTMONCEUX CASTLE \\
\hline uK & HAILSHAM BN27 IRP \\
\hline TEL: 323833171 & uk \\
\hline TLF: & TEL: \\
\hline TLX: & TLF: \\
\hline \multicolumn{2}{|c|}{ EML: ETOSTARLINK.RO. GREENWICH. AC. UK TLX: } \\
\hline \multirow[t]{2}{*}{ 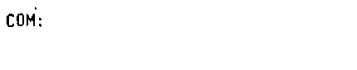 } & EML: \\
\hline & COM: 28 \\
\hline TERRIEN JEAN & TERRILE RICHARD JOHN \\
\hline 103 RUE DE VERSAILLES & JPL \\
\hline F 92410 VILLE D'AVRAY & MS 183501 \\
\hline FRANCE & 4BOO OAK GROVE OR \\
\hline $\begin{array}{llllll}T E L: & 1 & 47 & 09 & 1034\end{array}$ & PASADENA CA 91109 \\
\hline TLF: & USA \\
\hline TLX: & TEL: \\
\hline EML: & TLF: \\
\hline \multirow[t]{3}{*}{ COM: } & TLX: \\
\hline & EML: \\
\hline & COM: 16 \\
\hline
\end{tabular}

TERZIOES CHARALAMBOS OR

TESKE RICHARD G PROF DPT OF ASTRONOMY UNIVERSITY OF MICHIGAN DENNISON BLDG.

ANN ARBOR MI 481091090

USA

TEL: 3137643398

ILF:

ILX:

EML:

COM: 10

THADQEUS PATRICK PROF

CENTER FOR ASTROPHYSICS

HCO/SAO

60 GAROEN ST

CAMBRIDGE MA D213日

USA

TEL: 6174957340

TEF:

TLX: 921428 SATELLITE CAM

EML:

COM: 34,51

THEDDOSSIOU EFSTRATILS OR OPT OF PHYSICS

NTL UNIVERSITY OF ATHENS PANEPISTIMIOPOLIS

GR 15783 ZOGRAFOS

GREECE

TEL: 17243414

ILF:

TLX:

EML:

COM:
THAKUR RATNA KUMAR DR DPT OF PHYSICS UNIVERSITY OF RAVISHANKAR RAIPUR 492010

INOIA

TEL: 27064

ILF:

TLX:

EML:

COM: 28

THEVENIN FREOERIC DR OCA OBSERV DE NICE

BP 139

F 06003 NICE COK

FRANCE

TEL: 92003011

TLF:

TLX:

EML: THEVENIN⿴囗RONI51

COM: 29 
THIELEMANN FRIEORICH-KARL CENTER FOR ASTROPHYSICS HCO/SAO MS 10

60 GARDEN ST CAMBRIOGE MA 02138 USA

TEL: 6174954979

TLF: 6174955374

TLX:

EML: fktocfa.3.bitnet

COM: 35

THOLEN DAVIO J DR

INSTIUUTE FOR ASTRONOMY

UNIVERSITY OF HAWAII

2680 WOODLAWN DR

HONOLULU HI 96822

USA

TEL: $80 B \quad 956 \quad 6930$

TLF: $808 \cdot 9882790$

TLX: 8459 UHAST HR

EML: tholeneuhifa.ifa. hawait.edu

COM: $06,15,16 C, 20$

THOMAS JOHN H PROF

DPT MECH \& AEROSPACE SCI

UNIVERSITY OF ROCHESTER

ROCHESTER NY 14627

USA

TEL: $716 \quad 275 \quad 4083$

TLF :

TLX:

EML:

COM: 10,12

\section{THOMASSON PETER OR}

NRAL

JOORELL BANK

MACCLESFIELO SK11 9DL

UK

TEL: 47771321

TLX: 36149

EML:

TLF:

COM: 40

\section{THOMPSON LAIRD A OR}

OPT OF ASTRONOMY

UNIVERSITY OF ILLINOIS

1011 W SPRINGFIELO AVE

URBANA IL 61801

USA

TEL: 2173333090

TLF:

TLX:

EML:

COM: 28,47
THIELHEIM KLAUS O DR

abteilung mathem. physiK

UNIVERSITAET KIEL

OLSHAUSENSTR $40 / 60$

D 2300 KIEL 1

GERMANY

TEL: 4318803216

TLF :

TLX: 292979

EML:

COM: 33

IHOMAS CLAUDINE OR

BIPM

PAVILLON DE BRETEUIL.

F 92312 SEVRES CDX

FRANCE

TEL: $145 \quad 077073$

TLF: 145342021

$T L X: 631351$

EML: BI PMarRMEUL]

COM: 31

THOMAS PETER A OR

ASTRONOMY CENTRE

UNIVERSITY OF SUSSEX

FALMER

BRIGHTON BNI 9QH

UK

TEL: $273 \quad 60 \quad 6755 * 3099$

TLF: 273678097

TLX: B77159 BHVTXS.G

EML: PETERT SYMA. SUSSEX.AC.UK

COM: $2 B$

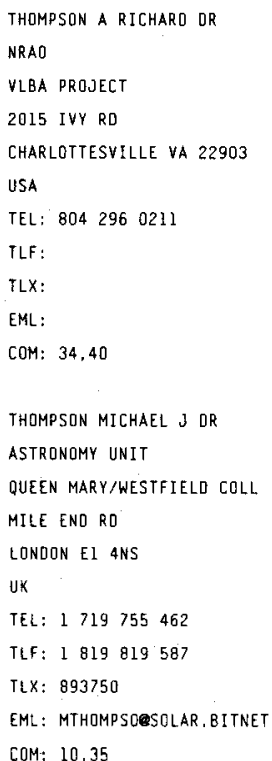

THIRY YVES R PROF

UNIVERSITE PARIS VI

4 PLACE JUSSIEU TOUR 66

F 75230 PARIS CDX 05

FRANCE

TEL: 143362525

TLF:

TLX:

EML:

COM: 07

THOMAS DAVIO V OR

OPT OF PHYSICS

UNIVERSITY OF EDINBURGH

MAYFIELO RO

EOINBURGH EH9 $3 \mathrm{JI}$

UK

TEL: 316505307

TLF: 316624712

TLX: 727442 UNIVEO G

EML:

COM: 08.24

THOMAS RICHARD N DR

1155 TIMBERLANE

PINEBROOK HILL

BOULOER CO 80302

USA

TEL: 3034439290

TLF:

TLX:

EML:

COM: 12.36

THOMPSON G I DR

7 MACDOWALL RD

EOINBURGH EH9 $3 \mathrm{HJ}$

UK

TEL:

TLF :

TLX:

EML:

COM:

THOMPSON RODGER I PROF

STEWARD OBSERVATORY

UNIVERSITY OF ARIZONA

TUCSON AZ E5721

USA

TEL: $602 \quad 621 \quad 6527$

TLF:

ILX: 467175

EML:

COM:
THOBURN CHRISTINE

ROYAL GREENWICH OBS

HERSTMONCEUX CASTLE

HAILSHAM BN27 IRP

UK

TEL: $323 \quad 833171$

TLF:

TLX: 87451

EML:

COM: 08

THOMAS HANS-CHR ISTOPH OR MPI FUER PHYSIK UND

ASTROPHYSIK

KARL-SCHWARZSCHILO-STR I

D B046 GARCHING MUENCHEN GERMANY

TEL:

TLF :

ILX:

EML:

COM: 35

THOMAS ROGER J DR

NASA/GSFC

CODE 682

GREENBELT MO 2071

USA

TEL: 3012867921

TLF:

TLX:

EML:

COM: 10.44

THOMPSON KEITH OR

OPT OF PHYSICS

MONASH UNIVERSITY

WELLINGTON RD

CLAYTON VIC 3168

AUSTRALIA

TEL: 35654000

TLF:

TLX: 32691 AA

EML:

COM: 27,42

THOMSEN BJARNE B LECT

INST OF PHYSICS \& ASTRDN UNIVERSITY OF AARHUS

NY MUNKEGABE

DK BOOO AARHUS $C$

DENMARK

TEL: $86 \quad 128899$

TLF: 86202711

TLX:

EML:

COM: 
THONNARQ NORBERT OR

ATOM SCIENCES

114 RIDGEWAY CENTER

OAK RIDGE TN 37830

USA

TEL: $615 \quad 483 \quad 1113$

TLF :

TLX:

EML:

COM: 28,34

THUAN TRINH XUAN DR

UNIVERSITY STATION

UNIVERSITY OF VIRGINIA

BOX 3818

CHARLOTTESVILLE VA 22903

USA

TEL: 804.9244894

ILF :

ILX:

EML:

COM: 28.47

TIMOTHY I GETHYN OR

CTR FOR SPACE SCIENCES \&

ASTROPHYSICS

STANFORL UNIV ERL 314

STANFORD CA 943054055

USA

TEL: 4154970059

TLF

TLX: 348402 STANFRD STNU

EML:

COM:

TIURI MARTII PROF

HELSINKI UNIV TECHNOLOGY

RADIO LABORATORY

GTAKAARI 5 A

SF 02150 ESPOO 15

FINLAND

TEL: 4512545

TLF:

TLX: 122771 RORTA SF

EML:

COM:

TOOORAN IOAN OR

ASTRONOMICAL OBSERVATORY

STR CIRESILOR 19

a 3400 CLUJ NAPOCA

RUMANIA

TEL:

TLF:

TLX:

EML:

COM: 25,42
THORME KIP S PROF

CALTECH

MS 13033

PASADENA CA 91125

USA

TEL: $213 \quad 356 \quad 4598$

TLF :

TLX: 675425

EML:

COM: 48

THUM CLEMENS OR
IRAM
AFO OIVINA PASTORA 7
BLOQUE 6/2B
E I8012 GRANADA
SPAIN
TEL: 5848 O4I3
TLF:
TLX: $7852 L$ IRAM E
EML:
COM: 40

TINBERGEN JAAP DR

KAPTEYN STERREWACHT

WERKGROEP

MENSINGHEWEG 20

NL 9301 KA RODEN

NE THERLANDS

TEL: $5908 \quad 19631$

TLF :

TLX: $53767^{\circ} \mathrm{KSWRO} \mathrm{NL}$

EML:

COM: 25

TJIN-A-DJIE HERMAN R E QR

KOEKOELAAN 106

NL 1403 EJ BUSSUM

NETHERLANOS

TEL: $2159 \quad 17076$

TLF :

EML:

COM: 27,35

TLX: 16460 FACWN NL

TOFANI GIANNI PROF

OSS ASTROFISICO

DI ARCETRI

LARGO E FERMI 5

I 50125 FIRENZE

ITALY

TEL: $55 \quad 275 \quad 2217$

TLF :

TEX: 572268 ARCETRI

EML:

COM: 40
THORSTENSEN JOHN R

DPT OF PHYSICS \& ASTRON

DARTMOUTH COLLEGE

HANOVER NH 03755

USA

TEL: $603 \quad 6462869$

TLF :

TLX:

EML:

COM:

TIFFT WILLIAM G PROF

STEWARD OBSERVATORY

UNTVERSITY OF ARIZONA

TUCSON AZ 85721

USA

TEL: 6026216532

TLF:

TLX: 467175

EML:

COM: 28,47

TING YEOU-TSWEN

ASTRONOMY SECTION

CENTRAL WEATHER BUREAU

64 KUNG YUEN RO

TAIPEI 100

CHINA R

TEL: $23713181 * 281$

TLF :

$T\lfloor X$ :

EML:

COM: 04

TLAMICHA ANTONIN DR

ASTRONOMICAL INSTITUTE

CZECH ACADEMY OF. SCIENCES

ONDREJOV OBSERVATORY

CS 25165 ONDREJOV

CZECHOSLOVAKIA

TEL: 20485201

TLF; 20485314

TLX: 121579 ASTR CZ

EML:

COM: 10,40

TOHLINE JOEL EDWARD

DPT PHYSTICS \& ASTRONOMY

LOUISIANA STATE' UNIV

BATON ROUGE LA 708034001

USA

TEL: 5043886851

TLF:

TLX: 559184

EML:

COM: 35
THRONSON HARLEY ANOREW JR OPT PHYSICS \& ASTRONOMY UNIVERSITY OF WYOMING LARAMIE WY 82072

USA

TEL: $307766 \quad 6150$

TLF :

TLX:

EML:

COM: 34

TIFREA EMILIA DR

ASTRONOMICAL OBSERVATORY

CUTITUL DE ARGENT 5

BOX 28

R 75212 BULAREST $2 B$

RUMANIA

TEL: $23-60-10$

TLF:

TLX: $09-26-29$

EML:

COM: 10

TIPLER FRANK JENNINGS OR

OPT OF PHYSICS

TULANE UNIVERSITY

NEW ORLEANS LA 70118

USA

TEL:

TLF :

TLX:

EML:

COM: 47

TOBIN WILLIAM

OPI OF PHYSICS

UNIVERSITY OF CANTERBURY

PRIVATE BAG

CHRISTCHURCH 1

NEW ZEALANO

TEL: $64 \quad 3 \quad 642531$

TLF: 64364299

TLX:

EML: w.tobinecanterbury. $8 c . n z$

COM: 33

TOKAREV YURIJ $\checkmark$ DR

RADIOPHYSICAL RESEARCH

INSTITUTE

LYADOY UL 25

603600 N NOVGOROD

RUSSIA

TEL: 978312360188

TLF: 978312369902

TLX:

EML:

COM: 34 


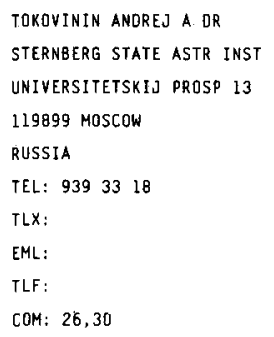

TOKUNAGA ALAN TAKASHI DR INSTITUTE FOR ASTRONOMY UNIVERSITY OF HAWAII 2680 WODDLAWN OR HONOLULLU HI 96822

USA

TEL: $808956 \quad 6691$

TLF :

TLX: 7238459 UHAST HR

EML: TOKunAgaegaliLEO.IFA. HAWAIt. ED EML:

COM: 25

TOMASKO MARTIN G OR
LUNAR \& PLANETARY LAB
UNIVERSITY OF ARIZONA
SPACE SCIENCES BLOG
TUCSON AZ 85721
USA
TEL: 6026216969
TLF:
TLX:
EML:

COM:

\section{TOMITA KENJI PROF}

UJI RESEARCH CENTER

YUKAWA INST THEORICAL PHY

KYOTO UNIVERSITY

UJI 611

JAPAN

TEL:

ILF:

TLX:

EML: tomitaryisunl.kyoto-u,ac.jp COM: 4 ?

\section{TONG FU}

PURPLE MOUNTAIN OBSERV

CAS

NANJING

CHINA PR

TEL: 2533921

ILF:

ILX: 34144 PMDAS CN

EML:

COM: $04 C, 07$

TOOMRE ALAR DR

DPT OF MATHEMATICS

MIT RM 2372

77 MASSACHUSETTS AVE

CAMBRIDGE MA 02139

USA

TEL: 6172534326

TLF:

TLX:

EML:

COM: 28,33
TOLBERT CHARLES R DR

UNIVERSITY STATION

UNIVERSITY OF VIRGINIA

80X 3818

CHARLOTTESVILLE VA 22903

USA

TEL: 8049247494

TLF:

ILX:

COM: 25.38 .40 .51

rOMBAUGH CLYOE W PROF

OPT OF ASTRONOMY

NEW MEXICO STATE UNIV

BOX 4500

LAS CRUCES NM 88003

USA

TEL: $505 \quad 646 \quad 2107$

TLF:

TLX:

EML:

COM: 16

TOMITA KOICHIRO MR

11-12 20 YOGA

SEtagataku

TOKYO 158

JAPAN

TEL: 037000066

TLF:

TLX:

EML:

COM: $15,20,22$

TONG YI

DPT OF ASTRONOMY

BEIJING NORMAL UNIVERSITY

19 XINJISKOW OUT ST .

BEIJING 100875

CHINA PR

TEL: $165 \quad 6531 * 6285$

TLF:

TLX:

EML:

COM: 28,33

TOOMRE JURI

JILA/DAG

UNIVERSITY OF COLORADO

BOX 440

BOULOER CO 803090440

USA

TEL: 3034927854

ILF:

ILX:

EML:

COM: $33,35,36$
TOLLER GARY N DR

\section{NASA/GSFC}

CODE 685.3

GREENBELT MO 20771

USA

TEL:

TLF :

TLX:

EML:

COM: 21

TOMIMATSU AKIRA OR DPT OF PHYSICS

NAGOYA UNIVERSITY

FUROCHO CHIKUSA KU

NAGOYA 464

JAPAN

TEL:

TLF:

ILX:

EML:

COM: 47,48

TOMOV ALEXANDER NIKOLOV OPT OF ASTRONOMY BULGarian aCAD SCIENCES 72 LENIN BLVO BG 1784 SOFIA BULLGARIA

TEL: 2758927

TLF :

TLX:

EML:

COM:

TONRY JOHN OR DPT OF MATHEMATICS MIT RM $6204 \mathrm{~A}$ 77 MASSACHUSETTS AVE CAMBR IOGE MA 02139 USA

TEL: $617253752 B$

TLF :

TLX:

EML: JTEALCOR.MIT.EQU

COM: $28,30,47$

TOPAKTAS LATIF A OR KING SAUO UNIVERSITY COLLEGE OF SCIENCE BOX 2455 RIYADH 11453 SAUDI ARABIA TEL:

TLF:

TLX:

EML:

COM: 


\begin{tabular}{|c|c|c|c|}
\hline TORAO MASAHISA & TORELLI M DR & TORNAMBE AMEOEO & TORO TIBOR PROF \\
\hline $410-11$ & OAR & IAS & INSTITUTE OF THEORETICAL \\
\hline SEMPUKU GA OKA 2-11-9 & VIA OEL PARCO MELLINI 84 & CNR & PHYSICS \\
\hline SUSONO CITY & I $00136 \mathrm{ROMA}$ & CP 67 & UNIVERSITY TIMISUARA \\
\hline JAPAN & ITALY & I 00044 FRASCATI & TIMISIJARA \\
\hline TEL: & TEL: 6347056 & ITALY & RUMANIA \\
\hline TLF: & $\dot{\mathrm{T}} \mathrm{LF}$ : & JEL: 69425655 & TEL: $40 \quad 6130823$ \\
\hline$T t x:$ & TLX: & TLF: & JLF: \\
\hline EML: & EML: & TLX: & TLX: \\
\hline \multirow[t]{2}{*}{ COM: 19} & COM: 12 & EML: & EML: \\
\hline & & COM: 37 & COM: 51 \\
\hline TOROSHLIDZE TEIMURAZ I OR & TORRA JOROI OR & TORRELLES JOSE M DR & TORRES CARLOS ALBERTO OR \\
\hline ABASTUMANI ASTROPHYSICAL & DPT FISICA & INST ASTROFISICA & OBSERVATORIO NACIONA \\
\hline OBSERVATORY & UNIVERSIDAD DE BARCELONA & DE ANDALUCIA APD 3004 & RUA CORONEL RENNO 07 \\
\hline GEORGIAN ACAD OF SCIENCES & AVD DIAGONAL 647 & PREFESOR ALBAREDA 1 & CP 21 \\
\hline 383762 ABASTUMANI & E 08028 BARCELONA & E 18080 GRANADA & 37500 ITAJUBA MG \\
\hline GEORGIA & SPAIN & SPAIN & BRAZIL \\
\hline TEL: & TEL: 33307311 & TEL: $58 \quad 121311$ & TEL: 356220788 \\
\hline TLF : & TLF : & TLF: $\begin{array}{llll}58 & 81 & 4530\end{array}$ & TLF: \\
\hline TLX: & TLX: & TLX: & TLX: $031-2603$ \\
\hline EML: & EML: & EML: 16488: :TORRELLES/TORRELLESTIAA & EML: \\
\hline COM: 21 & COM: 33 & COM: 34 & COM: 27.50 \\
\hline TORRES CARLOS DR & TORRES-PEIMBERT SILVIA OR & TORRICELLI GUIDETTA OR & TORROJA J PROF \\
\hline DPT OE ASTRONOMIA & INSTITUTO DE ASTRONOMIA & OSS ASTROFISICO & CATEQRA DE ASTRONOMIA \\
\hline UNIVERSIDAD DE CHILE & UNAM & DI ARCETRI & FAC DE CIENCIAS. \\
\hline CASILLA 35 0 & APOO POSTAL $70-264$ & LARGO E FERMI 5 & UNIVERSIDAD COMPLUTENSE \\
\hline SANT IAGO & 04510 MEXICO OF & I 50125 FIRENZE & E 28040 MADR IO \\
\hline CHILE & MEXICO & ITALY & SPAIN \\
\hline TEL: 22294101 & TEL: $905-548-5306$ & TEL: 552752260 & TEL: \\
\hline TLF: & ILF: & TLF : & TLF : \\
\hline TLX: 440001 & TLX: 1760155 CIC ME & TLX: 572268 & TLX: \\
\hline EML: & EML: & EML: & EML: \\
\hline coM: 20,50 & COM: 34,46 & COM: & COM: \\
\hline TOSA MAKOTO DR & TOSHIKI AIKAWA OR & TOSI MONICA & TOTH IMRE DR \\
\hline ASTRONOMICAL INSTITUTE & ASTRONOMICAL INSTITUTE & OSS ASIRONOMICO & KONKOLY OBSERVATORY \\
\hline TOHOKU UNIVERSITY & TOHOKU GAKUIN GNIVERSITY & UNIVERSITA DI BOLOGNA & THEGE U $13 / 17$ \\
\hline SENDAI AOBA & ICHINAZAKA IZUMI-KU & CP 596 & $B O X-87$ \\
\hline MIYAGI 980 & SENDAI 98131 & I 40100 BOLOGNA & H 1525 BUDAPEST \\
\hline JAPAN & JAPAN & ITALY & HUNGARY \\
\hline TEL: $0222-22-1800$ & TEL: $22 \quad 3751111 * 318$ & TEL: 51222956 & TEL: $1755866 / 75 \quad 4122$ \\
\hline TLF: & TLF: $22 \quad 375 \quad 4040$ & TLF: & TLF: 11569640 \\
\hline TLX: 852246 THUCOM J & Jtx: & ILX: 211664 INFN $80 \mathrm{I}$ & TLX: 227460 KONOB H \\
\hline EML: & EML: & EML: & EML: H697KNOQELLA.UUCP \\
\hline COM: 33 & COM: & COM: 34 & CDM: 15 \\
\hline TOTSUKA YOJI DR & TOUSEY RICHARD OR & TOUT CHRISTOPHER OR & TOVMASSIAN H M DR. \\
\hline INST COSMIC RAY RESEARCH & NAVAL RESEARCH LABORATORY & INSTITUTE OF ASIRONOMY & BYURAKAN ASTROPHYSICAL \\
\hline UNIVERSITY OF TOKYO & $\operatorname{COOE} 7140$ & THE OBSERVATORIES & OBSERVATORY \\
\hline MIDORICHO TANASHI & 4555 OVERLOOK AVE SW & MADINGLEY RD & 375433 ARMENIA \\
\hline TOKYO 188 & WASHINGTON OC 203755000 & CAMBRIOGE CB3. OHA & ARMENIA \\
\hline JAPAN & USA & UK & TEL: $88 \quad 52 \quad 28 \quad 3453$ \\
\hline TEL: $81 \quad 42 \quad 46 \quad 14131$ & TEL: 2027673441 & TEL: 223337548 & TLF : \\
\hline TLF: $8142 \quad 4681438$ & TLF: & TLF: 223337523 & Tex: \\
\hline TLX: 02822371 ICRTU J & TLX: & TLX: & EML: \\
\hline EML: TOTSUKAQJPNUTINS.BITNET & EML: & EML: CT23 PHOENIX.CAMBRIOGE.AC.UK & COM: $28,40,44.51$ \\
\hline COM: 48 & COM: 12,14 & COM: 42 & \\
\hline
\end{tabular}




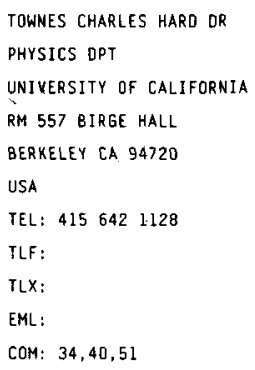

COM: $27,42,50$
TOYAMA KIYOTAKA

HOKKAIOO INFORMATION UNIV

NISHINOPPORO $59-2$

EBETSU 069

JAPAN

TEL: $81 \quad 11 \quad 3854411$

TLF: $81 \quad 11 \quad 384 \quad 0134$

TLX:

EML:

COM: $2 B$

TRAN MINH NGUYET DR OBSERVATOIRE DE PARIS SECTION DE MEUDON

F 92195 MEUDON PPL COX FRANCE

TEL: $145 \quad 07 \quad 7447$

TLF :

$T L X: 270912$

EML:

COM: 14

\section{TREDER H J PROF OR}

ZNTRLINST F ASTROPHYSIK

STERNWARTE BABELSBERG

ROSA-LUXEMBURG-STR 17A

D 1502 POTSDAM

GERMANY

TEL: 762225

TLF :

TLX: 1547.1

EML:

COM: 28,47

TREHAN SURINDAR $K$ PROF

OPT OF MATHEMATICS

UNIVERSITY OF PANJAB

CHANOIGARH $160 \quad 014$

INOIA

TEL: 29938

TLF :

TLX:

EML:

COM:

TREUMANN RUOOLF A OR
MPI F. PHYS \& ASTROPHYSIK
INST F EXTRATERR PHYSIK
D 8046 GARCHING MUENCHEN
GERMANY
TEL: 893299831
TLF:
TLX: 5215845 XTER 0
EML:
COM: 10

TOZER DAVID C OR

SCHODL OF PHYSICS

UNIVERSITY OF NEWCASTLE

NEWCASTLE/TYNE NEI 7RU

UK

TEL:

TLF :

TLX:

EML:

COM:

TRAN-MINH FRANCOISE OR OBSERVATOIRE DE PARIS SECTION DE MEUDON. DASGAL.

F 92195 MEUDON PPL CDX FRANCE

TEL: $\{45 \quad 077553$

ILF: $145 \quad 07 \quad 7469$

ILX: 201571

EML:

COM: 16

TREFFERS RICHARO R

ASTRONOMY DPT

UNIVERSITY OF CALIFORNIA

601 CAMPBELL HALL

BERKELEY CA 94720

USA

TEL: $415 \quad 642 \quad 4223$

TLF :

ILX:

EML:

COM: 34

TRELLIS MICHEL OR

OCA OBSERV OE NICE

BP 139

F 06003 NICE COX

FRANCE

TEL: 93890420

TLF:

TLX: 460004 OBSNICE F

EML:

COM: 10

\section{TREVESE DARIO}

ISTITUTO ASTRONOMICO

UNIVERSITA DI ROMA

VIA G M LANCISI 29

I 00161 ROMA

ITALY

TEL: $6 \quad 44 \quad 03734$

TLF: $6 \quad \mathbf{4 4 0} 3673$

TLX: 613255 INFNRO

EML:

COM: 47
TOZZI GIAN - PAOLO

OSS ASTROFISICO

DI ARCETRI

LARGO E FERMI 5

I 50125 FIRENZE

ITALY

TEL: 552752250

TLF:

TLX: 572268 ARCETR I

EML:

COM: 14

TRAUB WESLEY ARTHUR CENTER FOR ASTROPHYSICS

HCO/SAO

60 GARDEN ST

CAMBRIOGE MA 02138

USA

TEL: 6174957406

TLF:

TLX: $92142 B$ SATELLITE CAM

EML:

COM: 09,44

TREFFTZ ELEONORE E OR

MPI F PHYSIK UNO

ASTROPHYSIK

KARL-SCHWARZSCHILO-STR I

D 8046 GARCHING MUENCHEN

GERMANY

TEL: 8932990

ILF:

TLX: 524629 ASTRO D

EML:

COM: 14

TREMAINE SCOIT DUNCAN

CITA MCLENNAN LABS

UNIVERSITY OF TORONTO

60 SI GEORGE ST

TORONTO ON MES . IAL

CANADA

TEL: $416 \quad 978 \quad 6879$

TLF : $416 \quad 978 \quad 3921$

TLX:

EML :

COM: 28.47

TREXLER JAMES $H$ MR

1921 SO ABREGO OR

GREENYALLEY AZ $85614 \quad 1403$

USA

TEL:

TLF:

TLX:

EML:

COM 


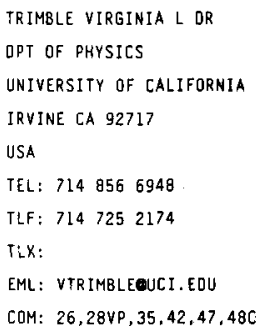

TRINCHIERI GINEVRA
OSS ASTROFISICO
OI ARCETRI
LARGO E FERMI 5
I 50125 FIRENZE
ITALY
TEL: 552752230
ILF:
TLX: 572268 ARCETR I
EML:
COM: 28

TRITTON KEITH P OR ROYAL GREENWICH OBS MAOINGLEY RD CAMBRIDGE CB3 OEZ UK

TEL: 223374000 TLF: 223374700 TLK: 817235 RGOSTR G EML:

COM: 40

TROITSKY $\checkmark$ SPDF DR
RADIOPHYSICAL RESEARCH
INSTITUTE
LYADOV UL $25 / 14$
603600 N NOVGOROD
RUSSIA
TEL: $36-04-40$
TLF:
TLX:
EML:
COM: $16,40,51$
TRUJILLO BUENO JAVIER OR
INST OE ASTROFISICA
DE CANARIAS
OBS DEL TEIDE
E 30200 LA LAGLNA
SPAIN
TEL: 2260 5266
TLF: 22 60 5210
TLX: 92640 IAC E
EML: IAC: :JTB
COM: 12

TRUSSONI EOOARDO IST OI COSMO GEOFISICA CNR

CORSO FIUME 4

I 10133 TORINO

ITALY

TEL: 1165 7694/8979

TLF

TLX: 2LLO4I INFNTO

EML:

COM: 48
TRIPATHI B M OR UTTAR PRADESH STATE DBSERVATORY

PO MANORA PEAK 263129 NAINITAL 263129

INDIA

TEL: $5942 \quad 2136$

TLF:

TLX: CABLE : ASTRONOMY

EML:

COM: 12

TRITTON SUSAN baRbARA

ROYAL OBSERYATORY

BLACKFORD HILL

EOINBURGH EK9 $3 \mathrm{HJ}$

UK

TEL: $316 \quad 673321$

TLF :

TLX: 72383 ROEDIN G

EML:

COM: 05

TROLAND THOMAS HUGH DPT PHYSICS \& ASTRONOMY UNIVERSITY OF KENTUCKY LEXINGTON KY 40506 USA

TEL: 6062578620

TLF:

ILX:

EML:

COM: 40

TRULSEN JAN K PROF

INST MATHS \& PHYSTCAL SCI UNIVERSITY OF TRGMSO

BOX 953

N 9001 TROMSD

NORWAY

TEL:

ILF:

ILX:

EML:

COM:

TRUTSE YU L DR

INST PHYSICS OF ATMOSPH ACADEMY OF SCIENCES

PYZHEVSKY 3

109017 MOSCOW

RUSSIA

TEL:

TLF :

TLX:

EML:

COM: 21
TRIPICCO MICHAEL J DR ASTRONOMY PROGRAM UNIVERSITY OF MARYLANO COLLEGE PARK MD 20742 USA

TEL: $3014051548^{\circ}$

TLF :

ILX:

EML: MIKETEASTRO.UMD. EOU COM: 37

TROCHE-BOGGINO A E OR INST OE CIENCIAS BASICAS UNIV NACIONAL DE ASUNCION CC 1039 - 1804

ASINC TON

paraguay

TEL:

TLF:

TLX:

EML:

COM: 46

TROTTET GERARD DR OBSERVATOIRE DE PARIS SEETION OE MEUDON DASOP F 92195 MEUDON PPL COX FRANCE

TEL: $145 \quad 077808$

TLF:

TLX:

EML:

COM: 10

TRUONG - BACH

OBSERVATOIRE DE PARIS SECTION OE MEUDON F 92195 MEUDON PPL CDX FRANCE

TEL: $145 \quad 077897$ TLF: $145 \quad 077893$ TLX: 270912 EML: TBACHEFREUSI COM: 40

TSAI CHANG-HSIEN OLRECTOR ASTRON SOCIETY OF CHINA

TAIPEI OBSERVATORY TAIPEI 104 CHINA $R$ TEL: TLF:

TLX:

EML: COM: 


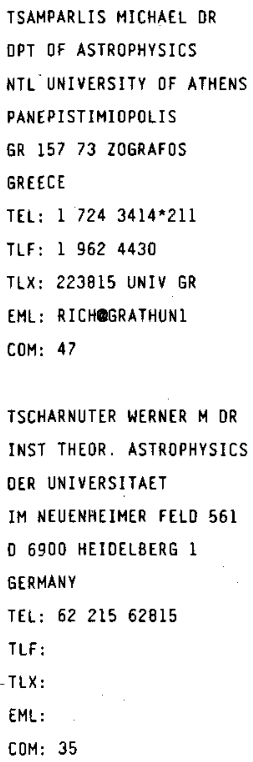

\begin{tabular}{|c|c|}
\hline ISAO MO PROF & TSAP TT TR \\
\hline NO $47 \operatorname{SEC} 3$ & CRIMEAN ASTROPHYS OBS \\
\hline HSIN-I RD & UKRAINIAN ACAD OF SCIENC \\
\hline TAIPEI 106 & NAUCHNY \\
\hline CHINA R & 334413 CRIMEA \\
\hline TEL: 27047795 & UKRAINE \\
\hline TLF : & TEL: 432945 \\
\hline ILX: & TLF: \\
\hline EML: & TLX: \\
\hline COM: 19 & EML: \\
\hline & COM: 12 \\
\hline ISEYTLIN NAUM M & TSIKONOI VASSILIKI PH O \\
\hline RADIOPHYSICAL RESEARCH & OPT OF PHYSICS \\
\hline INSTITUTE & UNIVERSITY OF IOANNINA \\
\hline LYADOV UL $25 / 14$ & GR 45332 IOANNINA \\
\hline 603600 N NOVGOROO & GREECE \\
\hline RUSSIA & TEL: $65 \quad 19 \quad 1084$ \\
\hline TEL: $36-01-29$ & TLF: \\
\hline ILF : & TLX: \\
\hline TEX: 1113 LUNA & EML: \\
\hline EML: & COM: \\
\hline COM: & \\
\hline TSIROPOULA GEORGIA OR & TSUBAKI TOKIO PROF \\
\hline ASTRONOMICAL INSTIIUTE & OPT OF EARTH SCIENCE \\
\hline NTL OBSERVATORY OF ATHENS & SHIGA UNIVERSITY \\
\hline$B 0 \times 20048$ & $2-5-1$ HIRATSU \\
\hline GR 11810 ATHENS & OHTSU 520 \\
\hline GREECE & JAPAN \\
\hline TEL: $1346 \quad 1191$ & TEL: $0775-37-0081$ \\
\hline TLF: & TLF: \\
\hline TLX: 215530 OBS.GR & TLX: \\
\hline EML & EML: \\
\hline 12 & COM: 10,12 \\
\hline
\end{tabular}

TSUCHIDA MASAYOSHI DR IAG

UNIVERSIDADE DE SAO PAULO aV Miguel Stefano 4200 04301 SAO PAULO SP BRAZIL

TEL: 115778599

TLF: 112763848

TLX: 36221 IAGM BR

EML: MASA\%IAGUSP POBRAPESP. BITNET

COM: 20

TSUNETA SAKU DR
INST OF ASTRONOMY
UNIVERSITY OF TOKYO
OSAWA MITAKA
TOKYO 181
JAPAN
TEL: 422324710
TLF:
TLX: 2822307 TAOMIK J
EML: STSUNETA: NASAMAIL
COM: 10

TSAY WEAN-SHUN DR INST, PHYSICS \& ASTRONOMY NTL CENTRAL UNIVERSITY CHUNG LI

TAIWAN 32054

CHINA $R$

TEL: $3422 \quad 7151 * 5335 / 5300$

TLF: 34251175

TLX:

EML: TSAYEPHYAST. DNET. NCU. EDU. TW COM: 26

TSINGANOS KANARIS OR DPT OF PHYSICS UNIVERSITY OF CRETE BOX 1527

GR 711 IO HERAKLION GREECE

TEL: $81 \quad 23 \quad 9757 * 154$

TLF: 81239735

TLX: 262860

EML: TŚINGANELESL, FORTH.GR COM: 10

TSUBOI MASATO OR NOBEYAMA RAOIO OBS NADJ MINAMIMAKI MURA NAGANO 38413 JAPAN

TEL: $81267 \quad 634314$

TLF: $81 \quad 26798 \quad 2927$

TLX: 3329005 NADNRO J EML: NRROTSUB JPNNRO.BITNET COM: 40

TSUJI TAKASHI INST OF ASTRONOMY UNIVERSITY OF TOKYO DSAWA MITAKA TOKYO 181

JAPAN

TEL: $0422-32-5111$

TLF :

TLX: 02822307 TAOMTK J

EML:

COM: $36 \mathrm{C}$

TSVETANOV ZLATAN IVANON ASTRONOMY PROGRAM UNIVERSITY OF MARYLANO COLLEge PARK MO 20742 USA

TEL: 3014540878 TLF: 3014542298 TLX: $710 \quad 8260352$ EML: ztsvetanoveastro.umd.edu COM: 
TSVETKOV MILCHO K DR DPT OF ASTRONOMY BULGARIAN ACAO SCIENCES 72 LENIN BLVO BG 1784 SOFIA BULGARIA

TEL: $275 \quad 8927$

TLF :

TLX: 23561 ECF BAN BG

EML:

COM: $05,27,37$

TUCHMAN YTZHAK

RACAH INST OF PHYSICS

HEBREW UNIV OF JERUSALEM

JERUSALEM 91904

ISRAEL

TEL: 2584417

ILF :

ILX: 25391 HUIL

EML:

COM :

TULL ROBERT G

ASTRONOMY DPT

UNIVERSITY OF TEXAS

RLM 15308

AUSTIN IX $78712 \quad 1083$

USA

TEL: $512 \quad 4713337$

TLF :

TLX: 910-874-1351

EML:

COM: 09

TUNG NGUYEN MAU OR

COMMITTEE FOR SPACE RES

AND APPLICATION

NGHIA DO-TU LIEM

HANOI

VIETNAM

TEL:

TLF:

ILX:

EML:

COM: 04

TURLO ZYGMUNT BR

INSTITUTE OF ASTRONOMY

$N$ COPERNICUS UNIVERSITY

UL CHOPINA $12 / 18$

PL 87 100 TORUN

POLAND

TEL:

TLF :

TLX:

EML:

COM: 40
TSVETKOV TSVETAN DR

OPT OF ASTRONOMY

UNIVERSITY OF SOFIA

ANTON IVANOV ST 5

BG 1126 SOFIA

bulgarla

TEL: 2544852

TLF:

TLX:

EML:

COM:

\section{TUCKER WALLACE H DR}

BOX 266

BONSALL CA 92003

USA

TEL: 6197287103

TLF:

EML:

COM:

TLX:

TULLY JOHN A OR
OCA OBSERV DE NICE
BP 139
F 06003 NICE CDX
FRANCE
TEL: 93890420
TLF:
TLX: 460004 OBSNICE F
EML:
COM:

TUOHY IAN R DR

BRITISH AEROSP. AUSTRALIA

14 PARK WAY

TECHNOLOGY PARK

SALISBURY 5095

AUSTRALIA

TEL: 834322111

TLF: $\theta 3496629$

TLX: 88342

EML:

COM:

TURNER BARRY E DR

NRAO

EDGEMONT RD

CHARLOTTESVILLE VA 22901

USA

TEL: $B 042960337$

TLX: $910-997-0174$

EML:

ILF:

COM: 34,40
TSVETKOVA KATIA

ASTRONOMICAL OBSERVATORY BULGARIAN ACAD SCIENCES

72 LENIN BLVI

BG 1784 SOFIA

BULGARIA

TEL: 2758927

TLF :

TLX: 23561 ECF BAN BG

EML:

COM: 37

TUEG HELMUT OR ALFRED-WEGENER INSTITUT FUR POLARFORSCHUNG

COLUMBUS CENTER

D 2850 BREMERHAVEN

GERMANY

TEL:

TLF :

TLX:

EML:

COM: 09

TULLY RICHARD BRENT OR INSTITUTE FOR ASTRONOMY UNIVERSITY OF HAWAII

2680 WOODLAWN DR

HONOLULU HI 96822

USA

TEL: 8089488606

TLF:

ILX: 723-8459 UHAST HR

EML:

COM: 28,47

TUDMINEN ILKKA $\checkmark$ OR

OBS \& ASTROPHYSICS LAB

UNIVERSITY OF HELSINKI

TAEHTITORNINMAKI

SF 00130 HELSINKI 13

FINLAND

TEL: $191^{\circ} 2946$

TLF :

TLX:

EML: TUDMINENEF INUH

COM: $10,29,35.36$

TURNER OAVID G DR

OPT OF ASTRONOMY

ST MARY'S UNIVERSITY

HALIFAX NS B3H 3 C3

CANADA

TEL: $902429 \quad 9780 * 2254$

TLF : 9024205561

TLX:

EML:

COM: 27,37
ISYGAN ANATOLII I PROF IOFFE PHYSICAL TECH INST ACADEMY OF SCIENCES POLYIECHNICHESKAYA UL 26 194021 ST PETERSBURG RUSSIA

TEL: $812 \quad 2479326$

TLF: $812 \quad 247 \quad 1963$

TLX: 121453 FIIAN SL

EML: VARSHEEO.PTI.SPB.SU

COM: 48

TUFEKCIOGLU ZEKI DR DPT OF ASTRONOMY KING ABOULAZIZ UNIV BOX 9028

JEDDAH 21413

SAUDI ARABIA

TEL:

TLF :

TLX:

EML:

COM:

TUNCA ZEYNEL DR FACULTY OF SCIENCE EGE UNIVERSITY

BOX 21

35100 BORNOVA IZMIR

TURKEY

TEL: 51 LO $0110 * 2332$

TLF:

TLX:

EML:

COM:

TURATTO MASSIMO DR OSS ASTRONOMICO DI PADOVA

VIC OELL OSSERVATORIO 5

I 35122 PADOVA

ITALY

TEL: $4966 \quad 1499$

TLF :

ILX: 432071

EML: SPAN: $39003:$ : TURATTO

COM:

TURNER EDWIN L DR PRINCETON UNIVERSITY OBS PEYTON HALL PRINCETON NJ DB544

USA

TEL: $609258 \quad 3577$

TLF: $608 \quad 258 \quad 1020$

TLX: 322409 ASTRO PRIN

EML: 21 teASTROUVAV. PRINCETON. EDU COM: $28,47,51$ 
TURNER KENNETH C DR

NTL SCIENCE FOUNDATION

DIV ASTRONOMICAL SCIENCES

1800 G ST NW

WASHINGTON DC 20550

USA

TEL:

TLF :

TLX:

EML: KTURNERENOTE.NSF,GOV

COM: $05 \mathrm{C}, 34,40,51$

TURTLE A J OR

DPT OF PHYSICS

UNIVERSITY OF SYONEY

SYONEY NSW 2006

AUSTRALIA

TEL: 2.6922222

TLF:

TLX: 26169 UNISYD

EML

COM: 40

TWORKOWSKI ANORZEJ S

SCHOOL OF MATHEMATICAL SC

QUEEN MARY/WESTFIELD COLL

MILE END RD

LONDON EL 4NS

uk

TEL: 19804822

TLF :

TLX: 893750

EML:

COM:

TYSON JOHN A OR

BELL LABS

RM 1D-316

600 MUUNTAIN AVE

MURRAY HILL NJ 07974

USA

TEL: 2015826028

ILF:

TLX: 138650 BELL LABS MUH

EML:

COM: $21,28,47$

UCHIOA JUICHI QR

TOHOKU GAKUEN UNIVERSITY

TAGAYO UNIVERSITY

MIYAGI 985

JAPAN

TEL:

TLF

TLX

EML:

COM: 35
TURNER MARTIN J L DR

OPT OF PHYSICS

UNIVERSITY OF LEICESTER

$X$-RAY ASTRONOMY GROUP

LEICESTER LEI TRH

uk

TEL: $533554 \quad 455$

TLF :

TLX: 341664 LUXRAY G

EML

COM:

TUTUKOV A V OR

INST OF ASTRONOMY

ACADEMY OF SCIENCES

PYATNITSKAYA UL 48

109017 MOSCOW

RUSSIA

TEL: $231-54-61$

TLF:

TLX: 412623 SCSTP SU

EML: :

COM: $27,35 \mathrm{C}, 42 \mathrm{C}$

TYLENDA ROMUALD DR

INSTITUTE OF ASTRONOMY

$N$ COPERNICUS UNIVERSITY

UL CHOPINA $12 \%$ I8

PL 87100 TORUN

POLAND

TEL: $26018 \times 10$

TLF:

$T L X: 552234$ ASTR PL

EML:

COM: 27

TYTLER DAVID DR

EASS

UCSO

C 011

LA JOLLA CA 920930216

USA

TEL: 6195343460

TLF :

TLX: 5106010681 CASS UD

EML:

COM: 47

UCHIDA YUTAKA PROF

DPT OF ASTRONOMY

UNIVERSITY OF TOKYO

BUNKYO KU

TOKYO 113

JAPAN

TEL:

TLF :

TLX:

EML:

COM: 12
TURNER MICHAEL S

ASTRONOMY ASTROPHYS CTR

UNIVERSITY OF CHICAGO

$5640 \mathrm{~S}$ ELLIS AVE

CHICAGO IL 60637

USA

TEL: 3129627974

TLF :

TLX: 6871133 UNCGO VW

EML:

COM: 47

TWAROG BRUCE A

OPT PHYSICS \& ASTRONOMY

UNIVERSITY OF RANSAS

LAWRENCE KS 66045

USA

TEL: $913 \quad 864 \quad 5163$

TiF:

TLX:

EML:

COM: 37

TYLER JR G LEONARD DR

RADAR ASTRONOMY INST

STANFORD UNIVERSITY

STANFORO CA 943054035

USA

TEL: $415 \quad 497 \quad 3535$

TLF :

TLX:

EML:

COM: 16

TZIOUMIS ANASTASIOS DR

AAO

ATNF

BOX 76

EPPING NSW 2121

AUSTRALIA

TEL: 28680222

TLF: 28680310

TLX: 26230 ASTRO

EML: ATZIOUMIOATNF.CSIRO.AL

COM: 40

UDALSKI ANORZEJ DR

ASTRONOMICAL OBSERVATORY

WARSAW UNIVERSITY

AL UJAZDOWSKIE 4

PL 00478 WARSAW

POLAND

TEL: 294011

TLF :

TLX: 817063 OAUW PL

EML: VDALSKIOPLWAVWGI. BITNET

COM:
TURON C OR

OBSERVATOLRE DE PARIS

SECTION DE MEUUON

DASGAL

F 92195 MEUOON PPL CDX

FRANCE

TEL: $145 \quad 07 \quad 7837$

TLF: $145 \quad 077878$

TLX:

EML: TURONEFRMEU5L/MESIOA: : TURON

COM: $08,24 \vee P, 33$

TWISS R O DR

C/O A.R. BOSCHI

96A HOLLAND ROOSCHI

LONDON W14 8BD

UK

TEL:

TLF :

TLX:

EML:

COM:

TYLKA ALLAN J DR

NAVAL RESEARCH LABORATORY

CODE 4154 GAMMA \& COSMIC

RAY ASTROPHYSICS BRANCH

WASHINGTON DC 203755000

USA

TEL: $202 \quad 767 \quad 2200$

TLF: 2027676473

TLX:

EML: $11335:$ : TYLKA

COM: 48

UBERTINI PIETRO

IAS

CNR

CP 67

I O0044 FRASCATI

ITALY

TEL: 69425132

TLF:

TLX: 610261 CNRFRA

EML:

COM:

UDAL'TSOV V A OR

LEBEOEV PHYSICAL INST

ACADEMY OF SCIENCES

LENINSKY PROSPEKT 53

117924 MOSCOW

RUSSIA

TEL: 1358560

TLF :

TLX: 411479 NEOD SU

EMt:

COM: 40 


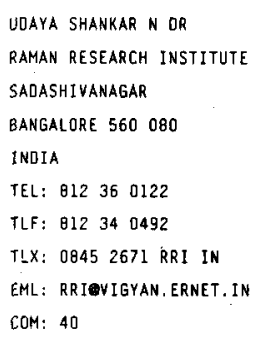

UENO SUEO PROF

KaNAZAWA TECHNOLOGY INST

7-1 OGIGAOKA

NONOTËHIMACHI

ISHIKAWA 921

JAPAN

TEL: $0762-48-1100$

TLF:

TLX: 5122456 KIY LCJ

EML:

COM: 36

ULICH BOBBY LEE

RAMAN AEROSPACE CORP

5055 E BROAOWAY BLVO

TUCSON AZ 85711

USA

TEL: 6027482038

TLF:

TLX:

EML:

COM: 09

ULRICH MARIE-HELENE O DR ESO

KARL - SCHWARSZCHILD-STR 2

O 8046 GARCHING MUENCHEN GERMANY

TEL: 8932006229

ILX: 52828222 EOD

EML:

TLF: 893202362

COM: $28 \mathrm{C}, 34,40$

UNDERHILL ANNE B OR 4696 WEST 10TH AVE \#301 VANCOUVER BC V6R 2.35

CANADA

TEL: 6042243552

TLF :

EML:

COM: $29,36,44$

TEX:

UNSOELD ALBRECHT PROF INST THEOR PHYS \& STERNW NEUE UNIV PHYSIK ZENTRUM OLSHAUSENST GEB N 610 D 2300 KIEL 1

GERMANY

TEL: 4318804205

TLF:

TLX:

EML:

COM
UESUGI AKIRA DR DPT OF ASTRONOMY KYOTO UNIVERSITY KITASHIRAKAWA SAKYO KU KYOTO 606 JAPAN

TEL:

TLE:

TLX:

EML:

COM: 05,36

ULMER MELVILLE P PROF OPT PHYSICS \& ASTRONOMY NORTHWESTERN UNIVERSITY DEARBORN OBSERVATORY EVANSTON IL 60208 USA

TEL: 3124915633

TLF:

TLX: 9102310040

EML:

COM:

ULRICH ROGER K PROF

OPT OF ASTRONOMY

UNIVERSITY OF CALIFORNIA 8931 MSB

LOS ANGELES CA 90024

USA

TEL: 2138254270

TLF:

TLX:

EML:

COM: 35

UNOERWOOD JAMES H OR

LAWRENCE BERKELEY LAB UNIVERSITY OF CALIFORNIA $X$ RAY OPTICS LAB 80101 BERKELEY CA 94720

USA

TEL: 4154854958

TLF:

TLX: 9103662037

EML:

COM: 10,44

UNWIN STEPHEN C

CALTECH

MS 10524

PASADENA CA 91125

USA

TEL: 2133564973

TLF:

TLX: 675425

EML:

COM: 40
UKITA NOBUHARU NOBEYAMA RADIO OBS NAOJ

MINAMIMAKI MURA

NAGANO $384 \quad 13$

JAPAN

TEL: 267-98-2831

TLF:

TLX: 3329005

EML:

COM: 40

ULMSCHNEIDER PETER PROF INST FUER THEORETISCHE ASTROPHYSIK

IM NEUENHEIMER FELD 561 0. 6900 HE IOELBERG 1

GERMANY

TEL: $62 \quad 215 \quad 62837$

TLF:

TLX: 461515 UNIHO D

EML: I9B@OHDURZI

COM: 36

UMEMURA MASAYUKI OR TOKYO ASTRONOMICAL OBS NAOJ

OSAWA MITAKA

TOKYO 181

JAPAN

TEL: $81 \quad 422 \quad 413731$

TLF: 81422413746

TLX: 028 22307 TAOMTK J

EML: UMEMURAOUME. MTK, NAO.AC.JP

COM: 47

UNGER STEPHEN DR ROYAL GREENWICH OBS HERSTMONCEUX CASTLE

HAILSHAM

EAST SUSSEX BN27 IRP

UK

TEL: 323833171

TLF:

TLX: 87451

EML: UK, AC, RGO. STAR

COM: 28,40

UOMOTO ALAN K DR DPT PHYSICS \& ASTRONOMY JOHNS HOPKINS UNIVERSITY CHARLES 34TH ST BALTIMORE MO 21218 USA TEL: 3013388594 TLF:

ILX:

EML: AUOSTSCI.BITNET COM: 
UPGREN ARTHUR R DR OPT OF ASTRONOMY VAN VLECK OBSERVATORY WESLEYAN UNIVERSITY MIODLETOWN CT 06457 USA

TEL: 2033479411

TLF:

TLX

EML:

COM: $24,26,33,37,45,50$

URASIN LIRIK A DR

ENGELHARDT ASTRONOMICAL

DBSERVATORY

OBSERVATORIA STATION

422526 XAZAN

RUSSIA

TEL: $32-48-27$

TLF:

TLX:

EML:

COM: 34

\section{URPO SEPPO I}

HELSINKI UNIV TECHNOLOGY

RADIO LABORATORY

OTAKAARI $5 \mathrm{~A}$

SF D2150 ESPOO 15

FINLAND

TEL: 4512548

TLF:

TLX: 122771 RORTA SF

EML:

COM: 10,40

\section{USOWICS JERZY BOGDAN DR}

INST OF RADIO ASTRONOMY

N COPERNICUS UNIVERSITY

UL CHOPINA $12 / 18$

PL 87100 TORIN

POLAND

TEL:

TLF :

TLX:

EML:

COM:

VAGHI SERGIO DR
ESA/ESTEC
PHA
BOX 299
NL 2200 AG NOOROW
NETHERLANDS
TEL: 171983453
TLF:
TLX: 39098
EML:
COM: 20

UPSON WALTER L II DR

PRINCETON UNIVERSITY OBS

PEYTON HALL

PRINCETON NJ OQ5.44

USA

TEL:

TLF:

TLX:

EML:

COM: 44

URBANIK MAREK OR

ASTRONOMICAL OBSERVATORY

JAGIELLONIAN UNIVERSITY

UL ORLA 171

PL 30244 KRAKOW

POLANG

TEL: $4812-221877$

TLF :

TLX: $0322297 \mathrm{UJ} P L$

EML:

COM: 28

\section{URRY CLAUOIA MEGAN DR}

STSCI

HOMEWOOO CAMPUS

3700 SAN MARTIN DR

BALTIMORE MO 21210

USA

TEL: 3013384593

TLF:

TLX: 6849101 STSCI

EML: SCIVAX: : CMU/CMU®STSCI, BITHET

COM:

UTSUMI KAZUHIKO OR

DPT OF ASTRONOMY

HIROSHIMA UNIVERSITY

HIGASHI SENDA MACHI

HIROSHIMA 730

JAPAN

TEL: $082-241-1221$

TLF:

$T L X$ :

EML:

COM: 29

\section{VAGNETTI FAUSTO}

DPT OI FISICA

II UNIVERSITA DI ROMA

VIA ORAZIO RAIMONDO

I 00173 ROMA

ITALY

TEL: $6 \quad 24990431$

TLF: 624990300

TLX: 626382 FIUNTV I

EML: VAGNETTIOVAXTOV. INFN. IT

COM: 47
UPTON E K L OR

DPT OF ASTRONOMY

UNIVERSITY OF CALIFORNIA

LOS ANGELES CA $900 \hat{24}$

USA

TEL:

TLF:

TLX:

EML:

COM:

URBARZ H OR

ASTRONOMISCHES INSTITUT

DER UNIVERSITAET

AUSSENSTELLE WEISSENAU

D 7980 RAVENSBURG/RASTHAL

GERMANY

TEL: 75161621

TLF :

TLX:

EML:

COM:

\section{USHER PETER D DR}

DPT OF ASTRONOMY

oEnNSYLVANIA STATE UNIV

507 DAVEY LAB

UNIVERSITY PARK PA 16802

USA

TEL: $814 \quad 865 \quad 3509$

TLF :

TLX: 842510 PENNSTBSTRCG

EML:

COM: 27

UUS UNDO OR

TARTU ASTROPHYSICAL OBS

ESTONIAN ACAD OF SCIENCES

202444 TARTU

ESTONIA

TEL:

TLF:

TLX:

EML:

COM: 12,35

VAIANA GIUSEPPE S QR

OSS ASTRONOMICO

UNIVERSITA OI PALERNO

PALAZZO DEI NORMANNI

I 90134 PALERMO

ITALY

TEL: $91 \quad 42 \quad 2588$

TLF :

TLX:

EML:

COM:
URAS SILVANO QR

ISTITUTO OI ASTRONOMIA

VIA OSPEDALE 72

I 09100 CAGLIARI

ITALY

TEL: $7071 \quad 1246$

TLF:

TLX: 790326 OSSAST I

EML:

COM:

URECHE VASILE QR

FAC OF MATHEMATICS

UNIVERSITY OF CLUJ-NAPOCA

STR M KOGALNICEANU 1

R 3400 CLUJ NAPOCA

RUMANIA

TEL: $951-16101 \% 11592$

TLF:

TLX:

EML:

COM: 25,42

USON JUAN M OR

NRAD

VLA

BOX O

SOCORRO NM $87801 \quad 0387$

USA

TEL: 5058357237

TLF :

TLX: 9109801710

EML:

COM: 40,47

VAGER ZEEV DR

DPT OF PHYSICS

WEIZMANN INSTITUTE OF SCI

BOX 26

REHOVOT 76100

ISRAEL

TEL:

ILF:

TLX:

EML:

COM:

VAIDYA P. C PROF

34. SHARDA NAGA

PALOI

AHMEDABAD 380007

INDIA

TEL: 272413322

TLF :

TLX:

EML:

COM: 47 
VAINSTEIN L A OR

LEBEOEV PHYSICAL INST

ACADEMY OF SCIENCES

LENINSKY PROSPEKT 53

117924 MOSCOW

RUSSIA

TEL: $135-22-50$

ILF:

$T L X$ :

EML

COM: 49

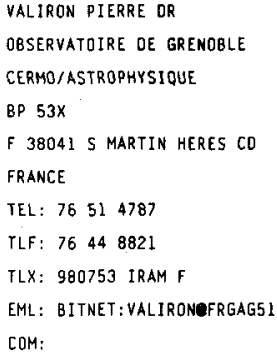

COM: 44
VAKILI FARROKH DR OCA OBSERY DU CALERN CAUSSOLS

F $06460 S$ VALLIER THIEY

FRAHCE

TEL: $93 \quad 42 \quad 6270$

TLF: 93092613

TLX: $461402 \mathrm{~F}$

EML: VAKILIOFRONI5

COM: 09,36

VALLEE JACOUES P OR
HERZBERG INST ASTROPHYS
NTL RESEARCH COUNCIL
100 SUSSEX DR
OTTAWA ON KLA OR6
CANADA
TEL: 6139936060
TLF: 6139526602
TLX: 0533715
EML:
COM: 40.51
VALTAOJA LEENA OR
TURKU UNIVE.RSITY OBS
TUORLA
SF 21500 PIIKKIO
FINLAND
IEL: 21435822
TLF: 21433767
TLX:
EML: LVALTAOJAEKONTU.UTU.FI
COM:

VAN ALBADA TJEERD S DR KAPTEYN ASTRONOMICAL INST B0X 800

NL 9700 AV GRONINGEN NETHERLANDS

TEL: 50116695

TLX: 53572 STARS NL

EML:

TLF:

COM: 28

VAN BLERKOM DAVIO J PROF DPT OF ASTRONOMY UNIV OF MASSACHUSETTS AMHERST MA 01002 USA

TEL:

TLF:

ILX:

ERL:

COM:
VALBOUSQUET ARMAND DR OBS DE STRASBOURG

11 RUE UNIVERSITE

F 67000 STRASBOURG FRANCE

TEL: $88 \quad 354300$

TLF : $88 \quad 250160$

TLX:

EML:

COM: $24,26,51$

VALNICEK BORIS DR ASTRONOMICAL INSTITUTE CZECH ACADEMY OF SCIENCES ONOREJOV OBSERVATORY CS 25165 ONOREJOV CZECHOSLOVAKIA

TEL: 20485201 TLF: 20485314

TLX: 121579

EML:

COM: $09,10,44$

VALTIER JEAN-CLAUDE OR OCA OBSERV DE NICE

BP 139

F 06003 NICE CDX

FRANCE

TEL: 93890420

TLF :

TLX: 460004 OBSNICE F

EML:

COM: 27.29

VAN ALLEN JAMES A PROF DPT PHYSICS \& ASTRONOMY UNIVERSITY OF IOWA IOWA CITY IA 52242 USA

TEL: 3193534531

TLF :

TLX:

EML:

COM: $20,16,21,49$

VAN BREQA IAN G OR ROYAL GREENWICH OBS HERSTMONCEUX CASTLE HAILSHAM BN27 IRP

UK

TEL: $323 \quad 833 \quad 171$

TLF:

TLX: 87451

EML:

COM:
VALENTIJN EOWIN A DR KAPTEYN ASTRONOMICAL INST

BOX 800

NL 9700 AV GRONINGEN NETHERLANDS

TEL: 50116695

TLF :

TLX:

EML:

COM: 28

VALSECCHI GIOYANNI B DR IAS REPARTO DI PLANETOLOGIA VIA OELL'UNIVERSITA 11 I OOL85 ROMA ITALY

TEL: 64456951

ILF :

TLX: [NR FRA 610261

EM: BITNET: GIOVANNIOIRMIAS

COM: $07,15,20$

VALTONEN MAURI J PROF TURXU UNIVERSITY OBS TUORLA

SF 21500 PIIKKIO

FINLAND

TEL: $21 \quad 435822$

TLF: 21433767

TLX: 62638 TYF SF

EML:

COM: $07,09,26,28,33,40,44$

VAN ALTENA WILLIAM F PROF YALE UNIVERSITY OBS

BDX 6666

NEW HAVEN CT 06511

USA

TEL: $203436 \quad 8318$

TLF : 2034325048

TLX: 9102508365

EML: VANALTENEYALASTRO

COM: $08,24 C, 26,37$

VAN BREUGEL WIL RADIO ASTRONOMY LAB UNIVERSITY OF CALIFORNIA 601 CAMPBELL HALL BERKELEY CA 94720 USA TEL: $415 \quad 6425275$

TLF :

TLX:

EML:

COM: 
VAN BUEREN HENDRIK G PROF MEIOOORNLAAN 13 NL 3461 - ES LINSCHOTEN NETHERLANDS

TEL: $34 \quad 60 \quad 15406$

TLF :

EML:

COM:

TLK:

VAN DE STADT HERMAN DR SPACE RESEARCH DPT $B 0 \times 800$

NL 9700 AV GRONINGEN NETHERLANDS

TEL: 50116695

TLX: 53572

EML:

TLF:

COM:

VAN DER BORGHT RENE PROF

31 THE PROMENAOE

ISLE OF CAPRI

SURFERS PARADISE 4217

AUSTRALIA

TEL: 385712

TLX:

EML:

TLF :

COM: 35

YAN DER KRUIT PIETER C DR KAPTEYN ASTRONOMICAL INST BOX 800

NL 97BQ AV GRONINGEN

NETHERLANDS

TEL: $50 \quad 63 \quad 4073$

TLX: 53572 STTARS NL

EML:

TLF:

COM: $28,33,40$

VAN DESSEL EOWIN LUDO DR OBSERVATOIRE ROVAL DE

BELGIQUE

AVE CIRCULAIRE 3

B 1180 BRUSSELS

BELGIUM

TEL: 26735366

ILF: 23749822

TLX: 21565 OBSBEL

EML:

COM: $26 \mathrm{C} .30$
VAN CITTERS GORDON W DR

NTL SCIENCE FOUNDATION

DIV ASTRONOMICAL SCIENCES

1800 G ST NW

WASHINGTON DC 20550

USA

TEL:

TLF:

TLX:

EML:

COM: 09

VAN DEN BERGH SIONEY PROF HERZBERG INST ASTROPHYS DOMINION ASTROPHYS OBS

5071 W SAANICH RO

VICTORIA BC V8X $4 M 6$

CANADA

TEL: $604 \quad 388 \quad 3924$

TLF: 6043630045

TLX: 0497295

EML:

COM: $28,37,50$

VAN DER HUCHT KAREL A DR

SPACE RESEARCH LABQRATORY SRON

SORBONNELAAN 2 .

NL 3584 CA UTRECHT

NETHERLANOS

TEL: 30535600

TLF: 30540860

TLX: 47224

EML:

COM: $26,29,44$

VAN DER LAAN H PROF DR

ESO

KARL SCHWARZSCHILDSTR. 2

D 8046 GARCHING MUENCHEN GERMANY

TEL: $89320 \quad 06227$

$T L F: 893202362$

TLX: 52828220 EO D

EML: HVDLAANOESOMCO. HQ. ESO.ORE

COM: $28,34,40,47$

VAN DIGGELEN J DR

OBSERVATORY UTRECHT

AETSVELDSELAAN 12

NL 1381 EA WEESP

NETHERLANDS

IEL:

TLF:

TLX:

EML:

COM:
VAN DE HULST H C PROF DR STERREWACHJ

BOX 9513

NL 2300 RA LEIDEN

NETHERLANDS

TEL: 71148333

TLF :

TLX: 39058

EML:

COM: $21,34,40,44$

VAN DEN HEUVEL EDWARD $P J$ ASTRONOMICAL INSTITUTE UNIVERSITY OF AMSTERDAM KRUISLAAN 403

NL 1098 SJ AMSTERDAM NETHERLANDS

TEL: 205257491

TLF: 205257484

TLX: 10262 HEF NL

EML:

COM: $35,38,42,48$

VAN DER HULST JAN M DR KAPTEYN ASTRONOMICAL INST BOX 800

NL 9700 AV GRONINGEN

NETHERLANDS

TEL: $50 \quad 63 \quad 4054$

TLF: 50634033

ILX: 53572 STARZ NL

EML: VDHULSTOHGRRUG5

COM: 28,400

VAN DER RAAY HERMAN B

OPT OF PHYSICS

UNIVERSITY OF BIRMINGHAM

BOX 363

BIRMINGHAM B15 2TT

IJK

TEL: $21472 \quad 1301$

TLF :

TIX: 228938 SPAPHY G

EML:

COM: 35

VAN OISHOECK EWINE F OR STERREWACHT

B0X 9513

NL 2300 RA LEIDEN

NETHERLANDS

TEL: $7127 \quad 5874 / 5835$

TLF: 71275819

TLX: 39058 ASTRO NL

EML:

COM: 34
VAN DE KAMP PETER

AMSTEL 244

NL 1017 AK AMSTERDAM

NETHERLANOS

TEL: $20 \quad 223377$

TLF:

TLX:

EML:

COM: $24,26,51$

VAN DEN OORD BERT H J OR STERREKUNDIG INSTITUTE

B0X 80000

NL 3500 TA UTRECHT

NETHERLANOS

TEL: 30535200

TLF : $30 \quad 53 \quad 1601$

TLX: 40048 FYLUT

EML: OORDOFYS.RUU.NL

COM: 10

VAN DER KLIS MICHIEL OR ASTRONOMICAL INSTITUTE UNIVERSITY OF AMSTERDAM KRUISLAAN 403 NL 1098 SJ AMSTERDAM NETHERLANDS

TEL: $205257498 / 7491 / 7492$

TLF: 205257484

TLX: 10262 HEF NL

EML: EARN/BITNET: A4LOMVDKEHASARAII COM:

VAN OER WALT D J OR

DPT OF PHYSICS

POTCHEFSTROOM UNIVERSITY

POTCHEFSTROOM

SOUTH AFRICA

TEL: $27 \quad 148 \quad 992408$

TLF: $27 \quad 148 \quad 2421$

$T L X: 346019$ SA

EML: FISJUDWPUKVML, PUK, AC.ZA

COM: 48

VAN DORN BRADT HALE DR CENTER FOR SPACE RESEARCH MIT RM 37581

BOX 165

CAMBRIDGE MA 02139

USA

TEL: 6172537550

TLF :

TLX; 921473 MITCAM

EML:

COM: 


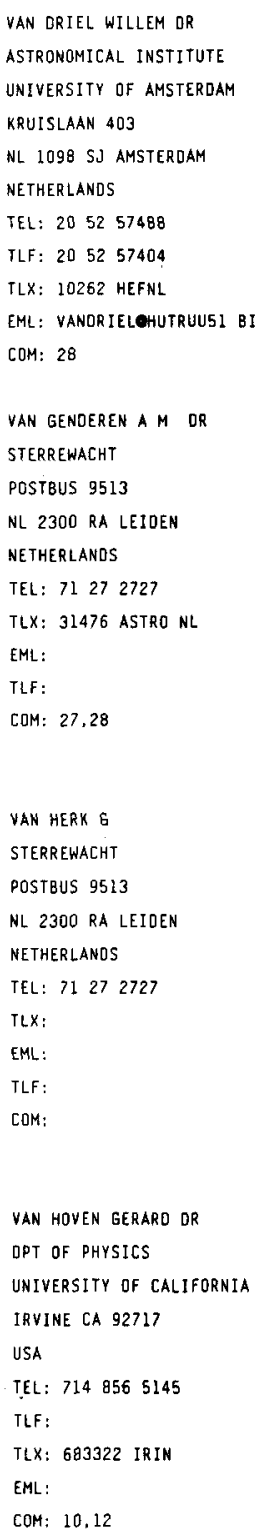

COH: 10,12

VAN PARAOIJS JOHANNES OR ASTRONOMICAL INSTITUTE UNIVERSITY OF AMSTERDAM KRUISLAAN 403 NL 1098 SJ AMSTEROAM NETHERLANOS

TEL: 205257491

TLF: 205257484

TLX: 10262 HEF NL

EML:

COM: 42
VAN DRIEL-GESZTELYI L OR STERREKUNDIG INSTITUTE BOX 80000

NL 3508 TA UTRECHT NETHERLANDS

TEL:

TLX:

EML:

TLF :

COM: 10

VAN GORKOM JACQUELINE H MRAO

BOX 0

SOCORRO NM $87801 \quad 0387$ USA

TEL: 5057724302

TLX: $910-997-0174$

EML:

TLF :

COM: $28,34,40$

VAN HORN HUEH M PROF OPT PHYSICS \& ASTRONOMY UNIVERSITY OF ROCHESTER ROCHESTER NY 14627 USA

TEL: $716 \quad 275 \quad 4344$

$T L F$ :

TiK:

EML:

COM: 35

VAN LEEUWEN FLOOR OR ROYAL GREENWICH OBS HERSTMONCEUX CASTLE HAILSHAM EAST SUSSEX BN27 IAP UK

TEL:

TLF :

TLX:

EML:

COM: 08

VAN REGEMORTER HENRI DR OBSERVATOIRE DE PARIS SECTION DE MEUDON

f 92195 MEUDON PPL COX FRANCE

TEL: $1145 \quad 077444$

TLF :

TLX: 201571

EML:

COM: 14,36
VAN DUINEN R J DR

FOKKER BV

SPACE OIVISION

$80 \times 7600$

NL 1117 ZJ SCHIPHOL

NETHERLANQS

IEL: 20.5442030

TLF :

$T L X: 12227$

EML:

COM: 44

VAN GRONINGEN ERNST DR ASTRONOMICAL OBSERVATORY BDX 515

S 75120 UPPSALA

SWEDEN

TEL:

TLF :

TLX:

EML:

COM:

VAN HOUTEN C J DR

STERREWACHT

POSTBUS 9513

NL 2300 RA LEIQEN

NETHERLANOS

TEL: 71272727

TLF :

TLX: 39058 ASTRO NL

EML:

COM: 20

VAN MOORSEL GUSTAAF DR

ESO

KARLSCHWARZSCHILO STR . 2

D 8046 GARCHING MUENCHEN GERMANY

TEL: 8932006362

TLF: 893202362

TLK: $52828222 E 0 \quad \mathrm{O}$

EML:

CDM: 28

VAN RENSBERGEN WALJER OR

IAAG

VRIJE UNIV BRUSSELS

CP 165

B 1050 BRUSSELS

BELGIUM

TEL: 26413497

TLF :

TLX:

EML:

COM: 14
VAN FLANDERN THOMAS OR

VF ASSOCIATES

6327. WESTERN AVE NW WASHINGTON DC 20015

USA

TEL: $202363 \quad 3860$

TLF :

TLX:

EML:

COM: $04,15,16,20,51$

VAN HAMME WALTER DPT PHYSICS \& ASTRONOMY FLORIDA ITL UNIVERSITY UNIVERSITY PARK MIAMI FL 33199 USA

TEL:

TLF:

TLX:

EML:

COM: 42

VAN HOUTEN-GROENEVELD I STERREWACHT

POSTBUS 9513

NL 2300 RA LEIDEN

NETHERLANOS

TEL: 71272727

TLF:

TLX: 39058 ASTRO NL

EML:

COM: 20

VAN NIEUWKOOP J OR IR PRINSESSELAAN 12

NL 7316 CN APELDOORN NETHERLANDS

TEL:

TLF:

$T L X$ :

EML:

COM: 40

VAN RIPER - KENNETH A. DR LOS ALAMOS NATIONAL LAB MS B $226 \times 6$ BOX 1663 LOS ALAMOS NM 87.545 USA TEL: $505 \quad 667 \quad 8104$ TLF :

TLX:

EML:

COM: 35,48 
VAN SPEYBROECK LEON P OR CENTER FOR ASTROPHYSICS HCO/SAD

GO GARDEN ST

CAMBRIDGE MA 02138 USA

TEL: 6174957233

TLF :

TLX

EML:

COM: 44

VANDERVOORT PETER O DR ASTRONOMY \& ASTROPHYS CTR UNIVERSITY OF CHICAGO $5640 \mathrm{~S}$ ELLIS AVE CHICAGO IL 60637 USA

TEL: 3129628209

TLF :

TLX:

EML:

COM: 33

VAPILLON LOIC J DR OBSERVATOIRE DE PARIS SECTION DE MEUDON F 92195 MEUDON PPL CDX FRANCE

TEL: $145 \quad 07 \quad 7623$

TLF :

$T L X: 201571$

EML:

COM:

VARMA RAM KUMAR PROF PHYSICAL RESEARCH LAB NAVRANGPURA

AHMEOABAO 380.009

INDIA

TEL: 272462129

TLF : 272445292

TLX: 0121397 PRL IN

EML:

COM: 28

VASILEVA GALINA J DR

PULKOVO OBSERVATORY

ACADEMY OF SCIENCES

10 KUTUZOV QUAY

196140 ST PETERSBURG

RUSSIA

TEL:

TLF

$T L X:$

EML:

COM: 12
VAN WOEROEN HUGO PROF DR KAPTEYN ASTRONOMICAL INST $B 0 \times 800$

NL 9700 AV GRONINGEN NETHERLANDS

TEL: 5011.6695

TLX: 53572 STARS NL

EML:

TLF

COM: $28,33,34,40$

VANYSEK VLADIHIR PROF

DPT OF ASTRONOMY

CHARLES UNIVERSITY

SVEOSKA $B$

CS 150 DO PRAHA 5

CZECHOSLOVAKIA

TEL: 2540395

TLF :

TLX: $121673 \mathrm{MFF}$

EML:

COM: $15,34,47$

VARDANIAN R A OR

BYURAKAN ASTROPHYSICAL

OBSERVATORY

378433 ARMENIA

ARMENIA

TEL: $8852 \quad 284142$

TLF :

T.LX

EML

COM: 25

VARSHALOVICH DIMITRTJ PR IOFFE PHYSICAL TECH INST. ACADEMY OF SCIENCES

POLYTECHNICHESKAYA UL 26 194021 ST PETERSBURG

RUSSIA

TEL: $247-22-55$

TLF :

TLX:

EML:

COM: $14,34,51$

VASU-MALLIK SUSHMA OR

INDIAN INSTITUTE OF

ASTROPHYSICS

KORAMANGALA

BANGALORE 560034

INOIA

IEL: $81256 \quad 9179 / 9180$

ILF:

ILX: 845763 IIAB IN

EML:

COM: 29,36

\author{
VANDEN BOUT PAUL A \\ NRAO \\ EOGEMONT RD \\ CHARLOTTESVILLE VA 22903 \\ USA \\ TEL: $B 042960241$ \\ TLF: \\ TLX: 910-997-0174 \\ EML: \\ COM: 34,40
}

YAN'T VEER FRANS OR OBSERVATOIRE DE PARIS

6l AVE OBSERVATOIRE

F 75014 PARIS

FRANCE

TEL: 140512221

TLF:

TLX: 270776 OBS $P$

EML:

COM: $10,36,42$

VAROAVAS ILIAS MIHAIL

OPT OF PHYSICS

UNIVERSITY OF CRETE

BOX 1527

GR 7 II 11 IRAKLION

GREECE

IEL: 81236589

TLF: 024167902

TLX: $26272 B$

EML:

COM: 36

VARVOGLIS H DR

DPT OF ASTRONOMY UNIVERSITY THESSALONIKI

GR 540 O6 THESSALONIKI

GREECE

TEL: $3199 \quad 1357$

TLF:

TLX: 412181

EML:

COM: 07

VATS HARI OM DR

PHYSICAL RESEAREH LAB

NAVRANGUYA

AHMEOABAD 9

INถIA

TEL: $272 \quad 46 \quad 2129$

TLF: 272445292

TLX : 01216397

EML: IPSAPRL, ERNET. IN

COM: 40
VANDENBERG DON DR

OPT OF PHYSICS

UNIVERSITY OF VICTORIA

BOX 1700

VICTORIA BC VEW $2 Y 2$

CANADA

TEL: 6047217739

TLF: 6047217715

TLX:

EML:

CON: $35 C, 37 C$

VAN'T VEER-MENNERET CL DR OBSERVATOIRE DE PARIS

GI AVE OBSERVATOIRE

F 75014 PARIS

FRANCE

TEL: 140512249

TLF :

TLX:

EML:

COM: 29,36

VARDYA MS OR

IIFR

HOMI BHABHA RD

COLABA

BOMBAY 400005

INDIA

TEL: $22 \quad 219 \quad 111 * 221$

TLF :

TLX: 011-3009 TIFR IN

EML:

COM: 35,36

VASHKOV'YAK SOF'YA N OR

STERNBERG STATE ASTR INST

UNIVERSITETSKIJ PROSP 13

119899 MOSCOW

RUSSIA

TEL: 139-37-64

TLF :。

TLX: 113037 JAPET

EML:

COM: 07

VAUCLAIR GERARD P DR

OBS MIDI PYRENEES

14 AVE E BELIN

F 31400 TOULOUSE CDX

FRANCE

TEL: $6125 \quad 2101$

TLF :

TLX: 530776

EML:

COM: $35 \mathrm{C}$ 
VAUCLAIR SYLVIE $D$ DR OBS MIDI PYRENEES

14 AVE E BELIN

F 31400 TOULOUSE COX

FRANCE

TEL: 61252101

TLF:

TLX:

EML:

COM: 46

VAVROVA ZOENKA OR
KLET OBSERVATORY
CESKE BUDEJOVICE
CS BEZRUCOVA A
CZECHOSLOVAKIA
TEL: 3373274
ILF:
TLX:
EML:
COM: 20

VECK NICHOLAS

MARCONI RESEARCH CENTRE

WEST HANNINGFIELD RD

GT BADDOW

CHELMSFORD ESSEX CMZ BHN uk

TEL: $245 \quad 733 \quad 31$

TLF: 24575244

TLX: 995016 GECRES $G$

EML: YEOQXa.gec-nrc. c0. ukeuel-cs

COM: 10

VEIS GEORGE PH O
GEOOESY LABORATORY
NATL TECHNICAL UNIVERSITY
PANEPIST IMLOPOLIS
GR 15773 Z0GRAFOS
GREECE
TEL: 17243414
TLF:
TLX:
EML:
COM:
VELLI MARCO OR
OBSERVATOIRE DE PARIS
SECTION OE MEUDON
OESPA
F 92195 MEUOON PPL COX
FRANCE
TEL: 145 07 7659
TLF: 145 O7 2806
TLX:
EML: VELLIOFRMEU51/17710: $:$ VELLI
COM: 10

VAUGHAN ALAN OR

SCHOOL OF MATHS/PHYSICS

COMPUTING AND ELECTRONICS

MACQUARIE UNIVERSITY

MACQUARIE 2109

AUSTRALIA

TEL: 28058904

TLF: 28058983

TLX: MACUNI AA 122377

EML: ALANVEMACASTRO.MPCS.MQ.0Z. AU

COM: 40

VAZ LUIZ PAULO RIBEIRO

OBSERVATORIO ASTRONOMICO

OEPTO OE FISICA-ICEX-UFMG

CP 702

30161 BELO HORIZONTE MG

BRAZIL

TEL: 314412541

TLF :

TLX: 312308 UFMG BR

EML:

COM: 42

VEEDER GLENN J DR

JPL

MS 183501

4800 OAK GROVE OR

PASADENA CA 91109

USA

TEL: $213 \quad 3547389$

ILF

TLX:

EML:

COM: 15

VEISMANN UNO OR

TARTU ASTROPHYSICAL OBS

ESTONIAN ACAD OF SCIENCES

202444 TARTU

ESTONIA

TEL:

TLF

TLX:

EML:

COM:

VELUSAMY T OR
TIFR/RADIO ASTRONOMY CT
BOX 8
UDHAGAMANDALAM 643001
TEL: $423 \quad 2651 / 2032$
TLF:
TLX: 8458488 TIFR IN
EML:
COM: $40 C$

VAUGHAN ARTHUR H OR

PERKIN-ELMER CORP

7421 ORANGEWOOD AVE

GARDEN GROVE CA 92641

USA

TEL: $714895 \quad 1667$

TLF:

TLX:

EML:

COM: $10,12,25,36$

VAZQUEZ MANUEL DR

INST DE ASTROFISICA

DE CANARIAS

OBS OEL TEIDE

E 38071 LA LAgUNA

SPAIN

TEL:

TLF :

TLX: 92640 IACE E

EML:

COM: 51

VEGA E IRENE OR

OBSERVATORIO ASTRONOMICO

PASEO DEL BOSQUE

1900 . LA PLATA (BS.AS.)

ARGENTINA

TEL: $2121730 \mathrm{~B}$

TLF:

TLX: 31151 BULAP AR

EML:

COM: 33

VEKSTEIN GREGORY DR

INSTITUTE OF NULLEAR PHYS

ACADEMY OF SCIENCES

SIBERIAN OIV

630090 NOVOSIBIRSK

RUSSIA

TEL: 3832359407

TLF: 3832352163

TLX: 133116 ATOM SU

EML: VEKSTEINOVXINPA. INP.NSK.SU

COM: 10

VENKATAKRISHNAN P DR

NASA/MSFC

CODE ES 52

HUNTSVILLE AL 35812

USA

TEL: 2055449404

TLF :

TLX:

EML:

COM: 12
VAUGLIN ISABELLE DR

OBSERVATOIRE DE LYON

AVE CHARLES ANDRE

F $69561 \mathrm{~S}$ GENIS LAVAL COX

FRANCE

TEL: 72399098

TLF: 72399791

TLX: 310926

EML: VAUGLINECASTOR. UNIV-LYONL.FR

COM: 28

VAZQUEZ RUBEN ANGEL OR

OBSERVATORIO ASTRONGMICO

LA PLATA

1900 LA PLATA (BS.AS.)

ARGENTINA

TEL: 21217308

TLF :

TLX: 31216 CESLA AR

EML: RVAZQUEZFFCAGLP, EDU, AR

COM: 37

VEILLET CHRISTIAN

OCA CERGA

AVE COPERNIC

F 06130 GRASSE

FRANCE

TEL: $93 \quad 36 \quad 5849$

TLF:

TLX: 470865

EML:

COM: $07,19,20,31$

VELKOV KIRIL

ASTRONDMICAL OBSERVATORY

BULGARIAN ACAD SCIENCES

72 LENIN BLVO

BG 1784 SOFIA

BULGARIA

TEL: 2758927

TLF :

TLX: 23561 ECF BQN BG

EML:

COM: 09,10

VENKATESAN DORASWAMY OR

DPT OF PHYSICS

UNIVERSITY OF EALGARY

2500 UNIVERSITY OR NW

CALGARY AB T2N IN4

CANADA

TEL: $403 \quad 9312366$

TLF: $403 \quad 289 \quad 3331$

TLX:

EML.

COM: 10 
VENTURA JOSEPH OR

DPT , RF PHYSICS

UNIVERSITY OF CRETE

BOX 1527

GR 71111 IRAKLION

GREECE

TEL: 81239757

TLF :

TLX: 262728

EML: VENTURA GREARN

COM:

VERDET JEAN-PIERRE OR

OBSERVATOIRE DE PARIS

61 AVE OBSERVATOIRE

F 75014 PARIS

FRANCE

TEL: 140512206

TLF :

TLX:

EME:

COM: 41

VERHEEST FRANK PROF
INST THEORET MECHANIKA
RIJKSUNIVERSITEIT GENT
KRIJGSLAAN 281
B 9000 GENT
BELGIUM
TEL: 91225715
TLF: 91240634
TLX: 12754 RUGENT B
EML: VERHEESTEIEOMECH. RUG. AC. BE
COM: $10,27,49$

\section{VERNIANI FRANCO PROF}

OPT OI FISICA

CNR

VIA IRNERIO 46

I 40126 BOLOENA

ITALY

TEL: 51260991

TLF:

TLX: 211664

EML:

COM: 22

\section{VERTER FRANCES DR}

NASA/GSFC

CODE 685

GREENBELT MD 20771

USA

TEL: 3012867860

TLF:

TLX:

EML:

COM: 40
VENUGOPAL $V$ R DR

TIFR/RADIO ASTRONOMY CTR

BOX 8

UDHAGAMANDALAM 643001

INDIA

TEL: 423 2651/2032

TLF :

TLX: 0853-241 RAC IN

EML:

COM: $33,40,51$

VERES FERENC

KONKOLY OBSERVATORY

THEGE U 13/17

BOX 67

H 1525 BUDAPEST

HUNGARY

TEL: 1755866

TLF : 1.569640

TLX: 227460

EML:

COM:

VERMA R P OR
TIFR
HOMI BHABHA RO
COLABA
BOMBAY 400005
INDIA
TEL: $22 \quad 219111$
TLF:
TLX: 0113009 TIFR IN
EML:
COM: 25

VERON MARIE-PAULE OR DHP

F 04870 S MICHEL OBS FRANCE

TEL: 92766368

TLF:

EML:

COM: 28

TLX: 410690 OHP F

VESECKY J F DR

RADAR ASTRONOMY INST STANFORD UNIVERSITY

233 DURAND

STANFORO CA $94305 \quad 4035$

USA

TEL:

ILF:

ILX:

EML:

COM:
VERBEEK PAUL DR

GEORGE MINNELAAN 50

B 9830 S MARTENS-LATEM

BELGIUM

TEL: 9 82 6119

TLF:

TLX:

EML:

COM:

Vergez madeleine or

OBS MIOI PYRENEES

9 R PONT DE LA MOUETTE

F 65200 BAGNERES BIGORRE

FRANCE

TEL: 62951969

TLF :

TLX: 531625 OBSPIC F

EML:

COM: 10

VERMA SATYA DEV DR

OPT PHYSICS \& SPACE SCI

UNIVERSITY SCHOOL OF SCI GUJARAT UNIVERSITY

AHMEDABAD 380009

INDIA

TEL: 272440920

TLF:

TLX:

EML:

COM:

VERON PHILIPPE OR

OHP

F $04870 S$ MICHEL OBS

FRANCE

TEL: $9276 \quad 6368$

TLF:

TLX: 410690 OHP F

EML:

COM: 28,40

VETESNIK MIROSLAV DR

DPT OF ASTROPHYSICS

PURKYNE UNIVERSITY

KOTLARSKA 2

CS 61137 BRNO

CZECHOSLOVAKIA

TEL: 5740500

TLF: 5740108

TLX:

EML: ASTRID®CSPUNI12, EARN

COM: $33, A 2$
VERBUNT FRANCISCUS OR

STERREKUNOIG INSTITUTE

$B 0 \times 80000$

NL 3508 TA UTRECHT

NETHERLANOS

TEL: 693299833

TLF :

TLX: 0521845 XTER D

EML: BITNET: FWVEDGAIPPIS

COM:

ERGMANO A PROF

OSS ASTRONOMICO DI TORINO

ST OSSERVATORIO 20

I 10025. PINO TORINESE

ITALY

TEL: $1181 \quad 1061$

TLF: $1184+128 \mathrm{i}$

TLX:

EML:

COM:

VERMA V K OR

UTTAR PRADESH STATE

OBSERVATORY

PO MANORA PEAK 263129

NAINITAL 263129

INOIA

TEL: $5942 \quad 2136$

ILF:

TIX: 59422401

EML:

COM: 10

VERSCHUUR GERRIT L PROF 4802 BROOKSTONE TERRACE

BOWIE MD 20715

USA

TEL:

TLF :

TLX:

EML:

COM: $33,34,40,51$

VETTOLANI GIAMPAOLO

IST DI RADIOASTRONOMIA

CNR

VIA IRNERIO 46

I $40 L 26$ BOLOGNA

ITALY

TEL: 51232856

TLF :

TLX: 211664 INFN BO I

EML:

COM: 47 


VEVERKA JOSEPH DR
CORNELL UNIVERSITY
312 SPACE SCI BLOG
ITHACA NY 14853
USA
TEL: 6072563507
TLX: 937478
EML:
TLF:
COM: 15,16
VICENTE RAIMUNDO O PROF
FACULOADE CIENCIAS LISBOA
RUA MESTRE AVIZ 30 R/C
P 1495 LISBOA
PORTUGAL
TEL: 2112666
TLX:
EML:
TLF:
COM: 19,31

VIEIRA MARTINS ROBERTO OR OBSERVATORIO NACIONAL RUA GL BRUCE $58 G$ SAO ERISTOVAO 20921 RIO DE JANEIRO RJ BRAZIL

TEL: 215807313

TLF: 215800332

TLX: $021-21288$

EML:

COM: 07,20

\section{VIIK TONU OR}

TARTU ASTROPHYSICAL OBS

ESTONIAN ACAD OF SCIENCES 202444 TARTU

ESTONIA

TEL: 41181

TLF:

ILX:

EML:

COM: 36

VILCHEZ MEDINA JOSBSM OR INST DE ASTROFISICA

DE CANARIAS

OBS DEL TEIDE

E 38200 LA LAGUNA

SPAIN

TEL: $22 \quad 262211$

TLF:

TLX: 92640

EML:

COM:

VIAL JEAN-CLAUDE
IAS
BP 10
F 91371 VERRIERES BUISSON
FRANCE
TEL: 164474217
TLF:
TLX: 600252
EML:
COM: $10,12,44$

VIDAL JEAN-LOUIS DR UNIVERSITE MONTPELLIER II USTL II

place eUgene bataillon

F 34095 MONTPELLIER COX 5 FRANCE

TEL: 67143901

TLF:

TLX: 490944 USTMONT

EML:

COM: 34

\section{VIETRI MARIO DR}

OSS ASTRONOMILD

1 OODAO MONTE CATONE

ITALY

TEL: 69449019

TLF :

EML: VIETRIOASTRMP. ASTRO.1T

COM:

TLX:

VILA SAMUEL C PROF
OPT OF ASTRONOMY
UNIV OF PENNSYLVANIA
33RD \& WALNUT STREETS
PHILADELPHIA PA 19104
USA
TEL: 2158985994
TLF:
TLX:
EML:
COM: 35
VILHENA OE MORAES R OR
INPE
CP 515
12200 S JOSE DOS CAMPOS
8RAZIL
TEL: 123229088
TLF:
TLX: OL173437 ZWO-24-73
EML:
COM: 07

VIALA YVES

OBSERVATOIRE DE PARIS

SECTION DE MEUDON

DENIRM

F 92195 MEUDON PPL COX

FRANCE

TEL: 145077912

TLF :

TLX: 270912

EML:

COM: 34

VIDAL NISSIM V OR

INST FOR SCIENCES \& TECHN

5 ARLOZOROV ST

giVAT SHMUEL 51905

ISRAEL

TEL: 35322490

ILF:

TLX:

EML:

COM: 48

VIGIER JEAN-PIERRE OR

INSIITUT H POINCARE

11 RUE $P \& M$ CURIE

F 75005 PARIS

FRANCE

TEL:

ILF :

TLX:

EML:

COM:

\section{VILAS FAITH OR}

NASA/JOHNSON SPACE CENTER

\section{COOE SN3}

HOUSTON TX 77058

USA

TEL: 7134835056

TLF:

TLX:

EMI: SN: :VILAS

COM: 25,40

VILHU OSMI OR

OBS \& ASTROPHYSICS LAB UNIVERSITY OF HELSINKI

TAEHT TTORNINMAKI

SF 00130 HELSINKI 13

FINLAND

TEL:

TLF:

TLX:

EML:

COM: $29,35,36,44$
VIALLEFOND FRANCOIS

oBservatoIRE de paris

SECTION OE MEUDON

DENIRM

F 92195 MEUDON PPL COX

FRANCE

TEL: 145077905

TLF: $1145: 07 \quad 7893$

TLX: 270912

EML: BITNET: FVIALLEFARREUSI

COM: 34

VIDAL-MADJAR ALFRED DR INSTITUT D'ASTROPHYSIQUE $98 B$ IS BD ARAGO

F 75014 PARIS

FRANCE

TEL: 143201425

TLF: 143298673

TLX:

EML:

COM: 34.44

VIGOTTI MARIO

IST OI RADIOASTRONOMIA

CNR

VIA IRNERIO 46

I 40126 BOLOGNA

ITALY

TEL: 51232856

TLF:

ILX:

EML:

COM:

VILAS-BOAS JOSE W DR

CRAAE/PTR ESCOLA POLI USP

CP 8174 CEP 05508

O1051 SAO PAULO SP

BRAZIL

TEL: 118155936

TLF: 118156289

TLX: 1180127 INPE BR

EML: JWOSYBOAФBRUSP.BITNET

COM: 40

VILKKI ERKKI U

4 PARC DE LA LONDE

f 76130 MONT SAINT AIGNAN

FRANCE.

TEL:

TLF :

TLX:

EML:

COM: 24 
VILKOVISKIJ EMMANUIL Y DR ASTROPHYSICAL INSTItUTE KAZAKH ACAD OF SCIENCES 480068 ALMA ATA KAZAKHSTAN

TEL:

ILF:

TEX:

EML:

COM: 35

VINER MELUYN R DR

OPT OF METALL ENGIN

QUEEN'S UNIVERSITY

KINGSTON ON K7L 3NG

CANADA

TEL:

TLF: 6135456463

TLX:

EML:

COM: 34,40

VIRGOPIA NICOLA PROF

DPT DI MATEMATICA

UNIV OI ROMA LA SAPIENZA

CITTA UNIVERSITARIA

I 00185 ROMA

ITALY

TEL:

TLF:

TLX:

EML:

COM:

VITINSKIJ YURTJ I DR

PULKOVO OBSERVATORY

aCADEMY OF SCIENCES

10 KUTUZZV QUAAY

196140 ST PETERSBURG

RUSSIA

TEL: 298-22-42

TLF:

TLX:

EML:

COM: 10,12

VITYAZEV VENEAMIN $\checkmark$ DR

ASTRONOMICAL OBSERVATORY

ST PETERSBURG UNIVERSITY

BIBLIOTECHNAJA PL 2

198904 ST PETERSBURG

RUSSIA

TEL;

TLF:

TLX:

EML:

COM:

VILLELA THYRSO NETO OR
INPE
CP 515
12201 S JOSE DOS CAMPOS
BRAZIL
TEL: $123418977 * 278$
TLF:
TLX:
EML:
COM:

VINLUAN RENATO

UNIVERSITY OF SOUTHERN

PHILIPPINES

OBRERO DAVAD CITY 9501

PHILIPPINES

TEL:

TLF:

$T L X:$

EML:

COM: 10

VISHNIAC ETHAN T
ASTRONOAY OPT
UNIVERSITY OF TEXAS
RLM $1 S 308$
AUSTIN $T \times \quad 787121083$
USA
TEL: 5124711429
TLF:
TLX:
EML:
COM: 47

VITON MAURICE OR

LAS

TRAVERSE OU SIPHON

LES TROIS LUCS

f 13012 MarSEILLE

FRANCE

TEL: 91055900

TLF: 91661855

ILX:

EML:

COM:

VIVERANAND M. DR

RAMAN RESEARCH INSTITUTE

SADASHIVANAGAR

BANGALORE 560080

INDIA

TEL: 812360122

ILF: 812340492

ILX: 8452671 RRI IN

EML:

COM: 40
VILMER NICOLE OR

observatoire oe paris

SECTION DE MEUDON

DASOP

F 92195 MEUDON PPL CDX

FRANCE

TEL: $145 \quad 077806$

TLF:

TLX: 200590

EML: SPAN:MEUDON: :VILMER

COM: 10,12

VINOD S KRISHAN MRS DR

INDIAN INSTITUTE OF

ASTROPHYSICS

KORAMANGALA

BANGALORE 560034

INOIA

TEL: $81256 \quad 6585 / 6497$

TLF:

TLX: 845763 IIAB IN

EML:

COM: $10.49 \mathrm{C}$

VISHVESHWARA C V PROF

RAMAN RESEARCH INSTITUTE

SADASHIVANAGAR

BANGALORE 560080

INDIA

TEL: 812360122

TLF: 812340492

TLX: 8452671 RRI IN

EML:

COM: 47

VITTONE ALBERTO ANGELO

OSS ASTRONOMICO

DI CAPODIMONTE

VIA MOIARIELLO 16

I $B 0131$ NAPOLI

ITALY

TEL: $8144 \quad 0101$

TLF:

TLX:

EML:

COM:

VIVEKANANDA RAO

CASA

UNIVERSITY OF OSMANIA

HYOERABAD 500007

INOLA

TEL: $85 \quad 1672$

ILF:

TLX:

EML:

COM:
YINCE ISTVAN

ASTRONOMICAL OBSERVATORY

VOLGINA 7

YU 11050 BEOGRAD

YUgOSLAVIA

TEL: $11419357 / 421875$

TLF:

TLX: 72610 AOB YU

EML: EAOPOOLEYUGSS2L.BITNET

COM:

\author{
VIOTTI ROBERTO DR \\ IAS \\ CNR \\ CP 67 \\ I 00044 FRASCATI \\ ITALY \\ TEL: 69425655 \\ TLF: 69416847 \\ TLX: 610261 CNRFRA \\ EML: VIOTTI IRMIAS.BITNET \\ COM: $27,29,44$
}

VISVANATHAN NATARAJAN DR

MOUNT STROMLO \& SIDING

SPRING OBSERVATORIES

PRIVATE BAG

WODEN PO ACT 2606

AUSTRALIA

TEL: 62881111

TLF :

ILX: 62270 TLG CANOPUS AA

EML:

CON: $25,28,34$

VITTORIO NICOLA

ISTITUTO ASTRONOMICO

UNIVERSITA OI ROMA

VIA G M LANCISI 29

I 00161 ROMA

ITALY

TEL:

TLF :

TLX:

EML:

COM:

VIVES TEODORO JOSE OR CTR ASTRON HISPAND ALEMAN REINA $669^{\circ} \mathrm{B}$

CORREOS 511

E 04002 ALMERIA

SPAIN

TEL: 230988

TLF :

TLX: 78812 DSAZ E

EML:

COM: 
VLACHOS DEMETRIUS G PROF OPT GEODESY \& SURVEYING UNIVERSITY THESSALONIKI FACULTY OF ENGINEERING GR 540 O6 THESSALONIKI GREECE

TEL: $3199 \quad 1520$

TLF :

TLX: 412181 AUTH GR

EML :

COM:

VOELK HEINRICH J PROF MPI FUER KERNPHYSIK POSTFACH 103980

D 6900 HEIDELBERG I GERMANY

TEL: $62 \quad 21516295$

TLX: 461666

EML:

TLF :

COM: 14,48

VOGT NIKOLAUS DR

GRUPO ASTROFIS PONTIFICA

UNIVERSTOAD CATOLICA

CASILLA 104

SANTIAGO 22

CHILE

TEL:

TLF:

$T L X:$

EML: NVGGTASTROUC. PUC.CL

COM: $27,29,51$

VOLLAND $H$ DR

ASTRONOMISCHES INSTITUT

DER UNIVERSITAET

AUF DEM HUEGEL 71

D 5300 BONN 1

GERMANY

TEL: $228 \quad 73 \quad 3674$

TLF :

TLX: 0886440 .

EML:

COM:

VON HOERNER SEBASTIAN DR

KRUMMENACKERSTR 186

D 7300 ESSLINGEN

GERMANY

TEL:

TLF:

EML:

COM: 51

TLX:
VLADT
OAT
BOX

BOX SUCC TRIESTE 5

VIA TIEPOLO 11

I 34131 TRIESTE

ITALY

TEL: $40 \quad 309342$

TLF:

TLX: 46!137 OAT I

EML:

COM: 29

VOGEL MANFRED DR

INSTITUT FUER ASTRONOMIE

ETH ZENTRUM

CH 8092 ZUERICH

SWITZERLANO

TEL: 12563806

TLF: 12620003

ILX: 817379 EHHG CH

EML: VOGELECZHETHSA

COM: 34

VOGT STEVEN SCOTT

LICK OBSERVATORY

UNIVERSITY OF CALIFORNIA

SANTA CRUZ CA 95064

USA

TEL: $408 \quad 4292844$

TLF :

TLX: $910-598-4408$

EML:

COM: 29

\section{VOLONTE SERGE DR}

ESA

8-10 RUE MARIO NIKIS

F 75738 PARIS CDX 15

FRANCE

TEL:

TLF :

TLX:

EML

COM: 12,14

VON WEIZSAECKER C F PROF

MAXIMILLIANSTR IS

D 8130 STARNBERG

GERMANY

TEL:

TLF:

EML:

COM:

TLX:
VLAOJMIROV SIMEON ASTRONOMICAL OBSERVATORY BULGARIAN ACAD SCIENCES BOX 15

BG 1309 SOFIA

BULGARIA

TEL: 2758927

TLF :

TLX: 23561 ECF BAN BG

EML:

COM: 09,46

VOGEL STUART NEWCOMBE DR ASTRONOMY PROGRAM UNIVERSITY OF MARYLAND COLLEGE PARK

MARYLANO 20742

USA

TEL:

TLF :

TLX:

EML:

COM: 40

VOIGT HANS H PROF

NIKOLAUSBERGER WEG 74

GEISMARLANDSTR 11

D 3400 GOETTINGEN

GERMANY

TEL: 55155879

TLF:

$T L X$ :

EML:

COM:

VON BORZESZKOWSKI H H DR EINSTEIN-LABORATOR IUM AKAD. WISSENSCHAFTEN DOR ROSA-LUXEMBURG-STR I7A O 1502 POTSOAM

GERMANY

TEL: 762225

TLF :

TLX:

EML:

COM: 47

VONDRAK JAN DR

ASTRONOMICAL INSTITUTE

CZECH ACADEMY SCIENCES

BUDECSKA 6

CS 12023 PRAHA 2

CZECHOSLOVAXIA

TEL: 2258757

TLF : 2255010

TLX: $66 \quad 222486$

EML: ASTDSSOCSEARN

COM: L LVP
VLAHOS LOUKAS DR DPT OF ASTROPHYSICS UNIVERSITY THESSALONIKI GR 540 O6 THESSALONIKI GREECE

TEL: 31991357

TLF:

TLX: 0412181 AVTH GR

EML:

COM:

VOGLIS NIKOS DR DPT OF ASTROPHYSICS NTL UNIVERSITY OF ATHENS PANEPISTIMIQPOLIS GR 157 93 ZOGRAFOS GREECE

TEL: 17243414

TLF :

TLX: 223815 UNIV GR

EML: node: GRATHUN1, userid: SPM7O

COM: 28,47

VOLK KEYIN OR

OPT OF PHYSICS \& ASTRON

UNIVERSITY OF CALGARY

2500 UNIVERSITY DR NW

CALGARY AB T2N LN4

CANADA

TEL: 4039312366

TLF: 4032893331

TLX:

EML:

COM: 34

VON OER HEIDE JOHANN OR

ALARDUSSTR 12

D 2000 HAMBURG 20

GERMANY

TEL: 404914016

TLF :

TLX:

EML:

COM: 08

VQRONTSOV-VEL' YAMINOV B A STERNBERG STATE ASTR INST

117234 MOSCOW

RUSSIA

TEL:

TLF :

TLX:

EML:

COM: 28,34 


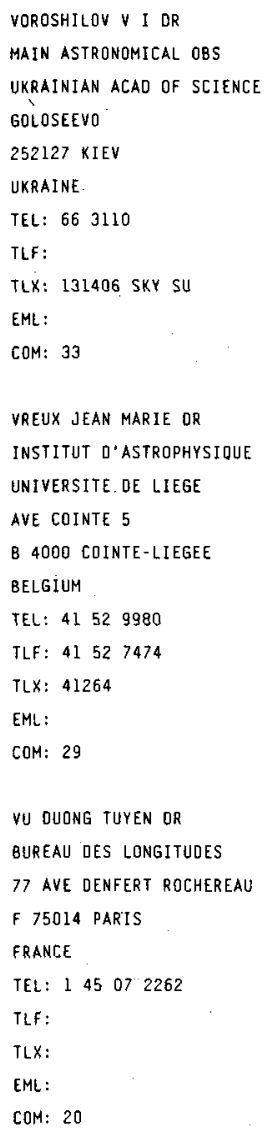

VORPAHL JOAN A OR
746 23RD ST
SANTA MONICA CA 90402
USA
TEL:
TLF:
EML:
COM:
TLX:

\begin{tabular}{|c|c|}
\hline VRSNAK BOJAN DR & VRTILEK JAN M OR \\
\hline HVAR OBSERVATORY & APPLIED SCIENCES OIV \\
\hline FACULTY OF GEODESY & HARVARO UNIVERSITY \\
\hline KACICEVA $26^{\circ}$ & 29 OXFORG ST \\
\hline YU 41000 ZAGREB & CAMBRIOGE MA 02138 \\
\hline YUGOSLAVIA & USA \\
\hline TEL: $41 \quad 442 \quad 600 * 335$ & TEL: $617495 \quad 0589$ \\
\hline TLF: $41 \quad 445 \quad 410$ & ILF: \\
\hline TLX: & ILX: \\
\hline EML: BOJAN.VRSNAK@UNI-FG.AC. MAIL. YU & EML: SPAN:CFARGZ::VRTILEK \\
\hline COM: 10 & COM: 21,28 \\
\hline
\end{tabular}

VUCETICH HECTOR OR COM: 21,28

VUILLEMIN ANDRE DR LAS

TRAVERSE DU SIPHON

LES TROIS LUCS

F 13012 MarseILle

FRANCE

TEL: $9105 \quad 5900$

TLF: $9166 \quad 1855$

TLX:

EML:

COM:

WACKERNAGEL H BEAT DR.

51 BROAOMOOR. HILLS OR COLORADO SPRINGS CO BO906 USA

TEL: 3035543801

TLF :

TLX:

EML

COM: 04,31

TLX: COM: 04,31

EML:

COM: 10

WAELKENS CHRISTOFFEL

ASTRONOMISCH INSTITUUT KATHOLIEKE UNIV LEUVEN CELESTIJNENLAAN $200 B$

B 3030 MEVERLEE

BELGIUM

TEL: 16200656

TLF:

TLX: 25715

EML:

COM: 27
VRBA FREDERICK J DR US NAVAL OBSERVATORY FLAGSTAFF STATION BOX 1149 FLAGSTAFF AZ 86002 USA TEL: 6027795132 TLF:

ILX:

EML:

COM: $09,25 \mathrm{C}, 34$

VRTILEK SAEQA DIL OR CENTER FOR ASTROPHYSICS HCO/SAO MS 4 60 GARDEN SI CAMBRIDGE HA 0213B USA TEL: 6174957400 TLF : 6174957356 TLX: 921428 SATELLITE CAM EML: SVRTILEKECFA COM: 44

VUJNOVIC VLADIS OR INSTITUTE OF PHYSICS UNIVERSITY OF ZAGREB BOX 304 YU 41001 ZAGREB YUGOSLAVIA

TEL: 41271211

TLF :

TLX: 22203 IFS YU

EML:

COM: 14,46

WADDINGTON C JAKE PROF DPT OF PHYSICS UNIVERSITY OF MINNESOTA 116 CHURCH ST SE MINNEAPOLIS MN 55455 USA

TEL: $612 \quad 6242566$

FLF :

TLX: $910-576-2955$

EML:

COM:

WAGNER ROBERT M DR LOWELL OBSERVATORY 1400 W MARS HILL RD $B 0 \times 1149$ FLAGSTAFF AZ B600L USA TEL: 6027790106 TLF :

TLX:

EML:

COM: 
WAGNER WILLIAM J DR NASA HEAQQUARTERS

CODE SS

SPACE PHYSICS DIV

WASHINGTON OC 20546

USA

TEL:

TLF:

TLX:

EML:

COM:

WAKO KOJIRO OR

INTL LATITUDE OBSERVATORY

NAOJ

HOSHIGAOKA MIZUSAWA SHI

IWATE 023

JAPAN

TEL:

TLF :

TLX:

EML:

COM:

WALKER ALISTAIR ROBIN DR

CERRO TOLOLO

INTERAMERICAN OBSERVATORY

CASILLA GO3

LA SERENA.

CHILE

TEL: 51213352

TLF: $51212466 * 342$

TLX: 621 3DI ALRA CT

EML:

COM: $09,15,25$

WALKER EOWARD N MR

ROYAL GREENWICH OBS

HERSTMONCEUX CASTLE

KAILSHAM BN27 IRP

UK

TEL: $323833 \quad 171$

$T L X: 87451$

EML:

TLF:

COM: 27

WALKER MERLE F PROF
LICK OBSERVATORY
UNIVERSITY OF CALIFORNIA
SANTA CRUZ CA 95064
USA
TEL: 4084292526
TLF:
ILX:
EML:
COM: $09,27,37,50$

WAGONER ROBERT $\checkmark$ PROF

VARIAN PHYSICS BLOG

STANFORD UNIVERISTY

STANFORD CA 94305

USA

TEL: 4157234561

TLF :

TLX: 348402

EML:

COM: 47

\author{
WALBORN NOLAN R OR \\ STSCI \\ HOMEWOOD CAMPUS \\ 3700 SAN MARTIN OR \\ BALTIMORE MO 21218 \\ USA \\ TEL: 3013384915 \\ TLF : \\ TLX: 6849101 STSCI UW \\ EML: \\ COM: $45 \mathrm{C}$
}

WALKER ALTA SHARON DR

US GEOLOGICAL SURVEY

927 NATIONAL CENTER

RESTON VA 22092

USA

TEL: $703 \quad 648 \quad 6387$

TLF: $703 \quad 648 \quad 6684$

TLX:

EML:

COM: 16

WALKER GORDON A H PROF

DPT GEOPHYS ASTRONOMY

UNIV OF BRITISH COLUMBIA

2075 WESBROOK PL

VANCOUVER BC VGT IW5

CANADA

TEL: 6042284133

TLF: 6042286047

TLX:

EML:

COM: $09,30,34,37,45$

WALKER RICHARO L

US NAVAL OBSERVATORY

FLAGSIAFF SIAIION

BOX 1149

FLAGSTAFF AZ 86002

USA

TEL: $602774 \quad 6623$

TLF :

TLX:

EML:

COM: 26,42
WAINWR IGHT JOHN DR DPT OF APPLIEO MATH UNIVERSITY OF WATERLOO WATERLOO ON N2L $3 G L$

\section{CANAOA}

TEL: 5198851211

TLF: 5197468115

TLX: 06955259

EML:

COM: 47

WALCH JEAN-JACQUES

OCÁ CERGA

AVE COPERNIC

F 06130 GRASSE

FRANCE

TEL: 93355849

TLF :

TLX: 47.0865 CERGA F

EML:

COM: 07

WALKER ARTHUR B C JR PROF CTR FOR SPACE SCIENCES \&

ASTROPHYSICS

STANFORD UNIV ERL 310

STANFORD CA 943054055

USA

TEL: $415497 \quad 1486$

TLF:

TLX:

EML:

COM:

WALKER HELEN J

RUTHERFORD APPLETON LAB

SPACE \& ASTROPHYSICS DIV BLDG R25/R68

CHILTON DIOCOT DXIL OOX

uk

TEL: 235821900

TLF: 235445848

TLX: 83159 RUTHLB E

EML:

COM:

WALKER ROBERT C OR

NRAO

BOX 0

SOCORRO NM $87801 \quad 0387$

USA

TEL: 5058357247

TLF:

TLX: $910988 \quad 1710$

EML: BITNET: CWALKERENRAD

COM: 40
WAKAMATSU KEN-ICHI DR FACULTY OF ENGINEER ING GIFU UNIVERSITY

GIFU 50111

JAPAN

TEL: 582-30-1111

TLF:

ILX:

EML:

COM: $2 B$

WALDMEIER MAX PROF OR SWISS FEOERAL OBSERVATORY

WIRZENWEID 15

CH 8053 ZUERICH

SWITZERLANO

TEL:

TLF :

TLX:

EML:

CDM: 10,12

WALKER DAVIO DOUGLAS DR OPT PHYSICS \& ASTRONOMY UNIVERSITY COLLEGE LONOON GOWER ST

LDNOON WCIE GBT

UKK

TEL: $1713 \quad 877 \quad 050 * 3510$

TLF: $1713807 \quad 145$

TLX: 28722

EML: ODWUK.AC.ULC.STARLINK

COM: 09

WALKER IAN WALTER

OPT OF ASTRONOMY

UNIVERSITY OF GLASGOW

GLASGOW G12 BQO

UK

TEL:

TLF :

TLX:

EML:

COM: 07

WALKER ROBERT M A PROF

DPT OF PHYSICS

WASHINGTON UNIVERSITY

BOX 1105

ST LOUIS MO 63130

USA

TEL: $\$ 314 \quad 8896225$

ILF:

TLX:

EML:

COM: 16 


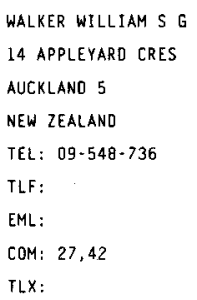

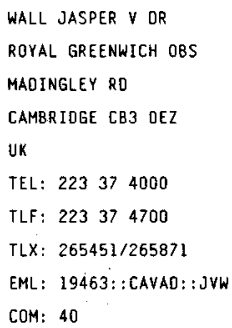

WALLENQUIST AAKE A E PROF ASTRONOMICAL OBSERVATORY NORRLANDSGATAN 340

S 75220 UPPSALA

SWEDEN

TEL: $18 \quad 135685$

TLX:

EML

TLF:

COM: 25,37

WALRAVEN TH DR

BOX 98

ORANGE FREESTATE 9850

SOUTH AFR ICA

TEL:

TLF :

EML:

COM: 25,27

TLX:

WALTER KURT PROF DR
ASTRONOMISCHES INSTITUT
OER UNIVERSITAET
WALDHAUSERSTR 64
D 7400 TUEBINGEN
GERMANY
TEL: 7071296126
TLF:
ILX: 7262714 ALT D
EML:
COM: 42
WAMSTEKER WILLEM DR
ESA IUE GROUNO STATION
VILSPA
APD 54065
E 28080 MADRID
SPAIN
TEL: 1 401 9661
TLF:
TLX: 42555
EML:
COM: $44 C$

WALLACE LLOYO V OR KITT PEAK NTL OBS BOX 26732 $950 \mathrm{~N}$ CHERRY AVE TUCSON AZ $85726 \quad 6732$ USA TEL: 6023275511 TLF :

ILX:

EML:

COM: 16,21

WALLERSTEIN GEORGE PROF DPT OF ASTRONOMY UNIVERSITY OF WASHINGTON FM 20 SEATTLE WA 98195

USA

TEL: 2065432888

TLF :

TLX:

EML:

COM: 27.29

WALSH DENNIS OR

NRAL

JOQRELL BANK

MACCLESFIELD SKII 9OL

UK

TEL: 47771321

TLF:

TLX: 36149

EML:

COM: 40,44

WALTERBOS RENE A M DR

DPT OF ASTRONOMY

NEW MEXICO STATE UNIY

B0X 4500

LAS CRUCES MM 88003

USA

TEL: 5056466522

TLF :

TLX:

EML:

COM: 28

WAN FOOK SUN DPT OF MATHEMATICS NTL UNIVERSITY SINGAPORE KENT RIDGE

SINGAPORE 0511

SINGAPORE

TEL: $772-2742$

TLF:

TLX:

EML:

COM:
WALLACE PATRICK T MR RUTHERFORO APPLETON LAB SPACE \& ASTROPHYSICS DIV BLDG R25/R6B CHILTON DIDCOT OXIL OQX UK

TEL: 235445472

TLF :

TLX: 83159

EML :

COM: $05,08,09$

WALLIS MAX $K$ DR DPT APPLIED MATHS/ASTRON UNIVERSITY COLLEGE

BOX 78

CAROIFF CF 1 IXL

UK

TEL: 222.44211

TLF:

TLX: 488635

EML:

COM: $15,21,51$

WALTER FREDERICK M DPT OF EARTH SPACE SCI ASTRONOMY PROGRAM SUNY AT STONY BROOX STONY BROOK NY 117942100 USA TEL: $516 \quad 6328221$ TLF :

TLX:

EML:

COM: 36

\section{WALTON NICHOLAS A DR} DPT PHYSICS \& ASIRONOMY UNIVERSITY COLLEGE LONDON GOWER ST LONDON WCIE GBT UK

TEL: $1713 \quad 877050 * 476$ TLF: $1713807 \quad 145$ TLX: 28722 UCPHYS $G$ EML: NAW UK.AC.UCL.STAR COM: 34

WAN LAI SHANGHAI OBSERVATORY CAS 80 NANOAN RD. SHANGHAI CHINA PR TEL: 21380696 TLF: TLX: 33164 SHAO CN EML COM: 24,37 
WAN TONG-SHAN

SHANGHAI DBSERVATORY

CAS

80 NANDAN RD

SHANGHAI

CHINA PR

TEL: $2138 \quad 6191$

TLF:

TLX: 33164 SHAO CN

EML:

COM: 40

WANG DE-CHANG

PURPLE MOUNTAIN OBSERV

CAS

NANJING

CHINA PR

TEL: $2564 \quad 6700 / 4205$

TLF:

TLX: 34144 PMONJ CN

EML:

COM: 22,41

WANG JING-SHENG

YUNNAN OBSERVATORY

CAS

BOX 110

KUNMING 72946 YUNHAN

CHINA PR

TEL: $B 712035$

TLF:

TLX: 64040 YUOBS CN

EML:

COM: 40

WANG SHOU-GUAN

BEIJING ASTRONOMICAL OBS

CAS

W SUBURB

BEIJING 100080

CHINA PR

TEL: $128 \quad 126$ I

TLF :

TLX: 22040 BADAS CN

EML:

COM: $38,40,48$

WANG YANAN
NANJING ASTRONOMICAL
INSTRUMENT FACTORY
BOX 846
NANJING
CHINA PR
TEL: 2546191
TLF:
TLX: 34136 GLYNJ C/O NAIF
EML:
COM: 09

WANAS M I PROF

DPT OF ASTRONOMY

FACULTY OF SCIENCES

CAIRO UNIVERSITY

GEZA ORMAN

EGYPT

TEL:

TLF :

TLX

EML:

COM: 48

WANG HAIMIN OR

CALTECH

MS 26433

PASADENA CA 91125

USA

TEL: $818 \quad 3563858$

TLF:

TLX:

EML: HAIMINESUNSOG CALTECH. EOU

COM: 10

WANG JING-XIU

BEIJING ASTRONOMICAL OBS

CAS

W SUBURB

BEIJING 100080

CHINA PR

TEL: 1281698

ILF:

TLX: 22040 BAOBS CN

EML:

COM: $10,12 \mathrm{C}$

WANG SHUI

DEPT EARTH \& SPACE SCI

UNIV SCIENCE \& TECHNOLOGY

HEFEI 230026 ANHUI

CHINA PR

TEL: $55133 \quad 1134 * 209$

TLF:

ILX: 4430

EML:

COM: 44

WANG YIMING

YUNNAN OBSERVATORY

CAS

BOX 110

KUNMING 72946 YUNNAN

CHINA PR

TEL: 8712035

TLF :

TLX:

EML:

COM: 09
WANG CHUAN - JIN

PURPLE MOUNTAIN OBSERV

CAS

NANJING

CHINA PR

TEL: 2546700

ILF :

TLX: 34144 PMONJ CN

EML:

COM: 25

WANG JIA-JI

SHANGHAI OBSERVATORY

CAS

BO NANDAN RO

SHANGHA]

CHINA PR

TEL: 21386191

TLF :

TLX: 33164 SHAO CN

EML:

COM: $24 \mathrm{C}$

WANG LAN-JUAN

SHANGHAI OBSERVATORY

CAS

80 NANDAN RD

SHANGHAI

CHINA PR

TEL: $2138 \quad 6191$

TLF

TLX: 33164 SHAO CN

EML:

COM: 09

WANG SHUNDE OR

BETJING ASTRONOMICAL OBS

CAS

W SUBURB

BEIJING 100080

CHINA PR

TEL: 12561264

ILF :

ILX: 22040 BAOAS CN

EML:

COM: 46.49

WANG YI-MING DR

NAVAL RESEARCH LABORATORY

COOE $4172 \mathrm{~W}$

455 '5 OVERLOOK AVE SW

WASHINGTON OC 203755000

USA

TEL: 2027676202

TLF:

TLX:

EML:

COM: $10,48,49$
WANG DEYU

PURPLE MOUNTAIN OBSERV

CAS

NANJING

CHINA $P R$

YEL: $25 \quad 42817 / 46700$

TLF :

TLX: 34144 PMONJ CN

EML:

COM: 48

WANG JIA-LONG

BEIJING ASTRONOMICAL OBS

CAS

W SUBURB

BEIJING 100080

CHINA PR

TEL:

TLF :

TLX: 22040 BAOBS CN

EML:

COM: 10

WANG RENCHUAN

CENTER FOR ASTROPHYSICS

UNIV SCIENCE \& TECHNOLOGY

HEFEI 230026 ANHUI

CHINA PR

TEL: 551331134

TLF :

ILX: 90028 USTC CN

EML:

[OM: 47

WANG SI-CHAD

PURPLE MOUNTAIN OBSERV

CAS

NANJING

CHINA PR

TEL: 2544205

ILF:

ILX: 34144 PMONJ CN

EML:

COM: 15

WANG ZHENG MING

SHAANXI OBSERVATORY

CAS

LINTONG XIAN

SHAANXI

CHINA PR

TEL: 332255

TLF :

TLX: 70121 CSAO CN

EML:

COM: 09,19 


WANG ZHEN-RU
OEPT OF ASTRONOMY
NANJING UNIVERSITY
NANJING
CHINA PR
TEL: 25 37551*2685
TLF:
TLX: 34151 PRCNU CN
EML:
COM: 48.
WARO MARTIN $30 H N$
INSTITUTE OF ASTRONOMY
THE OBSERVATORIES
MAOINGLEY RD
CAMBRIOGE CB3 OHA
UK
TEL:
TLF:
TLX:
EML:
COM: 28,42
WARES GORDON W OR
73 PERKINS ST
WEST NEWTON MA O2165
USA
TEL:
TLF:
EML:
COM: 14
TLX:

WARNER BRIAN PROF
OPT OF ASTRONGMY
INST OF THEOR PHYSICS
RONOEBOSCH 7700
SOUTH AFRICA
TEL: 6502391
TLF:
TLX: 521439
EML:
COM: 27,42

WARWICK JAMES W DR RADIOPHYSICS CORP 5475 WESTERN AVE BOULDER CO 80301 USA TEL: 3034479524 TLF:

TLX:

EML:

COM: 12.40

WANG ZHEN-YI
PURPLE MOUNTAIN OBSERV
CASERVATORY
NANJING
CHINA PR
TEL: 2546700
TLF:
TLX: 34144 PMONJ CN
EML:
COM: 12

WARO RICHATD A DR LAWRENCE LIVERMORE LAB L 23 A DIV BOX $80 \mathrm{~B}$

LIVERMORE CA 94550 USA

TEL: 4154232679

TLF:

TLX: $910-386-8339$ UCLLL

EML:

COM: 35

WARGAU WALTER F OR DPT OF MATHS \&ASTRONOMY UNIVERSITY OF $S$ AFRICA BOX 392

PRETORIA 0001

SOUTH AFRICA

TEL: $27-12-440-2133$

TLF:

TEX: 350068 TA UNISA TTX

EML:

COM: 42

$\begin{array}{ll}\text { WARNER JOHN W OR } & \text { WARNER PETER J DR } \\ \text { PERKIN ELMER CORP } & \text { MULLARS RADIO ASTRON OBS } \\ \text { MS } 892 & \text { CAVENDISH LABORATORY } \\ \text { LO0 WOOSTER HEIGHTS RD } & \text { MADINGLEY RD } \\ \text { DANBURY CT } 058107859 & \text { CAMBRIDGE CB3 OHE } \\ \text { USA } & \text { UK } \\ \text { TEL: } 2037967919 & \text { TEL: } 22366477 \\ \text { TLF: } & \text { TLF: } \\ \text { TLX: } & \text { TLX: } 81292 \text { CAVLAB G } \\ \text { EML: } & \text { EML: } \\ \text { COM: } 28,44 & \text { COM: } 40\end{array}$

WARWICK ROBERT S OR WASHIMI HARUICHI DR DPT OF PHYSICS UNIVERSITY OF LEICESTER UNIVERSITY RD LEICESTER LEI 7 RH uK TEL: 533554455 ILF:

ILX: 341664 LUXRAY G

EML:

COM:

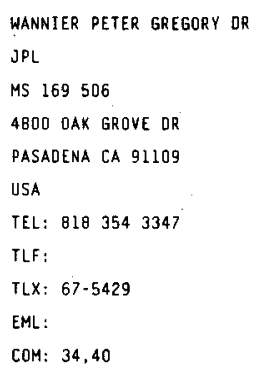

JPL

MS 183501

4800 DAK GRDVE DR

PASADENA CA 91109

USA

TEL:

TLF:

TLX:

EML:

COM:

WARMAN JOSEF OR INSTITUTO DE ASTRONOMIA UNAM APOO POSTAL 70-264 04510 MEXICO OF MEXICO

TEL:

TLF:

TLX:

EML:

COM:

INST FOR ATMOSPHERIC RES NAGOYA UNIVERSITY

3-13 HONOHARA

TOYOKAWA AICHI 442

JAPAN .

TEL: $05338-6-3154$

TLF:

TLX: 4322311

EML:

COM:
WARD WILLIAM A DR
WARO HENRY OR DPT OF PHYSICS \& ASTRON UNIVERSITY OF GLASGOW GLASGOW GL2 800 UK

TEL: $413398855 * 4705$ TLF: 413349029 TLX: 777070 UNIGLA EML: GWIOOUK, AC, GLA,PH, II COM: 09

WARDLE JOHN $F$ C PROF OPJ OF PHYSICS BRANOEIS UNIVERSITY WAL THAM MA 02154 USA TEL: 6176472889 TLF: TLX:

EML:

COM: 40

WARMELS REIN HERM OR ESO KARL SCHWARZSCHILDSTR 2 D 8046 GARCHING MUENCHEN GERMANY

TEL: $893200 \quad 6292$

TLF: $B 93202362$

TLX:

EML: RWARMELSEESO.ORG COM: 40

\section{WARREN WAYNE H JR OR} NASA/GSFC COOE 681 LAB ASTRON \& SOLAR PHYS GREENBELT MD 20771 USA

TEL:

ILF:

$T \perp x$ :

EML: W3WHWOSCFMVS, GSFC, NASA, GOV COM: $05 C, 25,37,45$

WASSERMAN LAWRENCE H DR LOWELL OBSERVATORY 1400 W MARS HILL RO BOX 1149 FLAGSTAFF AZ 86001 USA

TEL: 6027743358

TLF:

TLX:

EML:

COM: $16,20 \mathrm{C}, 24$ 
WASSON JOHN T

INST OF GEOPHYS \& PLANET UNIVERSITY OF CALIFORNIA LOS ANGELES CA 90024

USA

TEL: $213 \quad 8251986$

TLF:

TLX:

EML

COM: 15,16

WATERS LAURENS B F M DR SRON

BOX 800

NL 9700 AV GRONINGEN

NETHERLANDS

TEL: 50634090

TLX:

EML: RENSWRRUG.NL

TLF: 50634033

COM: 36

WATSON ROBERT DR

DPT OF PHYSICS

UNIVERSITY OF TASMANIA

GPO BOX 252C

HOBART TAS $700 !$

AUSTRALIA

TEL: 2202415

ILF: $2 \quad 202410$

ILX: AA 58150

EML:

COM: 27

WAYMAN PATRICK A PROF

OUNSINK OBSERVATORY

DIAS

DUBLIN 15

IRELAND

TEL: I 387911

TLF:

TLX: 31687 DIAS

EML:

COM: $05,33,50$

WEBB OAVIO F
PHILLIPS LAB/PHG
BOSTON COLLEGE
HANSCOM AFB MA 01731
USA
TEL: 6173773970
TLF: 6173773160
TLX:
EML: AFGLSC: : WEBB
COM: 10

WATANABE JUN-ICHI OR
TOKYO ASTRONOMICAL OBS
NAOJ
OSAWA MITAKA.
TOKYO 181
JAPAN
TEL: 81422413614
TLF: 81422413608
TLX: 02822307 TAOMTK J
EML: OWATANAECL.MTK.NAD.AC.JP
COM: 15

WATERWORTH MICHAEL OR

UNIVERSITY OF TASMANIA

GPO BOS 252C

HOBART TAS 7001

AUSTRALIA

TEL: 2202410

TLF :

TLX: 58150 AA

EML

COM: 29

WATSON WILLIAM D PROF

OPT OF PHYSICS

UNIVERSITY OF ILLINOIS

1110 W GREEN ST

URBANA IL 6IBOI

USA

TEL: 2173337240

TLF :

TLX

EML:

COM:

WDOWIAK THOMAS J DR

PHYSICS DPT

UNIVERSITY OF ALABAMA

BIRMINGHAM AL 35294

USA

TEL: $205934 \quad 4736$

TLF:

TLX: 888826 UAB BHM

EML:

COM: 15

WEBBER JOHN C OR

INTERFEROMETRICS INC

150 LEESBURG PIKE

VIENNA VA 22180

USA

TEL: 7037908500

TLF :

TLX:

EML:

COM:
WATANABE TAKASHI OR

INST FOR ATMOSPHERIC RES

NAGOYA UNIVERSITY

3-13 HONOHARA

TOYOKAWA 442

JAPAN

TEL: $05338 \quad 6 \quad 3154$

TLF : $05338 \quad 6 \quad 081]$

TLX: 4322311 RIANAG J

EML:

COM: 49

WATSON FREDERJCK GARNETT

UK SCHMIOT TELESCOPE

AAD

PRIVATE BAG

COONABARABRAN NSW 2357

AUSTRALIA

TEL: $68 \quad 421622$

TLF:

TLX: 63945 CANDPUS AA

EML:

COM: 09,51

WATT GRAEME DAVID

ROYAL OBSERVATORY

BLACKFORD HILL

EDINBURGH EH9 3HJ

uk

TEL:

TLF :

TLX:

EML:

COM: 34

WEAVER harOLL F PRof

ASTRONOMY DPT

UNIVERSITY OF CALIFORNIA

601 CAMPBELL HALL

BERKELEY CA 94720

USA

TEL:

TLF :

TLX: 820181 UCS AST

EML:

COM: $15,33,34,37$

WEBBINK RGNALD F OR

OPT OF ASTRONOMY

UNIVERSITY OF ILLINOIS

1011 W SPRINGFIELO AVE

URBANA IL 61801

USA

IEL: $217-333-9582$

ILF :

TLX: $910-245-2434$ AST

EML:

COM: $27,35,42 C$
WATANABE TETSUYA

TOKYO ASTRONONICAL OBS

NAOJ

OSAWA MITAKa

TOKYO 181

JAPAN

TEL: $422-32-5111$

TLF :

TLX:

EML:

COM: 36

WATSON MICHEAL G OR

DPT OF PHYSICS

UNIVERSITY OF LEICESTER

UNIVERSITY RD

LEICESTER LEI 7RH

UK

TEL: $533 \quad 523553$

TLF: $533 \quad 550 \quad 182$

TLX:

EML: NGWUK.AC.LE.STAR

COM:

WATTENBERG O PROF

LINDERHOFSTR 57

D 1147 BERLIN

GERMANY

TEL: $527 \cdot 7772$

TLF:

TLX:

EML:

COM: 41

WEAVER THOMAS A DR

LAWRENCE LIVERMORE LAB

L 17

BOX 808

LIVERMORE CA 94550

USA

TEL: $415 \quad 423 \quad 1850$

TLF :

TLX:

EML

COM: 35,48

WEBER STEPHEN VANCE

LAWRENCE LIVERMORE LAB

L 477

BOX 808

LIVERMORE CA 94550

USA

TEL: 4154225433

TLF:

TLX: $910-386-8339$

EML:

COM: 36 
WEBROVA LUDMILA OR ASTRONOMICAL INSTITUTE CZECH ACADEMY SCIENCES BUDECSKA 6

CS 12023 PRAHA 2 CZECHOSLOVAKIA

TEL: 2255287

ILF:

TLX: 122486

EML:

COM: 31

WEEKES TREVOR C DR FRED LAWRENCE WHIPPLE OBS HARVARO-SMITHSONIAN CTR AMADO AZ 856450097 USA

TEL: $602 \quad 6296741$

TLF :

TLX:

EML:

COM:

WEHLAU WILLIAM H PROF DPT OF ASTRONOMY UNIV OF WESTERN ONTARID LONDON ON N6A 3K7 CANADA

TEL: 5196793184

TLF: 5196613486

TLX: 0647134

EML:

COM: $27,29,42$

WEIDEMANN VOLKER PROF INST THEOR PHYS \& STERNW NEUE UNIV PHYSIK ZENTRUM OLSHAUSENST GEB N $61 \mathrm{C}$ D 2300 KIEL 1 GERMANY

TEL: $431880 \quad 4110$

TLF :

TLX: 292706

EML:

COM: 05,36

WEILER EDWARO J DR PRINCETON UNIVERSITY OBS PEYTON HALL

PRINCETON NJ 08544

USA

TEL:

TLF

TLX

EML

COM: $40,42,44$

WEBSTER AORIAN S OR
JOINT ASTRONOMY CENTER
UKIRT
665 KOMOHANA ST
HILO HI 96720
USA
TEL: 8089613756
TLF:
TLX: 633135
EML:
COM: 47,48

WEGNER GARY ALAN DPT OF PHYSICS \& ASTRON OARTMOUTH COLLEGE WILDER LABORATORY HANOVER NH 03755 USA

TEL: $603646 \quad 2359$

TLF :

TLX:

EML:

COM: 29,30

WEHRLE ANN ELIZABETH OR

JPL

MS $168 \quad 427$

4800 OAK GROVE OR

PASADENA CA 91109

USA

TEL: $818 \quad 354 \quad 1672$

TLF :

TLX

EML: DEIMOS: : AEW/AEWCITOEIMOI.CALT COM: 36

COM: $40,44,48$

WEIDERSCHILLING S J OR PLANETARY SCIENEE INST 2030 E SPEEDWAY

SUITE 201

TUCSON AZ 85719

USA

TEL: $602 \quad 8810.332$

TLF

TLX:

EML:

COM: 15,16

WEILER KURT W OR

NAYAL RESEARCH LABORATORY

COOE 4131

4555 OVERLOOK AVE SW WASHINGTON DC 203755000 USA

IEL: 2027670292

TLF:

ILX:

EML: SPAN: 11334::WEILER

COM: $28,34,40,44$
WEBSTER RACHEL.

CITA MCLENNAN LABS

UNIVERSITY OF TORONTO

6O ST GEORGE ST

TORONTO ON MSS IAI

CANADA

TEL: $416978 \quad 9496$

TLF: $416 \quad 978 \quad 3921$

TLX:

EML: BLTNET: WEBSTEREUTORPHYS

COM: 47

WEHINGER PETER A DR

DPT DF PHYSICS

ARIZONA STATE UNIVERSITY

ASTRONOMY PROGRAM

TEMPE AZ 8528?

USA

TEL: 6029654063

TLF :

TLX: 140289 HALLEY ASU UT

EML: BITNET: WEHINGEROASUCPS

COM: $15,28,29$

WEHRSE RAINER OR

INST F THEOR ASTROPHYSIK

IM NEUENHEIMER FELD 561

D 6900 HEIDELBERG 1

GERMANY

TEL: 6221562837

TLF :

TLX: 461515 UNIHD D

EML

365

MPI FUER RADIDASTRONOMIE

AUF DEM HUEGEL 69

O 5300 BONN 1

GERMANY

TEL: $228 \quad 525243$

TLF : $228 \quad 525229$

TLX: 886440

EML: P561GWEMPIFR-BONN,MPG.DE

COM: 40

WEILL GILBERT M OR

SPOT IMAGE CORPORATION

1697 PRESTON WHITE DRIVE

RESTON VA 220911326

USA

TEL: 7036202200

TLF :

TLX: 4993073

EML:

COM: 21
WEEDMAN DANIEL W PROF

DPT OF ASTRONOMY

PENNSYLVANIA STATE UNIV

525 DAVEY LAB

UNIVERSITY PARK PA 16802

USA

TEL: 8148650418

TLF :

FLX: 842510

EML:

COM: 28

WEHLAU AMELIA OR OPT OF ASTRONOMY UNIV OF WESTERN ONTARIO LONDON ON N6A $3 K 7$ CANADA

TEL: 5196793186

TLF: 5196613486

TLX: 0647134

EML:

COM: 27,37

WEI MINGZHI

BEIJING ASTRONOMICAL OBS CAS

W SUBURB

BEIJING 100080

CHINA PR

TEL: $\perp 28 \quad 1698$

ILF :

TLX: 22040 BAOBS CN

EML:

COM: 40

WEIGERT ALFREO PROF

HAMBURGER STERNWARTE

GOJENSBERGSWEG 112

0 2050 hamburg 80

GERMANY

TEL: 4072244112

TLF:

TEX: $2178 B 4$

EML:

COM: 35.42

WEIMER THEOPHILE P F OR OBSERVATOIRE DE PARIS

61 AVE OBSERVATOIRE

F 75014 PARIS

FRANCE

TEL: 143201210

TLF :

TlX:

EML.

COM: 16 


WEINBERG J I DR
SPACE ASTRONOMY LAB
UNIVERSITY OF FLORIDA
1810 NW 6TH ST
GAINESVILLE FL 32609
USA
TEL: 9043925450
TLF:
TLX: 8108252308 SPACELA
EML:
COM: $21,22,44$
WEISBERG JOEL MARK
OPT PHYSICS \& ASIRONOMY
CARLETON COLLEGE
NORTHFIELO MN 55057
USA
TEL: 5076634367
TLF:
TLX:
EML:
COM:

WEISS WERNER W OR INSTITUT FUER ASTRONOMIE DER UNIVERSITAET WIEN TUERKEMSCHANZSTR 17 A 1180 WIEN AUSTRIA

TEL: 1345360

TLF:

TLX: 116222 PHYSI A

EML: EARN: : "WEISS AVIA.UNA.AC.AT" COM: $09,25,27,29$

\begin{tabular}{|c|c|}
\hline $\begin{array}{l}\text { WELCH DDUGLAS \& OR } \\
\text { OPT OF PHYSICS \& ASTRON }\end{array}$ & $\begin{array}{l}\text { WELCH GARY A OR } \\
\text { DPT OF ASTRONOMY }\end{array}$ \\
\hline MCMASTE'R UNIVERSITY & ST MARY'S UNIVERSITY \\
\hline HAMILTON ON LAS $4 M I$ & HALIFAX NS B3H ЗСЗ \\
\hline CANADA & CANADA \\
\hline TEL: $4165259140 * 3186$ & TEL: 9024299780 \\
\hline TLF: $416 \quad 546 \quad 1252$ & TLF: $902 \quad 4205561$ \\
\hline$T L X: .0618347$ & TLX: \\
\hline EML: WELCH PHYSUN. PHYSICS.MCMASTER. & EML: \\
\hline COM: 27 & COM: 28 \\
\hline WELLGATE G BERNARD MR & WELLINGTON KELVIN DR \\
\hline CANEHEATH HOUSE & CSIRO \\
\hline ARLINGTON & DIVISION OF RADIOPHYSICS \\
\hline polegate bn26 6SJ & $80 \times 76$ \\
\hline uk & EPPING NSW 2121 \\
\hline TEL: & AUSTRALIA \\
\hline TLF: & TEL: 28680222 \\
\hline TLX: & TLF: \\
\hline EML: & TLX: $26230 \mathrm{AA}$ \\
\hline COM: & EML: KWELLINGARP.CSIROAU \\
\hline & COM: 40 \\
\hline
\end{tabular}

COOE ES 65
WEINBERGER RONALO DR INSTITUT FUER ASTRONOMIE TECHNIKERSTRASSE 25 A 6020 INNSBRUCK AUSTRIA

TEL: 52227485251

TLF:

TLX:

EML:

COM:

$$
\text { COM: } 47
$$

WEISHEIT JON $C$ OR OPT SPACE PHYS \& ASTRON RICE UNIVERSITY

\section{BOX 1892}

HOUSTON TX $77251 \quad 1892$

$$
\text { USA }
$$

TEL:

$$
\text { TLF: }
$$

TLX:

EML:

COM: 34,48

WEISSKOPF MARTIN CH DR

$$
\text { NASA/MSFC }
$$

HUNTSVILLE AL 35812

USA

TEL: 2054533238

ILF:

TLX:

EML:

COM: 48

WELCH GARY A DR

DPT OF ASTRONOMY

CANADA

TEL: 902429978

WEISS ACHIM DR ASTRGPHYSIK KARL-SCHWARZSCHILOSTR I GERMANY

TEL: 89329900

TLF: 8932993235

TLX: 524629 ASTRO D

EML: ACWOGAIPPIS

COM: 35

WEISSMAN PAUL ROBERT

JPL

MS 18 ถ่̀ 601

49OO OAK GRQVE DR

PASADENA CA 91109

USA

TEL: 8183542636

TLF:

TLK: 675429

EML.:

COM: 15,20

WELCH WILLIAM. J PROF

RADIO ASTRONOMY LAB

601 CAMPBELL HALL

BERKELEY CA 94720

$$
\text { USA }
$$

WEIS EOWARD W OR DPT OF ASTRONOMY VAN VLECK OBSERVATORY WESLEYAN UNIVERSITY MIDDLETOWN CT O645? USA TEL: 2033479411

TLF:

TLX:

EML:

COM: 26 MAX PLANCK INSTITUT FÜR D 8O46 GARCHING MUENCHEN

WEISS NIGEL O OR DPT APPLIED MATHS \& THEORETICAL PHYSICS SILVER STREeT CAMBRIDGE CB3 9EW. UK

TEL: 223351645

TLF:

TLX: 81240

EML:

COM: 12,35

UNIVERSITY OF CALIFDRNIA

TEL: $415 \quad 642 \quad 6679$

TLF:

TLX: 820181 UCB AST RAL

EML:

COM: 40,51

\section{WEISTROP DONNA OR}

DPT OF PHYSICS

UNIVERSITY OF NEYADA

4505 S MARYLANO PARKHAY

LAS VEGAS NV 89154

USA

TEL:

TLF:

TLK:

EML:

COM: 25.33

WELLER CHARLES S OR

DAVIO TAYLOR RES CENTER COOE 1402

BETHESDA MO 200845000

USA

TEL: $301 \quad 2271274$

TLF :

TEX:

EML:

COM: 49

\section{WELLMANN PETER PROF OR} INST FUER ASTRONOMIE \&

\section{ASTROPHYSIK}

SCHEINERSTR 1

0 8000 MUENCHEN 80

$$
\text { GERMANY }
$$

TEL:

TLF :

TLX:

EML:

COM: 36,42
WELLS DONALD C III DR NRAO

EDGEMONT RD CHARLOTTESVILLE VA 22901 USA

TEL: 8042960211

TLF:

TLX:

EML:

COM: 05 


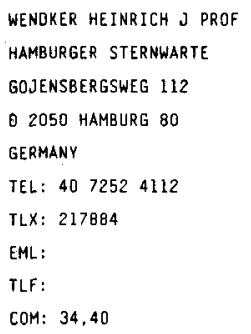

WENGER MARC

OBS DE STRASBOURG

11. RUE UNIVERSITE

F G7000 STRASBOURG FRANCE

TEL: $88 \quad 3.5 \quad 8219$

TLF: BB 250160

TLX:

EML: SIMBAD: :WENGER/WENGER IMBAD.U EML:

COM: 05

WESEMAEL FRANCOIS DR
OPT DE PHYSIQUE
UNIVERSITE DE 'MONTREAL
CP $612 B$ SUCC A
MONTREAL QC H3C 337
CANADA
TEL: 5143437355
TEF: 5143432071
TLX: 05562425 UOEMPHYSAS
EML:

EML :

COM :

WEST DONALO K DR

NASA/GSFC

CODE 684.1

GREENBELT MO 20771

USA

TEL:

TLF :

TLX:

EML:

COM: 42

WESTERHOUT GART OR

US NAVAL OBSERVATORY

SCIENTIFIC OIRECTOR

34 \& MASSACHUSETTS AVE NW

WASHINGTON OC 203925100

USA

TEL: $202 \quad 653 \quad 1513$

TLF:

TLX: $710 B 221970$

EML:

COM: $05 C, 08,24,33,40$

WETHERILL GEORGE W

DPT TERRESTR MAGNETISM

CARNEGIE INST WASHINGTON

5241 BRQAD BRANCH RO NW

WASHINGTON DC 20015

USA

TEL: 2029660863

TLF:

TLX: 440427 MAGN UI

EML:

COM: $15,16,22,51$
WENIGER SCHAME DR OBSERVATOIRE DE PARIS SECTION DE MEUDON F 92195 MEUDON PPL CDX FRANCE

TEL: 145347530

TLF :

$T L X:$

COM: $14,21,29$

WESSELINK ADRIAAN $J$ OR

143 FALLS RO

BETHANY CT 06525

USA

TEL: 2033933297

TLF:

ILX:

EML:

COM: $24,25,27,42$

WEST RICHARO M DR

ESO

KARL-SCHWARZSCHILD-STR 2

O 8046 GARCHING MUENCHEN GERMANY

TEL: 8932006276

TLF: 893202362

TLX: 52828220 ESO D

EML: ESOMC I::RICHARD

COM: 06YP, 09C, 20C, 46

WESTERLUND BENGT E PROF ASTRONOMICAL OBSERVATORY BOX 515

S 75120 UPPSALA

SWEDEN

TEL: $19 \quad 135157$

TLF:

TLX: 76024 UNIV UPPSS

EML:

COM: $28,33,45$

WEYMANN RAY J PROF

STEWARO OBSERVATORY

UNIVERSITY OF ARIZONA

TUCSON AZ 85721

USA

TEL: $602 \quad 6212375$

TLF:

TLX: 467175

EML:

COM: 34
WENTZEL DONAT G DR

ASTRONOMY PROGRAM

UNIVERSITY OF MARYLAND

COLLEGE PARK MO 20742

USA

TEL: 3014051518

TLF :

TLX:

EML: WENTZELEASTRO. UMO.EOU

COM: $10,12,46 \mathrm{C}, 46$

WESSELIUS PAUL R DR

SRON

$80 \times 800$

NL 9700 AV GRONINGEN

NETHERLANOS

TEL: $50 \quad 634 \quad 074$

ILF: $50 \quad 634033$

TLK:

EML: PAULEGUSPACE. RUG.NL

COM: $25,34,44,45$

WEST ROBERT ALAN

JPL

MS 183301

4BOD OAK GROVE OR

PASADENA CA 91109

USA

TEL: 8183540479

TLF :

TLX: 675429

EML:

COM: 16

WESTFOLD KEVIN C PROF MONASH UNIVERSITY

WELLINGTON RO

CLAYTON VIC 3168 AUSTRALIA

TLF :

TEL: 35413080

TLX: 32691 AA

EML:

COM: 40,48

WHEELER J CRAIG PROF

ASTRONOMY OPT

UNIVERSITY OF TEXAS

RLM 15308 AUSTIN TX 787121083

USA

TEL: $512 \quad 4714461$

TLF:

TLX: 910-874-1351

EML:

COM: $35,42,48$ 


\begin{tabular}{|c|c|}
\hline WHEELER JOHN A OR & WHIPPLE ARTHUR L OR \\
\hline OPT OF PHYSICS & MCOONALO OBSERVATORY \\
\hline PRINCETON UNIVERSITY & UNIVERSITY OF TEXAS \\
\hline JADWIN HALL & AUSIIN TX 787121351 \\
\hline PRINCETON NJ 08544 & USA \\
\hline USA & TEL: $512 \quad 471 \quad 6332$ \\
\hline TEL: 6092584400 & TLF: \\
\hline TLF: $609258 \quad 1124$ & ILX: \\
\hline$T\llcorner X: 4993512$ & EML: BITNET: asag 105๑uta3081 \\
\hline EML: DWNSOMSO.ANU.0Z.AU & COM: 07,20 \\
\hline COM: 47,48 & \\
\hline WHITE GLENN J & WHITE GRAEME LINOSAY DR \\
\hline DPT OF PHYSICS & CSIRO \\
\hline QUEEN MARY/WESTFIELO COLL & DIVISION OF RADIOPHYSICS \\
\hline MILE END RD & $80 \times 76$ \\
\hline LONOON EI 4NS & EPPING NSW 2121 \\
\hline UK & AUSTRALIA \\
\hline TEL: $1980 \quad 4811 * 4045$ & TEL: $2 \quad 868 \quad 0222 * 420$ \\
\hline TLF: & TLF : \\
\hline$T L X: 893750$ & $T L X: 26230$ ASTRO AA \\
\hline EML: & EML: \\
\hline COM: 34 & COM: 24,41 \\
\hline WHITE R STEPHEN PROF & WHITE RAYMONO E DR \\
\hline IGPP & STEWARD OBSERVATORY \\
\hline UNIVERSITY OF CALIFORNIA & UNIVERSITY OF ARIZONA \\
\hline RIYERSIDE CA 92521 & TUCSON AZ 85721 \\
\hline USA & USA \\
\hline TEL: 7147874503 & TEL: $602 \quad 621 \quad 6528$ \\
\hline TLF : & TLF : \\
\hline rLX: & $T L X: 467175$ \\
\hline EML: & EML: \\
\hline COM: & COM: 33,37 \\
\hline WHITE SIMON DAVIO MANION & WHITELOCK PATRICIA ANN OR \\
\hline STEWARD OBSERVATORY & SAAD \\
\hline UNIVERSITY OF ARIZONA & $80 \times 9$ \\
\hline TUCSON AZ 85721 & OBSERVATORY 7935 \\
\hline USA & SOUTH AFRICA \\
\hline TEL: 6026216530 & TEL: 470025 \\
\hline TLF : & TLF : \\
\hline TLX: & ILX: $57-20309$ \\
\hline EML: & EML: \\
\hline COM: 28,47 & COM: $27,33,34$ \\
\hline WHITMORE BRADLEY C & WHITNEY BALFOUR S \\
\hline STSCI & 1102 E MISSOURI \\
\hline HOMEWOOD CAMPUS & NORMAN OK 73071 \\
\hline 3700 SAN MARTIN DR & USA \\
\hline BALTIMORE MO 21218 & TEL: 4053213547 \\
\hline USA & TLF : \\
\hline TEL: $301 \quad 338 \quad 4713$ & EML: \\
\hline TLF: & COM: \\
\hline TLX: & $T L X:$ \\
\hline EML: & \\
\hline
\end{tabular}

WHIPPLE FRED L DR CENTER FOR ASTROPHYSICS HCO/SAO

60 GARDEN ST

CAMBRIDGE MA 02138

USA

TEL: 5174957200

TLF :

TLX:

EML:

COM: $15,20,22$

WHITE NATHANIEL M OR

LOWELL OBSERVATORY

1400 W MARS HILL RO

BOX 1149

FLAGSTAFF AZ 86001

USA

TEL: 6027743358

ILF:

TLX:

EML:

COM: 25

WHITE RICHARO E

DPT OF ASTRONOMY

SMITH COLLEGE

CEARK SCIENCE CENTER

NORTHAMPTON MA 01063

USA

TEL: 4135842700

TLF:

TLX

EML:

COM:

WHITEOAK J B DR

CSIRO

AUSTR TELESCOPE NTL FAC

BOX 76

EPPING NSW 2121

AUSTRALIA

TEL: 28680226

TLF: 28680310

$T L X: 26230$

EML: JWHITEOAOATNF. ESIRO. AU

COM: $33,34,40 \vee 9,50$

WHITNEY CHARLES A PROF

CENTER FOR ASTROPHYSICS

HCO/SAO

60 GARDEN ST

CAMBRTDGE MA 02138

USA

TEL : 6174957451

TLF :

TLX:

EML:

COM:
WHITAKER EWEN A

LUNAR \& PLANETARY LAB UNIVERSITY OF ARIZONA TUCSON AZ 85721

USA

TEL: $602 \quad 6212888$

TLF:

ILX: 910-952-1143

EML:

COM: 16,41

WHITE ORAN R OR

7590 ROAD 39

MANCOS CO 80307

USA

TEL: 3035337318

TLF :

$T L X$ :

EML:

COM:

WHITE RICHARD L

STSCI

HOMEWOOD CAMPUS

3700 SAN MARTIN DR

BALTIMORE MD 21218

USA

TEL: $301338 \quad 4797$

TLF :

TLX:

EML:

COM: 34,36

WHITFORD ALBERT E PROF L.ICK OBSERVATORY UNIVERSITY OF CALIFORNIA SANTA CRUZ CA 95064 USA

TEL: $408 \quad 4292149$

$T L F$ :

TLX:

EML:

COM: $2 B$

WHITROW GERALO JAMES PROF 41 HOME PARK RO WIMBLEOON LONDON SWI9 7HS UK TEL: 1947343467 TLF :

TLX:

EML :

COM: 41,47 
WHITTET BOUGLAS C B OR

DPT OF PHYSICS

RENSSELAER POLYTECHN INST

TROY NY $121 B 03590$

USA

TEL: $518276 \quad 6310$

TLF: $518 \quad 276 \quad 6680$

TLX:

EML:

COM: 33,34

WICKRAMASINGHE N C PROF OPT APPLIED MATHS/ASTRON UNIVERSITY COLLEGE

BOX 78

CARDIFF CFI LXL UK

TEL: $222442 \quad 11$

TLF:

TLX: 498635 ULIBCFG

EML:

COM: $34,36,40$

WIELEBINSKI RICHARO PROF MPI FUER RADIOASTRONOMIE AUF DEM HUEGEL 69

D 5300 BONN 1

GERMANY

TEL: 228525300

TLK: 886440

EML:

ILF :

COM: $25,28,33,40,51$

WIITA PAUL JOSEPH

DPT PHYSICS \& ASTRONOMY

GEORGIA STATE UNIVERSITY

ATLANTA GA 303033083

USA

TEL: $404 \quad 6582932$

TLF :

TLX:

EML:

COM: 28

WILO PAUL PROF

ASTRONOMISCHES INSTITUT

UNIVERSITAET BERN

SIDLERSTRASSE 5

CH 3012 BERN

SWITZERLANO

TEL: 31658596

TLF :

TLX: 32320 PHYBE CH

EML:

COM: 20,28

\section{WHITTLE D MARK DR \\ UNIVERSITY STATION \\ UNIVERSITY OF VIRGINIA \\ BOX 3818 \\ CHARLOTJESVILLE VA 22903 \\ USA \\ TEL: 8649244900 \\ TLF : \\ TLX: 910997 0174 NRAO \\ EML:}

COM:

WIDING KENNETH G DR

NAVAL RESEARCH LABORATORY

CODE 7144

4555 OVERLOOK AVE SW

WASHINGTON DC 203755000

USA

TEL: 2027672605

TLF :

TLX:

EML:

COM:

WIELEN ROLAND PROF DR

ASTRONOMISCHES-RECHEN

MOENCHHOFSTR 12-14

D 6900 HEIDELBERG !

GERMANY

TEL: 622149026

ILX: $46 ! 336$ ARIHO D

EML:

TLF :

COM: $04,05,08,28,33,37$

WI JNBERGEN JAN DR

LAB VOOR RUIMTEONDERZOEK

HOOGBOUW WSN

BOX BOO

NL 9700 AV GRONINGEN

NETHERLANDS

TEL: 50116660

TLF :

TLX: 53572 STARS NL

EML :

COM:

WILEEY ROBERT L PROF DR

ASTROPHYSICAL OBSERVATORY

N ARIZONA UNIVERSITY

FLAGSTAFF AZ 86011

USA

TEL: 6025232661

TLF :

TLX:

EML:

COM: 16
WHITWORTH ANTHONY PETER OPT APPLIED MATHS/ASTRON UNIVERSITY COLLEGE

BOX 78

CARDIFF CFI IXL

UK

TEL: $222442 \quad 11$

TLF :

TLX: 498635 ULIBCFG

EML:

COM: 34

WIĒDLING TOR DR

OSTRA VILLAVAGEN 15

$S 61136$ NYKOPING

SWEDEN

TEL:

TLF :

TLX:

EML:

CON:

WIESE WOLFGANG L DR

NTL BUREAU OF SATNDARDS

DIV 842 RM A267 BLOG 221

GAITHERSBURG MO 20899

USA

TEL: 3019753201

TLF : 3019753038

TLX: 898493

EML:

COM: $14 \mathrm{P}$

WILCOCK WILLIAM L PROF

SCHOOL OF PHYSICAL \&

MOLECULAR SCIENCES

UNIV COLLEGE OF N WALES

BANGOR GWYNEOO LL57 2UW

UK

TEL: $24835 \quad L 15$

TLF:

ILX: 61100

EML:

COM: 09

WILKENING LAUREL L DR

ADMIN BLDG 601

UNIVERSITY OF ARIZONA

TUCSON AZ 85721

USA

TEL: $602 \quad 626 \quad 3513$

TLF:

TLX:

EML:

COM: 15
WICKRAMASINGHE DT DR

AUSTRALIAN WTL UNIVERSITY

OPT OF APPLIED MATHS

BOX 4

CANBERRA ACT 2600

AUSTRALIA

TEL:

TLF;

TLX:

EML:

COM:

WIEHR EBERHARD DR

UNIVERSITAETS STERNWARTE

GEISMARLANDSTR 11

D 3400 GOETTINGEN

GERMANY

TEL: 551395053

TLF :

TLX: 96753

EML:

COM: 10

WIETH-KNUDSEN NIELS P DR

SVENO IROSTSVEJ 12

OK 1912 FREOERIKSBERG C

DENMARK

TEL: $3124 \quad 9131$

TLF :

TLX:

EML:

COM: 26.31

WILO JOHN PAUL OR

CSIRO

LIMESTONE AVE

BOX 225

DICKSON ACT 2602

AUSTRALIA

TEL:

TLF:

$T L X$ :

EML:

COM: $10,40,49$

WILKES BELINDA J

CENTER FOR ASTROPHYSICS

HCO/SAD

60 GARDEN ST

CAMBRIDGE MA 02138

USA

TEL: 6174957268

TLF :

TLX: 921428

EML:

COM: 


WILKINS GEORGE A OR
WINOWARO
HIGHER BROOK MEADOW
SIDFORD
SIOMOUTH OEVON EX10 9 SS
UK
TEL: 395579641
TLF:
TLX:
EML: G. A.WILKINSEUK. AC, EXETER
COM: B4, OSC,19,31
WILL CLIFFORD M DR
DPT OF PHYSICS
WASHINGTON UNIVERSITY
ST LOUIS MO 63130
USA
TEL: 314 889 6244
TLF:
TLX:
EML:
COM: 47,48

WILLIAMS DAVID A PROF DPT OF MATHEMATICS

UMIST

BOX 80

MANCHESTER M60 100

UK

TEL: 612363311

TLF:

ILX: 666094

EML:

COM: 34

WILLIAMS JOHN A OR
OPT OF PHYSICS
ALBION COLLEGE
ALBION MI 49224
USA
TEL: 5176295511
TLF:
TLX:
EML:
COM: 45

WILLIS ALLAN J DR

DPT PHYSICS \& ASTRONOMY

UNIVERSITY COLLEGE LONDON

GOWER ST

LONDON WC1E $68 T$

uk

TEL: 1713877050

TLF:

TLX: 28722

EML:

COM: 34,44
WILKINSON ALTHEA

DPT OF ASTRONOMY

UNIVERSITY OF MANCHESTER

MANCHESTER MI3 9PL

UK

TEL: $612737 \quad 121$

TLF:

TLX:

EML:

COM: 28

WILLIAMON RICHARO M

FERNBANK SCIENCE CENTER

I56 HEATON PARK ORIVE

I56 HEATON PARK ORIVE ATLANTA GA 30307

USA

TEL: $404 \quad 3784313$

TLF:

TLX:

EML:

COM: $27.42,46$

WILLIAMS GLEN A DR

DPT OF PHYSICS

CENTRAL MICHIGAN UNIV

MT PLEASANT MI 48058

USA

TEL: 5177743365

TLF :

TLX:

EML: 32NSQS

COM: 42

WILLIAMS PEREDUR M DR ROYAL OBSERVATORY

BLACKFORD HILL

EOINBURGH EH9 $3 \mathrm{HJ}$

UK

TEL: $316 \quad 673321$

TLX: 72383 ROEDIN G

EML:

TLF :

COM: 29

WILLIS ANTHONY GORGON DR DOMINION RAOIO ASTROPHYS OBSERVATORY

BOX 248

PENTICTON BC V2A 6K3

CANADA

TEL: 6044932277

TLF: 6044937767

TLX: 048 BB127

EML:

COM: 40
WILKINSON DAVID T PRINCETON UNIVERSITY

JADWIN HALL

BOX 708

PRINCETON NJ 08544

USA

TEL: 6094524406

TLF :

TLX:

EML:

COM: 47

WILLIAMS BARBARA A

OPT OF PHYSICS

UNIVERSITY OF DELAWARE

NEWARK DE 19716

USA

TEL: 3024516526

TLF:

TLX:

EML:

COM: 28

WILLIAMS IWAN P PROF

ASTRONOMY UNIT

QUEEN MARY/WESTFIELD COLL

MILE ENO RO

LONOON EL $4 N S$

UK

TEL: 1719755452

TLF:

TLX: 893750

EML: IPWUK.AC. QMW.MATHS

COM: $15,16,20,22$ VP. 51

WILLIAMS ROBERT E OR

EERRO TOLOLO

INTERAMERICAN OBSERVATORY

CASILLA 603

LA SERENA.

CHILE

TEL: 51213352

TLF : $51212466 * 342$

TLX: 645227 AURA CT

EML:

COM: $28,34,42$

WILLMORE A PETER PROF

SPACE RESEARCH OEPT

UNIVERSITY OF BIRMINGHAM

BOX 363

BIRMINGHAM B15 2TT

UK

TEL: $214 \quad 72 \quad 1301$

TLF :

TLX: 3399.38 SPAPHY G

EML:

COM:
WILKINSON PETER N OR

NRAL

JODRELL BANK

MACCLESFIELD SKII 9DL

UK

TEL: 47771321

TLF:

TLX: 36149

EML:

COM: 40

WILliams CAROL A

DPT OF MATHEMATICS

UNIVERSITY" OF S FLORIDA

TAMPA FL 33620.

USA

TEL: 8139742643

TLF:

TLX:

EML:

COM: $04,07,24$

\author{
WFLLIAMS JAMES G DR \\ JPL \\ MS 264700 \\ 4800 DAK GROVE DR \\ PASADENA CA 91109 \\ USA \\ TEL: 8183546466 \\ TLF: \\ TLX: $9105883269 \mathrm{JPL}$ \\ EML: \\ COM: $04,16,19,20$
}

WILLIAMS THEODORE B OR DFT PHYSICS \& ASTRONOMY RUTGERS UNIVERSITY

BOX 849

PISCATANAY NJ 088540849

USA

TEL: 2019322516

TLF:

FLX:

EML:

COM: 28

WILLNER STEVEN PAUL DR CENTER FOR ASTROPHYSICS HCO/SAO

60 GAROEN. ST

CAMBRIDGE MA 0213B

USA

TEL: 6174957123

TLF:

TLX: 92142B SATELLITE CAM

EML:

COM: 34,44 
WILLS BEVERLEY J OR

ASTRONOMY DPT

UNIVERSITY OF TEXAS

RLM 15308

AUSTIN TX 78712 1083

USA

TEL: $512 \quad 4713424$

TLF

TLX: $910-874-1351$

EML:

COM: 28,40

WILLSTROP ROOERICK V DR

INSTITUTE OF ASTRONOMY

THË OBSERVATORIES

MADINGLEY RO

CAMBRIDGE CB3 OHA

UK

TEL: 22362204

TLF:

TLX: 817297 ASTRON G

EML:

COM: 25,30

\section{WILSON CURTIS A}

ST JOHN'S COLLEGE

BOX 1671

ANNAPOLIS MD 21404

USA

TEL: 3012632371

TLF :

TLX:

EML:

COM: 41

\section{WILSON P DR}

INST ANGEWANOTE GEODAESIE

RICHARD-STRAUSS-ALLEE II

D 6000 FRANKFURT MAIN 70

GERMANY

IEL: 696333260

ILX: 413592

EML.

TLF:

COM: 196.21

WILSON ROBERT. PROF SIR

DPT PHYSICS \& ASTRONOMY

UNIVERSITY COLLEGE LONDON

GOWER ST

LONDON WCIE 6BT

UK

TEL: $1713807 \quad 154$

TLF:

ILX: 28722

EML:

COM: 14,44

\author{
WILLS DEREK DR \\ ASTRONOMY DPT \\ UNIVERSITY OF TEXAS \\ RLM 15308 \\ AUSTIN TX $78712 \quad 1083$ \\ USA \\ TEL: $512 \quad 4714461$ \\ TLF : \\ TLX: 910-874-1351 \\ EML: \\ COM: 28,40
}

WILSON ALBERT 6 DR

BOX 1871

SEBASTOPOL CA 95473

USA

TEL:

TLF :

EML:

COM: 28,47

$T\lfloor X:$

WILSON JAMES R OR

737 SGUTH M

LIVERMORE CA 94550

USA

TEL: $415 \quad 422 \quad 1659$

TLF :

EML:

COM: 48

TLX:

WILSON PETER R PROF

DPT OF APPLIED MAIHS

UNIVERSITY OF SYONEY

SYONEY NSW 2006

AUSTRALIA

TEL:

TLF :

TLX:

EML:

COM: $10,12 \mathrm{C}, 36$

WILSON ROBERT W DR

AT \& BELL LABORATORIES

HOH 2239

BOX 400

HOLMDEL NJ 07733

USA

TEL: 2019493803

TLF:

TLX:

EML:

COM: 34,40
WILLSON LEE ANNE DR

DPT OF PHYSICS

IOWA STATE UNIVERSITY

AMES IA 50011

USA

TEL: $515294 \quad 6765$

TLF :

TLX: 910-520-1157

EML:

COM: $27,35,36 \mathrm{C}$

WILSON ANDREW S OR

ASTRONOMY PROGRAM

UNIVERSITY BF MARYLAND

COLLEGE PARK MO 20742

USA

TEL: 3014546061

TLF :

TLX: 7108260352

EML:

COM: 40

WILSON LIONEL OR

ENV SCIENCE OPT

LANCASTER UNIVERSITY

LANCASTER LAL 4 YO

UK

TEL: $52465201 * 4075$

TLF :

ILX: 6511! LONCULG

EML:

COM: 27

WILSON RAYMOND N DR

ESO

KARL-SCHWARZSCHILD-STR 2

O 8046 GARCHING MUENCHEN

GERMANY

TEL: 8932006274

TLF: $B 93202362$

TLX: 52B2B2-D EO D

EML:

$\mathrm{COM}$ :

WILSON S J

OPT OF MATHEMATICS

NTL UNIVERSITY SINGAPORE

KENT RIDGE

SIMGAPORE 0511

SINGAPORE

TEL:

TLF :

TLX:

EML:

COM: 36
WILLSON ROBERT FREDERICK

DPT OF PHYSICS

TUFTS' UNIVERSITY

MEDFORD MO 02155

USA

TEL: 6176285000

TLF :

TLX:

EML:

COM: 40,51

WILSON BRIAN G PROF

UNIVERSITY OF QUEENSLAND

55 WALCOTT STREET

ST LUCIA OLD 4067

AUSTRALIA

TEL: 73772200

TLF :

TLX: 40315 UNIQLD AA

EML:

COM:

WILSON MICHAEL JOHN DR

DPT OF APPL MATHEMATICS

UNIVERSITY OF LEEOS

LEEOS L52 9JTT

UK

TEL:

TLF:

ILX:

EML:

COM:

WILSON ROBERT E PROF

OPT OF ASIRONOMY

UNIVERSITY OF FLORLDA

211 SSRB

GAINESUILLE FL 32611

USA

TEL: $904392 \quad 1182$

TLF :

TLX:

EML:

COM: 35,42

WILSON THOMAS L DR

MPI FUER RADIOASTRONOMIE

AUF OEM HUEGEL 69

D 5300 BONN 1

GERMANY

TEL: 228525378

TLF: 228525229

$T L X: 886440$

EML:

COM: $34,40,51$ 


WILSON WILLIAM J OR
JPL
MS 168327
4800 OAK GROVE OR
PASADENA CA 91109
USA
TEL: 8183545699
TLF:
TLX:
EML:
COM: 40
WINGET OONALD E
ASTRONOMY OPT
UNIVERSITY OF TEXAS
RLM 15 308
AUSTIN TX 787121083
USA
TEL: 5124714461
TLF:
TLX:
EML:
COM:

WINKLER GERNOT M R OR US NAVAL OBSERVATORY TIME SERVICE OPT 34 \& MASSACHUSETTS AVE NW WASHINGTON OC 203925100 USA

TEL: $202653 \quad 1520$

TLF :

TLX: 7108221970 NAVOBSY

EML:

COM: $04,19,31$

WINMEWISSER GISBERT OR
UNIVERSITAT ZU KOLN
I. PHYSI KALISCHES INST
UNIVERSITATSSTRASSE 14
O 5000 KOELN 41
GERMANY
TEL: 2114703567
TLF:
TLX:
EML:
COM: $14,34,40$
WIIT ADOLF N OR
OPT PHYSICS \& ASTRONOMY
UNIVERSITY OF TOLEDO
2801 W BANCROFT ST
TOLEOO OH 43606
USA
TEL: 4195372709
TLF:
TLX: 8104421633
EML:
COM: 21,34

WINCKLER JOHN R PROF SCHOOL OF PHYS \& ASTRON UNIVERSITY OF MINNESOTA 116 CHURCH ST SE MINNEAPOLIS MN 55455 USA TEL: 6123734688 TLF:

TLX:

EML:

COM:

WINIARSKI MACIEJ ASTRONOMICAL OBSERVATORY JAGIELLONIAN UNIVERSITY UL ORLA 171

PL 30244 KRAKOW

POLANO

TEL:

TLF :

TLX:

EML:

COM: 25

WINKLER KARL-HEINZ A DR LOS ALAMOS NATIONAL LAB MS B218 $\times$ OOT BOX 1663

LOS ALAMOS NM 87545

USA

TEL:

TLF:

TLX:

EML:

COM: 35

WIRAMIHARDJA SUHAROJA DR BOSSCHA OBSERVATORY

LEMBANG 40391

INDONESIA

TEL: 2296001

TLF : 22440699

EML:

COM:

TLX: 28324 BO

WITTEN LOUIS PROF
OPT OF PHYSICS
UNIVERSITY OF CINCINNATI
210 BRAUNSTEIN ML 11
CINCINNATI OH 452210111
USA
TEL: 5134756492
TLF:
TLX:
EML:
COM:

WINOHQRST ROGIER A OR

DPT OF PHYSICS

ARIZONA STATE UNIVERSITY

TEMPE AZ $85287 \quad 1504$

USA

TEL: 6029657143

TLF:

TLX: 1561058

EML:

COM: $09.28,40,47$

WINK JOERN ERHARD DR

IRAM

300 RUE DE LA PISCINE

F 38406 S MARTIN HERES CO fRANCE

TEL: 76423383

TLF:

TEX:

EML:

COM: 40

WINKLER PAUL FRANK DR

DPT OF PHYSICS

MIDDLEBURY COLLEGE

MIOOLEBURY VT 05753

USA

TEL: 8023883711

TLF:

TLX: 353249

EML:

COM:

WISNIEWSKI WIESLAW Z

LUNAR \& PLANETARY LAB UNIVERSITY OF ARIZONA TUCSON AZ 85721

USA

TEL: 6026216956

ILF:

ILX: $910-952-1143$

EML:

COM: $15,25,27$

WITTMANN AXEL O OR

UNIVERSITAETS - STERNWARTE

GEISMARLANDSR 11

D 3400 GOETTINGEN

GeRMANY

TEL: 551395042

TLF:

TLX: 96753

EML.:

COM: 10.12
WING ROBERT F PROF

OPT OF ASTRONOMY

OHIO STATE UNIVERSITY

174 W 18TH AVE

COLUMBUS OH. $43210 \quad 1106$

USA

TEL: 6144227876

TLF:

TLX:

EML:

COM: $27,29,45 C$

WINKLER CHR ISTOPH OR ESA/ESTEC

SSD

BOX 299

NL 2200 AG NOOROWIJK

NETHERLANOS

TEL: $17 \quad 1983591$

TLF: $17 \quad 1984690$

TLX: 39098

EML: CWINKLERGESTEC

COM: 44

WINNBERG AMDERS OR ONSALA SPACE OBSERVATORY GOETEBORG UNIVERSITY

5439 OO ONSALA

SHEDEN

TEL: 3006.0653

TLF:

TLX: 2400 INSPACE $S$

EML:

COM: 34,40

WITHBROE GEORGE $\downarrow$ DR

CENTER FOR ASTROPHYSICS

HCO/SAD

60 GARDEN ST

CAMBRIDGE MA 02138

USA

TEL: 6174957438

ILF:

ILX:

EML:

COM:

WITZEL ARNO OR

MPI FUER RADIOASTRONOMIE

AUF OEM HLEGEL 69

D 5300 BONN 1

GERMANY

TEL: 228525211

TLF:

TLX: 886440

EML:

COM: 40 


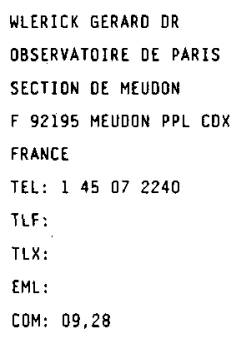

WNUK EOWIN

ASTRONOMICAL OBSERVATORY A MICKIEWICZ UNIVERSITY

UL SLONECZNA 36

PL 60286 POZNAN

POLAND

TEL:

TLF:

TLX:

EML:

\section{COM: 07}

WOLFE ARTHUR-M PROF
CASS
UCSO
C DII
LA JOLLA CA 920930216
USA
TEL: 6195347435
TLF: 6195346316
TLX:
EML: AWOLFEUCSD
COM:

WOLFSON RICHARO OR DPT OF PHYSICS MIODLEBURY COLLEGE MIODLEBÜY VT 05753 USA

TEL: $802 \quad 3883711$

TLF:

TLX:

EME: WOLFSONAMIDO BITNET COM: 10

WOLTJER LOOEWIJK PROF
OHP
F 04870 S MICHEL OBS
FRANCE
TEL: 92766368
TLF: 92766295
EML:
COM: $10,33,34,40,47,48 C$
TLX: 410690 OHP F

WOOD III H J DR ASTRONOMY OPT INDIANA UNIVERSITY SWAIN WEST 319 BLOOMINGTON IN 47405 USA

TEL:

TLF:

ILX:

EML:

COM: 29

$\begin{array}{ll}\text { WOEHL HUBERTUS OR } & \text { WOLF BERNHARO PH D } \\ \text { KIEPENHEUER INSTITUT } & \text { LANDESSTERNHARTE } \\ \text { FUER SONNENPHYSIK } & \text { KOENIGSTUHL } \\ \text { SCHOENECKSTRASSE } 6 & \text { D } 6900 \text { HEIDELBERG I } \\ \text { O } 7800 \text { FREIBURG BREISGAU } & \text { GERMANY } \\ \text { GERMANY } & \text { TEL: } 622110036 \\ \text { TEL: } 76132864 & \text { TLF: } \\ \text { TLF: } & \text { TLX: } \\ \text { TLX: } 7721552 \text { KIS } 0 & \text { EML: } \\ \text { EML: } & \text { COM: } 29\end{array}$

COM: $09,10,12,36$

WOLFENDALE ARNOLO W PROF

WOLFF SIONEY C OR KIIT PEAK NTL OBS BOX 26732

950 N CHERRY AVE TUCSON AZ $85726 \quad 6732$ USA

TEL: 6023275511

TLF:

TLX: 666-484 AURA NOAO

EML:

COM: 29,36

WOLSTENCROFT RAMON D DR ROYAL OBSERVATORY

BLACKFORD HILL EDINBURGH EH9 3HJ UK

TEL: 316673321

TLF :

$T L X: 72383$

EML:

COM: $21,34,48,51$

WOO JONG OK

KOREAN NTL UNIVERSITY

OF EDUCATION

CHUNGWON - GUN

CHUNGBUK 32023

KOREA R

TEL: $431 \quad 60 \quad 3712$

TLF :

TLX:

EML:

COM: 25,46

WOOD JANET H DR

ASTRONOMY DPT

UNIVERSITY OF TEXAS

RLM 15308

AUSTIN TX $78712 \quad 1083$

USA

TEL: $512 \quad 4713432$

TLF: 512471.6016

TLX: 9108741351

EML: EOU.UTEXAS.AS.ASTRO: : JHW

COM: 42
WOLSZCZAN ALEXANDER DR ARECIBO OBSERVATORY BOX 995 ARECIBO PR 00613 USA TEL: 8098782612 TLF:

TLX: 385638

EML:

COM: 34,40

WOOD DAVID B DR 6 TURNING MILL RD

LEXINGTON MA 02173

USA

TEL:

TLF:

TLX:

EML:

COM: 42

WOOD JOHN A OR CENTER FOR ASTROPHYSICS HCO/SAO 60 GARDEN ST CAMBRIDGE MA 0213B USA TEL: 6174957278 TLF: TLX: 92142B SATELLITE CAM EML: COM: $15,16,22$ 


WOOO PETER R OR
MOUNT STROMLO \& SIOING
SPRING OBSERVATORIES
PRIVATE BAG
WODEN PO ACT 2606
AUSTRALIA
TEL: 62 8 B 1111
ILF:
TLX: 62270 CANOPUS AA
EML:
COM: 27.35
WOOLF NEVILLE J
STEWARD OBSERVATORY
UNIVERSITY OF ARIZONA
TUCSON AZ 85721
USA
TEL:
TLF:
TLX:
EML:
COM: 34,50

WODTTEN HENRY ALWYN
NRAO
EOGEMONT RD
CHARLDTTESVILLE VA 22901
USA
TEL: 8042960211
TLX: $510-587-5482$
EML:
TLF:
COM: 34,40

WORRALL GOROON DR BIROSWOOD EAROISLEY HEREFOS UK TEL: TLF:

EML:

COM: TLX:

WRAY JAMES O OR
ASTRONOMY OPT
UNIVERSITY OF TEXAS
RLM $15 \quad 212$
AUSTIN TX $70712 \quad 1083$
USA
TEL:
TLF:
TLX:
EML:
COM: 44

WDOD ROGER DR ROYAL GREENWICH OBS HERSTMONCEUX CASTLE HAILSHAM BN27 IRP UK

TEL: $323833 \quad 171 * 3391$ TLX: 87451 RGOBSY G EML:

TLF:

COM:

WOOLFSON MICHAEL. M PROF OPT OF PHYSICS UNIVERSITY OF YORK HESLINGTON YORK YOL 500 UK

TEL: 90459861

TLF:

TLX: 57933 YORKULG

EML:

COM: $15,16,21,22$

WORDEN SIMON $P$ OR

6757 N $27 T H$ STREET

ARLINGTON VA 22213

USA

TEL:

TLF:

EML:

COM: 09.12

TLX:

WORSWICK SUSAN

ROYAL GREENWICH DBS

MAOINGLEY RO

CAMBRIDGE CB3 OEZ

UK

TEL: 223374000

TLF: 223374700

TLX: 265451/265871MONREFG

EML:

COM: 09

WRIGHT ALAN E OR
AAO
PRIVATE BAG
BOX 276
PARKES NSW 2970
AUSTRALIA
TEL: 68623677
TLF:
TLX: 63999 QASAR
EML: AWRIGHT ATNF. CSIRO. AU
COM: 05,40

WOODSWORTH ANDREW W. OR HERZBERG INST ASTROPHYS DOMINION ASTROPHYS OBS 5071. W SAANICH RO VICTORIA BC VBX $4 M 6$ CANADA

TEL: 6043880024

TLF: 6043630045

TLX: 0497295

EML:

COM: 40

WOOLSEY E G

1909 LAUDER ORIVE

OTTAWA ON KZA IA9

CANADA:

TEL:

TLF:

TLX:

EML:

COM:

WORLEY CHARLES E OR

US NAVAL OBSERVATORY

ASTRON \& ASTROPHYSICS DIV

34 \& MASSACHUSETTS AVE NW WASHINGTON OC 203925100 USA

TEL: 2026531588

TLF:

ILX:

EML: EOB@PHOBOS. USNO.NAVY.MIL COM: $\cdot 05,24,26 \mathrm{VP}$

WOSZCZYK ANDRZEJ PROF INSTITUTE OF ASTRONOMY N COPERNICUS UNIVERSITY UL CHOPINA $12 / 18$ PL 87 IOO TORUN POLAND

TEL: $2-60-18$

ILF :

TLX: 00552234 ASTR PL

EML:

COM: $15,16,50$

WRIGHT EDWARD L OR

DPT OF ASTRONOMY UNIVERSITY OF CALIFORNIA LOS ANGELES CA 90024 USA

TEL: 213 B25 5755

TLF :

TLX:

EML: BONNIE: :WRIGHT

COM: 34,47
WOODWARO PAUL R DR DPT OF ASTRONOMY UNIVERSITY OF MINNESOTA 116 CHURCH ST SE MINNEAPOLIS MN 55455 USA

TEL:

TLF:

TLX:

EML:

COM: 33,34

WOOSLEY S E PROF

LICK OBSERVATORY

UNIVERSITY OF CALIFORNIA SANTA CRUZ CA 95064

USA

TEL: 4084292976

TLF:

TLX:

EML:

COM: 28,35

WORRALL DIANA MARY CENTER FOR ASTROPHYSICS HCO/SAO

60 GARDEN ST

CAMBRIDGE MA D2138

USA

IEL: 6174957139

TLF:

ILX: 921428 SATELLITE CAM

EML:

COM: 28,48

WRAMDEMARK STIG S O OR

LUND OBSERVATORY

BOX 43

522100 LUNO

SWEDEN

TEL: $46 \quad 107303$

TLF:

TLX: 33199. OBSNOT S

EML:

COM: $25,33,37$

WRIGHT FRANCES W DR

DPT OF ASTRONOMY

HARVARD UNIVERSITY

60 GARDEN ST

CAMBRIDGE MA 02138

USA

TEL: 6174952647

TLF:

TLX:

EML:

COM: 27 
WRIGHT HELEN GREUTER THOMAS HOUSE APT 517 1330 MASSACHUSETIS AVE WASHINGTON OC 20005

USA

TEL:

TLF:

$T L X$ :

EML:

COM: 41

WRIXON GERARD T OR NTL MICROELECTRON RES CTR UNIVERSITY COLLEGE CORK CORK

IRELAND

TEL: 121508375

TLF:

TLX: 26050

EML:

COM:

WU HSIN-HENG OR

DPT OF PHYSICS

NIL CENTRAL UNIVERSITY

CHUNG \&I

CHINA $R$

TEL:

TLF:

TLX:

EML:

COM: $12,37,45$

WU MING-CHAN

YUNNAN OBSERVATORY

CAS

BOX. 110

KUNMING 72946 YUNNAN

CHINA PR

TEL: 8712035

TLF:

TLX: 64040 YJOBS CN

EML:

COM: 10,50

WU SHOU-XIAN

SHAANXI OBSERVATORY

CAS

LINTONG XIAN

SHAANXI

CHINA PR

TEL: 3355951

TLF

TLX: 7012L CSAO CN

EML:

COM: 19,31
WRIGHT JAMES $P$ DR

NTL SCIENCE FOUNDATION

OIV ASTRONOMICAL SCIENCES

1800 G ST NW

WASHINGTON DC 20550

USA

TEL: 2023577639

TLF :

TLX:

EML:

COM:

WROBEL JDAN MARIE OR

NRAO

BOX 0

SOCORRO NM $87801 \quad 0387$

USA

TEL: $505 \quad 8357000$

TLX: 9109881710

EML: JWROBELONRAO . EDU

TLF: 5058357027

COM: 28,40

WU HUAI -WEI

SHANGHAI OBSERVATORY

CAS

BO NANDAN RD

SHANGHAI

CHINA PR

TEL: $2138 \quad 6191$

TLF :

TLX: 33164 SHAO CN

EML:

COM: 40

WU NATLONG DR

BEIJING ASTRONOMICAL OBS

CAS

W SUBURB

BEIJING 10.0080

CHINA PR

TEL: $1256 \quad 1265$

TLF: 12561085

TLX: 22040

EML: BMAMAO ICA.BEIJING. CANET. CN

COM: 40

WU XINJI

OPT OF GEOPHYSICS

BEIJING UNIVERSITY

BEIJING 10007 !

CHINA $P R$

TEL: $128 \quad 2471 * 3929$

TLF:

$T\lfloor X: 22239$ PKUNI CN

EML:

COM: 40
WRIGHT KENNETH O OR

HERZBERG INST ASTROPHYS

DOMINION ASTROPHYS OBS

5071 W SAANICH RO

VICTORIA BC V8X $4 M 6$

CANADA

TEL: 6043883157

TLF: 6043630045

TLX: 0497295

EML:

COM: 36,42

WROBLEWSKI HERBERT OR

DPT DE ASTRONOMIA

UNIVERSIDAD DE CHILE

CASILLA 360

SANTIAGD

CHILE

TEL: 22294101

TLF :

TEX: 440001

EML:

COM: 20,24

WU LIAN-DA

PURPLE MOUNTAIN OBSERV

CAS

\section{NANJ ING}

CH!NA PR

TEL: 2532893

TLF :

TLX: 34144 PMONJ CN

EML:

COM: 07

WU SHENGYIN

BEIJING ASTRONOMICAL OBS

CAS

W SUBURB

BEIJING 100080

CHINA PR

TEL:

TLF:

TLX: 22040 BADAS CN

EML:

COM: 40

WU ZHIREN OR

SHANGHAL SCIENTIFICAL \&

TECH EOU PUBLISHING HOUSE

393 GUAN SHEN YAUN RO

SHANGHAI 200233

CHINA PR

TEL: 21365791

ILF :

ILX:

EML:

COM: 05
WRIGHT MELVYN C H DR

RADIO ASTRONOMY LAB

UNIVERSITY OF CALIFORNIA

601 CAMPBELL HALL

BERKELEY CA 94720

USA

TEL: $415 \quad 642 \quad 0420$

TLF:

TEX:

EML:

COM:

WII CHI CHAD OR

STSCI/CSC

HOMEWOOD CAMPUS

3700 SAN MARTIN DR

BALTIMORE MO 21218

USA

TEL: $301 \quad 338 \quad 4770$

TLF :

TLX: U.S.A.

EML:

COM: 34,44

WU LIN-XIANG

DPT OF GEOPHYSICS

BEIJING UNIVERSITY

BEIJING 100071

CHINA PR

TEL:

TLF:

TLX:

EML :

COM: $09,10,12$

WU SHI TSAN OR

SCHOOL OF ENGINEERING

UNIVERSITY OF ALABAMA

HUNTSVILLE AL 35899

USA

TEL: $205 \quad 895 \quad 6413$

TLF :

TLX:

EHL:

CIM: 10,49

WUNNER GUENTER

LEHRSTUHL F THEORET ASTRO

PHYSIK DER UNIV TUEBINGEN AUF OER MORGENSTELLE $12, \mathrm{C}$ D 7400 TUEBINGEN

GERMANY

TEL: $707 \quad 1292487$

TLF :

TLX; 7262714 AIT O

EML:

COM: 14,44 


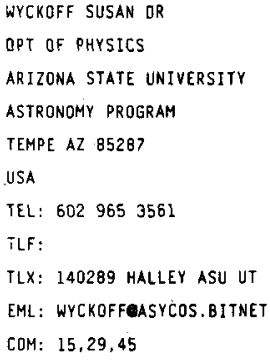

WYLLER ARNE A PROF

RRG LOT 14

ARROYO HONDO WEST

SANTA FE NM 87505

USA

TEL:

TLF: 5054735849

TLX:

EML:

COM: $09,12,36$

XANTHAKIS JOHN N PROF RES CENTER FOR ASTRONOMY ACADEMY OF ATHENS

14 ANAGNOSTOPOULOU ST GR 10673 ATHENS

GREECE

TEL: 13613589

TLF:

TLX:

EML:

COM: 10

XIA XIAOYANG DR

OPT OF PHYSICS

TIANJIN NORMAL UNIVERSITY

TIANJIN 300074

CHINA PR

TEL: 22716989

TLF :

TLX:

EML:

COM: 28

XIANG DELIN

PURPLE MOUNTAIN OBSERV

CAS

\section{NANJING}

CHINA PR

TEL: 2533738

TLF:

ILX: 34144 PMONJ [N

EML:

COM: 33.34

XIE LIANGYUN
INSTITUTE OF GEODEDY \&
GEOPHYSICS
XU OONG LL
WUCHANG HUBEI
CHINA PR
TEL:
ILF:
TLX:
EML:
COM: 08

WYNNE CHARLES 6 PROF INSTITUTE OF ASTRONOMY THE obSERVATORIES MADINGLEY RD CAMBRIDGE CB3 OHA UK

TEL: 2233375

TLF:

TLX: 817297 ASTRON G

EML:

COM: 09

XANTHOPOULOS B C DR

OPT OF PHYSICS

UNIVERSITY OF CRETE

BOX 1527

GR 711 11 IRAKLION

GREECE

TEL: 81235576

TLF :

TLX: 262728

EML:

COM: 47

XIA YI-FEI

DPT OF ASTRONOMY

NANJING UNIVERSITY

NANJING

CHINA PR

TEL: $25 \quad 34651 * 2882$

TLF :

TLX: 34151 PRCNU CN

EML:

COM: 08

XIANG SHOUPING

ASTROPHYSICS OIVISION

UNIV SCIENCE \& TECHNOLOGY

HEFEI 230026 ANHUI

CHINA PR

TEL: $55133 \quad 1134$

TLF:

TLX:

EML:

COM: 47

XIONG DA-RUN

PURPLE MOUNTAIN OBSERV

CAS

NANJING

CHINA PR

TEL: 25 42817

TLF:

ILX: 34144 PMONJ CN

EML:

COM: 27,35
WYNN-WILLIAMS C G DR INSTITUTE FOR ASTRONOMY UNIVERSITY OF HAWAII 2680 WOODLAWN OR HONOLULU HI 96822 USA

TEL: 808 948 B807

TLF:

ILX:

EML:

COM: 28.34

XI ZE-ZONG INST HISTORY NAT SCIENCE 1 GONG YUAN WEST RD BEIJING

CHINA PR

TEL: 1557180

TLF :

TLX:

EML:

COM: 41

XIA ZHIGUO OR INSTITUT. FUER ASTRONOMIE ETH ZENTRUM CH 8092 ZUERICH SWITZERLAND

TEL: 12563813

TLF:

TLX: 817379 EHHG CH EML:

COM: 40

XIAO NAI - YUAN DPT OF ASTRONOMY NANJING UNIVERSITY NANJING CHINA PR TEL: $2534651 * 2882$ TLF : TLX: 34151 PRCNU CN EML: COM: 19

XIRADAKI EVANGELIA DR OPT OF ASTROPHYSICS UNIVERSITY OF ATHENS PANEPISTIMIOPOLIS gR ATHENS

GREECE

TEL: $17235 \quad 122 / 9628306$ TLF:

ILX:

EML:

COM: 37 
XU AO-AO

DPT OF ASTRONOMY

NANJING UNIVERSITY

NANJING

CHINA PR

TEL:

TLF:

TLX: 34151 PRC NU CN

EML

COM: 10

XU PEI - YUAN

INST ELECTRONIC PHYSICS

SUST

JIA DING

SHANGHAI

CHINA PR

TEL: 21951602

TLF:

TLX:

EML:

COM: 40

$X U$ ZHI-CAI

PURPLE MOUNTAIN OBSERY

CAS

\section{NANJING}

CHINA PR

TEL: $25 \quad 46700$

TLF:

TLX:

EML:

COM: 40

YAKOVKIN N A DR

ASTRONOMICAL OBSERVATORY

KIEV STATE UNIVERSITY

OBSERVATORNAYA UL 3

252053 KIEV

IKRAINE

TEL:

TLF :

TLX:

EML:

COM: 10

YAMAKOSHI KAZUO
INST COSMIC RAY RESEARCH
UNIVERSITY OF TOKYO
MIOORICHO TANASHI
TOKYO 189
JAPAN
TEL: $0424-61-4131$
TLF:
TLX: 02822371 ICRTU J
EML:
COM: 21.22

XU BANG-XIN
OPT OF ASTRONOMY
NANJING UNIVERSITY
NANJING
CHINA PR
TEL:
TLF:
TLX:
EML:
COM: 08,31

XU PINXIN

PURPLE MOUNTAIN OBSERV

CAS

NANJING

CHINA PR

TEL: 2532893

TLF:

ILX: 34144 PMONJ CN

EML:

COM: 07.22

YABUSHITA SHIN A PROF

DPT APPLIED MATHS \& PHYS

KYOTO UNIVERSITY

SAKYO KU

KYOTO 606

JAPAN

TEL: 757512111

TLF :

TLX:

EML:

COM: $15,20,34$

YALLOP BERNARD D OR

ROYAL GREENWICH OBS

MADINGLEY RO

CAMBRIDGE CB3 OEZ

UK

TEL: $223 \quad 374735$

TLF: $223 \quad 374700$

TLX: 817235 RGOSTR G

EML: BDYUUK,AC.RGO,SRF

COM: 04P

YAMAMOTO TETSUO DR
ISAS
3-1-1 YOSHINODAI
SAGAMIHARA
KANAGAWA 229
JAPAN
TEL: 0427513911
TLF:
TLX: 24550 SPACETKY J
EML:
COM: 21

XU JIA-YAN

SHAANXI OBSERVATORY

CAS

IINTONG XIAN

SHAANXI

CHINA PR

TEL: 332255

TLF :

TLX:

EML:

COM: 19

XU TONG-QI

SHANGHAI OBSERVATORY

CAS

BO NANDAN RD

SHANGHAI 200030

CHINA PR

TEL: 21386191

ILF :

ILX: 33164 SHAO CN

EML:

COM: 08,19

YABUUTI KIYOSHI PROF

20 IANAKA HIGASKI

HINOKUCH MACHI

KYOTO 605

JAPAN

TEL:

TLF :

TLX:

EML:

COM: 41

YAMAGATA TOMOHIKO OR

TOKYO ASTRONOMICAL OBS

NAOJ

OSAWA MITAKA

TOKYO 181

JAPAN

TEL: 0422325111

TLF :

TLX: 02822307 TAOMTK J

EML:

COM: 28

YAMASAKI ATSUMA DR

DPT DF GEOSCIENCE

NATIONAL DEFENSE ACADEMY

HASHIRIMIZU

YOKOSUKA 239

JAPAN

TEL: $B 1 \quad 468 \quad 413810$

ILF: BL 468445902

TLX:

EML:

COM: $42 \mathrm{C}$
XU JI-HONG OR

URUMQI ASTRONOMICAL STAT

CAS

XINJIANG 830011

CHINA PR

TEL: 335757

TLF :

TLX: 79152 KWTWZ CN

EML: 830011

COM: 07

XU ZHENTAO

PURPLE MOUNTAIN OBSERV

CAS

NANJING

CHINA PR

TEL: 2531096

TLF:

TLX: 34144 PMONJ CN

EML:

COM: 10,41

YAHIL AMOS OR

DPT OF EARTH \& SPACE SCI

ASTRONOMY PROGRAM

SUNY AT STONY BROOK

STONY BROOK NY 117942100

USA

TEL: $516246 \quad 6545$

ILF :

TLX: $510-228-7767$

EML:

COM:

YAMAGUCHI SHICHIRO

FACULTY OF ENGINEERING

GIFU UNIVERSIIY

YANAGIDO

GIFU 50111

JAPAN

TEL: $582 \cdot 30-1111$

TLF :

TLX:

EML:

COM:

YAMASHITA KOJUN DR

ISAS

3-1-I YOSHINODAI

SAGAMIHARA

KANAGAWA 229

JAPAN

TEL: 427513911

TLF :

TLX:

EML:

COM: 21,44 
YAMASHITA YASUMASA PROF TOKYO ASTRONOHICAL OBS

NAOJ

OSAWA MITAKA

TOKYO 181

JAPAN

TEL:

ILF:

TLX: 2822307 TAOMTK I

EML:

COM: $25,29,45$

YANG JIAN

PURPLE MOUNTAIN OBSERV

CAS

NANJING

CHINA PR

TEL: 2546700

TLF :

TLX: 34144 PMONJ CN

EML:

COM: 40

\section{YANG TING-GAO}

SHAANXI ASTRONOMICAL POBS

BOX 18

LINTONG

WIAN

CHINA PR

TEL:

TLF :

ILX

EML:

COM: 24

YAO JIN-XING

PURPLE MOUNTAIN OBSERV

CAS

NANUING

CHINA PR

TEL: 2546700

TLF:

TLX:

EML:

COM: 10

YASUDA HARUD PROF DR

TOKYO ASTRONOMICAL OBS

NAOJ

OSAWA MITAKA

TOKYO 181

JAPAN

TEL:

TLF:

TLX:

EML:

COM: 08
YAMAZAKI AKIRA OR

HYOROGRAPHIC OPI

GEODESY GEOPHYSICS DIV

TSUKIJI 5 CHUO KU

TOKYO 104

JAPAN

TEL: $0.3-541-3811$

TLF:

TLX: 02522222 JAHYO J

EML:

COM: 04,08

YANG KE-JUN

GEQPHYSICAL INSIITUTE

UNIVERSITY OF ALASKA

FAIRBANKS AK 997750800

USA

TEL:

ILF:

TLX:

EML:

COM: 31

YANKULOVA IVANKA DR

DPT OF ASTRONOMY

UNIVERSITY OF SOFIA

ANTON IVANOV ST 5

BG 1126 SOFIA

BULGARIA

TEL: 2544852

TLF :

TLX:

EML:

COM:

YAO ZHENG-QIU

DPT OF SCIENTIFIC COOP

SSRG

BOX 4470

DAMASCUS

SYRIA

TEL:

TLF:

TLX:

EML:

COM: 09

VATSKIV YA S OR

MAIN ASTRONOMICAL OBS UKRAINIAN ACAD OF SCIENCE

GOLOSEEVO

252127 KIEV

UKRAINE

TEL: $66 \quad 3110$

TLF:

ILX: 131406 SKY SU

EML:

COM: 08.19
YAN LIN-SHAN

SHANGHAI OBSERVATORY

CAS

BO NANDAN RO

SHANGHAI

CHINA PR

TEL: 21386191

TLF :

TLX: 33164 SHAO CN

EML:

COM: 26

YANG LAN-TIAN

DPT OF PHYSICS

HUAZHONG NORMAL UNIV

WUHAN

CHINA PR

TEL: $75601 * 300 / 401$

TLF :

TLX:

EML:

COM: 47,48

YANOVITSKIJ EOGARD G DR

MAIN ASTRONOMICAL OBS

UKRAINIAN ACAO OF SCIENCE

GOLOSEEVO

252127 KIEV

UKRAINE

TEL: $66 \quad 3110$

TLF :

TLX: 131406 SKY SU

EML:

COM: 36

YAPLEE B $S$

8 CREST VIEW CT

ROCKVILLE MB 20854

USA

TEL: 3017620935

TLF:

TLX:

EML:

COM:

YAU KEVIN $X[O R$

DPT OF PHYSICS

UNIVERSITY OF OURHAM

SOUTH RO

DURHAM OHI 3LE

UK

TEL: 913742153

TLF: $913 \quad 743749$

TLX: 537351 DURLIB $G$

EML: YAUUUK, AC, OUR, STAR

COM: 41
YANG FUMIN

SHANGHAI OBSERVATORY

CAS

BO NANDAN RD

SHANGHAI

CHINA PR

TEL: 21386191

TLF :

TLX: 33164 SHAO CN

EML:

COM: 19

YANG SHI JIE

PURPLE MOUNTAIN OBSERV

CAS

NANJING

CHINA PR

TEL: 2546700

TLF:

TLX: 34144 PMO NJ CN

EML:

COM: 09

YAO BAO-AN

SHANGHAJ OBSERVATORY

CAS

80 NANDAN RD

SHANGHAI

CHINA PR

TEL: 21396191

TLF :

TLX: 33164 SHAO CN

EML:

COM: 27

YAROV-YARDVOJ MS DR

MATHEMATICS DPT

MVTU

VTORAYA BAUMANSKAYA 5

107005 MOSCOW

RUSSIA

TEL: 267-03-92

TLF :

TLX: 111572

EML:

COM: 07

YAVNEL ALEXANOER A DR

METEORITE COMMITTEE

ACADEMY OF SCIENCES

ULIANOVOJ M UL $3 \mathrm{~K} I$

117313 MOSCOW

RUSSIA

TEL: 1377538

TLF:

TLX:

EML:

COM: 15,22 
YE BINXUN

YUNNAN DBSERVATORY

CAS

BOX 110

KUNMING. 72946 YUNNAN

CHINA PR

TEL: 8712035

TLF :

TLX: 64040 YUOBS CN

EML

COM: 09

YEE HOWARO K C QR

DPT OF ASTRONOMY

UNIVERSITY OF TORONTO

60 ST GEORGE ST

TORONTO ON M5S IAI

CANAOA

TEL: $416 \quad 978 \quad 4833$

TLF: $416 \quad 978 \quad 3921$

TLX: 06986766

EML: HYEENTORPHYS BITAET

COM

YEOMANS DONALO $K$ OR

$J P L$

MS $301 \quad 150$ G

4800 OAK GROVE OR

PASADENA CA 91109

USA

TEL: $818 \quad 3542127$

TLF : $818393 \quad 1159$

TLX: 675429 JPL COMM PSO

EML: $5122:: 0 K Y$

COM: $15,20 \vee P, 22,41$

YIN JI - SHENG

BEIUING ASTRONOMICAL OBS

CAS

W SUBURB

BEIJING 100080

CHINA PR

IEL: 1281203

TLF :

TLX: 22040 BAOAS CN

EML

COM: 25

YOKOYAMA KOICHI OR

INTL LATITUDE OBSERVATORY

NAOJ

HOSHIGAOKA MIZUSAWA SHI

IWATE 023

JAPAN

TEL: $0197-24-7111$

TLF :

TEX: 837628

EML:

COM: 19
YE SHI-HUI

PURPLE MOUNTAIN OBSERY

CAS

NANJING

CHINA PR

TEL: 2546700

TLF :

TLX: 34144 PMONTJ CN

EML:

COM: 10

YEH TYAN BR

HIGH ALTITUDE OBSERVATORY

NCAR

BDX 3000

BOULDER CO 803073000

USA

TEL: 3034975401

TLF

TLX:

EML:

COM: 10,49

YI ZHAO-HUA

DPT OF ASTRONOMY

NANJING UNIVERSITY

NANJING

CHINA PR

TEL:

ILF :

TLX:

EML:

COM: 07

YIN QI-FENG

DPT OF GEOPHYSICS

BEIUING UNIVERSITY

BEIJING 100071

CHINA PR

TEL: $1 \quad 28 \quad 2471 * 3888$.

TLF :

TLX: 22239 PKUNY CN

EML:

COM: 40

YOKOYAMA TADASHI DR

UNIVERSIDADE ESTADUAL

PAULISTA

CP 178

13500 RIO CLARO

BRAZIL

TEL: 195340122

TLF :

TLX: 011-31870

EME:

COM: 07
YE SHU-HUA

SHANGH'AI OBSERVATORY

CAS

BO NANDAN RD

SHANGHAI 200030

CHINA PR

TEL: $21 \quad 386191$

TLF: 21384618

TLX: 33164 SHAO CN

EML:

COM: $08,19,31 \mathrm{C}, 38,40 \mathrm{C}, \mathrm{EC}$

YEIVIN Y PROF

SCHOOL OF PHYS \& ASTRON

TEL AVIV UNIVERSITY

RAMAT AVIV

TEL AVIV 69978

ISRAEL

TEL:

TLF

TLX:

EML:

COM:

YILMAZ FATMA OR

UNIVERSITY OBSERVATORY

UNIVERISTY OF ISTANBUL

34452 ISTANBUL

TURKEY

TEL:

ILF :

$T L X$

EML:

COM:

YOOER CHARLES $F$

JPL

MS 183150

4800 DAK GROVE DR

PASADENA CA 91109

USA

TEL: BIB 3542444

TLF :

TLX: $617-5429$

EML:

COM: 16

YONEYAMA TADAOKI DR

2-1-16 HIBARIGAOKA-KITA

HOYA-SHI

TOKYO 202

JAPAN

TEL:

TLF :

TLX:

EML:

COM:
YE WENWEI

INSTITUTE OF SEISMOLOGY

STATE SEISMO BUREAU

XIAO HONG SHAN WUHAN

HUBEI 230026 ANHUI

CHINA PR

TEL: 813401

TLF :

TLX:

EML:

COM:

YEN JUI-LIN PROF

DPT OF ELECTRICAL ENGIN

UNIVERSITY OF TORONTO

60 ST GEORGE ST

TORONTO ON MSS IA4

CANAOA

TEL: 4169788756

TLF: $416 \quad 978 \quad 3921$

TLX: 06986766

EML:

COM:

YILMAZ NIHAL OR

DPT OF ASTRONOMY

UNIVERSITY OF ANKARA

FEN FAKULTESI

06100 BESEVLER

TURKEY

TEL: 41236550

TLF :

TLX:

EML:

COM:

YOKOSAWA MASAYOSHI OR

DPT OF PHYSICS

IBARAKI UNIVERSITY

2-1-1 BUNKYO MITO

IBARAKI 310

JAPAN

TEL: $292 \quad 26 \quad 1621$

TLF:

$T L x$ :

EML:

COM:

YORK DONALD G OR

ASIRONOMY \& ASTROPHYS CTR

UNIVERSITY OF CHICAGO

5640 S ELLIS AVE

CHICAGO IL 60637

USA

TEL: 3129628930

TLF

TEX: 910-221-5617

EML:

COM: 34 


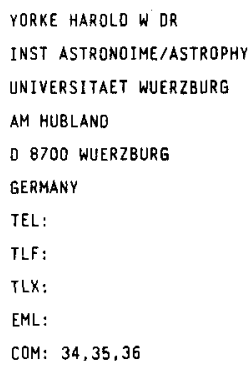

YOSHIDA HARUO

TOKYO ASTRONOMICAL OBS

NAOJ

OSAWA MITAKA

rokro 181

JAPAN

TEL: 422413614

TLF: 442413793

TLX: 02822307 TAOMTK

EML: YOSHIDAOC1. MTK. NAO.AC.JP

COM: 07

YOSHIMLRA MOTOHIKO OR
OPT OF PHYSICS
TOHOKU UNIVERSITY
ARAMAKI
SENDAI 980
JAPAN
TEL: 81222221800
TLF: 81222251891
TLX:
EML:
COH: 47
YOU JUNHAN
ASTROPHYSICS DIVISION
UHIV SCIENCE \& TECHNOLOGY
HEIFI 230026 ANHUI
CHINA PR.
TEL: 551331134
TLF:
TLX: 90028 USTC LN
EML:
COM: 48

COM: 48

YOUNG LOUISE GRAY DR
DPT OF ASTRONOMY
SAN DIEGO STATE UNIV
SAN OIEGO CA 92182
USA
TEL: 6192878890
TLF:
TLX:
EML:
COM: 14,16

YU KYUNG-LOH PROF DPT OF ASTRONOMY SEOUL NTL UNIVERSITY KWANAK KU SEOUL 151 KOREA $R$

TEL:

TLF:

TLX:

EML:

EOM: 08
YOSHIDA JUHZO PROF DPT OF PHYSICS KYOTO SANGYO UNIVERSITY KAMIGAMO

KYOTO 603

JAPAN

TEL: 757012151

TLF:

TLX: $5422661 \mathrm{kSUJ}$

EML:

COM: 07

YOSHIZAWA MASANORI DR TOKYO ASTRONOMICAL OBS NAOJ

OSAWA MITAKA

TOKYO 181

JAPAN

TEL: 422325111

TLF: 422321924

TLX: 2822307 TAOMTK

EML:

COM: $08 \mathrm{C}$

YOUNG ANDREW T DR

DPT OF ASTRONOMY

SAN DIEGO STATE UNIV

SAN DIEGO CA 92182.0334 USA

TEL: 6192655817

TLF :

TEX:

EML: ATYMINTAKA.SDSU. EDU

COM: $16,25 \mathrm{P}$

YOUNIS SAAD M

SARC

SCIENTIFIC RES COUNEIL

BOX 2441

JADIRIYAH BAGHDAD

IRAO

TEL: 17765127

TLF:

TLX: 2187 BATHILMI IK

EML:

COM: $24,33,34,40$

YU XIN ALFREO DR

DPT OF APLLIEO MATHS

HONG KONG PQLYTECHNIC

HUNG HOM

KOWLOON

HONG KONG

TEL: 37666951

TLF: 33629045

TLX:

EML:

COM:
YOSHII YUZURU DR TOKYO ASTRONOMICAL OBS NAOJ

OSAWA MITAKA

TOKYO LBI

JAPAN

TEL: 422325111

TLF:

TLX: 2822307 TAOMTK 3

EML:

COM: $33 \mathrm{C}, 47$

YOSS KENNETH M DR

OPI OF ASTRONOMY

UNIVERSITY OF ILLINOIS

1011 W SPRINGFIELD AVE URBANA IL 61801

USA

TEL: 217.3333295

TLF:

ILX:

EML:

COM: 30,45

YOUNG ARTHUR DR DPT OF ASTRONOMY SAN DIEgO STATE UNIV SAN DIEGO CA 92182 USA TEL: 6192656167 TLF:

TLX:

EML:

COM:

YOUSEF SHAHIAAZ M OR DPT OF ASTRONOMY FACULTY OF SCIENCES CAIRO UNIVERSITY GEZA EGYPT TEL:

TLF :

TEX:

EML:

COM:

YU YAN DR DPT OF ASTRONOMY UNIVERSITY OF ILLINOIS $1002 W$ GREEN ST URBANA IL 61801 USA TEL: 2172441187 TLF: TLX:

EML: YU®OENEB, ASTRO.UIUC, EDU COM: 14 
YUAN CHI PROF

DPT OF PHYSICS

CITY COLLEGE OF NY

138 ST CONVENE AVE

NEW YORK NY 10031

USA

TEL: 2126906823

TLF:

TLX:

EML:

COM: 33

YUMI SHIGERU PROF OR

KEYAKIDAI 1-12-2

KI YAMACHO

MIYAKIGUN

SAGA 84102

JAPAN

TEL:

TLF:

TLX:

EML:

COM: 19

ZACHARIADIS THEODOSIOS OR RES CENTER FOR ASTRONOMY ACADEMY OF ATHENS

14. ANAGNOSTQPOULOU ST GR 10673 ATHENS

GREECE

TEL:

TLF:

ILX:

EML: EXAKAZOGGRATHUN1

COM: 10

\section{ZADUNAISKY PEDRO E PROF}

UNIVERSIOAO BUENOS AIRES

FAC CIENCIAS EXACTAS MATH

CIUDAD UNIVERSITARIA PABI

1428 BUENOS AIRES

ARGENTINA

TEL:

TLF :

TLX:

EML:

COM: 20

ZAMBON GIULIO OR
25 URAWA ROAD
OUNCRAIG 6023
AUSTRALIA
TEL: 94478849
TLF:
EML:
COM:
TLX:

VUASÁ Manabu OR

OPT DF MATH \& PHYSICS

KINKI UNIVERSITY

HIGASHI

OSAKA 577

JAPAN

TEL:

TLF:

TLX:

EML:

COM: 07,20

YUN HONG-SIK PROF

DPT OF ASTRONOMY

SEOUL NTL UNIVERSITY

KWANAK KU

SEOUL 151

KOREA $R$

TEL: $877-2130 \times 2542$

TLF :

TiX:

EML:

COM: 10,12

ZACHARIAS NORBERT OR

HAMBURGER STERNWARTE

GOJENSBERGSWEG 112

D 2050 HAMBURG 80

GERMANY

TEL: $407252 \quad 4112$

TLX: 217884

EML: ZACHARIASODKRZ-HAMBURG.OBP.OE

TLF: 4072524190

COM: 24

ZAFIROPOULOS BASIL OR

OPT OF PHYSICS

UNIVERSITY OF PATRAS

GR 26110 RION

GREECE

TEL: 61991973

TLF: 61991909

TLX: 312447 UNPA GR

EML:

COM: 07

ZAMBRANO ALEJANDRO OR
REAL INST Y OBSERVATORIO
OE LA ARMAOA
CECILIO PUJAZON S/N
E 11110 SAN FERNANDO
SPAIN
TEL: 56883548
TLF: 56899302
TLX: 76108 IOM E
EML:
COM: 04

YUE ZENG-YUAN

OPT OF GEOPHYSICS

BEIJING UNIVERSITY

BEIJING 100071

CHINA PR

TEL:

TLF :

TLX:

EML:

COM:

YUNGELSON LEV R

INST OF ASTRONOMY

ACADEMY OF SCIENCES

PYATNITSKAYA UL 48

109017 MOSCOW

RUSSIA

TEL: $231-54-61$

TLF:

TEX: 412623 SLSTP SU

EML:

COM: 35

ZACHAROV IGOR OR

ASTRONOMICAL INSTITUTE

CZECH ACADEMY OF SCIENCES

ONDREJOV OBSERVATORY

CS 25165 ONDREJOV

CZECHOSLOVAKIA

TEL: 20485201

TLF : 20485314

TLX: 121579

EML:

COM: 09

ZAHN JEAN-PAUL DR

OBS MIOI PYRENEES

14 AVE E BELIN

F 31400 TOULOUSE COX

FRANCE

TEL: 6125.2101

TLF :

TLX: $530776 \mathrm{~F}$

EML:

EOM: 35.36

ZAMORANI GIOVANNI

IST DI RADIOASTRONOMIA

CNR

VIA IRNERIO 46

I 40126 BOLOGNA

ITALY

TEL: 51232856

TLF :

TLX: 211664 INFN BO I

EML:

COM: 47.48
YULDASHBAEV TAIMAS $S$

ASTRONOMICAL INSTITUTE

UZBEK ACADEMY OF SCIENCES

700052 TASHKENT

UZBEKISTAN

TEL:

TLF:

TLX:

EML:

COM:

ZABRISKIE F R PROF

RD 1

ALEXANORIA PA 16611

USA

TEL: $814 \quad 6694483$

TLF :

TLX:

EML:

COM:

ZACHILAS LOUKAS $Q R$

DPT OF CHEMISTRY

UNIVERSITY OF CRETE

B0X 1527

GR 71409 IRAKLION CRETE

GREECE

TEL: $B 1212453$

TLF: $\theta 1238468$

TLX:

EML: ZACHILASETALOS.CC. UCH. GR

COM: 33

ZAITSEV VALERII $\checkmark$ OR

INST OF APPLIEO PHYSICS

ACADEMY OF SCIENCES

ULYANOV UL 46

603600 N NOVGOROO

RUSSIA

TEL:

TLF:

TLX:

EML:

COM: 40

ZAMORANO JAIME DR

DPT DE ASTROFISICA

FAC C. FISICAS

UNIVERSIDAD COMPLUTENSE

E 28040 MADRID

SPAIN

TEL: 14495316

TLF :

TLX: 47272

EML:

COM: $2 B$ 


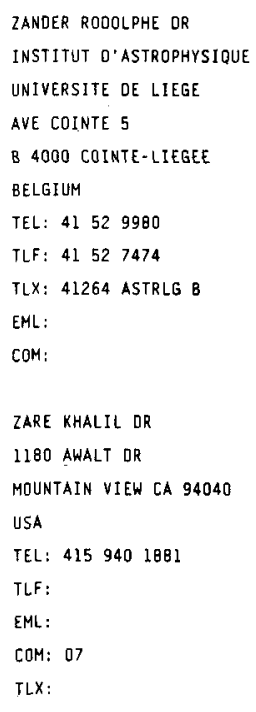

\author{
ZANINETII LORENZO \\ IST DI FISICA GENERALE \\ CORSO D AZEGLIO 46 \\ I 10125 TORINO \\ ITALY \\ TEL: 11657694 \\ TLX: 211041 INFNTO I \\ EML: \\ TLF: \\ COM:
}

ZARNECKI JAN CHARLES OR UNIT FOR SPACE SCIENCES UNIVERSITY OF KENT CANTERBURY CT2 7NR UK

TEL: 227764000 TLF: 227762616 TLX: 965449 UKLIB EML: jcz uk.ac.uke COM: 15,44

ZDANAVICIUS KAZIMERAS OR INST OF THEORET PHYS/ASTR LITHUENIAN ACAOEMY OF SCI POZELOS 54

232600 VILNIUS

LITHUANIA

TEL: $122 \quad 613440$

TLF :

TLX: 261141 LMI SU

EML:

COM: $45 \mathrm{C}$

\section{ZEIPPEN CLAUDÉ OR} OBSERVATOIRE OE PARIS SECTION DE MEUDON F 92195 MEUDON PPL COX FRANCE

TEL: $1 \quad 4507 \quad 7443$

TLF:

TLX: 201571

EML:

COM: 14

ZEL'MANOV A L DR
STERNBERG STATE ASTR INST
UNIVERSITETSKIJ PROSP 13
HI9899 MOSCOW
RUSSIA
TEL:
TLX:
EML:
TLF:
COM: 47

ZAPPALA ROSARIO ALDO OR IST DI ASTRONOMIA CITTA UNIVERSITARIA VIA A DORIA 6 I 95125 CATANiA IIALY

TEL: $33 \quad 0533 * 493$

TLF:

TLX: 970359 ASTRCT I

EML:

COM: 10

ZARRO OOMINIC M OR

NASA/GSFC

CODE 602.6

BLDG?

GREENBELT MO 20771

USA

TEL: 3012862039

ILF :

ILX: 89675

EML: SOLAR: : DZARRO

COM: 12

ZEALEY WILLIAM J DR

UNIVERSITY OF WOLLONGONG PHYSICS OEPT

BOX 1144

WOLLONGONG NSW 2500

AUSTRALIA

TEL: $42 \quad 270555$

ILF:

ILX: 29022 AA

EML:

COM: $09.34,46$

ZEKL HANS WILHELM

TON BELLER GMBH

BURGSTRASSE 22

D 6140 BENSHEIM 3

GERMANY

TEL: $625 \quad 173001$

TLF:

TLX: 468352

EML:

COM:

ZENG QIN DR

PURPLE MOUNTAIN OBSERV

CAS

NANJING

CHINA PR

TEL: $25 \quad 30 \quad 8516$

TLF:

TLX: 34144 PMONJ CN

EML:

COM: 14,34
ZAPPALA VINCENZO PROF OSS ASTRONOMICO OI TORINO

ST OSSERVATORIO 20

I loO25 PINO TORINESE

ITALY

TEL: $11 \quad 841067$

TLF: $1184 \quad 1281$

TLX: 213236 TO ASTR I

EML:

COM: $15 C, 20$

ZASOV ANATOLE $V$ OR

STERNBERG STATE ASTR INST

UNIVERSITETSKIJ PROSP 13 119899 MOSCOW

RUSSIA

TEL:

TLF:

TLX:

EML:

COM: $2 B$

ZEILIK MICHAEL II OR OPT PHYSICS \& ASTRONOMY UNIVERSITY OF NEW MEXICO BOO YALE BLVO NE ALBUDUERQUE NM 87131 USA

YEL: $505 \quad 2774442$

TLF:

TLX:

EML:

COM: $34,42,46$

ZELENKA ANTOINE QR DACHSLENBERGSTR 56

CH B1BO BUELACH SWITERLAND

TEL:

TLF:

ILX:

EML:

COM: 10,12

ZENSUS J-ANTON OR

NRAO

BOX 0

SOCORRO NM 878010387 USA

TEL: $505 \quad 835 \quad 7348$

TLF: 5058357027

TLX: $910-988-1710$

EML: BITNET: AZENSUSONRAO.EDU COM: 40 


\author{
ZERULL REINER H DR \\ RUHR-UNIVERSITAET BOCHUM \\ BEREICH EXTRATERR, PHYSIK \\ O 4630 BOCHUM 1 \\ GERMANY \\ TEL: 2347004576 \\ TLF: \\ ThX: 0825860 \\ EML: \\ COM: 21
}

ZHANF SHOUZHONG OR
414 WEST 120 ST
APT 401
NEW YORK NY 10027
USA
TEL: 21266664699
TLF:
TLK:
EML:
COM: 41

ZHANG ER-HO OR

ASTRONDMY OPT UNIVERSITY OF TEXAS

RLM 15220

AUSTIN IX 787121083

USA

TEL: 5124714462

TLF :

TLX:

EML:

COM: 42

ZHANG HUI

SHAANXI OBSERVATORY

CAS

LINTONG XIAN

SHAANXI

CHINA PR

TEL: 332255

TLF:

TLX: 70121 CSAO CM

EML:

COM: 0 OB

ZHANG PEIYU

PURPLE MOUNTAIN OBSERV

CAS

NANJING

CHINA PR

TEL: 2537521

TLF:

TLX: 34144 PMONJ CN

EML:

COM: 41
ZHAGAR YOURI H DR

ASTRONOMICAL OBSERVATORY

LATVIAN STATE UNIVERSITY

RAINIS BUL 19

226098 RIGA

LATVIA

TEL: 132223149

TLF:

TLX: 161171 TEMA SU

EML:

COM:

ZHANG BAI-RONG

YUNNAN OBSERVATORY

CAS

BOX 110

KUNMING 72946 YUNNAN

CHINA PR

TEL: 8712035

TLF :

TLX: 64040 YU0BS CN

EML:

COM: 10,50

ZHANG FU JUN

SHANGHAI OBSERVATORY

CAS

80 NANDAN RD

SHANGHAI

CHINA PR

TEL: $2138 \quad 6191$

TLF :

TLX:

EML:

COM: 40

ZHANG JIA-LU

ASTROPHYSICS DIVISION

UNIV SCIENCE \& TECHNOLOGY

HEFEI 230026 ANHUI

CHINA PR

TEL: $55133 \quad 1134$

TLF:

TLX: 9002B USTC CN

EML:

COM: 47,48

ZHANG SHENG-PAN

2 ASSINIBOINE ROAD

SUITE 720

OOWNSVIEW ON M3J LLI

CANADA

TEL:

TLF :

TLX:

EML:

COM: 07

\section{ZHAI DI - SHENG}

BEIJING ASTRONOMICAL OBS

CAS

W' SUBURB

BEIJING 100080

CHINA PR

TEL: 1281698

TLF:

TLX: 22040 BAOAS CN

EML:

COM: $42 C$

ZHANG BIN

OPT OF GEOPHYSICS

BEIJING UNIVERSITY

BEIJING 100071

CHINA PR

TEL:

TLF :

TLX:

EML:

COM: 33

ZHANG GUD-DONG

BEIJING ASTRONOMICAL OBS CAS

W SUBuRB

BEIJING 100080

CHINA PR

TEL:

TLF:

TLX:

EML:

COM: 19

ZHANG JIA-XIANG

PURPLE MOUNTAIN OBSERV

CAS

NANJING

CHINA PR

TEL: 2546700

TLF :

TLX: 34144 PMONJ CN

EML:

COM: $20 \mathrm{C}$

ZHANG XIU ZHONG

SHANGHAI OBSERVATORY

CAS

80 NANDAN RD

SHANGHAI 200030

CHINA PR

TEL: 21386191

ILF: 21384618

TLX: 33164 SHAO CN

EML:

COM: 09
ZHAI ZAOCHENG SHANGHAI OBSERVATORY

CAS

gO NANOAN RO

SHANGHAI

CHINA PR

TEL: 21386191

TLF:

TLX: 33164 SHAO CN

EML:

COM: 31

ZHANG CHENG-YUE OPT OF PHYSICS

UNIVERSITY OF CALGARY 2500 UNIVERSITY DR NW CALGARY AB T2N IN4

CANADA

TEL: 4032205385

TLF: $403 \quad 2203643$

ILX:

EML

COM: 34

ZHANG HE-QI

PURPLE MOUNTAIN OBSERV

CAS

NANUING

CHINA PR

TEL: 2546700

TLF :

TLX: 34144 PMONJ CN

EML:

COM: 10,48

ZHANG JINTONG

INSTITUTE OF GEODESY \& GEOPHYSICS

XU DONG LU

WUHAN HUBEI

CHINA PR

TEL:

TLF:

TLX:

EML

COM: 31,42

ZHANG YOUYI

PURPLE MOUNTAIN OBSERV

CAS

NANJING

CHINA PR

TEL: 2546700

TLF :

TLX: 34144 PMONJ CN

EML:

COM: 09 
ZHANG ZHEN-DA

DPT OF ASTRONOMY

NANJING UNIVERSITY

NANJING

CHINA PR

TEL: $2534651 * 2882$

TLF:

ILX: 34151 PRCNU CN

EML:

COM: 10

ZHAO MING

SHANGHAI OBSERVATORY

CAS

80 NANDAN RO

SHANGHAI

CHINA PR

TEL: 21386191

TLF :

TLX: 33164 SHAO CN

EML:

COM: 19

ZHEKOV SVETOZAR A DR

CTR LAB FOR SPACE RES

BULGARIAN ACAO SCIENCES

MOSKAVA ST 6

BG 1000 SOFIA

BULGARIA

TEL: 2883503

TLF :

TLX: 23351 IKIBAN BG

EML:

COM:

ZHENG JIA-OING

TUORLA OBSERVATORY

UNIVERSITY OF TURKU

SF 20520 TURKL

FINLAND

TEL:

TLF:

TLX:

EML:

COM: 07

ZHEVAKIN S A PROF DR RADIOPHYSICAL RESEARCH INSTITUTE

LYADOV UL 25/14

603600 N NOVGOROD

RUSSIA

TEL: $36-67-51$

TLF :

FLX:

EML:

COM: 35

ZHANG ZHEN-JIU
DPT OF PHYSICS
HUAZHONG NORMAL UNIV
WUHAN
CHINA PR
TEL: 75601
TLF:
TLX: 6908
EML:
COM: 47,48

ZHAD REN-YANG

BEIJING ASTRONOMICAL OBS CAS

W SUBURB

BEIJING 100080

CHINA PR

TEL:

TLF :

TLX:

EML

COM: 10

ZHELEZNIAKOV VLADIMIR V INST OF APPLIEO PHYSICS ACADEMY OF SCIENCES

ULYANOV UL 46

603600 N HOVGOROD

RUSSIA

TEL:

TLF :

TLX:

EML:

$\mathrm{COM}: 40$

ZHENG XUE-TANG

DPT OF APPLIED PHYSICS

EAST CHINA INSTITUTE OF.

TECHNOLOGY

NANJING 210014

CHINA PR

TEL:

TLF:

$T L X:$

EML:

COM: 07

ZHOU BIFANG OR

CENTER FOR ASTRONOMICAL

INSTRUMENTS RESEARCH

182 BANCANG

NANJING 210042

CHINA PR

TEL: $25 \quad 646191$

TLF :

TLX: 34025 ISSAS CN

EML:

COM: 09
ZHAO GANG

SHANGHAI OBSERVATORY

CAS

80 NANDAN RO

SHANGHAI

CHINA PR

TEL: $21 \quad 386191$

TLF:

TLX: 33164 SHAO CN

EML:

COM: 31

ZHARKOV VLADIMIR N DR

INST -PHYSICS OF THE EARTH

ACADEMY OF SCIENCES

GRUZINSKAYA 10

123342 MOSCOW

RUSSIA

TEL: 2545251

TLF:

TLX: 411196 IFZAN

EML:

CDM: 16

ZHELYAZKOV IVAN OR

FAC OF PHYSICS

UNIVERSITY OF SOFIA

ANTON IVANOV ST 5

BG 1126 SOF IA

BULGaRIA

TEL: 2544852

TLF :

TLX: 23296 SUKO R BE

EML:

COM: 10

ZHENG YING

PURPLE MOUNTAIN OBSERV

CAS

NANJING

CHINA PR

TEL: 2546700

TLF:

ILX: 34144 PMONJ CN

EML

COM: 31

ZHOU DAOQ1

DPT OF GEOPHYSICS

BEIJING UNIVERSITY

BEIJING 100071

CHINA PR

TEL: $1 \quad 28 \quad 2471 * 3888$

TLF :

TLX: 22239 PXUNI

EML

COM: $10,12,42$
ZHAO JUN-LIANG

SHANGHAI OBSERVATORY

CAS

80 NANDAN RD

SHANGHAI

CHINA PR

TEL: 21386191

TLF:

TLX: 33164 SHAO CN

EML:

COM: $33,37 \mathrm{C}$

ZHARKOVA VATENINA OR

OPT OF PHYSICS

KIEV STATE UNIVERSITY

GLUSHKOV PR 6

252022 KIEV

UKRAINE

TEL: 261212

ILF : 2274482

TLX: 131406 SKY SU

EML:

COM: 10

ZHENG DA-WEI

SHANGHAI OBSERVATORY

CAS

80 NANDAN RO

SHANGHAI

CHINA PR

TEL: 21 $38 \quad 6191$

TLF :

TLX: 33164 SHAO CN

EML':

COM: 19

ZHENG YI-JIA

BEIJING ASTRONOMICAL OBS

CAS

W SUBURB

BEIJING 100080

CHINA PR

TEL:

ILF :

TLX: 22040 BAOBS CN

EML:

CDM: 40

ZHOL HONG-NAN

DPT OF ASTRONOMY

NANJING UNIVERSITY

NANUING

CHINA PR

TEL: 25 34651*2882

TLF :

TLX: 34151 PRCNU CN

EML

COM: 07,42 
ZHOU TI-JIAN

DPT OF GEOPHYSICS

BEIJING UNIVERSITY

BEIJING 100071

CHINA PR

TEL: $1282471 * 388 B$

TLF:

TLX: 22239 PKUNI

EML:

COM: 40

ZHU NENGHONG
SHANGHAI OBSERVATORY
CAS
8O NANDAN RO
SHANGHAI
CHINA PR
TEL: 21386191
TLF:
TLX: 33164 SHAO CN
EML:
COM: 09
ZHU YAOZHONG OR
INST OF GEBOESY \& GEOPHYS
S4 XU OONG RD
WUCHANG
HUBEI
CHINA PR
TEL: 81 340L
TLF:
TLX:
EML:
COM: 19

COM: 19

ZHUGZHDA YUZEF D DR

IZMIRAN

ACADEMY OF SCIENCES

142092 TROITSK

RUSSIA

TEL:

TLF:

TLX:

EML:

COM: 10,12

ZINN ROBERT J OR
DPT OF ASTRONOMY
YALE UNIVERSITY
BOX 6666 '
NEW HAVEN CT 06520
USA
TEL: 2034363460
TLF!
TLX:
EML:
COM: 28,37

ZHOU YOU-YUAN

ASTROPHYSICS OIVISION

UNIV SCIENCE \& TECHNOLOGY

HEFEI 230026 ANHUI

CHINA PR

TEL: $55133 \quad 1134$

TLF :

TLX: 90028 USTC CN

EML:

COM: 28,47

ZHU SHI-CHANG
OPT OF PHYSICS
SHANGHAI TEACHERS UNIV
10 GILLIN RO
SHANGHAI
CHINA PR
TEL: 21384301
TLF:
TLX: 9016
EML:
COM: 47

ZHU YONG-HE

BEIJING ASTRONOMICAL OBS CAS

W SUBURB

BEIJING 100080

CHINA PR

TEL: $128 \quad 1698$

JLF :

TLX: 22040 BAOBS CN

EML:

COM: 19

ZIEBA STANISLAW DR ASTRONOMICAL OBSERVATORY JAGIELLONIAN UNIVERSITY

UL ORLA 171

PL 30244 KRAKOW

POLANO

TEL: 223856, 221877

TLF:

TLX: 0322297 UJ PL

EML:

COM: 40,47

ZINNECKER HANS
INSTITUT FUER ASTRONOMIE
\& ASTROPHYSIK
AM HUBLAND
D 8700 WLERZBURG
GERMANY
TEL: 9318885031
TLF: 931706297
TLX:
EML:
COM: $26 C$

ZHOU ZHEN-PL

PURPLE MOUNTAIN OBSERV

CAS

NANJING

CHINA PR

TEL: 2533738

TLF:

TLX: 34114 PMONTJ CN

EML:

COM: 34

ZHU WEN-YAO

SHANGHAI OBSERVATORY

CAS

80 NANDAN RO

SHANGHAI

CHINA PR

TEL: 2138.6191

TLF :

TLX: 33164 SHAO CN

EML:

COM: 07

ZHUANG OIXIANG

LAB FOR BASIC STANDARDS

NTL RESEARCH COUNCIL

OTTAWA ONT KIA ORG

CANADA

TEL:

TLF :

TLX:

EML:

COM: 31

ZIKIDES MICHAEL C OR

OPT OF ASTRONOMY

NTL UNIVERSITY OF ATHENS

PANEPISTIMIOPOLIS

GR 15771 2OGRAFOS

GREECE

TEL: I $724 \quad 3414$

ILF:

YLX:

EML:

COM:

ZIOLKOWSKI JANUSZ DR COPERNICUS ASTRON CENTER POLISH ACAO OF SCIENCES

UL BARTYCKA 18

PL OO 715 WARSAW

POLANO

TEL:

TLF:

TLX:

EML:

COM: 35,42
ZHU CI -SHENG

DPT OF ASTRONOMY

NANJING UNIVERSITY

NANJING

CHINA PR

TEL: $25 \quad 37551 * 2882$

TLF:

TLX: 34151 PRCNU CN

EML:

COM: 42

ZHU XINGFENG

ASTROPHYSICS DIVISION

UNIV SCIENCE \& TECHNOLOGY

HEFEI 230026 ANHUI

CHINA PR .

TEL: $55133 \quad 1134$

TLF :

TLX: 90028 USTC.CN

EML:

COM: 47

ZHUANG WEIFENG

BEIJING ASTRONOMICAL OBS

CAS

W SUBURB

BEIJING 100000

CHINA PR

TEL:

TLF :

TLX:

EML:

COM: $4 i$

ZIMMERMANN HELMUT OR

UNIVERSITAETS-STERNWARTE

SCHILLERGAESSCHEN 2

D G9OO JENA

GERMANY

TEL: 7827122

TLF :

TLX:

EML:

COM: $34 ; 46$

ZIOLKOWSKI KRZYSZTOF OR SPACE RESEARCH CENTER POLISH ACAD OF SCIENCES UL ORDONA 21

PL 01237 WARSAW

POLANO

TEL: 22410041

TLF:

$T L X:$

EML:

COM: 20 


ZIRIN HAROLO OR
CALTECH
MS 26433
PASADENA CA 91125
USA
TEL: B18 3563857
TLF:
TLX:
EML:
COM: $10,12,14$
ZLATEV SLAVEY
ASTRONOMICAL OBSERVATORY
OF KARDGALI
BG 6600 KARDGALI
BULGARIA
TEL: 36 I2 595
TLF:
TLX: 47421
EML:
COM:

\section{ZOREC JEAN DR}

INSTITUT O'ASTROPHYSIQUE

98BIS BD ARAGO

F 75014 PARTS

FRANCE

TEL: 143201425

TLF: 143298673

TLX:

EML:

COM: 29

ZOU ZHEN-LONG

BEIJING ASTRONOMICAL OBS

CAS

W SUBURB

BEIJING 100080

CHINA PR

TEL:

TLF:

TLX: 22040 BAOAS CN

EML:

COM: 47

ZUIDERWIJK EOWAROUS J

ROYAL GREENWICH OBS

MADINGLEY RO

CAMBRIDGE CB3 OEZ

UK

TEL: 223374868

TLF:

TLX:

EML:

COM: 42,47
ZIRKER JACK B OR

AIR FORCE GEOPHYSICS LAB

NTL SOLAR OBSERVATORY

SUNSPOT NM 88349

USA

TEL: 5054341390

TLF :

TLX:

EML:

COM: 12

ZLOBEC PAOLO DR

OAT

BOX SUCC TRIESTE 5

VIA TIEPOLO 11

I 34131 TRIESTE

ITALY

TEL: 40793921

TLF:

TLX: 461137 OAT I

EML:

COM: 10,40

ZOSIMOVICH IRINA $D$

INSTITUTE OF HISTORY

UKRAINIAN ACAD OF SCIENCE

KIROV UL 4

$252001 \mathrm{kIEV}$

UKRAINE

TEL: 290272

TLF:

TLX:

EML:

COM: 41

ts SOLDOS ENDRE DR

KONKOLY OBSERVATORY

THEGE U $13 / 17$

BOX 67

H 1525 BUDAPEST

HUNGARY

TEL: $175,5866 / 754122$

TLF: 11569640

TLX: 227460 KONOB H

EML: H697KONEELLLA. UUCP

COM: 27

ZVERKO JURAJ DR

ASTRONOMICAL INSTITUTE

SLOVAK ACADEMY SCIENCES

CS 059 60 TATRANSKA LOMNI

CZECHOSLOVAKIA

TEL: $96996 \quad 7866 / 7 / 8$

TLF: 969967656

TLX: 78277

EML:

COM: 29
ZITELLI VALENTINA OR OPT DI ASTRONOMIA UNIVERSITA DI BOLOGNA CP 596

I 40100 BOLOGNA

ITALY

TEL: 51259301

TLF:

TLX: 520634 INFN I

EML: SPAN: 37929

COM:

ZLOTNIK ELENA YA DR

INST OF APPLIED PHYSICS

ACADEMY OF SCIENCES

ULYANOV UL 46

603600 N NOVGOROD

RUSSIA

TEL: 7 \& $8312 \quad 363519$

TLF: 7 \& 8312362081

TLX: 412580 FIZIK SU

EML:

COM: 40

2OU HUI-CHENG

SHANGHAI OBSERVATORY

CAS

80 NANDAN RD

SHANGHAI

CHINA PR

TEL: $2138 \quad 6191$

TLF:

ILK: 331.64 SHAO CNCN

EML:

COM: 44

ZUCCARELLO FRANCESCA

IST DI ASTRONOMIA

CITIA UNIVERSITARIA

VIA A DORIA 6

I 95125 CATANIA

ITALY

TEL: 330533

TLF :

TLX: 970359 ASTRCT I

EML:

COM:

ZVOLAKKOVA JUDITA

ASTRONOMICAL INSTITUTE

SLOVAK ACADEMY SCIENCES

DUBRAVSKA 9

CS 84228 BRATISLAVA

CZECHOSLOVÁKIA

TEL: 737 5157

TLF:

TLX: 93373 SEIS

EML:

COM: 22
ZIZNOVSKY JOZEF DR ASTRONOMICAL INSTITUTE SLOVAK ACADEMY SCIENCES CS 05960 TATRANSKA LOMNI CZECHOSLOVAKIA

TEL: $969967866 / 7 / 8$

TLF: 969967656

TLX: 78277

EML:

COM: 25

ZOMBECK MARTIN $V$ OR CENTER FOR ASTROPHYSICS HCO/SAO

60 GARDEN ST

CAMBRIDGE MA 0213B

USA

TEL: 6174957227

TLF:

tLX: 921428 SATELLIte cam

EML:

COM: 44,48

ZOU YI-XIN

BEIJING ASTRONOMICAL OBS CAS

W SUBURB

BEIJING 100080

CHINA PR

TEL: $128 \quad 1261$

TLF:

TLX: 22040

EML:

COM: 10

ZUCKERMAN BEN M DR

DPT OF ASTRONOMY

UNIVERSITY OF CALIFORNIA

LOS ANGELES CA 90024

USA

TEL: 2138259338

TLF:

TLX: $910342 \quad 7597$

EML:

COM: $27,34,40,51$

ZWAAN CORNELIS PROF OR STERREKUNDIG INSTITUTE

BOX 80000

NL 3508 TA UTRECHT

NETHERLANDS

TEL: 30535223

TLF: 30535201

TLX: 40048 FYLUT NL

EML: ZWAANEFY.RUU.NL

COM: $10,12,36 \mathrm{C}$ 
ZWITTER TOMAZ

ASTRONOMICAL OBSERVATORY

UNIVERSITY OF E KARDELJ

JADRANSKA 19

YU 61110 LJUBLJANA.

YUGOSLAVIA

TEL: 61265061

TLF : $61217 \quad 281$

TLX

EML: ZWITTEREITSSISSA.BITNET

COM: 42 